Comparative Effectiveness Review Number 208

\title{
Treatment for Bipolar Disorder in Adults: A Systematic Review
}
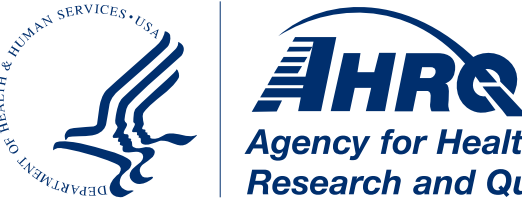

Agency for Healthcare Research and Quality
Effective Health

Care Program 


\title{
Comparative Effectiveness Review
}

Number 208

\section{Treatment for Bipolar Disorder in Adults: A Systematic Review}

\author{
Prepared for: \\ Agency for Healthcare Research and Quality \\ U.S. Department of Health and Human Services \\ 5600 Fishers Lane \\ Rockville, MD 20857 \\ www.ahrq.gov \\ Contract No. 290-2012-00016-I \\ Prepared by: \\ Minnesota Evidence-based Practice Center \\ Minneapolis, MN \\ Investigators: \\ Mary Butler, Ph.D., M.B.A. \\ Snezana Urosevic, Ph.D., L.P. \\ Priyanka Desai, M.H.P. \\ Scott R. Sponheim, Ph.D. \\ Jonah Popp, M.S., M.A. \\ Victoria A. Nelson, M.Sc. \\ Viengneesee Thao, M.P.H. \\ Benjamin Sunderlin, M.P.H.
}

AHRQ Publication No. 18-EHC012-EF

August 2018 


\section{Key Messages}

\section{Purpose of Review}

- To assess the effectiveness of drug and nondrug therapies for treating acute mania or depression symptoms and preventing relapse in adults with bipolar disorder (BD) diagnoses, including bipolar I disorder (BD-I), bipolar II disorder (BD-II), and other types.

\section{Key Messages}

- Acute mania treatment: Lithium, asenapine, cariprazine, olanzapine, quetiapine, risperidone, and ziprasidone may modestly improve acute mania symptoms in adults with BD-I. Participants on atypical antipsychotics, except for quetiapine, reported more extrapyramidal symptoms, and those on olanzapine reported more weight gain, compared with placebo.

- Maintenance treatment: Lithium may prevent relapse into acute episodes in adults with BD-I.

- Depression treatment: Evidence was insufficient for drug treatments for depressive episodes in adults with BD-I and BD-II.

- For adults with any BD type, cognitive behavioral therapy may be no better than other psychotherapies for improving acute bipolar symptoms and systematic/collaborative care may be no better than other behavioral therapies for preventing relapse of any acute symptoms.

- Stronger conclusions were prevented by high rates of participants dropping out. 
This report is based on research conducted by the Minnesota Evidence-based Practice Center (EPC) under contract to the Agency for Healthcare Research and Quality (AHRQ), Rockville, MD (Contract No. 290-2012-00016-I). The findings and conclusions in this document are those of the authors, who are responsible for its contents; the findings and conclusions do not necessarily represent the views of AHRQ. Therefore, no statement in this report should be construed as an official position of AHRQ or of the U.S. Department of Health and Human Services.

\section{None of the investigators have any affiliations or financial involvement that conflicts with the material presented in this report.}

The information in this report is intended to help health care decisionmakers—patients and clinicians, health system leaders, policymakers, and others-make well-informed decisions and thereby improve the quality of health care services. This report is not intended to be a substitute for the application of clinical judgment. Anyone who makes decisions concerning the provision of clinical care should consider this report in the same way as any medical reference and in conjunction with all other pertinent information, i.e., in the context of available resources and circumstances presented by individual patients.

This report is made available to the public under the terms of a licensing agreement between the author and the Agency for Healthcare Research and Quality. This report may be used and reprinted without permission except those copyrighted materials that are clearly noted in the report. Further reproduction of those copyrighted materials is prohibited without the express permission of copyright holders.

AHRQ or U.S. Department of Health and Human Services endorsement of any derivative products that may be developed from this report, such as clinical practice guidelines, other quality enhancement tools, or reimbursement or coverage policies, may not be stated or implied.

This report may periodically be assessed for the currency of conclusions. If an assessment is done, the resulting surveillance report describing the methodology and findings will be found on the Effective Health Care Program Web site at www.effectivehealthcare.ahrq.gov. Search on the title of the report.

Persons using assistive technology may not be able to fully access information in this report. For assistance contact epc@ahrq.hhs.gov.

Suggested citation: Butler M, Urosevic S, Desai P, Sponheim SR, Popp J, Nelson VA, Thao V, Sunderlin B. Treatment for Bipolar Disorder in Adults: A Systematic Review. Comparative Effectiveness Review No. 208. (Prepared by the Minnesota Evidence-based Practice Center under Contract No. 290-2012-00016-I.) AHRQ Publication No. 18-EHC012-EF. Rockville, MD: Agency for Healthcare Research and Quality; August 2018. Posted final reports are located on the Effective Health Care Program search page.

DOI: https://doi.org/10.23970/AHRQEPCCER208. 


\section{Preface}

The Agency for Healthcare Research and Quality (AHRQ), through its Evidence-based Practice Centers (EPCs), sponsors the development of systematic reviews to assist public- and private-sector organizations in their efforts to improve the quality of health care in the United States. These reviews provide comprehensive, science-based information on common, costly medical conditions, and new health care technologies and strategies.

Systematic reviews are the building blocks underlying evidence-based practice; they focus attention on the strength and limits of evidence from research studies about the effectiveness and safety of a clinical intervention. In the context of developing recommendations for practice, systematic reviews can help clarify whether assertions about the value of the intervention are based on strong evidence from clinical studies. For more information about AHRQ EPC systematic reviews, see www.effectivehealthcare.ahrq.gov/reference/purpose.cfm.

AHRQ expects that these systematic reviews will be helpful to health plans, providers, purchasers, government programs, and the health care system as a whole. Transparency and stakeholder input are essential to the Effective Health Care Program. Please visit the Web site (www.effectivehealthcare.ahrq.gov) to see draft research questions and reports or to join an email list to learn about new program products and opportunities for input.

If you have comments on this systematic review, they may be sent by mail to the Task Order Officer named below at: Agency for Healthcare Research and Quality, 5600 Fishers Lane, Rockville, MD 20857, or by email to epc@ahrq.hhs.gov.

Gopal Khanna, M.B.A.

Director

Agency for Healthcare Research and Quality

Stephanie Chang, M.D., M.P.H.

Director

Evidence-based Practice Center Program

Center for Evidence and Practice Improvement

Agency for Healthcare Research and Quality
Arlene S. Bierman, M.D., M.S.

Director

Center for Evidence and Practice Improvement

Agency for Healthcare Research and Quality

Aysegul Gozu, M.D., M.P.H.

Task Order Officer

Center for Evidence and Practice Improvement

Agency for Healthcare Research and Quality 


\section{Acknowledgments}

We wish to thank Kaci Parson, who helped as a research assistant; Jeannie Ouellette and Cheryl Cole-Hill for their help with editing and producing this report; James D. Neaton for his expertise in clinical trials and biostatics with regard to antipsychotics; and Aysegul Gozu and her colleagues at AHRQ for their helpful comments during the writing process. Special gratitude for Robert L. Kane in memoriam.

\section{Key Informants}

In designing the study questions, the EPC consulted several Key Informants who represent the end-users of research. The EPC sought the Key Informant input on the priority areas for research and synthesis. Key Informants are not involved in the analysis of the evidence or the writing of the report. Therefore, in the end, study questions, design, methodological approaches, and/or conclusions do not necessarily represent the views of individual Key Informants.

Key Informants must disclose any financial conflicts of interest greater than \$5,000 and any other relevant business or professional conflicts of interest. Because of their role as end-users, individuals with potential conflicts may be retained. The TOO and the EPC work to balance, manage, or mitigate any conflicts of interest.

The list of Key Informants who provided input into this report follows:

Benjamin G. Druss, M.D., M.P.H.

Rosalynn Carter Chair in Mental Health

Department of Health Policy and Management

Atlanta, GA

Brian Jost

NAMI Minnesota

St. Paul, MN

Amy M. Kilbourne, Ph.D., M.P.H.*

Director, VA Quality Enhancement Research Initiative (QUERI)

Professor of Psychiatry, University of Michigan Medical School

Ann Arbor, MI

Elinore F. McCance-Katz, M.D.

Chief Medical Officer

Substance Abuse and Mental Health Services

Washington, DC

Susan Carr Sonne, Pharm.D., B.C.P.P.

MUSC/Institute of Psychiatry

Charleston, SC 
Lauren M. Weinstock, Ph.D.*

Associate Professor

Alpert Medical School of Brown University

Providence, RI

\section{Technical Expert Panel}

In designing the study questions and methodology at the outset of this report, the EPC consulted several technical and content experts. Broad expertise and perspectives were sought. Divergent and conflicted opinions are common and perceived as healthy scientific discourse that results in a thoughtful, relevant systematic review. Therefore, in the end, study questions, design, methodologic approaches, and/or conclusions do not necessarily represent the views of individual technical and content experts.

Technical Experts must disclose any financial conflicts of interest greater than $\$ 5,000$ and any other relevant business or professional conflicts of interest. Because of their unique clinical or content expertise, individuals with potential conflicts may be retained. The TOO and the EPC work to balance, manage, or mitigate any potential conflicts of interest identified.

The list of Technical Experts who provided input to this report follows:

Mark S. Bauer, M.D.

Professor of Psychiatry

Harvard Medical School

Boston, MA

Laura J. Fochtmann, M.D., M.B.I.*

Distinguished Service Professor

Departments of Psychiatry, Pharmacological Sciences and Biomedical Informatics

Stony Brook University School of Medicine

Stony Brook, NY

Mark A. Frye, M.D.

Chair, Department of Psychiatry and Psychology

Mayo Clinic,

Rochester, MN

Susan Carr Sonne, Pharm.D., B.C.P.P.

MUSC/Institute of Psychiatry

Charleston, SC

Lauren M. Weinstock, Ph.D.*

Associate Professor

Alpert Medical School of Brown University

Providence, RI

*Provided input on Draft Report. 


\section{Peer Reviewers}

Prior to publication of the final evidence report, EPCs sought input from independent Peer Reviewers without financial conflicts of interest. However, the conclusions and synthesis of the scientific literature presented in this report do not necessarily represent the views of individual reviewers.

Peer Reviewers must disclose any financial conflicts of interest greater than $\$ 5,000$ and any other relevant business or professional conflicts of interest. Because of their unique clinical or content expertise, individuals with potential nonfinancial conflicts may be retained. The TOO and the EPC work to balance, manage, or mitigate any potential nonfinancial conflicts of interest identified.

David J. Bond, M.D., Ph.D.

Associate Professor

University of Minnesota

Minneapolis, MN

Matthew V. Rudorfer, M.D.

Program Chief

National Institute of Mental Health

Bethesda, MD 


\section{Treatment for Bipolar Disorder in Adults: A Systematic Review}

\section{Structured Abstract}

Objective. Assess the effect of drug and nondrug interventions for treating acute symptoms associated with bipolar disorder (BD) and preventing relapse.

Data sources. Ovid MEDLINE ${ }^{\circledR}$ and PsycINFO ${ }^{\circledR}$, the Cochrane Central Register of Controlled Trials, and Ovid Embase ${ }^{\circledR}$ bibliographic databases; hand searches of references of relevant systematic reviews through May 2017.

Review methods. Eligible studies included randomized controlled trials and prospective cohorts with comparator arms enrolling adults with bipolar disorder (BD) of any type with 3 weeks followup for acute mania, 3 months for depression, and 6 months for maintenance treatments. We excluded acute mania and depression studies with greater than 50 percent attrition.

Results. We synthesized evidence from 157 unique studies, 108 studies for 28 drugs, 49 studies for nondrug interventions. All drug study findings with at least low-strength evidence were based almost exclusively on adults with bipolar I disorder (BD-I). Asenapine, cariprazine, quetiapine, and olanzapine improved acute mania symptoms compared to placebo (low-strength evidence). However, improvements were of modest clinical significance, with values that were less than the minimally important difference, but still large enough that a reasonable proportion of participants likely received a benefit. Unpooled evidence indicated an overall beneficial effect of risperidone and ziprasidone on acute mania symptoms compared to placebo (low-strength evidence). Participants using atypical antipsychotics, except quetiapine, reported more extrapyramidal symptoms compared to placebo, and those using olanzapine reported more clinically significant weight gain. Lithium improved acute mania in the short term and prolonged time to relapse in the long term compared to placebo (low-strength evidence). No difference was found between olanzapine and divalproex/valproate for acute mania (low-strength evidence). For drugs not approved for BD, paliperidone improved acute mania compared to placebo (low-strength evidence), while topiramate and allopurinol showed no benefit (low-strength evidence). Further, lithium improved acute mania better than topiramate (low-strength evidence), although withdrawals for adverse events were lower for topiramate. Only lithium reached a minimally important difference for acute mania and maintenance treatment. All other drug comparisons to placebo or active controls for acute mania, depression, and maintenance had insufficient evidence. For psychosocial interventions, cognitive behavioral training (CBT) was no better for depression or mania symptoms than psychoeducation or other active psychosocial comparators (low-strength evidence). Systematic/collaborative care had no effect on relapse compared to inactive comparators (low-strength evidence).

Conclusions. We found no high- or moderate-strength evidence for any intervention to effectively treat any phase of any type of BD versus placebo or an active comparator. All antipsychotics approved by the Food and Drug Administration, except aripiprazole, had lowstrength evidence for benefit for acute mania in adults with BD-I. Lithium improved short-term for acute mania and resulted in longer time to relapse in the long term versus placebo in adults 
with BD-I. Aside from low-strength evidence showing CBT and systematic/collaborative care having no benefit for a few outcomes, evidence was insufficient for nondrug interventions.

Information on harms was limited across all studies. Future research examining BD treatments will require innovative ways to increase study completion rates. 


\section{Contents}

Evidence Summary .......................................................................................................................................

Chapter 1. Introduction ....................................................................................................... 1

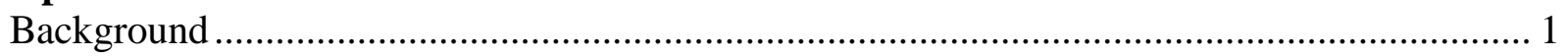

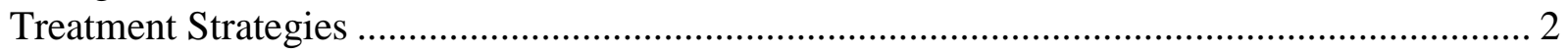

Scope and Key Questions ............................................................................................. 4

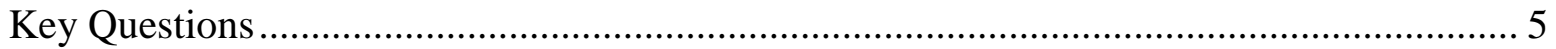

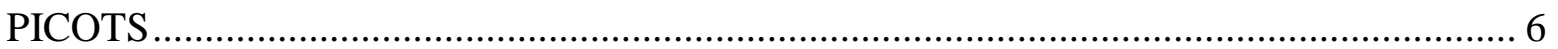

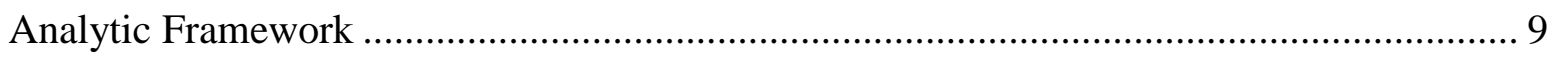

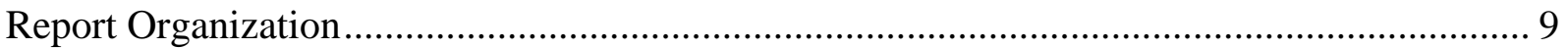

Chapter 2. Methods .............................................................................................................................. 10

Topic Refinement and Review Protocol ............................................................................ 10

Literature Search Strategy.................................................................................................. 10

Risk of Bias Assessment of Individual Studies .................................................................. 11

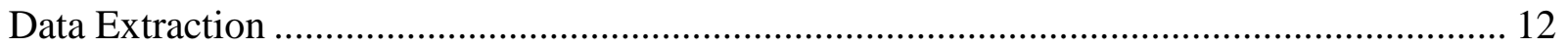

Data Synthesis............................................................................................................ 12

Strength of Evidence for Major Comparisons and Outcomes ................................................... 14

Applicability ................................................................................................................... 15

Peer Review and Public Commentary ……………………………………………………... 15

Chapter 3. Search Results .................................................................................................................. 16

Chapter 4. Drug Treatments for Acute Mania ............................................................................ 21

Antipsychotic Drugs for Acute Mania ............................................................................... 21

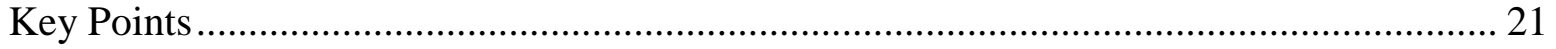

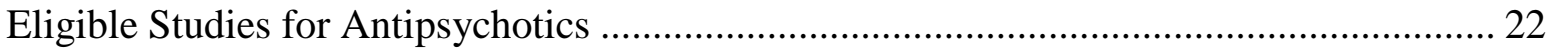

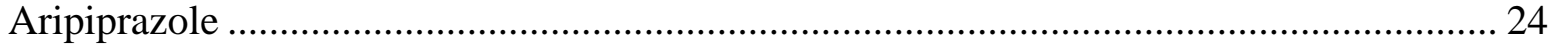

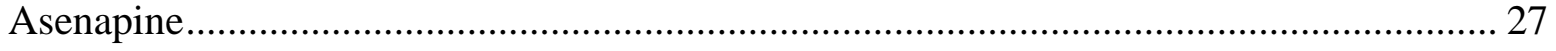

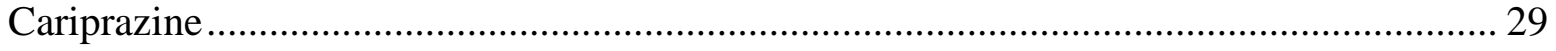

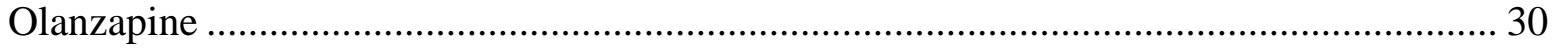

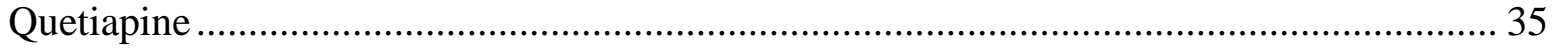

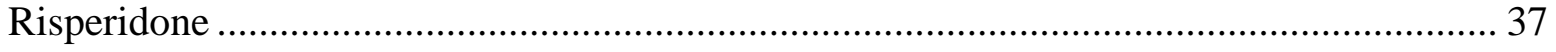

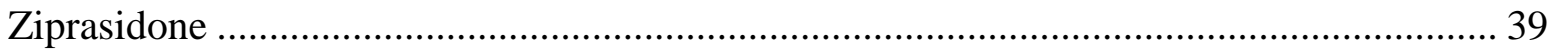

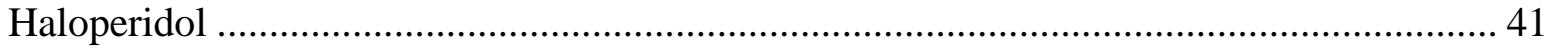

Mood Stabilizers for Acute Mania................................................................................... 42

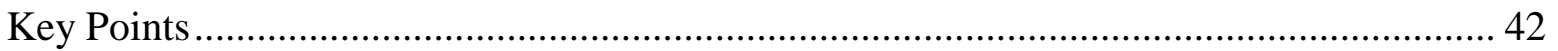

Eligible Studies for Mood Stabilizers .............................................................................. 43

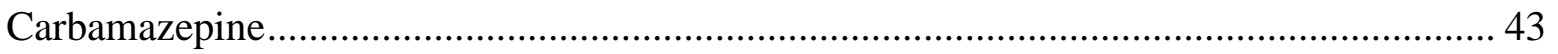

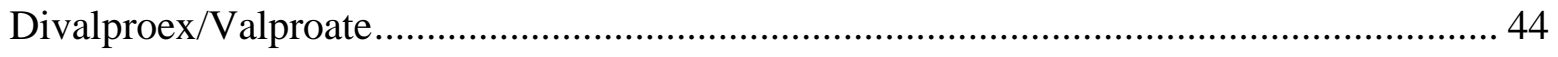

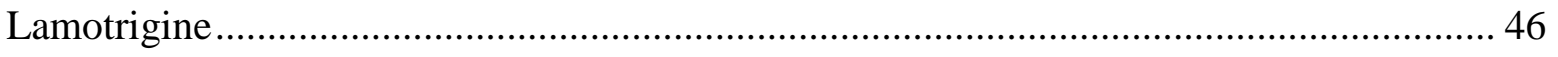

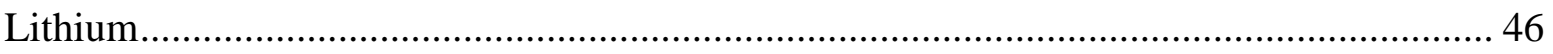


Drugs Not Approved by FDA for Acute Mania in Bipolar Disorder ................................... 48

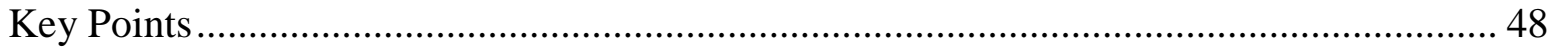

Eligible Studies for Drugs Not Approved by FDA....................................................... 48

Interpreting the Findings for Drugs for Acute Mania ......................................................... 53

Chapter 5. Drug Treatments for Depression....................................................................... 55

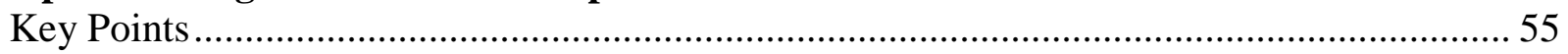

Eligible Studies for Depression Treatments .................................................................... 55

Drug Treatments for Depression Versus Placebo......................................................... 56

Drug Treatments for Depression Versus Active Control............................................... 57

Interpreting the Findings for Drug Treatments for Depression .......................................... 57

Chapter 6. Drug Treatments for Maintenance ...............................................................59

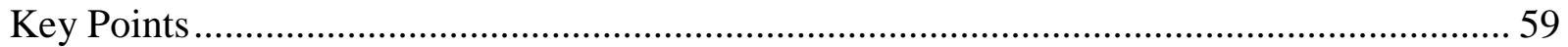

Eligible Studies for Maintenance Treatments ................................................................. 59

Single Drug Treatments for Maintenance ................................................................. 60

Combination Drug Treatment for Maintenance............................................................ 64

Interpreting the Findings for Drug Treatment for Maintenance ......................................... 69

Chapter 7. Psychosocial and Other Nondrug Treatments ..................................................... 70

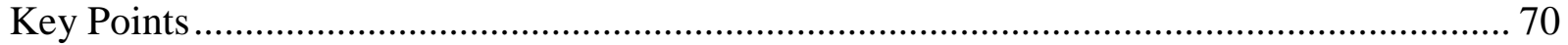

Eligible Studies for Psychosocial and Other Nondrug Treatments .................................... 70

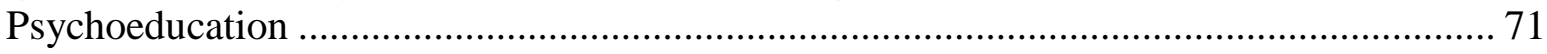

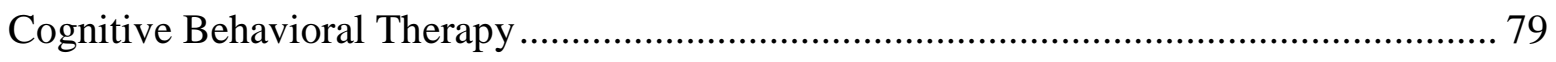

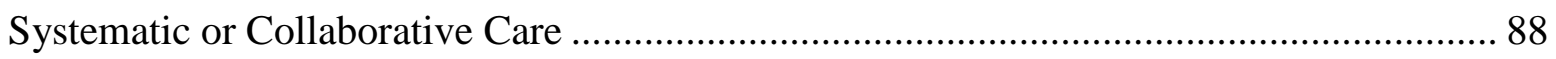

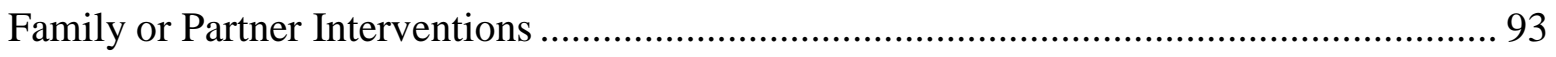

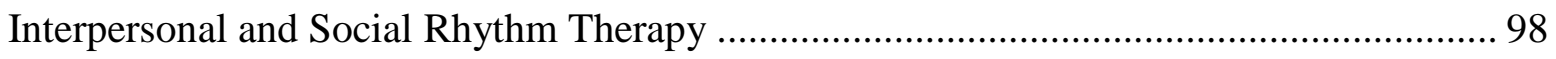

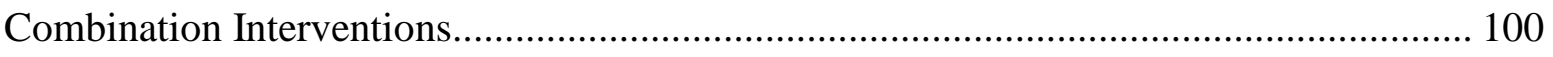

Systematic Treatment Enhancement Program for Bipolar Disorder (STEP-BD) Study and

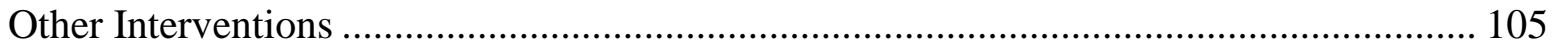

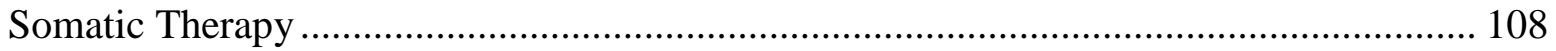

Interpreting the Findings for Psychosocial and Other Nondrug Treatments ........................ 109

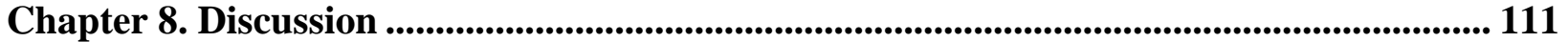

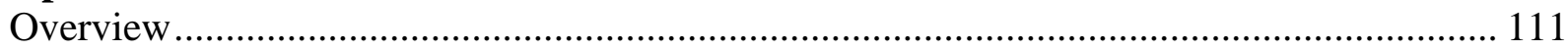

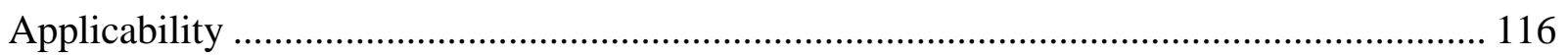

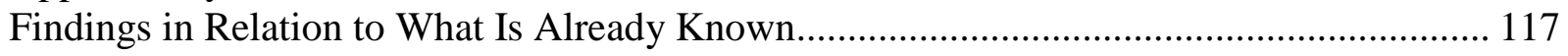

Limitations of the Comparative Effectiveness Review ................................................... 117

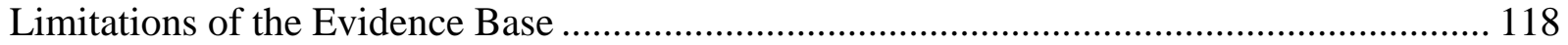

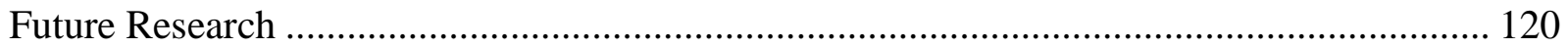

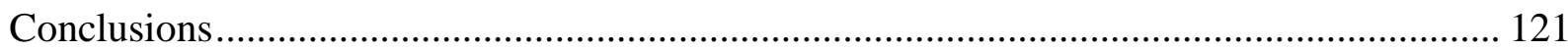

References......................................................................................................................................... 123

Abbreviations .................................................................................................................................. 142 


\section{Tables}

Table A. Summary of low-strength* evidence findings by intervention class ............................ 3

Table B. Interventions/comparators with insufficient strength of evidence ................................ 5

Table 1. FDA-approved medications for bipolar disorder.................................................... 3

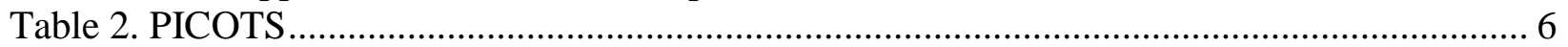

Table 3. Study inclusion criteria ................................................................................. 11

Table 4. Eligible unique studies by drug intervention and comparator .................................. 17

Table 5. Eligible unique studies by nondrug intervention and comparator ............................... 20

Table 6. Summary of findings with at least low-strength evidence for antipsychotic drug

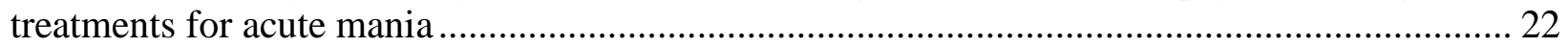

Table 7. Population and inclusion criteria for studies of aripiprazole alone for acute mania....... 24

Table 8. Population and inclusion criteria for aripiprazole plus mood stabilizers studies for acute

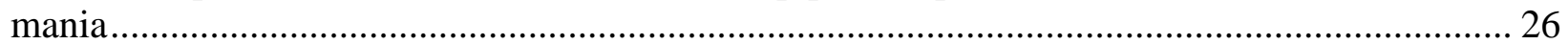

Table 9. Population and inclusion criteria for studies of asenapine alone for acute mania .......... 28

Table 10. Population and inclusion criteria for asenapine plus mood stabilizer studies for acute

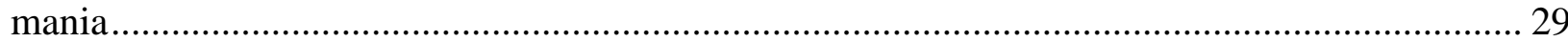

Table 11. Population and inclusion criteria for studies of cariprazine alone for acute mania ...... 29 Table 12. Population and inclusion criteria for studies of olanzapine alone for acute mania ...... 31 Table 13. Population and inclusion criteria for olanzapine plus mood stabilizers studies for acute

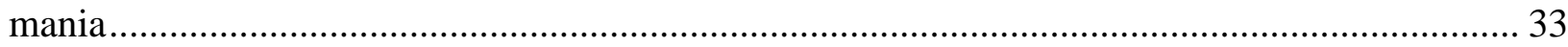

Table 14. Population and inclusion criteria for studies of quetiapine alone for acute mania....... 35 Table 15. Population and inclusion criteria for quetiapine plus mood stabilizer studies for acute

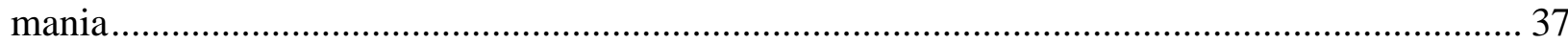

Table 16. Population and inclusion criteria for studies of risperidone alone for acute mania...... 38 Table 17. Population and inclusion criteria for risperidone plus mood stabilizer studies for acute

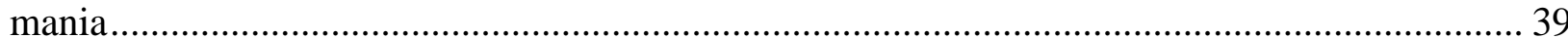

Table 18. Population and inclusion criteria for studies of ziprasidone alone for acute mania ..... 40 Table 19. Population and inclusion criteria for ziprasidone plus mood stabilizer studies for acute

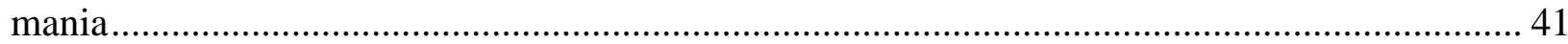

Table 20. Population and inclusion criteria for studies of haloperidol alone for acute mania ..... 42 Table 21. Summary of findings with at least low-strength evidence for mood stabilizers for acute

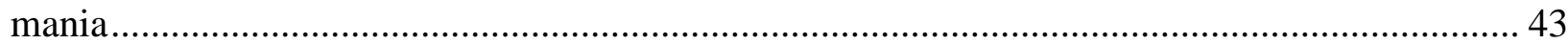

Table 22. Population and inclusion criteria for carbamazepine for acute mania........................ 44

Table 23. Population and inclusion criteria for divalproex/valproate for acute mania................ 45

Table 24. Population and inclusion criteria for lamotrigine for acute mania ............................ 46

Table 25. Population and inclusion criteria for lithium for acute mania .................................. 46

Table 26. Summary of findings with at least low-strength evidence for drugs not approved by

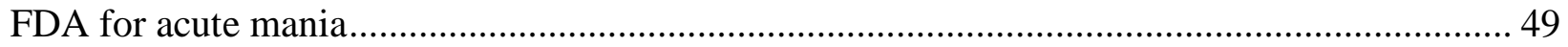

Table 27. Population and inclusion criteria for drugs not approved by FDA for acute mania..... 49

Table 28. Population and inclusion criteria for studies of drug treatments for depression .......... 55

Table 29. Summary of findings with at least low-strength evidence for maintenance studies.... 59

Table 30. Population and inclusion criteria for single drug studies for maintenance.................. 60

Table 31. Population and inclusion criteria for combination drug treatment for maintenance

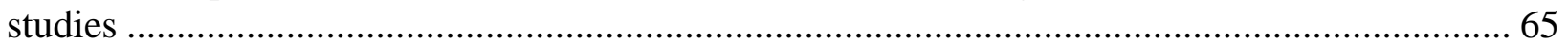

Table 32. Interventions, comparators, and outcomes for nondrug interventions........................ 71 
Table 33. Population and inclusion criteria for studies of psychoeducation versus inactive

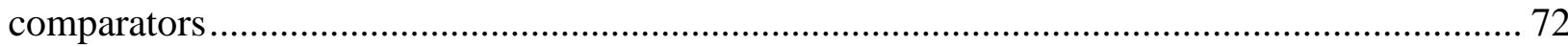

Table 34. Population and inclusion criteria for studies of psychoeducation versus active comparators... 78

Table 35. Summary of findings with at least low-strength evidence for cognitive behavioral

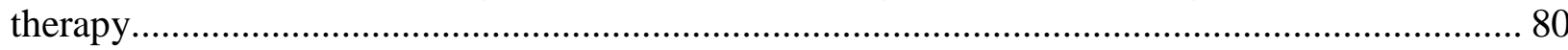

Table 36. Population and inclusion criteria for studies of CBT versus inactive comparators...... 81 Table 37. Population and inclusion criteria for studies of CBT versus active comparators ......... 86 Table 38. Summary of findings with at least low-strength evidence for cognitive behavioral

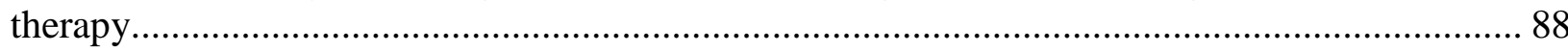
Table 39. Population and inclusion criteria for studies of systematic or collaborative care versus

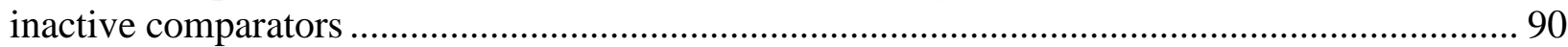

Table 40. Population and inclusion criteria for studies of FPI versus inactive comparators........ 93

Table 41. Population and inclusion criteria for studies of FPI versus active comparators ........... 96 Table 42. Population and inclusion criteria for studies of IPSRT versus inactive comparators... 99 Table 43. Population and inclusion criteria for studies of IPSRT versus active comparators.... 100 Table 44. Population and inclusion criteria for studies of combination interventions versus

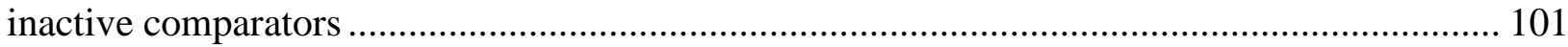
Table 45. Population and inclusion criteria for studies of combination interventions versus active comparators 104

Table 46. Population and inclusion criteria for studies of other psychosocial interventions ..... 106 Table 47. Population and inclusion criteria for studies examining a somatic therapy .............. 109 Table 48. Summary of low-strength* evidence findings by intervention class ........................ 111 Table 49. Interventions/comparators with insufficient strength of evidence for all outcomes (unless otherwise noted)

\section{Figures}

Figure 1. Analytic framework for treatment for bipolar disorder ......................................... 9

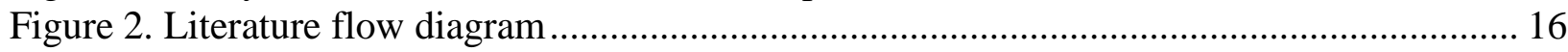

\section{Appendixes}

Appendix A. Search Strategies

Appendix B. Risk of Bias Assessment Tool

Appendix C. Outcomes

Appendix D. Excluded References

Appendix E. Antipsychotics for Mania

Appendix F. Mood Stabilizers for Mania

Appendix G. Other Drugs for Acute Mania

Appendix H. Drug Treatments for Depression

Appendix I. Drug Treatments for Maintenance

Appendix J. Psychoeducation

Appendix K. Cognitive Behavioral Therapy

Appendix L. Systematic or Collaborative Care

Appendix M. Family or Partner Interventions

Appendix N. Interpersonal and Social Rhythm Therapy (IPSRT)

Appendix O. Combination Interventions 
Appendix P. Systematic Treatment Enhancement Program for Bipolar Disorder (STEP-BD) and Other Psychosocial and Somatic Interventions

Appendix Q. Harms Tables 


\section{Evidence Summary}

\section{Background}

Bipolar disorder (BD), also known as manic-depressive illness, is a serious mental illness that causes unusual shifts in mood, energy, activity levels, and the inability to carry out day-today tasks. The Diagnostic and Statistical Manual of Mental Disorders, 5th Edition (DSM-5) recognizes a spectrum of bipolar diagnoses that differ in duration of bipolar episodes/periods and impairment: bipolar I disorder (BD-I), dipolar II disorder (BD-II), BD otherwise specified, and BD unspecified. Prevalence studies estimate about 1 percent of the population for BD-I, another 1 percent for BD-II, and up to 5 percent for the full spectrum of BD diagnoses, with relatively similar prevalence in men and women and across cultural and ethnic groups. ${ }^{1,2} \mathrm{BD}$ represents a significant individual and societal burden. Recurrent episodes of mania and depression can cause serious impairments in functioning, such as erratic work performance, increased divorce rates, and psychosocial morbidity. ${ }^{3,4}$ People with bipolar disorder account for between 3 and 14 percent of all suicides, and about 25 percent of bipolar disorder patients will attempt suicide. ${ }^{5}$ Further adding to the individual illness burden, 92 percent of individuals with BD experience another co-occurring psychiatric illness during their lifetime. ${ }^{6}$ Of all psychiatric conditions, BD is the most likely to co-occur with alcohol or drug abuse disorders. ${ }^{7}$

Treatment of BD generally begins with the goal of bringing a patient with mania or depression to symptomatic recovery and stable mood. Once the individual is stable, the goal progresses to reducing subthreshold symptoms and preventing relapse into full-blown episodes of mania and depression. Drug treatments have several purposes. Some drugs aim to reduce symptoms associated with acute manic or mixed mania/depression episodes, some aim to reduce acute depression symptoms, and others aim to reduce acute symptoms, maintain relatively symptom-free periods, and prevent relapsing to acute episodes. Given the chronic, relapsing/remitting course of bipolar disorder and the need for maintenance treatment in many patients, drugs begun for an acute mood episode (including mania) are often carried forward into maintenance therapy.

Nondrug psychosocial therapeutic approaches range from psychoeducational, cognitive behavioral, and family-focused therapies, to interpersonal social rhythm therapy, and are provided both in individual and group therapy modalities. Most psychosocial therapeutic approaches focus the treatment for individuals currently in the remission state of bipolar illness and often specifically exclude individuals currently in acute manic episodes. Other nondrug treatment forms range widely from electroconvulsive therapy to treatments for circadian rhythms (such as light boxes), to acupuncture, to repetitive transcranial magnetic stimulation.

This review provides a comprehensive up-to-date synthesis of the evidence on the effects of a broad range of BD interventions (drug and nondrug). We excluded botanicals and nutritional supplements. These are part of a broader class of remedies patients may take on their own for symptom relief.

The review addresses the benefits and harms of pharmacologic and nonpharmacologic treatment interventions for adults with any type of BD. Two additional questions regarding treatments to reduce metabolic change side effects of drug treatments, and how effects differ by patient characteristics, such as co-occurring substance abuse, were not answerable with the available literature. Reported results focus on Key Questions 1 and 2. 


\section{Methods}

The review used methods following Agency for Healthcare Research and Quality methods guidance. The protocol was posted June 23, 2014 at https://effectivehealthcare.ahrq.gov/searchfor-guides-reviews-and-reports/?pageaction=displayproduct\&productid=1926.

Eligible studies included randomized controlled trials and prospective cohorts with comparator arms enrolling adults with BD of any type with followup of 3 weeks for acute mania, 3 months for depression, and 6 months for maintenance treatments. We excluded studies with greater than 50 percent attrition (with the exception of maintenance studies with time-torelapse and withdrawal outcomes) because of potential systematic differences between patients who do not complete the study and those who do. That is, attrition may not be random and/or is likely due to BD or treatment-relevant factors.

We used published minimally important differences (MIDs) to interpret findings for the Young Mania Rating Scale (YMRS) (MID=6) and the Clinical Global Impressions (CGI) scale $(\mathrm{MID}=1){ }^{8}$ If a change in an outcome is at least equal to the MID, the interpretation that all participants benefitted from the intervention is clear. However, because the actual benefit each participant experiences lies somewhere along a distribution of benefits recorded for all the participants, changes less than an MID may also suggest that at least some of the participants benefitted from the intervention. ${ }^{9}$ We therefore followed a rule for interpretation that if an estimate of outcome is greater than 50 percent of the MID, it is possible that a reasonable proportion of participants received the benefit. Conversely, if the estimate is less than 50 percent of the MID, it is much less likely that an appreciable proportion received benefit from the treatment.

\section{Results}

We identified 6,116 unique publications through May 2017, of which 188 were eligible for our review; 123 publications of drug interventions, 65 publications for nondrug interventions. The publications comprised 67 unique drug studies for acute mania, seven drug studies for depression, 36 drug studies for maintenance, 48 for psychosocial therapies, and one study on repetitive transcranial magnetic stimulation. All acute mania treatment studies enrolled adults with BD-I; only two also explicitly included BD-II, and only one BD not otherwise specified (NOS). All depression treatment studies included adults with BD-II, while two also included BD-I. Fifteen of the 36 maintenance drug studies (42\%) included BD participants other than BD-I, but only five studies also included BD NOS. The nondrug studies were more inclusive in their included BD populations.

We found no high- or moderate-strength evidence for any intervention to effectively treat any type of BD compared to placebo or an active comparator. We found scattered evidence for some drug interventions that were assessed as low-strength for adults with BD-I, but none for adults with BD-II or BD-NOS. However, most manic symptom improvements were of modest clinical significance, with values that were less than the MID but still large enough that a reasonable proportion of participants likely received a benefit. Very few findings for psychosocial interventions were assessed as low strength.

Table A provides a summary of low-strength evidence findings from the results chapters detailing intervention results. A full reporting of results and evidence tables can be found in the full report. 
Table A. Summary of low-strength* evidence findings by intervention class

\begin{tabular}{|c|c|c|c|}
\hline Category & Intervention & $\begin{array}{l}\text { \# Studies/ } \\
\text { Design } \\
\text { (n Analyzed) } \\
\text { Timing } \\
\end{array}$ & $\begin{array}{l}\text { Findings } \\
\text { (Low Strength) }\end{array}$ \\
\hline \multirow[t]{13}{*}{$\begin{array}{l}\text { Antipsychotics } \\
\text { for acute mania }\end{array}$} & $\begin{array}{l}\text { Asenapine vs. } \\
\text { placebo }\end{array}$ & $\begin{array}{l}3 \mathrm{RCT}^{10-12} \\
(\mathrm{n}=936) \\
3 \text { weeks }\end{array}$ & $\begin{array}{l}\text { Response/Remission Rates: No difference } \\
\text { YMRS: Favors Asenapine, MD } 4.37 \text { (95\% CI 1.27, } \\
\text { 7.47; MID 6) } \\
\text { CGI-BP-S: Favors Asenapine, MD } 0.5(95 \% \mathrm{Cl} \\
\text { 0.29, 0.71; MID 1) } \\
\text { Withdrawal (AE, Lack of Efficacy, Overall): No } \\
\text { difference }\end{array}$ \\
\hline & $\begin{array}{l}\text { Cariprazine vs. } \\
\text { placebo }\end{array}$ & $\begin{array}{l}3 \mathrm{RCT}^{13-15} \\
(\mathrm{n}=1,047) \\
3 \text { weeks }\end{array}$ & $\begin{array}{l}\text { Response Rate: Favors Cariprazine, OR } 2.14 \text { (95\% } \\
\mathrm{CI} 1.08,4.23) \text {; NNT=5.6 } \\
\text { Remission Rate: Favors Cariprazine, OR1.95 (95\% } \\
\mathrm{CI} 1.45,2.63) \text {; NNT= } 7 \\
\text { YMRS: Favors Cariprazine, MD } 5.38 \text { (95\% Cl 1.84, } \\
\text { 8.92; MID 6) } \\
\text { CGI-BP-S: Favors Cariprazine, MD } 0.54(95 \% \mathrm{Cl} \\
\text { 0.35, 0.73; MID 1) } \\
\text { Withdrawal (AE, Lack of Efficacy, Overall): No } \\
\text { difference }\end{array}$ \\
\hline & \multirow[t]{2}{*}{$\begin{array}{l}\text { Olanzapine vs. } \\
\text { placebo }\end{array}$} & $\begin{array}{l}5 \mathrm{RCT}^{11,16-19} \\
(\mathrm{n}=1199) \\
3 \text { weeks }\end{array}$ & $\begin{array}{l}\text { Response Rate: Favors Olanzapine, OR } 1.99 \text { (95\% } \\
\text { CI 1.29, 3.08); NNT=6 } \\
\text { Remission Rate: Favors Olanzapine, OR } 1.75 \\
\text { (95\% Cl 1.19, 2.58); NNT=7.5 } \\
\text { YMRS: Favors Olanzapine, MD } 4.9(95 \% \text { Cl 2.34, } \\
\text { 7.45; MID 6) } \\
\text { Withdrawal (Lack of Efficacy, Overall): Favors } \\
\text { Olanzapine, MD } 0.42 \text { (95\% Cl 0.29,0.61) }\end{array}$ \\
\hline & & $\begin{array}{l}3 \mathrm{RCT} 16,18,19 \\
(\mathrm{n}=611) \\
3 \text { weeks }\end{array}$ & CGI-BP-S: No difference \\
\hline & \multirow{3}{*}{$\begin{array}{l}\text { Quetiapine vs. } \\
\text { placebo }\end{array}$} & $\begin{array}{c}4 \mathrm{RCT}^{20-23} \\
(\mathrm{n}=1,007) \\
3 \text { weeks }\end{array}$ & $\begin{array}{l}\text { Response Rate: Favors Quetiapine, OR } 2.07 \text { (95\% } \\
\mathrm{Cl} 1.39,3.09) \text {; NNT=6.2 } \\
\text { Withdrawal (Lack of Efficacy): Favors Quetiapine, } \\
\text { MD } 0.38(95 \% \mathrm{Cl} 0.23,0.63)\end{array}$ \\
\hline & & $\begin{array}{c}5 \mathrm{RCT}^{20-24} \\
(\mathrm{n}=699) \\
3 \text { weeks }\end{array}$ & $\begin{array}{l}\text { YMRS: Favors Quetiapine, MD } 4.92 \text { (95\% Cl 0.31, } \\
\text { 9.53; MID 6) }\end{array}$ \\
\hline & & $\begin{array}{l}5 \mathrm{RCT}^{20-24} \\
(\mathrm{n}=806) \\
3 \text { weeks }\end{array}$ & $\begin{array}{l}\text { CGI-BP-S: Favors Quetiapine, MD } 0.54(95 \% \mathrm{CI} \\
0.35,0.74 ; \text { MID 1) }\end{array}$ \\
\hline & $\begin{array}{l}\text { Risperidone vs. } \\
\text { placebo }\end{array}$ & $\begin{array}{l}2 \mathrm{RCT}^{25,}, 26 \\
(\mathrm{n}=584) \\
3 \text { weeks }\end{array}$ & $\begin{array}{l}\text { Response Rate, YMRS, and CGI: Favors } \\
\text { Risperidone (not pooled) }\end{array}$ \\
\hline & $\begin{array}{l}\text { Ziprasidone vs. } \\
\text { placebo }\end{array}$ & $\begin{array}{l}2 \mathrm{RCT}^{27,28} \\
(\mathrm{n}=402) \\
3 \text { weeks }\end{array}$ & $\begin{array}{l}\text { Response Rate, YMRS, and CGI: Favors } \\
\text { Ziprasidone (not pooled) }\end{array}$ \\
\hline & \multirow{4}{*}{$\begin{array}{l}\text { Olanzapine vs. } \\
\text { Divalproex/ } \\
\text { Valproate }\end{array}$} & $\begin{array}{c}2 \mathrm{RCTs}^{18,29} \\
(\mathrm{n}=635) \\
3 \text { weeks }\end{array}$ & Response and Remission: No difference \\
\hline & & $\begin{array}{c}3 \text { RCTs }^{18,29,30} \\
(n=750) \\
3 \text { weeks }\end{array}$ & YMRS: No difference \\
\hline & & $\begin{array}{c}\text { RCTs }^{18,29,30} \\
(n=578) \\
3 \text { weeks }\end{array}$ & CGI: No difference \\
\hline & & $\begin{array}{c}4 \mathrm{RCTs}^{18,29-31} \\
(\mathrm{n}=867) \\
3 \text { weeks }\end{array}$ & Withdrawals: No difference \\
\hline
\end{tabular}




\begin{tabular}{|c|c|c|c|}
\hline Category & Intervention & $\begin{array}{l}\text { \# Studies/ } \\
\text { Design } \\
\text { (n Analyzed) } \\
\text { Timing }\end{array}$ & $\begin{array}{l}\text { Findings } \\
\text { (Low Strength) }\end{array}$ \\
\hline \multirow[t]{3}{*}{$\begin{array}{l}\text { Mood stabilizers } \\
\text { treatments for } \\
\text { acute mania }\end{array}$} & \multirow{3}{*}{$\begin{array}{l}\text { Lithium vs. } \\
\text { placebo }\end{array}$} & $\begin{array}{c}1 \mathrm{RCT}^{21}+1 \mathrm{IPD}^{32} \\
(\mathrm{n}=325) \\
3 \text { weeks }\end{array}$ & $\begin{array}{l}\text { Remission and Response Rates: Favors Lithium } \\
\text { (not pooled) }\end{array}$ \\
\hline & & $\begin{array}{c}3 \mathrm{RCTs}^{21,32} \\
(\mathrm{n}=325) \\
3 \text { weeks }\end{array}$ & $\begin{array}{l}\text { YMRS: Favors Lithium, } \\
\text { MD } 5.81 \text { (95\% Cl 2.21, 9.4; MID 6) } \\
\text { Withdrawal (Overall): No difference }\end{array}$ \\
\hline & & $\begin{array}{l}1 \mathrm{IPD}^{32} \\
(\mathrm{n}=450) \\
3 \text { weeks }\end{array}$ & Withdrawal (Lack of Efficacy, AE): No difference \\
\hline \multirow[t]{6}{*}{$\begin{array}{l}\text { Other drug } \\
\text { treatments for } \\
\text { mania }\end{array}$} & $\begin{array}{l}\text { Paliperidone vs. } \\
\text { placebo }\end{array}$ & $\begin{array}{l}2 \mathrm{RCT}^{20,33} \\
(\mathrm{n}=763) \\
3 \text { weeks }\end{array}$ & $\begin{array}{l}\text { YMRS and Withdrawal (Lack of Efficacy): Favors } \\
\text { Paliperidone (possible dose response: No } \\
\text { difference at } 3 \text { and } 6 \mathrm{mg} \text {, benefit at } 12 \mathrm{mg} \text { or } \\
\text { median dosage of } 9 \mathrm{mg} \text { ) } \\
\text { Withdrawal (AE): No difference }\end{array}$ \\
\hline & $\begin{array}{l}\text { Topiramate vs. } \\
\text { placebo }\end{array}$ & $\begin{array}{l}1 \text { IPD }^{32} \\
(\mathrm{n}=876) \\
3 \text { weeks }\end{array}$ & $\begin{array}{l}\text { YMRS and Withdrawal (Lack of Efficacy): No } \\
\text { difference } \\
\text { Withdrawals (Overall): Favors Placebo, } 37.2 \% \text { vs. } \\
26.8 \%, p=0.005 \\
\text { Withdrawals (AE): Favors Placebo, } 6.04 \% \text { vs. } \\
2.84 \%, p=0.049\end{array}$ \\
\hline & \multirow{3}{*}{$\begin{array}{l}\text { Topiramate vs. } \\
\text { lithium }\end{array}$} & $\begin{array}{l}1 \mathrm{IPD}^{32} \\
(\mathrm{n}=453) \\
3 \text { weeks }\end{array}$ & $\begin{array}{l}\text { YMRS: Favors Lithium, MD } 6.14 \text { (95\% CI 3.94, } \\
\text { 8.34; MID 6) }\end{array}$ \\
\hline & & $\begin{array}{l}1 \mathrm{IPD}^{32} \\
(\mathrm{n}=453) \\
3 \text { weeks }\end{array}$ & Withdrawal (Overall, AE): No difference \\
\hline & & $\begin{array}{l}1 \text { IPD }^{32} \\
(\mathrm{n}=453) \\
3 \text { weeks }\end{array}$ & $\begin{array}{l}\text { Withdrawal (AE): Favors Topiramate, } 2.65 \% \text { vs. } \\
7.49 \%, p=0.019\end{array}$ \\
\hline & $\begin{array}{l}\text { Allopurinol + } \\
\text { lithium vs. } \\
\text { placebo + lithium }\end{array}$ & $\begin{array}{c}4 \mathrm{RCT}^{34-37} \\
(\mathrm{n}=355) \\
4 \text { weeks }\end{array}$ & YMRS, CGI, Withdrawal (Overall): No difference \\
\hline $\begin{array}{l}\text { Single drug } \\
\text { treatment for } \\
\text { maintenance }\end{array}$ & $\begin{array}{l}\text { Lithium vs. } \\
\text { placebo }\end{array}$ & $\begin{array}{c}6 \mathrm{RCT}^{38-43} \\
(\mathrm{n}=1579) \\
1 \text { to } 2 \text { years }\end{array}$ & Time to overall relapse: Favors Lithium \\
\hline \multirow[t]{2}{*}{$\begin{array}{l}\text { Psychosocial } \\
\text { interventions }\end{array}$} & $\begin{array}{l}\text { CBT vs. Active } \\
\text { Comparators }^{\star *}\end{array}$ & $\begin{array}{c}5 \text { RCTs }^{44-49} \\
(n=461) \\
6 \text { to } 12 \text { months }\end{array}$ & $\begin{array}{l}\text { Depression and Mania symptoms: No difference } \\
\text { between groups across range of time periods. }\end{array}$ \\
\hline & $\begin{array}{l}\text { Systematic or } \\
\text { Collaborative } \\
\text { Care vs. Inactive } \\
\text { Comparators }\end{array}$ & $\begin{array}{c}2 \mathrm{RCTS}^{50,51} \\
(\mathrm{n}=599) \\
7 \text { to } 12 \text { months }\end{array}$ & $\begin{array}{l}\text { Relapse Rate: No difference between groups } \\
\text { across different time periods. }\end{array}$ \\
\hline
\end{tabular}

*All findings are low-strength evidence based generally on moderate study limitations and imprecision. ** Active comparators are comparators such as a different psychosocial therapy or peer support. †Inactive comparators are comparators such as usual care, no intervention.

$\mathrm{AE}=$ adverse events; $\mathrm{CBT}=$ cognitive behavioral therapy; CGI =Clinical global impression; CGI-BP-S=Clinical global impression scale for bipolar severity; $\mathrm{CI}=$ confidence interval; IPD=individual patient data; $\mathrm{MD}=$ mean difference; $\mathrm{MID}=$ minimal important difference; $\mathrm{NNT}=$ number needed to treat; $\mathrm{OR}=$ odds ratio; RCT=randomized controlled trial; YMRS=Young mania rating scale

Asenapine, cariprazine, quetiapine, and olanzapine improved acute mania symptoms compared to placebo (low-strength evidence). However, improvements were of modest clinical significance, with values that were less than the MID, but still large enough that a reasonable proportion of participants likely received a benefit. Unpooled evidence indicated an overall beneficial effect of risperidone and ziprasidone on acute mania symptoms compared to placebo 
(low-strength evidence). Lithium improved acute mania in the short-term and prolonged time to relapse in the long-term compared to placebo (low-strength evidence). No difference was found between olanzapine and divalproex/valproate for acute mania (low-strength evidence). For drugs not approved for $\mathrm{BD}$, paliperidone also improved acute mania compared to placebo (lowstrength evidence), while topiramate and allopurinol showed no benefit (low-strength evidence). Further, lithium improved acute mania better than topiramate (low-strength evidence), although withdrawals for adverse events were lower for topiramate. Only lithium reached a minimally important difference for acute mania and maintenance treatment. All other drug comparisons to placebo or active controls for acute mania, depression, and maintenance had insufficient evidence.

Adverse events for drugs were variously reported and generally not with sufficient detail to allow pooling when multiple studies were available. When reported, all drug comparisons generally showed no differences between groups in serious adverse events. Participants using atypical antipsychotics as a single drug, except quetiapine, experienced more extrapyramidal symptoms compared to placebo. Participants using haloperidol experienced more extrapyramidal symptoms compared to other antipsychotics. Participants using olanzapine reported more clinically significant weight gain. Participants using carbamazepine reported more severe rash and number of adverse events compared to placebo.

For psychosocial interventions, cognitive behavioral training (CBT) was no better for depression or mania symptoms than psychoeducation or other active psychosocial comparators (low-strength evidence). Systematic/collaborative care had no effect on relapse compared to inactive comparators (low-strength evidence).

Table B provides a list of interventions and comparators with evidence that was insufficient to draw conclusions.

Table B. Interventions/comparators with insufficient strength of evidence

\begin{tabular}{|c|c|c|}
\hline Category & Drug & Comparator \\
\hline \multirow{17}{*}{$\begin{array}{l}\text { Antipsychotics } \\
\text { for mania }\end{array}$} & Aripiprazole & Placebo \\
\hline & Aripiprazole & Haloperidol \\
\hline & Aripiprazole plus Mood Stabilizer & Mood Stabilizer alone (placebo) \\
\hline & Aripiprazole plus Mood Stabilizers & Haloperidol plus Mood Stabilizer \\
\hline & Asenapine & Olanzapine \\
\hline & Asenapine plus Mood Stabilizer & Mood Stabilizer alone (placebo) \\
\hline & $\begin{array}{l}\text { Olanzapine (for withdrawal for adverse events } \\
\text { only) }\end{array}$ & Placebo \\
\hline & Olanzapine & Haloperidol or Lithium or Risperidone \\
\hline & Olanzapine plus Mood Stabilizer & Mood Stabilizer alone (placebo) \\
\hline & Olanzapine plus Mood Stabilizers & Mood Stabilizer alone (no placebo) \\
\hline & Quetiapine & Haloperidol or Lithium \\
\hline & Quetiapine plus Mood Stabilizers & Mood Stabilizer alone (placebo) \\
\hline & Risperidone & Haloperidol or Lithium \\
\hline & Risperidone plus Mood Stabilizers & Mood Stabilizer alone (placebo) \\
\hline & Ziprasidone plus Mood Stabilizers & Mood Stabilizer alone (placebo) \\
\hline & Ziprasidone plus Mood Stabilizer & Chlorpromazine plus Mood Stabilizer \\
\hline & Haloperidol & Placebo \\
\hline \multirow{7}{*}{$\begin{array}{l}\text { Mood Stabilizers } \\
\text { for mania }\end{array}$} & Carbamazepine & Placebo \\
\hline & Divalproex/Valproate & Placebo \\
\hline & Valproate & No Placebo \\
\hline & Lithium (for CGI only) & Placebo \\
\hline & Carbamazepine & Lithium or Valproate \\
\hline & Carbamazepine & Valporate \\
\hline & Lamotrigine & Lithium \\
\hline
\end{tabular}




\begin{tabular}{|c|c|c|}
\hline Category & Drug & Comparator \\
\hline & Lithium & Haloperidol or Divalproex \\
\hline \multirow[t]{14}{*}{$\begin{array}{l}\text { Other Drugs for } \\
\text { mania }\end{array}$} & $\begin{array}{l}\text { Paliperidone (for Remission, Response, CGI } \\
\text { Withdrawal (Overall)) }\end{array}$ & Placebo \\
\hline & $\begin{array}{l}\text { Allopurinol plus Lithium (for Response and } \\
\text { Remission) }\end{array}$ & Lithium alone (placebo) \\
\hline & Allopurinol plus Lithium & Dipyridamole plus Lithium \\
\hline & Celecoxib & Placebo \\
\hline & Dipyridamole plus Lithium & Lithium alone (placebo) \\
\hline & Donepezil plus Lithium & Lithium alone (placebo) \\
\hline & Endoxifen & Divalproex \\
\hline & Gabapentin plus Lithium & Lithium alone (placebo) \\
\hline & Oxcarbazepine & Divalproex \\
\hline & Paliperidone Extended Release & Olanzapine or Quetiapine \\
\hline & Paliperidone plus Mood Stabilizers & Mood Stabilizers alone (placebo) \\
\hline & Tamoxifen & Placebo \\
\hline & Topiramate plus Risperidone & Divalproex plus Risperidone \\
\hline & Topiramate and Mood Stabilizer & Mood Stabilizer alone (placebo) \\
\hline \multirow{6}{*}{$\begin{array}{l}\text { Drugs for } \\
\text { depression }\end{array}$} & Memantine plus Valproate & Valproate alone (placebo) \\
\hline & Lamotrigine plus Mood Stabilizers & Mood Stabilizers alone (placebo) \\
\hline & $\begin{array}{l}\text { Antidepressives (paraoxetine, bupropion, or } \\
\text { both) }\end{array}$ & Placebo \\
\hline & Sertraline & Lithium \\
\hline & Venlafaxine & Lithium \\
\hline & Lithium and OPT & OPT alone \\
\hline \multirow{29}{*}{$\begin{array}{l}\text { Drugs for } \\
\text { maintenance }\end{array}$} & Long-acting Injectable Aripiprazole & Placebo \\
\hline & Aripiprazole & Placebo \\
\hline & Aripiprazole plus Mood Stabilizer & Mood Stabilizer alone (placebo) \\
\hline & Carbamazepine & Lithium \\
\hline & Divalproex & Placebo \\
\hline & Divalproex plus Lithium & Lithium alone (placebo) \\
\hline & Fluoxetine & Placebo \\
\hline & Fluoxetine & Lithium \\
\hline & Gabapentin plus Mood Stabilizers & Mood Stabilizers alone (placebo) \\
\hline & Lamotrigine & Placebo \\
\hline & Lamotrigine for pregnant women & Discontinue Mood Stabilizers \\
\hline & Lamotrigine & Lithium \\
\hline & Lithium & Placebo \\
\hline & Lithium & Divalproex/Valproate \\
\hline & Olanzapine & Placebo \\
\hline & Olanzapine & Divalproex \\
\hline & Olanzapine & Lithium \\
\hline & Olanzapine plus Mood Stabilizers & Mood Stabilizers alone (placebo) \\
\hline & Oxcarbazepine plus Lithium & Lithium alone (placebo) \\
\hline & Paliperidone & Placebo \\
\hline & Paliperidone & Olanzapine \\
\hline & Perphenazine plus Mood Stabilizers & Mood Stabilizers alone (placebo) \\
\hline & Quetiapine & Placebo \\
\hline & Quetiapine & Lithium \\
\hline & Quetiapine plus Mood Stabilizers & Mood Stabilizers alone (placebo) \\
\hline & Quetiapine and Personalize Treatment & Lithium and Personalized Treatment \\
\hline & Risperidone & Placebo \\
\hline & Risperidone & Olanzapine \\
\hline & $\begin{array}{l}\text { Risperidone Long Acting Injectable and } \\
\text { Treatment as Usual }\end{array}$ & Placebo and Treatment as Usual \\
\hline
\end{tabular}




\begin{tabular}{|c|c|c|}
\hline Category & Drug & Comparator \\
\hline & Valproic Acid plus Aripiprazole & Lithium plus Aripiprazole \\
\hline & Venlafaxine & Lithium \\
\hline & Ziprasidone and Mood Stabilizers & Mood Stabilizers alone (placebo) \\
\hline \multirow{12}{*}{$\begin{array}{l}\text { Psychosocial } \\
\text { Interventions }\end{array}$} & Psychoeducation & Inactive ${ }^{\star}$ Comparators \\
\hline & Psychoeducation & Active $^{\star \star}$ Comparators \\
\hline & CBT & Inactive Comparators \\
\hline & $\begin{array}{l}\text { CBT (for Relapse, Global Function, Other } \\
\text { Measures of Function) }\end{array}$ & Active Comparators \\
\hline & $\begin{array}{l}\text { Systematic or Collaborative Care (for } \\
\text { Depression, Mania, Global Function, Other } \\
\text { Measures of Function) }\end{array}$ & Inactive Comparators \\
\hline & Family or Partner Interventions & Inactive Comparators \\
\hline & Family or Partner Interventions & Active Comparators \\
\hline & IPSRT & Inactive Comparators \\
\hline & IPSRT & Active Comparators \\
\hline & Combination Interventions & Inactive Comparators \\
\hline & Combination Interventions & Active Comparators \\
\hline & Other Psychosocial Interventions & Inactive Comparators \\
\hline Somatic & Repetitive transcranial magnetic stimulation & Sham stimulation \\
\hline
\end{tabular}

*Inactive comparators include usual care or no intervention. **Active comparators include a different psychosocial therapy, peer support, or similar.

CBT=cognitive behavioral therapy; CGI=Clinical Global Impression; IPSRT= Interpersonal and Social Rhythm Therapy;

OPT=Optimal Personalized Treatment

\section{Discussion}

This review found only low-strength evidence for treatments for adults with BD. All Food and Drug Administration-approved antipsychotics, except aripiprazole, improved mania symptoms when compared to placebo for acute mania in adults with BD-I. However, none of the drugs reached MID. Participants using atypical antipsychotics, except quetiapine, reported more extrapyramidal symptoms compared to placebo, and those using olanzapine reported more clinically significant weight gain. Lithium showed short-term benefit for acute mania and longer time to relapse to any mood episode in adults with BD-I versus placebo. Of all acute mania treatments, lithium treatment was closest to reaching a clinically meaningful difference for all the participants as measured by the MID. Evidence was generally insufficient for benefits from nondrug interventions for adults with BD. Low-strength evidence showed no effect for the effectiveness of CBT on bipolar symptoms and the efficacy of systematic/collaborative care on preventing relapse.

Our findings are consistent with other systematic reviews of treatments for bipolar; however, because we excluded studies with greater than 50 percent attrition rates, our findings are more conservative than those of other reviews. Similar to Cochrane reviews, we also found benefit for olanzapine and risperidone compared with placebo for mania, and benefit for lithium compared with placebo for maintenance. ${ }^{52-54}$ Cochrane reviews have reported benefit for several additional antipsychotics compared with placebo - aripipravole, haloperidol as single drug and added to mood stabilizers, and olanzapine or risperidone plus mood stabilizers - whereas we found evidence was insufficient. ${ }^{52,55-58}$ However, authors of these reviews consistently noted that issues with attrition and medication adherence may have impacted their results. Insufficient evidence for psychosocial interventions was consistent across all reviews. ${ }^{59,} 60$

Applicability of the review findings is challenging. The trials for drug treatments used restrictive exclusion criteria, making it difficult to determine whether the findings extend to 
adults with BD-II, or BD-I with a first manic episode, current comorbid substance use, pregnant or nursing women, or older adults (i.e., age 65 and over).

Conversely, most psychosocial trials provided too little information on the participant characteristics, limiting the ability to infer from the results. Mixtures of participants may mask patterns of effectiveness. With the current information, we cannot determine if or to what extent this contributed to the few findings of nonsignificance between groups.

Applicability is further challenged by high attrition rates. Trials with 20 to 50 percent attrition, such as were used in this review, at best provide an estimate of the efficacy or comparative effectiveness of a treatment for participants who comply with, tolerate, and, in some minimal sense, benefit from the treatment. However, at extremely high levels of attrition, even this interpretation is of limited value to clinicians. ${ }^{61}$ Likewise, the maintenance trials are most applicable to people with BD-I who respond to initial treatment.

Applicability is also hindered by lack of information about diagnostic accuracy. The accuracy of a diagnosis of BD, or study eligibility, depends on the interviews or screening tools, the criteria used to diagnose BD, and who performs the diagnostic assessment. Additional information and rigor in diagnostic assessment would generate a greater sense of confidence about who the study participants represent and, therefore, the populations to which the study results apply. Uncertainty and debate surround the question of whether the underlying mechanisms support the bipolar types as qualitatively and categorically different or as lying on a continuum of the same psychopathological dimensions. Meanwhile, the importance of diagnostic accuracy is further underscored by the great difficulty in accurately diagnosing the comorbid mental health conditions that were commonly treated as exclusion criteria in the studies we reviewed.

\section{Limitations}

Several inclusion criteria may have created limitations to the review findings. We only included studies if the populations were exclusively adults with BD, or if the bipolar subpopulation results were reported separately. Psychosocial treatments specific to depression or mania that combined participants with bipolar and nonbipolar diagnoses in analyses may have been missed and therefore not included in this review.

In addition to clearly reported outcomes for BD populations, we also required studies to be at least prospective cohort studies with comparator. This combination of inclusion criteria led to a number of observational studies being excluded, including those that looked at broad classes of drugs, or individual drugs across broad populations. Thus, harms information was essentially limited to RCTs or extensions of RCTs.

We also looked at minimum followup periods of 3 weeks for acute mania studies, 3 months for depression studies, and 6 months for maintenance studies. This criteria led to many studies, especially for depression treatment and other somatic treatments such as electroconvulsive therapy, being excluded for followup times that were too short. However, given the chronic nature of bipolar disorders, the clinical relevance of short followup studies is questionable. Moreover, evidence that a treatment reduces bipolar episode relapse rates likely requires followup longer than 12 months, because some individuals with bipolar disorder only experience episodes once or twice per year. 


\section{Research Needs}

Future studies of BD treatments need to consider innovative ways to increase study completion rates (e.g., use of technology for followup assessments and study reminders; "smart" bottles for assessing study drug adherence; multiple secondary contacts for participants and all-inclusive contact information from cell phones, email, to social media; flexible scheduling outside of business hours, availability at the last minute notice). More longitudinal data analysis techniques for intermittent followup would help, but that requires more effort to create data repositories that allow individual patient-level data pooling of these longitudinal studies. Such repositories could also help broaden inclusion criteria and allow for further subpopulation analyses. Future research should also enroll people with different patient characteristics and initial episodes and maintenance stages to fully understand the spectrum of responses. Attention should be given to addressing all states of the illness throughout the treatment stream.

\section{Conclusion}

We found no high or moderate-strength evidence for any intervention to effectively treat any phase of any type of BD compared to placebo or an active comparator. Low-strength evidence showed improved mania symptoms for all Food and Drug Administration-approved antipsychotics, except aripiprazole, when compared to placebo for adults with BD-I. Lowstrength evidence also showed benefit from lithium in the short-term for acute mania and longer time to relapse in the long-term versus placebo in adults with BD-I. Evidence was insufficient for most nondrug interventions. Aside from low-strength evidence showing CBT and systematic/collaborative care having no benefit for a few outcomes, evidence was insufficient for psychosocial interventions. We were unable to address questions on subpopulations or treatments to reduce the metabolic-related side effects of first-line drug treatments. Future studies of treatments for $\mathrm{BD}$ will require innovative ways to increase study completion rates.

\section{References}

1. Ferrari AJ, Stockings E, Khoo JP, et al. The prevalence and burden of bipolar disorder: findings from the Global Burden of Disease Study 2013. Bipolar Disord. 201601

Aug;18(5):440-50. doi: http://dx.doi.org/10.1111/bdi.12423. PMID: 611908711.

2. Gum A, King-Kallimanis B, Kohn R. Prevalence of mood, anxiety, and substance-abuse disorders for older Americans in the national comorbidity survey-replication. Am J Geriat Psychiatry. 2009;17:769-81.
3. Samame C, Martino DJ, Strejilevich SA. Social cognition in euthymic bipolar disorder: systematic review and meta-analytic approach. Acta Psychiat Scand. 2012;125(4):266-80.

4. Sole B, Martinez-Aran A, Torrent C, et al. Are bipolar II patients cognitively impaired? A systematic review. Psychol Med. 2011;41(9):1791-803.

5. Schaffer A, Isometsa ET, Tondo L, et al. Epidemiology, neurobiology and pharmacological interventions related to suicide deaths and suicide attempts in bipolar disorder: Part I of a report of the International Society for Bipolar Disorders Task Force on Suicide in Bipolar Disorder. Aust N Z J Psychiatry.49(9):785-802. PMID: 26185269. 
6. Merikangas K, Akiskal HS, Angst J, et al. Lifetime and 12-month prevalence of bipolar spectrum disorder in the National Comorbidity Survey replication. Arch Gen Psychiatry. 2007 May;64(5):543-52. PMID: 17485606.

7. Brady KT, Sonne S. The relationship between substance abuse and bipolar disorder. J Clin Psychiatry. 1995;56(Suppl 3):19-24. PMID: 7883738

8. Lukasiewicz M, Gerard S, Besnard A, et al. Young mania rating scale: how to interpret the numbers? Determination of a severity threshold and of the minimal clinically significant difference in the EMBLEM cohort. Int. J. Methods Psychiatr. Res. 2013;22(1):46-58. doi: 10.1002/mpr.1379. PMID: 23526724.

9. Johnston BC, Patrick DL, Thorlund K, et al. Patient-reported outcomes in meta-analyses Part 2: methods for improving interpretability for decision-makers. Health and Quality of Life Outcome. 2013;11(211)doi: doi:10.1186/14777525-11-211. PMID: 24359184.

10. McIntyre RS, Cohen M, Zhao J, et al. Asenapine in the treatment of acute mania in bipolar I disorder: a randomized, double-blind, placebocontrolled trial. J Affect Disord. 2010 Apr;122(12):27-38. doi:

http://dx.doi.org/10.1016/j.jad.2009.12.028. PMID: 20096936.

11. McIntyre RS, Cohen M, Zhao J, et al. A 3-week, randomized, placebo-controlled trial of asenapine in the treatment of acute mania in bipolar mania and mixed states.[Erratum appears in Bipolar Disord. 2010 May;12(3):350]. Bipolar Disord. 2009 Nov;11(7):673-86. doi: http://dx.doi.org/10.1111/j.13995618.2009.00748.x. PMID: 19839993.

12. Landbloom RL, Mackle M, Wu X, et al. Asenapine: Efficacy and safety of 5 and $10 \mathrm{mg}$ bid in a 3-week, randomized, double-blind, placebo-controlled trial in adults with a manic or mixed episode associated with bipolar I disorder. J Affect Disord. 2016 Jan 15;190:103-10. doi: http://dx.doi.org/10.1016/j.jad.2015.06.059. PMID: 26496015.

13. Calabrese JR, Keck PE, Jr., Starace A, et al. Efficacy and safety of low- and high-dose cariprazine in acute and mixed mania associated with bipolar I disorder: a double-blind, placebocontrolled study. J Clin Psychiatry. 2015 Mar;76(3):284-92. doi: http://dx.doi.org/10.4088/JCP.14m09081. PMID: 25562205.
14. Durgam S, Starace A, Li D, et al. The efficacy and tolerability of cariprazine in acute mania associated with bipolar I disorder: A phase II trial. Bipolar Disord. 2015 Feb;17(1):63-75. doi: http://dx.doi.org/10.1111/bdi.12238. PMID: 25056368 (pubmed) 2014-30788001(Psychinfo).

15. Sachs GS, Greenberg WM, Starace A, et al. Cariprazine in the treatment of acute mania in bipolar I disorder: a double-blind, placebocontrolled, phase III trial. J Affect Disord. 2015 Mar 15;174:296-302. doi: http://dx.doi.org/10.1016/j.jad.2014.11.018. PMID: 25532076.

16. Katagiri H, Takita $\mathrm{Y}$, Tohen M, et al. Efficacy and safety of olanzapine in the treatment of Japanese patients with bipolar I disorder in a current manic or mixed episode: a randomized, double-blind, placebo- and haloperidolcontrolled study. J Affect Disord. 2012 Feb;136(3):476-84. doi: http://dx.doi.org/10.1016/j.jad.2011.10.045. PMID: 22134043.

17. McIntyre RS. Aripiprazole for the maintenance treatment of bipolar I disorder: A review. Clin Ther. 2010;32 Suppl 1:S32-8. doi: http://dx.doi.org/10.1016/j.clinthera.2010.01.022. PMID: 20152551.

18. Tohen M, Vieta E, Goodwin GM, et al. Olanzapine versus divalproex versus placebo in the treatment of mild to moderate mania: a randomized, 12-week, double-blind study. J Clin Psychiatry. 2008 Nov;69(11):1776-89. PMID: 19014751.

19. Tohen M, Jacobs TG, Grundy SL, et al. Efficacy of olanzapine in acute bipolar mania: a doubleblind, placebo-controlled study. The Olanzipine HGGW Study Group.[Erratum appears in Arch Gen Psychiatry 2002 Jan;59(1):91]. Arch Gen Psychiatry. 2000 Sep;57(9):841-9. PMID: 10986547.

20. Vieta E, Nuamah IF, Lim P, et al. A randomized, placebo- and active-controlled study of paliperidone extended release for the treatment of acute manic and mixed episodes of bipolar I disorder. Bipolar Disord. 2010 May;12(3):23043. doi: http://dx.doi.org/10.1111/j.13995618.2010.00815.x. PMID: 20565430.

21. Bowden CL, Grunze H, Mullen J, et al. A randomized, double-blind, placebo-controlled efficacy and safety study of quetiapine or lithium as monotherapy for mania in bipolar disorder. $\mathrm{J}$ Clin Psychiatry. 2005 Jan;66(1):111-21. PMID: 15669897. 
22. McIntyre RS, Brecher M, Paulsson B, et al. Quetiapine or haloperidol as monotherapy for bipolar mania--a 12-week, double-blind, randomised, parallel-group, placebo-controlled trial. Eur Neuropsychopharmacol. 2005 Oct;15(5):573-85. PMID: 16139175.

23. Cutler AJ, Datto C, Nordenhem A, et al. Extended-release quetiapine as monotherapy for the treatment of adults with acute mania: a randomized, double-blind, 3-week trial. Clin Ther. 2011 Nov;33(11):1643-58. doi: http://dx.doi.org/10.1016/j.clinthera.2011.10.002. PMID: 22054797.

24. McElroy SL, Martens BE, Winstanley EL, et al. Placebo-controlled study of quetiapine monotherapy in ambulatory bipolar spectrum disorder with moderate-to-severe hypomania or mild mania. J Affect Disord. 2010 Jul;124(12):157-63. doi: http://dx.doi.org/10.1016/j.jad.2009.11.014. PMID: 19963274.

25. Khanna S, Vieta E, Lyons B, et al. Risperidone in the treatment of acute mania. Br J Psychiatry. 2005 2005;187(3):229-34. doi: http://dx.doi.org/10.1192/bjp.187.3.229. PMID: 16135859.

26. Smulevich AB, Khanna S, Eerdekens M, et al. Acute and continuation risperidone monotherapy in bipolar mania: a 3-week placebo-controlled trial followed by a 9-week double-blind trial of risperidone and haloperidol. Eur Neuropsychopharmacol. 2005 Jan;15(1):75-84. PMID: 15572276.

27. Potkin SG, Keck PE, Jr., Segal S, et al. Ziprasidone in acute bipolar mania: a 21-day randomized, double-blind, placebo-controlled replication trial. J Clin Psychopharmacol. 2005 Aug;25(4):301-10. PMID: 16012271.

28. Keck PE, Jr., Versiani M, Potkin S, et al. Ziprasidone in the treatment of acute bipolar mania: a three-week, placebo-controlled, doubleblind, randomized trial. Am J Psychiatry. 2003 Apr;160(4):741-8. PMID: 12668364.

29. Tohen M, Baker RW, Altshuler LL, et al. Olanzapine versus divalproex in the treatment of acute mania.[Erratum appears in Am J Psychiatry. 2005 Feb;7(1):102]. Am J of Psychiatry. 2002 Jun;159(6):1011-7. PMID: 12042191.
30. Zajecka JM, Weisler R, Sachs G, et al. A comparison of the efficacy, safety, and tolerability of divalproex sodium and olanzapine in the treatment of bipolar disorder. J Clinical Psychiatry. 2002 Dec;63(12):1148-55. PMID: 12523875.

31. Xu L, Lu Y, Yang Y, et al. Olanzapine-valproate combination versus olanzapine or valproate monotherapy in the treatment of bipolar imania: A randomized controlled study in a chinese population group. Neuropsychiatric Disease and Treatment. 201525 May;11:1265-71. doi: http://dx.doi.org/10.2147/NDT.S81146. PMID: 26060401 (pubmed) 2015078440 (embase).

32. Kushner SF, Khan A, Lane R, et al. Topiramate monotherapy in the management of acute mania: results of four double-blind placebo-controlled trials. Bipolar Disord. 2006 Feb;8(1):15-27. PMID: 16411977.

33. Berwaerts J, Xu H, Nuamah I, et al. Evaluation of the efficacy and safety of paliperidone extended-release in the treatment of acute mania: a randomized, double-blind, dose-response study. J Affect Disord. 2012 Jan;136(1-2):e51-60. doi: http://dx.doi.org/10.1016/j.jad.2010.06.030. PMID: 20624657.

34. Jahangard L, Soroush S, Haghighi M, et al. In a double-blind, randomized and placebo-controlled trial, adjuvant allopurinol improved symptoms of mania in in-patients suffering from bipolar disorder. Eur Neuropsychopharmacol. Jun. 2014 2, 2014(Pagination)doi: http://dx.doi.org/10.1016/j.euroneuro.2014.05.01 3. PMID: 24953766.

35. Weiser M, Burshtein S, Gershon AA, et al. Allopurinol for mania: A randomized trial of allopurinol versus placebo as add-on treatment to mood stabilizers and/or antipsychotic agents in manic patients with bipolar disorder. Bipolar Disord. 2014 2014;16(4):441-7. doi: http://dx.doi.org/10.1111/bdi.12202. PMID: 24712840.

36. Fan A, Berg A, Bresee C, et al. Allopurinol augmentation in the outpatient treatment of bipolar mania: a pilot study. Bipolar Disord. 2012 Mar;14(2):206-10. doi: http://dx.doi.org/10.1111/j.13995618.2012.01001.x. PMID: 22420596. 
37. Machado-Vieira R, Soares JC, Lara DR, et al. A double-blind, randomized, placebo-controlled 4week study on the efficacy and safety of the purinergic agents allopurinol and dipyridamole adjunctive to lithium in acute bipolar mania. $\mathrm{J}$ Clin Psychiatry. 2008 Aug;69(8):1237-45. PMID: 18681754.

38. Bowden CL, Calabrese JR, Sachs G, et al. A placebo-controlled 18-month trial of lamotrigine and lithium maintenance treatment in recently manic or hypomanic patients with bipolar I disorder.[Erratum appears in Arch Gen Psychiatry. 2004 Jul;61(7):680]. Arch Gen Psychiatry. 2003 Apr;60(4):392-400. PMID: 12695317.

39. Calabrese JR, Bowden CL, Sachs G, et al. A placebo-controlled 18-month trial of lamotrigine and lithium maintenance treatment in recently depressed patients with bipolar I disorder. J Clin Psychiatry. 2003 Sep;64(9):1013-24. PMID: 14628976.

40. Amsterdam JD, Shults J. Efficacy and safety of long-term fluoxetine versus lithium monotherapy of bipolar II disorder: a randomized, doubleblind, placebo-substitution study. Am J Psychiatry. $2010 \mathrm{Jul}$;67(7):792-800. doi: http://dx.doi.org/10.1176/appi.ajp.2009.0902028 4. PMID: 20360317.

41. Bowden CL, Calabrese JR, McElroy SL, et al. A randomized, placebo-controlled 12-month trial of divalproex and lithium in treatment of outpatients with bipolar I disorder. Divalproex Maintenance Study Group. Arch Gen Psychiatry. 2000 May;57(5):481-9. PMID: 10807488.

42. Prien RF, Point P, Caffey EM, et al. Prophylactic efficacy of lithium carbonate in manic-depressive illness: Report of the veterans administration and national institute of mental health collaborative study group. Arch Gen Psychiatry. 1973;28(3):337-41. doi: 10.1001/archpsyc.1973.01750330035006. PMID: 4569674.

43. Weisler RH, Nolen WA, Neijber A, et al. Continuation of quetiapine versus switching to placebo or lithium for maintenance treatment of bipolar I disorder (Trial 144: a randomized controlled study). J Clin Psychiatry. 2011 Nov;72(11):1452-64. doi: http://dx.doi.org/10.4088/JCP.11m06878. PMID: 22054050.
44. Castle D, White C, Chamberlain J, et al. Groupbased psychosocial intervention for bipolar disorder: randomised controlled trial. $\mathrm{Br} \mathrm{J}$ Psychiatry. 2010 May;196(5):383-8. doi: http://dx.doi.org/10.1192/bjp.bp.108.058263. PMID: 20435965.

45. Lam DH, Hayward P, Watkins ER, et al. Relapse prevention in patients with bipolar disorder: cognitive therapy outcome after 2 years. American Journal of Psychiatry. 2005 Feb;162(2):324-9. PMID: 15677598.

46. Lam DH, Watkins ER, Hayward P, et al. A randomized controlled study of cognitive therapy for relapse prevention for bipolar affective disorder: outcome of the first year. Archives of General Psychiatry. 2003 Feb;60(2):145-52. PMID: 12578431.

47. Jones SH, Smith G, Mulligan LD, et al. Recovery-focused cognitive-behavioural therapy for recent-onset bipolar disorder: randomised controlled pilot trial.[Erratum appears in $\mathrm{Br} \mathrm{J}$ Psychiatry. 2015 Feb;206(2):169]. Br J Psychiatry. 2015 Jan;206(1):58-66. doi: http://dx.doi.org/10.1192/bjp.bp.113.141259. PMID: 25213157.

48. Perich T, Manicavasagar V, Mitchell PB, et al. A randomized controlled trial of mindfulnessbased cognitive therapy for bipolar disorder. Acta Psychiatrica Scandinavica. 2013

May;127(5):333-43. doi: http://dx.doi.org/10.1111/acps.12033. PMID: 23216045.

49. Scott J, Paykel E, Morriss R, et al. Cognitivebehavioural therapy for severe and recurrent bipolar disorders: randomised controlled trial. $\mathrm{Br}$ J Psychiatry. 2006 Apr;188:313-20. PMID: 16582056.

50. Kessing LV, Hansen HV, Hvenegaard A, et al. Treatment in a specialised out-patient mood disorder clinic v. standard out-patient treatment in the early course of bipolar disorder: randomised clinical trial. Br J Psychiatry. 2013 Mar;202(3):212-9. doi: http://dx.doi.org/10.1192/bjp.bp.112.113548. PMID: 23349295.

51. Simon GE, Ludman EJ, Unutzer J, et al. Randomized trial of a population-based care program for people with bipolar disorder. Psychol Med. 2005 Jan;35(1):13-24. PMID: 15842025.

52. Rendell Jennifer M, Gijsman Harm J, Keck Paul $\mathrm{K}$, et al. Olanzapine alone or in combination for acute mania. Cochrane Database of Systematic Reviews: John Wiley \& Sons, Ltd; 2003. 
53. Rendell Jennifer M, Gijsman Harm J, Bauer Mark S, et al. Risperidone alone or in combination for acute mania. Cochrane Database of Systematic Reviews: John Wiley \& Sons, Ltd; 2006.

54. Burgess Sally SA, Geddes J, Hawton Keith KE, et al. Lithium for maintenance treatment of mood disorders. Cochrane Database of Systematic Reviews: John Wiley \& Sons, Ltd; 2001.

55. Rendell Jennifer M, Geddes J. Risperidone in long-term treatment for bipolar disorder. Cochrane Database of Systematic Reviews: John Wiley \& Sons, Ltd; 2006.

56. Brown R, Taylor Matthew J, Geddes J. Aripiprazole alone or in combination for acute mania. Cochrane Database of Systematic Reviews: John Wiley \& Sons, Ltd; 2013.

57. Cipriani A, Rendell Jennifer M, Geddes J. Olanzapine in long-term treatment for bipolar disorder. Cochrane Database of Systematic Reviews: John Wiley \& Sons, Ltd; 2009.
58. Cipriani A, Rendell Jennifer M, Geddes J. Haloperidol alone or in combination for acute mania. Cochrane Database of Systematic Reviews: John Wiley \& Sons, Ltd; 2006.

59. Swartz H, Swanson J. Psychotherapy for bipolar disorder in adults: a review of the evidence. Focus (Am Psychiatr Publ). 2014

Summer;12(3):251-66. doi: 10.1176/appi.focus.12.3.251. PMID: 26279641.

60. Reilly S, Planner C, Gask L, et al. Collaborative care approaches for people with severe mental illness. Cochrane Database of Systematic Reviews: John Wiley \& Sons, Ltd; 2013.

61. Council NR. The prevention and treatment of missing data in clinical trials: National Academies Press; 2011. 


\section{Chapter 1. Introduction}

\section{Background}

Bipolar disorder, also known as manic-depressive illness, is a serious mental illness that causes unusual shifts in mood, energy, activity levels, and the ability to carry out day-to-day tasks. The Diagnostic and Statistical Manual for Mental Disorders, $5^{\text {th }}$ Edition (DSM-5) ${ }^{1}$ defines mania and hypomania as bipolar episodes characterized by elation, or irritability, and increased energy, plus at least three additional symptoms (four if the predominant mood is irritability): increased pursuit of various goal-directed activities without concern for potential negative consequences (e.g., impulsive shopping, risky business undertakings, unsafe sexual behaviors); increased activity level or psychomotor restlessness; pressured speech or greater talkativeness; a subjective feeling that one's thoughts are racing or jumping from topic to topic; increased distractibility by stimuli in the environment; and exaggerated self-confidence, at times to the point of grandiose delusions (e.g., believing one has special abilities or powers). Bipolar depressive episodes are characterized by depressed mood or anhedonia (i.e., a lack of interest in pleasurable activities) and at least four additional symptoms: decreased energy; psychomotor slowing or psychomotor restlessness; changes in appetite and weight; sleep disturbance (from insomnia to hypersomnia); difficulty concentrating and/or inability to make everyday decisions; feelings of worthlessness and/or excessive guilt; and suicidal ideation and attempts.

Manic and depressive episodes can vary in symptom duration and severity as well as subsequent effects on everyday functioning; therefore, the DSM-5 recognizes a spectrum of bipolar diagnoses that differ in duration of bipolar episodes/periods and impairment: bipolar I, bipolar II, other specified bipolar and related disorder, and unspecified bipolar and related disorder. The latter two diagnoses were captured under the bipolar disorder not otherwise specified diagnosis in prior versions of the DSM (DSM-IV-TR; 2000) and in current research literature.

According to the DSM-5, bipolar I disorder is mainly defined by the presence of manic episodes that last at least seven days, or by manic symptoms severe enough to necessitate immediate hospital care. Mania symptoms must reflect a major change from the person's normal behavior and cause grave impairment. Usually, a person with bipolar I disorder also has depressive episodes, typically lasting at least two weeks, which significantly impair daily functioning or distress. Still, presence of depressive episodes is not necessary for bipolar I disorder diagnosis. Prior DSM versions and empirical literature also allowed for bipolar I disorder diagnosis based on the presence of mixed episodes, i.e., periods of one week or longer characterized by both manic and depressive symptoms. The DSM-5 omits language specifying that individuals meet the full criteria for both mania and a major depressive episode in favor of a new specifier, “with mixed features.” Mixed features is applicable to episodes of mania or hypomania when depressive features are present, and to episodes of depression when features of mania/hypomania are present. The associated symptom of psychosis can also shift the episode type from hypomania to mania.

Bipolar II disorder is defined by a pattern of depressive episodes alternating with hypomanic episodes, but no full-blown manic episodes. Diagnostic criteria specify that hypomanic episodes, lasting at least four days, must cause a change in functioning observable by others, but this change could be positive (i.e., more productive, social) and not impairing. Cyclothymic disorder is defined by many periods of hypomanic and depressive symptoms, but these symptoms do not reach the level of clinical hypomanic or depressive episodes. 
Bipolar disorder definitions for forms not reaching bipolar I and bipolar II disorder criteria have recently been reorganized and redefined. DSM-5 presented four different case scenarios for "other specified bipolar disorder," including a) history of major depressive episodes and hypomanic periods that meet episode criteria except for duration (i.e., last less than four days); b) history of major depressive episodes and hypomanic periods of sufficient duration but insufficient number of symptoms to meet episode criteria; c) history of hypomanic episodes without major depressive episodes; and d) criteria for cyclothymia met for less than two years. Other cases of failing to meet bipolar I or II disorder diagnoses would fit the unspecified bipolar disorder category. DSM-5 has also further specified that cyclothymic disorder cannot be comorbid/or jointly assigned along with bipolar I or II disorder (e.g., if an individual develops episodes, the diagnosis would change to the type that best reflects new symptoms).

Structured psychiatric interviews, such as the Structured Clinical Interview for the DSM-IV Axis I Disorders (SCID-I), or the MINI International Neuropsychiatric Interview (MINI), are widely used in research settings and provide reliable diagnoses when conducted by trained interviewers. $^{2}$ The structured interviews help differentiate bipolar disorders from other common diagnoses, such as borderline personality disorder, as well as between various bipolar disorder definitions. However, such structured interviews are not as common in regular clinical settings, where screening questionnaires and clinical interviews are more often used to identify new bipolar diagnoses or changes in patient symptoms.

Prevalence studies estimate about 1 percent of the population for bipolar I disorder, another 1 percent for bipolar II disorder, and up to 5 percent for the full spectrum of bipolar disorder diagnoses, with relatively similar prevalence in men and women, and across cultural and ethnic groups. ${ }^{3,4}$ Recurrent episodes of mania and depression can cause serious impairments in functioning, such as erratic work performance, increased divorce rates, and psychosocial morbidity. ${ }^{5,6}$ People with bipolar disorder account for between 3 and 14 percent of all suicides. ${ }^{7}$ About 25 percent of bipolar disorder patients will attempt suicide. ${ }^{7}$ The disease burden is heavy, with lifelong treatment requirements.

Further adding to the individual illness burden, 92 percent of individuals with bipolar disorder experience a co-occurring psychiatric illness during their lifetime. ${ }^{8}$ Substance abuse is a common comorbid condition; of all psychiatric conditions, bipolar disorder is the most likely to co-occur with alcohol or drug abuse. ${ }^{9}$ Thus, it is important to identify not just effective treatments for bipolar disorder alone, but effective treatments for individuals experiencing both bipolar symptoms and co-occurring substance abuse and other psychiatric illnesses.

Increasingly, empirical evidence supports disruption of specific neural circuits as a factor in bipolar disorders. However, the exact mechanisms that lead to onset of bipolar disorders are still not fully understood. This further complicates a search for effective treatments.

\section{Treatment Strategies}

Treatment generally begins with the goal of bringing a patient with mania, or disabling hypomania, or depression to symptomatic recovery and stable mood. Upon stabilization, maintenance treatment (both drug and nondrug options) aims to maintain euthymia (a nondepressed, reasonably positive mood), reduce any subthreshold symptoms (milder but still clinically significant symptoms), and prevent or delay relapse into full-blown episodes of mania and depression. Questions remain as to whether treatment effects differ by patient characteristics which may impact condition severity or treatment response. 
Drug treatments are used to reduce acute symptoms, maintain relatively symptom-free periods, and reduce risk of relapsing to acute episodes. Drug treatments approved by the Food and Drug Administration for bipolar treatment are summarized in Table 1. Lithium and many anticonvulsants are often also referred to as “mood stabilizers" based on their intended treatment effect rather than the drug's mechanism. Given the chronic, relapsing/remitting course of bipolar disorder and the need for maintenance treatment in many patients, drugs begun for an acute mood episode (including mania) are often carried forward as maintenance treatment.

Table 1. FDA-approved medications for bipolar disorder

\begin{tabular}{|c|c|c|c|c|c|c|}
\hline Drug Type & $\begin{array}{c}\text { Generic Name } \\
\text { First Date Approved }\end{array}$ & $\begin{array}{c}\text { FDA - Listed } \\
\text { Trade Name } \\
\text { (Pharmaceutical } \\
\text { Co.) }\end{array}$ & Manic & $\begin{array}{c}\text { Mixed } \\
\text { (Manial } \\
\text { Depression) }\end{array}$ & $\begin{array}{c}\text { Mainte- } \\
\text { nance }\end{array}$ & Depression \\
\hline Salts & $\begin{array}{l}\text { Lithium } \\
1970\end{array}$ & & $x$ & & $\mathrm{X}$ & \\
\hline \multirow[t]{9}{*}{$\begin{array}{l}\text { Atypical } \\
\text { Antipsychotics }\end{array}$} & $\begin{array}{l}\text { Aripiprazole } \\
2004\end{array}$ & Abilify (Otsuka) & $X$ & $\mathrm{X}$ & $\mathrm{X}$ & \\
\hline & $\begin{array}{l}\text { Asenapine } \\
2015\end{array}$ & $\begin{array}{l}\text { Saphris (Organon } \\
\text { Sub Merck) }\end{array}$ & $X$ & $X$ & & \\
\hline & $\begin{array}{l}\text { Cariprazine } \\
2015\end{array}$ & Vraylar (Forest & & & & \\
\hline & $\begin{array}{l}\text { Lurasidone } \\
2013\end{array}$ & $\begin{array}{l}\text { Latuda (Sunovion } \\
\text { Pharms) }\end{array}$ & & & & $\mathrm{X}$ \\
\hline & $\begin{array}{l}\text { Olanzapine* } \\
2000\end{array}$ & Zyprexa (Lilly) & $x$ & $\mathrm{X}$ & $\mathrm{X}$ & \\
\hline & $\begin{array}{l}\text { Olanzapine/fluoxetine } \\
\text { combination* } \\
2012\end{array}$ & Symbyax (Lilly) & & & & $x$ \\
\hline & $\begin{array}{l}\text { Quetiapine } \\
2004\end{array}$ & $\begin{array}{l}\text { Seroquel } \\
\text { (AstraZeneca) }\end{array}$ & $X$ & & & $x$ \\
\hline & $\begin{array}{l}\text { Risperidone } \\
2003\end{array}$ & $\begin{array}{l}\text { Risperdal } \\
\text { (Janssen Pharm) }\end{array}$ & $x$ & $\mathrm{X}$ & & \\
\hline & $\begin{array}{l}\text { Ziprasidone } \\
2004\end{array}$ & Geodon (Pfizer) & $\mathrm{X}$ & $\mathrm{X}$ & & \\
\hline \multirow[t]{4}{*}{ Anticonvulsants } & $\begin{array}{l}\text { Carbamazepine* } \\
2004\end{array}$ & $\begin{array}{l}\text { Carbetrol (Shire), } \\
\text { Epitol (TEVA), } \\
\text { Equetro (Validus } \\
\text { Pharms), Tegretol } \\
\text { (Novartis), Teril } \\
\text { (Taro) }\end{array}$ & $x$ & $\mathrm{X}$ & & \\
\hline & $\begin{array}{l}\text { Lamotrigine* } \\
2003\end{array}$ & $\begin{array}{l}\text { Lamictal } \\
\text { (GlaxoSmithKline) }\end{array}$ & & & $\mathrm{X}$ & \\
\hline & $\begin{array}{l}\text { Divalproex sodium* } \\
\text { or valproate } \\
1995\end{array}$ & $\begin{array}{l}\text { Depakote } \\
\text { (ABBVIE) }\end{array}$ & $x$ & & & \\
\hline & $\begin{array}{l}\text { Lamotrigine }^{*} \\
2003\end{array}$ & $\begin{array}{l}\text { Lamictal } \\
\text { (GlaxoSmithKline) }\end{array}$ & & & $\mathrm{X}$ & \\
\hline
\end{tabular}

${ }^{*}$ Generic forms are available. FDA=Food and Drug Administration

Pharmacologic treatment is challenging. Some drugs that alleviate depression may increase the risk of mania, hypomania, or rapid cycling (four or more episodes in 12 months), while some drugs that alleviate acute mania may increase the risk of rebound depressive episodes. Based on changes in the DSM-5 criteria, in individuals with no prior bipolar disorder diagnosis, drug treatment induced manic and hypomanic episodes that last longer than expected pharmacological effects are now considered "true" episodes and count towards a bipolar 
disorder diagnosis. All approved drugs have boxed warnings indicating serious or lifethreatening risks.

Nonpharmacologic or psychotherapeutic techniques are applied to enhance medication adherence, reduce episode relapse (maintenance), and improve social and occupational functioning, which are often impaired during and after acute bipolar episodes. The majority of bipolar disorder-specific psychotherapeutic approaches have been studied in the last 15 years. These psychosocial approaches range from psychoeducational, cognitive behavioral, and family-focused, to interpersonal social rhythm therapy, and are administered both individually and in groups. Most psychotherapeutic approaches focus the treatment for individuals currently in the remission state of bipolar illness and often specifically exclude individuals currently in acute manic episodes.

Other nondrug treatment forms are based on physical approaches. They range widely from electroconvulsive therapy to treatments for circadian rhythms, such as light boxes, to acupuncture.

\section{Scope and Key Questions}

Several systematic reviews have assessed the effects of bipolar disorder treatment. Available reviews, however, do not incorporate the broad range of interventions (pharmacologic, psychosocial, other nondrug treatments) or necessarily target guideline developers with the specific intention of improving the treatment of bipolar disorder. This review provides a comprehensive up-to-date synthesis of the evidence on the effects of bipolar disorder treatments. 


\section{Key Questions}

Key Question 1: What are the efficacy and comparative effectiveness of pharmacologic and nonpharmacologic treatments for adults with bipolar disorder?

a. How do pharmacologic treatments (monotherapy or combination therapies) affect patient centered outcomes when compared with placebo?

b. How do pharmacologic treatments (monotherapy or combination therapies) affect patient centered outcomes when compared with other active pharmacologic treatment?

c. How do behavioral health treatments (psychotherapy, psychosocial interventions) affect patient centered outcomes when compared with usual care?

d. How do behavioral health treatments (psychotherapy, psychosocial interventions, chronotherapy) affect patient centered outcomes when compared with other active treatment?

e. How do somatic treatments (electroconvulsive therapy (ECT), transcranial magnetic stimulation (TMS) affect patient-centered outcomes when compared with other active treatment?

f. How do comprehensive programs affect patient centered outcomes when compared with usual care?

Key Question 2: What are the harms from pharmacologic and nonpharmacologic treatments for adults with bipolar disorder?

a. What are the harms from pharmacologic treatments?

b. What are the harms from behavioral health treatments?

c. What are the harms from somatic treatments?

d. What are the harms from comprehensive programs? 
Key Question 3: What is the effectiveness of treatments to reduce the metabolic change (metabolic syndrome, glucose dysregulation, weight gain) side effects of first line pharmacologic treatments?

Key Question 4: Which patient characteristics predict the effectiveness and harms of pharmacologic and nonpharmacologic treatments for people with bipolar disorder, including disease-specific characteristics such as bipolar type, state severity, pediatric onset, new onset, treatment resistant, types of depression, and other comorbidities, and patient characteristics such as substance use, other psychiatric comorbidities, medical comorbidities, age, sex, race/ethnicity, socioeconomic status?

\section{PICOTS}

The Key Questions are further described with the populations, interventions, comparators, outcomes, timing, and settings (PICOTS) noted in Table 2.

Table 2. PICOTS

\begin{tabular}{|l|l|l|}
\hline PICOT & \multicolumn{1}{|c|}{ Included } & \multicolumn{1}{c|}{ Excluded } \\
\hline Population & $\begin{array}{l}\text { Adults, 18+ years old, with any bipolar disorder. } \\
\text { Includes pregnant women }\end{array}$ & $\begin{array}{l}\text { Pediatric patients with bipolar } \\
\text { disorder } \\
\text { Studies with samples of greater than } \\
\end{array}$ \\
& & $\begin{array}{l}\text { 25\% identified as schizoaffective } \\
\text { disorder with bipolar -type } \\
\text { symptoms. Schizoaffective disorder } \\
\text { is distinguished by longer periods of } \\
\text { psychotic symptoms than bipolar } \\
\text { disorder. }\end{array}$ \\
& & $\begin{array}{l}\text { Major affective disorder not } \\
\text { specifying unipolar depression } \\
\text { versus bipolar disorder }\end{array}$ \\
\hline
\end{tabular}




\begin{tabular}{|c|c|c|}
\hline PICOT & Included & Excluded \\
\hline Intervention & $\begin{array}{l}\text { Drug treatment } \\
\text { Manic episodes - lithium, anticonvulsants, } \\
\text { antipsychotics } \\
\text { Depressive or mixed episodes - lithium, } \\
\text { anticonvulsants, antipsychotics, antidepressants } \\
\text { Maintenance state - lithium, anticonvulsants, } \\
\text { antipsychotics, antidepressants } \\
\text { Combination therapy - } \\
\text { Two or more drugs begun simultaneously with } \\
\text { similar therapeutic goal; } \\
\text { Augmentation with a second drug to boost } \\
\text { response when patient's symptoms have only } \\
\text { partially remitted } \\
\text { Two drugs with different goals (such as acute } \\
\text { manic treatment added to maintenance drugs) } \\
\text { Psychosocial treatment } \\
\text { Individual or group psychotherapy } \\
\text { Cognitive behavioral therapy (CBT) } \\
\text { Family-focused therapy } \\
\text { Interpersonal and social rhythm therapy } \\
\text { Psychoeducation } \\
\text { Chronotherapy } \\
\text { Somatic treatment } \\
\text { Electroconvulsive therapy (ECT) } \\
\text { Transcranial magnetic stimulation (TMS) } \\
\text { Comprehensive programs - multicomponent } \\
\text { programs incorporating pharmacological, } \\
\text { psychological, and social components in an } \\
\text { integrated fashion. } \\
\text { Interventions to reduce side effects of medications } \\
\text { given for prolonged periods (metabolic syndrome, } \\
\text { glucose dysregulation, weight gain) (such as } \\
\text { verapamil, metformin) }\end{array}$ & $\begin{array}{l}\text { Over-the-counter botanicals, } \\
\text { nutritional supplements, } \\
\text { dietary approaches (including omega } \\
\text { 3) } \\
\text { Programs designed primarily as } \\
\text { improving drug adherence. }\end{array}$ \\
\hline $\begin{array}{l}\text { Comparator } \\
\text { groups }\end{array}$ & $\begin{array}{l}\text { Drug treatment - placebo, active comparator } \\
\text { Psychosocial or Somatic treatment - } \\
\text { placebo/sham, usual care, or active comparator } \\
\text { Comprehensive treatment - placebo or active } \\
\text { comparator } \\
\text { Interventions - placebo, waitlist, active } \\
\text { comparator, usual care }\end{array}$ & \\
\hline
\end{tabular}




\begin{tabular}{|c|c|c|}
\hline PICOT & Included & Excluded \\
\hline Outcomes & $\begin{array}{l}\text { Final health or patient-centered outcomes: } \\
\text { Reduction of episodes outcomes } \\
\text { Remission/Prevention of episodes } \\
\text { Increased time between episodes/Time to } \\
\text { remission } \\
\text { Reduced hospitalization } \\
\text { Reduction in self-harm } \\
\text { Reduction in suicide } \\
\text { Reduction in suicidal thoughts or self-harming } \\
\text { behaviors } \\
\text { Improved function } \\
\text { Improved social and occupational functioning } \\
\text { Change in disability } \\
\text { Health related quality of life } \\
\text { Severity reduction } \\
\text { Remission of co-occurring substance use disorder } \\
\text { Worsening of condition } \\
\text { Intermediate outcomes } \\
\text { Treatment response } \\
\text { Improved treatment adherence } \\
\text { Reduction of first line treatment side effects } \\
\text { (metabolic syndrome, glucose dysregulation, } \\
\text { weight gain) } \\
\text { Adverse effects of interventions } \\
\text { Switching states } \\
\text { Increase metabolic syndrome, glucose } \\
\text { dysregulations, weight gain } \\
\text { Reported adverse effects }\end{array}$ & $\begin{array}{l}\text { Time to drug effect } \\
\text { Drug tolerance studies; phase II } \\
\text { studies } \\
\text { All other intermediate outcomes, } \\
\text { such changes in physiologic } \\
\text { conditions }\end{array}$ \\
\hline Timing & $\begin{array}{l}\text { Acute mania/mixed episode: at least } 3 \text { weeks } \\
\text { treatment duration plus post-treatment followup (if } \\
\text { any) } \\
\text { Acute depression: at least } 3 \text { months treatment } \\
\text { duration plus post-treatment followup (if any) } \\
\text { Maintenance: at least } 6 \text { months treatment duration } \\
\text { plus post-treatment followup (if any) }\end{array}$ & \\
\hline Setting & $\begin{array}{l}\text { Inpatient and outpatient for mania or mixed } \\
\text { episodes, depression. Outpatient for depression or } \\
\text { maintenance (inpatient start allowed). }\end{array}$ & \\
\hline
\end{tabular}

PICOTS=Population, interventions, comparators, outcomes, timing, settings 


\section{Analytic Framework}

Figure 1. Analytic framework for treatment for bipolar disorder

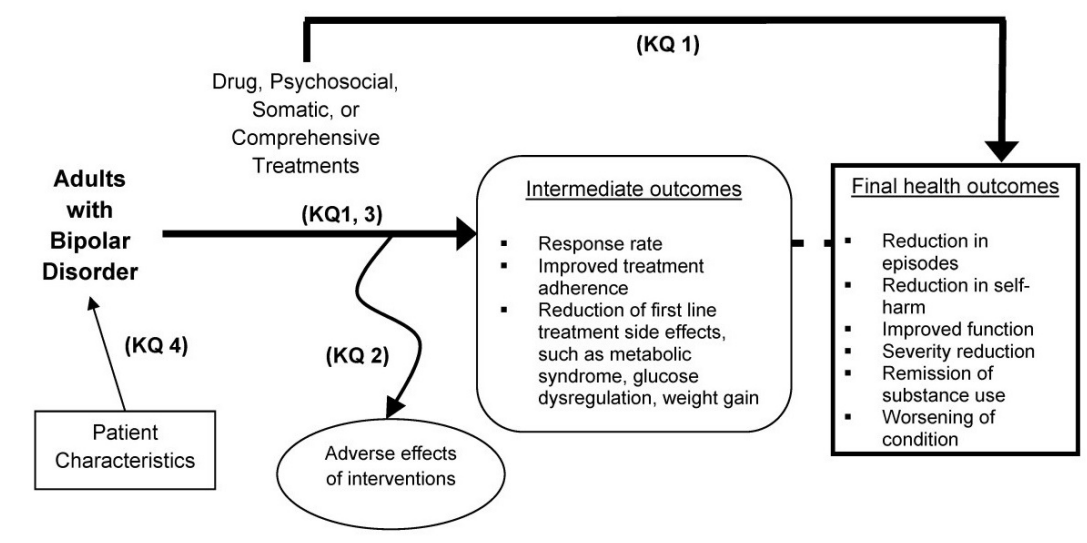

$\mathrm{KQ}=$ Key Question

\section{Report Organization}

As indicated by the structure of our Key Questions, we had originally planned to provide results for bipolar disorder subpopulations important to clinicians. However, we found that such detail was not forthcoming. Thus, rather than organizing the report by Key Question, this report presents the benefit and harms results organized by type of treatment. Chapter 4 gives results for drug treatments for mania. The chapter presents benefits and harms for antipsychotics, each drug first as monotherapy, then, if present, in combination with mood stabilizers, followed by results for mood stabilizers alone, and then other drugs. Chapter 5 presents benefits and harms for drug treatments for depression, Chapter 6 for maintenance treatment, and Chapter 7 for psychosocial therapies and other nondrug treatments. Since we were unable to provide information on subpopulations, we provide tables with details of the treated populations for each intervention. Each of the results chapters also includes a short discussion section for issues strictly related to the chapters' results. An overall discussion of themes cutting across all the chapters is provided in Chapter 8. 


\section{Chapter 2. Methods}

The methods for this Comparative Effectiveness Review (CER) follow the methods suggested in the Agency for Healthcare Research and Quality (AHRQ) Methods Guide for Effectiveness and Comparative Effectiveness Reviews (available at http://www.effectivehealthcare.ahrq.gov/methodsguide.cfm ); certain methods map to the Preferred Reporting Items for Systematic Reviews and Meta-Analysis (PRISMA) checklist. ${ }^{10}$ This section summarized the methods used.

\section{Topic Refinement and Review Protocol}

This report topic and preliminary Key Questions arose through a public process. Initially a panel of key informants, involving psychiatrists, psychologists, researchers, consumer advocates, and consumers, gave input on the Key Questions and population, interventions, comparators, outcomes, and timing (PICOT) to be examined. Key Questions, PICOT, and the analytic framework were posted for public comment from December 19, 2013 to January 10, 2014. In response to comments provided, we made several changes. We then drafted a protocol for the CER and recruited a technical expert panel to provide high-level content and methodological expertise feedback on the review protocol. The protocol was posted June 23, 2014 at https://effectivehealthcare.ahrq.gov/search-for-guides-reviews-andreports/?pageaction=displayproduct\&productid=1926.

\section{Literature Search Strategy}

We searched Ovid Medline, Ovid PsycInfo, Ovid Embase, and the Cochrane Central Register of Controlled Trials (CENTRAL) to identify previous randomized controlled trials and prospective cohort studies published and indexed in bibliographic databases. Our search strategy, which appears in Appendix A, included relevant medical subject headings and natural language terms for the concept of bipolar disorder. This concept was combined with filters to select randomized controlled trials (RCTs), observational studies, and systematic reviews. Dates for the search algorithm were 1994 to May 2017.We anticipated that older, established treatments would be covered by prior reviews, and we supplemented our searches with backward citation searches of relevant systematic reviews.

We conducted additional grey literature searching to identify relevant completed and ongoing studies. Relevant grey literature resources include trial registries and Food and Drug Administration databases. We searched ClinicalTrials.gov and the International Controlled Trials Registry Platform (ICTRP) for ongoing studies. We also reviewed Scientific Information Packets (SIPs) sent by manufacturers of relevant interventions. Grey literature search results were used to identify studies, outcomes, and analyses not reported in the published literature to assess publication and reporting bias and inform future research needs.

Studies were included in the review based on the PICOTS framework outlined in Table 2 and the study-specific inclusion criteria described in Table 3. 
Table 3. Study inclusion criteria

\begin{tabular}{|c|c|}
\hline Category & Criteria for Inclusion \\
\hline Study Enrollment & $\begin{array}{l}\text { Studies that enroll adults with any form of bipolar disorder (bipolar I, bipolar II, } \\
\text { bipolar otherwise specified, bipolar not otherwise specified, rapid cycling) using } \\
\text { any diagnostic process. } \\
\text { Studies that enroll bipolar disorder patients along with other patients with DSM- } \\
\text { V diagnoses were included if the patients with bipolar disorder are analyzed } \\
\text { separately. }\end{array}$ \\
\hline Study Design and Quality & $\begin{array}{l}\text { RCTs, nonrandomized controlled trials, and prospective cohort studies for each } \\
\text { population and treatment option. } \\
\text { Studies must have at least } 10 \text { participants per arm at the first relevant outcome } \\
\text { period. Except for studies reporting time to relapse as a primary outcome } \\
\text { (generally maintenance studies), Studies for acute mania or depression } \\
\text { treatments with greater than } 50 \% \text { attrition were excluded for fatally high risk of } \\
\text { bias. Follow-on studies of those excluded RCTs were also excluded. Studies } \\
\text { with greater than } 50 \% \text { attrition in the treatment arm were excluded if the control } \\
\text { arm did not incorporate some form of placebo or attention control; the lack of } \\
\text { such comparators creates a situation where the control arm would not be } \\
\text { subject to forces prompting a participant to withdraw from a study. Studies for } \\
\text { maintenance treatments with greater than } 50 \% \text { attrition were retained only if } \\
\text { time to relapse outcomes were available, and then only those outcomes and } \\
\text { withdrawal information were includable. } \\
\text { Prospective cohort studies must include a comparator and appropriate methods } \\
\text { to correct for selection bias. Observational studies that do not adequately report } \\
\text { study information to allow the abstraction of time sequences for treatment and } \\
\text { followup duration or have indeterminable numerators and denominators for } \\
\text { outcomes and adverse event rates were excluded at the abstraction phase. }\end{array}$ \\
\hline Time of Publication & $\begin{array}{l}1970 \text { and forward for trials of pharmacologic and somatic treatments. Lithium } \\
\text { was FDA approved in 1970. } 1994 \text { and forward for all other literature. This } \\
\text { corresponds with the period during which systematic reviews and evidence- } \\
\text { based research approaches have been applied to behavioral health. }\end{array}$ \\
\hline Publication type & Published in peer reviewed journals \\
\hline Language of Publication & English \\
\hline
\end{tabular}

DSM-5=Diagnostic and Statistical Manual for Mental Disorders, $5^{\text {th }}$ Edition; FDA=Food and Drug Administration; RCT=randomized controlled trial

We reviewed bibliographic database search results for studies relevant to our PICOTS framework and study-specific criteria. All studies identified at title and abstract as relevant by either of two independent investigator underwent full-text screening. Two investigators independently performed full-text screening to determine if inclusion criteria were met. Differences in screening decisions were resolved by consultation between investigators, and, if necessary, consultation with a third investigator.

\section{Risk of Bias Assessment of Individual Studies}

Risk of bias of eligible studies was assessed by two independent investigators using instruments specific to each study design. For RCTs, questionnaires developed from the Cochrane Risk of Bias ${ }^{11}$ tool were used. We developed an instrument for assessing risk of bias for observational studies based on the RTI Observational Studies Risk of Bias and Precision Item Bank $^{12}$ (Appendix B). We selected items most relevant in assessing risk of bias for this topic, including participant selection, attrition/incomplete outcome data, ascertainment of group assignment, and appropriateness of analytic methods. Study power was assessed in 'other sources of bias'in studies with data that were not eligible for pooling. For psychosocial intervention, the presence of treatment fidelity, that is, treatment definition and implementation, was also evaluated. Overall summary risk of bias assessments for each study were classified as 
low, moderate, or high based upon the collective risk of bias inherent in each domain and confidence that the results were believable given the study's limitations. When the two investigators disagreed, a third party was consulted to reconcile the summary judgment.

\section{Data Extraction}

For studies meeting inclusion criteria, one investigator abstracted relevant data into extraction forms created in Excel. Evidence tables were reviewed and verified for accuracy by a second investigator. Data fields included author, year of publication, setting, subject inclusion and exclusion criteria, intervention and control characteristics (intervention components, timing, frequency, duration), followup duration, participant baseline demographics, comorbidities; method of diagnosis, enrollment, and severity, descriptions and results of primary outcomes, adverse effects, study withdrawals, and study funding source.

For outcomes, only overall scale scores were reported for all measurement scales; subscales or individual items from scales were not abstracted. Abstracted outcomes included:

- Responders and/or remitters (for acute states) number and/or time to relapse (for maintenance), including definitions used in the studies,

- Symptoms scales; only one scale per state per study, following a "most reported" hierarchy,

- Global functioning (including social performance and quality of life for psychosocial studies),

- Utilization, such as emergency department use,

- Change in self-harm behaviors, including suicidality,

- Withdrawals; overall, due to lack of effect, and due to side effects,

- Serious adverse events; rates of extrapyramidal symptoms, switching, and weight gain of $>7$ percent.

Adverse events were treatment emergent, not treatment-related events. Harms were chosen based on an informal prioritization process with the help of the Technical Expert Panel (TEP). We focused on patient-centered harms and not on those that were already well-established.

For maintenance studies reporting time to relapse as the primary outcome but with greater than 50 percent attrition, only summary measures of time to relapse, overall withdrawal, withdrawal due to adverse events and adverse events were abstracted. We did not abstract symptom scales due to loss of participants over time. Time to relapse for any mood episode was primary unless the study was designed for a specific episode type; for example, the primary outcome of time to next depressive episode for bipolar II patients stabilized from depression.

As a courtesy to readers, we also abstracted limited information on studies excluded for greater than 50 percent attrition: study design, enrollment, intervention, and comparison (available in Appendix D).

\section{Data Synthesis}

We summarized the results into evidence tables and synthesized evidence for each unique population, comparison, and outcome combination. We emphasized patient-centered outcomes in the evidence synthesis. Results are organized by bipolar type and state (such as acute mania, acute depression, or euthymia). Where available, results by population subgroups were also provided. We used statistical differences between groups to assess effects. For outcomes with well-established minimum important differences (MIDs), we used the MID to aid interpretation. 
Appendix C provides a list of outcomes used in the available literatures, with associated MIDs where available.

Decisions for pooling were based on the homogeneity of study populations using inclusion criteria, specific interventions, and the ability to treat outcome measures as similar. When pooling was possible, we conducted meta-analyses using the random effects modeling approach. Continuous outcomes were summarized with precision-weighted mean differences (WMD) and/or standardized mean differences (SMD) and 95 percent confidence intervals (CIs). In our context, these were generally difference in difference estimates from each study. If a study did not report a standard error for the difference in difference estimate, we calculated it from a Pvalue or $\mathrm{CI}$ and the appropriate degrees of freedom. If neither a CI nor an exact P-value was given but an upper bound for the P-value was, e.g., $<0.05$, we used that to calculate an upper bound of the standard error. If the degrees of freedom of the relevant t-distribution was not given, we attempted to back it out of the study based upon the statistical methods that were used as long as we could confidently conclude that it was greater than 25 . Binary outcomes were summarized with precision-weighted log odds ratios (OR) and 95 percent CIs.

We used the restricted maximum likelihood estimator (REML) of the heterogeneity variance because, although simulation studies have shown it to suffer from negative bias ${ }^{13}$, it has generally performed comparatively well with regards to mean-square error ${ }^{14}$. We also used the Knapp-Hartung adjustment in order to avoid the potentially high inflation of the type-I error rate that can arise when dealing with small numbers of even moderately heterogeneous studies. ${ }^{15,16}$ We chose not to perform meta-analyses when only two studies were available to pool as, in this context, application of the Knapp-Hartung adjustment can diminish power to trivial levels and standard approaches can easily suffer from extreme inflation of type-I error. ${ }^{17}$

As a sensitivity analysis, we also performed all meta-analyses using fixed-effect models. These results are charitably interpreted as providing an estimate of the true average effect among completed trials and are presented along with the results derived from analyses using randomeffect models. ${ }^{18}$ However, we base our main conclusions on the random-effects set of results. All analyses were performed with $\mathrm{R}$ software ${ }^{19}$, using the metaphor package. ${ }^{18}$

We assessed the clinical and methodological heterogeneity to determine appropriateness of pooling data. ${ }^{20}$ When pooling was not appropriate due to lack of comparable studies or heterogeneity, qualitative synthesis was conducted.

Studies were grouped by treatment, bipolar type and/or bipolar state. Phases were grouped as: (1) acute mania or hypomania, including mixed, (2) acute depression, (3) any acute state (often for psychosocial maintenance studies), (4) euthymic or subsyndromal (generally for maintenance studies), and (5) nonspecific, that is, either euthymic, acute in any episode, or posthospitalization (these studies stated essentially any patient with bipolar disorder except acute mania). For drug studies treating patients for residual symptoms, patients were classified as nonresponders to standard treatment (usually noted in adjunctive drug studies). Studies were categorized as maintenance studies if the study inclusion criteria did not specify an acute episode at study entry.

Study outcomes were grouped by treatment duration or followup period. For acute mania treatment, outcomes were grouped by 3-4 weeks and then final measurement (generally 6 to 12 weeks) if available. Depression treatment studies are reported at 3 months and final endpoint. Maintenance study outcomes are reported at 6 months, 8-12 months, and "prolonged followup" of the final endpoint. 
Comparators for psychosocial studies were grouped as inactive (usual care or standardized care) or active (active head to head comparisons of psychosocial therapies including supportive therapy).

We conducted several sensitivity analyses where possible. In forest plots, outcomes in studies assessed as having a high risk of bias, or low to moderate risk of bias but at least 40 percent attrition, were presented in grey scale.

\section{Strength of Evidence for Major Comparisons and Outcomes}

The overall strength of evidence for primary outcomes within each comparison were evaluated based on four required domains: (1) study limitations (risk of bias); (2) directness (a single, direct link between intervention and outcome); (3) consistency (similarity of effect direction and size); and (4) precision (degree of certainty around an estimate). ${ }^{21}$ A fifth domain, reporting bias, was assessed when strength of evidence based upon the first four domains is moderate or high. ${ }^{21}$ Based on study design and conduct, study limitations were rated as low, moderate, or high. Consistency was rated as consistent, inconsistent, or unknown/not applicable (e.g., single study). Directness was rated as either direct or indirect. Precision was rated as precise or imprecise. Assessing strength of evidence for studies with null findings is especially challenging because several domains are designed to address differences. Although it is important to assess the strength of evidence for null findings (i.e. intervention and comparison yielded results that were not statistically different from each other), it is difficult. It is hard to assess effect size when there is no effect in studies that test for superiority; how does one establish a level of precision that provides confidence of no effect? This is especially true when populations, interventions, and comparators are not consistent, as is the case with much of the nondrug literature. We also downgraded precision when there was considerable attrition that was addressed through last-observation carried forward methods. Due to the large number of comparisons with findings of no effect, we assessed strength of evidence and formulated results cautiously. Based on these factors, the overall evidence for each outcome was rated as: ${ }^{21}$

High: Very confident that estimate of effect lies close to true effect. Few or no deficiencies in body of evidence, findings believed to be stable.

Moderate: Moderately confidence that estimate of effect lies close to true effect. Some deficiencies in body of evidence; findings likely to be stable, but some doubt.

Low: Limited confidence that estimate of effect lies close to true effect; major or numerous deficiencies in body of evidence. Additional evidence necessary before concluding that findings are stable or that estimate of effect is close to true effect.

Insufficient: No evidence, unable to estimate an effect, or no confidence in estimate of effect. No evidence is available or the body of evidence precludes judgment.

We assessed strength of evidence for validated scales (such as the Beck Depression Inventory, Young Mania Rating Scale, Hamilton Depression Rating Scale, Clinical Global Improvement Scale) and commonly used items that examine improved function (such as the Functional Assessment Short Test). We did not assess strength of evidence for less commonly measured items such as increased time between episodes or hospitalizations. Attempted suicide and other self-harming behaviors were also not assessed for strength of evidence due to the difficulty of defining and measuring such behaviors. 


\section{Applicability}

Applicability of studies was determined according to the PICOTS framework. Bipolar research generally draws from highly defined populations, resulting in samples that are often drawn from subpopulations rather than the bipolar populations at large. Thus, the ability to infer generalizability can be compromised. Applicability also deals with transportability of evidence for the type of treatment - level of treatment, treatment fidelity, skills of treatment agent, setting (and measurement) — and its fit to a particular treatment setting. Study characteristics that may affect applicability include, but are not limited to, the population from which the study participants are enrolled, diagnostic assessment processes, narrow eligibility criteria, and patient and intervention characteristics different than those described by population studies of bipolar disorder. $^{22}$ These applicability issues are present in the synthesis frameworks and sensitivity analyses described in more detail in the data synthesis section.

\section{Peer Review and Public Commentary}

Experts in bipolar disorder and systematic reviews were invited to provide external peer review of this systematic review; AHRQ and an associate editor also provided comments. The draft report was posted on the AHRQ website for 4 weeks to elicit public comment. We addressed all reviewer comments, revised the text as appropriate, and documented all responses in a disposition of comments report made available within 3 months of the Agency posting the final systematic review on the Effective Health Care website. 


\section{Chapter 3. Search Results}

We identified 6060 unique citations (Figure 2) to May 2017 from bibliographic databases addressing drug, psychosocial, or other nondrug treatments for bipolar disorder (BD). Fifty-six articles were added through hand search. An initial title and abstract review excluded 4,971 articles that were not related to drug or nondrug treatments for patients with BD. Full texts of 1,145 articles were reviewed to determine final inclusion. Appendix D provides a list of articles excluded at full text.

Figure 2. Literature flow diagram

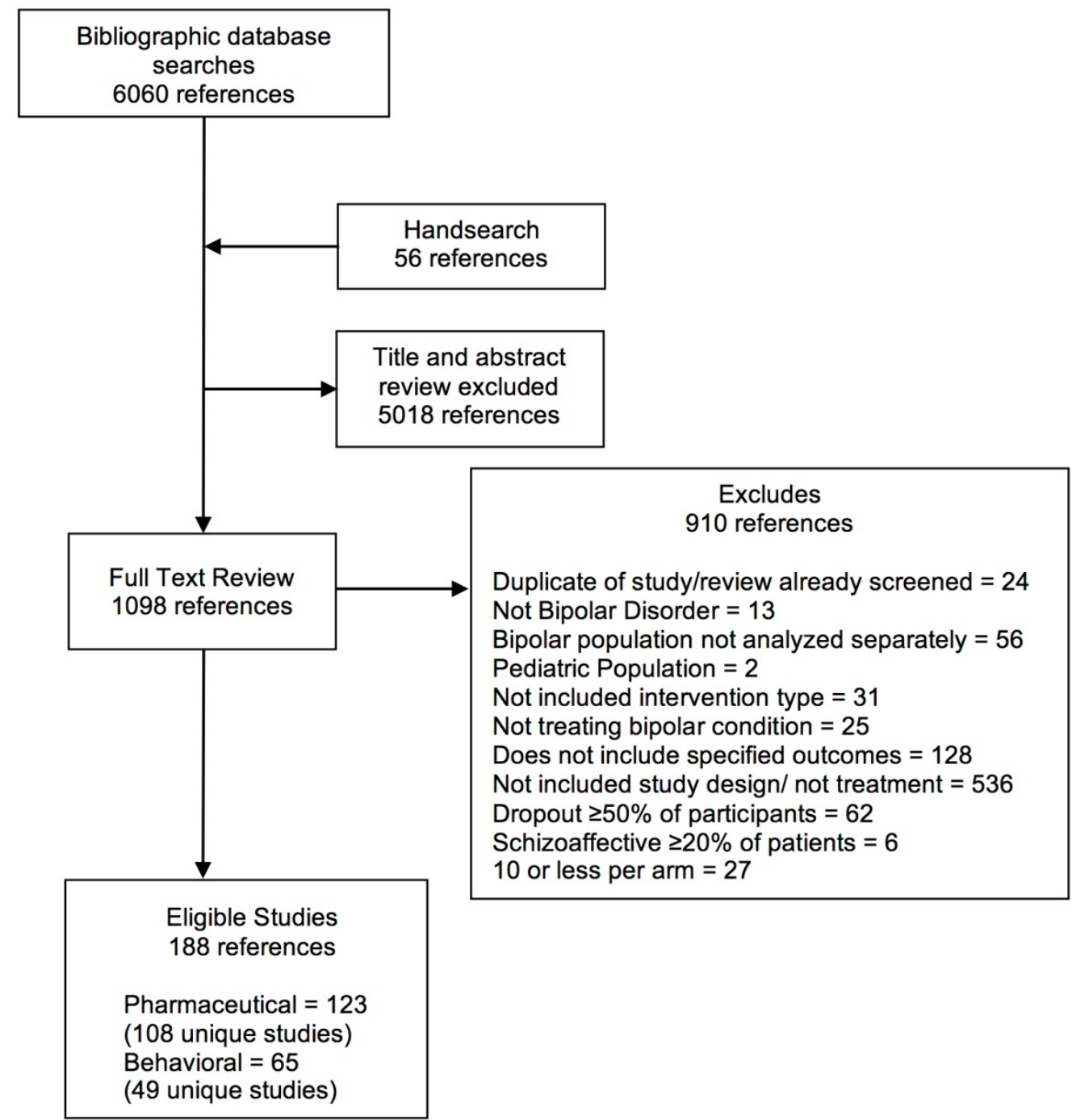

We identified 188 unique publications eligible for inclusion, including 123 studies of drug treatments and their associated harms, and 65 focused on psychosocial and other physical treatments. An additional 62 publications, 57 drug and 5 psychosocial, were excluded for attrition greater than 50 percent; brief abstracts of these studies are provided in Appendix D. 
Drug studies examined 28 separate drugs that were tested against 14 different types of comparators. These treatments and their comparators may have been single drug therapies or combination therapies of multiple drugs tested against either monotherapies or other multiple drug therapies. These then separated into 103 treatment comparisons, 59 of which had only one study contribute information. For Key Question 3, we found no studies meeting inclusion criteria that looked at treatments to reduce metabolic change side effects of drug treatments. Table 4 breaks the included studies down into each individual comparison for drug studies. Only five comparisons had four or more studies contributing. The populations tested in drug studies were overwhelmingly bipolar disorder I (BD-I) patients.

Table 4. Eligible unique studies by drug intervention and comparator

\begin{tabular}{|c|c|c|c|c|}
\hline Report Section & Treatment & Comparator & $\begin{array}{l}\text { Number } \\
\text { Unique } \\
\text { Studies }\end{array}$ & $\begin{array}{c}\text { Number } \\
\text { Unique } \\
\text { Studies } \\
\text { Not Solely } \\
\text { BD I } \\
\text { Population }\end{array}$ \\
\hline \multirow{29}{*}{$\begin{array}{l}\text { Antipsychotics } \\
\text { for Acute Mania } \\
\text { (Chapter 4) }\end{array}$} & \multirow[t]{2}{*}{ Aripiprazole } & Placebo & 3 & \\
\hline & & Haloperidol & 2 & \\
\hline & $\begin{array}{l}\text { Aripiprazole }+ \\
\text { Lithium/Divalproex /Valproate }\end{array}$ & $\begin{array}{l}\text { Placebo + Lithium/Divalproex } \\
\text { Nalproate }\end{array}$ & 2 & \\
\hline & Aripiprazole + Valproic Acid & Haloperidol + Valproic Acid & 1 & \\
\hline & \multirow[t]{2}{*}{ Asenapine } & Placebo & 3 & \\
\hline & & Olanzapine & 2 & \\
\hline & Asenapine + Lithium/Valproate & Placebo + Lithium/Valproate & 1 & \\
\hline & Cariprazine & Placebo & 3 & \\
\hline & Haloperidol & Placebo & 2 & \\
\hline & \multirow[t]{5}{*}{ Olanzapine } & Placebo & 5 & \\
\hline & & Haloperidol & 1 & \\
\hline & & Lithium & 3 & $1 \mathrm{NR}$ \\
\hline & & Risperidone & 1 & \\
\hline & & Divalproex/ Valproate & 4 & \\
\hline & Olanzapine + Lithium & Chlorpromazine + Lithium & 1 & \\
\hline & Olanzapine + carbamazepine & Placebo + Carbamazepine & 1 & \\
\hline & Olanzapine + Divalproex & No Placebo + Divalproex & 1 & \\
\hline & Olanzapine + Valproate & No Placebo + Valproate & 1 & \\
\hline & Olanzapine + Lithium/Valproate & Placebo + Lithium/Valproate & 1 & \\
\hline & \multirow[t]{3}{*}{ Quetiapine } & Placebo & 5 & $\begin{array}{l}1 \text { BD I, II, } \\
\text { NOS }\end{array}$ \\
\hline & & Haloperidol & 1 & \\
\hline & & Lithium & 2 & \\
\hline & $\begin{array}{l}\text { Quetiapine + Lithium or } \\
\text { Divalproex }\end{array}$ & Placebo + Lithium or Divalproex & 2 & \\
\hline & \multirow[t]{3}{*}{ Risperidone } & Placebo & 2 & \\
\hline & & Haloperidol & 2 & \\
\hline & & Lithium & 1 & \\
\hline & Risperidone + Mood Stabilizer & Placebo + Mood Stabilizer & 1 & \\
\hline & Ziprasidone & Placebo & 2 & \\
\hline & $\begin{array}{l}\text { Ziprasidone + Lithium or } \\
\text { Divalproex }\end{array}$ & Placebo + Lithium or Divalproex & 1 & \\
\hline \multirow{6}{*}{$\begin{array}{l}\text { Mood Stabilizer } \\
\text { for Acute Mania } \\
\text { (Chapter 4) }\end{array}$} & \multirow[t]{3}{*}{ Carbamazepine } & Placebo & 1 & \\
\hline & & Lithium & 2 & \\
\hline & & Valproate & 1 & \\
\hline & Divalproex & Placebo & 2 & \\
\hline & Lamotrigine & Lithium & 1 & \\
\hline & Lithium & Placebo & 2 & \\
\hline
\end{tabular}




\begin{tabular}{|c|c|c|c|c|}
\hline Report Section & Treatment & Comparator & $\begin{array}{l}\text { Number } \\
\text { Unique } \\
\text { Studies }\end{array}$ & $\begin{array}{c}\text { Number } \\
\text { Unique } \\
\text { Studies } \\
\text { Not Solely } \\
\text { BD I } \\
\text { Population }\end{array}$ \\
\hline & & Haloperidol & 1 & \\
\hline & & Valproate & 1 & \\
\hline & Olanzapine +Valproate & No placebo +Olanzapine & 1 & \\
\hline \multirow{17}{*}{$\begin{array}{l}\text { Other Drugs Not } \\
\text { FDA-Approved } \\
\text { for Acute Mania } \\
\text { (Chapter 4) }\end{array}$} & $\begin{array}{l}\text { Allopurinol + Lithium/Treatment } \\
\text { As Usual/Valproate }\end{array}$ & $\begin{array}{l}\text { Placebo + Lithium/Treatment As } \\
\text { Usual/Valproate }\end{array}$ & 3 & \\
\hline & $\begin{array}{l}\text { Allopurinol + Valproate/ } \\
\text { psychotropic medications }\end{array}$ & $\begin{array}{l}\text { Placebo + Valproate/ } \\
\text { psychotropic medications }\end{array}$ & 1 & \\
\hline & Celecoxib & Placebo & 1 & \\
\hline & \multirow[t]{2}{*}{ Dipyridamole + Lithium } & Placebo + Lithium & 1 & \\
\hline & & Allopurinol + Lithium & 1 & \\
\hline & Donepezil + Lithium & Placebo + Lithium & 1 & \\
\hline & Gabapentin + Lithium & Placebo + Lithium & 1 & \\
\hline & \multirow[t]{2}{*}{ Paliperdone ER } & Placebo & 2 & \\
\hline & & Quetiapine & 1 & \\
\hline & Paliperdone ER & Olanzapine & 1 & \\
\hline & $\begin{array}{l}\text { Paliperdone ER + Lithium or } \\
\text { Valproate }\end{array}$ & Placebo + Lithium or Valproate & 1 & \\
\hline & Tamoxifen & Placebo & 1 & \\
\hline & Endoxifen (tamoxifen derivative) & Divalproex & 1 & \\
\hline & \multirow[t]{2}{*}{ Topiramate } & Placebo & 1 & \\
\hline & & Lithium & 1 & \\
\hline & $\begin{array}{l}\text { Topiramate }+ \\
\text { Lithium/Divalproex }\end{array}$ & Placebo + Lithium/Divalproex & 1 & \\
\hline & Topiramate + Risperidone & Divalproex + Risperidone & 1 & \\
\hline \multirow[t]{7}{*}{$\begin{array}{l}\text { Treatment for } \\
\text { Acute } \\
\text { Depression } \\
\text { (Chapter 5) }\end{array}$} & $\begin{array}{l}\text { Antidepressant SSRI } \\
\text { (paroxetine or bupropion) + } \\
\text { Mood Stabilizer and/or } \\
\text { Psychosocial Interventions }\end{array}$ & $\begin{array}{l}\text { Placebo + Mood Stabilizer } \\
\text { and/or Psychosocial } \\
\text { Interventions }\end{array}$ & 1 & $1 \mathrm{BD}$ I, II \\
\hline & $\begin{array}{l}\text { Lamotrigine + Lithium or } \\
\text { Divalproex }\end{array}$ & Placebo + Lithium or Divalproex & 1 & $1 \mathrm{BD} I, \mathrm{II}$ \\
\hline & Memantine + Valproic Acid & Placebo + Valproic Acid & 1 & 1 BD II only \\
\hline & \multirow[t]{2}{*}{ Sertraline + Lithium } & Lithium & 1 & 1 BD II only \\
\hline & & Sertraline & 1 & 1 BD II only \\
\hline & Venlafaxine & Lithium & 2 & 2 BD II only \\
\hline & $\begin{array}{l}\text { Lithium + Optimal Personalized } \\
\text { Treatment }\end{array}$ & $\begin{array}{l}\text { No Placebo + Optimal } \\
\text { Personalized Treatment }\end{array}$ & 1 & $1 \mathrm{BD} \mathrm{I}, \mathrm{II}$ \\
\hline \multirow{14}{*}{$\begin{array}{l}\text { Maintenance } \\
\text { Treatments } \\
\text { (Chapter 6) }\end{array}$} & Aripiprazole (injectable) & Placebo & 1 & \\
\hline & Aripiprazole (oral) & Placebo & 1 & \\
\hline & Aripiprazole + Lamotrigine & Placebo + Lamotrigine & 1 & \\
\hline & $\begin{array}{l}\text { Aripiprazole }+ \\
\text { Lithium/Divalproex /Valproate }\end{array}$ & Placebo + Lithium/Valproate & 2 & \\
\hline & Carbamazepine & Lithium & 2 & $2 \mathrm{BD} I, \mathrm{II}$ \\
\hline & \multirow{2}{*}{ Fluoxetine } & Placebo & 1 & $1 \mathrm{BD}$ II \\
\hline & & Lithium & 1 & $1 \mathrm{BD}$ II \\
\hline & $\begin{array}{l}\text { Gabapentin + Lithium and/or } \\
\text { Divalproex and/or } \\
\text { Carbamazepine }\end{array}$ & $\begin{array}{l}\text { Placebo + Lithium and/or } \\
\text { Divalproex and/or } \\
\text { Carbamazepine }\end{array}$ & 1 & $1 \mathrm{BD}$ I, II \\
\hline & \multirow[t]{3}{*}{ Lamotrigine } & Placebo & 3 & $1 \mathrm{BD}$ I, II \\
\hline & & Mood stabilizer discontinuation & 1 & $\begin{array}{l}1 \text { BD I, II, } \\
\text { NOS }\end{array}$ \\
\hline & & Lithium & 2 & \\
\hline & Lithium & Placebo & 6 & \\
\hline & \multirow[t]{2}{*}{ Lithium + Valproate } & Lithium & 1 & \\
\hline & & Valproate & 1 & \\
\hline
\end{tabular}




\begin{tabular}{|c|c|c|c|c|}
\hline Report Section & Treatment & Comparator & $\begin{array}{l}\text { Number } \\
\text { Unique } \\
\text { Studies }\end{array}$ & $\begin{array}{c}\text { Number } \\
\text { Unique } \\
\text { Studies } \\
\text { Not Solely } \\
\text { BD I } \\
\text { Population }\end{array}$ \\
\hline & \multirow[t]{3}{*}{ Olanzapine } & Placebo & 2 & \\
\hline & & Divalproex & 1 & \\
\hline & & Lithium & 1 & \\
\hline & $\begin{array}{l}\text { Olanzapine + Lithium or } \\
\text { Valproate }\end{array}$ & Placebo + Lithium or Valproate & 1 & \\
\hline & Oxcarbazepine + Lithium & Placebo + Lithium & 1 & $1 \mathrm{BD} \mathrm{I}, \mathrm{II}$ \\
\hline & $\begin{array}{l}\text { Perphenazine + Lithium or } \\
\text { Carbamazepine or Valproate }\end{array}$ & $\begin{array}{l}\text { Placebo + Lithium or } \\
\text { Carbamazepine or Valproate }\end{array}$ & 1 & \\
\hline & \multirow[t]{2}{*}{ Quetiapine } & Placebo & 1 & \\
\hline & & Lithium & 1 & \\
\hline & $\begin{array}{l}\text { Quetiapine + Lithium or } \\
\text { Divalproex }\end{array}$ & Placebo + Lithium or Divalproex & 2 & \\
\hline & $\begin{array}{l}\text { Quetiapine + Adjunctive } \\
\text { Personalized Treatment }\end{array}$ & $\begin{array}{l}\text { Lithium + Adjunctive } \\
\text { Personalized Treatment }\end{array}$ & 1 & $1 \mathrm{BD} \mathrm{I}, \mathrm{II}$ \\
\hline & \multirow[t]{2}{*}{ Risperidone } & Placebo & 2 & \\
\hline & & Olanzapine & 1 & \\
\hline & \multirow{2}{*}{$\begin{array}{l}\text { Risperidone + Treatment as } \\
\text { Usual }\end{array}$} & Placebo + Treatment as Usual & 1 & $1 \mathrm{BD} \mathrm{I}, \mathrm{II}$ \\
\hline & & $\begin{array}{l}\text { No Placebo + Treatment as } \\
\text { Usual }\end{array}$ & 1 & $1 \mathrm{BD}$ I, II \\
\hline & Valproate & Lithium & 1 & \\
\hline & \multirow[t]{2}{*}{ Divalproex } & Placebo & 1 & \\
\hline & & Lithium & 2 & $1 \mathrm{BD} I, \mathrm{II}$ \\
\hline & Divalproex + Lithium & Placebo + Lithium & 1 & $1 \mathrm{BD} \mathrm{I}, \mathrm{II}$ \\
\hline & Valproic Acid + Aripiprazole & Lithium + Aripiprazole & 1 & \\
\hline & Venlafaxine & Lithium & 1 & $1 \mathrm{BD} \|$ \\
\hline & $\begin{array}{l}\text { Ziprasidone + Lithium or } \\
\text { Divalproex }\end{array}$ & Placebo + Lithium or Divalproex & 1 & \\
\hline
\end{tabular}

BD=bipolar disorder; ER=extended release; FDA=Food and Drug Administration; NR=not reported; NOS=not otherwise specified

Nondrug studies examined eight therapy approaches, seven of which were psychosocial intervention types: 1) psychoeducation, 2) cognitive behavioral therapy (CBT), 3) systematic/collaborative care, 4) family/partner interventions, 5) interpersonal and social rhythm therapy (IPSRT), 6) combination treatments (treatments that combined two or more psychosocial interventions, and 7) other psychosocial treatments (e.g. self-management via phone application support). A somatic therapy intervention, repetitive transcranial magnetic stimulation (rTMS), was examined as a nonpsychosocial, nondrug intervention. Each study represented a unique comparison due to differences in the structure of each intervention and control/comparator groups.

Table 5 provides the included studies for each individual comparison for nondrug studies. Comparators are categorized as inactive (e.g., usual care, no intervention) or active (e.g., a different psychosocial therapy, peer support) to indicate whether the studies were measuring the efficacy or effectiveness of the intervention. Since the nondrug studies were not as clearly delineated by BD states, the table further breaks down the studies by study enrollment criteria. For example, some studies may have required a particular BD state while other studies accepted BD participants in any state while still others may have excluded participants with a specific state. 
Table 5. Eligible unique studies by nondrug intervention and comparator

\begin{tabular}{|c|c|c|c|c|c|c|c|c|}
\hline cinguras & Comparator & Mania & Depression & Euthymic & $\begin{array}{l}\text { Any } \\
\text { State }\end{array}$ & $\begin{array}{l}\text { Non- } \\
\text { manic }\end{array}$ & $\begin{array}{l}\text { Current } \\
\text { Episode* }\end{array}$ & $\begin{array}{c}\text { Hypo } \\
- \\
\text { mani } \\
\text { c }\end{array}$ \\
\hline \multirow{2}{*}{ Psychoeducation } & Inactive & 0 & 0 & 8 & 2 & 0 & 0 & 0 \\
\hline & Active & 0 & 0 & 2 & 1 & 0 & 0 & 0 \\
\hline \multirow{2}{*}{ CBT } & Inactive & 0 & 0 & 6 & 3 & 0 & 0 & 0 \\
\hline & Active & 0 & 0 & 4 & 0 & 1 & 0 & 0 \\
\hline $\begin{array}{l}\text { Systematic or } \\
\text { Collaborative Care }\end{array}$ & Inactive & 0 & 0 & 2 & 5 & 0 & 0 & 0 \\
\hline \multirow{2}{*}{$\begin{array}{l}\text { Family or Partner } \\
\text { Interventions }\end{array}$} & Inactive & 0 & 0 & 1 & 0 & 0 & 1 & 0 \\
\hline & Active & 1 & 0 & 0 & 2 & 0 & 1 & 0 \\
\hline \multirow{2}{*}{ IPSRT } & Inactive & 0 & 0 & 0 & 0 & 0 & 1 & 0 \\
\hline & Active & 0 & 0 & 0 & 0 & 0 & 0 & 1 \\
\hline \multirow{2}{*}{$\begin{array}{l}\text { Combination } \\
\text { Therapy**}\end{array}$} & Inactive & 0 & 0 & 1 & 2 & 0 & 0 & 0 \\
\hline & Active & 0 & 0 & 1 & 1 & 0 & 0 & 0 \\
\hline Other Psychosocial & Active & 0 & 1 & 0 & 1 & 0 & 0 & 1 \\
\hline Somatic Therapy & Inactive & 0 & 1 & 0 & 0 & 0 & 0 & 0 \\
\hline
\end{tabular}

*Current episode means the enrolled participants may be currently experiencing any form of mania or depression, therefore not in a euthymic state. ${ }^{* *}$ Intervention is a combination of two or more psychosocial therapies.

$\mathrm{CBT}=$ cognitive behavioral therapy; IPSRT=interpersonal and social rhythm therapy 


\section{Chapter 4. Drug Treatments for Acute Mania}

We identified 71 publications of 67 unique studies for acute mania that examined 28 separate drugs tested against 14 different comparators. These treatments and their comparators may have been single drug therapies or combination therapies of multiple drugs tested against either placebo monotherapies or other multiple drug therapies. The 67 studies combined into 56 treatment comparisons, 35 of which had only one study contribute information. Only three comparisons had four or more studies contributing. An additional 54 studies were excluded due to attrition higher than 50 percent.

The high attrition studies (greater than 50\% were excluded because observed results among patients who complete a trial may not generalize to the entire patient population of interest if systematic differences between patients who do not complete the study and those who do (i.e., attrition is not random and/or likely due to bipolar disorder (BD) or treatment-relevant factors) occur. Moreover, if there are differential rates of attrition across study arms, or even similar rates but a different distribution of reasons for attrition, primary effect estimates and statistical inference can suffer from bias, potentially severe. The Last Observation Carried Forward (LOCF) method-by far the most common method used to address missing outcome data in the included studies - requires an assumption that the health-status of patients who dropped out of the trial would not have changed had future observations been recorded. This is a particularly heroic assumption in the context of withdrawal due to lack of efficacy or adverse events, not uncommon occurrences in the context of pharmaceutical treatment of patients with BD. ${ }^{23}$ When this assumption is inappropriate, use of LOCF methods can bias effect estimates. Moreover, estimates of standard errors will understate the true uncertainty surrounding effect estimates due to the added uncertainty of having to impute data, leading readers to believe the result is more precise than it actually is and potentially inflating the type-I error rate. ${ }^{24}$ This potential bias in the estimates of effect is even more problematic in studies with greater than 50 percent attrition that require imputing half or more of the data.

The results in this chapter for treatments for acute mania are organized by general drug category: antipsychotics, mood stabilizers, and drugs otherwise not specified. Within the antipsychotics section, results are presented by specific drug, then broken into single drugs compared to placebo or another drug, then, when appropriate, drug in combination with mood stabilizers compared to placebo or another drug. Likewise, the mood stabilizers and other drugs sections are presented first by single drug results followed by combination therapy results.

\section{Antipsychotic Drugs for Acute Mania}

\section{Key Points}

- Most antipsychotic drugs had few studies to contribute to findings. Studies for antipsychotics plus mood stabilizers were even more sparse and scattered.

- Low-strength evidence shows improved mania symptoms for all Food and Drug Administration (FDA)-approved antipsychotics, except aripiprazole, when compared to placebo for adults with bipolar I disorder (BD-I). For four of the antipsychotics we were able to provide a point estimate. However, most manic symptom improvements were of modest clinical significance, with values that were less than the minimally important difference (MID) but still large enough that a reasonable proportion of participants likely received a benefit. 
- Low-strength evidence showed no statistical differences in acute mania symptoms between olanzapine and divalproex/valproate.

- The ability to draw stronger conclusions for antipsychotics was hindered by high attrition rates.

- Evidence was insufficient to draw conclusions regarding antipsychotic drugs alone compared to placebo or antipsychotic drugs plus mood stabilizers compared with another drug for BD-I for the primary outcomes of interest (response, symptom scores, and function).

- When reported, all comparisons tended to show no differences between groups in serious adverse events. Participants using atypical antipsychotics, except quetiapine, reported experiencing more extrapyramidal symptoms compared to placebo. Participants using haloperidol reported experiencing more extrapyramidal symptoms compared to other antipsychotics. Participants using olanzapine reported experiencing more clinically significant weight gain.

\section{Eligible Studies for Antipsychotics}

Eight antipsychotic drugs were examined in 47 publications of 43 unique studies for BD patients experiencing acute manic events. Of these, seven are FDA approved for use in adults with BD experiencing mania: aripiprazole, asenapine, cariprazine, olanzapine, quetiapine, risperidone, and ziprasidone. An additional unpublished study for aripiprazole plus mood stabilizers was also included for metaanalysis. Haloperidol, an antipsychotic treatment available since the late 1950's and a World Health Organization listed essential medicine, was FDA approved in 1986 for schizophrenia (not for mania in adults with BD). All were examined as single drugs and all but cariprazine were also examined as a treatment combined with mood stabilizers. The populations tested were BD-I patients, which is understandable given the BD-I diagnosis requires history of just one episode of mania. Only one study (for quetiapine) included adults with bipolar II disorder (BD-II) or bipolar disorder not otherwise specificed (BD-NOS). No studies specifically assessed drug effectiveness in treatment of hypomania. The large majority of studies with usable outcomes were measured at 3 weeks duration.

Appendix E provides detailed evidence tables, summary risk of bias assessments, forest plots when appropriate, and assessments of strength of evidence for key comparisons and outcomes. A summary of findings with at least low-strength evidence for drug treatments for acute mania are provided in Table 6. Any intervention and comparison not listed in Table 6, or outcome not listed for an included intervention and comparison, was found to have an evidence base insufficient to draw conclusions.

Table 6. Summary of findings with at least low-strength evidence for antipsychotic drug treatments for acute mania

\begin{tabular}{|c|c|c|c|}
\hline Intervention & $\begin{array}{c}\text { \# Studies/ } \\
\text { Design } \\
\text { (n Analyzed) } \\
\text { Timing }\end{array}$ & Findings & $\begin{array}{l}\text { Strength of } \\
\text { Evidence }\end{array}$ \\
\hline $\begin{array}{l}\text { Asenapine } \\
\text { vs. placebo }\end{array}$ & $\begin{array}{c}3 \mathrm{RCT}^{25,2627} \\
(\mathrm{n}=936) \\
3 \text { weeks }\end{array}$ & $\begin{array}{l}\text { Response/Remission Rates: No difference } \\
\text { YMRS: Favors Asenapine, MD } 4.37 \text { (95\% CI } \\
\text { 1.27, 7.47; MID 6) } \\
\text { CGI-BP-S: Favors Asenapine, MD } 0.5 \text { (95\% } \\
\text { CI 0.29, 0.71; MID 1) } \\
\text { Withdrawal (AE, Lack of Efficacy, Overall): } \\
\text { No difference }\end{array}$ & $\begin{array}{l}\text { Low } \\
\text { (moderate study } \\
\text { limitations, } \\
\text { imprecise) }\end{array}$ \\
\hline
\end{tabular}




\begin{tabular}{|c|c|c|c|}
\hline Intervention & $\begin{array}{l}\text { \# Studies/ } \\
\text { Design } \\
\text { (n Analyzed) } \\
\text { Timing } \\
\end{array}$ & Findings & $\begin{array}{l}\text { Strength of } \\
\text { Evidence }\end{array}$ \\
\hline $\begin{array}{l}\text { Cariprazine } \\
\text { vs. placebo }\end{array}$ & $\begin{array}{c}3 \mathrm{RCT}^{28} 2930 \\
(\mathrm{n}=1,047) \\
3 \text { weeks }\end{array}$ & $\begin{array}{l}\text { Response Rate: Favors Cariprazine, OR } \\
2.14 \text { (95\% Cl 1.08, 4.23); NNT=5.6 } \\
\text { Remission Rate: Favors Cariprazine, } \\
\text { OR1.95 (95\% CI 1.45, 2.63); NNT= } 7 \\
\text { YMRS: Favors Cariprazine, MD } 5.38 \text { (95\% } \\
\mathrm{CI} 1.84,8.92 ; \text { MID 6) } \\
\text { CGI-BP-S: Favors Cariprazine, MD } 0.54 \\
\text { (95\% CI 0.35, 0.73; MID 1) } \\
\text { Withdrawal (AE, Lack of Efficacy, Overall): } \\
\text { No difference }\end{array}$ & $\begin{array}{l}\text { Low } \\
\text { (moderate study } \\
\text { limitations, } \\
\text { imprecise) }\end{array}$ \\
\hline \multirow[t]{2}{*}{$\begin{array}{l}\text { Olanzapine } \\
\text { vs. placebo }\end{array}$} & $\begin{array}{c}5 \mathrm{RCT}^{31} 3226 \\
3334 \\
(\mathrm{n}=1199) \\
3 \text { weeks }\end{array}$ & $\begin{array}{l}\text { Response Rate: Favors Olanzapine, OR } \\
1.99 \text { (95\% Cl 1.29, 3.08); NNT } 6 \\
\text { Remission Rate: Favors Olanzapine, OR } \\
1.75(95 \% \mathrm{Cl} 1.19,2.58) ; \text { NNT } 7.5 \\
\text { YMRS: Favors Olanzapine, MD } 4.9(95 \% \mathrm{Cl} \\
2.34,7.45 ; \text { MID 6) } \\
\text { Withdrawal (Lack of Efficacy, Overall): } \\
\text { Favors Olanzapine, MD } 0.42 \text { (95\% Cl } \\
0.29,0.61)\end{array}$ & $\begin{array}{l}\text { Low } \\
\text { (moderate study } \\
\text { limitations, } \\
\text { imprecise) }\end{array}$ \\
\hline & $\begin{array}{l}3 \mathrm{RCT}^{31} 3334 \\
(\mathrm{n}=611) \\
3 \text { weeks }\end{array}$ & CGI-BP-S: No difference & $\begin{array}{l}\text { Low } \\
\text { (moderate study } \\
\text { limitations, } \\
\text { imprecise) }\end{array}$ \\
\hline \multirow{3}{*}{$\begin{array}{l}\text { Quetiapine } \\
\text { vs. placebo }\end{array}$} & $\begin{array}{c}4 \mathrm{RCT}^{35} 3637 \\
38 \\
(\mathrm{n}=1,007) \\
3 \text { weeks }\end{array}$ & $\begin{array}{l}\text { Response Rate: Favors Quetiapine, OR } \\
2.07 \text { (95\% Cl 1.39, 3.09); NNT } 6.2 \\
\text { Withdrawal (Lack of Efficacy): Favors } \\
\text { Quetiapine, MD } 0.38(95 \% \mathrm{Cl} 0.23,0.63)\end{array}$ & $\begin{array}{l}\text { Low } \\
\text { (moderate study } \\
\text { limitations, } \\
\text { imprecise) }\end{array}$ \\
\hline & 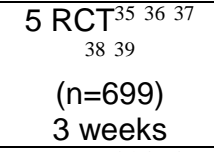 & $\begin{array}{l}\text { YMRS: Favors Quetiapine, MD } 4.92 \text { (95\% } \\
\text { CI 0.31, 9.53; MID 6) }\end{array}$ & $\begin{array}{l}\text { Low } \\
\text { (moderate study } \\
\text { limitations, } \\
\text { imprecise) }\end{array}$ \\
\hline & 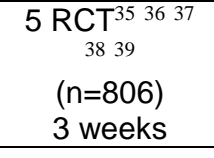 & $\begin{array}{l}\text { CGI-BP-S: Favors Quetiapine, MD } 0.54 \\
(95 \% \text { Cl 0.35, 0.74; MID 1) }\end{array}$ & $\begin{array}{l}\text { Low } \\
\text { (moderate study } \\
\text { limitations, } \\
\text { imprecise) }\end{array}$ \\
\hline $\begin{array}{l}\text { Risperidone } \\
\text { vs. placebo }\end{array}$ & $\begin{array}{c}2 \mathrm{RCT}^{40} 41 \\
(\mathrm{n}=584) \\
3 \text { weeks }\end{array}$ & $\begin{array}{l}\text { Response Rate, YMRS, and CGI: Favors } \\
\text { Risperidone (not pooled) }\end{array}$ & $\begin{array}{l}\text { Low } \\
\text { (moderate study } \\
\text { limitations, } \\
\text { imprecise) }\end{array}$ \\
\hline $\begin{array}{l}\text { Ziprasidone } \\
\text { vs. placebo }\end{array}$ & $\begin{array}{c}2 \mathrm{RCT}^{42,43} \\
(\mathrm{n}=402) \\
3 \text { weeks }\end{array}$ & $\begin{array}{l}\text { Response Rate, YMRS, and CGI: Favors } \\
\text { Ziprasidone (not pooled) }\end{array}$ & $\begin{array}{l}\text { Low } \\
\text { (moderate study } \\
\text { limitations, } \\
\text { imprecise) }\end{array}$ \\
\hline \multirow{3}{*}{$\begin{array}{l}\text { Olanzapine } \\
\text { vs. } \\
\text { Divalproex/ } \\
\text { Valproate }\end{array}$} & $\begin{array}{c}2 \text { RCTs }^{44} 33 \\
(n=635) \\
3 \text { weeks }\end{array}$ & Response and Remission: No difference & $\begin{array}{l}\text { Low } \\
\text { (moderate study } \\
\text { limitations, } \\
\text { imprecise) }\end{array}$ \\
\hline & $\begin{array}{c}3 \mathrm{RCTs}^{33,44,45} \\
(\mathrm{n}=750) \\
3 \text { weeks }\end{array}$ & YMRS: No difference & $\begin{array}{l}\text { Low } \\
\text { (moderate study } \\
\text { limitations, } \\
\text { imprecise) }\end{array}$ \\
\hline & $\begin{array}{c}3 \mathrm{RCTs}^{33,44,45} \\
(\mathrm{n}=578) \\
3 \text { weeks }\end{array}$ & CGI: No difference & $\begin{array}{l}\text { Low } \\
\text { (moderate study } \\
\text { limitations, } \\
\text { imprecise) }\end{array}$ \\
\hline
\end{tabular}




\begin{tabular}{|c|c|l|l|}
\hline Intervention & $\begin{array}{c}\text { \# Studies/ } \\
\text { Design } \\
\text { (n Analyzed) } \\
\text { Timing }\end{array}$ & Findings & \multicolumn{1}{c|}{$\begin{array}{c}\text { Strength of } \\
\text { Evidence }\end{array}$} \\
\hline & $\begin{array}{c}4 \text { RCTs } 33,44- \\
46 \\
(n=867) \\
3 \text { weeks }\end{array}$ & Withdrawals: No difference & $\begin{array}{l}\text { Low } \\
\text { (moderate study } \\
\text { limitations, } \\
\text { imprecise) }\end{array}$ \\
\hline
\end{tabular}

$\mathrm{AE}=$ adverse events; $\mathrm{CGI}=$ Clinical global impression; $\mathrm{CI}=$ confidence interval; $\mathrm{CGI}-\mathrm{BP}=\mathrm{Clinical}$ global impression scale, bipolar edition; $\mathrm{MD}=$ =mean difference; $\mathrm{MID}=$ minimally important difference; $\mathrm{n}=$ number; $\mathrm{NNT}=$ number needed to treat; $\mathrm{OR}=\mathrm{odds}$ ratio; RCT=randomized controlled trial; YMRS=Young mania rating scale

\section{Aripiprazole}

We identified four unique randomized controlled trials (RCTs) of aripiprazole, ${ }^{47-50}$ and two eligible publications reporting two unique RCTs of aripiprazole plus mood stabilizers for acute mania with at least 3 weeks followup. ${ }^{51,52}$ Three studies were assessed as moderate risk of bias and three were assessed as high. An additional 6 studies were excluded for attrition over 50 percent. ${ }^{53-58}$ All studies were funded by industry. Three studies compared aripiprazole to placebo $^{48-50}$ and two compared to haloperidol. ${ }^{47,48}$ Sample sizes ranged from 42 to 485 participants, and all participants were BD-I. One unpublished RCT was discoveredclinicaltrials.gov ID: NCT00046384—which compared aripiprazole with placebo, with a total of less than 60 patients. The trial was prematurely closed and allegedly did not produce results.

\section{Aripiprazole Alone}

Table 7 summarizes the population and major inclusion and exclusion criteria for each aripiprazole study for acute mania. Appendix E provides further detail.

Table 7. Population and inclusion criteria for studies of aripiprazole alone for acute mania

\begin{tabular}{|c|c|c|c|c|c|}
\hline $\begin{array}{l}\text { Author, Year } \\
\text { Single/Multisite } \\
\text { Location } \\
\text { Risk of Bias } \\
\text { (ROB) } \\
\text { PMID }\end{array}$ & $\begin{array}{l}\text { Intervention/ } \\
\text { Comparison }\end{array}$ & $\begin{array}{c}\text { BD Type; } \\
\text { Diagnostic } \\
\text { Criteria }\end{array}$ & Demographics & Current Episode & Key Exclusions \\
\hline $\begin{array}{l}\text { Kanba, 201449 } \\
\text { Multisite } \\
\text { Asia } \\
\text { High ROB } \\
22540407\end{array}$ & $\begin{array}{l}\text { I: } \\
\text { Aripiprazole } \\
\text { C: Placebo }\end{array}$ & $\begin{array}{l}\text { BD-I; } \\
\text { DSM-IV }\end{array}$ & $\begin{array}{l}\text { Mean Age } 38 \\
(18-75) \\
59 \% \text { Female } \\
\text { Race NR } \\
N=247\end{array}$ & $\begin{array}{l}\text { Manic/Mixed } \\
\text { episode; } \text { YMRS } \geq \\
20 ; \\
\text { Current episode }<4 \\
\text { weeks }\end{array}$ & $\begin{array}{l}\text { First Manic Episode } \\
\text { Schizoaffective } \\
\text { Neurological Disorders } \\
\text { Other Mental Health } \\
\text { Substance Abuse } \\
\text { Pregnant/Nursing }\end{array}$ \\
\hline $\begin{array}{l}\text { Young, } 2009^{48} \\
\text { Multisite } \\
\text { All Continents } \\
\text { Moderate ROB } \\
19118324\end{array}$ & $\begin{array}{l}\text { I: } \\
\text { Aripiprazole } \\
\text { C1: Placebo } \\
\text { C2: } \\
\text { Haloperidol }\end{array}$ & $\begin{array}{l}\text { BD-I; } \\
\text { DSM-IV }\end{array}$ & $\begin{array}{l}\text { Mean Age } 41 \\
(18-76) \\
56 \% \text { Female } \\
78 \% \text { White } \\
N=485\end{array}$ & $\begin{array}{l}\text { Manic/Mixed } \\
\text { (with/without } \\
\text { psychotic features) } \\
\text { in acute relapse; } \\
\text { YMRS } \geq 20 \text { and } \\
\text { MADRS } \leq 17 \text { at } \\
\text { baseline, }<25 \% \\
\text { decrease in YMRS } \\
\text { score and } \leq 4 \text { point } \\
\text { MADRS score } \\
\text { between screening } \\
\text { and baseline visits; } \\
\text { Current episode }<3 \\
\text { weeks }\end{array}$ & $\begin{array}{l}\text { First Manic Episode } \\
\text { Schizoaffective } \\
\text { Neurological Disorders } \\
\text { Other Mental Health } \\
\text { Taking Other Meds } \\
\text { Substance Abuse }\end{array}$ \\
\hline
\end{tabular}




\begin{tabular}{|c|c|c|c|c|c|}
\hline $\begin{array}{c}\text { Author, Year } \\
\text { Single/Multisite } \\
\text { Location } \\
\text { Risk of Bias } \\
\text { (ROB) } \\
\text { PMID }\end{array}$ & $\begin{array}{l}\text { Intervention/ } \\
\text { Comparison }\end{array}$ & $\begin{array}{c}\text { BD Type; } \\
\text { Diagnostic } \\
\text { Criteria }\end{array}$ & Demographics & Current Episode & Key Exclusions \\
\hline $\begin{array}{l}\text { Vieta, } 2005^{47} \\
\text { Multisite } \\
\text { NR } \\
\text { Moderate ROB } \\
16135860\end{array}$ & $\begin{array}{l}\text { I: } \\
\text { Aripiprazole } \\
\text { C: } \\
\text { Haloperidol }\end{array}$ & $\begin{array}{l}\text { BD-I; } \\
\text { DSM-IV }\end{array}$ & $\begin{array}{l}\text { Mean Age } 42 \\
\text { (NR) } \\
62 \% \text { Female } \\
\text { Race NR } \\
N=347\end{array}$ & $\begin{array}{l}\text { Manic/Mixed } \\
\text { episode; } \\
\text { YMRS } \geq 20 \text {; Current } \\
\text { episode }<4 \text { weeks }\end{array}$ & $\begin{array}{l}\text { Other Mental Health } \\
\text { Taking Other Meds } \\
\text { Substance Abuse }\end{array}$ \\
\hline $\begin{array}{l}\text { Sachs, } 2006^{50} \\
\text { Multisite } \\
\text { North America } \\
\text { High ROB } \\
16401666\end{array}$ & $\begin{array}{l}\text { I: } \\
\text { Aripiprazole } \\
\text { C: Placebo }\end{array}$ & $\begin{array}{l}\text { BD-I; } \\
\text { DSM-IV }\end{array}$ & $\begin{array}{l}\text { Mean Age } 39 \\
\text { (NR) } \\
51 \% \text { Female } \\
92 \% \text { White } \\
N=272\end{array}$ & $\begin{array}{l}\text { Manic/Mixed } \\
\text { episode; YMRS } \geq \\
\text { 20; } \\
\text { Current episode <4 } \\
\text { weeks }\end{array}$ & $\begin{array}{l}\text { First Manic Episode } \\
\text { Schizoaffective } \\
\text { Substance Abuse } \\
\text { Neurological Disorders } \\
\text { Taking Other Meds } \\
\text { Pregnant/Nursing } \\
\text { Labs/Other Conditions }\end{array}$ \\
\hline
\end{tabular}

$\mathrm{BD}=$ bipolar disorder; C=Comparison; DSM-IV=Diagnostic and Statistical Manual Fourth Edition; I=Intervention; MADRS= Montgomery-Asberg depression scale; N=number; NR=Not Reported; ROB=risk of bias; YMRS=Young Mania Rating Scale

\section{Aripiprazole Alone Versus Placebo}

Evidence was insufficient for all outcomes from three studies $(n=823)$ to address whether aripiprazole was better than placebo for acute mania in adults with BD-I, due to high study limitations and imprecise data. ${ }^{48-50}$ Following AHRQ methods, random effect models for pooling data, which allow one to extend the findings to the general population, largely showed no difference between groups in response rates, manic symptom improvement, or withdrawal rates. If fixed effect models are used, which only allows inferences for the specific participants in the specific studies, symptom improvements were seen. However, the improvement may not be clinically meaningful based on values that are less than the MID. The Young Mania Rating Scale (YMRS) mean difference of 3.85 (95\% CI 2.27, 5.44) is less than the MID of 6, and the Clinical Global Improvement (CGI) score mean difference of 0.44 (95\% CI $0.25,0.63$ ) is less than the MID of 1 . There were no differences between groups for serious adverse events.

\section{Aripiprazole Alone Versus Active Control}

Evidence was insufficient for all outcomes from two studies ( $n=674)$ to address whether aripiprazole was better than haloperidol for acute mania in adults with BD-I, due to mostly high study limitations and imprecise data. ${ }^{47,48}$ Studies reported no differences between groups for response or remission rates and mixed results as to which drug was favored. Akathisia and extrapyramidal symptoms were reported lower in participants using aripiprazole versus haloperidol; other harms or withdrawal outcomes were mixed.

\section{Aripiprazole Plus Mood Stabilizers}

Table 8 summarizes the bipolar type and major inclusion and exclusion criteria for each aripiprazole plus mood stabilizers study for acute mania. Appendix E provides further detail. 
Table 8. Population and inclusion criteria for aripiprazole plus mood stabilizers studies for acute mania

\begin{tabular}{|c|c|c|c|c|c|}
\hline $\begin{array}{c}\text { Author, Year } \\
\text { Single/Multisite } \\
\text { Location } \\
\text { Risk of Bias } \\
\text { (ROB) } \\
\text { PMID } \\
\end{array}$ & $\begin{array}{l}\text { Intervention/ } \\
\text { Comparison }\end{array}$ & $\begin{array}{c}\text { BD Type; } \\
\text { Diagnostic } \\
\text { Criteria }\end{array}$ & Demographics & Current Episode & Key Exclusions \\
\hline $\begin{array}{l}\text { NCT00665366 } \\
2013 \\
\text { Multisite } \\
3 \text { Continents } \\
\text { RoB NA }\end{array}$ & $\begin{array}{l}\text { I: } \\
\text { Aripiprazole } \\
\text { + lithium/ } \\
\text { valproate } \\
\text { C: Placebo + } \\
\text { lithium/ } \\
\text { valproate }\end{array}$ & $\begin{array}{l}\text { BD-I; } \\
\text { DSM NR }\end{array}$ & $\begin{array}{l}\text { Mean Age } 45 \\
(19-71) \\
54 \% \text { Female } \\
95 \% \text { White } \\
N=370\end{array}$ & $\begin{array}{l}\text { Mania/Manic/Mixed } \\
\text { episode; } \\
\text { YMRS } \geq 16\end{array}$ & $\begin{array}{l}\text { Schizoaffective } \\
\text { Substance Abuse } \\
\text { Other Mental Health } \\
\text { Neurological disorders } \\
\text { Pregnant/Nursing } \\
\text { Labs/Other Conditions }\end{array}$ \\
\hline $\begin{array}{l}\text { Jeong, } 201251 \\
\text { Multisite } \\
\text { South Korea } \\
\text { Low ROB } \\
22592508\end{array}$ & $\begin{array}{l}\text { I: } \\
\text { Aripiprazole } \\
+ \text { valproic } \\
\text { acid } \\
\text { C: } \\
\text { Haloperidol + } \\
\text { valproic acid }\end{array}$ & $\begin{array}{l}\text { BD-I; } \\
\text { DSM-IV }\end{array}$ & $\begin{array}{l}\text { Mean Age } 37 \\
\text { (NR) } \\
64 \% \text { Female } \\
\text { Race NR } \\
N=42\end{array}$ & $\begin{array}{l}\text { Mania; } \\
\text { YMRS } \geq 20\end{array}$ & $\begin{array}{l}\text { Schizoaffective } \\
\text { Substance Abuse } \\
\text { Other Mental Health } \\
\text { Taking Other Meds } \\
\text { Labs/Other Conditions }\end{array}$ \\
\hline $\begin{array}{l}\text { Vieta, } 200852 \\
\text { Multisite } \\
\text { NR } \\
\text { Moderate ROB } \\
18381903\end{array}$ & $\begin{array}{l}\text { I: } \\
\text { Aripiprazole } \\
+ \text { lithium/ } \\
\text { divalproex/ } \\
\text { valproate } \\
\text { C: Placebo + } \\
\text { lithium/ } \\
\text { divalproex/ } \\
\text { valproate }\end{array}$ & $\begin{array}{l}\text { BD-I; } \\
\text { DSM-IV }\end{array}$ & $\begin{array}{l}\text { Mean Age } 42 \\
(18-78) \\
52 \% \text { Female } \\
92 \% \text { White } \\
N=384\end{array}$ & $\begin{array}{l}\text { Manic/Mixed } \\
\text { episode; } \\
\text { Partial responders } \\
\text { to Lithium or } \\
\text { Valproate; YMRS } \geq \\
16 \text { with decrease of } \\
25 \% \text { between states }\end{array}$ & $\begin{array}{l}\text { Substance Abuse } \\
\text { Other Mental Health }\end{array}$ \\
\hline
\end{tabular}

BD=bipolar disorder; C=Comparison; DSM-IV=Diagnostic and Statistical Manual Fourth Edition; I=Intervention; MADRS= Montgomery-Asberg depression scale; N=number; NR=Not Reported; ROB=Risk of Bias; YMRS=Young Mania Rating Scale

\section{Aripiprazole Combination Versus Placebo}

Evidence was insufficient for all outcomes from two RCTs (n=752), one published and one unpublished, to address whether aripiprazole with a mood stabilizer (e.g., lithium, valproic acid, or divalproex) was better than placebo for acute mania in adults with BD-I, due to high study limitations, inconsistency, and imprecise data. ${ }^{52}$ Results were mixed, with the published study reporting aripiprazole plus mood stabilizers showed improvements in response, remission, mania symptoms, and CGI, while the unpublished study reported no difference between groups. Both studies reported no differences between groups in withdrawal rates, serious adverse events, or rates of akathisia.

\section{Aripiprazole Combination Versus Active Control}

Evidence was insufficient for all outcomes from one small RCT $(n=42)$ to address whether aripiprazole with mood stabilizers was better than haloperidol with mood stabilizer for acute mania in adults with BD-I, due to high study limitations, unknown consistency, and imprecise data. ${ }^{51}$ The study reported response rates in the 70 to 90 percent range but no differences between groups for mania symptom (YMRS) or CGI. Participants using aripiprazole reported experiencing more weight gain while participants using haloperidol reported experiencing more extrapyramidal symptoms. 


\section{Asenapine}

We identified three eligible publications reporting three unique RCTs of asenapine and one RCT examining asenapine with lithium or valproic acid for acute mania with at least 3 weeks followup. ${ }^{25-2759}$ Two studies were assessed as low to moderate risk of bias ${ }^{27} 59$ and two were assessed as high. ${ }^{25,} 26$ No additional studies were excluded for greater than 50 percent attrition. All were funded by industry. All interventions used a placebo comparator and two also compared to olanzapine. ${ }^{25,26}$ Sample sizes ranged from 324 to 489 and all followed participants with BD-I.

\section{Asenapine Alone}

Table 9 summarizes the bipolar type and major inclusion and exclusion criteria for each study of asenapine alone for acute mania. Appendix E provides further detail. 
Table 9. Population and inclusion criteria for studies of asenapine alone for acute mania

\begin{tabular}{|c|c|c|c|c|c|}
\hline $\begin{array}{l}\text { Author, Year } \\
\text { Single-Multisite } \\
\text { Local/Continent } \\
\text { Risk of Bias } \\
\text { PMID }\end{array}$ & $\begin{array}{l}\text { Intervention/ } \\
\text { Comparison }\end{array}$ & $\begin{array}{l}\text { BD Type; } \\
\text { Diagnostic } \\
\text { Criteria }\end{array}$ & Demographics & Current Episode & Key Exclusions \\
\hline $\begin{array}{l}\text { Landbloom, 2016 } \\
\text { Multisite } \\
\text { 3 Continents } \\
\text { Low ROB } \\
26496015\end{array}$ & $\begin{array}{l}\text { I: Asenapine } \\
\text { C: Placebo }\end{array}$ & $\begin{array}{l}\text { BD-I; } \\
\text { DSM-IV }\end{array}$ & $\begin{array}{l}\text { Mean Age } 44 \\
(18-77) \\
55 \% \text { Female } \\
\text { Race NR } \\
N=367\end{array}$ & $\begin{array}{l}\text { Mania; } \\
\text { Structured clinical } \\
\text { interview (MINI). } \\
\text { Episode began at } \\
\text { least } 1 \text { month } \\
\text { prior to screening. } \\
\text { YMRS } \geq 20\end{array}$ & $\begin{array}{l}\text { First Manic Episode } \\
\text { Schizoaffective } \\
\text { Substance Abuse } \\
\text { Other Mental Health } \\
\text { Taking Other Meds } \\
\text { Labs/Other } \\
\text { Conditions }\end{array}$ \\
\hline $\begin{array}{l}\text { Mclntyre, } 2010^{25} \\
\text { Multisite } \\
3 \text { Continents } \\
\text { High ROB } \\
20096936\end{array}$ & $\begin{array}{l}\text { I: Asenapine } \\
\text { C1: Placebo } \\
\text { C2: } \\
\text { Olanzapine }\end{array}$ & $\begin{array}{l}\text { BD-I; } \\
\text { DSM-IV }\end{array}$ & $\begin{array}{l}\text { Mean Age } 39 \\
(18-74) \\
47 \% \text { Female } \\
55 \% \text { White } \\
N=488\end{array}$ & $\begin{array}{l}\text { Manic/Mixed; } \\
\text { YMRS } \geq 20 ; \\
\text { Current episode } \\
\leq 3 \text { months }\end{array}$ & $\begin{array}{l}\text { First Manic Episode } \\
\text { Neurological } \\
\text { Disorders } \\
\text { Substance Abuse } \\
\text { Other Mental Health } \\
\text { Taking Other Meds } \\
\text { Lab/Other Conditions }\end{array}$ \\
\hline $\begin{array}{l}\text { McIntyre, } 2009^{26} \\
\text { Multisite } \\
3 \text { continents } \\
\text { High ROB } \\
19839993\end{array}$ & $\begin{array}{l}\text { I: Asenapine } \\
\text { C1: Placebo } \\
\text { C2: } \\
\text { Olanzapine }\end{array}$ & $\begin{array}{l}\text { BD-I; } \\
\text { DSM-IV }\end{array}$ & $\begin{array}{l}\text { Mean Age } 39 \\
(18-74) \\
43 \% \text { Female } \\
61 \% \text { White } \\
N=488\end{array}$ & $\begin{array}{l}\text { Manic/Mixed; } \\
\text { YMRS } \geq 20 ; \\
\text { Current episode } \\
\leq 3 \text { months }\end{array}$ & $\begin{array}{l}\text { First Manic Episode } \\
\text { Neurological } \\
\text { Disorders } \\
\text { Substance Abuse } \\
\text { Taking Other Meds } \\
\text { Labs/Other } \\
\text { Conditions }\end{array}$ \\
\hline
\end{tabular}

$\mathrm{BD}=$ bipolar disorder; $\mathrm{C}=$ Comparison; DSM-IV=Diagnostic and Statistical Manual Fourth Edition; I=Intervention; MADRS= Montgomery-Asberg depression scale; N=number; NR=Not Reported; ROB=Risk of Bias; YMRS=Young Mania Rating Scale

\section{Asenapine Alone Versus Placebo}

Low-strength evidence (moderate study limitations, imprecision) from three studies $(\mathrm{n}=956)$ showed asenapine improved mania symptoms (YMRS mean difference 4.37, 95\% CI 1.27, 7.47) and CGI (mean difference 0.5, 95\% CI 0.29, 0.71) compared to placebo after 3 weeks of treatment, although the improvement was about two-thirds the MID. ${ }^{25-27}$ Response and remission were not significantly different between groups. We found low-strength evidence that asenapine had a lower rate of withdrawal due to lack of efficacy than placebo (moderate study limitations, imprecision). However, low-strength evidence also showed that placebo had a lower rate of withdrawal due to adverse events than asenapine (moderate study limitations, imprecision). Overall withdrawal was less in the asenapine group, and favored asenapine over placebo, but results were not statistically significant. There were no differences between groups for serious adverse events, although participants with asenapine had significantly more extrapyramidal symptoms than those on placebo.

\section{Asenapine Alone Versus Active Control}

Evidence was insufficient for all outcomes from two studies $(n=763)$ to address whether asenapine was better than olanzapine for acute mania in adults with BD-I, due to high study limitations and imprecise data. ${ }^{25,26}$ Studies reported olanzapine showed a greater response rate but no differences in remissions. Serious adverse events were not different between arms, although participants using asenapine tended to withdraw at higher rates. 


\section{Asenapine Plus Mood Stabilizers}

Table 10 summarizes the bipolar type and major inclusion and exclusion criteria for each asenapine plus mood stabilizers study for acute mania. Appendix E provides further detail.

Table 10. Population and inclusion criteria for asenapine plus mood stabilizer studies for acute mania

\begin{tabular}{|l|l|l|l|l|l|}
\hline $\begin{array}{c}\text { Author, Year } \\
\text { Single-Multisite } \\
\text { Local/Continent } \\
\text { Risk of Bias } \\
\text { PMID }\end{array}$ & $\begin{array}{l}\text { Intervention/ } \\
\text { Comparison }\end{array}$ & $\begin{array}{l}\text { BD Type; } \\
\text { Diagnostic } \\
\text { Criteria }\end{array}$ & Demographics & Current Episode & Key Exclusions \\
\hline $\begin{array}{l}\text { Szegedi, 201259 } \\
\text { Multisite }\end{array}$ & $\begin{array}{l}\text { I: Asenapine + } \\
4 \text { Continents } \\
\text { lithium/ }\end{array}$ & $\begin{array}{l}\text { BD-l; } \\
\text { Nalproate }\end{array}$ & $\begin{array}{l}\text { Mean Age 39 } \\
(18-75)\end{array}$ & $\begin{array}{l}\text { Mania; } \\
\text { YMRS } \geq 20 \\
\text { Current episode } \leq 3 \\
\text { months }\end{array}$ & $\begin{array}{l}\text { Substance Abuse } \\
\text { Other Mental Health } \\
\text { Neurological } \\
\text { Disorders } \\
\text { Taking Other Meds } \\
\text { Pregnant/Nursing } \\
\text { Labs/Other } \\
\text { Conditions First Manic } \\
\text { Episode }\end{array}$ \\
\hline
\end{tabular}

BD=Bipolar Disorder; C=Comparison; DSM-IV=Diagnostic and Statistical Manual Fourth Edition; I=Intervention; N=number; NR=Not Reported; ROB=Risk of Bias; YMRS=Young Mania Rating Scale

\section{Asenapine Combination Versus Placebo}

Evidence from this single study of 324 participants with BD-I was rated as insufficient for all outcomes due to moderate study limitations, unknown consistency, and imprecision. The study reported the asenapine with lithium or valproate group showed improvement in manic symptom (YMRS) and CGI but no differences between groups for response or remission rates.

\section{Cariprazine}

We identified three eligible publications reporting three unique RCTs of cariprazine for acute mania with at least 3 weeks followup. ${ }^{28-30}$ All were assessed as low to moderate risk of bias and used a placebo comparator. No studies were excluded for greater than 50 percent attrition. All were funded by industry. Sample sizes ranged from 238 to 497 and all recruited participants with BD-I. Table 11 summarizes the bipolar type and major inclusion and exclusion criteria for each study. Appendix E provides further detail.

Table 11. Population and inclusion criteria for studies of cariprazine alone for acute mania

\begin{tabular}{|c|c|c|c|c|c|}
\hline $\begin{array}{c}\text { Author, Year } \\
\text { Single-Multisite } \\
\text { Local/Continent } \\
\text { Risk of Bias } \\
\text { PMID }\end{array}$ & $\begin{array}{l}\text { Intervention/ } \\
\text { Comparison }\end{array}$ & $\begin{array}{l}\text { BD Type; } \\
\text { Diagnostic } \\
\text { Criteria }\end{array}$ & Demographics & Current Episode & Key Exclusions \\
\hline $\begin{array}{l}\text { Calabrese, } \\
2015^{28} \text { Multisite } \\
3 \text { Continents } \\
\text { Low ROB } \\
\\
25562205\end{array}$ & $\begin{array}{l}\text { I: Cariprazine } \\
\text { C: Placebo }\end{array}$ & $\begin{array}{l}\text { BD-I; } \\
\text { DSM-IV }\end{array}$ & $\begin{array}{l}\text { Mean Age } 42 \\
(18-65) \\
47 \% \text { Female } \\
69 \% \text { White } \\
N=497\end{array}$ & $\begin{array}{l}\text { Manic/Mixed; } \\
\text { YMRS } \geq 20 \text { AND } \geq \\
4 \text { on two YMRS } \\
\text { items AND MADRS } \\
<18\end{array}$ & $\begin{array}{l}\text { First Manic Episode } \\
\text { Schizoaffective } \\
\text { Substance Abuse } \\
\text { Other Mental Health } \\
\text { Neurological Disorders } \\
\text { Taking Other Meds }\end{array}$ \\
\hline
\end{tabular}




\begin{tabular}{|c|c|c|c|c|c|}
\hline $\begin{array}{c}\text { Author, Year } \\
\text { Single-Multisite } \\
\text { Local/Continent } \\
\text { Risk of Bias } \\
\text { PMID }\end{array}$ & $\begin{array}{l}\text { Intervention/ } \\
\text { Comparison }\end{array}$ & $\begin{array}{c}\text { BD Type; } \\
\text { Diagnostic } \\
\text { Criteria }\end{array}$ & Demographics & Current Episode & Key Exclusions \\
\hline $\begin{array}{l}\text { Durgam, } 2015^{29} \\
\text { Multisite } \\
3 \text { Continents } \\
\text { Moderate ROB } \\
25056368\end{array}$ & $\begin{array}{l}\text { I: Cariprazine } \\
\text { C: Placebo }\end{array}$ & $\begin{array}{l}\text { BD-I; } \\
\text { DSM-IV }\end{array}$ & $\begin{array}{l}\text { Mean Age } 38 \\
(18-65) \\
67 \% \text { Female } \\
43 \% \text { White } \\
N=306\end{array}$ & $\begin{array}{l}\text { Manic/Mixed; } \\
\text { YMRS } \geq 20 \text { AND } \geq \\
4 \text { on two YMRS } \\
\text { items }\end{array}$ & $\begin{array}{l}\text { First Manic Episode } \\
\text { Schizoaffective } \\
\text { Substance Abuse } \\
\text { Other Mental Health } \\
\text { Neurological Disorders } \\
\text { Taking Other Meds } \\
\text { Pregnant/Nursing } \\
\text { Labs/Other Conditions }\end{array}$ \\
\hline $\begin{array}{l}\text { Sachs, } 2015^{30} \\
\text { Multisite } \\
2 \text { Continents } \\
\text { Moderate ROB } \\
25532076\end{array}$ & $\begin{array}{l}\text { I: Cariprazine } \\
\text { C: Placebo }\end{array}$ & $\begin{array}{l}\text { BD-I; } \\
\text { DSM-IV }\end{array}$ & $\begin{array}{l}\text { Mean Age } 36 \\
(18-65) \\
36 \% \text { Female } \\
21 \% \text { White } \\
N=312\end{array}$ & $\begin{array}{l}\text { Manic/Mixed; } \\
\text { YMRS } \geq 20 \text { YMRS } \\
\text { AND } \geq 4 \text { on two } \\
\text { YMRS items AND } \\
\text { MADRS }<18\end{array}$ & $\begin{array}{l}\text { Schizoaffective } \\
\text { Substance Abuse } \\
\text { Other Mental Health } \\
\text { Neurological Disorders } \\
\text { Taking Other Meds } \\
\text { Pregnant/Nursing } \\
\end{array}$ \\
\hline
\end{tabular}

$\mathrm{BD}=$ bipolar disorder; $\mathrm{C}=$ Comparison; DSM-IV=Diagnostic and Statistical Manual Fourth Edition; I=Intervention; MADRS=

Montgomery-Asberg depression scale; N=number; NR=Not Reported; ROB=Risk of Bias; YMRS=Young Mania Rating Scale

\section{Cariprazine Alone Versus Placebo}

Low-strength evidence (moderate study limitations, imprecision) from three studies $(\mathrm{n}=1,047)$ showed cariprazine improved response (OR 2.14, 95\% CI 1.08, 4.23) and remission (OR 1.95, 95\% CI 1.45, 2.63) rates, as well as mania symptoms (YMRS mean difference 5.38, 95\% CI 1.84, 8.92) and CGI-BP-S (mean difference 0.54, 95\% CI 0.35, 0.73), compared to placebo after 3 weeks of treatment. ${ }^{28-30}$ No differences were seen in withdrawal rates. There were no differences between groups for serious adverse events, although participants using cariprazine had more extrapyramidal symptoms than those using placebo.

\section{Olanzapine}

We identified 15 eligible publications reporting 13 unique RCTs of olanzapine and seven eligible publications reporting five unique RCTs of olanzapine with a mood stabilizer for acute mania with at least 3 weeks followup. ${ }^{25}$, 26, 31, 33, 34, 44-46, 60-72 Five were assessed as low risk of bias, five as moderate, and eight as high. Fourteen studies reported being funded by industry. An additional sixteen studies were excluded for greater than 50 percent attrition. ${ }^{73-88}$ Nine studies used a placebo comparator, while 14 used active comparators. Two studies of olanzapine with mood stabilizers did not use a placebo in place of olanzapine. ${ }^{46,69}$ Sample sizes ranged from 30 to 560 and most reported recruiting participants with BD-I; one study restricted participants to a current DSM-IV mixed episode. ${ }^{69}$

\section{Olanzapine Alone}

Table 12 summarizes the bipolar type and major inclusion and exclusion criteria for each study of olanzapine alone for acute mania. Appendix E provides further detail. 
Table 12. Population and inclusion criteria for studies of olanzapine alone for acute mania

\begin{tabular}{|c|c|c|c|c|c|}
\hline $\begin{array}{c}\text { Author, Year } \\
\text { Single-Multisite } \\
\text { Local/Continent } \\
\text { Risk of Bias } \\
\text { PMID } \\
\end{array}$ & $\begin{array}{l}\text { Intervention/ } \\
\text { Comparison }\end{array}$ & $\begin{array}{l}\text { BD Type; } \\
\text { Diagnostic } \\
\text { Criteria }\end{array}$ & Demographics & Current Episode & Key Exclusions \\
\hline $\begin{array}{l}\text { Xu, } 2015^{46} \\
\text { Singlesite } \\
\text { China } \\
\text { Low ROB } \\
26060401\end{array}$ & $\begin{array}{l}\text { I: Olanzapine } \\
\text { C1: Olanzapine } \\
\text { + Valproate } \\
\text { C2: Valproate }\end{array}$ & $\begin{array}{l}\text { BD-I; } \\
\text { DSM-IV }\end{array}$ & $\begin{array}{l}\text { Mean Age 31 } \\
(19-60) \\
52 \% \text { Female } \\
\text { Race NR } \\
N=120\end{array}$ & $\begin{array}{l}\text { Manic; } \\
\text { YMRS } \geq 17\end{array}$ & $\begin{array}{l}\text { Substance Abuse } \\
\text { Neurological } \\
\text { Disorders } \\
\text { Taking Other Meds } \\
\text { Pregnant/Nursing }\end{array}$ \\
\hline $\begin{array}{l}\text { Katagiri, } 2012^{31} \\
\text { Multisite } \\
\text { Japan } \\
\text { Moderate ROB } \\
22134043\end{array}$ & $\begin{array}{l}\text { I: Olanzapine } \\
\text { C1: Placebo } \\
\text { (Haloperidol arm } \\
\text { excluded for <10 } \\
\text { completers) }\end{array}$ & $\begin{array}{l}\text { BD-I; } \\
\text { DSM-IV }\end{array}$ & $\begin{array}{l}\text { Mean Age } 43 \\
(21-65) \\
55 \% \text { Female } \\
\text { Race NR } \\
\mathrm{N}=221\end{array}$ & $\begin{array}{l}\text { Manic or Mixed } \\
\text { Episode; } \\
\text { YMRS } \geq 20\end{array}$ & $\begin{array}{l}\text { Other Mental Health } \\
\text { Taking Other Meds } \\
\text { Labs/Other } \\
\text { Conditions }\end{array}$ \\
\hline $\begin{array}{l}\text { McIntyre, } 2010^{32} \\
\text { Multisite } \\
\text { 3 Continents } \\
\text { High ROB } \\
20096936\end{array}$ & $\begin{array}{l}\text { I: Olanzapine } \\
\text { C1: Placebo } \\
\text { C2: Asenapine }\end{array}$ & $\begin{array}{l}\text { BD-I; } \\
\text { DSM-IV }\end{array}$ & $\begin{array}{l}\text { Mean Age } 39 \\
(18-61) \\
47 \% \text { Female } \\
55 \% \text { White } \\
N=488\end{array}$ & $\begin{array}{l}\text { Manic or Mixed } \\
\text { Episode; } \\
\text { YMRS } \geq 20\end{array}$ & $\begin{array}{l}\text { First Manic Episode } \\
\text { Schizoaffective } \\
\text { Substance Abuse } \\
\text { Other Mental Health } \\
\text { Neurological } \\
\text { Disorders } \\
\text { Taking Other Meds } \\
\text { Pregnant/Nursing } \\
\text { Labs/Other } \\
\text { Conditions }\end{array}$ \\
\hline $\begin{array}{l}\text { Shafti, } 2010^{64} \\
\text { Singlesite } \\
\text { Iran } \\
\text { Moderate ROB } \\
19740546\end{array}$ & $\begin{array}{l}\text { I: Olanzapine } \\
\text { C: Lithium }\end{array}$ & $\begin{array}{l}\text { BD-I; } \\
\text { DSM-IV }\end{array}$ & $\begin{array}{l}\text { Mean Age NR } \\
100 \% \text { Female } \\
\text { Race NR } \\
N=40\end{array}$ & $\begin{array}{l}\text { Manic } \\
\text { (particulars not } \\
\text { described) }\end{array}$ & $\begin{array}{l}\text { Schizoaffective } \\
\text { Substance Abuse } \\
\text { Other Mental Health } \\
\text { Neurological } \\
\text { Disorders } \\
\text { Taking Other Meds } \\
\text { Labs/Other } \\
\text { Conditions }\end{array}$ \\
\hline $\begin{array}{l}\text { McIntyre, } 2009^{26} \\
\text { Multisite } \\
3 \text { Continents } \\
\text { High ROB } \\
19839993\end{array}$ & $\begin{array}{l}\text { I: Olanzapine } \\
\text { C1: Placebo } \\
\text { C2: Asenapine }\end{array}$ & $\begin{array}{l}\text { BD-I; } \\
\text { DSM-IV }\end{array}$ & $\begin{array}{l}\text { Mean Age } 40 \\
(18-74) \\
43 \% \text { Female } \\
61 \% \text { White } \\
N=489\end{array}$ & $\begin{array}{l}\text { Manic or Mixed } \\
\text { Episode; } \\
\text { YMRS } \geq 20\end{array}$ & $\begin{array}{l}\text { Schizoaffective } \\
\text { Substance Abuse } \\
\text { Other Mental Health } \\
\text { Neurological } \\
\text { Disorders } \\
\text { Taking Other Meds } \\
\text { Pregnant/Nursing } \\
\text { Labs/Other } \\
\text { Conditions }\end{array}$ \\
\hline $\begin{array}{l}\text { Niufan, } 2008^{61} \\
\text { Multisite } \\
\text { China } \\
\text { Low ROB } \\
17531327\end{array}$ & $\begin{array}{l}\text { I: Olanzapine } \\
\text { C: Lithium }\end{array}$ & $\begin{array}{l}\text { BD-I; } \\
\text { DSM-IV }\end{array}$ & $\begin{array}{l}\text { Mean Age } 33 \\
(18-72) \\
74 \% \text { Female } \\
\text { Race NR } \\
N=140\end{array}$ & $\begin{array}{l}\text { Manic or Mixed } \\
\text { Episode; } \\
\text { YMRS } \geq 20\end{array}$ & NR \\
\hline $\begin{array}{l}\text { Tohen, 2008b } \\
\text { Multisite } \\
3 \text { Continents } \\
\text { Low ROB } \\
19014751\end{array}$ & $\begin{array}{l}\text { I: Olanzapine } \\
\text { C1: Placebo } \\
\text { C2: Divalproex }\end{array}$ & $\begin{array}{l}\text { BD-I; } \\
\text { DSM-IV }\end{array}$ & $\begin{array}{l}\text { Mean Age } 40 \\
(18-65) \\
60 \% \text { Female } \\
\text { Race NR } \\
N=521\end{array}$ & $\begin{array}{l}\text { Manic or Mixed } \\
\text { Episode without } \\
\text { psychotic } \\
\text { features; } \\
\text { YMRS 20-30 } \\
\text { CGI-BP mania 3- } \\
4 \\
\end{array}$ & $\begin{array}{l}\text { Schizoaffective } \\
\text { Other Mental Health } \\
\text { Pregnant/Nursing }\end{array}$ \\
\hline
\end{tabular}




\begin{tabular}{|c|c|c|c|c|c|}
\hline $\begin{array}{c}\text { Author, Year } \\
\text { Single-Multisite } \\
\text { Local/Continent } \\
\text { Risk of Bias } \\
\text { PMID } \\
\end{array}$ & $\begin{array}{l}\text { Intervention/ } \\
\text { Comparison }\end{array}$ & $\begin{array}{l}\text { BD Type; } \\
\text { Diagnostic } \\
\text { Criteria }\end{array}$ & Demographics & Current Episode & Key Exclusions \\
\hline $\begin{array}{l}\text { Perlis, } 2006^{89} \\
\text { Multisite } \\
\text { US } \\
\text { High ROB } \\
17196055\end{array}$ & $\begin{array}{l}\text { I: Olanzapine } \\
\text { C: Risperidone }\end{array}$ & $\begin{array}{l}\text { BD-I; } \\
\text { DSM-IV }\end{array}$ & $\begin{array}{l}\text { Mean Age } 38 \\
(18-70) \\
55 \% \text { Female } \\
74 \% \text { White } \\
N=329\end{array}$ & $\begin{array}{l}\text { Manic; } \\
\text { YMRS } \geq 20\end{array}$ & $\begin{array}{l}\text { Substance Abuse } \\
\text { Labs/Other } \\
\text { Conditions }\end{array}$ \\
\hline $\begin{array}{l}\text { Zajecka, } 2002^{45} \\
\text { Multisite } \\
\text { US } \\
\text { Moderate ROB } \\
12523875 \\
12716270^{63}\end{array}$ & $\begin{array}{l}\text { I: Olanzapine } \\
\text { C: Divalproex }\end{array}$ & $\begin{array}{l}\text { BD-I; } \\
\text { DSM-IV }\end{array}$ & $\begin{array}{l}N=120 \\
\text { Age } 39(18-65) \\
46 \% \text { Female } \\
75 \% \text { White }\end{array}$ & $\begin{array}{l}\text { Mania; } \\
\text { SADS-C } \geq 25 \\
\text { with at least four } \\
\text { scale items rated } \\
\text { at least } 3 \text {. }\end{array}$ & $\begin{array}{l}\text { Schizoaffective; } \\
\text { Substance Abuse; } \\
\text { Other Mental Health } \\
\text { Conditions; } \\
\text { Neurological } \\
\text { Disorders; } \\
\text { Taking other } \\
\text { medications; } \\
\text { Pregnant/Nursing; } \\
\text { Labs/Other } \\
\text { conditions }\end{array}$ \\
\hline $\begin{array}{l}\text { Tohen, 200367 } \\
\text { Multisite } \\
4 \text { Continents } \\
\text { Moderate ROB } \\
14662554 \\
12177585^{65} \\
\end{array}$ & $\begin{array}{l}\text { I: Olanzapine } \\
\text { C: Haloperidol }\end{array}$ & $\begin{array}{l}\text { BD-I; } \\
\text { DSM-IV }\end{array}$ & $\begin{array}{l}\text { Mean Age } 40 \\
(18-80) \\
60 \% \text { Female } \\
\text { Race NR } \\
N=453\end{array}$ & $\begin{array}{l}\text { Manic; } \\
\text { YMRS } \geq 20\end{array}$ & $\begin{array}{l}\text { Substance Abuse } \\
\text { Labs/Other } \\
\text { Conditions }\end{array}$ \\
\hline $\begin{array}{l}\text { Tohen, 2002 } b^{44} \\
\text { Multisite } \\
\text { US } \\
\text { High ROB } \\
12042191\end{array}$ & $\begin{array}{l}\text { I: Olanzapine } \\
\text { C: Divalproex }\end{array}$ & $\begin{array}{l}\text { BD-I; } \\
\text { DSM-IV }\end{array}$ & $\begin{array}{l}\text { Mean Age } 41 \\
(18-75) \\
57 \% \text { Female } \\
81 \% \text { White } \\
N=251\end{array}$ & $\begin{array}{l}\text { Mania; } \\
\text { YMRS } \geq 20\end{array}$ & $\begin{array}{l}\text { Substance Abuse } \\
\text { Other Mental Health } \\
\text { Neurological } \\
\text { Disorders } \\
\text { Taking Other Meds } \\
\text { Pregnant/Nursing } \\
\text { Labs/Other } \\
\text { Conditions }\end{array}$ \\
\hline $\begin{array}{l}\text { Tohen, } 2000^{34} \\
\text { Multisite } \\
\text { US } \\
\text { High ROB } \\
10986547\end{array}$ & $\begin{array}{l}\text { I: Olanzapine } \\
\text { C: Placebo }\end{array}$ & $\begin{array}{l}\text { BD-I; } \\
\text { DSM-IV }\end{array}$ & $\begin{array}{l}\text { Mean Age } 39 \\
(18-69) \\
50 \% \text { Female } \\
80 \% \text { White } \\
N=115\end{array}$ & $\begin{array}{l}\text { Mixed Episode; } \\
\text { YMRS score } \geq \\
20\end{array}$ & $\begin{array}{l}\text { Substance Abuse; } \\
\text { Other Mental Health; } \\
\text { Neurological } \\
\text { Disorders; } \\
\text { Taking Other Meds; } \\
\text { Labs/Other } \\
\text { Conditions }\end{array}$ \\
\hline $\begin{array}{l}\text { Berk, } 1999^{60} \\
\text { Singlesite } \\
\text { South Africa } \\
\text { High ROB } \\
10565800 \\
\end{array}$ & $\begin{array}{l}\text { I: Olanzapine } \\
\text { C: Lithium }\end{array}$ & $\begin{array}{l}\text { NR; } \\
\text { DSM-IV }\end{array}$ & $\begin{array}{l}\text { Mean Age } 31 \\
(N R) \\
\text { Sex NR } \\
\text { Race NR } \\
N=30\end{array}$ & $\begin{array}{l}\text { Mania } \\
\text { No criteria } \\
\text { specific reported }\end{array}$ & $\begin{array}{l}\text { Other Mental Health; } \\
\text { Pregnant/Nursing; } \\
\text { Labs/Other } \\
\text { Conditions }\end{array}$ \\
\hline
\end{tabular}

BD=bipolar disorder; C=Comparison; CGI-BP-S=Clinical global impression scale, bipolar edition, severity; DSM-

IV=Diagnostic and Statistical Manual Fourth Edition; I=Intervention; MADRS= Montgomery-Asberg depression scale; $\mathrm{N}=$ number; NR=Not Reported; ROB=Risk of Bias; SADS-C=Schedule for Affective Disorders and Schizophrenia, change version; YMRS=Young Mania Rating Scale

\section{Olanzapine Alone Versus Placebo}

Low-strength evidence (moderate study limitations, imprecision) from five RCTs $(n=1,199)$ showed olanzapine was better for acute mania than placebo in response (OR 1.99, 95\% CI 1.29 to 3.08) and remission (OR 1.75, 95\% CI 1.19 to 2.58) rates. Mania symptom improvement was close to a MID (YMRS, mean difference 4.9, 95\% CI 2.34 to 7.45). ${ }^{25}$, 26, 31, 33, 34, 45 CGI trended 
toward improvement for olanzapine but did not reach significance. Low-strength evidence (moderate study limitations, imprecision) also showed overall withdrawal and withdrawal due to lack of effect were lower for olanzapine. Withdrawal for adverse events did not differ between groups. While serious adverse events did not differ by group, participants using olanzapine reported more extrapyramidal symptoms and weight gain (at least 7 percent increase) than those using placebo.

\section{Olanzapine Alone Versus Active Control}

Low-strength evidence (moderate study limitations, imprecision) from four RCTs $(\mathrm{n}=867)$ showed no statistically significant difference in outcomes between olanzapine versus divalproex or valproate for acute mania in adults with presumed BD-I. ${ }^{33,44-46,63,66}$ No differences were noted in response or remission rates $(n=635)$, mania symptoms (YMRS, $n=750)$, CGI $(n=578)$, or withdrawals ( $n=867)$. No differences were noted in serious adverse events. However, one study noted participants receiving olanzapine experienced more clinically important weight gain (at least $7 \%$ ) than those receiving divalproex; ${ }^{33}$ a trend toward greater weight gain in olanzapine groups was noted in the other studies as well.

Evidence was insufficient for all outcomes from three RCTs $(n=210)$ to address whether olanzapine was better for acute mania than lithium in adults with presumed BD-I, due to moderate study limitations, inconsistency, and imprecision. ${ }^{60,61,64}$ The studies reported mixed results for response, mania symptom improvement (YMRS), or CGI. Withdrawals and adverse events tended to show no differences between groups.

Evidence was also insufficient for all outcomes to address whether olanzapine was better than risperidone (one RCT, $n=329$ ), ${ }^{89}$ or haloperidol (one RCT, $n=453$ ), due to single studies of moderate to high study limitations and imprecision. ${ }^{67}$ The studies reported no differences between groups for response, remission, symptom improvement, function, or withdrawals over 3 weeks. No differences between groups were noted in serious adverse events. However, participants using olanzapine reported more weight gain while participants using haloperidol reported more akathisia.

Results for olanzapine versus asenapine were reported in the asenapine versus active comparator section above (e.g., evidence was insufficient for olanzapine compared to asenapine).

\section{Olanzapine Plus Mood Stabilizers}

Table 13 summarizes bipolar type and major inclusion and exclusion criteria for each olanzapine plus mood stabilizers study for acute mania. Appendix E provides further detail.

\section{Table 13. Population and inclusion criteria for olanzapine plus mood stabilizers studies for acute} mania

\begin{tabular}{|c|c|c|c|c|c|}
\hline $\begin{array}{l}\text { Author, Year } \\
\text { Single-Multisite } \\
\text { Local/Continent } \\
\text { Risk of Bias } \\
\text { PMID } \\
\end{array}$ & $\begin{array}{l}\text { Intervention/ } \\
\text { Comparison }\end{array}$ & $\begin{array}{c}\text { BD Type; } \\
\text { Diagnostic } \\
\text { Criteria }\end{array}$ & Demographics & Current Episode & Key Exclusions \\
\hline $\begin{array}{l}\text { Conus, } 2015^{68} \\
\text { Singlesite } \\
\text { Australia } \\
\text { Low ROB } \\
26485297\end{array}$ & $\begin{array}{l}\text { I: Olanzapine + } \\
\text { Lithium } \\
\text { C: } \\
\text { Chlorpromazine } \\
\text { + Lithium }\end{array}$ & $\begin{array}{l}\text { BD-I; } \\
\text { DSM-IV }\end{array}$ & $\begin{array}{l}\text { Mean Age } 22 \\
(15-28) \\
32 \% \text { Female } \\
\text { Race NR } \\
N=83\end{array}$ & $\begin{array}{l}\text { Manic or Mixed } \\
\text { Episode; } \\
\text { YMRS } \geq 20\end{array}$ & $\begin{array}{l}\text { Other Mental } \\
\text { Health } \\
\text { Neurological } \\
\text { Disorders } \\
\text { Taking Other Meds } \\
\text { Pregnant/Nursing } \\
\text { Labs/Other } \\
\text { Conditions }\end{array}$ \\
\hline
\end{tabular}




\begin{tabular}{|c|c|c|c|c|c|}
\hline $\begin{array}{c}\text { Author, Year } \\
\text { Single-Multisite } \\
\text { Local/Continent } \\
\text { Risk of Bias } \\
\text { PMID }\end{array}$ & $\begin{array}{l}\text { Intervention/ } \\
\text { Comparison }\end{array}$ & $\begin{array}{c}\text { BD Type; } \\
\text { Diagnostic } \\
\text { Criteria }\end{array}$ & Demographics & Current Episode & Key Exclusions \\
\hline $\begin{array}{l}\text { Xu, } 2015^{46} \\
\text { Singlesite } \\
\text { China } \\
\text { Low ROB } \\
26060401\end{array}$ & $\begin{array}{l}\text { I: Olanzapine + } \\
\text { Valproate } \\
\text { C1: No placebo } \\
\text { + Olanzapine } \\
\text { C2: No placebo } \\
\text { + Valproate }\end{array}$ & $\begin{array}{l}\text { BD-I; } \\
\text { DSM-IV }\end{array}$ & $\begin{array}{l}\text { Mean Age } 31 \\
(19-60) \\
52 \% \text { Female } \\
\text { Race NR } \\
N=114\end{array}$ & $\begin{array}{l}\text { Manic; } \\
\text { YMRS } \geq 17\end{array}$ & $\begin{array}{l}\text { Substance Abuse } \\
\text { Neurological } \\
\text { Disorders } \\
\text { Taking Other Meds } \\
\text { Pregnant/Nursing }\end{array}$ \\
\hline $\begin{array}{l}\text { Houston, } 2009^{69} \\
\text { Multisite } \\
\text { US and Puerto } \\
\text { Rico } \\
\text { High ROB } \\
19778495\end{array}$ & $\begin{array}{l}\text { I: Olanzapine + } \\
\text { Divalproex } \\
\text { C: Placebo } \\
+ \text { Divalproex }\end{array}$ & $\begin{array}{l}\text { BD-I; } \\
\text { DSM-IV }\end{array}$ & $\begin{array}{l}\text { Mean Age } 39 \\
(18-60) \\
59 \% \text { Female } \\
51 \% \text { White } \\
N=202\end{array}$ & $\begin{array}{l}\text { Mixed Episode; } \\
\text { YMRS } \geq 16 \\
\text { HDRS-21 } \\
\text { (inadequate } \\
\text { response to } \\
\text { divalproex) }\end{array}$ & $\begin{array}{l}\text { First Manic } \\
\text { Episode } \\
\text { Taking Other Meds } \\
\text { Labs/Other } \\
\text { Conditions }\end{array}$ \\
\hline $\begin{array}{l}\text { Tohen, } 2008 a^{70} \\
\text { Multisite } \\
3 \text { Continents } \\
\text { Moderate ROB } \\
18245032\end{array}$ & $\begin{array}{l}\text { I: Olanzapine + } \\
\text { Carbamazepine } \\
\text { C: Placebo + } \\
\text { Carbamazepine }\end{array}$ & $\begin{array}{l}\text { BD-I; } \\
\text { DSM-IV }\end{array}$ & $\begin{array}{l}\text { Mean Age } 41 \\
(18-65) \\
60 \% \text { Female } \\
\text { Race NR } \\
N=118\end{array}$ & $\begin{array}{l}\text { Manic or Mixed } \\
\text { Episode; } \\
\text { YMRS } \geq 20\end{array}$ & $\begin{array}{l}\text { Labs/Other } \\
\text { Conditions }\end{array}$ \\
\hline $\begin{array}{l}\text { Tohen, 2002a } \\
\text { Multisite } \\
\text { US and Canada } \\
\text { High ROB } \\
\\
11779284 \\
15337326^{71} \\
15572737^{72}\end{array}$ & $\begin{array}{l}\text { I: Olanzapine + } \\
\text { Lithium of } \\
\text { Valproate } \\
\text { C: Placebo + } \\
\text { Lithium or } \\
\text { Valproate }\end{array}$ & $\begin{array}{l}\text { BD-I; } \\
\text { DSM-IV }\end{array}$ & $\begin{array}{l}\text { Mean Age } 41 \\
(18-70) \\
52 \% \text { Female } \\
85 \% \text { White } \\
N=344\end{array}$ & $\begin{array}{l}\text { Manic or Mixed } \\
\text { Episode; } \\
\text { YMRS } \geq 16 \\
\text { (partially } \\
\text { nonresponsive to } \\
\text { lithium or } \\
\text { valproate) }\end{array}$ & $\begin{array}{l}\text { First Manic } \\
\text { Episode } \\
\text { Labs/Other } \\
\text { Conditions }\end{array}$ \\
\hline
\end{tabular}

BD=Bipolar Disorder; C=Comparison; DSM-IV=Diagnostic and Statistical Manual Fourth Edition; I=Intervention; N=number; $\mathrm{NR}=$ Not Reported; ROB=Risk of Bias; YMRS=Young Mania Rating Scale

\section{Olanzapine Combination Versus Placebo/No Placebo}

Evidence was insufficient for all outcomes from four RCTs to address whether olanzapine plus mood stabilizers was better for acute mania than mood stabilizers alone for adults with BDI, due to high study limitations and imprecision. Two studies examined olanzapine plus carbamazepine $(\mathrm{n}=118)^{70}$ or lithium/valproate $(\mathrm{n}=344) \cdot{ }^{66}$ The studies showed mixed results for response or remission rates, but both reported olanzapine improved symptoms. Two other studies examined olanzapine plus divalproex $(n=202)^{69}$ or valproate $(n=80)^{46}$ compared to the mood stabilizer alone without a placebo present. One study reporting response and remission rates reported results favoring olanzapine, while both reported improvements in mania symptoms. Participants receiving olanzapine reported greater frequency of clinically important weight gain. No other differences between groups were noted in serious adverse events.

\section{Olanzapine Combination Versus Active Control}

Evidence was insufficient for all outcomes from one RCT ( $\mathrm{n}=83$ ) to address whether olanzapine plus lithium was better for acute mania than chlorpromazine plus lithium for adults with BD-I, due to a single study and imprecision. ${ }^{68}$ The study reported no difference between groups for all outcomes including symptomatic recovery, response, remission, depressive symptoms, and CGI. No differences were noted in serious adverse events or clinically significant weight gain. 


\section{Quetiapine}

We identified six eligible publications reporting six unique RCTs of quetiapine and two eligible publications reporting two unique RCTs of quetiapine plus mood stabilizers for acute

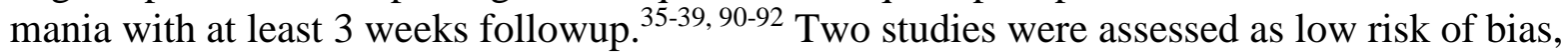
three as moderate, and three as high risk. Three additional studies were excluded for greater than 50 percent attrition. ${ }^{93,94,95}$ All studies were funded by industry. Five studies used placebos and three used active comparators. Sample sizes ranged from 39 to 493. All enrolled participants with BD-I; one study restricted participants to a current episode of mania but a history of manic or mixed episodes in the last 5 years. Another small study enrolled participants with mild to moderate hypomania or mild mania regardless of type of BD. ${ }^{39}$

\section{Quetiapine Alone}

Table 14 summarizes the bipolar type and major inclusion and exclusion criteria for each study of quetiapine alone for acute mania. Appendix E provides further detail.

Table 14. Population and inclusion criteria for studies of quetiapine alone for acute mania

\begin{tabular}{|c|c|c|c|c|c|}
\hline $\begin{array}{c}\text { Author, Year } \\
\text { Single-Multisite } \\
\text { Local/Continent } \\
\text { Risk of Bias } \\
\text { PMID } \\
\end{array}$ & $\begin{array}{l}\text { Intervention/ } \\
\text { Comparison }\end{array}$ & $\begin{array}{c}\text { BD Type; } \\
\text { Diagnostic } \\
\text { Criteria }\end{array}$ & Demographics & Current Episode & Key Exclusions \\
\hline $\begin{array}{l}\text { Cutler, } 2011^{38} \\
\text { Multisite } \\
\text { US } \\
\text { Low ROB } \\
22054797\end{array}$ & $\begin{array}{l}\text { I: Quetiapine } \\
\text { ER } \\
\text { C: Placebo }\end{array}$ & $\begin{array}{l}\text { BD-I; } \\
\text { DSM-IV }\end{array}$ & $\begin{array}{l}\text { Mean Age } 41 \\
(18-65) \\
40 \% \text { Female } \\
47 \% \text { White } \\
N=308\end{array}$ & $\begin{array}{l}\text { Mania; } \\
\text { YMRS } \geq 20 \\
\text { overall; YMRS } \geq 4 \\
\text { on at least } 2 \text { of } 4 \\
\text { specified mania } \\
\text { domains, and } \\
\text { CGI-BD-S } \geq 4\end{array}$ & $\begin{array}{l}\text { First Manic Episode } \\
\text { Schizoaffective } \\
\text { Substance Abuse } \\
\text { Other Mental Health } \\
\text { Labs/Other Conditions }\end{array}$ \\
\hline $\begin{array}{l}\text { McElroy, } 2010^{39} \\
\text { Singlesite } \\
\text { US } \\
\text { Moderate ROB } \\
19963274\end{array}$ & $\begin{array}{l}\text { I: Quetiapine } \\
\text { C: Placebo }\end{array}$ & $\begin{array}{l}\text { BD-I, II, } \\
\text { NOS; } \\
\text { DSM-IV }\end{array}$ & $\begin{array}{l}\text { Mean Age } 35 \\
(18-75) \\
51 \% \text { Female } \\
69 \% \text { White } \\
74 \% \text { BD-I } \\
21 \% \text { BD-II } \\
5 \% \text { BD-NOS } \\
N=41\end{array}$ & $\begin{array}{l}\text { Mild to moderate } \\
\text { hypomania or } \\
\text { mild mania; } \\
\text { CGI-BD } \geq 3 \text { AND } \\
<5\end{array}$ & $\begin{array}{l}\text { Substance Abuse } \\
\text { Other Mental Health } \\
\text { Neurological Disorders } \\
\text { Pregnant Nursing } \\
\text { Labs/Other Conditions }\end{array}$ \\
\hline $\begin{array}{l}\text { Vieta, } 2010^{35} \\
\text { Multisite } \\
3 \text { continents } \\
\text { High ROB } \\
20565430\end{array}$ & $\begin{array}{l}\text { I: Quetiapine } \\
\text { C: Placebo } \\
\text { (Paliperidone } \\
\text { arm } \\
\text { comparisons } \\
\text { in Other } \\
\text { Drugs } \\
\text { section) }\end{array}$ & $\begin{array}{l}\text { BD-I; } \\
\text { DSM-IV }\end{array}$ & $\begin{array}{l}\text { Mean Age } 39 \\
(18-65) 43 \% \\
\text { Female Race } \\
\text { NR } \\
\mathrm{N}=493\end{array}$ & $\begin{array}{l}\text { Mania; } \\
\text { YMRS } \geq 20\end{array}$ & $\begin{array}{l}\text { First Manic Episode } \\
\text { Schizoaffective } \\
\text { Substance Abuse } \\
\text { Neurological Disorders }\end{array}$ \\
\hline $\begin{array}{l}\text { Li, } 2008^{90} \\
\text { Multisite } \\
\text { China } \\
\text { Moderate ROB } \\
18028587\end{array}$ & $\begin{array}{l}\text { I: Quetiapine } \\
\text { C: Lithium }\end{array}$ & $\begin{array}{l}\text { BD-I; } \\
\text { CCMD-3 }\end{array}$ & $\begin{array}{l}\text { Mean Age } 33 \\
(18-65) \\
53 \% \text { Female } \\
\text { Race NR } \\
N=155\end{array}$ & $\begin{array}{l}\text { Mania; YMRS } \geq \\
20\end{array}$ & $\begin{array}{l}\text { Substance Abuse } \\
\text { Taking Other Meds } \\
\text { Pregnant/Nursing } \\
\text { Labs/Other Conditions }\end{array}$ \\
\hline
\end{tabular}




\begin{tabular}{|c|c|c|c|c|c|}
\hline $\begin{array}{c}\text { Author, Year } \\
\text { Single-Multisite } \\
\text { Local/Continent } \\
\text { Risk of Bias } \\
\text { PMID } \\
\end{array}$ & $\begin{array}{l}\text { Intervention/ } \\
\text { Comparison }\end{array}$ & $\begin{array}{c}\text { BD Type; } \\
\text { Diagnostic } \\
\text { Criteria }\end{array}$ & Demographics & Current Episode & Key Exclusions \\
\hline $\begin{array}{l}\text { Bowden, } 2005^{36} \\
\text { Multisite } \\
2 \text { Continents } \\
\text { High ROB } \\
15669897\end{array}$ & $\begin{array}{l}\text { I: Quetiapine } \\
\text { C1: Lithium } \\
\text { C2: Placebo }\end{array}$ & $\begin{array}{l}\text { BD-I; } \\
\text { DSM-IV }\end{array}$ & $\begin{array}{l}\text { Mean Age } 39 \\
(18-73) \\
42 \% \text { Female } \\
\text { Race NR } \\
\mathrm{N}=300\end{array}$ & $\begin{array}{l}\text { Mania; YMRS } \geq \\
20 \text { including score } \\
\text { of at least } 4 \text { on } 2 \\
\text { of the } 4 \text { double- } \\
\text { weighted items } \\
\text { (irritability, } \\
\text { speech, content, } \\
\text { and disruptive/ } \\
\text { aggressive } \\
\text { behavior), CGI } \geq \\
4\end{array}$ & $\begin{array}{l}\text { First Manic Episode } \\
\text { Substance Abuse } \\
\text { Taking Other Meds } \\
\text { Pregnant/Nursing } \\
\text { Labs/Other Conditions }\end{array}$ \\
\hline $\begin{array}{l}\text { Mclntyre, } 2005^{37} \\
\text { Multisite } \\
3 \text { Continents } \\
\text { Moderate ROB } \\
16139175\end{array}$ & $\begin{array}{l}\text { I: Quetiapine } \\
\text { C1: } \\
\text { Haloperidol } \\
\text { C2: Placebo }\end{array}$ & $\begin{array}{l}\text { BD-I; } \\
\text { DSM-IV }\end{array}$ & $\begin{array}{l}\text { Mean Age } 43 \\
(18-79) \\
63 \% \text { Female } \\
\text { Race NR } \\
N=299\end{array}$ & $\begin{array}{l}\text { Mania; } \\
\text { YMRS } \geq 20 \text { AND } \geq \\
4 \text { on at least } 2 \\
\text { YMRS subscales } \\
\text { AND } \geq 4 \text { on CGI- } \\
\text { BD-S }\end{array}$ & $\begin{array}{l}\text { First Manic Episode } \\
\text { Substance Abuse } \\
\text { Neurological Disorders } \\
\text { Taking Other Meds } \\
\text { Pregnant/Nursing } \\
\text { Labs/Other Conditions }\end{array}$ \\
\hline
\end{tabular}

$\mathrm{BD}=$ bipolar disorder; $\mathrm{C}=$ Comparison; $\mathrm{CCMD}=$ Chinese Classification and Diagnosis Criteria of Mental Disorder, $3^{\text {rd }}$ Version ; DSM-IV=Diagnostic and Statistical Manual Fourth Edition; CGI=Clinical global impression scale, bipolar edition, severity; I=Intervention; MADRS= Montgomery-Asberg depression scale; N=number; NR=Not Reported; ROB=Risk of Bias; YMRS=Young Mania Rating Scale

\section{Quetiapine Alone Versus Placebo}

Low-strength evidence (moderate study limitations, imprecision) from four RCTs showed improved response rates (OR 2.07, 95\% CI 1.39 to 3.09, $\mathrm{n}=1,007$ ) and manic symptom improvement close to the MID (YMRS, mean difference 4.92, 95\% CI 0.31 to 9.53, n=699) for participants receiving quetiapine. ${ }^{35-38}$ Evidence was insufficient to address remission rates $(\mathrm{n}=699)$ due to fewer studies of higher risk of bias contributing to the outcome. Low-strength evidence (moderate study limitations, imprecision) showed CGI improved for participants using quetiapine, but the improvement was about half the MID (mean difference 0.54 , 95\% CI 0.35 to $0.74, n=806$ ). Withdrawal due to lack of efficacy was lower for quetiapine but overall withdrawal and withdrawal due to adverse events did not differ between groups (low-strength, $\mathrm{n}=1,007$ ). Most studies reported no serious adverse events and no differences between groups for extrapyramidal symptoms. One small study $(\mathrm{n}=41)$ enrolling patients with mild to moderate hypomania or mild mania also found participants using quetiapine showed improvements in manic symptoms (YMRS) and CGI. ${ }^{39}$ Weight gain greater than 7 percent was infrequently reported but tended to be more common in participants using quetiapine.

\section{Quetiapine Alone Versus Active Control}

Evidence was insufficient for all outcomes from one RCT $(n=199)$ to address whether quetiapine was better for acute mania than haloperidol in adults with BD-I, due to a single study and imprecision. ${ }^{37}$ The study reported no differences between groups for response or remission rates, manic symptom improvement, CGI, or withdrawals. Participants using haloperidol reported more extrapyramidal symptoms; otherwise, no differences in serious adverse events were noted. 
Evidence was insufficient from two RCTs ( $\mathrm{n}=456)$ to address whether quetiapine was better for acute mania than lithium for in adults with BD-I, due to high study limitations, inconsistency, and imprecision. ${ }^{36,90}$ Both studies reported response and remission rates, and change in manic symptoms; one trial reported benefit with quetiapine ${ }^{90}$ and one reported no difference. ${ }^{36}$ One trial reported no difference in CGI. ${ }^{36}$ Both reported no difference in withdrawals or serious adverse events.

\section{Quetiapine Plus Mood Stabilizers}

Table 15 summarizes bipolar type and major inclusion and exclusion criteria for each quetiapine plus mood stabilizers study for acute mania. Appendix E provides further detail.

Table 15. Population and inclusion criteria for quetiapine plus mood stabilizer studies for acute mania

\begin{tabular}{|c|c|c|c|c|c|}
\hline $\begin{array}{l}\text { Author, Year } \\
\text { Single- } \\
\text { Multisite } \\
\text { Locall } \\
\text { Continent } \\
\text { Risk of Bias } \\
\text { PMID }\end{array}$ & $\begin{array}{l}\text { Intervention/ } \\
\text { Comparison }\end{array}$ & $\begin{array}{l}\text { BD Type; } \\
\text { Diagnostic } \\
\text { Criteria }\end{array}$ & Demographics & Current Episode & Key Exclusions \\
\hline $\begin{array}{l}\text { Yatham, } \\
2007^{91} \\
\text { Multisite } \\
4 \text { Continents } \\
\text { High ROB } \\
17519644\end{array}$ & $\begin{array}{l}\text { I: Quetiapine } \\
\text { + Lithium/ } \\
\text { Valproate } \\
\\
\text { C: Placebo + } \\
\text { Lithium/ } \\
\text { Valproate }\end{array}$ & $\begin{array}{l}\text { BD-I; } \\
\text { DSM-IV }\end{array}$ & $\begin{array}{l}\text { Mean Age } 40 \\
\text { (range NR) } \\
50 \% \text { Female } \\
\text { Race NR } \\
N=200\end{array}$ & $\begin{array}{l}\text { Mania; } \\
\text { YMRS } \geq 20 \text { AND } \geq 4 \\
\text { on at least } 2 \text { YMRS } \\
\text { subscales AND CGI- } \\
\text { BP-S } \geq 4\end{array}$ & $\begin{array}{l}\text { First Manic Episode } \\
\text { Taking Other Meds } \\
\text { Pregnant/Nursing }\end{array}$ \\
\hline $\begin{array}{l}\text { Yatham, } \\
2004^{92} \\
\text { Singlesite } \\
4 \text { continents } \\
\text { Moderate ROB } \\
15538120\end{array}$ & $\begin{array}{l}\text { I: Quetiapine } \\
\text { + Lithium/ } \\
\text { Valproate } \\
\text { C: Placebo + } \\
\text { Lithium/ } \\
\text { Valproate }\end{array}$ & $\begin{array}{l}\text { BD-I; } \\
\text { DSM-IV }\end{array}$ & $\begin{array}{l}\text { Mean Age NR } \\
(18-70) \\
47 \% \text { Female } \\
\text { Race NR } \\
N=370\end{array}$ & $\begin{array}{l}\text { Mania; } \\
\text { At least one manic or } \\
\text { mixed episode in } \\
\text { previous } 5 \text { years. } \\
\text { YMRS } \geq 20 \text {, including } \\
\text { score } \geq 4 \text { on two core } \\
\text { YMRS items; CGI- } \\
\text { BP } \geq 4\end{array}$ & $\begin{array}{l}\text { First Manic Episode } \\
\text { Substance Abuse } \\
\text { Other Mental Health } \\
\text { Pregnant/Nursing }\end{array}$ \\
\hline
\end{tabular}

BD=Bipolar Disorder; C=Comparison; CGI-BP-S=Clinical Global Impression scale, bipolar edition, severity; DSMIV=Diagnostic and Statistical Manual Fourth Edition; I=Intervention; N=number; NR=Not Reported; ROB=Risk of Bias; YMRS=Young Mania Rating Scale

\section{Quetiapine Combination Versus Placebo}

Evidence was insufficient for all outcomes from two RCTs $(n=570)$ to address whether quetipine plus mood stabilizers was better for acute mania than mood stabilizer alone in adults with BD-I, due to high study limitations and imprecise data. ${ }^{91,92}$ The studies reported quetiapine added to mood stabilizers improved response and remission rates, manic symptoms (YMRS), and CGI score. Both studies reported no differences between groups in withdrawal rates and serious adverse events, and results for extrapyramidal symptoms were mixed.

\section{Risperidone}

We identified three eligible publications reporting three unique RCTs of risperidone and one RCT for risperidone plus mood stabilizers for acute mania with at least 3 weeks followup. ${ }^{40,41,96}$ ${ }^{97}$ One study was assessed as low risk of bias and three studies were assessed as moderate. All 
were funded by industry. Four additional studies were excluded for greater than 50 percent attrition. ${ }^{84,94,98,99}$ Three studies used placebo comparators and two studies used active comparators. Sample sizes ranged from 45 to 438 and enrolled BD-I participants.

\section{Risperidone Alone}

Table 16 summarizes the bipolar type and major inclusion and exclusion criteria for each study of risperidone alone for acute mania. Appendix E provides further detail.

Table 16. Population and inclusion criteria for studies of risperidone alone for acute mania

\begin{tabular}{|c|c|c|c|c|c|}
\hline $\begin{array}{c}\text { Author, Year } \\
\text { Single-Multisite } \\
\text { Local/Continent } \\
\text { Risk of Bias } \\
\text { PMID } \\
\end{array}$ & $\begin{array}{l}\text { Intervention/ } \\
\text { Comparison }\end{array}$ & $\begin{array}{l}\text { BD Type; } \\
\text { Diagnostic } \\
\text { Criteria }\end{array}$ & Demographics & Current Episode & Key Exclusions \\
\hline $\begin{array}{l}\text { Khanna, } 2005^{40} \\
\text { Multisite } \\
\text { India } \\
\text { Moderate ROB } \\
16135859\end{array}$ & $\begin{array}{l}\text { I: } \\
\text { Risperidone } \\
\text { C:Placebo }\end{array}$ & $\begin{array}{l}\text { BD-I; } \\
\text { DSM-IV }\end{array}$ & $\begin{array}{l}\text { Mean Age } 35 \\
\text { (NR) } \\
38 \% \text { Female } \\
\text { Race NR } \\
N=290\end{array}$ & $\begin{array}{l}\text { Mania; } \\
\text { YMRS } \geq 20\end{array}$ & $\begin{array}{l}\text { Schizoaffective } \\
\text { Substance Abuse } \\
\text { Other Mental Health } \\
\text { Taking Other Meds }\end{array}$ \\
\hline $\begin{array}{l}\text { Smulevich, } \\
2005^{41} \\
\text { Multisite } \\
2 \text { Continents } \\
\text { Moderate ROB } \\
15572276\end{array}$ & $\begin{array}{l}\text { I: } \\
\text { Risperidone } \\
\text { C1: } \\
\text { Haloperidol } \\
\text { C2: Placebo }\end{array}$ & $\begin{array}{l}\text { BD-I; } \\
\text { DSM-IV }\end{array}$ & $\begin{array}{l}\text { Mean Age } 40 \\
(18-79) \\
49 \% \text { Female } \\
\text { Race NR } \\
N=438\end{array}$ & $\begin{array}{l}\text { Mania; } \\
\text { YMRS } \geq 20 \text { and } \\
\text { MADRS } \leq 20\end{array}$ & $\begin{array}{l}\text { First Manic Episode } \\
\text { Schizoaffective } \\
\text { Substance Abuse } \\
\text { Other Mental Health } \\
\text { Taking Other Meds }\end{array}$ \\
\hline $\begin{array}{l}\text { Segal, } 1998^{96} \\
\text { Singlesite } \\
\text { South Africa } \\
\text { Moderate ROB } \\
9617509\end{array}$ & $\begin{array}{l}\text { I: } \\
\text { Risperidone } \\
\text { C1: } \\
\text { Haloperidol } \\
\text { C2: Lithium }\end{array}$ & $\begin{array}{l}\text { BD-I, } \\
\text { DSM-IV }\end{array}$ & $\begin{array}{l}\text { Mean Age } 34 \\
(19-58) \\
78 \% \text { Female } \\
\text { Race NR } \\
N=45\end{array}$ & $\begin{array}{l}\text { Manic; } \\
\text { DSM-IV Criteria for } \\
\text { bipolar disorder, } \\
\text { manic phase }\end{array}$ & $\begin{array}{l}\text { Substance Abuse } \\
\text { Taking Other Meds } \\
\text { Pregnant/Nursing } \\
\text { Labs/Other } \\
\text { Conditions }\end{array}$ \\
\hline
\end{tabular}

BD=bipolar disorder; C=Comparison; DSM-IV=Diagnostic and Statistical Manual Fourth Edition; I=Intervention; MADRS= Montgomery-Asberg depression scale; N=number; NR=Not Reported; ROB=Risk of Bias; YMRS=Young Mania Rating Scale

\section{Risperidone Alone Versus Placebo}

Low-strength evidence (moderate study limitations, imprecision) from two studies $(\mathrm{n}=584)$ showed risperidone was better for acute mania than placebo for adults with BD-I. ${ }^{40,41}$ Although we were unable to conduct a meta-analysis based on the two studies, the finding in favor of risperidone was consistent across the studies for response rate, manic symptom improvement (YMRS), and CGI. No serious adverse events were reported. However, participants using risperidone experienced more extrapyramidal symptoms than those using placebo.

\section{Risperidone Alone Versus Active Control}

Evidence was insufficient for all outcomes to address whether risperidone performed better than an active comparator for acute mania, due to moderate study limitations, inconsistency, and imprecision. Findings were mixed from two studies comparing risperidone to haloperidol in adults with BD-I. ${ }^{41,96}$ Those who received risperidone also had lower total scores on the extrapyramidal symptom rating scale at 3 weeks compared to those who received haloperidol. 
Withdrawal rates were similar between groups. One study compared risperidone to lithium, finding no difference between groups in bipolar outcomes and extrapyramidal symptoms at 4 weeks. ${ }^{96}$ Withdrawal rates were not reported.

Results for risperidone versus olanzapine were reported in the olanzapine versus active comparator section above and were determined to yield insufficient evidence.

\section{Risperidone Plus Mood Stabilizers}

Table 17 summarizes bipolar type and major inclusion and exclusion criteria for each risperidone plus mood stabilizers study for acute mania. Appendix E provides further detail.

Table 17. Population and inclusion criteria for risperidone plus mood stabilizer studies for acute mania

\begin{tabular}{|c|c|c|c|c|c|}
\hline $\begin{array}{l}\text { Author, Year } \\
\text { Single- } \\
\text { Multisite } \\
\text { Local/Contin } \\
\text { ent } \\
\text { Risk of Bias } \\
\text { PMID }\end{array}$ & $\begin{array}{l}\text { Intervention/ } \\
\text { Comparison }\end{array}$ & $\begin{array}{c}\text { BD Type; } \\
\text { Diagnostic } \\
\text { Criteria }\end{array}$ & Demographics & $\begin{array}{l}\text { Current } \\
\text { Episode }\end{array}$ & Key Exclusions \\
\hline $\begin{array}{l}\text { Yatham, } \\
2003^{97} \\
\text { Multisite } \\
\text { 4 Continents } \\
\text { Low ROB } \\
12562742\end{array}$ & $\begin{array}{l}\text { I: Risperidone } \\
\text { + Lithium } \\
\text { or Divalproex } \\
\text { or } \\
\text { Carbamazepine } \\
\text { C: Placebo + } \\
\text { Lithium } \\
\text { or Divalproex } \\
\text { or } \\
\text { Carbamazepine }\end{array}$ & $\begin{array}{l}\text { BD-I; } \\
\text { DSM-IV }\end{array}$ & $\begin{array}{l}\text { Mean Age NR } \\
(19-65) \\
58 \% \text { Female } \\
\text { Race NR } \\
N=150\end{array}$ & $\begin{array}{l}\text { Mania; } \\
\text { YMRS } \geq 20\end{array}$ & $\begin{array}{l}\text { Schizoaffective } \\
\text { Substance Abuse } \\
\text { Other Mental Health } \\
\text { Pregnant/Nursing } \\
\text { Labs/Other Conditions }\end{array}$ \\
\hline
\end{tabular}

BD=Bipolar Disorder; C=Comparison; DSM-IV=Diagnostic and Statistical Manual Fourth Edition; I=Intervention; N=number; NR=Not Reported; ROB=Risk of Bias; YMRS=Young Mania Rating Scale

\section{Risperidone Combination Versus Placebo}

Evidence from this single study of 150 participants with BD-I was rated as insufficient due to unknown consistency and imprecision. The study reported that adding risperidone to mood stabilizers improved response rates and CGI and a trend toward reduced manic symptoms (YMRS). No differences were reported in adverse events; however, participants using risperidone experienced more extrapyramidal symptoms.

\section{Ziprasidone}

We identified two eligible publications reporting two unique RCTs of ziprasidone and one RCT of ziprasidone plus mood stabilizers for acute mania with at least 3 weeks followup. ${ }^{42,43,100}$ Two studies were assessed as moderate risk of bias, and one was high. All were funded by industry. Two additional studies were excluded for greater than 50 percent attrition. ${ }^{101,102}$ Sample sizes ranged from 197 to 680.

\section{Ziprasidone Alone}

Table 18 summarizes the bipolar type and major inclusion and exclusion criteria for each study of ziprasidone alone for acute mania. Appendix E provides further detail. 
Table 18. Population and inclusion criteria for studies of ziprasidone alone for acute mania

\begin{tabular}{|c|c|c|c|c|c|}
\hline $\begin{array}{c}\text { Author, Year } \\
\text { Single- } \\
\text { Multisite } \\
\text { Local/Contine } \\
\text { nt } \\
\text { Risk of Bias } \\
\text { PMID }\end{array}$ & $\begin{array}{l}\text { Intervention/ } \\
\text { Comparison }\end{array}$ & $\begin{array}{c}\text { BD Type; } \\
\text { Diagnostic } \\
\text { Criteria }\end{array}$ & Demographics & Current Episode & Key Exclusions \\
\hline $\begin{array}{l}\text { Potkin, } 2005^{42} \\
\text { RCT } \\
\text { Multisite } \\
2 \text { Continents } \\
\text { Moderate ROB } \\
16012271\end{array}$ & $\begin{array}{l}\text { I: Ziprasidone } \\
\text { C: Placebo }\end{array}$ & $\begin{array}{l}\text { BD-I; } \\
\text { DSM-IV }\end{array}$ & $\begin{array}{l}\text { Mean Age } 39 \\
(19-71) \\
49 \% \text { Female } \\
62 \% \text { White } \\
N=205\end{array}$ & $\begin{array}{l}\text { Mania; } \\
\text { YMRS } \geq 14 \text { with } \\
\text { score } \geq 2 \text { on four } \\
\text { items at screening } \\
\text { and admission }\end{array}$ & $\begin{array}{l}\text { Schizoaffective } \\
\text { Substance Abuse } \\
\text { Other Mental Health } \\
\text { Taking Other Meds } \\
\text { Pregnant/Nursing }\end{array}$ \\
\hline $\begin{array}{l}\text { Keck, } 2003^{43} \\
\text { RCT } \\
\text { Multisite } \\
2 \text { Continents } \\
\text { Moderate ROB } \\
12668364\end{array}$ & $\begin{array}{l}\text { I: Ziprasidone } \\
\text { C: Placebo }\end{array}$ & $\begin{array}{l}\text { BD-I; } \\
\text { DSM-IV }\end{array}$ & $\begin{array}{l}\text { Mean Age } 38 \\
(18-66) \\
46 \% \text { Female } \\
\text { Race NR } \\
N=197\end{array}$ & $\begin{array}{l}\text { Current Manic } \\
\text { episode; YMRS } \geq \\
14 \text { with score } \geq 2 \text { on } \\
\text { four items at } \\
\text { screening and } \\
\text { admission }\end{array}$ & $\begin{array}{l}\text { Schizoaffective } \\
\text { Substance Abuse } \\
\text { Other Mental Health } \\
\text { Taking Other Meds } \\
\text { Pregnant/Nursing } \\
\text { Labs/Other Conditions }\end{array}$ \\
\hline
\end{tabular}

BD=bipolar disorder; C=Comparison; DSM-IV=Diagnostic and Statistical Manual Fourth Edition; I=Intervention; MADRS= Montgomery-Asberg depression scale; N=number; NR=Not Reported; ROB=Risk of Bias; YMRS=Young Mania Rating Scale

\section{Ziprasidone Alone Versus Placebo}

Low-strength evidence (moderate study limitations, imprecision) from two studies ( $\mathrm{n}=402)$ showed ziprasidone was better for acute mania than placebo in adults with BD-I. ${ }^{42,43}$ Although we were unable to conduct a meta-analysis, the finding in favor of ziprasidone was consistent across the studies for response rate, manic symptom improvement (YMRS), and CGI. Withdrawal due to lack of effect also was lower for the ziprasidone group, while no differences were seen for overall withdrawal or adverse events. Serious adverse events were reported in one study, with no difference between groups. However, in one study participants using ziprasidone experienced more extrapyramidal symptoms than those using placebo. ${ }^{42}$

\section{Ziprasidone Plus Mood Stabilizers}

Table 19 summarizes bipolar type and major inclusion and exclusion criteria for each ziprasidone plus mood stabilizers study for acute mania. Appendix E provides further detail. 
Table 19. Population and inclusion criteria for ziprasidone plus mood stabilizer studies for acute mania

\begin{tabular}{|c|c|c|c|c|c|}
\hline $\begin{array}{l}\text { Author, Year } \\
\text { Single- } \\
\text { Multisite } \\
\text { Local/Contin } \\
\text { ent } \\
\text { Risk of Bias } \\
\text { PMID } \\
\end{array}$ & $\begin{array}{l}\text { Intervention/ } \\
\text { Comparison }\end{array}$ & $\begin{array}{c}\text { BD Type; } \\
\text { Diagnostic } \\
\text { Criteria }\end{array}$ & Demographics & $\begin{array}{l}\text { Current } \\
\text { Episode }\end{array}$ & Key Exclusions \\
\hline $\begin{array}{l}\text { Sachs, } \\
2012^{100} \\
\text { RCT } \\
\text { Multisite } \\
\text { US } \\
\text { High ROB } \\
23218157\end{array}$ & $\begin{array}{l}\text { I1: Low Dose } \\
\text { Ziprasidone } \\
40-80 \mathrm{mg} / \text { day + } \\
\text { Lithium/Valproate } \\
\text { 12:High Dose } \\
\text { Ziprasidone } \\
120-160 \mathrm{mg} / \text { day + } \\
\text { Lithium/Valproate } \\
\text { C: Placebo + } \\
\text { Lithium/Valproate }\end{array}$ & $\begin{array}{l}\text { BD-I; } \\
\text { DSM-IV }\end{array}$ & $\begin{array}{l}\text { Mean Age } 41 \\
\text { (NR) } \\
50 \% \text { Female } \\
65 \% \text { White } \\
N=680\end{array}$ & $\begin{array}{l}\text { Mania; } \\
\text { YMRS } \geq 18 \text { with } \\
25 \% \\
\text { improvement } \\
\text { between } \\
\text { screening and } \\
\text { baseline; current } \\
\text { episode } \leq 3 \\
\text { months }\end{array}$ & $\begin{array}{l}\text { First manic episode } \\
\text { Schizoaffective } \\
\text { Substance Abuse } \\
\text { Other Mental Health } \\
\text { Conditions } \\
\text { Taking Other Meds } \\
\text { Labs/Other Conditions }\end{array}$ \\
\hline
\end{tabular}

BD=Bipolar Disorder; C=Comparison; DSM-IV=Diagnostic and Statistical Manual Fourth Edition; I=Intervention; N=number; NR=Not Reported; ROB=Risk of Bias; YMRS=Young Mania Rating Scale

\section{Ziprasidone Combination Versus Placebo}

Evidence from a single study of 680 participants with BD-I was rated as insufficient due to a single high risk of bias study and imprecision. The study reported no differences between groups in manic symptom (YMRS) and CGI. No differences were reported in adverse events, however participants using high dose ziprasidone experienced more extrapyramidal symptoms.

\section{Haloperidol}

We identified two eligible publications reporting two unique RCTs of haloperidol for acute mania with at least 3 weeks followup. ${ }^{37,41}$ One study was assessed as moderate risk of bias ${ }^{41}$ and one was assessed as high. ${ }^{37}$ Both were funded by industry. One additional study was excluded for greater than 50 percent attrition. ${ }^{73}$ Both studies used a placebo comparator. Sample sizes ranged from 299 to 438 and recruited participants with BD-I.

\section{Haloperidol Alone}

Table 20 summarizes the bipolar type and major inclusion and exclusion criteria for each study. Appendix E provides further detail. 
Table 20. Population and inclusion criteria for studies of haloperidol alone for acute mania

\begin{tabular}{|c|c|c|c|c|c|}
\hline $\begin{array}{c}\text { Author, Year } \\
\text { Single-Multisite } \\
\text { Local/Continent } \\
\text { Risk of Bias } \\
\text { PMID }\end{array}$ & $\begin{array}{l}\text { Intervention/ } \\
\text { Comparison }\end{array}$ & $\begin{array}{c}\text { BD Type; } \\
\text { Diagnostic } \\
\text { Criteria }\end{array}$ & Demographics & Current Episode & Key Exclusions \\
\hline $\begin{array}{l}\text { Mclntyre, } 2005^{37} \\
\text { Multisite } \\
3 \text { Continents } \\
\text { Moderate } \\
\text { High ROB (for } \\
\text { haloperidol } \\
\text { comparisons) } \\
16139175\end{array}$ & $\begin{array}{l}\text { I: Haloperidol } \\
\text { C1: } \\
\text { Quetiapine } \\
\text { C2: Placebo }\end{array}$ & $\begin{array}{l}\text { BD-I; } \\
\text { DSM-IV }\end{array}$ & $\begin{array}{l}\text { Mean Age } 43 \\
(18-79) \\
63 \% \text { Female } \\
\text { Race NR } \\
N=299\end{array}$ & $\begin{array}{l}\text { Manic; } \\
\text { YMRS } \geq 20 \\
\text { CGI-BP } \geq 4\end{array}$ & $\begin{array}{l}\text { First Manic Episode } \\
\text { Substance Abuse } \\
\text { Other Mental Health } \\
\text { Neurological } \\
\text { disorders } \\
\text { Taking Other Meds } \\
\text { Pregnant/Nursing } \\
\text { Labs/Other } \\
\text { Conditions }\end{array}$ \\
\hline $\begin{array}{l}\text { Smulevich, } 2005^{41} \\
\text { Multisite } \\
\text { NR } \\
\text { Moderate ROB } \\
15572276\end{array}$ & $\begin{array}{l}\text { I: Haloperidol } \\
\text { C1: } \\
\text { Risperidone } \\
\text { C2: Placebo }\end{array}$ & $\begin{array}{l}\text { BD-I; } \\
\text { DSM-IV }\end{array}$ & $\begin{array}{l}\text { Mean Age } 40 \\
(18-83) \\
47 \% \text { Female } \\
65 \% \text { White } \\
N=438\end{array}$ & $\begin{array}{l}\text { Manic } \\
\text { YMRS } \geq 20 \text { AND } \\
\text { MADRS } \leq 20\end{array}$ & $\begin{array}{l}\text { Schizoaffective } \\
\text { Substance Abuse } \\
\text { Other Mental Health } \\
\text { Taking Other Meds } \\
\text { Labs/Other } \\
\text { Conditions }\end{array}$ \\
\hline
\end{tabular}

BD=Bipolar Disorder; C=Comparison; CGI-BP= clinical global impression scale, bipolar edition; DSM-IV=Diagnostic and Statistical Manual Fourth Edition; I=Intervention; MADRS=Montgomery-Asberg depression scale; N=number; NR=Not Reported; ROB=Risk of Bias; YMRS=Young Mania Rating Scale

\section{Haloperidol Versus Placebo}

Evidence was insufficient for all outcomes from two studies $(n=483)$ to address whether haloperidol was better for acute mania than placebo in participants with BD-I, due to high study limitations and imprecision. ${ }^{37,41}$ Studies reported results generally favored haloperidol. Neither study reported serious adverse events.

\section{Haloperidol Versus Active Control}

Results for haloperidol versus aripiprazole were reported in the aripiprazole versus active comparator section above and yielded insufficient evidence.

\section{Mood Stabilizers for Acute Mania}

\section{Key Points}

- $\quad$ Studies for mood stabilizers were sparse and scattered.

- Evidence was largely insufficient to draw conclusions regarding mood stabilizers compared to placebo or other drugs for BD-I for the primary outcomes of interest (response, symptom scores, and function).

- Low-strength evidence showed lithium increased response and remission rates and manic symptom improvement in BD-I participants with acute mania compared to placebo.

- When reported, all comparisons tended to show no differences between groups in serious adverse events. Participants using carbamazepine reported experiencing more severe rash and adverse events compared to placebo.

- The ability to draw stronger conclusions was hindered by high attrition rates. 


\section{Eligible Studies for Mood Stabilizers}

Four mood stabilizers, all FDA approved for use in patients with bipolar disorder experiencing mania, were examined in 12 publications of 12 unique studies for BD patients with acute mania. All were tested as single drugs: carbamazepine, divalproex/valproate, lamotrigine, and lithium. All studies enrolled adults with BD-I. Only one study (for lithium) also included adults with BD-II. There were no studies assessing drug effectiveness in treatment of hypomania. The large majority of studies with usable outcomes were measured at 3 weeks duration.

Appendix F provides detailed evidence tables, summary risk of bias assessments, forest plots when appropriate, and assessments of strength of evidence for key comparisons and outcomes. A summary of findings with at least low-strength evidence for mood stabilizers for acute mania are provided in Table 21. Any intervention and comparison not listed in Table 21, or outcome not listed for an included intervention and comparison, was found to have an evidence base insufficient to draw conclusions.

Table 21. Summary of findings with at least low-strength evidence for mood stabilizers for acute mania

\begin{tabular}{|c|c|c|c|}
\hline Intervention & $\begin{array}{c}\text { \# Studies/ } \\
\text { Design } \\
\text { (n Analyzed) } \\
\text { Timing }\end{array}$ & Findings & Strength of Evidence \\
\hline \multirow{3}{*}{$\begin{array}{l}\text { Lithium vs. } \\
\text { placebo }\end{array}$} & $\begin{array}{l}\text { 1 } \mathrm{RCT}^{36}+1 \\
\mathrm{IPD}^{103} \\
(\mathrm{n}=325) \\
3 \text { weeks }\end{array}$ & $\begin{array}{l}\text { Remission and Response Rates: Favors Lithium } \\
\text { (not pooled) }\end{array}$ & $\begin{array}{l}\text { Low } \\
\text { (moderate study } \\
\text { limitations, imprecise) }\end{array}$ \\
\hline & $\begin{array}{l}3 \mathrm{RCTS}^{36,103} \\
(\mathrm{n}=325) \\
3 \text { weeks }\end{array}$ & $\begin{array}{l}\text { YMRS: Favors Lithium, } \\
\text { MD } 5.81 \text { (95\% Cl 2.21, 9.4; MID=6) } \\
\text { Withdrawal (Overall): No difference }\end{array}$ & $\begin{array}{l}\text { Low } \\
\text { (moderate study } \\
\text { limitations, imprecise) }\end{array}$ \\
\hline & $\begin{array}{l}1 \mathrm{IPD}^{103} \\
(\mathrm{n}=450) \\
3 \text { weeks }\end{array}$ & Withdrawal (Lack of Efficacy, AE): No difference & $\begin{array}{l}\text { Low } \\
\text { (moderate study } \\
\text { limitations, imprecise) }\end{array}$ \\
\hline
\end{tabular}

$\mathrm{AE}=$ adverse events; $\mathrm{CI}=$ confidence interval; $\mathrm{IPD}=$ Individual patient data; $\mathrm{MD}=$ mean difference; $\mathrm{MID}=$ minimally important difference; $n=$ number; RCT=randomized controlled trial; YMRS=Young mania rating scale

\section{Carbamazepine}

We identified four RCTs examining carbamazepine for acute mania with at least 3 weeks followup. ${ }^{104-107}$ One study was moderate risk of bias and three were high. Three additional studies were excluded for greater than 50 percent attrition. ${ }^{108-110}$ Three studies were funded at least in part by industry. One study used placebo ${ }^{104}$ and three used active comparators. ${ }^{105-107}$ Sample sizes ranged from 30 to 443. All trials recruited participants with BD-I. Table 22 summarizes the bipolar type and major inclusion and exclusion criteria for each study. Appendix F provides further detail. 
Table 22. Population and inclusion criteria for carbamazepine for acute mania

\begin{tabular}{|c|c|c|c|c|c|}
\hline $\begin{array}{l}\text { Author, Year } \\
\text { Single-Multisite } \\
\text { Local/Continent } \\
\text { Risk of Bias } \\
\text { PMID }\end{array}$ & $\begin{array}{l}\text { Intervention/ } \\
\text { Comparison }\end{array}$ & $\begin{array}{l}\text { BD Type; } \\
\text { Diagnostic } \\
\text { Criteria }\end{array}$ & Demographics & Current Episode & Key Exclusions \\
\hline $\begin{array}{l}\text { Weisler, } 2006^{104} \\
\text { Multisite } \\
2 \text { Continents } \\
\text { High ROB } \\
16529527\end{array}$ & $\begin{array}{l}\text { I: } \\
\text { Carbamazepine } \\
\text { C: Placebo }\end{array}$ & $\begin{array}{l}\text { BD-I; } \\
\text { DSM-IV }\end{array}$ & $\begin{array}{l}\text { Mean Age } 38 \\
(18-76) \\
38 \% \text { Female } \\
59 \% \text { White } \\
N=443\end{array}$ & $\begin{array}{l}\text { Manic/Mixed; } \\
\text { YMRS } \geq 20\end{array}$ & Taking Other Meds \\
\hline $\begin{array}{l}\text { Vasudev, } \\
2000^{105} \\
\text { Singlesite } \\
\text { India } \\
\text { Moderate ROB } \\
10867972\end{array}$ & $\begin{array}{l}\text { I: } \\
\text { Carbamazepine } \\
\text { C: Valproate }\end{array}$ & $\begin{array}{l}\text { BD-I; } \\
\text { DSM-III }\end{array}$ & $\begin{array}{l}\text { Mean Age NR } \\
80 \% \text { Female } \\
\text { Race NR } \\
\mathrm{N}=30\end{array}$ & $\begin{array}{l}\text { Mania; } \\
\text { DSM-III-R criteria } \\
\text { for BD diagnosis; } \\
\text { YMRS } \geq 20\end{array}$ & $\begin{array}{l}\text { Substance Abuse } \\
\text { Neurological } \\
\text { Disorders } \\
\text { Taking other meds } \\
\text { Pregnant/Nursing }\end{array}$ \\
\hline $\begin{array}{l}\text { Small, } 1991^{106} \\
\text { Singlesite } \\
\text { US } \\
\text { High ROB } \\
1929761\end{array}$ & $\begin{array}{l}\text { I: } \\
\text { Carbamazepine } \\
\text { C: Lithium }\end{array}$ & $\begin{array}{l}\text { BD-I; } \\
\text { DSM-III }\end{array}$ & $\begin{array}{l}\text { Mean Age } 39 \\
(22-73) \\
38 \% \text { Female } \\
59 \% \text { White } \\
N=48\end{array}$ & $\begin{array}{l}\text { Manic/Mixed; } \\
\text { YMRS } \geq 20\end{array}$ & $\begin{array}{l}\text { First Manic Episode } \\
\text { Substance Abuse } \\
\text { Other Mental Health }\end{array}$ \\
\hline $\begin{array}{l}\text { Lerer, } 1987^{107} \\
\text { Singlesite } \\
\text { US } \\
\text { High ROB } \\
3546274\end{array}$ & $\begin{array}{l}\text { I: } \\
\text { Carbamazepine } \\
\text { C: Lithium }\end{array}$ & $\begin{array}{l}\text { BD-I; } \\
\text { DSM-III }\end{array}$ & $\begin{array}{l}\text { Mean Age } 41 \\
(23-65) \\
54 \% \text { Female } \\
\text { Race NR } \\
N=34\end{array}$ & $\begin{array}{l}\text { Mania (not } \\
\text { defined) }\end{array}$ & $\begin{array}{l}\text { Neurological } \\
\text { Disorders }\end{array}$ \\
\hline
\end{tabular}

BD =Bipolar Disorder; C=Comparison; DSM-IV=Diagnostic and Statistical Manual Fourth Edition; I=Intervention; =number; NR=Not Reported; ROB=risk of bias; YMRS=Young Mania Rating Scale

\section{Carbamazepine Alone Versus Placebo}

Evidence was insufficient for all outcomes from one pooled analysis $(n=443)$ of two high risk of bias trials to address whether carbamazepine was better for acute mania than placebo in adults with BD-I, due to high study limitations and imprecision. ${ }^{104}$ The study reported improvements in participants receiving carbamazepine in response rate, manic symptoms (YMRS) and CGI.

Withdrawal for lack of efficacy and adverse events was lower for carbamazepine, but not overall withdrawals. Participants using carbamazepine experienced more frequent severe rash.

\section{Carbamazepine Alone Versus Active Control}

Evidence was insufficient for all outcomes from two small RCTs $(n=82)$ for carbamazepine compared to lithium ${ }^{106,107}$ and one small RCT $(n=30)$ for carbamazepine versus valproate for acute mania in adults with BD-I, due to high study limitations and imprecision. ${ }^{105}$ When reported, the studies generally reported no differences between groups for response rates, manic symptoms, CGI, or withdrawal rates. Participants receiving carbamazepine reported more adverse events. Evidence was also insufficient from one small RCT $(n=30)$ for carbamazepine compared to valproate due to single study and imprecision.

\section{Divalproex/Valproate}

We identified two RCTs examining divalproex for acute mania with at least 3 weeks followup. ${ }^{33,111}$ One study was low risk of bias and one was high. Both studies were funded by 
industry. Two additional studies were excluded for greater than 50 percent attrition. ${ }^{112,113}$ Both studies used placebo and one used active comparators. Sample sizes ranged from 364 to 521. One small study examining valproate versus no placebo was also included. ${ }^{46}$ Seven additional valproate studies were excluded for greater than 50 percent attrition. ${ }^{114-120}$ Table 23 summarizes the bipolar type and major inclusion and exclusion criteria for each study. Appendix F provides details.

Table 23. Population and inclusion criteria for divalproex/valproate for acute mania

\begin{tabular}{|c|c|c|c|c|c|}
\hline $\begin{array}{c}\text { Author, Year } \\
\text { Single-Multisite } \\
\text { Local/Continent } \\
\text { Risk of Bias } \\
\text { PMID }\end{array}$ & $\begin{array}{l}\text { Intervention/ } \\
\text { Comparison }\end{array}$ & $\begin{array}{c}\text { BD Type; } \\
\text { Diagnostic } \\
\text { Criteria }\end{array}$ & Demographics & Current Episode & Key Exclusions \\
\hline $\begin{array}{l}\text { Xu, } 2015^{46} \\
\text { Singlesite } \\
\text { China } \\
\text { Low ROB } \\
26060401\end{array}$ & $\begin{array}{l}\text { I: Olanzapine } \\
\text { C1: Olanzapine } \\
\text { + Valproate } \\
\text { C2: Valproate }\end{array}$ & $\begin{array}{l}\text { BD-I; } \\
\text { DSM-IV }\end{array}$ & $\begin{array}{l}\text { Mean Age } 31 \\
(19-60) \\
52 \% \text { Female } \\
\text { Race NR } \\
N=120\end{array}$ & $\begin{array}{l}\text { Manic; } \\
\text { YMRS } \geq 17\end{array}$ & $\begin{array}{l}\text { Substance Abuse } \\
\text { Neurological } \\
\text { Disorders } \\
\text { Taking Other Meds } \\
\text { Pregnant/Nursing }\end{array}$ \\
\hline $\begin{array}{l}\text { Tohen, } 2008 b^{33} \\
\text { Multisite } \\
3 \text { Continents } \\
\text { Low ROB } \\
19014751\end{array}$ & $\begin{array}{l}\text { I: Divalproex } \\
\text { C1: Olanzapine } \\
\text { C2: Placebo }\end{array}$ & $\begin{array}{l}\text { BD-I; } \\
\text { DSM-IV }\end{array}$ & $\begin{array}{l}\text { Mean Age } 40 \\
(18-65) \\
49 \% \text { Female } \\
\text { Race NR } \\
N=521\end{array}$ & $\begin{array}{l}\text { Mania/Mixed; } \\
\text { YMRS } \geq 20 \text { and } \leq \\
30 ; \\
\text { CGI-BP-S mania } \\
\text { subscore } 3 \text { or } 4\end{array}$ & $\begin{array}{l}\text { Schizoaffective } \\
\text { Other Mental } \\
\text { Health Conditions } \\
\text { Pregnant/Nursing }\end{array}$ \\
\hline $\begin{array}{l}\text { Bowden, } 2006^{111} \\
\text { Multisite } \\
\text { US } \\
\text { High ROB } \\
17107240\end{array}$ & $\begin{array}{l}\text { I: Divalproex } \\
\text { C: Placebo }\end{array}$ & $\begin{array}{l}\text { BD-I; } \\
\text { DSM-IV }\end{array}$ & $\begin{array}{l}\text { Mean Age } 38 \\
(18-65) \\
43 \% \text { Female } \\
74 \% \text { White } \\
N=364\end{array}$ & $\begin{array}{l}\text { Mania; } \\
\text { Mania Rating Scale } \\
\text { (From SADS-C } \\
\text { interview) had to be } \\
\text { at least } 18 \text { with at } \\
\text { least } 4 \text { item scores } \\
>1 .\end{array}$ & $\begin{array}{l}\text { First Manic } \\
\text { Episode; } \\
\text { Schizoaffective; } \\
\text { Substance Abuse; } \\
\text { Other Mental } \\
\text { Health Conditions; } \\
\text { Taking other } \\
\text { Medications; }\end{array}$ \\
\hline
\end{tabular}

BD=Bipolar Disorder; C=Comparison; CGI-BP-S=Clinical Global Impression scale, bipolar edition, severity; DSM-

IV=Diagnostic and Statistical Manual Fourth Edition; I=Intervention; N=number; NR=Not Reported; ROB=risk of bias; SADS-

$\mathrm{C}=$ Schedule for Affective Disorders and Schizophrenia-Change version; YMRS=Young Mania Rating Scale

\section{Divalproex Alone Versus Placebo}

Evidence was insufficient for all outcomes from two RCTs $(n=670)$ to address whether divalproex sodium was better for acute mania than placebo in adults with BD-I, due to moderate to high study limitations, inconsistency, and imprecision. ${ }^{33,111}$ Results were mixed for response, remission, and symptoms at 3 weeks. Both studies reported no difference in CGI or function (Global Assessment Score (GAS)), withdrawal, or serious adverse events.

Evidence was also insufficient for all outcomes from one small study $(n=79)$ whether valproate plus olanzapine was better for acute mania than olanzapine alone in adults with BD-I, due to single study and imprecision. The study reported improvement in manic symptoms (YMRS) and CGI.

\section{Divalproex Alone Versus Active Control}

Results for divalproex versus olanzapine were reported in the olanzapine versus active comparator subsection of the antipsychotic section above (e.g., low-strength evidence for no difference in remission or response rates, or improvements in manic symptoms or function). 


\section{Lamotrigine}

We identified a single small, industry-funded, moderate risk of bias RCT examining lamotrigine for acute mania with at least 3 week followup. ${ }^{121}$ Seven additional studies were excluded for attrition over 50 percent. ${ }^{53,114,115,122-125}$ Table 24 summarizes the bipolar type and major inclusion and exclusion criteria. Appendix F provides details.

Table 24. Population and inclusion criteria for lamotrigine for acute mania

\begin{tabular}{|c|c|c|c|c|c|}
\hline $\begin{array}{l}\text { Author, Year } \\
\text { Single-Multisite } \\
\text { Local/Continent } \\
\text { Risk of Bias } \\
\text { PMID }\end{array}$ & $\begin{array}{l}\text { Intervention/ } \\
\text { Comparison }\end{array}$ & $\begin{array}{c}\text { BD Type; } \\
\text { Diagnostic } \\
\text { Criteria }\end{array}$ & Demographics & Current Episode & Key Exclusions \\
\hline $\begin{array}{l}\text { Ichim, } 2000^{121} \\
\text { Singlesite } \\
\text { South Africa } \\
\text { Moderate ROB } \\
10798820\end{array}$ & $\begin{array}{l}\text { I: Lamotrigine } \\
\text { C: Lithium }\end{array}$ & $\begin{array}{l}\text { BD-I; } \\
\text { DSM-IV }\end{array}$ & $\begin{array}{l}\text { Mean Age } 33 \\
(\mathrm{NR}) \\
47 \% \text { Female } \\
\text { Race NR } \\
\mathrm{N}=30\end{array}$ & Mania & $\begin{array}{l}\text { Substance Abuse } \\
\text { Taking Other } \\
\text { Meds } \\
\text { Pregnant/Nursing } \\
\text { Labs/Other } \\
\text { Conditions }\end{array}$ \\
\hline
\end{tabular}

BD=Bipolar Disorder; C=Comparison; DSM-IV=Diagnostic and Statistical Manual Fourth Edition; I=Intervention; N=number; NR=Not Reported; ROB=risk of bias

\section{Lamotrigine Alone Versus Active Control}

Evidence was insufficient for all outcomes to address whether lamotrigine was better for acute mania than lithium in adults with BD-I, due to single study and imprecision. The study reported no differences between group in bipolar symptoms or response. No serious adverse events were reported and withdrawal rates were similar between groups.

\section{Lithium}

We identified three RCTs ${ }^{36,96,126}$ and one meta-analysis that pooled individual patient data from four RCTs ${ }^{103}$ examining lithium for acute mania with at least 3 weeks followup. One study was low risk of bias, two moderate, and one high. Five additional studies were excluded for greater than 50 percent attrition. ${ }^{53,58,117,118,127}$ All studies were funded by industry. Two studies used placebo and all used active comparators. Sample sizes ranged from 45 to 876 . Table 25 summarizes the bipolar type and major inclusion and exclusion criteria for each study. Appendix F provides detail.

Table 25. Population and inclusion criteria for lithium for acute mania

\begin{tabular}{|c|c|c|c|c|c|}
\hline $\begin{array}{c}\text { Author, Year } \\
\text { Single/Multisite } \\
\text { Local/Continent } \\
\text { Risk of Bias } \\
\text { PMID }\end{array}$ & $\begin{array}{l}\text { Intervention/ } \\
\text { Comparison }\end{array}$ & $\begin{array}{c}\text { BD Type; } \\
\text { Diagnostic } \\
\text { Criteria }\end{array}$ & Demographics & Current Episode & Key Exclusions \\
\hline $\begin{array}{l}\text { Bowden, } 2010^{126} \\
\text { RCT } \\
\text { Multisite } \\
2 \text { Continents } \\
\text { Moderate ROB } \\
20101186\end{array}$ & $\begin{array}{l}\text { I: Lithium } \\
\text { C: Valproate }\end{array}$ & $\begin{array}{l}\text { BD-I: } \\
\text { DSM-IV }\end{array}$ & $\begin{array}{l}\text { Mean Age } 39 \\
(18-75) \\
\text { Female 59\% } \\
\text { Race NR } \\
\mathrm{N}=270\end{array}$ & $\begin{array}{l}\text { Mania; } \\
\text { YMRS } \geq 18\end{array}$ & $\begin{array}{l}\text { First Manic Episode } \\
\text { Substance Abuse } \\
\text { Pregnant/Nursing } \\
\text { Labs/Other Conditions } \\
\text { Other Mental Health }\end{array}$ \\
\hline
\end{tabular}




\begin{tabular}{|c|c|c|c|c|c|}
\hline $\begin{array}{c}\text { Author, Year } \\
\text { Single/Multisite } \\
\text { Local/Continent } \\
\text { Risk of Bias } \\
\text { PMID }\end{array}$ & $\begin{array}{l}\text { Intervention/ } \\
\text { Comparison }\end{array}$ & $\begin{array}{l}\text { BD Type; } \\
\text { Diagnostic } \\
\text { Criteria }\end{array}$ & Demographics & Current Episode & Key Exclusions \\
\hline $\begin{array}{l}\text { Kushner, } 2006^{103} \\
\text { Multisite } \\
4 \text { Continents } \\
\text { Low ROB } \\
16411977\end{array}$ & $\begin{array}{l}\text { I: Lithium } \\
\text { C1: Placebo } \\
\text { C2: } \\
\text { Topiramate }\end{array}$ & $\begin{array}{l}\text { BD-I; } \\
\text { DSM-IV }\end{array}$ & $\begin{array}{l}\text { Mean Age } 41 \\
18-82 \text { ) } \\
53 \% \text { Female } \\
77 \% \text { White } \\
N=876\end{array}$ & $\begin{array}{l}\text { Mania; } \\
\text { YMRS } \geq 20\end{array}$ & $\begin{array}{l}\text { Schizoaffective; } \\
\text { Substance Abuse; } \\
\text { Other Mental Health } \\
\text { Conditions; Taking } \\
\text { Other Medications; }\end{array}$ \\
\hline $\begin{array}{l}\text { Bowden, } 2005^{36} \\
\text { Multisite } \\
3 \text { Continents } \\
\text { High ROB } \\
15669897\end{array}$ & $\begin{array}{l}\text { I: Lithium } \\
\text { C1: Placebo } \\
\text { C2: } \\
\text { Quetiapine }\end{array}$ & $\begin{array}{l}\text { BD-I; } \\
\text { DSM-IV }\end{array}$ & $\begin{array}{l}\text { Mean Age } 39 \\
(18-73) \\
42 \% \text { Female } \\
\text { Race NR } \\
N=300\end{array}$ & $\begin{array}{l}\text { Mania; } \\
\text { YMRS } \geq 20 \\
\text { including score of } \\
\text { at least } 4 \text { on } 2 \text { of } \\
\text { the } 4 \text { double- } \\
\text { weighted items } \\
\text { (irritability, speech, } \\
\text { content, and } \\
\text { disruptive/aggressi } \\
\text { ve behavior); } \\
\text { CGI } \geq 4\end{array}$ & $\begin{array}{l}\text { First manic episode; } \\
\text { Substance Use; } \\
\text { Taking other } \\
\text { medications; } \\
\text { Pregnant/Nursing; } \\
\text { Labs/Other Conditions }\end{array}$ \\
\hline $\begin{array}{l}\text { Segal, } 1998^{96} \\
\text { Singlesite } \\
\text { South Africa } \\
\text { Moderate ROB } \\
9617509\end{array}$ & $\begin{array}{l}\text { I: Lithium } \\
\text { C1: } \\
\text { Haloperidol } \\
\text { C2: } \\
\text { Risperidone }\end{array}$ & $\begin{array}{l}\text { BD-I; } \\
\text { DSM-IV }\end{array}$ & $\begin{array}{l}\text { Mean Age } 34 \\
(19-58) \\
78 \% \text { Female } \\
\text { Race NR } \\
N=45\end{array}$ & $\begin{array}{l}\text { Mania; } \\
\text { DSM-IV criteria for } \\
\text { Bipolar Manic } \\
\text { Phase }\end{array}$ & $\begin{array}{l}\text { Substance Abuse; } \\
\text { Taking other } \\
\text { Medications; } \\
\text { Pregnant/Nursing; } \\
\text { Abnormal Lab Results }\end{array}$ \\
\hline
\end{tabular}

BD=Bipolar Disorder; C=Comparison; CGI=Clinical Global Impression; DSM-IV=Diagnostic and Statistical Manual Fourth Edition; I=Intervention; MADRS= Montgomery-Asberg depression rating scale; N=number; NR=Not Reported; ROB=risk of bias; YMRS=Young Mania Rating Scale

\section{Lithium Alone Versus Placebo}

Low-strength evidence (moderate study limitations, imprecision) from one RCT and one meta-analysis of independent data from 4 RCTs $(n=847)$ showed lithium increased response and remission rates in BD-I participants compared to placebo for acute mania. ${ }^{36,103}$ Using data available to pool from one RCT and two individual RCTs reported in the meta-analysis, lithium improved manic symptoms essentially to the level of MID (YMRS, MD 5.81, 95\% CI 2.21, 9.4; $\mathrm{n}=643$ ). Withdrawal rates did not differ by group. Serious adverse events were inconsistently reported and showed mixed results.

\section{Lithium Alone Versus Active Control}

Evidence was insufficient for all outcomes from one RCT $(n=270)$ to address whether lithium was better for acute mania than divalproex in adults with BD-I, due to a single study and imprecise data. ${ }^{126}$ The study reported response rate, symptoms (YMRS), CGI, and withdrawals did not differ between groups. One of two measures of remission showed benefit for divalproex. No differences in frequency of serious adverse events between groups were noted.

Evidence was insufficient for all outcomes from one small RCT $(n=30)$ to address whether lithium was better than haloperidol, due to a single study and imprecise data. ${ }^{96}$ The study reported no differences between groups in manic symptoms (YMRS) and CGI. Serious adverse events were not reported. 
Risperidone versus lithium and risperidone versus haloperidol comparisons are discussed in the risperidone subsection of the antipsychotics section above. Also discussed above in the antipsychotics section are olanzapine versus lithium and quetiapine versus lithium. Overall, while all comparisons were assessed as having insufficient evidence, studies generally reported no differences between the antipsychotic drug and lithium.

(The topiramate comparisons will be discussed in the following section.)

\section{Drugs Not Approved by FDA for Acute Mania in Bipolar Disorder}

\section{Key Points}

- Ten drugs were examined for acute mania in BD: allopurinol, celecoxib, donepezil, dipyridamole, endoxifen, gabapentin, paliperidone, tamoxifen, topiramate, and oxcarbazepine, some in combination with mood stabilizers.

- Low-strength evidence showed paliperidone improved manic symptoms over placebo in adults with BD-I, although the improvement was not a clinically important difference ( $\mathrm{n}=763)$. Participants using $12 \mathrm{mg}$ paliperidone reported more common akathisia and dystonia.

- Low-strength evidence showed topiramate was not significantly different from placebo for symptom improvement, and participants using placebo withdrew less for adverse events ( $n=876)$ in adults with BD-I. In addition, low-strength evidence showed lithium significantly improved manic symptoms compared to topiramate $(n=453)$ in adults with BD-I, although participants receiving lithium withdrew more for adverse events.

- Low-strength evidence showed allopurinol plus mood stabilizers/other psychotropic medications did not differ significantly from mood stabilizers alone for manic symptom or CGI improvement or overall withdrawals $(n=355)$ in adults with BD-I.

- Evidence was largely insufficient to draw conclusions for all other nonapproved FDA drugs for BD-I for the primary outcomes of interest (response, symptom scores, and function).

\section{Eligible Studies for Drugs Not Approved by FDA}

Sixteen unique studies examined nine other drugs for patients experiencing manic events. ${ }^{35}$, 103, 128-141 Four studies were assessed as low risk of bias, ${ }^{103,129,130,135}$ ten were moderate, ${ }^{128,132 \text {, }}$ 134, 136, 137, 139-141 and four were assessed as high. ${ }^{35,131,133,138}$ Three additional studies were excluded for greater than 50 percent attrition. ${ }^{142-144}$ Eight studies were funded or assisted by industry. ${ }^{35,103,128,135,138-141}$ All but three studies ${ }^{128,138,139}$ used a placebo comparator. Sample sizes ranged from 27 to 876. Appendix G provides detailed evidence tables, a summary of risk of bias assessments, and assessments of strength of evidence for key comparisons and outcomes. A summary of findings with at least low-strength evidence for other drugs not approved by FDA for acute mania are provided in Table 26. Any intervention and comparison not listed in Table 26, or outcome not listed for an included intervention and comparison, was found to have an evidence base insufficient to draw conclusions. 
Table 26. Summary of findings with at least low-strength evidence for drugs not approved by FDA for acute mania

\begin{tabular}{|c|c|c|c|}
\hline Intervention & $\begin{array}{l}\text { \# Studies/ } \\
\text { Design } \\
\text { (n Analyzed) } \\
\text { Timing } \\
\end{array}$ & Findings & $\begin{array}{l}\text { Strength of } \\
\text { Evidence }\end{array}$ \\
\hline $\begin{array}{l}\text { Paliperidone } \\
\text { vs. placebo }\end{array}$ & $\begin{array}{l}2 \mathrm{RCT}^{14035} \\
(\mathrm{n}=763) \\
3 \text { weeks }\end{array}$ & $\begin{array}{l}\text { YMRS and Withdrawal (Lack of Efficacy): Favors } \\
\text { Paliperidone (possible dose response: No difference at } 3 \\
\text { and } 6 \mathrm{mg} \text {, benefit at } 12 \mathrm{mg} \text { or median dosage of } 9 \mathrm{mg} \text { ) } \\
\text { Withdrawal (AE): No difference }\end{array}$ & $\begin{array}{l}\text { Low } \\
\text { (moderate study } \\
\text { limitations, } \\
\text { imprecise) }\end{array}$ \\
\hline $\begin{array}{l}\text { Topiramate } \\
\text { vs. placebo }\end{array}$ & $\begin{array}{l}1 \mathrm{IPD}^{103} \\
(\mathrm{n}=876) \\
3 \text { weeks }\end{array}$ & $\begin{array}{l}\text { YMRS and Withdrawal (Lack of Efficacy): No difference } \\
\text { Withdrawals (Overall): Favors Placebo, } 37.2 \% \text { vs. } 26.8 \% \text {, } \\
p=0.005 \\
\text { Withdrawals (AE): Favors Placebo, } 6.04 \% \text { vs. } 2.84 \% \text {, } \\
p=0.049\end{array}$ & $\begin{array}{l}\text { Low } \\
\text { (moderate study } \\
\text { limitations, } \\
\text { imprecise) }\end{array}$ \\
\hline \multirow{3}{*}{$\begin{array}{l}\text { Topiramate } \\
\text { vs. lithium }\end{array}$} & $\begin{array}{l}1 \text { IPD }^{103} \\
(\mathrm{n}=453) \\
3 \text { weeks }\end{array}$ & $\begin{array}{l}\text { YMRS: Favors Lithium, MD } 6.14 \text { (95\% Cl 3.94, 8.34; MID } \\
6)\end{array}$ & $\begin{array}{l}\text { Low } \\
\text { (moderate study } \\
\text { limitations, } \\
\text { imprecise) }\end{array}$ \\
\hline & $\begin{array}{l}1 \text { IPD } \\
(\mathrm{n}=453) \\
3 \text { weeks }\end{array}$ & Withdrawal (Overall, AE): No difference & $\begin{array}{l}\text { Low } \\
\text { (moderate study } \\
\text { limitations, } \\
\text { imprecise) }\end{array}$ \\
\hline & $\begin{array}{l}1 \text { IPD }^{103} \\
(n=453) \\
3 \text { weeks }\end{array}$ & $\begin{array}{l}\text { Withdrawal (AE): Favors Topiramate, } 2.65 \% \text { vs. } 7.49 \% \text {, } \\
p=0.019\end{array}$ & $\begin{array}{l}\text { Low } \\
\text { (moderate study } \\
\text { limitations, } \\
\text { imprecise) }\end{array}$ \\
\hline $\begin{array}{l}\text { Allopurinol + } \\
\text { lithium vs. } \\
\text { placebo + } \\
\text { lithium }\end{array}$ & $\begin{array}{l}4 \mathrm{RCT}^{130} 131 \\
132136 \\
(\mathrm{n}=355) \\
4 \text { weeks }\end{array}$ & YMRS, CGI, Withdrawal (Overall): No difference & $\begin{array}{l}\text { Low } \\
\text { (moderate study } \\
\text { limitations, } \\
\text { imprecise) }\end{array}$ \\
\hline
\end{tabular}

$\mathrm{AE}=$ adverse events; $\mathrm{CGI}=$ clinical global improvement; $\mathrm{CI}=$ confidence interval; $\mathrm{IPD}=\mathrm{Individual}$ patient data; $\mathrm{MD}=\mathrm{mean}$ difference; $\mathrm{MID}=$ minimally important difference; $\mathrm{n}=$ number; $\mathrm{RCT}=$ =randomized controlled trial; YMRS=Young mania rating scale

Table 27 summarizes the bipolar type and major inclusion and exclusion criteria for each study.

Table 27. Population and inclusion criteria for drugs not approved by FDA for acute mania

\begin{tabular}{|c|c|c|c|c|c|}
\hline $\begin{array}{c}\text { Author, Year } \\
\text { Single/-Multisite } \\
\text { Location } \\
\text { Risk of Bias } \\
\text { PMID }\end{array}$ & $\begin{array}{l}\text { Intervention/ } \\
\text { Comparison }\end{array}$ & $\begin{array}{l}\text { BD Type; } \\
\text { Diagnostic } \\
\text { Criteria }\end{array}$ & Demographics & Current Episode & Key Exclusions \\
\hline $\begin{array}{l}\text { Jahangard, } \\
2014^{130} \\
\text { Singlesite } \\
\text { Iran } \\
\text { Low ROB } \\
\\
24953766\end{array}$ & $\begin{array}{l}\text { I: Allopurinol + } \\
\text { valproate and } \\
\text { benzodiazapine } \\
\text { s } \\
\text { C: Placebo+ } \\
\text { valproate and } \\
\text { benzodiazapine } \\
\text { s }\end{array}$ & $\begin{array}{l}\text { BD-I; } \\
\text { DSM-IV }\end{array}$ & $\begin{array}{l}\text { Mean Age NR } \\
(18-40) \\
\text { Female NR } \\
\text { Race NR } \\
\mathrm{N}=60\end{array}$ & $\begin{array}{l}\text { Manic; } \\
\text { YMRS } \geq 28\end{array}$ & $\begin{array}{l}\text { Schizoaffective } \\
\text { Substance Abuse } \\
\text { Other Mental Health } \\
\text { Pregnant/Nursing }\end{array}$ \\
\hline
\end{tabular}




\begin{tabular}{|c|c|c|c|c|c|}
\hline $\begin{array}{c}\text { Author, Year } \\
\text { Single/-Multisite } \\
\text { Location } \\
\text { Risk of Bias } \\
\text { PMID }\end{array}$ & $\begin{array}{l}\text { Intervention/ } \\
\text { Comparison }\end{array}$ & $\begin{array}{c}\text { BD Type; } \\
\text { Diagnostic } \\
\text { Criteria }\end{array}$ & Demographics & Current Episode & Key Exclusions \\
\hline $\begin{array}{l}\text { Weiser, } 2014^{131} \\
\text { Multisite } \\
\text { Israel } \\
\text { High ROB } \\
24712840\end{array}$ & $\begin{array}{l}\text { I: Allopurinol + } \\
\text { mood stabilizers } \\
\text { C: Placebo+ } \\
\text { mood stabilizers }\end{array}$ & $\begin{array}{l}\text { BD-I; } \\
\text { DSM-IV }\end{array}$ & $\begin{array}{l}\text { Mean Age } 47 \\
(18-65) \\
66 \% \text { Female } \\
100 \% \text { White } \\
N=180\end{array}$ & $\begin{array}{l}\text { Manic; } \\
\text { Clinical Interview } \\
\text { in DSM-IV treated } \\
\text { with mood } \\
\text { stabilizer or } \\
\text { neuroleptics for } \\
\text { between } 3 \text { days } \\
\text { and } 2 \text { weeks. }\end{array}$ & None Specified \\
\hline $\begin{array}{l}\text { Fan, } 2012^{132} \\
\text { Singlesite } \\
\text { United States } \\
\text { Moderate ROB } \\
22420596\end{array}$ & $\begin{array}{l}\text { I: Allopurinol + } \\
\text { current } \\
\text { psychiatric } \\
\text { medications } \\
\text { C: Placebo+ } \\
\text { current } \\
\text { psychiatric }\end{array}$ & $\begin{array}{l}\text { BD-I; } \\
\text { DSM-IV }\end{array}$ & $\begin{array}{l}\text { Mean Age } 43 \\
\text { (NR) } \\
50 \% \text { Female } \\
63 \% \text { White } \\
N=27\end{array}$ & $\begin{array}{l}\text { Manic; } \\
\text { YMRS } \geq 14 \\
\text { partial response } \\
\text { to lithium, } \\
\text { valproate, } \\
\text { carbamazepine, } \\
\text { or atypical } \\
\text { antipsychotics }\end{array}$ & $\begin{array}{l}\text { Substance Abuse } \\
\text { Other Mental Health } \\
\text { Pregnant/Nursing } \\
\text { Labs/Other } \\
\text { Conditions }\end{array}$ \\
\hline $\begin{array}{l}\text { Machado-Vieira, } \\
2008^{136} \\
\text { Multisite } \\
\text { Brazil } \\
\text { Moderate ROB } \\
18681754\end{array}$ & $\begin{array}{l}\text { 11: Allopurinol + } \\
\text { Lithium } \\
\text { 12: Dipyridamole } \\
+ \text { Lithium } \\
\text { C: Placebo + } \\
\text { Lithium }\end{array}$ & $\begin{array}{l}\text { BD-I; } \\
\text { DSM-IV }\end{array}$ & $\begin{array}{l}\text { Mean Age } 29 \\
(18-65) \\
57 \% \text { Female } \\
\text { Race NR } \\
N=180\end{array}$ & $\begin{array}{l}\text { Manic; } \\
\text { YMRS } \geq 22\end{array}$ & $\begin{array}{l}\text { Schizoaffective } \\
\text { Substance abuse } \\
\text { Other mental health } \\
\text { Taking other meds } \\
\text { Labs/other } \\
\text { conditions }\end{array}$ \\
\hline $\begin{array}{l}\text { Arabzadeh, } \\
2015^{129} \\
\text { Multisite } \\
\text { Iran } \\
\text { Low ROB } \\
26291962\end{array}$ & $\begin{array}{l}\text { I: Celecoxib } \\
\text { C: Placebo }\end{array}$ & $\begin{array}{l}\text { BD-I; } \\
\text { DSM-IV }\end{array}$ & $\begin{array}{l}\text { Mean Age 31 } \\
(18-50) \\
35 \% \text { Female } \\
\text { Race NR } \\
N=48\end{array}$ & $\begin{array}{l}\text { Manic; } \\
\text { YMRS } \geq 20\end{array}$ & $\begin{array}{l}\text { Schizoaffective } \\
\text { Substance abuse } \\
\text { Other mental health } \\
\text { Taking other meds } \\
\text { Labs/other } \\
\text { conditions }\end{array}$ \\
\hline $\begin{array}{l}\text { Chen, 2013 }{ }^{137} \\
\text { Single Site } \\
\text { China } \\
\text { Moderate ROB } \\
23807849\end{array}$ & $\begin{array}{l}\text { I: Donepezil } \\
\text { +Lithium } \\
\text { C: Placebo + } \\
\text { Lithium }\end{array}$ & $\begin{array}{l}\text { BD-I; } \\
\text { NR }\end{array}$ & $\begin{array}{l}\text { Mean Age } 34 \\
(18-65) \\
40 \% \text { Female } \\
\text { Race NR } \\
N=30\end{array}$ & $\begin{array}{l}\text { Manic; } \\
\text { YMRS > } 20\end{array}$ & $\begin{array}{l}\text { Schizoaffective } \\
\text { Substance abuse } \\
\text { Other mental health } \\
\text { Taking other meds } \\
\text { Pregnant/nursing } \\
\text { Labs/other } \\
\text { conditions }\end{array}$ \\
\hline $\begin{array}{l}\text { Astaneh, } 2012^{133} \\
\text { Singlesite } \\
\text { Spain } \\
\text { High ROB } \\
22978083\end{array}$ & $\begin{array}{l}\text { I: Gabapentin + } \\
\text { Lithium } \\
\text { C: Placebo + } \\
\text { Lithium }\end{array}$ & $\begin{array}{l}\text { BD-I; } \\
\text { DSM-IV }\end{array}$ & $\begin{array}{l}\text { Mean Age NR } \\
\text { About 50\% } \\
\text { Female } \\
\text { Race NR } \\
\mathrm{N}=60\end{array}$ & $\begin{array}{l}\text { Manic; } \\
\text { Not Defined }\end{array}$ & Substance abuse \\
\hline $\begin{array}{l}\text { Ahmad, } 2016^{128} \\
\text { Multisite } \\
\text { India } \\
\text { Low ROB } \\
27346789\end{array}$ & $\begin{array}{l}\text { I Endoxifen } \\
\text { C: Divalproex }\end{array}$ & $\begin{array}{l}\text { BD-I } \\
\text { DSM-IV }\end{array}$ & $\begin{array}{l}\text { Mean Age } 37 \\
(18-65) \\
\text { Female NR } \\
\text { Race } 100 \% \\
\text { Asian } \\
\mathrm{N}=84\end{array}$ & $\begin{array}{l}\text { Manic/Mixed; } \\
\text { YMRS } \geq 20 \text { and } \\
\text { CGI-S } \geq 4\end{array}$ & $\begin{array}{l}\text { New diagnosis } \\
\text { Labs/other } \\
\text { conditions } \\
\text { Pregnant/nursing }\end{array}$ \\
\hline
\end{tabular}




\begin{tabular}{|c|c|c|c|c|c|}
\hline $\begin{array}{c}\text { Author, Year } \\
\text { Singlel-Multisite } \\
\text { Location } \\
\text { Risk of Bias } \\
\text { PMID }\end{array}$ & $\begin{array}{l}\text { Intervention/ } \\
\text { Comparison }\end{array}$ & $\begin{array}{c}\text { BD Type; } \\
\text { Diagnostic } \\
\text { Criteria }\end{array}$ & Demographics & Current Episode & Key Exclusions \\
\hline $\begin{array}{l}\text { Yildiz, } 2008^{134} \\
\text { Single site } \\
\text { Turkey } \\
\text { Moderate ROB } \\
18316672\end{array}$ & $\begin{array}{l}\text { I: Tamoxifen } \\
\text { C: Placebo }\end{array}$ & $\begin{array}{l}\text { BD-I; } \\
\text { DSM-IV }\end{array}$ & $\begin{array}{l}\text { Mean Age } 33 \\
(18-60) \\
52 \% \text { Female } \\
\text { Race NR } \\
\mathrm{N}=66\end{array}$ & $\begin{array}{l}\text { Manic; } \\
\text { YMRS } \geq 20\end{array}$ & $\begin{array}{l}\text { Schizoaffective } \\
\text { Substance abuse } \\
\text { Other mental health } \\
\text { Neurological } \\
\text { disorders } \\
\text { Taking other meds } \\
\text { Pregnant/nursing }\end{array}$ \\
\hline $\begin{array}{l}\text { Kushner, 2006 } \\
\text { Multisite } \\
6 \text { Continents } \\
\text { Low ROB } \\
16411977\end{array}$ & $\begin{array}{l}\text { I: Topiramate } \\
\text { C1: Placebo } \\
\text { C2: Lithium }\end{array}$ & $\begin{array}{l}\text { BD-I; } \\
\text { DSM-IV }\end{array}$ & $\begin{array}{l}\text { Mean Age } 41 \\
\text { (16 and up) } \\
51 \% \text { Female } \\
75 \% \text { White } \\
N=876\end{array}$ & $\begin{array}{l}\text { Manic/Mixed; } \\
\text { YMRS } \geq 20\end{array}$ & $\begin{array}{l}\text { First Manic Episode } \\
\text { Schizoaffective } \\
\text { Substance abuse } \\
\text { Other mental health } \\
\text { Taking other meds } \\
\text { Pregnant/nursing } \\
\text { Labs/other } \\
\text { conditions }\end{array}$ \\
\hline $\begin{array}{l}\text { Chengappa, } \\
2006^{135} \\
\text { Multisite } \\
\text { US } \\
\text { Low ROB } \\
17196048\end{array}$ & $\begin{array}{l}\text { I: Topiramate + } \\
\text { Valproate or } \\
\text { Lithium } \\
\text { C: Placebo + } \\
\text { Valproate or } \\
\text { Lithium }\end{array}$ & $\begin{array}{l}\text { BD-I; } \\
\text { DSM-IV }\end{array}$ & $\begin{array}{l}\text { Mean Age } 40 \\
(18-70) \\
56 \% \text { Female } \\
84 \% \text { White } \\
N=287\end{array}$ & $\begin{array}{l}\text { Manic/Mixed; } \\
\text { YMRS } \geq 18\end{array}$ & $\begin{array}{l}\text { Substance abuse } \\
\text { Other mental health } \\
\text { Neurological } \\
\text { disorders } \\
\text { Taking other meds } \\
\text { Pregnant/nursing } \\
\text { Labs/other } \\
\text { conditions }\end{array}$ \\
\hline $\begin{array}{l}\text { Bahk, } 2005^{138} \\
\text { Multisite } \\
\text { South Korea } \\
\text { High ROB } \\
15610953\end{array}$ & $\begin{array}{l}\text { I: Topiramate + } \\
\text { Risperidone } \\
\text { C: Divalproex + } \\
\text { Risperidone }\end{array}$ & $\begin{array}{l}\text { BD-I } \\
\text { DSM-IV }\end{array}$ & $\begin{array}{l}\text { Age } 37(18-65) \\
51 \% \text { Female } \\
\text { Race Asian } \\
N=74\end{array}$ & $\begin{array}{l}\text { Manic; } \\
\text { YMRS } \geq 20\end{array}$ & $\begin{array}{l}\text { Other mental health } \\
\text { Pregnant/nursing } \\
\text { Labs/other } \\
\text { conditions } \\
\text { Taking other meds }\end{array}$ \\
\hline $\begin{array}{l}\text { Berwaerts, } \\
2012^{139} \\
\text { Multisite } \\
5 \text { Continents } \\
\text { Moderate ROB } \\
22377512\end{array}$ & $\begin{array}{l}\text { I: Paliperidone } \\
\text { ER } \\
\text { C: Olanzapine }\end{array}$ & $\begin{array}{l}\text { BD-I; } \\
\text { DSM-IV }\end{array}$ & $\begin{array}{l}\text { Mean Age } 40 \\
(18-65) \\
52 \% \text { Female } \\
62 \% \text { White } \\
N=766\end{array}$ & $\begin{array}{l}\text { Manic/Mixed; } \\
\text { YMRS } \geq 20\end{array}$ & $\begin{array}{l}\text { First manic episode; } \\
\text { Schizoaffective; } \\
\text { Other mental } \\
\text { health; Neurological } \\
\text { disorders; } \\
\text { Taking other meds; } \\
\text { Pregnant/nursing; } \\
\text { Labs/Other } \\
\text { conditions }\end{array}$ \\
\hline $\begin{array}{l}\text { Berwaerts, } \\
20122^{140} \\
\text { Mutisite } \\
3 \text { Continents } \\
\text { Moderate ROB } \\
20624657\end{array}$ & $\begin{array}{l}\text { I: Paliperidone } \\
\text { ER } \\
\text { C: Placebo }\end{array}$ & $\begin{array}{l}\text { BD-I; } \\
\text { DSM-IV }\end{array}$ & $\begin{array}{l}\text { Mean Age } 40 \\
\text { (18-65); } \\
47 \% \text { Female } \\
50 \% \text { White } \\
N=469\end{array}$ & $\begin{array}{l}\text { Manic/Mixed; } \\
\text { YMRS } \geq 20 \text { with } 1 \\
\text { manic or mixed } \\
\text { episode in past } 3 \\
\text { years }\end{array}$ & $\begin{array}{l}\text { Schizoaffective; } \\
\text { Substance abuse; } \\
\text { Other Mental Health } \\
\text { Condition; } \\
\text { Taking other } \\
\text { medications; } \\
\text { Pregnant/Nursing }\end{array}$ \\
\hline $\begin{array}{l}\text { Vieta, } 2010^{35} \\
\text { Multisite } \\
\text { 4 Continents } \\
\text { High ROB } \\
20565430\end{array}$ & $\begin{array}{l}\text { I: Paliperidone } \\
\text { ER } \\
\text { C1: Placebo } \\
\text { C2: Quetiapine }\end{array}$ & $\begin{array}{l}\text { BD-I; } \\
\text { DSM-IV }\end{array}$ & $\begin{array}{l}\text { Mean Age } 39 \\
\text { (18-65) } \\
42 \% \text { Female } \\
68 \% \text { White } \\
N=493\end{array}$ & $\begin{array}{l}\text { Manic/Mixed; } \\
\text { YMRS } \geq 20\end{array}$ & $\begin{array}{l}\text { First Manic Episode } \\
\text { Schizoaffective } \\
\text { Substance abuse } \\
\text { Other mental health } \\
\text { Neurological } \\
\text { disorders }\end{array}$ \\
\hline
\end{tabular}




\begin{tabular}{|c|c|c|c|c|c|}
\hline $\begin{array}{c}\text { Author, Year } \\
\text { Single/-Multisite } \\
\text { Location } \\
\text { Risk of Bias } \\
\text { PMID }\end{array}$ & $\begin{array}{l}\text { Intervention/ } \\
\text { Comparison }\end{array}$ & $\begin{array}{c}\text { BD Type; } \\
\text { Diagnostic } \\
\text { Criteria }\end{array}$ & Demographics & Current Episode & Key Exclusions \\
\hline & & & & & $\begin{array}{l}\text { Labs/other } \\
\text { conditions }\end{array}$ \\
\hline $\begin{array}{l}\text { Berwaerts, } \\
2011^{141} \\
\text { Multisite } \\
3 \text { Continents } \\
\text { Moderate ROB } \\
20947174\end{array}$ & $\begin{array}{l}\text { I: Paliperidone } \\
\text { ER + Lithium } \\
\text { OR Valproate } \\
\text { C: Placebo + } \\
\text { Lithium OR } \\
\text { Valproate }\end{array}$ & $\begin{array}{l}\text { BD-I; } \\
\text { DSM-IV }\end{array}$ & $\begin{array}{l}\text { Mean Age } 40 \\
(18-65) \\
46 \% \text { Female } \\
77 \% \text { White } \\
N=300\end{array}$ & $\begin{array}{l}\text { Manic/Mixed; } \\
\text { YMRS } \geq 20\end{array}$ & $\begin{array}{l}\text { First Manic } \\
\text { Episode; } \\
\text { Schizoaffective; } \\
\text { Substance Abuse; } \\
\text { Other Mental Health } \\
\text { Conditions; } \\
\text { Neurological } \\
\text { Disorders; } \\
\text { Taking other } \\
\text { medications; } \\
\text { Pregnant/Nursing }\end{array}$ \\
\hline
\end{tabular}

BD=Bipolar Disorder; C=Comparison; CGI-S=Clinical Global Impression, severity; DSM-IV=Diagnostic and Statistical Manual Fourth Edition; ER=Extended Release; FDA=Federal Drug Administration; I=Intervention; N=number; NR=Not Reported; ROB=risk of bias; YMRS=Young Mania Rating Scale

\section{Drugs Not Approved by FDA Versus Placebo}

Twelve unique trials ${ }^{35,129-137,140,141}$ and one pooled analysis of a further four trials ${ }^{103}$ examined nine drugs versus placebo. Five studies examined the drugs as a single drug, ${ }^{35,103,129,}$ 134,140 while eight were added to mood stabilizers or other current psychiatric medications. ${ }^{130-133,}$ 135-137, 141 Studies ranged from 3 to 12 weeks long. All enrolled BD-I participants.

Low-strength evidence (moderate study limitations, imprecision) from two studies ( $n=763)$ showed paliperidone was better than placebo for improvement in mania symptoms (YMRS). ${ }^{35}$, ${ }^{140}$ However, for the highest dose of $12 \mathrm{mg}$ in the moderate risk of bias study, the improvement may not be clinically meaningful based on values that are less than the MID. The study reported YMRS mean difference of $3.4(\mathrm{p}=0.025)$, which is less than the MID of 6 . While a dose response was suggested, authors stated results were driven largely by participants in India, who comprised only 10 percent of the analysis set. Low-strength evidence (moderate study limitations, imprecision) showed no statistically significant differences between groups for withdrawal for lack of efficacy. Evidence for CGI and response and remission rates was insufficient due to moderate study limitations, inconsistency, and imprecision. With the exception of more common akathisia and dystonia EPS symptoms for $12 \mathrm{mg}$ paliperidone versus placebo, no differences in serious adverse events were noted Appendix G provides further detail.

Topiramate versus placebo was examined in a pooled analysis of four trials (n=876). ${ }^{103}$ Lowstrength evidence (high imprecision) showed no differences between topiramate and placebo for manic symptoms (YMRS) or withdrawal due to lack of efficacy for adults with BD-I. Additionally, overall withdrawals and withdrawals due to adverse events were lower in the placebo group (low-strength evidence, high imprecision). No differences in severe adverse events between groups were reported. Appendix G provides further detail. 
Evidence was insufficient for all outcomes for the two drugs celecoxib ${ }^{129}$ and tamoxifen, ${ }^{134}$ examined as single drug versus placebo for acute mania, due to single studies and imprecision.

For adjunctive medications, low-strength evidence (moderate study limitations, imprecision) from four RCTs $(\mathrm{n}=355)$ showed no significant differences between allopurinol plus mood stabilizers compared to mood stabilizers alone in manic symptoms (YMRS), CGI, or overall withdrawals. ${ }^{130-132,} 136$ Evidence was insufficient for response (high study limitations, imprecision) ${ }^{131,136}$ and remission (moderate study limitations, inconsistent, imprecision) rates. ${ }^{130,}$ ${ }^{136}$ No serious adverse event were reported. Appendix G provides further detail.

Evidence was insufficient for all outcomes for dipyridamole, ${ }^{136}$ donepezil,${ }^{137}$ or gabapentin ${ }^{133}$ plus lithium versus placebo largely due to single studies and imprecision. Evidence was also insufficient for all outcomes for one study of topiramate plus mood stabilizers versus mood stabilizers alone, although the general finding of no significant differences between groups was similar to the findings for topiramate as single drug. ${ }^{135}$ Likewise, one study of paliperidone plus mood stabilizers, while in itself providing insufficient evidence, repeated the general finding of no significant differences between groups observed in comparison of paliperidone as monotherapy versus placebo. ${ }^{141}$

\section{Drugs Not Approved by FDA Versus Active Control}

Six trials examined drugs versus active comparators in BD-I participants, each a unique comparison. ${ }^{35,103,136,138,139}$ Study sizes ranged from 30 to 388 and ran from 3 to 12 weeks.

Low-strength evidence (high imprecision) from a pooled analysis of individual patient data from two trials $(\mathrm{n}=453)$ of topiramate versus lithium for adults with BD-I with acute mania showed manic symptoms (YMRS) improved more with lithium and the difference was at the MID level (6.14, 95\% CI 3.94, 8.34). ${ }^{103}$ Overall withdrawals and withdrawals due to lack of efficacy did not differ between groups (low-strength evidence). However, less participants receiving topiramate withdrew due to adverse events ( $7 \%$ vs. $3 \%$ ). There were no differences in severe adverse events between lithium and topiramate groups.

Evidence was insufficient for all outcomes to address if endoxifen was better for acute mania in adults with BD-I than divalproex (unknown consistency, imprecise), ${ }^{128}$ or if paliperidone extended release was better than olanzapine ${ }^{139}$ or quetiapine (high study limitations, unknown consistency, imprecise). ${ }^{35}$

Evidence was insufficient for all outcomes to address if allopurinol plus lithium was better for acute mania in adults with BD-I than dipyridamole plus lithium (moderate study limitations, unknown consistency, imprecise), or if topiramate plus risperidone was better than divalproex plus risperidone (high study limitations, unknown consistency, imprecise). ${ }^{138}$

\section{Interpreting the Findings for Drugs for Acute Mania}

All FDA-approved antipsychotics, except aripiprazole, when compared to placebo improved mania symptoms for adults with BD-I (low-strength evidence). For four of the antipsychotics we were able to provide a point estimate. Lithium also reached low-strength evidence for improving mania symptoms, however, studies for carbamazepine, divalproex/valproate, and lamotrigine failed to reach sufficient evidence due to too few studies and imprecise results. Likewise, evidence was insufficient to draw conclusions for the efficacy of antipsychotics added to mood stabilizers.

Except for the finding that lithium improved mania symptoms better than topiramate (lowstrength evidence), evidence from studies of drugs compared to other drugs, whether as single 
drug or drug combinations, for treatment of acute mania was also insufficient to draw conclusions. Our ability to draw conclusions was hampered by the small number of studies and sample sizes to allow confidence in findings of no differences between groups. Study designs generally tested for superiority of one drug over the other, rather than noninferiority of the two drugs. With noninferiority tests, if the relative equivalence of the performance of two drugs is not demonstrated strongly enough, nonequivalence cannot be ruled out; that is, the treatment effects of the two drugs are too different.

Only two small studies attempted to address efficacy and harms for specific populations of interest, pregnant women with BD (lamotrigine), and BD patients with hypomania (quetiapine). Unfortunately, results for the effect of quetiapine treatment for patients with hypomania were not reported separately from patient with mild mania, thus no conclusions can be made. Similarly, the single observational study for pregnant women provided insufficient evidence to address whether lamotrigine provided benefits. Because of the weak evidence, there was little to be gained from the very few studies that did attempt post-hoc analysis of subgroups. Post-hoc analyses cannot reach the same level of strength of evidence due to the inherent higher study limitations from studies that generated low-strength evidence for main findings would . Given the generally high levels of attrition observed in the included studies, results of any subgroup analysis of such a restricted set are even more suspect.

Adverse events were somewhat consistently reported for extrapyramidal symptoms, and clinically significant weight gain of greater than 7 percent, but otherwise variably reported. The harms findings from the included placebo-controlled studies were consistent with information currently reported by FDA labels. While most studies reported no differences between groups in studies comparing drugs to drugs, we noted a general pattern of participants receiving atypical antipsychotics experiencing fewer extrapyramidal symptoms than participants receiving other medications.

The seventeen studies examining efficacy and comparing drugs to drugs of ten other medications, either as single drug or added to other psychiatric medications, largely yielded insufficient evidence due to a single study for each specific comparison, small sample sizes, and/or inconsistent findings.

There were a few exceptions, such as a low-strength evidence that lithium improved manic symptoms more than topiramate, although topiramate had lower rates of withdrawal due to adverse events than lithium. There was also low-strength evidence for no group differences in examined outcomes for topiramate versus placebo and allopurinol plus mood stabilizers/lithium/other psychiatric medications versus these other medications alone. Lowstrength evidence supported that paliperidone improved manic symptoms more than placebo, although the improvement was not clinically significant since it did not reach the MID.

Several issues impact the applicability of the studies. Over three quarters of the studies also excluded participants experiencing a first manic episode and most enrolled participants were 30 to 50 years of age. Moreover, given the inclusion criteria and actual participant characteristics, it is not clear if the current findings extend to populations with first manic episodes, current comorbid substance use, or pregnant or nursing women with BD I, or older adults with BD-I. 


\section{Chapter 5. Drug Treatments for Depression}

\section{Key Points}

- $\quad$ Evidence for treatment of depression in adults with bipolar disorder (BD) with at least 3 months followup was very sparse.

- The effects of four drugs compared with placebo: memantine, lamotrigine, or antidepressants (paroxetine, bupropion, or both) and two drugs compared with other drugs: sertraline or venlafaxine were examined for depression in BD.

- Evidence was largely insufficient to draw conclusions regarding the effects of drug treatments for depression in adults with BD for the primary outcomes of interest (relapse, symptom scores, and function).

\section{Eligible Studies for Depression Treatments}

We identified 11 eligible publications reporting seven unique randomized controlled trials (RCTs) of drug treatments for depression in adults with BD with at least 3 months followup. ${ }^{145-}$ 154 Two studies were assessed as moderate risk of bias and four were assessed as high. One additional study was excluded for greater than 50 percent attrition. ${ }^{155}$ All studies were funded in whole or part by government sources. No studies for lurasidone, olanzapine, or quetiapine, Federal Drug Administration (FDA)-approved for depression in BD, met the inclusion criteria of at least 3 months followup. Three interventions were compared to placebo ${ }^{147-150}$ and added to mood stabilizers while three were single drugs versus active comparators. ${ }^{33,151-154}$ Sample sizes ranged from 49 to 366. Also discussed is an additional RCT examining lithium in participants with BD with at least mild symptoms needing clinical care, as the majority of participants were experiencing depression symptoms. ${ }^{156}$

Table 28 summarizes the bipolar type and major inclusion and exclusion criteria for each study. Appendix H provides detailed evidence tables, a summary of risk of bias assessments, and assessments of strength of evidence for key comparisons and outcomes.

Table 28. Population and inclusion criteria for studies of drug treatments for depression

\begin{tabular}{|c|c|c|c|c|c|}
\hline $\begin{array}{c}\text { Author, Year } \\
\text { Single/-Multisite } \\
\text { Location } \\
\text { Risk of Bias } \\
\text { PMID }\end{array}$ & $\begin{array}{l}\text { Intervention/ } \\
\text { Comparison }\end{array}$ & $\begin{array}{c}\text { BD Type; } \\
\text { Diagnostic } \\
\text { Criteria }\end{array}$ & $\begin{array}{l}\text { Demo- } \\
\text { graphics }\end{array}$ & $\begin{array}{l}\text { Current } \\
\text { Episode }\end{array}$ & Key Exclusions \\
\hline $\begin{array}{l}\text { Lee, } 2014^{148,149} \\
\text { Multisite } \\
\text { China } \\
\text { High ROB } \\
24103632 \\
23870798\end{array}$ & $\begin{array}{l}\text { I: Memantine + } \\
\text { Valproic Acid } \\
\text { C: Placebo + } \\
\text { Valproic Acid }\end{array}$ & $\begin{array}{l}\text { Modified } \\
\text { BD-II; 2- } \\
\text { days } \\
\text { hypomanic } \\
\text { (versus } 4 \\
\text { in DSM-IV } \\
\text { criteria) }\end{array}$ & $\begin{array}{l}\text { Mean Age } \\
32 \text { (All } \\
\text { ages); } \\
49 \% \\
\text { Female; } \\
\text { Race } \\
\text { (Taiwan) } \\
\text { N=232 }\end{array}$ & $\begin{array}{l}\text { Depressed; } \\
\text { HAM-D } \geq 18\end{array}$ & $\begin{array}{l}\text { Schizoaffective } \\
\text { Substance abuse } \\
\text { Other mental health } \\
\text { Neurological } \\
\text { disorders } \\
\text { Taking other } \\
\text { medications }\end{array}$ \\
\hline $\begin{array}{l}\text { Kemp, } 2012^{147} \\
\text { Singlesite } \\
\text { US } \\
\text { Moderate ROB } \\
23107222\end{array}$ & $\begin{array}{l}\text { I: Lamotrigine + } \\
\text { mood stabilizer } \\
\text { C: Placebo + } \\
\text { mood stabilizer }\end{array}$ & $\begin{array}{l}\text { Rapid } \\
\text { cycling } \\
\text { BD-I or II } \\
\text { DSM-IV }\end{array}$ & $\begin{array}{l}\text { Mean Age } \\
38 \text { (16-65) } \\
56 \% \\
\text { Female; } \\
\text { White 92\% } \\
55 \% \text { BD-I } \\
45 \% \text { BD-II } \\
\text { N=49 }\end{array}$ & $\begin{array}{l}\text { Major } \\
\text { Depressive } \\
\text { Episode (did not } \\
\text { stabilize on open } \\
\text { treatment of } \\
\text { lithium and } \\
\text { divalproex) }\end{array}$ & $\begin{array}{l}\text { Substance Abuse } \\
\text { Other Mental Health } \\
\text { Pregnant/Nursing } \\
\text { Taking Other Meds } \\
\text { (nonresponsive to } \\
\text { lamotrigine } \\
\text { previously) }\end{array}$ \\
\hline
\end{tabular}




\begin{tabular}{|c|c|c|c|c|c|}
\hline $\begin{array}{c}\text { Author, Year } \\
\text { Single/-Multisite } \\
\text { Location } \\
\text { Risk of Bias } \\
\text { PMID }\end{array}$ & $\begin{array}{l}\text { Intervention/ } \\
\text { Comparison }\end{array}$ & $\begin{array}{c}\text { BD Type; } \\
\text { Diagnostic } \\
\text { Criteria }\end{array}$ & $\begin{array}{c}\text { Demo- } \\
\text { graphics }\end{array}$ & $\begin{array}{l}\text { Current } \\
\text { Episode }\end{array}$ & Key Exclusions \\
\hline $\begin{array}{l}\text { Sachs, } 2007^{150} \\
\text { Multisite } \\
\text { US } \\
\text { High ROB } \\
17392295\end{array}$ & $\begin{array}{l}\text { I: } \\
\text { antidepressant } \\
\text { (paroxetine, } \\
\text { bupropion, or } \\
\text { both) + mood } \\
\text { stabilizer } \\
\text { C: Placebo + } \\
\text { mood stabilizer }\end{array}$ & $\begin{array}{l}\text { BD-I or II } \\
\text { DMS-IV }\end{array}$ & $\begin{array}{l}\text { Mean Age } \\
40(18+) \\
57 \% \\
\text { Female } \\
\text { Race } 10 \% \\
\text { Nonwhite } \\
68 \% \text { BD-I } \\
32 \% \text { BD-II } \\
\mathrm{N}=366\end{array}$ & $\begin{array}{l}\text { Major } \\
\text { Depressive } \\
\text { Episode }\end{array}$ & $\begin{array}{l}\text { Substance Abuse } \\
\text { Other Mental Health } \\
\text { Taking Other Meds } \\
\text { Labs/Other } \\
\text { Conditions }\end{array}$ \\
\hline $\begin{array}{l}\text { Altshuler, } 2017^{151} \\
\text { Multisite } \\
\text { US } \\
\text { High ROB } \\
28135846\end{array}$ & $\begin{array}{l}\text { I: Sertraline } \\
\text { C1: Lithium } \\
\text { C2: Lithium + } \\
\text { Sertraline }\end{array}$ & $\begin{array}{l}\text { BD-II } \\
\text { DSM-IV }\end{array}$ & $\begin{array}{l}\text { Mean Age } \\
39(18-65) \\
\text { Female } \\
54 \% \\
\text { White } 97 \% \\
\text { BD-II 100\% } \\
\mathrm{N}=142\end{array}$ & $\begin{array}{l}\text { Current major } \\
\text { depressive } \\
\text { episode. } \\
\text { IDS-C } \geq 22 ; \mathrm{CGI}- \\
\mathrm{BD} \geq 3 \text { on } \\
\text { depression } \\
\text { subscale; } \\
\text { YMRS } \leq 8 ; \mathrm{CGI}- \\
\text { BD=1 on mania } \\
\text { severity subscale }\end{array}$ & $\begin{array}{l}\text { Substance Abuse } \\
\text { Other Mental Health } \\
\text { (Nonresponsive to } \\
\text { Lithium or Sertraline) }\end{array}$ \\
\hline $\begin{array}{l}\text { Amsterdam, } 2016^{152} \text {, } \\
153 \\
\text { Singlesite } \\
\text { US } \\
\text { Moderate ROB } \\
26892848 \\
26803764^{153}\end{array}$ & $\begin{array}{l}\text { I: Venlafaxine } \\
\text { C: Lithium }\end{array}$ & $\begin{array}{l}\text { BD-II } \\
\text { DSM-IV }\end{array}$ & $\begin{array}{l}\text { Mean Age } \\
43(18+) \\
\text { Female } \\
57 \% \\
\text { White } 73 \% \\
\text { BD-II 100\% } \\
\mathrm{N}=129\end{array}$ & $\begin{array}{l}\text { Current major } \\
\text { depressive } \\
\text { episode. HAM- } \\
D \geq 16\end{array}$ & $\begin{array}{l}\text { Substance Abuse } \\
\text { Other Mental Health } \\
\text { Pregnant/Nursing } \\
\text { Taking Other Meds } \\
\text { Labs/Other } \\
\text { Conditions } \\
\text { (Nonresponsive to } \\
\text { Venlafaxine or } \\
\text { Lithium) }\end{array}$ \\
\hline $\begin{array}{l}\text { Amsterdam, 2008 } \\
154 \\
\text { Singlesite } \\
\text { US } \\
\text { High ROB } \\
18344727 \\
18486235^{154}\end{array}$ & $\begin{array}{l}\text { I: Venlafaxine } \\
\text { C: Lithium }\end{array}$ & $\begin{array}{l}\text { BD-II } \\
\text { DSM-IV }\end{array}$ & $\begin{array}{l}\text { Mean Age } \\
37(18+) \\
\text { Female } \\
57 \% \\
\text { White } 82 \% \\
\text { BD-II 100\% } \\
\mathrm{N}=83\end{array}$ & $\begin{array}{l}\text { Current major } \\
\text { depressive } \\
\text { episode. HAM- } \\
D \geq 18\end{array}$ & $\begin{array}{l}\text { Substance Abuse } \\
\text { Other Mental Health } \\
\text { Pregnant/Nursing } \\
\text { Taking Other Meds } \\
\text { Labs/Other } \\
\text { Conditions } \\
\text { (Nonresponsive to } \\
\text { Venlafaxine or } \\
\text { Lithium) }\end{array}$ \\
\hline $\begin{array}{l}\text { Nierenberg, } 2013^{146,} \\
156 \\
\text { Multisite } \\
\text { US } \\
\text { Low ROB } \\
23288387 \\
19933719\end{array}$ & $\begin{array}{l}\text { I: Lithium + } \\
\text { Optimal } \\
\text { Personalized } \\
\text { Treatment } \\
\text { (OPT) } \\
\text { C: Optimal } \\
\text { Personalized } \\
\text { Treatment }\end{array}$ & $\begin{array}{l}\text { BD-I or II; } \\
\text { DSM-IV }\end{array}$ & $\begin{array}{l}\text { Mean Age } \\
39(18+) \\
57 \% \\
\text { Female } \\
75 \% \text { White } \\
76 \% \text { BD-I } \\
24 \% \text { BD-II } \\
\mathrm{N}=283\end{array}$ & $\begin{array}{l}\text { Currently } \\
\text { symptomatic (not } \\
\text { defined), } \\
\text { requiring a } \\
\text { change in } \\
\text { medication } \\
\text { (Mean YMRS } \\
\text { 12.5; MADRS } \\
\text { 22.5; CGI } \\
\text { severity } 4.3 \text { ) }\end{array}$ & $\begin{array}{l}\text { Current lithium use } \\
\text { Need for } \\
\text { hospitalization } \\
\text { Other Medical } \\
\text { Conditions } \\
\text { Pregnancy }\end{array}$ \\
\hline
\end{tabular}

BD=bipolar; C: Control; CGI=Clinical global impression scale; DSM-IV= Diagnostic and statistical manual, $4^{\text {th }}$ edition; HAMD= Hamilton Rating Scale for Depression; I=Intervention; IDS-C=Inventory of depressive symptomatology - clinician rated; MADRS= Montgomery Asberg depression rating scale; N=number; NR=not reported; ROB=risk of bias; YMRS=Young Mania Rating Scale.

\section{Drug Treatments for Depression Versus Placebo}

Strength of evidence from three RCTs was insufficient to draw conclusions for the effect of depression treatments compared to placebo in adults with BD due to single studies and 
imprecision. Each study with a placebo comparator addressed a different intervention for a different bipolar population; memantine versus placebo in adults with bipolar II disorder (BDII) ${ }^{148,}{ }^{149}$ lamotrigine versus placebo in adults with rapid cycling BD-II, ${ }^{147}$ and antidepressants versus placebo in adults with bipolar I disorder (BD-I) and BD-II. ${ }^{150}$ All three studies reported no significant differences between groups for all outcomes. Information on adverse events was reported in two studies. Both reported no differences between groups for severe adverse events or withdrawal due to a lack of response or clinical worsening. ${ }^{147,150}$

\section{Drug Treatments for Depression Versus Active Control}

Strength of evidence from three RCTs was assessed as insufficient to draw conclusions for depression treatments compared with other drugs in BD due to study limitations and imprecision. The three studies with active comparators addressed two comparisons for adults with BD-II and a current major depressive episode: a three arm study of sertraline versus lithium or lithium plus sertraline ${ }^{151}$ and two studies of venlafaxine versus lithium. ${ }^{150,153}$ Reported results were mixed for treatment response or remission. All three studies assessed switching to hypomanic or manic states but found no significant differences between groups. Only one unidentified serious adverse event was reported across the three studies' total of 354 participants.

\section{Lithium Plus OPT Versus OPT Alone}

One pragmatic RCT enrolled 283 participants with BD-I or II who were at least mildly symptomatic, with clinical need, and randomized them to receive moderate-dose lithium plus Optimized Personalized Treatment (OPT) or OPT alone. ${ }^{156}$ OPT follows the Texas Implementation of Medication Algorithm, so participants were commonly using medications other than lithium. While the population was not specifically identified as experiencing a manic state, and in fact 87 percent of the participants were experiencing a depressive state, the participants were not clinically stable and treatment was deemed necessary to stabilize mood. Evidence for all outcomes was deemed insufficient due to a single study with too small of a sample size to test the finding of no difference between groups in CGI or need for clinical treatment adjustment. The study was not designed to test for nonsignificance between groups. The study reported fewer participants in the lithium group needed less atyipcal antipsychotics than those in the OPT-only group (48.3 percent and 62.5 percent, respectively). Appendix H provides details.

\section{Interpreting the Findings for Drug Treatments for Depression}

Evidence for drug treatment for BD depression is insufficient to draw conclusions. Only six RCTs that examined five unique comparisons for bipolar depression met inclusion criteria. These studies differed in their diagnostic inclusion criteria, but tended to recruit predominantly individuals with BD II or mixed samples of BD-I and BD II without examining the effectiveness of treatments separately for each bipolar subtype. Given clinicians' concerns about treatmentinduced switching into hypomania/mania and other adverse events, it is notable that not all studies systematically reported adverse events. The few studies that did report adverse events tended to find no group differences. Additional evidence is necessary to draw definitive conclusions about adverse events of drug treatments for bipolar depression.

The degree and nature of the sparse and scattered studies is noteworthy. Often studies did not meet the review's inclusion criteria of at least three month followup for depression, including studies for lurasidone, olanzapine, or quetiapine, which are FDA-approved for depression in BD. 
Given the chronic nature of BD, it is doubtful that studies reporting effects for drugs with followup periods shorter than 3 months are clinically useful. 


\section{Chapter 6. Drug Treatments for Maintenance}

\section{Key Points}

- Evidence for maintenance treatments was scattered across 16 drugs administered alone or in combination therapy.

- Evidence was largely insufficient to draw conclusions regarding the effects of drug treatments for maintenance of euthymia in adults with bipolar disorders (BD) for the primary outcomes of interest (relapse, symptom scores, and function).

- Low-strength evidence showed longer time to recurrence of any mood state for bipolar I disorder (BD-I) patients receiving lithium compared to placebo $(n=1579)$ in followup up to 2 years. Participants receiving lithium reported more tremor than those receiving placebo.

- Evidence was insufficient for all other outcomes across all interventions.

\section{Eligible Studies for Maintenance Treatments}

We identified 44 eligible publications reporting 36 unique studies with at least 6 months followup. ${ }^{56,139,157-192}$ Twenty-one studies, seven of which were three-arm studies, examined a single drug treatment for maintenance, $56,82,99,117,124,139,160,161,164,165,167-173,176,178,180,181,184,187$,

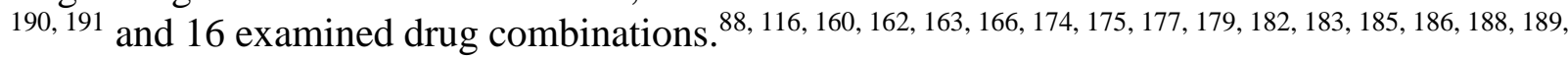

192 Drugs examined included: oral aripiprazole, long-acting injectable aripiprazole, divalproex/valproate, carbamazepine, fluoxetine, gabapentin, lamotrigine, lithium, olanzapine, oxcarbazepine, paliperidone, perphenazine, long-acting injectable risperidone, quetiapine, venlafaxine, and ziprasidone. Fourteen studies were assessed as low or moderate risk of bias and 22 were assessed as high, generally due to attrition. Of 36 unique studies, 27 were industry funded. An additional 15 studies were excluded due to attrition over 50 percent and not using time to relapse outcomes. ${ }^{74,75,81,83,86,87,95,115,119,120,123,125,193-196}$ Only two studies were not RCTs. ${ }^{176,185}$ Sample sizes ranged from 25 to 1226; 17 studies were below 200 participants, ranging from 25 to 175 . Study duration ranged from 6 months to 3 years, with 24 using followup of 6 months to 1 year.

Appendix I provides detailed evidence tables, summary risk of bias assessments, and assessments of strength of evidence for key comparisons and outcomes. A summary of findings with at least low-strength evidence for other drug treatments for maintenance are provided in Table 29. Any intervention and comparison not listed in Table 29, or outcome not listed for an included intervention and comparison, was found to have an evidence base insufficient to draw conclusions.

Table 29. Summary of findings with at least low-strength evidence for maintenance studies

\begin{tabular}{|l|l|l|l|}
\hline Intervention & $\begin{array}{l}\text { \# Studies/ Design } \\
\text { (n Analyzed) } \\
\text { Timing }\end{array}$ & \multicolumn{1}{|c|}{ Findings } & Strength of Evidence \\
\hline $\begin{array}{l}\text { Lithium vs. } \\
\text { placebo }\end{array}$ & $\begin{array}{l}\begin{array}{l}6 \mathrm{RCT}^{165} 167162164 \\
178\end{array} \\
(\mathrm{n}=1579) \\
1 \text { to 2 years }\end{array}$ & Time to overall relapse: Favors Lithium & $\begin{array}{l}\text { Low } \\
\text { (moderate study limitations, } \\
\text { imprecise) }\end{array}$ \\
\hline
\end{tabular}

n=number; RCT=randomized controlled trial 


\section{Single Drug Treatments for Maintenance}

Table 30 summarizes the bipolar type and major inclusion and exclusion criteria for single drug studies for maintenance. Appendix I provides details.

Table 30. Population and inclusion criteria for single drug studies for maintenance

\begin{tabular}{|c|c|c|c|c|c|}
\hline $\begin{array}{c}\text { Author, Year } \\
\text { Single-Multisite } \\
\text { Local/Continent } \\
\text { Risk of Bias } \\
\text { PMID }\end{array}$ & $\begin{array}{l}\text { Intervention/ } \\
\text { Comparison }\end{array}$ & $\begin{array}{c}\text { BD Type; } \\
\text { Diagnostic } \\
\text { Criteria }\end{array}$ & Demographics & Current Episode & Key Exclusions \\
\hline $\begin{array}{l}\text { Calabrese, } \\
2017^{191} \\
\text { Multisite } \\
4 \text { Continents } \\
\text { Moderate ROB } \\
\\
28146613\end{array}$ & $\begin{array}{l}\text { I: Long-acting } \\
\text { Aripiprazole } \\
\text { (monthly } \\
\text { injection) } \\
\text { C: Placebo }\end{array}$ & $\begin{array}{l}\text { BD-I; } \\
\text { DSM-IV }\end{array}$ & $\begin{array}{l}\text { Mean Age } 41 \\
(18-65) ; \\
\text { Female 58\%; } \\
\text { Race 54\% } \\
\text { Black/African } \\
\text { American 28\% } \\
\mathrm{N}=266\end{array}$ & $\begin{array}{l}\text { Initial manic episode } \\
\text { YMRS } \geq 20 \text { but then } \\
\text { met } Y M R S \leq 12 \text {, } \\
\text { MADRS } \leq 12 \text {, no } \\
\text { active suicidality }\end{array}$ & $\begin{array}{l}\text { Rapid Cycling } \\
\text { Refractory BD } \\
\text { First Manic } \\
\text { Episode } \\
\text { Substance } \\
\text { Abuse } \\
\text { Other Mental } \\
\text { Health } \\
\text { Labs/Other } \\
\text { Conditions }\end{array}$ \\
\hline $\begin{array}{l}\text { Keck, } 2006^{56} \\
\text { Multisite } \\
2 \text { Continents } \\
\text { Moderate ROB } \\
16669728\end{array}$ & $\begin{array}{l}\text { I Aripiprazole } \\
\text { C: Placebo }\end{array}$ & $\begin{array}{l}\text { BD-I } \\
\text { DSM-IV }\end{array}$ & $\begin{array}{l}\text { Mean Age } 40 \\
(18+) ; \\
\text { Female } 67 \% \\
\text { Race White } \\
56 \% \\
\text { Hispanic/Latino } \\
23 \% \\
\mathrm{~N}=161\end{array}$ & $\begin{array}{l}\text { Symptom stability: } \\
\text { YMRS } \leq 10 \text { and } \\
\text { MADRS } \leq 13 \text { for } 4 \\
\text { consecutive visits } \\
\text { over } 6 \text { weeks }\end{array}$ & $\begin{array}{l}\text { Substance } \\
\text { Abuse } \\
\text { Other Mental } \\
\text { Health } \\
\text { Labs/Other } \\
\text { Conditions } \\
\text { Pregnant/Nursin } \\
\text { g } \\
\text { Unresponsive to } \\
\text { Clozapine } \\
\text { ECT in last } 2 \\
\text { years }\end{array}$ \\
\hline $\begin{array}{l}\text { Greil, } 1997^{171} \\
\text { Multisite } \\
\text { Germany } \\
\text { High ROB } \\
9165384 \\
9864077^{170} \\
10529070^{169} \\
10529071^{168} \\
11093063^{190}\end{array}$ & $\begin{array}{l}\text { I: } \\
\text { Carbamazepine } \\
\text { C: Lithium }\end{array}$ & $\begin{array}{l}\text { BD-I or II; } \\
\text { DSM-IV }\end{array}$ & $\begin{array}{l}\text { Mean Age } 40 \\
(18-65) ; \\
\text { Female 57\%; } \\
\text { Race NR } \\
\text { BD I 58\% } \\
\text { BD-NOS 33\% } \\
\text { N=171 }\end{array}$ & $\begin{array}{l}\text { Remission from any } \\
\text { bipolar episode; GAS } \\
>70\end{array}$ & $\begin{array}{l}\text { First Manic } \\
\text { Episode } \\
\text { Substance } \\
\text { Abuse } \\
\text { Other Mental } \\
\text { Health } \\
\text { Neurological } \\
\text { Disorders }\end{array}$ \\
\hline $\begin{array}{l}\text { Hartong, } 2003^{173} \\
\text { Multisite } \\
\text { Netherlands } \\
\text { Low ROB } \\
12633122\end{array}$ & $\begin{array}{l}\text { I: } \\
\text { Carbamazepine } \\
\text { C: Lithium }\end{array}$ & $\begin{array}{l}\text { BD-I or II; } \\
\text { DSM-III }\end{array}$ & $\begin{array}{l}\text { Mean Age } 42 \\
(18+) ; \\
\text { Female 54\%; } \\
\text { Race NR } \\
\text { BD I 77\% } \\
\text { BD-II 23\% } \\
\text { N=98 }\end{array}$ & $\begin{array}{l}\text { Remission from any } \\
\text { bipolar episode, } \\
\text { according to Bech } \\
\text { Rafaelsen Mania or } \\
\text { Melancholia Scales }\end{array}$ & $\begin{array}{l}\text { First Manic } \\
\text { Episode }\end{array}$ \\
\hline
\end{tabular}




\begin{tabular}{|c|c|c|c|c|c|}
\hline $\begin{array}{c}\text { Author, Year } \\
\text { Single-Multisite } \\
\text { Local/Continent } \\
\text { Risk of Bias } \\
\text { PMID }\end{array}$ & $\begin{array}{l}\text { Intervention/ } \\
\text { Comparison }\end{array}$ & $\begin{array}{l}\text { BD Type; } \\
\text { Diagnostic } \\
\text { Criteria }\end{array}$ & Demographics & Current Episode & Key Exclusions \\
\hline $\begin{array}{l}\text { Bowden, } 2003^{165} \\
\text { Multisite } \\
3 \text { continents } \\
\text { Moderate ROB } \\
12695317\end{array}$ & $\begin{array}{l}\text { I: Lamotrigine } \\
\text { C1: Placebo } \\
\text { C2: Lithium }\end{array}$ & $\begin{array}{l}\text { BD-I; } \\
\text { DSM-IV }\end{array}$ & $\begin{array}{l}\text { Mean Age 41 } \\
(18+) ; \\
\text { Female 47\%; } \\
\text { Race NR } \\
\mathrm{N}=175\end{array}$ & $\begin{array}{l}\text { Lamotrigine } \\
\text { responders (CGI- } \\
\mathrm{S} \leq 3 \text { for at least } 4 \\
\text { continuous weeks), } \\
\text { after an open label } \\
\text { period: Manic; DSM- } \\
\text { IV Criteria for Mania } \\
\text { or Hypomania } \\
\text { currently or within } \\
\text { past } 60 \text { days with } \\
\text { previous episodes in } \\
\text { past } 3 \text { years. }\end{array}$ & $\begin{array}{l}\text { Other Mental } \\
\text { Health } \\
\text { Conditions }\end{array}$ \\
\hline $\begin{array}{l}\text { Calabrese, } \\
2003^{167} \\
\text { Multisite } \\
\text { 4 Continents } \\
\text { Moderate ROB } \\
14628976\end{array}$ & $\begin{array}{l}\text { I: Lamotrigine } \\
\text { C1: Placebo } \\
\text { C2: Lithium }\end{array}$ & $\begin{array}{l}\text { BD-I; } \\
\text { DSM-IV }\end{array}$ & $\begin{array}{l}\text { Mean Age } 43 \\
(18+) ; \\
\text { Female 56\%; } \\
\text { Race NR } \\
\mathrm{N}=410\end{array}$ & $\begin{array}{l}\text { Lamotrigine } \\
\text { responders (CGI- } \\
\mathrm{S} \leq 3 \text { for at least } 4 \\
\text { continuous weeks) } \\
\text { after an open label } \\
\text { period: depression; } \\
\text { DSM-IV criteria for } \\
\text { depression currently } \\
\text { or within past } 60 \\
\text { days with previous } \\
\text { depression and } \\
\text { mania episodes in } \\
\text { past } 3 \text { years. }\end{array}$ & $\begin{array}{l}\text { Other Mental } \\
\text { Health } \\
\text { Conditions }\end{array}$ \\
\hline $\begin{array}{l}\text { Calabrese, } \\
2000^{124} \\
\text { Multisite } \\
\text { US, Canada } \\
\text { High ROB } \\
11105737\end{array}$ & $\begin{array}{l}\text { I: Lamotrigine } \\
\text { C: Placebo }\end{array}$ & $\begin{array}{l}\text { Rapid } \\
\text { cycling } \\
\text { BD-I or II } \\
\text { DSM-IV }\end{array}$ & $\begin{array}{l}\text { Mean Age 38 } \\
(18+) ; \\
\text { Female 58\% } \\
\text { Race NR } \\
\text { BD I 70\% } \\
\text { BD-II 30\% } \\
\mathrm{N}=182\end{array}$ & $\begin{array}{l}\text { Rapid cyclers, } \\
\text { stabilized on } \\
\text { lamotrigine (no mood } \\
\text { episodes requiring } \\
\text { other drugs or ECT) }\end{array}$ & $\begin{array}{l}\text { Other Mental } \\
\text { Health } \\
\text { Conditions } \\
\text { Labs/Other } \\
\text { conditions }\end{array}$ \\
\hline $\begin{array}{l}\text { Amsterdam, } \\
2010^{162} \\
\text { Single-Site } \\
\text { US } \\
\text { Moderate ROB } \\
20360317\end{array}$ & $\begin{array}{l}\text { I: Fluoxetine } \\
\text { C1: Placebo } \\
\text { C2: Lithium }\end{array}$ & $\begin{array}{l}\text { BD-II; } \\
\text { DSM-IV }\end{array}$ & $\begin{array}{l}\text { Median Age } 38 \\
(18+) ; \\
\text { Sex NR; } \\
\text { Race NR } \\
N=81\end{array}$ & $\begin{array}{l}\text { Recovered; } \\
\text { HAM-D } \geq 16 \text { at } \\
\text { enrollment; } \\
\text { HAM-D } \leq 8 \text { after } 12 \\
\text { weeks of initial } \\
\text { Fluoxetine therapy at } \\
\text { 20-80mg/day) }\end{array}$ & $\begin{array}{l}\text { Substance abuse } \\
\text { Neurological } \\
\text { Disorders } \\
\text { Taking other } \\
\text { medications } \\
\text { Pregnant/Nursin } \\
\text { gLabs/Other } \\
\text { Conditions } \\
\end{array}$ \\
\hline $\begin{array}{l}\text { Calabrese, } \\
2005^{117} \\
\text { Single-site } \\
\text { US } \\
\text { Government } \\
\text { High ROB } \\
16263857\end{array}$ & $\begin{array}{l}\text { I: Divalproex } \\
\text { C: Lithium }\end{array}$ & $\begin{array}{l}\text { Rapid } \\
\text { cycling } \\
\text { BD-I or II } \\
\text { DSM-IV }\end{array}$ & $\begin{array}{l}\text { Mean Age } 37 \\
(18+) \\
\text { Female 52\% } \\
\text { White NR } \\
\text { BD I 60\% } \\
\text { BD-II 40\% } \\
\mathrm{N}=60\end{array}$ & $\begin{array}{l}\text { Responders to both } \\
\text { drugs } \\
\text { Rapid cycling; mood } \\
\text { episode in previous } 3 \\
\text { months }\end{array}$ & $\begin{array}{l}\text { Substance Use } \\
\text { Other Mental } \\
\text { Health } \\
\text { Conditions } \\
\text { Pregnant/Nursin } \\
\mathrm{g} \\
\text { Lab/other } \\
\text { conditions } \\
\text { Intolerant of } \\
\text { lithium }\end{array}$ \\
\hline
\end{tabular}




\begin{tabular}{|c|c|c|c|c|c|}
\hline $\begin{array}{c}\text { Author, Year } \\
\text { Single-Multisite } \\
\text { Local/Continent } \\
\text { Risk of Bias } \\
\text { PMID }\end{array}$ & $\begin{array}{l}\text { Interventionl } \\
\text { Comparison }\end{array}$ & $\begin{array}{l}\text { BD Type; } \\
\text { Diagnostic } \\
\text { Criteria }\end{array}$ & Demographics & Current Episode & Key Exclusions \\
\hline $\begin{array}{l}\text { Bowden, } 2000^{164} \\
\text { Multisite } \\
\text { US } \\
\text { Moderate ROB } \\
10807488 \\
12784116^{172}\end{array}$ & $\begin{array}{l}\text { I: Divalproex } \\
\text { C1: Placebo } \\
\text { C2: Lithium }\end{array}$ & $\begin{array}{l}\text { BD-I; } \\
\text { DSM-III }\end{array}$ & $\begin{array}{l}\text { Mean Age } 39.2 \\
(18-75) ; \\
\text { Female } 51 \% ; \\
\text { White } 94 \% \\
N=372\end{array}$ & $\begin{array}{l}\text { No episode at } \\
\text { randomization; } \\
\text { Scores of YMRS } \leq \\
\text { 11, DSS } \leq 13 \text {, GAS } \\
>60 ;\end{array}$ & $\begin{array}{l}\text { Substance } \\
\text { Abuse; Other } \\
\text { Mental Health } \\
\text { Conditions; } \\
\text { Taking Other } \\
\text { Medications; } \\
\text { Pregnant/Nursin } \\
\text { g }\end{array}$ \\
\hline $\begin{array}{l}\text { Newport, } 2008^{176} \\
\text { Single site } \\
\text { US } \\
\text { High ROB } \\
18402631\end{array}$ & $\begin{array}{l}\text { I: Lamotrigine } \\
\text { C: Discontinued } \\
\text { mood } \\
\text { stabilizers }\end{array}$ & $\begin{array}{l}\text { BD-I, II or } \\
\text { NOS; } \\
\text { DSM-IV }\end{array}$ & $\begin{array}{l}\text { Mean Age } \\
\text { Female 100\% } \\
\text { White 91\% } \\
\text { BD I 73\% } \\
\text { BD-II 23\% } \\
\text { BD-NOS 4\% } \\
\mathrm{N}=26\end{array}$ & $\begin{array}{l}\text { Euthymic; at } \\
\text { conception of current } \\
\text { pregnancy }\end{array}$ & $\begin{array}{l}\text { Labs/Other } \\
\text { Conditions }\end{array}$ \\
\hline $\begin{array}{l}\text { Prien, } 1973^{178} \\
\text { Multisite } \\
\text { US } \\
\text { High ROB } \\
4569674\end{array}$ & $\begin{array}{l}\text { I: Lithium } \\
\text { C: Placebo }\end{array}$ & $\begin{array}{l}\text { BD-I; } \\
\text { NR }\end{array}$ & $\begin{array}{l}\text { Median Age } 44 \\
(17-60) ; \\
\text { Sex NR; } \\
\text { Race NR } \\
N=205\end{array}$ & $\begin{array}{l}\text { No episode at } \\
\text { randomization; }\end{array}$ & $\begin{array}{l}\text { Neurological } \\
\text { Disorders; } \\
\text { Abnormal Lab } \\
\text { Results }\end{array}$ \\
\hline $\begin{array}{l}\text { Balance } \\
\text { Investigators, } \\
2010^{160} \\
\text { Multisite } \\
2 \text { Continents } \\
\text { Moderate ROB } \\
20092882\end{array}$ & $\begin{array}{l}\text { I: Lithium + } \\
\text { Valproate } \\
\text { C1: Lithium } \\
\text { C2: Valproate }\end{array}$ & $\begin{array}{l}\text { BD-I; } \\
\text { DSM-IV }\end{array}$ & $\begin{array}{l}\text { Mean Age } 43 \\
(16+) ; \\
\text { Female 49\%; } \\
\text { Race NR } \\
\text { (U.S.A and } \\
\text { Europe) } \\
\mathrm{N}=330\end{array}$ & $\begin{array}{l}\text { Not having acute } \\
\text { episode ; not defined }\end{array}$ & $\begin{array}{l}\text { Pregnant/Nursin } \\
\mathrm{g}\end{array}$ \\
\hline $\begin{array}{l}\text { Tohen, } 2006^{180} \\
\text { Multisite } \\
2 \text { Continents } \\
\text { Moderate ROB } \\
16449478\end{array}$ & $\begin{array}{l}\text { I: Olanzapine } \\
\text { C: Placebo }\end{array}$ & $\begin{array}{l}\text { BD-I; } \\
\text { DSM-IV }\end{array}$ & $\begin{array}{l}\text { Mean Age } 40 \\
(18+) ; \\
\text { Female } 39 \% ; \\
\text { White } 87 \% \\
N=361\end{array}$ & $\begin{array}{l}\text { Remission from } \\
\text { manic or mixed } \\
\text { episode; } \\
\text { YMRS } \leq 15 \text { and } \\
\text { HAM-D } \leq 8\end{array}$ & $\begin{array}{l}\text { First Manic } \\
\text { Episode }\end{array}$ \\
\hline $\begin{array}{l}\text { Tohen, } 2003^{82} \\
\text { Multisite } \\
\text { US } \\
\text { High ROB } \\
\text { 12832240 } \\
\text { Extension of } \\
\text { Tohen, 2002b }{ }^{44} \\
\text { 12042191 }\end{array}$ & $\begin{array}{l}\text { I: Olanzapine } \\
\text { C: Divalproex }\end{array}$ & $\begin{array}{l}\text { BD-I } \\
\text { DSM-IV }\end{array}$ & $\begin{array}{l}\text { Mean Age } 40 \\
(18-75) \\
\text { Female } 57 \% \\
\text { White } 82 \% \\
N=251\end{array}$ & $\begin{array}{l}\text { YMRS >19 } \\
\text { (time to relapse; not } \\
\text { clear what proportion } \\
\text { were stable) }\end{array}$ & $\begin{array}{l}\text { Substance Use } \\
\text { Pregnant/Nursin } \\
\mathrm{g} \\
\text { Labs/other } \\
\text { conditions }\end{array}$ \\
\hline $\begin{array}{l}\text { Vieta, } 2012^{184} \\
\text { Multisite } \\
4 \text { Continents } \\
\text { High ROB } \\
22503488\end{array}$ & $\begin{array}{l}\text { I: Olanzapine } \\
\text { C1: Placebo } \\
\text { C2: } \\
\text { Risperidone }\end{array}$ & $\begin{array}{l}\text { BD-I; } \\
\text { DSM-IV }\end{array}$ & $\begin{array}{l}\text { Mean Age } 37 \\
\text { (18-65); } \\
\text { Female 52\%; } \\
\text { White } 41 \% \\
\mathrm{~N}=398\end{array}$ & $\begin{array}{l}\text { No current episode; } \\
\text { responders from } \\
\text { Phase II Acute } \\
\text { (YMRS } \geq 20 \text { and } \\
\text { CGI-S } \geq 4 \text { ) or non- } \\
\text { acute (mood } \\
\text { episodes with YMRS } \\
<12 \text { and CGI-S } \leq 3 \text { ) }\end{array}$ & $\begin{array}{l}\text { First Manic } \\
\text { Episode } \\
\text { Schizoaffective } \\
\text { Other Mental } \\
\text { Health } \\
\text { Taking Other } \\
\text { Meds } \\
\text { Pregnant/Nursin } \\
\text { g }\end{array}$ \\
\hline
\end{tabular}




\begin{tabular}{|c|c|c|c|c|c|}
\hline $\begin{array}{c}\text { Author, Year } \\
\text { Single-Multisite } \\
\text { Local/Continent } \\
\text { Risk of Bias } \\
\text { PMID }\end{array}$ & $\begin{array}{l}\text { Intervention/ } \\
\text { Comparison }\end{array}$ & $\begin{array}{c}\text { BD Type; } \\
\text { Diagnostic } \\
\text { Criteria }\end{array}$ & Demographics & Current Episode & Key Exclusions \\
\hline $\begin{array}{l}\text { Tohen, } 2005^{181} \\
\text { Multisite } \\
5 \text { Continents } \\
\text { Moderate ROB } \\
15994710\end{array}$ & $\begin{array}{l}\text { I: Olanzapine } \\
\text { C: Lithium }\end{array}$ & $\begin{array}{l}\text { BD-I; } \\
\text { DSM-IV }\end{array}$ & $\begin{array}{l}\text { Mean Age } 42 \\
(18+) ; \\
\text { Sex 53\%; } \\
\text { White } 99 \% \\
\text { N=431 }\end{array}$ & $\begin{array}{l}\text { Met remission } \\
\text { criteria: including } \\
\text { YMRS } \leq 15 \text { and } \\
\text { HAM-D } \leq 8 \\
\text { After open-label: } \\
\text { Manic or Mixed } \\
\text { Episode } \\
\text { YMRS } \geq 20\end{array}$ & $\begin{array}{l}\text { Substance } \\
\text { Abuse } \\
\text { Other Mental } \\
\text { Health } \\
\text { Neurological } \\
\text { Disorders } \\
\text { Taking Other } \\
\text { Meds } \\
\text { Labs/Other } \\
\text { Conditions }\end{array}$ \\
\hline $\begin{array}{l}\text { Berwaerts, } \\
2012^{139} \\
\text { Multisite } \\
5 \text { Continents } \\
\text { High ROB } \\
\\
22377512\end{array}$ & $\begin{array}{l}\text { I: Paliperidone } \\
\text { extended } \\
\text { release } \\
\text { C: Placebo }\end{array}$ & $\begin{array}{l}\text { BD-I; } \\
\text { DSM-IV }\end{array}$ & $\begin{array}{l}\text { Mean Age } 40 \\
(18-65) ; \\
\text { Female } 55 \% \\
\text { White } 61 \% \\
\mathrm{~N}=383\end{array}$ & $\begin{array}{l}\text { Remission; YMRS } \\
\text { and MADRS } \leq 12 \text { for } \\
\text { last three weeks of } \\
\text { acute and } \\
\text { continuation } \\
\text { treatment study } \\
\text { phases }\end{array}$ & $\begin{array}{l}\text { First manic } \\
\text { episode } \\
\text { Schizoaffective } \\
\text { Substance abuse } \\
\text { Other mental } \\
\text { health } \\
\text { Neurological } \\
\text { disorders } \\
\text { Labs/other } \\
\text { conditions }\end{array}$ \\
\hline $\begin{array}{l}\text { Quiroz, } 2010^{99} \\
\text { Multisite } \\
3 \text { Continents } \\
\text { Moderate ROB } \\
20227682\end{array}$ & $\begin{array}{l}\text { I: Risperidone } \\
\text { long-acting } \\
\text { C: Placebo }\end{array}$ & $\begin{array}{l}\text { BD-I } \\
\text { DSM-IV }\end{array}$ & $\begin{array}{l}\text { Mean Age (18- } \\
65) \\
\text { Female 49\% } \\
\text { White } 80 \% \\
N=303\end{array}$ & $\begin{array}{l}\text { Responders to } \\
\text { Phase III: stable at } \\
\text { CGI-BP-S }<3\end{array}$ & $\begin{array}{l}\text { Substand abuse } \\
\text { Taking other } \\
\text { meds } \\
\text { Pregnant/nursing } \\
\text { Rapid cycling } \\
\text { Other mental } \\
\text { health } \\
\text { Labs/other } \\
\text { conditions }\end{array}$ \\
\hline $\begin{array}{l}\text { Amsterdam, } \\
2015^{161} \\
\text { Single site } \\
\text { US } \\
\text { High ROB } \\
26143402\end{array}$ & $\begin{array}{l}\text { I: Venlafaxine } \\
\text { C: Lithium }\end{array}$ & $\begin{array}{l}\text { BD-II; } \\
\text { DSM-IV }\end{array}$ & $\begin{array}{l}\text { Mean Age } 42 \\
(18+) ; \\
\text { Female } 54 \% \text {; } \\
\text { White } 17 \% \\
\mathrm{~N}=55\end{array}$ & $\begin{array}{l}\text { Responders to RCT } \\
\text { phase: } \geq 50 \% \\
\text { reduction in baseline } \\
\text { HAM-D + CGI-BP-S } \\
<3\end{array}$ & $\begin{array}{l}\text { Substance abuse } \\
\text { Neurological } \\
\text { disorders } \\
\text { Taking other } \\
\text { meds } \\
\text { Pregnant/nursing } \\
\text { Labs/other } \\
\text { conditions }\end{array}$ \\
\hline $\begin{array}{l}\text { Weisler, } 2011^{187} \\
\text { Multisite } \\
\text { RCT of } \\
\text { responders } \\
\text { Multisite } \\
5 \text { continents } \\
\text { Moderate ROB } \\
22054050\end{array}$ & $\begin{array}{l}\text { I: Quetiapine } \\
\text { C1: Placebo } \\
\text { C2: Lithium }\end{array}$ & $\begin{array}{l}\text { BD-I; } \\
\text { DSM-IV }\end{array}$ & $\begin{array}{l}\text { Mean Age } 40 \\
(18+) \\
\text { Female } 53 \% \\
\text { White } 63 \% \\
\mathrm{~N}=1226\end{array}$ & $\begin{array}{l}\text { Meeting stability } \\
\text { criteria of YMRS } \leq \\
12 \text { and MADRS } \leq 12 ; \\
\text { Current or previous } \\
\text { depression/mania/mi } \\
\text { xed episode at entry } \\
\text { or within past two } \\
\text { years }\end{array}$ & $\begin{array}{l}\text { Substance } \\
\text { Abuse } \\
\text { Other Mental } \\
\text { Health } \\
\text { Conditions } \\
\text { Pregnant/Nursin } \\
\text { g } \\
\text { Labs/Other } \\
\text { Conditions }\end{array}$ \\
\hline
\end{tabular}

$\mathrm{BD}=$ bipolar disorder; $\mathrm{C}=$ control; CGI-BP-S=clinical global impression scale, bipolar ediction, severity; DSM-IV= Diagnostic and statistical manual, $4^{\text {th }}$ edition; DSS=depression severity scale; ECT= electroconvulsive therapy; EX=extended release; GAS=Global Assessment Scale; HAM-D = Hamilton Rating Scale for Depression; I=intervention; MADRS= MontgomeryAsberg depression rating scale; $\mathrm{N}=$ number; $\mathrm{NR}=$ not reported; $\mathrm{RCT}=$ randomized controlled trial; $\mathrm{ROB}=$ risk of bias; YMRS=young mania rating scale.

\section{Single Drug for Maintenance Versus Placebo}

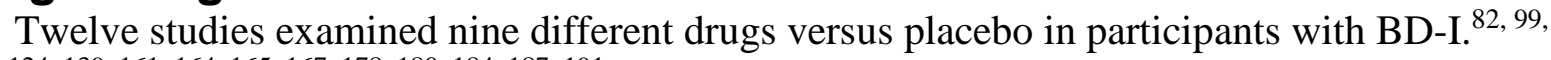
117, 124, 139, 161, 164, 165, 167, 178, 180, 184, 187, 191 Five studies also included bipolar II disorder (BD-II) 
participants. ${ }^{117,124,163,182,183}$ Sample sizes ranged from 26 to 1226 and followup lasted from 26 weeks to 3 years.

Low-strength evidence (moderate study limitations, imprecision) from six RCTs ( $\mathrm{n}=1579)$ showed that adults with BD-I receiving lithium over a 2 year period had longer time to recurrence of any mood state compared to those receiving a placebo. ${ }^{179,186}$ Since the time to event outcomes account for attrition, these were the only outcomes abstracted from these studies due to the high attrition rates. Evidence was insufficient for time to manic or depressive states due to mixed results. Participants receiving lithium reported more tremor than those receiving placebo. Otherwise serious adverse events did not differ by group. Appendix I provides details.

Evidence was insufficient for all outcomes to address whether ten drugs were better than placebo for maintenance in adults with BD: long-acting aripiprazole $(n=226),{ }^{191}$ aripiprazole $\left(n=1610,{ }^{56}\right.$ divalproex $(n=281),{ }^{165,}, 172$ fluoxetine $(n=55),{ }^{162}$ lamotrigine $(n=471 ; n=182$ rapid cycling), ${ }^{124,164,167}$ olanzapine $(n=855),{ }^{139}, 180,184$ paliperidone $(n=300),{ }^{139}$ quetiapine $(n=808),{ }^{187}$ and risperidone $(\mathrm{n}=353) .{ }^{99,184}$ Single studies, high study limitations, small sample sizes, and strong imprecision contributed to the insufficient strength of evidence rating. Except for divalproex, results were reported as favoring the interventions for time to overall relapse. Where reported, participants using placebo experience less frequent severe events of tremor than those using divalproex, or less parkinsonism than those using olanzapine; otherwise, serious adverse events were generally not different between groups. Appendix I provides details.

While providing insufficient evidence to draw conclusions, one observational study was noteworthy for examining lamotrigine use in 26 pregnant women, recruited before conception or during first trimester, with any BD type. Women chose to discontinue all mood stabilizers or to continue on lamotrigine only. While women who chose to continue lamotrigine were less likely to have an unplanned pregnancy than those who discontinued all treatment. Risk of relapse was 3/10 women using lamotrigine versus 16/16 women who discontinued treatment.

\section{Single Drug for Maintenance Versus Active Control}

Fourteen studies (20 publications) examined 10 different drugs versus another drug. ${ }^{139,157-162 \text {, }}$ 164, 165, 167-173, 178, 181, 184, 187, 190 Sample sizes ranged from 54 to 768 and followup lasted from 6 months to 3 years. Appendix I provides details.

Evidence was insufficient for all outcomes to address whether carbamazepine $(n=171),{ }^{168-171}$, 173, 190 divalproex ( $n=372, n=60$ rapid cycling), ${ }^{117,} 164,172$ fluoxetine $(n=54),{ }^{162}$ lamotrigine $(n=390),{ }^{165,167}$ olanzapine $(n=855),{ }^{181}$ quetiapine $(n=768),{ }^{187}$ valproate $(n=220),{ }^{160}$ and venlafaxine $(n=55)^{161}$ was better than lithium; paliperidone $(n=235)^{139}$ or risperidone $(n=263)^{184}$ was better than olanzapine; or olanzapine was better than divalproex $(n=251)^{82}$ for maintenance in adults with BD. Single studies, high study limitations, small sample sizes, and imprecision contributed to the insufficient strength of evidence rating. Results were mixed across the studies. With the exception of participants using divalproex showing less akathisia compared to those using lithium, no differences between groups were reported for serious adverse events.

\section{Combination Drug Treatment for Maintenance}

Table 31 summarizes bipolar type and major inclusion and exclusion criteria for combination drug therapy studies for maintenance. Appendix I provides details. 
Table 31. Population and inclusion criteria for combination drug treatment for maintenance studies

\begin{tabular}{|c|c|c|c|c|c|}
\hline $\begin{array}{c}\text { Author, Year } \\
\text { Singlel-Multisite } \\
\text { Location } \\
\text { Risk of Bias } \\
\text { PMID } \\
\end{array}$ & $\begin{array}{l}\text { Intervention/ } \\
\text { Comparison }\end{array}$ & $\begin{array}{c}\text { BD Type; } \\
\text { Diagnostic } \\
\text { Criteria }\end{array}$ & $\begin{array}{c}\text { Demo- } \\
\text { graphics }\end{array}$ & Current Episode & Key Exclusions \\
\hline $\begin{array}{l}\text { Woo, } 2011^{188} \\
\text { RCT } \\
\text { Multisite } \\
\text { Asia } \\
\text { High ROB } \\
22134973\end{array}$ & $\begin{array}{l}\text { I: Aripiprazole + } \\
\text { divalproex } \\
\text { C: Placebo + } \\
\text { divalproex }\end{array}$ & $\begin{array}{l}\text { BD-I; } \\
\text { DSM-IV }\end{array}$ & $\begin{array}{l}\text { Mean Age } 38 \\
(18-65) ; \\
\text { Female } 68 \% \\
\text { Asian } \geq 75 \% \\
\mathrm{~N}=83\end{array}$ & $\begin{array}{l}\text { Remission after } \\
\text { Manic/Mixed; } \\
\text { Initially } \\
\text { YMRS } \geq 20 \text {, then } \\
\text { YMRS } \leq 12 \text {, } \\
\text { MADRS } \leq 13 \text { at } \\
\text { randomization } \\
\text { after } 6 \text { weeks of } \\
\text { stabilization } \\
\text { treatment }\end{array}$ & $\begin{array}{l}\text { Schizoaffective } \\
\text { Substance abuse } \\
\text { Neurological } \\
\text { disorders } \\
\text { Taking other meds } \\
\text { Pregnant/nursing } \\
\text { Labs/other } \\
\text { conditions }\end{array}$ \\
\hline $\begin{array}{l}\text { Marcus, } 2011^{175} \\
\text { RCT } \\
\text { Multisite } \\
\text { NR } \\
\text { High ROB } \\
21443567\end{array}$ & $\begin{array}{l}\text { I: Aripiprazole + } \\
\text { lithium/valproate } \\
\text { C: Placebo + } \\
\text { lithium/valproate }\end{array}$ & $\begin{array}{l}\text { BD-I; } \\
\text { DSM-IV }\end{array}$ & $\begin{array}{l}\text { Mean Age } 39 \\
(18+) \\
\text { Female } 55 \% \\
\text { White } 68 \% \\
\mathrm{~N}=337\end{array}$ & $\begin{array}{l}\text { Remission after } \\
\text { Manic/Mixed; } \\
\text { Initially YMRS } \geq 16 \\
\text { and current } \\
\text { episode duration } \\
<2 \text { years; then } \\
\text { YMRS } \leq 12 \text {, } \\
\text { MADRS } \leq 12 \text { at } \\
\text { randomization } \\
\text { after } 12 \text { weeks of } \\
\text { stabilization } \\
\text { treatment }\end{array}$ & $\begin{array}{l}\text { First manic episode } \\
\text { Schizoaffective } \\
\text { Substance abuse } \\
\text { Other mental health } \\
\text { Neurological } \\
\text { disorders } \\
\text { Taking other meds } \\
\text { Labs/other } \\
\text { conditions }\end{array}$ \\
\hline $\begin{array}{l}\text { Carlson, } 2012^{192} \\
\text { RCT of responders } \\
\text { Multisite } \\
\text { US } \\
\text { Industry } \\
\text { High ROB } \\
22329471\end{array}$ & $\begin{array}{l}\text { I: Aripiprazole + } \\
\text { lamotrigine } \\
\text { C: Placebo + } \\
\text { lamotrigine }\end{array}$ & $\begin{array}{l}\text { BD-I; } \\
\text { DSM-IV }\end{array}$ & $\begin{array}{l}\text { Mean Age } 39 \\
(18+) \\
\text { Female } 65 \% \\
\text { White } 90 \% \\
\mathrm{~N}=351\end{array}$ & $\begin{array}{l}\text { Stabilization after } \\
\text { mania; } 8 \text { weeks at } \\
\text { YMRS } \leq 12 \text {, } \\
\text { MADRS } \leq 12 \text {. } \\
\text { Study entry manic } \\
\text { or mixed } \\
\text { YMRS } \geq 16 \text { in } \\
\text { previous } 3 \\
\text { months with or } \\
\text { without rapid } \\
\text { cycling (4 to } 7 \\
\text { mood episodes } \\
\text { per year) }\end{array}$ & $\begin{array}{l}\text { Substance abuse } \\
\text { Other Mental Health } \\
\text { Conditions } \\
\text { Pregnant/Nursing } \\
\text { Labs/other } \\
\text { conditions } \\
\text { First manic episode } \\
\text { Treatment } \\
\text { refractory } \\
\text { mania/mixed mania }\end{array}$ \\
\hline $\begin{array}{l}\text { Kemp, } 2009^{116} \\
\text { Single site } \\
\text { US } \\
\text { High ROB } \\
19192457\end{array}$ & $\begin{array}{l}\text { I: Divalproex + } \\
\text { lithium } \\
\text { C: Placebo + } \\
\text { lithium }\end{array}$ & $\begin{array}{l}\text { BD-I or II; } \\
\text { DSM-IV }\end{array}$ & $\begin{array}{l}\text { Mean Age } 36 \\
(16-65) \\
\text { Mean Age } 36 \\
\text { Female } 36 \% \\
\text { White } 82 \% \\
\text { BD I } 75 \% \\
\text { BD II 25\% } \\
N=31\end{array}$ & $\begin{array}{l}\text { Stable responders } \\
\text { (HAM-D score } \leq \\
\text { 20, YMRS score } \leq \\
\text { 12.5) Rapid } \\
\text { cycling, } \\
\text { substance use } \\
\text { disorder as } \\
\text { ascertained by } \\
\text { structured } \\
\text { interview; mood } \\
\text { episode in } \\
\text { previous } 3 \\
\text { months }\end{array}$ & $\begin{array}{l}\text { Labs/other } \\
\text { conditions } \\
\text { Pregnant/nursing }\end{array}$ \\
\hline
\end{tabular}




\begin{tabular}{|c|c|c|c|c|c|}
\hline $\begin{array}{c}\text { Author, Year } \\
\text { Singlel-Multisite } \\
\text { Location } \\
\text { Risk of Bias } \\
\text { PMID }\end{array}$ & $\begin{array}{l}\text { Intervention/ } \\
\text { Comparison }\end{array}$ & $\begin{array}{l}\text { BD Type; } \\
\text { Diagnostic } \\
\text { Criteria }\end{array}$ & $\begin{array}{c}\text { Demo- } \\
\text { graphics }\end{array}$ & Current Episode & Key Exclusions \\
\hline $\begin{array}{l}\text { Vieta, } 2006^{183} \\
\text { Multisite } \\
\text { Spain } \\
\text { High ROB } \\
16649836\end{array}$ & $\begin{array}{l}\text { I: Gabapentin + } \\
\text { mood stabilizers } \\
\text { C: Placebo + } \\
\text { mood stabilizezrs }\end{array}$ & $\begin{array}{l}\text { BD-I or II; } \\
\text { DSM-IV }\end{array}$ & $\begin{array}{l}\text { Mean Age 49 } \\
(18-75) \\
\text { Female 72\% } \\
\text { Race NR } \\
\text { BD I 76\% } \\
\text { BD II 24\% } \\
\mathrm{N}=25\end{array}$ & $\begin{array}{l}\text { Euthymic; } \\
\text { CGI-BP-M } \geq 4 ; \\
\text { HAMD } \leq 8 \\
\text { YMRS } \leq 4\end{array}$ & $\begin{array}{l}\text { Substance abuse } \\
\text { Pregnant/nursing } \\
\text { Labs/other } \\
\text { conditions }\end{array}$ \\
\hline $\begin{array}{l}\text { Tohen, 2004 } \\
\text { Multisite } \\
\text { US, Canada } \\
\text { Industry } \\
\text { High ROB } \\
\\
\text { 15056579 } \\
\text { extension of } \\
\text { Tohen, 2002a } \\
\text { 117796284 }\end{array}$ & $\begin{array}{l}\text { I: Olanzapine + } \\
\text { Lithium or } \\
\text { Valproate } \\
\text { C: Placebo + } \\
\text { Lithium or } \\
\text { Valproate }\end{array}$ & $\begin{array}{l}\text { BD-I; } \\
\text { DSM-IV }\end{array}$ & $\begin{array}{l}\text { Mean Age } 41 \\
(19-69) \\
48 \% \text { Female } \\
85 \% \text { White } \\
N=99\end{array}$ & $\begin{array}{l}\text { Responders to } \\
\text { olanzapine + } \\
\text { lithium or } \\
\text { valproate mania } \\
\text { and depression } \\
\text { no worse than } \\
\text { mild; }\end{array}$ & $\begin{array}{l}\text { First Manic Episode } \\
\text { Labs/Other } \\
\text { Conditions }\end{array}$ \\
\hline $\begin{array}{l}\text { Vieta, } 2008^{182} \\
\text { Multisite } \\
\text { Spain } \\
\text { Moderate ROB } \\
18346292\end{array}$ & $\begin{array}{l}\text { I: Oxcarbazepine } \\
\text { + Lithium } \\
\text { C: Placebo + } \\
\text { Lithium }\end{array}$ & $\begin{array}{l}\text { BD-I or II; } \\
\text { DSM-IV }\end{array}$ & $\begin{array}{l}\text { Mean Age 44 } \\
(18+) ; \\
\text { Female 66\% } \\
\text { Race NR } \\
\text { BD I 76\% } \\
\text { BD II 24\% } \\
\mathrm{N}=55\end{array}$ & $\begin{array}{l}\text { Euthymic; } \\
\text { YMRS } \leq 12 ; \\
\text { MADRS } \leq 20\end{array}$ & $\begin{array}{l}\text { Substance abuse } \\
\text { Other Mental Health } \\
\text { Conditions } \\
\text { Pregnant/Nursing } \\
\text { Labs/other } \\
\text { conditions }\end{array}$ \\
\hline $\begin{array}{l}\text { Zarate, } 2004^{189} \\
\text { Singlesite } \\
\text { US } \\
\text { High ROB } \\
14702269\end{array}$ & $\begin{array}{l}\text { l: Perphenazine + } \\
\text { mood stabilizers } \\
\text { C: Placebo + } \\
\text { mood stabilizers }\end{array}$ & $\begin{array}{l}\text { BD-I; } \\
\text { DSM-IV }\end{array}$ & $\begin{array}{l}\text { Mean Age 34 } \\
\text { (18-65); } \\
\text { Female78\% } \\
\text { White } 80 \% \\
\mathrm{~N}=37\end{array}$ & $\begin{array}{l}\text { Remission after } \\
\text { Manic /Mixed as } \\
\text { defined per DSM- } \\
\text { IV criteria } \\
\text { (Structured } \\
\text { Clinical Interview); } \\
\text { then euthymic by } \\
\text { week } 10 \text { at } \\
\text { randomization; } \\
\text { YMRS } \leq 10 ; \\
\text { HAM-D } \leq 10\end{array}$ & $\begin{array}{l}\text { Schizoaffective } \\
\text { Substance abuse } \\
\text { Other mental health } \\
\text { Labs/other } \\
\text { conditions }\end{array}$ \\
\hline $\begin{array}{l}\text { Suppes, } 2009^{179} \\
\text { Multisite } \\
\text { US/Canada } \\
\text { High ROB } \\
19289454\end{array}$ & $\begin{array}{l}\text { I: Quetiapine + } \\
\text { Lithium OR } \\
\text { Valproate } \\
\text { C: Placebo + } \\
\text { Lithium OR } \\
\text { Valproate }\end{array}$ & $\begin{array}{l}\text { BD-I; } \\
\text { DSM-IV }\end{array}$ & $\begin{array}{l}\text { Mean Age 40 } \\
(18+) \\
\text { Female 53\%; } \\
\text { White } 82 \% \\
\mathrm{~N}=623\end{array}$ & $\begin{array}{l}\text { Stabilization after } \\
\text { Mania; } \\
\text { Stable at } \\
\text { randomization } \\
\text { after Lithium or } \\
\text { Valproate; } \\
\text { YMRS and } \\
\text { MADRS } \leq 12 \\
\text { AND at least } 1 \\
\text { mood episode of } \\
\text { any type in past } 2 \\
\text { years and another } \\
6 \text { months prior to } \\
\text { randomization } \\
\end{array}$ & $\begin{array}{l}\text { First Manic Episode } \\
\text { Substance Abuse } \\
\text { Other Mental Health } \\
\text { Conditions } \\
\text { Pregnant/Nursing }\end{array}$ \\
\hline
\end{tabular}




\begin{tabular}{|c|c|c|c|c|c|}
\hline $\begin{array}{c}\text { Author, Year } \\
\text { Singlel-Multisite } \\
\text { Location } \\
\text { Risk of Bias } \\
\text { PMID }\end{array}$ & $\begin{array}{l}\text { Intervention/ } \\
\text { Comparison }\end{array}$ & $\begin{array}{c}\text { BD Type; } \\
\text { Diagnostic } \\
\text { Criteria }\end{array}$ & $\begin{array}{c}\text { Demo- } \\
\text { graphics }\end{array}$ & Current Episode & Key Exclusions \\
\hline $\begin{array}{l}\text { Vieta, } 2008^{186} \\
\text { Multisite } \\
\text { 4 Continents } \\
\text { Moderate ROB } \\
18579216\end{array}$ & $\begin{array}{l}\text { I: Quetiapine + } \\
\text { Lithium OR } \\
\text { Valproate } \\
\text { C: Placebo + } \\
\text { Lithium OR } \\
\text { Valproate }\end{array}$ & $\begin{array}{l}\text { BD-I; } \\
\text { DSM-IV }\end{array}$ & $\begin{array}{l}\text { Mean Age } 42 \\
(18+) ; \\
\text { Female 55\%; } \\
\text { White } 97 \% \\
\mathrm{~N}=706\end{array}$ & $\begin{array}{l}\text { Stabilization after } \\
\text { the latest episode } \\
\text { of any type } \\
\text { (mania, mixed, } \\
\text { depression) within } \\
\text { past } 26 \text { weeks, } \\
\text { then achieved } \\
\text { clinical stability } \\
\text { (YMRS and } \\
\text { MADRS } \leq 12 \text { ) } \\
\text { prior to } \\
\text { randomization, } \\
\text { subject to } \\
\text { specified time } \\
\text { periods }\end{array}$ & $\begin{array}{l}\text { Substance Abuse; } \\
\text { Other Mental Health } \\
\text { Conditions; } \\
\text { Taking Other Meds; } \\
\text { Pregnant/Nursing }\end{array}$ \\
\hline $\begin{array}{l}\text { Macfadden, } \\
2009^{174} \\
\text { Multisite } \\
\text { US/India } \\
\text { High ROB } \\
19922552\end{array}$ & $\begin{array}{l}\text { I: } \\
\text { Risperidone } \\
\text { (long-acting } \\
\text { injectable) + } \\
\text { Treatment As } \\
\text { Usual (mania } \\
\text { treatments, anti- } \\
\text { depressants, } \\
\text { etc.) } \\
\text { C: Placebo + } \\
\text { Treatment as } \\
\text { usual }\end{array}$ & $\begin{array}{l}\text { BD-I; } \\
\text { DSM-IV }\end{array}$ & $\begin{array}{l}\text { Mean Age } \\
38.9 \text { (18-63); } \\
\text { Female 28\%; } \\
\text { White 10\% } \\
\mathrm{N}=124\end{array}$ & $\begin{array}{l}\text { Any current phase } \\
\text { including } \\
\text { euthymic; } \\
4 \text { or more mood } \\
\text { episodes in past } \\
\text { year }\end{array}$ & $\begin{array}{l}\text { Substance Abuse; } \\
\text { Other Mental Health } \\
\text { Conditions; Taking } \\
\text { other Medications; } \\
\text { Abnormal Lab } \\
\text { Results }\end{array}$ \\
\hline $\begin{array}{l}\text { Bobo, 2011 } \\
\text { Single-site } \\
\text { US } \\
\text { High ROB } \\
22104634\end{array}$ & $\begin{array}{l}\text { I: } \\
\text { Risperidone + } \\
\text { treatment as } \\
\text { usual } \\
\text { C: Treatment as } \\
\text { usual }\end{array}$ & $\begin{array}{l}\text { BD-I or II; } \\
\text { DSM-IV }\end{array}$ & $\begin{array}{l}\text { Mean Age } \\
40.2(18-64) ; \\
\text { Female } 67 \% \text {; } \\
\text { White } 67 \% \\
\text { BD I 73\% } \\
\text { BD II } 27 \% \\
N=50\end{array}$ & $\begin{array}{l}\text { Any current } \\
\text { phase; } \\
\text { (Actual participant } \\
\text { profile: YMRS } \geq 8, \\
\text { HAM-D } \geq 8 \text { and } \\
\text { four or more } \\
\text { relapses in past } \\
\text { year with } 1 \text { event } \\
\text { in past } 6 \text { months) }\end{array}$ & $\begin{array}{l}\text { Schizoaffective; } \\
\text { Other Mental Health } \\
\text { Conditions; } \\
\text { Pregnant/Nursing }\end{array}$ \\
\hline $\begin{array}{l}\text { Bowden, } 2010^{159,} \\
166 \\
\text { Multisite } \\
\text { 3 Continents } \\
\text { High ROB } \\
20122373 \\
22999893\end{array}$ & $\begin{array}{l}\text { I: Ziprasidone + } \\
\text { Lithium or } \\
\text { valproate } \\
\text { C: Placebo + } \\
\text { Lithium or } \\
\text { valproate }\end{array}$ & $\begin{array}{l}\text { BD-I; } \\
\text { DSM-IV }\end{array}$ & $\begin{array}{l}\text { Mean Age } \\
38.9(18+) ; \\
\text { Female 54\%; } \\
\text { White 62\% } \\
\mathrm{N}=240\end{array}$ & $\begin{array}{l}\text { Stabilization after } \\
\text { Mania; Initial } \\
\text { YMRS } \geq 14 \text { with } \\
\text { score } \geq 2 \text { on at } \\
\text { least four items at } \\
\text { screening and } \\
\text { admission. } \\
\text { Followed by } \\
\text { stabilization: } \mathrm{CGI}- \\
\text { I } 3 \text { at least } 2 \\
\text { consecutive } \\
\text { weeks }\end{array}$ & $\begin{array}{l}\text { Substance Abuse; } \\
\text { Other Mental Health } \\
\text { Condition; } \\
\text { Pregnant/Nursing; } \\
\text { Labs/Other } \\
\text { Conditions }\end{array}$ \\
\hline $\begin{array}{l}\text { Balance } \\
\text { Investigators, } \\
2010^{160} \\
\text { Multisite } \\
2 \text { Continents } \\
\text { Moderate ROB } \\
20092882\end{array}$ & $\begin{array}{l}\text { I: Lithium + } \\
\text { Valproate } \\
\text { C1: Lithium } \\
\text { C2: Valproate }\end{array}$ & $\begin{array}{l}\text { BD-I; } \\
\text { DSM-IV }\end{array}$ & $\begin{array}{l}\text { Mean Age 43 } \\
(16+) ; \\
\text { Female 49\%; } \\
\text { Race NR } \\
\mathrm{N}=330\end{array}$ & $\begin{array}{l}\text { Not having acute } \\
\text { episode; Not } \\
\text { defined }\end{array}$ & Pregnant/Nursing \\
\hline
\end{tabular}




\begin{tabular}{|c|c|c|c|c|c|}
\hline $\begin{array}{c}\text { Author, Year } \\
\text { Single/-Multisite } \\
\text { Location } \\
\text { Risk of Bias } \\
\text { PMID }\end{array}$ & $\begin{array}{l}\text { Intervention/ } \\
\text { Comparison }\end{array}$ & $\begin{array}{l}\text { BD Type; } \\
\text { Diagnostic } \\
\text { Criteria }\end{array}$ & $\begin{array}{c}\text { Demo- } \\
\text { graphics }\end{array}$ & Current Episode & Key Exclusions \\
\hline $\begin{array}{l}\text { Nierenberg, } \\
2016^{157,158,177} \\
\text { Multisite } \\
\text { US } \\
\text { High ROB } \\
\text { 26845264 } \\
\text { NA } \\
\text { 24346608 }\end{array}$ & $\begin{array}{l}\text { I: Quetiapine + } \\
\text { personalized } \\
\text { treatment } \\
\text { C: Lithium + } \\
\text { personalized } \\
\text { treatment }\end{array}$ & $\begin{array}{l}\text { BD-I, II; } \\
\text { DSM-IV }\end{array}$ & $\begin{array}{l}\text { Mean Age 39 } \\
(18+) ; \\
\text { Female 59\%; } \\
\text { White 72\% } \\
\text { BD I 68\% } \\
\text { BD II NR } \\
\text { N=482 }\end{array}$ & Any current phase & $\begin{array}{l}\text { Pregnant/Nursing; } \\
\text { Labs/Other } \\
\text { Conditions }\end{array}$ \\
\hline $\begin{array}{l}\text { Vieta, } 2010^{185} \\
\text { Multisite } \\
\text { Spain } \\
\text { High ROB } \\
20429835\end{array}$ & $\begin{array}{l}\text { I: Valproate + } \\
\text { Aripirazole } \\
\text { C: Lithium + } \\
\text { Aripiprazole }\end{array}$ & $\begin{array}{l}\text { BD-I; } \\
\text { DSM-IV }\end{array}$ & $\begin{array}{l}\text { Mean Age } \\
\text { 43; } \\
\text { Female } 53 \% \text {; } \\
\text { White } 93 \% \\
\mathrm{~N}=283\end{array}$ & $\begin{array}{l}\text { Initial inclusion of } \\
\text { manic, partial } \\
\text { responders to } \\
\text { Lithium or } \\
\text { Valproate; Initial } \\
\text { YMRS } \geq 16 \text { with } \\
\text { decrease of } 25 \% \\
\text { between } \\
\text { treatment phases. } \\
\text { Patients eligible } \\
\text { for this extension } \\
\text { if investigator felt } \\
\text { the patient would } \\
\text { benefit from long- } \\
\text { term aripiprazole } \\
\text { treatment. }\end{array}$ & $\begin{array}{l}\text { Other Mental Health } \\
\text { Conditions; } \\
\text { Substance Abuse }\end{array}$ \\
\hline
\end{tabular}

$\mathrm{BD}=$ bipolar disorder; $\mathrm{C}=$ control; CGI-BP=Clinical global impression, bipolar edition; CGI-I=Clinical global impression, global improvement; DSM-IV= Diagnostic and statistical manual, $4^{\text {th }}$ edition; EX=extended release; HAM-D = Hamilton Rating Scale for Depression; I=intervention; MADRS= Montgomery-Asberg depression rating scale; NR=not reported; YMRS=Young mania rating scale.

\section{Combination Drug Therapy for Maintenance Versus Placebo}

Thirteen studies examined nine different combination therapies versus placebo in BD-I participants $^{88,116,163,166,174,175,179,182,183,186,188,189,192}$ Four studies also included BD-II participants. ${ }^{116,163,182,183}$ Sample sizes ranged from 25 to 706 and followup lasted from 26 weeks to 2 years.

Evidence was insufficient to address whether nine combinations performed better than placebo: aripiprazole plus mood stabilizers $(n=771),{ }^{175,}{ }^{188}$ divalproex plus lithium $(n=31),{ }^{116}$ gabapentin plus mood stabilizers $(n=25),{ }^{183}$ olanzapine plus mood stabilizers $(n=99) ; 8$ oxcarbazepine plus lithium $(n=55),{ }^{182}$ perphenazine plus mood stabilizers $(n=37),{ }^{189}$ quetiapine plus mood stabilizers $(\mathrm{n}=1329)$, long-acting injectable risperidone plus mood stabilizers $(n=174),{ }^{163,174}$ and ziprasidone plus mood stabilizers $(n=240) .{ }^{166}$ Single studies, high study limitations, small sample sizes, and imprecision contributed to the insufficient strength of evidence rating. Results were mixed across the studies and generally showed no differences between groups in withdrawals due to adverse events. Serious adverse events were also not different between groups.

\section{Combination Therapy for Maintenance Versus Active Control}

Three studies examined combination therapies versus active comparators in BD-I participants, each a unique, single study comparison. ${ }^{160,177,185}$ Only one study also enrolled 
participants with other types of BD. ${ }^{177}$ Sample sizes ranged from 283 to 482 and followup lasted from 24 weeks to 2 years.

Evidence was insufficient to address whether lithium plus valproate performed better than either lithium or valproate alone $(n=330),{ }^{160}$ quetiapine plus mood stabilizers performed better than lithium plus another mood stabilizer $(n=482),{ }^{177}$ or if aripiprazole plus valproate performed differently than aripiprazole plus lithium $(n=283),{ }^{185}$ generally due to high study limitations and imprecision. Overall, the trials reported no significant differences between groups. However, the three-group Balance study reported time to relapse hazard ratios favored lithium plus valproate over valproate alone, but did not significantly differ from lithium alone. Also, serious adverse events did not generally differ between groups. All studies reported at least one death, but not to significant differences between groups for such a rare outcome.

\section{Interpreting the Findings for Drug Treatment for Maintenance}

The current evidence for drug treatment for maintenance in BD is largely insufficient to draw conclusions for a number of reasons. First, 36 unique maintenance studies examined 16 different medications often resulting in a single study for a specific comparison for a specific followup duration. In addition, 22 of 36 of maintenance studies (61\%) were rated as having severe study limitations (high risk of bias). Second, the high rates of attrition often led to only one usable outcome measure - time to recurrence of a bipolar episode-since this metric accounted for high attrition rates by including information from participants who dropped out due to BD episode relapse. Moreover, 17 studies had small sample sizes of less than 200 participants and 24 studies (66\%) had followup between six to twelve months, precluding conclusions for long-term maintenance for most of examined treatments. Third, differences in current bipolar phase criteria across studies, ranging from any current phase (i.e., depression, hypomania, or euthymia), remission from mania, remission from any BD episode, or response or partial response to a specific acute episode treatment, made it difficult to determine for whom findings might apply.

Still, low-strength evidence showed a longer time to recurrence of any BD episode for lithium versus placebo treatment in adults with BD I during a two year followup. The evidence was insufficient for time to recurrence of depression or mania due to inconsistent findings. There was a greater rate of tremors but insufficient evidence for differences in other adverse events rates between lithium versus placebo treatment. In general, in single drug versus placebo comparisons, when reported, placebo showed less tremor than divalproex treatment and less parkinsonism signs than olanzapine treatment, but no differences in other serious adverse events. Also, comparisons between drugs and active comparators did not show differences in serious adverse events, except for less akathisia for divalproex than lithium treatment.

The nature of inclusion criteria and study populations limits the applicability of these findings for certain subpopulations of individuals with BD, such as individuals with BD II, older adults with any BD illness type, and individuals at the early stage of BD illness. For example, 20 studies included individuals with BD I only, while studies with multiple BD disorder subtypes did not report results separately by illness type. The majority of studies included younger adults with mean ages in 30s and early 40s. An additional eight studies excluded individuals experiencing first manic episode. Only two small studies looked at individuals with rapid cycling BD. Most studies did not examine whether the number of prior manic or depressive episodes affected the efficacy of drugs during maintenance phase treatment. 


\section{Chapter 7. Psychosocial and Other Nondrug Treatments}

\section{Key Points}

- Evidence was largely insufficient to draw conclusions regarding the effect of psychosocial interventions compared with either inactive or active comparators for bipolar disorders (BD) for the primary outcomes of interest (relapse, symptom scores, and function). This included the effect of interventions at specific phases (e.g., acute hypomania/mania or depression).

- Low-strength evidence showed no effect of cognitive behavioral therapy (CBT) on depression or mania symptoms when compared with an active comparator.

- Low-strength evidence showed no effect of systematic/collaborative care on relapse rates when compared with an inactive comparator.

- Evidence was insufficient for all other outcomes across all interventions.

- Evidence was insufficient to evaluate other nondrug interventions.

\section{Eligible Studies for Psychosocial and Other Nondrug Treatments}

We identified 63 eligible publications that reported 48 unique studies (50 unique comparisons) on psychosocial interventions for BD. We identified one eligible publication on somatic therapy. We excluded six studies during the screening process due to an attrition rate greater than 50 percent.

We analyzed the effect of interventions by category and grouped studies based on whether they used an inactive (i.e., usual care) or active comparator. Included studies on psychosocial therapy examined varied interventions ranging from psychoeducation, CBT, systematic or collaborative care, family or partner interventions (FPI), to interpersonal and social rhythm therapy (IPSRT). The one publication on somatic therapy examined repetitive transcranial magnetic stimulation (rTMS). Results are grouped by general outcome category: relapse, symptom scores (i.e., depression and mania symptoms), function, and additional outcomes (e.g., hospitalizations, suicide rates). None of the included studies reported harms, outside of limited information on self-harm and deaths reported by three studies. For the majority of included studies, the outcome reporting timepoints (6 months and beyond) represent the duration of the treatment and a followup period. For clarity, population/inclusion criteria tables include the number of sessions for psychosocial interventions and the length of time for the intervention (e.g., participants received 12 weekly sessions).

We did not aggregate or pool studies within intervention categories due to differences across studies in inclusion criteria, active components (e.g., individual, group, or internet-based therapy modality), scales used for outcome assessment, and outcome time points. Thus the majority of intervention/ comparator/outcome comparisons were based on single studies. Appendices J-P provide evidence tables, summary risk of bias assessments, assessments of strength of evidence for key comparisons and outcomes, and reporting for additional outcomes. We calculated effect size (Cohen's d) for individual studies in the appendix tables when sufficient data was available. Table 32 provides a matrix of nondrug interventions and comparators included in the review. 
Table 32. Interventions, comparators, and outcomes for nondrug interventions

\begin{tabular}{|c|c|c|c|c|c|c|c|}
\hline Intervention Type & Studies & $\begin{array}{l}\text { Low or } \\
\text { Moderate } \\
\text { ROB* }^{*}\end{array}$ & $\begin{array}{l}\text { High } \\
\text { ROB* }\end{array}$ & Relapse & $\begin{array}{c}\text { Symptom } \\
\text { Scores }\end{array}$ & Function & $\begin{array}{l}\text { Additional } \\
\text { Outcomes }\end{array}$ \\
\hline $\begin{array}{l}\text { Psychoeducation vs. Inactive } \\
\text { Control }\end{array}$ & 10 & 6 & 4 & 7 & 5 & 4 & 6 \\
\hline $\begin{array}{l}\text { Psychoeducation vs. Active } \\
\text { Control }\end{array}$ & 3 & 2 & 1 & 2 & 2 & 1 & 1 \\
\hline CBT vs. Inactive Control & 8 & 6 & 2 & 7 & 7 & 3 & 4 \\
\hline CBT vs. Active Control & 5 & 5 & 0 & 3 & 5 & 2 & 0 \\
\hline $\begin{array}{l}\text { Systematic/Collaborative Care } \\
\text { vs. Inactive Control }\end{array}$ & 6 & 6 & 0 & 2 & 5 & 4 & 3 \\
\hline $\begin{array}{l}\text { Systematic/Collaborative Care } \\
\text { vs. Active Control }\end{array}$ & 0 & NA & NA & NA & NA & NA & NA \\
\hline FPI vs. Inactive Control & 2 & 1 & 1 & 2 & 1 & 0 & 2 \\
\hline FPI vs.. Active Control & 4 & 4 & 0 & 2 & 3 & 1 & 1 \\
\hline IPSRT Inactive Control & 1 & 0 & 1 & 1 & 1 & 1 & 1 \\
\hline IPSRT vS. Active Control & 1 & 1 & 0 & 0 & 1 & 1 & 1 \\
\hline $\begin{array}{l}\text { Combination Interventions vs. } \\
\text { Inactive Control }\end{array}$ & 3 & 3 & 0 & 1 & 3 & 1 & 2 \\
\hline $\begin{array}{l}\text { Combination Interventions vs. } \\
\text { Active Control }\end{array}$ & 2 & 1 & 1 & 1 & 2 & 1 & 1 \\
\hline $\begin{array}{l}\text { Other Psychosocial } \\
\text { Interventions }\end{array}$ & 3 & 2 & 1 & 1 & 3 & 2 & 0 \\
\hline Somatic Therapy & 1 & 1 & 0 & 1 & 0 & 0 & 0 \\
\hline TOTAL & 49 & 35 & 9 & 26 & 36 & 20 & 20 \\
\hline
\end{tabular}

*Studies with multiple ROB ratings due to differences in reporting by outcome or across publications are categorized by their average ROB rating

CBT=Cognitive Behavioral Therapy; FPI=Family or Partner Interventions; IPSRT= Interpersonal and Social Rhythm Therapy; ROB=Risk of Bias

\section{Psychoeducation}

We identified 14 publications reporting 13 unique studies on psychoeducation as a treatment for BD. ${ }^{197-211}$ Appendix J provides details. We were unable to draw conclusions for psychoeducation interventions due to insufficient evidence.

\section{Psychoeducation Versus Inactive Control}

We identified 11 publications reporting 10 unique studies comparing psychoeducation interventions to inactive comparators. ${ }^{197-201,203-208}$ Six studies were rated low or moderate risk of

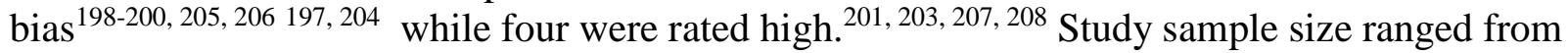
50 to 233. The majority of studies enrolled patients who were euthymic. Components of the psychoeducation included discussions about symptoms, medications, and relapse prevention. Formats for interventions included individual, group, and internet-based psychoeducation. Inactive comparisons included treatment as usual (including pharmacotherapy) and attention controls.

Table 33 provides a summary of inclusion/exclusion criteria and interventions and comparators. Appendix J provides details. 
Table 33. Population and inclusion criteria for studies of psychoeducation versus inactive comparators

\begin{tabular}{|c|c|c|c|c|c|}
\hline $\begin{array}{l}\text { Author, Year } \\
\text { Single-Multisite } \\
\text { Local/Continent } \\
\text { Risk of Bias } \\
\text { PMID }\end{array}$ & Intervention & Comparison & $\begin{array}{l}\text { Inclusion Criteria: } \\
\text { BD Type; Diagnostic } \\
\text { Criteria } \\
\text { Current Episode }\end{array}$ & Demographics & Key Exclusions \\
\hline $\begin{array}{l}\text { Barnes, } 2015^{197} \\
\text { Singlesite } \\
\text { Australia } \\
\text { Moderate ROB } \\
25554993\end{array}$ & $\begin{array}{l}\text { Internet-based psychoeducation } \\
\text { (Road to Recovery for Bipolar } \\
\text { Disorder) focused on managing } \\
\text { symptoms, medication, psychological } \\
\text { approaches, relationships, and } \\
\text { lifestyle. Participants had access to } \\
10 \text { sessions of cognitive behavioral } \\
\text { therapy as homework } \\
-20 \text { online sessions, first } 8 \text { sessions } \\
\text { weekly, } 9 \text { and } 10 \text { every } 2 \text {-week } \\
\text { period, and } 11-20 \text { were monthly }\end{array}$ & $\begin{array}{l}\text { Internet-based attention } \\
\text { control (Virtual Highway } \\
\text { for Bipolar Disorder) } \\
\\
-20 \text { online sessions, first } \\
8 \text { sessions weekly, } 9 \\
\text { and } 10 \text { every 2-week } \\
\text { period, and 11-20 were } \\
\text { monthly }\end{array}$ & $\begin{array}{l}\text { BD-I or II; DSM-IV } \\
\text { No current clinical } \\
\text { state excluded. } \\
\text { Severe episodes not } \\
\text { reported. }\end{array}$ & $\begin{array}{l}\text { Mean Age } 40(18- \\
58) \\
72 \% \text { Female } \\
\text { Race NR } \\
N=233\end{array}$ & $\begin{array}{l}\text { Labs/Other Medical } \\
\text { Conditions }\end{array}$ \\
\hline $\begin{array}{l}\text { Gumus, } 2015^{208} \\
\text { Singlesite } \\
\text { Turkey } \\
\text { High ROB } \\
26001717\end{array}$ & $\begin{array}{l}\text { Psychoeducation focused on illness } \\
\text { education, warning signs, medication } \\
\text { and side effects, and problem solving } \\
\text { skills as well as standard clinical } \\
\text { monitoring } \\
\text { - } 60 \text { minute sessions, once per week, } \\
\text { for } 4 \text { weeks }\end{array}$ & $\begin{array}{l}\text { Standard clinical follow } \\
\text { up (not described) } \\
\text {-Duration of study }\end{array}$ & $\begin{array}{l}\text { BD-I or II; DSM-IV } \\
\text { Euthymic/Maintenance }\end{array}$ & $\begin{array}{l}\text { Mean Age } 39(27- \\
52) \\
\text { Female } 48 \% \\
\text { Race NR } \\
\mathrm{N}=82\end{array}$ & Other Mental Health \\
\hline $\begin{array}{l}\text { de Barros } \\
\text { Pellegrinelli, } 2013^{201} \\
\text { Singlesite } \\
\text { Brazil } \\
\text { High ROB } \\
22943487\end{array}$ & $\begin{array}{l}\text { Psychoeducation consisting of } 15 \\
\text { min introduction, } 30 \text { min education, } \\
30 \text { min discussion and psychological } \\
\text { support, and } 15 \text { min for conclusion } \\
\text {-16 twice-weekly } 90 \text {-minute sessions }\end{array}$ & $\begin{array}{l}\text { Sessions promoting } \\
\text { relaxation consisting of } \\
\text { informal conversation } \\
\text { and relaxation using } \\
\text { three different types of } \\
\text { exercises } \\
\text {-16 twice-weekly } 90- \\
\text { minute sessions }\end{array}$ & $\begin{array}{l}\text { BD-I or II; DSM-IV } \\
\text { Euthymic/Maintenance }\end{array}$ & $\begin{array}{l}\text { Mean Age } 44(22- \\
66) \\
69 \% \text { Female } \\
\text { Race NR } \\
N=55\end{array}$ & $\begin{array}{l}\text { Schizoaffective; } \\
\text { Substance Abuse; } \\
\text { Other Mental Heath; } \\
\text { Neurological } \\
\text { Disorders }\end{array}$ \\
\hline
\end{tabular}




\begin{tabular}{|c|c|c|c|c|c|}
\hline $\begin{array}{c}\text { Author, Year } \\
\text { Single-Multisite } \\
\text { Local/Continent } \\
\text { Risk of Bias } \\
\text { PMID }\end{array}$ & Intervention & Comparison & $\begin{array}{c}\text { Inclusion Criteria: } \\
\text { BD Type; Diagnostic } \\
\text { Criteria } \\
\text { Current Episode }\end{array}$ & Demographics & Key Exclusions \\
\hline $\begin{array}{l}\text { Javadpour, } 2013^{203} \\
\text { Singlesite } \\
\text { Iran } \\
\text { High ROB } \\
23642977\end{array}$ & $\begin{array}{l}\text { Psychoeducation focusing on } \\
\text { understanding bipolar, familiarization } \\
\text { with symptoms understanding signs } \\
\text { of an episodes, awareness of causes } \\
\text { and prognosis, education about the } \\
\text { function, types and adverse side } \\
\text { effect of mood stabilizer medication, } \\
\text { functions, types and adverse effects } \\
\text { of anti-manic and antidepressant } \\
\text { medications, and risks of } \\
\text { discontinuing medications } \\
\text { - Eight 50-minute weekly session }\end{array}$ & $\begin{array}{l}\text { Standard } \\
\text { pharmacotherapy } \\
\text { (discretion of treating } \\
\text { psychiatrist of their } \\
\text { choice) }\end{array}$ & $\begin{array}{l}\text { BD type not specified } \\
\text { Euthymic/Maintenance }\end{array}$ & $\begin{array}{l}\text { Mean Age NR } \\
(18-60) \\
51 \% \text { Female } \\
\text { Race NR } \\
N=108\end{array}$ & First Manic Episode \\
\hline $\begin{array}{l}\text { Smith, } 2011^{206} \\
\text { Singlesite } \\
\text { United Kingdom } \\
\text { Low ROB } \\
22017225\end{array}$ & $\begin{array}{l}\text { Internet-based psychoeducation } \\
\text { focusing on causes, role of } \\
\text { medication, lifestyle changes, } \\
\text { relapse prevention and early } \\
\text { intervention, psychological } \\
\text { approaches, gender-specific } \\
\text { considerations, and advice for family } \\
\text { and careers } \\
\text { - Initial face-to-face meeting with } \\
\text { psychiatrist to learn how to use } \\
\text { program followed by four months of } \\
\text { every-other-week online } \\
\text { psychoeducation }\end{array}$ & $\begin{array}{l}\text { Treatment as usual: } \\
\text { Usual care delivered in } \\
\text { a collaborative model } \\
\text { between general } \\
\text { practitioners and } \\
\text { community mental } \\
\text { health teams. }\end{array}$ & $\begin{array}{l}\text { BD-I, II or NOS; DSM- } \\
\text { IV } \\
\text { Euthymic/Maintenance }\end{array}$ & $\begin{array}{l}\text { Mean Age } 44(22- \\
66) \\
62 \% \text { Female } \\
98 \% \text { White } \\
N=50\end{array}$ & $\begin{array}{l}\text { Neurological } \\
\text { Disorders }\end{array}$ \\
\hline $\begin{array}{l}\text { Colom, } 2009^{200} \\
\text { Colom, } 2003^{198} \\
\text { Singlesite } \\
\text { Spain } \\
\text { Low ROB } \\
12695318 \\
19252157\end{array}$ & $\begin{array}{l}\text { Group psychoeducation (and } \\
\text { pharmacologic treatment) } \\
\text { that focused on illness awareness, } \\
\text { treatment compliance, early } \\
\text { detection of prodromal symptoms } \\
\text { and recurrences, and life-style } \\
\text { regularity } \\
\text {-21 weekly } 90 \text {-minute sessions }\end{array}$ & $\begin{array}{l}\text { Standard pharmacologic } \\
\text { treatment and group } \\
\text { meetings with } \\
\text { psychologists without } \\
\text { any psychosocial } \\
\text { feedback (unless } \\
\text { necessary for patient } \\
\text { interaction) } \\
-20 \text { weekly group } \\
\text { sessions }\end{array}$ & $\begin{array}{l}\text { BD-I or II; DSM-IV } \\
\text { Euthymic/Maintenance }\end{array}$ & $\begin{array}{l}\text { Mean Age NR } \\
\text { (18-65) } \\
63 \% \text { Female } \\
\text { Race NR } \\
N=120\end{array}$ & $\begin{array}{l}\text { Other Mental Health; } \\
\text { Neurological } \\
\text { Disorders }\end{array}$ \\
\hline
\end{tabular}




\begin{tabular}{|c|c|c|c|c|c|}
\hline $\begin{array}{c}\text { Author, Year } \\
\text { Single-Multisite } \\
\text { Local/Continent } \\
\text { Risk of Bias } \\
\text { PMID }\end{array}$ & Intervention & Comparison & $\begin{array}{c}\text { Inclusion Criteria: } \\
\text { BD Type; Diagnostic } \\
\text { Criteria } \\
\text { Current Episode }\end{array}$ & Demographics & Key Exclusions \\
\hline $\begin{array}{l}\text { Sajatovic, } 2009^{205} \\
\text { Singlesite } \\
\text { United States } \\
\text { Low ROB } \\
19723732\end{array}$ & $\begin{array}{l}\text { Group psychoeducation (Life Goals } \\
\text { Program) focusing on illness } \\
\text { education, medication adherence, } \\
\text { management, goal setting, and } \\
\text { problem solving } \\
\text {-6 weekly sessions followed by } \\
\text { optional monthly group sessions }\end{array}$ & $\begin{array}{l}\text { Treatment as usual: } \\
\text { Treatment at community } \\
\text { mental health care } \\
\text { including medication } \\
\text { management and } \\
\text { psychosocial therapy } \\
\text { and counseling }\end{array}$ & $\begin{array}{l}\text { BD-I or II; DSM-IV } \\
\text { No current clinical } \\
\text { state excluded. } \\
\text { Severe episodes not } \\
\text { reported. }\end{array}$ & $\begin{array}{l}\text { Mean Age } 41 \text { (18- } \\
76) \\
68 \% \text { Female } \\
60 \% \text { White } \\
N=164\end{array}$ & Other Conditions \\
\hline $\begin{array}{l}\text { Colom, 2003b }{ }^{199} \\
\text { Singlesite } \\
\text { Spain } \\
\text { Low ROB } \\
14628987\end{array}$ & $\begin{array}{l}\text { Group psychoeducation (and } \\
\text { standard treatment) } \\
\text { focused on illness awareness, } \\
\text { treatment compliance, prodromal } \\
\text { symptoms and relapse, lifestyle } \\
\text { regularity, symptom monitoring, } \\
\text { treatment adherence, and illness } \\
\text { management skills. } \\
\text {-20 weekly group sessions for } 90 \\
\text { minutes }\end{array}$ & $\begin{array}{l}\text { Standard pharmacologic } \\
\text { treatment and group } \\
\text { meetings with } \\
\text { psychologists without } \\
\text { any psychosocial } \\
\text { feedback (unless } \\
\text { necessary for patient } \\
\text { interaction). Therapists } \\
\text { encouraged } \\
\text { communication between } \\
\text { patients. } \\
\text {-20 weekly group } \\
\text { sessions }\end{array}$ & $\begin{array}{l}\text { BD-I; DSM-IV } \\
\text { Euthymic/Maintenance }\end{array}$ & $\begin{array}{l}\text { Mean Age } 35(18- \\
57) \\
72 \% \text { Female } \\
N=50\end{array}$ & $\begin{array}{l}\text { Other Mental Health; } \\
\text { Neurological } \\
\text { Disorders; } \\
\text { Taking Other Meds }\end{array}$ \\
\hline $\begin{array}{l}\text { Weiss, } 2000^{207} \\
\text { Singlesite } \\
\text { United States } \\
\text { High ROB } \\
10847311\end{array}$ & $\begin{array}{l}\text { Psychoeducation focused on } \\
\text { acceptance, self-help, identifying and } \\
\text { fighting triggers, medication } \\
\text { adherence, coping skills, and } \\
\text { similarities between recovery and } \\
\text { relapse for bipolar and substance } \\
\text { abuse } \\
\text {-12-20 weekly group therapy, } 60 \\
\text { minutes per session }\end{array}$ & $\begin{array}{l}\text { Treatment as usual/No } \\
\text { treatment (not } \\
\text { described) with } 6 \\
\text { monthly assessments }\end{array}$ & $\begin{array}{l}\text { BD-I, II, or NOS; DSM- } \\
\text { IV } \\
\text { No current clinical } \\
\text { state excluded. } \\
\text { Severe episodes not } \\
\text { reported. }\end{array}$ & $\begin{array}{l}\text { Mean Age } 36 \text { (18- } \\
54) \\
49 \% \text { Female } \\
87 \% \text { White } \\
N=45\end{array}$ & $\begin{array}{l}\text { Neurological } \\
\text { Disorders; } \\
\text { Other Conditions } \\
\text { (which would preclude } \\
\text { attendance) }\end{array}$ \\
\hline
\end{tabular}




\begin{tabular}{|c|c|c|c|c|c|}
\hline $\begin{array}{c}\text { Author, Year } \\
\text { Single-Multisite } \\
\text { Local/Continent } \\
\text { Risk of Bias } \\
\text { PMID }\end{array}$ & Intervention & Comparison & $\begin{array}{l}\text { Inclusion Criteria: } \\
\text { BD Type; Diagnostic } \\
\text { Criteria } \\
\text { Current Episode }\end{array}$ & Demographics & Key Exclusions \\
\hline $\begin{array}{l}\text { Perry, } 1999^{204} \\
\text { Multisite } \\
\text { United Kingdom } \\
\text { Moderate ROB } \\
9888904\end{array}$ & $\begin{array}{l}\text { Psychoeducation (and routine } \\
\text { treatment) involving } 12 \text { individual } \\
\text { treatment sessions that focused on } \\
\text { identifying prodromal symptoms and } \\
\text { producing and rehearsing an action } \\
\text { plan once prodromes had been } \\
\text { recognized }\end{array}$ & $\begin{array}{l}\text { Treatment as usual: } \\
\text { Drug treatment, } \\
\text { monitoring of mood and } \\
\text { adherence to treatment, } \\
\text { education about } \mathrm{BD} \text {, } \\
\text { and inpatient care if } \\
\text { necessary. }\end{array}$ & $\begin{array}{l}\text { BD Type Not Specified } \\
\text { Maintenance }\end{array}$ & $\begin{array}{l}\text { Mean Age } 45(23- \\
67) \\
68 \% \text { Female } \\
91 \% \text { White } \\
N=69\end{array}$ & $\begin{array}{l}\text { Substance Abuse; } \\
\text { Neurological } \\
\text { Disorders }\end{array}$ \\
\hline
\end{tabular}

BD=Bipolar Disorder; DSM= Diagnostic and Statistical Manual of Mental Disorders; NOS= not otherwise specified; N=number; NR=not reported; ROB=risk of bias 


\section{Relapse}

Evidence was insufficient for the effect of psychoeducation on relapse when compared with an inactive comparator due to moderate study limitations, inconsistent findings, and imprecision. Seven studies enrolling 712 participants reported information on relapses. ${ }^{203,}$, 204, 206 197-200. Reported results regarding the number of relapses were mixed across studies rated low or moderate risk of bias. Two studies reported that participants who had received psychoeducation had fewer relapses of any type at 2 years than those who received an inactive comparator. ${ }^{198-200}$ Colom et al. also reported fewer relapses of any type for those that received psychoeducation at 5 years. ${ }^{200}$ However, Perry et al. ( $\mathrm{n}=69$ ) reported significance differences only for manic relapses at both 6 and 18 months, with fewer manic relapses in the psychoeducation group. Groups did not differ for depressive relapses at either outcome time point. ${ }^{204}$ Smith et al. $(\mathrm{n}=50)$ reported no difference between groups in the number of depressive or manic relapses at 10 months. ${ }^{206}$ Barnes et al. reported no difference in recurrence at 12 months. ${ }^{197}$ The study also reported no difference between groups in time to recurrence. ${ }^{197}$

Results were also mixed for studies rated high risk of bias. Javadpour et al. ( $\mathrm{n}=108)$ reported fewer recurrences in the psychoeducation group at 18 months. ${ }^{203}$ However, Gumus ( $\mathrm{n}=82$ ) reported no difference between groups in relapses at 12 months. ${ }^{208}$

\section{Symptom Scores}

Evidence was insufficient for depression and mania symptoms due to high study limitations and imprecision. Five studies enrolling 422 participants reported measures of symptom scores. ${ }^{201,203,205-207}$ All five studies, including three rated high risk of bias, reported no difference between groups in depression symptoms across a range of outcome time points (6 to 18 months). ${ }^{201,203,}$, 205-207.

Two low risk of bias studies reported no difference between groups in mania symptoms (at 6 or 12 months) ${ }^{205,}$, 206 The two high risk of bias studies also reported no difference between groups. ${ }^{201,203}$ Rated high risk of bias, Weiss et al. $(\mathrm{n}=45)$ reported statistically significant improvements in mania at 6 months for participants receiving psychoeducation group compared with the control group. ${ }^{207}$

\section{Function}

Evidence was insufficient for psychoeducation on all function outcomes due to moderate study limitations and strong imprecision. Four studies enrolling 446 participants reported measures of function. ${ }^{201,204-206}$ For global function, Sajatovic et al. $(\mathrm{n}=164)$ and Smith et al. $(n=50)$ found no difference between groups at their respective outcome time points (6 to 12 months). ${ }^{205,206}$ Rated high risk of bias, de Barros Pellegrinelli et al ( $\mathrm{n}=55$ ) also found no difference between groups at 12 months. ${ }^{201}$

Results for other measures of function were mixed. One low risk of bias study reported no difference between groups in measures of quality of life. ${ }^{206}$ One moderate risk of bias study found no difference between groups in social function at 6 months; however, at 18 months there was a better function in the intervention group. ${ }^{204}$

\section{Additional Outcomes}

Six studies reported data on hospitalizations. ${ }^{197-200,203,207,208}$ Four studies, including two rated high risk of bias, reported no difference between groups in number of hospitalizations across a range of time periods (12 months to 5 years). ${ }^{197,198,200,207,208}$ One low risk of bias study 
reported fewer hospitalizations for those who received psychoeducation at 2 years. ${ }^{197,}{ }^{199}$ Rated high risk of bias, Javadpour et al. $(n=108)$ reported fewer hospitalizations for those who received psychoeducation at 18 months. ${ }^{203}$

\section{Psychoeducation Versus Active Control}

We identified three studies on the effect of psychoeducation interventions compared with active comparators. $^{209-211}$ Two studies were rated moderate risk of bias, ${ }^{209,211}$ and one was rated high. ${ }^{210}$ Study sample size ranged from 85 to 304 . The majority of studies enrolled patients who were euthymic and used a group format for the intervention. Components of the psychoeducation included discussions about illness symptoms, medications, and recognition of early warning signs. Two studies examined the effect of different formats of psychoeducation (i.e., group vs. individual, guided vs. self-administered). ${ }^{209,210}$

Table 34 provides a summary of inclusion/exclusion criteria and interventions and comparators. Appendix J provides details. 
Table 34. Population and inclusion criteria for studies of psychoeducation versus active comparators

\begin{tabular}{|c|c|c|c|c|c|}
\hline $\begin{array}{c}\text { Author, Year } \\
\text { Single-Multisite } \\
\text { Local/Continent } \\
\text { Risk of Bias } \\
\text { PMID }\end{array}$ & Intervention & Comparison & $\begin{array}{c}\text { Inclusion Criteria: } \\
\text { BD Type; Diagnostic } \\
\text { Criteria } \\
\text { Current Episode }\end{array}$ & Demographics & $\begin{array}{c}\text { Key } \\
\text { Exclusions }\end{array}$ \\
\hline $\begin{array}{l}\text { Bilderbeck, } 2016^{209} \\
\text { United Kingdom } \\
\text { Singlesite } \\
\text { Moderate ROB } \\
27454410\end{array}$ & $\begin{array}{l}\text { Therapist facilitated psychoeducation } \\
\text { via manual focused on identifying the } \\
\text { relapse, reviewing risk factors, daily } \\
\text { sleep regulation, medications and } \\
\text { substance abuse; and mood } \\
\text { management planning. } \\
-5 \text { face to face sessions over } 12 \\
\text { weeks }\end{array}$ & $\begin{array}{l}\text { Self-administered } \\
\text { psychoeducation via manual } \\
\text { focused on identifying the } \\
\text { relapse, reviewing risk factors, } \\
\text { daily sleep regulation, } \\
\text { medications and substance } \\
\text { abuse; and mood } \\
\text { management planning. } \\
\text {-Manual access for } 12 \text { weeks }\end{array}$ & $\begin{array}{l}\text { BD I or II; DSM-IV } \\
\text { Euthymic/Maintenance }\end{array}$ & $\begin{array}{l}\text { Mean Age } 44 \\
(16-76) \\
\text { Female } 73 \% \\
\text { White } 93 \% \\
\mathrm{~N}=121\end{array}$ & $\begin{array}{l}\text { Labs/Other } \\
\text { Conditions }\end{array}$ \\
\hline $\begin{array}{l}\text { Kallestad, } 2016^{210} \\
\text { Singlesite } \\
\text { Norway } \\
\text { High ROB } \\
27253214\end{array}$ & $\begin{array}{l}\text { Group psychoeducation focused on } \\
\text { illness education, symptoms, early } \\
\text { detection, sleep, risk factors, stress } \\
\text { management, causes, work, social } \\
\text { rights/welfare system and } \\
\text { law/regulations } \\
\text {-Ten initial 90-minute sessions and } 8 \\
\text { booster sessions over next } 2 \text { years at } \\
\text { 3-montn intervals }\end{array}$ & $\begin{array}{l}\text { Individual psychoeducation } \\
\text { focused on } \\
\text { treatment, stress } \\
\text { management, sleep, } \\
\text { dysfunctional cognitions, and } \\
\text { other psychosocial factors } \\
\text { associated with increased risk } \\
\text { of relapse } \\
\text {-Three 1-hour weekly sessions }\end{array}$ & $\begin{array}{l}\text { BD I or II; DSM-IV } \\
\text { No current clinical } \\
\text { state excluded }\end{array}$ & $\begin{array}{l}\text { Mean Age } 38 \\
(19-64) \\
\text { Female } 54 \% \\
\text { Race NR } \\
\mathrm{N}=85\end{array}$ & $\begin{array}{l}\text { Labs/Other } \\
\text { Conditions; } \\
\text { Neurological } \\
\text { Disorders }\end{array}$ \\
\hline $\begin{array}{l}\text { Morriss, } 2016^{212} \\
\text { Multisite } \\
\text { United Kingdom } \\
\text { Moderate ROB } \\
27688021\end{array}$ & $\begin{array}{l}\text { Structured group psychoeducation } \\
\text { focused on life charting, recognition of } \\
\text { early warning signs, problem solving, } \\
\text { sleep hygiene, and care planning } \\
\text {-21 weekly sessions for } 2 \text { hours each } \\
\text { over a maximum of } 26 \text { weeks. }\end{array}$ & $\begin{array}{l}\text { Optimized unstructured group } \\
\text { support where participants set } \\
\text { the agenda at each meeting } \\
-21 \text { weekly sessions for } 2 \\
\text { hours each over a maximum } \\
\text { of } 26 \text { weeks }\end{array}$ & $\begin{array}{l}\text { BD I or II; DSM-IV } \\
\text { Euthymic/Maintenance }\end{array}$ & $\begin{array}{l}\text { Mean Age } 45 \\
(33-57) \\
\text { Female } 58 \% \\
\text { Race NR } \\
\mathrm{N}=304\end{array}$ & $\begin{array}{l}\text { Labs/Other } \\
\text { Conditions; } \\
\text { Other Mental } \\
\text { Health }\end{array}$ \\
\hline
\end{tabular}

BD=Bipolar Disorder; DSM= Diagnostic and Statistical Manual of Mental Disorders; NOS= not otherwise specified; N=number; NR=not reported; ROB=risk of bias 


\section{Relapse}

Evidence was insufficient on the effect of psychoeducation on relapse compared with an active comparator, due to moderate study limitations and strong imprecision. Two moderate risk of bias studies enrolling 425 participants reported information on relapses. ${ }^{209,} 211,212$ One study compares psychoeducation formats ${ }^{209}$ Both studies reported no difference between the psychoeducation interventions and active comparators in number of relapses. ${ }^{212} 209,211$ Morriss et al. $(n=121)$ also reported time to relapse, finding no difference between groups over 96 weeks. ${ }^{211,}$ 212

\section{Symptom Scores}

Evidence was insufficient on the effect of psychoeducation on relapse compared with an active comparator, due to moderate study limitations and strong imprecision. Two moderate risk of bias studies enrolling 425 participants reported information on symptom scores. ${ }^{209,} 212$ One study compares psychoeducation formats. ${ }^{209}$ Both studies reported no difference between the psychoeducation interventions and active comparators in depression and mania symptoms. ${ }^{209,} 212$

\section{Function}

Evidence was insufficient on the effect of psychoeducation on function compared with an active comparator due to high study limitations, unclear consistency, and imprecision. No studies reported measures of global function. One moderate risk of bias study enrolling 121 participants reported information on other measures of function. ${ }^{209}$ The study reported no difference between psychoeducation and the active comparator in social and occupational function at 96 weeks. ${ }^{209}$

\section{Additional Outcomes}

Two studies reported hospitalizations. ${ }^{209,} 210$ Rated moderate risk of bias, Bilderbeck et al. $(\mathrm{n}=121)$ found no difference between groups in hospitalizations at 12 months, but this study's active comparator was another format of psyhoeducation (i.e., self-administered via a manual). Rated high risk of bias, Kallestad et al. ( $\mathrm{n}=85$ ) reported that individuals who received group psychoeducation had a longer time to first hospital admission compared to individuals who received individual psychoeducation. ${ }^{210}$

\section{Cognitive Behavioral Therapy}

We identified 14 publications reporting 13 unique studies on CBT as a treatment for BD. ${ }^{213-}$ ${ }^{226}$ Appendix K provides evidence tables, summary risk of bias assessments, assessments of strength of evidence, and reporting for additional outcomes. A summary of findings with at least low-strength evidence for other drug treatments for maintenance are provided in Table 35. Any intervention and comparison not listed in Table 35, or outcome not listed for an included intervention and comparison, was found to have an evidence base insufficient to draw conclusions. 
Table 35. Summary of findings with at least low-strength evidence for cognitive behavioral therapy

\begin{tabular}{|l|l|l|l|}
\hline \multicolumn{1}{|c|}{ Intervention } & $\begin{array}{c}\text { \# Studies/ Design } \\
\text { (n Analyzed) } \\
\text { Timing }\end{array}$ & \multicolumn{1}{|c|}{ Findings } & Strength of Evidence \\
\hline $\begin{array}{l}\text { CBT vs. Active } \\
\text { Comparators* }^{2 \text { RCTs } 213,214,219,215,}\end{array}$ & $\begin{array}{l}210,221 \\
(\mathrm{n}=461) \\
6 \text { to } 12 \text { months }\end{array}$ & $\begin{array}{l}\text { Depression and Mania symptoms: No } \\
\text { difference between groups across range of } \\
\text { time periods. }\end{array}$ & $\begin{array}{l}\text { Low } \\
\text { (moderate study } \\
\text { limitations, imprecision) }\end{array}$ \\
\hline
\end{tabular}

* Active comparators are comparators such as a different psychosocial therapy or peer support.

$\mathrm{CBT}=$ cognitive behavioral therapy; $\mathrm{n}=$ number; $\mathrm{RCT}=$ randomized controlled trial

\section{Cognitive Behavioral Therapy Versus Inactive Control}

We identified nine publications reporting eight unique studies on the effect of CBT when compared with an inactive comparator yielding insufficient evidence for various outcomes. ${ }^{215}$, 218-220, 222-226 One study was rated low to high risk of bias due to differences in reporting of outcomes: low for pre-specified outcomes. ${ }^{215}$ Two studies were rated high risk of bias. ${ }^{222,225}$ Study sample sizes ranged from 52 to 253. The majority of studies enrolled patients without a current bipolar episode, while some did not exclude individuals based on the current clinical state except for acute mania. Components of the CBT interventions varied (e.g., group vs. individual; 8 vs. 20+ sessions); however, common elements included education about BD, identifying symptoms, and discussing strategies for management and coping. The length of interventions also varied ranging from 8 weeks to 6 months. Inactive comparisons were generally defined as "treatment as usual", which generally involved medication and variable contact with a provider. Five studies were rated low or moderate risk of bias. ${ }^{218-220,223,224,226}$

Table 36 summarizes the key characteristics of the studies. Appendix K provides details. 
Table 36. Population and inclusion criteria for studies of CBT versus inactive comparators

\begin{tabular}{|c|c|c|c|c|c|}
\hline $\begin{array}{l}\text { Author, Year } \\
\text { Single-Multisite } \\
\text { Local/Continent } \\
\text { Risk of Bias } \\
\text { PMID }\end{array}$ & Intervention & Comparison & $\begin{array}{c}\text { Inclusion Criteria: } \\
\text { BD Type; Diagnostic } \\
\text { Criteria } \\
\text { Current Episode }\end{array}$ & Demographics & Key Exclusions \\
\hline $\begin{array}{l}\text { Jones, } 2015^{220} \\
\text { Multisite } \\
\text { United Kingdom } \\
\text { Moderate ROB } \\
25213157\end{array}$ & $\begin{array}{l}\text { Individual CBT focused on recovery } \\
\text { approach, mood functioning, } \\
\text { understanding of diagnosis, recovery- } \\
\text { informed goals, relationships between } \\
\text { mood and progress towards recovery } \\
\text { goals, CBT techniques to cope, } \\
\text { functioning issues in relation to } \\
\text { recovery, development of recovery } \\
\text { plan, and sharing lessons from therapy } \\
\text { with stakeholders } \\
\text {-Total of } 18 \text { hours over } 6 \text { months; } \\
\text { weekly or biweekly } 45-60 \text { minute } \\
\text { sessions }\end{array}$ & $\begin{array}{l}\text { Treatment as usual: } \\
\text { Routine medication } \\
\text { (mood stabilizers, } \\
\text { antipsychotics, and } \\
\text { antidepressants) and } \\
\text { medical care from } \\
\text { clinician and community } \\
\text { mental health team. }\end{array}$ & $\begin{array}{l}\text { BD-I and BD-II; DSM- } \\
\text { IV } \\
\text { Euthymic/Maintenanc } \\
\text { e }\end{array}$ & $\begin{array}{l}\text { Mean Age } 39(18- \\
65) \\
70 \% \text { Female } \\
96 \% \text { White } \\
N=67\end{array}$ & Schizoaffective \\
\hline $\begin{array}{l}\text { Fava, 2011 } \\
\text { Singlesite } \\
\text { Italy } \\
\text { Low ROB } \\
21372621\end{array}$ & $\begin{array}{l}\text { CBT and well-being therapy focused } \\
\text { on patient's symptomatology, } \\
\text { monitoring of distress, strategies for } \\
\text { symptom management, } \\
\text { psychotherapeutic strategy for } \\
\text { enhancing well-being } \\
\text {-10 sessions every other week for } 45- \\
\text { minutes. }\end{array}$ & $\begin{array}{l}\text { Clinical Management: } \\
\text { Reviewed the patient's } \\
\text { clinical status and } \\
\text { provided the patient with } \\
\text { support and advice } \\
\text { according to protocol } \\
\text {-10 sessions every other } \\
\text { week for } 45 \text {-minutes. }\end{array}$ & $\begin{array}{l}\text { Cyclothymic; DSM-IV } \\
\text { No history of mania or } \\
\text { major depressive } \\
\text { disorder }\end{array}$ & $\begin{array}{l}\text { Mean Age } 40(18- \\
65) \\
55 \% \text { Female } \\
\text { Race NR } \\
N=62\end{array}$ & $\begin{array}{l}\text { First Manic Episode; } \\
\text { Schizoaffective; } \\
\text { Substance Abuse; } \\
\text { Other Mental Health; } \\
\text { Neurological Disorders; } \\
\text { Taking Other } \\
\text { Medications; } \\
\text { Pregnant/Nursing; } \\
\text { Labs/Other Conditions }\end{array}$ \\
\hline
\end{tabular}




\begin{tabular}{|c|c|c|c|c|c|}
\hline $\begin{array}{c}\text { Author, Year } \\
\text { Single-Multisite } \\
\text { Local/Continent } \\
\text { Risk of Bias } \\
\text { PMID }\end{array}$ & Intervention & Comparison & $\begin{array}{c}\text { Inclusion Criteria: } \\
\text { BD Type; Diagnostic } \\
\text { Criteria } \\
\text { Current Episode }\end{array}$ & Demographics & Key Exclusions \\
\hline $\begin{array}{l}\text { Gomes, } 2011^{222} \\
\text { Singlesite } \\
\text { Brazil } \\
\text { High ROB } \\
21372622\end{array}$ & $\begin{array}{l}\text { Group CBT focused on information } \\
\text { about BD and stabilized routine and } \\
\text { pharmacological issues; use of mood } \\
\text { graphs and stress vulnerability model, } \\
\text { cognitive and behavioral strategies to } \\
\text { manage depressive and manic } \\
\text { episodes; specific problems in BD; } \\
\text { techniques to improve relapse } \\
\text { prevention } \\
\text {-18 structured sessions, } 90 \text { minutes } \\
\text { each }\end{array}$ & $\begin{array}{l}\text { Treatment as usual: } \\
\text { Pharmacological } \\
\text { treatment }\end{array}$ & $\begin{array}{l}\text { BD-I and BD-II; DSM- } \\
\text { IV } \\
\text { Euthymic/Maintenanc } \\
\text { e }\end{array}$ & $\begin{array}{l}\text { Mean Age } 39 \text { (18- } \\
60) \\
76 \% \text { Female } \\
\text { Race NR } \\
\mathrm{N}=50\end{array}$ & $\begin{array}{l}\text { Substance Abuse; } \\
\text { Neurological Disorders }\end{array}$ \\
\hline $\begin{array}{l}\text { Castle, } 2010^{224} \\
\text { Multisite } \\
\text { Australia } \\
\text { Low ROB } \\
20435965\end{array}$ & $\begin{array}{l}\text { Group CBT focused on monitoring } \\
\text { mood and activities, assessing } \\
\text { prodromes, preventing relapse, and } \\
\text { setting specific, measurable, } \\
\text { achievable, realistic, time-framed goals } \\
-12 \text { weekly group sessions ( } 90 \\
\text { minutes) and } 3 \text { monthly booster } \\
\text { sessions with weekly telephone calls }\end{array}$ & $\begin{array}{l}\text { Treatment as usual (not } \\
\text { defined) and weekly } \\
\text { telephone calls }\end{array}$ & $\begin{array}{l}\text { BD-I, BD-II, BD NOS } \\
\text { DSM-IV-TR } \\
\text { Euthymic/Maintenanc } \\
\text { e }\end{array}$ & $\begin{array}{l}\text { Mean Age } 42 \text { (18- } \\
65) \\
76 \% \text { Female } \\
\text { Race NR } \\
\mathrm{N}=84\end{array}$ & $\begin{array}{l}\text { Schizoaffective; } \\
\text { Neurological Disorders; } \\
\text { Labs/Other Conditions }\end{array}$ \\
\hline $\begin{array}{l}\text { Ball, } 2006^{225} \\
\text { Singlesite } \\
\text { Australia } \\
\text { High ROB } \\
16566624\end{array}$ & $\begin{array}{l}\text { CBT focused on assessment, } \\
\text { psychoeducation, identifying early } \\
\text { warning signs, establishing stable } \\
\text { routines, identifying and modifying } \\
\text { cognitions, identifying and modifying } \\
\text { schemas } \\
-20 \text { weekly sessions, } 60 \text { minutes each }\end{array}$ & $\begin{array}{l}\text { Treatment as usual: } \\
\text { Regular sessions as } \\
\text { prescribed by patient's } \\
\text { medical practitioner }\end{array}$ & $\begin{array}{l}\text { BD-I and BD-II; DSM- } \\
\text { IV } \\
\text { Without a current } \\
\text { episode of severe } \\
\text { depression or mania }\end{array}$ & $\begin{array}{l}\text { Mean Age } 42 \text { (23- } \\
77) \\
58 \% \text { Female } \\
\text { Race NR } \\
N=52\end{array}$ & $\begin{array}{l}\text { Schizoaffective; Other } \\
\text { Mental Health; } \\
\text { Neurological Disorders; } \\
\text { Labs/Other Conditions }\end{array}$ \\
\hline
\end{tabular}




\begin{tabular}{|c|c|c|c|c|c|}
\hline $\begin{array}{c}\text { Author, Year } \\
\text { Single-Multisite } \\
\text { Local/Continent } \\
\text { Risk of Bias } \\
\text { PMID }\end{array}$ & Intervention & Comparison & $\begin{array}{l}\text { Inclusion Criteria: } \\
\text { BD Type; Diagnostic } \\
\text { Criteria } \\
\text { Current Episode }\end{array}$ & Demographics & Key Exclusions \\
\hline $\begin{array}{l}\text { Scott, } 2006^{215} \\
\text { Singlesite } \\
\text { United Kingdom } \\
\text { Low/High ROB (by } \\
\text { outcome) } \\
16582056\end{array}$ & $\begin{array}{l}\text { CBT focused on facilitating acceptance } \\
\text { of the need for treatment, reducing } \\
\text { variability in mood, managing } \\
\text { stressors, strategies to cope with } \\
\text { depression, identifying and modifying } \\
\text { dysfunctional automatic thoughts and } \\
\text { beliefs, improve medication adherence, } \\
\text { tackling substance misuse, teaching } \\
\text { early recognition of symptoms of } \\
\text { recurrence and coping techniques for } \\
\text { symptoms } \\
\text {-Weekly sessions for } 15 \text { weeks with } \\
\text { reduction in frequency from week } 16- \\
26 \text { - Two booster sessions at week } 32 \\
\text { and } 38 \text {. }\end{array}$ & $\begin{array}{l}\text { Treatment as usual: } \\
\text { Medication and contact } \\
\text { with key mental health } \\
\text { professionals when } \\
\text { appropriate. }\end{array}$ & $\begin{array}{l}\text { BD-I and BD-II } \\
\text { DSM-IV } \\
\text { Any Episode (25\% } \\
\text { depressed, 10\% } \\
\text { hypo/manic, remaining } \\
\text { without current } \\
\text { episode) }\end{array}$ & $\begin{array}{l}\text { Mean Age } 41 \text { (18- } \\
65) \\
65 \% \text { Female } \\
\text { Race NR } \\
N=253\end{array}$ & $\begin{array}{l}\text { First Manic Episode; } \\
\text { Substance Abuse; } \\
\text { Other Mental Health; } \\
\text { Neurological Disorders }\end{array}$ \\
\hline $\begin{array}{l}\text { Lam, } 2003^{218}, 2005^{219} \\
\text { Singlesite } \\
\text { United Kingdom } \\
\text { Moderate ROB } \\
12578431 \\
15677598\end{array}$ & $\begin{array}{l}\text { CBT focused on traditional cognitive } \\
\text { therapy for depression, diathesis-stress } \\
\text { model and need for pharmaceutical } \\
\text { and psychological therapy, mood } \\
\text { monitoring and prodromes, sleep } \\
\text { importance, and targeting extreme } \\
\text { striving attitudes and behavior } \\
-12 \text { to } 18 \text { individual } 60 \text {-minute sessions } \\
\text { in the first } 6 \text { months and } 2 \text { booster } \\
\text { sessions in the second } 6 \text { months. }\end{array}$ & $\begin{array}{l}\text { Minimal psychiatric care: } \\
\text { Mood stabilizers (at } \\
\text { appropriate level) and } \\
\text { regular outpatient } \\
\text { psychiatric follow up }\end{array}$ & $\begin{array}{l}\text { BD-I; DSM-IV } \\
\text { Euthymic/Maintenanc } \\
\text { e }\end{array}$ & $\begin{array}{l}\text { Mean Age } 44(22- \\
70) \\
56 \% \text { Female } \\
\text { Race NR } \\
N=103\end{array}$ & $\begin{array}{l}\text { First Manic Episode; } \\
\text { Schizoaffective; } \\
\text { Substance Abuse; } \\
\text { Other Mental Health }\end{array}$ \\
\hline
\end{tabular}

BD=Bipolar Disorder; CBT=cognitive behavioral therapy; DSM= Diagnostic and Statistical Manual of Mental Disorders; NOS=Not otherwise specified; N=number; NR=not reported; $\mathrm{ROB}=$ risk of bias 


\section{Relapse}

Evidence was insufficient for the effect of CBT interventions on relapse when compared with an inactive comparator, due to moderate study limitations, inconsistent findings, and imprecision. Seven studies enrolling 714 participants reported number of relapses. ${ }^{215,218-220,224,}$ 226225222 Among the studies rated low or moderate risk of bias, two studies reported that individuals who received CBT had significantly fewer relapses of any type compared to those that received an inactive comparator. ${ }^{218,219,} 224$ However, three studies showed no difference between groups in number of relapses. ${ }^{215,220,226}$ Jones et al. and reported longer times to recurrence for individuals who received CBT interventions. ${ }^{215}$

Two high risk of bias studies reported no difference between groups in number of relapses. 225222 Gomes et al. reported longer times to recurrence for individuals who received CBT interventions. ${ }^{222}$

\section{Symptom Scores}

Evidence was insufficient for the effect of CBT interventions on depression and mania symptoms when compared with an inactive comparator, due to moderate study limitations, inconsistent findings, and imprecision. Seven studies enrolling 716 participants provided information on symptom scores. ${ }^{223}$ 218, 219, 224, 220, 215, 226, 225 Results for depression symptoms were mixed for studies rated low or moderate risk of bias. Fava et al. (n=62) reported statistically significant improvements in depression for the CBT intervention group compared with an inactive comparator. ${ }^{223}$ Five studies found no difference between groups in depression at any time point. $^{218,219,224,220,215,226}$ Rated high risk of bias, Ball et al. $(n=52)$ reported a significant difference between groups in depression at 6 months. However, at 18 months there was no difference between groups. ${ }^{225}$

Similarly, evidence for mania symptoms was inconsistent among studies rated low or moderate risk of bias. Fava et al. $(n=62)$ reported statistically significant improvements in mania for the CBT intervention group compared with an inactive comparator. ${ }^{223}$ Lam et al. $(n=103)$ found no difference between groups at nearly all reported time points $(6,12,18$, and 24 months) with exception of final time point at 30 months, when an improvement was seen for the intervention group, although this finding may not have been corrected for multiple outcome tests. ${ }^{218,219}$ Four studies found no difference between groups in mania at any time point. ${ }^{215,220 \text {, }}$ 224, 226 Rated high risk of bias, Ball et al. found no difference between groups in mania at any time point. $^{225}$

\section{Function}

Evidence was insufficient for the effect of CBT interventions on all measures of function when compared with an inactive comparator due to moderate study limitations, unclear or inconsistent findings, and imprecision. Three studies enrolling 280 participants reported outcomes on function. ${ }^{218-220,} 225$ Rated high risk of bias, only Ball et al. $(n=52)$ assessed global function, finding no difference between groups at 6 and 18 months. ${ }^{225}$

Two moderate risk of bias studies reported other measures of function. Lam et al. ( $n=103)$ measured social function, finding no difference between groups at nearly all outcome time points $(12,18$, and 30 months), with the exception of 6 and 24 months, when a significant difference favored the intervention (which may not have been adjusted for multiple outcome tests). ${ }^{218,219}$. Jones et al. ( $n=67)$ measured both social function and quality of life, finding no difference between groups at 6 or 12 months. ${ }^{220}$ In addition, one high risk of bias study reported one 
measure of social function, cognitive function, and health and disability. The measure of health and disability showed a significant difference favoring the intervention at 6 months, but not at 18 months. There were no differences between groups for either time point for the other two measures. $^{225}$

\section{Additional Outcomes}

One high risk of bias study reported information on hospitalizations. Lam et al. (n=103) found that significantly more individuals in the control group were admitted for bipolar episodes compared to those who received CBT. ${ }^{218}$

\section{Cognitive Behavioral Therapy Versus Active Control}

We identified five unique studies enrolling a total of 461 participants examining the effect of CBT compared with active comparators. ${ }^{213,214,216,217,221}$ All five publications were rated as low or moderate risk of bias. ${ }^{213,214,216,217,221}$ The total sample sizes ranged from 58 to 204 participants. Populations across the studies varied; however the majority enrolled participants without a current bipolar episode. Components of the CBT interventions also varied; however, common elements included education about BD and relapse prevention. Active comparisons ranged from supportive therapy, group drug counseling, and psychoeducation

Table 37 summarizes the key characteristics of the studies. Appendix K provides details. 
Table 37. Population and inclusion criteria for studies of CBT versus active comparators

\begin{tabular}{|c|c|c|c|c|c|}
\hline $\begin{array}{c}\text { Author, Year } \\
\text { Single-Multisite } \\
\text { Local/Continent } \\
\text { Risk of Bias } \\
\text { PMID }\end{array}$ & Intervention & Comparison & $\begin{array}{c}\text { Inclusion Criteria: } \\
\text { BD Type; } \\
\text { Diagnostic } \\
\text { Criteria } \\
\text { Current Episode }\end{array}$ & Demographics & Key Exclusions \\
\hline $\begin{array}{l}\text { Harvey, } 2015^{221} \\
\text { Singlesite } \\
\text { United States } \\
\text { Moderate ROB } \\
25622197\end{array}$ & $\begin{array}{l}\text { CBT for insomnia focusing on } \\
\text { stimulus control, bed and sleep } \\
\text { associations, regularizing sleep } \\
\text { and wake times, sleep/circadian } \\
\text { education, relaxing wind down, } \\
\text { sleep-enhancing activities, and } \\
\text { devising a wake-up routine. The } \\
\text { module altered unhelpful beliefs } \\
\text { about sleep, bedtime worry, } \\
\text { rumination, and vigilance } \\
-8 \text { weekly } 50-60 \text { minute sessions } \\
\text { with behavioral module }\end{array}$ & $\begin{array}{l}\text { Psychoeducation sessions } \\
\text { that provided information } \\
\text { but no facilitation or plan for } \\
\text { behavior change. Sessions } \\
\text { focused on mood } \\
\text { regulation, the etiology of } \\
\text { bipolar disorders, } \\
\text { symptoms, prodromes, } \\
\text { medications, substance } \\
\text { use, diet, physical activity, } \\
\text { stress management, } \\
\text { relaxation, and self-esteem } \\
\text { and sleep in a social } \\
\text { context } \\
\text {-8 weekly } 50-60 \text { minute } \\
\text { sessions }\end{array}$ & $\begin{array}{l}\text { BD-I ; DSM-IV-TR } \\
\text { No current bipolar } \\
\text { episode } \\
\text { (interepisode) }\end{array}$ & $\begin{array}{l}\text { Mean Age } 37(18-62) \\
62 \% \text { Female } \\
64 \% \text { White } \\
N=58\end{array}$ & $\begin{array}{l}\text { Substance Abuse; } \\
\text { Other Mental Health; } \\
\text { Neurological Disorders; } \\
\text { Pregnant/Nursing; } \\
\text { Labs/Other Conditions }\end{array}$ \\
\hline $\begin{array}{l}\text { Meyer, } 2012^{217} \\
\text { Singlesite } \\
\text { Germany } \\
\text { Low ROB } \\
22099722\end{array}$ & $\begin{array}{l}\text { CBT focused on understanding of } \\
\text { BD, identifying early warning } \\
\text { symptoms, strategies for } \\
\text { management, communication } \\
\text { and problem solving skills } \\
-20 \text { sessions over } 9 \text { months, } 50 \text { - } \\
60 \text { minutes each }\end{array}$ & $\begin{array}{l}\text { Supportive Therapy: Client- } \\
\text { centered focus; whatever } \\
\text { problems the patient } \\
\text { presented were dealt with } \\
\text { by providing emotional } \\
\text { support and general advice } \\
\text {-20 sessions over } 9 \\
\text { months, 50-60 minutes } \\
\text { each. }\end{array}$ & $\begin{array}{l}\text { BD-I and BD-II; } \\
\text { DSM-IV } \\
\text { Euthymic/Maintena } \\
\text { nce }\end{array}$ & $\begin{array}{l}\text { Mean Age } 44(18-75) \\
50 \% \text { Female } \\
\text { Race NR } \\
\mathrm{N}=76\end{array}$ & $\begin{array}{l}\text { Schizoaffective; } \\
\text { Substance Abuse; } \\
\text { Other Mental Health; } \\
\text { Neurological Disorders; } \\
\text { Taking Other } \\
\text { Medications }\end{array}$ \\
\hline
\end{tabular}




\begin{tabular}{|c|c|c|c|c|c|}
\hline $\begin{array}{c}\text { Author, Year } \\
\text { Single-Multisite } \\
\text { Local/Continent } \\
\text { Risk of Bias } \\
\text { PMID }\end{array}$ & Intervention & Comparison & $\begin{array}{l}\text { Inclusion Criteria: } \\
\text { BD Type; } \\
\text { Diagnostic } \\
\text { Criteria } \\
\text { Current Episode }\end{array}$ & Demographics & Key Exclusions \\
\hline $\begin{array}{l}\text { Parikh, } 2012^{216} \\
\text { Multisite } \\
\text { Canada } \\
\text { Low ROB } \\
22795205\end{array}$ & $\begin{array}{l}\text { CBT including psychoeducation, } \\
\text { understanding of personal } \\
\text { warning signs for onset and } \\
\text { action plan, and cognitive } \\
\text { restructuring of dysfunctional } \\
\text { thoughts and assumptions } \\
-20 \text { individual } 50 \text {-minute sessions }\end{array}$ & $\begin{array}{l}\text { Group psychoeducation } \\
\text { using Life Goals manual; } \\
\text { focused on illness } \\
\text { recognition, treatment } \\
\text { approaches, and coping } \\
\text { strategies and the creation } \\
\text { of Personal Care Plan } \\
\text { including action plan for } \\
\text { both depression and mania } \\
-6 \text { sessions, } 90 \text { minutes } \\
\text { each session }\end{array}$ & $\begin{array}{l}\text { BD-I and BD-II } \\
\text { DSM-IV } \\
\text { Euthymic/Maintena } \\
\text { nce }\end{array}$ & $\begin{array}{l}\text { Mean Age } 40.9(18-64) \\
58 \% \text { Female } \\
\text { Race NR } \\
N=204\end{array}$ & $\begin{array}{l}\text { First Manic Episode; } \\
\text { Substance Abuse; } \\
\text { Other Mental Health; } \\
\text { Neurological Disorders; } \\
\text { Labs/Other Conditions }\end{array}$ \\
\hline $\begin{array}{l}\text { Weiss, } 2009^{214} \\
\text { Singlesite } \\
\text { United States } \\
\text { Low ROB } \\
19573999\end{array}$ & $\begin{array}{l}\text { Integrated group CBT on the } \\
\text { cognitive-behavioral relapse } \\
\text { prevention model which focuses } \\
\text { on the similarities between } \\
\text { recovery and relapse processes } \\
\text { in BD and substance abuse and } \\
\text { their interaction } \\
-12 \text { weekly } 60 \text {-minute sessions }\end{array}$ & $\begin{array}{l}\text { Group Drug Therapy: } \\
\text { Substance use disorders } \\
\text { therapy sessions that } \\
\text { focused on facilitating } \\
\text { abstinence, encouraging } \\
\text { mutual support, and } \\
\text { teaching new ways to cope } \\
\text { with substance-related } \\
\text { problems } \\
\text {-12 weekly } 60 \text {-minute } \\
\text { sessions }\end{array}$ & $\begin{array}{l}\text { BD-I, BD-II, and BD } \\
\text { NOS } \\
\text { DSM-IV } \\
\text { Non-manic }\end{array}$ & $\begin{array}{l}\text { Mean Age } 38(18-58) \\
41 \% \text { Female } \\
92 \% \text { White } \\
N=61\end{array}$ & $\begin{array}{l}\text { First Manic Episode; } \\
\text { Schizoaffective; Other } \\
\text { Mental Health; } \\
\text { Labs/Other Conditions }\end{array}$ \\
\hline $\begin{array}{l}\text { Weiss } 2007^{213} \\
\text { Singlesite } \\
\text { United States } \\
\text { Moderate ROB } \\
17202550\end{array}$ & $\begin{array}{l}\text { Integrated group CBT on } \\
\text { cognitive-behavioral relapse } \\
\text { prevention model which focuses } \\
\text { on the similarities between } \\
\text { recovery and relapse processes } \\
\text { in BD and substance abuse and } \\
\text { their interaction } \\
-20 \text { weekly } 60 \text {-minute sessions }\end{array}$ & $\begin{array}{l}\text { Group Drug Therapy: } \\
\text { Focused on facilitating } \\
\text { abstinence, encouraging } \\
\text { mutual support, and } \\
\text { teaching new ways to cope } \\
\text { with substance-related } \\
\text { problems } \\
\text {-20 weekly } 60 \text {-minute } \\
\text { sessions }\end{array}$ & $\begin{array}{l}\text { BD-I, BD-II, and BD } \\
\text { NOS; DSM-IV } \\
\text { Maintenance }\end{array}$ & $\begin{array}{l}\text { Mean Age } 41.9(22-65) \\
52 \% \text { Female } \\
94 \% \text { White } \\
N=62\end{array}$ & $\begin{array}{l}\text { First Manic Episode; } \\
\text { Schizoaffective; Other } \\
\text { Mental Health; } \\
\text { Labs/Other Conditions }\end{array}$ \\
\hline
\end{tabular}

BD=Bipolar Disorder; CBT=cognitive behavioral therapy; DSM= Diagnostic and Statistical Manual of Mental Disorders; N=number; NOS=Not Otherwise Specified; NR=not reported: $\mathrm{ROB}=$ risk of bias 


\section{Relapse}

Evidence was insufficient for the effect of CBT on relapse compared with an active comparator, due to moderate study limitations, inconsistent findings, and imprecision. Three low or moderate risk of bias studies enrolling 338 participants reported number of relapses. ${ }^{216,217,221}$ Meyer et al. $(n=76)$ reported no difference between groups in recurrence of any type of effective episode at both 9 and 30 months. Consistent with these findings, Parikh et al. (n=204) found no difference in the number of manic or depressive relapses over 72 weeks. Harvey et al. $(n=58)$ found that while individuals who received CBT had fewer hypomanic/manic relapses than those who received psychoeducational therapy, there was no difference between groups in depressive relapses.

\section{Symptom Scores}

Low-strength evidence (moderate study limitations, imprecision) showed no effect of CBT on depression or mania symptoms when compared to an active comparator. Five low or moderate risk of bias studies enrolling 461 participants provided information on symptom scores. ${ }^{213,214,216,217,221}$ All five included studies reported no difference between groups in depression or mania symptoms across a range of outcome timepoints. ${ }^{213,214,216,217,221}$

\section{Function}

Evidence was insufficient for all measures of function due to unclear consistency and strong imprecision. Two studies enrolling 134 participants reported outcomes for function. ${ }^{217}$, ${ }^{221}$ Rated low risk of bias, Meyer et al. $(n=76)$ reported a measure of global function, finding no difference between groups. Rated moderate risk of bias, Harvey et al. $(n=58)$ reported one measure of quality of life and one measure of disability. At 6-months of followup, groups did not differ for either measure.

\section{Systematic or Collaborative Care}

We identified eight publications reporting six unique studies on systematic or collaborative care for BD. ${ }^{227-234}$ Appendix L provides evidence tables, summary risk of bias assessments, assessments of strength of evidence, and reporting for additional outcomes. A summary of findings with at least low-strength evidence for other drug treatments for maintenance are provided in Table 38. Any intervention and comparison not listed in Table 38, or outcome not listed for an included intervention and comparison, was found to have an evidence base insufficient to draw conclusions.

Table 38. Summary of findings with at least low-strength evidence for cognitive behavioral therapy

\begin{tabular}{|c|c|c|c|}
\hline Intervention & $\begin{array}{c}\text { \# Studies/ Design } \\
\text { (n Analyzed) } \\
\text { Timing }\end{array}$ & Findings & Strength of Evidence \\
\hline $\begin{array}{l}\text { Systematic or } \\
\text { Collaborative Care } \\
\text { vs. Inactive } \\
\text { Comparators* }\end{array}$ & $\begin{array}{l}2 \mathrm{RCTS}^{228} 232 \\
(\mathrm{n}=599) \\
7 \text { to } 12 \text { months }\end{array}$ & $\begin{array}{l}\text { Relapse Rate: No difference between } \\
\text { groups across different time periods. }\end{array}$ & $\begin{array}{l}\text { Low } \\
\text { (moderate study } \\
\text { limitations, imprecision) }\end{array}$ \\
\hline
\end{tabular}

*Inactive comparators are comparators such as usual care, no intervention.

$\mathrm{n}=$ number; $\mathrm{RCT}=$ randomized controlled trial 


\section{Systematic or Collaborative Care Versus Inactive Control}

We identified eight publications reporting six unique studies on the effect of systematic or collaborative care compared with an inactive comparator. Four studies were rated as low or moderate risk of bias. ${ }^{227,229,230,233,234}$ Simon et al. was rated low risk of bias for all outcomes except symptom scores where it was rated high risk of bias. ${ }^{231,}{ }^{232}$ Kessing et al. was rated low risk of bias for the outcome of hospitalizations and high risk of bias for all other reported outcomes. ${ }^{228}$ Study sample sizes ranged from 61 to 441 . The majority of studies did not exclude individuals based on their current clinical state (e.g., acute depression, acute hypomania, euthymia). Components of the interventions included interaction with a care team and psychoeducation or CBT. Length of the intervention ranged from 6 months to two years. Inactive comparisons were generally defined as "treatment as usual", which included standard mental health care (including pharmacotherapy) with or without an added component for monitoring.

Table 39 summarizes the key characteristics of the studies. Appendix L provides details. 
Table 39. Population and inclusion criteria for studies of systematic or collaborative care versus inactive comparators

\begin{tabular}{|c|c|c|c|c|c|}
\hline $\begin{array}{c}\text { Author, Year } \\
\text { Single-Multisite } \\
\text { Local/Continent } \\
\text { Risk of Bias } \\
\text { PMID }\end{array}$ & Intervention & Comparison & $\begin{array}{c}\text { Inclusion Criteria: } \\
\text { BD Type; Diagnostic } \\
\text { Criteria } \\
\text { Current Episode }\end{array}$ & Demographics & Key Exclusions \\
\hline $\begin{array}{l}\text { Kessing, } 2013^{228} \\
\text { Multisite } \\
\text { Denmark } \\
\text { Low/High ROB } \\
\text { (based on outcome) } \\
23349295\end{array}$ & $\begin{array}{l}\text { Specialized outpatient care including a } \\
\text { medical evaluation, treatment plan, } \\
\text { pharmacological treatment, group } \\
\text { sessions consisting of } \\
\text { psychoeducation and discussions } \\
\text { about participants experiences and a } \\
\text { discharge group focused on identifying } \\
\text { early warning signs and } \\
\text { communication of signs to clinicians. } \\
\text {-Specialized care for } 2 \text { years including } \\
12 \text { sessions of psychoeducation (1.5 } \\
\text { hours per session) and 3-6 months of } \\
\text { discharge group }\end{array}$ & $\begin{array}{l}\text { Treatment as usual: } \\
\text { Standard outpatient } \\
\text { mental health services } \\
\text { included treatment with a } \\
\text { general practitioner, } \\
\text { psychiatrist, or community } \\
\text { mental health center. }\end{array}$ & $\begin{array}{l}\text { Manic Episode or BD-I, II, } \\
\text { or NOS; ICD-10 code: DF } \\
30.1-31 \\
\text { Non-specific (Recent } \\
\text { hospitalization for episode) }\end{array}$ & $\begin{array}{l}\text { Mean Age } 37(27- \\
48) \\
54 \% \text { Female } \\
\text { Race NR } \\
N=158\end{array}$ & $\begin{array}{l}\text { Neurological } \\
\text { Disorders; Other } \\
\text { Mental Health; } \\
\text { Labs/Other } \\
\text { Conditions }\end{array}$ \\
\hline $\begin{array}{l}\text { Kilbourne, } 2012^{229} \\
\text { Multisite } \\
\text { US } \\
\text { Low ROB } \\
23203358\end{array}$ & $\begin{array}{l}\text { Life Goals Collaborative Care } \\
\text { consisting of weekly group self- } \\
\text { management sessions (mixture of } \\
\text { motivational interviewing and cognitive } \\
\text { behavioral techniques) with care } \\
\text { management by interventionist and } \\
\text { providers } \\
\text {-Four 2-hour sessions of self- } \\
\text { management, } 6 \text { months of care } \\
\text { management }\end{array}$ & $\begin{array}{l}\text { Enhanced treatment as } \\
\text { Usual: Usual care and } \\
\text { monthly mailings on } \\
\text { mental health care and } \\
\text { referrals to primary care } \\
\text { services }\end{array}$ & $\begin{array}{l}\text { BD-I, II or NOS; NR } \\
\text { No current clinical state } \\
\text { excluded. Severe } \\
\text { episodes not reported. }\end{array}$ & $\begin{array}{l}\text { Mean Age } 43(18- \\
71) \\
61 \% \text { Female } \\
78 \% \text { White } \\
N=68\end{array}$ & $\begin{array}{l}\text { Neurological } \\
\text { Disorders; } \\
\text { Labs/Other } \\
\text { Conditions }\end{array}$ \\
\hline
\end{tabular}




\begin{tabular}{|c|c|c|c|c|c|}
\hline $\begin{array}{l}\text { Author, Year } \\
\text { Single-Multisite } \\
\text { Local/Continent } \\
\text { Risk of Bias } \\
\text { PMID }\end{array}$ & Intervention & Comparison & $\begin{array}{c}\text { Inclusion Criteria: } \\
\text { BD Type; Diagnostic } \\
\text { Criteria } \\
\text { Current Episode }\end{array}$ & Demographics & Key Exclusions \\
\hline $\begin{array}{l}\text { Kilbourne, } 2008^{230} \\
\text { Single-site } \\
\text { US } \\
\text { Moderate ROB } \\
18586993\end{array}$ & $\begin{array}{l}\text { Bipolar disorders medical care model } \\
\text { consisting of self-management } \\
\text { (adapted for Life Goals Program) } \\
\text { education, care management via nurse } \\
\text { care manager who coordinated with } \\
\text { providers regarding medical and } \\
\text { psychiatric care, and guideline } \\
\text { implementation training for providers } \\
\text {-Three sessions ( } 2 \text { hours) of self- } \\
\text { management program; } 6 \text { months of } \\
\text { care management }\end{array}$ & $\begin{array}{l}\text { Treatment as usual: } \\
\text { Routine care (as selected } \\
\text { by provider) without self- } \\
\text { management or care } \\
\text { management }\end{array}$ & $\begin{array}{l}\text { BD-I, II or NOS; NR } \\
\text { No current clinical state } \\
\text { excluded. Severe } \\
\text { episodes not reported. }\end{array}$ & $\begin{array}{l}\text { Mean Age } 55 \text { (39- } \\
71) \\
9 \% \text { Female } \\
90 \% \text { White } \\
\mathrm{N}=61\end{array}$ & $\begin{array}{l}\text { Substance Abuse; } \\
\text { Other Mental } \\
\text { Health; Labs/Other } \\
\text { Conditions }\end{array}$ \\
\hline $\begin{array}{l}\text { Bauer, } 2006^{227} \\
\text { Multisite } \\
\text { US } \\
\text { Low ROB } \\
16816277\end{array}$ & $\begin{array}{l}\text { Bipolar Disorders Program including } \\
\text { psychoeducation via the Life Goals } \\
\text { Program and care team consisting of } \\
\text { nurse care coordinator and } \\
\text { psychiatrist } \\
-3 \text { years of care via the program }\end{array}$ & $\begin{array}{l}\text { Treatment as usual: } \\
\text { Treatment based on } \\
\text { psychiatrist choice }\end{array}$ & $\begin{array}{l}\text { BD-I, II or NOS; DSM-IV } \\
\text { No current clinical state } \\
\text { excluded. Severe } \\
\text { episodes not reported. }\end{array}$ & $\begin{array}{l}\text { Mean Age } 47 \text { (26- } \\
66) \\
28 \% \text { Female } \\
29 \% \text { White } \\
N=330\end{array}$ & $\begin{array}{l}\text { Neurological } \\
\text { Disorders; } \\
\text { Labs/Other } \\
\text { Conditions }\end{array}$ \\
\hline $\begin{array}{l}\text { Simon, } 2006^{231} \\
\text { Simon, } 2005^{232} \\
\text { Low/High ROB } \\
\text { (based on outcome) } \\
15842025 \\
16651507\end{array}$ & $\begin{array}{l}\text { Systematic care consisting of } \\
\text { structured initial assessment and } \\
\text { planning, telephone monitoring, } \\
\text { coordinated mental health treatment } \\
\text { team, and psychoeducation. } \\
\text {-Services offered for } 24 \text { months post- } \\
\text { randomization }\end{array}$ & $\begin{array}{l}\text { Treatment as usual: } \\
\text { Services that are normally } \\
\text { available without any } \\
\text { additional care }\end{array}$ & $\begin{array}{l}\text { BD-I or II; DSM-IV } \\
\text { No current clinical state } \\
\text { excluded. Severe } \\
\text { episodes not reported. }\end{array}$ & $\begin{array}{l}\text { Mean Age } 44(20- \\
68) \\
68 \% \text { Female } \\
88 \% \text { White } \\
\mathrm{N}=441\end{array}$ & $\begin{array}{l}\text { Neurological } \\
\text { Disorders; } \\
\text { Labs/Other } \\
\text { Conditions }\end{array}$ \\
\hline
\end{tabular}

BD=Bipolar Disorder; DSM= Diagnostic and Statistical Manual of Mental Disorders; N=number; NOS=Not Otherwise Specified; N=number; NR=not reported; ROB=risk of bias 


\section{Relapse}

Low-strength evidence (moderate study limitations, imprecision) showed no effect for systematic/collaborative care on relapse. Two studies, one low risk of bias and one high risk of

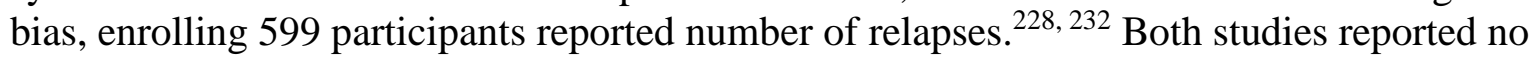
difference between groups in manic or depressive relapses at the reported outcome time points (1-3 years). ${ }^{228,232}$

\section{Symptom Scores}

Evidence was insufficient for the effect of systematic/collaborative care on depression and mania symptoms when compared with an inactive comparator due to moderate study limitations, inconsistent findings, and imprecision. Five studies enrolling 1,038 participants reported symptom scores. 227, 229-234

Among studies rated low or moderate risk of bias, one study reported no difference between groups in depression symptoms at 6 months. ${ }^{230}$ Kilbourne et al. ( $\left.n=68\right)$ reported no difference between groups across 6-12 months. ${ }^{229}$ Bauer et al. $(n=306)$ reported no difference between groups in depression at 3 years. However, while van der Voort et al. $(n=138)$ reported no difference between groups at 6 months, there was a statistically significant difference at 12 months, favoring the intervention. ${ }^{233}$ Rated high risk of bias, Simon et al. $(n=441)$ found that there were less depression symptoms in the collaborative care intervention group at 12 months. However, there was no difference between groups across the full 24-month follow-up period.

Four low or moderate risk of bias studies reported no difference between groups in mania symptoms at their respective outcome time points. ${ }^{227,229,230,233}$. Rated high risk of bias, Simon et al. $(n=441)$ reported no difference between group in mania at 12 months, but less mania symptoms in the systematic/collaborative care group across the full 24-month follow-up period. $^{231,232}$

\section{Function}

Evidence was insufficient for the effect of systematic/collaborative care on global function and other measures of function when compared with an inactive comparator due to unclear or inconsistent findings and strong imprecision. Four studies enrolling 597 participants reported measures of function. ${ }^{227,229,230,234}$

One low risk of bias study reported a measure of global function. The study reported no difference between groups at 6 months, but better function for those that received the systematic/collaborative care intervention at 12 months. ${ }^{234}$ Four low or moderate risk of bias studies reported additional measures of function. No difference was found between groups in quality of life at both 6 and 12 months. ${ }^{234}$ Similarly, no difference was found between groups in measures of mental function, physical function, and health and disability at 6 months. ${ }^{229,230}$ Based on the data from Kilbourne et al. ( $n=68)$, no differences occurred between groups in mental function and physical function at 12 months; however there was a difference in health and disability favoring the intervention. ${ }^{229}$ Bauer et al. $(n=330)$ found no difference between groups in physical function at 3 years, but reported a significant difference in mental function with better function in those who received the intervention. ${ }^{227}$

\section{Additional Outcomes}

Two low risk of bias studies reported additional outcomes related to hospitalizations. Simon et al. reported no difference between groups in number of psychiatric hospitalizations at 12 and 
24 months. ${ }^{231,232}$ However, Kessing et al. found that treatment with systematic or collaborative care resulted in a significant decrease in readmissions compared with the inactive comparator. In addition, the cumulative duration of readmissions was shorter the in intervention group. ${ }^{228}$ One low risk of bias study reported information on deaths and suicide rates. Bauer et al. $(\mathrm{n}=330)$ reported no differences between groups in number of deaths. The study reported that one person who received usual care attempted suicide. ${ }^{227}$

\section{Systematic or Collaborative Care Versus Active Control}

None of the eligible studies on systematic or collaborative care compared the intervention with an active comparator.

\section{Family or Partner Interventions}

We identified nine publications reporting six unique studies on the use of FPI as a treatment for BD. ${ }^{235-243}$ Appendix M provides evidence tables, summary risk of bias assessments, assessments of strength of evidence, and reporting for additional outcomes. We were unable to draw conclusions for FPI due to insufficient evidence.

\section{Family or Partner Interventions Versus Inactive Control}

We identified four publications reporting two unique studies on the effect of FPI compared with an inactive comparator. One study was rated low risk of bias. ${ }^{235}$ One study was rated moderate to high risk of bias due to differences reporting randomization and attrition across publications. ${ }^{238,239,242}$ Study sample sizes ranged from 58 to 92 . Subjects in one study were euthymic while the other study enrolled participants with a current episode (depressive, manic, or mixed). The FPI consisted of either 6 or 12 weekly sessions. Inactive comparator comparator included treatment as usual and pharmacotherapy.

Table 40 summarizes the key characteristics of the studies. Appendix M provides details.

Table 40. Population and inclusion criteria for studies of FPI versus inactive comparators

\begin{tabular}{|c|c|c|c|c|c|}
\hline $\begin{array}{l}\text { Author, } \\
\text { Year } \\
\text { Single- } \\
\text { Multisite } \\
\text { Locall } \\
\text { Continent } \\
\text { Risk of } \\
\text { Bias } \\
\text { PMID }\end{array}$ & Intervention & Comparison & $\begin{array}{c}\text { Inclusion } \\
\text { Criteria: } \\
\text { BD Type; } \\
\text { Diagnostic } \\
\text { Criteria } \\
\text { Current Episode }\end{array}$ & $\begin{array}{c}\text { Demographic } \\
\mathbf{s}\end{array}$ & $\begin{array}{c}\text { Key } \\
\text { Exclusion } \\
\mathrm{s}\end{array}$ \\
\hline $\begin{array}{l}\text { D'Souza, } \\
2010^{235} \\
\text { Singlesite } \\
\text { Australia } \\
\text { Low ROB } \\
19428117\end{array}$ & $\begin{array}{l}\text { Patient/companion } \\
\text { group psychoeducation } \\
\text { consisting of } \\
\text { discussion of } \\
\text { symptoms, } \\
\text { medications, and } \\
\text { warning signs, and } \\
\text { resources as well as } \\
\text { psychotherapy } \\
-12 \text { weekly sessions, } \\
90 \text { minutes each } \\
\text { session }\end{array}$ & $\begin{array}{l}\text { Treatment as usual: } \\
\text { Community based } \\
\text { case management } \\
\text { involving weekly } \\
\text { review with a } \\
\text { mental health } \\
\text { clinician and a } \\
\text { monthly medical } \\
\text { review } \\
\text {-Weekly sessions } \\
\text { for } 45 \text { minutes }\end{array}$ & $\begin{array}{l}\text { BD-I or II; MINI } \\
\text { Euthymic/Mainten } \\
\text { ance }\end{array}$ & $\begin{array}{l}\text { Mean Age } 41 \\
(19-60) \\
52 \% \text { Female } \\
\text { Race NR } \\
N=58\end{array}$ & $\begin{array}{l}\text { Substance } \\
\text { Abuse; } \\
\text { Other } \\
\text { Mental } \\
\text { Health; } \\
\text { Labs/Other } \\
\text { Conditions }\end{array}$ \\
\hline
\end{tabular}




\begin{tabular}{|c|c|c|c|c|c|}
\hline $\begin{array}{l}\text { Author, } \\
\text { Year } \\
\text { Single- } \\
\text { Multisite } \\
\text { Local/ } \\
\text { Continent } \\
\text { Risk of } \\
\text { Bias } \\
\text { PMID }\end{array}$ & Intervention & Comparison & $\begin{array}{c}\text { Inclusion } \\
\text { Criteria: } \\
\text { BD Type; } \\
\text { Diagnostic } \\
\text { Criteria } \\
\text { Current Episode }\end{array}$ & $\begin{array}{c}\text { Demographic } \\
\mathrm{s}\end{array}$ & $\begin{array}{c}\text { Key } \\
\text { Exclusion } \\
\text { s }\end{array}$ \\
\hline $\begin{array}{l}\text { Miller } \\
2008^{238} \\
\text { Solomon } \\
2008^{242} \\
\text { Miller } \\
2004^{239} \\
\text { Singlesite } \\
\text { US } \\
\text { Moderate/ } \\
\text { High ROB } \\
\\
15555694 \\
19032711 \\
18363424\end{array}$ & $\begin{array}{l}\text { Individual or group } \\
\text { family therapy } \\
\text { consisting of semi- } \\
\text { structured family } \\
\text { interventions. } \\
\text { Individual therapy was } \\
\text { based on McMaster } \\
\text { Model of Family } \\
\text { Function and group } \\
\text { therapy included } \\
\text { sessions focused on } \\
\text { signs and symptoms, } \\
\text { patient and family } \\
\text { perspectives, and } \\
\text { coping mechanisms. } \\
-6 \text { to } 10 \text { sessions of } \\
\text { family therapy, } 50 \\
\text { minutes per session } \\
\text { OR } \\
-6 \text { weekly group } \\
\text { sessions, } 90 \text { minutes } \\
\text { per session }\end{array}$ & $\begin{array}{l}\text { Pharmacotherapy: } \\
\text { Mood stabilizer with } \\
\text { other medications } \\
\text { as necessary }\end{array}$ & $\begin{array}{l}\text { BD-I; DSM-III } \\
\text { Current bipolar } \\
\text { mood episode. } \\
\text { Severe episodes } \\
\text { not reported }\end{array}$ & $\begin{array}{l}\text { Mean Age } 39 \\
(18-65) \\
57 \% \text { Female } \\
\text { Race NR } \\
\mathrm{N}=92\end{array}$ & $\begin{array}{l}\text { Substance } \\
\text { Abuse }\end{array}$ \\
\hline
\end{tabular}

BD=Bipolar Disorder; DSM= Diagnostic and Statistical Manual of Mental Disorders; FPI=Family or partner interventions; MINI=Mini-International Neuropsychatric Interview; N=number; NOS=Not otherwise specified; NR=not reported; ROB-risk of bias

\section{Relapse}

Evidence was insufficient for the effect of FPI on relapses when compared with an inactive comparator due to inconsistent findings and imprecision. Two studies enrolling 150 participants reported number of relapses. ${ }^{235,239} 242$ Evidence regarding the effect of FPI on relapses was mixed. At 15 months, D'Souza et al. reported fewer relapses for those who received FPI. ${ }^{235}$ Miller et al. ( $n=92)$, reported no difference between groups in the proportion of participants that experienced recovery or time to recovery across 28 months. ${ }^{239}$ In a high risk of bias publication, the study also reported that among the subset of 53 patients who recovered from their intake mood episode, there was no difference between those who received FPI (either individual or group) and the inactive comparator group in relapses across the 28 months of the study. ${ }^{242}$ There was also no difference between groups in time to recurrence. ${ }^{242}$

\section{Symptom Scores}

Evidence was insufficient for the effect of the FPI on depression and mania outcomes when compared with an inactive comparator due to unclear consistency and imprecision. One low risk of bias study enrolling 58 participants reported symptom scores and provided sufficient data to calculate effect sizes. ${ }^{235}$ D'Souza et al. found no difference between groups in depression 
symptoms at 15 months. ${ }^{235}$ However, a statistically significant difference in mania symptoms was reported at 15 months, with those that received FPI experiencing less symptoms. ${ }^{235}$

\section{Additional Outcomes}

One publication with high risk of bias reported information on hospitalizations. ${ }^{242}$ Among the subset of 53 patients who recovered from their intake mood episode, the study found that there was a significant difference between groups in frequency of hospitalizations. While the frequency of hospitalizations was relatively similar between those who received individual family therapy and those who received the inactive comparator, participants who received group family therapy had fewer hospitalizations. ${ }^{242}$

\section{Family or Partner Therapy Versus Active Control}

We identified five publications reporting four unique studies on the effect of FPI when compared with active comparator. Two studies were rated low risk of bias, ${ }^{236,237,240}$ and two were moderate. ${ }^{241,243}$ Study sample sizes ranged from 53 to 101. The studies did not exclude individuals based on the current clinical state (i.e., including euthymic state or a current depressive, hypomanic, manic, or mixed episode). Three of four studies included psychoeducation as a component of the intervention. In addition, three of the four studies had an intervention span of 9 months. Active comparators included family education with crisis management, treatment as usual with enhanced assessment and monitoring, and individual treatment.

Table 41 summarizes the key characteristics of the studies. Appendix M provides details. 
Table 41. Population and inclusion criteria for studies of FPI versus active comparators

\begin{tabular}{|c|c|c|c|c|c|}
\hline $\begin{array}{c}\text { Author, Year } \\
\text { Single-Multisite } \\
\text { Local/Continent } \\
\text { Risk of Bias } \\
\text { PMID }\end{array}$ & Intervention & Comparison & $\begin{array}{c}\text { Inclusion Criteria: } \\
\text { BD Type; Diagnostic } \\
\text { Criteria } \\
\text { Current Episode }\end{array}$ & Demographics & Key Exclusions \\
\hline $\begin{array}{l}\text { Wenze, } 2015^{243} \\
\text { Singlesite } \\
\text { US } \\
\text { Moderate ROB } \\
26117247\end{array}$ & $\begin{array}{l}\text { Integrated Treatment Adherence } \\
\text { Program based on a cognitive } \\
\text { behavioral approach focused on } \\
\text { transitioning patients from acute to } \\
\text { maintenance care using patient and } \\
\text { family or significant other meetings in } \\
\text { person and via telephone. } \\
\text {-3 individual in-person sessions, } 60 \\
\text { minutes per session; a } 60 \text { minute in- } \\
\text { person session with family session, } \\
\text { and } 11 \text { phone contacts held separately } \\
\text { with subject and designated family } \\
\text { member or significant other }\end{array}$ & $\begin{array}{l}\text { Enhanced Assessment } \\
\text { and Monitoring consisting } \\
\text { of treatment as usual with } \\
\text { enhanced monitoring } \\
\text { (battery of interview-rated } \\
\text { and self-report } \\
\text { assessments followed by } \\
\text { feedback letters) }\end{array}$ & $\begin{array}{l}\text { BD-I, II, or NOS; } \\
\text { DSM-IV } \\
\text { No current clinical } \\
\text { state excluded. } \\
\text { Severe episodes not } \\
\text { reported }\end{array}$ & $\begin{array}{l}\text { Mean Age } 47 \text { (24- } \\
68) \\
50 \% \text { Female } \\
90 \% \text { White } \\
\mathrm{N}=30\end{array}$ & $\begin{array}{l}\text { Other Mental Health; } \\
\text { Pregnant/Nursing; } \\
\text { Labs/Other Conditions }\end{array}$ \\
\hline $\begin{array}{l}\text { Miklowitz, } 2003^{236} \\
\text { Miklowitz, } 2000^{237} \\
\text { Multisite } \\
\text { US } \\
\text { Low ROB } \\
11018229 \\
12963672\end{array}$ & $\begin{array}{l}\text { Family-focused therapy with } \\
\text { pharmacotherapy consisting of } \\
\text { psychoeducation, developing } \\
\text { communication skills, and learning a } \\
\text { framework for defining problems and } \\
\text { implementing solutions. } \\
\text {-Up to } 21 \text { family or martial sessions } \\
\text { over } 9 \text { months, } 60 \text { minutes per session }\end{array}$ & $\begin{array}{l}\text { Family education ( } 2 \\
\text { sessions) and crisis } \\
\text { management consisting } \\
\text { of treatment as usual with } \\
\text { emergency counseling } \\
\text { sessions as needed and } \\
\text { monthly telephone calls } \\
\text { with patient }\end{array}$ & $\begin{array}{l}\text { BD-I; DSM-III } \\
\text { No current clinical } \\
\text { state excluded. } \\
\text { Severe episodes not } \\
\text { reported }\end{array}$ & $\begin{array}{l}\text { Mean Age } 36 \text { (18- } \\
56) \\
63 \% \text { Female } \\
\text { Race NR } \\
N=101\end{array}$ & $\begin{array}{l}\text { Substance Abuse; } \\
\text { Neurological Disorders; } \\
\text { Labs/Other Conditions }\end{array}$ \\
\hline
\end{tabular}




\begin{tabular}{|c|c|c|c|c|c|}
\hline $\begin{array}{c}\text { Author, Year } \\
\text { Single-Multisite } \\
\text { Local/Continent } \\
\text { Risk of Bias } \\
\text { PMID }\end{array}$ & Intervention & Comparison & $\begin{array}{c}\text { Inclusion Criteria: } \\
\text { BD Type; Diagnostic } \\
\text { Criteria } \\
\text { Current Episode }\end{array}$ & Demographics & Key Exclusions \\
\hline $\begin{array}{l}\text { Rea, } 2003^{240} \\
\text { Multisite } \\
\text { US } \\
\text { Low ROB } \\
12795572\end{array}$ & $\begin{array}{l}\text { Family-focused treatment (with } \\
\text { medication management) consisting of } \\
\text { psychoeducation, communication } \\
\text { enhancement training, and problem- } \\
\text { solving skills training } \\
-21 \text { therapy sessions over } 9 \text { months } \\
\text { (60 minutes per session) with } 1 \text { year of } \\
\text { medication management }\end{array}$ & $\begin{array}{l}\text { Individual treatment (with } \\
\text { medication management) } \\
\text { consisting of meeting a } \\
\text { therapist to receive } \\
\text { education about illness } \\
\text { and symptoms, discuss } \\
\text { problem-solving, and } \\
\text { establishing goals. } \\
-21 \text { therapy sessions over } \\
9 \text { months ( } 30 \text { minutes } \\
\text { per session) with } 1 \text { year } \\
\text { of medication } \\
\text { management }\end{array}$ & $\begin{array}{l}\text { BD-I, II, or NOS; } \\
\text { DSM-III } \\
\text { Manic }\end{array}$ & $\begin{array}{l}\text { Mean Age } 26 \\
(18=46) \\
57 \% \text { Female } \\
60 \% \text { White } \\
N=53\end{array}$ & $\begin{array}{l}\text { Substance Abuse; } \\
\text { Labs/Other Conditions }\end{array}$ \\
\hline $\begin{array}{l}\text { Simoneau. } 1999^{241} \\
\text { US } \\
\text { Multisite } \\
\text { Moderate ROB } \\
10609423\end{array}$ & $\begin{array}{l}\text { Family-focused therapy (with } \\
\text { medication management) consisting of } \\
\text { psychoeducation, communication- } \\
\text { enhancement training, and problem- } \\
\text { solving skills training } \\
\text {-21 sessions over } 9 \text { months }\end{array}$ & $\begin{array}{l}\text { Crisis management with } \\
\text { naturalistic follow-up (with } \\
\text { medication management) } \\
\text { consisting of two sessions } \\
\text { of home-based family } \\
\text { education, crisis } \\
\text { intervention as needed, } \\
\text { telephone counseling and } \\
\text { individual support } \\
\text { sessions as needed, and } \\
\text { monthly contacts. } \\
-9 \text { months of } \\
\text { management }\end{array}$ & $\begin{array}{l}\text { BD (Not Specified); } \\
\text { DSM-III } \\
\text { No current clinical } \\
\text { state excluded. } \\
\text { Severe episodes not } \\
\text { reported }\end{array}$ & $\begin{array}{l}\text { Mean Age } 34 \text { (18- } \\
57) \\
54 \% \text { Female } \\
\text { Race NR } \\
N=79\end{array}$ & $\begin{array}{l}\text { Substance Abuse; } \\
\text { Labs/Other Conditions }\end{array}$ \\
\hline
\end{tabular}

BD=Bipolar Disorder; DSM= Diagnostic and Statistical Manual of Mental Disorders; N=number; NOS=Not otherwise specified; NR=not reported; ROB=risk of bias 


\section{Relapse}

Evidence was insufficient for the effect of FPI on relapse due to strong imprecision. Two low or moderate risk of bias studies enrolling 154 participants reported information on relapses. ${ }^{236 \text {, }}$ 237, 240 Miklowitz et al. ( $n=101$ ) had no difference between groups at 12 months; however there were fewer relapses at 24 months in participants who received the family/partner therapy. ${ }^{236,237}$ In addition, Rea et al. (2003) $(n=53)$ reported no difference between groups in number of relapses at 12 months; however there was a significant difference between groups in the 1-year period post-treatment (24 months total) with fewer relapses reported for the FPI group. ${ }^{240}$

\section{Symptom Scores}

Evidence was insufficient for the effect of FPI on depression and mania symptoms, due to strong imprecision. Three low or moderate risk of bias studies enrolling 210 subjects reported symptom scores. Two studies were rated low risk of bias and two were rated moderate risk of bias. ${ }^{236,237,241,243}$ Only one study provided sufficient evidence to calculate effect sizes. Two studies reported a significant difference in depression symptoms at various time points, all favoring participants that received FPI. ${ }^{236,237,243}$ One study reported no difference in mania symptoms at 12 or 24 months. ${ }^{236,237}$ Wenze et al. $(n=30)$ reported a significant difference between groups at 6 months, with less manic symptoms in the group that received FPI. ${ }^{243}$ In addition, Simoneau et al. (1999) $(n=79)$ reported a generalized symptom score finding that participants who received FPI showed a greater improvement in BD symptoms than those who received the active comparator at one year post-treatment. ${ }^{241}$

\section{Function}

No measures of global function were reported. Evidence was insufficient for the effect of FPI on health and disability due to unclear consistency and imprecision. For other measures of function, one moderate risk of bias study of enrolling 30 participants reported that individuals who received the FPI had greater improvements in health and disability at 6 months than those who received the active comparator. ${ }^{243}$

\section{Additional Outcomes}

One moderate risk of bias study reported information on emergency room visits and hospitalizations. Wenze et al. $(n=30)$ reported that no significant difference between groups in either outcome at 6 months. ${ }^{243}$

\section{Interpersonal and Social Rhythm Therapy}

We identified seven publications reporting two unique studies on IPSRT as a treatment for BD. ${ }^{244-250}$ Appendix N provides evidence tables, summary risk of bias assessments, assessments of strength of evidence, and reporting for additional outcomes. We were unable to draw conclusions for IPSRT interventions due to insufficient evidence.

\section{Interpersonal and Social Rhythm Therapy Versus Inactive Control}

Five publications reported one high risk of bias study, the Maintenance Therapy in Bipolar Disorder trial, compared IPSRT to intensive clinical management. ${ }^{244-247,250}$ Total enrollment for the trial was 181 subjects. The trial randomized patients to an initial 12 weeks of either IPSRT or intensive clinical management. All participants received pharmacotherapy. Patients were then randomized again after 12 weeks to 2 years of additional monthly sessions of either IPSRT or 
intensive clinical management. Sample sizes across the publications ranged from 32 to 175 participants.

Table 42 describes the intervention and comparator. Appendix N provides details.

Table 42. Population and inclusion criteria for studies of IPSRT versus inactive comparators

\begin{tabular}{|c|c|c|c|c|c|}
\hline $\begin{array}{l}\text { Author, Year } \\
\text { Single-Multisite } \\
\text { Local/Continent } \\
\text { Risk of Bias } \\
\text { PMID }\end{array}$ & Intervention & Comparison & $\begin{array}{c}\text { Inclusion } \\
\text { Criteria: } \\
\text { BD Type; } \\
\text { Diagnostic } \\
\text { Criteria } \\
\text { Current } \\
\text { Episode }\end{array}$ & Demographics & Key Exclusions \\
\hline $\begin{array}{l}\text { Maintenance } \\
\text { Therapies in } \\
\text { Bipolar } \\
\text { Disorder } \\
\text { Frank, } 1997^{244} \\
\text { Frank, 1999247 } \\
\text { Rucci, 2002 } 250 \\
\text { Frank, 2005'245 } \\
\text { Frank, 2008 } 246 \\
\text { US } \\
\text { Multisite } \\
\text { High ROB } \\
\text { 9171907 } \\
\text { 10609422 } \\
12091194 \\
16143731 \\
18829872\end{array}$ & $\begin{array}{l}\text { IPSRT (acute, } \\
\text { maintenance, or } \\
\text { both) focused on } \\
\text { maintaining } \\
\text { regular daily } \\
\text { routines, } \\
\text { identification and } \\
\text { management of } \\
\text { potential triggers } \\
\text { and } \\
\text { interpersonal } \\
\text { psychotherapy. } \\
\text {-Acute weekly } \\
\text { treatment until } \\
\text { remission } \\
\text { followed by } \\
\text { biweekly } \\
\text { sessions for } 12 \\
\text { weeks and } \\
\text { monthly } \\
\text { treatment to } 24 \\
\text { months, } 45 \text { to } 55 \\
\text { minutes per } \\
\text { session }\end{array}$ & $\begin{array}{l}\text { Clinical } \\
\text { management } \\
\text { (acute, } \\
\text { maintenance, or } \\
\text { both) consisting } \\
\text { of medical } \\
\text { management of } \\
\text { BD (education, } \\
\text { review of } \\
\text { symptoms, } \\
\text { management of } \\
\text { adverse effects) } \\
\text { - Acute weekly } \\
\text { treatment until } \\
\text { remission } \\
\text { followed by } \\
\text { biweekly sessions } \\
\text { for } 12 \text { weeks and } \\
\text { monthly treatment } \\
\text { to } 24 \text { months, } 20 \\
\text { to } 25 \text { minutes per } \\
\text { session }\end{array}$ & $\begin{array}{l}\text { BD-I or } \\
\text { schizoaffective } \\
\text { disorder, } \\
\text { manic type; } \\
\text { DSM-IV } \\
\text { Depressive, } \\
\text { Manic, or } \\
\text { Mixed }\end{array}$ & $\begin{array}{l}\text { Mean Age } 35 \\
(18-55) \\
59 \% \text { Female } \\
94 \% \text { White } \\
N=38-175\end{array}$ & $\begin{array}{l}\text { Substance } \\
\text { Abuse; Other } \\
\text { Mental Health; } \\
\text { Pregnant/Nursing; } \\
\text { Labs/Other } \\
\text { Conditions }\end{array}$ \\
\hline
\end{tabular}

BD=Bipolar Disorder; DSM= Diagnostic and Statistical Manual of Mental Disorders; IPSRT=Interpersonal and Social Rhythm Therapy; N=number; NOS=Not otherwise specified; NR=not reported

Evidence was insufficient for all reported outcomes (relapse, depression symptoms, mania symptoms, and non-global function) due to high study limitations, unclear consistency, and imprecision. Overall, the assignment of maintenance treatment had no effect on outcomes. Results at 52 weeks showed no difference between groups in risk of recurrence. ${ }^{247}$ At 2 years, there was no difference between groups in the proportion achieving remission. ${ }^{246}$ After 2 years of acute and maintenance treatment, no difference was seen between groups in depression or mania symptoms. $^{244,245}$

Global functioning was not measured. For non-global measures of function, receiving IPSRT as an acute treatment appeared to improve occupational functioning compared with intensive clinical management. However, the difference was lost after 2 years of maintenance treatment; occupational functioning across groups was nearly identical. ${ }^{246}$ The study reported reductions in the suicide attempts compared to the period before study initiation; however, groups did not differ in the number of suicide attempts. ${ }^{250}$ 


\section{Interpersonal and Social Rhythm Therapy Versus Active Control}

Two publications reported one low risk of bias study, which compared IPSRT to specialist supportive care in 100 adolescents and young adults. ${ }^{248,249}$ The trial randomized participants to either IPSRT or specialist supportive care, the latter consisting of supportive psychotherapy and psychoeducation.

Table 43 describes the intervention and comparator. Appendix N provides details.

Table 43, Population and inclusion criteria for studies of IPSRT versus active comparators

\begin{tabular}{|c|c|c|c|c|c|}
\hline $\begin{array}{l}\text { Author, Year } \\
\text { Single-Multisite } \\
\text { Local/Continent } \\
\text { Risk of Bias } \\
\text { PMID }\end{array}$ & Intervention & Comparison & $\begin{array}{c}\text { Inclusion } \\
\text { Criteria: } \\
\text { BD Type; } \\
\text { Diagnostic } \\
\text { Criteria } \\
\text { Current } \\
\text { Episode }\end{array}$ & Demographics & $\begin{array}{c}\text { Key } \\
\text { Exclusions }\end{array}$ \\
\hline $\begin{array}{l}\text { Inder, } 2016^{249} \\
\text { Inder, } 2015^{248} \\
\text { Singlesite } \\
\text { New Zealand } \\
\text { Low ROB } \\
\\
25346391 \\
26698820\end{array}$ & $\begin{array}{l}\text { IPSRT consisting } \\
\text { of interpersonal } \\
\text { psychotherapy } \\
\text { with a focus on } \\
\text { social routines } \\
\text { and achieve of } \\
\text { goals. } \\
\text {-Weekly sessions } \\
\text { for } 3 \text { months, } \\
\text { fortnightly for up } \\
\text { to } 6 \text { months, and } \\
\text { then fortnightly to } \\
\text { monthly from } 6 \text { to } \\
18 \text { months } \\
\text { (frequency } \\
\text { tailored to patient } \\
\text { needs) }\end{array}$ & $\begin{array}{l}\text { Specialist } \\
\text { supportive care } \\
\text { consisting of } \\
\text { supportive } \\
\text { psychotherapy } \\
\text { and } \\
\text { psychoeducation } \\
\text {-Weekly sessions } \\
\text { for } 3 \text { months, } \\
\text { fortnightly for up } \\
\text { to } 6 \text { months, and } \\
\text { then fortnightly to } \\
\text { monthly from } 6 \text { to } \\
18 \text { months } \\
\text { (frequency } \\
\text { tailored to patient } \\
\text { needs) }\end{array}$ & $\begin{array}{l}\text { BD-I, II, or NOS; } \\
\text { DSM-IV } \\
\text { No current } \\
\text { clinical state } \\
\text { excluded. } \\
\text { Severe episodes } \\
\text { not reported }\end{array}$ & $\begin{array}{l}\text { Mean Age } 27 \\
(15-36) \\
76 \% \text { Female } \\
\text { Race NR } \\
N=100\end{array}$ & $\begin{array}{l}\text { Substance } \\
\text { Abuse }\end{array}$ \\
\hline
\end{tabular}

BD=Bipolar Disorder; DSM= Diagnostic and Statistical Manual of Mental Disorders; IPSRT=Interpersonal and Social Rhythm Therapy; N=number; NOS=Not otherwise specified; NR=not reported; ROB=risk of bias

Evidence was insufficient for all reported outcomes (depression, mania, and nonglobal function) due to unclear consistency and imprecision. The study did not report relapse. At 6 and 18 months, no difference was seen between groups in depression or mania symptoms. ${ }^{248}$ Global functioning was not measured. However, groups did not differ in social functioning at 6 months. ${ }^{248}$ At approximately 3 years (following an 18 month intervention and 18 month followup), the number of suicide attempts and other self-injury attempts was reduced from baseline. There was no information regarding differences between groups in self-injury attempts. ${ }^{249}$

\section{Combination Interventions}

We identified six publications reporting five unique studies on combinations of psychosocial interventions for BD. ${ }^{251-256}$ Appendix O provides evidence tables, summary risk of bias assessments, assessments of strength of evidence, and reporting for additional outcomes. We were unable to draw conclusions for combination interventions due to insufficient evidence. 


\section{Combination Interventions Versus Inactive Control}

Four publications reporting three unique studies examined the effect of combination interventions when compared with an inactive comparator. ${ }^{251-254}$ Two studies were rated low risk of bias, ${ }^{251-253}$ one was moderate, ${ }^{254}$ and one was high. ${ }^{253}$ Study sample sizes ranged from 40 to 122. Two studies did not exclude participants based on the current clinical state (i.e., including euthymic or a current manic, hypomanic, mixed or depressive episode). Components of the combination interventions used in the studies varied, with no consistency across the studies. One study used an online format. Intervention length ranged from 20 weeks to 6 months. Inactive comparisons were generally standard psychopharmacological treatment and clinical management without any form of psychotherapy.

Table 44 summarizes the key characteristics of the studies. Appendix O provides details.

Table 44. Population and inclusion criteria for studies of combination interventions versus inactive comparators

\begin{tabular}{|c|c|c|c|c|c|}
\hline $\begin{array}{l}\text { Author, Year } \\
\text { Single-Multisite } \\
\text { Local/Continent } \\
\text { Risk of Bias } \\
\text { PMID }\end{array}$ & Intervention & Comparison & $\begin{array}{c}\text { Inclusion } \\
\text { Criteria: } \\
\text { BD Type; } \\
\text { Diagnostic } \\
\text { Criteria } \\
\text { Current Episode }\end{array}$ & Demographics & $\begin{array}{c}\text { Key } \\
\text { Exclusions }\end{array}$ \\
\hline $\begin{array}{l}\text { Gonzalez-Isasi, } \\
2014^{251} \\
\text { Gonzalez-Isasi, } \\
2010^{252} \\
\text { Singlesite } \\
\text { Spain } \\
\text { Low ROB } \\
\\
20444503 \\
23276524\end{array}$ & $\begin{array}{l}\text { Group psychoeducation } \\
\text { and CBT consisting of } \\
\text { sessions about their } \\
\text { disorder, the } \\
\text { relationship between } \\
\text { thoughts and feelings, } \\
\text { anxiety control } \\
\text { techniques, cognitive re- } \\
\text { structuring, problem- } \\
\text { solving and self-esteem, } \\
\text { and social skills. } \\
\text {-20 weekly sessions, } 90 \\
\text { minutes each }\end{array}$ & $\begin{array}{l}\text { Standard } \\
\text { pharmacologi } \\
\text { c treatment } \\
\text { (mood } \\
\text { stabilizers, } \\
\text { antipsychotic } \\
\text { s, and/ or } \\
\text { benzodiapine } \\
\text { ) adjusted by } \\
\text { psychiatrist }\end{array}$ & $\begin{array}{l}\text { BD-I or II; DSM-IV } \\
\text { Euthymic or } \\
\text { Subsyndromal } \\
\text { (i.e., not meeting } \\
\text { current bipolar } \\
\text { episode full } \\
\text { criteria) }\end{array}$ & $\begin{array}{l}\text { Mean Age } 41 \\
(18-63) \\
48 \% \text { Female } \\
\text { Race NR } \\
N=40\end{array}$ & $\begin{array}{l}\text { Labs/Other } \\
\text { Conditions }\end{array}$ \\
\hline $\begin{array}{l}\text { Todd, } 2014^{254} \\
\text { Singlesite } \\
\text { UK } \\
\text { Moderate ROB } \\
25129531\end{array}$ & $\begin{array}{l}\text { Interactive, online } \\
\text { recovery informed self- } \\
\text { management } \\
\text { intervention (Living with } \\
\text { Bipolar) based on both } \\
\text { psychoeducation and } \\
\text { CBT. Ten interactive } \\
\text { modules to help } \\
\text { participants learn more } \\
\text { about bipolar } \\
\text { experiences, increase } \\
\text { self-esteem and self- } \\
\text { efficacy for managing } \\
\text { BD, increase ability to } \\
\text { self-manage, and } \\
\text { develop interpersonal } \\
\text { skills. Modules included } \\
\text { case studies and mood } \\
\text { checking tools. } \\
\text {-Access to program for } \\
6 \text { months }\end{array}$ & $\begin{array}{l}\text { Wait list } \\
\text { control } \\
\text { receiving } \\
\text { treatment as } \\
\text { usual } \\
\text { (general } \\
\text { practitioner } \\
\text { and/or } \\
\text { specialist } \\
\text { mental health } \\
\text { services). }\end{array}$ & $\begin{array}{l}\text { BD-I or II; Self- } \\
\text { report and MDQ } \\
\text { score } \\
\text { No current clinical } \\
\text { state excluded. } \\
\text { Severe episodes } \\
\text { not reported }\end{array}$ & $\begin{array}{l}\text { Mean Age } 43 \\
(21-65) \\
72 \% \text { Female } \\
89 \% \text { White } \\
N=122\end{array}$ & None \\
\hline
\end{tabular}




\begin{tabular}{|c|c|c|c|c|c|}
\hline $\begin{array}{c}\text { Author, Year } \\
\text { Single-Multisite } \\
\text { Local/Continent } \\
\text { Risk of Bias } \\
\text { PMID }\end{array}$ & Intervention & Comparison & $\begin{array}{c}\text { Inclusion } \\
\text { Criteria: } \\
\text { BD Type; } \\
\text { Diagnostic } \\
\text { Criteria } \\
\text { Current Episode }\end{array}$ & Demographics & $\begin{array}{c}\text { Key } \\
\text { Exclusions }\end{array}$ \\
\hline $\begin{array}{l}\text { Miklowitz } \\
2003^{253} \\
\text { Multisite } \\
\text { US } \\
\text { High ROB } \\
\\
12633127\end{array}$ & $\begin{array}{l}\text { Individual IPSRT and } \\
\text { family (or partner) } \\
\text { therapy. Individual } \\
\text { IPSRT consisted of } \\
\text { identifying interpersonal } \\
\text { problems, using Social } \\
\text { Rhythm Metric form, } \\
\text { managing symptoms } \\
\text { and identifying triggers, } \\
\text { and relapse prevention. } \\
\text { Family therapy involved } \\
\text { education about BD, } \\
\text { identification of triggers, } \\
\text { communication } \\
\text { enhancement, and } \\
\text { problem-solving. } \\
\text {-25 sessions of } \\
\text { individual therapy and } \\
25 \text { sessions of family- } \\
\text { focused therapy } \\
\text { (frequency adapted to } \\
\text { patient needs) }\end{array}$ & $\begin{array}{l}\text { Treatment as } \\
\text { usual: Crisis } \\
\text { management } \\
\text { (not } \\
\text { described, } \\
\text { comparison } \\
\text { group from } \\
\text { previous } \\
\text { clinical trial) }\end{array}$ & $\begin{array}{l}\text { BD-I or II; DSM-IV } \\
\text { No current clinical } \\
\text { state excluded. } \\
\text { Severe episodes } \\
\text { not reported }\end{array}$ & $\begin{array}{l}\text { Mean Age } 36 \\
(18-55) \\
60 \% \text { Female } \\
89 \% \text { White } \\
N=100\end{array}$ & $\begin{array}{l}\text { Substance } \\
\text { Abuse; } \\
\text { Neurological } \\
\text { Disorders }\end{array}$ \\
\hline
\end{tabular}

BD=Bipolar Disorder; CBT=Cognitive Behavioral Therapy; DSM= Diagnostic and Statistical Manual of Mental Disorders; IPSRT=Interpersonal and Social Rhythm Therapy; MDQ=Mood Disorder Questionnaire; N=number; NOS=Not otherwise specified; $\mathrm{NR}=$ not reported; $\mathrm{ROB}=$ risk of bias

\section{Relapse}

Evidence was insufficient for the effect of combination interventions on relapse when compared with an inactive comparator due to high study limitations, unclear consistency, and imprecision. A high risk of bias cohort study enrolling 100 participants reported number of relapses finding no difference between groups at 12 months. However, individuals who received the combination intervention had a longer time to recurrence than those who received the inactive comparator. ${ }^{253}$

\section{Symptom Scores}

Evidence was insufficient for the effect of combination interventions on depression and mania symptoms when compared with an inactive comparator, due to moderate study limitations and strong imprecision. Three low to high risk of bias studies (enrolling 262 participants) reported symptom scores. ${ }^{251-254}$ All three studies found that individuals receiving combination intervention had less depressive symptoms than those that received an inactive comparator..$^{252-254}$ Gonzalez-Isasi et al. ( $\mathrm{n}=40$ ) found that this trend continued long-term, finding a significant difference between groups at 5 years. ${ }^{251}$

Two low to high risk of bias studies reported results for mania symptoms. Rated low risk of bias, Gonzalez-Isasi et al. $(\mathrm{n}=40)$ reported a significant difference in mania symptoms at the initial outcome time points (11 and 17 months) and 5 years. ${ }^{251,252}$ Rated high risk of bias, Miklowitz et al. ( $\mathrm{n}=100)$ found no difference between groups at 12 months. ${ }^{253}$ 


\section{Function}

No measures of global function were reported. Evidence was insufficient for other measures of function due to moderate study limitations, unclear consistency, and imprecision. One moderate risk of bias study enrolling 122 participants reported a significant difference between groups in both quality of life and social functioning at 6 months, favoring the combination intervention. ${ }^{254}$

\section{Additional Outcomes}

Rated low risk of bias, Gonzalez-Isasi et al. $(n=-40)$ reported data on hospitalizations, finding fewer hospitalizations at 17 months for a combination intervention compared with an inactive comparator. However, there was no difference between groups in hospitalizations at 11 months or 5 years. $^{251,252}$

\section{Combination Interventions Versus Active Control}

Two studies compared combination interventions with an active comparator. ${ }^{255,256}$ One study was rated moderate risk of bias, ${ }^{256}$ the other study was high. ${ }^{255}$ Sample sizes ranged from 79 to 463. Included populations varied across the studies with two including participants in acute episodes. Components of the interventions and comparators also varied, with no consistency across the two studies.

Table 45 summarizes the key characteristics of the studies. Appendix O provides details. 
Table 45. Population and inclusion criteria for studies of combination interventions versus active comparators

\begin{tabular}{|c|c|c|c|c|c|}
\hline $\begin{array}{c}\text { Author, Year } \\
\text { Single-Multisite } \\
\text { Local/Continent } \\
\text { Risk of Bias } \\
\text { PMID }\end{array}$ & Intervention & Comparison & $\begin{array}{l}\text { Inclusion Criteria: } \\
\text { BD Type; } \\
\text { Diagnostic Criteria } \\
\text { Current Episode }\end{array}$ & Demographics & Key Exclusions \\
\hline $\begin{array}{l}\text { Fagiolini } 2009^{256} \\
\text { Multisite } \\
\text { US } \\
\text { Moderate ROB } \\
19500091\end{array}$ & $\begin{array}{l}\text { Enhanced clinical intervention and } \\
\text { specialized care for BD. Enhanced } \\
\text { clinical intervention consisted of } 10 \\
\text { basic elements plus specific modules } \\
\text { for young, elderly, and African } \\
\text { American patients. Elements } \\
\text { consisted of education (on disorder, } \\
\text { medications, sleep) and } \\
\text { management (review of symptoms, } \\
\text { discussion and management of side } \\
\text { effects, discussion of early warning } \\
\text { signs). Additional non-specific } \\
\text { support provided to both patient and } \\
\text { families. } \\
\text {-Weekly enhanced clinical sessions } \\
\text { for } 12 \text { weeks, then every other week } \\
\text { for } 8 \text { weeks, and then monthly for } \\
\text { remaining time or until they achieved } \\
\text { recurrence }\end{array}$ & $\begin{array}{l}\text { Specialized care for BD } \\
\text { consisting of a } \\
\text { manualized system of } \\
\text { clinical management } \\
\text { included assessment of } \\
\text { quality of life, } \\
\text { standardized } \\
\text { assessments of mood, } \\
\text { comprehensive medical } \\
\text { evaluations, frequent } \\
\text { visits with treatment } \\
\text { team, pharmacological } \\
\text { treatment and tracking } \\
\text { and monitoring of visits. }\end{array}$ & $\begin{array}{l}\text { BD-I, II, or NOS or } \\
\text { schizoaffective } \\
\text { bipolar subtype } \\
\text { disorder; DSM-IV for } \\
\text { adults, KSADS-PL } \\
\text { for adolescents } \\
\text { Any Episode }\end{array}$ & $\begin{array}{l}\text { Mean Age } 41(12- \\
75) \\
61 \% \text { Female } \\
83 \% \text { White } \\
N=463\end{array}$ & $\begin{array}{l}\text { Substance Abuse; } \\
\text { Other Mental Health; } \\
\text { Pregnant/Nursing; } \\
\text { Labs/Other Conditions }\end{array}$ \\
\hline $\begin{array}{l}\text { Zaretsky } 2008^{255} \\
\text { Multisite } \\
\text { Canada } \\
\text { High ROB } \\
18674402\end{array}$ & $\begin{array}{l}\text { Psychoeducation and CBT. CBT } \\
\text { was based on Basco and Rush } \\
\text { manual and emphasized } \\
\text { collaborative goal setting, cognitive } \\
\text { restructuring, problem-solving, and } \\
\text { enhancing interpersonal } \\
\text { communication. } \\
-7 \text { weekly, audiotaped individual } \\
\text { sessions of psychoeducation and } 13 \\
\text { weekly, audiotaped individual } \\
\text { sessions of CBT }\end{array}$ & $\begin{array}{l}\text { Psychoeducation based } \\
\text { on the first five chapters } \\
\text { of the Basco and Rush } \\
\text { CBT manual. } \\
-7 \text { weekly, audiotaped } \\
\text { individual sessions }\end{array}$ & $\begin{array}{l}\text { BD-I or II; NR } \\
\text { Euthymic/ } \\
\text { Maintenance }\end{array}$ & $\begin{array}{l}\text { Mean Age } 40(18- \\
62) \\
\text { Sex NR } \\
\text { Race NR } \\
N=293\end{array}$ & $\begin{array}{l}\text { Substance Abuse; } \\
\text { Schizoaffective; Other } \\
\text { Mental Health; } \\
\text { Neurological } \\
\text { Disorders; Labs/Other } \\
\text { Conditions }\end{array}$ \\
\hline
\end{tabular}

Affective Disorders and Schizophrenia; NOS=Not otherwise specified; NR=not reported; ROB=risk of bias 


\section{Relapse}

Evidence was insufficient for the effect of combination interventions on relapse due to high study limitations, unclear consistency, and imprecision. One high risk of bias study enrolling 79 participants reported no difference between groups in number of relapses. ${ }^{255}$

\section{Symptom Scores}

Evidence was insufficient for the effect of combination interventions on depression and mania symptoms due to high study limitations and imprecision. Two studies enrolling 542 participants reported symptom scores. ${ }^{255,256}$ One moderate risk of bias study reported no difference between groups at 18 months. ${ }^{256}$ However, a high risk of bias study reported a significant difference between groups in depressive symptoms at 12 months, favoring the combination intervention. ${ }^{255}$ One moderate risk of bias study, enrolling 463 participants, reported measures of mania. ${ }^{256}$ The study found no difference between groups at 18 months. ${ }^{256}$

\section{Function}

Evidence was insufficient for the effect of combination interventions on global function and other measures of function due to moderate study limitations, unclear consistency, and imprecision. One moderate risk of bias study enrolling 463 participants reported measures of function. ${ }^{256}$ Fagiolini et al. (2009) $(n=463)$ reported no differences between groups in global function at 18 months. The study also reported one measure of quality of life, with participants who received the combination intervention reporting better outcomes at 18 months. ${ }^{256}$

\section{Systematic Treatment Enhancement Program for Bipolar Disorder (STEP-BD) Study and Other Interventions}

Three studies examined either additional psychosocial interventions not previously described (e.g. self-monitoring), ${ }^{202,257}$ or represented a unique set of analyses based on a large scale, multisite study, the STEP-BD study. ${ }^{258-261}$ STEP-BD assessed effects of intensive, individual CBT, IPSRT, and Family-Focused Therapy in comparison with collaborative care. While STEP$\mathrm{BD}$ had three intervention arms, the primary aim of the study was to compare intensive psychotherapy to psychoeducation-based collaborative care. While, the authors did report some response outcomes by individual intervention arm (provided in Appendix P), the primary analysis of relapse/response and other outcomes like function are reported collapsed as only "intensive psychotherapy."

Table 46 describes the characteristics of these studies. Appendix P provides evidence tables, summary risk of bias assessments, assessments of strength of evidence, and reporting for additional outcomes. 
Table 46. Population and inclusion criteria for studies of other psychosocial interventions

\begin{tabular}{|c|c|c|c|c|c|}
\hline $\begin{array}{l}\text { Author, Year } \\
\text { Single-Multisite } \\
\text { Local/Continent } \\
\text { Risk of Bias } \\
\text { PMID }\end{array}$ & Intervention & Comparison & $\begin{array}{c}\text { Inclusion Criteria: } \\
\text { BD Type; } \\
\text { Diagnostic Criteria } \\
\text { Current Episode }\end{array}$ & Demographics & Key Exclusions \\
\hline $\begin{array}{l}\text { Deckersbach, } \\
2014^{259} \\
\text { Miklowitz, 2006258 } \\
\text { Miklowitz, 2007260 } \\
\text { Miklowitz, 2007b b61 } \\
\text { Multisite } \\
\text { US } \\
\text { Moderate/High ROB } \\
\text { (based on } \\
\text { outcomes/timing) } \\
\\
24077657 \\
16816280 \\
17728418 \\
17404119\end{array}$ & $\begin{array}{l}\text { Intensive psychotherapy consisting of } \\
\text { one of the following: 1) individual } \\
\text { CBT consisting of psychoeducation, } \\
\text { life events scheduling, cognitive } \\
\text { restructuring, problem-solving, } \\
\text { strategies for early detection, and } \\
\text { interventions for comorbidities, 2) } \\
\text { IPSRT consisting of selecting a } \\
\text { primary problem area and teaching } \\
\text { patients about the Social Rhythm } \\
\text { Metric and interpersonal problem } \\
\text { resolution, or 3) family-focused } \\
\text { therapy which encouraged patients } \\
\text { and relatives to develop a common } \\
\text { understanding, develop a relapse } \\
\text { prevention plan, participate in } \\
\text { communication enhancement } \\
\text { exercises, and identify and solve } \\
\text { problems related to illness or the } \\
\text { home environment. } \\
\text {-30 50-minute sessions over } 9 \\
\text { months }\end{array}$ & $\begin{array}{l}\text { Collaborative care } \\
\text { consisting of a reviewing } \\
\text { a psychoeducational } \\
\text { videotape and workbook } \\
\text { and developing a } \\
\text { treatment contract. } \\
\text { Worbook included } \\
\text { information about BD, } \\
\text { schedule management } \\
\text { and mood charting, } \\
\text { improving } \\
\text { communication skills, } \\
\text { and developing a } \\
\text { treatment contract. } \\
\text {-Three 50-minute } \\
\text { individual sessions }\end{array}$ & $\begin{array}{l}\text { BD-I or II; DSM-IV } \\
\text { Major Depressive } \\
\text { Episode }\end{array}$ & $\begin{array}{l}\text { Mean Age } 40(18- \\
62) \\
59 \% \text { Female } \\
91 \% \text { White } \\
N=293\end{array}$ & $\begin{array}{l}\text { Substance Abuse; } \\
\text { Other Mental Health; } \\
\text { Pregnant/Nursing; } \\
\text { Labs/Other Conditions }\end{array}$ \\
\hline
\end{tabular}




\begin{tabular}{|c|c|c|c|c|c|}
\hline $\begin{array}{c}\text { Author, Year } \\
\text { Single-Multisite } \\
\text { Local/Continent } \\
\text { Risk of Bias } \\
\text { PMID }\end{array}$ & Intervention & Comparison & $\begin{array}{c}\text { Inclusion Criteria: } \\
\text { BD Type; } \\
\text { Diagnostic Criteria } \\
\text { Current Episode }\end{array}$ & Demographics & Key Exclusions \\
\hline $\begin{array}{l}\text { Depp, } 2015^{202} \\
\text { Single-site } \\
\text { US } \\
\text { Low ROB } \\
25479050\end{array}$ & $\begin{array}{l}\text { Psychoeducation followed by use of } \\
\text { a smart phone that delivered } \\
\text { interactive elements via a mobile } \\
\text { web-based program that delivered } \\
\text { questionnaires and responses based } \\
\text { on symptoms or early warning signs } \\
-4 \text { sessions of psychoeducation } \\
\text { followed by smart intervention ( } 2 \\
\text { surveys per day) for } 10 \text { weeks }\end{array}$ & $\begin{array}{l}\text { Psychoeducation } \\
\text { followed by binder with } \\
\text { paper and pencil mood } \\
\text { charts. Monitored } \\
\text { remotely via cell phone } \\
\text { and had to turn in } \\
\text { completed charts at the } \\
\text { end of study. } \\
-4 \text { sessions of } \\
\text { psychoeducation } \\
\text { followed by mood charts } \\
\text { once per day for } 10 \\
\text { weeks }\end{array}$ & $\begin{array}{l}\text { BD Type Not } \\
\text { Specified; DSM-IV } \\
\text { Any Episode } \\
\text { (Without Severe } \\
\text { Affective Symptoms) }\end{array}$ & $\begin{array}{l}\text { Mean Age } 48(22- \\
74) \\
59 \% \text { Female } \\
70 \% \text { White } \\
\mathrm{N}=104\end{array}$ & $\begin{array}{l}\text { Substance Abuse; } \\
\text { Other Mental Health }\end{array}$ \\
\hline $\begin{array}{l}\text { Faurholt-Jepsen, } \\
2015^{257} \\
\text { Single-site } \\
\text { Denmark } \\
\text { Low ROB } \\
26220802\end{array}$ & $\begin{array}{l}\text { Smartphone with self-monitoring } \\
\text { system that documented mood, sleep } \\
\text { length, activity, medication taken, } \\
\text { irritability, cognitive problems, alcohol } \\
\text { consumption, stress, menstruation, } \\
\text { and early warning signs. Patients } \\
\text { could see visual representations of } \\
\text { data to self-monitor. System } \\
\text { included feedback loop with clinic } \\
\text { and contact with study nurse. } \\
-6 \text { months of self-monitoring }\end{array}$ & $\begin{array}{l}\text { Smartphone without } \\
\text { self-monitoring system } \\
\text { and nurse contact if } \\
\text { needed. } \\
-6 \text { months of smart } \\
\text { phone access }\end{array}$ & $\begin{array}{l}\text { BD Type Not } \\
\text { Specified; ICD-10 } \\
\text { and Schedules for } \\
\text { Clinical Assessment } \\
\text { in Neuropsychiatry } \\
\text { Any Episode (HDRS } \\
\leq 17 \text { and a YMRS } \\
\leq 17 \text { ) }\end{array}$ & $\begin{array}{l}\text { Mean Age } 29 \text { (18- } \\
60) \\
82 \% \text { Female } \\
\text { Race NR } \\
\mathrm{N}=78\end{array}$ & $\begin{array}{l}\text { Other Mental Health; } \\
\text { Pregnant/Nursing; } \\
\text { Labs/Other Conditions }\end{array}$ \\
\hline
\end{tabular}

BD=Bipolar Disorder; DSM= Diagnostic and Statistical Manual of Mental Disorders; HDRS=Hamilton depression rating scale; N=number; NOS=Not otherwise specified; $\mathrm{NR}=$ not reported; ROB=risk of bias; YRMS=Young mania rating scale. 
The STEP-BD study ( $\mathrm{n}=293)$ compared three different types of intensive psychosocial therapy to collaborative care. The study was rated moderate to high risk of bias study due to differences in reporting across outcomes (moderate for relapse, high for function). The study reported outcomes for relapse and function. Compared with the active comparator, a greater proportion of participants who received any type of intensive psychotherapy recovered. Compared with the other three intensive psychotherapy interventions, a greater proportion of those who received family therapy recovered. In addition, those who received family therapy had the shortest time to recovery. ${ }^{261}$ However, these differences between the intensive psychotherapy interventions were not statistically significant. ${ }^{261}$ In a subset of patients who had been assessed for functioning at baseline, those who received the intensive psychosocial intervention showed statistically significant improvements in overall function compared with those who received the active comparator at 9 months. ${ }^{260}$ Of the therapies for the intensive psychosocial intervention, the family therapy group showed the largest improvement (mean change from baseline) in function. 260

Two low risk of bias studies examined the use of mobile devices for self-monitoring and selfmanagement. Strength of evidence was insufficient for all reported outcomes due to strong imprecision. Depp et al. ( $\mathrm{n}=104)$ randomized individuals to either self-management via mobile device or mood monitoring via paper and pencil, each following four sessions of psychoeducation. ${ }^{202}$ The study reported no difference between groups in depression and mania symptoms at 6 months. Faurholt-Jepsen et al. $(\mathrm{n}=78)$ randomized participants to use a smart phone for daily monitoring (including receiving clinical feedback) or for normal purposes. The study reported no difference between groups in depression, mania, global function, or quality of life at 6 months. ${ }^{257}$

\section{Somatic Therapy}

One study examined a somatic therapy approach. Table 47 describes the characteristics of this study. Appendix P provides evidence tables, summary risk of bias assessments, assessments of strength of evidence, and reporting for additional outcomes.

Evidence was insufficient from one small, moderate risk of bias study $(n=46)$ that compared repetitive transcranial magnetic stimulation (rTMS) to sham stimulation. ${ }^{262}$ At 4 weeks, there was no difference between groups in response or remission rates, or depressive symptoms in individuals experiencing a depressive episode. ${ }^{262}$ 
Table 47. Population and inclusion criteria for studies examining a somatic therapy

\begin{tabular}{|c|c|c|c|c|c|}
\hline $\begin{array}{l}\text { Author, Year } \\
\text { Single- } \\
\text { Multisite } \\
\text { Local/ } \\
\text { Continent } \\
\text { Risk of Bias } \\
\text { PMID }\end{array}$ & Intervention & Comparison & $\begin{array}{c}\text { Inclusion } \\
\text { Criteria: } \\
\text { BD Type; } \\
\text { Diagnostic } \\
\text { Criteria } \\
\text { Current } \\
\text { Episode }\end{array}$ & Demographics & $\begin{array}{c}\text { Key } \\
\text { Exclusions }\end{array}$ \\
\hline $\begin{array}{l}\text { Fitzgerald, } \\
2016^{262} \\
\text { Singlesite } \\
\text { Australia } \\
\text { Moderate } \\
\text { ROB } \\
27016659\end{array}$ & $\begin{array}{l}\text { Repetitive transcranial } \\
\text { magnetic stimulation } \\
-20 \text { rTMS sessions for } \\
\text { four weeks }\end{array}$ & $\begin{array}{l}\text { Sham stimulation } \\
-20 \text { sham } \\
\text { sessions for four } \\
\text { weeks }\end{array}$ & $\begin{array}{l}\text { BD I or II; } \\
\text { DSM-IV } \\
\text { Depressive } \\
\text { Episode }\end{array}$ & $\begin{array}{l}\text { Mean Age } 46 \\
(33-59) \\
\text { Female } 57 \% \\
\text { Race NR } \\
\mathrm{N}=46\end{array}$ & $\begin{array}{l}\text { Labs/Other } \\
\text { Conditions; } \\
\text { Neurological } \\
\text { Disorder }\end{array}$ \\
\hline
\end{tabular}

BD=Bipolar Disorder; DSM= Diagnostic and Statistical Manual of Mental Disorders; N=number; NR=not reported; ROB=risk of bias; rTMS= repetitive transcranial magnetic stimulation

\section{Interpreting the Findings for Psychosocial and Other Nondrug Treatments}

We were largely unable to draw conclusions about the effect of psychosocial interventions for BD. In contrast to previous reviews and metaanalyses, we separately examined studies by both intervention category and comparator type (inactive and active). ${ }^{263}$ We also considered the variation of interventions within categories and the variation across the clinical states of enrolled subjects. This limited our ability to draw conclusions from the available evidence.

The evidence base had several limitations. Similar to the drug evidence, a substantial number of studies were excluded due to an attrition rate greater than 50 percent. While this limited the evidence, the findings from these studies were of questionable validity.

Lack of transparency was also a significant limitation. Reporting of outcome time points, number of participants in each arm, and loss to follow-up was at times unclear. Studies inconsistently used scales to measure symptoms of depression, mania, or function. Notably, some studies chose to measure global function while others measured quality of life or social function.

We were unable to assess the impact of interventions on specific phases of BD. The majority of psychosocial interventions studies did not exclude individuals based on their current clinical state, thus investigated mixed samples of individuals in acute hypomanic/manic, mixed, depression episodes, or euthymia. Studies investigating the mixed samples did not examine whether the baseline clinical state affected intervention effect. Additionally, in several instances we could not abstract sufficient data to calculate estimates or verify conclusions presented by studies. Multiple studies provided only test statistics for outcomes without additional data (e.g., means at baseline and outcome time points, mean difference at outcome time points).

The available evidence on other nondrug interventions such as acupuncture or light therapy that may be used to treat BD did not meet our inclusion criteria. Several studies were eliminated because they had high rates of attrition, sample sizes below 10 participants per arm, or did not meet timing criteria (e.g., 3 months for treatment of acute depression). High quality studies, with sufficient sample sizes and appropriate followup periods, are needed to determine whether these interventions benefit individuals with BD. 
It is possible that psychosocial interventions provide benefits not expressly or consistently measured in the reviewed literature. Many of the psychosocial interventions included common components of disease education, discussion of triggers, and coping mechanisms. If these common components are active ingredients of a therapeutic effect for psychosocial interventions, then the lack of difference seen in head-to-head comparisons of psychosocial interventions are not surprising; for example the low-strength evidence we found for no differences between CBT versus active comparator in reducing bipolar symptoms. Moreover, some of the outcomes assessed in psychosocial treatment literature, such as rates of relapses into manic or depressive episodes, require long followup intervals to adequately measure change in rates of events that for some patients occur only once every 6-12 months. In other words, true treatment effects may be obscured in studies with followup shorter than 12 months. Finally, studies inconsistently reported other relevant outcomes, such as adherence to drug treatment, which can be improved through educational efforts that help patients accept their diagnoses and improve their coping skills. ${ }^{264}$ 


\section{Chapter 8. Discussion}

\section{Overview}

The evidence base for treatments for bipolar disorder (BD) is sparse and scattered. While a large number of studies were identified, they mapped across a considerable number of treatments and comparators, ultimately yielding few for each actual comparison.

We found no high or moderate strength of evidence for any treatment during any phase of bipolar illness (i.e., acute mania, acute depression, or maintenance). For treatment of acute mania, low-strength evidence was found for atypical antipsychotics compared to placebo for improvements in response and possible remission rates, and improvements in manic symptoms and clinical global impressions. (Table 48) There was also low-strength evidence for improved response and remission rates, as well as manic symptom improvement, for lithium versus placebo. However, most manic symptom improvements were of modest clinical significance, with values that were less than the minimally important difference (MID) but still large enough that a reasonable proportion of participants likely received a benefit. For maintenance phase treatment, only lithium achieved low-strength evidence for benefit for the long-term (1-2 years). No treatments with even low-strength evidence showed favorable outcomes for treatment of depression. Across treatment phases, the large majority of drug comparisons, including almost all comparisons using active comparators, had insufficient evidence from which to draw conclusions.

Table 48. Summary of low-strength* evidence findings by intervention class

\begin{tabular}{|c|c|c|c|}
\hline Category & Intervention & $\begin{array}{c}\text { \# Studies/ } \\
\text { Design } \\
\text { (n analyzed) } \\
\text { Timing }\end{array}$ & Findings \\
\hline \multirow[t]{3}{*}{$\begin{array}{l}\text { Antipsychotics } \\
\text { acute mania }\end{array}$} & $\begin{array}{l}\text { Asenapine vs. } \\
\text { placebo }\end{array}$ & $\begin{array}{l}3 \mathrm{RCT} \\
(\mathrm{n}=936) \\
3 \text { weeks }\end{array}$ & $\begin{array}{l}\text { Response/Remission: No difference } \\
\text { YMRS: Favors Asenapine, MD } 4.37 \text { (95\% Cl } 1.27 \text {, } \\
\text { 7.47; MID 6) } \\
\text { CGI-BP-S: Favors Asenapine, MD } 0.5(95 \% \mathrm{Cl} \\
0.29,0.71 ; \text { MID 1) } \\
\text { Withdrawal (AE, Lack of Efficacy, Overall): No } \\
\text { difference }\end{array}$ \\
\hline & $\begin{array}{l}\text { Cariprazine vs. } \\
\text { placebo }\end{array}$ & $\begin{array}{l}3 \mathrm{RCT} \\
(\mathrm{n}=1,047) \\
3 \text { weeks }\end{array}$ & $\begin{array}{l}\text { Response Rate: Favors Cariprazine, OR } 2.14 \text { (95\% } \\
\mathrm{CI} 1.08,4.23) \text {; NNT=5.6 } \\
\text { Remission Rate: Favors Cariprazine, OR1.95 (95\% } \\
\mathrm{CI} 1.45,2.63) \text {; NNT= } 7 \\
\text { YMRS: Favors Cariprazine, MD } 5.38 \text { (95\% CI 1.84, } \\
\text { 8.92; MID 6) } \\
\text { CGI-BP-S: Favors Cariprazine, MD } 0.54(95 \% \mathrm{Cl} \\
\text { 0.35, 0.73; MID 1) } \\
\text { Withdrawal (AE, Lack of Efficacy, Overall): No } \\
\text { difference }\end{array}$ \\
\hline & $\begin{array}{l}\text { Olanzapine vs. } \\
\text { placebo }\end{array}$ & $\begin{array}{l}5 \text { RCT } \\
(n=1199) \\
3 \text { weeks }\end{array}$ & $\begin{array}{l}\text { Response: Favors Olanzapine, OR } 1.99(95 \% \mathrm{Cl} \\
1.29,3.08) ; \text { NNT=6 } \\
\text { Remission: Favors Olanzapine, OR } 1.75(95 \% \mathrm{Cl} \\
\text { 1.19, 2.58); NNT=7.5 } \\
\text { YMRS: Favors Olanzapine, MD } 4.9(95 \% \mathrm{Cl} 2.34 \text {, } \\
\text { 7.45; MID 6) } \\
\text { Withdrawal (Lack of Efficacy, Overall): Favors } \\
\text { Olanzapine, MD } 0.42(95 \% \mathrm{Cl} 0.29,0.61)\end{array}$ \\
\hline
\end{tabular}




\begin{tabular}{|c|c|c|c|}
\hline Category & Intervention & $\begin{array}{l}\text { \# Studies/ } \\
\text { Design } \\
\text { (n analyzed) } \\
\text { Timing } \\
\end{array}$ & Findings \\
\hline & & $\begin{array}{l}3 \text { RCT } \\
(n=611) \\
3 \text { weeks }\end{array}$ & CGI-BP-S: No difference \\
\hline & \multirow{3}{*}{$\begin{array}{l}\text { Quetiapine vs. } \\
\text { placebo }\end{array}$} & $\begin{array}{l}4 \mathrm{RCT} \\
(\mathrm{n}=1,007) \\
3 \text { weeks }\end{array}$ & $\begin{array}{l}\text { Response: Favors Quetiapine, OR } 2.07(95 \% \mathrm{Cl} \\
\text { 1.39, 3.09); NNT=6.2 } \\
\text { Withdrawal (Lack of Efficacy): Favors Quetiapine, } \\
\text { MD } 0.38(95 \% \mathrm{Cl} 0.23,0.63)\end{array}$ \\
\hline & & $\begin{array}{l}5 \mathrm{RCT} \\
(\mathrm{n}=699 \text { forest plot, } \\
1439 \text { total) } \\
3 \text { weeks }\end{array}$ & $\begin{array}{l}\text { YMRS: Favors Quetiapine, MD } 4.92 \text { (95\% Cl 0.31, } \\
\text { 9.53; MID 6) }\end{array}$ \\
\hline & & $\begin{array}{l}5 \mathrm{RCT} \\
(\mathrm{n}=806 \text { forest plot, } \\
1439 \text { total) } \\
3 \text { weeks }\end{array}$ & $\begin{array}{l}\text { CGI-BP-S: Favors Quetiapine, MD } 0.54(95 \% \text { Cl } \\
0.35,0.74 ; \text { MID 1) }\end{array}$ \\
\hline & $\begin{array}{l}\text { Risperidone vs. } \\
\text { placebo }\end{array}$ & $\begin{array}{l}\text { RCT } \\
(\mathrm{n}=584) \\
3 \text { weeks }\end{array}$ & $\begin{array}{l}\text { Response, YMRS, and CGI: Favors Risperidone } \\
\text { (not pooled) }\end{array}$ \\
\hline & $\begin{array}{l}\text { Ziprasidone vs. } \\
\text { placebo }\end{array}$ & $\begin{array}{l}2 \mathrm{RCT} \\
(\mathrm{n}=402) \\
3 \text { weeks }\end{array}$ & $\begin{array}{l}\text { Response, YMRS, and CGI: Favors Ziprasidone } \\
\text { (not pooled) }\end{array}$ \\
\hline & \multirow{4}{*}{$\begin{array}{l}\text { Olanzapine vs. } \\
\text { Divalproex/ } \\
\text { Valproate }\end{array}$} & $\begin{array}{l}2 \text { RCTs } \\
(n=635) \\
3 \text { weeks }\end{array}$ & Response and Remission: No differenceS \\
\hline & & $\begin{array}{l}3 \text { RCTs } \\
(n=750) \\
3 \text { weeks }\end{array}$ & YMRS: No difference \\
\hline & & $\begin{array}{l}3 \text { RCTs } \\
(n=578) \\
3 \text { weeks }\end{array}$ & CGI: No difference \\
\hline & & $\begin{array}{l}\text { 4 RCTs } \\
(n=867) \\
3 \text { weeks }\end{array}$ & Withdrawals: No difference \\
\hline \multirow[t]{3}{*}{$\begin{array}{l}\text { Mood stabilizers } \\
\text { treatments for } \\
\text { acute mania }\end{array}$} & \multirow{3}{*}{$\begin{array}{l}\text { Lithium vs. } \\
\text { placebo }\end{array}$} & $\begin{array}{l}1 \text { RCT + } 1 \text { IPD } \\
(n=325) \\
3 \text { weeks }\end{array}$ & $\begin{array}{l}\text { Remission and Response: Favors Lithium (not } \\
\text { pooled) }\end{array}$ \\
\hline & & $\begin{array}{l}3 \text { RCTs } \\
(n=325) \\
3 \text { weeks }\end{array}$ & $\begin{array}{l}\text { YMRS: Favors Lithium, } \\
\text { MD } 5.81 \text { (95\% Cl 2.21, 9.4; MID 6) } \\
\text { Withdrawal (Overall): No difference }\end{array}$ \\
\hline & & $\begin{array}{l}1 \text { IPD } \\
(n=450) \\
3 \text { weeks }\end{array}$ & Withdrawal (Lack of Efficacy, AE): No difference \\
\hline \multirow[t]{3}{*}{$\begin{array}{l}\text { Other drug } \\
\text { treatments for } \\
\text { mania }\end{array}$} & $\begin{array}{l}\text { Paliperidone vs. } \\
\text { placebo }\end{array}$ & $\begin{array}{l}2 \text { RCT } \\
(n=763) \\
3 \text { weeks }\end{array}$ & $\begin{array}{l}\text { YMRS and Withdrawal (Lack of Efficacy): Favors } \\
\text { Paliperidone (possible dose response: No } \\
\text { difference at } 3 \text { and } 6 \mathrm{mg} \text {, benefit at } 12 \mathrm{mg} \text { or } \\
\text { median dosage of } 9 \mathrm{mg} \text { ) (not pooled) } \\
\text { Withdrawal (AE): No difference }\end{array}$ \\
\hline & $\begin{array}{l}\text { Topiramate vs. } \\
\text { placebo }\end{array}$ & $\begin{array}{l}1 \text { IPD } \\
(n=876) \\
3 \text { weeks }\end{array}$ & $\begin{array}{l}\text { YMRS and Withdrawal (Lack of Efficacy): No } \\
\text { difference } \\
\text { Withdrawals (Overall): Favors Placebo, } 37.2 \% \text { vs. } \\
26.8 \%, p=0.005 \\
\text { Withdrawals (AE): Favors Placebo, } 6.04 \% \text { vs. } \\
2.84 \%, p=0.049\end{array}$ \\
\hline & $\begin{array}{l}\text { Topiramate vs. } \\
\text { lithium }\end{array}$ & $\begin{array}{l}1 \text { IPD } \\
(n=453) \\
3 \text { weeks }\end{array}$ & $\begin{array}{l}\text { YMRS: Favors Lithium, MD } 6.14 \text { (95\% Cl 3.94, } \\
\text { 8.34; MID 6) }\end{array}$ \\
\hline
\end{tabular}




\begin{tabular}{|c|c|c|c|}
\hline Category & Intervention & $\begin{array}{c}\text { \# Studies/ } \\
\text { Design } \\
\text { (n analyzed) } \\
\text { Timing }\end{array}$ & Findings \\
\hline & & $\begin{array}{l}1 \text { IPD } \\
(n=453) \\
3 \text { weeks }\end{array}$ & Withdrawal (Overall, AE): No difference \\
\hline & & $\begin{array}{l}1 \text { IPD } \\
(n=453) \\
3 \text { weeks }\end{array}$ & $\begin{array}{l}\text { Withdrawal (AE): Favors Topiramate, } 2.65 \% \text { vs. } \\
7.49 \%, p=0.019\end{array}$ \\
\hline & $\begin{array}{l}\text { Allopurinol + } \\
\text { lithium vs. } \\
\text { placebo + lithium }\end{array}$ & $\begin{array}{l}4 \text { RCT } \\
(n=355) \\
4 \text { weeks }\end{array}$ & YMRS, CGI, Withdrawal (Overall): No difference \\
\hline $\begin{array}{l}\text { Single drug for } \\
\text { maintenance }\end{array}$ & $\begin{array}{l}\text { Lithium vs. } \\
\text { placebo }\end{array}$ & $\begin{array}{l}6 \text { RCT } \\
(n=1579) \\
1 \text { to } 2 \text { years }\end{array}$ & Time to overall relapse: Favors Lithium \\
\hline \multirow[t]{2}{*}{$\begin{array}{l}\text { Psychosocial } \\
\text { Interventions }\end{array}$} & $\begin{array}{l}\text { CBT vs. Active } \\
\text { Comparators }^{\star *}\end{array}$ & $\begin{array}{l}5 \text { RCTs } \\
(n=461) \\
6 \text { to } 12 \text { months }\end{array}$ & $\begin{array}{l}\text { Depression and Mania symptoms: No difference } \\
\text { between groups across range of time periods. }\end{array}$ \\
\hline & $\begin{array}{l}\text { Systematic or } \\
\text { Collaborative } \\
\text { Care vs. Inactive } \\
\text { Comparators } †\end{array}$ & $\begin{array}{l}2 \text { RCTs } \\
(n=599) \\
7 \text { to } 12 \text { months }\end{array}$ & $\begin{array}{l}\text { Relapse: No difference between groups across } \\
\text { different time periods. }\end{array}$ \\
\hline
\end{tabular}

*All findings are low-strength evidence based generally on moderate study limitations and imprecision; details available in results section and appendixes. ** Active comparators are comparators such as a different psychosocial therapy or peer support. †Inactive comparators are comparators such as usual care, no intervention.

$\mathrm{AE}=$ adverse events; $\mathrm{CBT}=$ cognitive behavioral therapy; $\mathrm{CGI}=$ Clinical global impression; $\mathrm{IPD}=\mathrm{Individual}$ patient data;

$\mathrm{MD}=$ mean difference; $\mathrm{NS}=$ not significant; $\mathrm{OR}=$ odds ratio; RCT=randomized controlled trial; YMRS=Young mania rating scale

Similarly, only a few studies of psychosocial interventions reached low-strength evidence, finding no differences between particular psychosocial treatment approaches versus active comparators (e.g., another psychotherapeutic approach) for a subset of outcomes. Most comparisons had insufficient evidence to address whether the therapy of interest improves outcomes compared to either inactive (usual care) or active (another therapeutic approach) controls. However, the studies' inclusion criteria and limitations (see section below on limitations) preclude definitive conclusions about the effects of psychosocial interventions.

We were unable to draw a conclusion for several Food and Drug Administration (FDA)approved drugs for BD. One FDA-approved atypical antipsychotic, aripiprazole, had a limited number of studies and high risk of bias contributing to study limitations for mania treatment evidence. We noted that while a random effect model largely showed no difference between groups in response rates, manic symptom improvement, or withdrawal rates, if a fixed effect model is used, symptom improvements were seen, but at just over half the MID. Fixed effect models only allow inferences for the specific participants in the specific studies, not generalization to the larger applicable population. One FDA-approved drug, chlorpromazine, was used as a comparator in only one study and otherwise not examined. A typical (first generation) antipsychotic, chlorpromazine was approved by the FDA in 1957. The lack of chlorpromazine in the included literature reflects the treatment preference for a different typical antipsychotic, haloperidol, because of the sedative and blood pressure effects of chlorpromazine. Lurasidone, olanzapine, and quetiapine have been approved for depression in BD, based on 6 to 8 week studies, but no studies were identified with at least 3 months followup in this review.

Table 49 provides a list of all comparisons in this review for which we were unable to draw conclusions. Notably, the insufficiency of the evidence does not indicate that the examined 
approaches do not have therapeutic benefits, but rather that the scientific evidence is insufficient to draw any conclusions about their therapeutic effects.

Table 49. Interventions/comparators with insufficient strength of evidence for all outcomes (unless otherwise noted)

\begin{tabular}{|c|c|c|}
\hline Category & Drug & Comparator \\
\hline \multirow{17}{*}{$\begin{array}{l}\text { Antipsychotics } \\
\text { for mania }\end{array}$} & Aripiprazole & Placebo \\
\hline & Aripiprazole & Haloperidol \\
\hline & Aripiprazole plus Mood Stabilizer & Mood Stabilizer alone (placebo) \\
\hline & Aripiprazole plus Mood Stabilizers & Haloperidol plus Mood Stabilizer \\
\hline & Asenapine & Olanzapine \\
\hline & Asenapine plus Mood Stabilizer & Mood Stabilizer alone (placebo) \\
\hline & $\begin{array}{l}\text { Olanzapine (for withdrawal for adverse events } \\
\text { only) }\end{array}$ & Placebo \\
\hline & Olanzapine & Haloperidol or Lithium or Risperidone \\
\hline & Olanzapine plus Mood Stabilizer & Mood Stabilizer alone (placebo) \\
\hline & Olanzapine plus Mood Stabilizers & Mood Stabilizer alone (no placebo) \\
\hline & Quetiapine & Haloperidol or Lithium \\
\hline & Quetiapine plus Mood Stabilizers & Mood Stabilizer alone (placebo) \\
\hline & Risperidone & Haloperidol or Lithium \\
\hline & Risperidone plus Mood Stabilizers & Mood Stabilizer alone (placebo) \\
\hline & Ziprasidone plus Mood Stabilizers & Mood Stabilizer alone (placebo) \\
\hline & Ziprasidone plus Mood Stabilizer & Chlorpromazine plus Mood Stabilizer \\
\hline & Haloperidol & Placebo \\
\hline \multirow{8}{*}{$\begin{array}{l}\text { Mood Stabilizers } \\
\text { for mania }\end{array}$} & Carbamazepine & Placebo \\
\hline & Divalproex/Valproate & Placebo \\
\hline & Valproate & No Placebo \\
\hline & Lithium (for CGI only) & Placebo \\
\hline & Carbamazepine & Lithium or Valproate \\
\hline & Carbamazepine & Valporate \\
\hline & Lamotrigine & Lithium \\
\hline & Lithium & Haloperidol or Divalproex \\
\hline \multirow[t]{14}{*}{$\begin{array}{l}\text { Other Drugs for } \\
\text { mania }\end{array}$} & $\begin{array}{l}\text { Paliperidone (for Remission, Response, CGI } \\
\text { Withdrawal (Overall)) }\end{array}$ & Placebo \\
\hline & $\begin{array}{l}\text { Allopurinol plus Lithium (for Response and } \\
\text { Remission) }\end{array}$ & Lithium alone (placebo) \\
\hline & Allopurinol plus Lithium & Dipyridamole plus Lithium \\
\hline & Celecoxib & Placebo \\
\hline & Dipyridamole plus Lithium & Lithium alone (placebo) \\
\hline & Donepezil plus Lithium & Lithium alone (placebo) \\
\hline & Endoxifen & Divalproex \\
\hline & Gabapentin plus Lithium & Lithium alone (placebo) \\
\hline & Oxcarbazepine & Divalproex \\
\hline & Paliperidone Extended Release & Olanzapine or Quetiapine \\
\hline & Paliperidone plus Mood Stabilizers & Mood Stabilizers alone (placebo) \\
\hline & Tamoxifen & Placebo \\
\hline & Topiramate plus Risperidone & Divalproex plus Risperidone \\
\hline & Topiramate and Mood Stabilizer & Mood Stabilizer alone (placebo) \\
\hline \multirow{6}{*}{$\begin{array}{l}\text { Drugs for } \\
\text { depression }\end{array}$} & Memantine plus Valproate & Valproate alone (placebo) \\
\hline & Lamotrigine plus Mood Stabilizers & Mood Stabilizers alone (placebo) \\
\hline & $\begin{array}{l}\text { Antidepressives (paraoxetine, bupropion, or } \\
\text { both) }\end{array}$ & Placebo \\
\hline & Sertraline & Lithium \\
\hline & Venlafaxine & Lithium \\
\hline & Lithium and OPT & OPT alone \\
\hline \multirow{3}{*}{$\begin{array}{l}\text { Drugs for } \\
\text { maintenance }\end{array}$} & Long-acting Injectable Aripiprazole & Placebo \\
\hline & Aripiprazole & Placebo \\
\hline & Aripiprazole plus Mood Stabilizer & Mood Stabilizer alone (placebo) \\
\hline
\end{tabular}




\begin{tabular}{|c|c|c|}
\hline Category & Drug & Comparator \\
\hline & Carbamazepine & Lithium \\
\hline & Divalproex & Placebo \\
\hline & Divalproex plus Lithium & Lithium alone (placebo) \\
\hline & Fluoxetine & Placebo \\
\hline & Fluoxetine & Lithium \\
\hline & Gabapentin plus Mood Stabilizers & Mood Stabilizers alone (placebo) \\
\hline & Lamotrigine & Placebo \\
\hline & Lamotrigine for pregnant women & Discontinue Mood Stabilizers \\
\hline & Lamotrigine & Lithium \\
\hline & Lithium & Placebo \\
\hline & Lithium & Divalproex/Valproate \\
\hline & Olanzapine & Placebo \\
\hline & Olanzapine & Divalproex \\
\hline & Olanzapine & Lithium \\
\hline & Olanzapine plus Mood Stabilizers & Mood Stabilizers alone (placebo) \\
\hline & Oxcarbazepine plus Lithium & Lithium alone (placebo) \\
\hline & Paliperidone & Placebo \\
\hline & Paliperidone & Olanzapine \\
\hline & Perphenazine plus Mood Stabilizers & Mood Stabilizers alone (placebo) \\
\hline & Quetiapine & Placebo \\
\hline & Quetiapine & Lithium \\
\hline & Quetiapine plus Mood Stabilizers & Mood Stabilizers alone (placebo) \\
\hline & Quetiapine and Personalize Treatment & Lithium and Personalized Treatment \\
\hline & Risperidone & Placebo \\
\hline & Risperidone & Olanzapine \\
\hline & $\begin{array}{l}\text { Risperidone Long Acting Injectable and } \\
\text { Treatment as Usual }\end{array}$ & Placebo and Treatment as Usual \\
\hline & Valproic Acid plus Aripiprazole & Lithium plus Aripiprazole \\
\hline & Venlafaxine & Lithium \\
\hline & Ziprasidone and Mood Stabilizers & Mood Stabilizers alone (placebo) \\
\hline \multirow{12}{*}{$\begin{array}{l}\text { Psychosocial } \\
\text { Interventions }\end{array}$} & Psychoeducation & Inactive* Comparators \\
\hline & Psychoeducation & Active $^{\star *}$ Comparators \\
\hline & CBT & Inactive Comparators \\
\hline & $\begin{array}{l}\text { CBT (for Relapse, Global Function, Other } \\
\text { Measures of Function) }\end{array}$ & Active Comparators \\
\hline & $\begin{array}{l}\text { Systematic or Collaborative Care (for } \\
\text { Depression, Mania, Global Function, Other } \\
\text { Measures of Function) }\end{array}$ & Inactive Comparators \\
\hline & Family or Partner Interventions & Inactive Comparators \\
\hline & Family or Partner Interventions & Active Comparators \\
\hline & IPSRT & Inactive Comparators \\
\hline & IPSRT & Active Comparators \\
\hline & Combination Interventions & Inactive Comparators \\
\hline & Combination Interventions & Active Comparators \\
\hline & Other Psychosocial Interventions & Inactive Comparators \\
\hline Somatic & Repetitive transcranial magnetic stimulation & Sham stimulation \\
\hline
\end{tabular}

*Inactive comparators include usual care or no intervention. **Active comparators include a different psychosocial therapy, peer support, or similar.

CBT=cognitive behavioral therapy; CGI=Clinical Global Impression; IPSRT= Interpersonal and Social Rhythm Therapy;

OPT=Optimal Personalized Treatment

Adverse events in drug studies were somewhat consistently reported for extrapyramidal symptoms, and clinically significant weight gain of greater than 7 percent, but otherwise variably 
reported. The harms findings from the included placebo-controlled studies were consistent with information currently reported by FDA labels. (Please see Appendix Q for drug label information on FDA box warnings and serious adverse events.) While most studies reported no differences between groups, we noted participants using antipsychotics, except quetiapine, reported more extrapyramidal symptoms compared to placebo, and those using olanzapine reported more clinically significant weight gain. For mood stabilizers, participants using carbamazepine reported more severe rash and adverse events compared to placebo. In head-to-head studies, we noted a general pattern of participants receiving atypical antipsychotics fewer extrapyramidal symptoms than participants receiving haloperidol. Unfortunately, psychosocial studies generally did not report attempting to collect harms or other unintentional consequences of receiving psychosocial treatments.

Although we had originally anticipated parsing study findings across several populations and subgroups of interest to address Key Question 4, the vast majority drug treatment studies enrolled participants with bipolar I disorder (BD-I). This held even for maintenance trials as many were extensions of trials with participants who had responded to treatment for an acute manic episode. Given the low to insufficient strength of evidence assessments arising from high study limitations and attrition for the main study research questions, any post-hoc analysis for subgroups would be by definition high risk of bias and not sufficient to draw conclusions. We were therefore unable to address how treatments may differ across different BD populations and subgroups. Likewise, we also did not locate any studies specifically testing interventions in BD patients to address drug treatment side effects for Key Question 3.

\section{Applicability}

Applicability of the review findings is challenging. The trials for drug treatments used restrictive exclusion criteria. Over three quarters of the studies for mania also excluded participants experiencing a first manic episode. Moreover, given the inclusion criteria, it is not clear if the current findings extend to populations with bipolar II disorder (BD II), current comorbid substance use, pregnant or nursing women with BD I, or older adults (i.e., age 65 and over). Conversely, the psychosocial trials often did not provide detailed information on the participants and the lack of population description limits the ability to infer from the results. Such a mixed population may mask patterns of effect. With the current information, we cannot determine if or to what extent, this contributed to the few findings of nonsignificance between groups.

Factoring in the issue of high attrition, trials with 20 to 50 percent attrition, such as were used in this review, at best provide an estimate of the effect of a treatment for participants who adhere to, tolerate, and, in some minimal sense, benefit from the treatment. However, at extremely high levels of attrition, even this interpretation is of limited value to clinicians. ${ }^{265}$ If over 50 percent of patients do not finish treatment, and thus were not followed-up to the end of the trial, then the chances of the trial results being applicable to a new patient would be less than half. Applicability drops even further when we recognize the original randomized sample excludes many subpopulations and co-occuring conditions which reduces how much the sample represents people encountered in regular clinical practice, Likewise, the maintenance trials are most applicable to people with BD-I who respond to initial treatment. 


\section{Findings in Relation to What Is Already Known}

The findings of this review are consistent with other systematic reviews of treatments for $\mathrm{BD}$, although, given the attention this review paid to the role of attrition, more restricted in positive findings. Compared to published Cochrane reviews, our findings were generally consistent, although somewhat more conservative. We also found benefit for olanzapine and risperidone compared to placebo for mania, and benefit for lithium compared to placebo for maintenance. ${ }^{266-268}$ Cochrane reviews have reported benefit for several additional antipsychotics compared to placebo for which we found insufficient evidence (aripiprazole, haloperidol as single drug and added to mood stabilizers, and olanzapine or risperidone plus mood stabilizers). ${ }^{266,269-272}$ However, authors of these reports consistently noted issues with attrition and medication adherence may have impacted their results. Insufficient evidence for psychosocial interventions was consistent across all reviews. ${ }^{263,273}$

\section{Limitations of the Comparative Effectiveness Review}

There were several limitations of the review. The search strategy relied on previous published reviews to identify relevant studies published prior to 1994. The original date was chosen to reflect the change to DMS-IV diagnostic criteria for BD and to focus review resources on abstracting relevant studies rather than searches for ground that has been otherwise well-tread. We believe we have identified the relevant literature, but the possibility of missing a publication, particularly on lithium, remains.

Several inclusion criteria may also have created limitations. We only included studies if the populations were exclusively diagnosed with $\mathrm{BD}$, or if the bipolar subpopulation results were reported separately. While still relevant for drug treatments, psychosocial treatments in particular that were specific to depression or mania and combined in analyses participants with bipolar and nonbipolar diagnoses might not have been included in this review.

Excluding all outcomes except for time-to-event outcomes from studies with greater than 50 percent attrition hindered our ability to address outcomes of interest that require longer followup in studies of smaller sample sizes. However, as is noted in the section below on limitations of the evidence base, the missing data problems created by high attrition is a counterweight to this limitation. A recent overview of reviews from the International Society for Bipolar Disorders Task Force on Suicide in Bipolar Disorder found that while lithium or anticonvulsants are suggestive for preventing suicide attempts and deaths, more research is needed to before the effects can be confirmed. ${ }^{7}$

Literature on harms was essentially based on identified RCTs. We required studies to be at least prospective cohort studies with comparator arms and clearly reported for BD populations. This led to a number of observational studies being excluded, including observational studies that looked at broad classes of drugs, or individual drugs across broad populations.

We also chose minimum study followup periods of 3 weeks for acute mania studies, 3 months for depression studies, and 6 months for maintenance studies. Many studies for depression treatment and other somatic treatments, such as ECT or light therapy, were excluded due to too short of study followup. Given the chronic nature of $\mathrm{BD}$, the clinical relevance of studies reporting the effects of treatments with shorter followup periods is questionable. For example, if a treatment response to depression is not sustained, does it matter if the initial response to one treatment was faster than another? Moreover, in order to provide evidence that a treatment reduces bipolar episode relapse rates, a study followup longer than 12 months is likely 
needed to capture frequency of episodes that may occur once or twice per year for some individuals with bipolar disorder.

\section{Limitations of the Evidence Base}

Even though we excluded studies with greater than 50 percent attrition (unless the outcome was time to relapse), one of the great challenges we confronted in conducting this systematic review was deciding how to interpret trial results in the face of often very high attrition rates. In the case of trials evaluating pharmaceutical treatments for acute mania, it was very common for anywhere from 10 to70 percent of randomized patients to not complete treatment for even 3 weeks trials. In principle, treatment discontinuation need not lead to trial discontinuation, i.e., dropping out of the study and subsequent missing data. A National Research Council (NRC) report on missing data in randomized control trials stressed the importance of continuing to collect data on patients for whom treatment is discontinued, be it due to lack of efficacy, adverse events, or other reasons. ${ }^{259}$ Unfortunately, while the majority of reports did not explicitly comment on whether treatment discontinuation implied trial dropout, we were generally left with this impression given the common reliance on last-observation carried forward (LOCF) techniques and usage of terms like 'discontinued' and 'percent completing trial'. This means that many, if not most, trials had dropout rates (with subsequent missing data) ranging from 10 to 70 percent. Moreover, trials did not provide details about when in the trial period participants' last observations were observed, other than generally after baseline. Given the frequency of measurements in these trials, dropout as early as the first week cannot be ruled out.

It is well known that missing outcome data can pose a serious threat to both the internal and external validity of a trial. ${ }^{265,274}$ Some of this risk can be mitigated with appropriate analytic techniques. The appropriateness of different analytic methods depends upon the assumptions one makes, and the justifiability of these assumptions in the relevant context, about the missing-ness mechanism (the reason the data are missing and the relationship between observed and unobserved data). Ultimately every approach will require untestable assumptions. However, the aforementioned-panel recommends that some analytic approaches, including LOCF, ought to be avoided as their validity depends upon categorically unreasonable assumptions. The LOCF method, while easy, requires an assumption that the health-status of participants who dropped out of the trial would not have changed had future observations been recorded. When this assumption is inappropriate, use of LOCF methods can bias effect estimates. Moreover, estimates of standard errors will understate the true uncertainty surrounding effect estimates due to the added uncertainty of having to impute data, and this increases as the number of periods the value is carried forward increases. This can potentially inflate the type-I error rate. ${ }^{24}$

Several authors have proposed guidelines for acceptable levels of attrition in RCTs. One guideline suggested that anything greater than 5 percent was cause for concern, and anything greater than 20 percent represented a serious threat to validity. ${ }^{275}$ Although somewhat arbitrary, this is not without theoretical and empirical support. One simulation study found that, while there was only limited or even no bias in estimates of odds-ratios with attrition rates as high as 60 percent if the mechanism leading to the missing outcome data was unrelated to the value of the missing data (referred to as missing at random, or MAR), estimates were 'seriously biased' with even low levels of loss to follow-up when the mechanism for missing data was related to the value of the data (referred to as missing not at random, or MNAR). ${ }^{276}$ Missing at random is a hard assumption to make with a BD population. 
On the other hand, it has been argued that, taken in isolation, the overall amount of attrition in a trial is a poor measure of the level of threat missing data poses to the validity of a trial's conclusions. ${ }^{277}$ This is because the risk of bias also depends upon the size of the observed treatment effect, the reasons for attrition, the degree that attrition rates and reasons vary across arms, and many other factors that might be specific to a trial and intervention under study. Ideally trial reports would include a discussion of the results of sensitivity analyses performed to assess how, under a range of reasonable assumptions, observed levels of missing data might have influenced the primary results. However, such robustness-analyses were almost universally not performed. We were thus presented with the difficult task of trying to interpret the results of trials with often large percentages of missing outcome data and little to no information on how much risk this level of missing-ness posed to the validity of the trial's primary outcome estimates, statistical inference, and even qualitative conclusions.

We acknowledge the extreme difficulty inherent in studying and treating patients with BD (see below for future research suggestions). Still, while it is reasonable to question the wisdom of the decision made in many of the trials to discontinue patients from the trial once they stop treatment (due to lack of efficacy or adverse events), this problem is not limited to patients with BD. ${ }^{23,265}$

As a form of compromise, we used what we considered to be an extremely lenient set of criteria for evaluating risk-of-bias from attrition. First, we excluded any outcomes for which over 50 percent of the data was missing. In the context of pharmaceutical treatment of acute mania, if a trial had less than 50 percent attrition at 3 weeks but greater than 50 percent attrition after this, the former outcomes were included and the latter outcomes were excluded. Any trial with over 50 percent attrition by the first outcome was excluded entirely, but we present the attrition rates in the appendix. For studies with attrition rates between 40 to 50 percent, we considered the withdrawal rates to be a valid, poolable outcome but treated other outcomes and harms as suffering from a high-risk of bias. We note that this criterion did not apply to time-to-event outcomes in trials where patients were discontinued after the event was observed, e.g., patients discontinued from follow-up after suffering a mood-episode in a maintenance trial studying timeto-mood relapse.

The other major challenge of the evidence base was variability and potential accuracy of the diagnostic assessment methods during recruitment processes. Most studies used the DSM criteria current for the study period, but the methods and likely reliability of the patient ascertainment varied. Often, detailed information on diagnostic assessment and statistics reporting interrater reliability were lacking. Given the debate whether the underlying mechanisms support the bipolar types as qualitatively and categorically different or lay on a continuum of the same psychopathological dimensions, it would be important to include more standard information about lifetime history of bipolar episodes assessment. There is also great difficulty in accurately diagnosing comorbid mental health conditions that were commonly treated as exclusion criteria, which also speaks for the need of standardized diagnostic assessments and reporting of interrater reliability statistics. Additional information and rigor in diagnostic assessment would generate a greater sense of confidence about who the study participants represent.

Other common limitations of health and medical research were also present. Industry funding for drug treatments was the most common source of funding. Publication bias for antipsychotics, antidepressants, and psychosocial interventions for depressive disorders has been documented. ${ }^{278-281}$ Harms, particularly for drug trials, were variably and inconsistently reported 
in formats difficult to aggregate. Usual care was not well-described. Publications often incompletely reported study design and conduct.

\section{Future Research}

Since evidence-based medicine relies on three realms--evidence, clinical experience, and patient experience--insufficient evidence means decisions must be informed by the latter two realms. This is an unsatisfying position for both clinicians and patients. Additional research for pharmacological, psychosocial, and somatic treatment of various phases of bipolar disorders, especially maintenance and depression, is needed to provide stronger scientific evidence for clinical decisions in these instances. Since only low-strength evidence was reached for benefit or no difference between groups for any treatment, drug or psychosocial, essentially all Key Questions would benefit from further research.

Acknowledging the difficulty and unavoidable issue of withdrawal in BD treatment research, there are a few possible actions to take: (1) Examine clinical and demographic characteristics that may differentiate participants who withdraw from participants who complete, and incorporate these findings in caveats about potential conclusions of treatment effects. Increased awareness of the clinical and demographic predictors of withdrawal are likely to lead to new studies that can attempt to better address treatment for these specific subsets of population. (2) In combination with examining predictors of withdrawal, it is imperative to better assess reasons for withdrawal of consent and more systematically report reasons outside of side effects and lack of efficacy. Currently, often the reasons for withdrawal of consent are not provided, or are unsatisfyingly vague. (3) If high attrition rates exist in a study, performing sensitivity analyses to determine how different assumptions about missing data would affect the effect size and corresponding confidence intervals would be important prior to drawing conclusions based on the existing data. For example, if minor adjustments in the assumptions about the missing data (e.g., adjustments in symptom severity of potentially missing data) would eliminate the treatment effects in a particular study, this should lead researchers to be highly skeptical of such findings. (4) Assuming some indications that attrition was random, certain statistical techniques are more adapt at modeling missing data and not unduly influencing the results, such as average score/observation method or use of multilevel linear mixed modeling.

Future studies of BD treatments will require innovative ways to increase study completion rates (e.g., use of technology for followup assessments and study reminders; "smart” bottles, mobile apps, and pills for assessing study drug adherence; multiple secondary contacts for participants and all-inclusive contact information from cell phones, email, to social media; flexible scheduling outside of business hours, availability at the last minute notice). For example, more longitudinal data analysis techniques for intermittent follow-up would help, but that in turn generates the need for more effort to create data repositories that allow individual patient-level data pooling of these longitudinal studies. This also requires greater funding for research with longer study followup duration.

Future research also needs to attend to subpopulation analyses. It is clinically useful to know what treatments are more effective for patients with early (prior to age 18) versus later age of BD illness onset, older adult patients versus younger adult patients, patients with BD-I versus BD-II or bipolar disorder not otherwise specified (BD NOS), patients with comorbid substance use disorders versus without this comorbidity, or patients with specific demographic characteristics. The lack of evidence for specific subpopulations of patients with BD is a direct result of prevailing inclusion and exclusion criteria. For example, the majority of BD treatment studies 
have focused on individuals with BD-I diagnoses. While this practice is understandable for studies focusing on the mania treatment effect, it is less clear in cases of maintenance or depression treatment.

Future research should also endeavor to enroll people with different initial episodes and maintenance stages to fully understand the spectrum of responses. Attention should be given to addressing all states of the illness throughout the treatment stream. Different clinical states could use more attention. For example, is maintenance after acute mania versus a depression episode the same? Does maintenance after an acute episode differ from patients "off the street"? We need to understand the nature of the interventions within the context of clinical practice (cotreatments).

Psychosocial therapies also need to address whether people with BD can benefit from a generalized groups without manualized treatment, or if the treatments need to be specifically designed for BD. For certain psychosocial therapeutic treatments, particular bipolar states may not be as relevant. But where targets are based on theorized mechanisms that are likely to affect manic or depressive symptoms, the populations should match the mechanisms so the study can directly address the question.

Psychosocial studies were more likely than drug studies to have inclusive inclusion criteria, but still outcome results were often not reported separately by BD subtype. Failure to assess subjects based on the current clinical state (i.e., including individuals who are currently euthymic, in acute mania/hypomania, in a mixed state, or in acute depression) may have washed out any effects that interventions may have had for a subset of the sample (e.g., any improvements in depression symptoms for individuals in acute depression at the baseline).

With the possible exception of treatment of acute depressive episodes, most psychosocial interventions for people with BD are designed to be used in concert with other - generally pharmacologic - treatments, and not stand on their own as complete treatments of the syndrome. So perhaps it is unrealistic to look too closely at "effects on symptoms" of psychosocial and behavioral interventions in isolation. Beyond simply augmenting medication effects, behavioral interventions can enhance adherence to treatment, reduce family friction, and promote hopefulness in patients and their families and friends. Consistent collection and reporting of other relevant outcomes, such as adherence to drug treatment, which can be improved through educational efforts that help patients accept their diagnoses and improve their coping skills ${ }^{258}$ would be beneficial.

Consistent minimum outcome datasets for BD research (and report inter-rater reliability of measures used in the study) would help, including harms or unintended consequences for psychosocial interventions. Consistent minimum methodological rigor is also required at the journal level.

\section{Conclusions}

No high or moderate-strength evidence was found for any intervention to effectively treat any phase of any type of BD compared to placebo or an active comparator. Low-strength evidence showed improved mania symptoms for all FDA-approved antipsychotics, except aripiprazole, when compared to placebo for adults with BD-I. Participants using antipsychotics, except quetiapine, reported experiencing more extrapyramidal symptoms compared to placebo, and those using olanzapine reported experiencing more clinically significant weight gain. Lowstrength evidence also showed benefit from lithium in the short-term for acute mania and longer time to relapse in the long-term versus placebo in adults with BD-I. Evidence was insufficient for 
most nondrug interventions. Low-strength evidence showed no effect for CBT on bipolar symptoms compared with active comparators, or systematic/collaborative care on relapse compared with inactive comparators. We were unable to address questions on subpopulations or treatments to reduce the metabolic change side effects of first line drug treatments. Future studies of $\mathrm{BD}$ treatments will require innovative ways to increase study completion rates. 


\section{References}

1. American Psychiatric Association. Diagnostic and Statistical Manual of Mental Disorders. 5th ed. Arlington, VA: American Psychiatric Publishing; 2013.

2. Kotwicki R, Harvey PD. Systematic study of structured diagnostic prodecures in outpatient psychiatric rehabilitation: a three-year, threecohort study of the stability of psychiatric diagnoses. Innov Clin Neurosci. 2013 MayJun;10(5-6):14-9. PMID: 23882436.

3. Ferrari A, Baxter A, Whiteford H. A systematic review of the global distribution and availability of prevalence data for bipolar disorder. J Affect Disord. 2011;134(1-3):113.

4. Gum A, King-Kallimanis B, Kohn R. Prevalence of mood, anxiety, and substanceabuse disorders for older Americans in the national comorbidity survey-replication. Am J Geriatr Psychiatry. 2009;17:769-81.

5. Samame C, Martino DJ, Strejilevich SA. Social cognition in euthymic bipolar disorder: systematic review and metaanalytic approach. Acta Psychiatr Scand. 2012;125(4):266-80.

6. Sole B, Martinez-Aran A, Torrent C, et al. Are bipolar II patients cognitively impaired? A systematic review. Psychol Med. 2011;41(9):1791-803.

7. Schaffer A, Isometsa ET, Tondo L, et al. Epidemiology, neurobiology and pharmacological interventions related to suicide deaths and suicide attempts in bipolar disorder: Part I of a report of the International Society for Bipolar Disorders Task Force on Suicide in Bipolar Disorder. Aust N Z J Psychiatry.49(9):785-802. PMID: 26185269.

8. Merikangas K, Akiskal HS, Angst J, et al. Lifetime and 12-month prevalence of bipolar spectrum disorder in the National Comorbidity Survey replication. Arch Gen Psychiatry. 2007 May;64(5):543-52. PMID: 17485606.

9. Brady KT, Sonne S. The relationship between substance abuse and bipolar disorder. J Clin Psychiatry. 1995;56(Suppl 3):19-24. PMID: 7883738
10. Moher D, Liberati A, Tetzlaff J, et al. Preferred reporting items for systematic reviews and meta-analyses: the PRISMA statement. J Clin Epidemiol. 2009 Oct;62(10):1006-12. doi: 10.1016/j.jclinepi.2009.06.005. PMID: 19631508.

11. Higgins JPT, Altman D, Sterne J. Chapter 8: Assessing risk of bias in included studies. In: Higgins JPT, Green S, eds. Cochrane Handbook for Systematic Reviews of Interventions: Version 5.1.0. The Cochrane Collaboration; 2011.

12. Viswanath B, Jose SP, Squassina A, et al. Cellular models to study bipolar disorder: A systematic review. J Affect Disord. 201511 Jun;184:36-50. doi: http://dx.doi.org/10.1016/j.jad.2015.05.037. PMID: 2015111614.

13. Langan D, Higgins JP, Simmonds M. Comparative performance of heterogeneity variance estimators in meta-analysis: a review of simulation studies. Res Synth Methods. 2016 Apr 06doi: 10.1002/jrsm.1198. PMID: 27060925.

14. Sidik K, Jonkman JN. A comparison of heterogeneity variance estimators in combining results of studies. Stat Med. 2007 Apr 30;26(9):1964-81. doi: 10.1002/sim.2688. PMID: 16955539.

15. IntHout J, Ioannidis JP, Borm GF. The Hartung-Knapp-Sidik-Jonkman method for random effects meta-analysis is straightforward and considerably outperforms the standard DerSimonian-Laird method. BMC Med Res Methodol. 2014 Feb 18;14:25. doi: 10.1186/1471-2288-14-25. PMID: 24548571.

16. Rover C, Knapp G, Friede T. HartungKnapp-Sidik-Jonkman approach and its modification for random-effects metaanalysis with few studies. BMC Med Res Methodol. 2015 Nov 14;15:99. doi: 10.1186/s12874-015-0091-1. PMID: 26573817.

17. Gonnermann A, Framke T, Grosshennig A, et al. No solution yet for combining two independent studies in the presence of heterogeneity. Stat Med. $2015 \mathrm{Jul}$ 20;34(16):2476-80. doi: 10.1002/sim.6473. PMID: 26040434. 
18. Viechtbauer W. Conducting meta-analyses in $\mathrm{R}$ with the metafor package. J Stat Softw . 2010;36(3):1-48.

19. R Core Team. R: A language and environment for statistical computing. Vienna, Austria: R Foundation for Statistical Computing; 2016.

20. Fu R, Gartlehner G, Grant M, et al. Conducting quantitative synthesis when comparing medical interventions: AHRQ and the Effective Health Care Program. J Clin Epidemiol. 2011 Nov;64(11):1187-97. PMID: 21477993.

21. Berkman ND, Lohr K, Ansari M, et al. Grading the strength of a body of evidence when assessing health care interventions for the effective health care program of the Agency for Healthcare Research and Quality: An update. . Rockville, MD: Agency for Healthcare Research and Quality; 2013. (vol. AHRQ Publication No. 13(14)-EHC 130$\mathrm{EF}$ ).

22. Atkins D, Chang S, Gartlehner G, et al. Assessing the applicability of studies when comparing medical interventions Agency for Healthcare Research and Quality. January 2011.

23. Leon AC, Mallinckrodt CH, Chuang-Stein C, et al. Attrition in Randomized Controlled Clinical Trials: Methodological Issues in Psychopharmacology. Biol Psychiatry. 2006 6/1/;59(11):1001-5. doi: http://dx.doi.org/10.1016/j.biopsych.2005.10. $\underline{020}$.

24. Little RJ, D'Agostino R, Cohen ML, et al. The Prevention and Treatment of Missing Data in Clinical Trials. N Engl J Med. 2012;367(14):1355-60. doi: doi:10.1056/NEJMsr1203730. PMID: 23034025.

25. McIntyre RS, Cohen M, Zhao J, et al. Asenapine in the treatment of acute mania in bipolar I disorder: a randomized, doubleblind, placebo-controlled trial. J Affect Disord. 2010 Apr;122(1-2):27-38. doi: http://dx.doi.org/10.1016/j.jad.2009.12.028. PMID: 20096936.
26. McIntyre RS, Cohen M, Zhao J, et al. A 3week, randomized, placebo-controlled trial of asenapine in the treatment of acute mania in bipolar mania and mixed states.[Erratum appears in Bipolar Disord. 2010

May;12(3):350]. Bipolar Disord. 2009

Nov;11(7):673-86. doi: http://dx.doi.org/10.1111/j.13995618.2009.00748.x. PMID: 19839993.

27. Landbloom RL, Mackle M, Wu X, et al. Asenapine: Efficacy and safety of 5 and $10 \mathrm{mg}$ bid in a 3-week, randomized, doubleblind, placebo-controlled trial in adults with a manic or mixed episode associated with bipolar I disorder. J Affect Disord. 2016 Jan 15;190:103-10. doi: http://dx.doi.org/10.1016/j.jad.2015.06.059. PMID: 26496015.

28. Calabrese JR, Keck PE, Jr., Starace A, et al. Efficacy and safety of low- and high-dose cariprazine in acute and mixed mania associated with bipolar I disorder: a doubleblind, placebo-controlled study. J Clin Psychiatry. 2015 Mar;76(3):284-92. doi: http://dx.doi.org/10.4088/JCP.14m09081. PMID: 25562205.

29. Durgam S, Starace A, Li D, et al. The efficacy and tolerability of cariprazine in acute mania associated with bipolar I disorder: A phase II trial. Bipolar Disord. 2015 Feb;17(1):63-75. doi: http://dx.doi.org/10.1111/bdi.12238. PMID: 25056368 (pubmed) 2014-30788001(Psychinfo).

30. Sachs GS, Greenberg WM, Starace A, et al. Cariprazine in the treatment of acute mania in bipolar I disorder: a double-blind, placebocontrolled, phase III trial. J Affect Disord. 2015 Mar 15;174:296-302. doi: http://dx.doi.org/10.1016/j.jad.2014.11.018. PMID: 25532076.

31. Katagiri H, Takita Y, Tohen M, et al. Efficacy and safety of olanzapine in the treatment of Japanese patients with bipolar I disorder in a current manic or mixed episode: a randomized, double-blind, placebo- and haloperidol-controlled study. J Affect Disord. 2012 Feb;136(3):476-84. doi: http://dx.doi.org/10.1016/j.jad.2011.10.045. PMID: 22134043. 
32. McIntyre RS. Aripiprazole for the maintenance treatment of bipolar I disorder: A review. Clin Ther. 2010;32 Suppl 1:S32-8. doi:

http://dx.doi.org/10.1016/j.clinthera.2010.01. 022. PMID: 20152551.

33. Tohen M, Vieta E, Goodwin GM, et al. Olanzapine versus divalproex versus placebo in the treatment of mild to moderate mania: a randomized, 12-week, double-blind study. J Clin Psychiatry. 2008 Nov;69(11):1776-89. PMID: 19014751.

34. Tohen M, Jacobs TG, Grundy SL, et al. Efficacy of olanzapine in acute bipolar mania: a double-blind, placebo-controlled study. The Olanzipine HGGW Study Group.[Erratum appears in Arch Gen Psychiatry 2002 Jan;59(1):91]. Arch Gen Psychiatry. 2000 Sep;57(9):841-9. PMID: 10986547.

35. Vieta E, Nuamah IF, Lim P, et al. A randomized, placebo- and active-controlled study of paliperidone extended release for the treatment of acute manic and mixed episodes of bipolar I disorder. Bipolar Disord. 2010 May;12(3):230-43. doi: http://dx.doi.org/10.1111/j.13995618.2010.00815.x. PMID: 20565430.

36. Bowden CL, Grunze H, Mullen J, et al. A randomized, double-blind, placebo-controlled efficacy and safety study of quetiapine or lithium as monotherapy for mania in bipolar disorder. J Clin Psychiatry. 2005 Jan;66(1):111-21. PMID: 15669897.

37. McIntyre RS, Brecher M, Paulsson B, et al. Quetiapine or haloperidol as monotherapy for bipolar mania--a 12-week, double-blind, randomised, parallel-group, placebocontrolled trial. Eur Neuropsychopharmacol. 2005 Oct;15(5):573-85. PMID: 16139175.

38. Cutler AJ, Datto C, Nordenhem A, et al. Extended-release quetiapine as monotherapy for the treatment of adults with acute mania: a randomized, double-blind, 3-week trial. Clin Ther. 2011 Nov;33(11):1643-58. doi: http://dx.doi.org/10.1016/j.clinthera.2011.10. 002. PMID: 22054797.
39. McElroy SL, Martens BE, Winstanley EL, et al. Placebo-controlled study of quetiapine monotherapy in ambulatory bipolar spectrum disorder with moderate-to-severe hypomania or mild mania. J Affect Disord. 2010 Jul;124(1-2):157-63. doi: http://dx.doi.org/10.1016/j.jad.2009.11.014. PMID: 19963274.

40. Khanna S, Vieta E, Lyons B, et al. Risperidone in the treatment of acute mania. Br J Psychiatry. 2005 2005;187(3):229-34. doi: http://dx.doi.org/10.1192/bjp.187.3.229. PMID: 16135859.

41. Smulevich AB, Khanna S, Eerdekens M, et al. Acute and continuation risperidone monotherapy in bipolar mania: a 3-week placebo-controlled trial followed by a 9-week double-blind trial of risperidone and haloperidol. Eur Neuropsychopharmacol. 2005 Jan;15(1):75-84. PMID: 15572276.

42. Potkin SG, Keck PE, Jr., Segal S, et al. Ziprasidone in acute bipolar mania: a 21-day randomized, double-blind, placebo-controlled replication trial. J Clin Psychopharmacol. 2005 Aug;25(4):301-10. PMID: 16012271.

43. Keck PE, Jr., Versiani M, Potkin S, et al. Ziprasidone in the treatment of acute bipolar mania: a three-week, placebo-controlled, double-blind, randomized trial. Am J Psychiatry. 2003 Apr;160(4):741-8. PMID: 12668364.

44. Tohen M, Baker RW, Altshuler LL, et al. Olanzapine versus divalproex in the treatment of acute mania.[Erratum appears in Am J Psychiatry. 2005 Feb;7(1):102]. Am J Psychiatry. 2002 Jun;159(6):1011-7. PMID: 12042191.

45. Zajecka JM, Weisler R, Sachs G, et al. A comparison of the efficacy, safety, and tolerability of divalproex sodium and olanzapine in the treatment of bipolar disorder. J Clin Psychiatry. 2002 Dec;63(12):1148-55. PMID: 12523875.

46. Xu L, Lu Y, Yang Y, et al. Olanzapinevalproate combination versus olanzapine or valproate monotherapy in the treatment of bipolar imania: A randomized controlled study in a chinese population group. Neuropsychiatr Dis Treat. 201525 May;11:1265-71. doi: http://dx.doi.org/10.2147/NDT.S81146. PMID: 26060401 (pubmed) 2015078440 (embase). 
47. Vieta E, Bourin M, Sanchez R, et al. Effectiveness of aripiprazole v. haloperidol in acute bipolar mania: double-blind, randomised, comparative 12-week trial. $\mathrm{Br} \mathrm{J}$ Psychiatry. 2005 Sep;187:235-42. PMID: 16135860.

48. Young AH, Oren DA, Lowy A, et al. Aripiprazole monotherapy in acute mania: 12-week randomised placebo- and haloperidol-controlled study. Br J Psychiatry. 2009 Jan;194(1):40-8. doi: http://dx.doi.org/10.1192/bjp.bp.108.049965. PMID: 19118324.

49. Kanba S, Kawasaki H, Ishigooka J, et al. A placebo-controlled, double-blind study of the efficacy and safety of aripiprazole for the treatment of acute manic or mixed episodes in Asian patients with bipolar I disorder (the AMAZE study). World J Biol Psychiatry. 2014 2014;15(2):113-21. doi: http://dx.doi.org/10.3109/15622975.2012.669 047. PMID: 22540407.

50. Sachs G, Sanchez R, Marcus R, et al. Aripiprazole in the treatment of acute manic or mixed episodes in patients with bipolar I disorder: a 3-week placebo-controlled study. J Psychopharmacol. 2006 Jul;20(4):536-46. PMID: 16401666.

51. Jeong HG, Lee MS, Ko YH, et al. Combination treatment with aripiprazole and valproic acid for acute mania: an 8-week, single-blind, randomized controlled trial. Clin Neuropharmacol . 2012 MayJun;35(3):97-102. doi: http://dx.doi.org/10.1097/WNF.0b013e31825 60401. PMID: 22592508.

52. Vieta E, T'Joen C, McQuade RD, et al. Efficacy of adjunctive aripiprazole to either valproate or lithium in bipolar mania patients partially nonresponsive to valproate/lithium monotherapy: a placebo-controlled study. Am J Psychiatry. 2008 Oct;165(10):1316-25. doi:

http://dx.doi.org/10.1176/appi.ajp.2008.0710 1560. PMID: 18381903.

53. Suppes T, Eudicone J, McQuade R, et al. Efficacy and safety of aripiprazole in subpopulations with acute manic or mixed episodes of bipolar I disorder. J Affect Disord. 2008 Apr;107(1-3):145-54. PMID: 17904226.
54. Sachs GS, Gaulin BD, Gutierrez-Esteinou R, et al. Antimanic response to aripiprazole in bipolar I disorder patients is independent of the agitation level at baseline. J Clin Psychiatry. 2007 Sep;68(9):1377-83. PMID: 17915976.

55. El Mallakh RS, Vieta E, Rollin L, et al. A comparison of two fixed doses of aripiprazole with placebo in acutely relapsed, hospitalized patients with bipolar disorder I (manic or mixed) in subpopulations (CN138007). Eur Neuropsychopharmacol. 2010 Nov;20(11):776-83. doi: http://dx.doi.org/10.1016/j.euroneuro.2010.0 7.003. PMID: 20728318.

56. Keck PE, Jr., Calabrese JR, McQuade RD, et al. A randomized, double-blind, placebocontrolled 26-week trial of aripiprazole in recently manic patients with bipolar I disorder. J Clin Psychiatry. 2006 Apr;67(4):626-37. PMID: 16669728.

57. Keck PE, Jr., Marcus R, Tourkodimitris S, et al. A placebo-controlled, double-blind study of the efficacy and safety of aripiprazole in patients with acute bipolar mania. Am J Psychiatry. 2003 Sep;160(9):1651-8. PMID: 12944341.

58. Keck PE, Orsulak PJ, Cutler AJ, et al. Aripiprazole monotherapy in the treatment of acute bipolar I mania: a randomized, doubleblind, placebo- and lithium-controlled study. J Affect Disord. 2009 Jan;112(1-3):36-49. doi: http://dx.doi.org/10.1016/j.jad.2008.05.014. PMID: 18835043.

59. Szegedi A, Calabrese JR, Stet L, et al. Asenapine as adjunctive treatment for acute mania associated with bipolar disorder: results of a 12-week core study and 40-week extension. J Clin Psychopharmacol. 2012 Feb;32(1):46-55. doi: http://dx.doi.org/10.1097/JCP.0b013e31823f 872f. PMID: 22198448.

60. Berk M, Ichim L, Brook S. Olanzapine compared to lithium in mania: a double-blind randomized controlled trial. Int Clin Psychopharmacol. 1999 Nov;14(6):339-43. PMID: 10565800.

61. Niufan G, Tohen M, Qiuqing A, et al. Olanzapine versus lithium in the acute treatment of bipolar mania: a double-blind, randomized, controlled trial. J Affect Disord. 2008 Jan;105(1-3):101-8. PMID: 17531327. 
62. Perlis RH, Baker RW, Zarate CA, Jr., et al. Olanzapine versus risperidone in the treatment of manic or mixed States in bipolar I disorder: a randomized, double-blind trial. J Clin Psychiatry. 2006 Nov;67(11):1747-53. PMID: 17196055.

63. Revicki DA, Paramore LC, Sommerville $\mathrm{KW}$, et al. Divalproex sodium versus olanzapine in the treatment of acute mania in bipolar disorder: health-related quality of life and medical cost outcomes. J Clin Psychiatry. 2003 Mar;64(3):288-94. PMID: 12716270.

64. Shafti SS. Olanzapine vs. lithium in management of acute mania. J Affect Disord. 2010 May;122(3):273-6. doi: http://dx.doi.org/10.1016/j.jad.2009.08.013. PMID: 19740546.

65. Shi L, Namjoshi MA, Zhang F, et al. Olanzapine versus haloperidol in the treatment of acute mania: clinical outcomes, health-related quality of life and work status. Int Clin Psychopharmacol. 2002 Sep;17(5):227-37. PMID: 12177585.

66. Tohen M, Chengappa KN, Suppes T, et al. Efficacy of olanzapine in combination with valproate or lithium in the treatment of mania in patients partially nonresponsive to valproate or lithium monotherapy. Arch Gen Psychiatry. 2002 Jan;59(1):62-9. PMID: 11779284.

67. Tohen M, Goldberg JF, Gonzalez-Pinto Arrillaga AM, et al. A 12-week, double-blind comparison of olanzapine vs haloperidol in the treatment of acute mania. Arch Gen Psychiatry. 2003 Dec;60(12):1218-26. PMID: 14662554.

68. Conus P, Berk M, Cotton SM, et al. Olanzapine or chlorpromazine plus lithium in first episode psychotic mania: An 8-week randomised controlled trial. Eur Psychiatry. 2015 Nov;30(8):975-82. doi: http://dx.doi.org/10.1016/j.eurpsy.2015.09.00 9. PMID: 26485297.
69. Houston JP, Tohen M, Degenhardt EK, et al. Olanzapine-divalproex combination versus divalproex monotherapy in the treatment of bipolar mixed episodes: a double-blind, placebo-controlled study.[Erratum appears in J Clin Psychiatry. 2011 Aug;72(8):1157], [Erratum appears in J Clin Psychiatry. 2010 Jan;71(1):93]. J Clin Psychiatry. 2009 Nov;70(11):1540-7. doi: http://dx.doi.org/10.4088/JCP.08m04895yel. PMID: 19778495.

70. Tohen M, Bowden CL, Smulevich AB, et al. Olanzapine plus carbamazepine v. carbamazepine alone in treating manic episodes. Br J Psychiatry. 2008 Feb;192(2):135-43. doi: http://dx.doi.org/10.1192/bjp.bp.107.041301. PMID: 18245032.

71. Namjoshi MA, Risser R, Shi L, et al. Quality of life assessment in patients with bipolar disorder treated with olanzapine added to lithium or valproic acid. J Affect Disord. 2004;81(3):223-9. PMID: 15337326.

72. Baker RW, Brown E, Akiskal HS, et al. Efficacy of olanzapine combined with valproate or lithium in the treatment of dysphoric mania. Br J Psychiatry. 2004 Dec;185:472-8. PMID: 15572737.

73. Moreno RA, Hanna MM, Tavares SM, et al. A double-blind comparison of the effect of the antipsychotics haloperidol and olanzapine on sleep in mania. Braz J Med Biol Res. 2007 Mar;40(3):357-66. PMID: 17334533.

74. Tohen M, Sutton VK, Calabrese JR, et al. Maintenance of response following stabilization of mixed index episodes with olanzapine monotherapy in a randomized, double-blind, placebo-controlled study of bipolar 1 disorder. J Affect Disord. 2009 Jul;116(1-2):43-50. doi: http://dx.doi.org/10.1016/j.jad.2008.11.003. PMID: 19054570.

75. Vieta E, Calabrese JR, Hennen J, et al. Comparison of rapid-cycling and non-rapidcycling bipolar I manic patients during treatment with olanzapine: analysis of pooled data. J Clin Psychiatry. 2004 Oct;65(10):1420-8. PMID: 15491248.

76. Baker RW, Tohen M, Fawcett J, et al. Acute dysphoric mania: treatment response to olanzapine versus placebo. J Clin Psychopharmacol. 2003 Apr;23(2):132-7. PMID: 12640214. 
77. Baker RW, Milton DR, Stauffer VL, et al. Placebo-controlled trials do not find association of olanzapine with exacerbation of bipolar mania. J Affect Disord. 2003 Jan;73(1-2):147-53. PMID: 12507747.

78. Baldessarini RJ, Hennen J, Wilson M, et al. Olanzapine versus placebo in acute mania: treatment responses in subgroups. J Clin Psychopharmacol. 2003 Aug;23(4):370-6. PMID: 12920413.

79. Chengappa KN, Baker RW, Shao L, et al. Rates of response, euthymia and remission in two placebo-controlled olanzapine trials for bipolar mania. Bipolar Disord. 2003 Feb;5(1):1-5. PMID: 12656931.

80. Tohen M, Sanger TM, McElroy SL, et al. Olanzapine versus placebo in the treatment of acute mania. The Am J Psychiatry. 1999 1999;156(5):702-9. PMID: 10327902.

81. Ketter TA, Houston JP, Adams DH, et al. Differential efficacy of olanzapine and lithium in preventing manic or mixed recurrence in patients with bipolar I disorder based on number of previous manic or mixed episodes. J Clin Psychiatry. 2006 Jan;67(1):95-101. PMID: 16426094.

82. Tohen M, Ketter TA, Zarate CA, et al. Olanzapine versus divalproex sodium for the treatment of acute mania and maintenance of remission: a 47-week study. Am J Psychiatry. 2003 Jul;160(7):1263-71. PMID: 12832240.

83. Suppes T, Brown E, Schuh LM, et al. Rapid versus non-rapid cycling as a predictor of response to olanzapine and divalproex sodium for bipolar mania and maintenance of remission: post hoc analyses of 47-week data. J Affect Disord. 2005 Dec;89(1-3):69-77. PMID: 16253344.

84. Novick D, Reed C, Haro JM, et al. Comparison of olanzapine and risperidone in the EMBLEM Study: translation of randomized controlled trial findings into clinical practice. Int Clin Psychopharmacol. 2010 Sep;25(5):257-63. doi: http://dx.doi.org/10.1097/YIC.0b013e32833b 8fe4. PMID: 20531011.

85. Sanger TM, Tohen M, Vieta E, et al. Olanzapine in the acute treatment of bipolar I disorder with a history of rapid cycling. J Affect Disord. 2003 Jan;73(1-2):155-61. PMID: 12507748.
86. Katagiri H, Takita Y, Tohen M, et al. Safety and efficacy of olanzapine monotherapy and olanzapine with a mood stabilizer in 18-week treatment of manic/mixed episodes for Japanese patients with bipolar I disorder. Curr Med Res Opin. 2012 May;28(5):701-13. doi:

http://dx.doi.org/10.1185/03007995.2012.666 961. PMID: 22356118.

87. Brown E, Dunner DL, McElroy SL, et al. Olanzapine/fluoxetine combination vs. lamotrigine in the 6-month treatment of bipolar I depression. Int J

Neuropsychopharmcol . 2009 Jul;12(6):77382. doi:

http://dx.doi.org/10.1017/S14611457080097 35. PMID: 19079815.

88. Tohen M, Chengappa KN, Suppes T, et al. Relapse prevention in bipolar I disorder: 18month comparison of olanzapine plus mood stabiliser v. mood stabiliser alone. Br J Psychiatry. 2004 Apr;184:337-45. PMID: 15056579.

89. Perlis RH, Welge JA, Vornik LA, et al. Atypical antipsychotics in the treatment of mania: a meta-analysis of randomized, placebo-controlled trials. J Clin Psychiatry. 2006 Apr;67(4):509-16. PMID: 16669715.

90. Li H, Ma C, Wang G, et al. Response and remission rates in Chinese patients with bipolar mania treated for 4 weeks with either quetiapine or lithium: a randomized and double-blind study. Curr Med Res Opin. 2008 Jan;24(1):1-10. PMID: 18028587.

91. Yatham LN, Vieta E, Young AH, et al. A double blind, randomized, placebo-controlled trial of quetiapine as an add-on therapy to lithium or divalproex for the treatment of bipolar mania. Int Clin Psychopharmacol. 2007 Jul;22(4):212-20. PMID: 17519644.

92. Yatham LN, Paulsson B, Mullen J, et al. Quetiapine versus placebo in combination with lithium or divalproex for the treatment of bipolar mania.[Erratum appears in J Clin Psychopharmacol. 2005 Apr;25(2):201], [Erratum appears in J Clin Psychopharmacol. 2005 Feb;25(1):103]. J Clin Psychopharmacol. 2004 Dec;24(6):599-606. PMID: 15538120. 
93. Suppes T, Ketter TA, Gwizdowski IS, et al. First controlled treatment trial of bipolar II hypomania with mixed symptoms: quetiapine versus placebo. J Affect Disord. 2013 Aug 15;150(1):37-43. doi: http://dx.doi.org/10.1016/j.jad.2013.02.031. PMID: 23521871.

94. Nejtek VA, Avila M, Chen LA, et al. Do atypical antipsychotics effectively treat cooccurring bipolar disorder and stimulant dependence? A randomized, double-blind trial. J Clin Psychiatry. 2008 Aug;69(8):1257-66. PMID: 18681757.

95. Vieta E, Suppes T, Ekholm B, et al. Longterm efficacy of quetiapine in combination with lithium or divalproex on mixed symptoms in bipolar I disorder. J Affect Disord. 2012 Dec 15;142(1-3):36-44. doi: http://dx.doi.org/10.1016/j.jad.2012.04.014. PMID: 23062763.

96. Segal J, Berk M, Brook S. Risperidone compared with both lithium and haloperidol in mania: a double-blind randomized controlled trial. Clin Neuropharmacol 1998 May-Jun;21(3):176-80. PMID: 9617509.

97. Yatham LN, Grossman F, Augustyns I, et al. Mood stabilisers plus risperidone or placebo in the treatment of acute mania. International, double-blind, randomised controlled trial.[Erratum appears in Br J Psychiatry. 2003 Apr;182:369]. Br J Psychiatry. 2003 Feb;182:141-7. PMID: 12562742.

98. Hirschfeld RM, Keck PE, Jr., Kramer M, et al. Rapid antimanic effect of risperidone monotherapy: a 3-week multicenter, doubleblind, placebo-controlled trial. Am J Psychiatry. 2004 Jun;161(6):1057-65. PMID: 15169694.

99. Quiroz JA, Yatham LN, Palumbo JM, et al. Risperidone long-acting injectable monotherapy in the maintenance treatment of bipolar I disorder. Biol Psychiatry. $2010 \mathrm{Jul}$ 15;68(2):156-62. doi: http://dx.doi.org/10.1016/j.biopsych.2010.01. 015. PMID: 20227682.

100. Sachs GS, Vanderburg DG, Edman S, et al. Adjunctive oral ziprasidone in patients with acute mania treated with lithium or divalproex, part 1: results of a randomized, double-blind, placebo-controlled trial. J Clin Psychiatry. 2012 Nov;73(11):1412-9. doi: http://dx.doi.org/10.4088/JCP.11m07388. PMID: 23218157.
101. Vieta E, Ramey T, Keller D, et al. Ziprasidone in the treatment of acute mania: a 12-week, placebo-controlled, haloperidolreferenced study. J Psychopharmacol. 2010 Apr;24(4):547-58. doi: http://dx.doi.org/10.1177/0269881108099418 . PMID: 19074536.

102. Stahl S, Lombardo I, Loebel A, et al. Efficacy of ziprasidone in dysphoric mania: pooled analysis of two double-blind studies. $\mathrm{J}$ Affect Disord. 2010 Apr;122(1-2):39-45. doi: http://dx.doi.org/10.1016/j.jad.2009.06.023. PMID: 19616304.

103. Kushner SF, Khan A, Lane R, et al. Topiramate monotherapy in the management of acute mania: results of four double-blind placebo-controlled trials. Bipolar Disord. 2006 Feb;8(1):15-27. PMID: 16411977.

104. Weisler RH, Hirschfeld R, Cutler AJ, et al. Extended-release carbamazepine capsules as monotherapy in bipolar disorder : pooled results from two randomised, double-blind, placebo-controlled trials. CNS Drugs. 2006;20(3):219-31. PMID: 16529527.

105. Vasudev K, Goswami U, Kohli K. Carbamazepine and valproate monotherapy: feasibility, relative safety and efficacy, and therapeutic drug monitoring in manic disorder. Psychopharmacology. 2000 May;150(1):15-23. PMID: 10867972.

106. Small JG, Klapper MH, Milstein V, et al. Carbamazepine compared with lithium in the treatment of mania. Arch Gen Psychiatry. 1991 Oct;48(10):915-21. PMID: 1929761.

107. Lerer B, Moore N, Meyendorff E, et al. Carbamazepine versus lithium in mania: a double-blind study. J Clin Psychiatry. 1987 Mar;48(3):89-93. PMID: 3546274.

108. Weisler RH, Kalali AH, Cutler AJ, et al. Efficacy and safety of once- versus twicedaily carbamazepine extended-release capsules for the treatment of manic symptoms in patients with bipolar I disorder. Psychiatry. 2008;5(3):35-48. PMID: 22778707.

109. Weisler RH, Kalali AH, Ketter TA, et al. A multicenter, randomized, double-blind, placebo-controlled trial of extended-release carbamazepine capsules as monotherapy for bipolar disorder patients with manic or mixed episodes. J Clin Psychiatry. 2004 Apr;65(4):478-84. PMID: 15119909. 
110. El-Mallakh RS, Salem MR, Chopra AS, et al. Adverse event load in bipolar participants receiving either carbamazepine immediaterelease or extended-release capsules: a blinded, randomized study. Int Clin Psychopharmacol. 2009 May;24(3):145-9. doi:

http://dx.doi.org/10.1097/YIC.0b013e328329 b199. PMID: 19367153.

111. Bowden CL, Swann AC, Calabrese JR, et al. A randomized, placebo-controlled, multicenter study of divalproex sodium extended release in the treatment of acute mania. J Clin Psychiatry. 2006 Oct;67(10):1501-10. PMID: 17107240.

112. Hirschfeld RM, Bowden CL, Vigna NV, et al. A randomized, placebo-controlled, multicenter study of divalproex sodium extended-release in the acute treatment of mania. J Clin Psychiatry. 2010

Apr;71(4):426-32. doi: http://dx.doi.org/10.4088/JCP.08m04960yel. PMID: 20361904.

113. McElroy SL, Martens BE, Creech RS, et al. Randomized, double-blind, placebocontrolled study of divalproex extended release loading monotherapy in ambulatory bipolar spectrum disorder patients with moderate-to-severe hypomania or mild mania. J Clin Psychiatry. 2010 May;71(5):557-65. doi: http://dx.doi.org/10.4088/JCP.08m04854yel. PMID: 20361901.

114. Wang Z, Gao K, Kemp DE, et al. Lamotrigine adjunctive therapy to lithium and divalproex in depressed patients with rapid cycling bipolar disorder and a recent substance use disorder: a 12-week, doubleblind, placebo-controlled pilot study. Psychopharmacol Bull. 2010;43(4):5-21. PMID: 21240149.

115. Bowden CL, Singh V, Weisler R, et al. Lamotrigine vs. lamotrigine plus divalproex in randomized, placebo-controlled maintenance treatment for bipolar depression. Acta Psychiatr Scand. 2012 Nov;126(5):34250. doi: http://dx.doi.org/10.1111/j.16000447.2012.01890.x. PMID: 22708645.
116. Kemp DE, Gao K, Ganocy SJ, et al. A 6month, double-blind, maintenance trial of lithium monotherapy versus the combination of lithium and divalproex for rapid-cycling bipolar disorder and Co-occurring substance abuse or dependence. J Clin Psychiatry. 2009 Jan;70(1):113-21. PMID: 19192457.

117. Calabrese JR, Shelton MD, Rapport DJ, et al. A 20-month, double-blind, maintenance trial of lithium versus divalproex in rapidcycling bipolar disorder. Am J Psychiatry. 2005 Nov;162(11):2152-61. PMID: 16263857.

118. Bowden CL, Brugger AM, Swann AC, et al. Efficacy of divalproex vs lithium and placebo in the treatment of mania. The Depakote Mania Study Group.[Erratum appears in JAMA 1994 Jun 15;271(23):1830]. JAMA. 1994 Mar 2330;271(12):918-24. PMID: 8120960.

119. Oquendo MA, Galfalvy HC, Currier D, et al. Treatment of suicide attempters with bipolar disorder: a randomized clinical trial comparing lithium and valproate in the prevention of suicidal behavior.[Erratum appears in Am J Psychiatry. 2012

Feb;169(2):223]. Am J Psychiatry. 2011 Oct;168(10):1050-6. doi: http://dx.doi.org/10.1176/appi.ajp.2011.1101 0163. PMID: 21768611.

120. Salloum IM, Cornelius JR, Daley DC, et al. Efficacy of valproate maintenance in patients with bipolar disorder and alcoholism: a double-blind placebo-controlled study. Arch Gen Psychiatry. 2005 Jan;62(1):37-45. PMID: 15630071.

121. Ichim L, Berk M, Brook S. Lamotrigine compared with lithium in mania: a doubleblind randomized controlled trial. Ann Clin Psychiatry. 2000 Mar;12(1):5-10. PMID: 10798820.

122. Licht RW, Nielsen JN, Gram LF, et al. Lamotrigine versus lithium as maintenance treatment in bipolar I disorder: an open, randomized effectiveness study mimicking clinical practice. The 6th trial of the Danish University Antidepressant Group (DUAG-6). Bipolar Disord. 2010 Aug;12(5):483-93. doi: http://dx.doi.org/10.1111/j.13995618.2010.00836.x. PMID: 20712749. 
123. Bowden CL, Calabrese JR, Ketter TA, et al. Impact of lamotrigine and lithium on weight in obese and nonobese patients with bipolar I disorder. Am J Psychiatry. 2006 Jul;163(7):1199-201. PMID: 16816224.

124. Calabrese JR, Suppes T, Bowden CL, et al. A double-blind, placebo-controlled, prophylaxis study of lamotrigine in rapidcycling bipolar disorder. Lamictal 614 Study Group. J Clin Psychiatry. 2000 Nov;61(11):841-50. PMID: 11105737.

125. Goldberg JF, Calabrese JR, Saville BR, et al. Mood stabilization and destabilization during acute and continuation phase treatment for bipolar I disorder with lamotrigine or placebo. J Clin Psychiatry. 2009 Sep;70(9):1273-80. doi: http://dx.doi.org/10.4088/JCP.08m04381. PMID: 19689918.

126. Bowden CL, Mosolov S, Hranov L, et al. Efficacy of valproate versus lithium in mania or mixed mania: a randomized, open 12-week trial. Int Clin Psychopharmacol. 2010 Mar;25(2):60-7. doi: http://dx.doi.org/10.1097/YIC.0b013e328333 ac1b. PMID: 20101186.

127. Amsterdam JD, Luo L, Shults J. Efficacy and mood conversion rate during long-term fluoxetine v. lithium monotherapy in rapidand non-rapid-cycling bipolar II disorder. $\mathrm{Br}$ J Psychiatry. 2013 2013;202(4):301-6. doi: http://dx.doi.org/10.1192/bjp.bp.111.104711. PMID: 23099447.

128. Ahmad A, Sheikh S, Shah T, et al. Endoxifen, a New Treatment Option for Mania: A Double-Blind, Active-Controlled Trial Demonstrates the Antimanic Efficacy of Endoxifen. Clin Transl Sci . 201601 Oct;9(5):252-9. doi: http://dx.doi.org/10.1111/cts.12407. PMID: 27346789 PMID/611114504 Embase.

129. Arabzadeh S, Ameli N, Zeinoddini A, et al. Celecoxib adjunctive therapy for acute bipolar mania: A randomized, double-blind, placebo-controlled trial. Bipolar Disord. 2015 01 Sep;17(6):606-14. doi: http://dx.doi.org/10.1111/bdi.12324. PMID: 26291962 (pubmed) 2015329826 (embase).
130. Jahangard L, Soroush S, Haghighi M, et al. In a double-blind, randomized and placebocontrolled trial, adjuvant allopurinol improved symptoms of mania in in-patients suffering from bipolar disorder. Eur Neuropsychopharmacol. Jun. 2014 2, 2014(Pagination)doi: http://dx.doi.org/10.1016/j.euroneuro.2014.0 5.013. PMID: 24953766.

131. Weiser M, Burshtein S, Gershon AA, et al. Allopurinol for mania: A randomized trial of allopurinol versus placebo as add-on treatment to mood stabilizers and/or antipsychotic agents in manic patients with bipolar disorder. Bipolar Disord. 2014 2014;16(4):441-7. doi: http://dx.doi.org/10.1111/bdi.12202. PMID: 24712840.

132. Fan A, Berg A, Bresee C, et al. Allopurinol augmentation in the outpatient treatment of bipolar mania: a pilot study. Bipolar Disord. 2012 Mar;14(2):206-10. doi: http://dx.doi.org/10.1111/j.13995618.2012.01001.x. PMID: 22420596.

133. Astaneh AN, Rezaei O. Adjunctive treatment with gabapentin in bipolar patients during acute mania. Int J Psychiatry Med. 2012;43(3):261-71. PMID: 22978083.

134. Yildiz A, Guleryuz S, Ankerst DP, et al. Protein kinase $\mathrm{C}$ inhibition in the treatment of mania: a double-blind, placebo-controlled trial of tamoxifen. Arch Gen Psychiatry. 2008 Mar;65(3):255-63. doi: http://dx.doi.org/10.1001/archgenpsychiatry. 2007.43. PMID: 18316672.

135. Chengappa RKN, Schwarzman LK, Hulihan JF, et al. Adjunctive topiramate therapy in patients receiving a mood stabilizer for bipolar I disorder: a randomized, placebo-controlled trial. J Clin Psychiatry. 2006 Nov;67(11):1698-706. PMID: 17196048.

136. Machado-Vieira R, Soares JC, Lara DR, et al. A double-blind, randomized, placebocontrolled 4-week study on the efficacy and safety of the purinergic agents allopurinol and dipyridamole adjunctive to lithium in acute bipolar mania. J Clin Psychiatry. 2008 Aug;69(8):1237-45. PMID: 18681754. 
137. Chen J, Lu Z, Zhang M, et al. A randomized, 4-week double-blind placebo control study on the efficacy of donepezil augmentation of lithium for treatment of acute mania. Neuropsychiatr Dis Treat. 2013 17, 2013;9:839-45. PMID: 23807849.

138. Bahk WM, Shin YC, Woo JM, et al. Topiramate and divalproex in combination with risperidone for acute mania: a randomized open-label study. Prog Neuropsychopharmacol Biol Psychiatry. 2005 Jan;29(1):115-21. doi: 10.1016/j.pnpbp.2004.10.013. PMID: 15610953.

139. Berwaerts J, Melkote R, Nuamah I, et al. A randomized, placebo- and active-controlled study of paliperidone extended-release as maintenance treatment in patients with bipolar I disorder after an acute manic or mixed episode. J Affect Disord. 2012 May;138(3):247-58. doi: http://dx.doi.org/10.1016/j.jad.2012.01.047. PMID: 22377512.

140. Berwaerts J, Xu H, Nuamah I, et al. Evaluation of the efficacy and safety of paliperidone extended-release in the treatment of acute mania: a randomized, double-blind, dose-response study. J Affect Disord. 2012 Jan;136(1-2):e51-60. doi: http://dx.doi.org/10.1016/j.jad.2010.06.030. PMID: 20624657.

141. Berwaerts J, Lane R, Nuamah IF, et al. Paliperidone extended-release as adjunctive therapy to lithium or valproate in the treatment of acute mania: a randomized, placebo-controlled study. J Affect Disord. 2011 Mar;129(1-3):252-60. doi: http://dx.doi.org/10.1016/j.jad.2010.09.011. PMID: 20947174.

142. Vik A, Ravindran A, Shiah IS, et al. A double-blind, placebo-controlled study of adjunctive calcitonin nasal spray in acute refractory mania. Bipolar Disord. 2013 2013;15(4):359-64. PMID: 23551803.

143. Grunze H, Kotlik E, Costa R, et al. Assessment of the efficacy and safety of eslicarbazepine acetate in acute mania and prevention of recurrence: experience from multicentre, double-blind, randomised phase II clinical studies in patients with bipolar disorder I. J Affect Disord. 2015 Mar 15;174:70-82. doi: http://dx.doi.org/10.1016/j.jad.2014.11.013. PMID: 25484179.
144. Amsterdam JD, Garcia-Espana F, Fawcett J, et al. Efficacy and safety of fluoxetine in treating bipolar II major depressive episode. J Clin Psychopharmacol. 1998 Dec;18(6):43540. PMID: 9864074.

145. Amsterdam JD, Shults J. Comparison of short-term venlafaxine versus lithium monotherapy for bipolar II major depressive episode: a randomized open-label study. J Clin Psychopharmacol. 2008 Apr;28(2):17181. doi: 10.1097/JCP.0b013e318166c4e6. PMID: 18344727.

146. Nierenberg AA, Sylvia LG, Leon AC, et al. Lithium treatment -- moderate dose use study (LiTMUS) for bipolar disorder: rationale and design. Clinical Trials. 2009 Dec;6(6):63748. doi: http://dx.doi.org/10.1177/1740774509347399 . PMID: 19933719.

147. Kemp DE, Gao K, Fein EB, et al Lamotrigine as add-on treatment to lithium and divalproex: lessons learned from a double-blind, placebo-controlled trial in rapid-cycling bipolar disorder. Bipolar Disord. 2012 Nov;14(7):780-9. doi: http://dx.doi.org/10.1111/bdi.12013. PMID: 23107222.

148. Lee SY, Chen SL, Chang YH, et al. Genotype variant associated with add-on memantine in bipolar II disorder.[Erratum appears in Int J Neuropsychopharmacol. 2014 Jun;17(6):979 Note: Lu, Ru-Band [removed]; Hong, Jau-Shyong [added]]. Int J Neuropsychopharmcol . 2014 Feb;17(2):18997. doi:

http://dx.doi.org/10.1017/S14611457130008 25. PMID: 24103632.

149. Lee S-Y, Chen S-L, Chang Y-H, et al. Add-on memantine to valproate treatment increased HDL-C in bipolar II disorder. J Psychiatr Res. 2013 2013;47(10):1343-8. doi: http://dx.doi.org/10.1016/j.jpsychires.2013.0 6.017. PMID: 23870798.

150. Sachs GS, Nierenberg AA, Calabrese JR, et al. Effectiveness of adjuctive antidepressant treatment for bipolar depression. The N Engl J Med. 2007 2007;356(17):1711-22. doi: http://dx.doi.org/10.1056/NEJMoa064135. PMID: 17392295. 
151. Altshuler LL, Sugar CA, McElroy SL, et al. Switch rates during acute treatment for Bipolar II depression with Lithium, Sertraline, or the two combined: A randomized double-blind comparison. Am J Psychiatry. 2017 March;174(3):266-76. doi: http://dx.doi.org/10.1176/appi.ajp.2016.1504 0558. PMID: 28135846 PMID/614689619 Embase.

152. Amsterdam JD, Lorenzo-Luaces L, Soeller I, et al. Short-term venlafaxine v. lithium monotherapy for bipolar type II major depressive episodes: Effectiveness and mood conversion rate. Br J Psychiatry. 2016 April;208(4):359-65. doi: http://dx.doi.org/10.1192/bjp.bp.115.169375. PMID: 26892848 PMID/609914520 Embase.

153. Lorenzo-Luaces L, Amsterdam JD, Soeller I, et al. Rapid versus non-rapid cycling bipolar II depression: Response to venlafaxine and lithium and hypomanic risk. Acta Psychiatr Scand. 201601 Jun;133(6):459-69. doi: http://dx.doi.org/10.1111/acps.12557. PMID: 26803764 PMID/608565179 Embase.

154. Amsterdam JD, Wang CH, Shwarz M, et al. Venlafaxine versus lithium monotherapy of rapid and non-rapid cycling patients with bipolar II major depressive episode: a randomized, parallel group, open-label trial. J Affect Disord. 2009 Jan;112(1-3):219-30. doi:

http://dx.doi.org/10.1016/j.jad.2008.03.029. PMID: 18486235.

155. Ghaemi SN, Ostacher MM, El-Mallakh RS, et al. Antidepressant discontinuation in bipolar depression: a Systematic Treatment Enhancement Program for Bipolar Disorder (STEP-BD) randomized clinical trial of longterm effectiveness and safety. J Clin Psychiatry. 2010 Apr;71(4):372-80. doi: http://dx.doi.org/10.4088/JCP.08m04909gre. PMID: 20409444.

156. Nierenberg AA, Friedman ES, Bowden CL, et al. Lithium treatment moderate-dose use study (LiTMUS) for bipolar disorder: a randomized comparative effectiveness trial of optimized personalized treatment with and without lithium. Am J Psychiatry. 2013 Jan 1;170(1):102-10. doi: http://dx.doi.org/10.1176/appi.ajp.2012.1206 0751. PMID: 23288387.
157. Nierenberg AA, McElroy S, Ketter TA, et al. Bipolar CHOICE (Clinical Health Outcomes Initiative in Comparative Effectiveness): A pragmatic six month trial of lithium vs. quetiapine for bipolar disorder. Bipolar Disord. 2015 June;17:25-6. doi: http://dx.doi.org/10.1111/bdi.12306_15.

158. Nierenberg AA, Sylvia LG, Leon AC, et al. Clinical and Health Outcomes Initiative in Comparative Effectiveness for Bipolar Disorder (Bipolar CHOICE): a pragmatic trial of complex treatment for a complex disorder. Clinical Trials. 2014 Feb;11(1):11427. doi: http://dx.doi.org/10.1177/1740774513512184 . PMID: 24346608.

159. Bowden CL, Karayal ON, Schwartz JH, et al. Characterizing relapse prevention in bipolar disorder with adjunctive ziprasidone: clinical and methodological implications. J Affect Disord. 2013 Jan 10;144(1-2):171-5. doi:

http://dx.doi.org/10.1016/j.jad.2012.04.024. PMID: 22999893.

160. Balance investigators, Geddes JR, Goodwin GM, et al. Lithium plus valproate combination therapy versus monotherapy for relapse prevention in bipolar I disorder (BALANCE): a randomised open-label trial. Lancet. 2010 Jan 30;375(9712):385-95. doi: http://dx.doi.org/10.1016/S01406736(09)61828-6. PMID: 20092882.

161. Amsterdam JD, Lorenzo-Luaces L, Soeller I, et al. Safety and effectiveness of continuation antidepressant versus mood stabilizer monotherapy for relapse-prevention of bipolar II depression: A randomized, double-blind, parallel-group, prospective study. J Affect Disord. 201506 Jul;185:31-7. doi:

http://dx.doi.org/10.1016/j.jad.2015.05.070. PMID: 26143402 (pubmed) 2015175772 (embase).

162. Amsterdam JD, Shults J. Efficacy and safety of long-term fluoxetine versus lithium monotherapy of bipolar II disorder: a randomized, double-blind, placebosubstitution study. Am J Psychiatry. 2010 Jul;167(7):792-800. doi: http://dx.doi.org/10.1176/appi.ajp.2009.0902 0284. PMID: 20360317. 
163. Bobo WV, Epstein RA, Lynch A, et al. A randomized open comparison of long-acting injectable risperidone and treatment as usual for prevention of relapse, rehospitalization, and urgent care referral in community-treated patients with rapid cycling bipolar disorder. Clin Neuropharmacol . 2011 Nov-

Dec;34(6):224-33. doi: http://dx.doi.org/10.1097/WNF.0b013e31823 7709a. PMID: 22104634.

164. Bowden CL, Calabrese JR, McElroy SL, et al. A randomized, placebo-controlled 12month trial of divalproex and lithium in treatment of outpatients with bipolar I disorder. Divalproex Maintenance Study Group. Arch Gen Psychiatry. 2000 May;57(5):481-9. PMID: 10807488.

165. Bowden CL, Calabrese JR, Sachs G, et al. A placebo-controlled 18-month trial of lamotrigine and lithium maintenance treatment in recently manic or hypomanic patients with bipolar I disorder.[Erratum appears in Arch Gen Psychiatry. 2004 Jul;61(7):680]. Arch Gen Psychiatry. 2003 Apr;60(4):392-400. PMID: 12695317.

166. Bowden CL, Vieta E, Ice KS, et al. Ziprasidone plus a mood stabilizer in subjects with bipolar I disorder: a 6-month, randomized, placebo-controlled, double-blind trial. J Clin Psychiatry. 2010 Feb;71(2):1307. doi: http://dx.doi.org/10.4088/JCP.09m05482yel. PMID: 20122373.

167. Calabrese JR, Bowden CL, Sachs G, et al. A placebo-controlled 18-month trial of lamotrigine and lithium maintenance treatment in recently depressed patients with bipolar I disorder. J Clin Psychiatry. 2003 Sep;64(9):1013-24. PMID: 14628976.

168. Greil W, Kleindienst N. Lithium versus carbamazepine in the maintenance treatment of bipolar II disorder and bipolar disorder not otherwise specified. Int Clin Psychopharmacol. 1999 Sep;14(5):283-5. PMID: 10529071.

169. Greil W, Kleindienst N. The comparative prophylactic efficacy of lithium and carbamazepine in patients with bipolar I disorder. Int Clin Psychopharmacol. 1999 Sep;14(5):277-81. PMID: 10529070.
170. Greil W, Kleindienst N, Erazo N, et al. Differential response to lithium and carbamazepine in the prophylaxis of bipolar disorder. J Clin Psychopharmacol. 1998 Dec;18(6):455-60. PMID: 9864077.

171. Greil W, Ludwig-Mayerhofer W, Erazo N, et al. Lithium versus carbamazepine in the maintenance treatment of bipolar disorders--a randomised study. J Affect Disord. 1997 Apr;43(2):151-61. PMID: 9165384.

172. Gyulai L, Bowden CL, McElroy SL, et al. Maintenance efficacy of divalproex in the prevention of bipolar depression. Neuropsychopharmacology. 2003 Jul;28(7):1374-82. PMID: 12784116.

173. Hartong EG, Moleman P, Hoogduin CA, et al. Prophylactic efficacy of lithium versus carbamazepine in treatment-naive bipolar patients. J Clin Psychiatry. 2003 Feb;64(2):144-51. PMID: 12633122.

174. Macfadden W, Alphs L, Haskins JT, et al. A randomized, double-blind, placebocontrolled study of maintenance treatment with adjunctive risperidone long-acting therapy in patients with bipolar I disorder who relapse frequently. Bipolar Disord. 2009 Dec;11(8):827-39. doi: http://dx.doi.org/10.1111/j.13995618.2009.00761.x. PMID: 19922552.

175. Marcus R, Khan A, Rollin L, et al. Efficacy of aripiprazole adjunctive to lithium or valproate in the long-term treatment of patients with bipolar I disorder with an inadequate response to lithium or valproate monotherapy: a multicenter, double-blind, randomized study. Bipolar Disord. 2011 Mar;13(2):133-44. doi: http://dx.doi.org/10.1111/j.13995618.2011.00898.x. PMID: 21443567.

176. Newport DJ, Stowe ZN, Viguera AC, et al. Lamotrigine in bipolar disorder: efficacy during pregnancy. Bipolar Disord. 2008 May;10(3):432-6. doi: http://dx.doi.org/10.1111/j.13995618.2007.00565.x. PMID: 18402631.

177. Nierenberg AA, McElroy SL, Friedman ES, et al. Bipolar CHOICE (Clinical Health Outcomes Initiative in Comparative Effectiveness): a pragmatic 6-month trial of lithium versus quetiapine for bipolar disorder. J Clin Psychiatry. 2016 Jan;77(1):90-9. doi: http://dx.doi.org/10.4088/JCP.14m09349. PMID: 26845264. 
178. Prien RF, Point P, Caffey EM, et al. Prophylactic efficacy of lithium carbonate in manic-depressive illness: Report of the veterans administration and national institute of mental health collaborative study group. Arch Gen Psychiatry. 1973;28(3):337-41. doi: 10.1001/archpsyc.1973.01750330035006. PMID: 4569674.

179. Suppes T, Vieta E, Liu S, et al. Maintenance treatment for patients with bipolar I disorder: results from a north american study of quetiapine in combination with lithium or divalproex (trial 127). Am J Psychiatry. 2009 Apr;166(4):476-88. doi: http://dx.doi.org/10.1176/appi.ajp.2008.0802 0189. PMID: 19289454.

180. Tohen M, Calabrese JR, Sachs GS, et al. Randomized, placebo-controlled trial of olanzapine as maintenance therapy in patients with bipolar I disorder responding to acute treatment with olanzapine. Am J Psychiatry. 2006 Feb;163(2):247-56. PMID: 16449478.

181. Tohen M, Greil W, Calabrese JR, et al. Olanzapine versus lithium in the maintenance treatment of bipolar disorder: a 12-month, randomized, double-blind, controlled clinical trial. Am J Psychiatry. 2005 Jul;162(7):128190. PMID: 15994710.

182. Vieta E, Cruz N, Garcia-Campayo J, et al. A double-blind, randomized, placebocontrolled prophylaxis trial of oxcarbazepine as adjunctive treatment to lithium in the longterm treatment of bipolar I and II disorder. Int J Neuropsychopharmcol. 2008

Jun;11(4):445-52. doi: http://dx.doi.org/10.1017/S14611457080085 96. PMID: 18346292.

183. Vieta E, Manuel Goikolea J, MartinezAran A, et al. A double-blind, randomized, placebo-controlled, prophylaxis study of adjunctive gabapentin for bipolar disorder. J Clin Psychiatry. 2006 Mar;67(3):473-7. PMID: 16649836.

184. Vieta E, Montgomery S, Sulaiman AH, et al. A randomized, double-blind, placebocontrolled trial to assess prevention of mood episodes with risperidone long-acting injectable in patients with bipolar I disorder. Eur Neuropsychopharmacol. 2012 Nov;22(11):825-35. doi: http://dx.doi.org/10.1016/j.euroneuro.2012.0 3.004. PMID: 22503488.
185. Vieta E, Owen R, Baudelet C, et al. Assessment of safety, tolerability and effectiveness of adjunctive aripiprazole to lithium/valproate in bipolar mania: a 46week, open-label extension following a 6week double-blind study. Curr Med Res Opin. 2010 Jun;26(6):1485-96. doi: http://dx.doi.org/10.1185/0300799100377938 0. PMID: 20429835.

186. Vieta E, Suppes T, Eggens I, et al. Efficacy and safety of quetiapine in combination with lithium or divalproex for maintenance of patients with bipolar I disorder (international trial 126). J Affect Disord. 2008 Aug;109(3):251-63. doi: http://dx.doi.org/10.1016/j.jad.2008.06.001. PMID: 18579216.

187. Weisler RH, Nolen WA, Neijber A, et al. Continuation of quetiapine versus switching to placebo or lithium for maintenance treatment of bipolar I disorder (Trial 144: a randomized controlled study). J Clin Psychiatry. 2011 Nov;72(11):1452-64. doi: http://dx.doi.org/10.4088/JCP.11m06878. PMID: 22054050.

188. Woo YS, Bahk WM, Chung MY, et al. Aripiprazole plus divalproex for recently manic or mixed patients with bipolar I disorder: a 6-month, randomized, placebocontrolled, double-blind maintenance trial. Hum Psychopharmacol. 2011 Dec;26(8):54353. doi: http://dx.doi.org/10.1002/hup.1240. PMID: 22134973.

189. Zarate CA, Jr., Tohen M. Double-blind comparison of the continued use of antipsychotic treatment versus its discontinuation in remitted manic patients. Am J Psychiatry. 2004 Jan;161(1):169-71. PMID: 14702269.

190. Kleindienst N, Greil W. Differential efficacy of lithium and carbamazepine in the prophylaxis of bipolar disorder: results of the MAP study. Neuropsychobiology. 2000;42 Suppl 1:2-10. PMID: 11093063.

191. Calabrese JR, Sanchez R, Jin N, et al. Efficacy and safety of aripiprazole oncemonthly in the maintenance treatment of bipolar i disorder: A double-blind, placebocontrolled, 52-week randomized withdrawal study. J Clin Psychiatry. 2017 March;78(3):324-31. doi: http://dx.doi.org/10.4088/JCP.16m11201. PMID: 28146613 PMID/615221995 Embase. 
192. Carlson BX, Ketter TA, Sun W, et al. Aripiprazole in combination with lamotrigine for the long-term treatment of patients with bipolar I disorder (manic or mixed): a randomized, multicenter, double-blind study (CN138-392). Bipolar Disord. 2012 Feb;14(1):41-53. doi: http://dx.doi.org/10.1111/j.13995618.2011.00974.x. PMID: 22329471.

193. Masand PS, Eudicone J, Pikalov A, et al. Criteria for defining symptomatic and sustained remission in bipolar I disorder: a post-hoc analysis of a 26-week aripiprazole study (study CN138-010). Psychopharmacol Bull. 2008;41(2):12-23. PMID: 18668014.

194. Keck PE, Jr., Calabrese JR, McIntyre RS, et al. Aripiprazole monotherapy for maintenance therapy in bipolar I disorder: a 100-week, double-blind study versus placebo. J Clin Psychiatry. 2007 Oct;68(10):1480-91. PMID: 17960961.

195. El-Mallakh RS, Marcus R, Baudelet C, et al. A 40-week double-blind aripiprazole versus lithium follow-up of a 12-week acute phase study (total 52 weeks) in bipolar I disorder. J Affect Disord. 2012

Feb;136(3):258-66. doi: http://dx.doi.org/10.1016/j.jad.2011.11.043. PMID: 22209190.

196. Solomon DA, Ristow WR, Keller MB, et al. Serum lithium levels and psychosocial function in patients with bipolar I disorder. Am J Psychiatry. 1996 Oct;153(10):1301-7. PMID: 8831438.

197. Barnes CW, Hadzi-Pavlovic D, Wilhelm $\mathrm{K}$, et al. A web-based preventive intervention program for bipolar disorder: outcome of a 12-months randomized controlled trial. J Affect Disord. 2015 Mar 15;174:485-92. doi: http://dx.doi.org/10.1016/j.jad.2014.11.038. PMID: 25554993.

198. Colom F, Vieta E, Martinez-Aran A, et al. A randomized trial on the efficacy of group psychoeducation in the prophylaxis of recurrences in bipolar patients whose disease is in remission. Arch Gen Psychiatry. 2003 Apr;60(4):402-7. PMID: 12695318.

199. Colom F, Vieta E, Reinares M, et al. Psychoeducation efficacy in bipolar disorders: beyond compliance enhancement. J Clin Psychiatry. 2003 Sep;64(9):1101-5. PMID: 14628987.
200. Colom F, Vieta E, Sanchez-Moreno J, et al. Group psychoeducation for stabilised bipolar disorders: 5-year outcome of a randomised clinical trial.[Erratum appears in $\mathrm{Br} \mathrm{J}$ Psychiatry. 2009 Jun;194(6):571]. Br J Psychiatry. 2009 Mar;194(3):260-5. doi: http://dx.doi.org/10.1192/bjp.bp.107.040485. PMID: 19252157.

201. de Barros Pellegrinelli K, de OCLF, Silval $\mathrm{KI}$, et al. Efficacy of psychoeducation on symptomatic and functional recovery in bipolar disorder. Acta Psychiatr Scand. 2013 Feb;127(2):153-8. doi: http://dx.doi.org/10.1111/acps.12007. PMID: 22943487.

202. Depp CA, Ceglowski J, Wang VC, et al. Augmenting psychoeducation with a mobile intervention for bipolar disorder: a randomized controlled trial. J Affect Disord. 2015 Mar 15;174:23-30. doi: http://dx.doi.org/10.1016/j.jad.2014.10.053. PMID: 25479050.

203. Javadpour A, Hedayati A, Dehbozorgi GR, et al. The impact of a simple individual psycho-education program on quality of life, rate of relapse and medication adherence in bipolar disorder patients. Asian J Psychiatr. 2013 Jun;6(3):208-13. doi: http://dx.doi.org/10.1016/j.ajp.2012.12.005. PMID: 23642977.

204. Perry A, Tarrier N, Morriss R, et al. Randomised controlled trial of efficacy of teaching patients with bipolar disorder to identify early symptoms of relapse and obtain treatment. BMJ. 1999 Jan 16;318(7177):14953. PMID: 9888904.

205. Sajatovic M, Davies MA, Ganocy SJ, et al. A comparison of the life goals program and treatment as usual for individuals with bipolar disorder. Psychiatr Serv. 2009 Sep;60(9):1182-9. doi: http://dx.doi.org/10.1176/appi.ps.60.9.1182. PMID: 19723732.

206. Smith DJ, Griffiths E, Poole R, et al. Beating Bipolar: exploratory trial of a novel Internet-based psychoeducational treatment for bipolar disorder. Bipolar Disord. 2011 Aug-Sep;13(5-6):571-7. doi: http://dx.doi.org/10.1111/j.13995618.2011.00949.x. PMID: 22017225. 
207. Weiss RD, Griffin ML, Greenfield SF, et al. Group therapy for patients with bipolar disorder and substance dependence: results of a pilot study. J Clin Psychiatry. 2000 May;61(5):361-7. PMID: 10847311.

208. Gumus F, Buzlu S, Cakir S. Effectiveness of individual psychoeducation on recurrence in bipolar disorder; a controlled study. Archives of psychiatric nursing. 201501 Jun;29(3):174-9. doi: http://dx.doi.org/10.1016/j.apnu.2015.01.005. PMID: 26001717.

209. Bilderbeck AC, Atkinson LZ, McMahon HC, et al. Psychoeducation and online mood tracking for patients with bipolar disorder: A randomised controlled trial. J Affect Disord. 201615 Nov;205:245-51. doi: http://dx.doi.org/10.1016/j.jad.2016.06.064. PMID: 27454410 PMID/612460558 Embase.

210. Kallestad H, Wullum E, Scott J, et al. The long-term outcomes of an effectiveness trial of group versus individual psychoeducation for bipolar disorders. J Affect Disord. 2016;202:32-8. doi: http://dx.doi.org/10.1016/j.jad.2016.05.043. PMID: 27253214 PMID/611342382 Embase.

211. Camacho EM, Ntais D, Jones S, et al. Costeffectiveness of structured group psychoeducation versus unstructured group support for bipolar disorder: Results from a multi-centre pragmatic randomised controlled trial. J Affect Disord. 201715 Mar;211:2736. doi:

http://dx.doi.org/10.1016/j.jad.2017.01.005. PMID: 614007566.

212. Morriss R, Lobban F, Riste L, et al. Clinical effectiveness and acceptability of structured group psychoeducation versus optimised unstructured peer support for patients with remitted bipolar disorder (PARADES): a pragmatic, multicentre, observer-blind, randomised controlled superiority trial. The Lancet Psychiatry. 2016 01 Nov;3(11):1029-38. doi: http://dx.doi.org/10.1016/S22150366\%2816\%2930302-9. PMID: 27688021.

213. Weiss RD, Griffin ML, Kolodziej ME, et al. A randomized trial of integrated group therapy versus group drug counseling for patients with bipolar disorder and substance dependence. Am J Psychiatry. 2007 Jan;164(1):100-7. PMID: 17202550.
214. Weiss RD, Griffin ML, Jaffee WB, et al. A "community-friendly" version of integrated group therapy for patients with bipolar disorder and substance dependence: a randomized controlled trial. Drug Alcohol Depend. 2009 Oct 1;104(3):212-9. doi: http://dx.doi.org/10.1016/j.drugalcdep.2009.0 4.018. PMID: 19573999.

215. Scott J, Paykel E, Morriss R, et al. Cognitive-behavioural therapy for severe and recurrent bipolar disorders: randomised controlled trial. Br J Psychiatry. 2006 Apr;188:313-20. PMID: 16582056.

216. Parikh SV, Zaretsky A, Beaulieu S, et al. A randomized controlled trial of psychoeducation or cognitive-behavioral therapy in bipolar disorder: a Canadian Network for Mood and Anxiety treatments (CANMAT) study [CME]. J Clin Psychiatry. 2012 Jun;73(6):803-10. doi: http://dx.doi.org/10.4088/JCP.11m07343. PMID: 22795205.

217. Meyer TD, Hautzinger M. Cognitive behaviour therapy and supportive therapy for bipolar disorders: relapse rates for treatment period and 2-year follow-up. Psychol Med. 2012 Jul;42(7):1429-39. doi: http://dx.doi.org/10.1017/S00332917110025 22. PMID: 22099722.

218. Lam DH, Watkins ER, Hayward P, et al. A randomized controlled study of cognitive therapy for relapse prevention for bipolar affective disorder: outcome of the first year. Arch Gen Psychiatry. 2003 Feb;60(2):14552. PMID: 12578431.

219. Lam DH, Hayward P, Watkins ER, et al. Relapse prevention in patients with bipolar disorder: cognitive therapy outcome after 2 years. Am J Psychiatry. 2005 Feb;162(2):324-9. PMID: 15677598.

220. Jones SH, Smith G, Mulligan LD, et al. Recovery-focused cognitive-behavioural therapy for recent-onset bipolar disorder: randomised controlled pilot trial.[Erratum appears in Br J Psychiatry. 2015 Feb;206(2):169]. Br J Psychiatry. 2015 Jan;206(1):58-66. doi: http://dx.doi.org/10.1192/bjp.bp.113.141259. PMID: 25213157. 
221. Harvey AG, Soehner AM, Kaplan KA, et al. Treating insomnia improves mood state, sleep, and functioning in bipolar disorder: a pilot randomized controlled trial. J Consult Clin Psychol. 2015 Jun;83(3):564-77. doi: 10.1037/a0038655. PMID: 25622197.

222. Gomes BC, Abreu LN, Brietzke E, et al. A randomized controlled trial of cognitive behavioral group therapy for bipolar disorder. Psychother Psychosom. 2011;80(3):144-50. doi: http://dx.doi.org/10.1159/000320738. PMID: 21372622.

223. Fava GA, Rafanelli C, Tomba E, et al. The sequential combination of cognitive behavioral treatment and well-being therapy in cyclothymic disorder. Psychother Psychosom. 2011;80(3):136-43. doi: http://dx.doi.org/10.1159/000321575. PMID: 21372621.

224. Castle D, White C, Chamberlain J, et al. Group-based psychosocial intervention for bipolar disorder: randomised controlled trial. Br J Psychiatry. 2010 May;196(5):383-8. doi: http://dx.doi.org/10.1192/bjp.bp.108.058263. PMID: 20435965.

225. Ball JR, Mitchell PB, Corry JC, et al. A randomized controlled trial of cognitive therapy for bipolar disorder: focus on longterm change. J Clin Psychiatry. 2006 Feb;67(2):277-86. PMID: 16566624.

226. Perich T, Manicavasagar V, Mitchell PB, et al. A randomized controlled trial of mindfulness-based cognitive therapy for bipolar disorder. Acta Psychiatr Scand. 2013 May;127(5):333-43. doi: http://dx.doi.org/10.1111/acps.12033. PMID: 23216045.

227. Bauer MS, McBride L, Williford WO, et al. Collaborative care for bipolar disorder: Part II. Impact on clinical outcome, function, and costs. Psychiatr Serv. 2006 Jul;57(7):937-45. PMID: 16816277.

228. Kessing LV, Hansen HV, Hvenegaard A, et al. Treatment in a specialised out-patient mood disorder clinic v. standard out-patient treatment in the early course of bipolar disorder: randomised clinical trial. $\mathrm{Br} \mathrm{J}$ Psychiatry. 2013 Mar;202(3):212-9. doi: http://dx.doi.org/10.1192/bjp.bp.112.113548. PMID: 23349295.
229. Kilbourne AM, Goodrich DE, Lai Z, et al. Life Goals Collaborative Care for patients with bipolar disorder and cardiovascular disease risk. Psychiatr Serv. 2012

Dec;63(12):1234-8. doi: http://dx.doi.org/10.1176/appi.ps.201100528. PMID: 23203358.

230. Kilbourne AM, Post EP, Nossek A, et al. Improving medical and psychiatric outcomes among individuals with bipolar disorder: a randomized controlled trial. Psychiatr Serv. 2008 Jul;59(7):760-8. doi: http://dx.doi.org/10.1176/appi.ps.59.7.760. PMID: 18586993.

231. Simon GE, Ludman EJ, Bauer MS, et al. Long-term effectiveness and cost of a systematic care program for bipolar disorder. Arch Gen Psychiatry. 2006 May;63(5):500-8. PMID: 16651507.

232. Simon GE, Ludman EJ, Unutzer J, et al. Randomized trial of a population-based care program for people with bipolar disorder. Psychol Med. 2005 Jan;35(1):13-24. PMID: 15842025.

233. van der Voort TY, van Meijel B, Goossens PJ, et al. Collaborative care for patients with bipolar disorder: randomised controlled trial. Br J Psychiatry. 2015 Mar 19doi: 10.1192/bjp.bp.114.152520. PMID: 25792695.

234. van der Voort TY, van Meijel B, Hoogendoorn AW, et al. Collaborative care for patients with bipolar disorder: Effects on functioning and quality of life. J Affect Disord. 2015 Mar 11;179(1):14-22. doi: 10.1016/j.jad.2015.03.005. PMID: 25841077.

235. D'Souza R, Piskulic D, Sundram S. A brief dyadic group based psychoeducation program improves relapse rates in recently remitted bipolar disorder: a pilot randomised controlled trial. J Affect Disord. 2010 Jan;120(1-3):272-6. doi: http://dx.doi.org/10.1016/j.jad.2009.03.018. PMID: 19428117.

236. Miklowitz DJ, George EL, Richards JA, et al. A randomized study of family-focused psychoeducation and pharmacotherapy in the outpatient management of bipolar disorder. Arch Gen Psychiatry. 2003 Sep;60(9):90412. PMID: 12963672. 
237. Miklowitz DJ, Simoneau TL, George EL, et al. Family-focused treatment of bipolar disorder: 1-year effects of a psychoeducational program in conjunction with pharmacotherapy. Biol Psychiatry. 2000 Sep 15;48(6):582-92. PMID: 11018229.

238. Miller IW, Keitner GI, Ryan CE, et al. Family treatment for bipolar disorder: family impairment by treatment interactions. J Clin Psychiatry. 2008 May;69(5):732-40. PMID: 18363424.

239. Miller IW, Solomon DA, Ryan CE, et al. Does adjunctive family therapy enhance recovery from bipolar I mood episodes? J Affect Disord. 2004 Nov 1;82(3):431-6. PMID: 15555694.

240. Rea MM, Tompson MC, Miklowitz DJ, et al. Family-focused treatment versus individual treatment for bipolar disorder: results of a randomized clinical trial. J Consult Clin Psychol. 2003 Jun;71(3):48292. PMID: 12795572.

241. Simoneau TL, Miklowitz DJ, Richards JA, et al. Bipolar disorder and family communication: effects of a psychoeducational treatment program. J Abnorm Psychol. 1999 Nov;108(4):588-97. PMID: 10609423.

242. Solomon DA, Keitner GI, Ryan CE, et al. Preventing recurrence of bipolar I mood episodes and hospitalizations: family psychotherapy plus pharmacotherapy versus pharmacotherapy alone. Bipolar Disord. 2008 Nov;10(7):798-805. doi: http://dx.doi.org/10.1111/j.13995618.2008.00624.x. PMID: 19032711.

243. Wenze SJ, Gaudiano BA, Weinstock LM, et al. Adjunctive psychosocial intervention following Hospital discharge for Patients with bipolar disorder and comorbid substance use: A pilot randomized controlled trial. Psychiatry Res. 2015 Aug 30;228(3):516-25. doi: 10.1016/j.psychres.2015.06.005. PMID: 26117247.

244. Frank E, Hlastala S, Ritenour A, et al. Inducing lifestyle regularity in recovering bipolar disorder patients: results from the maintenance therapies in bipolar disorder protocol. Biol Psychiatry. 1997 Jun 15;41(12):1165-73. PMID: 9171907.
245. Frank E, Kupfer DJ, Thase ME, et al. Twoyear outcomes for interpersonal and social rhythm therapy in individuals with bipolar I disorder. Arch Gen Psychiatry. 2005 Sep;62(9):996-1004. PMID: 16143731.

246. Frank E, Soreca I, Swartz HA, et al. The role of interpersonal and social rhythm therapy in improving occupational functioning in patients with bipolar I disorder. Am J Psychiatry. 2008 Dec;165(12):1559-65. doi: http://dx.doi.org/10.1176/appi.ajp.2008.0712 1953. PMID: 18829872.

247. Frank E, Swartz HA, Mallinger AG, et al. Adjunctive psychotherapy for bipolar disorder: effects of changing treatment modality. J Abnorm Psychol. 1999 Nov;108(4):579-87. PMID: 10609422.

248. Inder ML, Crowe MT, Luty SE, et al. Randomized, controlled trial of Interpersonal and Social Rhythm Therapy for young people with bipolar disorder. Bipolar Disord. 2015 Mar;17(2):128-38. doi: 10.1111/bdi.12273. PMID: 25346391.

249. Inder ML, Crowe MT, Luty SE, et al. Prospective rates of suicide attempts and nonsuicidal self-injury by young people with bipolar disorder participating in a psychotherapy study. Aust N Z J Psychiatry. 2016 Feb;50(2):167-73. doi: http://dx.doi.org/10.1177/0004867415622268 . PMID: 26698820.

250. Rucci P, Frank E, Kostelnik B, et al. Suicide attempts in patients with bipolar I disorder during acute and maintenance phases of intensive treatment with pharmacotherapy and adjunctive psychotherapy. Am J Psychiatry. 2002 Jul;159(7):1160-4. PMID: 12091194.

251. Gonzalez Isasi A, Echeburua E, Liminana JM, et al. Psychoeducation and cognitivebehavioral therapy for patients with refractory bipolar disorder: A 5-year controlled clinical trial. Eur Psychiatry. Dec. 2014 March 2014 doi: http://dx.doi.org/10.1016/j.eurpsy.2012.11.00 2. PMID: 23276524.

252. Isasi AG, Echeburua E, Liminana JM, et al. How effective is a psychological intervention program for patients with refractory bipolar disorder? A randomized controlled trial. J Affect Disord. 2010 Oct;126(1-2):80-7. doi: http://dx.doi.org/10.1016/j.jad.2010.03.026. PMID: 20444503. 
253. Miklowitz DJ, Richards JA, George EL, et al. Integrated family and individual therapy for bipolar disorder: results of a treatment development study. J Clin Psychiatry. 2003 Feb;64(2):182-91. PMID: 12633127.

254. Todd NJ, Jones SH, Hart A, et al. A webbased self-management intervention for Bipolar Disorder 'living with bipolar': a feasibility randomised controlled trial. J Affect Disord. 2014 Dec;169:21-9. doi: 10.1016/j.jad.2014.07.027. PMID: 25129531.

255. Zaretsky A, Lancee W, Miller C, et al. Is cognitive-behavioural therapy more effective than psychoeducation in bipolar disorder? Canadian Journal of Psychiatry - Revue Canadienne de Psychiatrie. 2008 Jul;53(7):441-8. PMID: 18674402.

256. Fagiolini A, Frank E, Axelson DA, et al. Enhancing outcomes in patients with bipolar disorder: results from the Bipolar Disorder Center for Pennsylvanians Study. Bipolar Disord. 2009 Jun;11(4):382-90. doi: http://dx.doi.org/10.1111/j.13995618.2009.00700.x. PMID: 19500091.

257. Faurholt-Jepsen M, Frost M, Ritz C, et al. Daily electronic self-monitoring in bipolar disorder using smartphones - the MONARCA I trial: a randomized, placebocontrolled, single-blind, parallel group trial. Psychol Med. 2015 Oct;45(13):2691-704. doi: http://dx.doi.org/10.1017/S00332917150004 10. PMID: 26220802.

258. Miklowitz DJ, Otto MW, Wisniewski SR, et al. Psychotherapy, symptom outcomes, and role functioning over one year among patients with bipolar disorder. Psychiatr Serv. 2006 Jul;57(7):959-65. PMID: 16816280.

259. Deckersbach T, Peters AT, Sylvia L, et al. Do comorbid anxiety disorders moderate the effects of psychotherapy for bipolar disorder? Results from STEP-BD. Am J Psychiatry. 2014 Feb;171(2):178-86. doi: http://dx.doi.org/10.1176/appi.ajp.2013.1302 0225. PMID: 24077657.

260. Miklowitz DJ, Otto MW, Frank E, et al. Intensive psychosocial intervention enhances functioning in patients with bipolar depression: results from a 9-month randomized controlled trial. Am J Psychiatry. 2007 Sep;164(9):1340-7. PMID: 17728418.
261. Miklowitz DJ, Otto MW, Frank E, et al. Psychosocial treatments for bipolar depression: a 1-year randomized trial from the Systematic Treatment Enhancement Program. Arch Gen Psychiatry. 2007 Apr;64(4):419-26. PMID: 17404119.

262. Fitzgerald PB, Hoy KE, Elliot D, et al. A negative double-blind controlled trial of sequential bilateral rTMS in the treatment of bipolar depression. J Affect Disord. 201601 Jul;198:158-62. doi: http://dx.doi.org/10.1016/j.jad.2016.03.052. PMID: 27016659 PMID/609157504 Embase.

263. Swartz H, Swanson J. Psychotherapy for bipolar disorder in adults: a review of the evidence. Focus (Am Psychiatr Publ). 2014 Summer;12(3):251-66. doi: 10.1176/appi.focus.12.3.251. PMID: 26279641.

264. Greenhouse WJ, Meyer B, Johnson SL. Coping and medication adherence in bipolar disorder. J Affect Disord. 2000 Sep;59(3):237-41. PMID: 10854641.

265. Council NR. The prevention and treatment of missing data in clinical trials: National Academies Press; 2011.

266. Rendell Jennifer M, Gijsman Harm J, Keck Paul K, et al. Olanzapine alone or in combination for acute mania. Cochrane Database of Systematic Reviews: John Wiley \& Sons, Ltd; 2003.

267. Rendell Jennifer M, Gijsman Harm J, Bauer Mark S, et al. Risperidone alone or in combination for acute mania. Cochrane Database of Systematic Reviews: John Wiley \& Sons, Ltd; 2006.

268. Burgess Sally SA, Geddes J, Hawton Keith $\mathrm{KE}$, et al. Lithium for maintenance treatment of mood disorders. Cochrane Database of Systematic Reviews: John Wiley \& Sons, Ltd; 2001.

269. Rendell Jennifer M, Geddes J. Risperidone in long-term treatment for bipolar disorder. Cochrane Database of Systematic Reviews: John Wiley \& Sons, Ltd; 2006.

270. Brown R, Taylor Matthew J, Geddes J. Aripiprazole alone or in combination for acute mania. Cochrane Database of Systematic Reviews: John Wiley \& Sons, Ltd; 2013. 
271. Cipriani A, Rendell Jennifer M, Geddes J. Olanzapine in long-term treatment for bipolar disorder. Cochrane Database of Systematic Reviews: John Wiley \& Sons, Ltd; 2009.

272. Cipriani A, Rendell Jennifer M, Geddes J. Haloperidol alone or in combination for acute mania. Cochrane Database of Systematic Reviews: John Wiley \& Sons, Ltd; 2006.

273. Reilly S, Planner C, Gask L, et al. Collaborative care approaches for people with severe mental illness. Cochrane Database of Systematic Reviews: John Wiley \& Sons, Ltd; 2013.

274. Friedman LM, Furberg C, DeMets DL, et al. Fundamentals of clinical trials: Springer; 1998.

275. Sackett DL, Strauss SE, Richardson WS, et al. Evidence-based medicine: how to practice and teach EBM. Edinburgh: Churchill Livingstone; 2000.

276. Kristman V, Manno M, Cote P. Loss to follow-up in cohort studies: how much is too much? Eur J Epidemiol. 2004;19(8):751-60. PMID: 15469032.

277. Amico KR. Percent total attrition: a poor metric for study rigor in hosted intervention designs. Am J Public Health. 2009 Sep;99(9):1567-75. doi: 10.2105/ajph.2008.134767. PMID: 19608965.
278. Sen S, Prabhu M. Reporting bias in industry-supported medication trials presented at the American Psychiatric Association meeting. J Clin

Psychopharmacol. 2012 Jun;32(3):435. doi: https://dx.doi.org/10.1097/JCP.0b013e31825 3d737. PMID: 22561482.

279. Turner EH, Matthews AM, Linardatos E, et al. Selective publication of antidepressant trials and its influence on apparent efficacy. N Engl J Med. 2008 Jan 17;358(3):252-60. doi: https://dx.doi.org/10.1056/NEJMsa065779. PMID: 18199864.

280. Turner EH, Knoepflmacher D, Shapley L. Publication bias in antipsychotic trials: an analysis of efficacy comparing the published literature to the US Food and Drug Administration database. PLoS Medicine / Public Library of Science. 2012;9(3):e1001189. doi: https://dx.doi.org/10.1371/journal.pmed.1001 189. PMID: 22448149.

281. Driessen E, Hollon SD, Bockting CL, et al. Does Publication Bias Inflate the Apparent Efficacy of Psychological Treatment for Major Depressive Disorder? A Systematic Review and Meta-Analysis of US National Institutes of Health-Funded Trials. PLoS ONE [Electronic Resource]. 2015;10(9):e0137864. doi: https://dx.doi.org/10.1371/journal.pone.0137 864. PMID: 26422604. 


\section{Abbreviations}

\begin{tabular}{|c|c|}
\hline AHRQ & Agency for Healthcare Research and Quality \\
\hline BMI & Body Mass Index \\
\hline $\mathrm{BD}$ & Bipolar Disorder \\
\hline BPD & Borderline Personality Disorder \\
\hline $\mathrm{C}$ & Comparison \\
\hline CBT & Cognitive Behavioral Therapy \\
\hline CCMD-3 & $\begin{array}{l}\text { Chinese Classification and Diagnosis Criteria of Mental Disorder, } \\
3^{\text {rd }} \text { Version }\end{array}$ \\
\hline CENTRAL & Cochrane Central Register of Controlled Trials \\
\hline CER & Comparative Effectiveness Review \\
\hline CGI & Clinical Global Impression \\
\hline CGI-BP & Clinical Global Impressions-Bipolar Questionnaire \\
\hline CGI-BP-S & $\begin{array}{l}\text { Clinical Global Impressions-Bipolar Questionnaire Severity } \\
\text { Subscale }\end{array}$ \\
\hline CGI-I & Clinical Global Impressions-Improvement \\
\hline CI & Confidence Interval \\
\hline DSM & Diagnostic Statistical Manual of Mental Disorders \\
\hline DSS & Depressive Syndrome Scale \\
\hline ECT & Electroconvulsive Therapy \\
\hline ER & Extended Release \\
\hline ESRS & Extrapyramidal Symptom Rating Scale \\
\hline FDA & Food and Drug Administration \\
\hline FPI & Family or Partner Interventions \\
\hline FTP & Family Therapy Plus Pharmacotherapy \\
\hline GAS & Global Assessment Scale \\
\hline HAM-D & Hamilton Scale for Depression \\
\hline HDRS & Hamilton Depression Rating Scale \\
\hline $\mathrm{I}$ & Intervention \\
\hline ICD-10 & $\begin{array}{l}\text { International Statistical Classification of Diseases and Related } \\
\text { Health Problems }-10^{\text {th }} \text { Revision }\end{array}$ \\
\hline ICTRP & International Controlled Trials Registry Platform \\
\hline IDS-C & Inventory of Depressive Symptoms (Clinician-rated) \\
\hline IPSRT & Interpersonal and Social Rhythm Therapy \\
\hline KSADS-PL & $\begin{array}{l}\text { Kiddie Schedule for Affective Disorders and Schizophrenia- } \\
\text { Present and Lifetime Version }\end{array}$ \\
\hline KQ & Key Question \\
\hline LOCF & Last Observation Carried Forward \\
\hline MADRS & Montgomery-Asberg Depression Rating Scale \\
\hline MAR & Missing at Random \\
\hline MD & Mean Difference \\
\hline MDQ & Mood Disorder Questionnaire \\
\hline MIDs & Minimum Important Differences \\
\hline MINI & MINI International Neuropsychiatric Interview \\
\hline NA & Not Applicable \\
\hline
\end{tabular}




$\begin{array}{ll}\text { NOS } & \text { Not Otherwise Specified } \\ \text { NR } & \text { Not Reported } \\ \text { NRC } & \text { National Research Council } \\ \text { OR } & \text { Odds Ratio } \\ \text { PICOTS } & \text { Populations, Interventions, Comparisons, Outcomes, Timing, and } \\ & \text { Setting } \\ \text { PRISMA } & \text { Preferred Reporting Items for Systematic Reviews and Meta- } \\ & \text { Analyses } \\ \text { RCT } & \text { Randomized Controlled Trial } \\ \text { REML } & \text { Restricted Maximum Likelihood Estimator } \\ \text { ROB } & \text { Risk of Bias } \\ \text { RTI } & \text { Research Triangle Institute } \\ \text { SADS-C } & \text { Schedule for Affective Disorders and Schizophrenia, change } \\ & \text { version } \\ \text { SCID-I } & \text { Structured Clinical Interview for the DSM-IV Axis I Disorders } \\ \text { SE } & \text { Standard Error } \\ \text { SIPs } & \text { Scientific Information Packets } \\ \text { SMD } & \text { Standardized Mean Differences } \\ \text { SOE } & \text { Strength of Evidence } \\ \text { STEP-BD } & \text { Bipolar Disorder Systematic Treatment Enhancement Program } \\ \text { TEP } & \text { Technical Expert Panel } \\ \text { WHOQOL-BREF } & \text { World Health Organization Quality of Life-Short Version } \\ \text { WMD } & \text { Weighted Mean Differences } \\ \text { YMRS } & \text { Young Mania Rating Scale } \\ & \end{array}$




\section{Appendix A. Search Strategies}

\section{Database: Ovid MEDLINE(R) Search Strategy: RCTs}

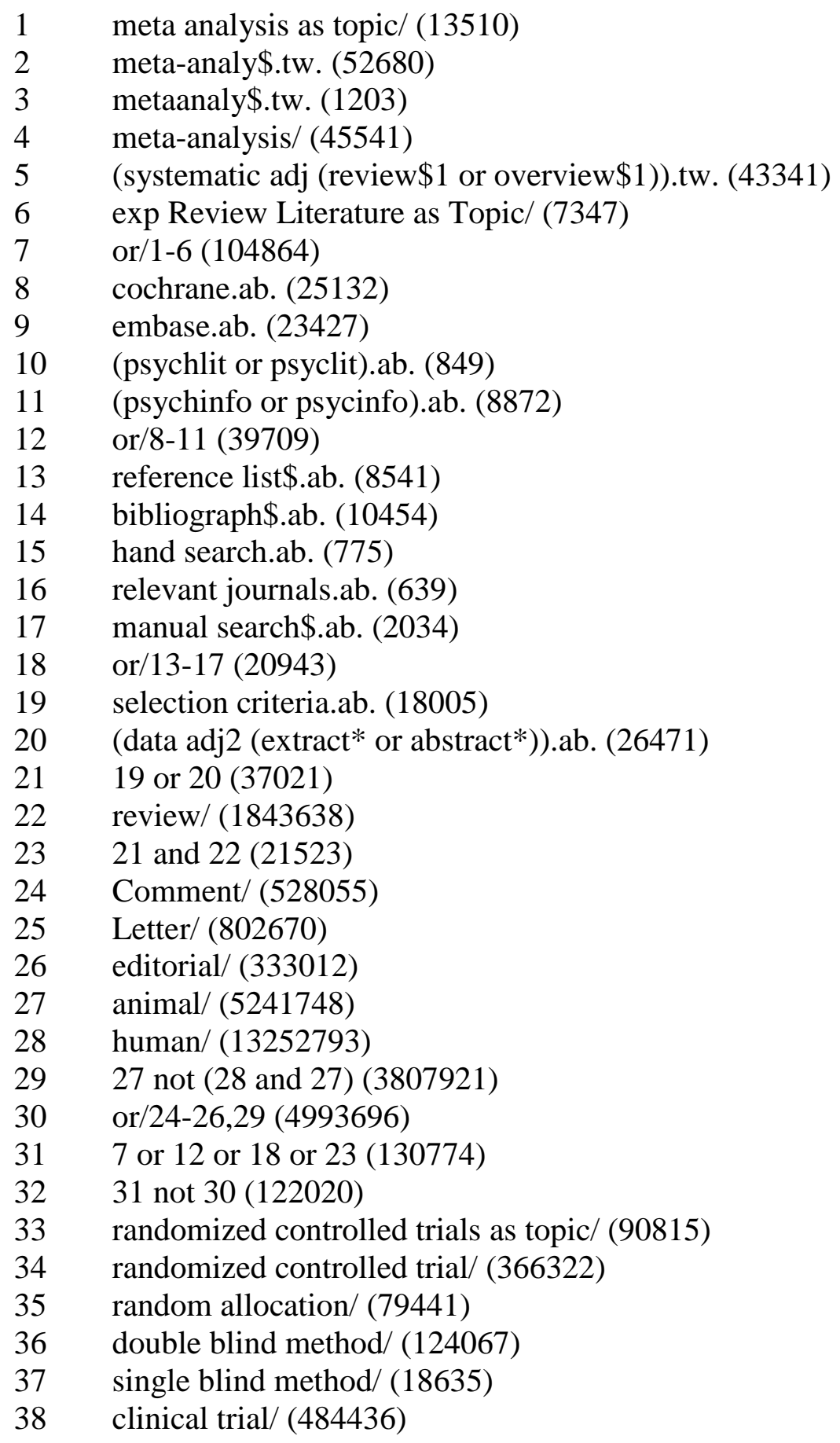


39 clinical trial, phase i.pt. (13833)

40 clinical trial, phase ii.pt. (22208)

41 clinical trial, phase iii.pt. (8628)

42 clinical trial, phase iv.pt. (905)

43 controlled clinical trial.pt. (87769)

44 randomized controlled trial.pt. (366322)

45 multicenter study.pt. (167455)

$46 \quad$ clinical trial.pt. (484436)

47 exp clinical trials as topic/ (276232)

48 or/33-47 (1007391)

49 (clinical adj trial\$).tw. (193529)

$50 \quad$ ((singl\$ or doubl\$ or treb\$ or tripl\$) adj (blind\$3 or mask\$3)).tw. (121456)

51 placebos/ (32313)

52 placebo\$.tw. (148340)

53 randomly allocated.tw. (15301)

54 (allocated adj2 random\$).tw. (17733)

55 or/49-54 (386436)

$56 \quad 48$ or $55(1124628)$

57 case report.tw. (180193)

58 case report.tw. (180193)

59 letter/ (802670)

60 historical article/ (298584)

61 or/57-60 (1270322)

6256 not 61 (1095429)

63 exp cohort studies/ (1320622)

64 cohort\$.tw. (244490)

$65 \quad$ controlled clinical trial.pt. (87769)

66 epidemiological methods/ (29547)

67 limit 66 to $\mathrm{yr}=1971-1983$ (5333)

68 or/63-65,67 (1486088)

69 (ae or to or po or co).fs. (3071650)

70 side effect\$.ti,ab. (162368)

71 side effect\$.ti,ab. (162368)

72 ((adverse or undesireable or harm\$ or serious or toxic) adj3 (effect\$ or reaction\$ or event\$ or outcome\$)).ti,ab. (277104)

73 exp product surveillance, postmarketing/ (10888)

74 exp adverse drug reaction reporting systems/ (5477)

75 exp clinical trials, phase iv/ (221)

76 exp poisoning/ (129396)

77 exp substance-related disorders/ (220844)

78 exp drug toxicity/ (86909)

79 exp abnormalities, drug induced/ (13732)

80 exp drug monitoring/ (13980)

81 exp drug hypersensitivity/ (37576) 
82 (toxicity or complication\$ or noxious or tolerability).ti,ab. (807144)

83 exp postoperative complication/ (410502)

84 exp intraoperative complications/ (39444)

85 or/69-84 (4065205)

86 exp Bipolar disorder/ (30924)

87 bipolar*.ti. (15477)

88 cyclothymia.ti. (77)

$89 \quad$ (rapid adj cycl*).ti. (416)

90 (mania or hypomania or manic or hypomanic).ti. (5588)

91 or/86-90 (35187)

$92 \quad 32$ and $91(799)$

$93 \quad 62$ and 91 (4169)

$94 \quad 68$ and 85 and 91 (1916)

95 limit 92 to yr="1994-Current" (788)

96 limit 93 to yr="1994-Current" (3286) RCTs

97 limit 94 to yr="1994-Current" (1518)

9895 not 96 (522) Systematic reviews

$99 \quad 97$ not (95 or 96) (989) Cohort harms

$100 \quad$ (68 and 91) not 85 (3060)

101 limit 100 to $\mathrm{yr}=" 1994-C u r r e n t " ~(2188)$ Cohort benefits

102101 not (95 or 96 or 97) (1704)

103 limit 96 to "all child (0 to 18 years)" (726)

104 limit 96 to "all adult (19 plus years)" (2056)

$105 \quad 103$ and $104(474)$

106103 not $105(252)$

10796 not 106 (3034) RCTs without pediatric-only

108 limit 98 to "all child (0 to 18 years)" (70)

109 limit 98 to "all adult (19 plus years)" (161)

110108 and $109(46)$

111108 not $110(24)$

11298 not 111 (498) Systematic reviews without pediatric-only

113 limit 99 to "all child (0 to 18 years)" (274)

114 limit 99 to "all adult (19 plus years)" (843)

$115 \quad 113$ and $114(208)$

$116 \quad 113$ not $115(66)$

11799 not 116 (923) Cohort harms without pediatric-only

118 limit 102 to "all child (0 to 18 years)" (607)

119 limit 102 to "all adult (19 plus years)" (1360)

$120 \quad 118$ and $119(459)$

121118 not $120(148)$

122102 not 121 (1556) Cohort benefits without pediatric-only

123 (resection or prostate or radiofrequency or sealer or ablation or hip or fibrillation).ab. (396573)

124107 not 123 (2855) RCTs without pediatric-only or resection, ablation, etc. 
125112 not 123 (495) Systematic reviews without pediatric-only or resection, ablation, etc.

126117 not 123 (800) Cohort harms without pediatric-only or resection, ablation, etc.

127122 not 123 (1515) Cohort benefits without pediatric-only or resection, ablation, etc.

\section{Database: Embase Classic+Embase}

1 retracted article/ (6992)

2 (random $\$$ or placebo $\$$ or single blind $\$$ or double blind $\$$ or triple blind $\$$ ).ti,ab. (964464)

3 (animal\$ not human\$).sh,hw. (3892889)

4 (book or conference paper or editorial or letter or review).pt. not exp randomized controlled trial/ (4026686)

51 or $2(971306)$

65 not (3 or 4) (788381)

7 exp cohort analysis/ (159852)

8 exp longitudinal study/ (65122)

9 exp prospective study/ (251233)

10 exp follow up/ (749090)

11 cohort\$.tw. (363883)

127 or 8 or 9 or 10 or 11 (1281760)

13 exp case-control study/ (90165)

14 (case\$ and control\$).tw. (421076)

1513 or $14(455349)$

16 (case\$ and series).tw. (156704)

17 exp review/ (2051725)

18 (literature adj3 review\$).ti,ab. (210224)

19 exp meta analysis/ (76123)

20 exp "Systematic Review"/ (64783)

2117 or 18 or 19 or 20 (2235229)

22 (medline or embase or pubmed or cinahl or amed or psychlit or psychinfo or scisearch or cochrane).ti,ab. (99517)

23 retracted article/ (6992)

2422 or $23(106462)$

2521 and 24 (78850)

26 (systematic\$ adj2 (review\$ or overview)).ti,ab. (66304)

27 (meta?anal\$ or meta anal\$ or metaanal\$ or metanal\$).ti,ab. (75185)

2825 or 26 or 27 (159362)

29 (ae or si or to or co).fs. (3025512)

30 (safe or safety).ti,ab. (597602)

31 side effect\$.ti,ab. (238444) 
32 ((adverse or undesireable or harm\$ or serious or toxic) adj3 (effect\$ or reaction\$ or event\$ or outcome\$)).ti,ab.

(406794)

33 exp adverse drug reaction/ (358463)

34 exp drug toxicity/ (77722)

35 exp intoxication/ (328776)

36 exp drug safety/ (221042)

37 exp drug monitoring/ (40454)

38 exp drug hypersensitivity/ (49258)

39 exp postmarketing surveillance/ (22410)

40 exp phase iv clinical trial/ (1496)

41 (toxicity or complication\$ or noxious or tolerability).ti,ab. (1146485)

42 exp postoperative complication/ (478856)

43 exp peroperative complication/ (19583)

44 or/29-43 (4747913)

45 exp *bipolar disorder/ (21744)

46 (manic and depress*).ti. (2122)

47 (bipolar and (disorder or depress*)).ti. (14239)

$48 \quad 45$ or 46 or $47(25031)$

$49 \quad 28$ and $48(966)$

5049 not (3 or 4$)(463)$

51 limit 50 to yr="2010 -Current" (238)

52 limit 51 to (book or conference abstract or conference proceeding or "conference review" or editorial or erratum or letter or note or short survey or trade journal) (105)

5351 not 52 (133) Systematic Reviews

$54 \quad 6$ and 48 (1987)

5554 not $52(1952)$ RCTs

$56 \quad 12$ and 48 (2527)

5756 not (3 or 4) (2224)

$58 \quad 57$ not $51(2195)$

$59 \quad 44$ and 48 (5891)

6058 and 59 (417) Cohort Harms 


\section{Database: PsycINFO}

\section{Search Strategy: RCTs}

1 retracted article/ (6992)

2 (random\$ or placebo\$ or single blind\$ or double blind\$ or triple blind\$).ti,ab. (964464)

3 (animal\$ not human\$).sh,hw. (3892889)

4 (book or conference paper or editorial or letter or review).pt. not exp randomized controlled trial/ (4026686)

51 or $2(971306)$

65 not (3 or 4) (788381)

7 exp cohort analysis/ (159852)

8 exp longitudinal study/ (65122)

$9 \quad$ exp prospective study/ (251233)

10 exp follow up/ (749090)

118 cohort\$.tw. (363883)

1297 or 8 or 9 or 10 or 11 (1281760)

13 exp case-control study/ (90165)

1410 (case\$ and control\$).tw. (421076)

1513 or $14(455349)$

1611 (case\$ and series).tw. (156704)

1712 exp review/ (2051725)

1813 (literature adj3 review\$).ti,ab. (210224)

1914 exp meta analysis/ (76123)

20 exp "Systematic Review"/ (64783)

211512 or 13 or 14 (2235229)

2216 (medline or embase or pubmed or cinahl or amed or psychlit or psychinfo or scisearch or cochrane).ti,ab. (99517)

2317 retracted article/ (6992)

241816 or $17(106462)$

251915 and 18 (78850)

2620 (systematic\$adj2 (review\$ or overview)).ti,ab. (66304)

2721 (meta?anal\$ or meta anal\$ or metaanal\$ or metanal\$).ti,ab. (75185)

282219 or 20 or $21(159362)$

29 (ae or si or to or co).fs. (3025512)

3023 (safe or safety).ti,ab. (597602)

3124 side effect\$.ti,ab. (238444)

3225 ((adverse or undesireable or harm\$ or serious or toxic) adj3 (effect\$ or reaction\$ or event\$ or outcome\$)).ti,ab.

(406794)

33 exp adverse drug reaction/ (358463)

34 exp drug toxicity/ (77722)

3526 exp intoxication/ (328776)

36 exp drug safety/ (221042) 
37 exp drug monitoring/ (40454)

38 exp drug hypersensitivity/ (49258)

39 exp postmarketing surveillance/ (22410)

40 exp phase iv clinical trial/ (1496)

4127 (toxicity or complication\$ or noxious or tolerability).ti,ab. (1146485)

42 exp postoperative complication/ (478856)

43 exp peroperative complication/ (19583)

4428 or/23-27(4747913)

4529 exp *bipolar disorder/ (21744)

4630 (manic and depress*).ti. (2122)

4731 (bipolar and (disorder or depress*)).ti. (14239)

483229 or 30 or $31(25031)$

493322 and 32 (966)

503433 not (3 or 4) (463)

5135 limit 34 to $\mathrm{yr}=" 2015 "(238)$

5236 limit 35 to (book or conference abstract or conference proceeding or "conference review" or editorial or erratum

or letter or note or short survey or trade journal) (105)

$5337 \quad 35$ not 36 (133) Systematic Reviews

$5438 \quad 6$ and $32(1987)$

553938 not 36 (1952) RCTs

$5640 \quad 9$ and $32(2527)$

574140 not (3 or 4$)(2224)$

584241 not $35(2195)$

$5943 \quad 28$ and 32 (5891)

604442 and 43 (417) Cohort Harms 


\section{Appendix B. Risk of Bias Assessment Tool}

\begin{tabular}{|c|c|c|c|c|}
\hline Question & Response & & Criteria & Justification \\
\hline & \multicolumn{3}{|c|}{ Internal Validity } & \\
\hline \multirow[t]{3}{*}{$\begin{array}{l}\text { 1. Is the study design } \\
\text { prospective, } \\
\text { retrospective, or } \\
\text { mixed? }\end{array}$} & Prospective & $\square$ & $\begin{array}{l}\text { Outcome has not occurred at } \\
\text { the time the study is initiated } \\
\text { and information is collected over } \\
\text { time to assess relationships with } \\
\text { the outcome. }\end{array}$ & \\
\hline & Mixed & $\square$ & $\begin{array}{l}\text { Studies in which one group is } \\
\text { studied prospectively and the } \\
\text { other retrospectively. }\end{array}$ & \\
\hline & Retrospective & $\square$ & $\begin{array}{l}\text { Analyzes data from past } \\
\text { records. }\end{array}$ & \\
\hline \multirow{3}{*}{$\begin{array}{l}\text { 2. Are } \\
\text { inclusion/exclusion } \\
\text { criteria clearly } \\
\text { stated? }\end{array}$} & Yes & $\square$ & & \\
\hline & Partially & $\square$ & $\begin{array}{l}\text { Some, but not all, criteria stated } \\
\text { or some not clearly stated. }\end{array}$ & \\
\hline & No & L & & \\
\hline \multirow{3}{*}{$\begin{array}{l}\text { 3. Are baseline } \\
\text { characteristics } \\
\text { measured using valid } \\
\text { and reliable } \\
\text { measures and } \\
\text { equivalent in both } \\
\text { groups? }\end{array}$} & Yes & $\bar{\square}$ & & \\
\hline & No & $\square$ & & \\
\hline & Uncertain & $\square$ & Could not be ascertained. & \\
\hline \multirow{3}{*}{$\begin{array}{l}\text { 4. Is the level of } \\
\text { detail describing the } \\
\text { intervention } \\
\text { adequate? }\end{array}$} & Yes & $\square$ & $\begin{array}{l}\text { Intervention described included } \\
\text { adequate service details }\end{array}$ & \\
\hline & Partially & $\square$ & Some of the above features. & \\
\hline & No & $\square$ & None of the above features. & \\
\hline $\begin{array}{l}\text { 5. Is the selection of } \\
\text { the comparison } \\
\text { group appropriate? }\end{array}$ & Yes & $\square$ & $\begin{array}{l}\text { Considering bipolar type, } \\
\text { diagnostic assessment, other } \\
\text { patient characteristics }\end{array}$ & \\
\hline \multirow{3}{*}{$\begin{array}{l}\text { 6. Did researchers } \\
\text { isolate the impact } \\
\text { from a concurrent } \\
\text { intervention or an } \\
\text { unintended exposure } \\
\text { that might bias } \\
\text { results? }\end{array}$} & Yes & $\square$ & $\begin{array}{l}\text { Accounted for concurrent } \\
\text { informal care. }\end{array}$ & \\
\hline & Partially & $\square$ & & \\
\hline & No & $\square$ & & \\
\hline \multirow{3}{*}{$\begin{array}{l}\text { 7. Any attempt to } \\
\text { balance the } \\
\text { allocation between } \\
\text { the groups (e.g. } \\
\text { stratification, } \\
\text { matching, propensity } \\
\text { scores)? }\end{array}$} & Yes & $\square$ & (if yes, what was used?) & \\
\hline & No & $\square$ & & \\
\hline & Uncertain & $\square$ & Could not be ascertained. & \\
\hline $\begin{array}{l}\text { 8. Were outcomes } \\
\text { assessors blinded? }\end{array}$ & & $\square$ & Who were outcome assessors? & \\
\hline \multirow{2}{*}{$\begin{array}{l}\text { 9. Are outcomes } \\
\text { assessed using valid } \\
\text { and reliable } \\
\text { measures, } \\
\text { implemented } \\
\text { consistently across }\end{array}$} & Yes & $\square$ & $\begin{array}{l}\text { Measure valid and reliable } \\
\text { (i.e. objective measures, well } \\
\text { validated scale, provider report); } \\
\text { and equivalent across groups. }\end{array}$ & \\
\hline & Partially & $\square$ & $\begin{array}{l}\text { Some of the above features } \\
\text { (partially validated scale) }\end{array}$ & \\
\hline
\end{tabular}

B-1 


\begin{tabular}{|c|c|c|c|}
\hline Question & Respons & Criteria & Justification \\
\hline \multirow[t]{2}{*}{$\begin{array}{l}\text { all study } \\
\text { participants? }\end{array}$} & No & $\begin{array}{l}\text { None of the above features. } \\
\text { (self-report, scales with lower } \\
\text { validity, reliability); not } \\
\text { equivalent across groups }\end{array}$ & \\
\hline & Uncertain & Could not be ascertained. & \\
\hline \multirow{3}{*}{$\begin{array}{l}\text { 10. Is the length of } \\
\text { follow-up the same } \\
\text { for all groups? }\end{array}$} & Yes & & \\
\hline & No & & \\
\hline & Uncertain & Could not be ascertained. & \\
\hline \multirow{3}{*}{$\begin{array}{l}\text { 11. Did attrition result } \\
\text { in a difference in } \\
\text { group characteristics } \\
\text { between baseline } \\
\text { and follow-up? }\end{array}$} & Yes & $\begin{array}{l}\text { (measurement period of interest } \\
\text { if repeated measures) }\end{array}$ & \\
\hline & No & & \\
\hline & Uncertain & $\begin{array}{l}\text { Could not be ascertained (i.e. } \\
\text { retrospective designs where } \\
\text { eligible at baseline could not be } \\
\text { determined) }\end{array}$ & \\
\hline \multirow{3}{*}{$\begin{array}{l}\text { 12. If baseline } \\
\text { characteristics are } \\
\text { not similar, does the } \\
\text { analysis control for } \\
\text { baseline differences } \\
\text { between groups? }\end{array}$} & Yes & & \\
\hline & No & & \\
\hline & Uncertain & $\begin{array}{l}\text { Could not be ascertained (i.e. } \\
\text { retrospective designs where } \\
\text { eligible at baseline could not be } \\
\text { determined) }\end{array}$ & \\
\hline \multirow{4}{*}{$\begin{array}{l}\text { 13. Are confounding } \\
\text { and/or effect } \\
\text { modifying variables } \\
\text { assessed using valid } \\
\text { and reliable } \\
\text { measures across all } \\
\text { study participants? }\end{array}$} & Yes & & \\
\hline & No & & \\
\hline & Uncertain & $\begin{array}{l}\text { Could not be ascertained (i.e. } \\
\text { retrospective designs where } \\
\text { eligible at baseline could not be } \\
\text { determined) }\end{array}$ & \\
\hline & NA & $\begin{array}{l}\text { No confounders or effect } \\
\text { modifiers included in the study. }\end{array}$ & \\
\hline \multirow{4}{*}{$\begin{array}{l}\text { 14. Were the } \\
\text { important } \\
\text { confounding and } \\
\text { effect modifying } \\
\text { variables taken into } \\
\text { account in the design } \\
\text { and/or analysis (e.g. } \\
\text { through matching, } \\
\text { stratification, } \\
\text { interaction terms, } \\
\text { multivariate analysis, } \\
\text { or other statistical } \\
\text { adjustment)? }\end{array}$} & Yes & & \\
\hline & Partially & $\begin{array}{l}\text { Some variables taken into } \\
\text { account or adjustment achieved } \\
\text { to some extent. }\end{array}$ & \\
\hline & No & $\begin{array}{l}\text { Not accounted for or not } \\
\text { identified. }\end{array}$ & \\
\hline & Uncertain & Could not be ascertained & \\
\hline $\begin{array}{l}\text { 15. Are the statistical } \\
\text { methods used to }\end{array}$ & Yes & $\begin{array}{l}\text { Statistical techniques used must } \\
\text { be appropriate to the data. }\end{array}$ & \\
\hline
\end{tabular}

B-2 


\begin{tabular}{|c|c|c|c|}
\hline Question & Respons & Criteria & Justification \\
\hline \multirow{3}{*}{$\begin{array}{l}\text { assess the primary } \\
\text { outcomes } \\
\text { appropriate to the } \\
\text { data? }\end{array}$} & Partially & & \\
\hline & No & & \\
\hline & Uncertain & Could not be ascertained & \\
\hline \multirow{3}{*}{$\begin{array}{l}\text { 16. Are reports of the } \\
\text { study free of } \\
\text { suggestion of } \\
\text { selective outcome } \\
\text { reporting? }\end{array}$} & Yes & & \\
\hline & No & $\begin{array}{l}\text { Not all prespecified outcomes } \\
\text { reported, subscales not } \\
\text { prespecified reported, outcomes } \\
\text { reported incompletely. }\end{array}$ & \\
\hline & Uncertain & Could not be ascertained. & \\
\hline \multirow{4}{*}{$\begin{array}{l}\text { 17. Funding source } \\
\text { identified }\end{array}$} & No & & \multirow{3}{*}{$\begin{array}{l}\text { Industry, government, } \\
\text { university, Foundation } \\
\text { (funded by what money } \\
\text { source?) }\end{array}$} \\
\hline & Yes & Who provided funding? & \\
\hline & Uncertain & & \\
\hline & \multicolumn{2}{|c|}{ Overall Assessment } & \\
\hline \multirow[t]{3}{*}{$\begin{array}{l}\text { 18. Overall Risk of } \\
\text { Bias assessment }\end{array}$} & Low & $\begin{array}{l}\text { Results are believable taking } \\
\text { study limitations into } \\
\text { consideration }\end{array}$ & \\
\hline & Moderate & $\begin{array}{l}\text { Results are probably believable } \\
\text { taking study limitations into } \\
\text { consideration }\end{array}$ & \\
\hline & High & $\begin{array}{l}\text { Results are uncertain taking } \\
\text { study limitations into } \\
\text { consideration }\end{array}$ & \\
\hline
\end{tabular}

B-3 


\section{Appendix C. Outcomes}

Appendix Table C1. Outcomes used in bipolar treatment studies

\begin{tabular}{|c|c|c|c|}
\hline Outcome/measure & $\begin{array}{l}\text { Version (if } \\
\text { applicable) }\end{array}$ & $\begin{array}{c}\text { Operationalization (other than total score, if } \\
\text { applicable) }\end{array}$ & Source \\
\hline $\begin{array}{l}\text { Altman Self Rating } \\
\text { Mania Scale (ASRM) }\end{array}$ & & & $\begin{array}{l}\text { Altman EG, Hedeker D, Peterson JL, Davis } \\
\text { JM: The Altman Self-Rating Mania Scale. Biol } \\
\text { Psychiatry 1997, 42:948-955. }\end{array}$ \\
\hline $\begin{array}{l}\text { Beck Anxiety } \\
\text { Inventory (BAl) }\end{array}$ & & & $\begin{array}{l}\text { Beck AT, Epstein N, Brown G, et al. An } \\
\text { inventory for measuring clinical anxiety: } \\
\text { psychometric properties. J Consult Clin } \\
\text { Psychol. 1988;56(6):893-897. }\end{array}$ \\
\hline \multirow[t]{2}{*}{$\begin{array}{l}\text { Beck Depression } \\
\text { Inventory (BDI) }\end{array}$} & BDI & & $\begin{array}{l}\text { Beck AT, Ward CH, Mendelson M, et al. An } \\
\text { inventory for measuring depression. Arch } \\
\text { Gen Psychiatry. 1961;4(6):561-571. }\end{array}$ \\
\hline & BDI-II & & $\begin{array}{l}\text { Beck AT, Steer RA, Brown GK. Manual for } \\
\text { Beck Depression Inventory II (BDI-II). } \\
\text { San Antonio, TX: Psychological Corp; } 1996 .\end{array}$ \\
\hline \multicolumn{4}{|l|}{$\begin{array}{l}\text { Beck Scale for } \\
\text { Suicide Ideation }\end{array}$} \\
\hline $\begin{array}{l}\text { Bipolar Self-efficacy } \\
\text { Scale }\end{array}$ & & & $\begin{array}{l}\text { Centre for Clinical Interventions (2008) } \\
\text { Bipolar } \\
\text { Self Efficacy Scale. Centre for Clinical } \\
\text { Interventions, Perth, Australia. }\end{array}$ \\
\hline $\begin{array}{l}\text { Brief Psychiatric } \\
\text { Rating Scale (BPRS) }\end{array}$ & & & $\begin{array}{l}\text { Overall J, Gorham D. The brief psychiatric } \\
\text { rating scale. Psychol Rep 1962; 10: 799-812. }\end{array}$ \\
\hline $\begin{array}{l}\text { Brief Symptom } \\
\text { Inventory (BSI) }\end{array}$ & & & $\begin{array}{l}\text { Beurs E de, Zitman FG: De Brief Symptom } \\
\text { Inventory (BSI): De betrouwbaarheid en } \\
\text { validiteit van een handzaam alternatief voor } \\
\text { de SCL-90. Maandblad Geestelijke } \\
\text { Volksgezondheid 2006, 61:120-141. }\end{array}$ \\
\hline \multirow[t]{2}{*}{$\begin{array}{l}\text { Clinical Global } \\
\text { Impressions }\end{array}$} & CGI & Minimally important difference: 1 point & $\begin{array}{l}\text { Lukasiewicz et al. Young mania rating scale: } \\
\text { how to interpret the numbers? International } \\
\text { Journal of Methods in Psychiatric Research. } \\
\text { Int. J. Methods Psychiatr. Res. 22(1): 46-58 } \\
\text { (2013). DOI: } 10.1002 / \text { mpr.1379 }\end{array}$ \\
\hline & $\begin{array}{l}\text { Scale for Bipolar } \\
\text { Disorder (CGI-BP) }\end{array}$ & mania score only (20728318) & $\begin{array}{l}\text { Spearing MK, Post RM, Leverich GS, et al. } \\
\text { Modification of the Clinical Global } \\
\text { Impressions (CGI) Scale for use in bipolar }\end{array}$ \\
\hline
\end{tabular}




\begin{tabular}{|c|c|c|c|}
\hline Outcome/measure & $\begin{array}{l}\text { Version (if } \\
\text { applicable) }\end{array}$ & $\begin{array}{c}\text { Operationalization (other than total score, if } \\
\text { applicable) }\end{array}$ & Source \\
\hline & $\begin{array}{l}\text { Bipolar disorder- } \\
\text { Severity (CGI-BP-S) }\end{array}$ & mania score only (18835043) & $\begin{array}{l}\text { illness (BP): the CGI-BP. Psychiatry Res } \\
\text { 1997; 73:159-171. }\end{array}$ \\
\hline & $\begin{array}{l}\text { Severity of IIIness } \\
\text { Scale (CGI-S) }\end{array}$ & & \multirow{3}{*}{$\begin{array}{l}\text { Guy, W. (Ed.), 1976. ECDEU Assessment } \\
\text { Manual for Psychopharmacology: Publication } \\
\text { ADM 76-338. US Department of Health, } \\
\text { Education, and Welfare, Washington, DC, pp. } \\
218-222\end{array}$} \\
\hline & $\begin{array}{l}\text { Improvement scale } \\
(\mathrm{CGI}-\mathrm{I})\end{array}$ & & \\
\hline & $\begin{array}{l}\text { Change scale (CGI- } \\
\text { C) }\end{array}$ & & \\
\hline $\begin{array}{l}\text { Functioning } \\
\text { Assessment Short } \\
\text { Test }\end{array}$ & & & $\begin{array}{l}\text { Rosa AR, Sánchez-Moreno J, Martínez-Arán } \\
\text { A, Salamero M, Torrent C, Reinares M, } \\
\text { Comes M, Colom F, Van Riel W, Ayuso- } \\
\text { Mateos JL, Kapczinski F, Vieta E: Validity and } \\
\text { reliability of the } \\
\text { Functioning Assessment Short Test (FAST) in } \\
\text { bipolar disorder. Clin Pract Epidemiol Ment } \\
\text { Health 2007; 3:5 }\end{array}$ \\
\hline $\begin{array}{l}\text { Global Assessment } \\
\text { of Functioning }\end{array}$ & & & $\begin{array}{l}\text { Diagnostic and Statistical Manual ofMental } \\
\text { Disorders-Text Revision (DSM-IV-TR). } \\
\text { 4th ed. Washington, DC: American } \\
\text { Psychiatric Association; } 2000 .\end{array}$ \\
\hline $\begin{array}{l}\text { Hamilton Anxiety } \\
\text { Rating Scale (HARS) }\end{array}$ & & & $\begin{array}{l}\text { Hamilton M. The assessment of anxiety } \\
\text { states by rating. Br J Med Psychol. } \\
\text { 1959;32(1):50-55. }\end{array}$ \\
\hline \multirow[t]{3}{*}{$\begin{array}{l}\text { Hamilton Depression } \\
\text { Rating Scale }\end{array}$} & $\begin{array}{l}\text { HAM-D (also referred } \\
\text { to as HDRS) }\end{array}$ & & \multirow[t]{2}{*}{$\begin{array}{l}\text { Hamilton, M., 1960. A rating scale for } \\
\text { depression. J. Neurol. Neurosurg. Psychiatry } \\
\text { 3, 62-66. }\end{array}$} \\
\hline & HAMD-17 & & \\
\hline & HSRD-25 & & $\begin{array}{l}\text { Thase ME, Carpenter L, Kupfer DJ, Frank EF. } \\
\text { Clinical significance of reversed vegetative } \\
\text { subtypes of recurrent major depression. } \\
\text { Psychopharmacol Bull 1991; } 27: 17-22 .\end{array}$ \\
\hline
\end{tabular}




\begin{tabular}{|c|c|c|c|}
\hline Outcome/measure & $\begin{array}{l}\text { Version (if } \\
\text { applicable) }\end{array}$ & $\begin{array}{l}\text { Operationalization (other than total score, if } \\
\text { applicable) }\end{array}$ & Source \\
\hline & HAMD-28 & $\begin{array}{l}\text { "with embedded 'typical' HAM-D } 17 \text { and 'atypical' HAM-D } \\
\text { 17-R symptom cores" (18486235); } \\
\text { Proportion of responders with a >50\% reduction in } \\
\text { baseline HAM-D (18486235); } \\
\text { Proportion of remitters with a final HAM-D score <=8 } \\
\text { (18486235) }\end{array}$ & $\begin{array}{l}\text { Williams, J.B.W., 1988. A structured interview } \\
\text { guide for the Hamilton Depression Rating } \\
\text { Scale. Arch. Gen. Psychiatry 45, 742-747. } \\
\text { Reimherr, F.W., Amsterdam, J.D., Fawcett, } \\
\text { J., Quitkin, F.M., Rosenbaum, J., Beasley, C., } \\
\text { Michelson, D., Roback, P., Sundell, K., 1998. } \\
\text { Optimal length of continuation therapy: a } \\
\text { prospective assessment during fluoxetine } \\
\text { long-term treatment. Am. J. Psychiatry 55, } \\
\text { 1247-1253. }\end{array}$ \\
\hline $\begin{array}{l}\text { Inventory for } \\
\text { Depressive } \\
\text { Symptomology (IDS) }\end{array}$ & & & $\begin{array}{l}\text { Rush AJ, Gullion CM, Basco MR, et al. The } \\
\text { Inventory of Depressive Symptomatology } \\
\text { (IDS): psychometric properties. Psychol Med } \\
\text { 1996; 26:477-486. }\end{array}$ \\
\hline $\begin{array}{l}\text { Life Chart Method } \\
\text { (NIMH-LCM) }\end{array}$ & & & $\begin{array}{l}\text { Denicoff KD, Leverich GS, Nolen WA, Rush } \\
\text { AJ, McElroy SL, Keck PE, et al: Validation of } \\
\text { the prospective NIMH-Life-Chart Method } \\
\text { (NIMH-LCM-p) for longitudinal assessment of } \\
\text { bipolar illness. Psychol Med 2000, 30:1391- } \\
\text { 1397. }\end{array}$ \\
\hline $\begin{array}{l}\text { Longitudinal Interval } \\
\text { Follow-up Evaluation }\end{array}$ & & & $\begin{array}{l}\text { Keller MB, Lavori PW, Friedman B, et al. The } \\
\text { Longitudinal Interval Follow-up Evaluation. A } \\
\text { comprehensive method for assessing } \\
\text { outcome in prospective longitudinal studies. } \\
\text { Arch Gen Psychiatry 1987;44:540-548. }\end{array}$ \\
\hline $\begin{array}{l}\text { Montgomery-Asberg } \\
\text { Depression Rating } \\
\text { Scale (MADRS) }\end{array}$ & & Individual item scores (22868059) & $\begin{array}{l}\text { Montgomery, S.A., Asberg, M., 1979. A new } \\
\text { depression scale designed to be sensitive to } \\
\text { change. Br. J. Psychiatry } 134,382-389 \text {. }\end{array}$ \\
\hline $\begin{array}{l}\text { Positive and } \\
\text { Negative Syndrome } \\
\text { Scale (PANSS) }\end{array}$ & & Cognitive and Hostility subscales (18835043) & $\begin{array}{l}\text { Kay, S.R., Fiszbein, A., Opler, L.A., } 1987 . \\
\text { The positive and negative syndrome scale for } \\
\text { schizophrenia. Schizophr. Bull. 13, 261-276. }\end{array}$ \\
\hline $\begin{array}{l}\text { Quality of Life, } \\
\text { Enjoyment, and } \\
\text { Satisfaction } \\
\text { Questionnaire }\end{array}$ & & & $\begin{array}{l}\text { Endicott J, Nee J, Harrison W, et al. Quality of } \\
\text { Life Enjoyment and Satisfaction } \\
\text { Questionnaire: a new measure. } \\
\text { Psychopharmacol Bull 1993; 29:321-326. }\end{array}$ \\
\hline
\end{tabular}




\begin{tabular}{|c|c|c|c|}
\hline Outcome/measure & $\begin{array}{l}\text { Version (if } \\
\text { applicable) }\end{array}$ & $\begin{array}{l}\text { Operationalization (other than total score, if } \\
\text { applicable) }\end{array}$ & Source \\
\hline $\begin{array}{l}\text { Quality of Life Scale } \\
\text { of the World Health } \\
\text { Organization Quality } \\
\text { of Life Assessment }\end{array}$ & WHOQOL-BREF & & $\begin{array}{l}\text { Fleck MP, Louzada S, Xavier M et al. } \\
\text { Application of the Portuguese version of the } \\
\text { abbreviated instrument of quality life } \\
\text { WHOQOL-bref. Rev Saude Publica } \\
\text { 2000;34:178-183. }\end{array}$ \\
\hline $\begin{array}{l}\text { Quick Inventory of } \\
\text { Depressive } \\
\text { Symptoms (QIDS) }\end{array}$ & $\begin{array}{l}\text { Self-report (QIDS- } \\
\text { SR) }\end{array}$ & & $\begin{array}{l}\text { Rush AJ, Trivedi MH, Ibrahim HM, et al. The } \\
\text { 16-Item Quick Inventory of Depressive } \\
\text { Symptomatology (QIDS), clinician rating } \\
\text { (QIDS-C), and self-report (QIDS-SR): a } \\
\text { psychometric evaluation in patients with } \\
\text { chronic major depression. Biol Psychiatry } \\
\text { 2003;54:573-583. }\end{array}$ \\
\hline $\begin{array}{l}\text { Rathus } \\
\text { Assertiveness } \\
\text { Schedule (RAS) }\end{array}$ & & & $\begin{array}{l}\text { Rathus, S.A., 1973. A } 30 \text { item schedule for } \\
\text { assessing assertiveness. Behavior Therapy } \\
4, \\
398-406 .\end{array}$ \\
\hline $\begin{array}{l}\text { Sheehan Disability } \\
\text { Scale (SDS) }\end{array}$ & & & $\begin{array}{l}\text { In Sheehan DV. The Anxiety Disease. New } \\
\text { York, NY: Charles Scribner; 1983:151. }\end{array}$ \\
\hline $\begin{array}{l}\text { Short-Form Health } \\
\text { Survey }\end{array}$ & SF-12v2 & & $\begin{array}{l}\text { Ware J. (1994) SF-36 Health Survey Manual } \\
\text { and } \\
\text { Interpretation Guide. The Health Institute, } \\
\text { Boston, MA. }\end{array}$ \\
\hline $\begin{array}{l}\text { Social Adjustment } \\
\text { Scale Self Report } \\
\text { (SAS-SR) }\end{array}$ & & & $\begin{array}{l}\text { Weissman MM, Bothwell S. Assessment of } \\
\text { social adjustment by patient self-report. Arch } \\
\text { Gen Psychiatry 1976;33:1111-1115. }\end{array}$ \\
\hline $\begin{array}{l}\text { STEP-BD Clinical } \\
\text { Monitoring Form }\end{array}$ & & & $\begin{array}{l}\text { Sachs GS, Guille C, McMurrich SL. A clinical } \\
\text { monitoring form for mood disorders. Bipolar } \\
\text { Disord. 2002;4:323-327. }\end{array}$ \\
\hline $\begin{array}{l}\text { STEP-BD Affective } \\
\text { Disorders Evaluation }\end{array}$ & & & $\begin{array}{l}\text { Sachs GS, Thase ME, Otto MW, et al. } \\
\text { Rationale, design, and methods of the } \\
\text { systematic treatment enhancement program } \\
\text { for bipolar disorder (STEP-BD). Biol } \\
\text { Psychiatry. 2003;53:1028-1042. }\end{array}$ \\
\hline Symptom Check List & SCL-90 & Used only depression and mania subscales (22070452) & $\begin{array}{l}\text { Derogatis L. \& Cleary P. (1977) Confirmation } \\
\text { of } \\
\text { the dimensional structure of the SCL-90: a } \\
\text { study } \\
\text { in construct validation. Journal of Clinical } \\
\text { Psychology 33, 981-989. }\end{array}$ \\
\hline
\end{tabular}




\begin{tabular}{|c|c|c|c|}
\hline Outcome/measure & $\begin{array}{l}\text { Version (if } \\
\text { applicable) }\end{array}$ & $\begin{array}{l}\text { Operationalization (other than total score, if } \\
\text { applicable) }\end{array}$ & Source \\
\hline \multirow[t]{2}{*}{$\begin{array}{l}\text { Young Mania Rating } \\
\text { Scale }\end{array}$} & YMRS & $\begin{array}{l}\text { Proportion of patients with YMRS >=8 at any time during } \\
\text { treatment }(23609385) \\
\text { Individual item scores }(22868059,22134043)\end{array}$ & $\begin{array}{l}\text { Young, R.C., Biggs, J.T., Ziegler, V.E., } \\
\text { Meyer, D.A., 1978. A rating scale for mania: } \\
\text { reliability, validity and sensitivity. Br. J. } \\
\text { Psychiatry } 61,638-642 .\end{array}$ \\
\hline & & Minimally important difference: 6 points & $\begin{array}{l}\text { Lukasiewicz et al. Young mania rating scale: } \\
\text { how to interpret the numbers? International } \\
\text { Journal of Methods in Psychiatric Research. } \\
\text { Int. J. Methods Psychiatr. Res. 22(1): 46-58 } \\
\text { (2013). DOI: } 10.1002 / \mathrm{mpr} .1379\end{array}$ \\
\hline $\begin{array}{l}\text { Change in mood } \\
\text { status }\end{array}$ & & $\begin{array}{l}\text { "Participants had syndromal or subsyndromal depressive } \\
\text { symptoms, as defined in the DSM-IV-TR or STEP-BD, } \\
\text { respectively. Subsyndromal symptoms consisted of } \\
\text { having }>2 \text { pervasive DSM-IV-TR depressive symptoms } \\
\text { but not meeting criteria for a DSM-IV-TR syndromal } \\
\text { depressive, hypomanic, manic, or mixed episode" } \\
(21318192)\end{array}$ & \\
\hline
\end{tabular}




\begin{tabular}{|c|c|c|c|}
\hline Outcome/measure & $\begin{array}{l}\text { Version (if } \\
\text { applicable) }\end{array}$ & $\begin{array}{l}\text { Operationalization (other than total score, if } \\
\text { applicable) }\end{array}$ & Source \\
\hline Relapse & & $\begin{array}{l}\text { "(1) a YMRS score higher than } 14 \text { or a MADRS score } \\
\text { higher than 15; (2) } 20 \% \text { or greater increase in the YMRS } \\
\text { or MADRS scores from the previous study visit for } \\
\text { patients with a MADRS score of } 10 \text { or higher or a YMRS } \\
\text { score of } 8 \text { or higher at the current study visit; (3) urgent } \\
\text { care visit/referral (psychiatric hospitalization; emergency } \\
\text { department visit; or referral for respite care, partial } \\
\text { hospitalization, or intensive outpatient treatment) due to } \\
\text { worsening mood symptoms; (4) a Clinical Global } \\
\text { Impression-Severity of Illness score of } 4 \text { or higher; (5) } \\
\text { syndromal relapse (Diagnostic and Statistical Manual of } \\
\text { Mental Disorders, 4th Edition, } \\
\text { Text Revision criteria for manic, hypomanic, major } \\
\text { depressive, or mixed episode met); (6) withdrawal from } \\
\text { the study due to inefficacy; and (7) necessary clinical } \\
\text { medication adjustments (NCAs) [change in medication } \\
\text { treatment intended to prevent } \\
\text { worsening of clinical symptoms, and included addition of } \\
\text { a new mood stabilizer, antipsychotic, or antidepressant } \\
\text { [other than the experimental treatment]; substitution of } \\
\text { an existing bipolar medication with a new drug; and/or a } \\
20 \% \text { or greater change in dose of any current medication } \\
\text { (excluding routine increases in dose due to titration).]." } \\
\text { (22104634) } \\
\text { "Relapse or recurrence of a manic or depressive episode } \\
\text { defined as a score >=4 (at least moderate symptoms) on } \\
\text { the CGI-BP severity of depression or mania scale" } \\
\text { (21320258) }\end{array}$ & \\
\hline
\end{tabular}




\begin{tabular}{|c|c|c|c|}
\hline Outcome/measure & $\begin{array}{l}\text { Version (if } \\
\text { applicable) }\end{array}$ & $\begin{array}{l}\text { Operationalization (other than total score, if } \\
\text { applicable) }\end{array}$ & Source \\
\hline $\begin{array}{l}\text { First recurrence of } \\
\text { any mood symptoms }\end{array}$ & & $\begin{array}{l}\text { "YMRS } \geq 15 \text { and Clinical Global Impression- } \\
\text { Bipolar Disorder-Severity of Illness Scale (CGI-BP-S) for } \\
\text { Mania } \geq 4 \text {; YMRS < } 15 \text {, MADRS } \geq 16 \text { and CGI-BP-S for } \\
\text { depression } \geq 4 \text {; voluntary or involuntary hospitalization for } \\
\text { any mood symptoms; therapeutic intervention } \\
\text { to prevent or treat an impending mood episode (i.e., use } \\
\text { of benzodiazepines for }>3 \text { consecutive days; } \\
\text { supplementation with an antipsychotic, antidepressant, } \\
\text { or mood stabilizer; another therapeutic measure [e.g., } \\
\text { psychotherapy or electroconvulsive therapy]); any other } \\
\text { clinically relevant event suggestive of a recurrent mood } \\
\text { episode." (22377512) } \\
\text { "ITT analysis of the prevalence .... of modd symptoms } \\
\text { (comparing any depressive or manic symptoms vs. none } \\
\text { over the first year)" (20409444) }\end{array}$ & \\
\hline Time to recurrence & & $\begin{array}{l}\text { "time (days) elapsed between baseline and the } \\
\text { emergence of a new acute episode according to DSM- } \\
\text { IV criteria and scores above or equal to } 20 \text { on the YMRS } \\
\text { for manic recurrence; above or equal to } 12 \text { for } \\
\text { hypomanic recurrence; above or equal to } 17 \text { on the } \\
\text { HRSD-17 for depressive recurrence; and above or equal } \\
\text { to } 20 \text { on the YMRS and } 12 \text { on the HRSD-17 for mixed } \\
\text { recurrence." (19252157) } \\
\text { "any of the following: } 1 \text { ) fulfilled DSM-IV-TR criteria for } \\
\text { a manic, hypomanic, mixed, or depressive episode; } 2 \text { ) } \\
\text { required treatment intervention with any mood stabilizer, } \\
\text { antipsychotic medication (other than study drug), } \\
\text { benzodiazepine (beyond the dosage allowed), or } \\
\text { antidepressant medication; } 3 \text { ) hospitalization for any } \\
\text { bipolar mood episode; } 4 \text { ) had YMRS score }>12 \text {, } \\
\text { Montgomery Assberg Depression Rating Scale (MADRS) } \\
\text { score }>12 \text {, or CGI-S scale score }>4 \text { at any visit; or } 5 \text { ) } \\
\text { needed an increase in risperidone LAI dosage or } \\
\text { supplementation with oral risperidone". (20227682) } \\
\text { Weeks to first new episode, weeks to first depressive } \\
\text { episode, weeks to first manic episode (20409444) }\end{array}$ & \\
\hline $\begin{array}{l}\text { Number of } \\
\text { recurrences }\end{array}$ & & See "time to recurrence" definition for 19252157 & \\
\hline Time spent ill & & $\begin{array}{l}\text { Prospectively measured number of days participants met } \\
\text { criteria listed under "time to recurrence" for } 19252157\end{array}$ & \\
\hline
\end{tabular}




\begin{tabular}{|c|c|c|c|}
\hline Outcome/measure & $\begin{array}{l}\text { Version (if } \\
\text { applicable) }\end{array}$ & $\begin{array}{c}\text { Operationalization (other than total score, if } \\
\text { applicable) }\end{array}$ & Source \\
\hline $\begin{array}{l}\text { Emergent } \\
\text { depression }\end{array}$ & & $\begin{array}{l}\text { MADRS Total score }>=18 \text { and }>=4 \text { point increase from } \\
\text { baseline for any two consecutive assessments } \\
(18835043)\end{array}$ & \\
\hline $\begin{array}{l}\text { Full syndromal } \\
\text { depressive relapse }\end{array}$ & & $\begin{array}{l}\text { Increase in baseline HAM-D score to }>=14 \text { and met } \\
\text { criteria for a major depressive episode ( } 20360317)\end{array}$ & $\begin{array}{l}\text { Reimherr FW, Amsterdam JD, Quitkin FM, } \\
\text { Rosenbaum JF, Fava M, Zajecka J, Beasley } \\
\text { DM Jr, Michelson D, Roback P, Sundell K: } \\
\text { Optimal length of continuation therapy in } \\
\text { depression: a prospective assessment during } \\
\text { long-term fluoxetine treatment. Am J } \\
\text { Psychiatry 1998; 155:1247-1253 }\end{array}$ \\
\hline $\begin{array}{l}\text { Severe depressive } \\
\text { symptoms }\end{array}$ & & BDI score > 18 & \\
\hline $\begin{array}{l}\text { Subsyndromal } \\
\text { depressive episode }\end{array}$ & & $\begin{array}{l}\text { Any increase in baseline HAM-D score without meeting } \\
\text { criteria for major depressive episode (20360317) }\end{array}$ & \\
\hline $\begin{array}{l}\text { Switch to depressive } \\
\text { symptoms }\end{array}$ & & $\begin{array}{l}\text { HAMD-17>=13 in patients with HAMD-17<=7 at baseline } \\
(22134043)\end{array}$ & \\
\hline $\begin{array}{l}\text { Time to first } \\
\text { recurrence of } \\
\text { depressive } \\
\text { symptoms }\end{array}$ & & $\begin{array}{l}\text { YMRS }<15, \text { MADRS } \geq 16 \text { and CGI-BP-S for } \\
\text { depression } \geq 4(22377512) \\
\text { "how long the MADRS score was } \\
\text { maintained at }<50 \% \text { of its baseline value after } \\
\text { patients reached this level for the first time during } \\
\text { phase } 1 \text { or } 2 . \text {." ( } 21320258)\end{array}$ & \\
\hline Hypomania & & $\begin{array}{l}\text { DSM-IV criteria: episode lasting >=4 days with >=4 } \\
\text { symptoms (20360317, } 23609385) ; \\
\text { YMRS score cut-points of >=8 and >=12 at any study } \\
\text { visit ( } 20360317)\end{array}$ & \\
\hline Moderate mania & & YMRS $>6$ & \\
\hline $\begin{array}{l}\text { Remission of manic } \\
\text { symptoms }\end{array}$ & & YMRS<=12 (22134043) & \\
\hline $\begin{array}{l}\text { Subsyndromal } \\
\text { hypomania }\end{array}$ & & $\begin{array}{l}\text { Episode lasting }<=3 \text { days with }>=4 \text { symptoms } \\
(20360317,23609385) \\
\text { Episode lasting }>=4 \text { days with }<=3 \text { symptoms } \\
\text { (20360317, } 23609385) \\
\text { Episode lasting }<=3 \text { days with }<=3 \text { symptoms } \\
\text { (23609385) }\end{array}$ & \\
\hline $\begin{array}{l}\text { Switch to } \\
\text { mania/hypomania }\end{array}$ & & $\begin{array}{l}\text { "Need to discontinue antidepressant medication because } \\
\text { of the emergence of manic symptoms" (19358785) }\end{array}$ & \\
\hline
\end{tabular}




\begin{tabular}{|c|c|c|c|}
\hline Outcome/measure & $\begin{array}{l}\text { Version (if } \\
\text { applicable) }\end{array}$ & $\begin{array}{c}\text { Operationalization (other than total score, if } \\
\text { applicable) }\end{array}$ & Source \\
\hline $\begin{array}{l}\text { Time to first } \\
\text { recurrence of manic } \\
\text { symptoms }\end{array}$ & & $\begin{array}{l}\text { YMRS } \geq 15 \text { and Clinical Global Impression- } \\
\text { Bipolar Disorder-Severity of Illness Scale (CGI-BP-S) for } \\
\text { Mania } \geq 4(22377512)\end{array}$ & \\
\hline $\begin{array}{l}\text { Adherence to } \\
\text { treatment }\end{array}$ & & $\begin{array}{l}\text { "Adherence was assessed by the combination } \\
\text { of an adherence-focused interview with the individual, an } \\
\text { adherence-focused interview with significant first-degree } \\
\text { relatives or a partner and by analyses of plasma } \\
\text { concentrations of mood stabilisers" (19252157) } \\
\text { "Adherence with study medication was evaluated with } \\
\text { returned capsule count. Patients who missed >=5 } \\
\text { consecutive days of study medication were considered } \\
\text { nonadherent and discontinued from the trial." } \\
\text { (20361901) }\end{array}$ & $\begin{array}{l}\text { Fully described in Simon GE, Ludman EJ, } \\
\text { Bauer MS, Unutzer J, Operskalski B. Long- } \\
\text { term effectiveness and cost of a systematic } \\
\text { care program for bipolar disorder. Arch Gen } \\
\text { Psychiatry 2006; 63: 500-8. }\end{array}$ \\
\hline Hospitalizations & & Hospitalized "owing to recurrence" (19252157) & \\
\hline $\begin{array}{l}\text { Necessary clinical } \\
\text { adjustments }\end{array}$ & & $\begin{array}{l}\text { "a proxy for the clinical burden of a treatment strategy } \\
\text { and capture the treatment changes that result from lack } \\
\text { of efficacy or safety issues. They consist of all } \\
\text { medication adjustments necessary to respond to clinical } \\
\text { need, such as exacerbation of mood symptoms, } \\
\text { emergence of a mood episode, persistence of } \\
\text { symptoms, or adjustments because of adverse effects. } \\
\text { Necessary clinical adjustments do not include } \\
\text { planned dosage titrations or decreases in dosage based } \\
\text { on the assessment of positive responses or the } \\
\text { judgment that a medication is no longer required" } \\
\text { (23288387) }\end{array}$ & $\begin{array}{l}\text { Nierenberg AA, Sylvia LG, Leon AC, Reilly- } \\
\text { Harrington NA, Ketter TA, Calabrese JR, } \\
\text { Thase ME, Bowden CL, Friedman ES, } \\
\text { Ostacher MJ, Novak L, Iosifescu DV; Litmus } \\
\text { Study Group: Lithium Treatment Moderate- } \\
\text { Dose Use Study (LiTMUS) for bipolar } \\
\text { disorder: rationale and design. Clin Trials } \\
\text { 2009; 6:637-648 }\end{array}$ \\
\hline $\begin{array}{l}\text { Response to } \\
\text { treatment }\end{array}$ & Positive & $\begin{array}{l}\text { 50\%+ improvement on IDS from baseline or 2+ point } \\
\text { decrease in CGI-BP from baseline (19358785) } \\
>=50 \% \text { reduction in total HAMD-17 and final HAMD-17 } \\
\text { score >=9 at treatment week } 10 \text { ( } 23609385) \\
>=50 \% \text { reduction from baseline in YMRS and MADRS } \\
(22868059) \\
>=50 \% \text { reduction from baseline in YMRS }(20947174, \\
22377512,20624657,22134043,20361901,20728318, \\
18835043) \\
50 \%+\text { improvement on HDRS, BDI, QIDS-SR, or CGI-I } \\
(22579164) \\
>=50 \% \text { change in HDRS score (22213770) } \\
\text { CGI-BP improvement of depression score of } 1 \text { or } 2 \text { (very } \\
\text { much improved or much improved) (21320258) }\end{array}$ & \\
\hline
\end{tabular}




\begin{tabular}{|c|c|c|c|}
\hline Outcome/measure & $\begin{array}{l}\text { Version (if } \\
\text { applicable) }\end{array}$ & $\begin{array}{l}\text { Operationalization (other than total score, if } \\
\text { applicable) }\end{array}$ & Source \\
\hline & Partial & $\begin{array}{l}\text { Pt judged partial improvement, but did not meet criteria } \\
\text { for "positive response" (19358785) }\end{array}$ & \\
\hline & Nonresponse & $\begin{array}{l}<50 \% \text { reduction in HAMD-17 by treatment week } 10 \\
(23609385) \\
\text { Exiting study or }<50 \% \text { improvement on HDRS } \\
(22213770)\end{array}$ & \\
\hline Remission & & $\begin{array}{l}\text { IDS }<12 \text { OR CGI-BP depression severity of } 1 \\
(19358785) \\
>=50 \% \text { reduction in total HAMD-17 and final HAMD-17 } \\
\text { score }<=8 \text { at treatment week } 6,8 \text {, or } 10 \text {, maintained for } 2 \\
\text { weeks }(23609385) \\
\text { YMRS }<=12 \text { and HAMD-17 }<=7(22134043) \\
\text { YMRS }<=12 \text { and MADRS }<=10(22868059) \\
\text { YMRS }<=12 \text { and MADRS }<=12(22377512) \\
\text { YMRS }<=12 \text { (20947174, } 20624657,18835043) \\
\text { CGI-I outcome "remission", HDRS }<=7 \text {, QIDS-SR }<=5 \text {, } \\
\text { or BDI <=9 (22579164) } \\
\text { HDRS }<8(22213770) \\
\text { CGI-BP-S }<2(23288387)\end{array}$ & \\
\hline Laboratory tests & & Serum Lithium levels (23288387) & \\
\hline $\begin{array}{l}\text { Time to early } \\
\text { discontinuation from } \\
\text { study medication }\end{array}$ & & $\begin{array}{l}\text { "Time to early discontinuation from study medication for } \\
\text { any reason (including recurrence), before termination of } \\
\text { the study when at least } 114 \text { recurrences were observed, } \\
\text { was also analyzed." }\end{array}$ & \\
\hline $\begin{array}{l}\text { Time to onset of } \\
\text { therapeutic effect }\end{array}$ & & $\begin{array}{l}\text { "the first time point at which [the treatment group] was } \\
\text { statistically significantly different from the [comparison } \\
\text { treatment] for the change from baseline in the YMRS } \\
\text { total score and remained different thereafter until } \\
\text { endpoint" (20947174, 20624657) }\end{array}$ & \\
\hline
\end{tabular}




\title{
Appendix D. Excluded References
}

\author{
Excluded References ${ }^{1-957}$
}

1. Aarre TF, Dahl AA. Pharmacotherapy for bipolar depression: A review of the evidence. Current Psychiatry Reviews. 2008 2008;4(3):145-56. doi: http://dx.doi.org/10.2174/157340008785829913. Ineligible study design

2. Agosti V, Stewart JW. Efficacy and safety of antidepressant monotherapy in the treatment of bipolar-II depression. International Clinical Psychopharmacology. 2007 Sep;22(5):309-11. PMID: 17690600 Ineligible study design

3. Akhondzadeh S, Mohajari H, Reza Mohammadi M, et al. Ritanserin as an adjunct to lithium and haloperidol for the treatment of medication-naive patients with acute mania: a double blind and placebo controlled trial. BMC Psychiatry. 2003 Jun 19;3:7. PMID: 12816549 Bipolar not analyzed separately

4. Akiskal HS, Akiskal KK, Lancrenon S, et al. Validating the soft bipolar spectrum in the French National EPIDEP Study: the prominence of BP-II 1/2. Journal of Affective Disorders. 2006 Dec;96(3):207-13. PMID: 16647762 Ineligible study design

5. Albert U, Maina G, Aguglia A, et al. Vagus nerve stimulation for treatment-resistant mood disorders: a long-term naturalistic study. BMC Psychiatry. 2015 Mar 31;15:64. doi: https://dx.doi.org/10.1186/s12888015-0435-8. PMID: 25884606 Less than 11 subjects per arm

6. Alda M, McKinnon M, Blagdon R, et al. Methylene blue treatment for residual symptoms of bipolar disorder: Randomised crossover study. British Journal of Psychiatry. 2017 January;210(1):54-60. doi: http://dx.doi.org/10.1192/bjp.bp.115.173930. PMID: 614051189 Ineligible intervention

7. Allan SJ, Kavanagh GM, Herd RM, et al. The effect of inositol supplements on the psoriasis of patients taking lithium: a randomized, placebo-controlled trial. British Journal of Dermatology. 2004

May;150(5):966-9. PMID: 15149510 Not bipolar disorder

8. Allen MH, Hirschfeld RM, Wozniak PJ, et al. Linear relationship of valproate serum concentration to response and optimal serum levels for acute mania. American Journal of Psychiatry. 2006 Feb;163(2):2725. PMID: 16449481 No eligible outcomes reported

9. Altinbas K, Ozerdem A, Prieto ML, et al. A multinational study to pilot the modified Hypomania Checklist (mHCL) in the assessment of mixed depression. Journal of Affective Disorders. 2014 Jan;152-154:478-82. doi: http://dx.doi.org/10.1016/j.jad.2013.07.032. PMID: 24070907 Ineligible study design

10. Altshuler LL, Frye MA, Gitlin MJ. Acceleration and augmentation strategies for treating bipolar depression. Biological Psychiatry. 2003 Apr 15;53(8):691-700. PMID: 12706955 Ineligible study design

11. Altshuler LL, Suppes T, Black DO, et al. Lower switch rate in depressed patients with bipolar II than bipolar I disorder treated adjunctively with second-generation antidepressants. American Journal of Psychiatry. 2006 Feb;163(2):313-5. PMID: 16449487 No eligible outcomes reported 
12. Amann B, Born C, Crespo JM, et al. Lamotrigine: when and where does it act in affective disorders? A systematic review. Journal of Psychopharmacology. 2011 Oct;25(10):1289-94. doi: http://dx.doi.org/10.1177/0269881110376695. PMID: 20823080 Ineligible study design

13. Amann B, Grunze H, Vieta E, et al. Antiepileptic drugs and mood stability. Clinical EEG \& Neuroscience: Official Journal of the EEG \& Clinical Neuroscience Society (ENCS). 2007 Apr;38(2):116-23. PMID: 17515177 Ineligible study design

14. Amrollahi Z, Rezaei F, Salehi B, et al. Double-blind, randomized, placebo-controlled 6-week study on the efficacy and safety of the tamoxifen adjunctive to lithium in acute bipolar mania. Journal of Affective Disorders. 2011 Mar;129(1-3):327-31. doi: http://dx.doi.org/10.1016/j.jad.2010.08.015. PMID: 20843556 Bipolar not analyzed separately

15. Amsterdam JD, Brunswick DJ. Antidepressant monotherapy for bipolar type II major depression. Bipolar Disorders. 2003 Dec;5(6):388-95. PMID: 14636362 Ineligible study design

16. Amsterdam JD, Garcia-Espana F, Fawcett J, et al. Efficacy and safety of fluoxetine in treating bipolar II major depressive episode. Journal of Clinical Psychopharmacology. 1998 Dec;18(6):435-40. PMID: 9864074 Over $\mathbf{5 0 \%}$ dropout rate

17. Amsterdam JD, Lorenzo-Luaces L, DeRubeis RJ. Step-wise loss of antidepressant effectiveness with repeated antidepressant trials in bipolar II depression. Bipolar Disorders. 201601 Nov;18(7):563-70. doi: http://dx.doi.org/10.1111/bdi.12442. PMID: 613301128 Duplicate reference

18. Amsterdam JD, Luo L, Shults J. Efficacy and mood conversion rate during long-term fluoxetine v. lithium monotherapy in rapid- and non-rapid-cycling bipolar II disorder. The British Journal of Psychiatry. 2013 2013;202(4):301-6. doi: http://dx.doi.org/10.1192/bjp.bp.111.104711. PMID: 23099447 Over 50\% dropout rate

19. Amsterdam JD, Shults J. Fluoxetine monotherapy of bipolar type II and bipolar NOS major depression: a double-blind, placebo-substitution, continuation study. International Clinical Psychopharmacology. 2005 Sep;20(5):257-64. PMID: 16096516 Fewer than 11 subjects per arm

20. Anand A, Gunn AD, Barkay G, et al. Early antidepressant effect of memantine during augmentation of lamotrigine inadequate response in bipolar depression: a double-blind, randomized, placebo-controlled trial. Bipolar Disorders. 2012 Feb;14(1):64-70. doi: http://dx.doi.org/10.1111/j.1399-5618.2011.00971.x. PMID: 22329473 No eligible outcomes reported

21. Anfang MK, Pope HG, Jr. Treatment of neuroleptic-induced akathisia with nicotine patches. Psychopharmacology. 1997 1997;134(2):153-6. doi: http://dx.doi.org/10.1007/s002130050436. No eligible outcomes reported

22. Anonymous. Treatment of special populations with the atypical antipsychotics. Collaborative Working Group on Clinical Trial Evaluations. Journal of Clinical Psychiatry. 1998;59 Suppl 12:46-52. PMID: 9766620 Ineligible study design

23. Anonymous. Emerging therapies for bipolar depression. Journal of Clinical Psychiatry. 2006 Jul;67(7):1140-51. PMID: 17107231 Ineligible study design 
24. Anonymous. Asenapine: a less effective, yet, more dangerous neuroleptic! Prescrire International. 2012 Oct;21(131):229-32. PMID: 23185842 Ineligible study design

25. Anonymous. Correction: Recovery-focused cognitive-behavioural therapy for recent-onset bipolar disorder: Randomised controlled pilot trial (The British Journal of Psychiatry 206 (58-66)). British Journal of Psychiatry. 201501 Feb;206(2):169. doi: http://dx.doi.org/10.1192/bjp.206.2.169. PMID: 602333099 Duplicate reference

26. Arbaizar B, Dierssen-Sotos T, Gomez-Acebo I, et al. Aripiprazole in major depression and mania: metaanalyses of randomized placebo-controlled trials. General Hospital Psychiatry. 2009 Sep-Oct;31(5):478-83. doi: http://dx.doi.org/10.1016/j.genhosppsych.2009.05.005. PMID: 19703642 Ineligible study design

27. Azorin JM, Kaladjian A. An update on the treatment of bipolar depression. Expert Opinion on Pharmacotherapy. 2009 Feb;10(2):161-72. doi: http://dx.doi.org/10.1517/14656560802653172. PMID: 19236191 Ineligible study design

28. Azorin JM, Sapin C, Weiller E. Effect of asenapine on manic and depressive symptoms in bipolar I patients with mixed episodes: results from post hoc analyses. Journal of Affective Disorders. $2013 \mathrm{Feb}$ 15;145(1):62-9. doi: http://dx.doi.org/10.1016/j.jad.2012.07.013. PMID: 22868059 Ineligible study design

29. Azorin J-M, Bowden CL, Garay RP, et al. Possible new ways in the pharmacological treatment of bipolar disorder and comorbid alcoholism. Neuropsychiatric Disease and Treatment. 2010 3, 2010;6(1):37-46.

\section{Ineligible study design}

30. Baek JH, Bernstein EE, Nierenberg AA. One-carbon metabolism and bipolar disorder. Australian and New Zealand Journal of Psychiatry. 2013 2013;47(11):1013-8. doi: http://dx.doi.org/10.1177/0004867413502091. Ineligible study design

31. Baethge C. A bold meta-analysis on suicidality in bipolar disorder. Bipolar Disorders. 2015 Feb;17(1):17-8. doi: http://dx.doi.org/10.1111/bdi.12263. PMID: 25346160 Ineligible study design

32. Baethge C, Baldessarini RJ, Mathiske-Schmidt K, et al. Long-term combination therapy versus monotherapy with lithium and carbamazepine in 46 bipolar I patients. Journal of Clinical Psychiatry. 2005 Feb;66(2):174-82. PMID: 15705002 No eligible outcomes reported

33. Baethge C, Smolka MN, Gruschka P, et al. Does prophylaxis-delay in bipolar disorder influence outcome? Results from a long-term study of 147 patients. Acta Psychiatrica Scandinavica. 2003 Apr;107(4):260-7. PMID: 12662248 Ineligible study design

34. Bailine S, Fink M, Knapp R, et al. Electroconvulsive therapy is equally effective in unipolar and bipolar depression. Acta Psychiatrica Scandinavica. 2010 Jun;121(6):431-6. doi: http://dx.doi.org/10.1111/j.16000447.2009.01493.x. PMID: 19895623 No eligible outcomes reported

35. Bailine SH, Rifkin A, Kayne E, et al. Comparison of bifrontal and bitemporal ECT for major depression. American Journal of Psychiatry. 2000 Jan;157(1):121-3. PMID: 10618025 Bipolar not analyzed separately 
36. Baker RW, Goldberg JF, Tohen M, et al. The impact of response to previous mood stabilizer therapy on response to olanzapine versus placebo for acute mania. Bipolar Disorders. 2002 Feb;4(1):43-9. PMID: 12047494 Ineligible study design

37. Baker RW, Milton DR, Stauffer VL, et al. Placebo-controlled trials do not find association of olanzapine with exacerbation of bipolar mania. Journal of Affective Disorders. 2003 Jan;73(1-2):147-53. PMID: 12507747 Over $\mathbf{5 0 \%}$ dropout rate

38. Baker RW, Tohen M, Fawcett J, et al. Acute dysphoric mania: treatment response to olanzapine versus placebo. Journal of Clinical Psychopharmacology. 2003 Apr;23(2):132-7. PMID: 12640214 Over 50\% dropout rate

39. Baldassano C. Pharmacologic treatment of bipolar disorder. Cns Spectrums. 2010 Feb;15(2 Suppl 3):10-3; discussion 7. PMID: 20414160 Ineligible study design

40. Baldassano CF, Ballas CA, O'Reardon JP. Rethinking the treatment paradigm for bipolar depression: the importance of long-term management. Cns Spectrums. 2004 Sep;9(9 Suppl 9):11-8. PMID: 15361807

\section{Ineligible study design}

41. Baldessarini RJ, Faedda GL, Offidani E, et al. Antidepressant-associated mood-switching and transition from unipolar major depression to bipolar disorder: a review. Journal of Affective Disorders. 2013 May 15;148(1):129-35. doi: http://dx.doi.org/10.1016/j.jad.2012.10.033. PMID: 23219059 Ineligible study design

42. Baldessarini RJ, Hennen J, Wilson M, et al. Olanzapine versus placebo in acute mania: treatment responses in subgroups. Journal of Clinical Psychopharmacology. 2003 Aug;23(4):370-6. PMID: 12920413 Over $50 \%$ dropout rate

43. Baldessarini RJ, Pompili M, Tondo L, et al. Antidepressants and Suicidal Behavior: Are We Hurting Or Helping? [References]. Clinical Neuropsychiatry: Journal of Treatment Evaluation. 2005 2005;2(1):73-5. Ineligible study design

44. Baldessarini RJ, Tondo L. Does lithium treatment still work? Evidence of stable responses over three decades. Archives of General Psychiatry. 2000 Feb;57(2):187-90. PMID: 10665622 Ineligible study design

45. Baldessarini RJ, Tondo L, Davis P, et al. Decreased risk of suicides and attempts during long-term lithium treatment: a meta-analytic review.[Erratum appears in Bipolar Disord. 2007 May;9(3):314]. Bipolar Disorders. 2006 Oct;8(5 Pt 2):625-39. PMID: 17042835 Ineligible study design

46. Ballard ED, Vande Voort JL, Luckenbaugh DA, et al. Acute risk factors for suicide attempts and death: prospective findings from the STEP-BD study. Bipolar Disorders. 201601 Jun;18(4):363-72. doi: http://dx.doi.org/10.1111/bdi.12397. PMID: 611080830 Not treating bipolar

47. Ban TA. Fifty years chlorpromazine: a historical perspective. Neuropsychiatric disease and treatment. 2007;3(4):495-500. PMID: 19300578 Ineligible study design

48. Baptista T, Rangel N, Fernandez V, et al. Metformin as an adjunctive treatment to control body weight and metabolic dysfunction during olanzapine administration: a multicentric, double-blind, placebo-controlled 
trial. Schizophrenia Research. 2007 Jul;93(1-3):99-108. PMID: 17490862 Bipolar not analyzed separately

49. Barak Y, Kimhi R, Weizman R. Is selectivity for serotonin uptake associated with a reduced emergence of manic episodes in depressed patients? International Clinical Psychopharmacology. 2000 Jan;15(1):53-6.

PMID: 10836288 Not bipolar disorder

50. Barbini B, Colombo C, Benedetti F, et al. The unipolar-bipolar dichotomy and the response to sleep deprivation. Psychiatry Research. 1998 Jun 2;79(1):43-50. PMID: 9676825 Ineligible study design

51. Barekatain M, Jahangard L, Haghighi M, et al. Bifrontal versus bitemporal electroconvulsive therapy in severe manic patients. Journal of ECT. 2008 Sep;24(3):199-202. doi:

http://dx.doi.org/10.1097/YCT.0b013e3181624b5d. PMID: 18772704 No eligible outcomes reported

52. Basu R, Brar JS, Chengappa KNR, et al. The prevalence of the metabolic syndrome in patients with schizoaffective disorder - bipolar subtype. Bipolar Disorders. 2004 2004;6(4):314-8. doi: http://dx.doi.org/10.1111/j.1399-5618.2004.00126.x. Ineligible study design

53. Battaglia J, Lindborg SR, Alaka K, et al. Calming versus sedative effects of intramuscular olanzapine in agitated patients. American Journal of Emergency Medicine. 2003 May;21(3):192-8. PMID: 12811711

\section{Ineligible study design}

54. Bauer IE, Galvez JF, Hamilton JE, et al. Lifestyle interventions targeting dietary habits and exercise in bipolar disorder: A systematic review. Journal of Psychiatric Research. 201601 Mar;74:1-7. doi: http://dx.doi.org/10.1016/j.jpsychires.2015.12.006. PMID: 608386673 Ineligible study design

55. Bauer M, Rasgon N, Grof P, et al. Do antidepressants influence mood patterns? A naturalistic study in bipolar disorder. European Psychiatry: the Journal of the Association of European Psychiatrists. 2006 Jun;21(4):262-9. PMID: 16782312 No eligible outcomes reported

56. Bauer M, Ritter P, Grunze H, et al. Treatment options for acute depression in bipolar disorder. Bipolar Disorders. 2012 May;14 Suppl 2:37-50. doi: http://dx.doi.org/10.1111/j.1399-5618.2012.00991.x. PMID: 22510035 Ineligible study design

57. Bauer MS. An evidence-based review of psychosocial treatments for bipolar disorder. Psychopharmacology Bulletin. 2001;35(3):109-34. PMID: 12397882 Ineligible study design

58. Bauer MS, Mitchner L. What is a "mood stabilizer"? An evidence-based response. American Journal of Psychiatry. 2004 Jan;161(1):3-18. PMID: 14702242 Ineligible study design

59. Bauer MS, Wisniewski SR, Marangell LB, et al. Are antidepressants associated with new-onset suicidality in bipolar disorder? A prospective study of participants in the Systematic Treatment Enhancement Program for Bipolar Disorder (STEP-BD). Journal of Clinical Psychiatry. 2006 Jan;67(1):48-55. PMID: 16426088 No eligible outcomes reported

60. Bech P, Vendsborg PB, Rafaelsen OJ. Lithium maintenance treatment of manic-melancholic patients: its role in the daily routine. Acta Psychiatr Scand. 1976 Jan;53(1):70-81. PMID: 1251757 No eligible outcomes reported 
61. Belete H. Use of physical restraints among patients with bipolar disorder in Ethiopian Mental Specialized Hospital, outpatient department: cross-sectional study. International Journal of Bipolar Disorders. 201701 Dec;5 (1) (no pagination)(17)doi: http://dx.doi.org/10.1186/s40345-017-0084-6. PMID: 614975526

\section{Ineligible intervention}

62. Bellantuono C, Barraco A, Rossi A, et al. The management of bipolar mania: a national survey of baseline data from the EMBLEM study in Italy. BMC Psychiatry. 2007;7:33. PMID: 17640381 Ineligible study design

63. Bellivier F, Belzeaux R, Scott J, et al. Anticonvulsants and suicide attempts in bipolar I disorders. Acta Psychiatrica Scandinavica. 201701 May;135(5):470-8. doi: http://dx.doi.org/10.1111/acps.12709. PMID: 614392589 Not treating bipolar

64. Belmaker RH. Patient history must be incorporated into any guidelines. Bipolar Disorders. 2009 2009;11(7):772. doi: http://dx.doi.org/10.1111/j.1399-5618.2009.00743.x. Ineligible study design

65. Benedetti F, Barbini B, Campori E, et al. Dopamine agonist amineptine prevents the antidepressant effect of sleep deprivation. Psychiatry Research. 1996 Dec 20;65(3):179-84. PMID: 9029666 Ineligible study design

66. Benedetti F, Barbini B, Campori E, et al. Sleep phase advance and lithium to sustain the antidepressant effect of total sleep deprivation in bipolar depression: new findings supporting the internal coincidence model? Journal of Psychiatric Research. 2001 Nov-Dec;35(6):323-9. PMID: 11684139 Ineligible study design

67. Benedetti F, Campori E, Barbini B, et al. Dopaminergic augmentation of sleep deprivation effects in bipolar depression. Psychiatry Research. 2001 Nov 30;104(3):239-46. PMID: 11728613 Ineligible study design

68. Benedetti F, Colombo C, Pontiggia A, et al. Morning light treatment hastens the antidepressant effect of citalopram: A placebo-controlled trial. Journal of Clinical Psychiatry. 2003 2003;64(6):648-53. doi: http://dx.doi.org/10.4088/JCP.v64n0605. No eligible outcomes reported

69. Berk M. Lamotrigine and the treatment of mania in bipolar disorder. European Neuropsychopharmacology. 1999 Aug;9 Suppl 4:S119-23. PMID: 10524838 Duplicate reference

70. Berk M, Copolov DL, Dean O, et al. N-acetyl cysteine for depressive symptoms in bipolar disorder--a double-blind randomized placebo-controlled trial. Biological Psychiatry. 2008 Sep 15;64(6):468-75. doi: http://dx.doi.org/10.1016/j.biopsych.2008.04.022. PMID: 18534556 Ineligible intervention

71. Berk M, Ng F, Wang WV, et al. Going up in smoke: tobacco smoking is associated with worse treatment outcomes in mania. Journal of Affective Disorders. 2008 Sep;110(1-2):126-34. doi: http://dx.doi.org/10.1016/j.jad.2008.01.018. PMID: 18280579 No eligible outcomes reported

72. Berk M, Tiller JWG, Zhao J, et al. Effects of asenapine in bipolar I patients meeting proxy criteria for moderate-to-severe mixed major depressive episodes: A post hoc analysis. Journal of Clinical Psychiatry. 201501 Jun 2015;76(6):728-34. doi: http://dx.doi.org/10.4088/JCP.13m08827. PMID: 605004625 No eligible outcomes reported 
73. Bernardo M, Dodd S, Gama CS, et al. Effects of N-acetylcysteine on substance use in bipolar disorder: A randomised placebo-controlled clinical trial. Acta Neuropsychiatrica. 2009 2009;21(6):285-91. doi: http://dx.doi.org/10.1111/j.1601-5215.2009.00397.x. No eligible outcomes reported

74. Bersani FS, Minichino A, Bernabei L, et al. Prefronto-cerebellar tDCS enhances neurocognition in euthymic bipolar patients. Findings from a placebo-controlled neuropsychological and psychophysiological investigation. Journal of Affective Disorders. 201701 Feb;209:262-9. doi: http://dx.doi.org/10.1016/j.jad.2016.11.037. PMID: 613575159 Ineligible study design

75. Bersudsky Y. Phenytoin: an anti-bipolar anticonvulsant? International Journal of Neuropsychopharmacology. 2006 Aug;9(4):479-84. PMID: 16202184 Duplicate reference

76. Bersudsky Y, Applebaum J, Gaiduk Y, et al. Valnoctamide as a valproate substitute with low teratogenic potential in mania: a double-blind, controlled, add-on clinical trial. Bipolar Disorders. 2010 Jun;12(4):37682. doi: http://dx.doi.org/10.1111/j.1399-5618.2010.00828.x. PMID: 20636634 Bipolar not analyzed separately

77. Beynon S, Soares-Weiser K, Woolacott N, et al. Psychosocial interventions for the prevention of relapse in bipolar disorder: systematic review of controlled trials. British Journal of Psychiatry. 2008 Jan;192(1):5-11. doi: http://dx.doi.org/10.1192/bjp.bp.107.037887. PMID: 18174500 Ineligible study design

78. Beynon S, Soares-Weiser K, Woolacott N, et al. Pharmacological interventions for the prevention of relapse in bipolar disorder: a systematic review of controlled trials. Journal of Psychopharmacology. 2009 Jul;23(5):574-91. doi: http://dx.doi.org/10.1177/0269881108093885. PMID: 18635701 Ineligible study design

79. Bhana N, Perry CM. Olanzapine: a review of its use in the treatment of bipolar I disorder. CNS Drugs. 2001;15(11):871-904. PMID: 11700151 Ineligible study design

80. Bhugra D, Ayonrinde O, Butler G, et al. A randomised controlled trial of assertive outreach vs. treatment as usual for black people with severe mental illness. Epidemiology \& Psychiatric Science. 2011 Mar;20(1):839. PMID: 21657119 Bipolar not analyzed separately

81. Biel MG, Peselow E, Mulcare L, et al. Continuation versus discontinuation of lithium in recurrent bipolar illness: a naturalistic study. Bipolar Disorders. 2007 Aug;9(5):435-42. PMID: 17680913 No eligible outcomes reported

82. Bjorgvinsson T, Kertz SJ, Bigda-Peyton JS, et al. Effectiveness of cognitive behavior therapy for severe mood disorders in an acute psychiatric naturalistic setting: A benchmarking study. Cognitive Behaviour Therapy. 2014 2014;43(3):209-20. doi: http://dx.doi.org/10.1080/16506073.2014.901988. Bipolar not analyzed separately

83. Bobo WV, Reilly-Harrington NA, Ketter TA, et al. Effect of adjunctive benzodiazepines on clinical outcomes in lithium- or quetiapine-treated outpatients with bipolar I or II disorder: results from the Bipolar CHOICE trial. Journal of Affective Disorders. 2014 Jun;161:30-5. doi: http://dx.doi.org/10.1016/j.jad.2014.02.046. PMID: 24751304 Ineligible study design

84. Bobo WV, Reilly-Harrington NA, Ketter TA, et al. Complexity of illness and adjunctive benzodiazepine use in outpatients with bipolar I or II disorder: results from the Bipolar CHOICE study. Journal of Clinical 
Psychopharmacology. 2015 Feb;35(1):68-74. doi: http://dx.doi.org/10.1097/JCP.0000000000000257. PMID: 25514063 No eligible outcomes reported

85. Bogart GT, Chavez B. Safety and efficacy of quetiapine in bipolar depression. Annals of Pharmacotherapy. 2009 Nov;43(11):1848-56. doi: http://dx.doi.org/10.1345/aph.1M193. PMID: 19809011 Ineligible study design

86. Boland EM, Stange JP, Molz Adams A, et al. Associations between sleep disturbance, cognitive functioning and work disability in Bipolar Disorder. Psychiatry Research. 2015 Dec 15;230(2):567-74. doi: http://dx.doi.org/10.1016/j.psychres.2015.09.051. PMID: 26474660 Not treating bipolar

87. Bonafede M, Locklear JC, Wahlqvist $\mathrm{P}$, et al. Impact of once-daily extended-release quetiapine fumarate on hospitalization length in patients with acute bipolar mania. Journal of Comparative Effectiveness Research. 2015 Jan;4(1):51-9. doi: http://dx.doi.org/10.2217/cer.14.48. PMID: 25168473 No eligible outcomes reported

88. Bond DJ, Lam RW, Yatham LN. Divalproex sodium versus placebo in the treatment of acute bipolar depression: a systematic review and meta-analysis. Journal of Affective Disorders. 2010 Aug;124(3):22834. doi: http://dx.doi.org/10.1016/j.jad.2009.11.008. PMID: 20044142 Ineligible study design

89. Bond DJ, Pratoomsri W, Yatham LN. Depot antipsychotic medications in bipolar disorder: a review of the literature. Acta Psychiatrica Scandinavica, Supplementum. 2007(434):3-16. PMID: 17688458 Ineligible study design

90. Bonnin CM, Torrent C, Arango C, et al. Functional remediation in bipolar disorder: 1-year follow-up of neurocognitive and functional outcome. British Journal of Psychiatry. 2016 January;208(1):87-93. doi: http://dx.doi.org/10.1192/bjp.bp.114.162123. PMID: 608072552 Duplicate reference

91. Bonnin CM, Torrent C, Arango C, et al. Functional remediation in bipolar disorder: 1-year follow-up of neurocognitive and functional outcome. British Journal of Psychiatry. 2016 Jan;208(1):87-93. doi: http://dx.doi.org/10.1192/bjp.bp.114.162123. PMID: 26541692 Ineligible study design

92. Bos EH, Merea R, van den Brink E, et al. Mindfulness training in a heterogeneous psychiatric sample: outcome evaluation and comparison of different diagnostic groups. Journal of Clinical Psychology. 2014 Jan;70(1):60-71. doi: http://dx.doi.org/10.1002/jclp.22008. PMID: 23801545 Ineligible study design

93. Bottlender R, Rudolf D, Strauss A, et al. Mood-stabilisers reduce the risk of developing antidepressantinduced maniform states in acute treatment of bipolar I depressed patients. Journal of Affective Disorders. 2001 Mar;63(1-3):79-83. PMID: 11246083 No eligible outcomes reported

94. Bourin M, Lambert O, Guitton B. Treatment of acute mania--from clinical trials to recommendations for clinical practice. Human Psychopharmacology. 2005 Jan;20(1):15-26. PMID: 15568205 Ineligible study design

95. Bowden C, Boyer P. Treatment pathways for bipolar disorder in the USA and Europe: convergence or divergence? European Psychiatry: the Journal of the Association of European Psychiatrists. 2003 Dec;18 Suppl 1:19s-24s. PMID: 23573637 Ineligible study design 
96. Bowden C, Maier W. Bipolar disorder and personality disorder. European Psychiatry: the Journal of the Association of European Psychiatrists. 2003 Dec;18 Suppl 1:9s-12s. PMID: 23573635 Ineligible study design

97. Bowden CL. Dosing strategies and time course of response to antimanic drugs. Journal of Clinical Psychiatry. 1996;57 Suppl 13:4-9; discussion 10-2. PMID: 8970500 Ineligible study design

98. Bowden CL. Key treatment studies of lithium in manic-depressive illness: efficacy and side effects. Journal of Clinical Psychiatry. 1998;59 Suppl 6:13-9; discussion 20. PMID: 9674932 Ineligible study design

99. Bowden CL. Efficacy of lithium in mania and maintenance therapy of bipolar disorder. Journal of Clinical Psychiatry. 2000;61 Suppl 9:35-40. PMID: 10826659 Ineligible study design

100. Bowden CL. The ability of lithium and other mood stabilizers to decrease suicide risk and prevent relapse. Current Psychiatry Reports. 2000 Dec;2(6):490-4. PMID: 11123000 Ineligible study design

101. Bowden CL. Novel treatments for bipolar disorder. Expert Opinion on Investigational Drugs. 2001 Apr;10(4):661-71. PMID: 11281816 Ineligible study design

102. Bowden CL. Acute and maintenance treatment with mood stabilizers. International Journal of Neuropsychopharmacology. 2003 Sep;6(3):269-75. PMID: 12974993 Ineligible study design

103. Bowden CL. Valproate. Bipolar Disorders. 2003 Jun;5(3):189-202. PMID: 12780873 Ineligible study design

104. Bowden CL. Treatment options for bipolar depression. Journal of Clinical Psychiatry. 2005;66 Suppl 1:3-6. PMID: 15693745 Ineligible study design

105. Bowden CL. Anticonvulsants in bipolar disorders: current research and practice and future directions. Bipolar Disorders. 2009 Jun;11 Suppl 2:20-33. doi: http://dx.doi.org/10.1111/j.1399-5618.2009.00708.x. PMID: 19538683 Ineligible study design

106. Bowden CL. Pharmacological treatments for bipolar disorder: Present recommendations and future prospects. Manji, Husseini K [Ed]. 2011 Ineligible study design

107. Bowden CL, Brugger AM, Swann AC, et al. Efficacy of divalproex vs lithium and placebo in the treatment of mania. The Depakote Mania Study Group.[Erratum appears in JAMA 1994 Jun 15;271(23):1830]. JAMA. 1994 Mar 23-30;271(12):918-24. PMID: 8120960 Over 50\% dropout rate

108. Bowden CL, Calabrese JR, Ketter TA, et al. Impact of lamotrigine and lithium on weight in obese and nonobese patients with bipolar I disorder. American Journal of Psychiatry. 2006 Jul;163(7):1199-201. PMID: 16816224 Over 50\% dropout rate

109. Bowden CL, Janicak PG, Orsulak P, et al. Relation of serum valproate concentration to response in mania. American Journal of Psychiatry. 1996 Jun;153(6):765-70. PMID: 8633687 Ineligible study design

110. Bowden CL, Ketter TA, Sachs GS, et al. Focus on bipolar disorder treatment. Journal of Clinical Psychiatry. 2005 Dec;66(12):1598-609. PMID: 16401164 Ineligible study design 
111. Bowden CL, Mintz J, Tohen M. Multi-state outcome analysis of treatments (MOAT): application of a new approach to evaluate outcomes in longitudinal studies of bipolar disorder. Molecular Psychiatry. 2016 Feb;21(2):237-42. doi: http://dx.doi.org/10.1038/mp.2015.21. PMID: 25778474 Duplicate reference

112. Bowden CL, Singh V. Valproate in bipolar disorder: 2000 onwards. Acta Psychiatrica Scandinavica, Supplementum. 2005(426):13-20. PMID: 15833096 Ineligible study design

113. Bowden CL, Singh V. Lamotrigine (Lamictal IR) for the treatment of bipolar disorder. Expert Opinion on Pharmacotherapy. 2012 Dec;13(17):2565-71. doi: http://dx.doi.org/10.1517/14656566.2012.741590. PMID: 23140205 Ineligible study design

114. Bowden CL, Singh V, Weisler R, et al. Lamotrigine vs. lamotrigine plus divalproex in randomized, placebo-controlled maintenance treatment for bipolar depression. Acta Psychiatrica Scandinavica. 2012 Nov;126(5):342-50. doi: http://dx.doi.org/10.1111/j.1600-0447.2012.01890.x. PMID: 22708645 Over $50 \%$ dropout rate

115. Bradford DW, Cunningham NT, Slubicki MN, et al. An evidence synthesis of care models to improve general medical outcomes for individuals with serious mental illness: a systematic review. Journal of Clinical Psychiatry. 2013 Aug;74(8):e754-64. doi: http://dx.doi.org/10.4088/JCP.12r07666. PMID: 24021516 Ineligible study design

116. Brambilla P, Barale F, Soares JC. Perspectives on the use of anticonvulsants in the treatment of bipolar disorder. International Journal of Neuropsychopharmacology. 2001 Dec;4(4):421-46. PMID: 11806868 Ineligible study design

117. Brambilla P, Barale F, Soares JC. Atypical antipsychotics and mood stabilization in bipolar disorder. Psychopharmacology. 2003 Apr;166(4):315-32. PMID: 12607072 Ineligible study design

118. Bridle C, Palmer S, Bagnall AM, et al. A rapid and systematic review and economic evaluation of the clinical and cost-effectiveness of newer drugs for treatment of mania associated with bipolar affective disorder. Health Technology Assessment (Winchester, England). 2004 May;8(19):iii-iv, 1-187. PMID: 15147609 Ineligible study design

119. Brown E, Dunner DL, McElroy SL, et al. Olanzapine/fluoxetine combination vs. lamotrigine in the 6month treatment of bipolar I depression. International Journal of Neuropsychopharmacology. 2009 Jul;12(6):773-82. doi: http://dx.doi.org/10.1017/S1461145708009735. PMID: 19079815 Over 50\% dropout rate

120. Brown ES. Management of comorbid bipolar disorder and substance abuse. Journal of Clinical Psychiatry. 2006 Aug;67(8):e05. PMID: 17107268 Ineligible study design

121. Brown ES, Gabrielson B. A randomized, double-blind, placebo-controlled trial of citicoline for bipolar and unipolar depression and methamphetamine dependence. Journal of Affective Disorders. 2012 Dec 20;143(1-3):257-60. doi: http://dx.doi.org/10.1016/j.jad.2012.05.006. PMID: 22974472 Not treating bipolar

122. Brown ES, Garza M, Carmody TJ. A randomized, double-blind, placebo-controlled add-on trial of quetiapine in outpatients with bipolar disorder and alcohol use disorders. Journal of Clinical Psychiatry. 2008 May;69(5):701-5. PMID: 18312058 No eligible outcomes reported 
123. Brown ES, Gorman AR, Hynan LS. A randomized, placebo-controlled trial of citicoline add-on therapy in outpatients with bipolar disorder and cocaine dependence. Journal of Clinical Psychopharmacology. 2007 Oct;27(5):498-502. PMID: 17873684 Not treating bipolar

124. Brown ES, Nejtek VA, Perantie DC, et al. Quetiapine in bipolar disorder and cocaine dependence. Bipolar Disorders. 2002 Dec;4(6):406-11. PMID: 12519101 Fewer than 11 subjects per arm

125. Brown ES, Todd JP, Hu LT, et al. A randomized, double-blind, placebo-controlled trial of citicoline for cocaine dependence in bipolar i disorder. American Journal of Psychiatry. 201501 Oct;172(10):1014-21. doi: http://dx.doi.org/10.1176/appi.ajp.2015.14070857. PMID: 2015417503 Ineligible study design

126. Buchheit J, Uhring J, Sergent P, et al. Can preoperative CRP levels predict infections of bipolar hemiarthroplasty performed for femoral neck fracture? A retrospective, multicenter study. European journal of orthopaedic surgery \& traumatologie. 2015 Jan;25(1):117-21. doi: http://dx.doi.org/10.1007/s00590-0141449-5. PMID: 24719083 Not bipolar disorder

127. Buckley PF. Update on the treatment and management of schizophrenia and bipolar disorder. Cns Spectrums. 2008 Feb;13(2 Suppl 1):1-10; quiz 1-2. PMID: 18227747 Ineligible study design

128. Buckley PF, Paulsson B, Brecher M. Treatment of agitation and aggression in bipolar mania: efficacy of quetiapine. Journal of Affective Disorders. 2007;100 Suppl 1:S33-43. PMID: 17376537 No eligible outcomes reported

129. Burdick KE, Braga RJ, Gopin CB, et al. Dopaminergic influences on emotional decision making in euthymic bipolar patients. Neuropsychopharmacology. 2014 Jan;39(2):274-82. doi:

http://dx.doi.org/10.1038/npp.2013.177. PMID: 23884342 No eligible outcomes reported

130. Burgess S, Geddes J, Hawton K, et al. Lithium for maintenance treatment of mood disorders. Cochrane Database of Systematic Reviews. 2001(3):CD003013. PMID: 11687035 Ineligible study design

131. Busby KK, Sajatovic M. Patient, treatment, and systems-level factors in bipolar disorder nonadherence: A summary of the literature. CNS Neuroscience \& Therapeutics. 2010 2010;16(5):308-15. doi: http://dx.doi.org/10.1111/j.1755-5949.2010.00191.x. Ineligible study design

132. Busch AB, He Y, Zelevinsky K, et al. Predicting Participation in Psychiatric Randomized Controlled Trials: Insights From the STEP-BD. Psychiatric Services. 2015 Aug 1;66(8):817-23. doi: http://dx.doi.org/10.1176/appi.ps.201300557. PMID: 25828873 Not treating bipolar

133. Bushe C, Shaw M. Prevalence of hyperprolactinaemia in a naturalistic cohort of schizophrenia and bipolar outpatients during treatment with typical and atypical antipsychotics. Journal of Psychopharmacology. 2007 Sep;21(7):768-73. PMID: 17606473 Ineligible study design

134. Calabrese J, Rajagopalan K, Ng-Mak D, et al. Effect of lurasidone on meaningful change in health-related quality of life in patients with bipolar depression. International Clinical Psychopharmacology. 201613 Apr;31(3):147-54. doi: http://dx.doi.org/10.1097/YIC.0000000000000116. PMID: 607489863 No eligible outcomes reported

135. Calabrese JR, Goldberg JF, Ketter TA, et al. Recurrence in bipolar I disorder: a post hoc analysis excluding relapses in two double-blind maintenance studies. Biological Psychiatry. 2006 Jun 1;59(11):1061-4. PMID: 16769295 Ineligible study design 
136. Calabrese JR, Rapport DJ, Shelton MD, et al. Clinical studies on the use of lamotrigine in bipolar disorder. Neuropsychobiology. 1998 Oct;38(3):185-91. PMID: 9778607 Ineligible study design

137. Calabrese JR, Rapport DJ, Youngstrom EA, et al. New data on the use of lithium, divalproate, and lamotrigine in rapid cycling bipolar disorder. European Psychiatry: the Journal of the Association of European Psychiatrists. 2005 Mar;20(2):92-5. PMID: 15797691 Duplicate reference

138. Calabrese JR, Shelton MD, Rapport DJ, et al. Long-term treatment of bipolar disorder with lamotrigine. Journal of Clinical Psychiatry. 2002;63 Suppl 10:18-22. PMID: 12392349 Ineligible study design

139. Calabrese JR, Sullivan JR, Bowden CL, et al. Rash in multicenter trials of lamotrigine in mood disorders: clinical relevance and management. Journal of Clinical Psychiatry. 2002 Nov;63(11):1012-9. PMID: 12444815 Bipolar not analyzed separately

140. Calabrese JR, Vieta E, Shelton MD. Latest maintenance data on lamotrigine in bipolar disorder. European Neuropsychopharmacology. 2003 Aug;13 Suppl 2:S57-66. PMID: 12957721 Ineligible study design

141. Camm AJ, Karayal ON, Meltzer H, et al. Ziprasidone and the corrected QT interval: a comprehensive summary of clinical data. CNS Drugs. 2012 Apr 1;26(4):351-65. doi: http://dx.doi.org/10.2165/11599010000000000-00000. PMID: 22452529 Ineligible study design

142. Cardoso Tde A, Campos Mondin T, Reyes AN, et al. Biological Rhythm and Bipolar Disorder: TwelveMonth Follow-Up of a Randomized Clinical Trial. Journal of Nervous \& Mental Disease. 2015 Oct;203(10):792-7. doi: http://dx.doi.org/10.1097/NMD.0000000000000369. PMID: 26348588 Over $\mathbf{5 0 \%}$ dropout rate

143. Carey TS, Williams JW, Jr., Oldham JM, et al. Gabapentin in the treatment of mental illness: the echo chamber of the case series. Journal of Psychiatric Practice. 2008 Mar;14 Suppl 1:15-27. doi: http://dx.doi.org/10.1097/01.pra.0000333584.75741.45. PMID: 19034206 Ineligible study design

144. Carney SM, Goodwin GM. Lithium - a continuing story in the treatment of bipolar disorder. Acta Psychiatrica Scandinavica, Supplementum. 2005(426):7-12. PMID: 15833095 Ineligible study design

145. Carta MG, Hardoy MC, Hardoy MJ, et al. The clinical use of gabapentin in bipolar spectrum disorders. Journal of Affective Disorders. 2003 Jun;75(1):83-91. PMID: 12781355 Ineligible study design

146. Cassano GB, Heinze G, Loo H, et al. A double-blind comparison of tianeptine, imipramine and placebo in the treatment of major depressive episodes. European Psychiatry. 1996;11(5):254-9. doi: http://dx.doi.org/10.1016/0924-9338\%2896\%2982332-7. Bipolar not analyzed separately

147. Castle DJ. Bipolar mixed states: still mixed up? Current Opinion in Psychiatry. 2014 Jan;27(1):38-42. doi: http://dx.doi.org/10.1097/YCO.0000000000000029. PMID: 24270474 Ineligible study design

148. Cavazzoni PA, Berg PH, Kryzhanovskaya LA, et al. Comparison of treatment-emergent extrapyramidal symptoms in patients with bipolar mania or schizophrenia during olanzapine clinical trials. Journal of Clinical Psychiatry. 2006 Jan;67(1):107-13. PMID: 16426096 Ineligible study design 
149. Cazorla P, Zhao J, Mackle M, et al. Asenapine effects on individual Young Mania Rating Scale items in bipolar disorder patients with acute manic or mixed episodes: A pooled analysis. Neuropsychiatric Disease and Treatment. 2013 28, 2013;9:409-13. No eligible outcomes reported

150. Ceron-Litvoc D, Soares BG, Geddes J, et al. Comparison of carbamazepine and lithium in treatment of bipolar disorder: a systematic review of randomized controlled trials. Human Psychopharmacology. 2009 Jan;24(1):19-28. doi: http://dx.doi.org/10.1002/hup.990. PMID: 19053079 Ineligible study design

151. Cerullo MA, Strakowski SM. A systematic review of the evidence for the treatment of acute depression in bipolar I disorder. Cns Spectrums. 2013 Aug;18(4):199-208. doi:

http://dx.doi.org/10.1017/S1092852913000102. PMID: 23507138 Ineligible study design

152. Chafetz L, White M, Collins-Bride G, et al. Clinical trial of wellness training: health promotion for severely mentally ill adults. Journal of Nervous \& Mental Disease. 2008 Jun;196(6):475-83. doi: http://dx.doi.org/10.1097/NMD.0b013e31817738de. PMID: 18552625 Bipolar not analyzed separately

153. Chan HY, Jou SH, Juang YY, et al. A single-blind, comparative study of zotepine versus haloperidol in combination with a mood stabilizer for patients with moderate-to-severe mania. Psychiatry \& Clinical Neurosciences. 2010 Apr;64(2):162-9. doi: http://dx.doi.org/10.1111/j.1440-1819.2010.02066.x. PMID: 20447012 Fewer than 11 subjects per arm

154. Chandler D, Meisel J, Hu TW, et al. Client outcomes in a three-year controlled study of an integrated service agency model. Psychiatric Services. 1996 Dec;47(12):1337-43. PMID: 9117472 Bipolar not analyzed separately

155. Chandramouli J. Newer anticonvulsant drugs in neuropathic pain and bipolar disorder. Journal of Pain \& Palliative Care Pharmacotherapy. 2002;16(4):19-37. PMID: 14635823 Ineligible study design

156. Chapel S, Chiu YY, Hsu J, et al. Lurasidone Dose Response in Bipolar Depression: A Population Doseresponse Analysis. Clinical Therapeutics. 2016 Jan 1;38(1):4-15. doi: http://dx.doi.org/10.1016/j.clinthera.2015.11.013. PMID: 26730454 Ineligible study design

157. Chen D-G. Meta-analysis for psychiatric research using free software R. Shanghai Archives of Psychiatry. 2015 Jun;27(3):195-9. PMID: 2015-36530-010 Ineligible study design

158. Chen G, Henter ID, Manji HK. Looking ahead: Electroretinographic anomalies, glycogen synthase kinase3, and biomarkers for neuropsychiatric disorders. Biological Psychiatry. 2014 15, 2014;76(2):86-8. doi: http://dx.doi.org/10.1016/j.biopsych.2014.05.005. Ineligible study design

159. Chen PS, Chang HH, Huang CC, et al. A longitudinal study of the association between the GNB3 C825T polymorphism and metabolic disturbance in bipolar II patients treated with valproate. Pharmacogenomics Journal. 201701 Mar;17(2):155-61. doi: http://dx.doi.org/10.1038/tpj.2015.96. PMID: 608296835

\section{Ineligible intervention}

160. Chen Y, Bobo WV, Watts K, et al. Comparative effectiveness of switching antipsychotic drug treatment to aripiprazole or ziprasidone for improving metabolic profile and atherogenic dyslipidemia: a 12-month, prospective, open-label study. Journal of Psychopharmacology. 2012 Sep;26(9):1201-10. doi: http://dx.doi.org/10.1177/0269881111430748. PMID: 22234928 Bipolar not analyzed separately 
161. Chengappa KN, Baker RW, Shao L, et al. Rates of response, euthymia and remission in two placebocontrolled olanzapine trials for bipolar mania. Bipolar Disorders. 2003 Feb;5(1):1-5. PMID: 12656931

\section{Over $50 \%$ dropout rate}

162. Chengappa KN, Levine J, Gershon S, et al. Inositol as an add-on treatment for bipolar depression. Bipolar Disorders. 2000 Mar;2(1):47-55. PMID: 11254020 No eligible outcomes reported

163. Chiang KJ, Tsai JC, Liu D, et al. Efficacy of cognitive-behavioral therapy in patients with bipolar disorder: A metaanalysis of randomized controlled trials. PLoS ONE. 2017 May;12 (5) (no pagination)(e0176849)doi: http://dx.doi.org/10.1371/journal.pone.0176849. PMID: 615955928 Ineligible study design

164. Chiesa A, Chierzi F, De Ronchi D, et al. Quetiapine for bipolar depression: a systematic review and metaanalysis. International Clinical Psychopharmacology. 2012 Mar;27(2):76-90. doi: http://dx.doi.org/10.1097/YIC.0b013e32834e4c56. PMID: 22107783 Ineligible study design

165. Chou JC, Czobor P, Charles O, et al. Acute mania: haloperidol dose and augmentation with lithium or lorazepam. Journal of Clinical Psychopharmacology. 1999 Dec;19(6):500-5. PMID: 10587284 Over $50 \%$ dropout rate

166. Chou JC, Czobor P, Tuma I, et al. Pretreatment plasma HVA and haloperidol response in acute mania. Journal of Affective Disorders. 2000 Jul;59(1):55-9. PMID: 10814771 No eligible outcomes reported

167. Chou JCY. Review and update of the American Psychiatric Association practice guideline for bipolar disorder. Primary Psychiatry. 2004 2004;11(9):73-84. Ineligible study design

168. Chou JCY, Czobor P, Tuma I, et al. Pretreatement plasma HVA and haloperidol response in acute mania. Journal of Affective Disorders. 2000 2000;59(1):55-9. doi: http://dx.doi.org/10.1016/S01650327\%2899\%2900134-2. No eligible outcomes reported

169. Chue P, Kovacs CS. Safety and tolerability of atypical antipsychotics in patients with bipolar disorder: prevalence, monitoring and management. Bipolar Disorders. 2003;5 Suppl 2:62-79. PMID: 14700015 Ineligible study design

170. Chwieduk CM, Scott LJ. Asenapine: a review of its use in the management of mania in adults with bipolar I disorder. CNS Drugs. 2011 Mar;25(3):251-67. doi: http://dx.doi.org/10.2165/11206700-00000000000000. PMID: 21323396 Ineligible study design

171. Ciapparelli A, Dell'Osso L, Tundo A, et al. Electroconvulsive therapy in medication-nonresponsive patients with mixed mania and bipolar depression. Journal of Clinical Psychiatry. 2001 Jul;62(7):552-5. PMID:

\section{Ineligible study design}

172. Cipriani A, Barbui C, Rendell J, et al. Clinical and regulatory implications of active run-in phases in longterm studies for bipolar disorder. Acta Psychiatrica Scandinavica. 2014 May;129(5):328-42. doi: http://dx.doi.org/10.1111/acps.12223. PMID: 24289821 Ineligible study design

173. Cipriani A, Barbui C, Salanti G, et al. Comparative efficacy and acceptability of antimanic drugs in acute mania: a multiple-treatments meta-analysis. Lancet. 2011 Oct 8;378(9799):1306-15. doi: http://dx.doi.org/10.1016/S0140-6736(11)60873-8. PMID: 21851976 Ineligible study design 
174. Cipriani A, Hawton K, Stockton S, et al. Lithium in the prevention of suicide in mood disorders: updated systematic review and meta-analysis. BMJ. 2013;346:f3646. doi: http://dx.doi.org/10.1136/bmj.f3646.

\section{PMID: 23814104 Ineligible study design}

175. Cipriani A, Rendell J, Geddes JR. Olanzapine in the long-term treatment of bipolar disorder: a systematic review and meta-analysis. Journal of Psychopharmacology. 2010 Dec;24(12):1729-38. doi: http://dx.doi.org/10.1177/0269881109106900. PMID: 19828571 Ineligible study design

176. Cipriani A, Rendell JM, Geddes J. Olanzapine in long-term treatment for bipolar disorder. Cochrane Database of Systematic Reviews. 2009(1):CD004367. doi: http://dx.doi.org/10.1002/14651858.CD004367.pub2. PMID: 19160237 Ineligible study design

177. Cipriani A, Rendell JM, Geddes JR. Haloperidol alone or in combination for acute mania. Cochrane Database of Systematic Reviews. 2006(3):CD004362. PMID: 16856043 Ineligible study design

178. Citrome L. Asenapine for schizophrenia and bipolar disorder: a review of the efficacy and safety profile for this newly approved sublingually absorbed second-generation antipsychotic. International Journal of Clinical Practice. 2009 Dec;63(12):1762-84. doi: http://dx.doi.org/10.1111/j.1742-1241.2009.02228.x. PMID: 19840150 Ineligible study design

179. Citrome L. Ziprasidone HCI capsules for the adjunctive maintenance treatment of bipolar disorder in adults. Expert Review of Neurotherapeutics. 2010 2010;10(7):1031-7. doi: http://dx.doi.org/10.1586/ern.10.66. Duplicate reference

180. Citrome L, Holt RI, Walker DJ, et al. Weight gain and changes in metabolic variables following olanzapine treatment in schizophrenia and bipolar disorder. Clinical Drug Investigation. 2011;31(7):455-82. doi: http://dx.doi.org/10.2165/11589060-000000000-00000. PMID: 21495734 Ineligible study design

181. Citrome L, Kantrowitz JT. Olanzapine dosing above the licensed range is more efficacious than lower doses: Fact or fiction? [References]. Expert Review of Neurotherapeutics. 2009 2009;9(7):1045-58. doi: http://dx.doi.org/10.1586/ern.09.54. Ineligible study design

182. Citrome L, Ketter TA, Cucchiaro J, et al. Clinical assessment of lurasidone benefit and risk in the treatment of bipolar I depression using number needed to treat, number needed to harm, and likelihood to be helped or harmed. Journal of Affective Disorders. Oct. 2013 28, 2013(Pagination)doi: http://dx.doi.org/10.1016/j.jad.2013.10.040. No eligible outcomes reported

183. Clarkin JF, Carpenter D, Hull J, et al. Effects of psychoeducational intervention for married patients with bipolar disorder and their spouses. Psychiatric Services. 1998 Apr;49(4):531-3. PMID: 9550248 Bipolar not analyzed separately

184. Colom F, Pacchiarotti I, Vieta E. Treatment arsenal for bipolar disorders: The role of psychoeducation in good clinical practice. Psichiatria e Psicoterapia. 2006 2006;25(1):3-6. Ineligible study design

185. Colom F, Vieta E, Martinez A, et al. What is the role of psychotherapy in the treatment of bipolar disorder? Psychotherapy \& Psychosomatics. 1998;67(1):3-9. PMID: 9491434 Ineligible study design 
186. Colom F, Vieta E, Sanchez-Moreno J, et al. Psychoeducation for bipolar II disorder: an exploratory, 5-year outcome subanalysis. Journal of Affective Disorders. 2009 Jan;112(1-3):30-5. doi:

http://dx.doi.org/10.1016/j.jad.2008.03.023. PMID: 18486237 Less than 11 subjects per arm

187. Colom F, Vieta E, Sanchez-Moreno J, et al. Stabilizing the stabilizer: group psychoeducation enhances the stability of serum lithium levels. Bipolar Disorders. 2005;7 Suppl 5:32-6. PMID: 16225558 No eligible outcomes reported

188. Colom F, Vieta E, Sanchez-Moreno J, et al. Psychoeducation in bipolar patients with comorbid personality disorders. Bipolar Disorders. 2004 Aug;6(4):294-8. PMID: 15225146 Ineligible intervention

189. Colombo C, Benedetti F, Barbini B, et al. Rate of switch from depression into mania after therapeutic sleep deprivation in bipolar depression. Psychiatry Research. 1999 Jun 30;86(3):267-70. PMID: 10482346

\section{Ineligible study design}

190. Colombo C, Lucca A, Benedetti F, et al. Total sleep deprivation combined with lithium and light therapy in the treatment of bipolar depression: replication of main effects and interaction. Psychiatry Research. 2000 Jul 24;95(1):43-53. PMID: 10904122 Ineligible study design

191. Connolly KR, Helmer A, Cristancho MA, et al. Effectiveness of transcranial magnetic stimulation in clinical practice post-FDA approval in the United States: results observed with the first 100 consecutive cases of depression at an academic medical center. Journal of Clinical Psychiatry. 2012 Apr;73(4):e567-73. doi: http://dx.doi.org/10.4088/JCP.11m07413. PMID: 22579164 Ineligible study design

192. Cook JA, Copeland ME, Jonikas JA, et al. Results of a randomized controlled trial of mental illness selfmanagement using Wellness Recovery Action Planning. Schizophrenia Bulletin. 2012 Jun;38(4):881-91. doi: http://dx.doi.org/10.1093/schbul/sbr012. PMID: 21402724 Bipolar not analyzed separately

193. Cookson J, Elliott B. The use of anticonvulsants in the aftermath of mania. Journal of Psychopharmacology. 2006 Mar;20(2 Suppl):23-30. PMID: 16551669 Ineligible study design

194. Correll CU. The role of antipsychotics and mood stabilizers in the treatment of bipolar disorder. Giornale Italiano di Psicopatologia / Italian Journal of Psychopathology. 2011 2011;17(3):341-51. Ineligible study design

195. Correll CU, Sheridan EM, DelBello MP. Antipsychotic and mood stabilizer efficacy and tolerability in pediatric and adult patients with bipolar I mania: a comparative analysis of acute, randomized, placebocontrolled trials. Bipolar Disorders. 2010 Mar;12(2):116-41. doi: http://dx.doi.org/10.1111/j.1399-

5618.2010.00798.x. PMID: 20402706 Ineligible study design

196. Coryell W. Maintenance treatment in bipolar disorder: a reassessment of lithium as the first choice. Bipolar Disorders. 2009 Jun;11 Suppl 2:77-83. doi: http://dx.doi.org/10.1111/j.1399-5618.2009.00712.x. PMID: 19538687 Ineligible study design

197. Coryell W, Leon AC, Turvey C, et al. The significance of psychotic features in manic episodes: a report from the NIMH collaborative study. Journal of Affective Disorders. 2001 Dec;67(1-3):79-88. PMID: 11869754 Bipolar not analyzed separately 
198. Coryell W, Winokur G, Solomon D, et al. Lithium and recurrence in a long-term follow-up of bipolar affective disorder. Psychological Medicine. 1997 Mar;27(2):281-9. PMID: 9089821 No eligible outcomes reported

199. Costa RT, Cheniaux E, Range BP, et al. Group cognitive behavior therapy for bipolar disorder can improve the quality of life. Brazilian Journal of Medical \& Biological Research. 2012 Sep;45(9):862-8. PMID: 22735175 No eligible outcomes reported

200. Costa RT, Cheniaux E, Rosaes PA, et al. The effectiveness of cognitive behavioral group therapy in treating bipolar disorder: a randomized controlled study. Revista Brasileira de Psiquiatria. 2011 Jun;33(2):144-9. PMID: 21829907 No eligible outcomes reported

201. Cousins DA, Young AH. The armamentarium of treatments for bipolar disorder: a review of the literature. International Journal of Neuropsychopharmacology. 2007 Jun;10(3):411-31. PMID: 17176493

\section{Ineligible study design}

202. Cramer JA, Rosenheck R. Enhancing medication compliance for people with serious mental illness. Journal of Nervous \& Mental Disease. 1999 Jan;187(1):53-5. PMID: 9952254 No eligible outcomes reported

203. Crane CA, Hawes SW, Devine S, et al. Axis I psychopathology and the perpetration of intimate partner violence. Journal of Clinical Psychology. 2014 Mar;70(3):238-47. doi: http://dx.doi.org/10.1002/jclp.22013. PMID: 23824500 Not treating bipolar

204. Crowe M, Inder M, Carlyle D, et al. Nurse-led delivery of specialist supportive care for bipolar disorder: a randomized controlled trial. Journal of Psychiatric \& Mental Health Nursing. 2012 Jun;19(5):446-54. doi: http://dx.doi.org/10.1111/j.1365-2850.2011.01822.x. PMID: 22070452 Over 50\% dropout rate

205. Cruz N, Sanchez-Moreno J, Torres F, et al. Efficacy of modern antipsychotics in placebo-controlled trials in bipolar depression: a meta-analysis. International Journal of Neuropsychopharmacology. 2010 Feb;13(1):5-14. doi: http://dx.doi.org/10.1017/S1461145709990344. PMID: 19638254 Ineligible study design

206. Cuomo I, Motta P, Fini C, et al. The efficacy of asenapine in the treatment of bipolar disorder: A naturalistic longitudinal study indicating a favourable response in patients with substance abuse comorbidity. European Psychiatry. 201531 Mar;30:1152. Ineligible study design

207. Curtin F, Schulz P. Clonazepam and lorazepam in acute mania: a Bayesian meta-analysis. Journal of Affective Disorders. 2004 Mar;78(3):201-8. PMID: 15013244 Ineligible study design

208. Daglas R, Cotton SM, Allott K, et al. A single-blind, randomised controlled trial on the effects of lithium and quetiapine monotherapy on the trajectory of cognitive functioning in first episode mania: A 12-month follow-up study. European Psychiatry: the Journal of the Association of European Psychiatrists. 2016 Jan;31:20-8. doi: http://dx.doi.org/10.1016/j.eurpsy.2015.09.460. PMID: 26655594 No eligible

\section{outcomes reported}

209. Dalum HS, Korsbek L, Mikkelsen JH, et al. Illness management and recovery (IMR) in Danish community mental health centres. Trials [Electronic Resource]. 2011;12:195. doi: http://dx.doi.org/10.1186/1745-621512-195. PMID: 21849024 Ineligible study design 
210. Dardennes R, Even C, Bange F, et al. Comparison of carbamazepine and lithium in the prophylaxis of bipolar disorders. A meta-analysis. British Journal of Psychiatry. 1995 Mar;166(3):378-81. PMID: 7788131 Ineligible study design

211. Datto C, Pottorf WJ, Feeley L, et al. Bipolar II compared with bipolar I disorder: Baseline characteristics and treatment response to quetiapine in a pooled analysis of five placebo-controlled clinical trials of acute bipolar depression. Annals of General Psychiatry. 2016 March 11;15 (1) (no pagination)(9)doi: http://dx.doi.org/10.1186/s12991-016-0096-0. PMID: 608914705 No eligible outcomes reported

212. Dauphinais DR, Rosenthal JZ, Terman M, et al. Controlled trial of safety and efficacy of bright light therapy vs. negative air ions in patients with bipolar depression. Psychiatry Research. $2012 \mathrm{Mar}$ 30;196(1):57-61. doi: http://dx.doi.org/10.1016/j.psychres.2012.01.015. PMID: 22424890 Ineligible study design

213. de Azevedo Cardoso T, de Azambuja Farias C, Mondin TC, et al. Brief psychoeducation for bipolar disorder: Impact on quality of life in young adults in a 6-month follow-up of a randomized controlled trial. Psychiatry Research. 2014 30, 2014;220(3):896-902. doi: http://dx.doi.org/10.1016/j.psychres.2014.09.013. PMID: 25300245 Over 50\% dropout rate

214. De Azevedo Cardoso T, Mondin TC, Reyes AN, et al. Biological rhythm and bipolar disorder: Twelvemonth follow-up of a randomized clinical trial. Journal of Nervous and Mental Disease. 2015;203(10):7927. doi: http://dx.doi.org/10.1097/NMD.000000000000369. PMID: 2015431675 Over 50\% dropout rate

215. De Fruyt J, Deschepper E, Audenaert K, et al. Second generation antipsychotics in the treatment of bipolar depression: a systematic review and meta-analysis. Journal of Psychopharmacology. 2012 May;26(5):60317. doi: http://dx.doi.org/10.1177/0269881111408461. PMID: 21940761 Ineligible study design

216. de Macedo-Soares MB, Moreno RA, Rigonatti SP, et al. Efficacy of Electroconvulsive Therapy in Treatment-Resistant Bipolar Disorder: A Case Series. The Journal of ECT. 2005 2005;21(1):31-4. doi: http://dx.doi.org/10.1097/01.yct.0000148621.88104.f1. Ineligible study design

217. Deberdt W, Winokur A, Cavazzoni PA, et al. Amantadine for weight gain associated with olanzapine treatment. European Neuropsychopharmacology. 2005 2005;15(1):13-21. doi: http://dx.doi.org/10.1016/j.euroneuro.2004.03.005. PMID: 15572269 Bipolar not analyzed separately

218. Deckersbach T, Holzel BK, Eisner LR, et al. Mindfulness-based cognitive therapy for nonremitted patients with bipolar disorder. CNS Neuroscience \& Therapeutics. 2012 Feb;18(2):133-41. doi: http://dx.doi.org/10.1111/j.1755-5949.2011.00236.x. PMID: 22070469 Ineligible study design

219. Deckersbach T, Nierenberg AA, Kessler R, et al. RESEARCH: Cognitive rehabilitation for bipolar disorder: An open trial for employed patients with residual depressive symptoms. CNS Neuroscience \& Therapeutics. 2010 Oct;16(5):298-307. doi: http://dx.doi.org/10.1111/j.1755-5949.2009.00110.x. PMID: 19895584 Ineligible study design

220. Deckersbach T, Nierenberg AA, McInnis MG, et al. Baseline disability and poor functioning in bipolar disorder predict worse outcomes: results from the Bipolar CHOICE study. Journal of Clinical Psychiatry. 2016 Jan;77(1):100-8. doi: http://dx.doi.org/10.4088/JCP.14m09210. PMID: 26845265 Ineligible study design 
221. DeLeon A, Patel NC, Crismon ML. Aripiprazole: a comprehensive review of its pharmacology, clinical efficacy, and tolerability. Clinical Therapeutics. 2004 May;26(5):649-66. PMID: 15220010 Ineligible study design

222. Dell'Osso B, Mundo E, D'Urso N, et al. Augmentative repetitive navigated transcranial magnetic stimulation (rTMS) in drug-resistant bipolar depression. Bipolar Disorders. 2009 Feb;11(1):76-81. doi: http://dx.doi.org/10.1111/j.1399-5618.2008.00651.x. PMID: 19133969 Ineligible study design

223. Dell'osso B, Timtim S, Hooshmand F, et al. Superior chronic tolerability of adjunctive modafinil compared to pramipexole in treatment-resistant bipolar disorder. Journal of Affective Disorders. 2013 Aug 15;150(1):130-5. doi: http://dx.doi.org/10.1016/j.jad.2012.11.030. PMID: 23261131 Ineligible study design

224. Demant KM, Vinberg M, Kessing LV, et al. Effects of short-term cognitive remediation on cognitive dysfunction in partially or fully remitted individuals with bipolar disorder: Results of a randomised controlled trial. PLoS ONE. 201512 Jun;10(6)doi: http://dx.doi.org/10.1371/journal.pone.0127955. PMID: 2015178422 No eligible outcomes reported

225. Denicoff KD, Smith-Jackson EE, Disney ER, et al. Comparative prophylactic efficacy of lithium, carbamazepine, and the combination in bipolar disorder. Journal of Clinical Psychiatry. 1997 Nov;58(11):470-8. PMID: 9413412 Ineligible study design

226. Dennehy EB, Schnyer R, Bernstein IH, et al. The safety, acceptability, and effectiveness of acupuncture as an adjunctive treatment for acute symptoms in bipolar disorder. Journal of Clinical Psychiatry. 2009 Jun;70(6):897-905. doi: http://dx.doi.org/10.4088/JCP.08m04208. PMID: 19422756 Less than 11 subjects per arm

227. Depp CA, Bowie CR, Mausbach BT, et al. Current smoking is associated with worse cognitive and adaptive functioning in serious mental illness. Acta Psychiatrica Scandinavica. 2015 May;131(5):333-41. doi: http://dx.doi.org/10.1111/acps.12380. PMID: 25559296 Ineligible study design

228. Depp CA, Lebowitz BD, Patterson TL, et al. Medication adherence skills training for middle-aged and elderly adults with bipolar disorder: development and pilot study. Bipolar Disorders. 2007 Sep;9(6):636-45. PMID: 17845279 Ineligible study design

229. Depp CA, Mausbach B, Granholm E, et al. Mobile interventions for severe mental illness: design and preliminary data from three approaches. Journal of Nervous \& Mental Disease. 2010 Oct;198(10):715-21. doi: http://dx.doi.org/10.1097/NMD.0b013e3181f49ea3. PMID: 20921861 Ineligible study design

230. Der-Hacopian E. Motivations for alcohol consumption among individuals diagnosed with bipolar disorder. Dissertation Abstracts International: Section B: The Sciences and Engineering. 2014;74(8-B(E)

\section{Ineligible study design}

231. Derry S, Moore RA. Atypical antipsychotics in bipolar disorder: systematic review of randomised trials. BMC Psychiatry. 2007;7:40. PMID: 17705840 Ineligible study design

232. Deshauer D, Fergusson D, Duffy A, et al. Re-evaluation of randomized control trials of lithium monotherapy: a cohort effect. Bipolar Disorders. 2005 Aug;7(4):382-7. PMID: 16026492 Ineligible study design 
233. Dogan S, Sabanciogullari S. The effects of patient education in lithium therapy on quality of life and compliance. Archives of Psychiatric Nursing. 2003 Dec;17(6):270-5. PMID: 14685951 Ineligible study design

234. Dolberg OT, Dannon PN, Schreiber S, et al. Transcranial magnetic stimulation in patients with bipolar depression: a double blind, controlled study. Bipolar Disorders. 2002;4 Suppl 1:94-5. PMID: 12479689

\section{Ineligible study design}

235. Dopheide JA, Wincor MZ. Gabapentin and lamotrigine in the treatment of bipolar disorder. Journal of the American Pharmaceutical Association. 1998 Sep-Oct;38(5):632-4. PMID: 9782699 Ineligible study design

236. Drake RE, Frey W, Bond GR, et al. Assisting Social Security Disability Insurance beneficiaries with schizophrenia, bipolar disorder, or major depression in returning to work. American Journal of Psychiatry. 2013 Dec 1;170(12):1433-41. doi: http://dx.doi.org/10.1176/appi.ajp.2013.13020214. PMID: 23929355 Ineligible intervention

237. Dratcu L, Bobmanuel S, Davies W, et al. A UK panel consensus on the initiation of aripiprazole for the treatment of bipolar mania. International Journal of Psychiatry in Clinical Practice. 2012 Oct;16(4):244-58. doi: http://dx.doi.org/10.3109/13651501.2012.709865. PMID: 22809129 Ineligible study design

238. Dubovsky SL. Group therapy effective for bipolar disorder. Education-based therapy may help avert relapses. Health News. 2003 Jun;9(6):4. PMID: 12793397 Ineligible study design

239. Dubovsky SL. Treatment of bipolar depression. Psychiatric Clinics of North America. 2005 Jun;28(2):34970, vii. PMID: 15826736 Ineligible study design

240. Ducharme S, Murray ED, Seiner SJ, et al. Retrospective Analysis of the Short-Term Safety of ECT in Patients With Neurological Comorbidities: A Guide for Pre-ECT Neurological Evaluations. Journal of Neuropsychiatry \& Clinical Neurosciences. 2015;27(4):311-21. doi: http://dx.doi.org/10.1176/appi.neuropsych.14080195. PMID: 25658682 No eligible outcomes reported

241. Dukart J, Regen F, Kherif F, et al. Electroconvulsive therapy-induced brain plasticity determines therapeutic outcome in mood disorders. Proceedings of the National Academy of Sciences of the United States of America. 2014 Jan 21;111(3):1156-61. doi: http://dx.doi.org/10.1073/pnas.1321399111. PMID: 24379394 Bipolar not analyzed separately

242. Duncan D, McConnell HW, Taylor D. Lamotrigine in bipolar affective disorder. Psychiatric Bulletin. 1998 1998;22(10):630-2. doi: http://dx.doi.org/10.1192/pb.22.10.630. Ineligible study design

243. Dunner DL. Safety and tolerability of emerging pharmacological treatments for bipolar disorder. Bipolar Disorders. 2005 2005;7(4):307-25. doi: http://dx.doi.org/10.1111/j.1399-5618.2005.00235.x. Ineligible study design

244. Durgam S, Earley W, Lipschitz A, et al. An 8-Week Randomized, Double-Blind, Placebo-Controlled Evaluation of the Safety and Efficacy of Cariprazine in Patients With Bipolar I Depression. American Journal of Psychiatry. 2016 Mar 1;173(3):271-81. doi: http://dx.doi.org/10.1176/appi.ajp.2015.15020164. PMID: 26541814 Ineligible study design 
245. Earley W, Durgam S, Lu K, et al. Tolerability of cariprazine in the treatment of acute bipolar I mania: A pooled post hoc analysis of 3 phase II/III studies. Journal of Affective Disorders. 2017 01 Jun;215:205-12. doi: http://dx.doi.org/10.1016/j.jad.2017.03.032. PMID: 614950830 Duplicate reference

246. Ebert D, Jaspert A, Murata H, et al. Initial lithium augmentation improves the antidepressant effects of standard TCA treatment in non-resistant depressed patients. Psychopharmacology. 1995 Mar;118(2):223-5. PMID: 7617812 No eligible outcomes reported

247. Eden Evins A, Demopulos C, Nierenberg A, et al. A double-blind, placebo-controlled trial of adjunctive donepezil in treatment-resistant mania. Bipolar Disorders. 2006 Feb;8(1):75-80. PMID: 16411983 Fewer than 11 subjects per arm

248. Eden Evins A, Demopulos C, Yovel I, et al. Inositol augmentation of lithium or valproate for bipolar depression. Bipolar Disorders. 2006 Apr;8(2):168-74. PMID: 16542187 Ineligible study design

249. Ekman M, Lindgren P, Miltenburger C, et al. Cost effectiveness of quetiapine in patients with acute bipolar depression and in maintenance treatment after an acute depressive episode. Pharmacoeconomics. 2012 Jun 1;30(6):513-30. doi: http://dx.doi.org/10.2165/11594930-000000000-00000. PMID: 22591130 Ineligible study design

250. El Haddad M, Houben R, Berte B, et al. Bipolar electrograms characteristics at the left atrial-pulmonary vein junction: Toward a new algorithm for automated verification of pulmonary vein isolation. Heart Rhythm. 2015 Jan;12(1):21-31. doi: http://dx.doi.org/10.1016/j.hrthm.2014.08.030. PMID: 25240694 Not bipolar disorder

251. El Mallakh RS, Vieta E, Rollin L, et al. A comparison of two fixed doses of aripiprazole with placebo in acutely relapsed, hospitalized patients with bipolar disorder I (manic or mixed) in subpopulations (CN138007). European Neuropsychopharmacology. 2010 Nov;20(11):776-83. doi: http://dx.doi.org/10.1016/j.euroneuro.2010.07.003. PMID: 20728318 Over 50\% dropout rate

252. El-Mallakh R, Vohringer P, Ostacher M, et al. Erratum: Antidepressants worsen rapid-cycling course in bipolar disorder: A STEP-BD randomized clinical trial (Journal of Affective Disorders (2015)). Journal of Affective Disorders. 201615 Jan;190:895. doi: http://dx.doi.org/10.1016/j.jad.2015.08.015. PMID: 605789134 Fewer than 11 subjects per arm

253. El-Mallakh R, Weisler RH, Townsend MH, et al. Bipolar II disorder: current and future treatment options. Annals of Clinical Psychiatry. 2006 Oct-Dec;18(4):259-66. PMID: 17162626 Ineligible study design

254. El-Mallakh RS. Medication adherence and the use of long-acting antipsychotics in bipolar disorder. Journal of Psychiatric Practice. 2007 Mar;13(2):79-85. PMID: 17414683 Ineligible study design

255. El-Mallakh RS, Ghaemi SN, Sagduyu K, et al. Antidepressant-associated chronic irritable dysphoria (ACID) in STEP-BD patients. Journal of Affective Disorders. 2008 Dec;111(2-3):372-7. doi: http://dx.doi.org/10.1016/j.jad.2008.03.025. PMID: 18565592 No eligible outcomes reported

256. El-Mallakh RS, Ketter TA, Weisler RH, et al. Switching from other agents to extended-release carbamazepine in acute mania. Psychopharmacology Bulletin. 2008;41(1):52-8. PMID: 18362871 Ineligible study design 
257. El-Mallakh RS, Marcus R, Baudelet C, et al. A 40-week double-blind aripiprazole versus lithium follow-up of a 12-week acute phase study (total 52 weeks) in bipolar I disorder. Journal of Affective Disorders. 2012 Feb;136(3):258-66. doi: http://dx.doi.org/10.1016/j.jad.2011.11.043. PMID: 22209190 Over 50\% dropout rate

258. El-Mallakh RS, Salem MR, Chopra A, et al. A blinded, randomized comparison of immediate-release and extended-release carbamazepine capsules in manic and depressed bipolar subjects. Annals of Clinical Psychiatry. 2010 Feb;22(1):3-8. PMID: 201969776 Pediatric

259. El-Mallakh RS, Salem MR, Chopra AS, et al. Adverse event load in bipolar participants receiving either carbamazepine immediate-release or extended-release capsules: a blinded, randomized study. International Clinical Psychopharmacology. 2009 May;24(3):145-9. doi: http://dx.doi.org/10.1097/YIC.0b013e328329b199. PMID: 19367153 Over 50\% dropout rate

260. El-Mallakh RS, Vohringer PA, Ostacher MM, et al. Antidepressants worsen rapid-cycling course in bipolar depression: A STEP-BD randomized clinical trial. Journal of Affective Disorders. 2015 Sep;184:318-21. doi: http://dx.doi.org/10.1016/j.jad.2015.04.054. PMID: 2015-34966-047 Ineligible intervention

261. El-Mallakh RS, Vohringer PA, Ostacher MM, et al. Antidepressants worsen rapid-cycling course in bipolar depression: A STEP-BD randomized clinical trial. Journal of Affective Disorders. 201504 Jul;184:318-21. doi: http://dx.doi.org/10.1016/j.jad.2015.04.054. PMID: 605067425 Fewer than 11 subjects per arm

262. Elmslie JL, Porter RJ, Joyce PR, et al. Carnitine does not improve weight loss outcomes in valproatetreated bipolar patients consuming an energy-restricted, low-fat diet. Bipolar Disorders. 2006 Oct;8(5 Pt 1):503-7. PMID: 17042889 Ineligible study design

263. Emilien G, Maloteaux JM, Seghers A, et al. Lithium compared to valproic acid and carbamazepine in the treatment of mania: a statistical meta-analysis. European Neuropsychopharmacology. 1996 Aug;6(3):24552. PMID: 8880085 Ineligible study design

264. Emilien G, Septien L, Brisard C, et al. Bipolar disorder: how far are we from a rigorous definition and effective management? Progress in Neuro-Psychopharmacology \& Biological Psychiatry. 2007 Jun 30;31(5):975-96. PMID: 17459551 Ineligible study design

265. Endicott J, Rajagopalan K, Minkwitz M, et al. A randomized, double-blind, placebo-controlled study of quetiapine in the treatment of bipolar I and II depression: improvements in quality of life. International Clinical Psychopharmacology. 2007 Jan;22(1):29-37. PMID: 17159457 No eligible outcomes reported

266. Engstrom C, Astrom M, Nordqvist-Karlsson B, et al. Relationship between prophylactic effect of lithium therapy and family history of affective disorders. Biological Psychiatry. 1997 Sep 15;42(6):425-33. PMID: 9285078 Ineligible study design

267. Ernst CL, Goldberg JF. Antidepressant properties of anticonvulsant drugs for bipolar disorder. Journal of Clinical Psychopharmacology. 2003 Apr;23(2):182-92. PMID: 12640220 Ineligible study design

268. Evans S, Newton R, Higgins S. Nutritional intervention to prevent weight gain in patients commenced on olanzapine: a randomized controlled trial. Australian \& New Zealand Journal of Psychiatry. 2005 Jun;39(6):479-86. PMID: 15943650 Bipolar not analyzed separately 
269. Evins AE. Efficacy of newer anticonvulsant medications in bipolar spectrum mood disorders. Journal of Clinical Psychiatry. 2003;64 Suppl 8:9-14. PMID: 12892536 Ineligible study design

270. Fagan CS, Carmody TJ, McClintock SM, et al. The effect of cognitive functioning on treatment attendance and adherence in comorbid bipolar disorder and cocaine dependence. Journal of Substance Abuse Treatment. 2015 Feb;49:15-20. doi: http://dx.doi.org/10.1016/j.jsat.2014.06.008. PMID: 2014-33120-001 Ineligible study design

271. Fagiolini A, De Filippis S, Azzarelli O, et al. Intramuscular aripiprazole for the treatment of agitation in schizophrenia and bipolar disorder: From clinical research to clinical practice. Journal of Psychopathology / Giornale di Psicopatologia. 2013 2013;19(1):34-41. Ineligible study design

272. Fagiolini A, Frank E, Cherry CR, et al. Clinical indicators for the use of antidepressants in the treatment of bipolar I depression. Bipolar Disorders. 2002 Oct;4(5):277-82. PMID: 12479658 Ineligible study design

273. Fagiolini A, Frank E, Houck PR, et al. Prevalence of obesity and weight change during treatment in patients with bipolar I disorder. Journal of Clinical Psychiatry. 2002 Jun;63(6):528-33. PMID: 12088166 Ineligible study design

274. Fajutrao L, Paulsson B, Liu S, et al. Cost-effectiveness of quetiapine plus mood stabilizers compared with mood stabilizers alone in the maintenance therapy of bipolar I disorder: results of a Markov model analysis. Clinical Therapeutics. 2009 Jun;31 Pt 1:1456-68. doi: http://dx.doi.org/10.1016/j.clinthera.2009.06.009. PMID: 19698903 Ineligible study design

275. Farren CK, Mc Elroy S. Treatment response of bipolar and unipolar alcoholics to an inpatient dual diagnosis program. Journal of Affective Disorders. 2008 Mar;106(3):265-72. PMID: 17707085 Not treating bipolar

276. Faurholt-Jepsen M, Munkholm K, Frost M, et al. Electronic self-monitoring of mood using IT platforms in adult patients with bipolar disorder: A systematic review of the validity and evidence. BMC Psychiatry. 201615 Jan;16 (1) (no pagination)(7)doi: http://dx.doi.org/10.1186/s12888-016-0713-0. PMID: 607740398 Ineligible study design

277. Fava GA, Bartolucci G, Rafanelli C, et al. Cognitive-behavioral management of patients with bipolar disorder who relapsed while on lithium prophylaxis. Journal of Clinical Psychiatry. 2001 Jul;62(7):556-9. PMID: 11488368 Ineligible study design

278. Fava GA, Ruini C, Rafanelli C. Sequential treatment of mood and anxiety disorders. Journal of Clinical Psychiatry. 2005 Nov;66(11):1392-400. PMID: 16420076 Ineligible study design

279. Fenton C, Scott LJ. Risperidone: a review of its use in the treatment of bipolar mania. CNS Drugs. 2005;19(5):429-44. PMID: 15907153 Ineligible study design

280. Ferrier IN. Lamotrigine and gabapentin: Alternatives in the treatment of bipolar disorder. Neuropsychobiology. 1998;38(3):192-7. doi: http://dx.doi.org/10.1159/000026536. Ineligible study design 
281. Ferrier IN. Developments in mood stabilisers. British Medical Bulletin. 2001;57:179-92. PMID: 11719917 Ineligible study design

282. Fiorillo A, Del Vecchio V, Luciano M, et al. Efficacy of psychoeducational family intervention for bipolar I disorder: A controlled, multicentric, real-world study. Journal of Affective Disorders. 2015 1, 2015;172:291-9. doi: http://dx.doi.org/10.1016/j.jad.2014.10.021. PMID: 25451428 No eligible outcomes reported

283. Flood C, Byford S, Henderson C, et al. Joint crisis plans for people with psychosis: economic evaluation of a randomised controlled trial. BMJ: British Medical Journal. 2006 2006;333(7571):729. doi: http://dx.doi.org/10.1136/bmj.38929.653704.55. Ineligible study design

284. Forester B, Sajatovic M, Tsai J, et al. Long-term treatment with lurasidone in older adults with bipolar depression: Results of a 6 month open-label study. European Psychiatry. 201531 Mar;30:1134. PMID: 71930814 Ineligible study design

285. Forester B, Sajatovic M, Tsai J, et al. Efficacy and safety of long-term treatment with lurasidone in older adults with bipolar depression: Results of a 6 month open-label study. American Journal of Geriatric Psychiatry. 2015 March;1):S170-S1. PMID: 71814372 Ineligible study design

286. Forester BP, Harper DG, Georgakas J, et al. Antidepressant effects of open label treatment with coenzyme Q10 in geriatric bipolar depression. Journal of Clinical Psychopharmacology. 2015 Jun;35(3):338-40. doi: http://dx.doi.org/10.1097/JCP.0000000000000326. PMID: 2015-19762-024 Ineligible study design

287. Forleo GB, Di Biase L, Bharmi R, et al. Hospitalization rates and associated cost analysis of cardiac resynchronization therapy with an implantable defibrillator and quadripolar vs. bipolar left ventricular leads: a comparative effectiveness study. Europace. 2015 Jan;17(1):101-7. doi: http://dx.doi.org/10.1093/europace/euu290. PMID: 25371428 Not bipolar disorder

288. Fornaro M, McCarthy MJ, De Berardis D, et al. Adjunctive agomelatine therapy in the treatment of acute bipolar II depression: A preliminary open label study. Neuropsychiatric Disease and Treatment. 2013 15, 2013;9:243-51. Ineligible study design

289. Fornaro M, Stubbs B, De Berardis D, et al. Atypical antipsychotics in the treatment of acute bipolar depression with mixed features: A systematic review and exploratory meta-analysis of placebo-controlled clinical trials. International Journal of Molecular Sciences. 2016;17(2)doi: http://dx.doi.org/10.3390/ijms17020241. PMID: 608342330 Ineligible study design

290. Fountoulakis KN. The contemporary face of bipolar illness: complex diagnostic and therapeutic challenges. Cns Spectrums. 2008 Sep;13(9):763-74, 77-9. PMID: 18849896 Ineligible study design

291. Fountoulakis KN, Gonda X, Vieta E, et al. Treatment of psychotic symptoms in bipolar disorder with aripiprazole monotherapy: A meta-analysis. Annals of General Psychiatry. 2009 31, 2009;8:27. doi: http://dx.doi.org/10.1186/1744-859X-8-27. Ineligible study design

292. Fountoulakis KN, Kasper S, Andreassen O, et al. Efficacy of pharmacotherapy in bipolar disorder: a report by the WPA section on pharmacopsychiatry. European Archives of Psychiatry \& Clinical Neuroscience. 2012 Jun;262 Suppl 1:1-48. doi: http://dx.doi.org/10.1007/s00406-012-0323-x. PMID: 22622948

\section{Ineligible study design}


293. Fountoulakis KN, Kontis D, Gonda X, et al. Treatment of mixed bipolar states. International Journal of Neuropsychopharmacology. 2012 Aug;15(7):1015-26. doi: http://dx.doi.org/10.1017/S1461145711001817. PMID: 22217434 Ineligible study design

294. Fountoulakis KN, Kontis D, Gonda X, et al. A systematic review of the evidence on the treatment of rapid cycling bipolar disorder. Bipolar Disorders. 2013 Mar;15(2):115-37. doi:

http://dx.doi.org/10.1111/bdi.12045. PMID: 23437958 Ineligible study design

295. Fountoulakis KN, Vieta E. Treatment of bipolar disorder: a systematic review of available data and clinical perspectives. International Journal of Neuropsychopharmacology. 2008 Nov;11(7):999-1029. doi: http://dx.doi.org/10.1017/S1461145708009231. PMID: 18752718 Ineligible study design

296. Fountoulakis KN, Vieta E. Efficacy and safety of aripiprazole in the treatment of bipolar disorder: A systematic review. Annals of General Psychiatry. 2009 27, 2009;8:16. doi: http://dx.doi.org/10.1186/1744859X-8-16. Ineligible study design

297. Fountoulakis KN, Vieta E, Schmidt F. Aripiprazole monotherapy in the treatment of bipolar disorder: a meta-analysis. Journal of Affective Disorders. 2011 Oct;133(3):361-70. doi: http://dx.doi.org/10.1016/j.jad.2010.10.018. PMID: 21040979 Ineligible study design

298. Frangou S, Lewis M, McCrone P. Efficacy of ethyl-eicosapentaenoic acid in bipolar depression: randomised double-blind placebo-controlled study. British Journal of Psychiatry. 2006 Jan;188:46-50. PMID: 16388069 Ineligible intervention

299. Frank E, Wallace ML, Hall M, et al. An integrated risk reduction intervention can reduce body mass index in individuals being treated for bipolar I disorder: Results from a randomized trial. Bipolar Disorders. 2015 Jun;17(4):424-37. doi: http://dx.doi.org/10.1111/bdi.12283. PMID: 2015-25546-007 No eligible outcomes reported

300. Frecska E, Kovacs AI, Balla P, et al. The message of the survival curves: I. Composite analysis of longterm treatment studies in bipolar disorder. Neuropsychopharmacologia Hungarica. 2012 Sep;14(3):155-64. PMID: 22987729 Ineligible study design

301. Fredman SJ, Baucom DH, Boeding SE, et al. Relatives' emotional involvement moderates the effects of family therapy for bipolar disorder. Journal of Consulting and Clinical Psychology. 2015 Feb;83(1):81-91. doi: http://dx.doi.org/10.1037/a0037713. PMID: 2014-37304-001 Ineligible study design

302. Freeman MP, Stoll AL. Mood stabilizer combinations: a review of safety and efficacy. American Journal of Psychiatry. 1998 Jan;155(1):12-21. PMID: 9433333 Ineligible study design

303. Frye MA, Altshuler LL. Selection of initial treatment for bipolar disorder, manic phase. Modern Problems of Pharmacopsychiatry. 1997;25:88-113. PMID: 9344372 Ineligible study design

304. Frye MA, Amchin J, Bauer M, et al. Randomized, placebo-controlled, adjunctive study of armodafinil for bipolar I depression: implications of novel drug design and heterogeneity of concurrent bipolar maintenance treatments. International Journal of Bipolar Disorders. 201507 Dec;3(1)doi: http://dx.doi.org/10.1186/s40345-015-0034-0. Ineligible study design

305. Frye MA, Eudicone J, Pikalov A, et al. Aripiprazole efficacy in irritability and disruptive-aggressive symptoms: Young Mania Rating Scale line analysis from two, randomized, double-blind, placebo- 
controlled trials. Journal of Clinical Psychopharmacology. 2008 2008;28(2):243-5. doi: http://dx.doi.org/10.1097/JCP.0b013e31816745f7. Ineligible study design

306. Frye MA, Helleman G, McElroy SL, et al. Correlates of treatment-emergent mania associated with antidepressant treatment in bipolar depression. American Journal of Psychiatry. 2009 Feb;166(2):164-72. doi: http://dx.doi.org/10.1176/appi.ajp.2008.08030322. PMID: 19015231 No eligible outcomes reported

307. Frye MA, Ketter TA, Kimbrell TA, et al. A placebo-controlled study of lamotrigine and gabapentin monotherapy in refractory mood disorders. Journal of Clinical Psychopharmacology. 2000 2000;20(6):60714. doi: http://dx.doi.org/10.1097/00004714-200012000-00004. Bipolar not analyzed separately

308. Frye MA, McElroy SL, Prieto ML, et al. Clinical risk factors and serotonin transporter gene variants associated with antidepressant-induced mania. Journal of Clinical Psychiatry. 2015 Feb;76(2):174-80. doi: http://dx.doi.org/10.4088/JCP.14m09127. PMID: 25611077 No eligible outcomes reported

309. Frye MA, Yatham LN, Calabrese JR, et al. Incidence and time course of subsyndromal symptoms in patients with bipolar I disorder: an evaluation of 2 placebo-controlled maintenance trials. Journal of Clinical Psychiatry. 2006 Nov;67(11):1721-8. PMID: 17196051 Ineligible study design

310. Fu DJ, Turkoz I, Bossie CA, et al. Rapid onset of treatment effects on psychosis, depression, and mania in patients with acute exacerbation of schizoaffective disorder following treatment with oral extended-release paliperidone. Journal of Affective Disorders. 2016 Mar 15;193:381-90. doi: http://dx.doi.org/10.1016/j.jad.2015.12.060. PMID: 26802315 Over 20\% schizoaffective

311. Furey ML, Drevets WC. Antidepressant efficacy of the antimuscarinic drug scopolamine: a randomized, placebo-controlled clinical trial. Archives of General Psychiatry. 2006 Oct;63(10):1121-9. PMID: 17015814 Bipolar not analyzed separately

312. Furey ML, Khanna A, Hoffman EM, et al. Scopolamine produces larger antidepressant and antianxiety effects in women than in men. Neuropsychopharmacology. 2010 Nov;35(12):2479-88. doi: http://dx.doi.org/10.1038/npp.2010.131. PMID: 20736989 Bipolar not analyzed separately

313. Furey ML, Nugent AC, Speer AM, et al. Baseline mood-state measures as predictors of antidepressant response to scopolamine. Psychiatry Research. 2012 30, 2012;196(1):62-7. doi: http://dx.doi.org/10.1016/j.psychres.2012.01.003. Ineligible study design

314. Gagne GG, Jr., Furman MJ, Carpenter LL, et al. Efficacy of continuation ECT and antidepressant drugs compared to long-term antidepressants alone in depressed patients. American Journal of Psychiatry. 2000 Dec;157(12):1960-5. PMID: 11097961 Ineligible study design

315. Gajwani P, Kemp DE, Muzina DJ, et al. Acute treatment of mania: an update on new medications. Current Psychiatry Reports. 2006 Dec;8(6):504-9. PMID: 17094930 Ineligible study design

316. Gallagher P, Malik N, Newham J, et al. Antiglucocorticoid treatments for mood disorders. Cochrane Database of Systematic Reviews. 2008(1):CD005168. doi: http://dx.doi.org/10.1002/14651858.CD005168.pub2. PMID: 18254070 Not treating bipolar

317. Galvez V, Alonzo A, Martin D, et al. Hypomania induction in a patient with bipolar II disorder by transcranial direct current stimulation (tDCS). Journal of ECT. 2011 Sep;27(3):256-8. doi: http://dx.doi.org/10.1097/YCT.0b013e3182012b89. PMID: 21206371 Ineligible study design 
318. Gao K, Calabrese JR. Newer treatment studies for bipolar depression. Bipolar Disorders. 2005;7 Suppl 5:13-23. PMID: 16225556 Ineligible study design

319. Gao K, Fang F, Wang Z, et al. Subjective Versus Objective Weight Gain during Acute Treatment with Second-Generation Antipsychotics in Schizophrenia and Bipolar Disorder. Journal of Clinical Psychopharmacology. 201601 Dec;36(6):637-42. doi: http://dx.doi.org/10.1097/JCP.0000000000000596. PMID: 612817049 Ineligible study design

320. Gao K, Gajwani P, Elhaj O, et al. Typical and atypical antipsychotics in bipolar depression. Journal of Clinical Psychiatry. 2005 Nov;66(11):1376-85. PMID: 16420074 Ineligible study design

321. Gao K, Kemp DE, Fein E, et al. Number needed to treat to harm for discontinuation due to adverse events in the treatment of bipolar depression, major depressive disorder, and generalized anxiety disorder with atypical antipsychotics. Journal of Clinical Psychiatry. 2011 Aug;72(8):1063-71. doi: http://dx.doi.org/10.4088/JCP.09r05535gre. PMID: 21034695 Ineligible study design

322. Gao K, Kemp DE, Ganocy SJ, et al. Antipsychotic-induced extrapyramidal side effects in bipolar disorder and schizophrenia: a systematic review. Journal of Clinical Psychopharmacology. 2008 Apr;28(2):203-9. doi: http://dx.doi.org/10.1097/JCP.0b013e318166c4d5. PMID: 18344731 Ineligible study design

323. Gao K, Kemp DE, Ganocy SJ, et al. Treatment-emergent mania/hypomania during antidepressant monotherapy in patients with rapid cycling bipolar disorder. Bipolar Disorders. 2008 Dec;10(8):907-15. doi: http://dx.doi.org/10.1111/j.1399-5618.2008.00637.x. PMID: 19594506 No eligible outcomes reported

324. Gao K, Muzina D, Gajwani P, et al. Efficacy of typical and atypical antipsychotics for primary and comorbid anxiety symptoms or disorders: a review. Journal of Clinical Psychiatry. 2006 Sep;67(9):132740. PMID: 17017818 Ineligible study design

325. Gao K, Pappadopulos E, Karayal ON, et al. Risk for adverse events and discontinuation due to adverse events of ziprasidone monotherapy relative to placebo in the acute treatment of bipolar depression, mania, and schizophrenia. Journal of Clinical Psychopharmacology. 2013 Jun;33(3):425-31. doi: http://dx.doi.org/10.1097/JCP.0b013e3182917f3f. PMID: 23609405 Duplicate reference

326. Garfinkel PE, Stancer HC, Persad E. A comparison of haloperidol, lithium carbonate and their combination in the treatment of mania. Journal of Affective Disorders. 1980 12//;2(4):279-88. doi: http://dx.doi.org/10.1016/0165-0327(80)90029-4. PMID: 6450787 Fewer than 11 subjects per arm

327. Gaudiano BA, Miller IW. Anxiety disorder comobidity in Bipolar I Disorder: relationship to depression severity and treatment outcome. Depression \& Anxiety. 2005;21(2):71-7. PMID: 15786484 Ineligible study design

328. Gaudiano BA, Uebelacker LA, Miller IW. Impact of remitted substance use disorders on the future course of bipolar I disorder: findings from a clinical trial. Psychiatry Research. 2008 Jul 15;160(1):63-71. doi: http://dx.doi.org/10.1016/j.psychres.2007.05.014. PMID: 18514326 Ineligible study design

329. Geddes JR, Burgess S, Hawton K, et al. Long-term lithium therapy for bipolar disorder: systematic review and meta-analysis of randomized controlled trials. American Journal of Psychiatry. 2004 Feb;161(2):21722. PMID: 14754766 Ineligible study design 
330. Geddes JR, Calabrese JR, Goodwin GM. Lamotrigine for treatment of bipolar depression: independent meta-analysis and meta-regression of individual patient data from five randomised trials. British Journal of Psychiatry. 2009 Jan;194(1):4-9. doi: http://dx.doi.org/10.1192/bjp.bp.107.048504. PMID: 19118318 No eligible outcomes reported

D2331. Geddes JR, Gardiner A, Rendell J, et al. Comparative evaluation of quetiapine plus lamotrigine combination versus quetiapine monotherapy (and folic acid versus placebo) in bipolar depression (CEQUEL): A 2 x 2 factorial randomised trial. The Lancet Psychiatry. 201601 Jan;3(1):31-9. doi: http://dx.doi.org/10.1016/S2215-0366\%2815\%2900450-2. PMID: 26687300 PMID/607320783 Embase Over $50 \%$ dropout rate

332. Gedge L, Lazowski L, Murray D, et al. Effects of quetiapine on sleep architecture in patients with unipolar or bipolar depression. Neuropsychiatric Disease and Treatment. 2010 10, 2010;6:501-8. Ineligible study design

333. Gentile S. Extrapyramidal adverse events associated with atypical antipsychotic treatment of bipolar disorder. Journal of Clinical Psychopharmacology. 2007 Feb;27(1):35-45. PMID: 17224710 Ineligible study design

334. George MS, Nahas Z, Molloy M, et al. A controlled trial of daily left prefrontal cortex TMS for treating depression. Biological Psychiatry. 2000 2000;48(10):962-70. doi: http://dx.doi.org/10.1016/S00063223\%2800\%2901048-9. No eligible outcomes reported

335. George MS, Padberg F, Schlaepfer TE, et al. Controversy: Repetitive transcranial magnetic stimulation or transcranial direct current stimulation shows efficacy in treating psychiatric diseases (depression, mania, schizophrenia, obsessive-complusive disorder, panic, posttraumatic stress disorder). Brain Stimulation. 2009 Jan;2(1):14-21. doi: http://dx.doi.org/10.1016/j.brs.2008.06.001. PMID: 20633399 Ineligible study design

336. Gergerlioglu HS, Savas HA, Bulbul F, et al. Changes in nitric oxide level and superoxide dismutase activity during antimanic treatment.[Erratum appears in Prog Neuropsychopharmacol Biol Psychiatry. 2007 Aug 15;31(6):1345]. Progress in Neuro-Psychopharmacology \& Biological Psychiatry. 2007 Apr 13;31(3):697-702. PMID: 17303295 Ineligible study design

337. Gergerlioglu HS, Savas HA, Celik A, et al. Atypical antipsychotic usage-related higher serum leptin levels and disabled lipid profiles in euthymic bipolar patients. Neuropsychobiology. 2006;53(2):108-12. PMID:

\section{Ineligible study design}

338. Ghadiri Vasfi M, Moradi-Lakeh M, Esmaeili N, et al. Efficacy of aftercare services for people with severe mental disorders in Iran: a randomized controlled trial. Psychiatric Services. 2015 Apr 1;66(4):373-80. doi: http://dx.doi.org/10.1176/appi.ps.201400111. PMID: 25828982 Over 20\% schizoaffective

339. Ghaemi SN, Goodwin FK. Use of atypical antipsychotic agents in bipolar and schizoaffective disorders: review of the empirical literature. Journal of Clinical Psychopharmacology. 1999 Aug;19(4):354-61. PMID: 10440464 Ineligible study design

340. Ghaemi SN, Hsu DJ, Rosenquist KJ, et al. Extrapyramidal side effects with atypical neuroleptics in bipolar disorder. Progress in Neuro-Psychopharmacology \& Biological Psychiatry. 2006 Mar;30(2):209-13.

PMID: 16412546 No eligible outcomes reported 
341. Ghaemi SN, Lenox MS, Baldessarini RJ. Effectiveness and safety of long-term antidepressant treatment in bipolar disorder. Journal of Clinical Psychiatry. 2001 Jul;62(7):565-9. PMID: 11488370 Ineligible study design

342. Ghaemi SN, Ostacher MM, El-Mallakh RS, et al. Antidepressant discontinuation in bipolar depression: a Systematic Treatment Enhancement Program for Bipolar Disorder (STEP-BD) randomized clinical trial of long-term effectiveness and safety. Journal of Clinical Psychiatry. 2010 Apr;71(4):372-80. doi: http://dx.doi.org/10.4088/JCP.08m04909gre. PMID: 20409444 Over 50\% dropout rate

343. Ghaemi SN, Wingo AP, Filkowski MA, et al. Long-term antidepressant treatment in bipolar disorder: meta-analyses of benefits and risks. Acta Psychiatrica Scandinavica. 2008 Nov;118(5):347-56. doi: http://dx.doi.org/10.1111/j.1600-0447.2008.01257.x. PMID: 18727689 Ineligible study design

344. Giannini AJ, Nakoneczie AM, Melemis SM, et al. Magnesium oxide augmentation of verapamil maintenance therapy in mania. Psychiatry Research. 2000 Feb 14;93(1):83-7. PMID: 10699232 Ineligible intervention

345. Gijsman HJ, Geddes JR, Rendell JM, et al. Antidepressants for bipolar depression: a systematic review of randomized, controlled trials. American Journal of Psychiatry. 2004 Sep;161(9):1537-47. PMID: 15337640 Ineligible study design

346. Gildengers A, Tatsuoka C, Bialko C, et al. Correlates of treatment response in depressed older adults with bipolar disorder. Journal of Geriatric Psychiatry \& Neurology. 2012 Mar;25(1):37-42. doi: http://dx.doi.org/10.1177/0891988712436685. PMID: 22467845 Ineligible study design

347. Gitlin M, Frye MA. Maintenance therapies in bipolar disorders. Bipolar Disorders. 2012 May;14 Suppl 2:51-65. doi: http://dx.doi.org/10.1111/j.1399-5618.2012.00992.x. PMID: 22510036 Ineligible study design

348. Gliddon E, Lauder S, Berk L, et al. Evaluating discussion board engagement in the MoodSwings online self-help program for bipolar disorder: protocol for an observational prospective cohort study. BMC Psychiatry. 2015 Oct 14;15:243. doi: http://dx.doi.org/10.1186/s12888-015-0630-7. PMID: 26462799

\section{Ineligible study design}

349. Gnanadesikan M, Freeman MP, Gelenberg AJ. Alternatives to lithium and divalproex in the maintenance treatment of bipolar disorder. Bipolar Disorders. 2003 Jun;5(3):203-16. PMID: 12780874 Ineligible study design

350. Goikolea JM, Colom F, Torres I, et al. Lower rate of depressive switch following antimanic treatment with second-generation antipsychotics versus haloperidol. Journal of Affective Disorders. 2013 Jan 25;144(3):191-8. doi: http://dx.doi.org/10.1016/j.jad.2012.07.038. PMID: 23089129 Ineligible study design

351. Goldberg JF. What psychotherapists should know about pharmacotherapies for bipolar disorder. Journal of Clinical Psychology. 2007 May;63(5):475-90. PMID: 17417812 Ineligible study design

352. Goldberg JF, Bowden CL, Calabrese JR, et al. Six-month prospective life charting of mood symptoms with lamotrigine monotherapy versus placebo in rapid cycling bipolar disorder. Biological Psychiatry. 2008 Jan 1;63(1):125-30. PMID: 17543894 No eligible outcomes reported 
353. Goldberg JF, Burdick KE, Endick CJ. Preliminary randomized, double-blind, placebo-controlled trial of pramipexole added to mood stabilizers for treatment-resistant bipolar depression. American Journal of Psychiatry. 2004 Mar;161(3):564-6. PMID: 14992985 Fewer than 11 subjects per arm

354. Goldberg JF, Calabrese JR, Saville BR, et al. Mood stabilization and destabilization during acute and continuation phase treatment for bipolar I disorder with lamotrigine or placebo. Journal of Clinical Psychiatry. 2009 Sep;70(9):1273-80. doi: http://dx.doi.org/10.4088/JCP.08m04381. PMID: 19689918 Over $50 \%$ dropout rate

355. Goldberg JF, Nassir Ghaemi S. Benefits and limitations of antidepressants and traditional mood stabilizers for treatment of bipolar depression. Bipolar Disorders. 2005;7 Suppl 5:3-12. PMID: 16225555 Ineligible study design

356. Goldberg JF, Perlis RH, Ghaemi SN, et al. Adjunctive antidepressant use and symptomatic recovery among bipolar depressed patients with concomitant manic symptoms: findings from the STEP-BD. American Journal of Psychiatry. 2007 Sep;164(9):1348-55. PMID: 17728419 Ineligible study design

357. Goldberg JF, Truman CJ. Antidepressant-induced mania: an overview of current controversies. Bipolar Disorders. 2003 Dec;5(6):407-20. PMID: 14636364 Ineligible study design

358. Goldstein MJ, Miklowitz DJ. Family intervention for persons with bipolar disorder. Hatfield, Agnes B [Ed]. 1994 Ineligible study design

359. Gonzalez Arnold J, Salcedo S, Ketter TA, et al. An exploratory study of responses to low-dose lithium in African Americans and Hispanics. Journal of Affective Disorders. 2015 Jun 1;178:224-8. doi: http://dx.doi.org/10.1016/j.jad.2015.02.035. PMID: 25827507 Over 50\% dropout rate

360. Gonzalez JM, Bowden CL, Berman N, et al. One-year treatment outcomes of African-American and Hispanic patients with bipolar I or II disorder in STEP-BD. Psychiatric Services. 2010 Feb;61(2):164-72. doi: http://dx.doi.org/10.1176/appi.ps.61.2.164. PMID: 20123822 Ineligible study design

361. Gonzalez JM, Prihoda TJ. A case study of psychodynamic group psychotherapy for bipolar disorder. American Journal of Psychotherapy. 2007;61(4):405-22. Ineligible study design

362. Gonzalez S, Artal J, Gomez E, et al. Early intervention in bipolar disorder: the Jano program at Hospital Universitario Marques de Valdecilla. Actas Espanolas de Psiquiatria. 2012 Mar-Apr;40(2):51-6. PMID: 22508069 Ineligible study design

363. Gonzalez-Pinto A, Vieta E, Reed C, et al. Effectiveness of olanzapine monotherapy and olanzapine combination treatment in the long term following acute mania--results of a two year observational study in bipolar disorder (EMBLEM). Journal of Affective Disorders. 2011 Jun;131(1-3):320-9. doi: http://dx.doi.org/10.1016/j.jad.2010.11.037. PMID: 21195486 Ineligible study design

364. Goodnick PJ. Bipolar depression: a review of randomised clinical trials. Expert Opinion on Pharmacotherapy. 2007 Jan;8(1):13-21. PMID: 17163803 Ineligible study design

365. Goodwin FK. Rationale for long-term treatment of bipolar disorder and evidence for long-term lithium treatment. Journal of Clinical Psychiatry. 2002;63 Suppl 10:5-12. PMID: 12392347 Ineligible study design 
366. Goodwin G, Vieta E. Effective maintenance treatment--breaking the cycle of bipolar disorder. European Psychiatry: the Journal of the Association of European Psychiatrists. 2005 Aug;20(5-6):365-71. PMID: 16122915 Ineligible study design

367. Goodwin GM, Bowden CL, Calabrese JR, et al. A pooled analysis of 2 placebo-controlled 18-month trials of lamotrigine and lithium maintenance in bipolar I disorder. Journal of Clinical Psychiatry. 2004 Mar;65(3):432-41. PMID: 15096085 Ineligible study design

368. Goodwin GM, Geddes JR. Latest maintenance data on lithium in bipolar disorder. European Neuropsychopharmacology. 2003 Aug;13 Suppl 2:S51-5. PMID: 12957720 Ineligible study design

369. Gopal S, Steffens DC, Kramer ML, et al. Symptomatic remission in patients with bipolar mania: results from a double-blind, placebo-controlled trial of risperidone monotherapy. Journal of Clinical Psychiatry. 2005 Aug;66(8):1016-20. PMID: 16086617 No eligible outcomes reported

370. Goss AJ, Kaser M, Costafreda SG, et al. Modafinil augmentation therapy in unipolar and bipolar depression: a systematic review and meta-analysis of randomized controlled trials. Journal of Clinical Psychiatry. 2013 Nov;74(11):1101-7. doi: http://dx.doi.org/10.4088/JCP.13r08560. PMID: 24330897

\section{Ineligible study design}

371. Gouliaev G, Licht RW, Vestergaard P, et al. Treatment of manic episodes: zuclopenthixol and clonazepam versus lithium and clonazepam. Acta Psychiatrica Scandinavica. 1996 Feb;93(2):119-24. PMID: 8686481 Bipolar not analyzed separately

372. Grande I, Hidalgo-Mazzei D, Nieto E, et al. Asenapine prescribing patterns in the treatment of manic inand outpatients: Results from the MANACOR study. European Psychiatry. 2015 Jun;30(4):528-34. doi: http://dx.doi.org/10.1016/j.eurpsy.2015.01.003. PMID: 2015-06676-001 No eligible outcomes reported

373. Grande I, Kapczinski F, Stertz L, et al. Peripheral brain-derived neurotrophic factor changes along treatment with extended release quetiapine during acute mood episodes: an open-label trial in drug-free patients with bipolar disorder. Journal of Psychiatric Research. 2012 Nov;46(11):1511-4. doi: http://dx.doi.org/10.1016/j.jpsychires.2012.08.017. PMID: 22939945 No eligible outcomes reported

374. Grande I, Vieta E. Pharmacotherapy of acute mania: Monotherapy or combination therapy with mood stabilizers and antipsychotics? CNS Drugs. 2015 Mar;29(3):221-7. doi: http://dx.doi.org/10.1007/s40263015-0235-1. PMID: 2015-14036-005 Ineligible study design

375. Greenhalgh J, Knight C, Hind D, et al. Clinical and cost-effectiveness of electroconvulsive therapy for depressive illness, schizophrenia, catatonia and mania: systematic reviews and economic modelling studies. Health Technology Assessment (Winchester, England). 2005 Mar;9(9):1-156, iii-iv. PMID: 15774232

\section{Ineligible study design}

376. Gregory VL, Jr. Cognitive-behavioral therapy for depression in bipolar disorder: a meta-analysis. Journal of Evidence-Based Social Work. 2010 Jul;7(4):269-79. doi: http://dx.doi.org/10.1080/15433710903176088. PMID: 20799127 Ineligible study design 
377. Grisaru N, Chudakov B, Yaroslavsky Y, et al. Transcranial magnetic stimulation in mania: a controlled study. American Journal of Psychiatry. 1998 Nov;155(11):1608-10. PMID: 9812128 Ineligible study design

378. Grunhaus L, Hirschman S, Dolberg OT, et al. Coadministration of melatonin and fluoxetine does not improve the 3-month outcome following ECT. Journal of ECT. 2001 Jun;17(2):124-8. PMID: 11417923

\section{Ineligible intervention}

379. Grunhaus L, Schreiber S, Dolberg OT, et al. Response to ECT in major depression: are there differences between unipolar and bipolar depression? Bipolar Disorders. 2002;4 Suppl 1:91-3. PMID: 12479688

\section{Ineligible study design}

380. Grunze H. Lithium in the acute treatment of bipolar disorders-a stocktaking. European Archives of Psychiatry \& Clinical Neuroscience. 2003 Jun;253(3):115-9. PMID: 12904974 Ineligible study design

381. Grunze H. Reevaluating therapies for bipolar depression. Journal of Clinical Psychiatry. 2005;66 Suppl 5:17-25. PMID: 16038598 Ineligible study design

382. Grunze H, Kotlik E, Costa R, et al. Assessment of the efficacy and safety of eslicarbazepine acetate in acute mania and prevention of recurrence: experience from multicentre, double-blind, randomised phase II clinical studies in patients with bipolar disorder I. Journal of Affective Disorders. 2015 Mar 15;174:70-82. doi: http://dx.doi.org/10.1016/j.jad.2014.11.013. PMID: 25484179 Over $50 \%$ dropout rate

383. Grunze H, Vieta E, Goodwin GM, et al. The World Federation of Societies of Biological Psychiatry (WFSBP) guidelines for the biological treatment of bipolar disorders: update 2009 on the treatment of acute mania.[Erratum appears in World J Biol Psychiatry. 2009;10(3):255 Note: Dosage error in article text]. World Journal of Biological Psychiatry. 2009;10(2):85-116. doi: http://dx.doi.org/10.1080/15622970902823202. PMID: 19347775 Ineligible study design

384. Grunze H, Vieta E, Goodwin GM, et al. The World Federation of Societies of Biological Psychiatry (WFSBP) Guidelines for the Biological Treatment of Bipolar Disorders: Update 2010 on the treatment of acute bipolar depression. World Journal of Biological Psychiatry. 2010 Mar;11(2):81-109. doi: http://dx.doi.org/10.3109/15622970903555881. PMID: 20148751 Ineligible study design

385. Grunze H, Vieta E, Goodwin GM, et al. The World Federation of Societies of Biological Psychiatry (WFSBP) guidelines for the biological treatment of bipolar disorders: update 2012 on the long-term treatment of bipolar disorder. World Journal of Biological Psychiatry. 2013 Apr;14(3):154-219. doi: http://dx.doi.org/10.3109/15622975.2013.770551. PMID: 23480132 Ineligible study design

386. Grunze H, Walden J. Relevance of new and newly rediscovered anticonvulsants for atypical forms of bipolar disorder. Journal of Affective Disorders. 2002 Dec;72 Suppl 1:S15-21. PMID: 12589899

\section{Ineligible study design}

387. Gu N, Wang G, Tan Q, et al. Efficacy and safety of quetiapine extended release monotherapy in bipolar depression: A multi-center, randomized, double-blind, placebo-controlled trial. Psychopharmacology. 2016 01 Apr;233(7):1289-97. doi: http://dx.doi.org/10.1007/s00213-016-4215-z. PMID: 608645719 No

\section{eligible outcomes reported}


388. Gutierrez MJ, Scott J. Psychological treatment for bipolar disorders--a review of randomised controlled trials. European Archives of Psychiatry \& Clinical Neuroscience. 2004 Apr;254(2):92-8. PMID: 15146338 Ineligible study design

389. Hadjipavlou G, Mok H, Yatham LN. Pharmacotherapy of bipolar II disorder: a critical review of current evidence. Bipolar Disorders. 2004 Feb;6(1):14-25. PMID: 14996137 Ineligible study design

390. Haghighi M, Bajoghli H, Bigdelou G, et al. Assessment of cognitive impairments and seizure characteristics in electroconvulsive therapy with and without sodium valproate in manic patients. Neuropsychobiology. 2013;67(1):14-24. doi: http://dx.doi.org/10.1159/000343490. PMID: 23221898 No eligible outcomes reported

391. Halaris A. Antiinflammatory augmentation strategy reverses treatment resistant bipolar depression. Biological Psychiatry. 201501 May;1):297S. PMID: 71846905 Ineligible study design

392. Harvey PD, Hassman H, Mao L, et al. Cognitive functioning and acute sedative effects of risperidone and quetiapine in patients with stable bipolar I disorder: a randomized, double-blind, crossover study. Journal of Clinical Psychiatry. 2007 Aug;68(8):1186-94. PMID: 17854242 No eligible outcomes reported

393. Hayes JF, Marston L, Walters K, et al. Lithium vs. valproate vs. olanzapine vs. quetiapine as maintenance monotherapy for bipolar disorder: a population-based UK cohort study using electronic health records. World Psychiatry. 2016 February;15(1):53-8. doi: http://dx.doi.org/10.1002/wps.20298. PMID: 611189425 No eligible outcomes reported

394. Hayes RD, Downs J, Chang CK, et al. The effect of clozapine on premature mortality: an assessment of clinical monitoring and other potential confounders. Schizophrenia Bulletin. 2015 May;41(3):644-55. doi: http://dx.doi.org/10.1093/schbul/sbu120. PMID: 25154620 Over 20\% schizoaffective

395. Hayes RD, Downs J, Chang C-K, et al. The effect of clozapine on premature mortality: An assessment of clinical monitoring and other potential confounders. Schizophrenia Bulletin. 2015 May;41(3):644-55. doi: http://dx.doi.org/10.1093/schbul/sbu120. PMID: 2015-16497-014 Bipolar not analyzed separately

396. Henderson C, Flood C, Leese M, et al. Effect of joint crisis plans on use of compulsory treatment in psychiatry: single blind randomised controlled trial. BMJ. 2004 Jul 17;329(7458):136. PMID: 15240438 Bipolar not analyzed separately

397. Hennen J, Perlis RH, Sachs G, et al. Weight gain during treatment of bipolar I patients with olanzapine. Journal of Clinical Psychiatry. 2004 Dec;65(12):1679-87. PMID: 15641874 Over 50\% dropout rate

398. Henriksen TE, Skrede S, Fasmer OB, et al. Blue-blocking glasses as additive treatment for mania: A randomized placebo-controlled trial. Bipolar Disorders. 201601 May;18(3):221-32. doi: http://dx.doi.org/10.1111/bdi.12390. PMID: 610504287 Ineligible study design

399. Himmerich H, Koethe D, Schuld A, et al. Plasma levels of leptin and endogenous immune modulators during treatment with carbamazepine or lithium. Psychopharmacology. 2005 May;179(2):447-51. PMID: 15565432 Bipolar not analyzed separately

400. Hirano J, Takamiya A, Yamagata B, et al. Frontal and temporal cortical functional recovery after electroconvulsive therapy for depression: A longitudinal functional near-infrared spectroscopy study. Journal of Psychiatric Research. 201701 Aug;91:26-35. doi: http://dx.doi.org/10.1016/j.jpsychires.2017.02.018. PMID: 614736995 Ineligible study design 
401. Hirota T, Kishi T. Adenosine hypothesis in schizophrenia and bipolar disorder: A systematic review and meta-analysis of randomized controlled trial of adjuvant purinergic modulators. Schizophrenia Research. 2013 2013;149(1-3):88-95. doi: http://dx.doi.org/10.1016/j.schres.2013.06.038. Ineligible study design

402. Hirschfeld RM, Baker JD, Wozniak P, et al. The safety and early efficacy of oral-loaded divalproex versus standard-titration divalproex, lithium, olanzapine, and placebo in the treatment of acute mania associated with bipolar disorder. Journal of Clinical Psychiatry. 2003 Jul;64(7):841-6. PMID: 12934987 No eligible outcomes reported

403. Hirschfeld RM, Bowden CL, Vigna NV, et al. A randomized, placebo-controlled, multicenter study of divalproex sodium extended-release in the acute treatment of mania. Journal of Clinical Psychiatry. 2010 Apr;71(4):426-32. doi: http://dx.doi.org/10.4088/JCP.08m04960yel. PMID: 20361904 Over 50\% dropout rate

404. Hirschfeld RM, Kasper S. A review of the evidence for carbamazepine and oxcarbazepine in the treatment of bipolar disorder. International Journal of Neuropsychopharmacology. 2004 Dec;7(4):507-22. PMID: 15458610 Ineligible study design

405. Hirschfeld RM, Keck PE, Jr., Kramer M, et al. Rapid antimanic effect of risperidone monotherapy: a 3week multicenter, double-blind, placebo-controlled trial. American Journal of Psychiatry. 2004 Jun;161(6):1057-65. PMID: 15169694 Over 50\% dropout rate

406. Hirschfeld RM, Weisler RH, Raines SR, et al. Quetiapine in the treatment of anxiety in patients with bipolar I or II depression: a secondary analysis from a randomized, double-blind, placebo-controlled study. Journal of Clinical Psychiatry. 2006 Mar;67(3):355-62. PMID: 16649820 No eligible outcomes reported

407. Hirschowitz J, Kolevzon A, Garakani A. The pharmacological treatment of bipolar disorder: the question of modern advances. Harvard Review of Psychiatry. 2010 Sep-Oct;18(5):266-78. doi: http://dx.doi.org/10.3109/10673229.2010.507042. PMID: 20825264 Ineligible study design

408. Hlastala SA, Frank E, Mallinger AG, et al. Bipolar depression: an underestimated treatment challenge. Depression \& Anxiety. 1997;5(2):73-83. PMID: 9262937 Ineligible study design

409. Hollander E, Pallanti S, Allen A, et al. Does sustained-release lithium reduce impulsive gambling and affective instability versus placebo in pathological gamblers with bipolar spectrum disorders? American Journal of Psychiatry. 2005 Jan;162(1):137-45. PMID: 15625212 Not treating bipolar

410. Holloway F, Carson J. Intensive case management for the severely mentally ill. Controlled trial. British Journal of Psychiatry. 1998 Jan;172:19-22. PMID: 9534826 Bipolar not analyzed separately

411. Holmes MK, Erickson K, Luckenbaugh DA, et al. A comparison of cognitive functioning in medicated and unmedicated subjects with bipolar depression. Bipolar Disorders. 2008 Nov;10(7):806-15. doi: http://dx.doi.org/10.1111/j.1399-5618.2008.00628.x. PMID: 19032712 No eligible outcomes reported 
412. Holtzheimer PE, Kelley ME, Gross RE, et al. Subcallosal cingulate deep brain stimulation for treatmentresistant unipolar and bipolar depression. Archives of General Psychiatry. 2012 Feb;69(2):150-8. doi: http://dx.doi.org/10.1001/archgenpsychiatry.2011.1456. PMID: 22213770 Ineligible study design

413. Honig A, Arts BM, Ponds RW, et al. Lithium induced cognitive side-effects in bipolar disorder: a qualitative analysis and implications for daily practice. International Clinical Psychopharmacology. 1999 May;14(3):167-71. PMID: 10435769 No eligible outcomes reported

414. Honig A, Hofman A, Rozendaal N, et al. Psycho-education in bipolar disorder: effect on expressed emotion. Psychiatry Research. 1997 Aug 29;72(1):17-22. PMID: 9355815 No eligible outcomes reported

415. Hopkins HS, Gelenberg AJ. Serum lithium levels and the outcome of maintenance therapy of bipolar disorder. Bipolar Disorders. 2000 Sep;2(3 Pt 1):174-9. PMID: 11256684 Ineligible study design

416. Houston JP, Ahl J, Meyers AL, et al. Reduced suicidal ideation in bipolar I disorder mixed-episode patients in a placebo-controlled trial of olanzapine combined with lithium or divalproex. Journal of Clinical Psychiatry. 2006 Aug;67(8):1246-52. PMID: 16965203 No eligible outcomes reported

417. Huang CC, Wei IH. Unexpected interaction between quetiapine and valproate in patients with bipolar disorder. General Hospital Psychiatry. 2010 Jul-Aug;32(4):446.e1-2. doi: http://dx.doi.org/10.1016/j.genhosppsych.2009.06.005. PMID: 20633751 Ineligible study design

418. Hurley SC. Lamotrigine update and its use in mood disorders. Annals of Pharmacotherapy. 2002 May;36(5):860-73. PMID: 11978166 Ineligible study design

419. Husain MI, Chaudhry IB, Rahman RR, et al. Pilot study of a culturally adapted psychoeducation (CaPE) intervention for bipolar disorder in Pakistan. International Journal of Bipolar Disorders. 201701 Dec;5 (1) (no pagination)(3)doi: http://dx.doi.org/10.1186/s40345-017-0074-8. PMID: 614403784 Ineligible study design

420. Issakidis C, Sanderson K, Teesson M, et al. Intensive case management in Australia: A randomized controlled trial. Acta Psychiatrica Scandinavica. 1999 1999;99(5):360-7. doi: http://dx.doi.org/10.1111/j.1600-0447.1999.tb07242.x. Bipolar not analyzed separately

421. Ives-Deliperi VL, Howells F, Stein DJ, et al. The effects of mindfulness-based cognitive therapy in patients with bipolar disorder: A controlled functional MRI investigation. Journal of Affective Disorders. 2013 25, 2013;150(3):1152-7. doi: http://dx.doi.org/10.1016/j.jad.2013.05.074. No eligible outcomes reported

422. Jackson HJ, McGorry PD, Killackey E, et al. Acute-phase and 1-year follow-up results of a randomized controlled trial of CBT versus Befriending for first-episode psychosis: the ACE project. Psychological Medicine. 2008 May;38(5):725-35. PMID: 18005494 Bipolar not analyzed separately

423. Jahangard L, Haghighi M, Bigdelou G, et al. Comparing efficacy of ECT with and without concurrent sodium valproate therapy in manic patients. Journal of ECT. 2012 Jun;28(2):118-23. doi: http://dx.doi.org/10.1097/YCT.0b013e31824b64b5. PMID: 22531205 No eligible outcomes reported 
424. Jainer AK, King M, Sridharan S, et al. New Perspectives in the Treatment of Bipolar Affective Disorder. International Medical Journal. 2005 2005;12(4):247-50. Ineligible study design

425. Janicak PG, Sharma RP, Pandey G, et al. Verapamil for the treatment of acute mania: a double-blind, placebo-controlled trial. American Journal of Psychiatry. 1998 Jul;155(7):972-3. PMID: 9659868 Not treating bipolar

426. Jefferson JW. Lamotrigine in psychiatry: pharmacology and therapeutics. Cns Spectrums. 2005 Mar;10(3):224-32. PMID: 15744223 Ineligible study design

427. Jensen HV, Davidsen K, Toftegaard L, et al. Double-blind comparison of the side-effect profiles of dasily versus alternate-day dosing schedules in lithium maintenance treatment of manic-depressive disorder. Journal of Affective Disorders. 1996 1996;36(3-4):89-93. doi: http://dx.doi.org/10.1016/01650327\%2895\%2900052-6. No eligible outcomes reported

428. Jensen HV, Holm J, Davidsen K, et al. Urinary excretion of albumin and transferrin in lithium maintenance treatment: daily versus alternate-day lithium dosing schedule. Psychopharmacology. 1995 Dec;122(3):31720. PMID: 8748403 No eligible outcomes reported

429. Jensen HV, Plenge P, Mellerup ET, et al. Lithium prophylaxis of manic-depressive disorder: daily lithium dosing schedule versus every second day. Acta Psychiatrica Scandinavica. 1995 Jul;92(1):69-74. PMID: 7572251 Bipolar not analyzed separately

430. Jeste DV, Dolder CR. Treatment of non-schizophrenic disorders: Focus on atypical antipsychotics. Journal of Psychiatric Research. 2004 2004;38(1):73-103. doi: http://dx.doi.org/10.1016/S00223956\%2803\%2900094-3. Ineligible study design

431. Jeste DV, Dolder CR, Nayak GV, et al. Atypical antipsychotics in elderly patients with dementia or schizophrenia: Review of recent literature. Harvard Review of Psychiatry. 2005 2005;13(6):340-51. doi: http://dx.doi.org/10.1080/10673220500433247. Ineligible study design

432. Jiang Y, McCombs JS, Park SH. A Retrospective Cohort Study of Acute Kidney Injury Risk Associated with Antipsychotics. CNS Drugs. 201701 Apr;31(4):319-26. doi: http://dx.doi.org/10.1007/s40263-0170421-4. PMID: 614799558 Bipolar not analyzed separately

433. Jindal RD, Thase ME. Integrating psychotherapy and pharmacotherapy to improve outcomes among patients with mood disorders. Psychiatric Services. 2003 Nov;54(11):1484-90. PMID: 14600307

\section{Ineligible study design}

434. Joas E, Karanti A, Song J, et al. Pharmacological treatment and risk of psychiatric hospital admission in bipolar disorder. British Journal of Psychiatry. 2017 March;210(3):197-202. doi:

http://dx.doi.org/10.1192/bjp.bp.116.187989. No eligible outcomes reported

435. Joffe RT. The use of thyroid supplements to augment antidepressant medication. Journal of Clinical Psychiatry. 1998;59 Suppl 5:26-9; discussion 30-1. PMID: 9635545 Ineligible study design

436. Johnson SL, Fulford D. Preventing mania: a preliminary examination of the GOALS Program. Behavior Therapy. 2009 Jun;40(2):103-13. doi: http://dx.doi.org/10.1016/j.beth.2008.03.002. PMID: 19433142 Ineligible study design 
437. Jones RM, Thompson C, Bitter I. A systematic review of the efficacy and safety of second generation antipsychotics in the treatment of mania. European Psychiatry: the Journal of the Association of European Psychiatrists. 2006 Jan;21(1):1-9. PMID: 16487905 Ineligible study design

438. Jones S. Psychotherapy of bipolar disorder: A review. Journal of Affective Disorders. 2004 2004;80(23):101-14. doi: http://dx.doi.org/10.1016/S0165-0327\%2803\%2900111-3. Ineligible study design

439. Jones S, McGrath E, Hampshire K, et al. A randomised controlled trial of time limited CBT informed psychological therapy for anxiety in bipolar disorder. BMC Psychiatry. 2013;13:54. doi: http://dx.doi.org/10.1186/1471-244X-13-54. PMID: 23414176 Not treating bipolar

440. Jones S, Mulligan LD, Law H, et al. A randomised controlled trial of recovery focused CBT for individuals with early bipolar disorder. BMC Psychiatry. 2012;12:204. doi: http://dx.doi.org/10.1186/1471-244X-12204. PMID: 23171304 Ineligible study design

441. Jones SH, Smith G, Mulligan LD, et al. Recovery-focused cognitive-behavioural therapy for recent-onset bipolar disorder: Randomized controlled pilot trial. British Journal of Psychiatry. 201501 Jan;206(1):5866. doi: http://dx.doi.org/10.1192/bjp.bp.113.141259. PMID: 601137212 Duplicate reference

442. Juruena MF, Ottoni GL, Machado-Vieira R, et al. Bipolar I and II disorder residual symptoms: oxcarbazepine and carbamazepine as add-on treatment to lithium in a double-blind, randomized trial. Progress in Neuro-Psychopharmacology \& Biological Psychiatry. 2009 Feb 1;33(1):94-9. doi: http://dx.doi.org/10.1016/j.pnpbp.2008.10.012. PMID: 19007842 Ineligible study design

443. Justo LP, Soares BG, Calil HM. Family interventions for bipolar disorder. Cochrane Database of Systematic Reviews. 2007(4):CD005167. PMID: 17943843 Ineligible study design

444. Kakkar AK, Rehan HS, Unni KE, et al. Comparative efficacy and safety of oxcarbazepine versus divalproex sodium in the treatment of acute mania: a pilot study. European Psychiatry: the Journal of the Association of European Psychiatrists. 2009 Apr;24(3):178-82. doi: http://dx.doi.org/10.1016/j.eurpsy.2008.12.014. PMID: 19324530 Over 50\% dropout rate

445. Kantrowitz JT, Halberstam B, Gangwisch J. Single-dose ketamine followed by daily D-cycloserine in treatment-resistant bipolar depression. Journal of Clinical Psychiatry. 2015 Jun;76(6):737-8. doi: http://dx.doi.org/10.4088/JCP.14109527. PMID: 2015-34824-030 Ineligible study design

446. Kaptsan A, Yaroslavsky Y, Applebaum J, et al. Right prefrontal TMS versus sham treatment of mania: a controlled study. Bipolar Disorders. 2003 Feb;5(1):36-9. PMID: 12656936 Bipolar not analyzed separately

447. Kasper S, Calabrese JR, Johnson G, et al. International consensus group on the evidence-based pharmacologic treatment of bipolar I and II depression. Journal of Clinical Psychiatry. 2008 2008;69(10):1632-46. doi: http://dx.doi.org/10.4088/JCP.v69n1014. Ineligible study design

448. Katagiri H, Takita Y, Tohen M, et al. Safety and efficacy of olanzapine monotherapy and olanzapine with a mood stabilizer in 18-week treatment of manic/mixed episodes for Japanese patients with bipolar I disorder. Current Medical Research \& Opinion. 2012 May;28(5):701-13. doi: http://dx.doi.org/10.1185/03007995.2012.666961. PMID: 22356118 Over 50\% dropout rate

449. Katagiri H, Tohen M, McDonnell DP, et al. Efficacy and safety of olanzapine for treatment of patients with bipolar depression: Japanese subpopulation analysis of a randomized, double-blind, placebo-controlled 
study. BMC Psychiatry. 2013;13:138. doi: http://dx.doi.org/10.1186/1471-244X-13-138. PMID: 23672672 Ineligible study design

450. Katz MM, Maas JW, Frazer A, et al. Drug-induced actions on brain neurotransmitter systems and changes in the behaviors and emotions of depressed patients. Neuropsychopharmacology. 1994 Oct;11(2):89-100. PMID: 7530963 Ineligible study design

451. Keck PE, Jr. Long-term therapy of bipolar illness. Journal of Family Practice. 2003 Mar;Suppl:S18-21. PMID: 12676080 Ineligible study design

452. Keck PE, Jr. Defining and improving response to treatment in patients with bipolar disorder. Journal of Clinical Psychiatry. 2004;65 Suppl 15:25-9. PMID: 15554793 Ineligible study design

453. Keck PE, Jr. Bipolar depression: A new role for atypical antipsychotics? [References]. Bipolar Disorders. 2005;7(Suppl4):34-40. doi: http://dx.doi.org/10.1111/j.1399-5618.2005.00213.x. Ineligible study design

454. Keck PE, Jr., Bowden CL, Meinhold JM, et al. Relationship between serum valproate and lithium levels and efficacy and tolerability in bipolar maintenance therapy. International Journal of Psychiatry in Clinical Practice. 2005 2005;9(4):271-7. doi: http://dx.doi.org/10.1080/13651500500305622. PMID: 24930925 Duplicate reference

455. Keck PE, Jr., Calabrese JR, McIntyre RS, et al. Aripiprazole monotherapy for maintenance therapy in bipolar I disorder: a 100-week, double-blind study versus placebo. Journal of Clinical Psychiatry. 2007 Oct;68(10):1480-91. PMID: 17960961 Over 50\% dropout rate

456. Keck PE, Jr., Corya SA, Altshuler LL, et al. Analyses of treatment-emergent mania with olanzapine/fluoxetine combination in the treatment of bipolar depression. Journal of Clinical Psychiatry. 2005 May;66(5):611-6. PMID: 15889948 Ineligible study design

457. Keck PE, Jr., Marcus R, Tourkodimitris S, et al. A placebo-controlled, double-blind study of the efficacy and safety of aripiprazole in patients with acute bipolar mania. American Journal of Psychiatry. 2003 Sep;160(9):1651-8. PMID: 12944341 Over 50\% dropout rate

458. Keck PE, Jr., McElroy SL. Outcome in the pharmacologic treatment of bipolar disorder. Journal of Clinical Psychopharmacology. 1996 Apr;16(2 Suppl 1):15S-23S. PMID: 8707996 Ineligible study design

459. Keck PE, Jr., McElroy SL. Carbamazepine and valproate in the maintenance treatment of bipolar disorder. Journal of Clinical Psychiatry. 2002;63 Suppl 10:13-7. PMID: 12392348 Ineligible study design

460. Keck PE, Jr., McElroy SL. Divalproex in the treatment of bipolar disorder. Psychopharmacology Bulletin. 2003;37 Suppl 2:67-73. PMID: 15021862 Ineligible study design

461. Keck PE, Jr., McElroy SL. New approaches in managing bipolar depression. Journal of Clinical Psychiatry. 2003;64 Suppl 1:13-8. PMID: 12625800 Ineligible study design

462. Keck PE, Jr., McElroy SL, Strakowski SM. Anticonvulsants and antipsychotics in the treatment of bipolar disorder. Journal of Clinical Psychiatry. 1998;59 Suppl 6:74-81; discussion 2. PMID: 9674940

\section{Ineligible study design}


463. Keck PE, Jr., McElroy SL, Strakowski SM, et al. Antipsychotics in the treatment of mood disorders and risk of tardive dyskinesia. Journal of Clinical Psychiatry. 2000;61 Suppl 4:33-8. PMID: 10739329

\section{Ineligible study design}

464. Keck PE, Jr., Mendlwicz J, Calabrese JR, et al. A review of randomized, controlled clinical trials in acute mania. Journal of Affective Disorders. 2000 Sep;59 Suppl 1:S31-S7. PMID: 11121825 Ineligible study design

465. Keck PE, Jr., Nelson EB, McElroy SL. Advances in the pharmacologic treatment of bipolar depression. Biological Psychiatry. 2003 Apr 15;53(8):671-9. PMID: 12706953 Ineligible study design

466. Keck PE, Orsulak PJ, Cutler AJ, et al. Aripiprazole monotherapy in the treatment of acute bipolar I mania: a randomized, double-blind, placebo- and lithium-controlled study. Journal of Affective Disorders. 2009 Jan;112(1-3):36-49. doi: http://dx.doi.org/10.1016/j.jad.2008.05.014. PMID: 18835043 Over 50\% dropout rate

467. Keck PE, Jr., Strawn JR, McElroy SL. Pharmacologic treatment considerations in co-occurring bipolar and anxiety disorders. Journal of Clinical Psychiatry. 2006;67 Suppl 1:8-15. PMID: 16426111 Ineligible study design

468. Keck PE, Jr., Versiani M, Warrington L, et al. Long-term safety and efficacy of ziprasidone in subpopulations of patients with bipolar mania. Journal of Clinical Psychiatry. 2009 Jun;70(6):844-51. PMID: 19573482 No eligible outcomes reported

469. Kelly T, Lieberman DZ. The Utility of Low-Dose Aripiprazole for the Treatment of Bipolar II and Bipolar NOS Depression. Journal of Clinical Psychopharmacology. 201701 Feb;37(1):99-101. doi: http://dx.doi.org/10.1097/JCP.0000000000000636. PMID: 613690731 No eligible outcomes reported

470. Kemp DE, Calabrese JR, Eudicone JM, et al. Predictive value of early improvement in bipolar depression trials: a post-hoc pooled analysis of two 8-week aripiprazole studies. Psychopharmacology Bulletin. 2010;43(2):5-27. PMID: 21052040 Ineligible study design

471. Kemp DE, Muzina DJ, McIntyre RS, et al. Bipolar depression: trial-based insights to guide patient care. Dialogues in Clinical Neuroscience. 2008;10(2):181-92. PMID: 18689288 Ineligible study design

472. Kemp DE, Sylvia LG, Calabrese JR, et al. General medical burden in bipolar disorder: Findings from the LiTMUS comparative effectiveness trial. Acta Psychiatrica Scandinavica. 2014 2014;129(1):24-34. doi: http://dx.doi.org/10.1111/acps.12101. Ineligible study design

473. Kessing LV, Gerds TA, Feldt-Rasmussen B, et al. Use of lithium and anticonvulsants and the rate of chronic kidney disease a nationwide population-based study. JAMA Psychiatry. 2015 December;72(12):1182-91. doi: http://dx.doi.org/10.1001/jamapsychiatry.2015.1834. PMID: 607152515

\section{Ineligible study design}

474. Kessing LV, Hansen HV, Christensen EM, et al. The effects of centralised and specialised combined pharmacological and psychological intervention compared with decentralised and non-specialised treatment in the early course of severe unipolar and bipolar affective disorders--design of two randomised clinical trials. Trials [Electronic Resource]. 2011;12(1):32. doi: http://dx.doi.org/10.1186/1745-6215-12-32. PMID: 21291564 Ineligible study design 
475. Kessing LV, Hansen HV, Christensen EM, et al. Do young adults with bipolar disorder benefit from early intervention? Journal of Affective Disorders. 2014 Jan;152-154:403-8. doi: http://dx.doi.org/10.1016/j.jad.2013.10.001. PMID: 24268595 No eligible outcomes reported

476. Kessler U, Vaaler AE, Schoyen $\mathrm{H}$, et al. The study protocol of the Norwegian randomized controlled trial of electroconvulsive therapy in treatment resistant depression in bipolar disorder. BMC Psychiatry. 2010;10:16. doi: http://dx.doi.org/10.1186/1471-244X-10-16. PMID: 20178636 Ineligible study design

477. Ketter TA, Amchin J, Frye MA, et al. Long-term safety and efficacy of armodafinil in bipolar depression: A 6-month open-label extension study. Journal of Affective Disorders. 201601 Jun;197:51-7. doi: http://dx.doi.org/10.1016/j.jad.2016.02.050. PMID: 608872019 No eligible outcomes reported

478. Ketter TA, Brooks JO, 3rd, Hoblyn JC, et al. Long-term effectiveness of quetiapine in bipolar disorder in a clinical setting. Journal of Psychiatric Research. 2010 Oct;44(14):921-9. doi: http://dx.doi.org/10.1016/j.jpsychires.2010.02.005. PMID: 20378127 No eligible outcomes reported

479. Ketter TA, Greist JH, Graham JA, et al. The effect of dermatologic precautions on the incidence of rash with addition of lamotrigine in the treatment of bipolar I disorder: a randomized trial. Journal of Clinical Psychiatry. 2006 Mar;67(3):400-6. PMID: 16649826 Not bipolar disorder

480. Ketter TA, Houston JP, Adams DH, et al. Differential efficacy of olanzapine and lithium in preventing manic or mixed recurrence in patients with bipolar I disorder based on number of previous manic or mixed episodes. Journal of Clinical Psychiatry. 2006 Jan;67(1):95-101. PMID: 16426094 Over 50\% dropout rate

481. Ketter TA, Jenkins JB, Schroeder DH, et al. Carbamazepine but not valproate induces bupropion metabolism. Journal of Clinical Psychopharmacology. 1995 Oct;15(5):327-33. PMID: 8830063

\section{Ineligible study design}

482. Ketter TA, Jones M, Paulsson B. Rates of remission/euthymia with quetiapine monotherapy compared with placebo in patients with acute mania. Journal of Affective Disorders. 2007;100 Suppl 1:S45-53. PMID: 17383011 Duplicate reference

483. Ketter TA, Post RM, Parekh PI, et al. Addition of monoamine oxidase inhibitors to carbamazepine: preliminary evidence of safety and antidepressant efficacy in treatment-resistant depression. Journal of Clinical Psychiatry. 1995 Oct;56(10):471-5. PMID: 7559374 Ineligible study design

484. Ketter TA, Sarma K, Silva R, et al. LURASIDONE in the LONG-TERM TREATMENT of PATIENTS with BIPOLAR DISORDER: A 24-WEEK OPEN-LABEL EXTENSION STUDY. Depression and Anxiety. 201601 May;33(5):424-34. doi: http://dx.doi.org/10.1002/da.22479. PMID: 608756629 No eligible outcomes reported

485. Ketter TA, Wang PW, Chandler RA, et al. Dermatology precautions and slower titration yield low incidence of lamotrigine treatment-emergent rash. Journal of Clinical Psychiatry. 2005 May;66(5):642-5. PMID: 1588953 Ineligible study design 
486. Ketter TA, Yang R, Frye MA. Adjunctive armodafinil for major depressive episodes associated with bipolar i disorder. Journal of Affective Disorders. 201501 Aug;181:87-91. doi: http://dx.doi.org/10.1016/j.jad.2015.04.012. PMID: 2015012361 Ineligible study design

487. Khan A, Ginsberg LD, Asnis GM, et al. Effect of lamotrigine on cognitive complaints in patients with bipolar I disorder. Journal of Clinical Psychiatry. 2004 Nov;65(11):1483-90. PMID: 15554760 Duplicate reference

488. Kikkawa A, Kitamura Y, Aiba T, et al. Correlation between the efficacy of lamotrigine and the serum lamotrigine level during the remission phase of acute bipolar II depression: A naturalistic and unblinded prospective pilot study. Biological and Pharmaceutical Bulletin. 2017;40(4):413-8. doi:

http://dx.doi.org/10.1248/bpb.b16-00725. PMID: 615121800 Ineligible study design

489. Kilbourne AM, Goodrich DE, Lai Z, et al. Randomized controlled trial to assess reduction of cardiovascular disease risk in patients with bipolar disorder: the Self-Management Addressing Heart Risk Trial (SMAHRT). Journal of Clinical Psychiatry. 2013 Jul;74(7):e655-62. doi: http://dx.doi.org/10.4088/JCP.12m08082. PMID: 23945460 Ineligible intervention

490. Kleindienst N, Greil W. Inter-episodic morbidity and drop-out under carbamazepine and lithium in the maintenance treatment of bipolar disorder. Psychological Medicine. 2002 Apr;32(3):493-501. PMID: 11989994 Over $20 \%$ schizoaffective

491. Kleindienst N, Greil W. Are illness concepts a powerful predictor of adherence to prophylactic treatment in bipolar disorder? Journal of Clinical Psychiatry. 2004 Jul;65(7):966-74. PMID: 15291686 Ineligible study design

492. Kleindienst N, Severus WE, Greil W. Are serum lithium levels related to the polarity of recurrence in bipolar disorders? Evidence from a multicenter trial. International Clinical Psychopharmacology. 2007 May;22(3):125-31. PMID: 17414737 Ineligible study design

493. Kluwe-Schiavon B, Viola TW, Levandowski ML, et al. A systematic review of cognitive rehabilitation for bipolar disorder. Trends in Psychiatry and Psychotherapy. 2015 October;37(4):194-201. doi: http://dx.doi.org/10.1590/2237-6089-2015-0006. PMID: 607358592 Ineligible study design

494. Kohler-Forsberg O, Sylvia L, Thase M, et al. Nonsteroidal anti-inflammatory drugs (NSAIDs) and paracetamol do not affect 6-month mood-stabilizing treatment outcome among 482 patients with bipolar disorder. Depression and Anxiety. 201701 Mar;34(3):281-90. doi: http://dx.doi.org/10.1002/da.22601. PMID: 614328413 Ineligible intervention

495. Konovalov S, Muralee S, Tampi RR. Anticonvulsants for the treatment of behavioral and psychological symptoms of dementia: A literature review. International Psychogeriatrics. 2008 2008;20(2):293-308. doi: http://dx.doi.org/10.1017/S1041610207006540. Ineligible study design

496. Koukopoulos A, De Chiara L, Koukopoulos AE, et al. Three-year, naturalistic, mirror-image assessment of adding memantine to the treatment of 30 treatment-resistant patients with bipolar disorder. The Journal of clinical psychiatry. 201501 Jan;76(1):e91-e7. doi: http://dx.doi.org/10.4088/JCP.13m08956. PMID:

25650685 No eligible outcomes reported

497. Kramlinger KG, Phillips KA, Post RM. Rash complicating carbamazepine treatment. Journal of Clinical Psychopharmacology. 1994 Dec;14(6):408-13. PMID: 7884021 Not treating bipolar 
498. Kruger S, Trevor Young L, Braunig P. Pharmacotherapy of bipolar mixed states. Bipolar Disorders. 2005 Jun;7(3):205-15. PMID: 15898959 Ineligible study design

499. Kulkarni J, Berk M, Wang W, et al. A four week randomised control trial of adjunctive medroxyprogesterone and tamoxifen in women with mania. Psychoneuroendocrinology. 2014 May;43:5261. doi: http://dx.doi.org/10.1016/j.psyneuen.2014.02.004. PMID: 24703170 Over 20\% schizoaffective

500. Kulkarni J, Garland KA, Scaffidi A, et al. A pilot study of hormone modulation as a new treatment for mania in women with bipolar affective disorder. Psychoneuroendocrinology. 2006 May;31(4):543-7. PMID: 16356651 Less than 11 subjects per arm

501. Kusalic M, Engelsmann F. Renal reactions to changes of lithium dosage. Neuropsychobiology. 1996;34(3):113-6. PMID: 8916067 No eligible outcomes reported

502. Kusumakar V, Yatham LN, Haslam DR, et al. Treatment of mania, mixed state, and rapid cycling. Canadian Journal of Psychiatry - Revue Canadienne de Psychiatrie. 1997 Aug;42 Suppl 2:79S-86S. PMID: 9288440 Ineligible study design

503. Ladea M, Sinca MC, Barbu CM, et al. Clinical and therapeutical aspects of bipolar disorder: The switch on depakine chrono $<$ sup $><$ /sup $>$ from other valproate treatments - Retrospective data collection. Farmacia. 201506 Aug;63(3):446-52. PMID: 605514200 No eligible outcomes reported

504. Lahera G, Benito A, Montes JM, et al. Social cognition and interaction training (SCIT) for outpatients with bipolar disorder. Journal of Affective Disorders. 2013 Mar 20;146(1):132-6. doi: http://dx.doi.org/10.1016/j.jad.2012.06.032. PMID: 22840617 Ineligible study design

505. Lam DH, Burbeck R, Wright K, et al. Psychological therapies in bipolar disorder: the effect of illness history on relapse prevention - a systematic review. Bipolar Disorders. 2009 Aug;11(5):474-82. doi: http://dx.doi.org/10.1111/j.1399-5618.2009.00724.x. PMID: 19624386 Ineligible study design

506. Lam DH, McCrone P, Wright K, et al. Cost-effectiveness of relapse-prevention cognitive therapy for bipolar disorder: 30-month study. British Journal of Psychiatry. 2005 Jun;186:500-6. PMID: 15928361

\section{Ineligible intervention}

507. Landin-Romero R, Novo P, Vicens V, et al. EMDR therapy modulates the default mode network in a subsyndromal, traumatized bipolar patient. Neuropsychobiology. 2013;67(3):181-4. doi: http://dx.doi.org/10.1159/000346654. PMID: 23548794 Ineligible study design

508. Lao KSJ, He Y, Wong ICK, et al. Tolerability and Safety Profile of Cariprazine in Treating Psychotic Disorders, Bipolar Disorder and Major Depressive Disorder: A Systematic Review with Meta-Analysis of Randomized Controlled Trials. CNS Drugs. 201601 Nov;30(11):1043-54. doi: http://dx.doi.org/10.1007/s40263-016-0382-z. PMID: 611873767 Ineligible study design

509. Larsen ER, Saric K. Pregnancy and bipolar disorder: the risk of recurrence when discontinuing treatment with mood stabilisers: a systematic review. Acta Neuropsychiatrica. 201617 Nov:1-8. doi: http://dx.doi.org/10.1017/neu.2016.60. PMID: 613273686 Ineligible study design

510. Latalova K. Insight in bipolar disorder. Psychiatric Quarterly. 2012 Sep;83(3):293-310. doi: http://dx.doi.org/10.1007/s11126-011-9200-4. PMID: 22101737 Ineligible study design 
511. Lauder S, Chester A, Castle D, et al. A randomized head to head trial of MoodSwings.net.au: an Internet based self-help program for bipolar disorder. Journal of Affective Disorders. 2015 Jan 15;171:13-21. doi: http://dx.doi.org/10.1016/j.jad.2014.08.008. PMID: 25282145 Over 50\% dropout rate

512. Leadbetter R, Messenheimer J, Bentley B, et al. Mood-stabilizing properties of lamotrigine: A review of data from controlled clinical trials. Psychiatric Annals. 2002 2002;32(12):766-72. Ineligible study design

513. Lee AA, Laurent SM, Wykes TL, et al. Genetic attributions and mental illness diagnosis: effects on perceptions of danger, social distance, and real helping decisions. Social Psychiatry \& Psychiatric Epidemiology. 2014 May;49(5):781-9. doi: http://dx.doi.org/10.1007/s00127-013-0764-1. PMID: 24068437 Ineligible study design

514. Lee JY, Harvey AG. Memory for therapy in bipolar disorder and Comorbid insomnia. Journal of Consulting and Clinical Psychology. 2015;83(1):92-102. doi: http://dx.doi.org/10.1037/a0037911. PMID: 2015868969 Ineligible study design

515. Leibenluft E, Turner EH, Feldman-Naim S, et al. Light therapy in patients with rapid cycling bipolar disorder: preliminary results. Psychopharmacology Bulletin. 1995;31(4):705-10. PMID: 8851643 Less than 11 subjects per arm

516. Lemoine P, Fondarai J, Faivre T. Valpromide increases amplitude of heart rate circadian rhythm in remitted bipolar and unipolar disorders. A placebo-controlled study. European Psychiatry. 2000 2000;15(7):424-32. doi: http://dx.doi.org/10.1016/S0924-9338\%2800\%2900513-7. PMID: 11112935 No eligible

\section{outcomes reported}

517. Lepkifker E, Iancu I, Horesh N, et al. Lithium therapy for unipolar and bipolar depression among the middle-aged and older adult patient subpopulation. Depression \& Anxiety. 2007;24(8):571-6. PMID:

\section{No eligible outcomes reported}

518. Leverich GS, Altshuler LL, Frye MA, et al. Risk of switch in mood polarity to hypomania or mania in patients with bipolar depression during acute and continuation trials of venlafaxine, sertraline, and bupropion as adjuncts to mood stabilizers. American Journal of Psychiatry. 2006 Feb;163(2):232-9. PMID: 16449476 No eligible outcomes reported

519. Levin JB, Sams J, Tatsuoka C, et al. Use of automated medication adherence monitoring in bipolar disorder research: pitfalls, pragmatics, and possibilities. Therapeutic Advances in Psychopharmacology. 201502 Apr;5(2):76-87. doi: http://dx.doi.org/10.1177/2045125314566807. PMID: 2015886937 Ineligible study design

520. Levin JB, Tatsuoka C, Cassidy KA, et al. Trajectories of medication attitudes and adherence behavior change in non-adherent bipolar patients. Comprehensive Psychiatry. 2015 Apr;58:29-36. doi: http://dx.doi.org/10.1016/j.comppsych.2014.11.023. PMID: 25617964 Ineligible study design

521. Levine J, Barak Y, Kofman O, et al. Follow-up and relapse analysis of an inositol study of depression. Israel Journal of Psychiatry \& Related Sciences. 1995;32(1):14-21. PMID: 7622343 Bipolar not analyzed separately 
522. Levine J, Shectman T, Lefkifker E, et al. Inositol may reverse lithium-induced polydipsia but not polyuria. Human Psychopharmacology: Clinical and Experimental. 1997 1997;12(5):459-65. doi: http://dx.doi.org/10.1002/\%28SICI\%291099-1077\%28199709/10\%2912:5\%3C459::AIDHUP889\%3E3.0.CO;2-K. No eligible outcomes reported

523. Levy NA, Janicak PG. Calcium channel antagonists for the treatment of bipolar disorder. Bipolar Disorders. 2000 Jun;2(2):108-19. PMID: 11252650 Ineligible study design

524. Li DJ, Tseng PT, Chen YW, et al. Significant treatment effect of bupropion in patients with bipolar disorder but similar phase-shifting rate as other antidepressants: A meta-analysis following the PRISMA guidelines. Medicine (United States). 2016;95(13):e3165. doi: http://dx.doi.org/10.1097/MD.0000000000003165. PMID: 609693558 Ineligible study design

525. Licht RW. Drug treatment of mania: a critical review. Acta Psychiatrica Scandinavica. 1998 Jun;97(6):38797. PMID: 9669508 Ineligible study design

526. Licht RW, Gijsman H, Nolen WA, et al. Are antidepressants safe in the treatment of bipolar depression? A critical evaluation of their potential risk to induce switch into mania or cycle acceleration. Acta Psychiatrica Scandinavica. 2008 Nov;118(5):337-46. doi: http://dx.doi.org/10.1111/j.16000447.2008.01237.x. PMID: 18754834 Ineligible study design

527. Licht RW, Nielsen JN, Gram LF, et al. Lamotrigine versus lithium as maintenance treatment in bipolar I disorder: an open, randomized effectiveness study mimicking clinical practice. The 6th trial of the Danish University Antidepressant Group (DUAG-6). Bipolar Disorders. 2010 Aug;12(5):483-93. doi: http://dx.doi.org/10.1111/j.1399-5618.2010.00836.x. PMID: 20712749 Over 50\% dropout rate

528. Licht RW, Vestergaard P, Brodersen A. Long-term outcome of patients with bipolar disorder commenced on lithium prophylaxis during hospitalization: a complete 15-year register-based follow-up. Bipolar Disorders. 2008 Feb;10(1):79-86. doi: http://dx.doi.org/10.1111/j.1399-5618.2008.00499.x. PMID: 18199244 No eligible outcomes reported

529. Licht RW, Vestergaard P, Kessing LV, et al. Psychopharmacological treatment with lithium and antiepileptic drugs: suggested guidelines from the Danish Psychiatric Association and the Child and Adolescent Psychiatric Association in Denmark. Acta Psychiatrica Scandinavica, Supplementum. 2003(419):1-22. PMID: 12974784 Ineligible study design

530. Lipkovich I, Citrome L, Perlis R, et al. Early predictors of substantial weight gain in bipolar patients treated with olanzapine. Journal of Clinical Psychopharmacology. 2006 Jun;26(3):316-20. PMID: 16702898 Ineligible study design

531. Lipkovich IA, Houston JP, Ahl J. Identifying patterns in treatment response profiles in acute bipolar mania: a cluster analysis approach. BMC Psychiatry. 2008;8:65. doi: http://dx.doi.org/10.1186/1471-244X-8-65. PMID: 18664256 Ineligible study design

532. Lobban F, Taylor L, Chandler C, et al. Enhanced relapse prevention for bipolar disorder by community mental health teams: cluster feasibility randomised trial. British Journal of Psychiatry. 2010 Jan;196(1):5963. doi: http://dx.doi.org/10.1192/bjp.bp.109.065524. PMID: 20044662 Ineligible study design

533. Loebel A, Siu C, Pikalov A, et al. Remission in lurasidone-treated patients with bipolar i depression: Posthoc analysis of a 6-week, placebo-controlled trial followed by a 6-month extension. European Psychiatry. 201531 Mar;30:1148. PMID: 71930816 Ineligible study design 
534. Loebel A, Siu C, Rajagopalan K, et al. Recovery in bipolar depression: Post-hoc analysis of a placebocontrolled lurasidone trial followed by a long-term continuation study. Journal of Affective Disorders. 2015 17 Aug;186:376-82. doi: http://dx.doi.org/10.1016/j.jad.2015.07.033. PMID: 2015301525 No eligible outcomes reported

535. Lombardo I, Sachs G, Kolluri S, et al. Two 6-week, randomized, double, blind, placebo-controlled studies of ziparsidone in outpatients with bipolar I depression: Did baseline characteristics impact trial outcome?

[References]. Journal of Clinical Psychopharmacology. 2012 2012;32(4):470-8. doi: http://dx.doi.org/10.1097/JCP.0b013e31825ccde5. No eligible outcomes reported

536. Loo CK, Sainsbury K, Sheehan P, et al. A comparison of RUL ultrabrief pulse (0.3 ms) ECT and standard RUL ECT. International Journal of Neuropsychopharmacology. 2008 2008;11(7):883-90. doi: http://dx.doi.org/10.1017/S1461145708009292. No eligible outcomes reported

537. Lorberg B, Youssef NA, Bhagwagar Z. Lamotrigine-associated rash: To rechallenge or not to rechallenge? [References]. International Journal of Neuropsychopharmacology. 2009 2009;12(2):257-65. doi: http://dx.doi.org/10.1017/S1461145708009504. Ineligible study design

538. Lynch D, Laws KR, McKenna PJ. Cognitive behavioural therapy for major psychiatric disorder: does it really work? A meta-analytical review of well-controlled trials. Psychological Medicine. 2010 Jan;40(1):924. doi: http://dx.doi.org/10.1017/S003329170900590X. PMID: 19476688 Ineligible study design

539. Lyseng-Williamson KA, Perry CM. Aripiprazole: in acute mania associated with bipolar I disorder. CNS Drugs. 2004;18(6):367-76; discussion 77-8. PMID: 15089107 Ineligible study design

540. Machado-Vieira R. Purinergic system in the treatment of bipolar disorder: Uric acid levels as a screening test in mania. Journal of Clinical Psychopharmacology. 2012 2012;32(5):735-6. doi: http://dx.doi.org/10.1097/JCP.0b013e318268391d. Ineligible study design

541. Machado-Vieira R, Andreazza AC, Viale CI, et al. Oxidative stress parameters in unmedicated and treated bipolar subjects during initial manic episode: a possible role for lithium antioxidant effects. Neuroscience Letters. 2007 Jun 21;421(1):33-6. PMID: 17548157 Ineligible study design

542. Machado-Vieira R, Zarate CA, Jr. Proof of concept trials in bipolar disorder and major depressive disorder: a translational perspective in the search for improved treatments. Depression \& Anxiety. 2011 Apr;28(4):267-81. doi: http://dx.doi.org/10.1002/da.20800. PMID: 21456037 Ineligible study design

543. Macritchie K, Geddes JR, Scott J, et al. Valproate for acute mood episodes in bipolar disorder. Cochrane Database of Systematic Reviews. 2003(1):CD004052. PMID: 12535506 Ineligible study design

544. Macritchie KA, Geddes JR, Scott J, et al. Valproic acid, valproate and divalproex in the maintenance treatment of bipolar disorder. Cochrane Database of Systematic Reviews. 2001(3):CD003196. PMID: 11687047 Ineligible study design

545. Magalhaes PV, Dean OM, Bush AI, et al. N-acetylcysteine for major depressive episodes in bipolar disorder. Revista Brasileira de Psiquiatria. 2011 Dec;33(4):374-8. PMID: 22189927 Ineligible intervention 
546. Magalhaes PV, Dean OM, Bush AI, et al. N-acetyl cysteine add-on treatment for bipolar II disorder: a subgroup analysis of a randomized placebo-controlled trial. Journal of Affective Disorders. 2011 Mar;129(1-3):317-20. doi: http://dx.doi.org/10.1016/j.jad.2010.08.001. PMID: 20800897 Ineligible intervention

547. Magalhaes PV, Dean OM, Bush AI, et al. A preliminary investigation on the efficacy of N-acetyl cysteine for mania or hypomania. Australian \& New Zealand Journal of Psychiatry. 2013 Jun;47(6):564-8. doi: http://dx.doi.org/10.1177/0004867413481631. PMID: 23493756 Ineligible intervention

548. Maidment I. Update on the use of new anticonvulsants as mood stabilisers. Psychiatric Bulletin. 1999 1999;23(9):554-8. doi: http://dx.doi.org/10.1192/pb.23.9.554. Ineligible study design

549. Maidment ID. Gabapentin treatment for bipolar disorders. Annals of Pharmacotherapy. 2001 Oct;35(10):1264-9. PMID: 11675857 Ineligible study design

550. Maidment ID. The use of topiramate in mood stabilization. Annals of Pharmacotherapy. 2002 JulAug;36(7-8):1277-81. PMID: 12086564 Ineligible study design

551. Maj M. Selection of the initial drug(s) in the treatment of bipolar disorder, depressed phase. Modern Problems of Pharmacopsychiatry. 1997;25:66-77. PMID: 9344370 Ineligible study design

552. Maj M. The impact of lithium prophylaxis on the course of bipolar disorder: a review of the research evidence. Bipolar Disorders. 2000 Jun;2(2):93-101. PMID: 11252656 Ineligible study design

553. Malhi GS, Adams D, Berk M. Is lithium in a class of its own? A brief profile of its clinical use. Australian \& New Zealand Journal of Psychiatry. 2009 Dec;43(12):1096-104. doi: http://dx.doi.org/10.3109/00048670903279937. PMID: 20001408 Ineligible study design

554. Malhi GS, Adams D, Berk M. Medicating mood with maintenance in mind: bipolar depression pharmacotherapy. Bipolar Disorders. 2009 Jun;11 Suppl 2:55-76. doi: http://dx.doi.org/10.1111/j.13995618.2009.00711.x. PMID: 19538686 Ineligible study design

555. Malhi GS, Bargh DM, Cashman E, et al. The clinical management of bipolar disorder complexity using a stratified model. Bipolar Disorders. 2012 May;14 Suppl 2:66-89. doi: http://dx.doi.org/10.1111/j.13995618.2012.00993.x. PMID: 22510037 Ineligible study design

556. Malhi GS, Berk M, Bourin M, et al. A typical mood stabilizers: a "typical role for atypical antipsychotics. Acta Psychiatrica Scandinavica, Supplementum. 2005(426):29-38. PMID: 16104066 Ineligible study design

557. Mallinger AG, Thase ME, Haskett R, et al. Verapamil augmentation of lithium treatment improves outcome in mania unresponsive to lithium alone: preliminary findings and a discussion of therapeutic mechanisms. Bipolar Disorders. 2008 Dec;10(8):856-66. doi: http://dx.doi.org/10.1111/j.13995618.2008.00636.x. PMID: 19594501 Fewer than 11 subjects per arm

558. Marcus R. Aripiprazole improved time to any mood episode when added to mood stabilizer. Psychiatric Annals. 2011 2011;41(7):353. Ineligible study design 
559. Martin DM, Chan H, Green MJ, et al. Transcranial direct current stimulation (tDCS) to remediate cognitive function in euthymic bipolar disorder patients. Brain Stimulation. 2015 March-April;8 (2):367-8. PMID: 71911914 Ineligible study design

560. Martinez JM, Marangell LB, Simon NM, et al. Baseline predictors of serious adverse events at one year among patients with bipolar disorder in STEP-BD. Psychiatric Services. 2005 Dec;56(12):1541-8. PMID: 16339616 Ineligible study design

561. Martinotti G, Andreoli S, Di Nicola M, et al. Quetiapine decreases alcohol consumption, craving, and psychiatric symptoms in dually diagnosed alcoholics. Human Psychopharmacology: Clinical and Experimental. 2008 2008;23(5):417-24. doi: http://dx.doi.org/10.1002/hup.944. Ineligible study design

562. Martinotti G, Sepede G, Signorelli M, et al. Efficacy and safety of fluoxetine monotherapy in bipolar depression: a systematic review. Expert Opinion on Pharmacotherapy. 2013 Jun;14(8):1065-75. doi: http://dx.doi.org/10.1517/14656566.2013.783014. PMID: 23527943 Ineligible study design

563. Masand PS, Eudicone J, Pikalov A, et al. Criteria for defining symptomatic and sustained remission in bipolar I disorder: a post-hoc analysis of a 26-week aripiprazole study (study CN138-010). Psychopharmacology Bulletin. 2008;41(2):12-23. PMID: 18668014 Over 50\% dropout rate

564. Mazza M, Di Nicola M, Martinotti G, et al. Oxcarbazepine in bipolar disorder: a critical review of the literature. Expert Opinion on Pharmacotherapy. 2007 Apr;8(5):649-56. PMID: 17376019 Ineligible study design

565. McCloud TL, Caddy C, Jochim J, et al. Ketamine and other glutamate receptor modulators for depression in bipolar disorder in adults. The Cochrane database of systematic reviews. 2015;9:CD011611. doi: http://dx.doi.org/10.1002/14651858.CD011611.pub2. PMID: 609677719 Ineligible study design

566. McClure D, Greenman SC, Koppolu SS, et al. A Pilot Study of Safety and Efficacy of Cranial Electrotherapy Stimulation in Treatment of Bipolar II Depression. Journal of Nervous \& Mental Disease. 2015 Nov;203(11):827-35. doi: http://dx.doi.org/10.1097/NMD.0000000000000378. PMID: 26414234 Less than 11 subjects per arm

567. McCormack PL, Wiseman LR. Olanzapine: a review of its use in the management of bipolar I disorder. Drugs. 2004;64(23):2709-26. PMID: 15537371 Ineligible study design

568. McDonell MG, Srebnik D, Angelo F, et al. Randomized controlled trial of contingency management for stimulant use in community mental health patients with serious mental illness. American Journal of Psychiatry. 2013 Jan 1;170(1):94-101. PMID: 23138961 Bipolar not analyzed separately

569. McElroy SL, Keck PE, Jr. Pharmacologic agents for the treatment of acute bipolar mania. Biological Psychiatry. 2000 Sep 15;48(6):539-57. PMID: 11018226 Ineligible study design

570. McElroy SL, Martens BE, Creech RS, et al. Randomized, double-blind, placebo-controlled study of divalproex extended release loading monotherapy in ambulatory bipolar spectrum disorder patients with moderate-to-severe hypomania or mild mania. Journal of Clinical Psychiatry. 2010 May;71(5):557-65. doi: http://dx.doi.org/10.4088/JCP.08m04854yel. PMID: 20361901 Over 50\% dropout rate 
571. McElroy SL, Winstanley E, Mori N, et al. A randomized, placebo-controlled study of zonisamide to prevent olanzapine-associated weight gain. Journal of Clinical Psychopharmacology. 2012 Apr;32(2):16572. doi: http://dx.doi.org/10.1097/JCP.0b013e3182488758. PMID: 22367654 Not treating bipolar

572. McElroy SL, Winstanley EL, Martens B, et al. A randomized, placebo-controlled study of adjunctive ramelteon in ambulatory bipolar I disorder with manic symptoms and sleep disturbance. International Clinical Psychopharmacology. 2011 Jan;26(1):48-53. doi: http://dx.doi.org/10.1097/YIC.0b013e3283400d35. PMID: 20861739 Fewer than 11 subjects per arm

573. McElroy SL, Zarate CA, Cookson J, et al. A 52-week, open-label continuation study of lamotrigine in the treatment of bipolar depression. Journal of Clinical Psychiatry. 2004 Feb;65(2):204-10. PMID: 15003074 No eligible outcomes reported

574. McFarlane WR, Dixon L, Lukens E, et al. Family psychoeducation and schizophrenia: A review of the literature. Journal of Marital and Family Therapy. 2003 2003;29(2):223-45. doi: http://dx.doi.org/10.1111/j.1752-0606.2003.tb01202.x. Ineligible study design

575. McGee V, Whittingham P. Society for Neuroscience--38th Annual Meeting--Therapeutic approaches for Alzheimer's disease, schizophrenia and bipolar disorder. Idrugs. 2009 Jan;12(1):14-6. PMID: 19127498

\section{Ineligible study design}

576. McGirr A, Vohringer PA, Ghaemi SN, et al. Safety and efficacy of adjunctive second-generation antidepressant therapy with a mood stabiliser or an atypical antipsychotic in acute bipolar depression: a systematic review and meta-analysis of randomised placebo-controlled trials. The Lancet Psychiatry. 2016 01 Dec;3(12):1138-46. doi: http://dx.doi.org/10.1016/S2215-0366\%2816\%2930264-4. PMID: 613441082

\section{Ineligible study design}

577. McIntyre R, Katzman M. The role of atypical antipsychotics in bipolar depression and anxiety disorders. Bipolar Disorders. 2003;5 Suppl 2:20-35. PMID: 14700010 Ineligible study design

578. McIntyre RS. Aripiprazole for the maintenance treatment of bipolar I disorder: A review. Clinical Therapeutics. 2010;32 Suppl 1:S32-8. doi: http://dx.doi.org/10.1016/j.clinthera.2010.01.022. PMID: 20152551 Ineligible study design

579. McIntyre RS, Cohen M, Zhao J, et al. Asenapine versus olanzapine in acute mania: a double-blind extension study.[Erratum appears in Bipolar Disord. 2010 Feb;12(1):112]. Bipolar Disorders. 2009 Dec;11(8):815-26. doi: http://dx.doi.org/10.1111/j.1399-5618.2009.00749.x. PMID: 19832806 No eligible outcomes reported

580. McIntyre RS, Cohen M, Zhao J, et al. Asenapine for long-term treatment of bipolar disorder: a doubleblind 40-week extension study. Journal of Affective Disorders. 2010 Nov;126(3):358-65. doi: http://dx.doi.org/10.1016/j.jad.2010.04.005. PMID: 20537396 Ineligible study design

581. McIntyre RS, Konarski JZ, Jones M, et al. Quetiapine in the treatment of acute bipolar mania: efficacy across a broad range of symptoms. Journal of Affective Disorders. 2007;100 Suppl 1:S5-14. PMID: 17391773 Duplicate reference

582. McIntyre RS, Mancini DA, McCann S, et al. Valproate, bipolar disorder and polycystic ovarian syndrome. Bipolar Disorders. 2003 Feb;5(1):28-35. PMID: 12656935 Ineligible study design 
583. McIntyre RS, Mancini DA, McCann S, et al. Topiramate versus bupropion SR when added to mood stabilizer therapy for the depressive phase of bipolar disorder: a preliminary single-blind study. Bipolar Disorders. 2002 Jun;4(3):207-13. PMID: 12180276 Ineligible study design

584. McIntyre RS, Soczynska JK, Lewis GF, et al. Managing psychiatric disorders with antidiabetic agents: translational research and treatment opportunities. Expert Opinion on Pharmacotherapy. 2006 Jul;7(10):1305-21. PMID: 16805717 Ineligible study design

585. McIntyre RS, Soczynska JK, Woldeyohannes HO, et al. Aripiprazole: pharmacology and evidence in bipolar disorder. Expert Opinion on Pharmacotherapy. 2007 May;8(7):1001-9. PMID: 17472545 Ineligible study design

586. McNamara B, Ray JL, Arthurs OJ, et al. Transcranial magnetic stimulation for depression and other psychiatric disorders. Psychological Medicine. 2001 2001;31(7):1141-6. doi: http://dx.doi.org/10.1017/S0033291701004378. Ineligible study design

587. Meador KJ. Newer anticonvulsants: dosing strategies and cognition in treating patients with mood disorders and epilepsy. Journal of Clinical Psychiatry. 2003;64 Suppl 8:30-4. PMID: 12892539

\section{Ineligible study design}

588. Mech AW. High-dose ziprasidone monotherapy in bipolar I disorder patients with depressed or mixed episodes. Journal of Clinical Psychopharmacology. 2008 2008;28(2):240-1. doi: http://dx.doi.org/10.1097/JCP.0b013e31816745de. Ineligible study design

589. Mei-Dan E, Ray JG, Vigod SN. Perinatal outcomes among women with bipolar disorder: a populationbased cohort study. American Journal of Obstetrics \& Gynecology. 2015 Mar;212(3):367.e1-8. doi: http://dx.doi.org/10.1016/j.ajog.2014.10.020. PMID: 25446660 Ineligible intervention

590. Meltzer HY. Focus on the metabolic consequences of long-term treatment with olanzapine, quetiapine and risperidone: Are there differences? [References]. International Journal of Neuropsychopharmacology. 2005 2005;8(2):153-6. doi: http://dx.doi.org/10.1017/S1461145705005183. Ineligible study design

591. Meltzer HY, Bonaccorso S, Bobo WV, et al. A 12-month randomized, open-label study of the metabolic effects of olanzapine and risperidone in psychotic patients: influence of valproic acid augmentation. Journal of Clinical Psychiatry. 2011 Dec;72(12):1602-10. doi: http://dx.doi.org/10.4088/JCP.10m05997. PMID:

\section{Bipolar not analyzed separately}

592. Meric G, Gracitelli GC, Gortz S, et al. Fresh osteochondral allograft transplantation for bipolar reciprocal osteochondral lesions of the knee. American Journal of Sports Medicine. 2015 Mar;43(3):709-14. doi: http://dx.doi.org/10.1177/0363546514562549. PMID: 25573390 Not bipolar disorder

593. Michalak EE, Guiraud-Diawara A, Sapin C. Asenapine treatment and health-related quality of life in patients experiencing bipolar I disorder with mixed episodes: post-hoc analyses of pivotal trials. Current Medical Research \& Opinion. 2014 Apr;30(4):711-8. doi: http://dx.doi.org/10.1185/03007995.2013.874988. PMID: 24329543 No eligible outcomes reported

594. Miklowitz DJ. Adjunctive psychotherapy for bipolar disorder: state of the evidence. American Journal of Psychiatry. 2008 Nov;165(11):1408-19. doi: http://dx.doi.org/10.1176/appi.ajp.2008.08040488. PMID: 18794208 Ineligible study design 
595. Miklowitz DJ. Psychosocial interventions for bipolar disorder: A critical review of evidence for efficacy. Yatham, Lakshmi N [Ed]; Kusumakar, Vivek [Ed]. (2009). 2009 pp;Bipolar disorder:A clinician's guide to treatment management (2nd ed.). (pp. 575-90). xvi. Ineligible study design

596. Milano W, Grillo F, Del Mastro A, et al. Appropriate intervention strategies for weight gain induced by olanzapine: a randomized controlled study. Advances in Therapy. 2007 Jan-Feb;24(1):123-34. PMID: 17526469 No eligible outcomes reported

597. Millar A, Espie CA, Scott J. The sleep of remitted bipolar outpatients: a controlled naturalistic study using actigraphy. Journal of Affective Disorders. 2004 Jun;80(2-3):145-53. PMID: 15207927 Not treating bipolar

598. Miller IW, Uebelacker LA, Keitner GI, et al. Longitudinal course of bipolar I disorder. Comprehensive Psychiatry. 2004 Nov-Dec;45(6):431-40. PMID: 15526253 Ineligible study design

599. Minnai GP, Salis PG, Oppo R, et al. Effectiveness of maintenance electroconvulsive therapy in rapidcycling bipolar disorder. Journal of ECT. 2011 Jun;27(2):123-6. doi: http://dx.doi.org/10.1097/YCT.0b013e3181dbf797. PMID: 20559148 Less than 11 subjects per arm

600. Mishory A, Winokur M, Bersudsky Y. Prophylactic effect of phenytoin in bipolar disorder: a controlled study. Bipolar Disorders. 2003 Dec;5(6):464-7. PMID: 14636372 Over 50\% dropout rate

601. Mishory A, Yaroslavsky Y, Bersudsky Y, et al. Phenytoin as an antimanic anticonvulsant: a controlled study. American Journal of Psychiatry. 2000 Mar;157(3):463-5. PMID: 10698828 Bipolar not analyzed separately

602. Miskowiak KW, Vinberg M, Macoveanu J, et al. Effects of Erythropoietin on Hippocampal Volume and Memory in Mood Disorders. Biological Psychiatry. 2015 Aug 15;78(4):270-7. doi: http://dx.doi.org/10.1016/j.biopsych.2014.12.013. PMID: 25641635 Bipolar not analyzed separately

603. Mitchell PB, Malhi GS. The expanding pharmacopoeia for bipolar disorder. Annual Review of Medicine. 2002;53:173-88. PMID: 11818469 Ineligible study design

604. Miziou S, Tsitsipa E, Moysidou S, et al. Psychosocial treatment and interventions for bipolar disorder: A systematic review. Annals of General Psychiatry. 2015;07doi: http://dx.doi.org/10.1186/s12991-015-0057z. PMID: 605105502 Ineligible study design

605. Mohan TS, Tharyan P, Alexander J, et al. Effects of stimulus intensity on the efficacy and safety of twiceweekly, bilateral electroconvulsive therapy (ECT) combined with antipsychotics in acute mania: a randomised controlled trial. Bipolar Disorders. 2009 Mar;11(2):126-34. doi: http://dx.doi.org/10.1111/j.1399-5618.2009.00668.x. PMID: 19267695 Bipolar not analyzed separately

606. Mokhber N, Lane CJ, Azarpazhooh MR, et al. Anticonvulsant treatments of dysphoric mania: A trial of gabapentin, lamotrigine and carbamazepine in Iran. Neuropsychiatric Disease and Treatment. 2008;4(1B):227-34. No eligible outcomes reported 
607. Moller HJ, Grunze H, Broich K. Do recent efficacy data on the drug treatment of acute bipolar depression support the position that drugs other than antidepressants are the treatment of choice? A conceptual review. European Archives of Psychiatry \& Clinical Neuroscience. 2006 Feb;256(1):1-16. PMID: 16078087

\section{Ineligible study design}

608. Moncrieff J. Lithium revisited. A re-examination of the placebo-controlled trials of lithium prophylaxis in manic-depressive disorder. British Journal of Psychiatry. 1995 Nov;167(5):569-73; discussion 73-4.

PMID: 8564310 Ineligible study design

609. Moore DJ, Poquette A, Casaletto KB, et al. Individualized Texting for Adherence Building (iTAB): Improving antiretroviral dose timing among HIV-infected persons with co-occurring bipolar disorder. AIDS and Behavior. 2015 Mar;19(3):459-71. doi: http://dx.doi.org/10.1007/s10461-014-0971-0. PMID: 2014-55785-001 Not bipolar disorder

610. Moreno RA, Hanna MM, Tavares SM, et al. A double-blind comparison of the effect of the antipsychotics haloperidol and olanzapine on sleep in mania. Brazilian Journal of Medical \& Biological Research. 2007 Mar;40(3):357-66. PMID: 17334533 Over 50\% dropout rate

611. Morriss RK, Faizal MA, Jones AP, et al. Interventions for helping people recognise early signs of recurrence in bipolar disorder. Cochrane Database of Systematic Reviews. 2007(1):CD004854. PMID: 17253526 Ineligible study design

612. Morriss RK, Lobban F, Jones S, et al. Pragmatic randomised controlled trial of group psychoeducation versus group support in the maintenance of bipolar disorder. BMC Psychiatry. 2011;11:114. doi: http://dx.doi.org/10.1186/1471-244X-11-114. PMID: 21777426 Ineligible intervention

613. Mueser KT, Pratt SI, Bartels SJ, et al. Randomized trial of social rehabilitation and integrated health care for older people with severe mental illness. Journal of Consulting \& Clinical Psychology. 2010 Aug;78(4):561-73. doi: http://dx.doi.org/10.1037/a0019629. PMID: 20658812 Bipolar not analyzed separately

614. Muller-Oerlinghausen B, Retzow A, Henn FA, et al. Valproate as an adjunct to neuroleptic medication for the treatment of acute episodes of mania: a prospective, randomized, double-blind, placebo-controlled, multicenter study. European Valproate Mania Study Group. Journal of Clinical Psychopharmacology. 2000 Apr;20(2):195-203. PMID: 10770458 Bipolar not analyzed separately

615. Mundo E, Cattaneo E, Zanoni S, et al. The use of atypical antipsychotics beyond psychoses: Efficacy of quetiapine in bipolar disorder. Neuropsychiatric Disease and Treatment. 2006;2(2):139-48. doi: http://dx.doi.org/10.2147/nedt.2006.2.2.139. Ineligible study design

616. Muralidharan K, Ali M, Silveira LE, et al. Efficacy of second generation antipsychotics in treating acute mixed episodes in bipolar disorder: A meta-analysis of placebo-controlled trials. Journal of Affective Disorders. 2013 5, 2013;150(2):408-14. doi: http://dx.doi.org/10.1016/j.jad.2013.04.032. Ineligible study design

617. Murray G, Leitan ND, Berk M, et al. Online mindfulness-based intervention for late-stage bipolar disorder: pilot evidence for feasibility and effectiveness. Journal of Affective Disorders. 2015 Jun 1;178:46-51. doi: http://dx.doi.org/10.1016/j.jad.2015.02.024. PMID: 25795535 Ineligible study design 
618. Muzina DJ, Calabrese JR. Maintenance therapies in bipolar disorder: focus on randomized controlled trials. Australian \& New Zealand Journal of Psychiatry. 2005 Aug;39(8):652-61. PMID: 16050919 Ineligible study design

619. Muzina DJ, Elhaj O, Gajwani P, et al. Lamotrigine and antiepileptic drugs as mood stabilizers in bipolar disorder. Acta Psychiatrica Scandinavica, Supplementum. 2005(426):21-8. PMID: 15833097 Ineligible study design

620. Muzina DJ, El-Sayegh S, Calabrese JR. Antiepileptic drugs in psychiatry-focus on randomized controlled trial. Epilepsy Research. 2002 Jun;50(1-2):195-202. PMID: 12151129 Ineligible study design

621. Muzina DJ, Momah C, Eudicone JM, et al. Aripiprazole monotherapy in patients with rapid-cycling bipolar I disorder: an analysis from a long-term, double-blind, placebo-controlled study. International Journal of Clinical Practice. 2008 May;62(5):679-87. doi: http://dx.doi.org/10.1111/j.1742-1241.2008.01735.x. PMID: 18373615 Fewer than 11 subjects per arm

622. Nahas Z, Kozel FA, Li X, et al. Left prefrontal transcranial magnetic stimulation (TMS) treatment of depression in bipolar affective disorder: a pilot study of acute safety and efficacy. Bipolar Disorders. 2003 Feb;5(1):40-7. PMID: 12656937 Ineligible study design

623. Nahas Z, Teneback C, Chae JH, et al. Serial vagus nerve stimulation functional MRI in treatment-resistant depression. Neuropsychopharmacology. 2007 Aug;32(8):1649-60. PMID: 17203016 No eligible outcomes reported

624. Nakamura K, Iga J, Matsumoto N, et al. Risk of bipolar disorder and psychotic features in patients initially hospitalised with severe depression. Acta Neuropsychiatrica. 2015 Apr;27(2):113-8. doi: http://dx.doi.org/10.1017/neu.2014.42. PMID: 25529988 Not treating bipolar

625. Narendran R, Young CM, Valenti AM, et al. Olanzapine therapy in treatment-resistant psychotic mood disorders: a long-term follow-up study. Journal of Clinical Psychiatry. 2001 Jul;62(7):509-16. PMID: 11488360 Bipolar not analyzed separately

626. Nasrallah HA, Brecher M, Paulsson B. Placebo-level incidence of extrapyramidal symptoms (EPS) with quetiapine in controlled studies of patients with bipolar mania. Bipolar Disorders. 2006 Oct;8(5 Pt 1):46774. PMID: 17042884 Duplicate reference

627. Nejtek VA, Avila M, Chen LA, et al. Do atypical antipsychotics effectively treat co-occurring bipolar disorder and stimulant dependence? A randomized, double-blind trial. Journal of Clinical Psychiatry. 2008 Aug;69(8):1257-66. PMID: 18681757 Over 50\% dropout rate

628. Nemeroff CB. Use of atypical antipsychotics in refractory depression and anxiety. Journal of Clinical Psychiatry. 2005;66 Suppl 8:13-21. PMID: 16336032 Ineligible study design

629. Nemeroff CB, Evans DL, Gyulai L, et al. Double-blind, placebo-controlled comparison of imipramine and paroxetine in the treatment of major depression. The American Journal of Psychiatry. 2001 2001;158(6):906-12. doi: http://dx.doi.org/10.1176/appi.ajp.158.6.906. No eligible outcomes reported

630. Nery FG, Monkul ES, Hatch JP, et al. Celecoxib as an adjunct in the treatment of depressive or mixed episodes of bipolar disorder: a double-blind, randomized, placebo-controlled study. Human 
Psychopharmacology. 2008 Mar;23(2):87-94. doi: http://dx.doi.org/10.1002/hup.912. PMID: 18172906 No eligible outcomes reported

631. Newcomer JW, Tsai J, Pikalov A, et al. Effect of lurasidone on metabolic parameters in patients with bipolar depression. European Psychiatry. 201531 Mar;30:1140. PMID: 71931686 Ineligible study design

632. Ng F, Dodd S, Berk M. Atypical antipsychotics for bipolar disorder: Overblown or blown over? [References]. Clinical Psychopharmacology and Neuroscience. 2007 2007;5(2):53-64. Ineligible study design

633. Nguyen LN, Guthrie SK. Risperidone treatment of bipolar mania. Annals of Pharmacotherapy. 2006 Apr;40(4):674-82. PMID: 16569811 Ineligible study design

634. Niciu MJ, Xu AJ, Lundin NB, et al. Lithium and valproate levels do not correlate with ketamine's antidepressant efficacy in treatment-resistant bipolar depression. Biological Psychiatry. 201501

May;1):130S. PMID: 71846495 Ineligible study design

635. Niedermier JA, Nasrallah HA. Clinical correlates of response to valproate in geriatric inpatients. Annals of Clinical Psychiatry. 1998 Dec;10(4):165-8. PMID: 9988057 Ineligible study design

636. Nierenberg AA. An analysis of the efficacy of treatments for bipolar depression. Journal of Clinical Psychiatry. 2008;69 Suppl 5:4-8. PMID: 19265634 Ineligible study design

637. Nierenberg AA, Adler LA, Peselow E, et al. Trazodone for antidepressant-associated insomnia. The American Journal of Psychiatry. 1994 1994;151(7):1069-72. Bipolar not analyzed separately

638. Nierenberg AA, Alpert JE, Gardner-Schuster EE, et al. Vagus nerve stimulation: 2-year outcomes for bipolar versus unipolar treatment-resistant depression. Biological Psychiatry. 2008 Sep 15;64(6):455-60. doi: http://dx.doi.org/10.1016/j.biopsych.2008.04.036. PMID: 18571625 Less than 11 subjects per arm

639. Nivoli AM, Murru A, Vieta E. Lithium: still a cornerstone in the long-term treatment in bipolar disorder? Neuropsychobiology. 2010;62(1):27-35. doi: http://dx.doi.org/10.1159/000314307. PMID: 20453532 Ineligible study design

640. No authorship i. 2nd Biennial Conference of the International Society for Bipolar Disorders: Edinburgh, Scotland, 2-4 August 2006. Bipolar Disorders. 2006 2006;8(Suppl 1):1. Ineligible study design

641. Nolen WA, Bloemkolk D. Treatment of bipolar depression, a review of the literature and a suggestion for an algorithm. Neuropsychobiology. 2000;42 Suppl 1:11-7. PMID: 11093064 Ineligible study design

642. Nolen WA, Kupka RW, Hellemann G, et al. Tranylcypromine vs. lamotrigine in the treatment of refractory bipolar depression: a failed but clinically useful study. Acta Psychiatrica Scandinavica. 2007

May;115(5):360-5. PMID: 17430413 No eligible outcomes reported

643. Nolen WA, Weisler RH. The association of the effect of lithium in the maintenance treatment of bipolar disorder with lithium plasma levels: a post hoc analysis of a double-blind study comparing switching to lithium or placebo in patients who responded to quetiapine (Trial 144). Bipolar Disorders. 2013 
Feb;15(1):100-9. doi: http://dx.doi.org/10.1111/bdi.12027. PMID: 23228201 No eligible outcomes reported

644. Novick D, Gonzalez-Pinto A, Haro JM, et al. Translation of randomised controlled trial findings into clinical practice: comparison of olanzapine and valproate in the EMBLEM study. Pharmacopsychiatry. 2009 Jul;42(4):145-52. doi: http://dx.doi.org/10.1055/s-0028-1128115. PMID: 19585393 Ineligible study design

645. Novick D, Reed C, Haro JM, et al. Comparison of olanzapine and risperidone in the EMBLEM Study: translation of randomized controlled trial findings into clinical practice. International Clinical Psychopharmacology. 2010 Sep;25(5):257-63. doi: http://dx.doi.org/10.1097/YIC.0b013e32833b8fe4. PMID: 20531011 Over 50\% dropout rate

646. Obrocea GV, Dunn RM, Frye MA, et al. Clinical predictors of response to lamotrigine and gabapentin monotherapy in refractory affective disorders. Biological Psychiatry. 2002 2002;51(3):253-60. doi: http://dx.doi.org/10.1016/S0006-3223\%2801\%2901206-9. Ineligible study design

647. O'Donnell M, Parker G, Proberts M, et al. A study of client-focused case management and consumer advocacy: The Community and Consumer Service Project. Australian and New Zealand Journal of Psychiatry. 1999 1999;33(5):684-93. doi: http://dx.doi.org/10.1046/j.1440-1614.1999.00629.x. Bipolar not analyzed separately

648. Oertel-Knochel V, Reuter J, Reinke B, et al. Association between age of disease-onset, cognitive performance and cortical thickness in bipolar disorders. Journal of Affective Disorders. 2015 Mar 15;174:627-35. doi: http://dx.doi.org/10.1016/j.jad.2014.10.060. PMID: 25577157 Ineligible intervention

649. O'Garro-Moore JK, Adams AM, Abramson LY, et al. Anxiety comorbidity in bipolar spectrum disorders: the mediational role of perfectionism in prospective depressive symptoms. Journal of Affective Disorders. 2015 Mar 15;174:180-7. doi: http://dx.doi.org/10.1016/j.jad.2014.11.024. PMID: 25499686 Ineligible intervention

650. Ohtani T, Nishimura Y, Takahashi K, et al. Association between longitudinal changes in prefrontal hemodynamic responses and social adaptation in patients with bipolar disorder and major depressive disorder. Journal of Affective Disorders. 2015 May 1;176:78-86. doi: http://dx.doi.org/10.1016/j.jad.2015.01.042. PMID: 25702603 Ineligible intervention

651. O'Malley AJ, Zelevinsky K, He Y, et al. Do Patients at Sites With High RCT Enrollment Propensity Have Better Outcomes? Medical Care. 2015 Nov;53(11):989-95. doi: http://dx.doi.org/10.1097/MLR.0000000000000429. PMID: 26465127 Not treating bipolar

652. Ongur D, Lewandowski KE. Brain activation changes in psychotic disorders in response to targeted cognitive training. Biological Psychiatry. 201501 May;1):10S. PMID: 71846196 Ineligible study design

653. Oostervink F, Nolen WA, Kok RM, et al. Two years' outcome of acute mania in bipolar disorder: different effects of age and age of onset. International Journal of Geriatric Psychiatry. 2015 Feb;30(2):201-9. doi: http://dx.doi.org/10.1002/gps.4128. PMID: 24798245 Ineligible intervention 
654. Oquendo MA, Galfalvy HC, Currier D, et al. Treatment of suicide attempters with bipolar disorder: a randomized clinical trial comparing lithium and valproate in the prevention of suicidal behavior.[Erratum appears in Am J Psychiatry. 2012 Feb;169(2):223]. American Journal of Psychiatry. 2011

Oct;168(10):1050-6. doi: http://dx.doi.org/10.1176/appi.ajp.2011.11010163. PMID: 21768611 Over $\mathbf{5 0 \%}$ dropout rate

655. Oral TE. Treatment of acute mania. Neuroendocrinology Letters. 2005 Aug;26 Suppl 1:9-25. PMID: 16361986 Ineligible study design

656. Ostacher M, Ng-Mak D, Patel P, et al. Lurasidone compared to other atypical antipsychotic monotherapies for bipolar depression: A systematic review and network meta-analysis. World Journal of Biological Psychiatry. 201706 Mar:1-11. doi: http://dx.doi.org/10.1080/15622975.2017.1285050. PMID: 614711266 Ineligible study design

657. Ostacher MJ, Nierenberg AA, Rabideau D, et al. A clinical measure of suicidal ideation, suicidal behavior, and associated symptoms in bipolar disorder: Psychometric properties of the Concise Health Risk Tracking Self-Report (CHRT-SR). Journal of Psychiatric Research. 2015 Dec;71:126-33. doi: http://dx.doi.org/10.1016/j.jpsychires.2015.10.004. PMID: 26476489 Ineligible study design

658. Ostinelli EG, Cavallotti S, Castelnovo A, et al. Asenapine in the Treatment of Acute Mania: A Real-World Observational Study With 6 Months Follow-Up. Journal of Clinical Psychopharmacology. 2015 Oct;35(5):553-8. doi: http://dx.doi.org/10.1097/JCP.0000000000000374. PMID: 26252438 Fewer than

\section{1 subjects per arm}

659. Ouyang WC, Hsu MC, Yeh IN, et al. Efficacy and safety of combination of risperidone and haloperidol with divalproate in patients with acute mania. International Journal of Psychiatry in Clinical Practice. 2012 Sep;16(3):178-88. doi: http://dx.doi.org/10.3109/13651501.2011.644564. PMID: 22404731 Over 20\% schizoaffective

660. Owen RT. Extended-release carbamazepine for acute bipolar mania: a review. Drugs of Today. 2006 May;42(5):283-9. PMID: 16801991 Ineligible study design

661. Owen RT. Olanzapine/fluoxetine combination for bipolar depression and other mood disorders: a review. Drugs of Today. 2006 Mar;42(3):185-92. PMID: 16628260 Ineligible study design

662. Pacchiarotti I, Valenti M, Colom F, et al. Differential outcome of bipolar patients receiving antidepressant monotherapy versus combination with an antimanic drug. Journal of Affective Disorders. 2011 Mar;129(13):321-6. doi: http://dx.doi.org/10.1016/j.jad.2010.07.036. PMID: 20817267 Ineligible study design

663. Pae CU, Kim TS, Kim JJ, et al. Long-term treatment of adjunctive quetiapine for bipolar mania. Progress in Neuro-Psychopharmacology \& Biological Psychiatry. 2005 Jun;29(5):763-6. PMID: 15951090

\section{Ineligible study design}

664. Pae CU, Nassir Ghaemi S, Kim TS, et al. Rapid titration versus conventional titration of quetiapine in the treatment of bipolar mania: a preliminary trial. International Clinical Psychopharmacology. 2005

Nov;20(6):327-30. PMID: 16192842 Ineligible study design

665. Pae CU, Patkar AA, Gilmer W, et al. Predictors of response to ziprasidone: Results from a 6-week randomized double-blind, placebo-controlled trial for acute depressive mixed state. Pharmacopsychiatry. 2012 2012;45(4):152-5. doi: http://dx.doi.org/10.1055/s-0031-1297984. Ineligible study design 
666. Pande AC, Crockatt JG, Janney CA, et al. Gabapentin in bipolar disorder: a placebo-controlled trial of adjunctive therapy. Gabapentin Bipolar Disorder Study Group. Bipolar Disorders. 2000 Sep;2(3 Pt 2):24955. PMID: 11249802 Ineligible study design

667. Pappadopulos E, Newcomer JW, Kolluri S. Changes in weight, plasma lipids, and glucose in adults treated with ziprasidone: a comprehensive analysis of pfizer-initiated clinical trials. Journal of Clinical Psychiatry. 2012 Jun;73(6):e742-8. doi: http://dx.doi.org/10.4088/JCP.10r06802. PMID: 22795213 Not treating bipolar

668. Parikh SV, Hawke LD, Zaretsky A, et al. Psychosocial interventions for bipolar disorder and coping style modification: similar clinical outcomes, similar mechanisms? Canadian Journal of Psychiatry - Revue Canadienne de Psychiatrie. 2013 Aug;58(8):482-6. PMID: 23972110 No eligible outcomes reported

669. Parikh SV, LeBlanc SR, Ovanessian MM. Advancing bipolar disorder: key lessons from the Systematic Treatment Enhancement Program for Bipolar Disorder (STEP-BD). Canadian Journal of Psychiatry Revue Canadienne de Psychiatrie. 2010 Mar;55(3):136-43. PMID: 20370963 Ineligible study design

670. Park LT, Lener MS, Hopkins M, et al. A double-blind, placebo-controlled, pilot study of riluzole monotherapy for acute bipolar depression. Journal of Clinical Psychopharmacology. 2017;37(3):355-8. doi: http://dx.doi.org/10.1097/JCP.0000000000000693. PMID: 615006763 No eligible outcomes reported

671. Patel R, Reiss P, Shetty H, et al. Do antidepressants increase the risk of mania and bipolar disorder in people with depression? A retrospective electronic case register cohort study. BMJ Open. 2015 Dec 14;5(12):e008341. doi: http://dx.doi.org/10.1136/bmjopen-2015-008341. PMID: 26667012 Not treating bipolar

672. Patino LR, Rummelhoff ER, Blom T, et al. A double-blind placebo-controlled study of exenatide for the treatment of weight gain associated with olanzapine in overweight or obese adults with bipolar disorder, major depressive disorder, schizophrenia or schizoaffective disorder. Biological Psychiatry. 201501 May;1):132S. PMID: 71846499 Ineligible study design

673. Patkar AA, Pae CU, Vohringer PA, et al. A 13-week, randomized double-blind, placebo-controlled, crossover trial of ziprasidone in bipolar spectrum disorder. Journal of Clinical Psychopharmacology. 201513 Jun;35(3):319-23. doi: http://dx.doi.org/10.1097/JCP.0000000000000323. PMID: 25882763 (pubmed) 2015037995 (embase) Not treating bipolar

674. Pazzaglia PJ, George MS, Post RM, et al. Nimodipine increases CSF somatostatin in affectively ill patients. Neuropsychopharmacology. 1995 Aug;13(1):75-83. PMID: 8526973 Bipolar not analyzed separately

675. Pazzaglia PJ, Post RM, Ketter TA, et al. Nimodipine monotherapy and carbamazepine augmentation in patients with refractory recurrent affective illness. Journal of Clinical Psychopharmacology. 1998 Oct;18(5):404-13. PMID: 9790159 Bipolar not analyzed separately

676. Perlis RH, Adams DH, Fijal B, et al. Genetic association of treatment response with olanzapine/fluoxetine combination or lamotrigine in bipolar I depression. Journal of Clinical Psychiatry. 2010 2010;71(5):599605. doi: http://dx.doi.org/10.4088/JCP.08m04632gre. Ineligible study design 
677. Perlis RH, Sachs GS, Lafer B, et al. Effect of abrupt change from standard to low serum levels of lithium: a reanalysis of double-blind lithium maintenance data. American Journal of Psychiatry. 2002 Jul;159(7):1155-9. PMID: 12091193 Ineligible study design

678. Perlis RH, Welge JA, Vornik LA, et al. Atypical antipsychotics in the treatment of mania: a meta-analysis of randomized, placebo-controlled trials. Journal of Clinical Psychiatry. 2006 Apr;67(4):509-16. PMID: 16669715 Ineligible study design

679. Peters S, Pontin E, Lobban F, et al. Involving relatives in relapse prevention for bipolar disorder: a multiperspective qualitative study of value and barriers. BMC Psychiatry. 2011;11:172. doi: http://dx.doi.org/10.1186/1471-244X-11-172. PMID: 22044486 Ineligible study design

680. Petrakis I, Ralevski E, Nich C, et al. Naltrexone and disulfiram in patients with alcohol dependence and current depression. Journal of Clinical Psychopharmacology. 2007 Apr;27(2):160-5. PMID: 17414239 Not bipolar disorder

681. Petrakis IL, Nich C, Ralevski E. Psychotic spectrum disorders and alcohol abuse: a review of pharmacotherapeutic strategies and a report on the effectiveness of naltrexone and disulfiram. Schizophrenia Bulletin. 2006 Oct;32(4):644-54. PMID: 16887890 Bipolar not analyzed separately

682. Petty F, Rush AJ, Davis JM, et al. Plasma GABA predicts acute response to divalproex in mania. Biological Psychiatry. 1996 Feb 15;39(4):278-84. PMID: 8645774 No eligible outcomes reported

683. Pfennig A, Bschor T, Falkai P, et al. The diagnosis and treatment of bipolar disorder: recommendations from the current s3 guideline. Deutsches Arzteblatt International. 2013 Feb;110(6):92-100. doi: http://dx.doi.org/10.3238/arztebl.2013.0092. PMID: 23451001 Ineligible study design

684. Pies R. Combining lithium and anticonvulsants in bipolar disorder: a review. Annals of Clinical Psychiatry. 2002 Dec;14(4):223-32. PMID: 12630658 Ineligible study design

685. Pikalov A, Tsai J, Mao Y, et al. Long-term use of lurasidone in patients with bipolar disorder: safety and effectiveness over 2 years of treatment. International Journal of Bipolar Disorders. 201701 Dec;5 (1) (no pagination)(9)doi: http://dx.doi.org/10.1186/s40345-017-0075-7. PMID: 614687852 No eligible

\section{outcomes reported}

686. Pillay SS, Stoll AL, Weiss MK, et al. EEG abnormalities before clozapine therapy predict a good clinical response to clozapine. Annals of Clinical Psychiatry. 1996 Mar;8(1):1-5. PMID: 8743641 Ineligible study design

687. Pini S, Abelli M, Cassano GB. The role of quetiapine in the treatment of bipolar disorder. Expert Opinion on Pharmacotherapy. 2006 May;7(7):929-40. PMID: 16634715 Ineligible study design

688. Pompili M, Serafini G, Del Casale A, et al. Improving adherence in mood disorders: the struggle against relapse, recurrence and suicide risk. Expert Review of Neurotherapeutics. 2009 2009;9(7):985-1004. doi: http://dx.doi.org/10.1586/ern.09.62. Ineligible study design 
689. Pompili M, Venturini P, Innamorati M, et al. The role of asenapine in the treatment of manic or mixed states associated with bipolar I disorder. Neuropsychiatric Disease and Treatment. 2011 6, 2011;7(1):25965. Ineligible study design

690. Poole R, Simpson SA, Smith DJ. Internet-based psychoeducation for bipolar disorder: a qualitative analysis of feasibility, acceptability and impact. BMC Psychiatry. 2012;12:139. doi: http://dx.doi.org/10.1186/1471244X-12-139. PMID: 22971042 Ineligible study design

691. Poolsup N, Li Wan Po A, de Oliveira IR. Systematic overview of lithium treatment in acute mania. Journal of Clinical Pharmacy \& Therapeutics. 2000 Apr;25(2):139-56. PMID: 10849192 Ineligible study design

692. Pope M, Dudley R, Scott J. Determinants of social functioning in bipolar disorder. Bipolar Disorders. 2007 2007;9(1-2):38-44. doi: http://dx.doi.org/10.1111/j.1399-5618.2007.00323.x. Ineligible study design

693. Popovic D, Reinares M, Amann B, et al. Number needed to treat analyses of drugs used for maintenance treatment of bipolar disorder. Psychopharmacology. 2011 Feb;213(4):657-67. doi: http://dx.doi.org/10.1007/s00213-010-2056-8. PMID: 21052983 Ineligible study design

694. Popovic D, Torrent C, Goikolea JM, et al. Clinical implications of predominant polarity and the polarity index in bipolar disorder: a naturalistic study. Acta Psychiatrica Scandinavica. 2014 May;129(5):366-74. doi: http://dx.doi.org/10.1111/acps.12179. PMID: 23865756 No eligible outcomes reported

695. Post RM, Altshuler LL, Frye MA, et al. Rate of switch in bipolar patients prospectively treated with second-generation antidepressants as augmentation to mood stabilizers. Bipolar Disorders. 2001 Oct;3(5):259-65. PMID: 11912569 Ineligible study design

696. Post RM, Altshuler LL, Leverich GS, et al. Mood switch in bipolar depression: comparison of adjunctive venlafaxine, bupropion and sertraline.[Erratum appears in Br J Psychiatry. 2006 Dec;189:569]. British Journal of Psychiatry. 2006 Aug;189:124-31. PMID: 16880481 Ineligible study design

697. Post RM, Denicoff KD, Leverich GS, et al. Drug-induced switching in bipolar disorder: Epidemiology and therapeutic implications. CNS Drugs. 1997 1997;8(5):352-65. doi: http://dx.doi.org/10.2165/00023210199708050-00002. Ineligible study design

698. Post RM, Frye MA, Denicoff KD, et al. Emerging trends in the treatment of rapid cycling bipolar disorder: a selected review. Bipolar Disorders. 2000 Dec;2(4):305-15. PMID: 11252642 Ineligible study design

699. Post RM, Ketter TA, Denicoff K, et al. The place of anticonvulsant therapy in bipolar illness. Psychopharmacology. 1996 Nov;128(2):115-29. PMID: 8956373 Ineligible study design

700. Post RM, Ketter TA, Pazzaglia PJ, et al. Rational polypharmacy in the bipolar affective disorders. Epilepsy Research - Supplement. 1996;11:153-80. PMID: 9294735 Ineligible study design

701. Post RM, Leverich GS, Nolen WA, et al. A re-evaluation of the role of antidepressants in the treatment of bipolar depression: data from the Stanley Foundation Bipolar Network. Bipolar Disorders. 2003

Dec;5(6):396-406. PMID: 14636363 Ineligible study design 
702. Praharaj SK, Jana AK, Goyal N, et al. Metformin for olanzapine-induced weight gain: a systematic review and meta-analysis. British Journal of Clinical Pharmacology. 2011 Mar;71(3):377-82. doi:

http://dx.doi.org/10.1111/j.1365-2125.2010.03783.x. PMID: 21284696 Ineligible study design

703. Praharaj SK, Ram D, Arora M. Efficacy of high frequency (rapid) suprathreshold repetitive transcranial magnetic stimulation of right prefrontal cortex in bipolar mania: a randomized sham controlled study. Journal of Affective Disorders. 2009 Oct;117(3):146-50. doi: http://dx.doi.org/10.1016/j.jad.2008.12.020. PMID: 19178948 Ineligible study design

704. Pratoomsri W, Yatham LN, Bond DJ, et al. Oxcarbazepine in the treatment of bipolar disorder: a review. Canadian Journal of Psychiatry - Revue Canadienne de Psychiatrie. 2006 Jul;51(8):540-5. PMID: 16933591 Ineligible study design

705. Proudfoot J, Parker G, Manicavasagar V, et al. Effects of adjunctive peer support on perceptions of illness control and understanding in an online psychoeducation program for bipolar disorder: a randomised controlled trial. Journal of Affective Disorders. 2012 Dec 15;142(1-3):98-105. doi: http://dx.doi.org/10.1016/j.jad.2012.04.007. PMID: 22858215 Ineligible study design

706. Proudfoot JG, Jayawant A, Whitton AE, et al. Mechanisms underpinning effective peer support: a qualitative analysis of interactions between expert peers and patients newly-diagnosed with bipolar disorder. BMC Psychiatry. 2012;12:196. doi: http://dx.doi.org/10.1186/1471-244X-12-196. PMID: 23140497 Ineligible study design

707. Rabheru K. Maintenance electroconvulsive therapy (M-ECT) after acute response: examining the evidence for who, what, when, and how? Journal of ECT. 2012 Mar;28(1):39-47. doi: http://dx.doi.org/10.1097/YCT.0b013e3182455758. PMID: 22330700 Ineligible study design

708. Rajagopalan K, Bacci ED, Ng-Mak D, et al. Effects on health-related quality of life in patients treated with lurasidone for bipolar depression: Results from two placebo controlled bipolar depression trials. BMC Psychiatry. 201623 May;16 (1) (no pagination)(157)doi: http://dx.doi.org/10.1186/s12888-016-0865-y. PMID: 610427781 No eligible outcomes reported

709. Rakofsky JJ, Dunlop BW. Treating nonspecific anxiety and anxiety disorders in patients with bipolar disorder: a review. Journal of Clinical Psychiatry. 2011 Jan;72(1):81-90. doi: http://dx.doi.org/10.4088/JCP.09r05815gre. PMID: 21208580 Ineligible study design

710. Rapinesi C, Bersani FS, Kotzalidis GD, et al. Maintenance deep transcranial magnetic stimulation sessions are associated with reduced depressive relapses in patients with unipolar or bipolar depression. Frontiers in Neurology. 2015;6(FEB)doi: http://dx.doi.org/10.3389/fneur.2015.00016. PMID: 2015896165 Bipolar not analyzed separately

711. Rasgon N. The relationship between polycystic ovary syndrome and antiepileptic drugs: a review of the evidence. Journal of Clinical Psychopharmacology. 2004 Jun;24(3):322-34. PMID: 15118487 Ineligible study design

712. Rasgon NL, Altshuler LL, Gudeman D, et al. Medication status and polycystic ovary syndrome in women with bipolar disorder: a preliminary report. Journal of Clinical Psychiatry. 2000 Mar;61(3):173-8. PMID: 10817101 No eligible outcomes reported 
713. Redden L, Pritchett Y, Robieson W, et al. Suicidality and divalproex sodium: Analysis of controlled studies in multiple indications. Annals of General Psychiatry. 2011 18, 2011;10:1. doi:

http://dx.doi.org/10.1186/1744-859X-10-1. Ineligible study design

714. Reinares M, Colom F, Sanchez-Moreno J, et al. Impact of caregiver group psychoeducation on the course and outcome of bipolar patients in remission: a randomized controlled trial.[Erratum appears in Bipolar Disord. 2008 Jul;10(5):657]. Bipolar Disorders. 2008 Jun;10(4):511-9. doi: http://dx.doi.org/10.1111/j.1399-5618.2008.00588.x. PMID: 18452447 Ineligible study design

715. Reinares M, Rosa AR, Franco C, et al. A systematic review on the role of anticonvulsants in the treatment of acute bipolar depression. International Journal of Neuropsychopharmacology. 2013 Mar;16(2):485-96. doi: http://dx.doi.org/10.1017/S1461145712000491. PMID: 22575611 Ineligible study design

716. Reinares M, Vieta E, Colom F, et al. Impact of a psychoeducational family intervention on caregivers of stabilized bipolar patients. Psychotherapy \& Psychosomatics. 2004 Sep-Oct;73(5):312-9. PMID: 15292629 Not treating bipolar

717. Rendell JM, Geddes JR. Risperidone in long-term treatment for bipolar disorder. Cochrane Database of Systematic Reviews. 2006(4):CD004999. PMID: 17054229 Ineligible study design

718. Rendell JM, Gijsman HJ, Bauer MS, et al. Risperidone alone or in combination for acute mania. Cochrane Database of Systematic Reviews. 2006(1):CD004043. PMID: 16437472 Ineligible study design

719. Rendell JM, Gijsman HJ, Keck P, et al. Olanzapine alone or in combination for acute mania. Cochrane Database of Systematic Reviews. 2003(3):CD004040. PMID: 12918000 Ineligible study design

720. Riemann D, Konig A, Hohagen F, et al. How to preserve the antidepressive effect of sleep deprivation: A comparison of sleep phase advance and sleep phase delay. European Archives of Psychiatry \& Clinical Neuroscience. 1999;249(5):231-7. PMID: 10591988 Ineligible study design

721. Rifkin A, Doddi S, Karajgi B, et al. Dosage of haloperidol for mania. British Journal of Psychiatry. 1994 Jul;165(1):113-6. PMID: 7953013 Bipolar not analyzed separately

722. Rihmer Z, Gonda X. The effect of pharmacotherapy on suicide rates in bipolar patients. CNS Neuroscience \& Therapeutics. 2012 Mar;18(3):238-42. doi: http://dx.doi.org/10.1111/j.1755-5949.2011.00261.x. PMID: 22070662 Ineligible study design

723. Rosa AR, Fountoulakis K, Siamouli M, et al. Is anticonvulsant treatment of mania a class effect? Data from randomized clinical trials. CNS Neuroscience \& Therapeutics. 2011 Jun;17(3):167-77. doi: http://dx.doi.org/10.1111/j.1755-5949.2009.00089.x. PMID: 20015083 Ineligible study design

724. Rosenblat JD, Kakar R, Berk M, et al. Anti-inflammatory agents in the treatment of bipolar depression: A systematic review and meta-analysis. Bipolar Disorders. 201601 Mar;18(2):89-101. doi: http://dx.doi.org/10.1111/bdi.12373. PMID: 609161232 Ineligible study design

725. Rouillon F, Gorwood P. The use of lithium to augment antidepressant medication. Journal of Clinical Psychiatry. 1998;59 Suppl 5:32-9; discussion 40-1. PMID: 9635546 Ineligible study design

726. Royal A, New Zealand College of Psychiatrists Clinical Practice Guidelines Team for Bipolar D. Australian and New Zealand clinical practice guidelines for the treatment of bipolar disorder. Australian \& 
New Zealand Journal of Psychiatry. 2004 May;38(5):280-305. PMID: 15144505 Ineligible study design

727. Ruchlewska A, Mulder CL, Smulders R, et al. The effects of crisis plans for patients with psychotic and bipolar disorders: a randomised controlled trial. BMC Psychiatry. 2009;9:41. doi: http://dx.doi.org/10.1186/1471-244X-9-41. PMID: 19589145 Ineligible study design

728. Rusner M, Berg M, Begley C. Bipolar disorder in pregnancy and childbirth: A systematic review of outcomes. BMC Pregnancy and Childbirth. 201628 Oct;16 (1) (no pagination)(331)doi: http://dx.doi.org/10.1186/s12884-016-1127-1. PMID: 612941750 Ineligible study design

729. Sacchetti E, Galluzzo A, Valsecchi P. Oral ziprasidone in the treatment of patients with bipolar disorders: a critical review. Expert Review of Clinical Pharmacology. 2011 Mar;4(2):163-79. doi: http://dx.doi.org/10.1586/ecp.10.139. PMID: 22115400 Ineligible study design

730. Sachs G, Bowden C, Calabrese JR, et al. Effects of lamotrigine and lithium on body weight during maintenance treatment of bipolar I disorder. Bipolar Disorders. 2006 Apr;8(2):175-81. PMID: 16542188 No eligible outcomes reported

731. Sachs G, Chengappa KN, Suppes T, et al. Quetiapine with lithium or divalproex for the treatment of bipolar mania: a randomized, double-blind, placebo-controlled study. Bipolar Disorders. 2004 Jun;6(3):213-23. PMID: 15117400 Duplicate reference

732. Sachs GS, Gardner-Schuster EE. Adjunctive treatment of acute mania: a clinical overview. Acta Psychiatrica Scandinavica, Supplementum. 2007(434):27-34. PMID: 17688460 Ineligible study design

733. Sachs GS, Gaulin BD, Gutierrez-Esteinou R, et al. Antimanic response to aripiprazole in bipolar I disorder patients is independent of the agitation level at baseline. Journal of Clinical Psychiatry. 2007 Sep;68(9):1377-83. PMID: 17915976 Over 50\% dropout rate

734. Sachs GS, Grossman F, Ghaemi SN, et al. Combination of a mood stabilizer with risperidone or haloperidol for treatment of acute mania: a double-blind, placebo-controlled comparison of efficacy and safety. American Journal of Psychiatry. 2002 Jul;159(7):1146-54. PMID: 12091192 Over 50\% dropout rate

735. Sachs GS, Lafer B, Stoll AL, et al. A double-blind trial of bupropion versus desipramine for bipolar depression. Journal of Clinical Psychiatry. 1994 Sep;55(9):391-3. PMID: 7929019 Not treating bipolar

736. Sachs GS, Thase ME. Bipolar disorder therapeutics: maintenance treatment. Biological Psychiatry. 2000 Sep 15;48(6):573-81. PMID: 11018228 Ineligible study design

737. Sajatovic M, Calabrese JR, Mullen J. Quetiapine for the treatment of bipolar mania in older adults. Bipolar Disorders. 2008 Sep;10(6):662-71. doi: http://dx.doi.org/10.1111/j.1399-5618.2008.00614.x. PMID: 18837860 Ineligible study design

738. Sajatovic M, Davies M, Bauer MS, et al. Attitudes regarding the collaborative practice model and treatment adherence among individuals with bipolar disorder. Comprehensive Psychiatry. 2005 Jul-Aug;46(4):272-7. PMID: 16175758 Ineligible study design 
739. Sajatovic M, Dines P, Fuentes-Casiano E, et al. Asenapine in the treatment of older adults with bipolar disorder. International Journal of Geriatric Psychiatry. 201501 Jul;30(7):710-9. doi: http://dx.doi.org/10.1002/gps.4213. PMID: 2015118224 Ineligible study design

740. Sajatovic M, Elhaj O, Youngstrom EA, et al. Treatment adherence in individuals with rapid cycling bipolar disorder: Results from a clinical-trial setting. Journal of Clinical Psychopharmacology. 2007 2007;27(4):412-4. doi: http://dx.doi.org/10.1097/01.jcp.0000280310.50871.ff. Ineligible study design

741. Sajatovic M, Gildengers A, Al Jurdi RK, et al. Multisite, open-label, prospective trial of lamotrigine for geriatric bipolar depression: A preliminary report. Bipolar Disorders. 2011 2011;13(3):294-302. doi: http://dx.doi.org/10.1111/j.1399-5618.2011.00923.x. PMID: 21676132 Ineligible study design

742. Sajatovic M, Levin J, Tatsuoka C, et al. Customized adherence enhancement for individuals with bipolar disorder receiving antipsychotic therapy. Psychiatric Services. 2012 Feb 1;63(2):176-8. doi: http://dx.doi.org/10.1176/appi.ps.201100133. PMID: 22302337 Ineligible study design

743. Sajatovic M, Ramsay E, Nanry K, et al. Lamotrigine therapy in elderly patients with epilepsy, bipolar disorder or dementia. International Journal of Geriatric Psychiatry. 2007 Oct;22(10):945-50. PMID: 17326238 Ineligible study design

744. Sakinofsky I. Treating suicidality in depressive illness. Part 2: does treatment cure or cause suicidality? Canadian Journal of Psychiatry - Revue Canadienne de Psychiatrie. 2007 Jun;52(6 Suppl 1):85S-101S. PMID: 17824355 Ineligible study design

745. Saksa JR, Baker CB, Woods SW. Mood-stabilizer-maintained, remitted bipolar patients: taper and discontinuation of adjunctive antipsychotic medication. General Hospital Psychiatry. 2004 MayJun;26(3):233-6. PMID: 15121352 Ineligible study design

746. Salloum IM, Cornelius JR, Daley DC, et al. Efficacy of valproate maintenance in patients with bipolar disorder and alcoholism: a double-blind placebo-controlled study. Archives of General Psychiatry. 2005 Jan;62(1):37-45. PMID: 15630071 Over 50\% dropout rate

747. Salloum IM, Cornelius JR, Douaihy A, et al. Patient characteristics and treatment implications of marijuana abuse among bipolar alcoholics: results from a double blind, placebo-controlled study. Addictive Behaviors. 2005 Oct;30(9):1702-8. PMID: 16098680 Ineligible study design

748. Salvi V, Fagiolini A, Swartz HA, et al. The use of antidepressants in bipolar disorder. Journal of Clinical Psychiatry. 2008 Aug;69(8):1307-18. PMID: 18681751 Ineligible study design

749. Sampogna G, Del Vecchio V, Luciano M, et al. Efficacy of psychoeducational family intervention in bipolar I disorder: Results from a multicenter, randomized, controlled trial. European Psychiatry. 201531 Mar;30:554. PMID: 71931264 Ineligible study design

750. Sanchez-Moreno J, Bonnin C, Gonzalez-Pinto A, et al. Do patients with bipolar disorder and subsyndromal symptoms benefit from functional remediation? A 12-month follow-up study. European Neuropsychopharmacology. 2017 April;27(4):350-9. doi: http://dx.doi.org/10.1016/j.euroneuro.2017.01.010. PMID: 614182952 Ineligible study design 
751. Sanchez-Moreno J, Martinez-Aran A, Gadelrab HF, et al. The role and impact of contextual factors on functioning in patients with bipolar disorder. Disability \& Rehabilitation. 2010;32 Suppl 1:S94-S104. doi: http://dx.doi.org/10.3109/09638288.2010.520810. PMID: 20883145 Ineligible study design

752. Sanford M, Keating GM. Quetiapine: a review of its use in the management of bipolar depression. CNS Drugs. 2012 May 1;26(5):435-60. doi: http://dx.doi.org/10.2165/11203840-000000000-00000. PMID: 22519923 Ineligible study design

753. Sanger TM, Tohen M, Vieta E, et al. Olanzapine in the acute treatment of bipolar I disorder with a history of rapid cycling. Journal of Affective Disorders. 2003 Jan;73(1-2):155-61. PMID: 12507748 Over 50\% dropout rate

754. Saroukhani S, Emami-Parsa M, Modabbernia A, et al. Aspirin for treatment of lithium-associated sexual dysfunction in men: Randomized double-blind placebo-controlled study. Bipolar Disorders. 2013 2013;15(6):650-6. doi: http://dx.doi.org/10.1111/bdi.12108. No eligible outcomes reported

755. Sarris J, Lake J, Hoenders R. Bipolar disorder and complementary medicine: current evidence, safety issues, and clinical considerations. Journal of Alternative \& Complementary Medicine. 2011 Oct;17(10):881-90. doi: http://dx.doi.org/10.1089/acm.2010.0481. PMID: 22010777 Ineligible study design

756. Schaffer A, Zuker P, Levitt A. Randomized, double-blind pilot trial comparing lamotrigine versus citalopram for the treatment of bipolar depression. Journal of Affective Disorders. 2006 Nov;96(1-2):95-9. PMID: 16820213 Fewer than 11 subjects per arm

757. Scherk H, Pajonk FG, Leucht S. Second-generation antipsychotic agents in the treatment of acute mania: a systematic review and meta-analysis of randomized controlled trials. Archives of General Psychiatry. 2007 Apr;64(4):442-55. PMID: 17404121 Ineligible study design

758. Schottle D, Huber CG, Bock T, et al. Psychotherapy for bipolar disorder: a review of the most recent studies. Current Opinion in Psychiatry. 2011 Nov;24(6):549-55. doi: http://dx.doi.org/10.1097/YCO.0b013e32834b7c5f. PMID: 21918448 Ineligible study design

759. Scogin F, Morthland M, Kaufman A, et al. Improving quality of life in diverse rural older adults: a randomized trial of a psychological treatment. Psychology \& Aging. 2007 Dec;22(4):657-65. doi: http://dx.doi.org/10.1037/0882-7974.22.4.657. PMID: 18179286 Not bipolar disorder

760. Scott J, Colom F. Psychosocial treatments for bipolar disorders. Psychiatric Clinics of North America. 2005 Jun;28(2):371-84. PMID: 15826737 Ineligible study design

761. Scott J, Colom F, Vieta E. A meta-analysis of relapse rates with adjunctive psychological therapies compared to usual psychiatric treatment for bipolar disorders. International Journal of Neuropsychopharmacology. 2007 Feb;10(1):123-9. PMID: 16787554 Ineligible study design

762. Scott J, Etain B. Which psychosocial interventions in bipolar depression? Encephale. 2011 Dec;37 Suppl 3:S214-7. doi: http://dx.doi.org/10.1016/S0013-7006(11)70056-2. PMID: 22212878 Ineligible study design

763. Searson R, Mansell W, Lowens I, et al. Think Effectively About Mood Swings (TEAMS): a case series of cognitive-behavioural therapy for bipolar disorders. Journal of Behavior Therapy \& Experimental 
Psychiatry. 2012 Jun;43(2):770-9. doi: http://dx.doi.org/10.1016/j.jbtep.2011.10.001. PMID: 22104659

\section{Ineligible study design}

764. Selten JP, Lundberg M, Rai D, et al. Risks for nonaffective psychotic disorder and bipolar disorder in young people with autism spectrum disorder: a population-based study. JAMA Psychiatry. 2015 May;72(5):483-9. doi: http://dx.doi.org/10.1001/jamapsychiatry.2014.3059. PMID: 258067976

\section{Pediatric}

765. Seo HJ, Chiesa A, Lee SJ, et al. Safety and tolerability of lamotrigine: results from 12 placebo-controlled clinical trials and clinical implications. Clinical Neuropharmacology. 2011 Jan-Feb;34(1):39-47. doi: http://dx.doi.org/10.1097/WNF.0b013e3182055c07. PMID: 21242744 Ineligible study design

766. Serafini G, Pompili M, Del Casale A, et al. Duloxetine versus venlafaxine in the treatment of unipolar and bipolar depression. Clinica Terapeutica. 2010;161(4):321-7. PMID: 20931154 Bipolar not analyzed separately

767. Serra G, Koukopoulos A, De Chiara L, et al. Three-year, naturalistic, mirror-image assessment of adding memantine to the treatment of 30 treatment-resistant patients with bipolar disorder. Journal of Clinical Psychiatry. 2015 Jan;76(1):e91-7. doi: http://dx.doi.org/10.4088/JCP.13m08956. PMID: 25650685

\section{Ineligible study design}

768. Serra G, Koukopoulos A, De Chiara L, et al. Features preceding diagnosis of bipolar versus major depressive disorders. Journal of Affective Disorders. 2015 Mar 1;173:134-42. doi: http://dx.doi.org/10.1016/j.jad.2014.10.050. PMID: 25462407 Ineligible study design

769. Severus WE, Grunze H, Kleindienst N, et al. Is the prophylactic antidepressant efficacy of lithium in bipolar I disorder dependent on study design and lithium level? Journal of Clinical Psychopharmacology. 2005 Oct;25(5):457-62. PMID: 16160621 Ineligible study design

770. Severus WE, Kleindienst N, Seemuller F, et al. What is the optimal serum lithium level in the long-term treatment of bipolar disorder--a review? Bipolar Disorders. 2008 Mar;10(2):231-7. doi: http://dx.doi.org/10.1111/j.1399-5618.2007.00475.x. PMID: 18271901 Ineligible study design

771. Shafti SS, Shahveisi B. Comparison between lithium and valproate in the treatment of acute mania. Journal of Clinical Psychopharmacology. 2008 2008;28(6):718-20. doi: http://dx.doi.org/10.1097/JCP.0b013e31818ce5ba. Ineligible study design

772. Shakeri J, Khanegi M, Golshani S, et al. Effects of omega-3 supplement in the treatment of patients with bipolar I disorder. International Journal of Preventive Medicine. 2016(pagination)doi: http://dx.doi.org/10.4103/2008-7802.182734. PMID: 610426806 Ineligible intervention

773. Shan GW, Makmor-Bakry M, Omar MS. Long term use of lithium and factors associated with treatment response among patients with bipolar disorder. Psychiatria Danubina. 2016 Jun;28(2):146-53. PMID:

\section{No eligible outcomes reported}

774. Shansis FM, Reche M, Capp E. Evaluating response to mood stabilizers in patients with mixed depression: A study of agreement between three different mania rating scales and a depression rating scale. Journal of Affective Disorders. 201601 Jun;197:1-7. doi: http://dx.doi.org/10.1016/j.jad.2016.02.064. PMID:

608820721 No eligible outcomes reported 
775. Sharma V, Sharma P. Peripartum-onset of obsessive-compulsive disorder in women with bipolar disorder A case series. Journal of Obsessive-Compulsive and Related Disorders. 2015 July 01;6:120-3. doi: http://dx.doi.org/10.1016/j.jocrd.2015.07.002. PMID: 2015198049 Ineligible study design

776. Sharma V, Yatham LN, Haslam DR, et al. Continuation and prophylactic treatment of bipolar disorder. Canadian Journal of Psychiatry - Revue Canadienne de Psychiatrie. 1997 Aug;42 Suppl 2:92S-100S. PMID: 9288442 Ineligible study design

777. Shashidhara M, Sushma BR, Viswanath B, et al. Comorbid obsessive compulsive disorder in patients with bipolar-I disorder. Journal of Affective Disorders. 2015 Mar 15;174:367-71. doi: http://dx.doi.org/10.1016/j.jad.2014.12.019. PMID: 25545603 Ineligible intervention

778. Shelton RC, Stahl SM. Risperidone and paroxetine given singly and in combination for bipolar depression. Journal of Clinical Psychiatry. 2004 Dec;65(12):1715-9. PMID: 15641878 Fewer than 11 subjects per arm

779. Shen GH, Sylvia LG, Alloy LB, et al. Lifestyle regularity and cyclothymic symptomatology. Journal of Clinical Psychology. 2008 Apr;64(4):482-500. doi: http://dx.doi.org/10.1002/jclp.20440. PMID: 18322928 No eligible outcomes reported

780. Shi L, Namjoshi MA, Swindle R, et al. Effects of olanzapine alone and olanzapine/fluoxetine combination on health-related quality of life in patients with bipolar depression: secondary analyses of a double-blind, placebo-controlled, randomized clinical trial.[Erratum appears in Clin Ther. 2004 Nov;26(11):1934]. Clinical Therapeutics. 2004 Jan;26(1):125-34. PMID: 14996525 Ineligible study design

781. Shi L, Schuh LM, Trzepacz PT, et al. Improvement of Positive and Negative Syndrome Scale cognitive score associated with olanzapine treatment of acute mania. Current Medical Research \& Opinion. 2004 Sep;20(9):1371-6. PMID: 15383185 No eligible outcomes reported

782. Shine B, McKnight RF, Leaver L, et al. Long-term effects of lithium on renal, thyroid, and parathyroid function: a retrospective analysis of laboratory data. Lancet. 2015 Aug 1;386(9992):461-8. doi: http://dx.doi.org/10.1016/S0140-6736(14)61842-0. PMID: 26003379 Ineligible study design

783. Shopsin B, Gershon S, Thompson H, et al. Psychoactive drugs in mania. A controlled comparison of lithium carbonate, chlorpromazine, and haloperidol. Arch Gen Psychiatry. 1975 Jan;32(1):34-42. PMID: 1089401 Fewer than 11 subjects per arm

784. Sidor MM, Macqueen GM. Antidepressants for the acute treatment of bipolar depression: a systematic review and meta-analysis. Journal of Clinical Psychiatry. 2011 Feb;72(2):156-67. doi: http://dx.doi.org/10.4088/JCP.09r05385gre. PMID: 21034686 Ineligible study design

785. Sienaert P, Vansteelandt K, Demyttenaere K, et al. Ultra-brief pulse ECT in bipolar and unipolar depressive disorder: differences in speed of response. Bipolar Disorders. 2009 Jun;11(4):418-24. doi: http://dx.doi.org/10.1111/j.1399-5618.2009.00702.x. PMID: 19500095 No eligible outcomes reported

786. Sikdar S, Kulhara P, Avasthi A, et al. Combined chlorpromazine and electroconvulsive therapy in mania. British Journal of Psychiatry. 1994 Jun;164(6):806-10. PMID: 7952988 Bipolar not analyzed separately 
787. Silva MT, Zimmermann IR, Galvao TF, et al. Olanzapine plus fluoxetine for bipolar disorder: a systematic review and meta-analysis. Journal of Affective Disorders. 2013 Apr 25;146(3):310-8. doi: http://dx.doi.org/10.1016/j.jad.2012.11.001. PMID: 23218251 Ineligible study design

788. Simhandl C, Konig B, Amann BL. A prospective 4-year naturalistic follow-up of treatment and outcome of 300 bipolar I and II patients. Journal of Clinical Psychiatry. 2014 Mar;75(3):254-62; quiz 63. doi: http://dx.doi.org/10.4088/JCP.13m08601. PMID: 24717379 Ineligible study design

789. Simhandl C, Radua J, Konig B, et al. The prevalence and effect of life events in 222 bipolar I and II patients: a prospective, naturalistic 4 year follow-up study. Journal of Affective Disorders. 2015 Jan 1;170:166-71. doi: http://dx.doi.org/10.1016/j.jad.2014.08.043. PMID: 25240845 Ineligible intervention

790. Simon NM, Otto MW, Wisniewski SR, et al. Anxiety disorder comorbidity in bipolar disorder patients: data from the first 500 participants in the Systematic Treatment Enhancement Program for Bipolar Disorder (STEP-BD). American Journal of Psychiatry. 2004 Dec;161(12):2222-9. PMID: 15569893 No eligible outcomes reported

791. Simons W, Dierick M. Transcranial magnetic stimulation as a therapeutic tool in psychiatry. World Journal of Biological Psychiatry. 2005;6(1):6-25. PMID: 16097402 Ineligible study design

792. Simpson S, Barnes E, Griffiths E, et al. The Bipolar Interactive Psychoeducation (BIPED) study: trial design and protocol. BMC Psychiatry. 2009;9:50. doi: http://dx.doi.org/10.1186/1471-244X-9-50. PMID: 19674448 Ineligible study design

793. Singh JB, Zarate CA, Jr. Pharmacological treatment of psychiatric comorbidity in bipolar disorder: a review of controlled trials. Bipolar Disorders. 2006 Dec;8(6):696-709. PMID: 17156156 Ineligible study design

794. Small JG, Klapper MH, Marhenke JD, et al. Lithium combined with carbamazepine or haloperidol in the treatment of mania. Psychopharmacology Bulletin. 1995;31(2):265-72. PMID: 7491378 Over 50\% dropout rate

795. Smith LA, Cornelius V, Warnock A, et al. Effectiveness of mood stabilizers and antipsychotics in the maintenance phase of bipolar disorder: a systematic review of randomized controlled trials. Bipolar Disorders. 2007 Jun;9(4):394-412. PMID: 17547586 Ineligible study design

796. Smith LA, Cornelius V, Warnock A, et al. Acute bipolar mania: a systematic review and meta-analysis of co-therapy vs. monotherapy. Acta Psychiatrica Scandinavica. 2007 Jan;115(1):12-20. PMID: 17201861 Ineligible study design

797. Smith LA, Cornelius V, Warnock A, et al. Pharmacological interventions for acute bipolar mania: a systematic review of randomized placebo-controlled trials. Bipolar Disorders. 2007 Sep;9(6):551-60. PMID: 17845269 Ineligible study design

798. Soares-Weiser K, Bravo Vergel Y, Beynon S, et al. A systematic review and economic model of the clinical effectiveness and cost-effectiveness of interventions for preventing relapse in people with bipolar disorder. Health Technology Assessment (Winchester, England). 2007 Oct;11(39):iii-iv, ix-206. PMID: 17903393 Ineligible study design 
799. Sokolski KN. Adjunctive aripiprazole in bipolar I depression. Annals of Pharmacotherapy. 2007 Jan;41(1):35-40. PMID: 17190849 Ineligible study design

800. Solmi M, Veronese N, Zaninotto L, et al. Lamotrigine compared to placebo and other agents with antidepressant activity in patients with unipolar and bipolar depression: A comprehensive meta-analysis of efficacy and safety outcomes in short-term trials. CNS Spectrums. 201601 Oct;21(5):403-18. doi: http://dx.doi.org/10.1017/S1092852916000523. PMID: 612523889 Ineligible study design

801. Solomon DA, Keitner GI, Miller IW, et al. Course of illness and maintenance treatments for patients with bipolar disorder. Journal of Clinical Psychiatry. 1995 Jan;56(1):5-13. PMID: 7836345 Ineligible study design

802. Solomon DA, Keitner GI, Ryan CE, et al. Lithium plus valproate as maintenance polypharmacy for patients with bipolar I disorder: a review. Journal of Clinical Psychopharmacology. 1998 Feb;18(1):38-49. PMID: 9472841 Ineligible study design

803. Solomon DA, Ristow WR, Keller MB, et al. Serum lithium levels and psychosocial function in patients with bipolar I disorder. American Journal of Psychiatry. 1996 Oct;153(10):1301-7. PMID: 8831438 Over $50 \%$ dropout rate

804. Solomon DA, Ryan CE, Keitner GI, et al. A pilot study of lithium carbonate plus divalproex sodium for the continuation and maintenance treatment of patients with bipolar I disorder. J Clin Psychiatry. 1997 Mar;58(3):95-9. PMID: 9108809 Fewer than 11 subjects per arm

805. Spaulding T, Westlund R, Thomason C, et al. Adjunctive treatment for mood stabilization of patients with bipolar I disorder treated with lamotrigine. Cns Spectrums. 2006 Sep;11(9):711-6; quiz 9. PMID: 16946696 Ineligible study design

806. Spina E, Perugi G. Antiepileptic drugs: indications other than epilepsy. Epileptic Disorders. 2004 Jun;6(2):57-75. PMID: 15246950 Ineligible study design

807. Srisurapanont M, Yatham LN, Zis AP. Treatment of acute bipolar depression: a review of the literature. Canadian Journal of Psychiatry - Revue Canadienne de Psychiatrie. 1995 Nov;40(9):533-44. PMID: 8574989 Ineligible study design

808. Srivastava S, Ketter TA. Clinical relevance of treatments for acute bipolar disorder: balancing therapeutic and adverse effects. Clinical Therapeutics. 2011 Dec;33(12):B40-8. doi: http://dx.doi.org/10.1016/j.clinthera.2011.11.020. PMID: 22177379 Ineligible study design

809. Stahl S, Lombardo I, Loebel A, et al. Efficacy of ziprasidone in dysphoric mania: pooled analysis of two double-blind studies. Journal of Affective Disorders. 2010 Apr;122(1-2):39-45. doi: http://dx.doi.org/10.1016/j.jad.2009.06.023. PMID: 19616304 Over 50\% dropout rate

810. Stange JP, Sylvia LG, da Silva Magalhaes PV, et al. Extreme attributions predict transition from depression to mania or hypomania in bipolar disorder. Journal of Psychiatric Research. 2013 2013;47(10):1329-36. doi: http://dx.doi.org/10.1016/j.jpsychires.2013.05.016. Ineligible study design

811. Stedman M, Pettinati HM, Brown ES, et al. A double-blind, placebo-controlled study with quetiapine as adjunct therapy with lithium or divalproex in bipolar I patients with coexisting alcohol dependence. Alcoholism: Clinical \& Experimental Research. 2010 Oct;34(10):1822-31. doi: 
http://dx.doi.org/10.1111/j.1530-0277.2010.01270.x. PMID: 20626727 No eligible outcomes

reported

812. Stoner SC, Nelson LA, Lea JW, et al. Historical review of carbamazepine for the treatment of bipolar disorder. Pharmacotherapy:The Journal of Human Pharmacology \& Drug Therapy. 2007 Jan;27(1):68-88. PMID: 17192163 Ineligible study design

813. Stoner SC, Pace HA. Asenapine: a clinical review of a second-generation antipsychotic. Clinical Therapeutics. 2012 May;34(5):1023-40. doi: http://dx.doi.org/10.1016/j.clinthera.2012.03.002. PMID: 22494521 Ineligible study design

814. Storosum JG, Wohlfarth T, Gispen-de Wied CC, et al. Suicide risk in placebo-controlled trials of treatment for acute manic episode and prevention of manic-depressive episode. American Journal of Psychiatry. 2005 Apr;162(4):799-802. PMID: 15800158 Ineligible study design

815. Suppes T. Review of the use of topiramate for treatment of bipolar disorders. Journal of Clinical Psychopharmacology. 2002 Dec;22(6):599-609. PMID: 12454560 Ineligible study design

816. Suppes T, Brown E, Schuh LM, et al. Rapid versus non-rapid cycling as a predictor of response to olanzapine and divalproex sodium for bipolar mania and maintenance of remission: post hoc analyses of 47-week data. Journal of Affective Disorders. 2005 Dec;89(1-3):69-77. PMID: 16253344 Over 50\% dropout rate

817. Suppes T, Eudicone J, McQuade R, et al. Efficacy and safety of aripiprazole in subpopulations with acute manic or mixed episodes of bipolar I disorder. Journal of Affective Disorders. 2008 Apr;107(1-3):145-54. PMID: 17904226 Over 50\% dropout rate

818. Suppes T, Kelly DI, Hynan LS, et al. Comparison of two anticonvulsants in a randomized, single-blind treatment of hypomanic symptoms in patients with bipolar disorder. Australian \& New Zealand Journal of Psychiatry. 2007 May;41(5):397-402. PMID: 17464731 Fewer than 11 subjects per arm

819. Suppes T, Ketter TA, Gwizdowski IS, et al. First controlled treatment trial of bipolar II hypomania with mixed symptoms: quetiapine versus placebo. Journal of Affective Disorders. 2013 Aug 15;150(1):37-43. doi: http://dx.doi.org/10.1016/j.jad.2013.02.031. PMID: 23521871 Over 50\% dropout rate

820. Suppes T, Marangell LB, Bernstein IH, et al. A single blind comparison of lithium and lamotrigine for the treatment of bipolar II depression. Journal of Affective Disorders. 2008 Dec;111(2-3):334-43. doi: http://dx.doi.org/10.1016/j.jad.2008.02.004. PMID: 18358540 Over 50\% dropout rate

821. Suppes T, Rush AJ, Dennehy EB, et al. Texas Medication Algorithm Project, phase 3 (TMAP-3): clinical results for patients with a history of mania. Journal of Clinical Psychiatry. 2003 Apr;64(4):370-82. PMID: 12716236 Ineligible intervention

822. Suppes T, Rush AJ, Jr., Kraemer HC, et al. Treatment algorithm use to optimize management of symptomatic patients with a history of mania. Journal of Clinical Psychiatry. 1998 Feb;59(2):89-96; quiz 78. PMID: 9501899 Ineligible study design

823. Suppes T, Webb A, Paul B, et al. Clinical outcome in a randomized 1-year trial of clozapine versus treatment as usual for patients with treatment-resistant illness and a history of mania. American Journal of Psychiatry. 1999 Aug;156(8):1164-9. PMID: 10450255 Bipolar not analyzed separately 
824. Sussman N, Mullen J, Paulsson B, et al. Rates of remission/euthymia with quetiapine in combination with lithium/divalproex for the treatment of acute mania. Journal of Affective Disorders. 2007;100 Suppl 1:S5563. PMID: 17383736 Duplicate reference

825. Swainston Harrison T, Keating GM. Extended-release carbamazepine capsules : in bipolar I disorder. CNS Drugs. 2005;19(8):709-16. PMID: 16097852 Ineligible study design

826. Swann AC, Bowden CL, Calabrese JR, et al. Differential effect of number of previous episodes of affective disorder on response to lithium or divalproex in acute mania. American Journal of Psychiatry. 1999

Aug;156(8):1264-6. PMID: 10450271 Ineligible study design

827. Swann AC, Bowden CL, Calabrese JR, et al. Mania: differential effects of previous depressive and manic episodes on response to treatment. Acta Psychiatrica Scandinavica. 2000 Jun;101(6):444-51. PMID: 10868467 Ineligible study design

828. Swann AC, Bowden CL, Morris D, et al. Depression during mania. Treatment response to lithium or divalproex. Archives of General Psychiatry. 1997 Jan;54(1):37-42. PMID: 9006398 Bipolar not analyzed separately

829. Swann AC, Petty F, Bowden CL, et al. Mania: gender, transmitter function, and response to treatment. Psychiatry Research. 1999 Oct 18;88(1):55-61. PMID: 10641586 No eligible outcomes reported

830. Swartz HA, Frank E. Psychotherapy for bipolar depression: a phase-specific treatment strategy? Bipolar Disorders. 2001 Feb;3(1):11-22. PMID: 11256459 Ineligible study design

831. Swartz HA, Frank E, Cheng Y. A randomized pilot study of psychotherapy and quetiapine for the acute treatment of bipolar II depression. Bipolar Disorders. 2012 Mar;14(2):211-6. doi: http://dx.doi.org/10.1111/j.1399-5618.2012.00988.x. PMID: 22420597 Less than 11 subjects per arm

832. Sylvia LG, Ametrano RM, Nierenberg AA. Exercise treatment for bipolar disorder: potential mechanisms of action mediated through increased neurogenesis and decreased allostatic load. Psychotherapy \& Psychosomatics. 2010;79(2):87-96. doi: http://dx.doi.org/10.1159/000270916. PMID: 20051706

\section{Ineligible study design}

833. Sylvia LG, Peters AT, Deckersbach T, et al. Nutrient-based therapies for bipolar disorder: A systematic review. Psychotherapy and Psychosomatics. 2012 2012;82(1):10-9. doi: http://dx.doi.org/10.1159/000341309. Ineligible study design

834. Sylvia LG, Rabideau DJ, Nierenberg AA, et al. The effect of personalized guideline-concordant treatment on quality of life and functional impairment in bipolar disorder. Journal of Affective Disorders. 2014 1, 2014;169:144-8. doi: http://dx.doi.org/10.1016/j.jad.2014.08.019. PMID: 25194782 Ineligible intervention

835. Sylvia LG, Shelton RC, Kemp DE, et al. Medical burden in bipolar disorder: Findings from the Clinical and Health Outcomes Initiative in Comparative Effectiveness for Bipolar Disorder study (Bipolar CHOICE). Bipolar Disorders. 201501 Mar;17(2):212-23. doi: http://dx.doi.org/10.1111/bdi.12243. Not treating bipolar 
836. Szegedi A, Zhao J, McIntyre RS. Early improvement as a predictor of acute treatment outcome in manic or mixed episodes in bipolar-1 disorder: A pooled, post hoc analysis from the asenapine development program. Journal of Affective Disorders. 2013 25, 2013;150(3):745-52. doi: http://dx.doi.org/10.1016/j.jad.2013.01.024. Ineligible study design

837. Szegedi A, Zhao J, van Willigenburg A, et al. Effects of asenapine on depressive symptoms in patients with bipolar I disorder experiencing acute manic or mixed episodes: a post hoc analysis of two 3-week clinical trials. BMC Psychiatry. 2011;11:101. doi: http://dx.doi.org/10.1186/1471-244X-11-101. PMID: 21689438 Ineligible study design

838. Szentagotai A, David D. The efficacy of cognitive-behavioral therapy in bipolar disorder: a quantitative meta-analysis. Journal of Clinical Psychiatry. 2010 Jan;71(1):66-72. doi: http://dx.doi.org/10.4088/JCP.08r04559yel. PMID: 19852904 Ineligible study design

839. Szuba MP, Amsterdam JD. Rapid Antidepressant Response After Nocturnal TRH Administration in Patients With Bipolar Type I and Bipolar Type II Major Depression. Journal of Clinical Psychopharmacology. 2005 2005;25(4):325-30. doi: http://dx.doi.org/10.1097/01.jcp.0000169037.17884.79. No eligible outcomes reported

840. Tada M, Uchida H, Mizushima J, et al. Antidepressant dose and treatment response in bipolar depression: Reanalysis of the Systematic Treatment Enhancement Program for Bipolar Disorder (STEP-BD) data. Journal of Psychiatric Research. 201501 Sep;68:151-6. doi: http://dx.doi.org/10.1016/j.jpsychires.2015.06.015. PMID: 2015230798 Ineligible intervention

841. Tamayo JM, Mazzotti G, Tohen M, et al. Outcomes for Latin American versus White patients suffering from acute mania in a randomized, double-blind trial comparing olanzapine and haloperidol. Journal of Clinical Psychopharmacology. 2007 Apr;27(2):126-34. PMID: 17414234 Duplicate reference

842. Tamayo JM, Mejia-Rodriguez D, Navarro-Montoya AM, et al. Therapy of No-Type I bipolar spectrum disorders: A systematic review. Current Psychiatry Reviews. 2013 2013;9(1):41-50. Ineligible study design

843. Tamayo JM, Sutton VK, Mattei MA, et al. Effectiveness and safety of the combination of fluoxetine and olanzapine in outpatients with bipolar depression: an open-label, randomized, flexible-dose study in Puerto Rico. Journal of Clinical Psychopharmacology. 2009 Aug;29(4):358-61. doi: http://dx.doi.org/10.1097/JCP.0b013e3181ad223f. PMID: 19593175 Ineligible study design

844. Tamayo JM, Zarate CA, Jr., Vieta E, et al. Level of response and safety of pharmacological monotherapy in the treatment of acute bipolar I disorder phases: a systematic review and meta-analysis. International Journal of Neuropsychopharmacology. 2010 Jul;13(6):813-32. doi: http://dx.doi.org/10.1017/S1461145709991246. PMID: 20128953 Ineligible study design

845. Tarr GP, Glue P, Herbison P. Comparative efficacy and acceptability of mood stabilizer and second generation antipsychotic monotherapy for acute mania--a systematic review and meta-analysis. Journal of Affective Disorders. 2011 Nov;134(1-3):14-9. doi: http://dx.doi.org/10.1016/j.jad.2010.11.009. PMID: 21145595 Ineligible study design

846. Thase ME. Quetiapine monotherapy for bipolar depression. Neuropsychiatric Disease and Treatment. 2008;4(1A):21-31. doi: http://dx.doi.org/10.2147/NDT.S1162. Ineligible study design 
847. Thase ME, Bowden CL, Nashat M, et al. Aripiprazole in bipolar depression: a pooled, post-hoc analysis by severity of core depressive symptoms. International Journal of Psychiatry in Clinical Practice. 2012 Jun;16(2):121-31. doi: http://dx.doi.org/10.3109/13651501.2011.632680. PMID: 22296512 Ineligible study design

848. Thase ME, Jonas A, Khan A, et al. Aripiprazole monotherapy in nonpsychotic bipolar I depression: results of 2 randomized, placebo-controlled studies.[Erratum appears in J Clin Psychopharmacol. 2009

Feb;29(1):38]. Journal of Clinical Psychopharmacology. 2008 Feb;28(1):13-20. doi: http://dx.doi.org/10.1097/jcp.0b013e3181618eb4. PMID: 18204335 No eligible outcomes reported

849. Thase ME, Macfadden W, Weisler RH, et al. Efficacy of Quetiapine Monotherapy in Bipolar I and II Depression: A Double-blind, Placebo-controlled Study (The BOLDER II Study). Journal of Clinical Psychopharmacology. 2006 2006;26(6):600-9. doi: http://dx.doi.org/10.1097/01.jcp.0000248603.76231.b7. No eligible outcomes reported

850. Thirthalli J, Prasad MK, Gangadhar BN. Electroconvulsive therapy (ECT) in bipolar disorder: A narrative review of literature. Asian Journal of Psychiatry. 2012 2012;5(1):11-7. doi: http://dx.doi.org/10.1016/j.ajp.2011.12.002. Ineligible study design

851. Todd NJ, Solis-Trapala I, Jones SH, et al. An online randomised controlled trial to assess the feasibility, acceptability and potential effectiveness of 'Living with Bipolar': a web-based self-management intervention for bipolar disorder: trial design and protocol. Contemporary Clinical Trials. 2012 Jul;33(4):679-88. doi: http://dx.doi.org/10.1016/j.cct.2012.02.011. PMID: 22387150 Ineligible study design

852. Tohen M, Baker RW, Altshuler LL, et al. Olanzapine versus divalproex in the treatment of acute mania. The American Journal of Psychiatry. 2002 2002;159(6):1011-7. doi: http://dx.doi.org/10.1176/appi.ajp.159.6.1011. Duplicate reference

853. Tohen M, Sanger TM, McElroy SL, et al. Olanzapine versus placebo in the treatment of acute mania. The American Journal of Psychiatry. 1999 1999;156(5):702-9. PMID: 10327902 Over 50\% dropout rate

854. Tohen M, Sniadecki J, Sutton VK, et al. Number needed to treat or harm analyses of olanzapine for maintenance treatment of bipolar disorder. Journal of Clinical Psychopharmacology. 2009 Dec;29(6):5208. doi: http://dx.doi.org/10.1097/JCP.0b013e3181bfe128. PMID: 19910715 Ineligible study design

855. Tohen M, Sutton VK, Calabrese JR, et al. Maintenance of response following stabilization of mixed index episodes with olanzapine monotherapy in a randomized, double-blind, placebo-controlled study of bipolar 1 disorder. Journal of Affective Disorders. 2009 Jul;116(1-2):43-50. doi: http://dx.doi.org/10.1016/j.jad.2008.11.003. PMID: 19054570 Over 50\% dropout rate

856. Tohen M, Zarate CA, Jr. Antipsychotic agents and bipolar disorder. Journal of Clinical Psychiatry. 1998;59 Suppl 1:38-48; discussion 9. PMID: 9448668 Ineligible study design

857. Tolliver BK, Desantis SM, Brown DG, et al. A randomized, double-blind, placebo-controlled clinical trial of acamprosate in alcohol-dependent individuals with bipolar disorder: a preliminary report. Bipolar Disorders. 2012 Feb;14(1):54-63. doi: http://dx.doi.org/10.1111/j.1399-5618.2011.00973.x. PMID: 22329472 No eligible outcomes reported 
858. Tolliver BK, McRae AL, Verduin ML, et al. Reversible elevation of triglycerides in dual-diagnosis patients taking aripiprazole: A case series. Journal of Clinical Psychopharmacology. 2008 2008;28(4):464-7. doi: http://dx.doi.org/10.1097/JCP.0b013e31817efb99. PMID: 18626281 Ineligible study design

859. Tondo L, Baldessarini RJ, Floris G. Long-term clinical effectiveness of lithium maintenance treatment in types I and II bipolar disorders. British Journal of Psychiatry - Supplementum. 2001 Jun;41:s184-90. PMID: 11450181 Ineligible study design

860. Torrent C, Bonnin Cdel M, Martinez-Aran A, et al. Efficacy of functional remediation in bipolar disorder: a multicenter randomized controlled study. American Journal of Psychiatry. 2013 Aug 1;170(8):852-9. doi: http://dx.doi.org/10.1176/appi.ajp.2012.12070971. PMID: 23511717 Ineligible study design

861. Torrey EF, Davis JM. Adjunct treatments for schizophrenia and bipolar disorder: what to try when you are out of ideas. Clinical Schizophrenia \& Related Psychoses. 2012 Jan;5(4):208-16. doi: http://dx.doi.org/10.3371/CSRP.5.4.5. PMID: 22182458 Ineligible study design

862. Tsai AC, Rosenlicht NZ, Jureidini JN, et al. Aripiprazole in the maintenance treatment of bipolar disorder: a critical review of the evidence and its dissemination into the scientific literature. PLoS Medicine / Public Library of Science. 2011 May;8(5):e1000434. doi: http://dx.doi.org/10.1371/journal.pmed.1000434. PMID: 21559324 Ineligible study design

863. Tseng PT, Chen YW, Tu KY, et al. Light therapy in the treatment of patients with bipolar depression: A meta-analytic study. European Neuropsychopharmacology. 201601 Jun;26(6):1037-47. doi: http://dx.doi.org/10.1016/j.euroneuro.2016.03.001. PMID: 609105359 Ineligible study design

864. Tundo A, Calabrese JR, Proietti L, et al. Variation in response to short-term antidepressant treatment between patients with continuous and non-continuous cycling bipolar disorders. Journal of Affective Disorders. 2015 Mar 15;174:126-30. doi: http://dx.doi.org/10.1016/j.jad.2014.11.036. PMID: 25497468 Not treating bipolar

865. Ukaegbu C, Banks JB, Carter NJ. What drugs are best for bipolar depression? [References]. The Journal of Family Practice. 2008 2008;57(9):606-8. Ineligible study design

866. Ulcickas Yood M, Delorenze G, Quesenberry CP, Jr., et al. Epidemiologic study of aripiprazole use and the incidence of suicide events. Pharmacoepidemiology \& Drug Safety. 2010 Nov;19(11):1124-30. doi: http://dx.doi.org/10.1002/pds.2047. PMID: 20925132 Bipolar not analyzed separately

867. Ummar S, Dorai B, Ramanathan S. Distressing cutaneous lesion among bipolar affective disorder patients on lithium therapy: A retrospective cross-sectional study. Indian Journal of Psychiatry. 2016 OctoberDecember;58(4):383-6. doi: http://dx.doi.org/10.4103/0019-5545.196708. PMID: 614018225 No eligible outcomes reported

868. Unholzer S, Haen E. Retrospective analysis of therapeutic drug monitoring data for treatment of bipolar disorder with lamotrigine.[Erratum appears in Pharmacopsychiatry. 2015 Nov;48(7):296; PMID: 26630654]. Pharmacopsychiatry. 2015 Sep;48(6):211-4. doi: http://dx.doi.org/10.1055/s-0035-1559635. PMID: 26252722 Ineligible study design

869. Valenti M, Benabarre A, Garcia-Amador M, et al. Electroconvulsive therapy in the treatment of mixed states in bipolar disorder. European Psychiatry: the Journal of the Association of European Psychiatrists. 2008 Jan;23(1):53-6. doi: http://dx.doi.org/10.1016/j.eurpsy.2007.10.011. PMID: 18191551 Ineligible study design 
870. Valenti M, Pacchiarotti I, Undurraga J, et al. Risk factors for rapid cycling in bipolar disorder. Bipolar Disorders. 201501 Aug;17(5):549-59. doi: http://dx.doi.org/10.1111/bdi.12288. PMID: 2015769954 Ineligible study design

871. van der Loos ML, Mulder P, Hartong EG, et al. Efficacy and safety of two treatment algorithms in bipolar depression consisting of a combination of lithium, lamotrigine or placebo and paroxetine. Acta Psychiatrica Scandinavica. 2010 Sep;122(3):246-54. doi: http://dx.doi.org/10.1111/j.1600-0447.2009.01537.x. PMID: 20136801 No eligible outcomes reported

872. van der Loos ML, Mulder P, Hartong EG, et al. Long-term outcome of bipolar depressed patients receiving lamotrigine as add-on to lithium with the possibility of the addition of paroxetine in nonresponders: a randomized, placebo-controlled trial with a novel design. Bipolar Disorders. 2011 Feb;13(1):111-7. doi: http://dx.doi.org/10.1111/j.1399-5618.2011.00887.x. PMID: 21320258 No eligible outcomes reported

873. van der Loos ML, Mulder PG, Hartong EG, et al. Efficacy and safety of lamotrigine as add-on treatment to lithium in bipolar depression: a multicenter, double-blind, placebo-controlled trial. Journal of Clinical Psychiatry. 2009 Feb;70(2):223-31. PMID: 19200421 Ineligible study design

874. van der Voort TY, van Meijel B, Goossens PJ, et al. Collaborative care for patients with bipolar disorder: a randomised controlled trial. BMC Psychiatry. 2011;11:133. doi: http://dx.doi.org/10.1186/1471-244X-11133. PMID: 21849078 Ineligible study design

875. Van Dijk S, Jeffrey J, Katz MR. A randomized, controlled, pilot study of dialectical behavior therapy skills in a psychoeducational group for individuals with bipolar disorder. Journal of Affective Disorders. 2013 Mar 5;145(3):386-93. doi: http://dx.doi.org/10.1016/j.jad.2012.05.054. PMID: 22858264 Ineligible intervention

876. Van Lieshout RJ, MacQueen GM. Efficacy and acceptability of mood stabilisers in the treatment of acute bipolar depression: systematic review. British Journal of Psychiatry. 2010 Apr;196(4):266-73. doi: http://dx.doi.org/10.1192/bjp.bp.108.057612. PMID: 20357301 Ineligible study design

877. Vasudev A, Macritchie K, Rao SK, et al. Tiagabine for acute affective episodes in bipolar disorder. Cochrane Database of Systematic Reviews. 2012;12:CD004694. doi: http://dx.doi.org/10.1002/14651858.CD004694.pub3. PMID: 23235614 Ineligible study design

878. Vasudev A, Macritchie K, Rao SN, et al. Tiagabine in the maintenance treatment of bipolar disorder. Cochrane Database of Systematic Reviews. 2011(12):CD005173. doi: http://dx.doi.org/10.1002/14651858.CD005173.pub3. PMID: 22161389 Ineligible study design

879. Vasudev A, Macritchie K, Vasudev K, et al. Oxcarbazepine for acute affective episodes in bipolar disorder. Cochrane Database of Systematic Reviews. 2011(12):CD004857. doi: http://dx.doi.org/10.1002/14651858.CD004857.pub2. PMID: 22161387 Ineligible study design

880. Vasudev A, Macritchie K, Watson S, et al. Oxcarbazepine in the maintenance treatment of bipolar disorder. Cochrane Database of Systematic Reviews. 2008(1):CD005171. doi: http://dx.doi.org/10.1002/14651858.CD005171.pub2. PMID: 18254071 Ineligible study design 
881. Vasudev K, Macritchie K, Geddes J, et al. Topiramate for acute affective episodes in bipolar disorder. Cochrane Database of Systematic Reviews. 2006(1):CD003384. PMID: 16437453 Ineligible study design

882. Vazquez GH, Tondo L, Undurraga J, et al. Overview of antidepressant treatment of bipolar depression. International Journal of Neuropsychopharmacology. 2013 Aug;16(7):1673-85. doi: http://dx.doi.org/10.1017/S1461145713000023. PMID: 23428003 Ineligible study design

883. Vedula SS, Bero L, Scherer RW, et al. Outcome reporting in industry-sponsored trials of gabapentin for off-label use. New England Journal of Medicine. 2009 Nov 12;361(20):1963-71. doi: http://dx.doi.org/10.1056/NEJMsa0906126. PMID: 19907043 Ineligible study design

884. Veeh J, Kopf J, Kittel-Schneider S, et al. Cognitive remediation for bipolar patients with objective cognitive impairment: a naturalistic study. International Journal of Bipolar Disorders. 201701 Dec;5 (1) (no pagination)(8)doi: http://dx.doi.org/10.1186/s40345-017-0079-3. PMID: 615340856 Ineligible study design

885. Versiani M, Cheniaux E, Landeira-Fernandez J. Efficacy and safety of electroconvulsive therapy in the treatment of bipolar disorder: a systematic review. Journal of ECT. 2011 Jun;27(2):153-64. doi: http://dx.doi.org/10.1097/YCT.0b013e3181e6332e. PMID: 20562714 Ineligible study design

886. Vieta E, Calabrese JR, Hennen J, et al. Comparison of rapid-cycling and non-rapid-cycling bipolar I manic patients during treatment with olanzapine: analysis of pooled data. Journal of Clinical Psychiatry. 2004 Oct;65(10):1420-8. PMID: 15491248 Over 50\% dropout rate

887. Vieta E, Durgam S, Lu K, et al. Effect of cariprazine across the symptoms of mania in bipolar I disorder: Analyses of pooled data from phase II/III trials. European Neuropsychopharmacology. 2015 Nov;25(11):1882-91. doi: http://dx.doi.org/10.1016/j.euroneuro.2015.08.020. PMID: 26419293 Duplicate reference

888. Vieta E, Goldberg JF, Mullen J, et al. Quetiapine in the treatment of acute mania: target dose for efficacious treatment. Journal of Affective Disorders. 2007;100 Suppl 1:S23-31. PMID: 17382403 Ineligible study design

889. Vieta E, Gunther O, Locklear J, et al. Effectiveness of psychotropic medications in the maintenance phase of bipolar disorder: a meta-analysis of randomized controlled trials. International Journal of Neuropsychopharmacology. 2011 Sep;14(8):1029-49. doi: http://dx.doi.org/10.1017/S1461145711000885. PMID: 21733231 Ineligible study design

890. Vieta E, Locklear J, Gunther O, et al. Treatment options for bipolar depression: a systematic review of randomized, controlled trials. Journal of Clinical Psychopharmacology. 2010 Oct;30(5):579-90. doi: http://dx.doi.org/10.1097/JCP.0b013e3181f15849. PMID: 20814319 Ineligible study design

891. Vieta E, Martinez-Aran A, Goikolea JM, et al. A randomized trial comparing paroxetine and venlafaxine in the treatment of bipolar depressed patients taking mood stabilizers. Journal of Clinical Psychiatry. 2002 Jun;63(6):508-12. PMID: 12088162 Ineligible study design

892. Vieta E, Mullen J, Brecher M, et al. Quetiapine monotherapy for mania associated with bipolar disorder: combined analysis of two international, double-blind, randomised, placebo-controlled studies. Current Medical Research \& Opinion. 2005 Jun;21(6):923-34. PMID: 15969892 Duplicate reference 
893. Vieta E, Ramey T, Keller D, et al. Ziprasidone in the treatment of acute mania: a 12-week, placebocontrolled, haloperidol-referenced study. Journal of Psychopharmacology. 2010 Apr;24(4):547-58. doi: http://dx.doi.org/10.1177/0269881108099418. PMID: 19074536 Over 50\% dropout rate

894. Vieta E, Suppes T, Ekholm B, et al. Long-term efficacy of quetiapine in combination with lithium or divalproex on mixed symptoms in bipolar I disorder. Journal of Affective Disorders. 2012 Dec 15;142(13):36-44. doi: http://dx.doi.org/10.1016/j.jad.2012.04.014. PMID: 23062763 Over 50\% dropout rate

895. Vik A, Ravindran A, Shiah IS, et al. A double-blind, placebo-controlled study of adjunctive calcitonin nasal spray in acute refractory mania. Bipolar Disorders. 2013 2013;15(4):359-64. PMID: 23551803 Over $50 \%$ dropout rate

896. Vik A, Yatham LN. Calcitonin and bipolar disorder: a hypothesis revisited. Journal of Psychiatry \& Neuroscience. 1998 Mar;23(2):109-17. PMID: 9549251 Ineligible study design

897. Viktorin A, Ryden E, Thase ME, et al. The risk of treatment-emergent mania with methylphenidate in bipolar disorder. American Journal of Psychiatry. 201701 Apr;174(4):341-8. doi: http://dx.doi.org/10.1176/appi.ajp.2016.16040467. PMID: 27690517 PMID/615229531 Embase No eligible outcomes reported

898. Visser HM, Van Der Mast RC. Bipolar disorder, antidepressants and induction of hypomania or mania. A systematic review. World Journal of Biological Psychiatry. 2005;6(4):231-41. PMID: 16272078

\section{Ineligible study design}

899. Vita A, De Peri L, Siracusano A, et al. Efficacy and tolerability of asenapine for acute mania in bipolar I disorder: Meta-analyses of randomized-controlled trials. International Clinical Psychopharmacology. 2013 2013;28(5):219-27. doi: http://dx.doi.org/10.1097/YIC.0b013e32836290d2. Ineligible study design

900. Vohringer PA, Ostacher MJ, El-Mallakh RS, et al. Antidepressants in Type II Versus Type i Bipolar Depression: A Randomized Discontinuation Trial. Journal of Clinical Psychopharmacology. 201512 Oct;35(5):605-8. doi: http://dx.doi.org/10.1097/JCP.0000000000000384. PMID: 2015371706 Over 50\% dropout rate

901. Walton SA, Berk M, Brook S. Superiority of lithium over verapamil in mania: a randomized, controlled, single-blind trial. Journal of Clinical Psychiatry. 1996 Nov;57(11):543-6. PMID: 8968305 Not treating bipolar

902. Wang PW, Ketter TA. Clinical use of carbamazepine for bipolar disorders. Expert Opinion on Pharmacotherapy. 2005 Dec;6(16):2887-902. PMID: 16318439 Ineligible study design

903. Wang PW, Ketter TA, Becker OV, et al. New anticonvulsant medication uses in bipolar disorder. Cns Spectrums. 2003 Dec;8(12):930-2, 41-7. PMID: 14978468 Ineligible study design

904. Wang Z, Gao K, Kemp DE, et al. Lamotrigine adjunctive therapy to lithium and divalproex in depressed patients with rapid cycling bipolar disorder and a recent substance use disorder: a 12-week, double-blind, placebo-controlled pilot study. Psychopharmacology Bulletin. 2010;43(4):5-21. PMID: 21240149 Over $50 \%$ dropout rate 
905. Warrington L, Lombardo I, Loebel A, et al. Ziprasidone for the treatment of acute manic or mixed episodes associated with bipolar disorder. CNS Drugs. 2007;21(10):835-49. PMID: 17850172 Ineligible study design

906. Watson S, Gallagher P, Porter RJ, et al. A randomized trial to examine the effect of mifepristone on neuropsychological performance and mood in patients with bipolar depression. Biological Psychiatry. 2012 Dec 1;72(11):943-9. doi: http://dx.doi.org/10.1016/j.biopsych.2012.05.029. PMID: 22770649 No eligible outcomes reported

907. Weber B, Jermann F, Gex-Fabry M, et al. Mindfulness-based cognitive therapy for bipolar disorder: a feasibility trial. European Psychiatry: the Journal of the Association of European Psychiatrists. 2010 Oct;25(6):334-7. doi: http://dx.doi.org/10.1016/j.eurpsy.2010.03.007. PMID: 20561769 No eligible outcomes reported

908. Weisler RH. Carbamazepine extended-release capsules in bipolar disorder. Neuropsychiatric Disease and Treatment. 2006 2006;2(1):3-11. Ineligible study design

909. Weisler RH, Kalali AH, Cutler AJ, et al. Efficacy and safety of once- versus twice-daily carbamazepine extended-release capsules for the treatment of manic symptoms in patients with bipolar I disorder. Psychiatry. 2008 2008;5(3):35-48. PMID: 22778707 Over 50\% dropout rate

910. Weisler RH, Kalali AH, Ketter TA, et al. A multicenter, randomized, double-blind, placebo-controlled trial of extended-release carbamazepine capsules as monotherapy for bipolar disorder patients with manic or mixed episodes. Journal of Clinical Psychiatry. 2004 Apr;65(4):478-84. PMID: 15119909 Over 50\% dropout rate

911. Weisler RH, Keck PE, Jr., Swann AC, et al. Extended-release carbamazepine capsules as monotherapy for acute mania in bipolar disorder: a multicenter, randomized, double-blind, placebo-controlled trial.[Erratum appears in J Clin Psychiatry. 2005 May;66(5):659]. Journal of Clinical Psychiatry. 2005 Mar;66(3):323-30. PMID: 15766298 Duplicate reference

912. Welge JA, Keck PE, Jr., Meinhold JM. Predictors of response to treatment of acute bipolar manic episodes with divalproex sodium or placebo in 2 randomized, controlled, parallel-group trials. Journal of Clinical Psychopharmacology. 2004 Dec;24(6):607-12. PMID: 15538121 Ineligible study design

913. Welten CC, Koeter MW, Wohlfarth TD, et al. Does Insight Affect the Efficacy of Antipsychotics in Acute Mania?: An Individual Patient Data Regression Meta-Analysis. Journal of Clinical Psychopharmacology. 2016 Feb;36(1):71-6. doi: http://dx.doi.org/10.1097/JCP.0000000000000435. PMID: 26647231 No eligible outcomes reported

914. Welten CCM, Koeter MWJ, Wohlfarth TD, et al. Early nonresponse in the antipsychotic treatment of acute mania: A criterion for reconsidering treatment? Results from an individual patient data Meta-Analysis. Journal of Clinical Psychiatry. 2016 September;77(9):e1117-e23. doi: http://dx.doi.org/10.4088/JCP.15r10051. PMID: 612499219 Ineligible study design

915. Wesseloo R, Liu X, Clark CT, et al. Risk of postpartum episodes in women with bipolar disorder after lamotrigine or lithium use during pregnancy: A population-based cohort study. Journal of Affective Disorders. 201715 Aug;218:394-7. doi: http://dx.doi.org/10.1016/j.jad.2017.04.070. PMID: 616031023 No eligible outcomes reported 
916. Wheeler AL, Wessa M, Szeszko PR, et al. Further neuroimaging evidence for the deficit subtype of schizophrenia: a cortical connectomics analysis. JAMA Psychiatry. 2015 May;72(5):446-55. doi: http://dx.doi.org/10.1001/jamapsychiatry.2014.3020. PMID: 25786193 Not bipolar disorder

917. Whiskey E, Taylor D. Pramipexole in unipolar and bipolar depression. Psychiatric Bulletin. 2004 2004;28(12):438-40. doi: http://dx.doi.org/10.1192/pb.28.12.438. Ineligible study design

918. Wilcox J. Divalproex sodium in the treatment of aggressive behavior. Annals of Clinical Psychiatry. 1994 Mar;6(1):17-20. PMID: 7951640 Bipolar not analyzed separately

919. Williams JM, Alatiq Y, Crane C, et al. Mindfulness-based Cognitive Therapy (MBCT) in bipolar disorder: preliminary evaluation of immediate effects on between-episode functioning. Journal of Affective Disorders. 2008 Apr;107(1-3):275-9. PMID: 17884176 Bipolar not analyzed separately

920. Wilson KC, Scott M, Abou-Saleh M, et al. Long-term effects of cognitive-behavioural therapy and lithium therapy on depression in the elderly. British Journal of Psychiatry. 1995 Nov;167(5):653-8. PMID: 8564323 Not bipolar disorder

921. Wilting I, Heerdink ER, Mersch PP, et al. Association between lithium serum level, mood state, and patient-reported adverse drug reactions during long-term lithium treatment: a naturalistic follow-up study. Bipolar Disorders. 2009 Jun;11(4):434-40. doi: http://dx.doi.org/10.1111/j.1399-5618.2009.00699.x. PMID: 19500096 Bipolar not analyzed separately

922. Winsberg ME, DeGolia SG, Strong CM, et al. Divalproex therapy in medication-naive and moodstabilizer-naive bipolar II depression. Journal of Affective Disorders. 2001 Dec;67(1-3):207-12. PMID: 11869770 Ineligible study design

923. Woo YS, Bahk WM, Jon DI, et al. Rash in adult patients receiving lamotrigine to treat bipolar I disorder in Korea: a multicenter, prospective, naturalistic, open-label trial. Progress in Neuro-Psychopharmacology \& Biological Psychiatry. 2009 Oct 1;33(7):1147-52. doi: http://dx.doi.org/10.1016/j.pnpbp.2009.06.010. PMID: 19540298 Ineligible study design

924. Woo YS, Shim IH, Wang HR, et al. A diagnosis of bipolar spectrum disorder predicts diagnostic conversion from unipolar depression to bipolar disorder: a 5-year retrospective study. Journal of Affective Disorders. 2015 Mar 15;174:83-8. doi: http://dx.doi.org/10.1016/j.jad.2014.11.034. PMID: 25486276

\section{Ineligible study design}

925. Worthington MA, El-Mallakh RS. A naturalistic retrospective review of weight gain in bipolar patients treated with second generation antipsychotics. Journal of Clinical Psychopharmacology. 2015 Apr;35(2):192-3. doi: http://dx.doi.org/10.1097/JCP.0000000000000271. PMID: 2015-13623-015

\section{Ineligible study design}

926. Wu CS, Hsieh MH, Tang $\mathrm{CH}$, et al. Comparative effectiveness of long-acting injectable risperidone vs. long-acting injectable first-generation antipsychotics in bipolar disorder. Journal of Affective Disorders. 2016 Jun;197:189-95. doi: https://dx.doi.org/10.1016/j.jad.2016.03.043. PMID: 26994437 Ineligible study design

927. Wu CS, Wang SC, Yeh IJ, et al. Comparative risk of seizure with use of first- And Second-Generation antipsychotics in patients with Schizophrenia and mood disorders. Journal of Clinical Psychiatry. 2016 May;77(5):e573-e9. doi: http://dx.doi.org/10.4088/JCP.15m09898. PMID: 610733712 Ineligible intervention 
928. Wu F, Laber EB, Lipkovich IA, et al. Who will benefit from antidepressants in the acute treatment of bipolar depression? A reanalysis of the STEP-BD study by Sachs et al. 2007, using Q-learning. International Journal of Bipolar Disorders. 2015;3(1)doi: http://dx.doi.org/10.1186/s40345-014-0018-5. PMID: 2015897901 No eligible outcomes reported

929. Wu JC, Kelsoe JR, Schachat C, et al. Rapid and sustained antidepressant response with sleep deprivation and chronotherapy in bipolar disorder. Biological Psychiatry. 2009 Aug 1;66(3):298-301. doi: http://dx.doi.org/10.1016/j.biopsych.2009.02.018. PMID: 19358978 Ineligible study design

930. Xia G, Gajwani P, Muzina DJ, et al. Treatment-emergent mania in unipolar and bipolar depression: focus on repetitive transcranial magnetic stimulation. International Journal of Neuropsychopharmacology. 2008 Feb;11(1):119-30. PMID: 17335643 Ineligible study design

931. Xu AJ, Niciu MJ, Lundin NB, et al. Lithium and valproate levels do not correlate with ketamine's antidepressant efficacy in treatment-resistant bipolar depression. Neural Plasticity. 2015;2015(858251)doi: http://dx.doi.org/10.1155/2015/858251. PMID: 2015155220 Ineligible study design

932. Yaroslavsky Y, Grisaru N, Chudakov B, et al. Is TMS therapeutic in mania as well as in depression? Electroencephalography \& Clinical Neurophysiology - Supplement. 1999;51:299-303. PMID: 10590963 Not bipolar disorder

933. Yatham LN. Mood stabilization and the role of antipsychotics. International Clinical Psychopharmacology. 2002 Aug;17 Suppl 3:S21-7. PMID: 12570068 Ineligible study design

934. Yatham LN. A clinical review of aripiprazole in bipolar depression and maintenance therapy of bipolar disorder. Journal of Affective Disorders. 2011 Jan;128 Suppl 1:S21-8. doi: http://dx.doi.org/10.1016/S0165-0327(11)70005-2. PMID: 21220077 Ineligible study design

935. Yatham LN, Binder C, Riccardelli R, et al. Risperidone in acute and continuation treatment of mania. Int Clin Psychopharmacol. 2003 Jul;18(4):227-35. doi: 10.1097/01.yic.0000074990.54339.e3. PMID: 12817157 Ineligible study design

936. Yatham LN, Calabrese JR, Kusumakar V. Bipolar depression: criteria for treatment selection, definition of refractoriness, and treatment options. Bipolar Disorders. 2003 Apr;5(2):85-97. PMID: 12680897 Ineligible study design

937. Yatham LN, Fountoulakis KN, Rahman Z, et al. Efficacy of aripiprazole versus placebo as adjuncts to lithium or valproate in relapse prevention of manic or mixed episodes in bipolar I patients stratified by index manic or mixed episode. Journal of Affective Disorders. 2013 May;147(1-3):365-72. doi: http://dx.doi.org/10.1016/j.jad.2012.11.042. PMID: 23290791 Duplicate reference

938. Yatham LN, Kusumakar V, Calabrese JR, et al. Third generation anticonvulsants in bipolar disorder: a review of efficacy and summary of clinical recommendations. Journal of Clinical Psychiatry. 2002 Apr;63(4):275-83. PMID: 12000201 Ineligible study design

939. Yatham LN, Vieta E, Goodwin GM, et al. Agomelatine or placebo as adjunctive therapy to a mood stabiliser in bipolar I depression: randomised double-blind placebo-controlled trial. British Journal of Psychiatry. 2016 Jan;208(1):78-86. doi: http://dx.doi.org/10.1192/bjp.bp.114.147587. PMID: 25999335 Ineligible study design 
940. Ye BY, Jiang ZY, Li X, et al. Effectiveness of cognitive behavioral therapy in treating bipolar disorder: An updated meta-analysis with randomized controlled trials. Psychiatry and Clinical Neurosciences. 201601 Aug;70(8):351-61. doi: http://dx.doi.org/10.1111/pcn.12399. PMID: 611542365 Ineligible study design

941. Yildiz A, Vieta E, Leucht S, et al. Efficacy of antimanic treatments: meta-analysis of randomized, controlled trials. Neuropsychopharmacology. 2011 Jan;36(2):375-89. doi: http://dx.doi.org/10.1038/npp.2010.192. PMID: 20980991 Ineligible study design

942. Yoldi-Negrete M, Flores-Ramos M, Rodriguez-Ramirez AM, et al. The use of quetiapine for comorbid bipolar and obsessive compulsive disorders. Current Psychopharmacology. 201501 Aug;4(2):103-11. PMID: 608569783 Ineligible study design

943. Young AH, Cookson J, Elliott B, et al. Managing the aftermath of mania - Newcastle, 2 September 2005: Consensus Meeting Statement. Journal of Psychopharmacology. 2006 Mar;20(2 Suppl):51-4. PMID: 16551673 Ineligible study design

944. Young AH, Gallagher P, Watson S, et al. Improvements in neurocognitive function and mood following adjunctive treatment with mifepristone (RU-486) in bipolar disorder. Neuropsychopharmacology. 2004 Aug;29(8):1538-45. PMID: 15127079 Ineligible study design

945. Young AH, Geddes JR, Macritchie K, et al. Tiagabine in the maintenance treatment of bipolar disorders. Cochrane Database of Systematic Reviews. 2006(3):CD005173. PMID: 16856081 Ineligible study design

946. Young AH, Geddes JR, Macritchie K, et al. Tiagabine in the treatment of acute affective episodes in bipolar disorder: efficacy and acceptability. Cochrane Database of Systematic Reviews. 2006(3):CD004694. PMID: 16856056 Ineligible study design

947. Young AH, McElroy SL, Olausson B, et al. A randomised, placebo-controlled 52-week trial of continued quetiapine treatment in recently depressed patients with bipolar I and bipolar II disorder. The World Journal of Biological Psychiatry. 2014 2014;15(2):96-112. doi: http://dx.doi.org/10.3109/15622975.2012.665177. PMID: 22404704 Ineligible study design

948. Young LT, Joffe RT, Robb JC, et al. Double-blind comparison of addition of a second mood stabilizer versus an antidepressant to an initial mood stabilizer for treatment of patients with bipolar depression. American Journal of Psychiatry. 2000 Jan;157(1):124-6. PMID: 10618026 No eligible outcomes reported

949. Young RC, Schulberg HC, Gildengers AG, et al. Conceptual and methodological issues in designing a randomized, controlled treatment trial for geriatric bipolar disorder: GERI-BD. Bipolar Disorders. 2010 Feb;12(1):56-67. doi: http://dx.doi.org/10.1111/j.1399-5618.2009.00779.x. PMID: 20148867 Ineligible study design

950. Zarate CA, Jr. Antipsychotic drug side effect issues in bipolar manic patients. Journal of Clinical Psychiatry. 2000;61 Suppl 8:52-61; discussion 2-3. PMID: 10811244 Ineligible study design

951. Zarate CA, Jr., Payne JL, Singh J, et al. Pramipexole for bipolar II depression: a placebo-controlled proof of concept study. Biological Psychiatry. 2004 Jul 1;56(1):54-60. PMID: 15219473 Ineligible study design 
952. Zarate CA, Jr., Singh JB, Carlson PJ, et al. Efficacy of a protein kinase C inhibitor (tamoxifen) in the treatment of acute mania: a pilot study.[Erratum appears in Bipolar Disord. 2007 Dec;9(8):932]. Bipolar Disorders. 2007 Sep;9(6):561-70. PMID: 17845270 Fewer than 11 subjects per arm

953. Zarate CA, Jr., Tohen M, Baldessarini RJ. Clozapine in severe mood disorders. Journal of Clinical Psychiatry. 1995 Sep;56(9):411-7. PMID: 7665540 Ineligible study design

954. Zaretsky AE, Rizvi S, Parikh SV. How well do psychosocial interventions work in bipolar disorder? [References]. The Canadian Journal of Psychiatry / La Revue canadienne de psychiatrie. 2007 2007;52(1):14-21. Ineligible study design

955. Zerjav-Lacombe S, Tabarsi E. Lamotrigine: a review of clinical studies in bipolar disorders. Canadian Journal of Psychiatry - Revue Canadienne de Psychiatrie. 2001 May;46(4):328-33. PMID: 11387788 Ineligible study design

956. Zeschel E, Bingmann T, Bechdolf A, et al. Temperament and prodromal symptoms prior to first manic/hypomanic episodes: results from a pilot study. Journal of Affective Disorders. 2015 Mar 1;173:3944. doi: http://dx.doi.org/10.1016/j.jad.2014.10.031. PMID: 25462394 Ineligible study design

957. Zupancic ML. Role of atypical antipsychotics in rapid cycling bipolar disorder: a review of the literature. Annals of Clinical Psychiatry. 2011 May;23(2):141-9. PMID: 21547275 Ineligible study design 


\begin{tabular}{|c|c|c|c|}
\hline $\begin{array}{l}\text { Author, Year } \\
\text { PMID }\end{array}$ & $\begin{array}{l}\text { Interventionl } \\
\text { Comparison }\end{array}$ & N; BD Type & Study Description \\
\hline $\begin{array}{l}\text { Masand, } 2008^{188} \\
18668014\end{array}$ & $\begin{array}{l}\text { I: Aripiprazole } \\
\text { C: Placebo }\end{array}$ & $\begin{array}{l}\mathrm{n}=161 \\
\mathrm{BD} \text { I }\end{array}$ & $\begin{array}{l}\text { post hoc analysis of Keck, } 2006 \\
\text { (J Clin Psychiatry 2006; } 67: 626- \\
637)^{2} \text { analyzing remission criteria } \\
26 \text { week RCT of responders }\end{array}$ \\
\hline $\begin{array}{l}\text { Suppes, } 2008^{817} \\
17904226\end{array}$ & $\begin{array}{l}\text { I: Aripiprazole } \\
\text { C: Placebo }\end{array}$ & $\begin{array}{l}\mathrm{n}=534 \\
\mathrm{BD} \text { I }\end{array}$ & $\begin{array}{l}\text { post hoc pooled analysis of } \\
\text { Sachs, } 2007 \text { (J. } \\
\text { Psychopharmacol. } \\
20,536-546)^{4} \text { and Keck, } 2003 \\
\text { (Am. J. Psychiatry } \\
\text { 160, 1651-1658) by disease } \\
\text { traits }^{5} 3 \text { week RCT }\end{array}$ \\
\hline $\begin{array}{l}\text { Sachs, } 2007^{733} \\
17915976\end{array}$ & $\begin{array}{l}\text { I: Aripiprazole } \\
\text { C: Placebo }\end{array}$ & $\begin{array}{l}n=513 \\
B D \text { I }\end{array}$ & $\begin{array}{l}\text { post hoc pooled analysis of Keck } \\
\text { 2003 (Am J Psychiatry 2003; } \\
\text { 160: } 1651-1658)^{5} \text { and Sachs, } \\
\text { 2007 (J Psychopharmacol 2006; } \\
\text { 20: } 536-546)^{4} \text { analyzed by } \\
\text { agitation level } 3 \text { week RCT }\end{array}$ \\
\hline $\begin{array}{l}\text { Keck, } 2007^{455} \\
17960961\end{array}$ & $\begin{array}{l}\text { I: Aripiprazole } \\
\text { C: Placebo }\end{array}$ & $\begin{array}{l}\mathrm{n}=66 \\
\mathrm{BD} \text { । }\end{array}$ & $\begin{array}{l}\text { extension of included } 26 \text { week } \\
\text { RCT in Keck, } 2006 \text { (J Clin } \\
\text { Psychiatry 2006; 67:626-637) }\end{array}$ \\
\hline $\begin{array}{l}\text { Keck, } 2003^{457} \\
12944341\end{array}$ & $\begin{array}{l}\text { I: Aripiprazole } \\
\text { C: Placebo }\end{array}$ & $\begin{array}{l}n=262 \\
B D \text { I }\end{array}$ & 3 week RCT \\
\hline $\begin{array}{l}\text { El Mallakh, } 2010^{251} \\
20728318\end{array}$ & $\begin{array}{l}\text { I: Aripiprazole (high } \\
\text { dose) } \\
\text { C1: Aripiprazole (low } \\
\text { dose) } \\
\text { C2: Placebo }\end{array}$ & $\begin{array}{l}\mathrm{n}=401 \\
\mathrm{BD} \text { I }\end{array}$ & 3 week RCT \\
\hline $\begin{array}{l}\text { Keck, } 2009^{466} \\
18835043\end{array}$ & $\begin{array}{l}\text { I: Aripiprazole } \\
\text { C1: Lithium } \\
\text { C2: Placebo }\end{array}$ & $\begin{array}{l}\mathrm{n}=181 \\
\mathrm{BD} \text { I }\end{array}$ & $\begin{array}{l}3 \text { week RCT } \\
+9 \text { week extension of } \\
\text { responders }\end{array}$ \\
\hline $\begin{array}{l}\text { El-Mallakh, } 2012^{257} \\
22209190\end{array}$ & $\begin{array}{l}\text { I: Aripiprazole } \\
\text { C1: Lithium } \\
\text { C2: Placebo }\end{array}$ & $\begin{array}{l}\mathrm{n}=99 \\
\mathrm{BD} \text { । }\end{array}$ & $\begin{array}{l}40 \text { week extension of excluded } \\
12 \text { week study in Keck, } 2009 \text { (J } \\
\text { Aff Disorders } 112(1-3), 36-49)\end{array}$ \\
\hline $\begin{array}{l}\text { Moreno, } 2007^{610} \\
17334533\end{array}$ & $\begin{array}{l}\text { I: Haloperidol } \\
\text { C: Olanzapine }\end{array}$ & $\begin{array}{l}\mathrm{n}=12 \\
\mathrm{BD} \text { । }\end{array}$ & $\begin{array}{l}6 \text { week RCT; } \\
\text { sleep study }\end{array}$ \\
\hline $\begin{array}{l}\text { Tohen, } 2009^{855} \\
19054570\end{array}$ & $\begin{array}{l}\text { I: Olanzapine } \\
\text { C: Placebo }\end{array}$ & $\begin{array}{l}\mathrm{n}=121 \\
\mathrm{BD} \text { I }\end{array}$ & $\begin{array}{l}\text { Subgroup analysis of Tohen } 2006 \\
12\end{array}$ \\
\hline $\begin{array}{l}\text { Vieta, } 2004^{866} \\
15491248 \\
15641874^{397}\end{array}$ & $\begin{array}{l}\text { I: Olanzapine } \\
\text { C: Placebo }\end{array}$ & $\begin{array}{l}\mathrm{n}=254 \text { RCT; } \\
\mathrm{n}=113 \text { extension } \\
; \\
\mathrm{BD} \text { I }\end{array}$ & $\begin{array}{l}\text { post hoc pooled analysis of } \\
\text { Tohen, 1999 (Am J Psychiatry } \\
\text { 156:702-709) }{ }^{14} \text { and Tohen, 2000 } \\
\text { (Arch gen Psychiatry 57:841-849) } \\
\text { comparing rapid and non-rapid } \\
\text { cyclers. Year-long extension not } \\
\text { cited and could not be located. } \\
\text { 3-4 week RCT + } 1 \text { year extension } \\
\text { (maintenance) }\end{array}$ \\
\hline $\begin{array}{l}\text { Baker, } 2003^{38} \\
12640214\end{array}$ & $\begin{array}{l}\text { I: Olanzapine } \\
\text { C: Placebo }\end{array}$ & $\begin{array}{l}\mathrm{n}=68 ; \\
\text { BD I with severe } \\
\text { dysphoric mania }\end{array}$ & $\begin{array}{l}3 \text { week RCT } \\
\text { pooling of Tohen, } 1999\left(\mathrm{Am} . \mathrm{J}^{14}\right. \\
\text { Psychiatry } 156(5), 702-709)^{14} \\
\text { and Tohen, } 2000 \text { (Arch. General } \\
\text { Psychiatry } 57 \text { (9), 841-849) }\end{array}$ \\
\hline
\end{tabular}




\begin{tabular}{|c|c|c|c|}
\hline $\begin{array}{l}\text { Author, Year } \\
\text { PMID }\end{array}$ & $\begin{array}{c}\text { Intervention/ } \\
\text { Comparison }\end{array}$ & N; BD Type & Study Description \\
\hline $\begin{array}{l}\text { Baker, } 2003^{37} \\
12507747\end{array}$ & $\begin{array}{l}\text { I: Olanzapine } \\
\text { C: Placebo }\end{array}$ & $\begin{array}{l}n=254 \\
B D \text { I }\end{array}$ & $\begin{array}{l}3 \text { week RCT } \\
\text { pooling of Tohen, } 1999(\mathrm{Am} . \mathrm{J} \\
\text { Psychiatry } 156(5), 702-709)^{14} \\
\text { and Tohen, } 2000 \text { (Arch. General } \\
\text { Psychiatry } 57 \text { (9), 841-849) }\end{array}$ \\
\hline $\begin{array}{l}\text { Baldessarini, } 2003^{42} \\
12920413\end{array}$ & $\begin{array}{l}\text { I: Olanzapine } \\
\text { C: Placebo }\end{array}$ & $\begin{array}{l}\mathrm{n}=254 \\
\mathrm{BD} \text { । }\end{array}$ & $\begin{array}{l}\text { 3-4 weeks RCT } \\
\text { pooling of Tohen, } 1999(\mathrm{Am} . \mathrm{J} \\
\text { Psychiatry } 156(5), 702-709)^{14} \\
\text { and Tohen, } 2000 \text { (Arch. General } \\
\text { Psychiatry } 57 \text { (9), 841-849) }\end{array}$ \\
\hline $\begin{array}{l}\text { Chengappa, } 2003^{161} \\
12656931\end{array}$ & $\begin{array}{l}\text { I: Olanzapine } \\
\text { C: Placebo }\end{array}$ & $\begin{array}{l}n=246 ; \\
B D \text { । }\end{array}$ & $\begin{array}{l}3 \text { week RCT } \\
\text { pooling of Tohen, } 1999 \text { (Am. J } \\
\text { Psychiatry } 156 \text { (5), 702-709) } \\
\text { and Tohen, } 2000 \text { (Arch. General } \\
\text { Psychiatry } 57 \text { (9), 841-849) with } \\
\text { revised definitions for response } \\
\text { and remission) }\end{array}$ \\
\hline $\begin{array}{l}\text { Tohen, } 1999^{853} \\
10327902\end{array}$ & $\begin{array}{l}\text { I: Olanzapine } \\
\text { C: Placebo }\end{array}$ & $\begin{array}{l}\mathrm{n}=139 \\
\mathrm{BD} \text { । }\end{array}$ & 3 week RCT \\
\hline $\begin{array}{l}\text { Ketter, } 2006^{480} \\
16426094\end{array}$ & $\begin{array}{l}\text { I: Olanzapine } \\
\text { C: Lithium }\end{array}$ & $\begin{array}{l}\mathrm{n}=431 \\
\mathrm{BD} \text { I }\end{array}$ & $\begin{array}{l}\text { post hoc analysis of Tohen, } 2005 \\
\text { (Am J Psychiatry 2005; } 162 \text { : } \\
\text { 1281-1290) by number of } \\
\text { previous mood episodes } 12 \\
\text { months RCT }\end{array}$ \\
\hline $\begin{array}{l}\text { Suppes, } 2005^{816} \\
16253344\end{array}$ & $\begin{array}{l}\text { I: Olanzapine } \\
\text { C: Valproate }\end{array}$ & $\begin{array}{l}n=251 \\
B D \text { I }\end{array}$ & $\begin{array}{l}\text { Post hoc analysis of Tohen, } 2003 \\
\text { (Am. J. Psychiatry } 160,1272- \\
1276)^{21} \text { concerning rapid cycling } \\
47 \text { week RCT (maintenance) }\end{array}$ \\
\hline $\begin{array}{l}\text { Novick, } 2010^{645} \\
20531011 \\
\text { modelled after Perlis, } \\
2006 \text { (J Clin } \\
\text { Psychiatry 67: 1747- } \\
\text { 1753) }\end{array}$ & $\begin{array}{l}\text { I: Olanzapine } \\
\text { C: Risperidone }\end{array}$ & $\begin{array}{l}n=245 \\
B D \text { I }\end{array}$ & $\begin{array}{l}12 \text { week acute observational } \\
+2 \text { year observational }\end{array}$ \\
\hline $\begin{array}{l}\text { Suppes, } 2013^{819} \\
23521871\end{array}$ & $\begin{array}{l}\text { I: Quetiapine } \\
\text { C: Placebo }\end{array}$ & $\begin{array}{l}\mathrm{n}=81 ; \\
\mathrm{BD} \text { II }\end{array}$ & 8 week RCT \\
\hline $\begin{array}{l}\text { Nejtek, } 2008^{627} \\
18681757\end{array}$ & $\begin{array}{l}\text { I: Quetiapine } \\
\text { C: Risperidone }\end{array}$ & $\begin{array}{l}\mathrm{n}=96 \\
\mathrm{BD} \text { I or II }\end{array}$ & 20 week RCT; stimulant users \\
\hline $\begin{array}{l}\text { Hirschfeld, } 2004^{405} \\
15169694\end{array}$ & $\begin{array}{l}\text { I: Risperidone } \\
\text { C: Placebo }\end{array}$ & $\begin{array}{l}n=262 \\
B D \text { I }\end{array}$ & 3 week RCT \\
\hline $\begin{array}{l}\text { Sanger, } 2003^{753} \\
12507748\end{array}$ & $\begin{array}{l}\text { I: Olanzapine } \\
\text { C: Placebo }\end{array}$ & $\begin{array}{l}\mathrm{N}=45 \\
\mathrm{BD} \mathrm{I} ; \text { rapid } \\
\text { cycling }\end{array}$ & 3 week RCT \\
\hline $\begin{array}{l}\text { Stahl, } 2010^{809} \\
19616304\end{array}$ & $\begin{array}{l}\text { I: Ziprasidone } \\
\text { C: Placebo }\end{array}$ & $\begin{array}{l}\mathrm{n}=181 \\
\mathrm{BD} \text { I }\end{array}$ & $\begin{array}{l}\text { post hoc pooled analysis of Keck, } \\
2003 \text { (Am. J. Psychiatry } 160, \\
741-748 \text { ) and Potkin, } 2005 \text { (J. } \\
\text { Clin. Psychopharmacol. } 25,301- \\
\text { 310) in dysphoric mania } 3 \text { week } \\
\text { RCT }\end{array}$ \\
\hline $\begin{array}{l}\text { Vieta, } 2010^{893} \\
19074536\end{array}$ & $\begin{array}{l}\text { I: Ziprasidone } \\
\text { C1: Haloperidol } \\
\text { C2: Placebo }\end{array}$ & $\begin{array}{l}\mathrm{n}=438 \\
\mathrm{BD} \text { । }\end{array}$ & $\begin{array}{l}3 \text { week } 3 \text { arm RCT } \\
+9 \text { week } 2 \text { arm extension of } \\
\text { responders }\end{array}$ \\
\hline
\end{tabular}


Abbreviations: $\mathrm{BP}=$ bipolar disorder; $\mathrm{C}=$ comparison; $\mathrm{I}=$ intervention; NS=not significant; $\mathrm{PMID}=\mathrm{PubMed}$ Identification Number; RCT=randomized controlled trial

\begin{tabular}{|c|c|c|c|}
\hline $\begin{array}{c}\text { Author, Year } \\
\text { PMID }\end{array}$ & $\begin{array}{l}\text { Interventionl } \\
\text { Comparison }\end{array}$ & N; BD Type & Study Description \\
\hline $\begin{array}{l}\text { Chou, 1999165 } \\
10587284\end{array}$ & $\begin{array}{l}\text { I: Haloperidol (high or low } \\
\text { dose) + Lithium } \\
\text { C: Haloperidol (high or low } \\
\text { dose) + Lorazepam }\end{array}$ & $\begin{array}{l}\mathrm{n}=63 \\
\mathrm{BD} \text { । }\end{array}$ & 3 week RCT \\
\hline $\begin{array}{l}\text { Katagiri, } 2012^{448} \\
22356118 \\
\\
\text { extension of } 6 \text { week } \\
\text { RCT (not cited, unable } \\
\text { to locate) }\end{array}$ & $\begin{array}{l}\text { I: Olanzapine + Lithium } \\
\text { C1: Olanzapine + Valproate } \\
\text { C2: Olanzapine }\end{array}$ & $\begin{array}{l}\mathrm{n}=139 ; \\
\mathrm{BD} \text { । }\end{array}$ & $\begin{array}{l}18 \text { week observational } \\
\text { (maintenance) }\end{array}$ \\
\hline $\begin{array}{l}\text { Brown, } 2009^{119} \\
19079815 \\
\text { Continuation of Brown, } \\
2006 \text { (J Clin Psychiatry } \\
60,79-88 \text { ) }\end{array}$ & $\begin{array}{l}\text { I: Olanzapine + Fluoxetine } \\
\text { (antidepressant) } \\
\text { C: Lamotrigine }\end{array}$ & $\begin{array}{l}n=410 ; \\
B D \text { I }\end{array}$ & $\begin{array}{l}25 \text { week RCT depression } \\
\text { (not proper time-to-event } \\
\text { and post-hoc) }\end{array}$ \\
\hline $\begin{array}{l}\text { Vieta, } 2012^{894} \\
23062763\end{array}$ & $\begin{array}{l}\text { I: Quetiapine + Lithium OR } \\
\text { Valproate } \\
\text { C: Placebo + Lithium OR } \\
\text { Valproate }\end{array}$ & $\begin{array}{l}\mathrm{n}=445 \\
\mathrm{BD} \text { । }\end{array}$ & $\begin{array}{l}\text { post hoc pooled analysis of } \\
\text { Suppes, } 2009 \text { (Am J } \\
\text { Psychiatry 166, 476-488) } \\
\text { and Vieta, 2008 (J Aff } \\
\text { Disorders 109, 251-263) of } \\
\text { those with mixed episodes } \\
104 \text { week RCT } \\
\text { (maintenance) }\end{array}$ \\
\hline
\end{tabular}

Abbreviations: $\mathrm{BP}=$ bipolar disorder; $\mathrm{C}=$ comparison; I=intervention; NS=not significant; $\mathrm{PMID=PubMed}$ Identification Number; RCT=randomized controlled trial

\begin{tabular}{|c|c|c|c|}
\hline $\begin{array}{l}\text { Author, Year } \\
\text { PMID }\end{array}$ & $\begin{array}{l}\text { Intervention/ } \\
\text { Comparison }\end{array}$ & N; BD Type & Study Description \\
\hline $\begin{array}{l}\text { Weisler, } 2008^{909} \\
22778707\end{array}$ & $\begin{array}{l}\text { I: Carbamazepine ER (once- } \\
\text { daily) } \\
\text { C: Carbamazepine ER (twice- } \\
\text { daily) }\end{array}$ & $\begin{array}{l}\mathrm{n}=111 ; \\
\mathrm{BD} \text { I }\end{array}$ & 12 week RCT \\
\hline $\begin{array}{l}\text { Weisler, } 2004^{910} \\
15119909\end{array}$ & $\begin{array}{l}\text { I: Carbamazepine ER } \\
\text { C: Placebo }\end{array}$ & $\begin{array}{l}n=204 ; \\
B D I\end{array}$ & 3 week RCT \\
\hline $\begin{array}{l}\text { El-Mallakh, } 2009^{259} \\
19367153\end{array}$ & $\begin{array}{l}\text { I: Carbamazepine ER } \\
\text { C: Carbamazepine }\end{array}$ & $\begin{array}{l}\mathrm{n}=41 ; \\
\mathrm{BD} \text { I or II }\end{array}$ & 3 month RCT \\
\hline $\begin{array}{l}\text { Licht, } 2010^{527} \\
20712749\end{array}$ & $\begin{array}{l}\text { I: Lamotrigine } \\
\text { C: Lithium }\end{array}$ & $\begin{array}{l}n=155 \\
B D \text { I }\end{array}$ & 6 month RCT \\
\hline $\begin{array}{l}\text { Suppes, } 2008^{820} \\
18358540\end{array}$ & $\begin{array}{l}\text { I: Lamotrigine } \\
\text { C: Lithium }\end{array}$ & $\begin{array}{l}\mathrm{n}=102 ; \\
\mathrm{BD} \text { II }\end{array}$ & 16 week RCT \\
\hline $\begin{array}{l}\text { Bowden, } 2006^{108} \\
16816224\end{array}$ & $\begin{array}{l}\text { I: Lamotrigine } \\
\text { C1: Lithium }\end{array}$ & $\begin{array}{l}\mathrm{n}=254 ; \\
\text { BD I, obese } \\
\text { vs. non- } \\
\text { obese }\end{array}$ & $\begin{array}{l}\text { pools two studies from } \\
\text { Goodwin, 2004 (J Clin } \\
\text { Psychiatry (65) 432- } \\
\text { 441)18 month RCT } \\
\text { (maintenance) }\end{array}$ \\
\hline
\end{tabular}




\begin{tabular}{|c|c|c|c|}
\hline $\begin{array}{l}\text { Author, Year } \\
\text { PMID }\end{array}$ & $\begin{array}{l}\text { Intervention/ } \\
\text { Comparison }\end{array}$ & N; BD Type & Study Description \\
\hline $\begin{array}{l}\text { Wang, } 2010^{904} \\
21240149\end{array}$ & $\begin{array}{l}\text { I: Lamotrigine + Lithium AND } \\
\text { Valproate } \\
\text { C: Placebo + Lithium AND } \\
\text { Valproate }\end{array}$ & $\begin{array}{l}\mathrm{n}=36 \\
\mathrm{BD} \text { I or II }\end{array}$ & $\begin{array}{l}12 \text { week RCT; } \\
\text { substance users with } \\
\text { recent depression and } \\
\text { rapid cycling }\end{array}$ \\
\hline $\begin{array}{l}\text { Bowden, } 2012^{114} \\
22708645\end{array}$ & $\begin{array}{l}\text { I: Lamotrigine + Valproate } \\
\text { C: Lamotrigine }\end{array}$ & $\begin{array}{l}\mathrm{n}=164 \\
\text { (treatment); } \\
\mathrm{n}=86 \\
\text { (randomized); } \\
\text { BD I or II }\end{array}$ & $\begin{array}{l}8 \text { week treatment } \\
+8 \text { month RCT of } \\
\text { responders } \\
\text { (maintenance) }\end{array}$ \\
\hline $\begin{array}{l}\text { Goldberg, 2009354 } \\
19689918 \\
\text { pooled post hoc } \\
\text { analysis of Bowden, } \\
2003 \text { (Arch Gen Psych } \\
\text { 2003; 60: 392-400) and } \\
\text { Calabrese, 2003 (J Clin } \\
\text { Psych 2003; 64: 1013- } \\
\text { 1024) }\end{array}$ & $\begin{array}{l}\text { I: Lamotrigine } \\
\text { C1: Lithium } \\
\text { C2: Placebo }\end{array}$ & $\begin{array}{l}\mathrm{n}=966 \\
\text { observational; } \\
\mathrm{n}=463 \mathrm{RCT} ; \\
\text { BD I }\end{array}$ & $\begin{array}{l}\text { 8-16 week observational } \\
\text { treatment phase with } \\
\text { Lamotrigine }+ \\
18 \text { month RCT } \\
\text { (maintenance) }\end{array}$ \\
\hline $\begin{array}{l}\text { Solomon, } 1996^{803} \\
8831438\end{array}$ & $\begin{array}{l}\text { I: Lithium, High Dose } \\
\text { C: Lithium, Low Dose }\end{array}$ & $\begin{array}{l}\mathrm{n}=94 ; \\
\mathrm{BD} \text { । }\end{array}$ & $\begin{array}{l}\geq 2 \text { year RCT } \\
\text { (maintenance) }\end{array}$ \\
\hline $\begin{array}{l}\text { Small, } 1995^{794} \\
7491378\end{array}$ & $\begin{array}{l}\text { I: Lithium + Carbamazepine } \\
\text { C: Lithium + Haloperidol }\end{array}$ & $\begin{array}{l}\mathrm{n}=33 \\
\mathrm{BD} \text { । }\end{array}$ & 8 week RCT \\
\hline $\begin{array}{l}\text { Kakkar, } 2009^{444} \\
19324530\end{array}$ & $\begin{array}{l}\text { I: Oxcarbazepine } \\
\text { C: Valproate }\end{array}$ & $\begin{array}{l}n=60 ; \\
B D ~ I\end{array}$ & 12 week RCT \\
\hline $\begin{array}{l}\text { Bowden, } 1994^{107} \\
8120960\end{array}$ & $\begin{array}{l}\text { I: Valproate } \\
\text { C1: Lithium } \\
\text { C2: Placebo }\end{array}$ & $\begin{array}{l}\mathrm{n}=179 \\
\mathrm{BD} \text { । }\end{array}$ & 3 week RCT \\
\hline $\begin{array}{l}\text { Hirschfeld, } 2010^{403} \\
20361904\end{array}$ & $\begin{array}{l}\text { I: Divalproex } \\
\text { C: Placebo }\end{array}$ & $\begin{array}{l}n=225 \\
B D \text { I }\end{array}$ & 3 week RCT \\
\hline $\begin{array}{l}\text { McElroy, } 2010^{570} \\
20361901\end{array}$ & $\begin{array}{l}\text { I: Divalproex } \\
\text { C: Placebo }\end{array}$ & $\begin{array}{l}\mathrm{n}=62 ; \\
\text { BD I, II or } \\
\text { NOS }\end{array}$ & 8 week RCT \\
\hline $\begin{array}{l}\text { Oquendo, } 2011^{654} \\
21768611\end{array}$ & $\begin{array}{l}\text { I: Valproate + Various Adjuncts } \\
\text { C: Lithium + Various Adjunct }\end{array}$ & $\begin{array}{l}\text { n=98; } \\
\text { BD I, II, or } \\
\text { NOS }\end{array}$ & $\begin{array}{l}2.5 \text { year RCT; } \\
\text { study of suicide }\end{array}$ \\
\hline $\begin{array}{l}\text { Salloum, } 2005^{746} \\
15630071\end{array}$ & $\begin{array}{l}\text { I: Valproate + Lithium + Dual } \\
\text { diagnosis recovery counseling } \\
\text { C1: Lithium + Dual diagnosis } \\
\text { recovery counseling }\end{array}$ & $\begin{array}{l}\mathrm{n}=59 \\
\mathrm{BD} \text { । }\end{array}$ & $\begin{array}{l}24 \text { week RCT; } \\
\text { alcoholism } \\
\text { (maintenance) }\end{array}$ \\
\hline
\end{tabular}

Abbreviations: $\mathrm{BP}=$ bipolar disorder; $\mathrm{C}=$ comparison; I=intervention; NS=not significant; $\mathrm{PMID}=\mathrm{PubMed}$ Identification Number; RCT=randomized controlled trial 


\begin{tabular}{|c|c|c|c|}
\hline $\begin{array}{c}\text { Author, Year } \\
\text { PMID }\end{array}$ & $\begin{array}{l}\text { Intervention/ } \\
\text { Comparison }\end{array}$ & N; BD Type & Study Description \\
\hline $\begin{array}{l}\text { Ghaemi, } 2010^{342} \\
20409444 \\
2015371706^{900}\end{array}$ & $\begin{array}{l}\text { I: Antidepressant + Mood } \\
\text { Stabilizer } \\
\text { C: Mood Stabilizer }\end{array}$ & $\begin{array}{l}\mathrm{n}=70 ; \\
\text { BD NR }\end{array}$ & $\begin{array}{l}3 \text { year RCT; } \\
\text { antidepressant } \\
\text { discontinuation study }\end{array}$ \\
\hline $\begin{array}{l}\text { Geddes, } 2016^{331} \\
26687300\end{array}$ & $\begin{array}{l}\text { I: Lamotrigine + Quetiapine } \\
\text { C: Placebo + Quetiapine }\end{array}$ & $\begin{array}{l}\mathrm{n}=202 ; \\
\mathrm{BD} \text { I or } \\
\text { II }\end{array}$ & 12 week RCT \\
\hline $\begin{array}{l}\text { Gonzalez Arnold, } \\
2015^{359} \\
25827507\end{array}$ & $\begin{array}{l}\text { I: Optimized Personal } \\
\text { Treatment (OPT) + Lithium } \\
\text { C1: OPT }\end{array}$ & $\begin{array}{l}\mathrm{n}=283 \\
\text { BD I or II }\end{array}$ & $\begin{array}{l}6 \text { month RCT; } \\
\text { racial disparity study }\end{array}$ \\
\hline $\begin{array}{l}\text { Vik, } 2013^{895} \\
23551803\end{array}$ & $\begin{array}{l}\text { I: Calcitonin + Mood } \\
\text { Stabilizer And/Or } \\
\text { Antipsychotic } \\
\text { C: Placebo + Mood Stabilizer } \\
\text { And/Or Antipsychotic }\end{array}$ & $\begin{array}{l}\mathrm{n}=46 \\
\mathrm{BD} \text { I }\end{array}$ & 3 week RCT \\
\hline $\begin{array}{l}\text { Mishory, } 2003^{600} \\
14636372\end{array}$ & $\begin{array}{l}\text { I: Phenytoin } \\
\text { C: Placebo }\end{array}$ & $\begin{array}{l}\mathrm{n}=23 \\
\mathrm{BD} \text { I }\end{array}$ & $\begin{array}{l}6 \text { month observational } \\
\text { crossover }\end{array}$ \\
\hline $\begin{array}{l}\text { Grunze, } 2015^{382} \\
25484179\end{array}$ & $\begin{array}{l}\text { I: Eslicarbazepine acetate } \\
\text { (various dose arms) } \\
\text { C: Placebo }\end{array}$ & $\begin{array}{l}\mathrm{n}=200 \text { placebo } \\
\text { controlled } \mathrm{RCT} \text {; } \\
\mathrm{n}=87 \text { dose } \mathrm{RCT} \text {; } \\
\mathrm{BD} \text { I }\end{array}$ & $\begin{array}{l}3 \text { week placebo } \\
\text { controlled RCT } \\
+6 \text { month RCT of } \\
\text { responders without } \\
\text { placebo control for dose } \\
\text { effects }\end{array}$ \\
\hline $\begin{array}{l}\text { Amsterdam, } 1998^{16} \\
9864074\end{array}$ & $\begin{array}{l}\text { I: Fluoxetine } \\
\text { C: Placebo }\end{array}$ & $\begin{array}{l}\text { n=89, } 12 \text { week } \\
n=28,50 \text { week } \\
\text { extension; } \\
\text { BDII vs. unipolar; }\end{array}$ & $\begin{array}{l}12 \text { week observational } \\
\text { treatment phase } \\
+50 \text { week RCT of } \\
\text { remitters }\end{array}$ \\
\hline $\begin{array}{l}\text { Amsterdam, } 2013^{18} \\
23099447\end{array}$ & $\begin{array}{l}\text { I: Fluoxetine } \\
\text { C1: Lithium } \\
\text { C2: Placebo }\end{array}$ & $\begin{array}{l}\mathrm{n}=81 \\
\mathrm{BD} \text { II }\end{array}$ & $\begin{array}{l}\text { post hoc analysis of } \\
\text { Amsterdam, 2010 (Am J } \\
\text { Psychiatry 2010; 167: } \\
\text { 792-800) comparing } \\
\text { rapid and non-rapid } \\
\text { cycling patients } \\
\end{array}$ \\
\hline
\end{tabular}

Abbreviations: $\mathrm{BP}=$ bipolar disorder; $\mathrm{C}=$ comparison; $\mathrm{I}=$ =intervention; $\mathrm{NS}=$ not significant; $\mathrm{PMID}=\mathrm{PubMed}$ Identification Number; RCT=randomized controlled trial

\section{Appendix Table D5. Studies of psychosocial therapy excluded for withdrawal rates $>50 \%$}

\begin{tabular}{|c|c|c|c|}
\hline $\begin{array}{c}\text { Author, Year } \\
\text { PMID }\end{array}$ & $\begin{array}{l}\text { Intervention/ } \\
\text { Comparison }\end{array}$ & N; BD Type & Study Description \\
\hline $\begin{array}{l}\text { Cardoso Tde, } 2010^{142,} \\
213,214 \\
26348588 \\
25300245 \\
2015431675\end{array}$ & $\begin{array}{l}\text { I: Psychoeducationon biological } \\
\text { rhythm } \\
\text { C: Treatment as usual }\end{array}$ & $\begin{array}{l}\mathrm{n}=61 ; \\
\mathrm{BD}\end{array}$ & $\begin{array}{l}6 \text { month RCT in } \\
\text { young adults 18-29 }\end{array}$ \\
\hline $\begin{array}{l}\text { Crowe, } 2012^{204} \\
22070452\end{array}$ & $\begin{array}{l}\text { I: Nurse-led supportive care } \\
\text { C: Usual care }\end{array}$ & $\begin{array}{l}\mathrm{n}=36 \\
\mathrm{BD}\end{array}$ & $\begin{array}{l}9 \text { month RCT } \\
\text { adults } 18+\end{array}$ \\
\hline $\begin{array}{l}\text { Lauder, } 2015^{511} \\
25282145\end{array}$ & $\begin{array}{l}\text { I: Website-based interactive } \\
\text { program MoodSwings-Plus } \\
\text { C: Website-based program } \\
\text { MoodSwings }\end{array}$ & $\begin{array}{l}\mathrm{n}=156 ; \\
\mathrm{BD} \text { I or II }\end{array}$ & 12 month RCT \\
\hline
\end{tabular}


Abbreviations: $\mathrm{BP}=$ bipolar disorder; $\mathrm{C}=$ comparison; $\mathrm{I}=$ =intervention; NS=not significant; $\mathrm{PMID}=\mathrm{PubMed}$ Identification Number; RCT=randomized controlled trial 


\section{Appendix E. Antipsychotics for Mania}

\section{Section 1. Aripiprazole for Acute Mania}

Appendix Table E1. Characteristics of eligible studies: aripiprazole for acute mania

\begin{tabular}{|c|c|c|c|c|c|c|}
\hline $\begin{array}{l}\text { Study, Year } \\
\text { Design } \\
\text { Location } \\
\text { Funder } \\
\text { Risk of Bias } \\
\text { PMID }\end{array}$ & $\begin{array}{c}\text { \# Randomized } \\
\text { Age (mean) } \\
\text { Sex (\% Female) } \\
\text { Race (\% White) } \\
\text { Diagnosis } \\
\text { (\% BP-I, II, NOS) } \\
\text { Setting }\end{array}$ & $\begin{array}{c}\text { Inclusions } \\
\text { Key Exclusions }\end{array}$ & $\begin{array}{c}\text { Intervention } \\
\text { Dosage }\end{array}$ & $\begin{array}{c}\text { Comparison } \\
\text { Dosage }\end{array}$ & Follow-up Duration & $\begin{array}{c}\text { Outcomes } \\
\text { Reported } \\
\text { Withdrawal (\%) at } \\
\text { endpoint }\end{array}$ \\
\hline $\begin{array}{l}\text { Kanba, } 20141 \\
\text { RCT } \\
\text { Multisite } \\
\text { Asia } \\
\text { Industry } \\
\text { RoB High } \\
22540407\end{array}$ & $\begin{array}{l}\mathrm{N}=258 \\
\text { Mean Age 38 } \\
\text { Female 59\% } \\
\text { Japanese 32\% } \\
\text { Korean/Chinese 43\% } \\
\text { Other 25\% } \\
\text { BP-I 100\% } \\
\text { Inpatient }\end{array}$ & $\begin{array}{l}\text { Manic/Mixed episode; } \\
\text { YMRS } \geq 20 ; \\
\text { Current episode <4 } \\
\text { weeks } \\
\text { First Manic Episode } \\
\text { Schizoaffective } \\
\text { Neurological } \\
\text { Disorders Other } \\
\text { Mental Health } \\
\text { Substance Abuse } \\
\text { Pregnant/Nursing }\end{array}$ & \begin{tabular}{|l} 
Aripiprazole \\
$24 \mathrm{mg} / \mathrm{day}$ \\
$(22.9 \mathrm{mg} / \mathrm{day})$
\end{tabular} & Placebo & 3 weeks & $\begin{array}{l}\text { YMRS } \\
\text { CGI-BP-S } \\
\text { Response } \\
\text { Adverse Events } \\
\text { Withdrawal 47\% }\end{array}$ \\
\hline
\end{tabular}




\begin{tabular}{|c|c|c|c|c|c|c|}
\hline $\begin{array}{l}\text { Study, Year } \\
\text { Design } \\
\text { Location } \\
\text { Funder } \\
\text { Risk of Bias } \\
\text { PMID }\end{array}$ & $\begin{array}{c}\text { \# Randomized } \\
\text { Age (mean) } \\
\text { Sex (\% Female) } \\
\text { Race (\% White) } \\
\text { Diagnosis } \\
\text { (\% BP-I, II, NOS) } \\
\text { Setting }\end{array}$ & $\begin{array}{l}\text { Inclusions } \\
\text { Key Exclusions }\end{array}$ & $\begin{array}{l}\text { Intervention } \\
\text { Dosage }\end{array}$ & $\begin{array}{c}\text { Comparison } \\
\text { Dosage }\end{array}$ & Follow-up Duration & $\begin{array}{c}\text { Outcomes } \\
\text { Reported } \\
\text { Withdrawal (\%) at } \\
\text { endpoint }\end{array}$ \\
\hline $\begin{array}{l}\text { Young, } 20092 \\
\text { RCT } \\
\text { Multisite } \\
\text { All Continents } \\
\text { Industry } \\
\text { RoB Moderate } \\
19118324\end{array}$ & $\begin{array}{l}\mathrm{N}=332 \\
\text { Mean Age } 41 \\
\text { Female } 57 \% \\
\text { White } 78 \% \\
\text { BP-I 100\% } \\
\text { Inpatient (weeks 1-2) } \\
\text { Outpatient (weeks 3- } \\
\text { 12) }\end{array}$ & $\begin{array}{l}\text { Manic/Mixed in acute } \\
\text { relapse; } \\
\text { YMRS } \geq 20 \text { and } \\
\text { MADRS } \leq 17 \text { at } \\
\text { baseline, }<25 \% \\
\text { decrease in YMRS } \\
\text { score and } \leq 4 \text { point } \\
\text { MADRS score } \\
\text { between screening } \\
\text { and baseline visits; } \\
\text { Current episode < } 3 \\
\text { weeks } \\
\\
\text { First Manic Episode } \\
\text { Schizoaffective } \\
\text { Neurological } \\
\text { Disorders Other } \\
\text { Mental Health } \\
\text { Taking Other Meds } \\
\text { Substance Abuse }\end{array}$ & $\begin{array}{l}\text { Aripiprazole } \\
15-30 \mathrm{mg} / \text { day } \\
(22.0 \mathrm{mg} / \text { day })\end{array}$ & $\begin{array}{l}\text { C1: Placebo } \\
\text { C2: Haloperidol } \\
\text { 5-15 mg/day } \\
\text { (7.4 mg/day) }\end{array}$ & $\begin{array}{l}12 \text { weeks for } \\
\text { aripiprazole and } \\
\text { haloperidol; } 3 \text { weeks } \\
\text { for placebo }\end{array}$ & $\begin{array}{l}\text { YMRS } \\
\text { CGI-BP-S } \\
\text { Response } \\
\text { Adverse Events } \\
\text { EPS } \\
\text { Withdrawal } 27 \% \text { at } 3 \\
\text { weeks }\end{array}$ \\
\hline
\end{tabular}




\begin{tabular}{|c|c|c|c|c|c|c|}
\hline $\begin{array}{l}\text { Study, Year } \\
\text { Design } \\
\text { Location } \\
\text { Funder } \\
\text { Risk of Bias } \\
\text { PMID }\end{array}$ & $\begin{array}{c}\text { \# Randomized } \\
\text { Age (mean) } \\
\text { Sex (\% Female) } \\
\text { Race (\% White) } \\
\text { Diagnosis } \\
\text { (\% BP-I, II, NOS) } \\
\text { Setting }\end{array}$ & $\begin{array}{c}\text { Inclusions } \\
\text { Key Exclusions }\end{array}$ & $\begin{array}{l}\text { Intervention } \\
\text { Dosage }\end{array}$ & $\begin{array}{c}\text { Comparison } \\
\text { Dosage }\end{array}$ & Follow-up Duration & $\begin{array}{c}\text { Outcomes } \\
\text { Reported } \\
\text { Withdrawal (\%) at } \\
\text { endpoint }\end{array}$ \\
\hline $\begin{array}{l}\text { Sachs, } 20063 \\
\text { RCT } \\
\text { Multisite } \\
\text { North America } \\
\text { Industry } \\
\text { RoB High } \\
16401666\end{array}$ & $\begin{array}{l}\mathrm{N}=272 \\
\text { Mean Age 39 } \\
\text { Female 51\% } \\
\text { White } 72 \% \\
\text { BP-I 100\% } \\
\text { Inpatient (weeks 1-2) } \\
\text { Outpatient (week 3) }\end{array}$ & \begin{tabular}{|l|} 
Manic/Mixed episode; \\
YMRS $\geq 20 ;$ \\
Current episode < 4 \\
weeks \\
First Manic Episode \\
Schizoaffective \\
Substance Abuse \\
Neurological \\
Disorders \\
Taking Other Meds \\
Pregnant/Nursing \\
Labs/Other \\
Conditions
\end{tabular} & $\begin{array}{l}\text { Aripiprazole } \\
15-30 \mathrm{mg} / \text { day } \\
(27.7 \mathrm{mg} / \text { day })\end{array}$ & Placebo & 3 weeks & $\begin{array}{l}\text { YMRS } \\
\text { CGI-BP-S } \\
\text { Response } \\
\text { Adverse Events } \\
\text { EPS } \\
\text { Withdrawal 47\% }\end{array}$ \\
\hline $\begin{array}{l}\text { Vieta, } 20054 \\
\text { RCT } \\
\text { Multisite } \\
\text { Not Disclosed } \\
\text { Industry } \\
\text { RoB Moderate } \\
16135860\end{array}$ & $\begin{array}{l}\mathrm{N}=347 \\
\text { Mean Age } 42 \\
\text { Female 28\% } \\
\text { Race NR } \\
\text { BP-I 100\% } \\
\text { Inpatient or } \\
\text { Outpatient }\end{array}$ & $\begin{array}{l}\text { Manic/Mixed; } \\
\text { YMRS } \geq 20 ; \\
\text { Current episode < } 4 \\
\text { weeks } \\
\text { Other Mental Health } \\
\text { Taking Other Meds } \\
\text { Substance Abuse }\end{array}$ & \begin{tabular}{|l|} 
Aripiprazole \\
$15-30 \mathrm{mg} /$ day
\end{tabular} & $\begin{array}{l}\text { C1: Placebo } \\
\text { C2: Haloperidol } \\
\text { 10-15 mg/day }\end{array}$ & $\begin{array}{l}3 \text { weeks (with } \\
\text { withdrawal < 50\%; } 12 \\
\text { weeks total) }\end{array}$ & $\begin{array}{l}\text { YMRS } \\
\text { CGI-BP-S } \\
\text { Response } \\
\text { Adverse Events } \\
\text { EPS } \\
\text { Withdrawal 34\% }\end{array}$ \\
\hline
\end{tabular}

Abbreviations: AIMS=Abnormal Involuntary Movement Scale; BARS=Barnes Akathisia Scale; BAS=Behavioral Approach System; BMI=Body Mass Index; BP=bipolar

disorder; BPRS=Brief Psychiatric Rating Scale; C=comparison; CGI= Clinical Global Impressions; CGI-I=Clinical Global Impressions-Improvement; CGI-S =CGI-Severity; CGIBP=Clinical Global Impressions Scale-Bipolar; CGI-BP-C= Clinical Global Impressions, Bipolar, Change Scale; CGI-BP-S=Clinical Global Impressions, Bipolar, Severity Scale; C-SSRS=Columbia Suicide Severity Rating Scale; DAI-10=Drug Attitutde Inventory, 10 question version; DIEPSS=Drug-Induced Extra-Pyramidal Symptoms Scale; DSM=Diagnostic and Statistical Manual of Mental Disorders; EPS=extrapyramidal symptoms; ER=Extended Release; ESRS=Extrapyramidal Symptom Rating Scale;

GAF=General Assessment of Functioning Scale; GAS=Global Assessment Scale; HAM-A=Hamilton Scale for Anxiety; HAM-D=Hamilton Scale for Depression; HRQL=Healthrelated quality of life; HRQOL=Health-related quality of life; I=intervention; IDS=Inventory for Depressive Symptoms; LIFE= Longitudinal Interval Follow-up Evaluation; MADRS=Montgomery-Asberg Depression Rating Scale; MAS=Bech-Rafaelsen Mania Rating Scale; MRS=Mania Rating Scale; MSRS=Manic state rating scale; NOS=not 
otherwise specified; NR=not reported; PANSS=Positive and Negative Syndrome Scale; PRS=Polygenic Risk Scores; PGWB=Psychological General Well-Being Index;

PMID=PubMed Identification Number; PRS=Polygenic Risk Scores; Q-LES-Q=Quality of Life Enjoyment and Satisfaction Questionnaire; RCT=randomized controlled trial; ROB=risk of bias; SADS-C= Schedule for Affective Disorders and Schizophrenia-Change version; SAE=Serious Adverse Events; SAS=Simpson Angus Scale; SF-12=12-Item

Short Form Health Survey; SF-36=36-Item Short Form Health Survey; SLICE=Streamlined Longitudinal Interview Clinical Evaluation; T=Trial; YMRS = Young Mania Rating Scale

\section{Appendix Table E2. Summary risk of bias assessments: aripiprazole for acute mania}

\begin{tabular}{|l|l|l|l|}
\hline \multicolumn{1}{|c|}{ Drug } & \multicolumn{1}{|c|}{$\begin{array}{c}\text { Study } \\
\text { Funding Source } \\
\text { PMID }\end{array}$} & $\begin{array}{c}\text { Overall Risk of } \\
\text { Bias Assessment }\end{array}$ & Rationale \\
\hline Aripiprazole & $\begin{array}{l}\text { Kanba, 2014 } \\
\text { Industry } \\
22540407\end{array}$ & High & High dropout rate (47\% overall); Randomization and blinding procedures not disclosed. \\
\cline { 2 - 4 } & $\begin{array}{l}\text { Young, 2009 } \\
\text { Industry } \\
19118324\end{array}$ & High & Moderate dropout rate (28\%); Randomization and blinding procedures not disclosed. \\
\cline { 2 - 5 } & $\begin{array}{l}\text { Sachs, 2006 } \\
\text { Industry } \\
16401666\end{array}$ & High & High withdrawal rate (47\%), randomization and blinding procedures not disclosed \\
\cline { 2 - 5 } & $\begin{array}{l}\text { Vieta, 20056 } \\
\text { Industry } \\
16135860\end{array}$ & Moderate & $\begin{array}{l}\text { Blinding not described, moderate dropout level (34\%), not balanced between the groups. } \\
\text { Groups may not be comparable at time of analysis. }\end{array}$ \\
\hline
\end{tabular}

Abbreviations: ITT=Intention to Treat; PMID=PubMed Identification Number; LOCF=last observation carried forward 


\section{Aripirazole Forest Plots}

Outcomes in studies assessed as having a high risk of bias, or low to moderate risk of bias but at least 40 percent attrition, are presented in grey tones. Both fixed-effect models and random-effects models are presented. We calculated fixed-effect models to provide a charitable estimate of the average effect among completed trials. However, we base our main conclusions on the random-effects models.

Appendix Figure E1. Aripiprazole vs. placebo - response

Odds Ratio of Response ( $>50 \%$ Reduction in YMRS) at 3 Weeks

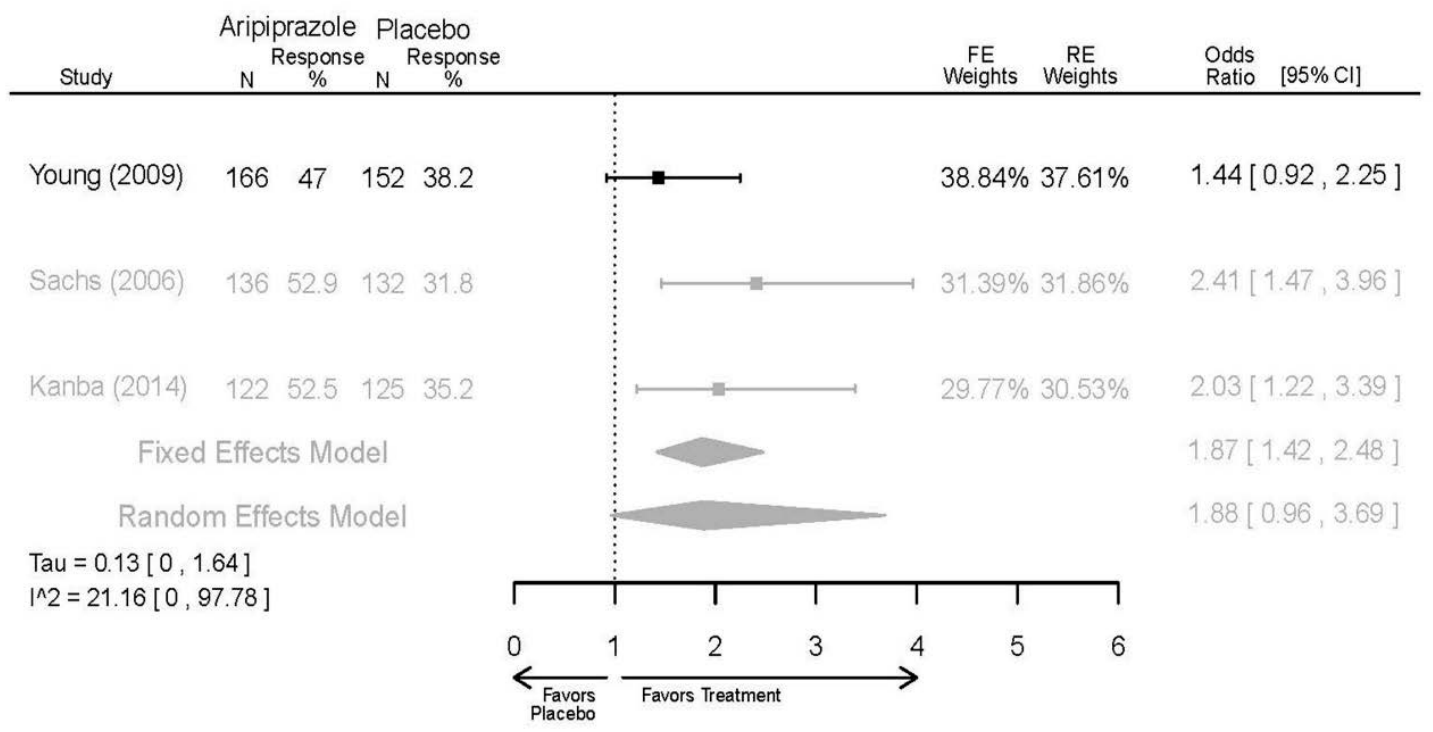


Appendix Figure E2. Aripiprazole vs. placebo - YMRS

Difference in Mean Change in YMRS

from Baseline to 3 Weeks

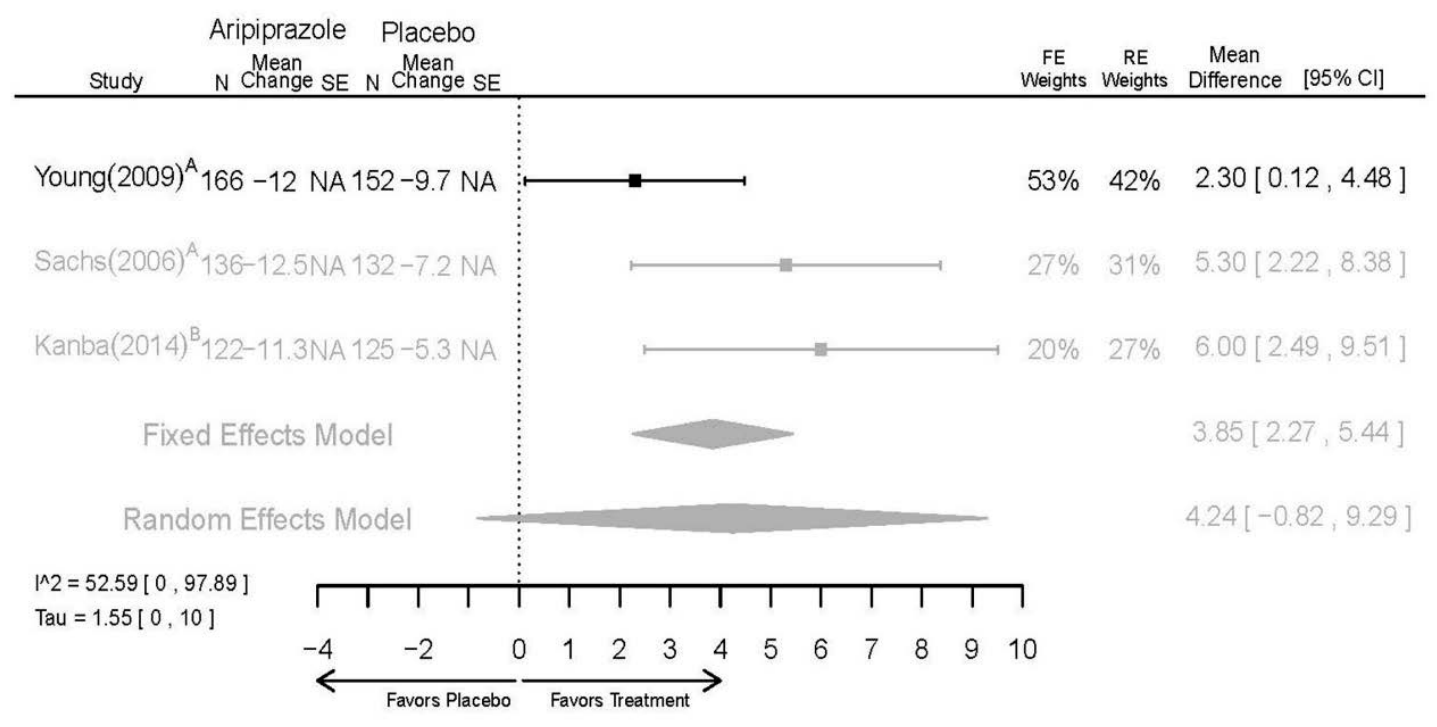

Appendix Figure E3. Aripiprazole vs. placebo - CGI-BP-S

Difference in Mean Change in CGI-BP-S (Overall)

from Baseline to 3 Weeks

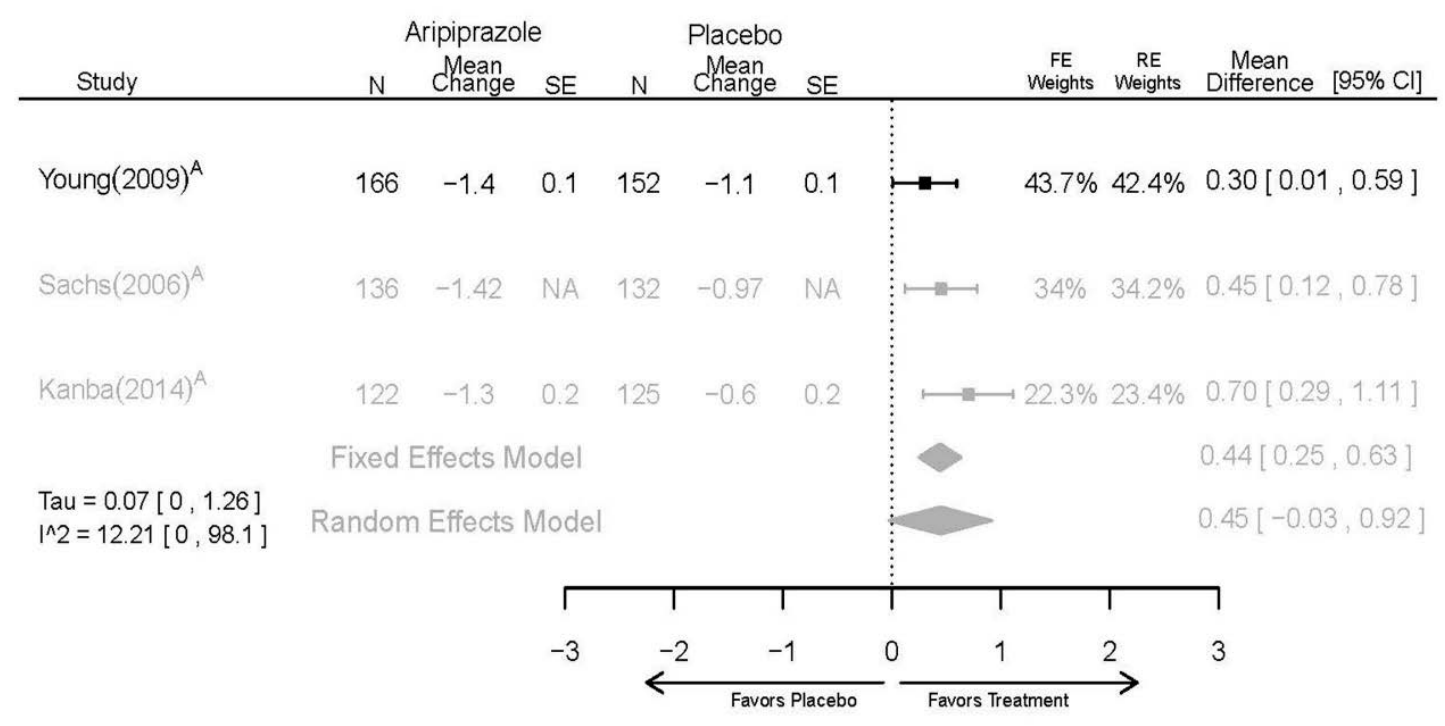

E-6 
Appendix Figure E4. Aripiprazole vs. placebo - withdrawal lack of efficacy

Odds Ratio of Withdrawal due to Lack of Efficacy at 3 Weeks

\begin{tabular}{|c|c|c|c|c|c|c|c|c|}
\hline Study & \multicolumn{2}{|c|}{ Aripiprazole } & $\begin{array}{l}\text { Pla } \\
\mathrm{N}\end{array}$ & $\begin{array}{l}\text { ebo } \\
\text { Withdrawal } \\
\%\end{array}$ & & $\begin{array}{c}\mathrm{FE} \\
\text { Weights }\end{array}$ & $\begin{array}{c}\mathrm{RE} \\
\text { Weights }\end{array}$ & 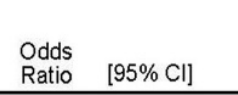 \\
\hline Young (2009) & 166 & 5.4 & 152 & 9.2 & & $21.21 \%$ & $24.29 \%$ & $0.57[0.24,1.35]$ \\
\hline Sachs (2006) & 136 & 8.8 & 132 & 21.2 & $\mapsto$ & $30.43 \%$ & $31.96 \%$ & $0.36[0.17,0.74]$ \\
\hline \multirow[t]{3}{*}{ Kanba (2014) } & 122 & 23 & 125 & 28 & & $48.36 \%$ & $43.75 \%$ & $0.77[0.43,1.36]$ \\
\hline & \multicolumn{2}{|c|}{ Fixed Effects Model } & & & & & & $0.57[0.38,0.85]$ \\
\hline & \multicolumn{2}{|c|}{ Random Effects Model } & & & & & & $0.56[0.21,1.5]$ \\
\hline \multirow[t]{2}{*}{$\begin{array}{l}\text { Tau }=0.23[0,2 \\
\wedge \wedge=27.98[0, s\end{array}$} & & & & & Г & $T$ & $T$ & 7 \\
\hline & & & & & 0 & 2 & 3 & 4 \\
\hline
\end{tabular}

Appendix Figure E5. Aripiprazole vs. placebo - withdrawal adverse events Odds Ratio of Withdrawal due to Adverse Events at 3 Weeks

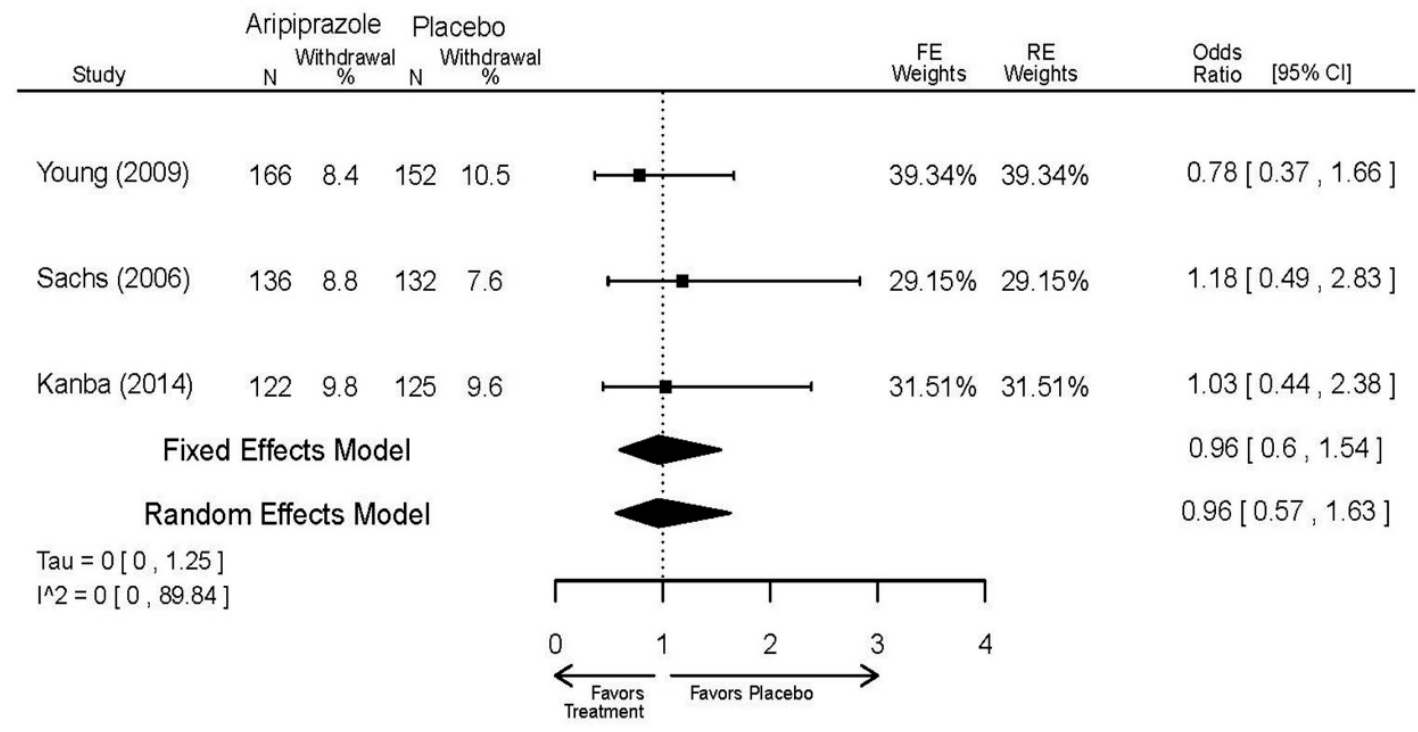


Appendix Figure E6. Aripiprazole vs. placebo - overall withdrawal

\section{Odds Ratio of Overall Withdrawal}

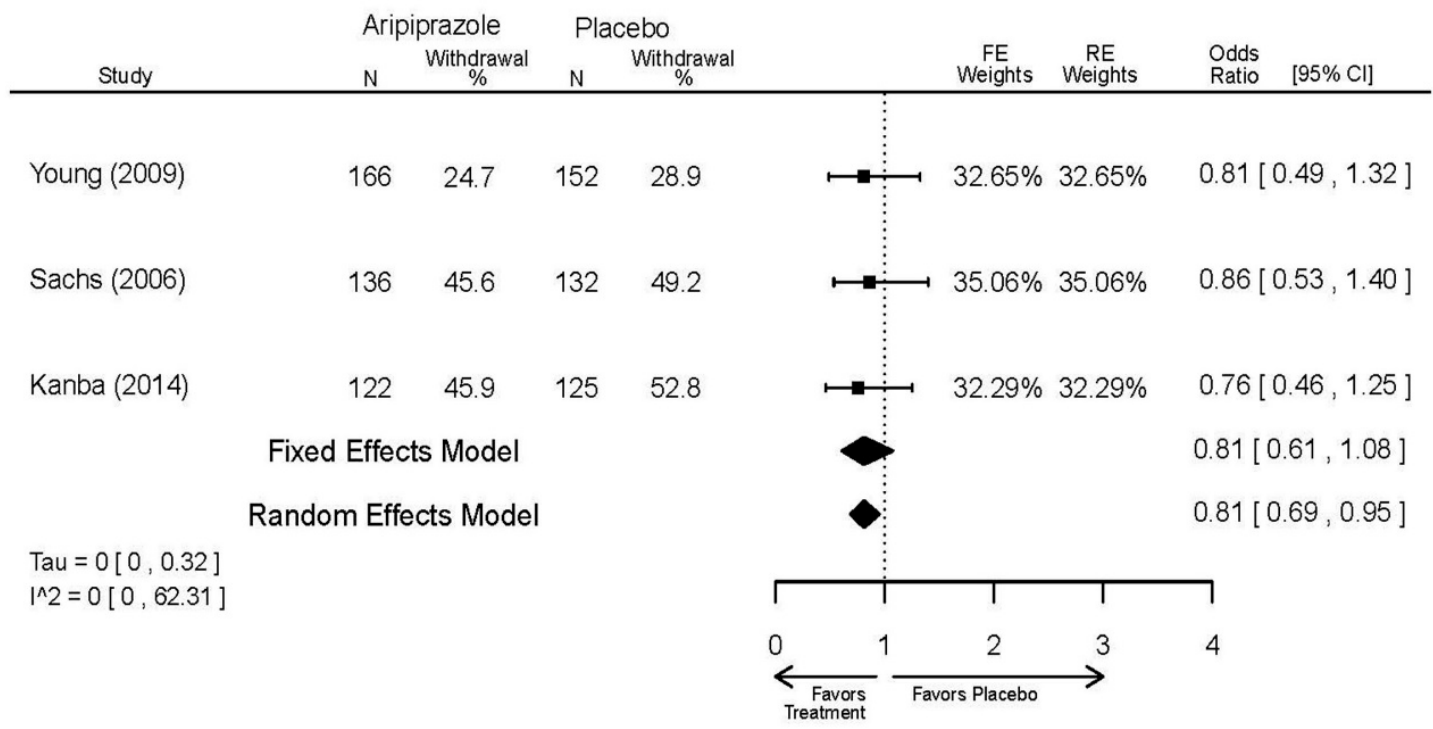

Appendix Figure E7. Aripiprazole vs. placebo - harms - akasthesia

\section{Odds Ratio of Akathasia}

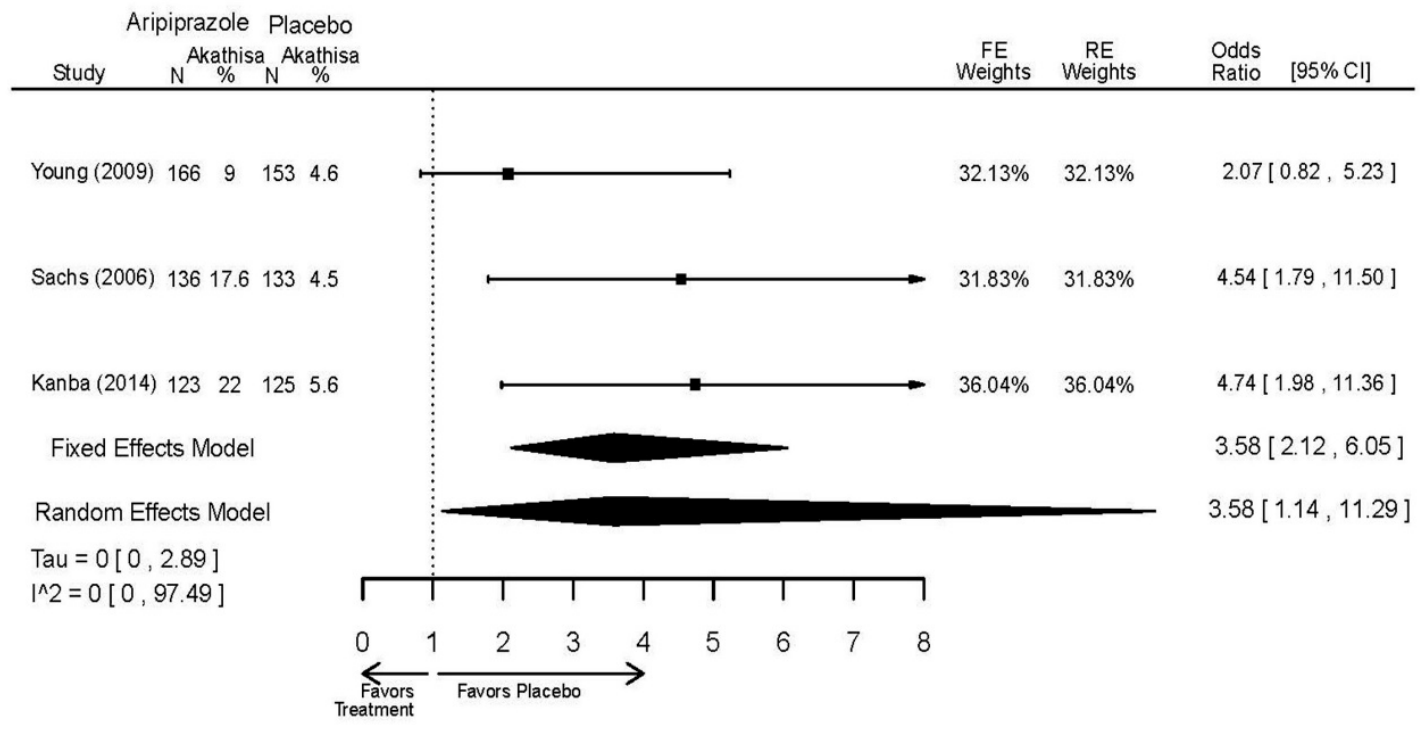


Appendix Figure E8. Aripiprazole vs. placebo - harms - emergent depression

\section{Odds Ratio of Emergent Depression}

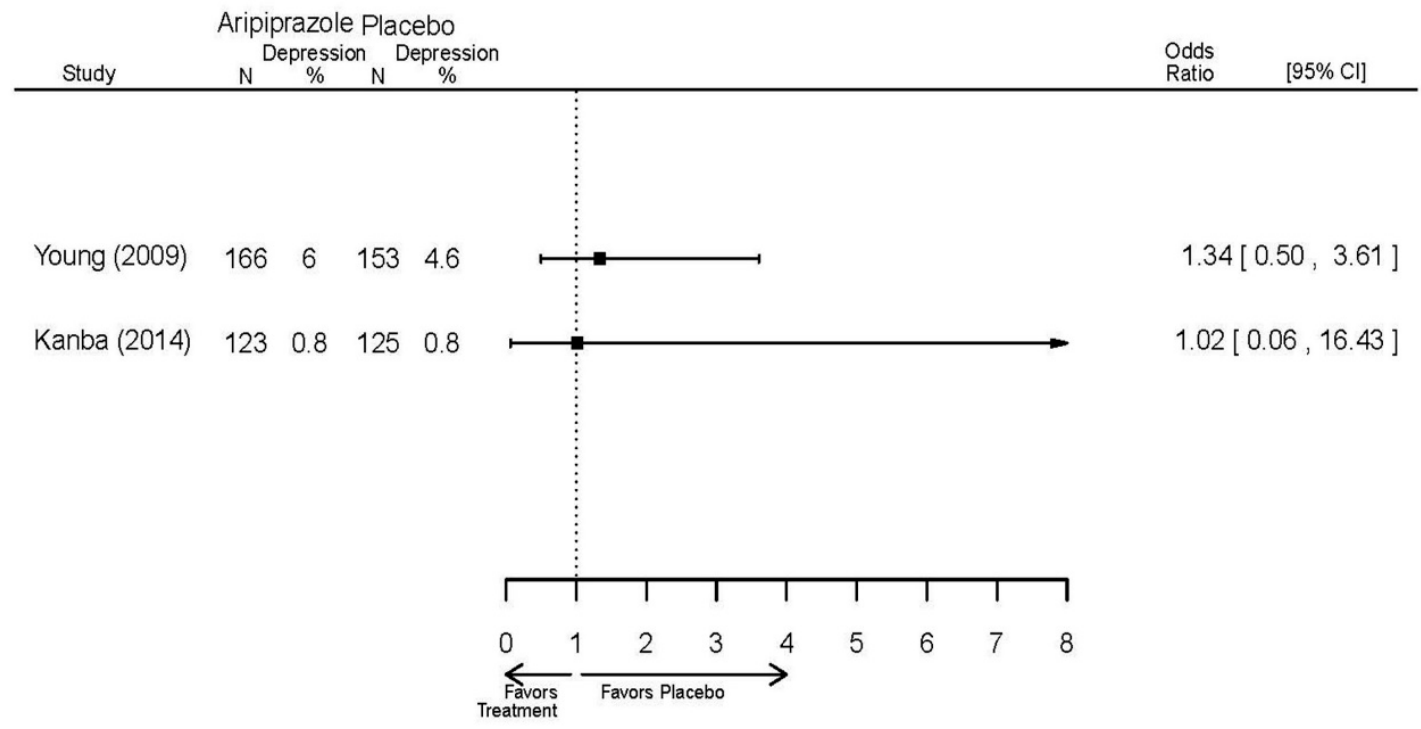

Appendix Figure E9. Aripiprazole vs. placebo - harms - emergent manic episode

\section{Odds Ratio of Manic Reaction or Relapse}

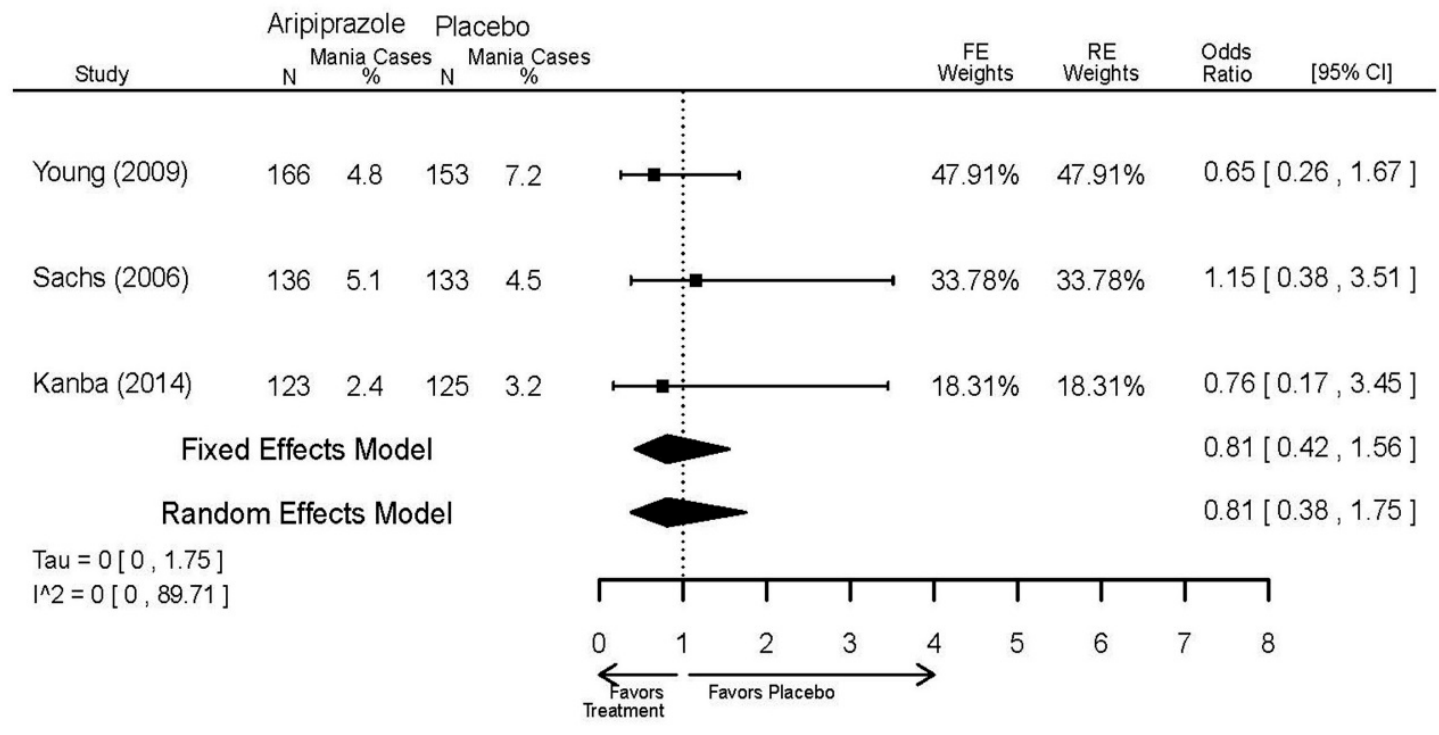


Appendix Figure E10. Aripiprazole vs. placebo - harms - serious adverse event

Odds Ratio of Experiencing a Serious Adverse Event Within 3 Weeks

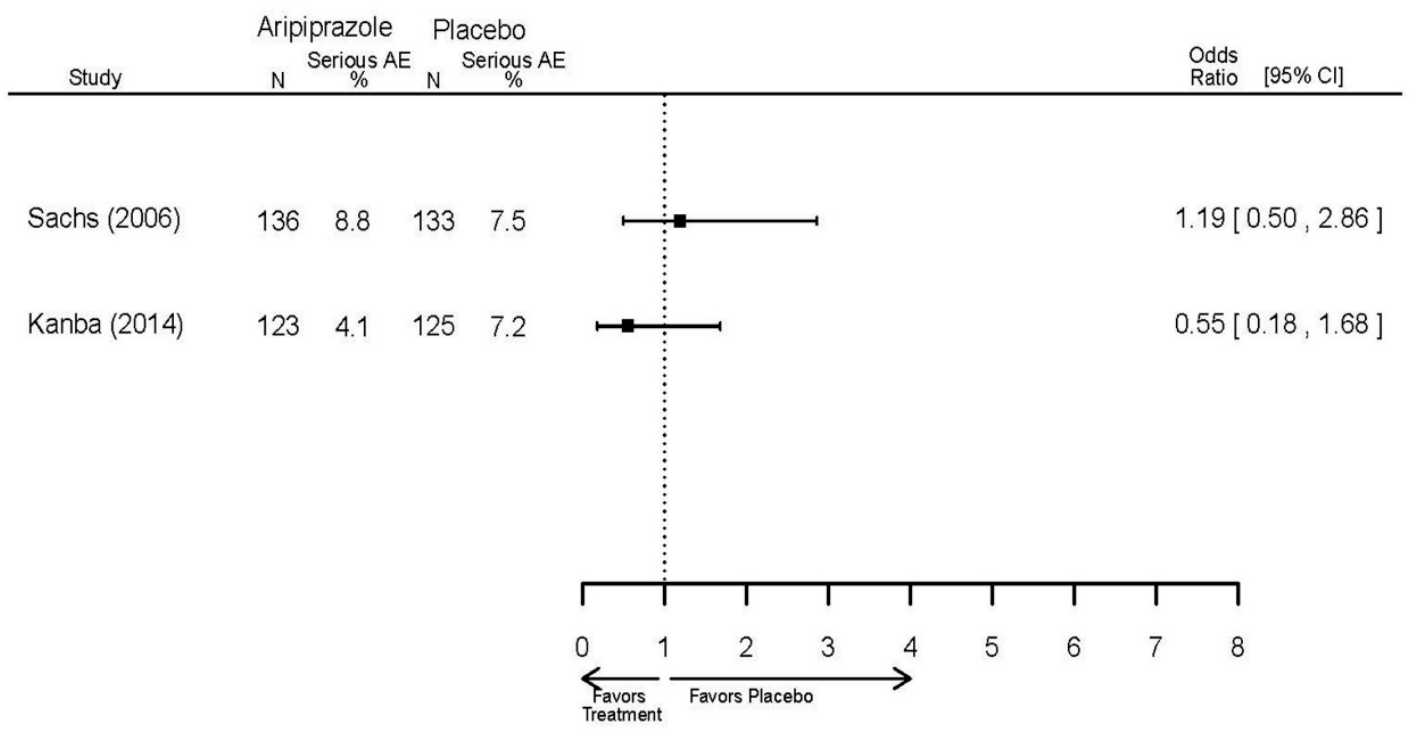

Appendix Figure E11. Aripiprazole vs. placebo - harms - weight gain

Odds Ratio of a $>7 \%$ increase in weight

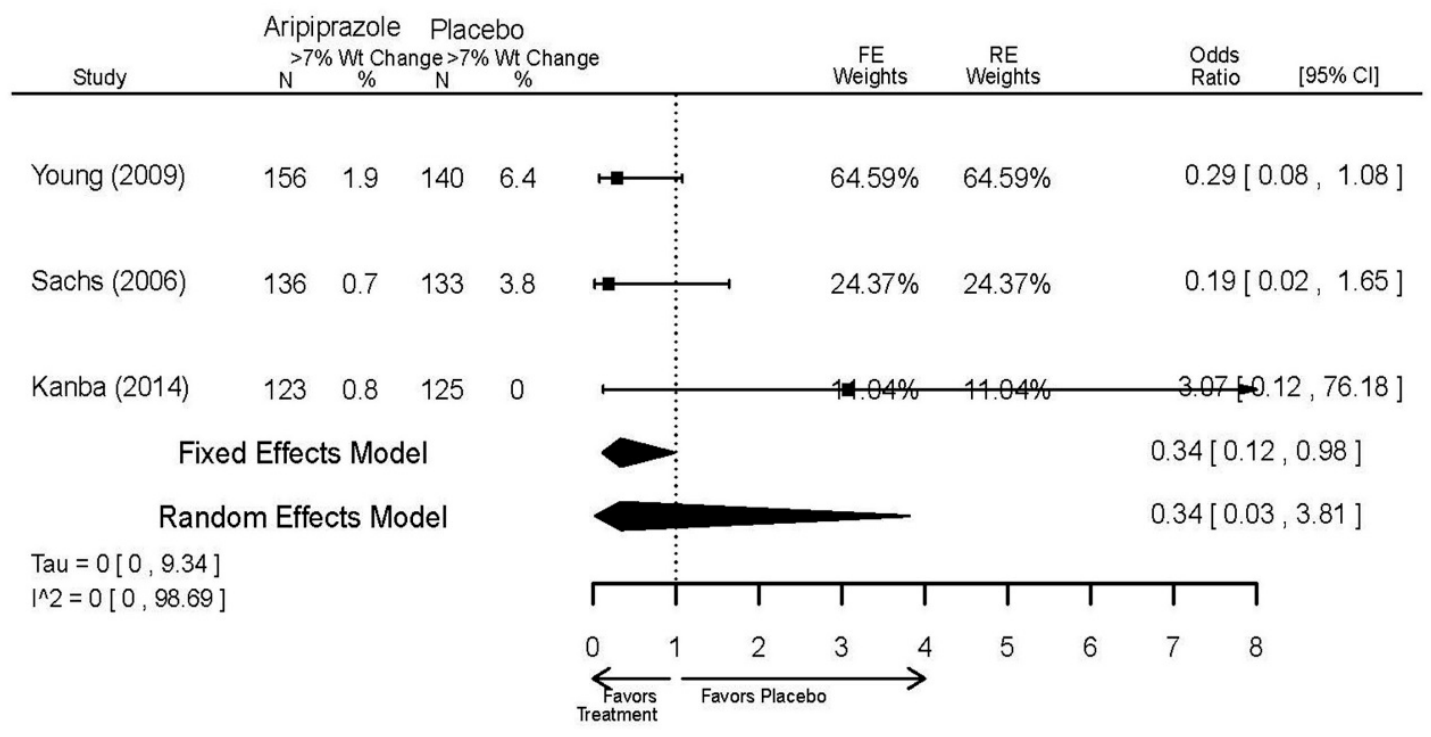


Appendix Figure E12. Aripiprazole vs. haloperidol - response

Odds Ratio of Response ( $>50 \%$ Reduction in YMRS) at 3 Weeks

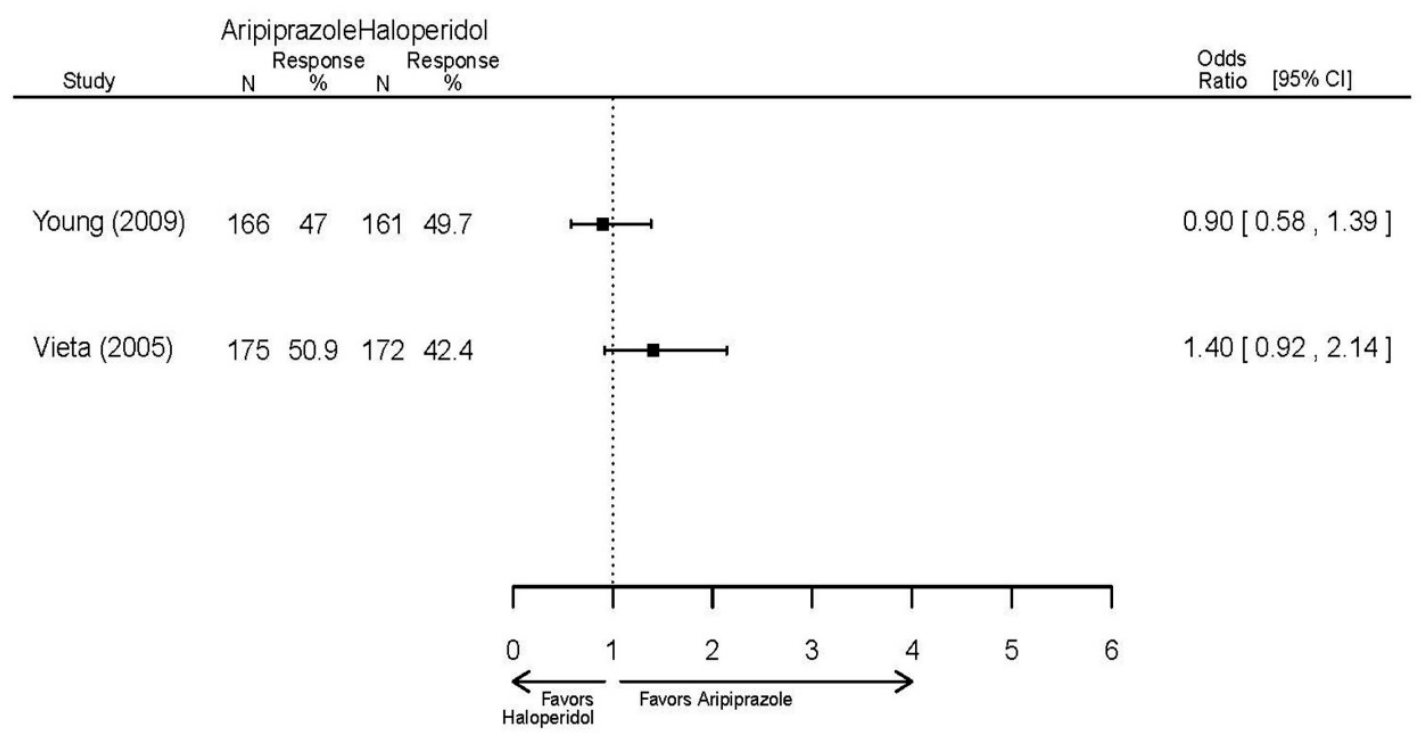

Appendix Figure E13. Aripiprazole vs. haloperidol - remission

Odds Ratio of Remission (YMRS drops to at least 12) at 3 Weeks

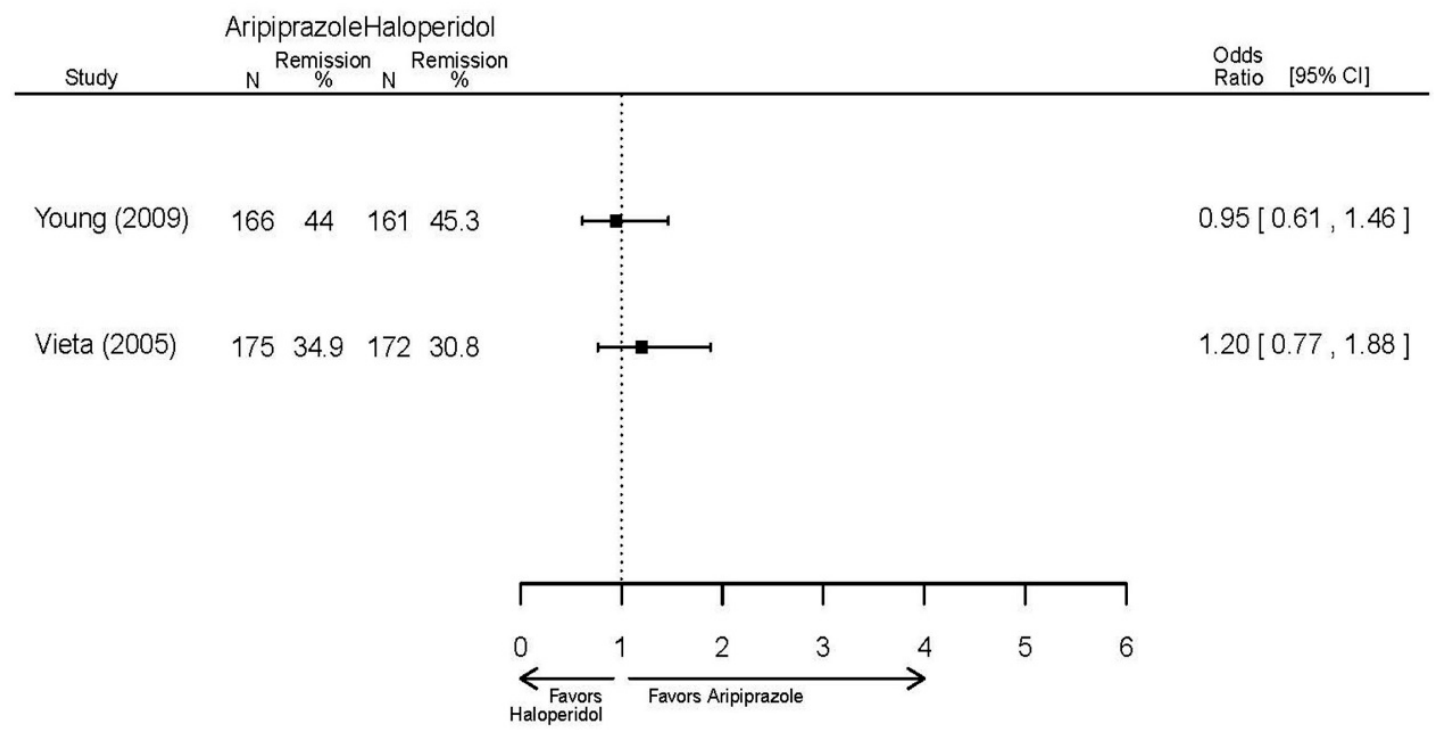


Appendix Figure E14. Aripiprazole vs. haloperidol - withdrawal - adverse events

Odds Ratio of Withdrawal due to Adverse Events at Week 3

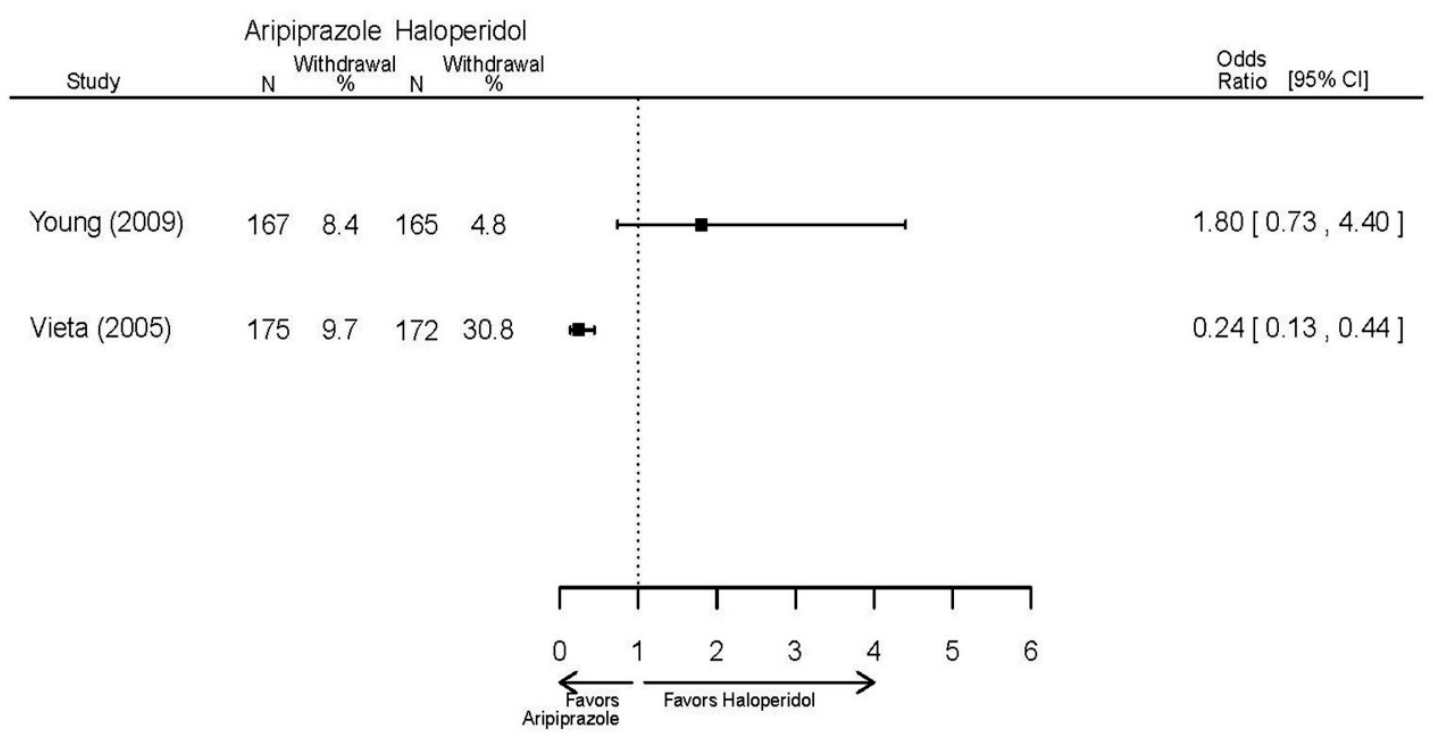

Appendix Figure E15. Aripiprazole vs. haloperidol - withdrawal - overall

Odds Ratio of Withdrawal due to All Causes at Week 3

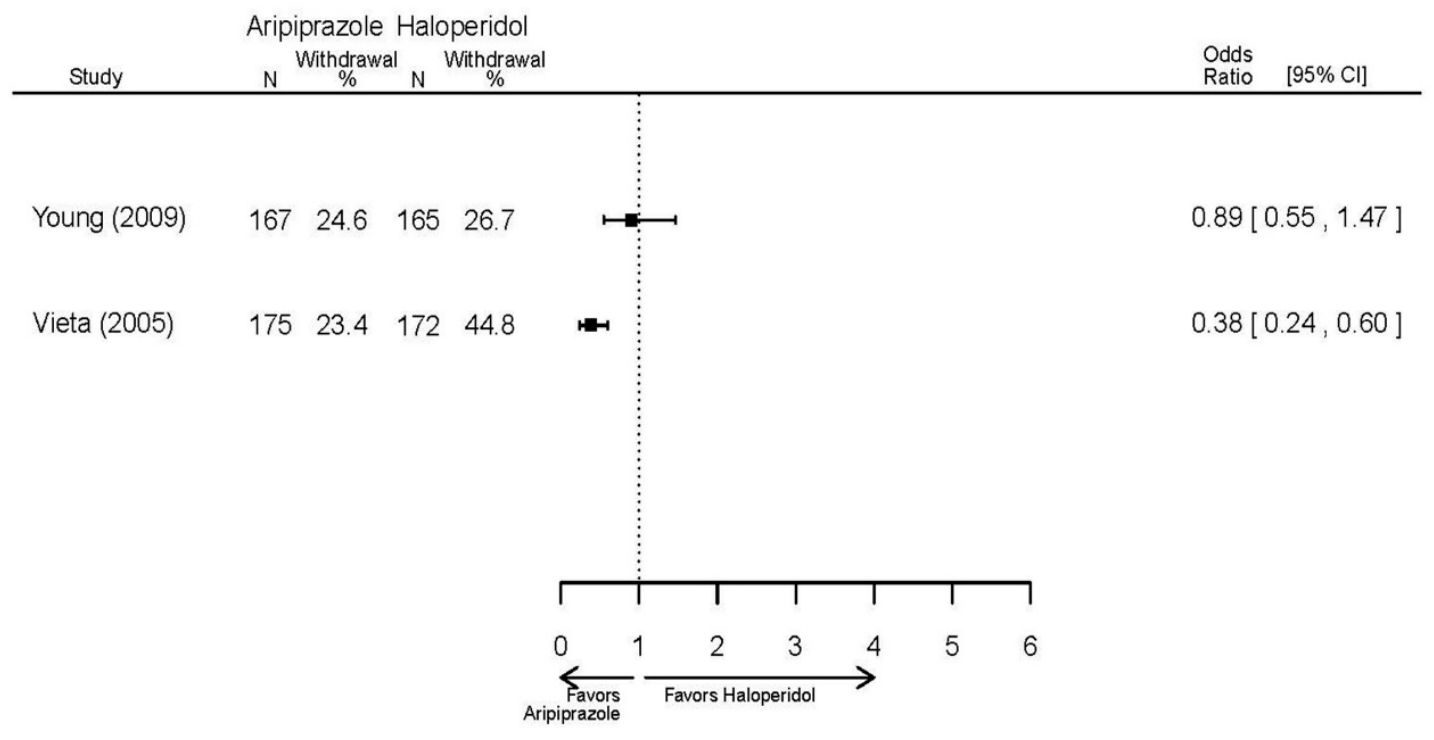


Appendix Figure E16. Aripiprazole vs. haloperidol - harms - akathisia

Odds Ratio of Akathisia

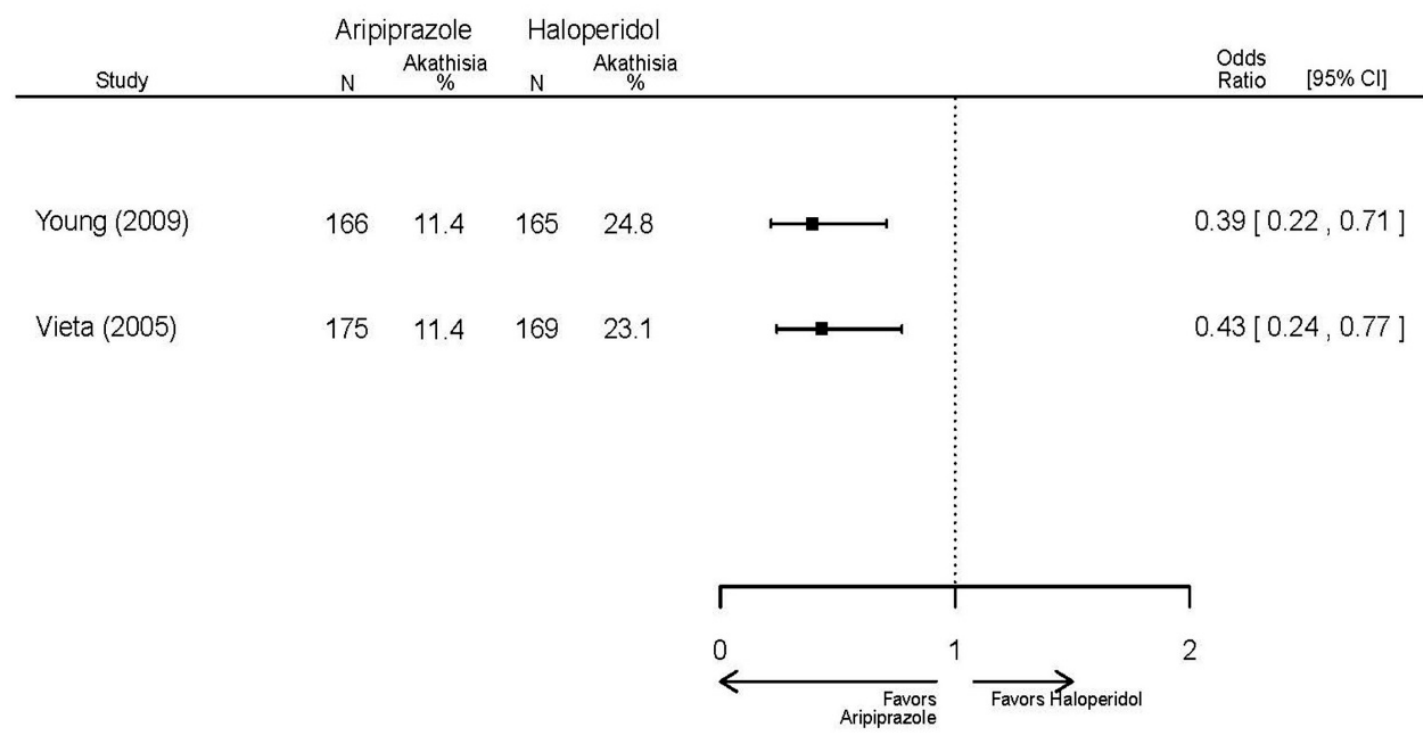

Appendix Figure E17. Aripiprazole vs. haloperidol - harms - emergent depression Odds Ratio of Emergent Depression

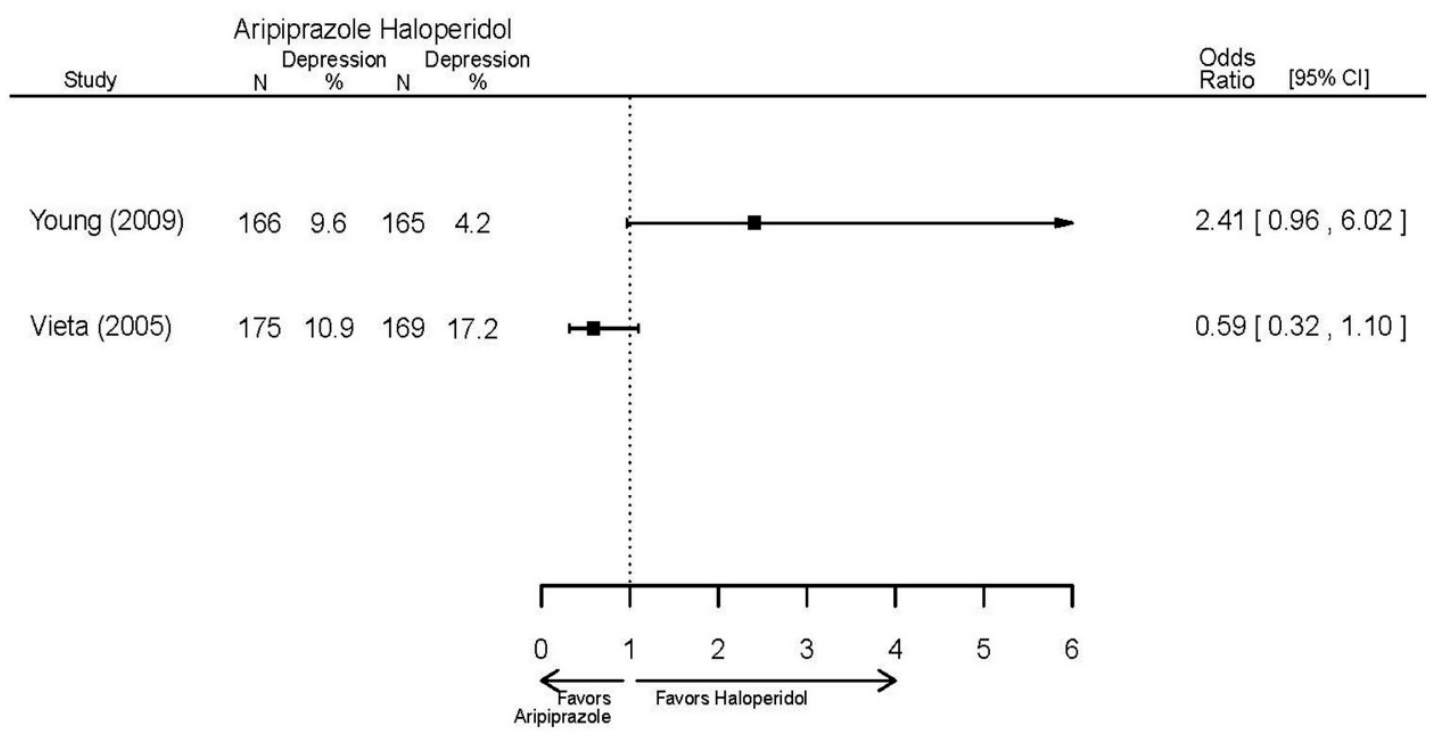


Appendix Figure E18. Aripiprazole vs. haloperidol - harms - extrapyramidal symptoms Odds Ratio of Extrapyramidal Symptoms

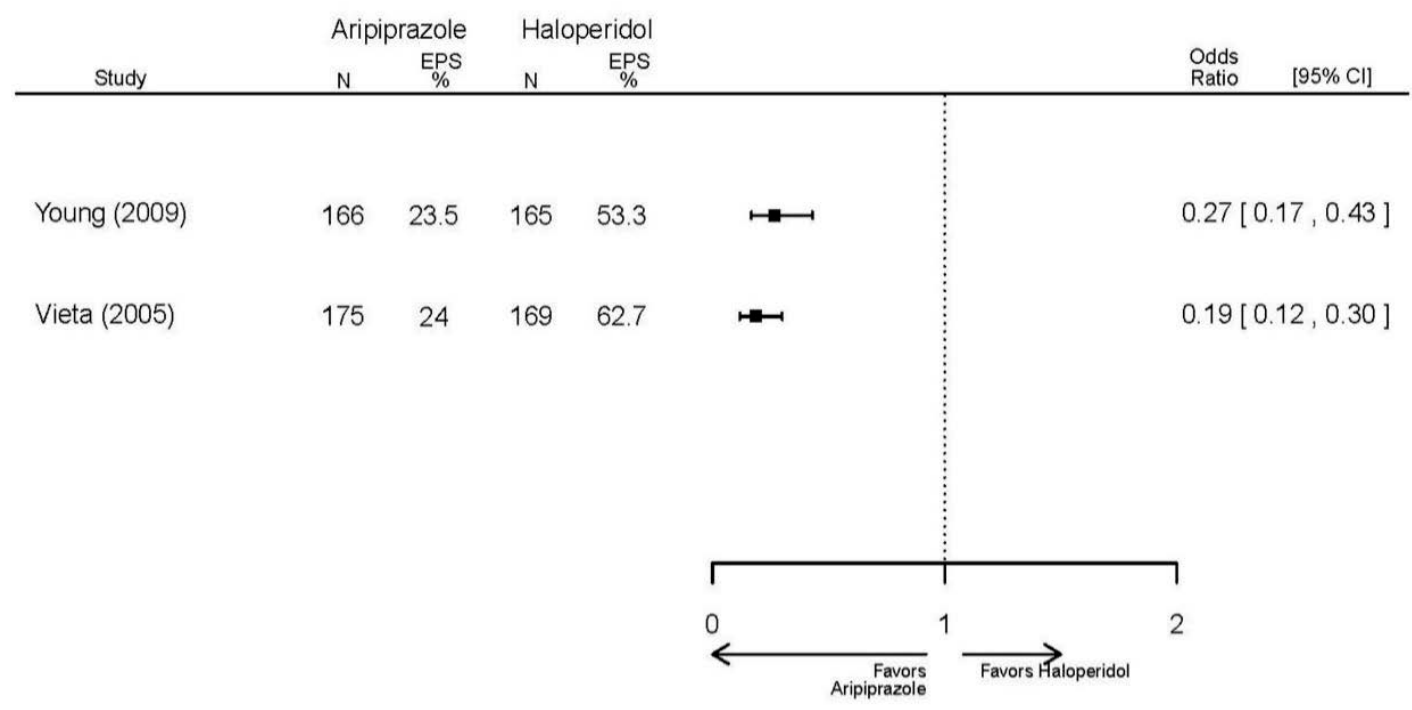

Appendix Figure E19. Aripiprazole vs. haloperidol - harms - serious adverse events Odds Ratio of Serious Adverse Events at Last Measurement

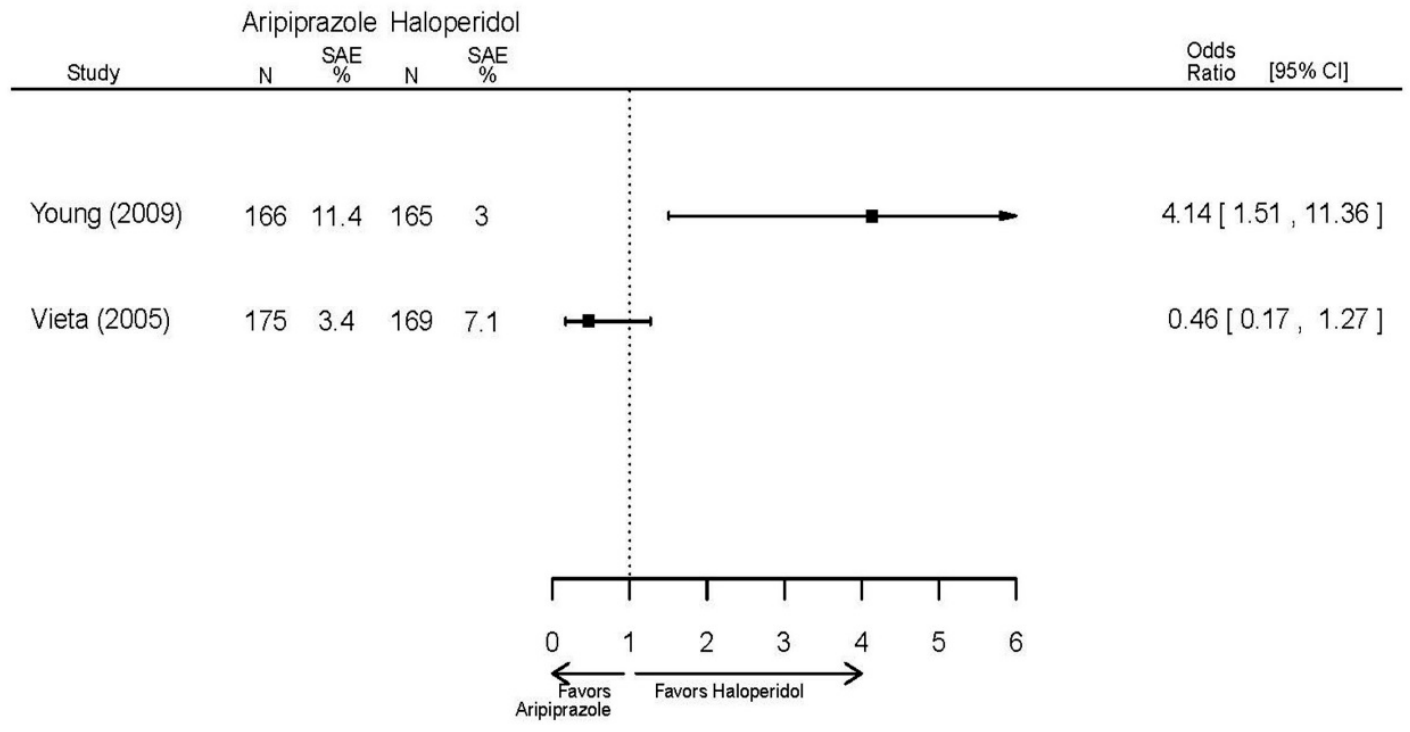




\begin{tabular}{|c|c|c|c|c|c|c|}
\hline Drug & $\begin{array}{l}\text { Study } \\
\text { PMID } \\
\text { RoB } \\
\end{array}$ & Responder/Remitter & Symptom & Function & Other & AE \\
\hline \multirow[t]{3}{*}{ Aripiprazole } & $\begin{array}{l}\text { Kanba, } 2014^{1} \\
22540407 \\
\text { Moderate }\end{array}$ & $\begin{array}{l}\text { See forest plot E1 } \\
\text { above for Response. }\end{array}$ & $\begin{array}{l}\text { See forest plot E2 } \\
\text { above for YMRS. }\end{array}$ & NR & $\begin{array}{l}\text { See forest plots E4, } \\
\text { E5, E6 above for } \\
\text { Withdrawals. }\end{array}$ & $\begin{array}{l}\text { See forest plots E7, E8, } \\
\text { E9, E10, E11 above for } \\
\text { Adverse Effects. } \\
\text { Very Serious AE } \\
3 \text { weeks } \\
1 \text { death during trial, } \\
\text { unrelated to study } \\
\text { medication }\end{array}$ \\
\hline & $\begin{array}{l}\text { Young, } 2009^{2} \\
19118324 \\
\text { Moderate }\end{array}$ & $\begin{array}{l}\text { Remission } \\
3 \text { weeks } \\
\text { NS } \\
\text { Aripiprazole }=73 / 166 \\
\text { Placebo }=56 / 152 \\
\text { OR }=1.35(95 \% \mathrm{Cl} \\
0.86,2.11) \text { p } 0.20\end{array}$ & $\begin{array}{l}\text { See forest plot E2 } \\
\text { above for YMRS. }\end{array}$ & NR & $\begin{array}{l}\text { See forest plots E4, } \\
\text { E5, E6 above for } \\
\text { Withdrawals. }\end{array}$ & $\begin{array}{l}\text { See forest plots E7, E8, } \\
\text { E9, E10, E11 above for } \\
\text { Adverse Effects. } \\
\text { Very Serious AE } \\
3 \text { weeks } \\
1 \text { non-fatal suicide } \\
\text { attempt, unclear which } \\
\text { study arm } \\
\text { SAE } \\
3 \text { weeks } \\
\text { Aripoprazole=19/166 } \\
\text { Placebo=NR } \\
\text { No statistical test } \\
\text { reported } \\
\text { Extrapyramidal } \\
\frac{\text { Symptoms }}{3 \text { weeks }} \\
\text { Aripoprazole=39/166 } \\
\text { Placebo=NR } \\
\text { No statistical test } \\
\text { reported }\end{array}$ \\
\hline & $\begin{array}{l}\text { Sachs. } 2006^{5} \\
16401666 \\
\text { Moderate }\end{array}$ & $\begin{array}{l}\text { See forest plot E1 } \\
\text { above for response. }\end{array}$ & $\begin{array}{l}\text { See forest plot E2 } \\
\text { above for YMRS. }\end{array}$ & NR & $\begin{array}{l}\text { See forest plots E4, } \\
\text { E5, E6 above for } \\
\text { Withdrawals. }\end{array}$ & $\begin{array}{l}\text { See forest plots E7, E8, } \\
\text { E9, E10, E11 above for } \\
\text { Adverse Effects. }\end{array}$ \\
\hline
\end{tabular}


Abbreviations: AE=Adverse Events; ANCOVA=Analysis of Covariance; CGI=Clinical Global Impressions Scale; CI=Confidence Interval; EPS=extrapyramidal symptoms; ESRS=Extrapyrimidal Symptom Rating Scale; GAS=Global Assessment Scale; MADRS=Montgomery-Asberg Depression Rating Scale; NR=Not reported; NS=not significant; OR=Odds Ratio; PMID=PubMed Identification Number; ROB=Risk of Bias; SAE=Serious Adverse Events; YMRS = Young Mania Rating Scale

\section{Appendix Table E4. Strength of evidence assessment: aripiprazole versus placebo for acute mania}

\begin{tabular}{|c|c|c|c|c|c|c|c|c|}
\hline Comparison & Outcome & $\begin{array}{l}\text { \# Studies/ } \\
\text { Design } \\
\text { (n analyzed) }\end{array}$ & $\begin{array}{c}\text { Finding or } \\
\text { Summary } \\
\text { Statistic }\end{array}$ & $\begin{array}{c}\text { Study } \\
\text { Limitations }\end{array}$ & Consistency & Directness & Precision & $\begin{array}{c}\text { Overall } \\
\text { Gradel } \\
\text { Conclusion }\end{array}$ \\
\hline $\begin{array}{l}\text { Aripiprazole } \\
\text { vs. placebo }\end{array}$ & $\begin{array}{l}\text { Response } 3 \text { wks } \\
\text { YMRS } 3 \text { wks } \\
\text { CGI-BP-S } 3 \text { wks } \\
\text { Overall withdrawal } \\
\text { Withdrawal lack of } \\
\text { efficacy } \\
\text { Withdrawal } \\
\text { adverse events }\end{array}$ & $\begin{array}{l}3 \mathrm{RCT} \\
(\mathrm{n}=823)\end{array}$ & See forest plots & High & Consistent & Direct & Imprecise & Insufficient \\
\hline
\end{tabular}

Abbreviations: CGI-BP=Clinical Global Impressions Scale for Bipolar Disorder; NS=not significant; RCT=randomized controlled trial; YMRS = Young Mania Rating Scale Notes:

1. Publication bias for antipsychotics, antidepressants, and behavioral interventions for depressive disorders is suspected.

2. Data were generally imprecise due to missing data from high attrition rates, which was commonly dealt with by Last Observation Carried Forward (LOCF). LOCF requires an assumption that the health status of patients who dropped out of the trial would not have changed had future observations been recorded, a strong assumption in the context of bipolar disorder research. 
Appendix Table E5. Outcomes summary: aripiprazole versus active comparator for acute mania

\begin{tabular}{|c|c|c|c|c|c|c|}
\hline Comparison & $\begin{array}{c}\text { Study } \\
\text { PMID } \\
\text { RoB }\end{array}$ & Responder/Remitter & Symptom & Function & Other & $\overline{A E}$ \\
\hline $\begin{array}{l}\text { Aripiprazole } \\
\text { (15 or } 30 \\
\text { mg/day) vs.. } \\
\text { Haliperidol (5- } \\
15 \text { mg/day) }\end{array}$ & $\begin{array}{l}\text { Young, 2009² } \\
\text { 19118324 } \\
\text { Moderate } \\
\text { High (12 } \\
\text { weeks) }\end{array}$ & See forest plot above. & $\begin{array}{l}\frac{Y \text { YMS, Mean }}{\text { Change }} \\
3 \text { weeks } \\
\text { NS } \\
\text { Aripiprazole }=-12.0 \\
\text { Haloperidol }=-12.8 \\
\text { No statistical test } \\
\text { reported } \\
12 \text { weeks } \\
\text { NS } \\
\text { Aripiprazole }=-17.2 \\
\text { Haloperidol }=-17.8 \\
\text { No statistical test } \\
\text { reported } \\
\\
\text { CGI-BP-Sev, Mean } \\
\text { Change } \\
\text { NS weeks } \\
\text { Aripiprazole }=-1.4 \\
\text { Haloperidol =-1.5 } \\
\text { No statistical test } \\
\text { reported } \\
12 \text { weeks } \\
\text { NS } \\
\text { Aripiprazole =-2.1 } \\
\text { Haloperidol }=-2.2 \\
\text { No statistical test } \\
\text { reported }\end{array}$ & NR & $\begin{array}{l}\text { Overall Withdrawal } \\
12 \text { weeks } \\
\text { NS } \\
\text { Aripiprazole }=72 / 167 \\
\text { Haloperidol=70/165 } \\
\text { OR }=1.03(95 \% \mathrm{Cl} \\
0.67,1.59) ; \mathrm{P}=0.90 \\
\\
\text { Withdrawal Due to } \\
\text { Lack of Efficacy } \\
\text { weeks } \\
\text { Aripiprazole }=9 / 167 \\
\text { Haloperidol=10/165 } \\
\text { OR }=0.88(95 \% \mathrm{Cl} \\
0.35,2.23) \\
\mathrm{p}=0.79 \\
12 \text { weeks } \\
\text { NS } \\
\text { Aripiprazole }=13 / 167 \\
\text { Haloperidol=11/165 } \\
\text { OR = } 1.18(.51,2.72) ; \\
\mathrm{P}=.694 \\
\text { Withdrawal Due to } \\
\frac{\text { AES }}{12 \text { weeks }} \\
\text { NS } \\
\text { Aripiprazole }=24 / 167 \\
\text { Haloperidol }=18 / 165 \\
\text { OR=1.37 }(.71,2.63) ; \\
\mathrm{P}=0.34\end{array}$ & $\begin{array}{l}\text { Very Serious AEs } \\
12 \text { weeks } \\
1 \text { patient in haloperidol } \\
\text { group suffered liver } \\
\text { damage, potentially } \\
\text { attributable to } \\
\text { haloperidol } \\
\text { Normalized Weight } \\
\text { Change ( } 7 \% \text { change) } \\
12 \text { weeks } \\
\text { NS } \\
\text { Aripiprazole }=5.1 \% \\
\text { Haloperidol }=5.8 \% \\
\text { OR = 0.87 } \\
\text { No statistical test } \\
\text { reported } \\
\text { Cases of Depression } \\
3 \text { weeks } \\
\text { Favors Comparator } \\
\text { Aripiprazole }=10 / 166 \\
\text { Haloperidol= } 3 / 165 \\
\text { OR = 3.33 }(0.98,15.83) \\
\text {; } \mathrm{P}=0.049 \\
\text { Cases of Manic } \\
\text { Reaction or Relapse at } \\
\text { Last Measurement } \\
12 \text { weeks } \\
\text { Favors Comparator } \\
\text { Aripiprazole }=8 / 166 \\
\text { Haloperidol= } 1 / 165 \\
\text { OR = } 7.36(1.30, \\
186.76) ; P=0.037 \\
\end{array}$ \\
\hline
\end{tabular}




\begin{tabular}{|c|c|c|c|c|c|c|}
\hline Comparison & $\begin{array}{c}\text { Study } \\
\text { PMID } \\
\text { RoB }\end{array}$ & Responder/Remitter & Symptom & Function & Other & $\mathrm{AE}$ \\
\hline & $\begin{array}{l}\text { Vieta, } 2005^{6} \\
16135860 \\
\text { Moderate }\end{array}$ & See forest plot above. & $\begin{array}{l}\text { YMRS } \\
3 \text { weeks } \\
\text { NS } \\
\text { Aripiprazole }-15.7 \\
\text { Haloperidol }=-15.7 \\
\text { No statistical test } \\
\text { reported } \\
\\
\text { CGI-BP-Sev } \\
3 \text { weeks } \\
\text { NS } \\
\text { Aripiprazole }-2.0 \\
\text { Haloperidol }=-1.9 \\
\text { No statistical test } \\
\text { reported }\end{array}$ & $\mathrm{NR}$ & See forest plot above. & See forest plot above. \\
\hline
\end{tabular}


Appendix Table E6. Strength of evidence assessment: aripiprazole versus active comparator for acute mania

\begin{tabular}{|c|c|c|c|c|c|c|c|c|}
\hline Comparison & Outcome & $\begin{array}{l}\text { \# Studies/ } \\
\text { Design } \\
\text { (n analyzed) }\end{array}$ & $\begin{array}{l}\text { Finding or } \\
\text { Summary } \\
\text { Statistic }\end{array}$ & $\begin{array}{c}\text { Study } \\
\text { Limitations }\end{array}$ & Consistency & Directness & Precision & $\begin{array}{c}\text { Overall } \\
\text { Gradel } \\
\text { Conclusion }\end{array}$ \\
\hline $\begin{array}{l}\text { Aripiprazole } \\
\text { vs. haloperidol }\end{array}$ & $\begin{array}{l}\text { Response } 3 \text { wk } \\
\text { Remission } 3 \text { wk } \\
\text { YMRS } 3 \text { wk } \\
\text { Withdrawal }\end{array}$ & $\begin{array}{l}2 \text { RCTs } \\
(n=674)\end{array}$ & See table above & High & Consistent & Direct & Imprecise & Insufficient \\
\hline
\end{tabular}

Abbreviations: CGI-BP=Clinical Global Impressions Scale for Bipolar Disorder; NS=not significant; RCT=randomized controlled trial; YMRS = Young Mania Rating Scale

Notes:

1. Publication bias for antipsychotics, antidepressants, and behavioral interventions for depressive disorders is suspected.

2. Data were generally imprecise due to missing data from high attrition rates, which was commonly dealt with by Last Observation Carried Forward (LOCF). LOCF requires an

assumption that the health status of patients who dropped out of the trial would not have changed had future observations been recorded, a strong assumption in the context of

bipolar disorder research. 


\section{Section 2. Asenapine for Acute Mania}

Appendix Table E7. Characteristics of eligible studies: asenapine for acute mania

\begin{tabular}{|c|c|c|c|c|c|c|}
\hline $\begin{array}{l}\text { Study, Year } \\
\text { Design } \\
\text { Location } \\
\text { Funder } \\
\text { Risk of Bias } \\
\text { PMID }\end{array}$ & $\begin{array}{c}\text { \# Randomized } \\
\text { Age (mean) } \\
\text { Sex (\% Female) } \\
\text { Race (\% White) } \\
\text { Diagnosis } \\
\text { (\% BP-I, II, NOS) } \\
\text { Setting }\end{array}$ & $\begin{array}{l}\text { Inclusions } \\
\text { Key Exclusions }\end{array}$ & $\begin{array}{c}\text { Intervention } \\
\text { Dosage }\end{array}$ & $\begin{array}{c}\text { Comparison } \\
\text { Dosage }\end{array}$ & Follow-up Duration & $\begin{array}{c}\text { Outcomes } \\
\text { Reported } \\
\text { Withdrawal (\%) at } \\
\text { endpoint }\end{array}$ \\
\hline $\begin{array}{l}\text { Landbloom, } 2016^{7} \\
\text { RCT } \\
\text { Multisite } \\
3 \text { Continents } \\
\text { Industry } \\
\text { RoB Moderate } \\
26496015\end{array}$ & \begin{tabular}{|l}
$\mathrm{N}=367$ \\
Mean Age 44 \\
Female 55\% \\
Race NR \\
BP-I 100\% \\
Inpatient (week 1) \\
Outpatient (weeks 2- \\
3
\end{tabular} & $\begin{array}{l}\text { Mania; } \\
\text { Structured clinical } \\
\text { interview (MINI). } \\
\text { Episode began at } \\
\text { least } 1 \text { month prior to } \\
\text { screening. } \\
\text { YMRS } \geq 20 \\
\\
\text { First Manic Episode } \\
\text { Schizoaffective } \\
\text { Substance Abuse } \\
\text { Other Mental Health } \\
\text { Taking Other Meds } \\
\text { Labs/Other } \\
\text { Conditions }\end{array}$ & $\begin{array}{l}\text { Asenapine } \\
\text { T1: } 5 \mathrm{mg} \text { BID } \\
\text { T2: 10mg BID }\end{array}$ & Placebo & 3 weeks & $\begin{array}{l}\text { Response (50\% } \\
\text { change YMRS } \\
\text { scores) } \\
\text { Remission (YMRS) } \\
\text { YMRS } \\
\text { CGI-BP-S } \\
\text { PANSS } \\
\text { MADRS } \\
\text { SAES } \\
\text { EPS } \\
\text { Withdrawal 28\% }\end{array}$ \\
\hline $\begin{array}{l}\text { Mclntyre, } 2010^{8} \\
\text { RCT } \\
\text { Multisite } \\
3 \text { Continents } \\
\text { Industry } \\
\text { RoB High } \\
20096936\end{array}$ & \begin{tabular}{|l|}
$\mathrm{N}=488$ \\
Mean Age 39 \\
Female $47 \%$ \\
White $55 \%$ \\
BP-I $100 \%$ \\
\\
Inpatient (week 1) \\
Outpatient (weeks 2- \\
3, subject to \\
investigator discretion \\
and successful \\
passing of InterSePT \\
Scale for Suicidal \\
Thinking criteria)
\end{tabular} & $\begin{array}{l}\text { Manic/Mixed; } \\
\text { YMRS } \geq 20 ; \\
\text { Current episode } \leq 3 \\
\text { months } \\
\\
\text { First Manic Episode } \\
\text { Neurological } \\
\text { Disorders } \\
\text { Substance Abuse } \\
\text { Other Mental Health } \\
\text { Taking Other Meds } \\
\text { Lab/Other Conditions }\end{array}$ & $\begin{array}{l}\text { Asenapine } \\
10-20 \text { mg/day (18.4 } \\
\text { mg/day average) } \\
\mathrm{N}=185\end{array}$ & $\begin{array}{l}\text { C1: Placebo } \\
\text { n=98 } \\
\text { C2: Olanzapine } \\
\text { 5-20 mg/day } \\
n=205\end{array}$ & 3 weeks & $\begin{array}{l}\text { CGI-BP-S } \\
\text { MADRS } \\
\text { Remission (YMRS } \leq \\
12 \text { ) } \\
\text { Remission Rate } \\
\text { (YMRS } \leq 12 \text { ) } \\
\text { Response (50\% } \\
\text { decrease in YMRS) } \\
\text { YMRS } \\
\text { Withdrawal 34\% }\end{array}$ \\
\hline
\end{tabular}




\begin{tabular}{|c|c|c|c|c|c|c|}
\hline $\begin{array}{l}\text { Study, Year } \\
\text { Design } \\
\text { Location } \\
\text { Funder } \\
\text { Risk of Bias } \\
\text { PMID }\end{array}$ & $\begin{array}{c}\text { \# Randomized } \\
\text { Age (mean) } \\
\text { Sex (\% Female) } \\
\text { Race (\% White) } \\
\text { Diagnosis } \\
\text { (\% BP-I, II, NOS) } \\
\text { Setting }\end{array}$ & $\begin{array}{c}\text { Inclusions } \\
\text { Key Exclusions }\end{array}$ & $\begin{array}{l}\text { Intervention } \\
\text { Dosage }\end{array}$ & $\begin{array}{c}\text { Comparison } \\
\text { Dosage }\end{array}$ & Follow-up Duration & $\begin{array}{c}\text { Outcomes } \\
\text { Reported } \\
\text { Withdrawal (\%) at } \\
\text { endpoint }\end{array}$ \\
\hline $\begin{array}{l}\text { McIntyre, } 2009^{9} \\
\text { RCT } \\
\text { Multisite } \\
3 \text { continents } \\
\text { Industry } \\
\text { RoB High } \\
19839993\end{array}$ & $\begin{array}{l}\text { N = 489 } \\
\text { Mean Age } 39 \\
\text { Female } 43 \% \\
\text { White } 61 \% \\
\text { BP-I } 100 \% \\
\\
\text { Inpatient (week 1) } \\
\text { Outpatient (weeks 2- } \\
\text { 3, subject to } \\
\text { investigator discretion } \\
\text { and successful } \\
\text { passing of InterSePT } \\
\text { Scale for Suicidal } \\
\text { Thinking criteria) }\end{array}$ & $\begin{array}{l}\text { Manic/Mixed; } \\
\text { YMRS } \geq 20 ; \\
\text { Current episode } \leq 3 \\
\text { months } \\
\\
\text { First Manic Episode } \\
\text { Neurological } \\
\text { Disorders } \\
\text { Substance Abuse } \\
\text { Taking Other Meds } \\
\text { Labs/Other } \\
\text { Conditions }\end{array}$ & $\begin{array}{l}\text { Asenapine } \\
10-20 \text { mg/day (18.2 } \\
\text { mg/day) } \\
\mathrm{N}=104\end{array}$ & $\begin{array}{l}\text { C1: Placebo } \\
n=194 \\
\\
\text { C2: Olanzapine } \\
\text { 5-20 mg/day } \\
n=190\end{array}$ & 3 weeks & $\begin{array}{l}\text { AIMS } \\
\text { BARS } \\
\text { CGI-BP-S } \\
\text { MADRS } \\
\text { Remission (YMRS } \\
\leq 12 \text { ) } \\
\text { Response (50\% } \\
\text { decrease in YMRS) } \\
\text { Simpson-Angus } \\
\text { Scale (SAS) } \\
\text { YMRS } \\
\text { Withdrawal 31\% }\end{array}$ \\
\hline $\begin{array}{l}\text { Calabrese, } 2015^{10} \\
\text { RCT } \\
\text { Multisite } \\
3 \text { Continents } \\
\text { Industry } \\
\text { RoB Low } \\
25562205\end{array}$ & $\begin{array}{l}\mathrm{N}=497 \\
\text { Mean Age } 42 \\
\text { Female } 47 \% \\
\text { White } 69 \% \\
\text { BP-I 100\% } \\
\\
\text { Inpatient (weeks 1-2) } \\
\text { Outpatient (week 3, } \\
\text { subject to inspector } \\
\text { discretion) }\end{array}$ & $\begin{array}{l}\text { Manic/Mixed; } \\
\text { YMRS } \geq 20 \text { AND } \\
\geq 4 \text { on two YMRS } \\
\text { items; } \\
\text { MADRS < } 18 \\
\\
\text { First Manic Episode } \\
\text { Schizoaffective } \\
\text { Substance Abuse } \\
\text { Other Mental Health } \\
\text { Neurological } \\
\text { Disorders } \\
\text { Taking Other Meds }\end{array}$ & $\begin{array}{l}\text { Cariprazine } \\
\text { I1: 3-6 mg/day } \\
\text { I2: 6-12 mg/day }\end{array}$ & Placebo & 3 weeks & $\begin{array}{l}\text { CGI-S } \\
\text { SAS } \\
\text { YMRS } \\
\text { Withdrawal 11\% }\end{array}$ \\
\hline
\end{tabular}




\begin{tabular}{|c|c|c|c|c|c|c|}
\hline $\begin{array}{l}\text { Study, Year } \\
\text { Design } \\
\text { Location } \\
\text { Funder } \\
\text { Risk of Bias } \\
\text { PMID }\end{array}$ & $\begin{array}{c}\text { \# Randomized } \\
\text { Age (mean) } \\
\text { Sex (\% Female) } \\
\text { Race (\% White) } \\
\text { Diagnosis } \\
\text { (\% BP-I, II, NOS) } \\
\text { Setting }\end{array}$ & $\begin{array}{c}\text { Inclusions } \\
\text { Key Exclusions }\end{array}$ & $\begin{array}{l}\text { Intervention } \\
\text { Dosage }\end{array}$ & $\begin{array}{c}\text { Comparison } \\
\text { Dosage }\end{array}$ & Follow-up Duration & $\begin{array}{c}\text { Outcomes } \\
\text { Reported } \\
\text { Withdrawal (\%) at } \\
\text { endpoint }\end{array}$ \\
\hline $\begin{array}{l}\text { Durgam, } 2015^{11} \\
\text { RCT } \\
\text { Multisite } \\
3 \text { Continents } \\
\text { Industry } \\
\text { RoB Moderate } \\
25056368\end{array}$ & $\begin{array}{l}\mathrm{N}=238 \\
\text { Mean Age } 38 \\
\text { Female } 67 \% \\
\text { White } 43 \% \\
\text { BP-I 100\% } \\
\\
\text { Inpatient (weeks 1-2) } \\
\text { Outpatient (week 3, } \\
\text { subject to inspector } \\
\text { discretion) }\end{array}$ & $\begin{array}{l}\text { Manic/Mixed; } \\
\text { YMRS } \geq 20 \text { AND } \\
\geq 4 \text { on two YMRS } \\
\text { items } \\
\text { First Manic Episode } \\
\text { Schizoaffective } \\
\text { Substance Abuse } \\
\text { Other Mental Health } \\
\text { Neurological } \\
\text { Disorders } \\
\text { Taking Other Meds } \\
\text { Pregnant/Nursing } \\
\text { Labs/Other } \\
\text { Conditions }\end{array}$ & $\begin{array}{l}\text { Cariprazine } \\
\text { 3-12 mg/day }\end{array}$ & Placebo & 3 weeks & $\begin{array}{l}\text { YMRS } \\
\text { MADRS } \\
\text { CGI-S } \\
\text { CGI-I } \\
\text { PANSS } \\
\text { AIMS } \\
\text { BARS } \\
\text { SAS } \\
\\
\text { Withdrawal } 37 \%\end{array}$ \\
\hline
\end{tabular}

Abbreviations: AIMS=Abnormal Involuntary Movement Scale; BARS=Barnes Akathisia Scale; BAS=Behavioral Approach System; BMI=Body Mass Index; BP=bipolar

disorder; BPRS=Brief Psychiatric Rating Scale; C=comparison; CGI= Clinical Global Impressions; CGI-I=Clinical Global Impressions-Improvement; CGI-S =CGI-Severity; CGIBP=Clinical Global Impressions Scale-Bipolar; CGI-BP-C= Clinical Global Impressions, Bipolar, Change Scale; CGI-BP-S=Clinical Global Impressions, Bipolar, Severity Scale; C-SSRS=Columbia Suicide Severity Rating Scale; DAI-10=Drug Attitutde Inventory, 10 question version; DIEPSS=Drug-Induced Extra-Pyramidal Symptoms Scale;

DSM=Diagnostic and Statistical Manual of Mental Disorders; EPS=extrapyramidal symptoms; ER=Extended Release; ESRS=Extrapyramidal Symptom Rating Scale;

GAF=General Assessment of Functioning Scale; GAS=Global Assessment Scale; HAM-A=Hamilton Scale for Anxiety; HAM-D=Hamilton Scale for Depression; HRQL=Healthrelated quality of life; HRQOL=Health-related quality of life; I=intervention; IDS=Inventory for Depressive Symptoms; LIFE= Longitudinal Interval Follow-up Evaluation; MADRS=Montgomery-Asberg Depression Rating Scale; MAS=Bech-Rafaelsen Mania Rating Scale; MRS=Mania Rating Scale; MSRS=Manic state rating scale; NOS=not otherwise specified; NR=not reported; PANSS=Positive and Negative Syndrome Scale; PRS=Polygenic Risk Scores; PGWB=Psychological General Well-Being Index; PMID=PubMed Identification Number; PRS=Polygenic Risk Scores; Q-LES-Q=Quality of Life Enjoyment and Satisfaction Questionnaire; RCT=randomized controlled trial; ROB=risk of bias; SADS-C= Schedule for Affective Disorders and Schizophrenia-Change version; SAE=Serious Adverse Events; SAS=Simpson Angus Scale; SF-12=12-Item Short Form Health Survey; SF-36=36-Item Short Form Health Survey; SLICE=Streamlined Longitudinal Interview Clinical Evaluation; T=Trial; YMRS = Young Mania Rating Scale 
Appendix Table E8. Summary risk of bias assessments: asenapine for acute mania

\begin{tabular}{|c|l|l|l|}
\hline Drug & \multicolumn{1}{|c|}{$\begin{array}{c}\text { Study } \\
\text { Funding Source } \\
\text { PMID }\end{array}$} & $\begin{array}{c}\text { Overall Risk of } \\
\text { Bias Assessment }\end{array}$ & \\
\hline Asenapine & $\begin{array}{l}\text { McIntyre, 201012 } \\
\text { Industry } \\
20096936\end{array}$ & High & Rationale \\
\cline { 2 - 4 } & $\begin{array}{l}\text { McIntyre, 20099 } \\
\text { Industry } \\
19839993\end{array}$ & High & $\begin{array}{l}\text { Randomization and blinding procedures not described. Patients discharged from inpatient } \\
\text { care at differing times and study doesn't include this as a point of analysis as a possible } \\
\text { confounder. Withdrawal 31\%. }\end{array}$ \\
\cline { 2 - 5 } & $\begin{array}{l}\text { Landbloom, 2016 } \\
\text { Industry } \\
26496015\end{array}$ & Low & No sources of bias identified. \\
\hline
\end{tabular}

Abbreviations: ITT=Intention to Treat; PMID=PubMed Identification Number; LOCF=last observation carried forward 


\section{Asenapine Forest Plots}

Outcomes in studies assessed as having a high risk of bias, or low to moderate risk of bias but at least 40 percent attrition, are presented in grey tones. Both fixed-effect models and random-effects models are presented. We calculated fixed-effect models to provide a charitable estimate of the average effect among completed trials. However, we base our main conclusions on the random-effects models.

Appendix Figure E20. Asenapine vs. placebo - response

Odds Ratio of Response ( $>50 \%$ Reduction in YMRS) at 3 Weeks

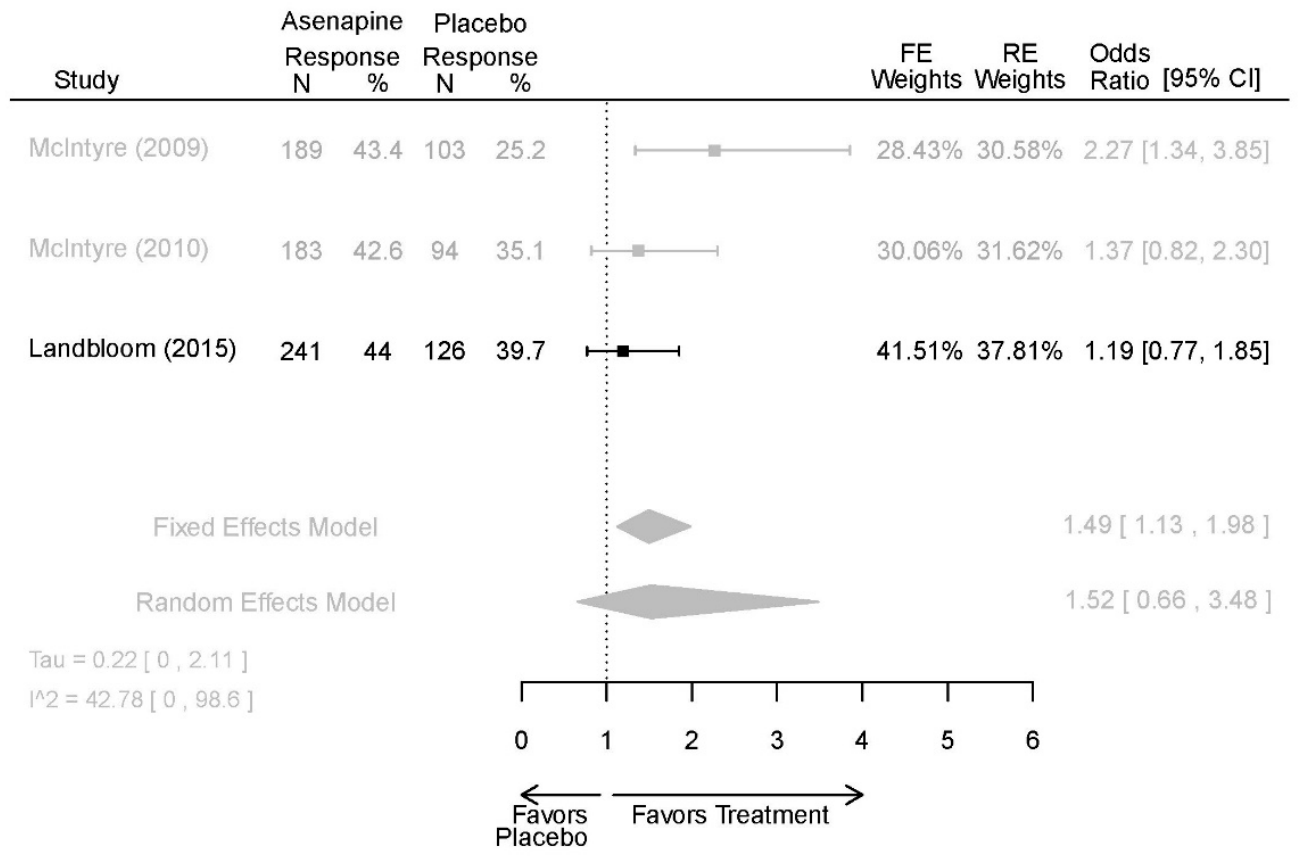


Appendix Figure E21. Asenapine vs. placebo - remission

Odds Ratio of Remission (YMRS $<=12$ ) at 3 Weeks

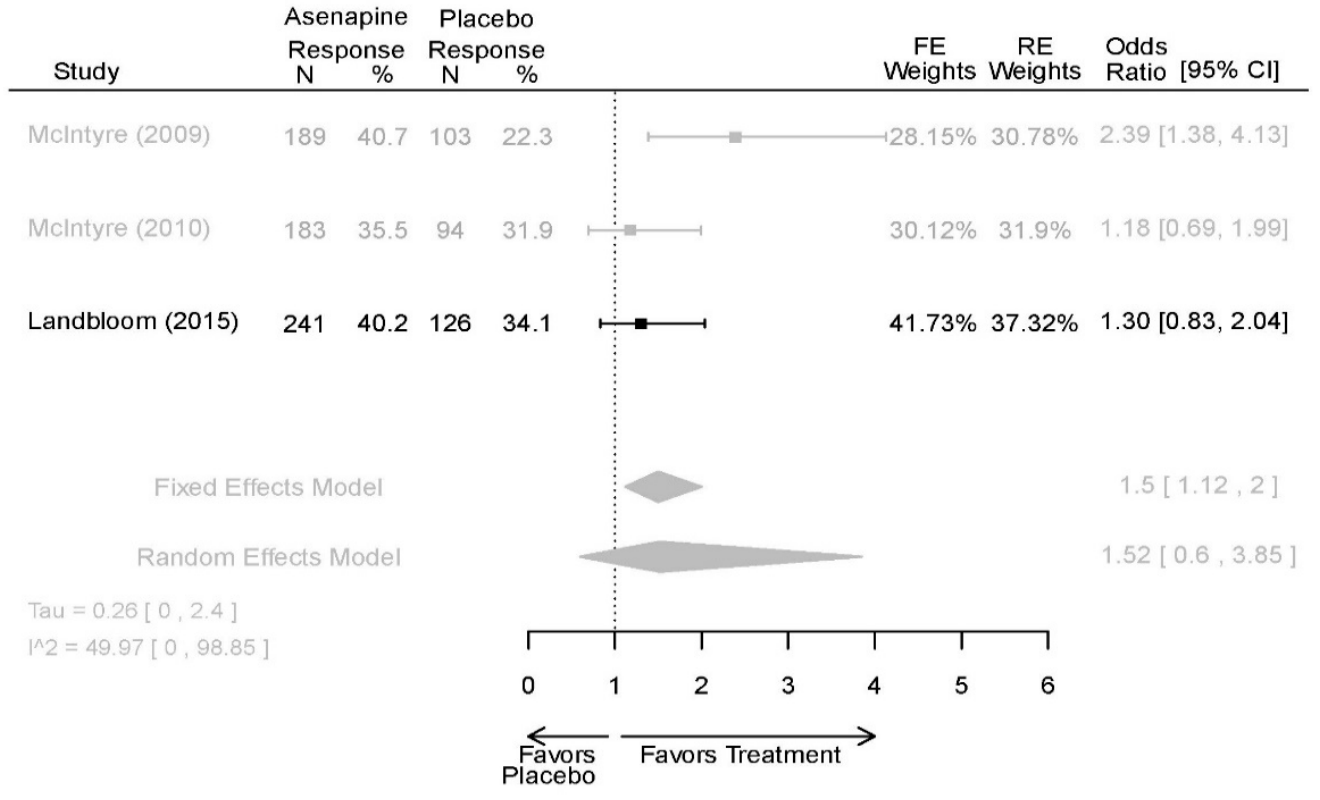

Appendix Figure E22. Asenapine vs. placebo - YMRS

Difference in Mean Change in YMRS from Baseline to 3 Weeks

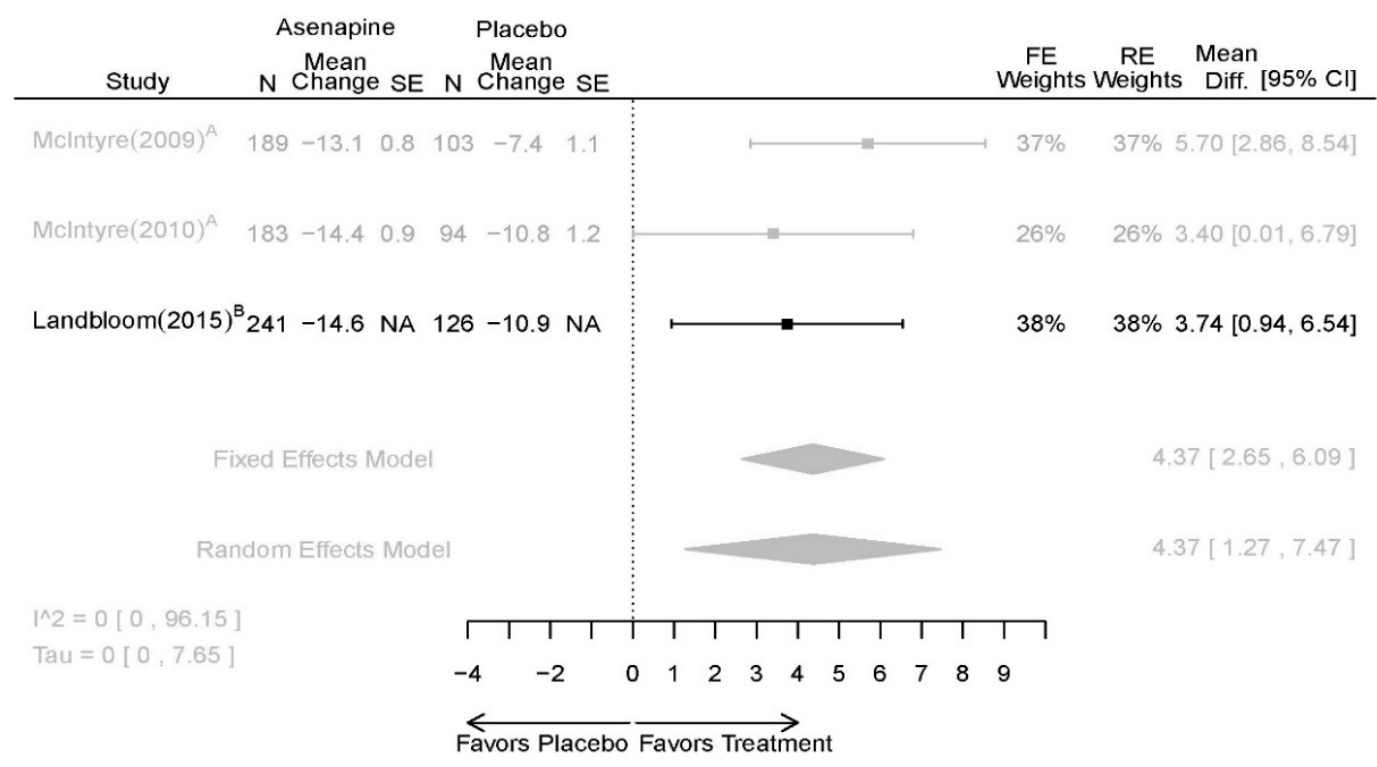


Appendix Figure E23. Asenapine vs. placebo - CGI-BP-S

Difference in Mean Change in CGI-BP-S (Overall) from Baseline to 3 Weeks

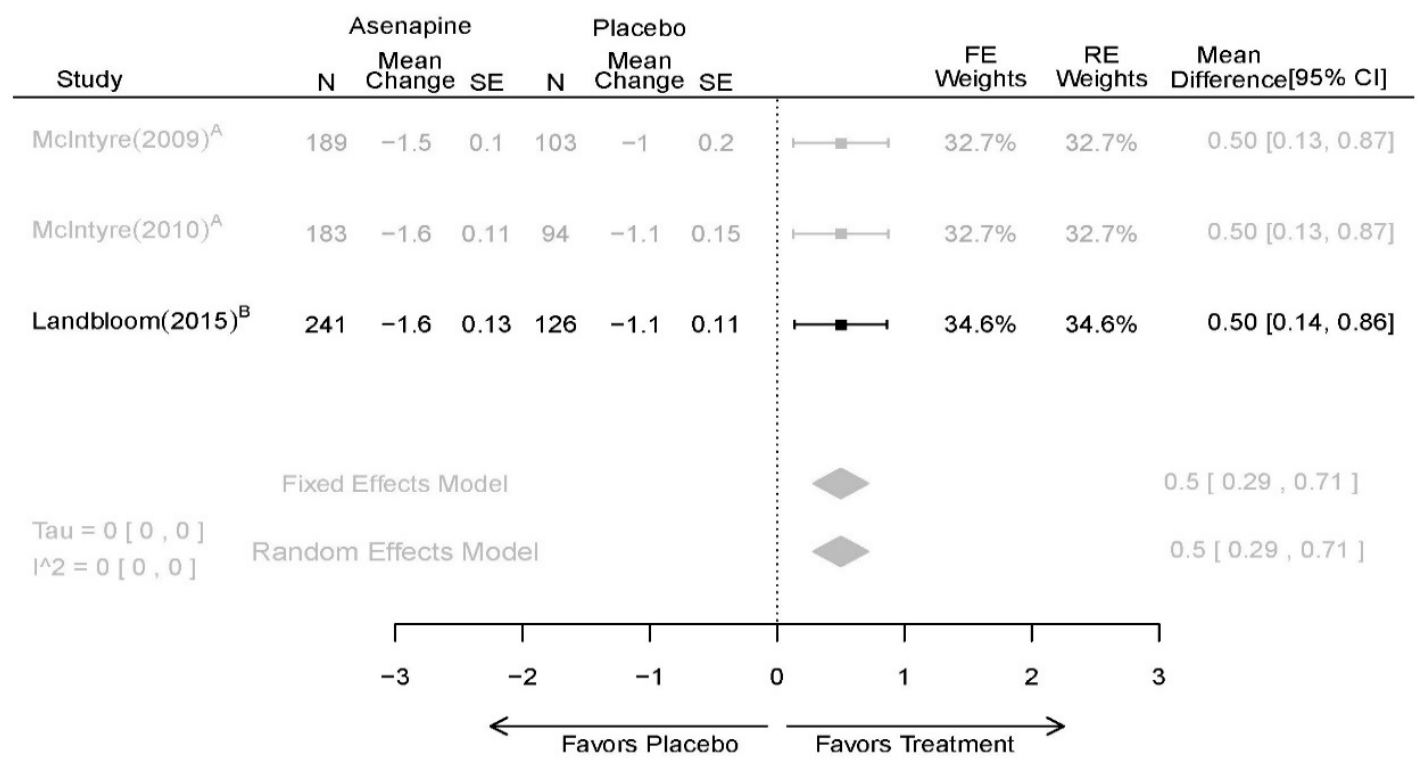

Appendix Figure E24. Asenapine vs. placebo - withdrawal lack of efficacy

Odds Ratio of Withdrawal due to Lack of Efficacy at 3 Weeks

\begin{tabular}{|c|c|c|c|c|c|c|c|c|c|}
\hline Study & $\begin{array}{l}\text { Ase } \\
\text { With } \\
\mathrm{N}\end{array}$ & $\begin{array}{c}\text { pine } \\
\text { awal } \\
\%\end{array}$ & $\begin{array}{c}\text { Pla } \\
\text { With } \\
\mathrm{N} \\
\end{array}$ & $\begin{array}{l}\text { ebo } \\
\text { rawal } \\
\%\end{array}$ & & $\begin{array}{c}\mathrm{FE} \\
\text { Weights } \\
\end{array}$ & $\begin{array}{c}\mathrm{RE} \\
\text { Weights }\end{array}$ & \multicolumn{2}{|c|}{$\begin{array}{l}\text { Odds } \\
\text { Ratio }[95 \% \mathrm{Cl}]\end{array}$} \\
\hline Mclntyre (2009) & 108 & 14.8 & 106 & 16 & & $40.63 \%$ & $36.71 \%$ & 0.91 & {$[0.43,1.91]$} \\
\hline Mclntyre (2010) & 75 & 18.7 & 73 & 19.2 & & 一 $33.07 \%$ & $33.47 \%$ & 0.97 & {$[0.42,2.20]$} \\
\hline Landbloom (2015) & 248 & 3.2 & 122 & 9.8 & $\mapsto$ & $26.3 \%$ & $29.82 \%$ & 0.31 & {$[0.12,0.77]$} \\
\hline \multicolumn{5}{|c|}{ Fixed Effects Model } & & & & \multicolumn{2}{|c|}{$0.7[0.43,1.12]$} \\
\hline \multicolumn{5}{|c|}{ Random Effects Model } & & 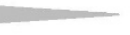 & & \multicolumn{2}{|c|}{$0.67[0.14,3.2]$} \\
\hline \multicolumn{5}{|c|}{ Tau $=0.44[0,4.05]$} & & & & & \\
\hline \multirow{2}{*}{\multicolumn{5}{|c|}{$1^{\wedge} 2=52.37[0,98.93]$}} & Г & T & 7 & & \\
\hline & & & & & 0 & 3 & 4 & & \\
\hline
\end{tabular}


Appendix Figure E25. Asenapine vs. placebo - withdrawal adverse events

Odds Ratio of Withdrawal due to Adverse Events at 3 Weeks

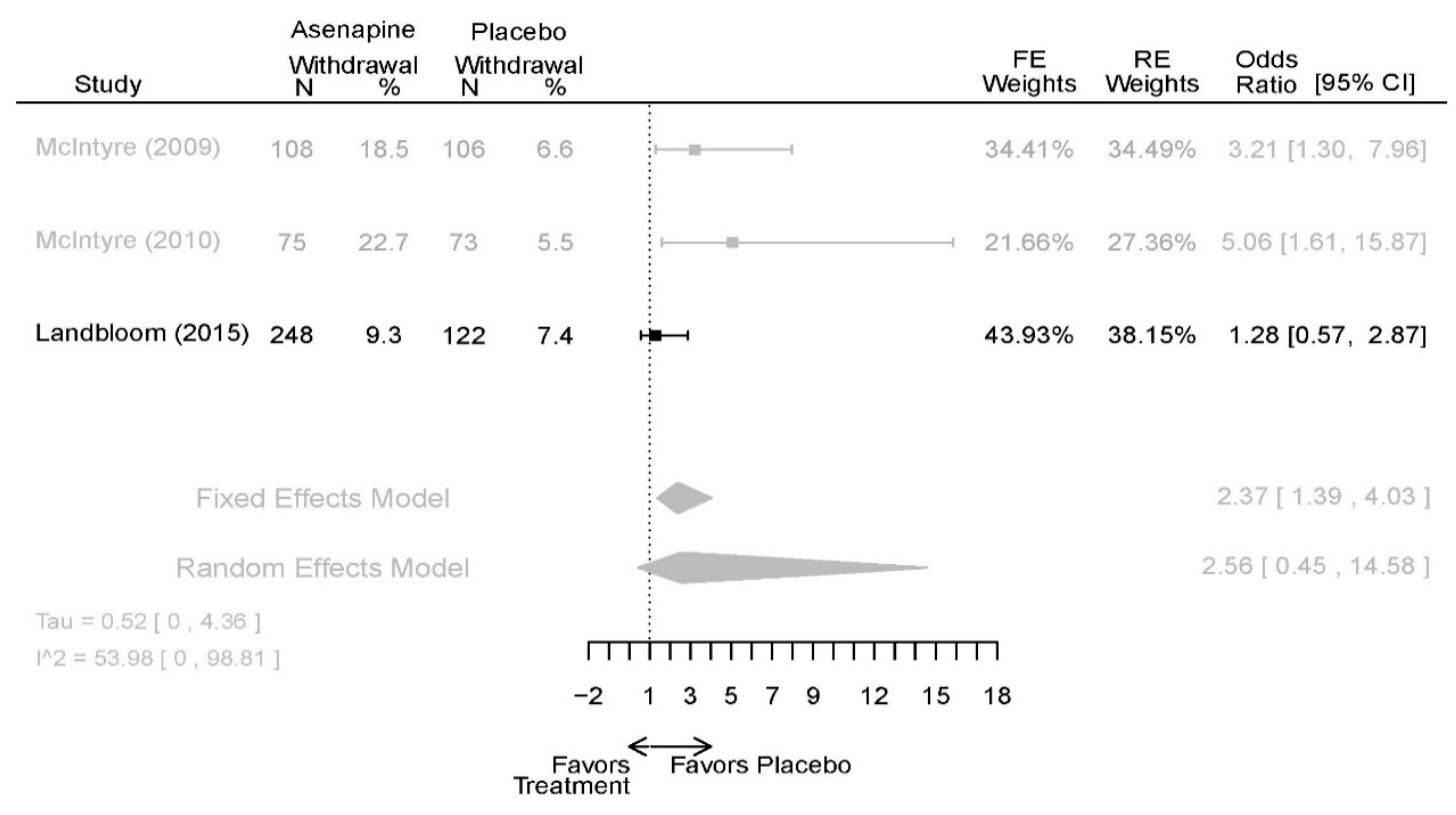

Appendix Figure E26. Asenapine vs. placebo - overall withdrawal

Odds Ratio of Overall Withdrawal

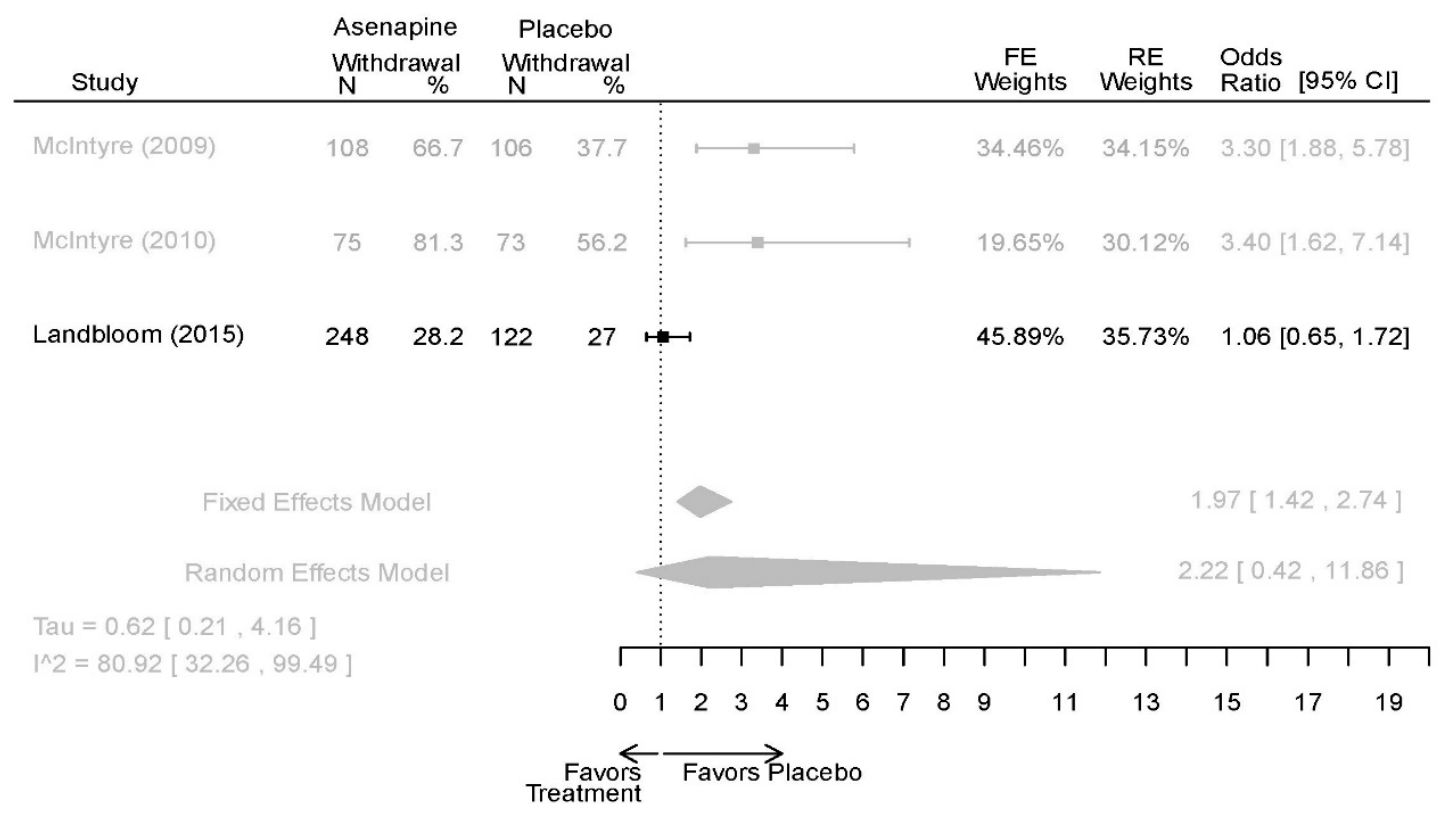


Appendix Table E9. Outcomes summary: asenapine versus placebo for acute mania

\begin{tabular}{|c|c|c|c|c|c|c|}
\hline Drug & $\begin{array}{r}\text { Study } \\
\text { PMID } \\
\text { RoB } \\
\end{array}$ & Responder/Remitter & Symptom & Function & Other & $\mathrm{AE}$ \\
\hline \multirow[t]{2}{*}{ Asenapine } & $\begin{array}{l}\text { Mclntyre, } \\
2010^{12} \\
\text { Industry } \\
20096936\end{array}$ & $\begin{array}{l}\text { See forest plot E20 } \\
\text { above for response. }\end{array}$ & $\begin{array}{l}\text { See forest plot E22 } \\
\text { above for YMRS. }\end{array}$ & $\begin{array}{l}\text { See forest plot E23 } \\
\text { above for CGI. }\end{array}$ & $\begin{array}{l}\text { See forest plots E24, } \\
\text { E25, E26 above for } \\
\text { Withdrawals. } \\
1 \text { suicide in asenapine }\end{array}$ & $\begin{array}{l}\text { SAE } \\
\text { Placebo: } 3.8 \% \\
\text { Asenapine: } 1.5 \% \\
\text { NS } \\
\text { EPS }(<1) \\
\text { Placebo: } 3.1 \% \\
\text { Asenapine: } 10.3 \% \\
\text { p=0.03 } \\
\text { Weight gain } \geq 7 \% \\
\text { Placebo: } 1.2 \% \\
\text { Asenapine: } 7.2 \% \\
\text { p=0.03 }\end{array}$ \\
\hline & $\begin{array}{l}\text { Mclntyre, } \\
2009^{9} \\
\text { Industry } \\
19839993\end{array}$ & $\begin{array}{l}\text { See forest plot E20 } \\
\text { above for response. }\end{array}$ & $\begin{array}{l}\text { See forest plot E22 } \\
\text { above for YMRS. }\end{array}$ & $\begin{array}{l}\text { See forest plot E32 } \\
\text { above for CGI. }\end{array}$ & $\begin{array}{l}\text { See forest plots E24, } \\
\text { E25, E26 above for } \\
\text { Withdrawals. }\end{array}$ & $\begin{array}{l}\text { SAE } \\
\text { Placebo: } 3.8 \% \\
\text { Asenapine: } 1.5 \% \\
\text { NS } \\
\text { EPS }(<1) \\
\text { Placebo: } 3.1 \% \\
\text { Asenapine: } 10.3 \% \\
\text { p=0.03 } \\
\text { Weight gain } \geq 7 \% \\
\text { Placebo: } 1.2 \% \\
\text { Asenapine: } 7.2 \% \\
\text { p=0.03 }\end{array}$ \\
\hline
\end{tabular}




\begin{tabular}{|c|c|c|c|c|c|c|}
\hline Drug & $\begin{array}{l}\text { Study } \\
\text { PMID } \\
\text { RoB }\end{array}$ & Responder/Remitter & Symptom & Function & Other & $\overline{A E}$ \\
\hline & $\begin{array}{l}\text { Landbloom, } \\
2016^{7} \\
\text { Industry } \\
26496015\end{array}$ & $\begin{array}{l}\text { See forest plot E20 } \\
\text { above for response. }\end{array}$ & $\begin{array}{l}\text { See forest plot E22 } \\
\text { above for YMRS. }\end{array}$ & $\begin{array}{l}\text { See forest plot E32 } \\
\text { above for CGI. }\end{array}$ & $\begin{array}{l}\text { See forest plots E24, } \\
\text { E25, E26 above for } \\
\text { Withdrawals. } \\
\text { Suicide Ideation } \\
\text { Placebo: } 5 / 126 \\
5 \mathrm{mg} \text { Asenapine: } \\
4 / 122 \\
10 \mathrm{mg} \text { Asenapine: } \\
1 / 129 \\
\text { NS }\end{array}$ & $\begin{array}{l}\text { SAE } \\
\text { "Most... were psychiatric } \\
\text { disorders class" } \\
\text { Placebo: } 2 / 126 \\
5 \text { mg Asenapine: } 3 / 122 \\
10 \text { mg Asenapine: } 1 / 119 \\
\text { NS } \\
\text { EPS } \\
\text { Placebo: } 6 / 126 \\
5 \text { mg Asenapine: } 8 / 122 \\
10 \text { mg Asenapine: } \\
25 / 129 \\
\text { p<0.0001 } 10 \text { mg }\end{array}$ \\
\hline
\end{tabular}

Abbreviations: AE=Adverse Events; ANCOVA=Analysis of Covariance; CGI=Clinical Global Impressions Scale; CI=Confidence Interval; EPS=extrapyramidal symptoms; ESRS=Extrapyrimidal Symptom Rating Scale; GAS=Global Assessment Scale; MADRS=Montgomery-Asberg Depression Rating Scale; NR=c; NS=not significant; OR=Odds Ratio; PMID=PubMed Identification Number; ROB=Risk of Bias; SAE=Serious Adverse Events; YMRS = Young Mania Rating Scale

\section{Appendix Table E10. Strength of evidence assessment: asenapine versus placebo for acute mania}

\begin{tabular}{|c|c|c|c|c|c|c|c|c|}
\hline Comparison & Outcome & $\begin{array}{l}\text { \# Studies/ } \\
\text { Design } \\
\text { (n analyzed) }\end{array}$ & $\begin{array}{l}\text { Finding or } \\
\text { Summary } \\
\text { Statistic }\end{array}$ & $\begin{array}{c}\text { Study } \\
\text { Limitations }\end{array}$ & Consistency & Directness & Precision & $\begin{array}{c}\text { Overall } \\
\text { Gradel } \\
\text { Conclusion }\end{array}$ \\
\hline \multirow{4}{*}{$\begin{array}{l}\text { Asenapine vs. } \\
\text { placebo }\end{array}$} & $\begin{array}{l}\text { Response } \\
\text { Remission } \\
3 \text { wks } \\
\end{array}$ & $\begin{array}{l}3 \text { RCT } \\
(n=936)\end{array}$ & NS & Moderate & Consistent & Direct & Imprecise & Low \\
\hline & YMRS 3 wks & $\begin{array}{l}3 \mathrm{RCT} \\
(\mathrm{n}=936)\end{array}$ & $\begin{array}{l}\text { Favors } \\
\text { Asenapine. MD } \\
4.37(1.27,7.47)\end{array}$ & Moderate & Consistent & Direct & Imprecise & Low \\
\hline & CGI-BP-S 3 wk & $\begin{array}{l}3 \mathrm{RCT} \\
(\mathrm{n}=936)\end{array}$ & $\begin{array}{l}\text { Favors } \\
\text { Asenapine } \mathrm{MD} \\
0.5(0.29,0.71)\end{array}$ & Moderate & Consistent & Direct & Imprecise & Low \\
\hline & $\begin{array}{l}\text { Withdrawal - AE, } \\
\text { Lack of Efficacy, } \\
\text { Overall }\end{array}$ & $\begin{array}{l}3 \text { RCT } \\
(n=936)\end{array}$ & NS & Moderate & Consistent & Direct & Imprecise & Low \\
\hline
\end{tabular}

Abbreviations: CGI-BP=Clinical Global Impressions Scale for Bipolar Disorder; NS=not significant; RCT=randomized controlled trial; YMRS = Young Mania Rating Scale

Notes:

1. Publication bias for antipsychotics, antidepressants, and behavioral interventions for depressive disorders is suspected.

2. Data were generally imprecise due to missing data from high attrition rates, which was commonly dealt with by Last Observation Carried Forward (LOCF). LOCF requires an 
assumption that the health status of patients who dropped out of the trial would not have changed had future observations been recorded, a strong assumption in the context of bipolar disorder research. 
Appendix Table E11. Outcomes summary: asenapine versus active comparator for acute mania

\begin{tabular}{|c|c|c|c|c|c|c|}
\hline Comparison & $\begin{array}{l}\text { Study } \\
\text { PMID } \\
\text { RoB }\end{array}$ & Responder/Remitter & Symptom & Function & Other & $\mathrm{AE}$ \\
\hline \multirow[t]{2}{*}{$\begin{array}{l}\text { Asenapine vs. } \\
\text { olanzapine }\end{array}$} & $\begin{array}{l}\text { Mclntyre, } \\
2009^{9} \\
19839993\end{array}$ & $\begin{array}{l}\frac{\text { Response }}{3 \text { weeks }} \\
\text { Asenapine } 42.3 \% \\
\text { Olanzapine } 50 \% \\
\frac{\text { Remission }}{3 \text { weeks }} \\
\text { Asenapine } 40.2 \% \\
\text { Olanzapine } 39.4 \% \\
\text { NS }\end{array}$ & $\begin{array}{l}\frac{\text { YMRS }}{3 \text { weeks }} \\
\text { Least square mean } \\
\text { Asenapine }-10.8 \text { SD } \\
0.8 \text { (effect size } 0.45 \text { ) } \\
\text { Olanzapine }-12.6 \\
\text { SD } 0.8 \text { (effect size } \\
0.70 \text { ) }\end{array}$ & $\begin{array}{l}\text { CGI } \\
3 \text { weeks } \\
\text { Least square mean } \\
\text { Asenapine }-1.2 \mathrm{SD} \\
0.01 \\
\text { Olanzapine }-1.4 \mathrm{SD} \\
0.01\end{array}$ & $\begin{array}{l}\text { NR } \\
\text { Overall Withdrawal } \\
\text { Asenapine } 37.1 \% \\
\text { Olanzapine } 30.9 \% \\
\text { Withdrawal Lack of } \\
\text { Efficacy: } \\
\text { Asenapine } 8.2 \% \\
\text { Olanzapine 5.8\% } \\
\text { Withdrawal AE } \\
\text { Asenapine } 10.3 \% \\
\text { Olanzapine } 4.2 \% \\
\end{array}$ & $\begin{array}{l}\text { Serious Adverse Events } \\
3 \text { weeks } \\
0 \text { in all arms } \\
\frac{\text { Deaths }}{3 \text { weeks }} \\
0 \text { in all arms } \\
\frac{\text { EPS }}{3 \text { weeks }} \\
2.9 \% \text { placebo } \\
7.2 \% \text { Asenapine } \\
7.9 \% \text { Olanzapine }\end{array}$ \\
\hline & $\begin{array}{l}\text { Mclntyre, } \\
2010^{12} \\
20096936\end{array}$ & $\begin{array}{l}\frac{\text { Response }}{3 \text { weeks }} \\
\text { Asenapine } 42.6 \% \\
\text { Olanzapine } 54.7 \% \\
\frac{\text { Remission }}{3 \text { weeks }} \\
\text { Asenapine } 35.5 \% \\
\text { Olanzapine } 46.3 \% \\
\text { NS }\end{array}$ & $\begin{array}{l}\frac{\text { YMRS }}{3 \text { weeks }} \\
\text { Least square mean } \\
\text { Asenapine }-11.5 \text { SD } \\
0.8 \text { (effect size } 0.32 \text { ) } \\
\text { Olanzapine }-14.6 \\
\text { SD } 0.8 \text { (effect size } \\
0.63 \text { ) }\end{array}$ & $\begin{array}{l}\text { CGI } \\
3 \text { weeks } \\
\text { Least square mean } \\
\text { Asenapine }-1.2 \mathrm{SD} \\
0.10 \\
\text { Olanzapine }-1.5 \mathrm{SD} \\
0.09\end{array}$ & $\begin{array}{l}\text { NR } \\
\text { Overall Withdrawal } \\
\text { Asenapine } 33.0 \% \\
\text { Olanzapine } 21.5 \% \\
\text { Withdrawal Lack of } \\
\text { Efficacy: } \\
\text { Asenapine } 7.6 \% \\
\text { Olanzapine } 6.3 \% \\
\text { Withdrawal AE } \\
\text { Asenapine } 9.2 \% \\
\text { Olanzapine } 3.4 \% \\
\end{array}$ & $\begin{array}{l}\text { Serious Adverse Events } \\
3 \text { weeks } \\
1 \text { Asenapine } \\
\frac{\text { Deaths }}{3 \text { weeks }} \\
1 \text { Asenapine - suicide } \\
\frac{\text { EPS }}{3 \text { weeks }} \\
3.1 \% \text { placebo } \\
10.3 \% \text { Asenapine } \\
6.8 \% \text { Olanzapine }\end{array}$ \\
\hline
\end{tabular}

Abbreviations: CGI=Clinical Global Impressions; EPS=extrapyramidal symptoms; NR=not reported; NS=not significant; RCT=randomized controlled trial; YMRS = Young

Mania Rating Scale 


\section{Appendix Table E12. Strength of evidence assessment: asenapine versus active comparator for acute mania}

\begin{tabular}{|c|c|c|c|c|c|c|c|c|}
\hline Comparison & Outcome & $\begin{array}{l}\text { \# Studies/ } \\
\text { Design } \\
\text { (n analyzed) }\end{array}$ & $\begin{array}{c}\text { Finding or } \\
\text { Summary } \\
\text { Statistic }\end{array}$ & $\begin{array}{c}\text { Study } \\
\text { Limitations }\end{array}$ & Consistency & Directness & Precision & $\begin{array}{c}\text { Overall } \\
\text { Gradel } \\
\text { Conclusion }\end{array}$ \\
\hline $\begin{array}{l}\text { Asenapine vs. } \\
\text { olanzapine }\end{array}$ & $\begin{array}{l}\text { Response } 3 \text { wk } \\
\text { Remission } 3 \text { wk } \\
\text { YMRS } 3 \text { wk } \\
\text { CGI } \\
\text { Withdrawal }\end{array}$ & $\begin{array}{l}2 \text { RCTs } \\
(n=763)\end{array}$ & See table above & High & Consistent & Direct & Imprecise & Insufficient \\
\hline
\end{tabular}

Abbreviations: CGI-BP=Clinical Global Impressions Scale for Bipolar Disorder; NS=not significant; RCT=randomized controlled trial; YMRS = Young Mania Rating Scale Notes:

1. Publication bias for antipsychotics, antidepressants, and behavioral interventions for depressive disorders is suspected.

2. Data were generally imprecise due to missing data from high attrition rates, which was commonly dealt with by Last Observation Carried Forward (LOCF). LOCF requires an

assumption that the health status of patients who dropped out of the trial would not have changed had future observations been recorded, a strong assumption in the context of bipolar disorder research. 


\section{Section 3. Cariprazine for Acute Mania}

Appendix Table E13. Characteristics of eligible studies: cariparazine for acute mania

\begin{tabular}{|c|c|c|c|c|c|c|}
\hline $\begin{array}{l}\text { Study, Year } \\
\text { Design } \\
\text { Location } \\
\text { Funder } \\
\text { Risk of Bias } \\
\text { PMID }\end{array}$ & $\begin{array}{c}\text { \# Randomized } \\
\text { Age (mean) } \\
\text { Sex (\% Female) } \\
\text { Race (\% White) } \\
\text { Diagnosis } \\
\text { (\% BP-I, II, NOS) } \\
\text { Setting }\end{array}$ & $\begin{array}{c}\text { Inclusions } \\
\text { Key Exclusions }\end{array}$ & $\begin{array}{c}\text { Intervention } \\
\text { Dosage }\end{array}$ & $\begin{array}{c}\text { Comparison } \\
\text { Dosage }\end{array}$ & Follow-up Duration & $\begin{array}{c}\text { Outcomes } \\
\text { Reported } \\
\text { Withdrawal (\%) at } \\
\text { endpoint }\end{array}$ \\
\hline $\begin{array}{l}\text { Calabrese, } 201510 \\
\text { RCT } \\
\text { Multisite } \\
3 \text { Continents } \\
\text { Industry } \\
\text { RoB Low } \\
25562205\end{array}$ & $\begin{array}{l}\mathrm{N}=497 \\
\text { Mean Age } 42 \\
\text { Female } 47 \% \\
\text { White } 69 \% \\
\text { BP-I } 100 \% \\
\text { Inpatient (weeks 1-2) } \\
\text { Outpatient (week 3, } \\
\text { subject to inspector } \\
\text { discretion) }\end{array}$ & $\begin{array}{l}\text { Manic/Mixed; } \\
\text { YMRS } \geq 20 \text { AND } \\
\geq 4 \text { on two YMRS } \\
\text { items; } \\
\text { MADRS < } 18 \\
\\
\text { First Manic Episode } \\
\text { Schizoaffective } \\
\text { Substance Abuse } \\
\text { Other Mental Health } \\
\text { Neurological } \\
\text { Disorders } \\
\text { Taking Other Meds }\end{array}$ & $\begin{array}{l}\text { Cariprazine } \\
\text { I1: 3-6 mg/day } \\
\text { I2: } 6-12 \text { mg/day }\end{array}$ & Placebo & 3 weeks & $\begin{array}{l}\text { CGI-S } \\
\text { SAS } \\
\text { YMRS } \\
\text { Withdrawal 11\% }\end{array}$ \\
\hline $\begin{array}{l}\text { Durgam, } 201511 \\
\text { RCT } \\
\text { Multisite } \\
3 \text { Continents } \\
\text { Industry } \\
\text { RoB Moderate } \\
25056368\end{array}$ & $\begin{array}{l}\text { N }=238 \\
\text { Mean Age } 38 \\
\text { Female } 67 \% \\
\text { White } 43 \% \\
\text { BP-I 100\% } \\
\text { Inpatient (weeks 1-2) } \\
\text { Outpatient (week 3, } \\
\text { subject to inspector } \\
\text { discretion) }\end{array}$ & $\begin{array}{l}\text { Manic/Mixed; } \\
\text { YMRS } \geq 20 \text { AND } \\
\geq 4 \text { on two YMRS } \\
\text { items } \\
\text { First Manic Episode } \\
\text { Schizoaffective } \\
\text { Substance Abuse } \\
\text { Other Mental Health } \\
\text { Neurological } \\
\text { Disorders } \\
\text { Taking Other Meds } \\
\text { Pregnant/Nursing } \\
\text { Labs/Other } \\
\text { Conditions }\end{array}$ & $\begin{array}{l}\text { Cariprazine } \\
\text { 3-12 mg/day }\end{array}$ & Placebo & 3 weeks & $\begin{array}{l}\text { YMRS } \\
\text { MADRS } \\
\text { CGI-S } \\
\text { CGI-I } \\
\text { PANSS } \\
\text { AIMS } \\
\text { BARS } \\
\text { SAS } \\
\text { Withdrawal } 37 \%\end{array}$ \\
\hline
\end{tabular}




\begin{tabular}{|c|c|c|c|c|c|c|}
\hline $\begin{array}{c}\text { Study, Year } \\
\text { Design } \\
\text { Location } \\
\text { Funder } \\
\text { Risk of Bias } \\
\text { PMID }\end{array}$ & $\begin{array}{c}\text { \# Randomized } \\
\text { Age (mean) } \\
\text { Sex (\% Female) } \\
\text { Race (\% White) } \\
\text { Diagnosis } \\
\text { (\% BP-I, II, NOS) } \\
\text { Setting }\end{array}$ & $\begin{array}{c}\text { Inclusions } \\
\text { Key Exclusions }\end{array}$ & $\begin{array}{c}\text { Intervention } \\
\text { Dosage }\end{array}$ & $\begin{array}{c}\text { Comparison } \\
\text { Dosage }\end{array}$ & Follow-up Duration & $\begin{array}{c}\text { Outcomes } \\
\text { Reported } \\
\text { Withdrawal (\%) at } \\
\text { endpoint }\end{array}$ \\
\hline \begin{tabular}{|l} 
Sachs, 201513 \\
RCT \\
Multisite \\
2 Continents \\
Industry \\
RoB Moderate \\
25532076
\end{tabular} & $\begin{array}{l}\mathrm{N}=312 \\
\text { Mean Age 36 } \\
\text { Female 36\% } \\
\text { White } 21 \% \\
\text { BP-I 100\% } \\
\text { Inpatient (weeks 1-2) } \\
\text { Outpatient (week 3, } \\
\text { subject to inspector } \\
\text { discretion) }\end{array}$ & $\begin{array}{l}\text { Manic/Mixed; } \\
\text { YMRS } \geq 20 \text { AND } \\
\geq 4 \text { on two YMRS } \\
\text { items; MADRS <18 } \\
\text { Schizoaffective } \\
\text { Substance Abuse } \\
\text { Other Mental Health } \\
\text { Neurological } \\
\text { Disorders } \\
\text { Taking Other Meds } \\
\text { Pregnant/Nursing } \\
\end{array}$ & \begin{tabular}{|l|} 
Cariprazine \\
3-12 mg/day
\end{tabular} & Placebo & 3 weeks & $\begin{array}{l}\text { YMRS } \\
\text { CGI-S } \\
\text { CGI-I } \\
\text { MADRS } \\
\text { PANSS } \\
\text { C-SSRS } \\
\text { AIMS } \\
\text { BARS } \\
\text { SAS } \\
\text { Withdrawal 31\% } \\
\end{array}$ \\
\hline
\end{tabular}

Abbreviations: AIMS=Abnormal Involuntary Movement Scale; BARS=Barnes Akathisia Scale; BAS=Behavioral Approach System; BMI=Body Mass Index; BP=bipolar

disorder; BPRS=Brief Psychiatric Rating Scale; C=comparison; CGI= Clinical Global Impressions; CGI-I=Clinical Global Impressions-Improvement; CGI-S =CGI-Severity; CGIBP=Clinical Global Impressions Scale-Bipolar; CGI-BP-C= Clinical Global Impressions, Bipolar, Change Scale; CGI-BP-S=Clinical Global Impressions, Bipolar, Severity Scale; C-SSRS=Columbia Suicide Severity Rating Scale; DAI-10=Drug Attitutde Inventory, 10 question version; DIEPSS=Drug-Induced Extra-Pyramidal Symptoms Scale;

DSM=Diagnostic and Statistical Manual of Mental Disorders; EPS=extrapyramidal symptoms; ER=Extended Release; ESRS=Extrapyramidal Symptom Rating Scale;

GAF=General Assessment of Functioning Scale; GAS=Global Assessment Scale; HAM-A=Hamilton Scale for Anxiety; HAM-D=Hamilton Scale for Depression; HRQL=Healthrelated quality of life; HRQOL=Health-related quality of life; I=intervention; IDS=Inventory for Depressive Symptoms; LIFE= Longitudinal Interval Follow-up Evaluation; MADRS=Montgomery-Asberg Depression Rating Scale; MAS=Bech-Rafaelsen Mania Rating Scale; MRS=Mania Rating Scale; MSRS=Manic state rating scale; NOS=not otherwise specified; NR=not reported; PANSS=Positive and Negative Syndrome Scale; PRS=Polygenic Risk Scores; PGWB=Psychological General Well-Being Index; PMID=PubMed Identification Number; PRS=Polygenic Risk Scores; Q-LES-Q=Quality of Life Enjoyment and Satisfaction Questionnaire; RCT=randomized controlled trial; ROB=risk of bias; SADS-C= Schedule for Affective Disorders and Schizophrenia-Change version; SAE=Serious Adverse Events; SAS=Simpson Angus Scale; SF-12=12-Item Short Form Health Survey; SF-36=36-Item Short Form Health Survey; SLICE=Streamlined Longitudinal Interview Clinical Evaluation; T=Trial; YMRS = Young Mania Rating Scale 
Appendix Table E14. Summary risk of bias assessments: cariprazine for acute mania

\begin{tabular}{|c|l|l|l|}
\hline \multicolumn{1}{|c|}{ Drug } & \multicolumn{1}{c|}{$\begin{array}{c}\text { Study } \\
\text { Funding Source } \\
\text { PMID }\end{array}$} & $\begin{array}{c}\text { Overall Risk of } \\
\text { Bias Assessment }\end{array}$ & Rationale \\
\hline Cariprazine & $\begin{array}{l}\text { Durgam, 201511 } \\
\text { Industry } \\
25056368\end{array}$ & Moderate & $\begin{array}{l}\text { The large dropout rate is likely to create some bias. Lack of disclosure of methods to allocate } \\
\text { and protect the blind also increases the risk. }\end{array}$ \\
\cline { 2 - 4 } & $\begin{array}{l}\text { Calabrese, 2015 } \\
\text { Industry } \\
25562205\end{array}$ & Low & $\begin{array}{l}\text { Procedures for concealing allocation and blinding participants and providers are not } \\
\text { described, but, the study appears to have been well executed, fully reported, and } \\
\text { investigators have taken steps to ensure bias was minimized, like pattern mixture modeling. }\end{array}$ \\
\cline { 2 - 5 } & $\begin{array}{l}\text { Sachs, 2015 } \\
\text { Industry } \\
25532076\end{array}$ & Moderate & $\begin{array}{l}\text { A moderately high dropout rate combined with a lack of disclosure for the methods of } \\
\text { allocation and concealment create strong conditions where bias may be present. }\end{array}$ \\
\hline
\end{tabular}

Abbreviations: ITT=Intention to Treat; PMID=PubMed Identification Number; LOCF=last observation carried forward 


\section{Cariprazine Forest Plots}

Outcomes in studies assessed as having a high risk of bias, or low to moderate risk of bias but at least 40 percent attrition, are presented in grey tones. Both fixed-effect models and random-effects models are presented. We calculated fixed-effect models to provide a charitable estimate of the average effect among completed trials. However, we base our main conclusions on the random-effects models.

Appendix Figure E27. Cariprazine vs. placebo - response

Odds Ratio of Response ( $>50 \%$ Reduction in YMRS) at 3 Weeks

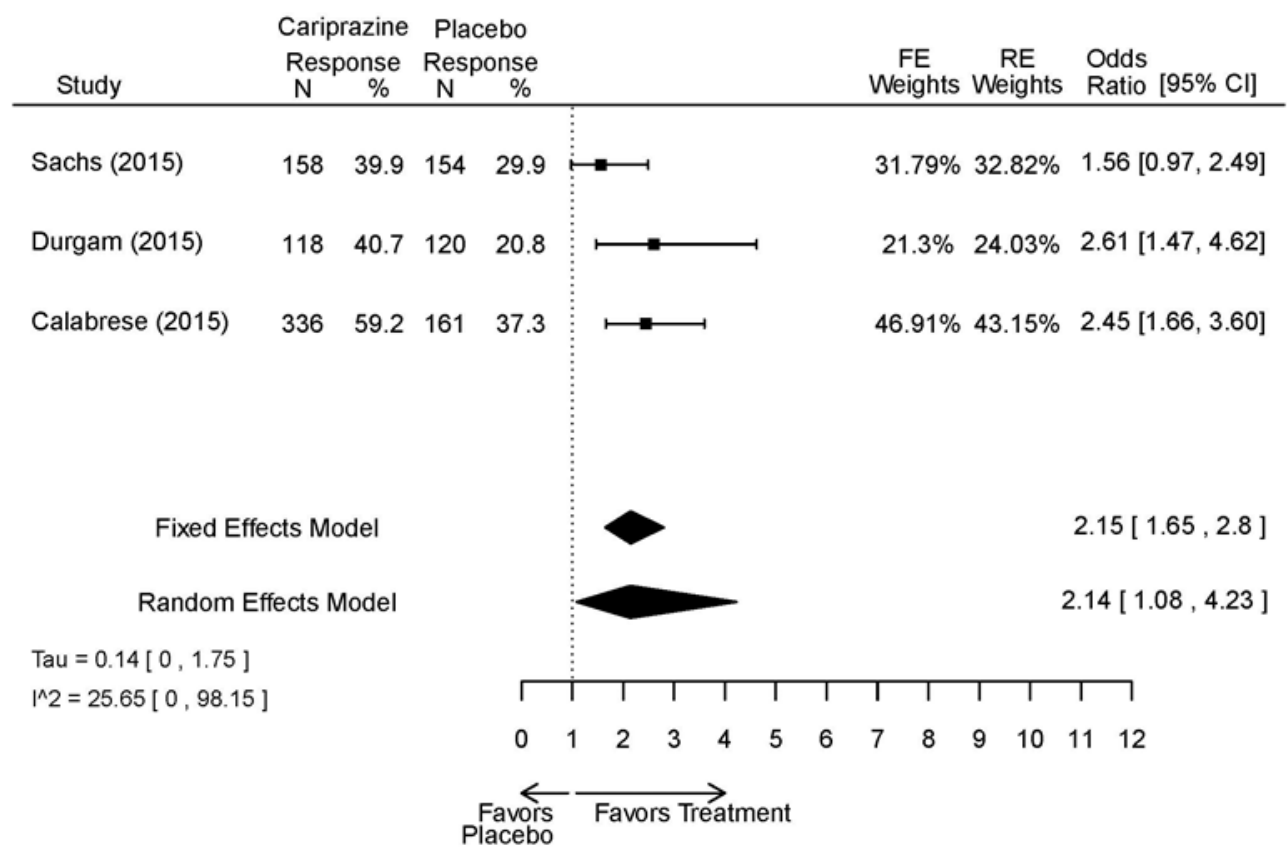


Appendix Figure E28. Cariprazine vs. placebo - remission

Odds Ratio of Remission (YMRS $<=12$ ) at 3 Weeks

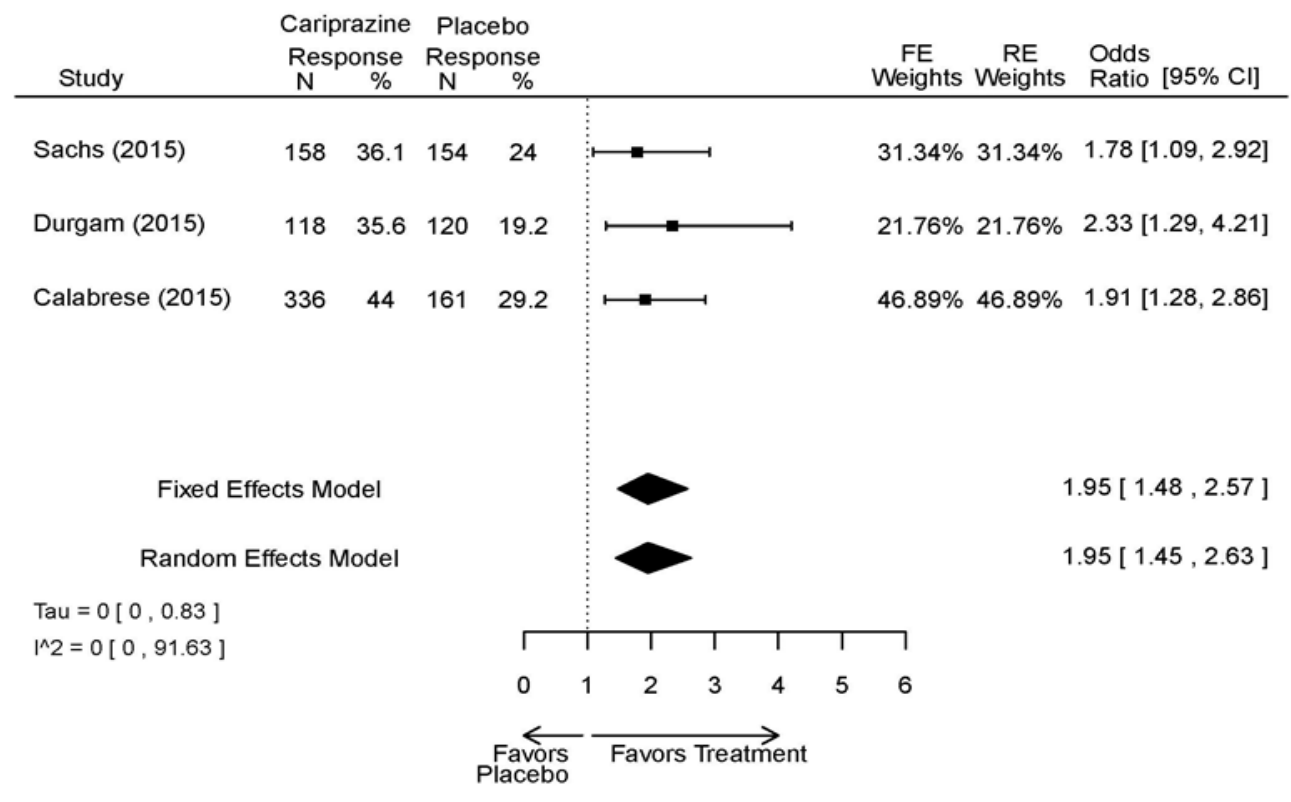

Appendix Figure E29. Cariprazine vs. placebo - YMRS

Difference in Mean Change in YMRS from Baseline to 3 Weeks

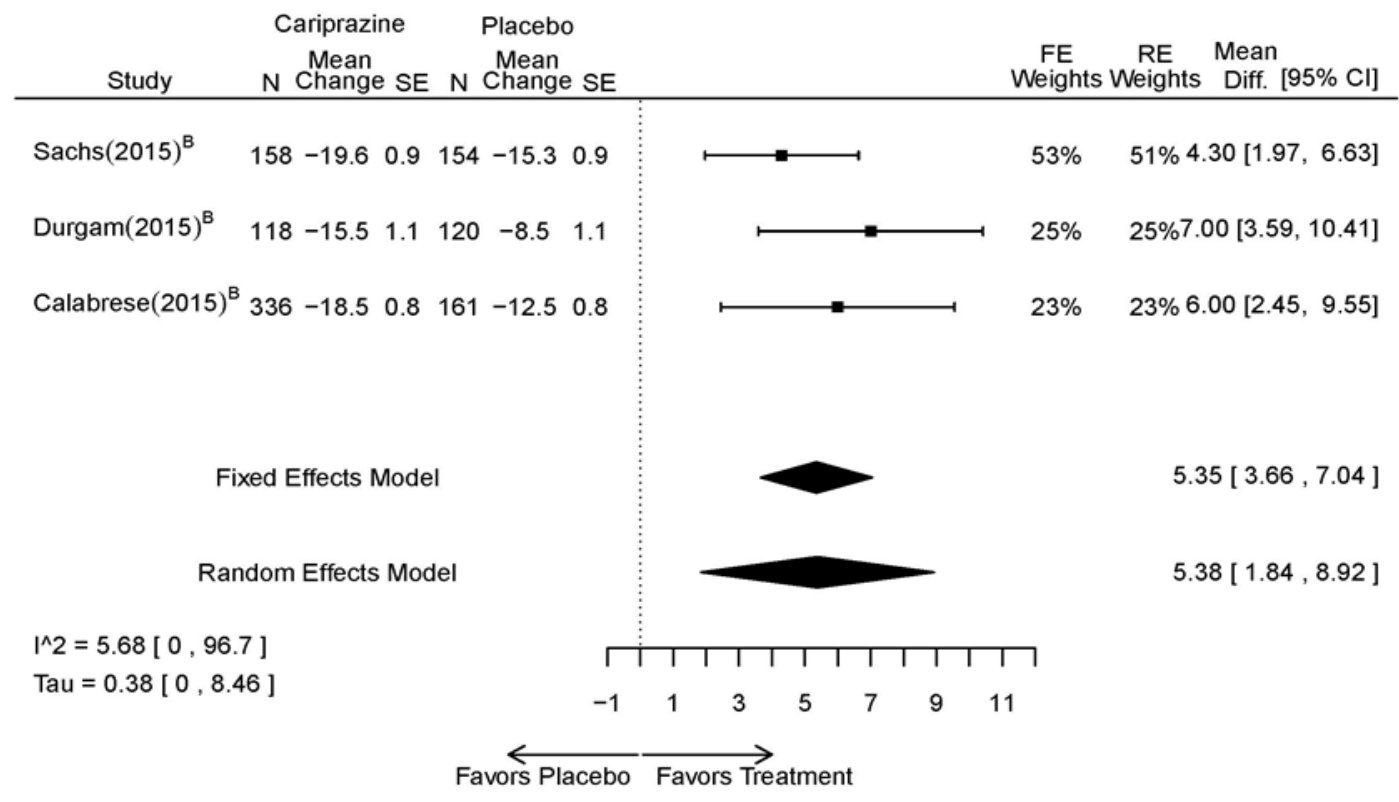


Appendix Figure E30. Cariprazine vs. placebo - CGI-BP-S

Difference in Mean Change in CGI-BP-S (Overall) from Baseline to 3 Weeks

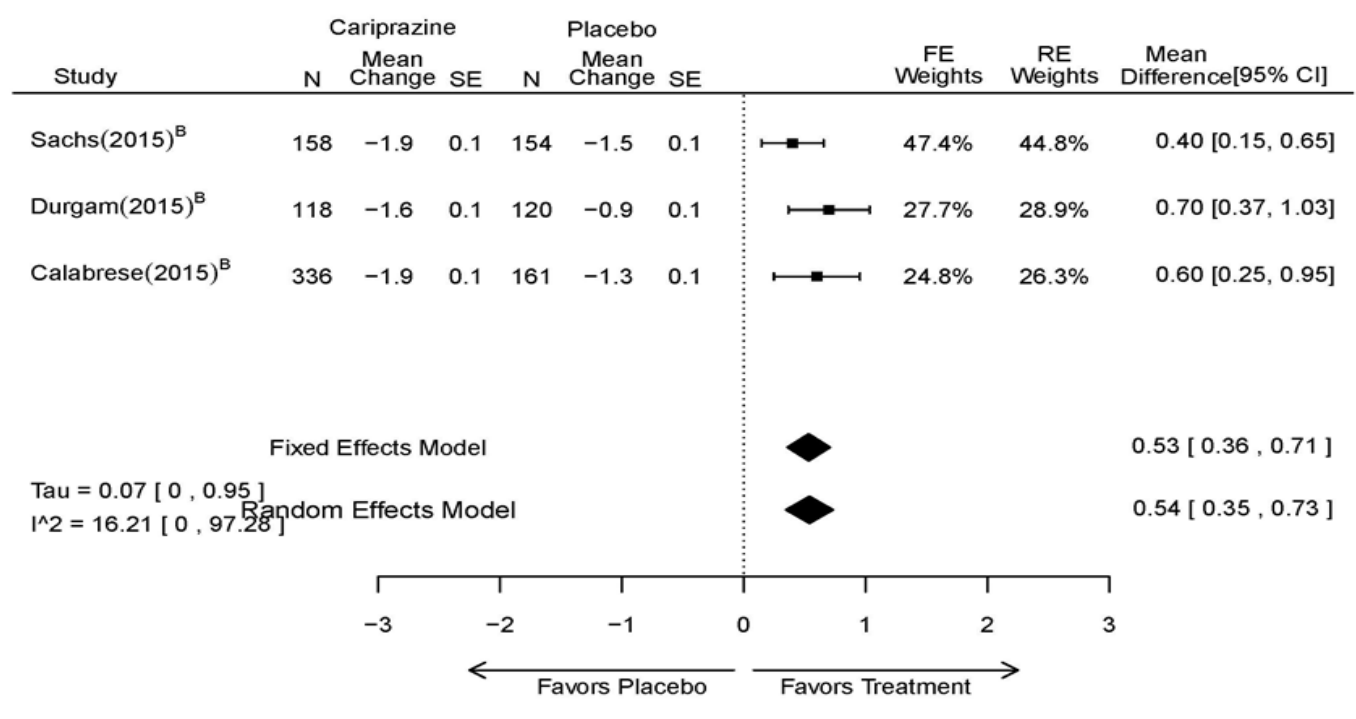

Appendix Figure E31. Cariprazine vs. placebo - withdrawal lack of efficacy

Odds Ratio of Withdrawal due to Lack of Efficacy at 3 Weeks

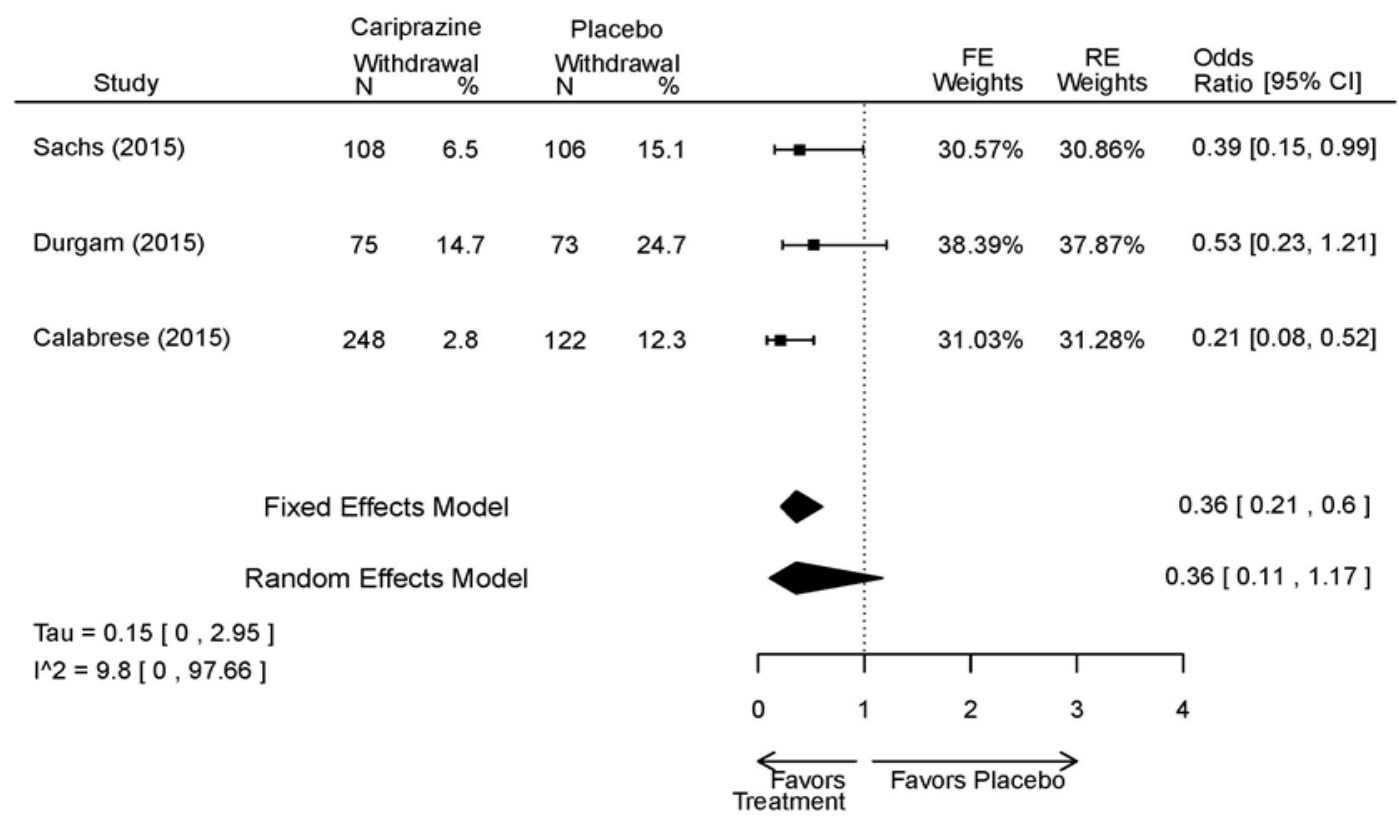


Appendix Figure E32. Cariprazine vs. placebo - withdrawal adverse events

Odds Ratio of Withdrawal due to Adverse Events at 3 Weeks

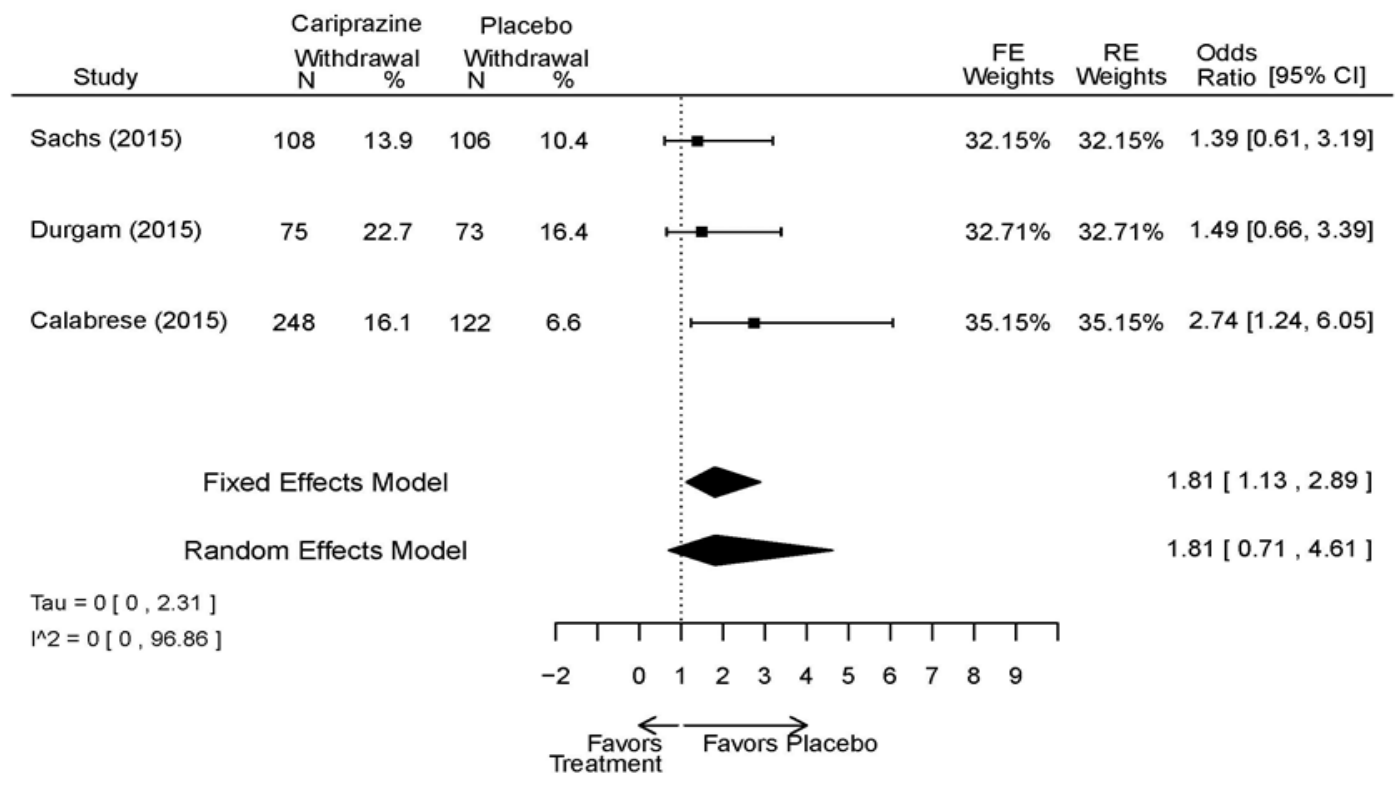

Appendix Figure E33. Cariprazine vs. placebo - overall withdrawal

Odds Ratio of Overall Withdrawal

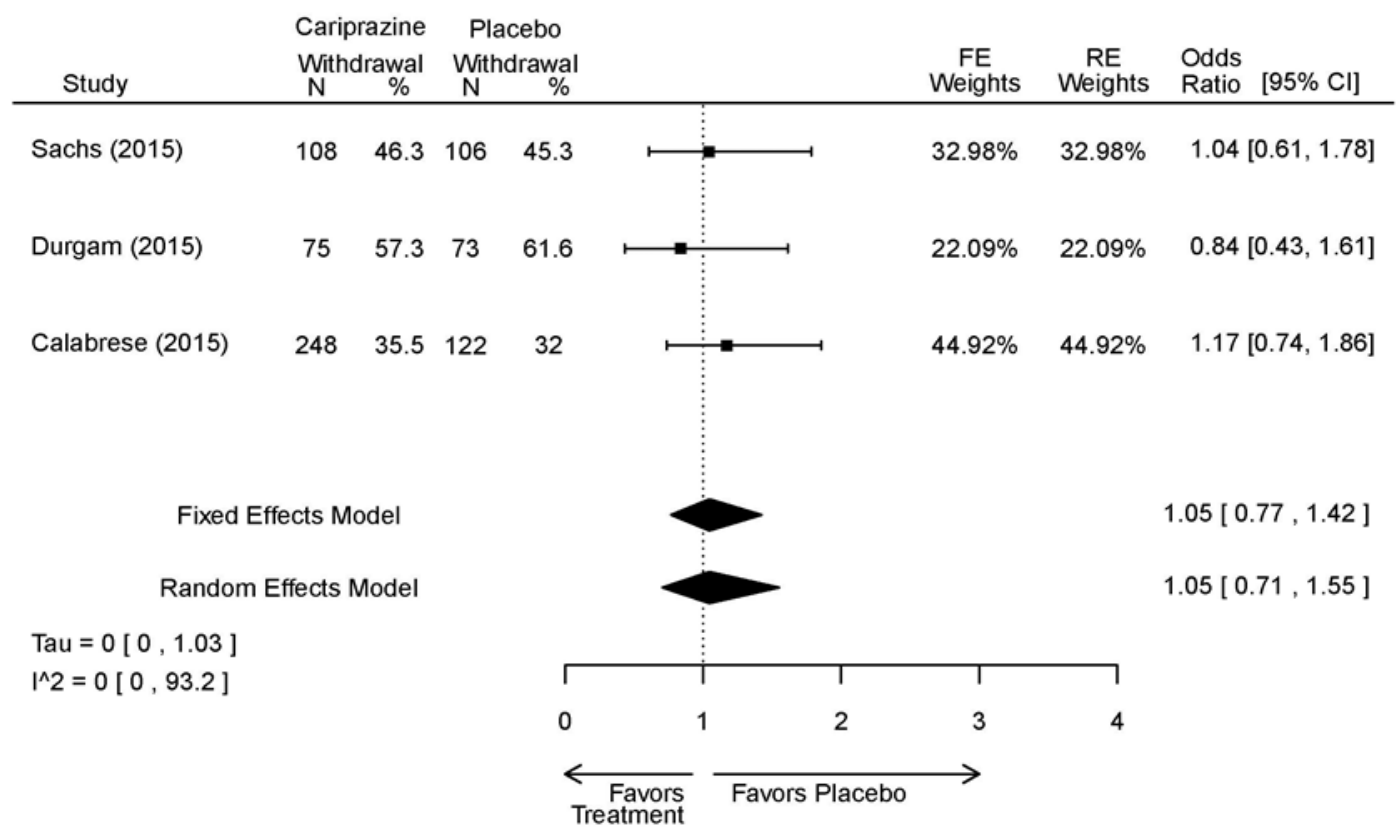




\begin{tabular}{|c|c|c|c|c|c|c|}
\hline Drug & $\begin{array}{l}\text { Study } \\
\text { PMID } \\
\text { RoB } \\
\end{array}$ & Responder/Remitter & Symptom & Function & Other & AE \\
\hline \multirow[t]{2}{*}{ Cariprazine } & $\begin{array}{l}\text { Durgam, } \\
2015^{11} \\
\text { Industry } \\
25056368\end{array}$ & $\begin{array}{l}\text { See forest plot E27 } \\
\text { above for response. }\end{array}$ & $\begin{array}{l}\text { See forest plot E29 } \\
\text { above for YMRS. }\end{array}$ & $\begin{array}{l}\text { See forest plot E30 } \\
\text { above for CGI. }\end{array}$ & $\begin{array}{l}\text { See forest plots E31, } \\
\text { E32, E33 above for } \\
\text { Withdrawals. }\end{array}$ & $\begin{array}{l}\text { SAE } \\
\text { Placebo: } 5 \text { patients } \\
\text { Cariprazine: } 4 \text { patients } \\
\text { No suicide attempts } \\
\text { No difference between } \\
\text { groups in suicide } \\
\text { ideation } \\
\text { EPS } \\
\text { Placebo: 1\% } \\
\text { Cariprazine groups: } \\
\text { 11\% or 14\% } \\
\text { Akasthesia } \\
\text { Placebo: } 4 \% \\
\text { Cariprazine groups:20\% } \\
\text { or 23\% }\end{array}$ \\
\hline & $\begin{array}{l}\text { Calabrese, } \\
2015^{10} \\
\text { Industry } \\
25562205\end{array}$ & $\begin{array}{l}\text { See forest plot E27 } \\
\text { above for response. }\end{array}$ & $\begin{array}{l}\text { See forest plot E29 } \\
\text { above for YMRS. }\end{array}$ & $\begin{array}{l}\text { See forest plot E30 } \\
\text { above for CGI. }\end{array}$ & $\begin{array}{l}\text { See forest plots E31, } \\
\text { E32, E33 above for } \\
\text { Withdrawals. }\end{array}$ & $\begin{array}{l}\text { SAE } \\
\text { Placebo: } 0 \text { patients } \\
\text { Cariprazine: } 4 \text { patients } \\
\text { No suicide attempts } \\
\text { I placebo patients } \\
\text { reported suicide ideation } \\
\text { EPS } \\
\text { Placebo: } 1 \text { patient } \\
\text { Cariprazine: } 19 \text { patients } \\
\text { Akasthesia } \\
\text { Placebo: } 7 \text { patient } \\
\text { Cariprazine: } 26 \text { patients }\end{array}$ \\
\hline
\end{tabular}




\begin{tabular}{|c|c|c|c|c|c|c|}
\hline Drug & $\begin{array}{l}\text { Study } \\
\text { PMID } \\
\text { RoB }\end{array}$ & Responder/Remitter & Symptom & Function & Other & $\overline{A E}$ \\
\hline & $\begin{array}{l}\text { Sachs, } 2015^{13} \\
\text { Industry } \\
25532076\end{array}$ & $\begin{array}{l}\text { See forest plot E27 } \\
\text { above for response. }\end{array}$ & $\begin{array}{l}\text { See forest plot E29 } \\
\text { above for YMRS. }\end{array}$ & $\begin{array}{l}\text { See forest plot E30 } \\
\text { above for CGI. }\end{array}$ & $\begin{array}{l}\text { See forest plots E31, } \\
\text { E32, E33 above for } \\
\text { Withdrawals. }\end{array}$ & $\begin{array}{l}\text { SAE } \\
\text { Placebo: } 3 \text { patients } \\
\text { Cariprazine: } 5 \text { patients } \\
\text { No suicide attempts } \\
\text { No difference between } \\
\text { groups in suicide } \\
\text { ideation } \\
\text { EPS } \\
\text { Placebo: } 6 \text { patients } \\
\text { Cariprazine: } 30 \text { patients } \\
\text { Akasthesia } \\
\text { Placebo: } 8 \text { patient } \\
\text { Cariprazine: } 36 \text { patients }\end{array}$ \\
\hline
\end{tabular}

Abbreviations: $\mathrm{AE}=$ Adverse Events; ANCOVA=Analysis of Covariance; CGI=Clinical Global Impressions Scale; CI=Confidence Interval; EPS=extrapyramidal symptoms; ESRS=Extrapyrimidal Symptom Rating Scale; GAS=Global Assessment Scale; MADRS=Montgomery-Asberg Depression Rating Scale; NR=Not reported; NS=not significant; OR=Odds Ratio; PMID=PubMed Identification Number; ROB=Risk of Bias; SAE=Serious Adverse Events; YMRS = Young Mania Rating Scale

\section{Appendix Table E16. Strength of evidence assessment: cariprazine versus placebo for acute mania}

\begin{tabular}{|c|c|c|c|c|c|c|c|c|}
\hline Comparison & Outcome & $\begin{array}{c}\text { \# Studies/ } \\
\text { Design } \\
\text { (n analyzed) }\end{array}$ & $\begin{array}{c}\text { Finding or } \\
\text { Summary } \\
\text { Statistic }\end{array}$ & $\begin{array}{c}\text { Study } \\
\text { Limitations }\end{array}$ & Consistency & Directness & Precision & $\begin{array}{c}\text { Overall } \\
\text { Gradel } \\
\text { Conclusion }\end{array}$ \\
\hline \multirow{3}{*}{$\begin{array}{l}\text { Cariprazine } \\
\text { vs. placebo }\end{array}$} & Response 3 wks & $\begin{array}{l}3 \mathrm{RCT} \\
(n=1,047)\end{array}$ & $\begin{array}{l}\text { Favors } \\
\text { Cariprazine } \\
\text { OR } 2.14(95 \% \\
\text { Cl } 1.08,4.23)\end{array}$ & Moderate & Consistent & Direct & Imprecise & Low \\
\hline & Remission 3 wks & $\begin{array}{l}3 \mathrm{RCT} \\
(\mathrm{n}=1,047)\end{array}$ & $\begin{array}{l}\text { Favors } \\
\text { Cariprazine } \\
\text { OR } 1.95(95 \% \\
\text { Cl 1.45, 2.63) }\end{array}$ & Moderate & Consistent & Direct & Imprecise & Low \\
\hline & YMRS 3 wks & $\begin{array}{l}3 \text { RCT } \\
(n=1,047)\end{array}$ & $\begin{array}{l}\text { Favors } \\
\text { Cariprazine } \\
\text { MD 5.38 (95\% } \\
\text { Cl 1.84, 8.92) }\end{array}$ & Moderate & Consistent & Direct & Imprecise & Low \\
\hline
\end{tabular}




\begin{tabular}{|c|c|c|c|c|c|c|c|c|}
\hline Comparison & Outcome & $\begin{array}{c}\text { \# Studies/ } \\
\text { Design } \\
\text { (n analyzed) }\end{array}$ & $\begin{array}{l}\text { Finding or } \\
\text { Summary } \\
\text { Statistic }\end{array}$ & $\begin{array}{c}\text { Study } \\
\text { Limitations }\end{array}$ & Consistency & Directness & Precision & $\begin{array}{c}\text { Overall } \\
\text { Gradel } \\
\text { Conclusion }\end{array}$ \\
\hline & CGI-BP-S 3 wk & $\begin{array}{l}3 \mathrm{RCT} \\
(\mathrm{n}=1,047)\end{array}$ & $\begin{array}{l}\text { Favors } \\
\text { Cariprazine } \\
\text { MD } 0.54(95 \% \\
\text { Cl } 0.35,0.73) \\
\end{array}$ & Moderate & Consistent & Direct & Imprecise & Low \\
\hline & $\begin{array}{l}\text { Withdrawal - AE, } \\
\text { Lack of Efficacy, } \\
\text { Overall }\end{array}$ & $\begin{array}{l}3 \mathrm{RCT} \\
(\mathrm{n}=1,047)\end{array}$ & NS & Moderate & Consistent & Direct & Imprecise & Low \\
\hline
\end{tabular}

Abbreviations: CGI-BP=Clinical Global Impressions Scale for Bipolar Disorder; NS=not significant; RCT=randomized controlled trial; YMRS = Young Mania Rating Scale Notes:

1. Publication bias for antipsychotics, antidepressants, and behavioral interventions for depressive disorders is suspected.

2. Data were generally imprecise due to missing data from high attrition rates, which was commonly dealt with by Last Observation Carried Forward (LOCF). LOCF requires an assumption that the health status of patients who dropped out of the trial would not have changed had future observations been recorded, a strong assumption in the context of bipolar disorder research.

\section{Section 4. Haloperidol for Acute Mania}

Appendix Table E17. Characteristics of eligible studies: haloperidol for acute mania

\begin{tabular}{|c|c|c|c|c|c|c|}
\hline $\begin{array}{l}\text { Study, Year } \\
\text { Design } \\
\text { Location } \\
\text { Funder } \\
\text { Risk of Bias } \\
\text { PMID }\end{array}$ & $\begin{array}{c}\text { \# Randomized } \\
\text { Age (mean) } \\
\text { Sex (\% Female) } \\
\text { Race (\% White) } \\
\text { Diagnosis } \\
\text { (\% BP-I, II, NOS) } \\
\text { Setting }\end{array}$ & $\begin{array}{l}\text { Inclusions } \\
\text { Key Exclusions }\end{array}$ & $\begin{array}{l}\text { Intervention } \\
\text { Dosage }\end{array}$ & $\begin{array}{c}\text { Comparison } \\
\text { Dosage }\end{array}$ & Follow-up Duration & $\begin{array}{c}\text { Outcomes } \\
\text { Reported } \\
\text { Withdrawal (\%) at } \\
\text { endpoint }\end{array}$ \\
\hline $\begin{array}{l}\text { Mclntyre, } 2005^{14} \\
\text { RCT } \\
\text { Multisite } \\
3 \text { Continents } \\
\text { Industry } \\
\text { Moderate } \\
16139175\end{array}$ & $\begin{array}{l}\mathrm{N}=299 \\
\text { Mean Age } 42 \\
\text { Female 63\% } \\
\text { Race NR } \\
\text { BP-I 100\% } \\
\text { Inpatient }\end{array}$ & $\begin{array}{l}\text { Manic } \\
\text { YMRS } \geq 20 \\
\text { CGI-BP } \geq 4 \\
\\
\text { First Manic Episode } \\
\text { Substance Abuse } \\
\text { Neurological } \\
\text { Disorders } \\
\text { Taking Other Meds } \\
\text { Pregnant/Nursing } \\
\text { Labs/Other } \\
\text { Conditions }\end{array}$ & $\begin{array}{l}\text { Haloperidol } \\
\text { Starting } 2 \mathrm{mg} / \text { day up } \\
\text { to } 8 \mathrm{mg} / \text { day }\end{array}$ & $\begin{array}{l}\text { C1: Placebo } \\
\text { C2: Quetiapine } \\
\text { 100mg/day } \\
\text { increasing by } 100 \mathrm{mg} \\
\text { up to } 800 \mathrm{mg} / \text { day }\end{array}$ & 12 weeks & $\begin{array}{l}\text { Remission Rates } \\
\text { Adverse Events } \\
\text { Efficacy } \\
\text { YMRS } \\
\text { CGI } \\
\text { PANSS } \\
\text { MADRS } \\
\text { GAS } \\
\text { Withdrawal } 50 \%\end{array}$ \\
\hline
\end{tabular}




\begin{tabular}{|c|c|c|c|c|c|c|}
\hline $\begin{array}{l}\text { Study, Year } \\
\text { Design } \\
\text { Location } \\
\text { Funder } \\
\text { Risk of Bias } \\
\text { PMID }\end{array}$ & $\begin{array}{c}\text { \# Randomized } \\
\text { Age (mean) } \\
\text { Sex (\% Female) } \\
\text { Race (\% White) } \\
\text { Diagnosis } \\
\text { (\% BP-I, II, NOS) } \\
\text { Setting }\end{array}$ & $\begin{array}{c}\text { Inclusions } \\
\text { Key Exclusions }\end{array}$ & $\begin{array}{c}\text { Intervention } \\
\text { Dosage }\end{array}$ & $\begin{array}{c}\text { Comparison } \\
\text { Dosage }\end{array}$ & Follow-up Duration & $\begin{array}{c}\text { Outcomes } \\
\text { Reported } \\
\text { Withdrawal (\%) at } \\
\text { endpoint }\end{array}$ \\
\hline $\begin{array}{l}\text { Smulevich, } 2005^{15} \\
\text { RCT } \\
\text { Multisite } \\
\text { Location NR } \\
\text { Industry } \\
\text { Moderate } \\
15572276\end{array}$ & $\begin{array}{l}\mathrm{N}=438 \\
\text { Mean Age } 40 \\
\text { Female } 47 \% \\
\text { White } 65 \% \\
\text { BP-I } 100 \% \\
\text { Outpatient }\end{array}$ & \begin{tabular}{|l} 
Manic \\
YMRS $\geq 20$ \\
MADRS $\leq 20$ \\
\\
First Manic Episode \\
Schizoaffective \\
Substance Abuse \\
Other Mental Health \\
Taking Other Meds
\end{tabular} & $\begin{array}{l}\text { Haloperidol } \\
\text { Initiated at } 4 \mathrm{mg} / \text { day } \\
\text { increased by } \\
2 \mathrm{mg} / \text { day up to } 12 \\
\mathrm{mg} / \text { day }\end{array}$ & $\begin{array}{l}\text { C1: Placebo } \\
\text { C2: Risperidone } \\
\text { Initiated at } 2 \mathrm{mg} / \text { day } \\
\text { increased by } \\
\text { 1mg/day up to } \\
6 \mathrm{mg} / \text { day }\end{array}$ & $\begin{array}{l}12 \text { weeks (12 week } \\
\text { outcomes excluded } \\
\text { due to attrition of } \\
\text { Haloperidol arm) }\end{array}$ & \begin{tabular}{|l|} 
Efficacy \\
YMRS \\
CGI \\
GAS \\
MADRS \\
BPRS \\
Extrapyramidal \\
symptoms \\
ESRS \\
Withdrawal \\
$48 \%$ at 12 weeks \\
$12 \%$ at 3 weeks
\end{tabular} \\
\hline
\end{tabular}

Abbreviations: AIMS=Abnormal Involuntary Movement Scale; BARS=Barnes Akathisia Scale; BAS=Behavioral Approach System; BMI=Body Mass Index; BP=bipolar disorder; BPRS=Brief Psychiatric Rating Scale; C=comparison; CGI= Clinical Global Impressions; CGI-I=Clinical Global Impressions-Improvement; CGI-S =CGI-Severity; CGIBP=Clinical Global Impressions Scale-Bipolar; CGI-BP-C= Clinical Global Impressions, Bipolar, Change Scale; CGI-BP-S=Clinical Global Impressions, Bipolar, Severity Scale; C-SSRS=Columbia Suicide Severity Rating Scale; DAI-10=Drug Attitutde Inventory, 10 question version; DIEPSS=Drug-Induced Extra-Pyramidal Symptoms Scale; DSM=Diagnostic and Statistical Manual of Mental Disorders; EPS=extrapyramidal symptoms; ER=Extended Release; ESRS=Extrapyramidal Symptom Rating Scale; GAF=General Assessment of Functioning Scale; GAS=Global Assessment Scale; HAM-A=Hamilton Scale for Anxiety; HAM-D=Hamilton Scale for Depression; HRQL=Healthrelated quality of life; HRQOL=Health-related quality of life; I=intervention; IDS=Inventory for Depressive Symptoms; LIFE= Longitudinal Interval Follow-up Evaluation; MADRS=Montgomery-Asberg Depression Rating Scale; MAS=Bech-Rafaelsen Mania Rating Scale; MRS=Mania Rating Scale; MSRS=Manic state rating scale; NOS=not otherwise specified; NR=not reported; PANSS=Positive and Negative Syndrome Scale; PRS=Polygenic Risk Scores; PGWB=Psychological General Well-Being Index; PMID=PubMed Identification Number; PRS=Polygenic Risk Scores; Q-LES-Q=Quality of Life Enjoyment and Satisfaction Questionnaire; RCT=randomized controlled trial; ROB=risk of bias; SADS-C= Schedule for Affective Disorders and Schizophrenia-Change version; SAE=Serious Adverse Events; SAS=Simpson Angus Scale; SF-12=12-Item Short Form Health Survey; SF-36=36-Item Short Form Health Survey; SLICE=Streamlined Longitudinal Interview Clinical Evaluation; T=Trial; YMRS = Young Mania Rating Scale 
Appendix Table E18. Summary risk of bias assessments: haloperidol for acute mania

\begin{tabular}{|l|l|l|l|}
\hline \multicolumn{1}{|c|}{ Drug } & \multicolumn{1}{|c|}{$\begin{array}{c}\text { Study } \\
\text { Funding Source } \\
\text { PMID }\end{array}$} & $\begin{array}{c}\text { Overall Risk of } \\
\text { Bias Assessment }\end{array}$ & \\
\hline Haloperidol & $\begin{array}{l}\text { McIntyre, 2005 } \\
\text { Industry } \\
16139175\end{array}$ & High & $\begin{array}{l}\text { High dropout rates (46\% overall) create a likelihood of bias, lacks some core information on } \\
\text { how allocation was concealed and blinding of treatment staff and raters was maintained. }\end{array}$ \\
\cline { 2 - 4 } & $\begin{array}{l}\text { Smulevich, 2005 } \\
\text { Industry } \\
15572276\end{array}$ & Moderate & $\begin{array}{l}\text { Open drugs given to some participants. Pools results for blinded and unblinded without } \\
\text { establishing similarity of groups }\end{array}$ \\
\cline { 2 - 4 } & $\begin{array}{l}\text { Sachs, } 2002^{17} \\
\text { Industry } \\
12091192\end{array}$ & High & $\begin{array}{l}\text { Lacks randomization and blinding procedures. High dropout rates across all arms (46\% } \\
\text { overall) }\end{array}$ \\
\cline { 2 - 4 } & $\begin{array}{l}\text { Vieta, 2010 } \\
\text { Industry } \\
20565430\end{array}$ & High & Large dropout rate among all study arms, across all time periods; raters may not be blinded \\
\hline
\end{tabular}

Abbreviations: ITT=Intention to Treat; PMID=PubMed Identification Number; LOCF=last observation carried forward 


\section{Haloperidol Forest Plots}

Outcomes in studies assessed as having a high risk of bias, or low to moderate risk of bias but at least 40 percent attrition, are presented in grey tones. Both fixed-effect models and random-effects models are presented. We calculated fixed-effect models to provide a charitable estimate of the average effect among completed trials. However, we base our main conclusions on the random-effects models.

Appendix Figure E34. Haloperidol vs. placebo - response

Odds Ratio of Response ( $>50 \%$ Reduction in YMRS) at 3 Weeks

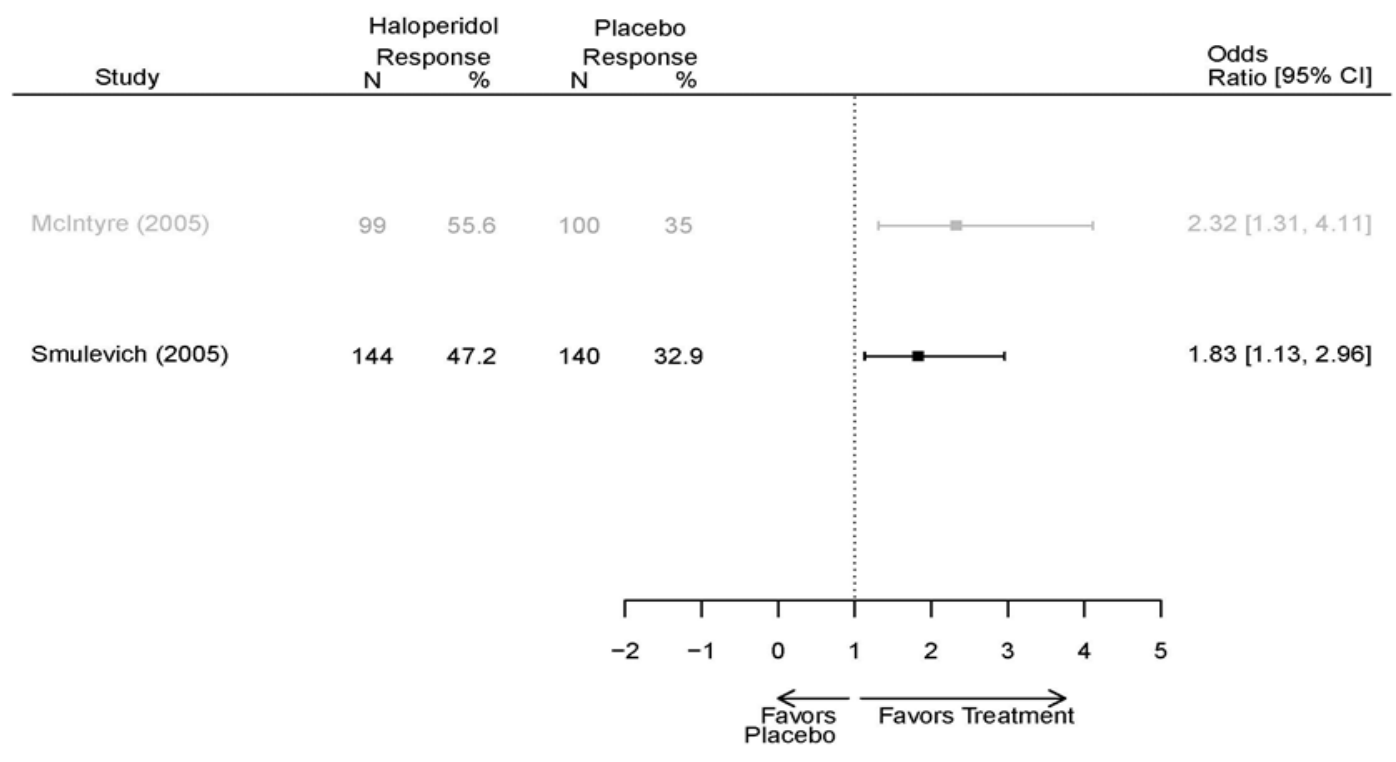


Appendix Figure E35. Haloperidol vs. placebo - YMRS

Difference in Mean Change in YMRS from Baseline to Last Measurement

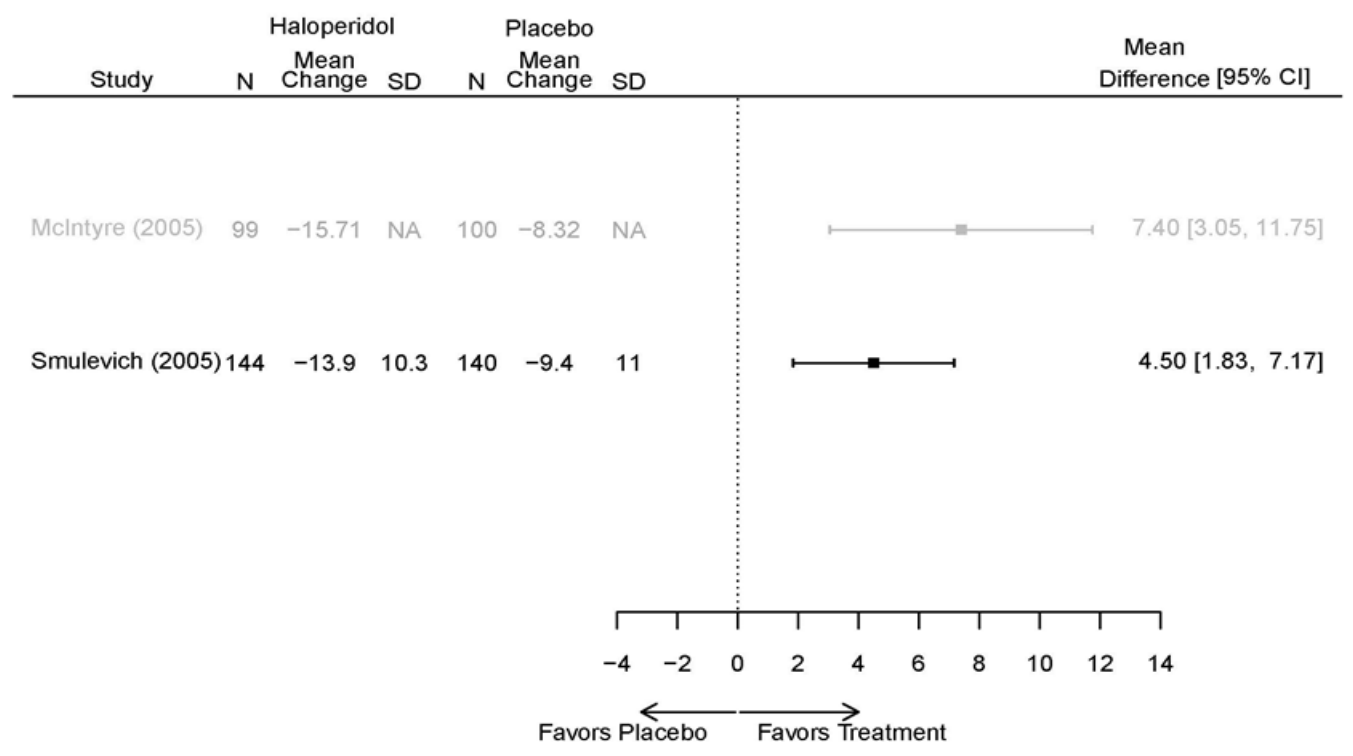

Appendix Figure E36. Haloperidol vs. placebo - CGI-BP-S

Difference in Mean Change in CGI-BP-S (Overall)

from Baseline to Last Measurement

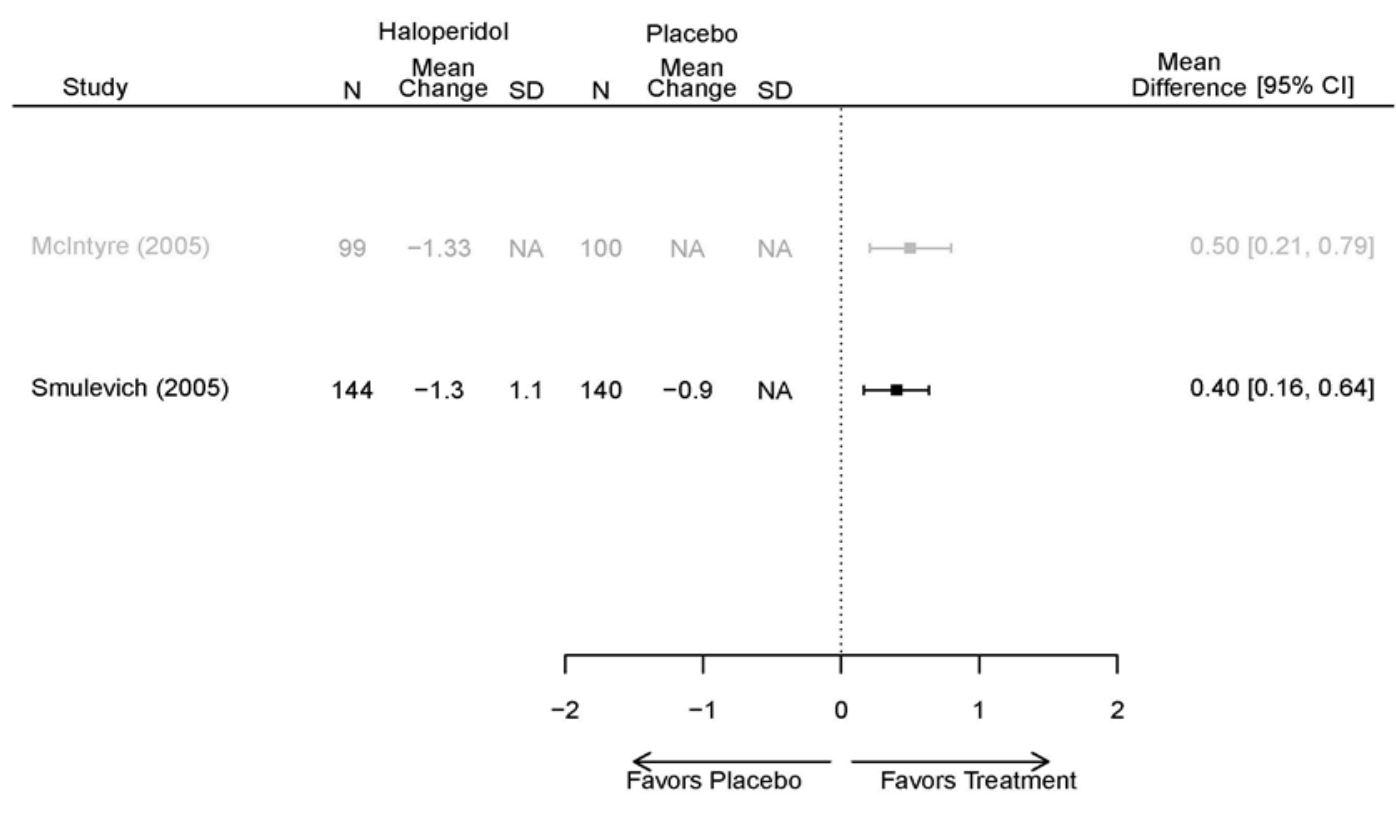


Appendix Figure E37. Haloperidol vs. placebo - withdrawal lack of efficacy Odds Ratio of Withdrawal due to Lack of Efficacy at 3 Weeks

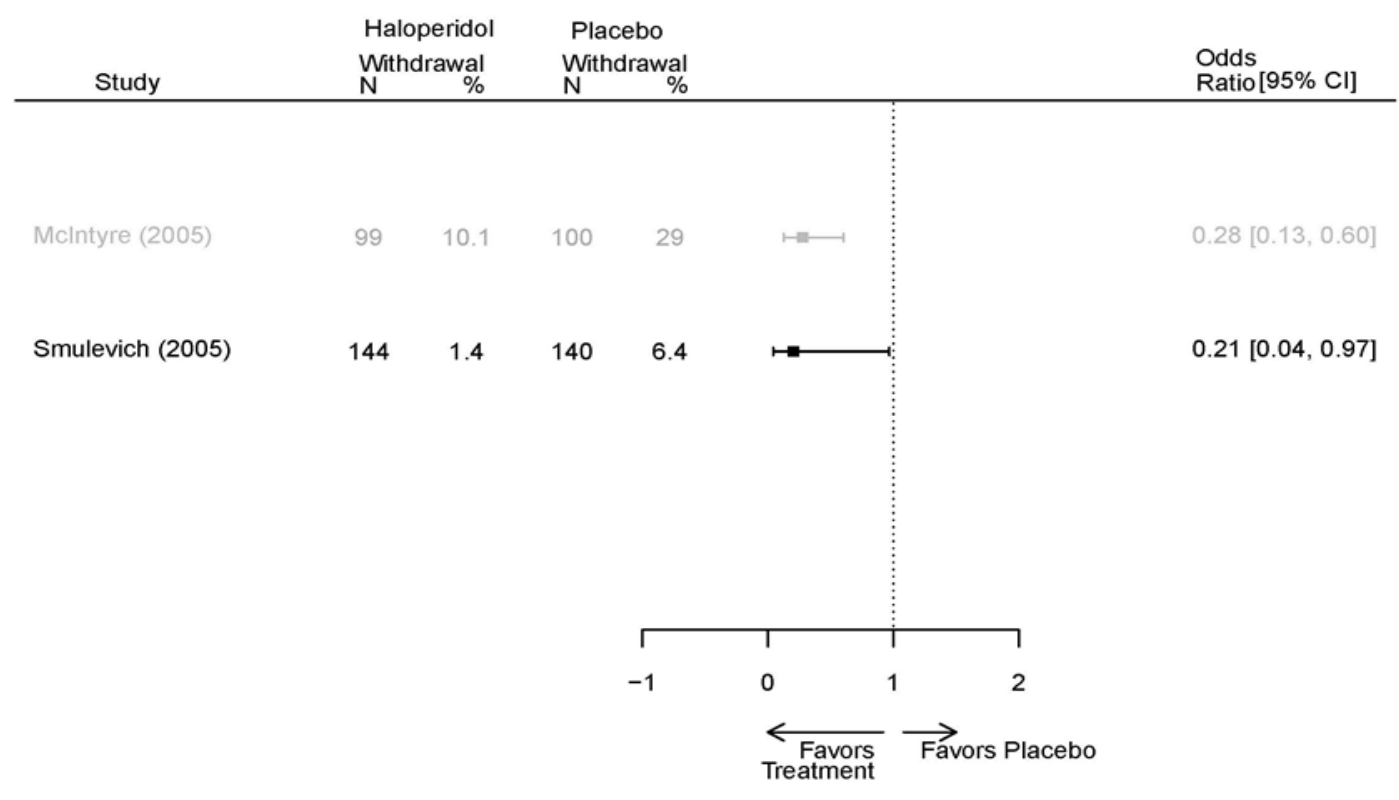

Appendix Figure E38. Haloperidol vs. placebo - withdrawal adverse events

Odds Ratio of Withdrawal due to Adverse Events at 3 Weeks

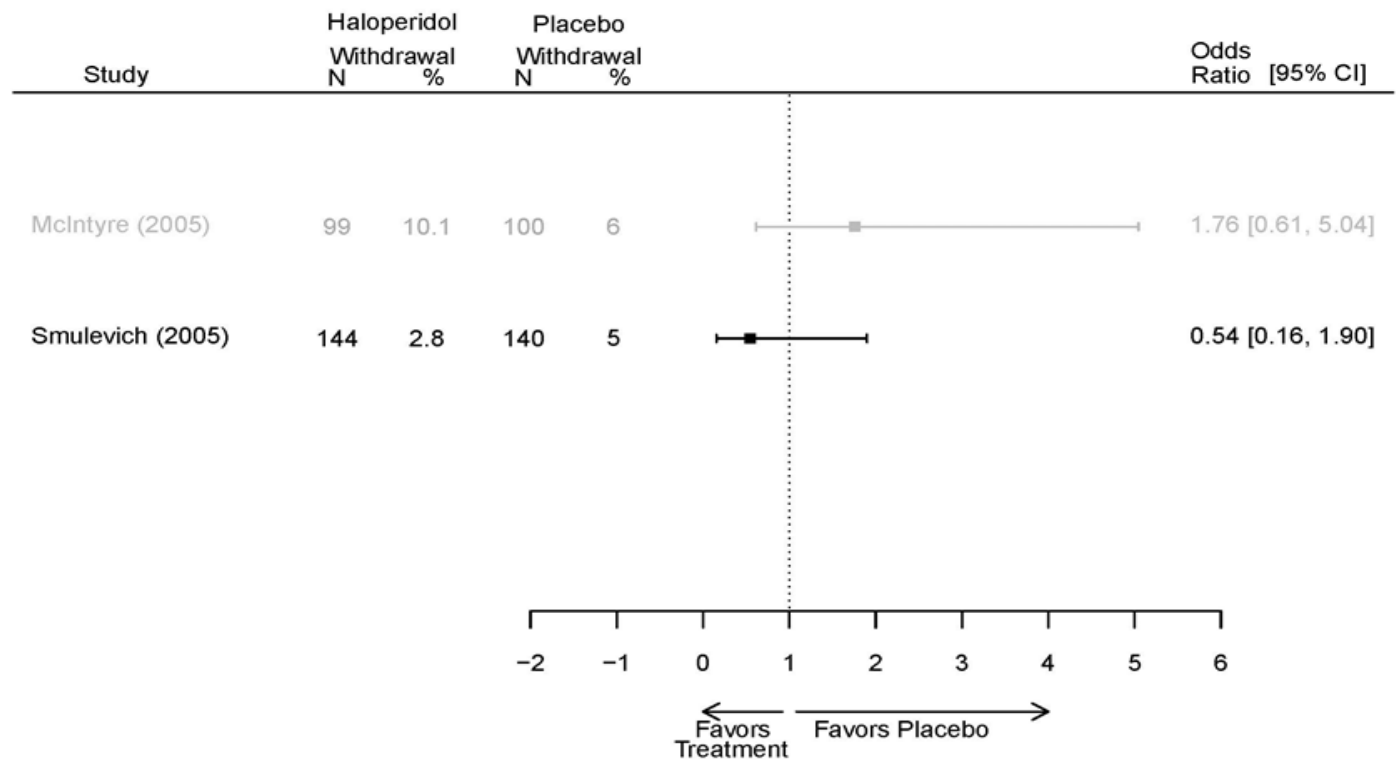


Appendix Figure E39. Haloperidol vs. placebo - overall withdrawal

Odds Ratio of Overall Withdrawal

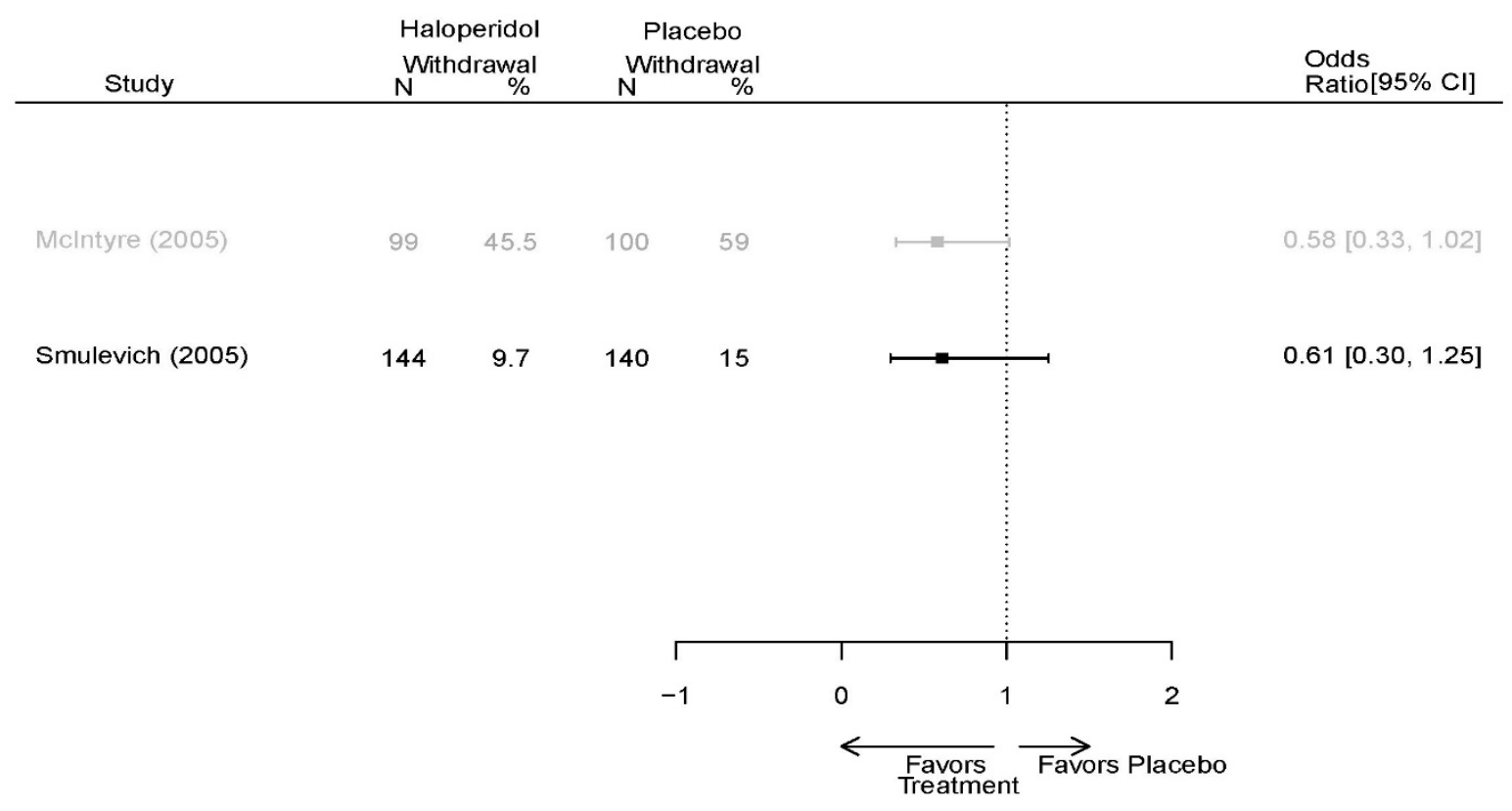




\begin{tabular}{|c|c|c|c|c|c|c|}
\hline Drug & $\begin{array}{l}\text { Study } \\
\text { PMID } \\
\text { RoB } \\
\end{array}$ & Responder/Remitter & Symptom & Function & Other & $\mathrm{AE}$ \\
\hline \multirow[t]{2}{*}{ Haloperidol } & $\begin{array}{l}\text { Mclntyre, } \\
2005^{14} \\
16139175 \\
\text { High }\end{array}$ & $\begin{array}{l}\text { Response } \\
\text { See forest plot E34 } \\
\text { above for response } \\
\frac{\text { Remission }}{\text { NS }}\end{array}$ & $\begin{array}{l}\text { See forest plot E35 } \\
\text { above for YMRS }\end{array}$ & $\begin{array}{l}\text { See forest plot E36 } \\
\text { for } \mathrm{CGI}\end{array}$ & $\begin{array}{l}\text { See forest plot E37, } \\
\text { E38, E39 above for } \\
\text { Withdrawals }\end{array}$ & $\begin{array}{l}\text { SAE } \\
\text { No reported serious } \\
\text { events } \\
\text { EPS } \\
\text { Haloperidol } 35.4 \% \\
\text { Placebo } 5.9 \% \\
\text { P<0.001 } \\
\text { Weight gain } 7 \% \\
\text { NS }\end{array}$ \\
\hline & $\begin{array}{l}\text { Smulevich, } \\
2005^{15} \\
15572276 \\
\text { Moderate }\end{array}$ & $\begin{array}{l}\text { Response } \\
\text { See forest plot E34 } \\
\text { above for response } \\
\text { Remission }\end{array}$ & $\begin{array}{l}\text { See forest plot E35 } \\
\text { above for YMRS }\end{array}$ & $\begin{array}{l}\begin{array}{l}\text { See forest plot E36 } \\
\text { for CGI }\end{array} \\
\frac{\text { GAS }}{3 \text { week }} \\
\text { Placebo -10.3(1.7) } \\
\text { Haloperidol - } \\
13.9(10.3) \\
\text { No Statistical Tests } \\
\text { reported } \\
\text { GAS } \\
12 \text { week } \\
\text { Placebo } \\
\text { Haloperidol } \\
\text { No Statistical Tests } \\
\text { reported }\end{array}$ & $\begin{array}{l}\text { See forest plot E37, } \\
\text { E38, E39 above for } \\
\text { Withdrawals }\end{array}$ & No reported SAE \\
\hline
\end{tabular}

Abbreviations: AE=Adverse Events; ANCOVA=Analysis of Covariance; CGI=Clinical Global Impressions Scale; CI=Confidence Interval; EPS=extrapyramidal symptoms; ESRS=Extrapyrimidal Symptom Rating Scale; GAS=Global Assessment Scale; MADRS=Montgomery-Asberg Depression Rating Scale; NR=Not reported; NS=not significant; OR=Odds Ratio; PMID=PubMed Identification Number; ROB=Risk of Bias; SAE=Serious Adverse Events; YMRS = Young Mania Rating Scale 
Appendix Table E20. Strength of evidence assessment: Haloperidol versus placebo for acute mania

\begin{tabular}{|c|c|c|c|c|c|c|c|c|}
\hline Comparison & Outcome & $\begin{array}{l}\text { \# Studies/ } \\
\text { Design } \\
\text { (n analyzed) }\end{array}$ & $\begin{array}{c}\text { Finding or } \\
\text { Summary } \\
\text { Statistic }\end{array}$ & $\begin{array}{c}\text { Study } \\
\text { Limitations }\end{array}$ & Consistency & Directness & Precision & $\begin{array}{c}\text { Overall } \\
\text { Gradel } \\
\text { Conclusion }\end{array}$ \\
\hline $\begin{array}{l}\text { Haloperidol } \\
\text { vs. placebo }\end{array}$ & $\begin{array}{l}\text { Relapse } 3 \text { wks } \\
\text { YMRS } \\
\text { CGI-BP-S } \\
\text { Withdrawals }\end{array}$ & $\begin{array}{l}2 \text { RCTs } \\
(n=483)\end{array}$ & See forest plots & High & Consistent & Direct & Imprecise & Insufficient \\
\hline
\end{tabular}

Abbreviations: CGI-BP=Clinical Global Impressions Scale for Bipolar Disorder; NS=not significant; RCT=randomized controlled trial; YMRS = Young Mania Rating Scale Notes:

1. Publication bias for antipsychotics, antidepressants, and behavioral interventions for depressive disorders is suspected.

2. Data were generally imprecise due to missing data from high attrition rates, which was commonly dealt with by Last Observation Carried Forward (LOCF). LOCF requires an assumption that the health status of patients who dropped out of the trial would not have changed had future observations been recorded, a strong assumption in the context of

bipolar disorder research.

\section{Section 5. Olanzapine for Acute Mania}

Appendix Table E21. Characteristics of eligible studies: olanzapine for acute mania

\begin{tabular}{|c|c|c|c|c|c|c|}
\hline $\begin{array}{c}\text { Study, Year } \\
\text { Design } \\
\text { Location } \\
\text { Funder } \\
\text { Risk of Bias } \\
\text { PMID }\end{array}$ & $\begin{array}{c}\text { \# Randomized } \\
\text { Age (mean) } \\
\text { Sex (\% Female) } \\
\text { Race (\% White) } \\
\text { Diagnosis } \\
\text { (\% BP-I, II, NOS) } \\
\text { Setting }\end{array}$ & $\begin{array}{c}\text { Inclusions } \\
\text { Key Exclusions }\end{array}$ & $\begin{array}{c}\text { Intervention } \\
\text { Dosage }\end{array}$ & $\begin{array}{c}\text { Comparison } \\
\text { Dosage }\end{array}$ & Follow-up Duration & $\begin{array}{c}\text { Outcomes } \\
\text { Reported } \\
\text { Withdrawal (\%) at } \\
\text { endpoint }\end{array}$ \\
\hline $\begin{array}{l}\text { Xu, } 2015^{19} \\
\text { RCT } \\
\text { Single-site } \\
\text { Government } \\
\text { RoB Low } \\
26060401\end{array}$ & $\begin{array}{l}\mathrm{N}=120 \\
\text { Mean Age } 31 \\
\text { Female } 52 \% \\
\text { Race NR } \\
\text { BP-I 100\% } \\
\text { Setting NR }\end{array}$ & $\begin{array}{l}\text { First manic; } \\
\text { YMRS } \geq 17 \\
\text { Substance Abuse } \\
\text { Neurological } \\
\text { Disorders } \\
\text { Taking Other Meds } \\
\text { Pregnant/Nursing }\end{array}$ & $\begin{array}{l}\text { Olanzapine } \\
10 \text { mg/day } \\
\text { Flexible dosing } \\
5-20 \text { mg/day }\end{array}$ & $\begin{array}{l}\text { C1: Olanzapine } \\
10 \text { mg/day }+ \\
\text { Valproate } 600 \\
\text { mg/day } \\
\text { C2: Valproate } 600 \\
\text { mg/day alone }\end{array}$ & 4 weeks & $\begin{array}{l}\text { Efficacy } \\
\text { YMRS } \\
\text { CGI-BP } \\
\text { Adverse events } \\
\text { Extrapyramidal } \\
\text { symptoms } \\
\text { SAS } \\
\text { Withdrawal 5\% } \\
\end{array}$ \\
\hline
\end{tabular}




\begin{tabular}{|c|c|c|c|c|c|c|}
\hline $\begin{array}{l}\text { Study, Year } \\
\text { Design } \\
\text { Location } \\
\text { Funder } \\
\text { Risk of Bias } \\
\text { PMID }\end{array}$ & $\begin{array}{c}\text { \# Randomized } \\
\text { Age (mean) } \\
\text { Sex (\% Female) } \\
\text { Race (\% White) } \\
\text { Diagnosis } \\
\text { (\% BP-I, II, NOS) } \\
\text { Setting }\end{array}$ & $\begin{array}{c}\text { Inclusions } \\
\text { Key Exclusions }\end{array}$ & $\begin{array}{l}\text { Intervention } \\
\text { Dosage }\end{array}$ & $\begin{array}{c}\text { Comparison } \\
\text { Dosage }\end{array}$ & Follow-up Duration & $\begin{array}{c}\text { Outcomes } \\
\text { Reported } \\
\text { Withdrawal (\%) at } \\
\text { endpoint }\end{array}$ \\
\hline $\begin{array}{l}\text { Katagiri, } 2012^{20} \\
\text { RCT } \\
\text { Single Site } \\
\text { Japan } \\
\text { Industry } \\
\text { RoB Moderate } \\
\\
22134043\end{array}$ & $\begin{array}{l}\mathrm{N}=221 \\
\text { Mean Age } 45 \\
\text { Female 55\% } \\
\text { Race NR } \\
\text { BP-I 100\% } \\
\text { Outpatient }\end{array}$ & $\begin{array}{l}\text { Manic or Mixed } \\
\text { Episode } \\
\text { YMRS } \geq 20 \\
\text { Other Mental Health } \\
\text { Taking Other Meds } \\
\text { Labs/Other } \\
\text { Conditions }\end{array}$ & $\begin{array}{l}\text { Olanzapine } \\
\text { Initiated at } 10 \mathrm{mg} / \text { day } \\
(5-20 \mathrm{mg} / \text { day })\end{array}$ & $\begin{array}{l}\text { Placebo } \\
\text { (Haloperidol arm not } \\
\text { used <10 per arm } \\
\text { completed) }\end{array}$ & $\begin{array}{l}3 \text { weeks } \\
\text { (6 week not } \\
\text { abstracted due to } \\
\text { attrition) }\end{array}$ & $\begin{array}{l}\text { Adverse Events } \\
\text { Extrapyramidal } \\
\text { symptoms } \\
\text { DIEPSS } \\
\text { Efficacy } \\
\text { YMRS } \\
\text { HAM-D } \\
\text { CGI } \\
\text { Withdrawal } 41 \% \text { at } 3 \\
\text { weeks } \\
52 \% \text { at } 6 \text { weeks }\end{array}$ \\
\hline $\begin{array}{l}\text { Vieta, } 2012^{21} \\
\text { RCT } \\
\text { Multisite } \\
4 \text { Continents } \\
\text { Industry } \\
\text { RoB High } \\
22503488\end{array}$ & $\begin{array}{l}\mathrm{N}=560 \\
\text { Mean Age } 37 \\
\text { Female } 52 \% \\
\text { White } 41 \% \\
\text { BP-I 100\% } \\
\text { Outpatient }\end{array}$ & $\begin{array}{l}\text { Manic or Mixed } \\
\text { Episode; } \\
\text { Acute (YMRS } \geq 20 \\
\text { and CGI-S } \geq 4) \text { or } \\
\text { non-acute (mood } \\
\text { episodes with YMRS } \\
<12 \text { and CGI-S } \leq 3 \text { ) }\end{array}$ & $\begin{array}{l}\text { Olanzapine } \\
10 \text { mg/day }\end{array}$ & $\begin{array}{l}\text { C1: Placebo } \\
\text { C2: Risperidone long- } \\
\text { acting injectable (25- } \\
50 \mathrm{mg})\end{array}$ & $\begin{array}{l}12 \text { weeks (18 month } \\
\text { extension for } \\
\text { participants without } \\
\text { mood recurrence) }\end{array}$ & $\begin{array}{l}\text { Response (YMRS } \leq \\
\text { 19) } \\
\text { Time to first } \\
\text { recurrence of mood } \\
\text { symptoms } \\
\text { Efficacy } \\
\text { YMRS } \\
\text { CGI-S } \\
\text { MADRS } \\
\text { Adverse events } \\
\text { Extrapyramidal } \\
\text { symptoms } \\
\text { ESRS } \\
\text { Withdrawal 29\% }\end{array}$ \\
\hline
\end{tabular}




\begin{tabular}{|c|c|c|c|c|c|c|}
\hline $\begin{array}{l}\text { Study, Year } \\
\text { Design } \\
\text { Location } \\
\text { Funder } \\
\text { Risk of Bias } \\
\text { PMID }\end{array}$ & $\begin{array}{c}\text { \# Randomized } \\
\text { Age (mean) } \\
\text { Sex (\% Female) } \\
\text { Race (\% White) } \\
\text { Diagnosis } \\
\text { (\% BP-I, II, NOS) } \\
\text { Setting }\end{array}$ & $\begin{array}{c}\text { Inclusions } \\
\text { Key Exclusions }\end{array}$ & $\begin{array}{l}\text { Intervention } \\
\text { Dosage }\end{array}$ & $\begin{array}{c}\text { Comparison } \\
\text { Dosage }\end{array}$ & Follow-up Duration & $\begin{array}{c}\text { Outcomes } \\
\text { Reported } \\
\begin{array}{c}\text { Withdrawal (\%) at } \\
\text { endpoint }\end{array}\end{array}$ \\
\hline $\begin{array}{l}\text { McIntyre, } 2010^{8} \\
\text { RCT } \\
\text { Multisite } \\
3 \text { Continents } \\
\text { Industry } \\
\text { RoB High } \\
20096936\end{array}$ & $\begin{array}{l}\mathrm{N}=488 \\
\text { Mean Age } 39 \\
\text { Female } 47 \% \\
\text { White } 55 \% \\
\text { BP-I 100\% } \\
\text { Inpatient and } \\
\text { Outpatient }\end{array}$ & $\begin{array}{l}\text { Manic or Mixed } \\
\text { Episode; } \\
\text { YMRS } \geq 20 \\
\text { First Manic Episode } \\
\text { Schizoaffective } \\
\text { Substance Abuse } \\
\text { Other Mental Health } \\
\text { Neurological } \\
\text { Disorders } \\
\text { Taking Other Meds } \\
\text { Pregnant/Nursing } \\
\text { Labs/Other } \\
\text { Conditions }\end{array}$ & $\begin{array}{l}\text { Olanzapine } \\
5-20 \mathrm{mg} / \mathrm{day}\end{array}$ & $\begin{array}{l}\text { C1: Placebo } \\
\text { C2: Asenapine } \\
\text { 5-10 mg/2 times daily }\end{array}$ & 3 weeks & $\begin{array}{l}\text { Response ( } \geq 50 \% \\
\text { YMRS reduction) } \\
\text { Time to response } \\
\text { (days from baseline } \\
\text { to } \geq 50 \% \text { YMRS } \\
\text { reduction) } \\
\text { Remission ( } \leq 12 \\
\text { YMRS) } \\
\text { Efficacy } \\
\text { YMRS } \\
\text { CGI (BP and mania } \\
\text { subscales) } \\
\text { MADRS } \\
\text { Adverse events } \\
\text { Extrapyramidal } \\
\text { symptoms } \\
\text { SAS } \\
\text { BAS } \\
\text { AIMS } \\
\text { Withdrawal 34\% }\end{array}$ \\
\hline $\begin{array}{l}\text { Shafti, } 2010 \\
\text { RCT } \\
\text { Iran } \\
\text { Funding NR } \\
\text { RoB Moderate } \\
19740546\end{array}$ & $\begin{array}{l}\mathrm{N}=40 \\
\text { Age NR } \\
\text { Female 100\%; } \\
\text { Race NR } \\
\text { BP-I 100\% } \\
\text { Inpatient }\end{array}$ & $\begin{array}{l}\text { Manic } \\
\text { (not described) } \\
\text { Schizoaffective } \\
\text { Substance Abuse } \\
\text { Other Mental Health } \\
\text { Neurological } \\
\text { Disorders } \\
\text { Taking Other Meds } \\
\text { Labs/Other } \\
\text { Conditions }\end{array}$ & $\begin{array}{l}\text { Olanzapine } \\
5 \mathrm{mg} / \text { day }\end{array}$ & $\begin{array}{l}\text { Lithium } \\
300 \mathrm{mg} / \text { day }\end{array}$ & 12 weeks & $\begin{array}{l}\text { MSRS } \\
\text { CGI-S } \\
\text { Adverse events } \\
\text { Extrapyramidal } \\
\text { symptoms } \\
\text { Withdrawal 13\% }\end{array}$ \\
\hline
\end{tabular}




\begin{tabular}{|c|c|c|c|c|c|c|}
\hline $\begin{array}{l}\text { Study, Year } \\
\text { Design } \\
\text { Location } \\
\text { Funder } \\
\text { Risk of Bias } \\
\text { PMID }\end{array}$ & $\begin{array}{c}\text { \# Randomized } \\
\text { Age (mean) } \\
\text { Sex (\% Female) } \\
\text { Race (\% White) } \\
\text { Diagnosis } \\
\text { (\% BP-I, II, NOS) } \\
\text { Setting }\end{array}$ & $\begin{array}{c}\text { Inclusions } \\
\text { Key Exclusions }\end{array}$ & $\begin{array}{l}\text { Intervention } \\
\text { Dosage }\end{array}$ & $\begin{array}{c}\text { Comparison } \\
\text { Dosage }\end{array}$ & Follow-up Duration & $\begin{array}{c}\text { Outcomes } \\
\text { Reported } \\
\text { Withdrawal (\%) at } \\
\text { endpoint }\end{array}$ \\
\hline $\begin{array}{l}\text { McIntyre, } 2009 \\
\text { RCT } \\
\text { Multisite } \\
\text { 3 Continents } \\
\text { Industry } \\
\text { RoB High } \\
19839993\end{array}$ & $\begin{array}{l}\mathrm{N}=489 \\
\text { Mean Age } 40 \\
\text { Female } 43 \% \\
\text { White } 61 \% \\
\text { BP-I } 100 \% \\
\\
\text { Inpatient and } \\
\text { Outpatient }\end{array}$ & $\begin{array}{l}\text { Manic or Mixed } \\
\text { Episode; } \\
\text { YMRS } \geq 20 \\
\text { Schizoaffective } \\
\text { Substance Abuse } \\
\text { Other Mental Health } \\
\text { Neurological } \\
\text { Disorders } \\
\text { Taking Other Meds } \\
\text { Pregnant/Nursing } \\
\text { Labs/Other } \\
\text { Conditions }\end{array}$ & $\begin{array}{l}\text { Olanzapine } \\
\text { 5-20 mg/day }\end{array}$ & $\begin{array}{l}\text { C1: Placebo } \\
\text { C2: Asenapine 5-10 } \\
\text { mg/2 times daily }\end{array}$ & 3 weeks & $\begin{array}{l}\text { Response (YMRS, } \\
\text { cutoff NR) } \\
\text { Remission (YMRS, } \\
\text { cutoff NR) } \\
\text { Efficacy } \\
\text { YMRS } \\
\text { CGI (multiple } \\
\text { subscales) } \\
\text { MADRS } \\
\text { Adverse events } \\
\text { Extrapyramidal } \\
\text { symptoms } \\
\text { SAS } \\
\text { BAS } \\
\text { AIMS } \\
\text { Withdrawal } 31 \% \\
\end{array}$ \\
\hline $\begin{array}{l}\text { Niufan, } 2008^{22} \\
\text { RCT } \\
\text { Multisite } \\
\text { China } \\
\text { Industry } \\
\text { RoB Low } \\
17531327\end{array}$ & $\begin{array}{l}\mathrm{N}=140 \\
\text { Mean Age } 33 \\
\text { Female 74\% } \\
\text { Race NR } \\
\text { Diagnosis NR } \\
\text { Outpatient }\end{array}$ & $\begin{array}{l}\text { Manic or Mixed } \\
\text { Episode; } \\
\text { YMRS } \geq 20 \\
\text { NR }\end{array}$ & \begin{tabular}{|l} 
Olanzapine \\
$15 \mathrm{mg} /$ day \\
Flexible dosing \\
$5-20 \mathrm{mg} / \mathrm{day}$
\end{tabular} & $\begin{array}{l}\text { Lithium Carbonate } \\
300-600 \mathrm{mg} / \mathrm{day} \\
\text { Flexible dosing } \\
600-1800 \mathrm{mg} / \text { day } \\
\text { divided dose }\end{array}$ & 4 weeks & $\begin{array}{l}\text { Efficacy } \\
\text { YMRS } \\
\text { CGI } \\
\text { BPRS } \\
\text { MADRS } \\
\text { Extrapyramidal } \\
\text { symptoms } \\
\text { SAS } \\
\text { Withdrawal 15\% }\end{array}$ \\
\hline
\end{tabular}




\begin{tabular}{|c|c|c|c|c|c|c|}
\hline $\begin{array}{l}\text { Study, Year } \\
\text { Design } \\
\text { Location } \\
\text { Funder } \\
\text { Risk of Bias } \\
\text { PMID }\end{array}$ & $\begin{array}{c}\text { \# Randomized } \\
\text { Age (mean) } \\
\text { Sex (\% Female) } \\
\text { Race (\% White) } \\
\text { Diagnosis } \\
\text { (\% BP-I, II, NOS) } \\
\text { Setting }\end{array}$ & $\begin{array}{c}\text { Inclusions } \\
\text { Key Exclusions }\end{array}$ & $\begin{array}{c}\text { Intervention } \\
\text { Dosage }\end{array}$ & $\begin{array}{c}\text { Comparison } \\
\text { Dosage }\end{array}$ & Follow-up Duration & $\begin{array}{c}\text { Outcomes } \\
\text { Reported } \\
\text { Withdrawal (\%) at } \\
\text { endpoint }\end{array}$ \\
\hline $\begin{array}{l}\text { Tohen, 2008b } 23 \\
\text { RCT } \\
3 \text { Continents } \\
\text { Industry } \\
\text { RoB Low } \\
19014751\end{array}$ & \begin{tabular}{|l}
$\mathrm{N}=521$ \\
Mean Age 40 \\
Female 49\% \\
Race NR \\
Diagnosis NR \\
Inpatient and \\
Outpatient
\end{tabular} & $\begin{array}{l}\text { Manic or Mixed } \\
\text { Episode; } \\
\text { YMRS 20-30 } \\
\text { CGI-BP mania 3-4 } \\
\text { Schizoaffective } \\
\text { Other Mental Health } \\
\text { Pregnant/Nursing }\end{array}$ & $\begin{array}{l}\text { Olanzapine } \\
5-20 \mathrm{mg} / \mathrm{day}\end{array}$ & $\begin{array}{l}\text { C1: Placebo } \\
\text { C2: Divalproex 500- } \\
\text { 2500 mg/2-3 times } \\
\text { daily }\end{array}$ & 12 weeks & $\begin{array}{l}\text { Response ( } \geq 50 \% \\
\text { YMRS reduction) } \\
\text { Time to response } \\
\text { (days from baseline } \\
\text { to } \geq 50 \% \text { YMRS } \\
\text { reduction) } \\
\text { Remission ( } \leq 12 \\
\text { YMRS) } \\
\text { Efficacy } \\
\text { YMRS } \\
\text { CGI (multiple } \\
\text { subscales) } \\
\text { MADRS } \\
\text { Adverse events } \\
\text { Extrapyramidal } \\
\text { symptoms } \\
\text { SAS } \\
\text { BAS } \\
\text { AIMS } \\
\text { Withdrawal 26\% }\end{array}$ \\
\hline $\begin{array}{l}\text { Perlis, 2006 } \\
\text { RCT } \\
\text { Multisite } \\
\text { US } \\
\text { Industry } \\
\text { RoB High } \\
17196055\end{array}$ & $\begin{array}{l}\mathrm{N}=329 \\
\text { Mean Age } 38 \\
\text { Female 55\% } \\
\text { White } 74 \% \\
\text { BP-I 100\% } \\
\text { Outpatient }\end{array}$ & $\begin{array}{l}\text { Manic; } \\
\text { YMRS } \geq 20 \\
\\
\text { Substance Abuse } \\
\text { Labs/Other } \\
\text { Conditions }\end{array}$ & $\begin{array}{l}\text { Olanzapine } \\
5-20 \mathrm{mg} / \mathrm{day}\end{array}$ & $\begin{array}{l}\text { Risperidone } \\
1-6 \text { mg/day }\end{array}$ & 3 weeks & \begin{tabular}{|l|} 
Efficacy \\
YMRS \\
MADRS \\
CGI \\
HAM-D \\
DAl-10 \\
PGWB \\
SF-12 \\
Extrapyramidal \\
symptoms \\
BAS \\
Simpson-Angus \\
Withdrawal 27\%
\end{tabular} \\
\hline
\end{tabular}

E-54 


\begin{tabular}{|c|c|c|c|c|c|c|}
\hline $\begin{array}{l}\text { Study, Year } \\
\text { Design } \\
\text { Location } \\
\text { Funder } \\
\text { Risk of Bias } \\
\text { PMID }\end{array}$ & $\begin{array}{c}\text { \# Randomized } \\
\text { Age (mean) } \\
\text { Sex (\% Female) } \\
\text { Race (\% White) } \\
\text { Diagnosis } \\
\text { (\% BP-I, II, NOS) } \\
\text { Setting }\end{array}$ & $\begin{array}{c}\text { Inclusions } \\
\text { Key Exclusions }\end{array}$ & $\begin{array}{l}\text { Intervention } \\
\text { Dosage }\end{array}$ & $\begin{array}{c}\text { Comparison } \\
\text { Dosage }\end{array}$ & Follow-up Duration & $\begin{array}{c}\text { Outcomes } \\
\text { Reported } \\
\begin{array}{c}\text { Withdrawal (\%) at } \\
\text { endpoint }\end{array}\end{array}$ \\
\hline \begin{tabular}{|l|} 
Revicki, $2003^{25}$ \\
RCT \\
Multisite \\
US \\
Industry \\
RoB Moderate \\
12716270 \\
Secondary analysis of \\
Zajecka 2002 [PMID \\
12523875]
\end{tabular} & $\begin{array}{l}\mathrm{N}=52 \\
\text { Mean Age } 38 \\
\text { Female } 56 \% \\
\text { White } 81 \% \\
\text { BP-I } 100 \% \\
\\
\text { Inpatient and } \\
\text { outpatient }\end{array}$ & $\begin{array}{l}\text { Manic; } \\
\text { YMRS } \geq 25\end{array}$ & $\begin{array}{l}\text { Olanzapine } \\
20 \text { mg/day }\end{array}$ & $\begin{array}{l}\text { Divalproex } \\
20 \mathrm{mg} / \mathrm{kg} / \mathrm{day} \text { up to } \\
\text { an additional } 1000 \\
\mathrm{mg} / \text { day }\end{array}$ & $\begin{array}{l}12 \text { weeks (6 and } 12 \\
\text { week outcomes } \\
\text { excluded due to } \\
\text { attrition) }\end{array}$ & $\begin{array}{l}\text { Efficacy } \\
\text { YMRS } \\
\text { Quality of Life } \\
\text { HRQL } \\
\text { Q-LES-Q } \\
\text { Medical Resource } \\
\text { Use } \\
\text { Cost } \\
\\
\text { Withdrawal (base } \\
\text { study Zajecka, 2002 } \\
\text { reports withdrawal of } \\
\text { 69\% at 12 weeks, } \\
\text { Revicki reports 35\% } \\
\text { withdrawal at 3 } \\
\text { weeks) } \\
\end{array}$ \\
\hline $\begin{array}{l}\text { Tohen, } 2003^{26} \\
\text { RCT } \\
\text { Multisite } \\
\text { 4 Continents } \\
\text { Industry } \\
\\
\text { RoB Moderate } \\
\\
14662554 \\
12177585^{27}\end{array}$ & \begin{tabular}{|l|}
$\mathrm{N}=453$ \\
Mean Age 40 \\
Female $60 \%$ \\
Race NR \\
BP-I $100 \%$ \\
Inpatient or outpatient
\end{tabular} & $\begin{array}{l}\text { Manic; } \\
\text { YMRS } \geq 20 \\
\text { Substance Abuse } \\
\text { Labs/Other } \\
\text { Conditions }\end{array}$ & $\begin{array}{l}\text { Olanzapine } \\
15 \text { mg/day } \\
\text { flex dosing } \\
5,10,15, \text { or } 20 \\
\text { mg/day }\end{array}$ & $\begin{array}{l}\text { Haloperidol } \\
10 \text { mg/day } \\
\text { flex dosing } \\
3,5,10 \text {, or } 15 \mathrm{mg} / \text { day }\end{array}$ & 12 weeks & $\begin{array}{l}\text { Illness Severity } \\
\text { YMRS } \\
\text { HAM-D } \\
\text { Quality of Life } \\
\text { SF-36 } \\
\text { Extrapyramidal } \\
\text { Symptoms } \\
\text { AIMS } \\
\text { BAS } \\
\text { Remission } \\
\text { Adverse Events } \\
\\
\text { Withdrawal 43\% }\end{array}$ \\
\hline
\end{tabular}




\begin{tabular}{|c|c|c|c|c|c|c|}
\hline $\begin{array}{l}\text { Study, Year } \\
\text { Design } \\
\text { Location } \\
\text { Funder } \\
\text { Risk of Bias } \\
\text { PMID }\end{array}$ & $\begin{array}{c}\text { \# Randomized } \\
\text { Age (mean) } \\
\text { Sex (\% Female) } \\
\text { Race (\% White) } \\
\text { Diagnosis } \\
\text { (\% BP-I, II, NOS) } \\
\text { Setting }\end{array}$ & $\begin{array}{c}\text { Inclusions } \\
\text { Key Exclusions }\end{array}$ & $\begin{array}{l}\text { Intervention } \\
\text { Dosage }\end{array}$ & $\begin{array}{c}\text { Comparison } \\
\text { Dosage }\end{array}$ & Follow-up Duration & $\begin{array}{c}\text { Outcomes } \\
\text { Reported } \\
\text { Withdrawal (\%) at } \\
\text { endpoint }\end{array}$ \\
\hline $\begin{array}{l}\text { Tohen, } 2002 b^{28} \\
\text { RCT } \\
\text { Multisite } \\
\text { US } \\
\text { Industry } \\
\text { RoB High } \\
12042191\end{array}$ & $\begin{array}{l}\mathrm{N}=251 \\
\text { Mean Age } 41 \\
\text { Female } 57 \% \\
\text { White } 81 \% \\
\text { BP-I 100\% } \\
\text { Outpatient }\end{array}$ & $\begin{array}{l}\text { Mania; } \\
\text { YMRS } \geq 20 \\
\text { Substance Abuse } \\
\text { Other Mental Health } \\
\text { Neurological } \\
\text { Disorders } \\
\text { Taking Other Meds } \\
\text { Pregnant/Nursing } \\
\text { Labs/Other } \\
\text { Conditions }\end{array}$ & $\begin{array}{l}\text { Olanzapine } \\
15 \mathrm{mg} / \text { day with } \\
\text { flexible dosing from } \\
5-20 \mathrm{mg} / \mathrm{day}\end{array}$ & $\begin{array}{l}\text { Divalproex } \\
750 \mathrm{mg} / \text { day with } \\
\text { flexible dosing from } \\
500-2500 \mathrm{mg} / \text { day }\end{array}$ & 3 weeks & $\begin{array}{l}\text { Efficacy } \\
\text { YMRS } \\
\text { HAM-D } \\
\text { Remission } \\
\text { Adverse Events } \\
\text { Extrapyramidal } \\
\text { symptom } \\
\text { Withdrawal 34\% }\end{array}$ \\
\hline $\begin{array}{l}\text { Tohen, } 2000^{29} \\
\text { RTC } \\
\text { Multisite } \\
\text { US } \\
\text { Industry } \\
\text { RoB High } \\
10986547\end{array}$ & $\begin{array}{l}\mathrm{N}=115 \\
\text { Mean Age } 39 \\
\text { Female } 50 \% \\
\text { White } 80 \% \\
\text { Diagnosis NR } \\
\text { Inpatient (week 1) } \\
\text { Outpatient (after } \\
\text { week } 1 \text { if CGI-BP } \leq 3 \text { ) }\end{array}$ & $\begin{array}{l}\text { Mixed Episode; } \\
\text { YMRS score } \geq 20 \\
\text { Substance Abuse; } \\
\text { Other Mental Health; } \\
\text { Neurological } \\
\text { Disorders; } \\
\text { Taking Other Meds; } \\
\text { Labs/Other } \\
\text { Conditions }\end{array}$ & $\begin{array}{l}\text { Olanzapine } \\
15 \text { mg/day (adjusted } \\
\text { between 5-20 } \\
\text { mg/day) }\end{array}$ & Placebo & 4 weeks & $\begin{array}{l}\text { Symptom Severity } \\
\text { YMRS } \\
\text { HAM-D } \\
\text { CGI } \\
\text { PANSS } \\
\text { Safety } \\
\text { EPS } \\
\text { Adverse Events } \\
\text { Withdrawal 49\% }\end{array}$ \\
\hline $\begin{array}{l}\text { Berk, } 1999^{30} \\
\text { RCT } \\
\text { Single-Site } \\
\text { South Africa } \\
\text { University } \\
\text { RoB High } \\
10565800\end{array}$ & $\begin{array}{l}\mathrm{N}=30 \\
\text { Mean Age } 31 \\
\text { Sex NR } \\
\text { Race NR } \\
\text { Diagnosis NR } \\
\text { Inpatient }\end{array}$ & $\begin{array}{l}\text { Mania (no other } \\
\text { inclusion criteria } \\
\text { provided) } \\
\text { Other Mental Health; } \\
\text { Pregnant/Nursing; } \\
\text { Labs/Other } \\
\text { Conditions }\end{array}$ & $\begin{array}{l}\text { Olanzapine } \\
10 \text { mg/day }\end{array}$ & $\begin{array}{l}\text { Lithium } \\
\text { 400mg twice /day }\end{array}$ & 4 weeks & $\begin{array}{l}\text { Psychiatric Condition } \\
\text { PRS } \\
\text { CGI } \\
\text { MAS } \\
\text { Functioning } \\
\text { GAF } \\
\text { Side Effects } \\
\text { SAS } \\
\text { Withdrawal NR }\end{array}$ \\
\hline
\end{tabular}

Abbreviations: AIMS=Abnormal Involuntary Movement Scale; BARS=Barnes Akathisia Scale; BAS=Behavioral Approach System; BMI=Body Mass Index; BP=bipolar

disorder; BPRS=Brief Psychiatric Rating Scale; C=comparison; CGI= Clinical Global Impressions; CGI-I=Clinical Global Impressions-Improvement; CGI-S =CGI-Severity; CGIBP=Clinical Global Impressions Scale-Bipolar; CGI-BP-C= Clinical Global Impressions, Bipolar, Change Scale; CGI-BP-S=Clinical Global Impressions, Bipolar, Severity Scale; C-SSRS=Columbia Suicide Severity Rating Scale; DAI-10=Drug Attitutde Inventory, 10 question version; DIEPSS=Drug-Induced Extra-Pyramidal Symptoms Scale; 
DSM=Diagnostic and Statistical Manual of Mental Disorders; EPS=extrapyramidal symptoms; ER=Extended Release; ESRS=Extrapyramidal Symptom Rating Scale;

GAF=General Assessment of Functioning Scale; GAS=Global Assessment Scale; HAM-A=Hamilton Scale for Anxiety; HAM-D=Hamilton Scale for Depression; HRQL=Healthrelated quality of life; HRQOL=Health-related quality of life; I=intervention; IDS=Inventory for Depressive Symptoms; LIFE= Longitudinal Interval Follow-up Evaluation; MADRS=Montgomery-Asberg Depression Rating Scale; MAS=Bech-Rafaelsen Mania Rating Scale; MRS=Mania Rating Scale; MSRS=Manic state rating scale; NOS=not otherwise specified; NR=not reported; PANSS=Positive and Negative Syndrome Scale; PRS=Polygenic Risk Scores; PGWB=Psychological General Well-Being Index;

PMID=PubMed Identification Number; PRS=Polygenic Risk Scores; Q-LES-Q=Quality of Life Enjoyment and Satisfaction Questionnaire; RCT=randomized controlled trial; ROB=risk of bias; SADS-C= Schedule for Affective Disorders and Schizophrenia-Change version; SAE=Serious Adverse Events; SAS=Simpson Angus Scale; SF-12=12-Item Short Form Health Survey; SF-36=36-Item Short Form Health Survey; SLICE=Streamlined Longitudinal Interview Clinical Evaluation; T=Trial; YMRS = Young Mania Rating Scale

\begin{tabular}{|c|c|c|c|}
\hline Drug & $\begin{array}{l}\text { Study } \\
\text { Funding Source } \\
\text { PMID }\end{array}$ & $\begin{array}{c}\text { Overall Risk of } \\
\text { Bias Assessment }\end{array}$ & Rationale \\
\hline \multirow[t]{9}{*}{ Olanzapine } & $\begin{array}{l}\text { Xu, } 2015^{19} \\
\text { Government } \\
26060401 \\
\end{array}$ & Low & Well-constructed, described, and reported study. 5\% dropout. \\
\hline & $\begin{array}{l}\text { Katagiri, } 2012^{31} \\
\text { Industry } \\
22134043\end{array}$ & Moderate & $\begin{array}{l}\text { Appears to be completely reported and generally well executed, however, dropout rates and } \\
\text { LOCF create substantial possibility for bias. }\end{array}$ \\
\hline & $\begin{array}{l}\text { Vieta, } 2012^{32} \\
\text { Industry } \\
22503488\end{array}$ & High & $\begin{array}{l}\text { High - blinding and randomization procedures not well described. Period II results are biased } \\
\text { by the drug assignment being open label. Period three efficacy scores are likely to be biased } \\
\text { by the large non-completer rate. }\end{array}$ \\
\hline & $\begin{array}{l}\text { McIntyre, } 2010^{12} \\
\text { Industry } \\
20096936\end{array}$ & High & Randomization and blinding procedures not described. 34\% dropout. \\
\hline & $\begin{array}{l}\text { Shafti, } 2010^{33} \\
\text { Funding NR } \\
19740546\end{array}$ & Moderate & Randomization and blinding procedures not described. 13\% dropout \\
\hline & $\begin{array}{l}\text { Mclntyre, } 2009^{9} \\
\text { Industry } \\
19839993\end{array}$ & High & $\begin{array}{l}\text { Randomization and blinding procedures not described. Patients discharged from hospital at } \\
\text { differing times and doesn't account for this as a possible confounder. } 31 \% \text { overall dropout } \\
\text { with high differential dropout between olanzapine and other groups. }\end{array}$ \\
\hline & $\begin{array}{l}\text { Niufan, } 2008^{22} \\
\text { Industry } \\
17531327\end{array}$ & Low & Randomization procedure not described. Medication and rater blinded. 16\% dropout. \\
\hline & $\begin{array}{l}\text { Tohen, } 2008 b^{23} \\
\text { Industry } \\
19014751\end{array}$ & Low & Well-constructed and described study. No obvious sources of bias present. $26 \%$ dropout. \\
\hline & $\begin{array}{l}\text { Perlis, } 2006^{34} \\
\text { Industry } \\
17196055\end{array}$ & High & Randomization and blinding procedure not described. 27\% dropout. \\
\hline
\end{tabular}




\begin{tabular}{|c|c|c|c|}
\hline Drug & $\begin{array}{l}\text { Study } \\
\text { Funding Source } \\
\text { PMID }\end{array}$ & $\begin{array}{c}\text { Overall Risk of } \\
\text { Bias Assessment }\end{array}$ & Rationale \\
\hline & $\begin{array}{l}\text { Revicki, } 2003^{25} \\
\text { Industry } \\
12716270\end{array}$ & Moderate & $\begin{array}{l}\text { Randomization and blinding procedures not described. } 35 \% \text { before } 3 \text { weeks, 52/120 } \\
\text { complete } 12 \text { week study. Study notes consistency in traits between dropouts and those who } \\
\text { complete, which may be an indication that outcomes may be less biased. }\end{array}$ \\
\hline & $\begin{array}{l}\text { Tohen, } 2003^{26} \\
\text { Industry } \\
14662554\end{array}$ & Moderate & Randomization procedure not described. $43 \%$ dropout. \\
\hline & $\begin{array}{l}\text { Shi, } 2002^{27} \\
\text { Industry } \\
12177585\end{array}$ & High & $\begin{array}{l}\text { Randomization procedure not described, although does note "randomization codes". States } \\
\text { that } 166 \text { olanz and } 141 \text { halo complete } 6 \text { weeks and } 140 \text { olanz and } 116 \text { halo complete } 12 \\
\text { weeks. The counts of patients who complete the follow-up assessments do not match these } \\
\text { numbers, in some cases quite substantially. Missing patients not accounted for. Described in } \\
\text { the methods that only patients who completed the questionnaire and provided data about the } \\
\text { change from baseline to endpoint were included -- not ITT. }\end{array}$ \\
\hline & $\begin{array}{l}\text { Tohen, } 2002 b^{28} \\
\text { Industry } \\
12042191\end{array}$ & High & Randomization and blinding procedures not described. 33\% dropout. \\
\hline & $\begin{array}{l}\text { Tohen, } 2000^{29} \\
\text { Industry } \\
10986547\end{array}$ & High & $\begin{array}{l}\text { Blinding procedures not described. Responders allowed to leave hospital, non-responders } \\
\text { were not. } 49 \% \text { Dropout. }\end{array}$ \\
\hline & $\begin{array}{l}\text { Berk, } 1999^{30} \\
\text { University } \\
10565800\end{array}$ & High & $\begin{array}{l}\text { Randomization and blinding procedures not described. Does not describe handling of } \\
\text { participants who drop out. Notes that the groups were matched at baseline by education, } \\
\text { marital status, ethnicity, employment status but offers no details on these demographic rates. }\end{array}$ \\
\hline
\end{tabular}

Abbreviations: ITT=Intention to Treat; PMID=PubMed Identification Number; LOCF=last observation carried forward 


\section{Olanzapine Forest Plots}

Outcomes in studies assessed as having a high risk of bias, or low to moderate risk of bias but at least 40 percent attrition, are presented in grey tones. Both fixed-effect models and random-effects models are presented. We calculated fixed-effect models to provide a charitable estimate of the average effect among completed trials. However, we base our main conclusions on the random-effects models.

Appendix Figure E40. Olanzapine vs. placebo - response

Odds Ratio of Response ( $>50 \%$ Reduction in YMRS) at 3 Weeks

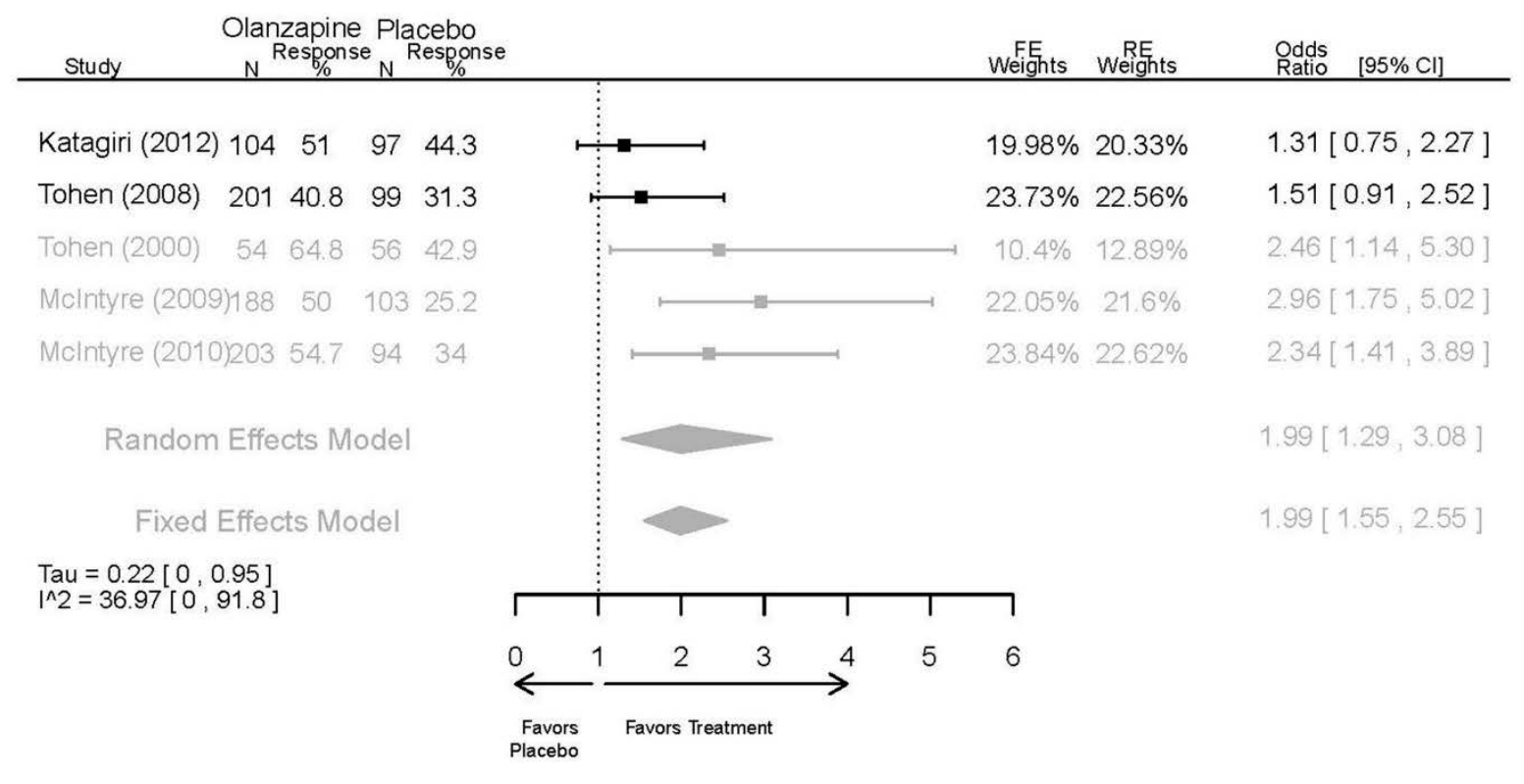


Appendix Figure E41. Olanzapine vs. placebo - remission

\section{Odds Ratio of Remission (YMRS $=12$ ) at 3 Weeks}

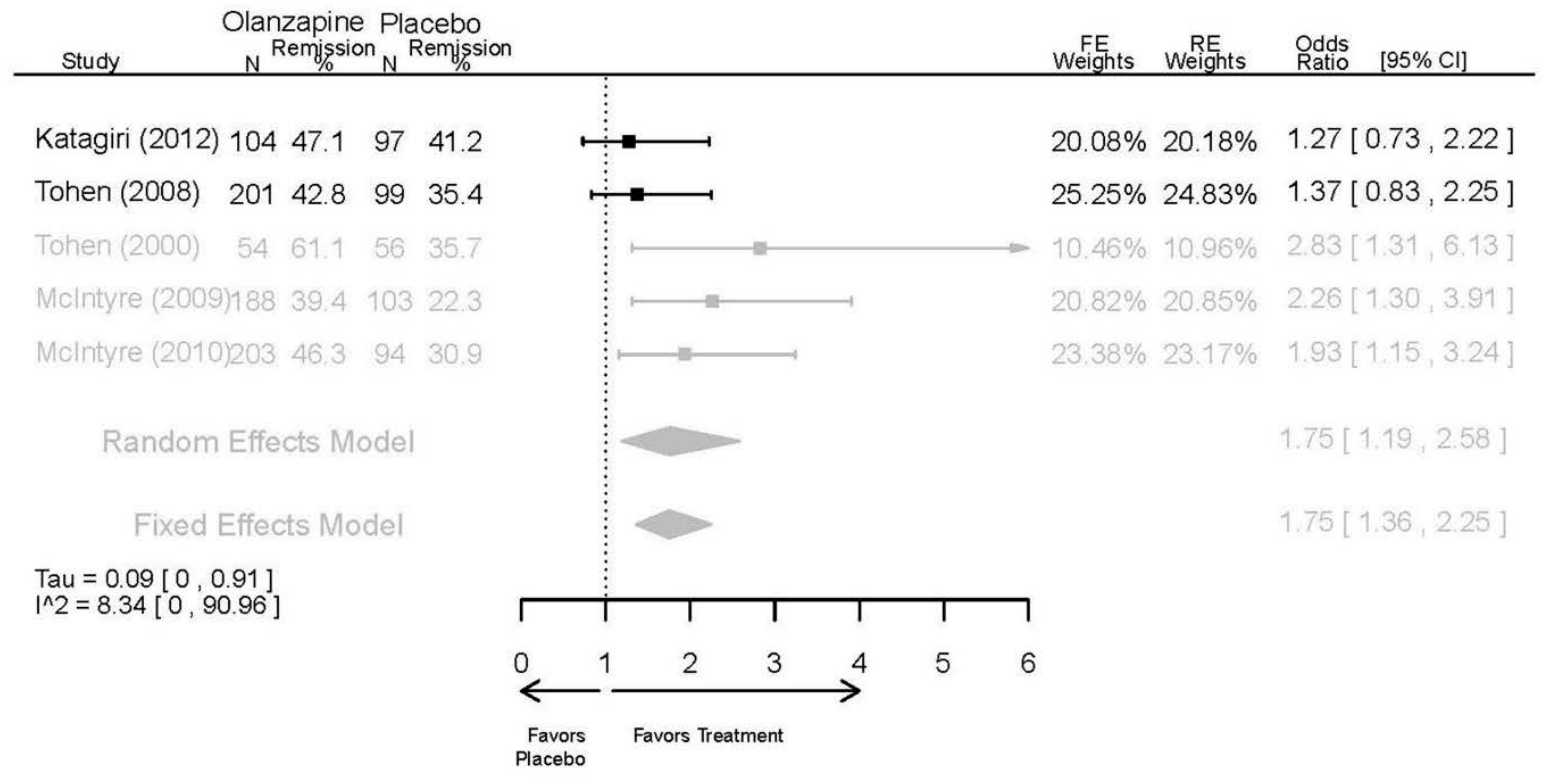

Appendix Figure E42. Olanzapine vs. placebo - YMRS

Difference in Mean Change in YMRS

from Baseline to 3 Weeks

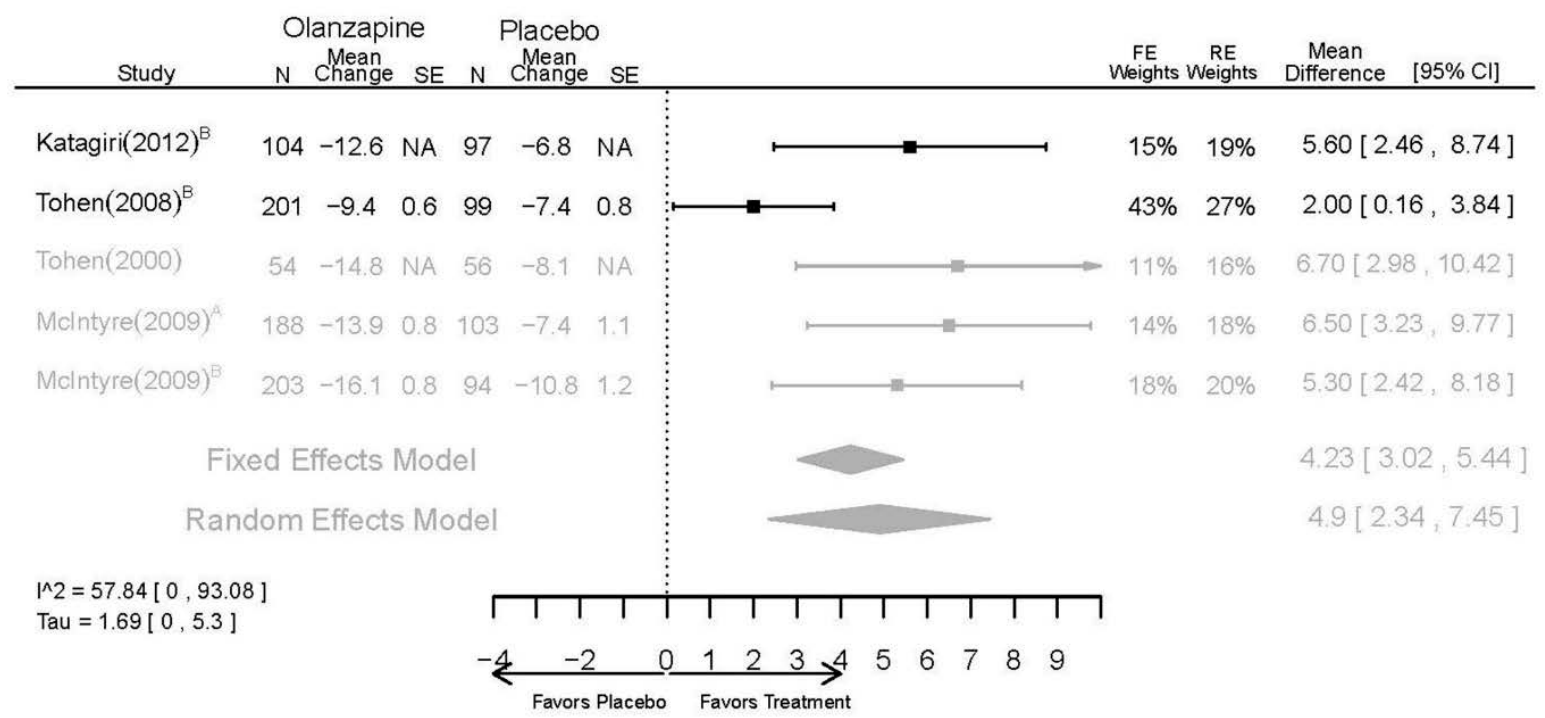




\section{Appendix Figure E43. Olanzapine vs. placebo - CGI}

Difference in Mean Change in CGI-BP-S (Overall) from Baseline to 3 Weeks

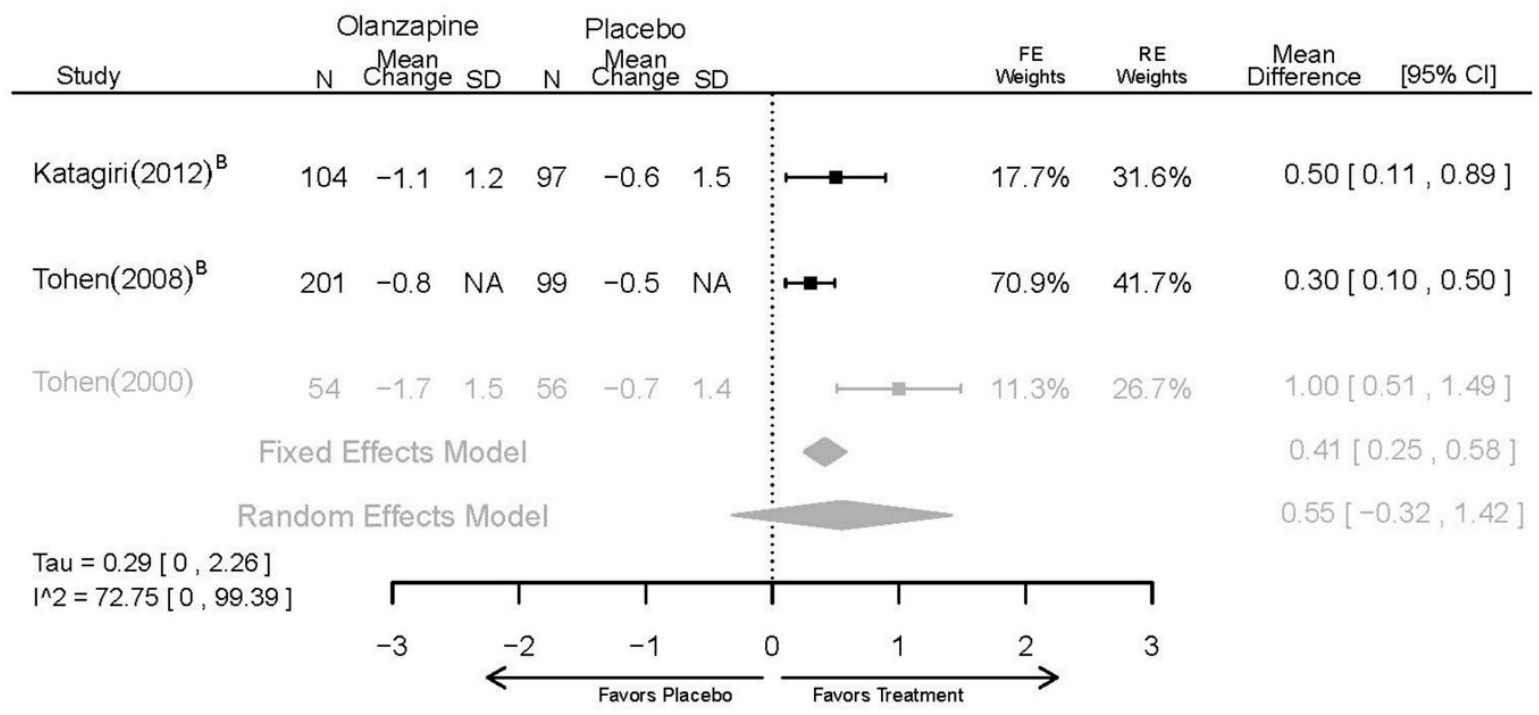

Appendix Figure E44. Olanzapine vs. placebo - overall withdrawal Odds Ratio of Withdrawal due to All Causes at 3 Weeks

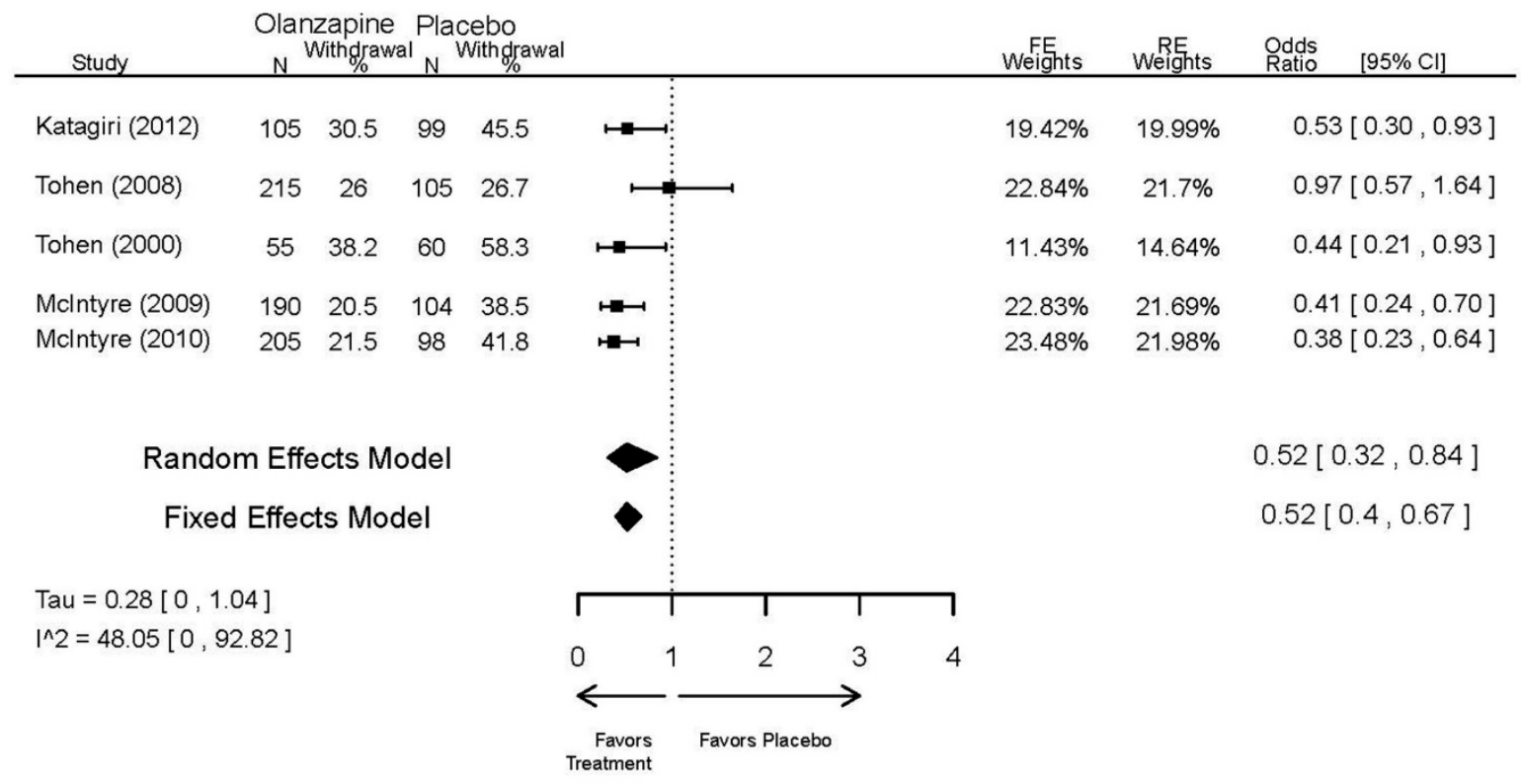


Appendix Figure E45. Olanzapine vs. placebo - withdrawal - lack of efficacy

Odds Ratio of Withdrawal due to Lack of Efficacy at 3 Weeks

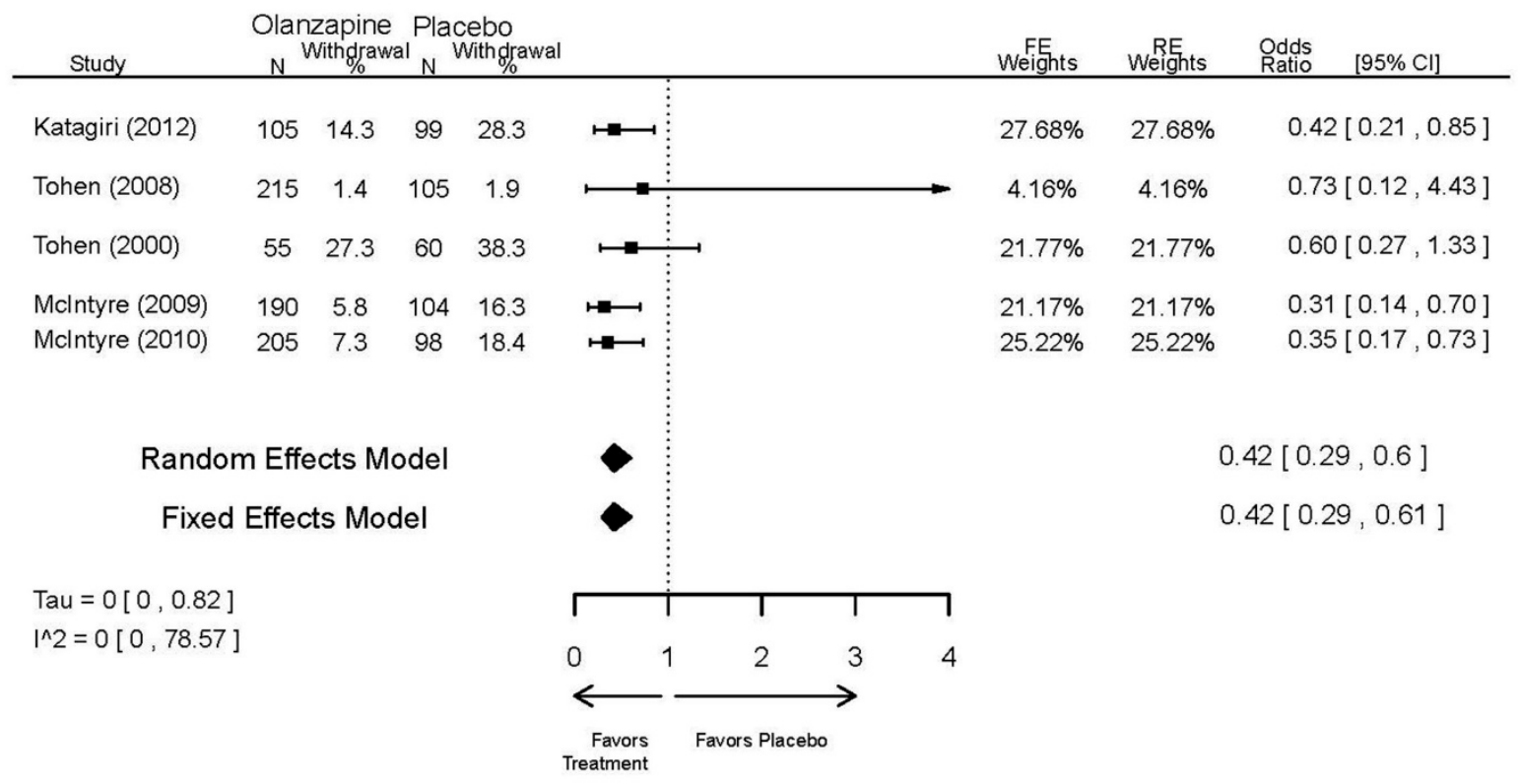

Appendix Figure E46. Olanzapine vs. placebo - withdrawal - adverse events

Odds Ratio of Withdrawal due to Adverse Events at 3 Weeks

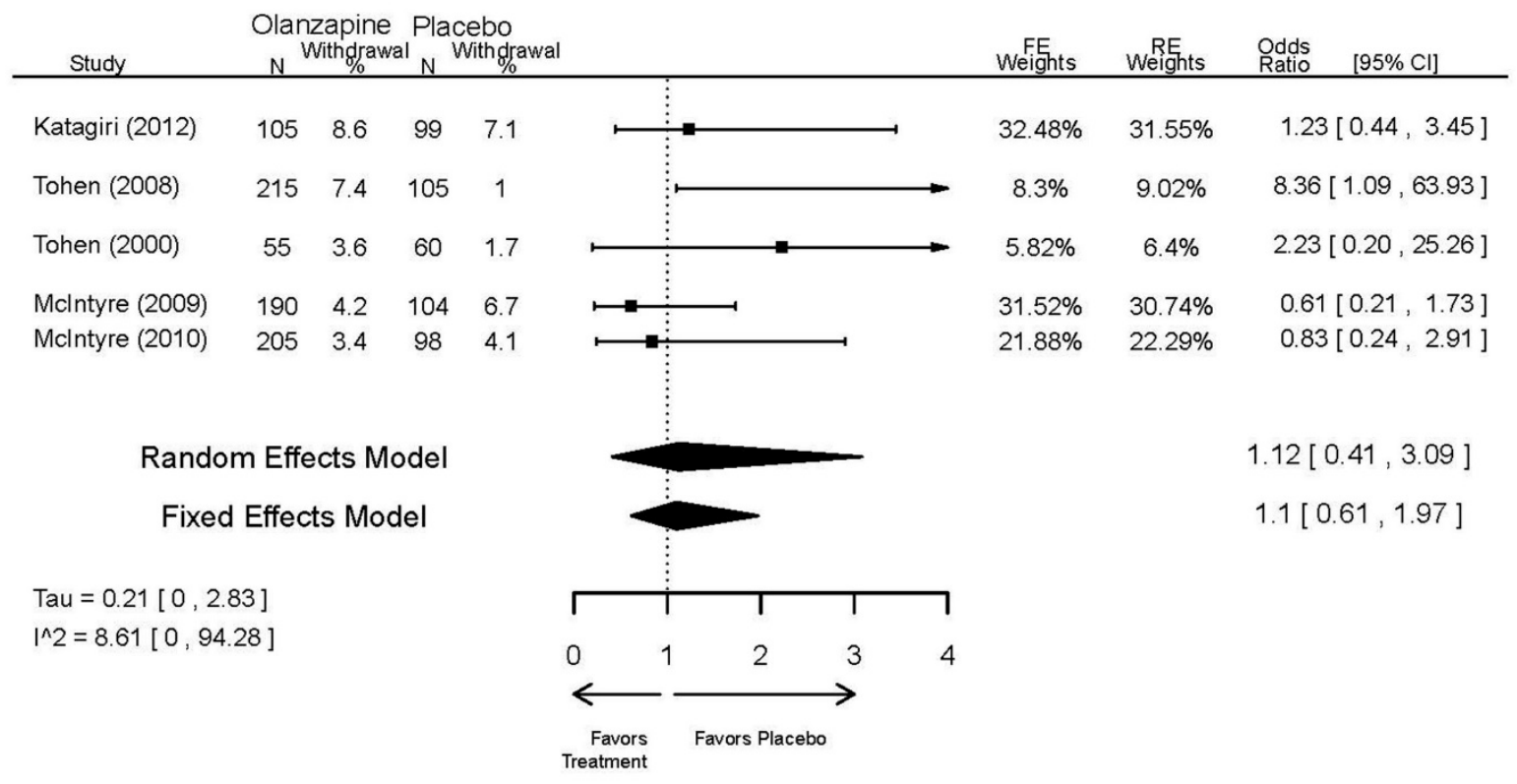


Appendix Figure E47. Olanzapine vs. placebo - serious adverse events

Odds Ratio of Serious Adverse Events at 3 Weeks

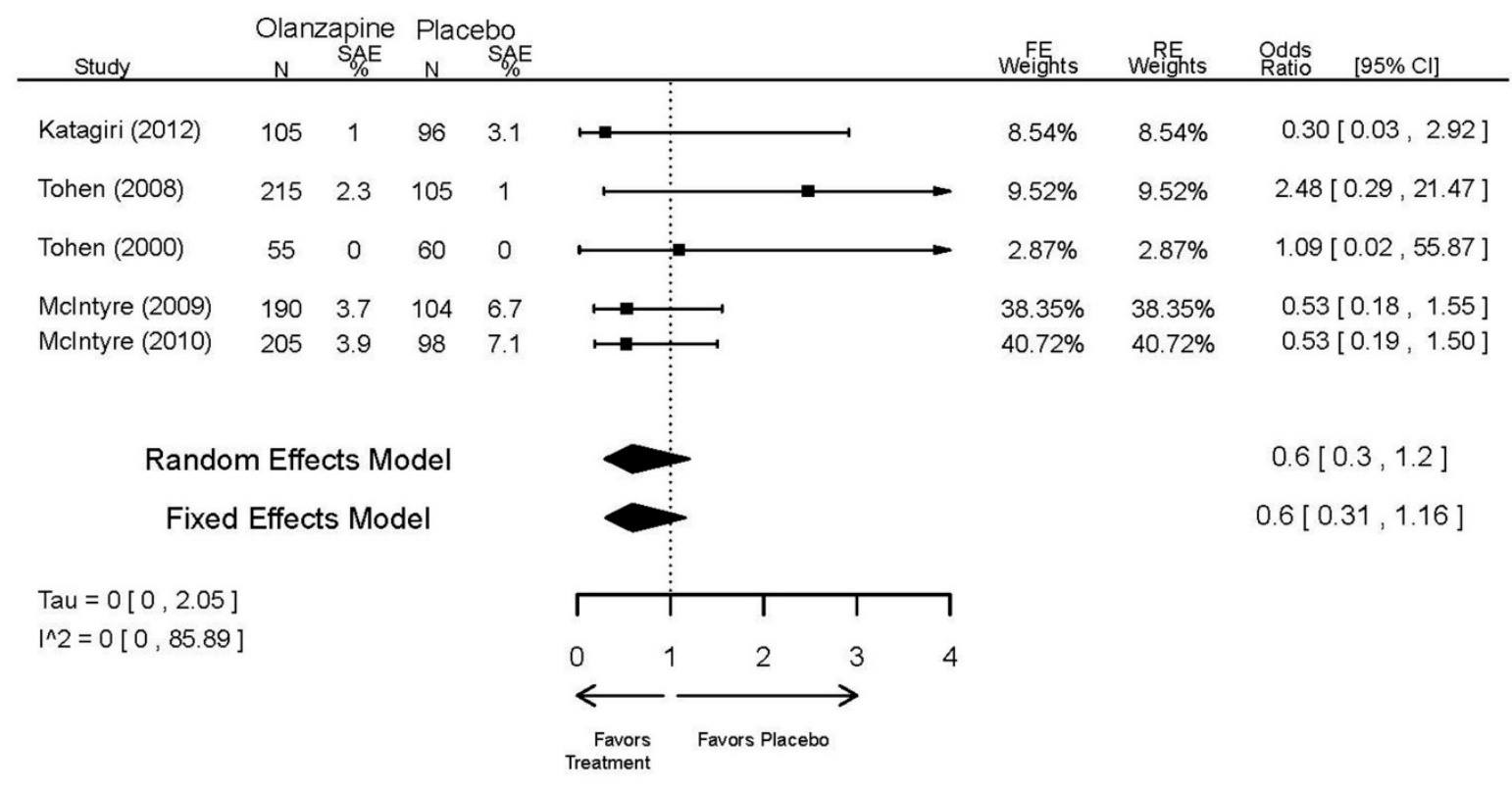

Appendix Figure E48. Olanzapine vs. placebo - EPS

Odds Ratio of Extrapyramidal Symptoms at 3 Weeks

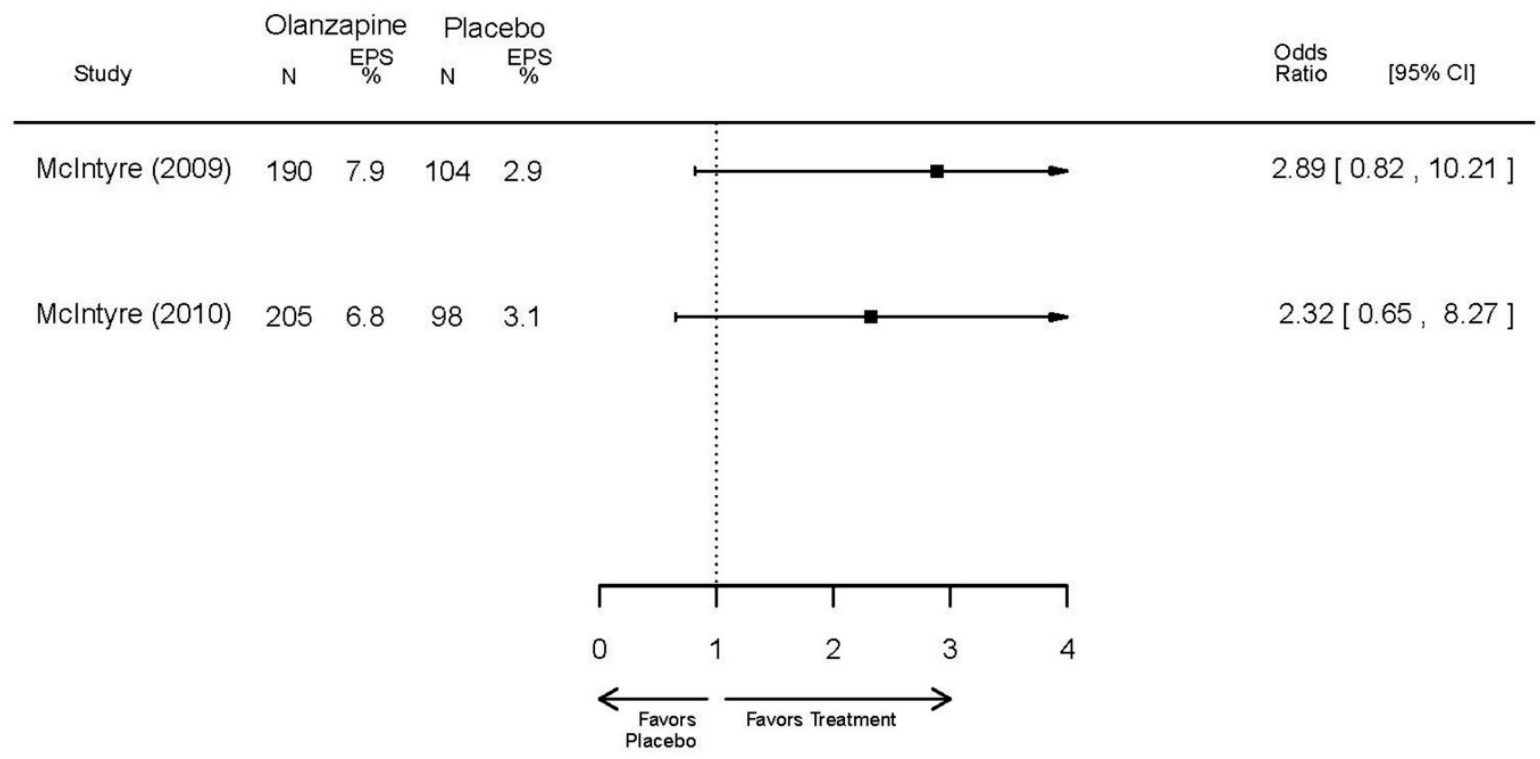


Appendix Figure E49. Olanzapine vs. placebo - emergent depression Odds Ratio of Weight Gain of Emergent or Worsening Depression

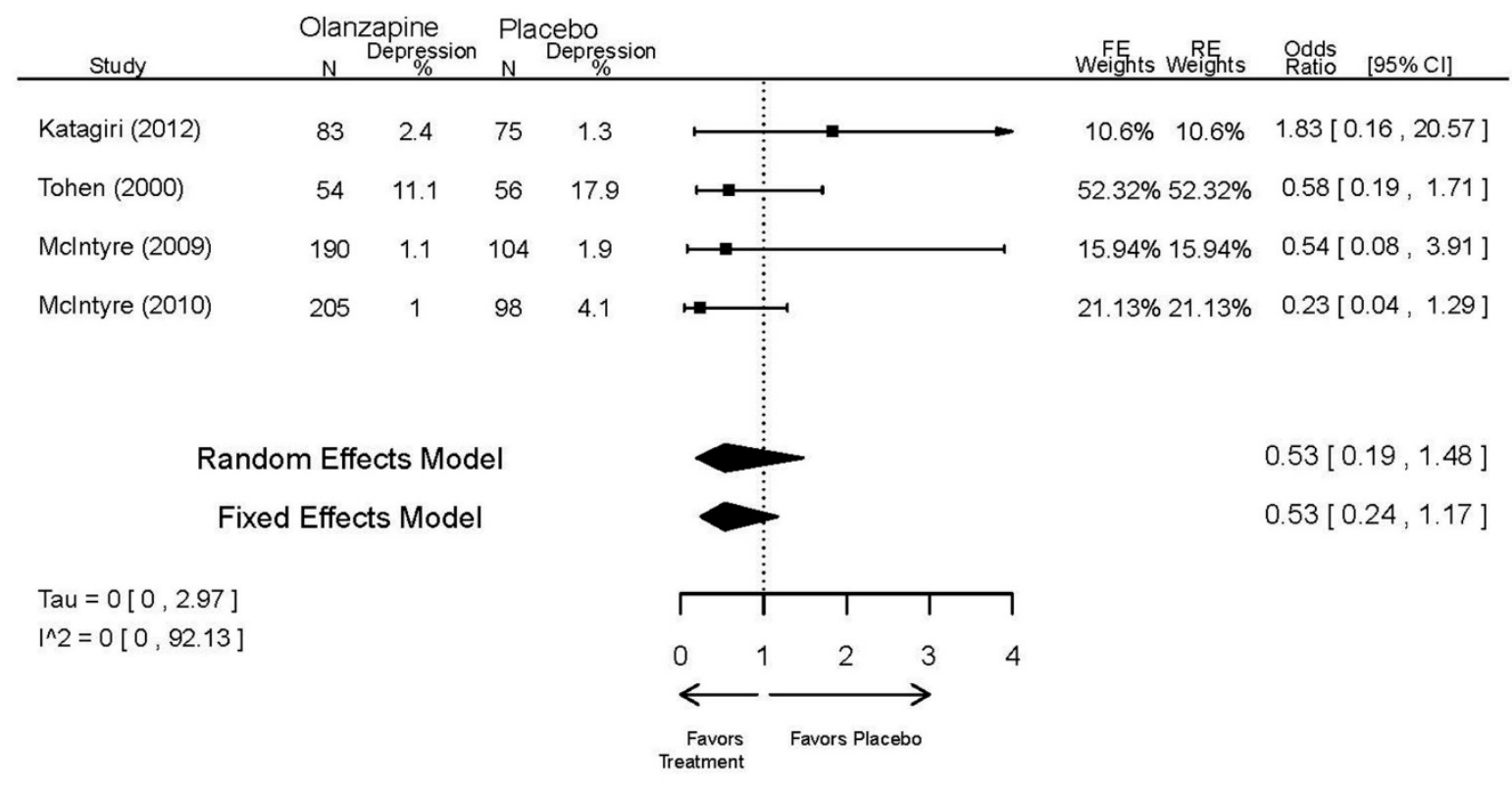

Appendix Figure E50. Olanzapine vs. placebo - weight gain

Odds Ratio of Weight Gain of at least $7 \%$ at 3 Weeks

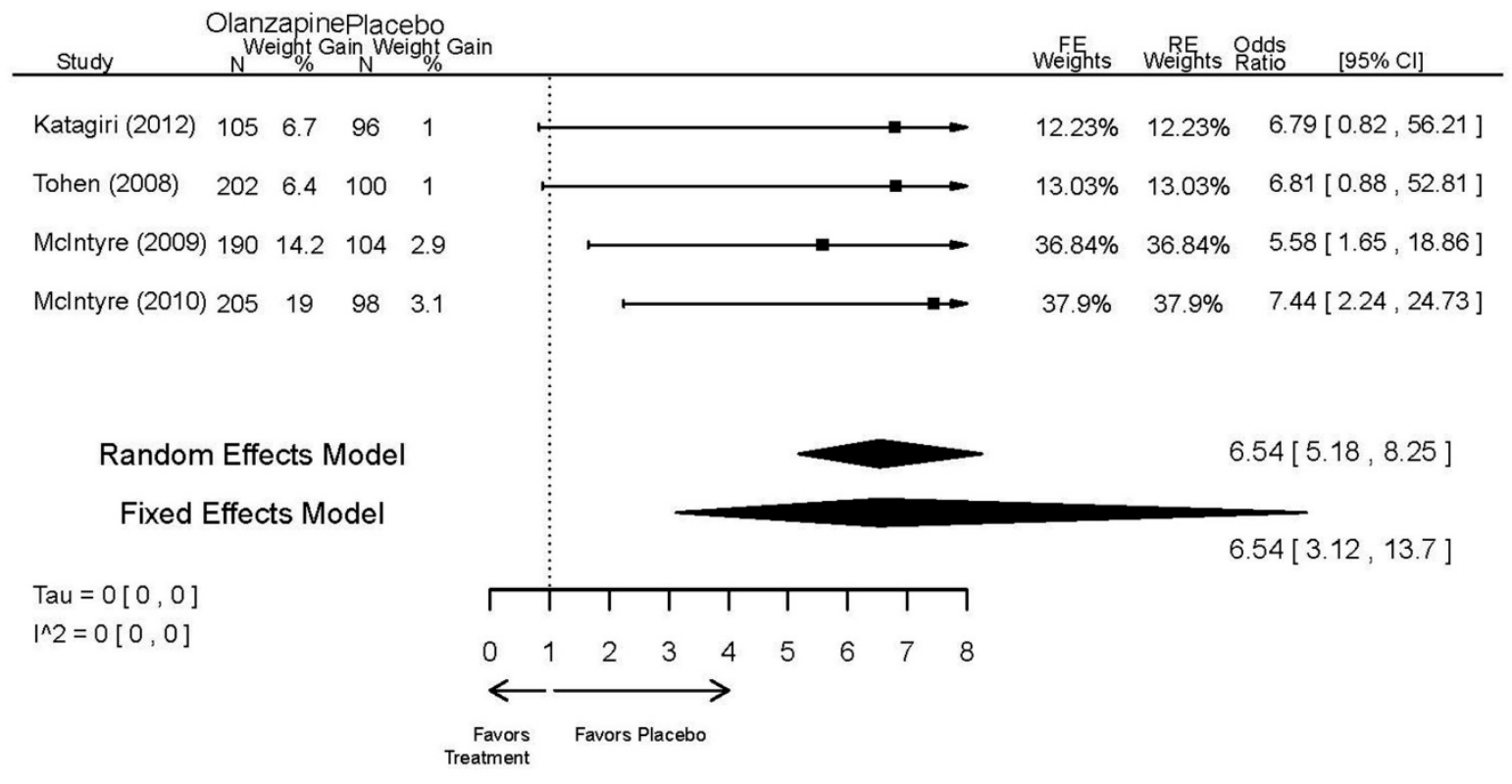


Appendix Figure E51. Olanzapine vs. lithium - response

Odds Ratio of Response ( $>50 \%$ Reduction in Mania Scale) at 3 Weeks

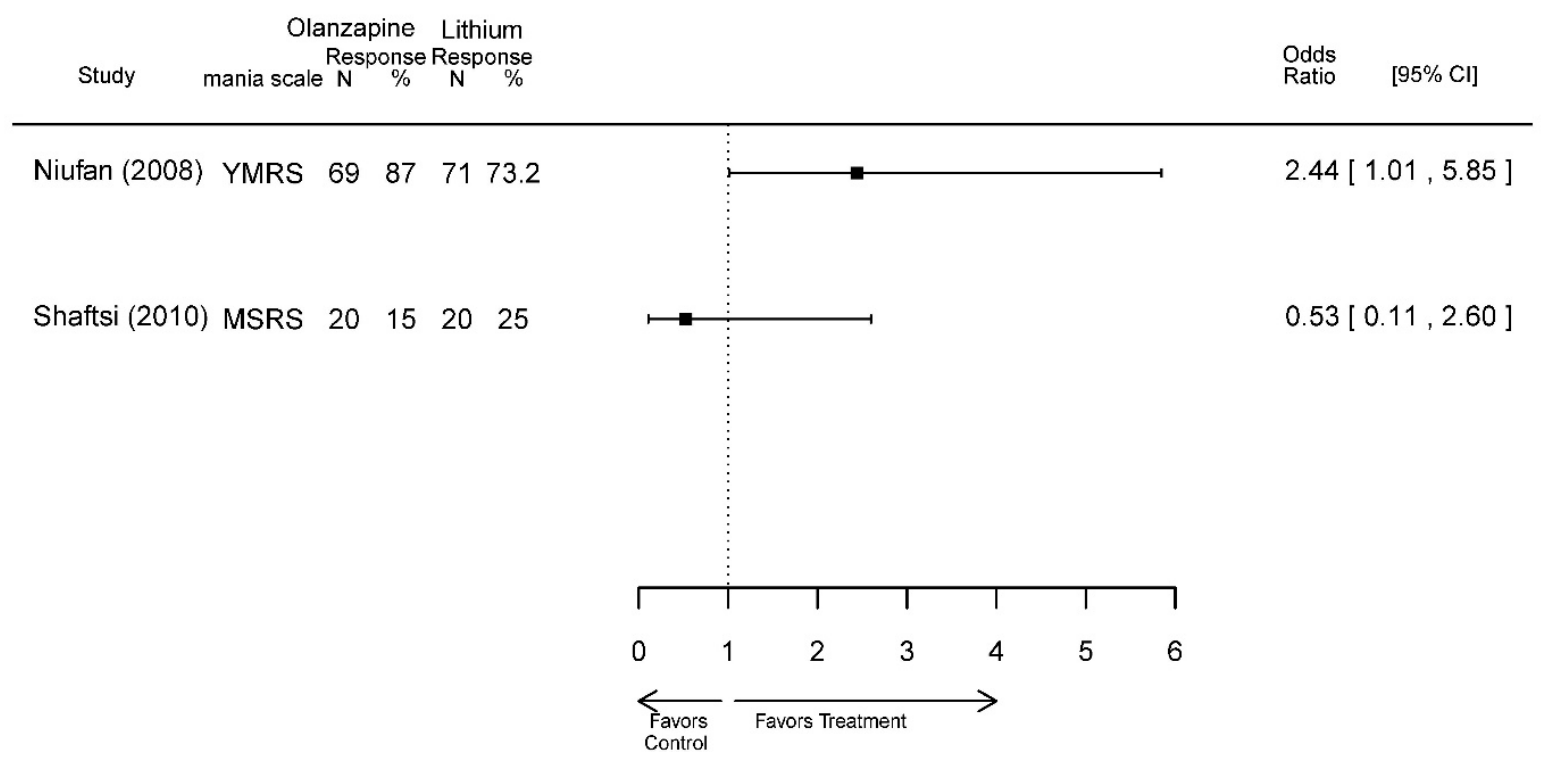

Appendix Figure E52. Olanzapine vs. lithium - overall withdrawal

Odds Ratio of Overall Withdrawal

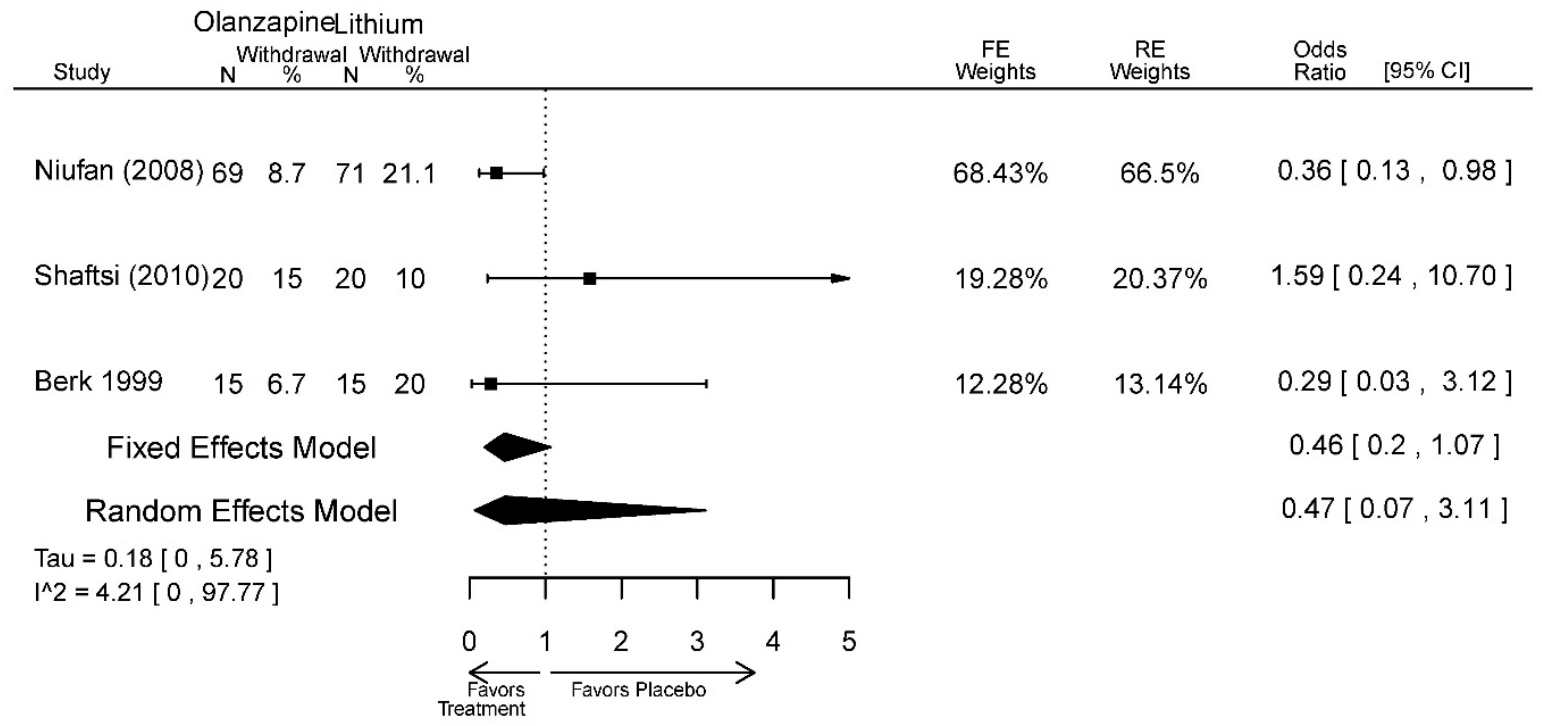


Appendix Figure E53. Olanzapine vs. lithium - withdrawal - adverse events

Odds Ratio of Withdrawal due to Adverse Events at Last Measurement

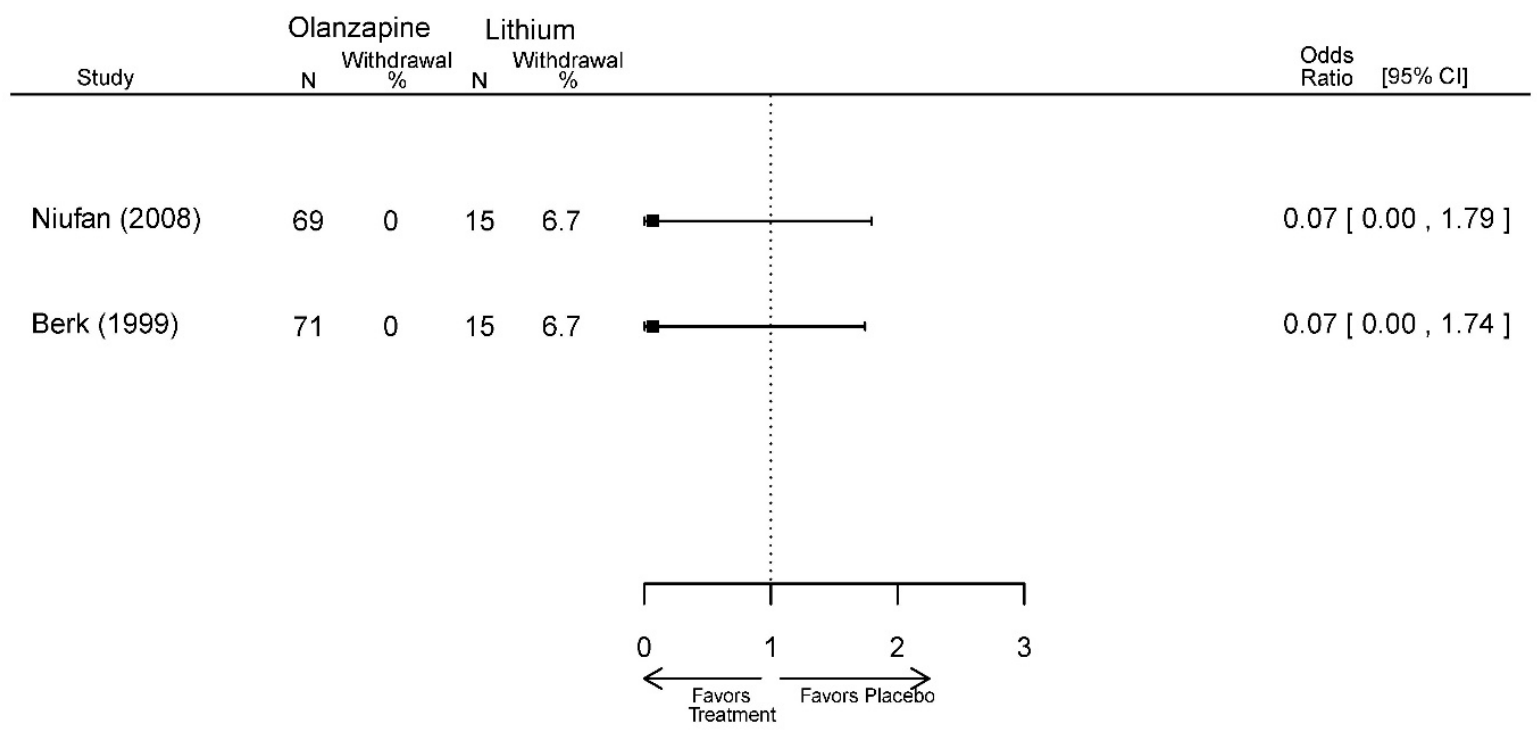

Appendix Figure E54. Olanzapine vs. divalproex/valproate - response

Odds Ratio of Response ( $>50 \%$ Reduction in Mania Scale) at 3 Weeks

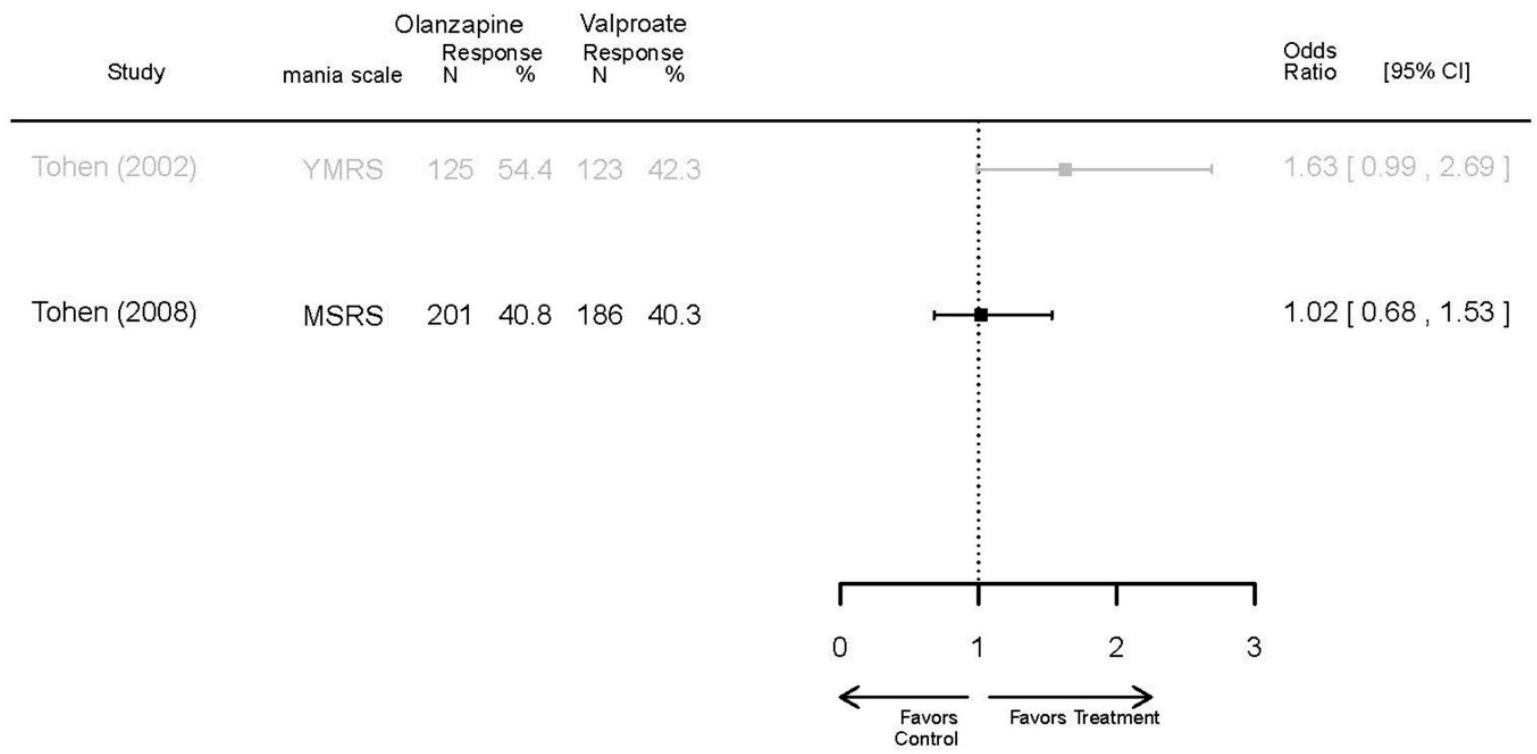


Appendix Figure E55. Olanzapine vs. divalproex/valproate - remission Odds Ratio of Remission (YMRS 12 or less) at 3 Weeks

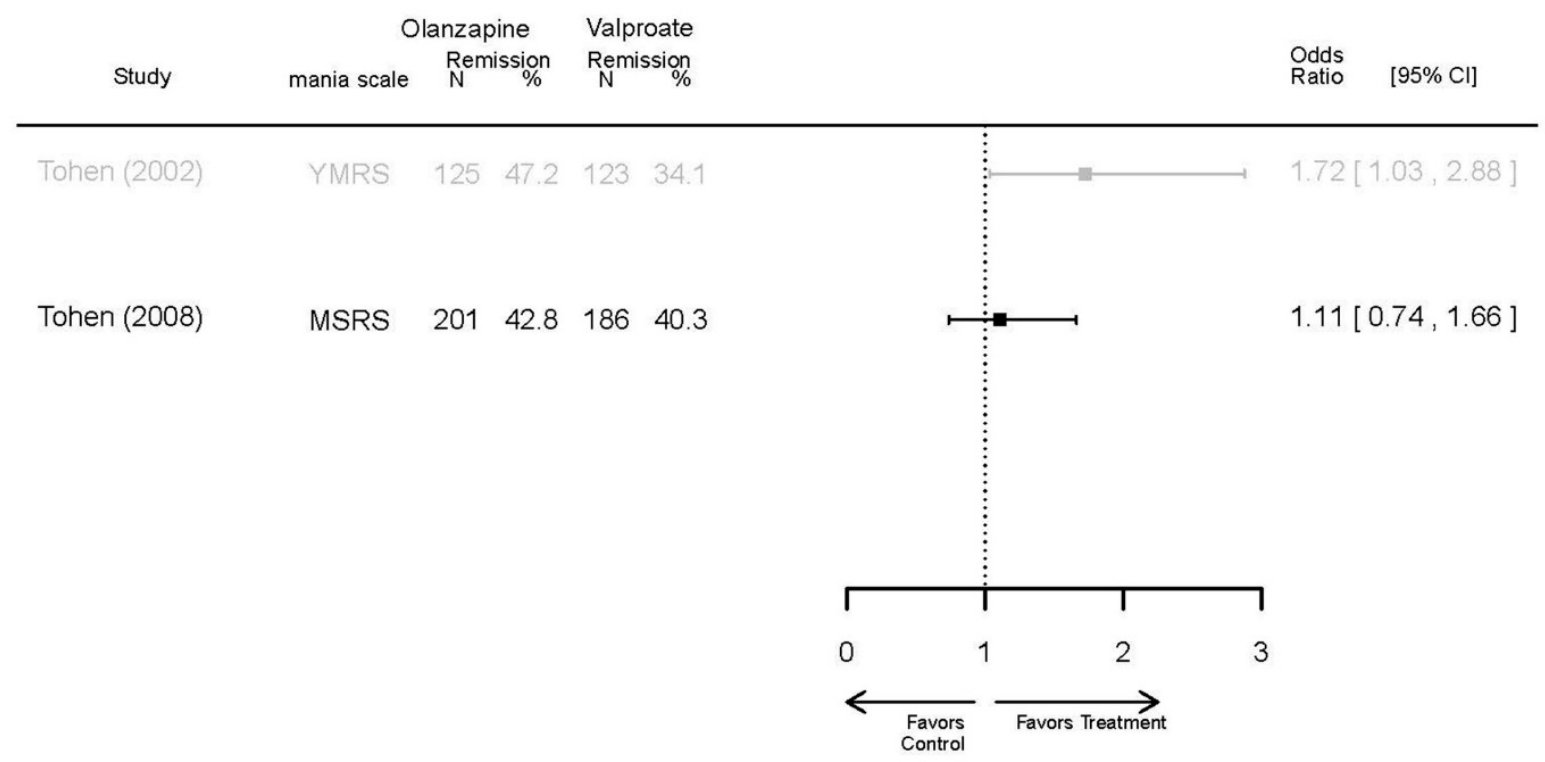

Appendix Figure E56. Olanzapine vs. divalproex/valproate - YMRS

Difference in Mean Change in YMRS

from Baseline to 3 Weeks

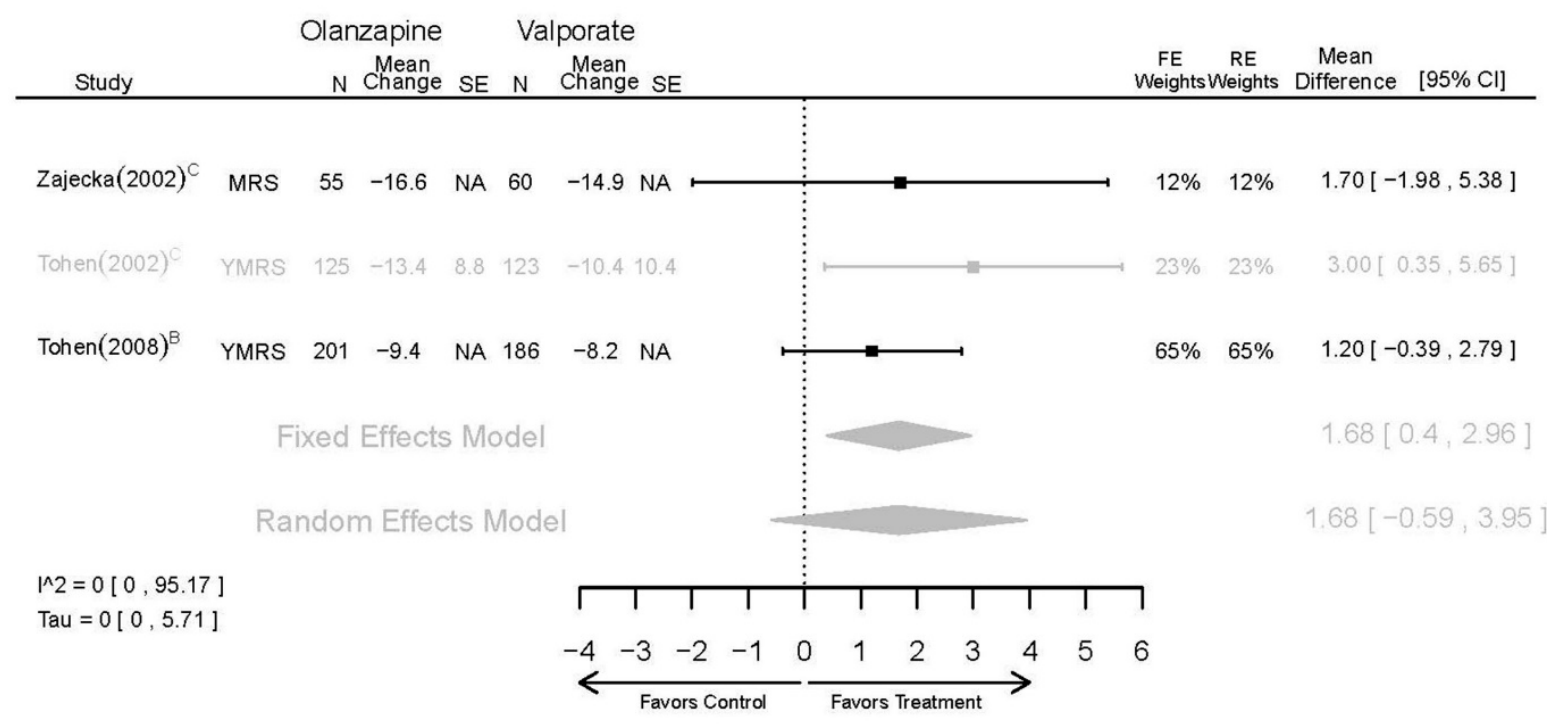


Appendix Figure E57. Olanzapine vs. divalproex/valproate - CGI

Difference in Mean Change in CGI-BP-S (Overall)

from Baseline to 3 Weeks

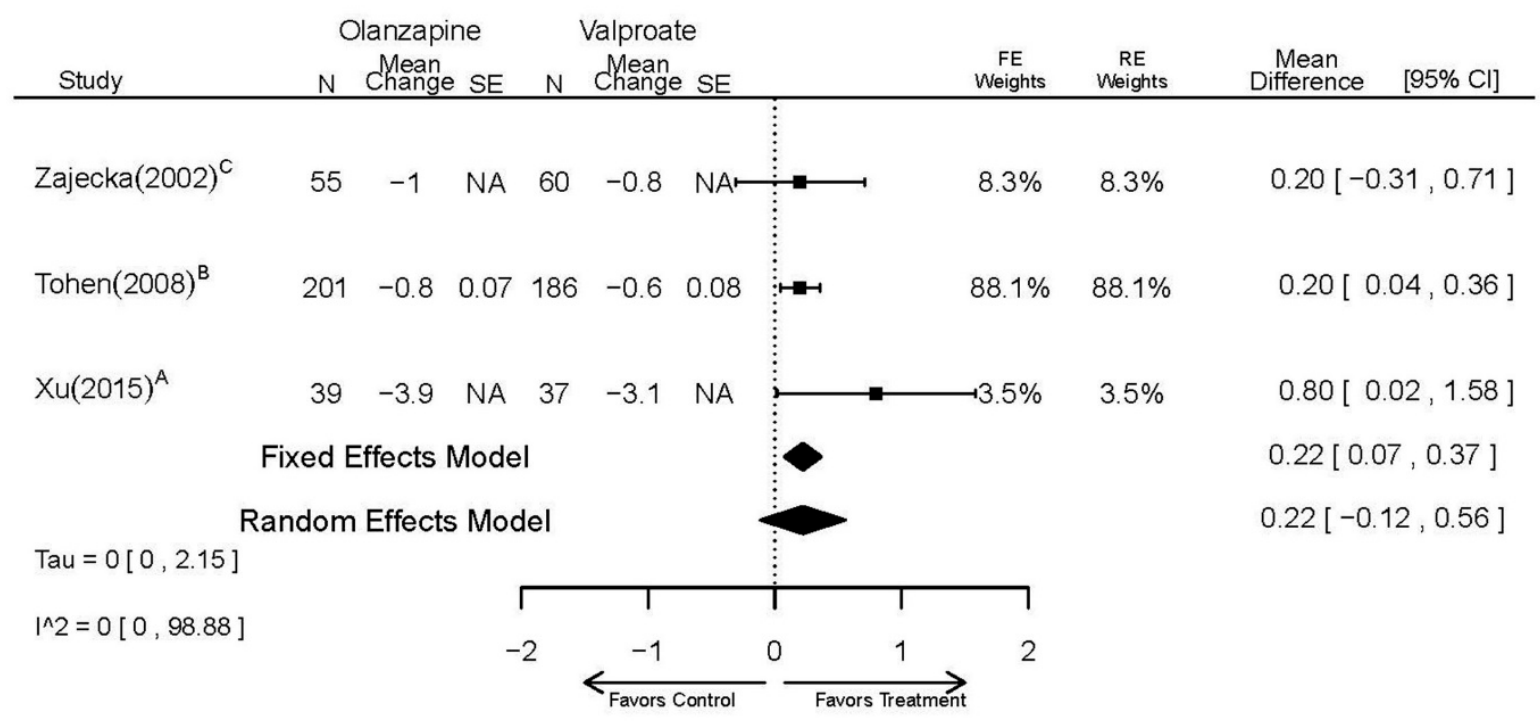

Appendix Figure E58. Olanzapine vs. divalproex/valproate - overall withdrawal Odds Ratio of Withdrawal due to All Causes at 3 Weeks

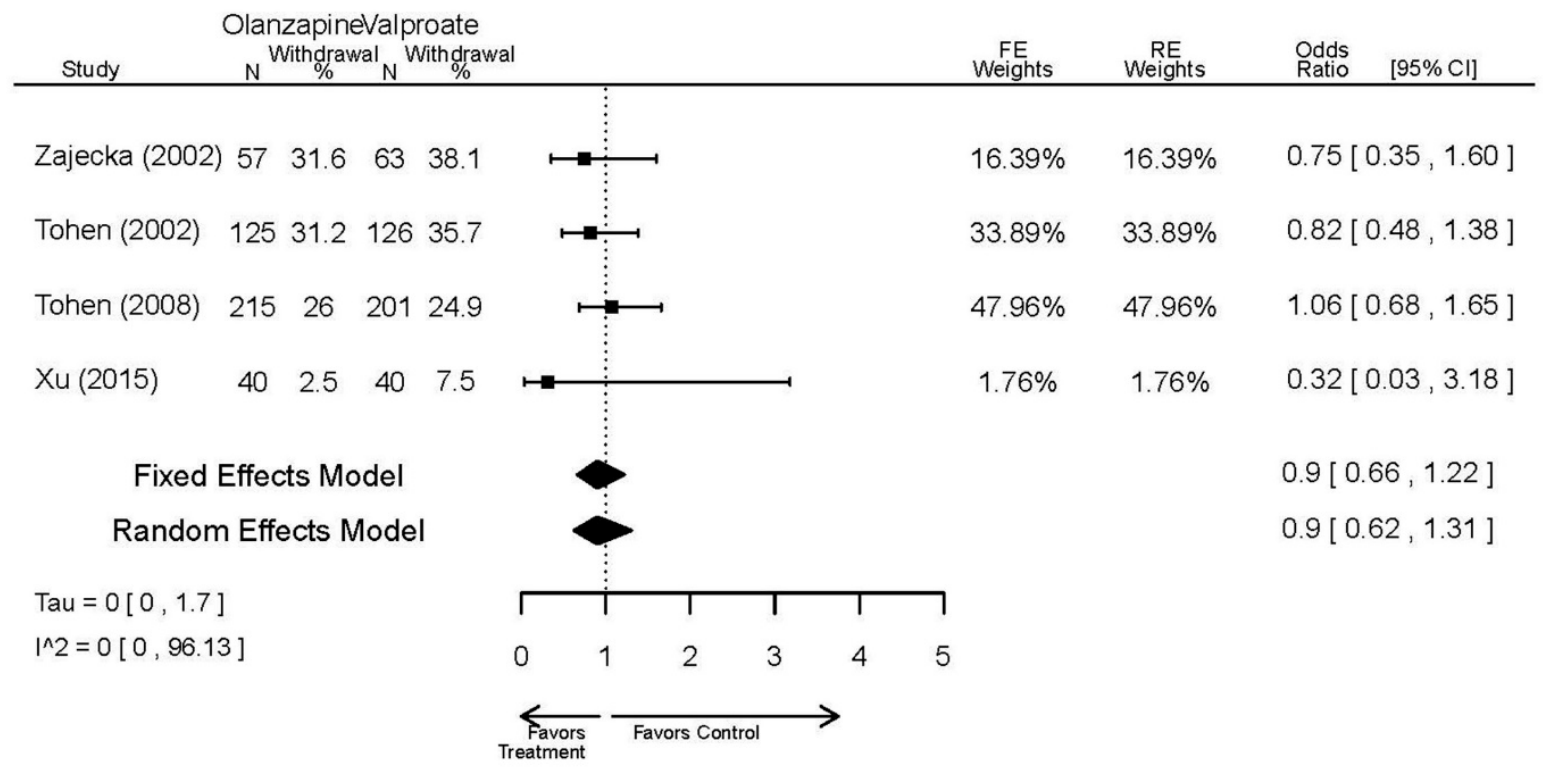


Appendix Figure E59. Olanzapine vs. divalproex/valproate - withdrawal - lack of efficacy Odds Ratio of Withdrawal due to Lack of Efficacy at 3 Weeks

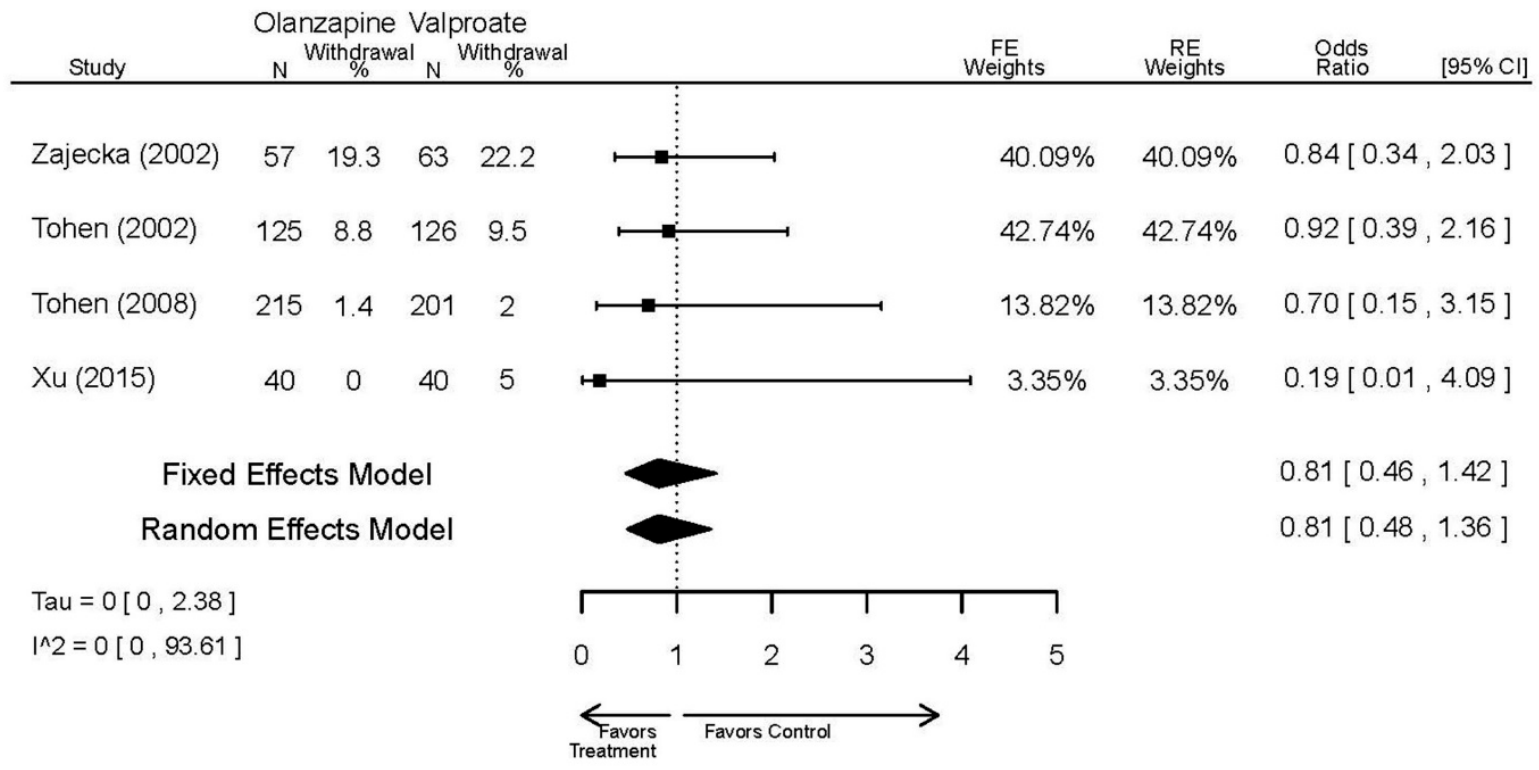

Appendix Figure E60. Olanzapine vs. divalproex/valproate - withdrawal - adverse events Odds Ratio of Withdrawal due to Adverse Events at 3 Weeks

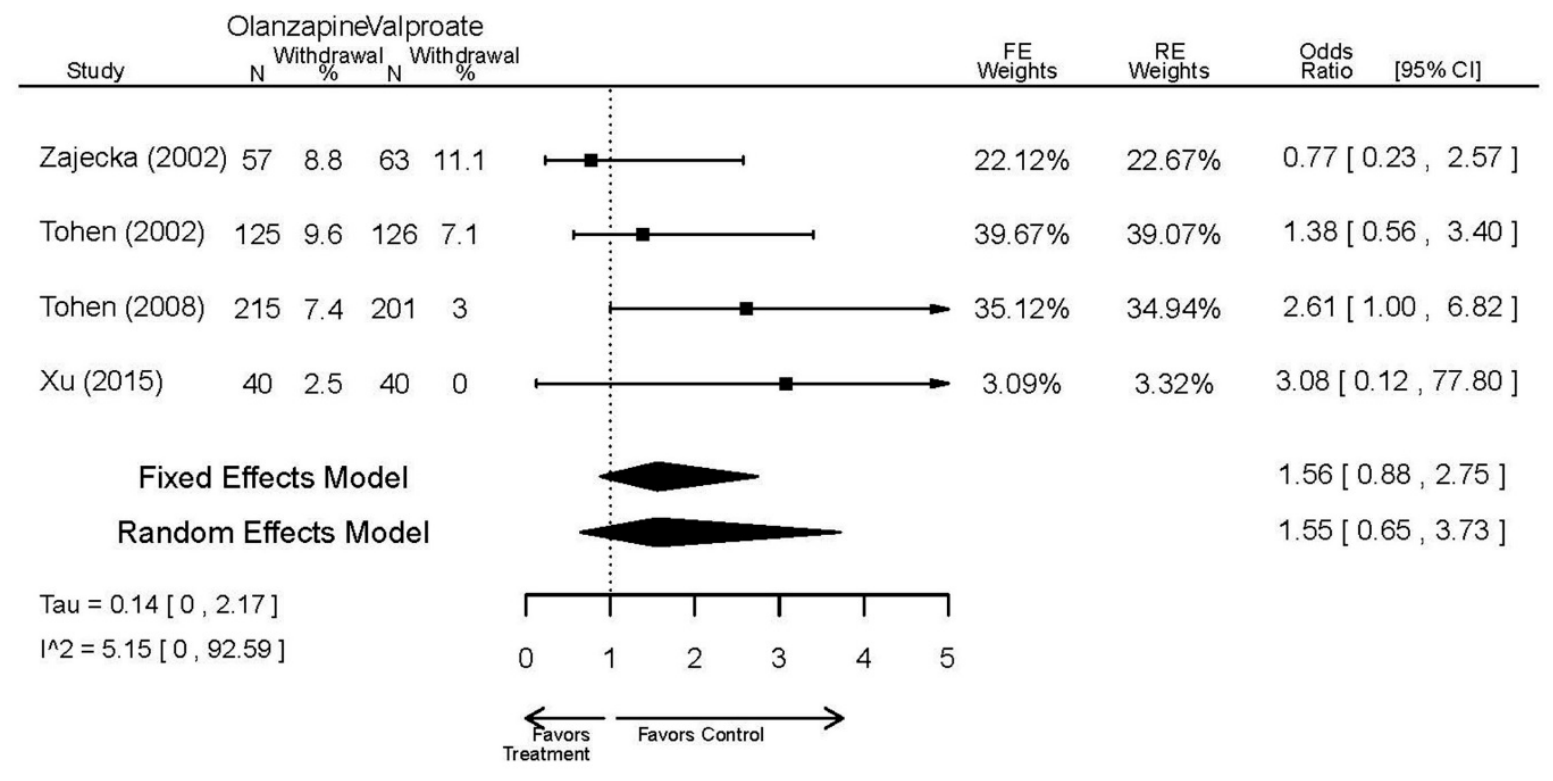


Appendix Figure E61. Olanzapine vs. divalproex/valproate - SAE

Odds Ratio of Serious Adverse Events at 3 Weeks

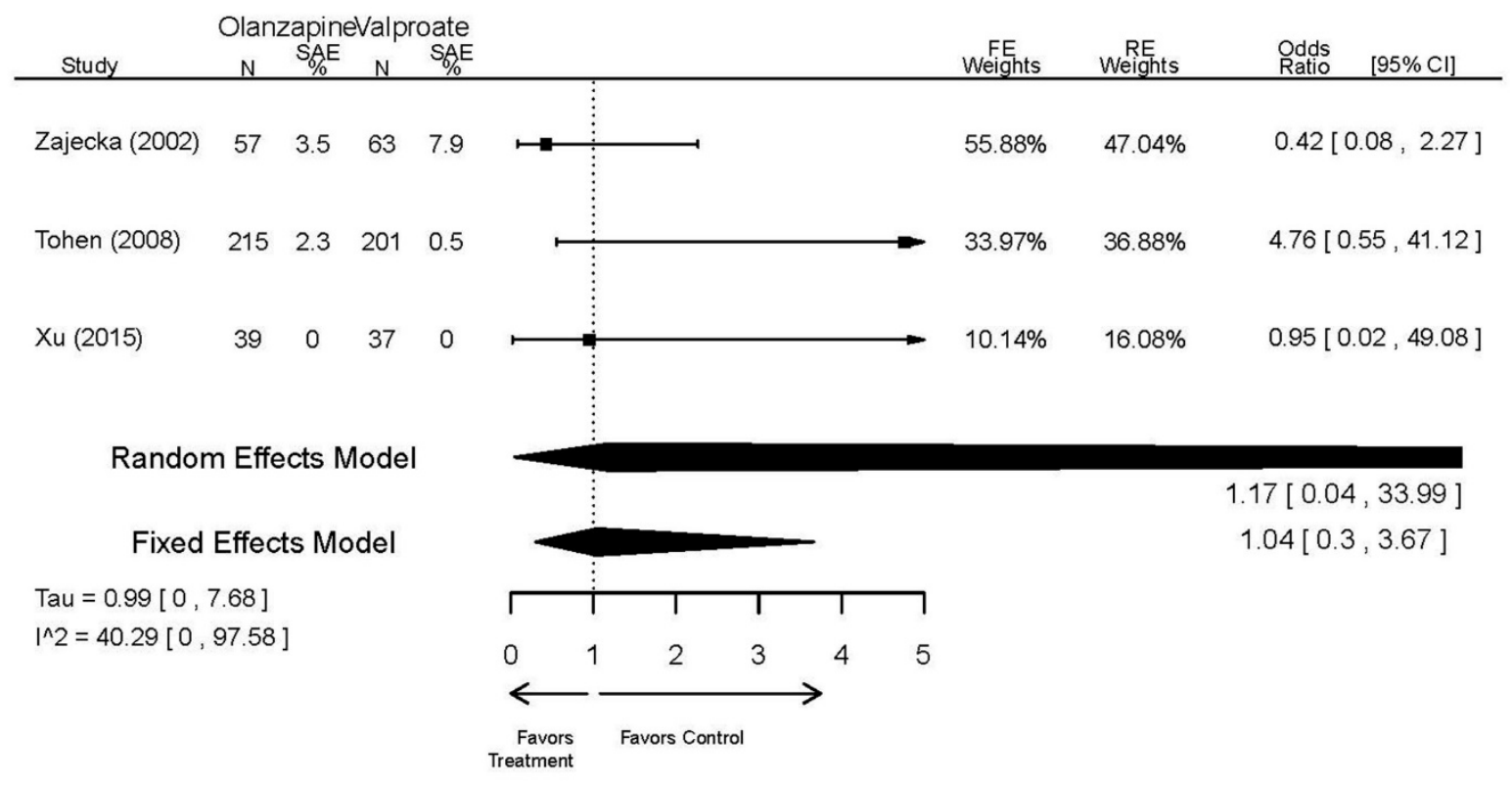




\begin{tabular}{|c|c|c|c|c|c|c|}
\hline Drug & $\begin{array}{l}\text { Study } \\
\text { PMID } \\
\text { RoB }\end{array}$ & Responder/Remitter & Symptom & Function & Other & $\mathrm{AE}$ \\
\hline \multirow[t]{5}{*}{ Olanzapine } & $\begin{array}{l}\text { Katagiri, } 2012^{20} \\
22134043 \\
\text { Moderate }\end{array}$ & $\begin{array}{l}\text { See forest plot E40 } \\
\text { above for response. }\end{array}$ & $\begin{array}{l}\text { See forest plot E42 } \\
\text { above for YMRS. }\end{array}$ & $\begin{array}{l}\text { See forest plot E43 } \\
\text { above for CGI. }\end{array}$ & $\begin{array}{l}\text { See forest plot E44, } \\
\text { E45, E46 above for } \\
\text { Withdrawals. }\end{array}$ & $\begin{array}{l}\text { See forest plots E47, } \\
\text { E48, E49, E50 above for } \\
\text { Adeverse Effects. }\end{array}$ \\
\hline & $\begin{array}{l}\text { McIntyre, } \\
2010^{8} \\
20096936 \\
\text { High }\end{array}$ & $\begin{array}{l}\text { See forest plot E40 } \\
\text { above for response. }\end{array}$ & $\begin{array}{l}\text { See forest plot E42 } \\
\text { above for YMRS. }\end{array}$ & $\begin{array}{l}\text { See forest plot E43 } \\
\text { above for CGI. }\end{array}$ & $\begin{array}{l}\text { See forest plot E44, } \\
\text { E45, E46 above for } \\
\text { Withdrawals. }\end{array}$ & $\begin{array}{l}\text { See forest plots E41, } \\
\text { E42, E43, E44 above for } \\
\text { Adeverse Effects. }\end{array}$ \\
\hline & $\begin{array}{l}\text { McIntyre, } \\
2009^{9} \\
19839993 \\
\text { High }\end{array}$ & $\begin{array}{l}\text { See forest plot E40 } \\
\text { above for response. }\end{array}$ & $\begin{array}{l}\text { See forest plot E42 } \\
\text { above for YMRS. }\end{array}$ & $\begin{array}{l}\text { See forest plot E43 } \\
\text { above for CGI. }\end{array}$ & $\begin{array}{l}\text { See forest plot E44, } \\
\text { E45, E46 above for } \\
\text { Withdrawals. }\end{array}$ & $\begin{array}{l}\text { See forest plots E47, } \\
\text { E48, E49, E50 above for } \\
\text { Adeverse Effects. }\end{array}$ \\
\hline & $\begin{array}{l}\text { Tohen, } 2008 b^{28} \\
19014751\end{array}$ & $\begin{array}{l}\text { See forest plot E40 } \\
\text { above for response. }\end{array}$ & $\begin{array}{l}\text { See forest plot E42 } \\
\text { above for YMRS. }\end{array}$ & $\begin{array}{l}\text { See forest plot E43 } \\
\text { above for CGI. }\end{array}$ & $\begin{array}{l}\text { See forest plot E44, } \\
\text { E45, E46 above for } \\
\text { Withdrawals. }\end{array}$ & $\begin{array}{l}\text { See forest plots E47, } \\
\text { E48, E49, E50 above for } \\
\text { Adeverse Effects. } \\
\text { Suicidal Ideation } \\
\text { Olanzapine: } 1 \text { case } \\
\text { Placebo: } 0 \text { cases }\end{array}$ \\
\hline & $\begin{array}{l}\text { Tohen, } 2000^{29} \\
10986547 \\
\text { High }\end{array}$ & $\begin{array}{l}\text { See forest plot E40 } \\
\text { above for response. }\end{array}$ & $\begin{array}{l}\text { See forest plot E42 } \\
\text { above for YMRS. }\end{array}$ & $\begin{array}{l}\text { See forest plot E43 } \\
\text { above for CGI. }\end{array}$ & $\begin{array}{l}\text { See forest plot E44, } \\
\text { E45, E46 above for } \\
\text { Withdrawals. }\end{array}$ & $\begin{array}{l}\text { See forest plots E47, } \\
\text { E48, E49, E50 above for } \\
\text { Adeverse Effects. }\end{array}$ \\
\hline
\end{tabular}

Abbreviations: AE=Adverse Events; ANCOVA=Analysis of Covariance; CGI=Clinical Global Impressions Scale; CI=Confidence Interval; EPS=extrapyramidal symptoms; ESRS=Extrapyrimidal Symptom Rating Scale; GAS=Global Assessment Scale; MADRS=Montgomery-Asberg Depression Rating Scale; NR=Not reported; NS=not significant; OR=Odds Ratio; PMID=PubMed Identification Number; ROB=Risk of Bias; SAE=Serious Adverse Events; YMRS = Young Mania Rating Scale 
Appendix Table E24. Strength of evidence assessment: olanzapine versus placebo for acute mania

\begin{tabular}{|c|c|c|c|c|c|c|c|c|}
\hline Comparison & Outcome & $\begin{array}{c}\text { \# Studies/ } \\
\text { Design } \\
\text { (n analyzed) }\end{array}$ & $\begin{array}{l}\text { Finding or } \\
\text { Summary } \\
\text { Statistic }\end{array}$ & $\begin{array}{c}\text { Study } \\
\text { Limitations }\end{array}$ & Consistency & Directness & Precision & $\begin{array}{c}\text { Overall } \\
\text { Gradel } \\
\text { Conclusion }\end{array}$ \\
\hline \multirow{6}{*}{$\begin{array}{l}\text { Olanzapine } \\
\text { vs. placebo }\end{array}$} & Response 3 wks & $\begin{array}{l}5 \text { RCTs } \\
(n=1199)\end{array}$ & $\begin{array}{l}\text { Favors } \\
\text { Olanzapine } \\
\text { OR } 1.99(95 \% \\
\text { Cl 1.29, 3.08) } \\
\end{array}$ & Moderate & Consistent & Direct & Imprecise & Low \\
\hline & Remission 3 wks & $\begin{array}{l}5 \text { RCTs } \\
(n=1199)\end{array}$ & $\begin{array}{l}\text { Favors } \\
\text { Olanzapine } \\
\text { OR } 1.75(95 \% \\
\text { Cl 1.19, 2.58) } \\
\end{array}$ & Moderate & Consistent & Direct & Imprecise & Low \\
\hline & YMRS 3 wks & $\begin{array}{l}5 \text { RCTs } \\
(n=1199)\end{array}$ & $\begin{array}{l}\text { Favors } \\
\text { Olanzapine } \\
\text { MD } 4.9(95 \% \mathrm{Cl} \\
2.34,7.45) \\
\end{array}$ & Moderate & Consistent & Direct & Imprecise & Low \\
\hline & CGI-BP-S 3 wks & $\begin{array}{l}3 \text { RCTs } \\
(n=611)\end{array}$ & NS & Moderate & Consistent & Direct & Imprecise & Low \\
\hline & $\begin{array}{l}\text { Withdrawal - Lack } \\
\text { of Efficacy, Overall }\end{array}$ & $\begin{array}{l}5 \text { RCTs } \\
(n=1199)\end{array}$ & $\begin{array}{l}\text { Favors } \\
\text { Olanzapine }\end{array}$ & Moderate & Consistent & Direct & Imprecise & Low \\
\hline & Withdrawal - AE & $\begin{array}{l}5 \text { RCTs } \\
(n=1199)\end{array}$ & NS & Moderate & Consistent & Indirect & Imprecise & Insufficient \\
\hline
\end{tabular}

Abbreviations: CGI-BP=Clinical Global Impressions Scale for Bipolar Disorder; NS=not significant; RCT=randomized controlled trial; YMRS = Young Mania Rating Scale

Notes:

1. Publication bias for antipsychotics, antidepressants, and behavioral interventions for depressive disorders is suspected.

2. Data were generally imprecise due to missing data from high attrition rates, which was commonly dealt with by Last Observation Carried Forward (LOCF). LOCF requires an assumption that the health status of patients who dropped out of the trial would not have changed had future observations been recorded, a strong assumption in the context of bipolar disorder research. 
Appendix Table E25. Outcomes summary: olanzapine versus active comparator for acute mania

\begin{tabular}{|c|c|c|c|c|c|c|}
\hline Comparison & $\begin{array}{l}\text { Study } \\
\text { PMID } \\
\text { RoB }\end{array}$ & Responder/Remitter & Symptom & Function & Other & $\overline{A E}$ \\
\hline $\begin{array}{l}\text { Olanzapine vs. } \\
\text { Haloperidol }\end{array}$ & $\begin{array}{l}\text { Tohen, } 2003^{26} \\
14662554 \\
\text { Moderate } \\
\text { Shi, } 2002^{27} \\
12177585 \\
\text { High }\end{array}$ & $\begin{array}{l}\text { Response } \\
6 \text { weeks } \\
\text { NS } \\
\text { Olanzapine=169/234 } \\
\text { Haloperidol=163/219 } \\
P=0.67 \\
12 \text { weeks } \\
\text { NS } \\
\text { Olanzapine=226/234 } \\
\text { Haloperidol=206/219 } \\
P=0.42 \\
\text { Remission } \\
6 \text { weeks } \\
\text { NS } \\
\text { OR = 1.27 (95\% Cl } \\
0.88,1.84) \\
P=0.15 \\
12 \text { weeks } \\
\text { NS } \\
1.37(95 \% \mathrm{Cl} 0.94 \\
1.99) \\
P=0.08\end{array}$ & $\begin{array}{l}\frac{Y M R S}{6 \text { weeks }} \\
\text { Favors Haloperidol } \\
\text { Difference in } \\
\text { Difference=-2.2 } \\
(95 \% \mathrm{Cl}-4.2,-0.2) \\
p=0.03 \\
12 \text { weeks } \\
\text { NS } \\
\text { Difference in } \\
\text { Difference=-0.3 } \\
(95 \% \mathrm{Cl}-2.0,1.4) \\
p=0.72\end{array}$ & NR & $\begin{array}{l}\text { Overall Withdrawal } \\
12 \text { weeks } \\
\text { NS } \\
\text { Olanzapine }=94 / 234 \\
\text { Haloperidol=103/219 } \\
p=0.15 \\
\text { Withdrawal due to Aes } \\
12 \text { weeks } \\
\text { NS } \\
\text { Olanzapine }=19 / 234 \\
\text { Haloperidol=25/219 } \\
p=0.27 \\
\text { Withdrawal, Lack of } \\
\text { Efficacy } \\
12 \text { weeks } \\
\text { NS } \\
\text { Olanzapine }=35 / 234 \\
\text { Haloperidol=33/219 }\end{array}$ & $\begin{array}{l}\frac{\text { Normalized Weight }}{\text { Change }} \\
12 \text { weeks } \\
\text { Favors Olanzapine } \\
\text { Olanzapine }=94 / 229 \\
\text { Haloperidol }=34 / 211 \\
p<0.001 \\
\text { Emergent Depression } \\
12 \text { weeks } \\
\mathrm{NS} \\
\mathrm{p}=0.10 \\
\text { Akathisia } \\
12 \text { weeks } \\
\text { Favors Haloperidol } \\
\mathrm{p}<0.001\end{array}$ \\
\hline
\end{tabular}




\begin{tabular}{|c|c|c|c|c|c|c|}
\hline Comparison & $\begin{array}{l}\text { Study } \\
\text { PMID } \\
\text { RoB }\end{array}$ & Responder/Remitter & Symptom & Function & Other & $\mathrm{AE}$ \\
\hline $\begin{array}{l}\text { Olanzapine vs. } \\
\text { Lithium }\end{array}$ & $\begin{array}{l}\text { Niufan, } 2008^{22} \\
17531327 \\
\text { Low }\end{array}$ & $\begin{array}{l}\text { See forest plot above } \\
\frac{\text { Remission }}{4 \text { weeks }} \\
\text { Olanzapine }=57 / 69 \\
\text { Lithium }=50 / 71\end{array}$ & $\begin{array}{l}\text { YMRS } \\
4 \text { weeks } \\
\text { Difference in } \\
\text { Difference (SE)=-4.5 } \\
(1.8) \\
\mathrm{p}=0.013 \\
\text { Favors Olanzapine } \\
\frac{\text { CGI-BP }}{4 \text { weeks }} \\
\text { Difference in } \\
\text { Difference (SE)=-0.6 } \\
(0.2) \\
\mathrm{p}=0.009 \\
\text { Favors Olanzapine } \\
\text { MADRS } \\
4 \text { weeks } \\
\text { Difference in } \\
\text { Difference (SE)=-NS }\end{array}$ & NR & See forest plot above & $\begin{array}{l}\text { Severe Harms } \\
4 \text { weeks } \\
\text { NS } \\
\text { Olanzapine }=0 / 69 \\
\text { Lithium }=0 / 71 \\
\text { EP Symptoms } \\
4 \text { weeks } \\
\text { NS } \\
\text { Olanzapine }=1 / 69 \\
\text { Lithium }=2 / 71 \\
\\
\text { Normalized Weight } \\
\text { Change } \\
\text { 4 weeks } \\
\text { Olanzapine }=11 / 69 \\
\text { Lithium }=2 / 71 \\
\\
\text { Emergent Mood } \\
\text { Episodes } \\
4 \text { weeks } \\
\text { NS } \\
\text { Olanzapine=0/69 } \\
\text { Lithium }=0 / 71\end{array}$ \\
\hline
\end{tabular}




\begin{tabular}{|c|c|c|c|c|c|c|}
\hline Comparison & $\begin{array}{c}\text { Study } \\
\text { PMID } \\
\text { RoB }\end{array}$ & Responder/Remitter & Symptom & Function & Other & $\mathrm{AE}$ \\
\hline & $\begin{array}{l}\text { Shafti, } 2010^{33} \\
19740546 \\
\text { Moderate }\end{array}$ & See forest plot above & 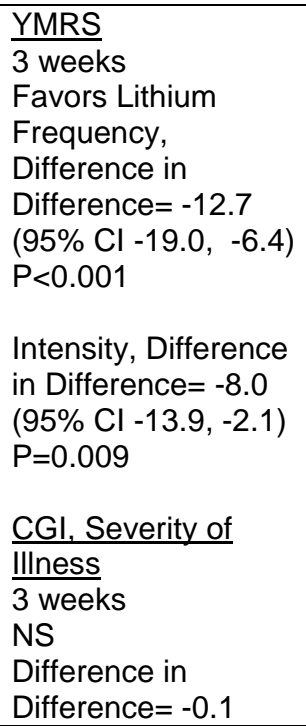 & NR & See forest plot above & NR \\
\hline & $\begin{array}{l}\text { Berk, 199930 } \\
10565800 \\
\text { High }\end{array}$ & NR & $\begin{array}{l}\text { Mania Scale } \\
4 \text { weeks } \\
\text { NS } \\
\text { Difference in } \\
\text { Difference }=3.1 \\
\mathrm{P}=0.32 \\
\frac{\text { CGI-BP }}{4 \text { weeks }} \\
\text { Favors Olanzapine } \\
\text { Difference in } \\
\text { Difference }=0.5 \\
\mathrm{P}=0.03\end{array}$ & $\mathrm{NR}$ & $\begin{array}{l}\text { See forest plot above } \\
\text { Withdrawal, Lack of } \\
\text { Efficacy } \\
4 \text { weeks } \\
\text { NS } \\
\text { Olanzapine }=1 / 15 \\
\text { Lithium }=0 / 15\end{array}$ & NR \\
\hline
\end{tabular}




\begin{tabular}{|c|c|c|c|c|c|c|}
\hline Comparison & $\begin{array}{l}\text { Study } \\
\text { PMID } \\
\text { RoB }\end{array}$ & Responder/Remitter & Symptom & Function & Other & $\mathrm{AE}$ \\
\hline $\begin{array}{l}\text { Olanzapine vs. } \\
\text { Risperidone }\end{array}$ & $\begin{array}{l}\text { Perlis, } 2006^{24} \\
17196055 \\
\text { High }\end{array}$ & $\begin{array}{l}\frac{\text { Response }}{3 \text { weeks }} \\
\text { NS } \\
\text { OR }=1.12(95 \% \mathrm{Cl} \\
0.72,1.75) \\
P=0.65 \\
\text { Remission } \\
3 \text { weeks } \\
\text { NS } \\
\text { OR }=1.57(95 \% \mathrm{Cl} 1.0 \\
2.51) \\
P=0.06\end{array}$ & $\begin{array}{l}\frac{\text { YMRS }}{3 \text { weeks }} \\
\text { NS } \\
\text { Olanzapine }=-15.0 \\
\text { Risperidone }=-16.6\end{array}$ & $\begin{array}{l}\text { CGI-BP } \\
3 \text { weeks } \\
\text { NS } \\
\text { Olanzapine=-1.6 } \\
\text { Risperidone=-1.5 }\end{array}$ & $\begin{array}{l}\text { Overall Withdrawal } \\
3 \text { weeks } \\
\text { Favors Risperidone } \\
\text { Olanzapine }=35 / 165 \\
\text { Risperidone }=54 / 164 \\
\text { OR }=0.55(95 \% \mathrm{Cl} \\
0.33,0.90) \\
\mathrm{P}=0.019 \\
\text { Withdrawal due to Aes } \\
3 \text { weeks } \\
\text { NS } \\
\text { Olanzapine }=9 / 165 \\
\text { Risperidone }=14 / 164 \\
\text { OR }=0.62(95 \% \mathrm{Cl} \\
0.25,1.48) \\
\mathrm{P}=0.29 \\
\text { Withdrawal, Lack of } \\
\text { Efficacy } \\
3 \text { weeks } \\
\text { NS } \\
\text { Olanzapine }=7 / 165 \\
\text { Risperidone }=7 / 164\end{array}$ & $\begin{array}{l}\text { EP Symptoms } \\
3 \text { weeks } \\
\text { NS } \\
\text { Olanzapine }=23 / 165 \\
\text { Risperidone }=37 / 164 \\
P=0.06 \\
\text { Emergent Depression } \\
3 \text { weeks } \\
\text { Olanzapine }=2 / 165 \\
\text { Akathisia } \\
3 \text { weeks } \\
\text { NS } \\
\text { Olanzapine }=13 / 165 \\
\text { Risperidone }=17 / 164 \\
P=0.45 \\
\text { Suicidality } \\
3 \text { weeks } \\
3 \text { patients in the } \\
\text { Risperidone arm were } \\
\text { discontinued for } \\
\text { suicidality }\end{array}$ \\
\hline $\begin{array}{l}\text { Olanzapine vs. } \\
\text { Divalproex/ } \\
\text { Valproate }\end{array}$ & $\begin{array}{l}\text { Xu, } 2015^{19} \\
26060401 \\
\text { Low }\end{array}$ & NR & $\begin{array}{l}\text { YMRS \% decrease } \\
4 \text { weeks } \\
\text { Olanzapine } 75.2 \\
(15.08) \\
\text { Valproate } 55.11 \\
(5.72) \\
\text { Favors Olanzapine } \\
\text { p<0.01 }\end{array}$ & NR & See forest plot above & See forest plot above \\
\hline
\end{tabular}




\begin{tabular}{|c|c|c|c|c|c|c|}
\hline Comparison & $\begin{array}{l}\text { Study } \\
\text { PMID } \\
\text { RoB } \\
\end{array}$ & Responder/Remitter & Symptom & Function & Other & $\overline{A E}$ \\
\hline & $\begin{array}{l}\text { Tohen, } 2008^{23} \\
19014751 \\
\text { Low }\end{array}$ & See forest plot above & $\begin{array}{l}\text { See forest plot } \\
\text { above }\end{array}$ & NR & See forest plot above & $\begin{array}{l}\frac{\text { Normalized Weight }}{\text { Change }} \\
4 \text { weeks } \\
\text { Olanzapine=13/202 } \\
\text { Divalproex=5/188 } \\
\text { Suicide Ideation } \\
3 \text { weeks } \\
\text { NS } \\
\text { Olanzapine=1/215 } \\
\text { Divalproex }=0 / 201 \\
12 \text { weeks } \\
\text { NS } \\
\text { Olanzapine }=2 / 215 \\
\text { Divalproex }=1 / 201 \\
\text { Weight gain }>7 \% \\
\text { Favored Divalproex } \\
\text { p=0.002 }\end{array}$ \\
\hline & $\begin{array}{l}\text { Zajecka, } \\
2002^{35} \\
12523875 \\
\\
\text { Revicki, } 2003^{25} \\
12716270 \\
\text { Moderate }\end{array}$ & NR & $\begin{array}{l}\text { See forest plot } \\
\text { above } \\
\text { YMRS } \\
4 \text { weeks } \\
\text { Favors Olanzapine } \\
\text { \% Reduction (SD) } \\
\text { Olanzapine: } 75.2 \% \\
\text { (15.1\%) } \\
\text { Divalproex: } 55.2 \% \\
(5.7 \%) \\
\text { P<0.001 }\end{array}$ & NR & See forest plot above & $\begin{array}{l}\text { See forest plot above } \\
\text { Emergent Depression } \\
4 \text { weeks } \\
\text { NS } \\
\text { Olanzapine: } 1 / 57 \\
\text { Divalproex: } 1 / 63 \\
\text { Deaths } \\
4 \text { weeks } \\
1 \text { Olanzapine-treated } \\
\text { patient died from } \\
\text { diabetic ketoacidosis }\end{array}$ \\
\hline & $\begin{array}{l}\text { Tohen, } 2002^{28} \\
12042191 \\
\text { High }\end{array}$ & See forest plot above & $\begin{array}{l}\text { See forest plot } \\
\text { above }\end{array}$ & NR & See forest plot above & $\begin{array}{l}\text { See forest plot above } \\
\text { EPS } \\
\text { No difference between } \\
\text { groups }\end{array}$ \\
\hline
\end{tabular}


Abbreviations: $\mathrm{AE}=$ Adverse Events; ANCOVA=Analysis of Covariance; CGI=Clinical Global Impressions Scale; CI=Confidence Interval; EPS=extrapyramidal symptoms; ESRS=Extrapyrimidal Symptom Rating Scale; GAS=Global Assessment Scale; MADRS=Montgomery-Asberg Depression Rating Scale; NR=Not reported; NS=not significant; OR=Odds Ratio; PMID=PubMed Identification Number; ROB=Risk of Bias; SAE=Serious Adverse Events; YMRS = Young Mania Rating Scale 
Appendix Table E26. Strength of evidence assessment: olanzapine versus active comparator for acute mania

\begin{tabular}{|c|c|c|c|c|c|c|c|c|}
\hline Comparison & Outcome & $\begin{array}{l}\text { \# Studies/ } \\
\text { Design } \\
\text { (n analyzed) }\end{array}$ & $\begin{array}{l}\text { Finding or } \\
\text { Summary } \\
\text { Statistic }\end{array}$ & $\begin{array}{c}\text { Study } \\
\text { Limitations }\end{array}$ & Consistency & Directness & Precision & $\begin{array}{c}\text { Overall } \\
\text { Gradel } \\
\text { Conclusion }\end{array}$ \\
\hline $\begin{array}{l}\text { Olanzapine } \\
\text { vs. haloperidol }\end{array}$ & $\begin{array}{l}\text { Response } 6,12 \text { wks } \\
\text { Remission } 6,12 \\
\text { wks } \\
\text { YMRS } 6,12 \text { wks } \\
\text { Withdrawals }\end{array}$ & $\begin{array}{l}1 \mathrm{RCT} \\
(\mathrm{n}=453)\end{array}$ & See table above & Moderate & Unknown & Direct & Imprecise & Insufficient \\
\hline \multirow{2}{*}{$\begin{array}{l}\text { Olanzapine } \\
\text { vs. Lithium }\end{array}$} & Response 4 wks & $\begin{array}{l}2 \text { RCTs } \\
(n=180)\end{array}$ & $\begin{array}{l}\text { See forest plot } \\
\text { above }\end{array}$ & Moderate & Inconsistent & Direct & Imprecise & Insufficient \\
\hline & $\begin{array}{l}\text { YMRS } 4 \text { wks } \\
\text { CGI } 4 \text { wks } \\
\text { Withdrawal }\end{array}$ & $\begin{array}{l}3 \text { RCT } \\
(n=210)\end{array}$ & See table above & Moderate & Inconsistent & Direct & Imprecise & Insufficient \\
\hline $\begin{array}{l}\text { Olanzapine } \\
\text { vs. } \\
\text { Risperidone }\end{array}$ & $\begin{array}{l}\text { Response } 3 \text { wk } \\
\text { Remission } 3 \text { wk } \\
\text { YMRS } 3 \text { wk } \\
\text { CGl } \\
\text { Withdrawals }\end{array}$ & $\begin{array}{l}1 \mathrm{RCT} \\
(\mathrm{n}=329)\end{array}$ & See table above & High & Unknown & Direct & Imprecise & Insufficient \\
\hline \multirow{4}{*}{$\begin{array}{l}\text { Olanzapine } \\
\text { vs. } \\
\text { Divalproex/ } \\
\text { Valproate }\end{array}$} & $\begin{array}{l}\text { Response } \\
\text { Remission }\end{array}$ & $\begin{array}{l}2 \text { RCTs } \\
(n=635)\end{array}$ & NS & Moderate & Consistent & Direct & Imprecise & Low \\
\hline & YMRS & $\begin{array}{l}3 \text { RCTs } \\
(n=750)\end{array}$ & NS & Moderate & Consistent & Direct & Imprecise & Low \\
\hline & CGI & $\begin{array}{l}3 \text { RCTs } \\
(n=578)\end{array}$ & NS & Moderate & Consistent & Direct & Imprecise & Low \\
\hline & Withdrawals & $\begin{array}{l}4 \text { RCTs } \\
(n=867)\end{array}$ & NS & Moderate & Consistent & Direct & Imprecise & Low \\
\hline
\end{tabular}

Abbreviations: CGI-BP=Clinical Global Impressions Scale for Bipolar Disorder; NS=not significant; RCT=randomized controlled trial; YMRS = Young Mania Rating Scale

Notes:

1. Publication bias for antipsychotics, antidepressants, and behavioral interventions for depressive disorders is suspected.

2. Data were generally imprecise due to missing data from high attrition rates, which was commonly dealt with by Last Observation Carried Forward (LOCF). LOCF requires an

$$
\text { E-79 }
$$


assumption that the health status of patients who dropped out of the trial would not have changed had future observations been recorded, a strong assumption in the context of bipolar disorder research.

\section{Section 6. Quetiapine for Acute Mania}

Appendix Table E27. Characteristics of eligible studies: quetiapine for acute mania

\begin{tabular}{|c|c|c|c|c|c|c|}
\hline $\begin{array}{c}\text { Study, Year } \\
\text { Design } \\
\text { Location } \\
\text { Funder } \\
\text { Risk of Bias } \\
\text { PMID }\end{array}$ & $\begin{array}{c}\text { \# Randomized } \\
\text { Age (mean) } \\
\text { Sex (\% Female) } \\
\text { Race (\% White) } \\
\text { Diagnosis } \\
\text { (\% BP-I, II, NOS) } \\
\text { Setting }\end{array}$ & $\begin{array}{l}\text { Inclusions } \\
\text { Key Exclusions }\end{array}$ & $\begin{array}{l}\text { Intervention } \\
\text { Dosage }\end{array}$ & $\begin{array}{c}\text { Comparison } \\
\text { Dosage }\end{array}$ & Follow-up Duration & $\begin{array}{c}\text { Outcomes } \\
\text { Reported } \\
\text { Withdrawal (\%) at } \\
\text { endpoint }\end{array}$ \\
\hline $\begin{array}{l}\text { Cutler, } 2011^{36} \\
\text { RCT } \\
\text { Multisite } \\
\text { US } \\
\text { Industry } \\
\text { RoB Low } \\
22054797\end{array}$ & $\begin{array}{l}\mathrm{N}=316 \\
\text { Mean Age } 41 \\
\text { Female } 40 \% \\
\text { White } 47 \% \\
\text { BP-I } 100 \% \\
\text { Inpatient (days 1-4, } \\
\text { minimum) } \\
\text { Outpatient (at } \\
\text { inspectors discretion) }\end{array}$ & $\begin{array}{l}\text { Mania; YMRS } \geq 20 \\
\text { overall, YMRS } \geq 4 \text { on } \\
\text { at least } 2 \text { of } 4 \\
\text { specifed mania } \\
\text { domains, and CGI- } \\
\text { BP-S } \geq 4 \\
\text { First Manic Episode } \\
\text { Schizoaffective } \\
\text { Substance Abuse } \\
\text { Other Mental Health } \\
\text { Labs/Other } \\
\text { Conditions }\end{array}$ & $\begin{array}{l}\text { Quetiapine ER } \\
\text { 300-800 mg/day } \\
\text { (603.8 mg/day mean) }\end{array}$ & Placebo & 3 Weeks & $\begin{array}{l}\text { CGI-BP-S } \\
\text { CGI-BP-C } \\
\text { MADRS } \\
\text { Remission } \\
\text { (YMRS } 12 \text { ) } \\
\text { Response (YMRS } \\
50 \% \text { decrease) } \\
\text { YMRS } \\
\text { Withdrawal 29\% }\end{array}$ \\
\hline
\end{tabular}




\begin{tabular}{|c|c|c|c|c|c|c|}
\hline $\begin{array}{l}\text { Study, Year } \\
\text { Design } \\
\text { Location } \\
\text { Funder } \\
\text { Risk of Bias } \\
\text { PMID }\end{array}$ & $\begin{array}{c}\text { \# Randomized } \\
\text { Age (mean) } \\
\text { Sex (\% Female) } \\
\text { Race (\% White) } \\
\text { Diagnosis } \\
\text { (\% BP-I, II, NOS) } \\
\text { Setting }\end{array}$ & $\begin{array}{c}\text { Inclusions } \\
\text { Key Exclusions }\end{array}$ & $\begin{array}{l}\text { Intervention } \\
\text { Dosage }\end{array}$ & $\begin{array}{c}\text { Comparison } \\
\text { Dosage }\end{array}$ & Follow-up Duration & $\begin{array}{c}\text { Outcomes } \\
\text { Reported } \\
\begin{array}{c}\text { Withdrawal (\%) at } \\
\text { endpoint }\end{array}\end{array}$ \\
\hline $\begin{array}{l}\text { McElroy, } 2010^{37} \\
\text { Singlesite } \\
\text { US } \\
\text { Industry } \\
\text { RoB Low } \\
19963274\end{array}$ & $\begin{array}{l}\mathrm{N}=41 \\
\text { Mean Age } 35 \\
\text { Female } 51 \% \\
\text { White } 69 \% \\
\text { BP-I } 74 \% \\
\text { BP-II 21\% } \\
\text { BP-NOS 5\% } \\
\text { Outpatient }\end{array}$ & $\begin{array}{l}\text { Mild to moderate } \\
\text { hypomania or mild } \\
\text { mania; } \\
\text { CGI-BP } \geq 3 \text { AND <5 } \\
\text { Substance Abuse } \\
\text { Other Mental Health } \\
\text { Neurological } \\
\text { Disorders } \\
\text { Pregnant Nursing } \\
\text { Labs/Other } \\
\text { Conditions }\end{array}$ & $\begin{array}{l}\text { Quetiapine } \\
50-800 \mathrm{mg} / \text { day } \\
\text { (232 mg/day mean) }\end{array}$ & Placebo & 8 weeks & $\begin{array}{l}\text { CGI-BP-S } \\
\text { GAF } \\
\text { HAM-A } \\
\text { IDS } \\
\text { Remission } \\
\quad \text { (YMRS } \leq 7 \text { at week } \\
\text { 8, CGI-BP Overall } \leq 2 \\
\text { at week 8, } \\
\text { Improvement or no } \\
\text { change in IDS score } \\
\text { from baseline to week } \\
\text { 8) } \\
\text { Response } \\
\text { (YMRS 50\% } \\
\text { decrease) } \\
\text { YMRS } \\
\\
\text { Withdrawal } 36 \% \\
\end{array}$ \\
\hline $\begin{array}{l}\text { Vieta, } 2010^{18} \\
\text { RCT } \\
\text { Multisite } \\
3 \text { continents } \\
\text { Industry } \\
\text { RoB High } \\
20565430\end{array}$ & \begin{tabular}{|l|} 
N $=493$ \\
Mean Age 39 \\
Female 42\% \\
Race NR \\
BP-I $100 \%$ \\
\\
Inpatient (1 week) \\
Outpatient (weeks 2- \\
3, subject to inspector \\
discretion)
\end{tabular} & $\begin{array}{l}\text { Mania; } \\
\text { YMRS } \geq 20 \\
\text { First Manic Episode } \\
\text { Schizoaffective } \\
\text { Substance Abuse } \\
\text { Neurological } \\
\text { Disorders }\end{array}$ & $\begin{array}{l}\text { Quetiapine } \\
400-800 \mathrm{mg} / \mathrm{day} \\
(600 \mathrm{mg} / \text { day mean })\end{array}$ & $\begin{array}{l}\text { Placebo } \\
\text { (Palidperidone arm } \\
\text { discussed in Other } \\
\text { Drugs section) }\end{array}$ & 3 weeks & $\begin{array}{l}\text { Duration of Episode } \\
\text { YMRS } \\
\text { GAF } \\
\text { PANSS } \\
\text { CGI-BP-S } \\
\text { SAS } \\
\text { AIMS } \\
\text { MADRS } \\
\text { Withdrawal } 28 \%\end{array}$ \\
\hline
\end{tabular}




\begin{tabular}{|c|c|c|c|c|c|c|}
\hline $\begin{array}{l}\text { Study, Year } \\
\text { Design } \\
\text { Location } \\
\text { Funder } \\
\text { Risk of Bias } \\
\text { PMID }\end{array}$ & $\begin{array}{c}\text { \# Randomized } \\
\text { Age (mean) } \\
\text { Sex (\% Female) } \\
\text { Race (\% White) } \\
\text { Diagnosis } \\
\text { (\% BP-I, II, NOS) } \\
\text { Setting }\end{array}$ & $\begin{array}{c}\text { Inclusions } \\
\text { Key Exclusions }\end{array}$ & $\begin{array}{l}\text { Intervention } \\
\text { Dosage }\end{array}$ & $\begin{array}{c}\text { Comparison } \\
\text { Dosage }\end{array}$ & Follow-up Duration & $\begin{array}{c}\text { Outcomes } \\
\text { Reported } \\
\text { Withdrawal (\%) at } \\
\text { endpoint }\end{array}$ \\
\hline $\begin{array}{l}\text { Li, 2008 } \\
\text { RCT } \\
\text { Multisite } \\
\text { China } \\
\text { Industry } \\
\text { RoB Moderate } \\
18028587\end{array}$ & $\begin{array}{l}\text { N = 154 } \\
\text { Mean Age } 33 \\
\text { Female 53\% } \\
\text { Race NR } \\
\text { Diagnosis NR } \\
\\
\text { Inpatient (weeks 1-2) } \\
\text { Outpatient (week 2-4, } \\
\text { subject to inspector } \\
\text { discretion) }\end{array}$ & $\begin{array}{l}\text { Mania; } \\
\text { YMRS } \geq 20 \\
\text { Substance Abuse } \\
\text { Taking Other Meds } \\
\text { Pregnant/Nursing } \\
\text { Labs/Other } \\
\text { Conditions }\end{array}$ & $\begin{array}{l}\text { Quetiapine } \\
100-800 \mathrm{mg} / \text { day } \\
\text { (648.2 mg/day mean) }\end{array}$ & $\begin{array}{l}\text { Lithium } \\
250-2000 \mathrm{mg} / \text { day } \\
\text { (target serum level } \\
0.6-1.2 \mathrm{mmol} / \mathrm{L})(0.80 \\
\mathrm{mmol} / \mathrm{L} \text { average) }\end{array}$ & 4 weeks & $\begin{array}{l}\text { MADRS } \\
\text { PANSS } \\
\text { Remission (various } \\
\text { definitions) } \\
\text { Response (YMRS } \\
50 \% \text { decrease) } \\
\text { Weight } \\
\text { YMRS } \\
\text { Withdrawal } 12 \%\end{array}$ \\
\hline $\begin{array}{l}\text { Bowden, } 2005^{39} \\
\text { RCT } \\
\text { Multisite } \\
\text { 2 Continents } \\
\text { Industry } \\
\text { RoB High } \\
15669897\end{array}$ & $\begin{array}{l}\mathrm{N}=302 \\
\text { Mean Age } 39 \\
\text { Female } 24 \% \\
\text { Race NR } \\
\text { BP-I 100\% } \\
\text { Inpatient }\end{array}$ & $\begin{array}{l}\text { Mania; } \\
\text { YMRS } \geq 20 \text { including } \\
\text { score of at least } 4 \text { on } \\
2 \text { of the } 4 \text { double- } \\
\text { weighted items } \\
\text { (irritability, speech, } \\
\text { content, and } \\
\text { disruptive/aggressive } \\
\text { behavior), CGI } \geq 4 \\
\\
\text { First Manic Episode } \\
\text { Substance Abuse } \\
\text { Taking Other Meds } \\
\text { Pregnant/Nursing } \\
\text { Labs/Other } \\
\text { Conditions }\end{array}$ & \begin{tabular}{|l} 
Quetiapine \\
$100-800 \mathrm{mg} /$ day
\end{tabular} & $\begin{array}{l}\text { Placebo } \\
\text { Lithium } \\
0.6-1.4 \mathrm{mEq} / \mathrm{L} \\
\text { (mean } 0.80 \mathrm{mEq} / \mathrm{L} \text { ) }\end{array}$ & 12 weeks & $\begin{array}{l}\text { CGI-BP-S Overall } \\
\text { Global Assessment } \\
\text { Scale (GAS) } \\
\text { MADRS } \\
\text { PANSS (positive) } \\
\text { Remission } \\
\text { (YMRS } 12 \text { ) } \\
\text { Response (50\% } \\
\text { decrease in YMRS) } \\
\text { YMRS } \\
\text { Withdrawal 48\% }\end{array}$ \\
\hline
\end{tabular}

Abbreviations: AIMS=Abnormal Involuntary Movement Scale; BARS=Barnes Akathisia Scale; BAS=Behavioral Approach System; BMI=Body Mass Index; BP=bipolar

disorder; BPRS=Brief Psychiatric Rating Scale; C=comparison; CGI= Clinical Global Impressions; CGI-I=Clinical Global Impressions-Improvement; CGI-S =CGI-Severity; CGIBP=Clinical Global Impressions Scale-Bipolar; CGI-BP-C= Clinical Global Impressions, Bipolar, Change Scale; CGI-BP-S=Clinical Global Impressions, Bipolar, Severity Scale; C-SSRS=Columbia Suicide Severity Rating Scale; DAI-10=Drug Attitutde Inventory, 10 question version; DIEPSS=Drug-Induced Extra-Pyramidal Symptoms Scale;

DSM=Diagnostic and Statistical Manual of Mental Disorders; EPS=extrapyramidal symptoms; ER=Extended Release; ESRS=Extrapyramidal Symptom Rating Scale;

GAF=General Assessment of Functioning Scale; GAS=Global Assessment Scale; HAM-A=Hamilton Scale for Anxiety; HAM-D=Hamilton Scale for Depression; HRQL=Health- 
related quality of life; HRQOL=Health-related quality of life; I=intervention; IDS=Inventory for Depressive Symptoms; LIFE= Longitudinal Interval Follow-up Evaluation; MADRS=Montgomery-Asberg Depression Rating Scale; MAS=Bech-Rafaelsen Mania Rating Scale; MRS=Mania Rating Scale; MSRS=Manic state rating scale; NOS=not otherwise specified; NR=not reported; PANSS=Positive and Negative Syndrome Scale; PRS=Polygenic Risk Scores; PGWB=Psychological General Well-Being Index;

PMID=PubMed Identification Number; PRS=Polygenic Risk Scores; Q-LES-Q=Quality of Life Enjoyment and Satisfaction Questionnaire; RCT=randomized controlled trial; ROB=risk of bias; SADS-C= Schedule for Affective Disorders and Schizophrenia-Change version; SAE=Serious Adverse Events; SAS=Simpson Angus Scale; SF-12=12-Item Short Form Health Survey; SF-36=36-Item Short Form Health Survey; SLICE=Streamlined Longitudinal Interview Clinical Evaluation; T=Trial; YMRS = Young Mania Rating Scale

\section{Appendix Table E28. Summary risk of bias assessments: quetiapine for acute mania}

\begin{tabular}{|c|c|c|c|}
\hline Drug & $\begin{array}{c}\text { Study } \\
\text { Funding Source } \\
\text { PMID }\end{array}$ & $\begin{array}{l}\text { Overall Risk of } \\
\text { Bias Assessment }\end{array}$ & Rationale \\
\hline \multirow[t]{6}{*}{ Quetiapine } & $\begin{array}{l}\text { McElroy, } 2010^{37} \\
\text { Industry } \\
19963274\end{array}$ & Moderate & No specific sources of bias identified. Attrition rate $36 \%$ \\
\hline & $\begin{array}{l}\text { Cutler, 201136 } \\
\text { Industry } \\
22054797 \\
\end{array}$ & Low & Blinding not described. No other sources of bias identified. \\
\hline & $\begin{array}{l}\text { Vieta, } 2010^{18} \\
\text { Industry } \\
20565430 \\
\end{array}$ & High & Blinding not described; large dropout in placebo group (41\%). \\
\hline & $\begin{array}{l}\mathrm{Li}, 2008^{38} \\
\text { Industry } \\
18028587 \\
\end{array}$ & Moderate & Randomization and blinding procedure not described. \\
\hline & $\begin{array}{l}\text { Bowden, } 2005^{39} \\
\text { Industry } \\
15669897\end{array}$ & High & $\begin{array}{l}\text { Randomization and blinding procedure not described; }>50 \% \text { dropout in placebo group at day } \\
84 ; 33 \% \text { at } 3 \text { weeks }\end{array}$ \\
\hline & $\begin{array}{l}\text { McIntyre, } 2005^{14} \\
\text { Industry } \\
16139175\end{array}$ & Moderate & $\begin{array}{l}\text { Dropout rate for quetiapine and placebo in } 30 \%-40 \% \text { range, lacks some core information on } \\
\text { how allocation was concealed and blinding of treatment staff and raters was maintained. } \\
\text { Author notes may be underpowered for quetiapine vs. haloperidol comparison. }\end{array}$ \\
\hline
\end{tabular}

Abbreviations: ITT=Intention to Treat; PMID=PubMed Identification Number; LOCF=last observation carried forward 


\section{Quetiapine Forest Plots}

Outcomes in studies assessed as having a high risk of bias, or low to moderate risk of bias but at least 40 percent attrition, are presented in grey tones. Both fixed-effect models and random-effects models are presented. We calculated fixed-effect models to provide a charitable estimate of the average effect among completed trials. However, we base our main conclusions on the random-effects models.

Appendix Figure E62. Quetiapine vs. placebo - response

Odds Ratio of Response ( $>50 \%$ Reduction in YMRS) at 3 Weeks

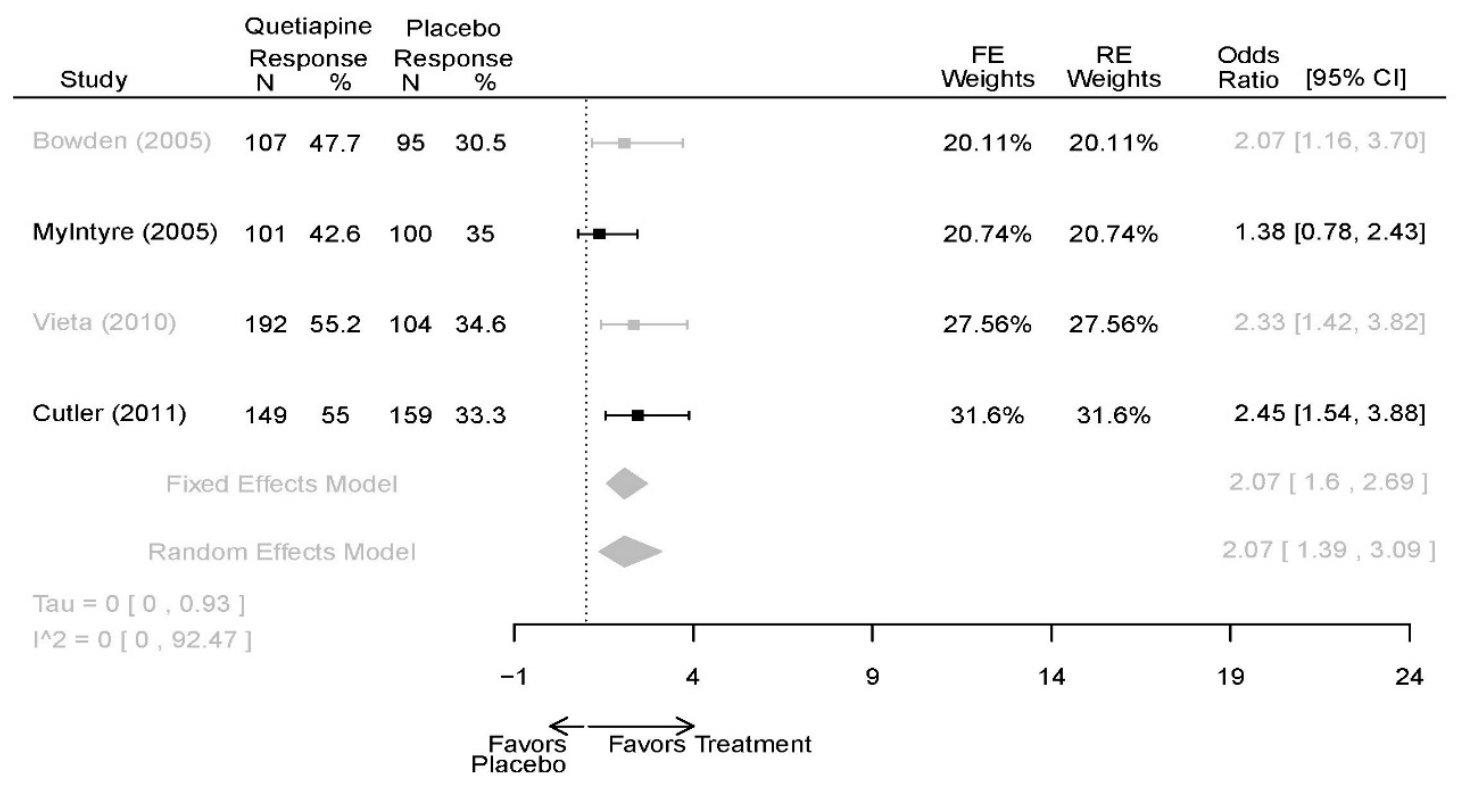


Appendix Figure E63. Quetiapine vs. placebo - remission

Odds Ratio of Remission (YMRS $<=12$ ) at 3 Weeks

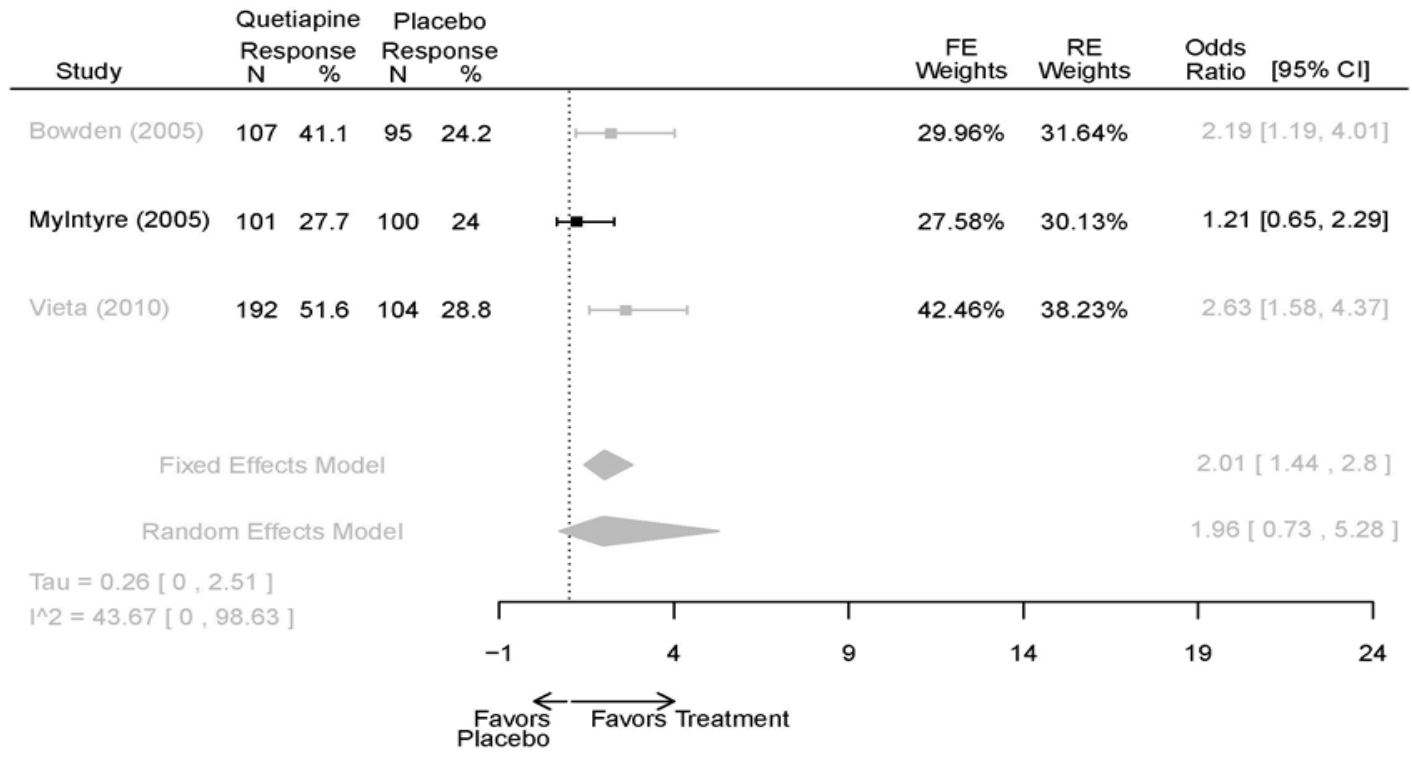

Appendix Figure E64. Quetiapine vs. placebo - YMRS

Difference in Mean Change in YMRS from Baseline to 3 Weeks

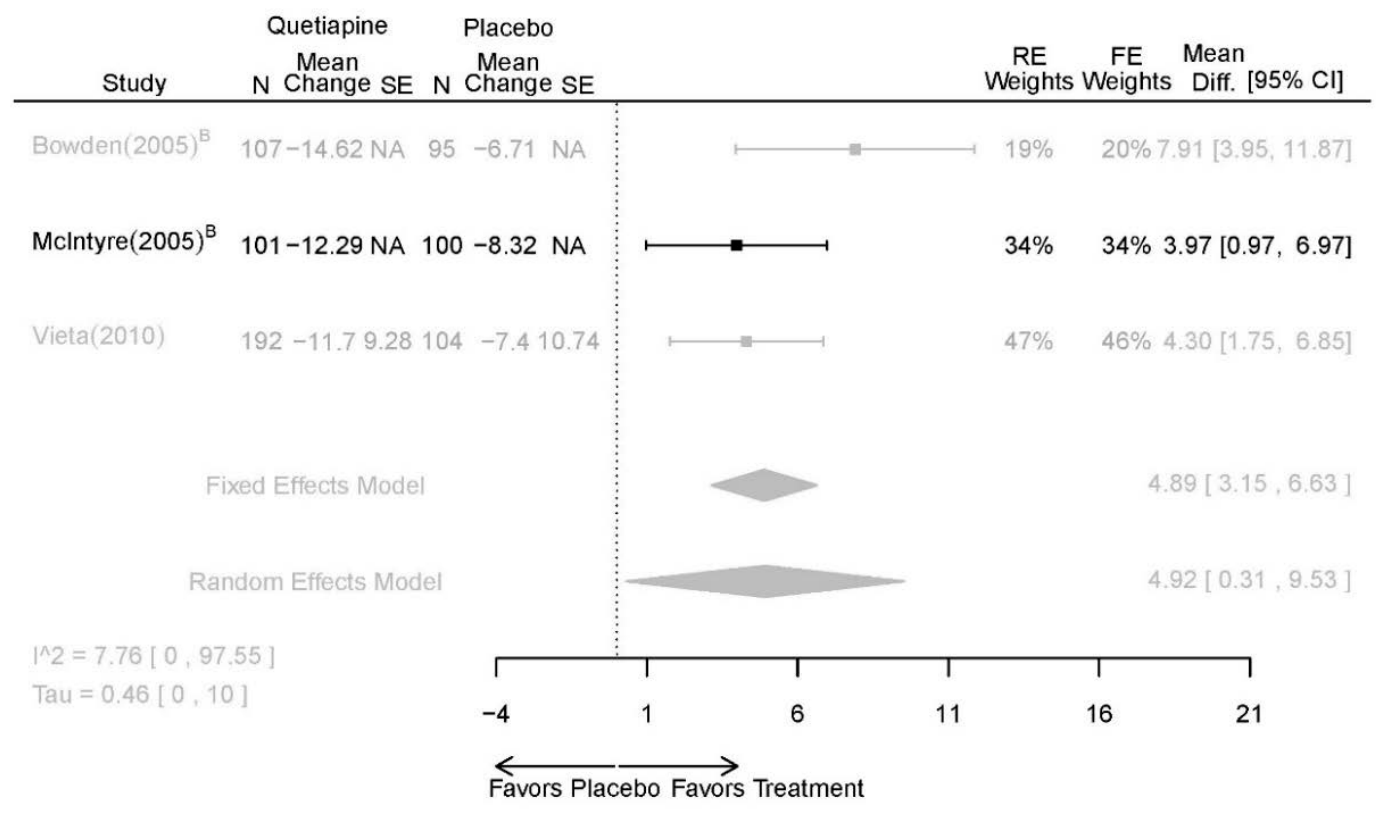


Appendix Figure E65. Quetiapine vs. placebo - CGI

Difference in Mean Change in CGI-BP-S (Overall) from Baseline to 3 Weeks

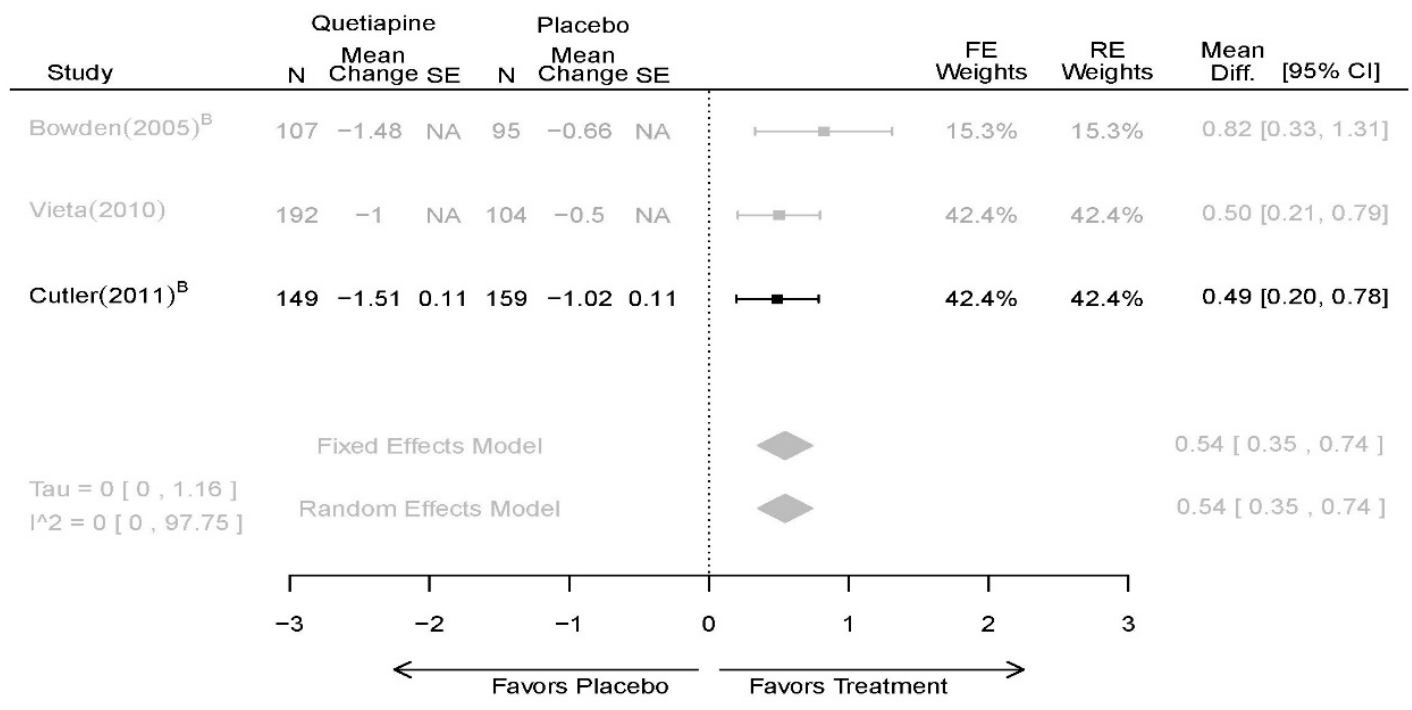

Appendix Figure E66. Quetiapine vs. placebo - overall withdrawal

Odds Ratio of Overall Withdrawal

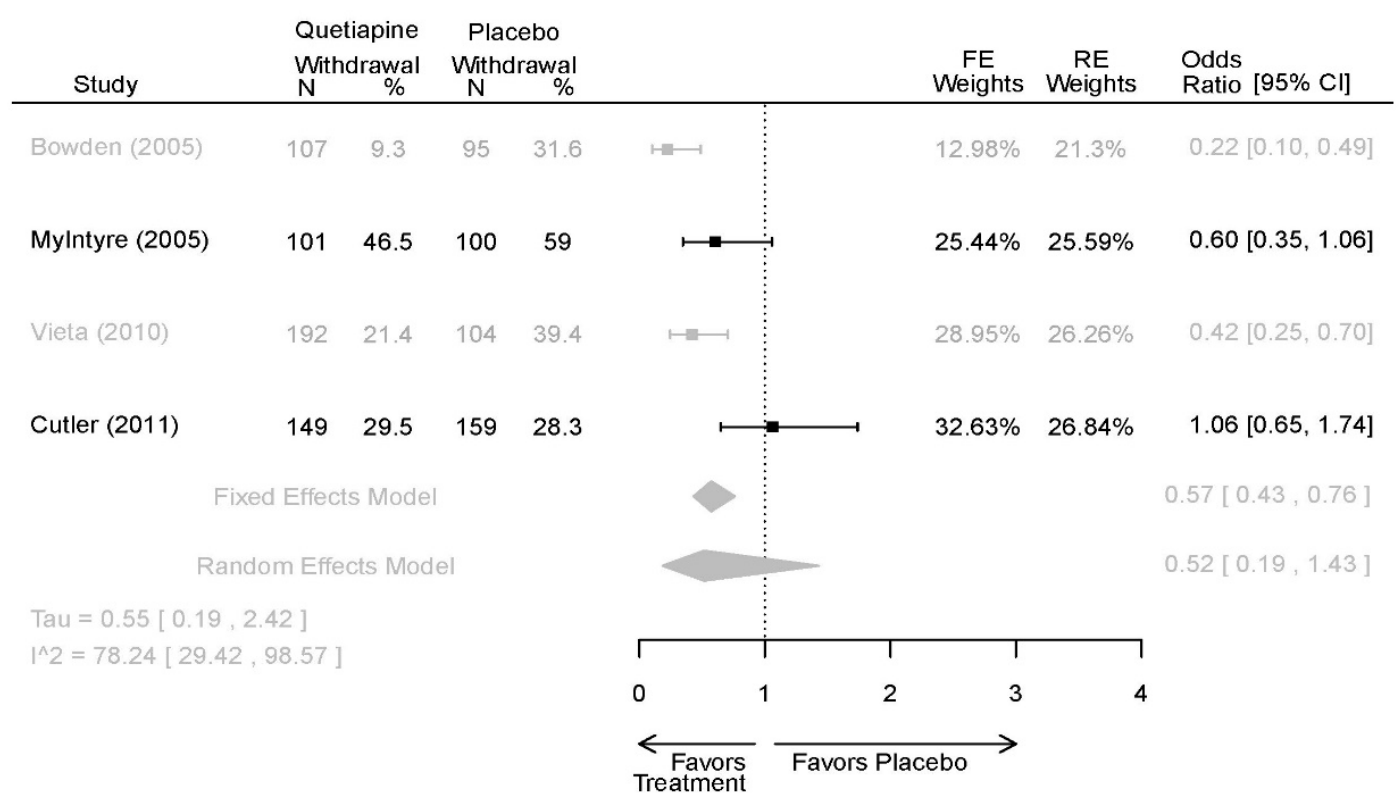


Appendix Figure E67. Quetiapine vs. placebo - withdrawal lack of efficacy Odds Ratio of Withdrawal due to Lack of Efficacy at 3 Weeks

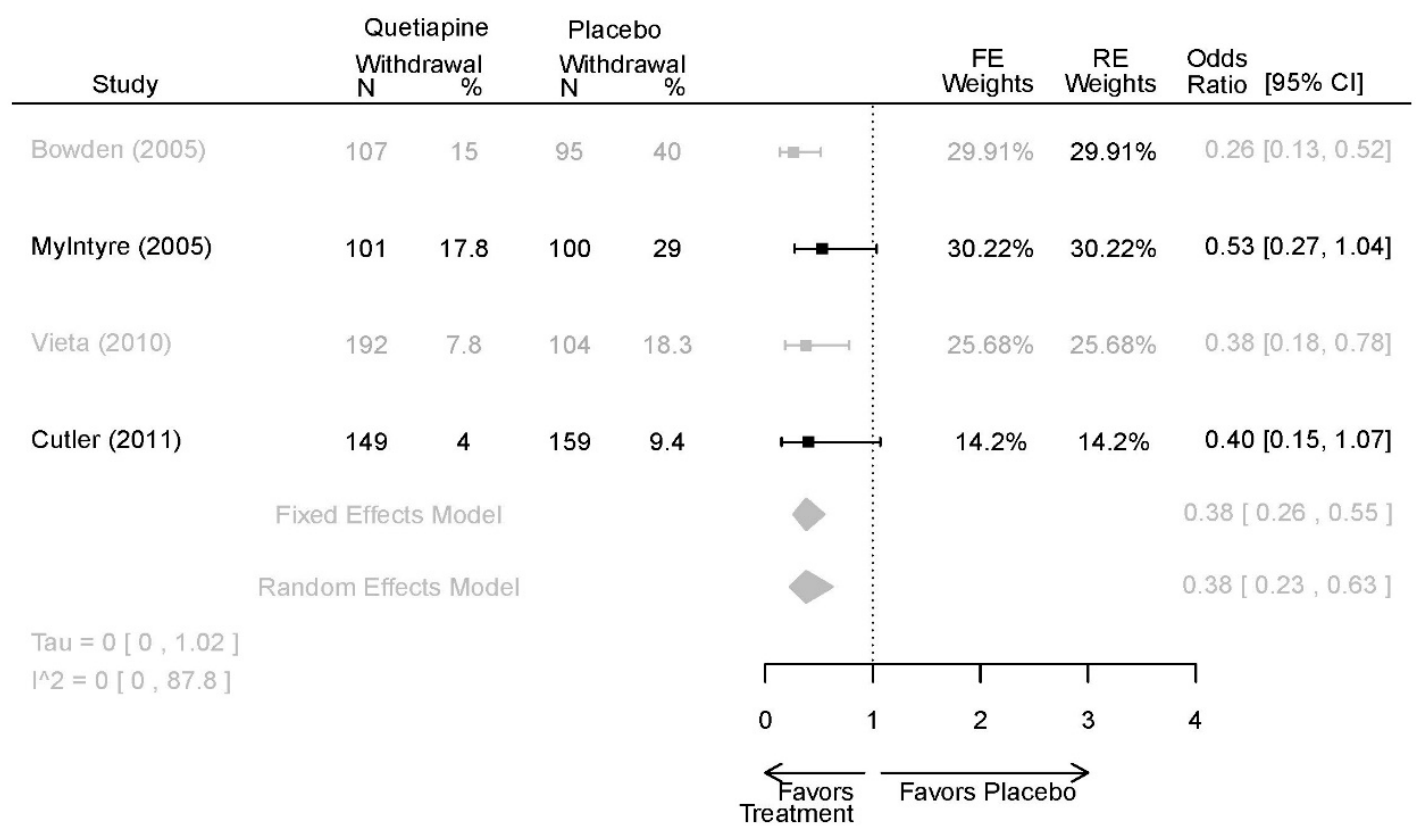

Appendix Figure E68. Quetiapine vs. placebo - withdrawal adverse events Odds Ratio of Withdrawal due to Adverse Events at 3 Weeks

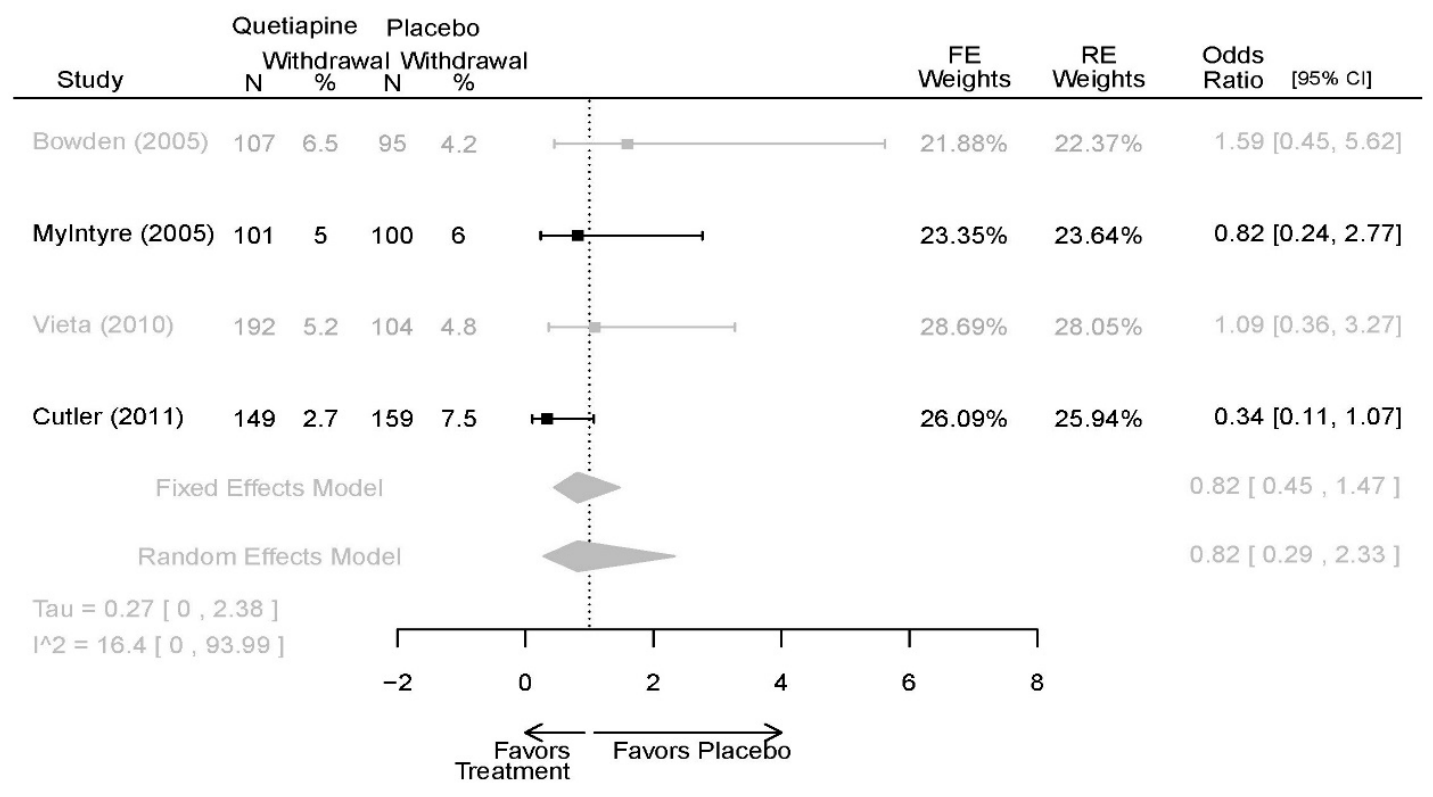




\begin{tabular}{|c|c|c|c|c|c|c|}
\hline Drug & $\begin{array}{l}\text { Study } \\
\text { PMID } \\
\text { RoB }\end{array}$ & Responder/Remitter & Symptom & Function & Other & AE \\
\hline \multirow[t]{3}{*}{ Quetiapine } & $\begin{array}{l}\text { McElroy, } \\
2010^{37} \\
19963274 \\
\text { Moderate }\end{array}$ & $\begin{array}{l}\text { Response } \\
8 \text { weeks } \\
\text { Completers only } \\
\text { reported } \\
\text { Remission } \\
8 \text { weeks } \\
\text { Completers only } \\
\text { reported }\end{array}$ & $\begin{array}{l}\text { YMRS } \\
8 \text { weeks } \\
\text { Regression model } \\
\text { NS }(p=0.06)\end{array}$ & $\begin{array}{l}\text { CGI } \\
8 \text { weeks } \\
\text { Regression model } \\
\text { Favors Quetiapine } \\
(p<0.001) \\
\text { GAF } \\
8 \text { weeks } \\
\text { Regression model } \\
\text { NS }\end{array}$ & $\begin{array}{l}\text { Overall Withdrawal } \\
\text { Quetiapine } 6 / 21 \\
\text { Placebo } 8 / 20 \\
\text { Withdrawal Lack of } \\
\text { efficacy } \\
\text { Quetiapine } 0 / 21 \\
\text { Placebo } 2 / 20 \\
\text { Withdrawal AE } \\
\text { Quetiapine } 2 / 21 \\
\text { Placebo } 1 / 20\end{array}$ & 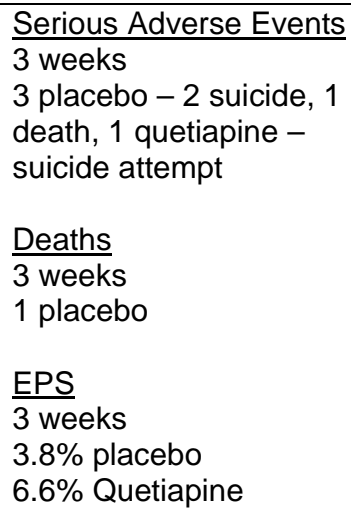 \\
\hline & $\begin{array}{l}\text { Cutler, } 2011^{36} \\
22054797 \\
\text { Low }\end{array}$ & $\begin{array}{l}\text { See forest plot E62 } \\
\text { above for Response. }\end{array}$ & $\begin{array}{l}\text { See forest plot E64 } \\
\text { above for YMRS. }\end{array}$ & $\begin{array}{l}\text { See forest plot E65 } \\
\text { above for } \mathrm{CGI}\end{array}$ & $\begin{array}{l}\text { See forest plots E66, } \\
\text { E67, E68 above for } \\
\text { withdrawals }\end{array}$ & $\begin{array}{l}\text { SAE } \\
\text { Quetiapine } 4.0 \% \\
\text { Placebo } 8.1 \% \\
\frac{\text { Deaths }}{3 \text { placebo - } 2 \text { suicide, } 1} \\
\text { death, } 1 \text { quetiapine - } \\
\text { suicide attempt } \\
\frac{\text { EPS }}{3 \text { weeks }} \\
3.8 \% \text { placebo } \\
6.6 \% \text { Quetiapine }\end{array}$ \\
\hline & $\begin{array}{l}\text { Vieta, } 2010^{18} \\
20565430 \\
\text { High }\end{array}$ & $\begin{array}{l}\text { See forest plot E562 } \\
\text { above for Response. }\end{array}$ & $\begin{array}{l}\text { See forest plot E64 } \\
\text { above for YMRS. }\end{array}$ & $\begin{array}{l}\text { See forest plot E65 } \\
\text { above for } \mathrm{CGI}\end{array}$ & $\begin{array}{l}\text { See forest plots E66, } \\
\text { E67, E68 above for } \\
\text { withdrawals }\end{array}$ & $\begin{array}{l}\text { SAE } \\
\text { Reported no difference } \\
1 \text { suicide reported } \\
\frac{\text { Akathisia }}{\text { Quetiapine } 6(3 \%)} \\
\text { Placebo } 3(3 \%)\end{array}$ \\
\hline
\end{tabular}




\begin{tabular}{|c|c|c|c|c|c|c|}
\hline Drug & $\begin{array}{l}\text { Study } \\
\text { PMID } \\
\text { RoB }\end{array}$ & Responder/Remitter & Symptom & Function & Other & $\overline{A E}$ \\
\hline & $\begin{array}{l}\text { Bowden, } \\
2005^{39} \\
15669897 \\
\text { High }\end{array}$ & $\begin{array}{l}\text { See forest plot E62 } \\
\text { above for Response. }\end{array}$ & $\begin{array}{l}\text { See forest plot E64 } \\
\text { above for YMRS. }\end{array}$ & $\begin{array}{l}\text { See forest plot E65 } \\
\text { above for } \mathrm{CGI}\end{array}$ & $\begin{array}{l}\text { See forest plots E66, } \\
\text { E67, E68 above for } \\
\text { withdrawals }\end{array}$ & $\begin{array}{l}\frac{\text { SAE }}{\text { None reported }} \\
\frac{\text { EPS }}{\text { Reported no difference }} \\
\text { Weight gain }>7 \% \\
\text { Quetiapine more } \\
\text { frequent }(\mathrm{p}=0.008)\end{array}$ \\
\hline & $\begin{array}{l}\text { Mclntyre, } \\
2005^{14} \\
16139175 \\
\\
\text { Moderate }\end{array}$ & $\begin{array}{l}\text { See forest plot E62 } \\
\text { above for Response. }\end{array}$ & $\begin{array}{l}\frac{\text { YMRS }}{3 \text { weeks }} \\
\text { ANCOVA model } \\
\text { Favors Quetiapine } \\
p=.01 \\
\text { Results sustained at } \\
12 \text { weeks }\end{array}$ & $\begin{array}{l}\text { CGI } \\
3 \text { weeks } \\
\text { ANCOVA model } \\
\text { Favors Quetiapine } \\
(p<0.05) \\
\text { Results sustained at } \\
12 \text { weeks }\end{array}$ & $\begin{array}{l}\text { See forest plots E66, } \\
\text { E67, E68 above for } \\
\text { withdrawals }\end{array}$ & $\begin{array}{l}\text { SAE } \\
\text { None reported } \\
\text { EPS } \\
\text { Quetiapine } 13(12.7 \%) \\
\text { Placebo } 16(15.8 \%) \\
\text { NS } \\
\text { Weight gain }>7 \% \\
\text { Quetiapine } 12.8 \% \\
\text { Placebo } 4 \% \\
\text { NS }\end{array}$ \\
\hline
\end{tabular}

Abbreviations: AE=Adverse Events; ANCOVA=Analysis of Covariance; CGI=Clinical Global Impressions Scale; CI=Confidence Interval; EPS=extrapyramidal symptoms; ESRS=Extrapyrimidal Symptom Rating Scale; GAS=Global Assessment Scale; MADRS=Montgomery-Asberg Depression Rating Scale; NR=Not reported; NS=not significant; OR=Odds Ratio; PMID=PubMed Identification Number; ROB=Risk of Bias; SAE=Serious Adverse Events; YMRS = Young Mania Rating Scale 
Appendix Table E30. Strength of evidence assessment: quetiapine versus placebo for acute mania

\begin{tabular}{|c|c|c|c|c|c|c|c|c|}
\hline Comparison & Outcome & $\begin{array}{l}\text { \# Studies/ } \\
\text { Design } \\
\text { (n analyzed) }\end{array}$ & $\begin{array}{l}\text { Finding or } \\
\text { Summary } \\
\text { Statistic }\end{array}$ & $\begin{array}{c}\text { Study } \\
\text { Limitations }\end{array}$ & Consistency & Directness & Precision & $\begin{array}{l}\text { Overall } \\
\text { Gradel } \\
\text { Conclusion }\end{array}$ \\
\hline \multirow{6}{*}{$\begin{array}{l}\text { Quetiapine vs. } \\
\text { placebo }\end{array}$} & Response 3 wks & $\begin{array}{l}4 \mathrm{RCT} \\
(\mathrm{n}=1,007)\end{array}$ & $\begin{array}{l}\text { Favors } \\
\text { Quetiapine } \\
\text { OR } 2.07 \text { (95\% } \\
\text { Cl 1.39, 3.09) } \\
\end{array}$ & Moderate & Consistent & Direct & Imprecise & Low \\
\hline & Remission 3 wks & $\begin{array}{l}3 \text { RCT } \\
(n=699)\end{array}$ & NS & High & Consistent & Direct & Imprecise & Insufficient \\
\hline & YRMS 3 wks & $\begin{array}{l}5 \mathrm{RCT} \\
\text { ( } \mathrm{n}=699 \text { forest plot, } \\
1439 \text { total) }\end{array}$ & $\begin{array}{l}\text { Favors } \\
\text { Quetiapine } \\
\text { MD 4.92 (95\% } \\
\text { Cl 0.31, 9.53) }\end{array}$ & Moderate & Inconsistent & Direct & Imprecise & Low \\
\hline & CGI-BP-S 3 wk & $\begin{array}{l}5 \mathrm{RCT} \\
\text { ( } \mathrm{n}=806 \text { forest plot, } \\
1439 \text { total) }\end{array}$ & $\begin{array}{l}\text { Favors } \\
\text { Quetiapine } \\
\text { Mean Difference } \\
0.54(95 \% \mathrm{Cl} \\
0.35,0.74) \\
\end{array}$ & Moderate & Consistent & Direct & Imprecise & Low \\
\hline & $\begin{array}{l}\text { Withdrawal - AE, } \\
\text { Overall }\end{array}$ & $\begin{array}{l}4 \mathrm{RCT} \\
(\mathrm{n}=1,007)\end{array}$ & NS & Moderate & Consistent & Indirect & Imprecise & Insufficient \\
\hline & $\begin{array}{l}\text { Withdrawal -Lack } \\
\text { of Efficacy }\end{array}$ & $\begin{array}{l}4 \mathrm{RCT} \\
(\mathrm{n}=1,007)\end{array}$ & $\begin{array}{l}\text { Favors } \\
\text { Quetiapine } \\
\text { Mean Difference } \\
0.38(95 \% \mathrm{Cl} \\
0.23,0.63)\end{array}$ & Moderate & Consistent & Direct & Imprecise & Low \\
\hline
\end{tabular}

Abbreviations: CGI-BP=Clinical Global Impressions Scale for Bipolar Disorder; NS=not significant; RCT=randomized controlled trial; YMRS = Young Mania Rating Scale

Notes:

1. Publication bias for antipsychotics, antidepressants, and behavioral interventions for depressive disorders is suspected.

2. Data were generally imprecise due to missing data from high attrition rates, which was commonly dealt with by Last Observation Carried Forward (LOCF). LOCF requires an

assumption that the health status of patients who dropped out of the trial would not have changed had future observations been recorded, a strong assumption in the context of

bipolar disorder research. 
Appendix Table E31. Outcomes summary: quetiapine versus active comparator for acute mania

\begin{tabular}{|c|c|c|c|c|c|c|}
\hline Comparison & $\begin{array}{l}\text { Study } \\
\text { PMID } \\
\text { RoB } \\
\end{array}$ & Responder/Remitter & Symptom & Function & Other & $\overline{A E}$ \\
\hline $\begin{array}{l}\text { Quetiapine vs. } \\
\text { haloperidol }\end{array}$ & $\begin{array}{l}\text { Mclntyre, } \\
2005^{16} \\
16139175 \\
\\
\text { Moderate }\end{array}$ & $\begin{array}{l}\frac{\text { Response }}{3 \text { weeks }} \\
\text { Quetiapine } 42.6 \% \\
\text { Haloperidol } 56.1 \% \\
\text { NS } \\
\frac{\text { Remission }}{3 \text { weeks }} \\
\text { Quetiapine } 27.7 \% \\
\text { Haloperidol } 36.7 \% \\
\text { NS }\end{array}$ & $\begin{array}{l}\text { YMRS Change } \\
3 \text { week } \\
\text { Quetiapine }-12.29 \\
\text { Haloperidol -15.71 } \\
\text { No Statistical Tests } \\
\text { reported }\end{array}$ & $\begin{array}{l}\text { CGI-BP-S Change } \\
3 \text { week } \\
\text { Quetiapine }-1.02 \\
\text { Haloperidol }-1.33 \\
\text { No Statistical Tests } \\
\text { reported } \\
\frac{\text { GAS }}{12 \text { weeks }} \\
\text { Favors quetiapine } \\
\text { against placebo } \\
\text { p }<.001 \\
12 \text { weeks } \\
\text { Favors haloperidol } \\
\text { against placebo } \\
p<.001\end{array}$ & $\begin{array}{l}\text { NR } \\
\text { Overall Withdrawal } \\
\text { Quetiapine 55/102 } \\
\text { Haloperidol 45/99 } \\
\text { Withdrawal Lack of } \\
\text { Efficacy } \\
\text { Quetiapine 18/102 } \\
\text { Haloperidol 10/99 } \\
\text { Withdrawal AE } \\
\text { Quetiapine 5/102 } \\
\text { Haloperidol 10/99 }\end{array}$ & $\begin{array}{l}\frac{\text { Serious Adverse Events }}{12 \text { weeks }} \\
0 \text { in both arms } \\
\frac{\text { Deaths }}{12 \text { weeks }} \\
0 \text { in both arms } \\
\frac{\text { EPS }}{12 \text { weeks }} \\
12.7 \% \text { Quetiapine } \\
59.6 \% \text { Haloperidol }\end{array}$ \\
\hline $\begin{array}{l}\text { Quetiapine vs. } \\
\text { lithium }\end{array}$ & $\begin{array}{l}\text { Bowden, } 2005 \\
15669897\end{array}$ & $\begin{array}{l}\frac{\text { Response }}{12 \text { weeks }} \\
\text { Quetiapine } 72.0 \% \\
\text { Lithium } 75.5 \% \\
\text { No Statistical Tests } \\
\text { reported } \\
\text { Remission } \\
12 \text { weeks } \\
\text { Quetiapine } 69.2 \% \\
\text { Lithium } 72.4 \% \\
\text { No Statistical Tests } \\
\text { reported }\end{array}$ & $\begin{array}{l}\text { YMRS Change } \\
12 \text { weeks } \\
\text { Quetiapine }-20.28 \\
\text { Lithium -20.76 } \\
\text { NS }\end{array}$ & $\begin{array}{l}\text { CGI-BP-S Change } \\
12 \text { weeks } \\
\text { Quetiapine }-2.20 \\
\text { Lithium }-2.18 \\
\text { No Statistical Tests } \\
\text { reported } \\
\text { GAS Change } \\
12 \text { weeks } \\
\text { Quetiapine } 26.35 \\
\text { Lithium NR } \\
\text { No Statistical Tests } \\
\text { reported }\end{array}$ & NR & $\begin{array}{l}\frac{\text { Serious Adverse Events }}{12 \text { weeks }} \\
0 \text { in both arms } \\
\frac{\text { Deaths }}{12 \text { weeks }} \\
0 \text { in both arms } \\
\text { EPS } \\
12 \text { weeks } \\
9.3 \% \text { placebo } \\
13.1 \% \text { Quetiapine } \\
\text { NR Lithium }\end{array}$ \\
\hline
\end{tabular}




\begin{tabular}{|c|c|c|c|c|c|c|}
\hline Comparison & $\begin{array}{l}\text { Study } \\
\text { PMID } \\
\text { RoB }\end{array}$ & Responder/Remitter & Symptom & Function & Other & $\mathrm{AE}$ \\
\hline & $\begin{array}{l}\mathrm{Li}, 2008 \\
18028587 \\
\\
\text { Moderate }\end{array}$ & $\begin{array}{l}\frac{\text { Response }}{4 \text { weeks }} \\
\text { Favors quetiapine } \\
\text { Quetiapine } 77.9 \% \\
\text { Lithium } 59.7 \% \\
p=0.013 \\
\text { Remission } \\
4 \text { weeks } \\
\text { Favors quetiapine } \\
\text { Quetiapine } 70.1 \% \\
\text { Lithium } 48.1 \% \\
p=0.007\end{array}$ & $\begin{array}{l}\text { YMRS Change } \\
4 \text { weeks } \\
\text { Quetiapine }-18.2 \\
\text { (10.4) } \\
\text { Lithium }-15.9(12.2) \\
\text { NS }\end{array}$ & NR & NR & $\begin{array}{l}\text { Serious Adverse Events } \\
4 \text { weeks } \\
0 \text { in both arms } \\
\text { Deaths } \\
4 \text { weeks } \\
0 \text { in both arms } \\
\text { EPS } \\
4 \text { weeks } \\
5.1 \% \text { Quetiapine } \\
6.5 \% \text { lithium }\end{array}$ \\
\hline
\end{tabular}

Abbreviations: AE=Adverse Events; ANCOVA=Analysis of Covariance; CGI=Clinical Global Impressions Scale; CI=Confidence Interval; EPS=extrapyramidal symptoms;

ESRS=Extrapyrimidal Symptom Rating Scale; GAS=Global Assessment Scale; MADRS=Montgomery-Asberg Depression Rating Scale; NR=Not reported; NS=not significant; OR=Odds Ratio; PMID=PubMed Identification Number; ROB=Risk of Bias; SAE=Serious Adverse Events; YMRS = Young Mania Rating Scale

\section{Appendix Table E32. Strength of evidence assessment: quetiapine versus active comparator for acute mania}

\begin{tabular}{|c|c|c|c|c|c|c|c|c|}
\hline Comparison & Outcome & $\begin{array}{l}\text { \# Studies/ } \\
\text { Design } \\
\text { (n analyzed) }\end{array}$ & $\begin{array}{l}\text { Finding or } \\
\text { Summary } \\
\text { Statistic }\end{array}$ & $\begin{array}{c}\text { Study } \\
\text { Limitations }\end{array}$ & Consistency & Directness & Precision & $\begin{array}{c}\text { Overall } \\
\text { Gradel } \\
\text { Conclusion }\end{array}$ \\
\hline $\begin{array}{l}\text { Quetiapine vs. } \\
\text { haloperidol }\end{array}$ & $\begin{array}{l}\text { Response } \\
\text { Remission } \\
\text { YMRS } \\
\text { CGI } \\
\text { Withdrawals }\end{array}$ & $\begin{array}{l}1 \mathrm{RCT} \\
(\mathrm{n}=199)\end{array}$ & See table above & Moderate & Unknown & Direct & Imprecise & Insufficient \\
\hline $\begin{array}{l}\text { Quetiapine vs. } \\
\text { lihtium }\end{array}$ & $\begin{array}{l}\text { Response } \\
\text { Remission } \\
\text { YMRS } \\
\text { Withdrawals }\end{array}$ & $\begin{array}{l}2 \text { RCTs } \\
(n=456)\end{array}$ & See table & High & Inconsistent & Direct & Imprecise & Insufficient \\
\hline
\end{tabular}

Abbreviations: CGI-BP=Clinical Global Impressions Scale for Bipolar Disorder; NS=not significant; RCT=randomized controlled trial; YMRS = Young Mania Rating Scale

Notes:

1. Publication bias for antipsychotics, antidepressants, and behavioral interventions for depressive disorders is suspected.

2. Data were generally imprecise due to missing data from high attrition rates, which was commonly dealt with by Last Observation Carried Forward (LOCF). LOCF requires an assumption that the health status of patients who dropped out of the trial would not have changed had future observations been recorded, a strong assumption in the context of bipolar disorder research. 


\section{Section 7. Risperidone for Acute Mania}

Appendix Table E33. Characteristics of eligible studies: Risperidone for acute mania

\begin{tabular}{|c|c|c|c|c|c|c|}
\hline $\begin{array}{l}\text { Study, Year } \\
\text { Design } \\
\text { Location } \\
\text { Funder } \\
\text { Risk of Bias } \\
\text { PMID }\end{array}$ & $\begin{array}{c}\text { \# Randomized } \\
\text { Age (mean) } \\
\text { Sex (\% Female) } \\
\text { Race (\% White) } \\
\text { Diagnosis } \\
\text { (\% BP-I, II, NOS) } \\
\text { Setting }\end{array}$ & $\begin{array}{c}\text { Inclusions } \\
\text { Key Exclusions }\end{array}$ & $\begin{array}{c}\text { Intervention } \\
\text { Dosage }\end{array}$ & $\begin{array}{c}\text { Comparison } \\
\text { Dosage }\end{array}$ & Follow-up Duration & $\begin{array}{c}\text { Outcomes } \\
\text { Reported } \\
\text { Withdrawal (\%) at } \\
\text { endpoint }\end{array}$ \\
\hline $\begin{array}{l}\text { Smulevich, } 200515 \\
\text { RCT } \\
\text { Multisite } \\
2 \text { Continents } \\
\text { Industry } \\
\text { RoB Moderate } \\
15572276\end{array}$ & $\begin{array}{l}\mathrm{N}=438 \\
\text { Mean Age } 40 \\
\text { Female } 47 \% \\
\text { White } 65 \% \\
\text { BP-I } 100 \% \\
\text { Inpatient }\end{array}$ & $\begin{array}{l}\text { Mania; } \\
\text { YMRS } \geq 20 \text { and } \\
\text { MADRS } \leq 20 \\
\text { First Manic Episode } \\
\text { Schizoaffective } \\
\text { Substance Abuse } \\
\text { Other Mental Health } \\
\text { Taking Other Meds }\end{array}$ & $\begin{array}{l}\text { Risperidone } \\
1-6 \text { mg/day (mean } \\
4.2 \text { mg/day) }\end{array}$ & $\begin{array}{l}\text { C1: Placebo } \\
\text { C2: Haloperidol } \\
\text { 2-12 mg/day (mean } \\
8.0 \mathrm{mg} / \text { day) }\end{array}$ & $\begin{array}{l}12 \text { weeks (12 week } \\
\text { outcomes excluded } \\
\text { due to attrition }\end{array}$ & \begin{tabular}{|l} 
BPRS \\
CGI-S \\
GAS \\
MADRS \\
YMRS \\
\\
Withdrawal \\
$48 \%$ at 12 weeks \\
$12 \%$ at 3 weeks
\end{tabular} \\
\hline $\begin{array}{l}\text { Khanna, } 200540 \\
\text { RCT } \\
\text { Multisite } \\
\text { India } \\
\text { Industry } \\
\text { RoB Moderate } \\
16135859\end{array}$ & $\begin{array}{l}\mathrm{N}=290 \\
\text { Mean Age } 35 \\
\text { Female } 38 \% \\
\text { Race NR } \\
\text { BP-I 100\% } \\
\text { Inpatient }\end{array}$ & $\begin{array}{l}\text { Mania; } \\
\text { YMRS } \geq 20 \\
\text { Schizoaffective } \\
\text { Substance Abuse } \\
\text { Other Mental Health } \\
\text { Taking Other Meds }\end{array}$ & \begin{tabular}{|l|} 
Risperidone \\
1-6 mg/day
\end{tabular} & Placebo & 3 weeks & \begin{tabular}{|l|} 
CGI-S \\
MADRS \\
PANSS \\
YMRS \\
Withdrawal 20\%
\end{tabular} \\
\hline
\end{tabular}




\begin{tabular}{|c|c|c|c|c|c|c|}
\hline 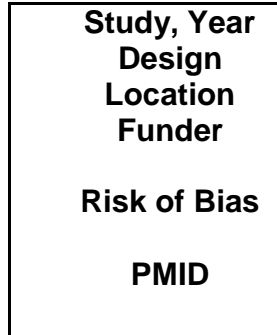 & $\begin{array}{c}\text { \# Randomized } \\
\text { Age (mean) } \\
\text { Sex (\% Female) } \\
\text { Race (\% White) } \\
\text { Diagnosis } \\
\text { (\% BP-I, II, NOS) } \\
\text { Setting }\end{array}$ & $\begin{array}{c}\text { Inclusions } \\
\text { Key Exclusions }\end{array}$ & $\begin{array}{c}\text { Intervention } \\
\text { Dosage }\end{array}$ & $\begin{array}{c}\text { Comparison } \\
\text { Dosage }\end{array}$ & Follow-up Duration & $\begin{array}{c}\text { Outcomes } \\
\text { Reported } \\
\text { Withdrawal (\%) at } \\
\text { endpoint }\end{array}$ \\
\hline $\begin{array}{l}\text { Segal, } 199841 \\
\text { RCT } \\
\text { Singlesite } \\
\text { South Africa } \\
\text { Industry/University } \\
\text { RoB Moderate } \\
9617509\end{array}$ & $\begin{array}{l}\mathrm{N}=45 \\
\text { Mean Age 34 } \\
\text { Female 78\% } \\
\text { Race NR } \\
\text { BP-I 100\% } \\
\text { Inpatient }\end{array}$ & $\begin{array}{l}\text { Mania; } \\
\text { DSM-IV criteria for } \\
\text { Bipolar Manic Phase } \\
\\
\text { Substance Abuse } \\
\text { Taking Other Meds } \\
\text { Pregnant/Nursing } \\
\text { Labs/Other } \\
\text { Conditions }\end{array}$ & \begin{tabular}{|l|} 
Risperidone \\
6 mg/day
\end{tabular} & $\begin{array}{l}\text { C1: Placebo } \\
\text { C2: Haloperidol } \\
10 \text { mg/day } \\
\text { C3: Lithium } \\
0.6-1.2 \mathrm{mmol} / \mathrm{L}\end{array}$ & 4 weeks & $\begin{array}{l}\text { BPRS } \\
\text { CGI Unknown Scale - } \\
\text { Not reported whether } \\
\text { global improvement } \\
\text { or severity scale is } \\
\text { being reported } \\
\text { GAF } \\
\text { MRS } \\
\text { Seclusion - Hours of } \\
\text { eclusion - Proportion } \\
\text { of patients needing } \\
\text { SAS } \\
\text { Withdrawal NR }\end{array}$ \\
\hline
\end{tabular}

Abbreviations: AIMS=Abnormal Involuntary Movement Scale; BARS=Barnes Akathisia Scale; BAS=Behavioral Approach System; BMI=Body Mass Index; BP=bipolar

disorder; BPRS=Brief Psychiatric Rating Scale; C=comparison; CGI= Clinical Global Impressions; CGI-I=Clinical Global Impressions-Improvement; CGI-S =CGI-Severity; CGIBP=Clinical Global Impressions Scale-Bipolar; CGI-BP-C= Clinical Global Impressions, Bipolar, Change Scale; CGI-BP-S=Clinical Global Impressions, Bipolar, Severity Scale; C-SSRS=Columbia Suicide Severity Rating Scale; DAI-10=Drug Attitutde Inventory, 10 question version; DIEPSS=Drug-Induced Extra-Pyramidal Symptoms Scale;

DSM=Diagnostic and Statistical Manual of Mental Disorders; EPS=extrapyramidal symptoms; ER=Extended Release; ESRS=Extrapyramidal Symptom Rating Scale;

GAF=General Assessment of Functioning Scale; GAS=Global Assessment Scale; HAM-A=Hamilton Scale for Anxiety; HAM-D=Hamilton Scale for Depression; HRQL=Healthrelated quality of life; HRQOL=Health-related quality of life; I=intervention; IDS=Inventory for Depressive Symptoms; LIFE= Longitudinal Interval Follow-up Evaluation; MADRS=Montgomery-Asberg Depression Rating Scale; MAS=Bech-Rafaelsen Mania Rating Scale; MRS=Mania Rating Scale; MSRS=Manic state rating scale; NOS=not otherwise specified; NR=not reported; PANSS=Positive and Negative Syndrome Scale; PRS=Polygenic Risk Scores; PGWB=Psychological General Well-Being Index; PMID=PubMed Identification Number; PRS=Polygenic Risk Scores; Q-LES-Q=Quality of Life Enjoyment and Satisfaction Questionnaire; RCT=randomized controlled trial; ROB=risk of bias; SADS-C= Schedule for Affective Disorders and Schizophrenia-Change version; SAE=Serious Adverse Events; SAS=Simpson Angus Scale; SF-12=12-Item Short Form Health Survey; SF-36=36-Item Short Form Health Survey; SLICE=Streamlined Longitudinal Interview Clinical Evaluation; T=Trial; YMRS = Young Mania Rating Scale 
Appendix Table E34. Summary risk of bias assessments: risperidone for acute mania

\begin{tabular}{l} 
Appendix Table E34. Summary risk of bias assessments: risperidone for acute mania \\
\begin{tabular}{|l|l|l|l|}
\hline \multicolumn{1}{|c|}{ Drug } & \multicolumn{1}{|c|}{$\begin{array}{c}\text { Study } \\
\text { Funding Source } \\
\text { PMID }\end{array}$} & $\begin{array}{c}\text { Overall Risk of } \\
\text { Bias Assessment }\end{array}$ & Rationale \\
\hline Risperidone & $\begin{array}{l}\text { Smulevich, 2005 } \\
\text { Industry } \\
15572276\end{array}$ & Moderate & $\begin{array}{l}\text { Randomization and blinding procedures not well-described. Some participants treated in } \\
\text { open-label fashion. 12\% dropout. }\end{array}$ \\
\cline { 2 - 4 } & $\begin{array}{l}\text { Khanna, 200540 } \\
\text { Industry } \\
16135859\end{array}$ & Moderate & $\begin{array}{l}\text { Randomization and blinding procedures not described. Handling of data from missing } \\
\text { persons not described. 20\% dropout. }\end{array}$ \\
\cline { 2 - 4 } & $\begin{array}{l}\text { Segal, 1998 } \\
\text { Industry/University } \\
9617509\end{array}$ & Moderate & $\begin{array}{l}\text { Randomization not described, patients assigned consecutively which infers both a lack of } \\
\text { randomization and a likelihood of a lack of allocation concealment. }\end{array}$ \\
\hline
\end{tabular} \\
\hline
\end{tabular}

Abbreviations: ITT=Intention to Treat; PMID=PubMed Identification Number; LOCF=last observation carried forward 


\section{Risperidone Forest Plots}

Outcomes in studies assessed as having a high risk of bias, or low to moderate risk of bias but at least 40 percent attrition, are presented in grey tones. Both fixed-effect models and random-effects models are presented. We calculated fixed-effect models to provide a charitable estimate of the average effect among completed trials. However, we base our main conclusions on the random-effects models.

Appendix Figure E69. Risperidone vs. placebo - response

Odds Ratio of Response ( $>50 \%$ Reduction in YMRS) at 3 Weeks

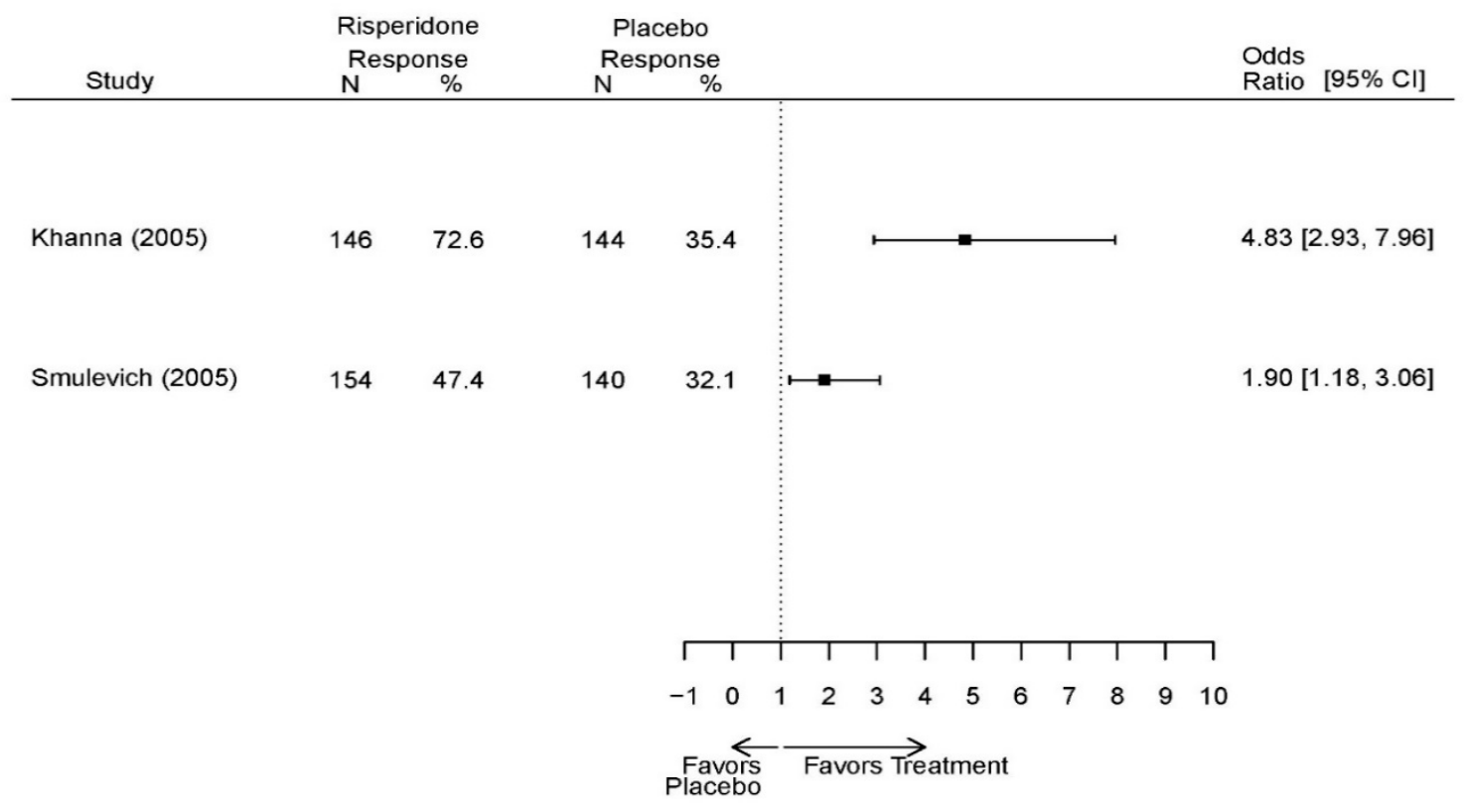


Appendix Figure E70. Risperidone vs. placebo - YMRS

Difference in Mean Change in YMRS from Baseline to Last Measurement

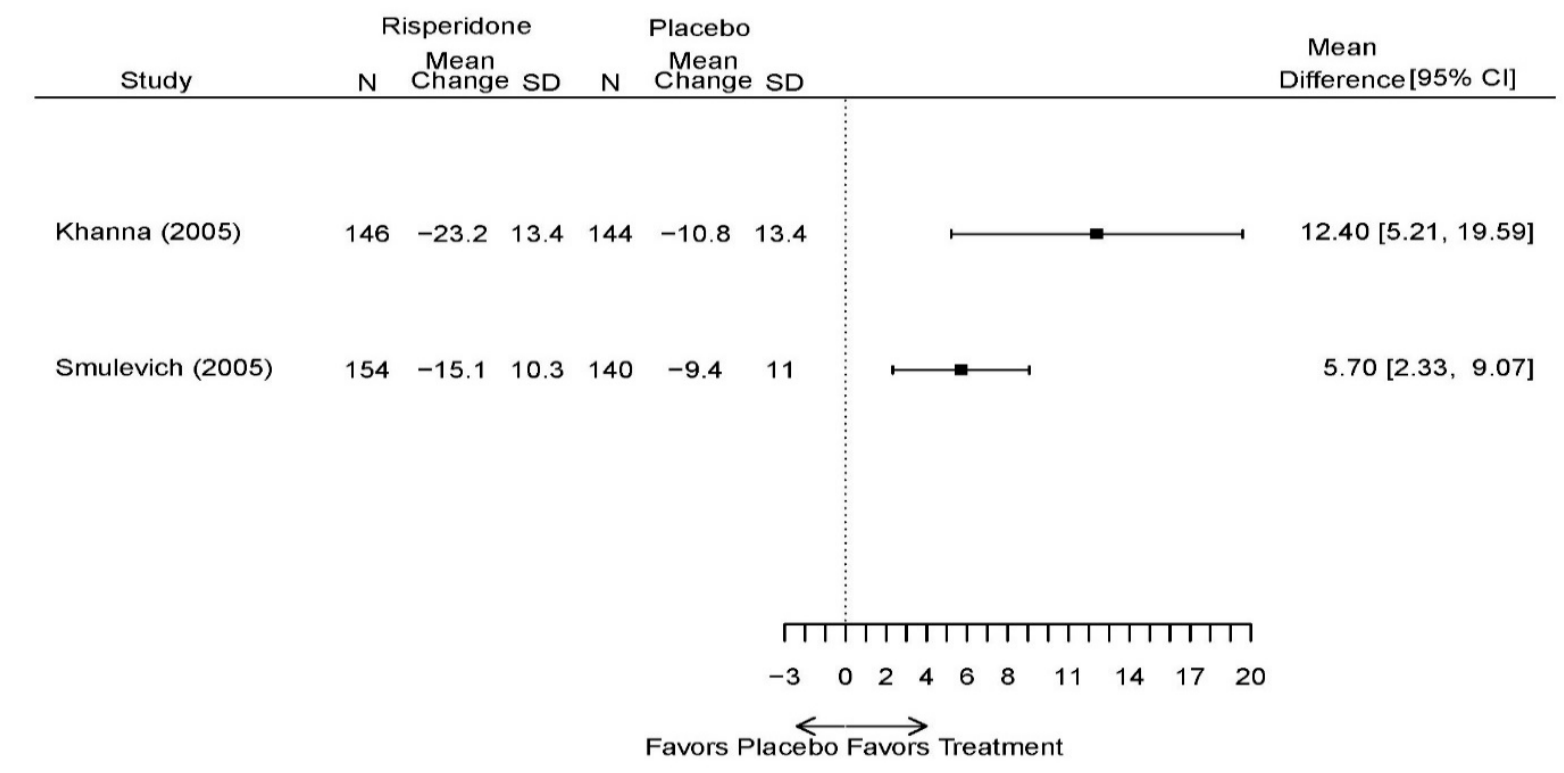

Appendix Figure E71. Risperidone vs. placebo - CGI

Difference in Mean Change in CGI-BP-S (Overall)

from Baseline to Last Measurement

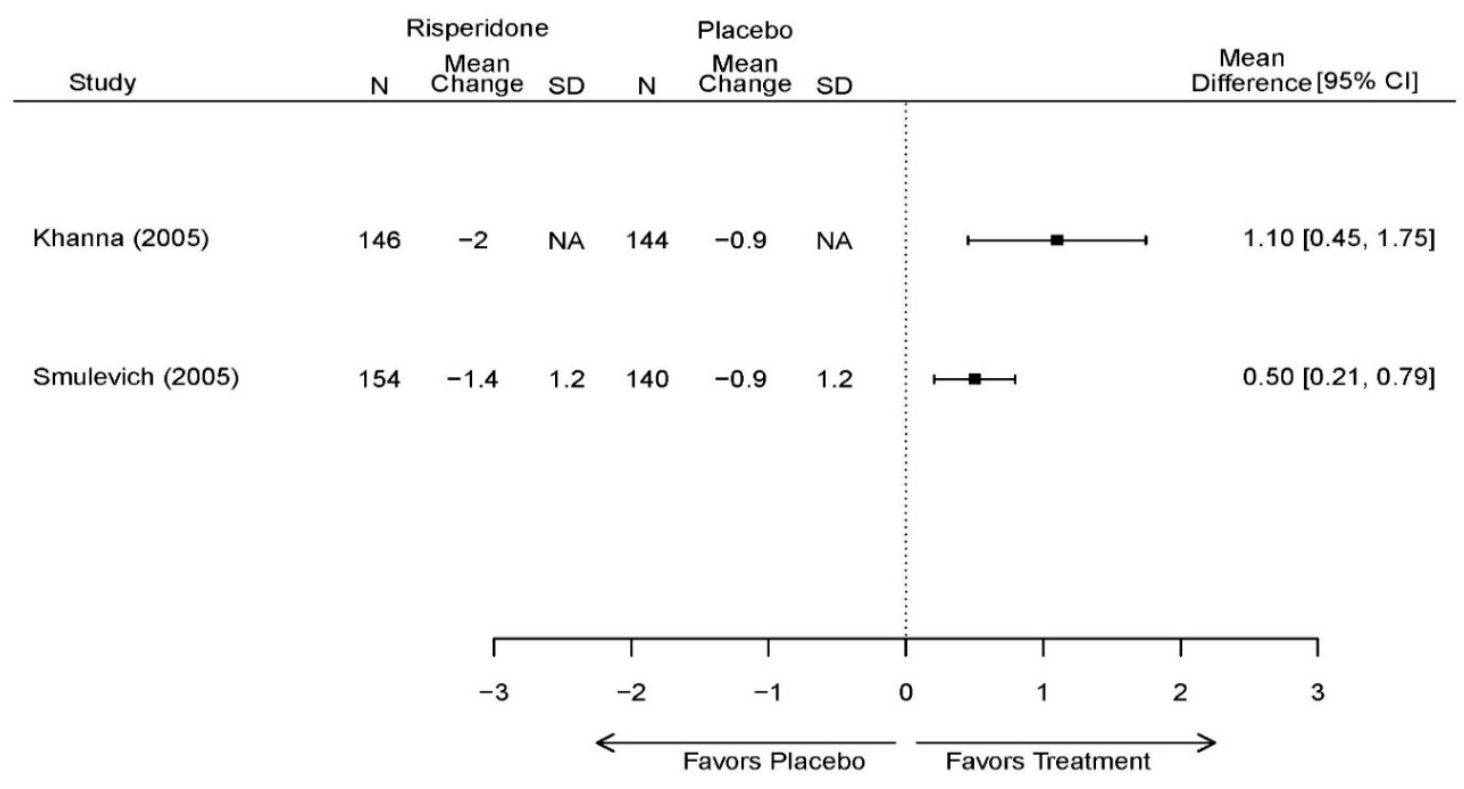


Appendix Figure E72. Risperidone vs. placebo - overall withdrawal

Odds Ratio of Overall Withdrawal

\begin{tabular}{|c|c|c|c|c|c|c|c|}
\hline Study & \multicolumn{2}{|c|}{$\begin{array}{l}\text { Risperidone } \\
\text { Withdrawal }\end{array}$} & \multicolumn{2}{|c|}{$\begin{array}{c}\text { Placebo } \\
\text { Withdrawal }\end{array}$} & & & $\begin{array}{l}\text { Odds } \\
\text { Ratio [95\% Cl] }\end{array}$ \\
\hline Khanna (2005) & 146 & 11 & 144 & 29.2 & $\mapsto$ & & $0.30[0.16,0.56]$ \\
\hline \multirow[t]{4}{*}{ Smulevich (2005) } & 154 & 11 & 140 & 15 & & & $0.70[0.35,1.39]$ \\
\hline & & $\Gamma$ & & & $T$ & $T$ & $T$ \\
\hline & & -2 & & & 0 & 2 & 3 \\
\hline & & & & & $\leftarrow \frac{F_{r}}{T r e}$ & & \\
\hline
\end{tabular}

Appendix Figure E73. Risperidone vs. placebo - withdrawal - adverse events

Odds Ratio of Withdrawal due to Adverse Events at 3 Weeks

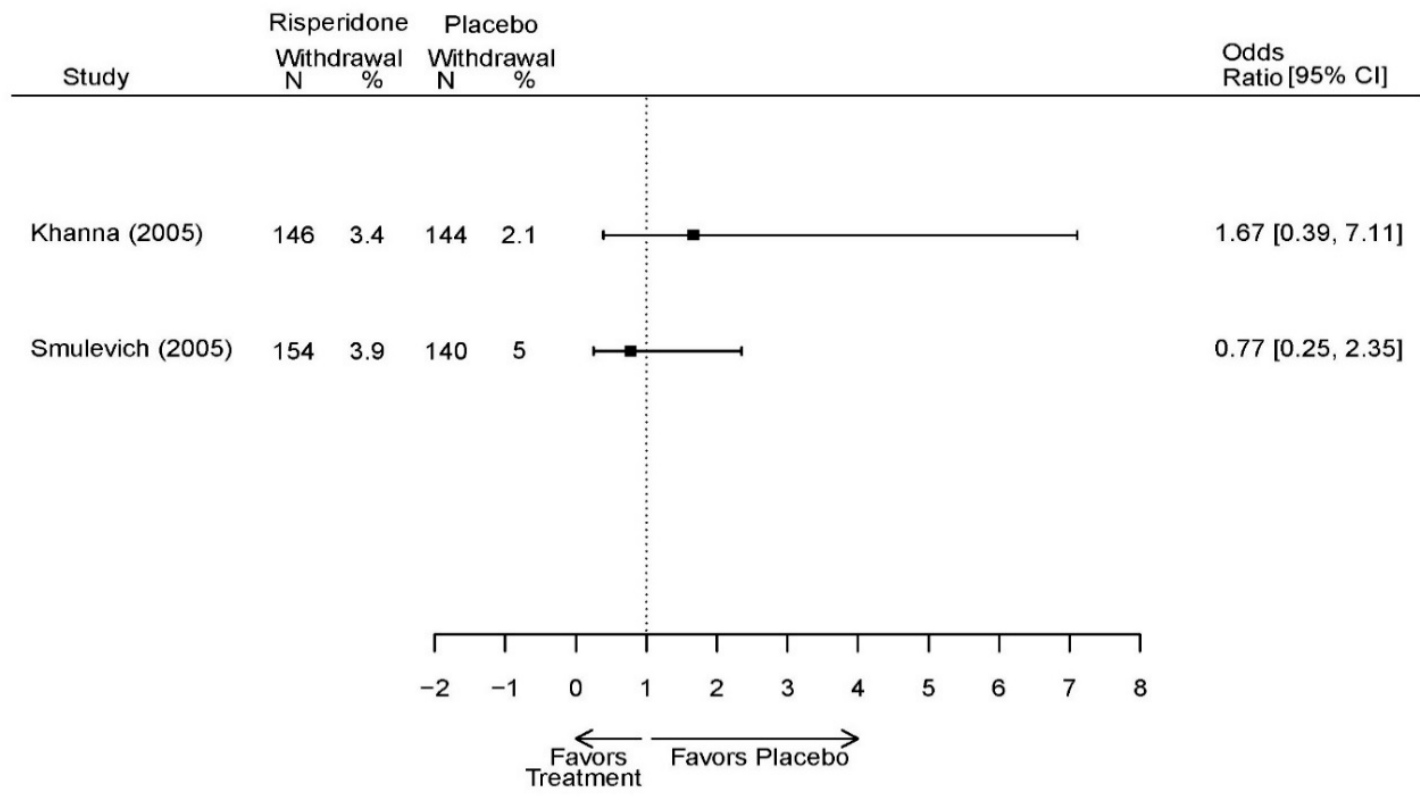




\begin{tabular}{|c|c|c|c|c|c|c|}
\hline Drug & $\begin{array}{l}\text { Study } \\
\text { PMID } \\
\text { RoB }\end{array}$ & Responder/Remitter & Symptom & Function & Other & AE \\
\hline \multirow[t]{2}{*}{ Risperidone } & $\begin{array}{l}\text { Khanna, } \\
2005^{40} \\
16135859 \\
\\
\text { Moderate }\end{array}$ & $\begin{array}{l}\text { See forest plot E69 } \\
\text { above for Response }\end{array}$ & $\begin{array}{l}\text { See forest plot E70 } \\
\text { above for YMRS } \\
\text { MADRS } \\
3 \text { weeks } \\
\text { Mean change } \\
\text { Risperidone: }-3.2 \\
(0.4) \\
\text { Placebo: }-2.5,(0.34) \\
\text { P<0.001) }\end{array}$ & $\begin{array}{l}\text { See forest plot E70 } \\
\text { above for CGI }\end{array}$ & $\begin{array}{l}\text { See forest plot E72, } \\
\text { E73 above for } \\
\text { Withdrawal }\end{array}$ & $\begin{array}{l}\text { Serious Adverse Events } \\
3 \text { weeks } \\
0 \text { in both arms } \\
\frac{\text { Deaths }}{3 \text { weeks }} \\
0 \text { in both arms } \\
\frac{\text { EPS }}{3 \text { weeks }} \\
6.0 \% \text { placebo } \\
36.0 \% \text { Risperidone }\end{array}$ \\
\hline & $\begin{array}{l}\text { Smulevich, } \\
2005^{15} \\
15572276 \\
\text { Moderate }\end{array}$ & $\begin{array}{l}\text { See forest plot E69 } \\
\text { above for Response }\end{array}$ & $\begin{array}{l}\text { See forest plot E70 } \\
\text { above for YMRS }\end{array}$ & $\begin{array}{l}\text { See forest plot E70 } \\
\text { above for CGI } \\
\frac{\text { GAS }}{3 \text { week }} \\
\text { Risperidone - } \\
17.1(1.8) \\
\text { Placebo -10.3(1.7) } \\
\text { No Statistical Tests } \\
\text { reported }\end{array}$ & $\begin{array}{l}\text { See forest plot E72, } \\
\text { E73 above for } \\
\text { Withdrawal }\end{array}$ & $\begin{array}{l}\text { No reported SAE } \\
\text { EPS } \\
\text { Mean ESRS score } \\
\text { increases greater for } \\
\text { haloperidol than } \\
\text { risperidone }(p<0.001)\end{array}$ \\
\hline
\end{tabular}

Abbreviations: AE=Adverse Events; ANCOVA=Analysis of Covariance; CGI=Clinical Global Impressions Scale; CI=Confidence Interval; EPS=extrapyramidal symptoms; ESRS=Extrapyrimidal Symptom Rating Scale; GAS=Global Assessment Scale; MADRS=Montgomery-Asberg Depression Rating Scale; NR=Not reported; NS=not significant; OR=Odds Ratio; PMID=PubMed Identification Number; ROB=Risk of Bias; SAE=Serious Adverse Events; YMRS = Young Mania Rating Scale 
Appendix Table E36. Strength of evidence assessment: risperidone versus placebo for acute mania

\begin{tabular}{|c|c|c|c|c|c|c|c|c|}
\hline Comparison & Outcome & $\begin{array}{l}\text { \# Studies/ } \\
\text { Design } \\
\text { (n analyzed) }\end{array}$ & $\begin{array}{l}\text { Finding or } \\
\text { Summary } \\
\text { Statistic }\end{array}$ & $\begin{array}{c}\text { Study } \\
\text { Limitations }\end{array}$ & Consistency & Directness & Precision & $\begin{array}{c}\text { Overall } \\
\text { Gradel } \\
\text { Conclusion }\end{array}$ \\
\hline $\begin{array}{l}\text { Risperidone } \\
\text { vs. placebo }\end{array}$ & $\begin{array}{l}\text { Response } 3 \text { wks } \\
\text { YMRS } 3 \text { wks } \\
\text { CGI } 3 \text { wks }\end{array}$ & $\begin{array}{l}2 \text { RCTs } \\
(n=584)\end{array}$ & $\begin{array}{l}\text { Favors } \\
\text { Risperidone }\end{array}$ & Moderate & Consistent & Direct & Imprecise & Low \\
\hline
\end{tabular}

Abbreviations: CGI-BP=Clinical Global Impressions Scale for Bipolar Disorder; NS=not significant; RCT=randomized controlled trial; YMRS = Young Mania Rating Scale

Notes:

1. Publication bias for antipsychotics, antidepressants, and behavioral interventions for depressive disorders is suspected.

2. Data were generally imprecise due to missing data from high attrition rates, which was commonly dealt with by Last Observation Carried Forward (LOCF). LOCF requires an

assumption that the health status of patients who dropped out of the trial would not have changed had future observations been recorded, a strong assumption in the context of

bipolar disorder research.

\begin{tabular}{|c|c|c|c|c|c|c|}
\hline Drub & $\begin{array}{c}\text { Study } \\
\text { PMID } \\
\text { RoB }\end{array}$ & Responder/Remitter & Symptom & Function & Other & AE \\
\hline $\begin{array}{l}\text { Risperidone vs. } \\
\text { haloperidol }\end{array}$ & $\begin{array}{l}\text { Smulevich, } \\
2005^{15} \\
15572276 \\
\text { Moderate }\end{array}$ & $\begin{array}{l}\text { Response } \\
3 \text { weeks } \\
\text { Risperidone } 48 \% \\
\text { Haloperidol } 47 \% \\
\text { NS }\end{array}$ & $\begin{array}{l}\text { YMRS Change } \\
3 \text { week } \\
\text { Risperidone - } \\
15.1(10.3) \\
\text { Haloperidol - } \\
13.9(10.3) \\
\text { NS }\end{array}$ & $\begin{array}{l}\text { CGI-S Change } \\
3 \text { week } \\
\text { Risperidone -1.4(1.2) } \\
\text { Haloperidol -1.3(1.1) } \\
\text { NS } \\
\frac{\text { GAS }}{3 \text { week }} \\
\text { Risperidone - } \\
17.1(1.8) \\
\text { Haloperidol - } \\
13.9(10.3) \\
\text { NS }\end{array}$ & $\begin{array}{l}\text { Withdrawal lack of } \\
\text { efficacy } \\
\text { Risperidone } 4 \% \\
\text { Haloperidol } 3 \% \\
\text { Withdrawal adverse } \\
\text { events } \\
\text { Risperidone 3\% } \\
\text { Haloperidol 1\% }\end{array}$ & $\begin{array}{l}\text { Serious Adverse Events } \\
12 \text { weeks } \\
0 \text { in both arms } \\
\frac{\text { Deaths }}{12 \text { weeks }} \\
0 \text { in both arms } \\
\frac{\text { EPS }}{N R}\end{array}$ \\
\hline
\end{tabular}




\begin{tabular}{|c|c|c|c|c|c|c|}
\hline Drub & $\begin{array}{l}\text { Study } \\
\text { PMID } \\
\text { RoB } \\
\end{array}$ & Responder/Remitter & Symptom & Function & Other & $\mathrm{AE}$ \\
\hline & $\begin{array}{l}\text { Segal, } 1998^{41} \\
9617509 \\
\text { Moderate }\end{array}$ & $\underline{\mathrm{NR}}$ & $\begin{array}{l}\frac{\text { MRS }}{4 \text { weeks }} \\
\text { NS }\end{array}$ & $\begin{array}{l}\frac{\mathrm{CGI}}{4 \text { weeks }} \\
\mathrm{NS} \\
\frac{\mathrm{GAF}}{4 \text { weeks }} \\
\mathrm{NS}\end{array}$ & NR & $\begin{array}{l}\text { Serious Adverse Events } \\
4 \text { weeks } \\
0 \text { in both arms } \\
\frac{\text { Deaths }}{4 \text { weeks }} \\
0 \text { in both arms } \\
\frac{\text { EPS }}{N R}\end{array}$ \\
\hline
\end{tabular}

Abbreviations: AE=Adverse Events; CI=Confindence Interval; CGI=Clinical Global Impressions Scale; CGI-BP=Clinical Global Impressions Scale, Bipolar; CGI-BP-S=Clinical Global Impressions, Bipolar, Severity Scale; CGI-S=Clinical Global Impressions; Severity Scale; EPS=extrapyramidal symptoms; GAF=Global Assessment of Functioning Scale; GAS=Global Assessment Scale; MADRS=Montgomery-Asberg Syndrome Scale; MRS=Mania Rating Scale; NR=not reported; NS=not significant; OR=Odds Ratio;

PMID=PubMed Identification Number; RoB=Risk of Bias; SD=Standard Deviation; SE=standard error; YMRS = Young Mania Rating Scale

Appendix Table E38. Strength of evidence assessment: risperidone versus active comparator for acute mania

\begin{tabular}{|c|c|c|c|c|c|c|c|c|}
\hline Comparison & Outcome & $\begin{array}{l}\text { \# Studies/ } \\
\text { Design } \\
\text { (n analyzed) }\end{array}$ & $\begin{array}{l}\text { Finding or } \\
\text { Summary } \\
\text { Statistic }\end{array}$ & $\begin{array}{c}\text { Study } \\
\text { Limitations }\end{array}$ & Consistency & Directness & Precision & $\begin{array}{c}\text { Overall } \\
\text { Gradel } \\
\text { Conclusion }\end{array}$ \\
\hline $\begin{array}{l}\text { Risperidone } \\
\text { vs. haloperidol }\end{array}$ & $\begin{array}{l}\text { Response } 3 \text { wks } \\
\text { YMRS } 3 \text { wks } \\
\text { CGI-S } 3 \text { wks }\end{array}$ & $\begin{array}{l}2 \text { RCTs } \\
(n=438)\end{array}$ & See table above & Moderate & Consistent & Direct & Imprecise & Insufficient \\
\hline $\begin{array}{l}\text { Risperidone } \\
\text { vs. lithium }\end{array}$ & $\begin{array}{l}\text { YMRS } 4 \text { wks } \\
\text { CGI } 4 \text { wks } \\
\text { GAF } 4 \text { wks }\end{array}$ & $\begin{array}{l}1 \mathrm{RCT} \\
(\mathrm{n}=45)\end{array}$ & See table above & Moderate & Unknown & Direct & Imprecise & Insufficient \\
\hline
\end{tabular}

Abbreviations: CGI-BP=Clinical Global Impressions Scale for Bipolar Disorder; NS=not significant; RCT=randomized controlled trial; YMRS = Young Mania Rating Scale Notes:

1. Publication bias for antipsychotics, antidepressants, and behavioral interventions for depressive disorders is suspected.

2. Data were generally imprecise due to missing data from high attrition rates, which was commonly dealt with by Last Observation Carried Forward (LOCF). LOCF requires an assumption that the health status of patients who dropped out of the trial would not have changed had future observations been recorded, a strong assumption in the context of bipolar disorder research. 


\section{Section 8. Ziprasidone for Acute Mania}

Appendix Table E39. Characteristics of eligible studies: ziprasidone drug treatments for acute mania by year then first author

\begin{tabular}{|c|c|c|c|c|c|c|}
\hline $\begin{array}{c}\text { Study, Year } \\
\text { Design } \\
\text { Location } \\
\text { Funder } \\
\text { Risk of Bias } \\
\text { PMID }\end{array}$ & $\begin{array}{c}\text { \# Randomized } \\
\text { Age (mean) } \\
\text { Sex (\% Female) } \\
\text { Race (\% White) } \\
\text { Diagnosis } \\
\text { (\% BP-I, II, NOS) } \\
\text { Setting }\end{array}$ & $\begin{array}{c}\text { Inclusions } \\
\text { Key Exclusions }\end{array}$ & $\begin{array}{c}\text { Intervention } \\
\text { Dosage }\end{array}$ & $\begin{array}{c}\text { Comparison } \\
\text { Dosage }\end{array}$ & Follow-up Duration & $\begin{array}{c}\text { Outcomes } \\
\text { Reported } \\
\text { Withdrawal (\%) at } \\
\text { endpoint }\end{array}$ \\
\hline $\begin{array}{l}\text { Potkin, } 200542 \\
\text { RCT } \\
\text { Multisite } \\
2 \text { Continents } \\
\text { Industry } \\
\text { RoB Low } \\
16012271\end{array}$ & $\begin{array}{l}\mathrm{N}=206 \\
\text { Mean Age } 40 \\
\text { Female } 49 \% \\
\text { White } 62 \% \\
\text { BP-I 100\% } \\
\text { Inpatient }\end{array}$ & $\begin{array}{l}\text { Mania; } \\
\text { Mania Rating Scale } \\
\text { (SADS-C interview } \\
\text { derived; Spitzer, } \\
1978) \geq 14 \text { with score } \\
\geq 2 \text { on four items at } \\
\text { screening and } \\
\text { admission } \\
\\
\text { Schizoaffective } \\
\text { Substance Abuse } \\
\text { Other Mental Health } \\
\text { Taking Other Meds } \\
\text { Pregnant/Nursing }\end{array}$ & \begin{tabular}{|l} 
Ziprasidone \\
80-160 mg/day (112 \\
mg/day mean)
\end{tabular} & Placebo & 3 weeks & $\begin{array}{l}\text { BMI or Weight } \\
\text { CGI-I } \\
\text { CGI-S } \\
\text { GAF } \\
\text { HAM-D } \\
\text { MADRS } \\
\text { MRS } \\
\text { PANSS } \\
\text { Withdrawal } 41 \%\end{array}$ \\
\hline $\begin{array}{l}\text { Keck, } 200343 \\
\text { RCT } \\
\text { Multisite } \\
2 \text { Continents } \\
\text { Industry } \\
\text { RoB Moderate } \\
12668364\end{array}$ & $\begin{array}{l}\mathrm{N}=210 \\
\text { Mean Age } 38 \\
\text { Female 46\% } \\
\text { Race NR } \\
\text { BP-I 100\% } \\
\text { Inpatient }\end{array}$ & $\begin{array}{l}\text { Mania; } \\
\text { Mania Rating Scale } \\
\text { (SADS-C interview } \\
\text { derived; Spitzer, } \\
1978) \geq 14 \text { with score } \\
\geq 2 \text { on four items at } \\
\text { screening and } \\
\text { admission } \\
\\
\text { Schizoaffective } \\
\text { Substance Abuse } \\
\text { Other Mental Health } \\
\text { Taking Other Meds } \\
\text { Pregnant/Nursing } \\
\text { Labs/Other } \\
\text { Conditions }\end{array}$ & \begin{tabular}{|l} 
Ziprasidone \\
$40-80 \mathrm{mg}$ bid \\
(mean $\approx 130 \mathrm{mg} /$ day $)$
\end{tabular} & Placebo & 3 weeks & $\begin{array}{l}\text { MRS } \\
\text { CGI-S } \\
\text { CGI-I } \\
\text { PANSS } \\
\text { GAF } \\
\text { Adverse Events } \\
\text { AIMS } \\
\text { Withdrawal 50\% }\end{array}$ \\
\hline
\end{tabular}

E-102 
Abbreviations: AIMS=Abnormal Involuntary Movement Scale; BARS=Barnes Akathisia Scale; BAS=Behavioral Approach System; BMI=Body Mass Index; BP=bipolar disorder; BPRS=Brief Psychiatric Rating Scale; C=comparison; CGI= Clinical Global Impressions; CGI-I=Clinical Global Impressions-Improvement; CGI-S =CGI-Severity; CGIBP=Clinical Global Impressions Scale-Bipolar; CGI-BP-C= Clinical Global Impressions, Bipolar, Change Scale; CGI-BP-S=Clinical Global Impressions, Bipolar, Severity Scale; C-SSRS=Columbia Suicide Severity Rating Scale; DAI-10=Drug Attitutde Inventory, 10 question version; DIEPSS=Drug-Induced Extra-Pyramidal Symptoms Scale;

DSM=Diagnostic and Statistical Manual of Mental Disorders; EPS=extrapyramidal symptoms; ER=Extended Release; ESRS=Extrapyramidal Symptom Rating Scale;

GAF=General Assessment of Functioning Scale; GAS=Global Assessment Scale; HAM-A=Hamilton Scale for Anxiety; HAM-D=Hamilton Scale for Depression; HRQL=Healthrelated quality of life; HRQOL=Health-related quality of life; I=intervention; IDS=Inventory for Depressive Symptoms; LIFE= Longitudinal Interval Follow-up Evaluation; MADRS=Montgomery-Asberg Depression Rating Scale; MAS=Bech-Rafaelsen Mania Rating Scale; MRS=Mania Rating Scale; MSRS=Manic state rating scale; NOS=not otherwise specified; NR=not reported; PANSS=Positive and Negative Syndrome Scale; PRS=Polygenic Risk Scores; PGWB=Psychological General Well-Being Index;

PMID=PubMed Identification Number; PRS=Polygenic Risk Scores; Q-LES-Q=Quality of Life Enjoyment and Satisfaction Questionnaire; RCT=randomized controlled trial; ROB=risk of bias; SADS-C= Schedule for Affective Disorders and Schizophrenia-Change version; SAE=Serious Adverse Events; SAS=Simpson Angus Scale; SF-12=12-Item Short Form Health Survey; SF-36=36-Item Short Form Health Survey; SLICE=Streamlined Longitudinal Interview Clinical Evaluation; T=Trial; YMRS = Young Mania Rating Scale

\section{Appendix Table E40. Summary risk of bias assessments: ziprasidone for acute mania by year then first author}

\begin{tabular}{|l|l|l|l|}
\hline \multicolumn{1}{|c|}{ Drug } & \multicolumn{1}{|c|}{$\begin{array}{c}\text { Study } \\
\text { Funding Source } \\
\text { PMID }\end{array}$} & $\begin{array}{c}\text { Overall Risk of } \\
\text { Bias Assessment }\end{array}$ & \\
\hline Ziprasidone & $\begin{array}{l}\text { Keck, 200343 } \\
\text { Industry } \\
12668364\end{array}$ & Moderate & All core sources of bias appear to have been addressed, however, almost 50\% dropout. \\
\cline { 2 - 4 } & $\begin{array}{l}\text { Potkin, 2005 } \\
\text { Industry } \\
16012271\end{array}$ & Low & No sources of bias identified. Well disclosed and reported study. \\
& & \\
\hline
\end{tabular}

Abbreviations: ITT=Intention to Treat; PMID=PubMed Identification Number; LOCF=last observation carried forward 


\section{Ziprasidone Forest Plots}

Outcomes in studies assessed as having a high risk of bias, or low to moderate risk of bias but at least 40 percent attrition, are presented in grey tones. Both fixed-effect models and random-effects models are presented. We calculated fixed-effect models to provide a charitable estimate of the average effect among completed trials. However, we base our main conclusions on the random-effects models.

Appendix Figure E74. Ziprasidone vs. placebo - response

Odds Ratio of Response (> 50\% Reduction in YMRS) at 3 Weeks

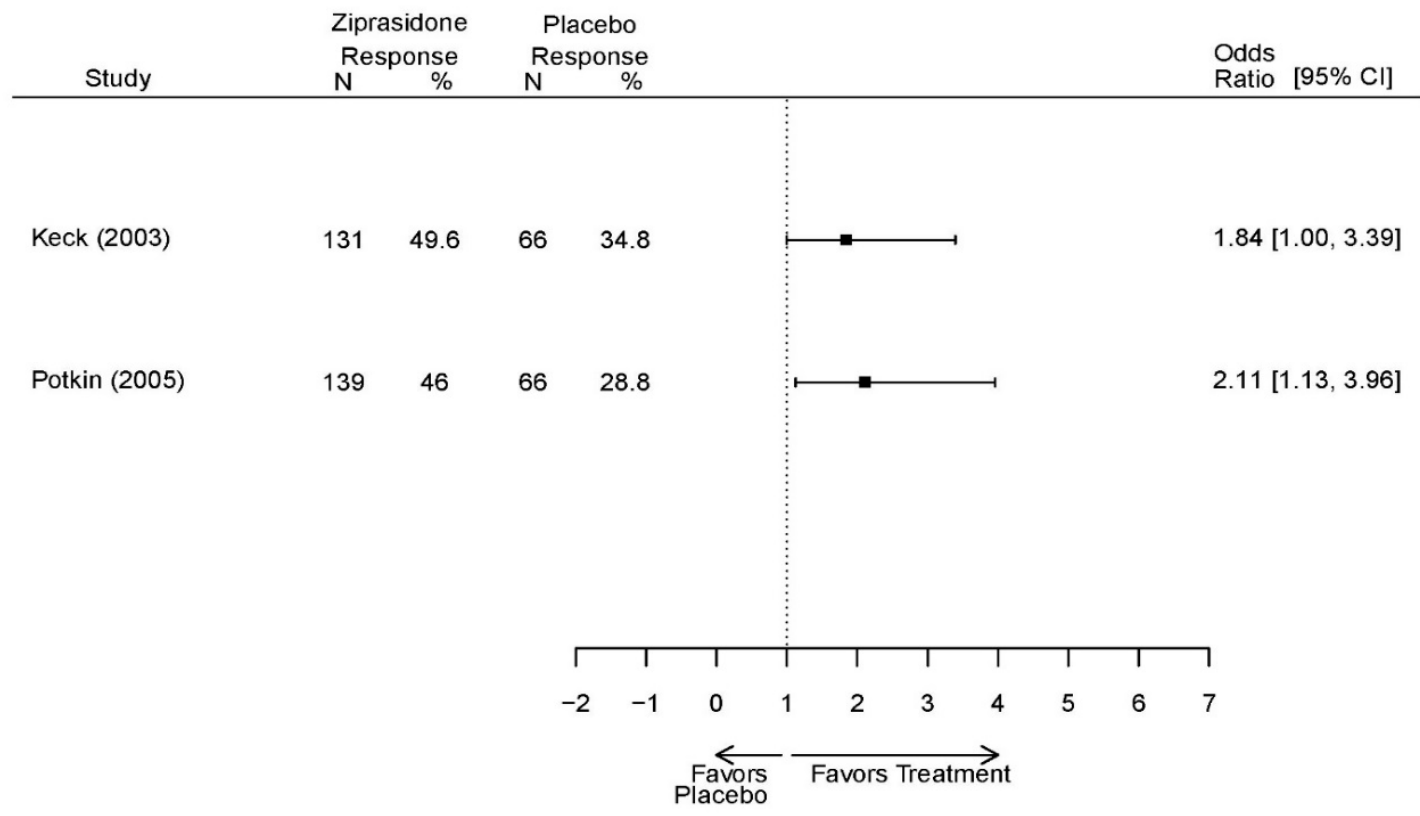


Appendix Figure E75. Ziprasidone vs. placebo - CGI

Difference in Mean Change in CGI-BP-S (Overall)

from Baseline to Last Measurement

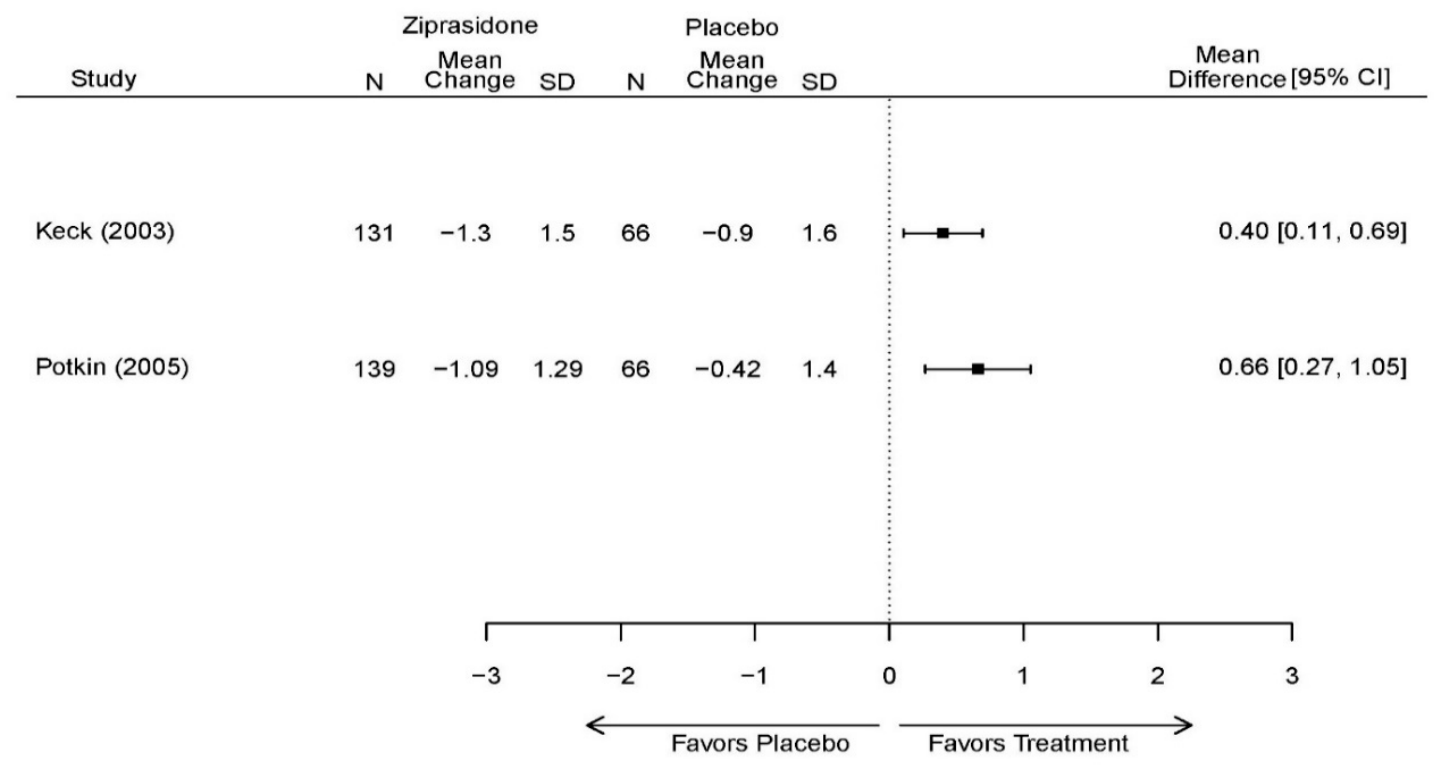

Appendix Figure E76 Ziprasidone vs. placebo - overall withdrawal

Odds Ratio of Overall Withdrawal

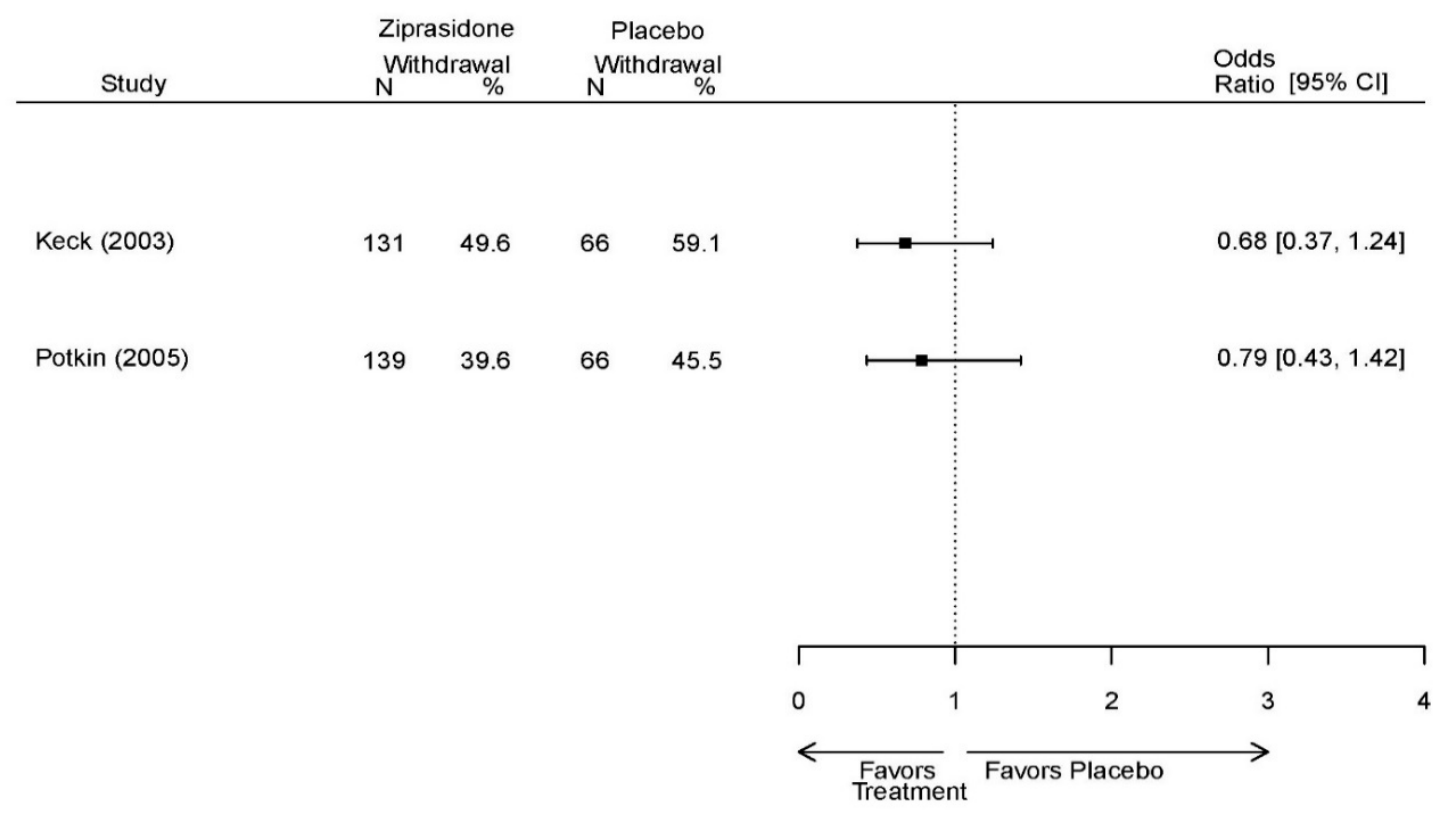

E-105 
Appendix Figure E77. Ziprasidone vs. placebo - withdrawal - lack of efficacy Odds Ratio of Withdrawal due to Lack of Efficacy at 3 Weeks

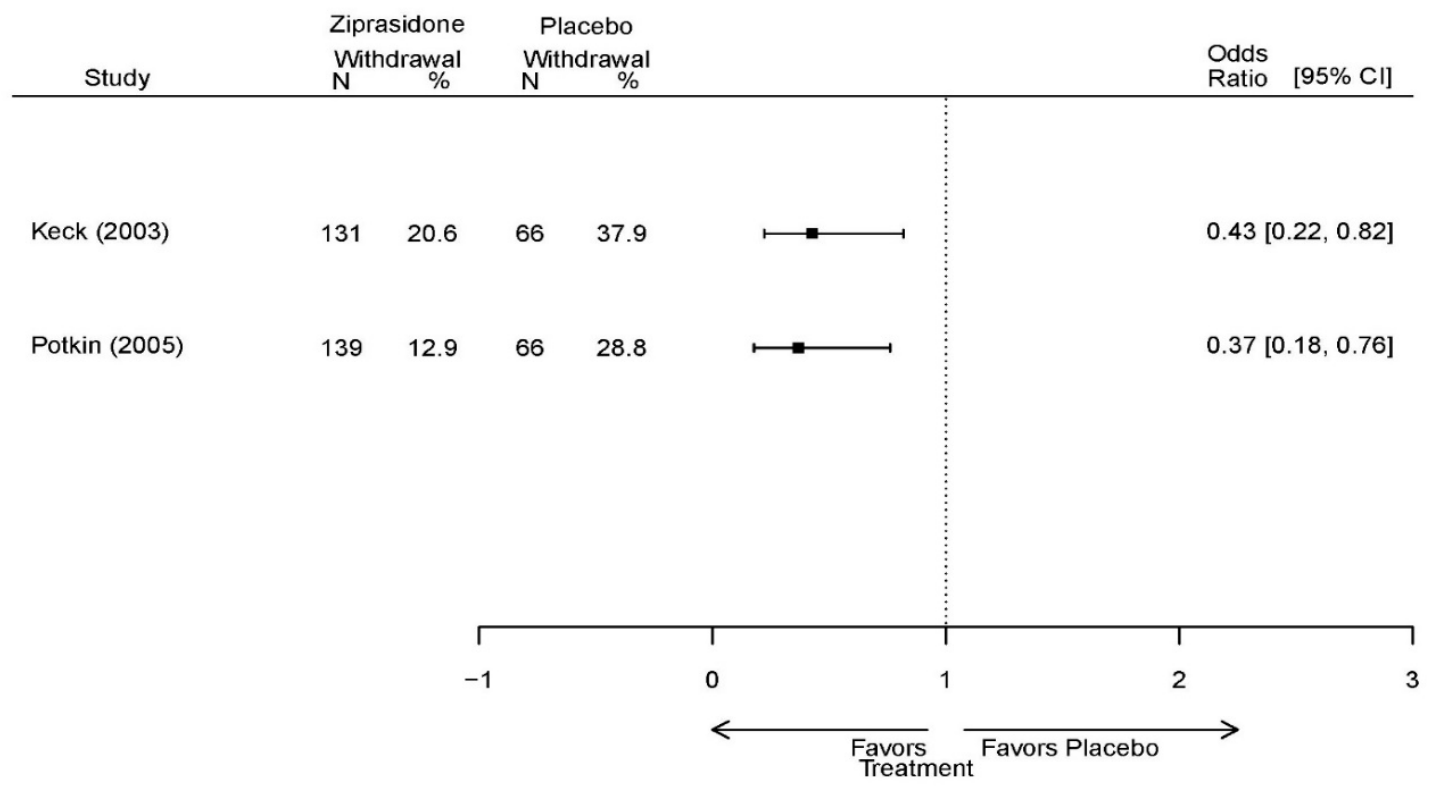

Appendix Figure E78. Ziprasidone vs. placebo - withdrawal - adverse events Odds Ratio of Withdrawal due to Adverse Events at 3 Weeks

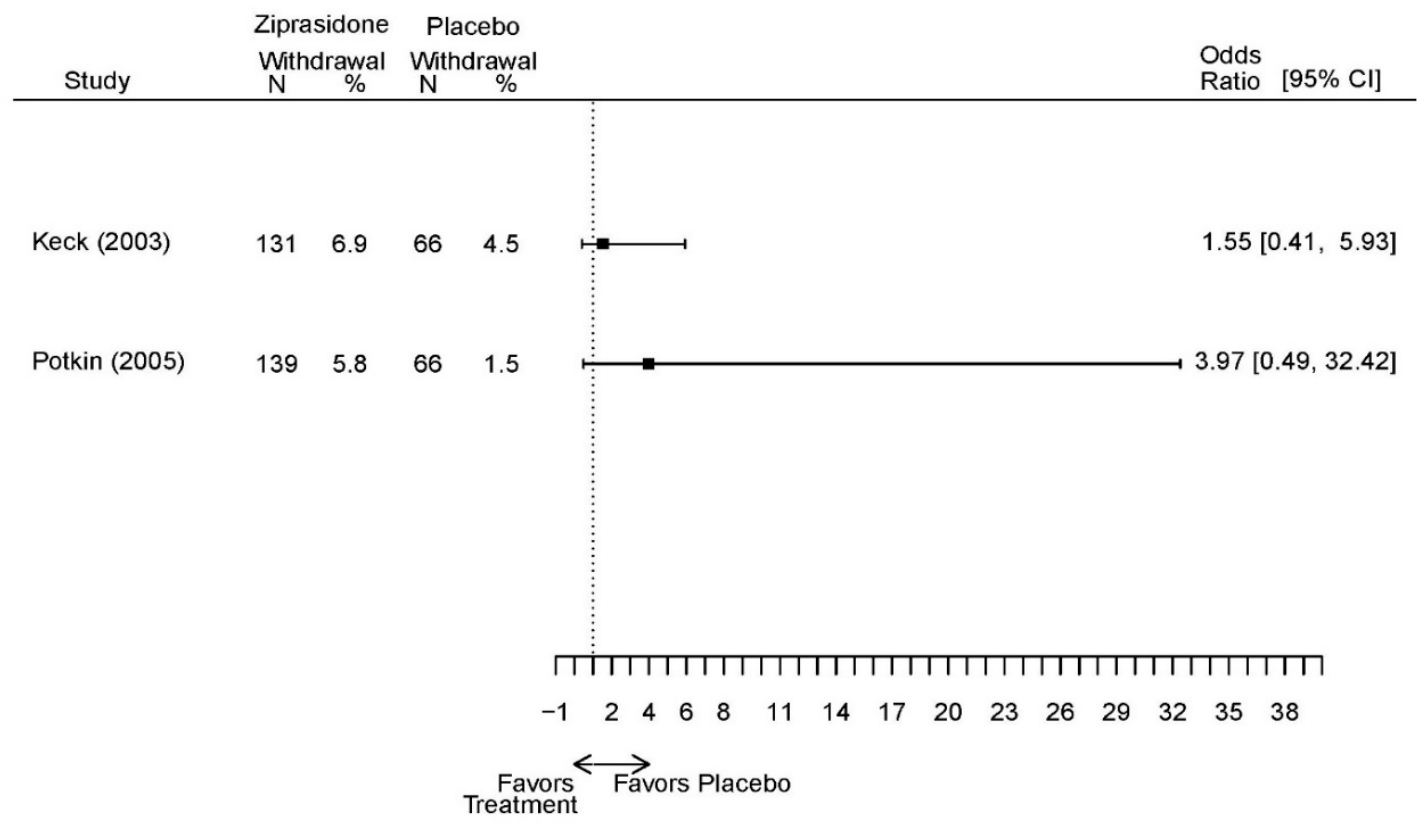




\begin{tabular}{|c|c|c|c|c|c|c|}
\hline Drug & $\begin{array}{l}\text { Study } \\
\text { PMID } \\
\text { RoB }\end{array}$ & Responder/Remitter & Symptom & Function & Other & AE \\
\hline \multirow[t]{2}{*}{ Ziprasidone } & $\begin{array}{l}\text { Keck, } 2003^{43} \\
12668364 \\
\text { Moderate }\end{array}$ & $\begin{array}{l}\text { See forest plot E74 } \\
\text { above for Response }\end{array}$ & $\begin{array}{l}\text { See forest plot } \\
\text { above }\end{array}$ & $\begin{array}{l}\text { See forest plot E75 } \\
\text { above for CGI } \\
\text { GAF } \\
3 \text { weeks } \\
F=10.35, d f=1,156, \\
p<0.005 \\
\text { Favors intervention }\end{array}$ & $\begin{array}{l}\text { See forest plots } 76 \text {, } \\
77 \text {, and } 78 \text { above }\end{array}$ & $\begin{array}{l}\frac{\text { Serious Adverse Events }}{3 \text { weeks }} \\
0 \text { in both arms } \\
\frac{\text { Deaths }}{3 \text { weeks }} \\
0 \text { in both arms } \\
\frac{\text { EPS }}{N R}\end{array}$ \\
\hline & $\begin{array}{l}\text { Potkin, } 2005^{42} \\
16012271 \\
\text { Moderate }\end{array}$ & $\begin{array}{l}\text { See forest plot E74 } \\
\text { above for Response }\end{array}$ & $\begin{array}{l}\text { See forest plot } \\
\text { above }\end{array}$ & $\begin{array}{l}\text { See forest plot E75 } \\
\text { above for CGI } \\
\text { GAF } \\
3 \text { weeks } \\
\text { Favors intervention } \\
p \leq .001 \\
\text { No statistical test } \\
\text { reported }\end{array}$ & $\begin{array}{l}\text { See plots } 76,77, \text { and } \\
78 \text { above }\end{array}$ & $\begin{array}{l}\text { Serious Adverse Events } \\
3 \text { weeks } \\
1 \text { Ziprasidone } \\
\frac{\text { Deaths }}{3 \text { weeks }} \\
1 \text { Ziprasidone - Suicide } \\
\frac{\text { EPS }}{3 \text { weeks }} \\
1.5 \% \text { placebo } \\
10.8 \% \text { Ziprasidone }\end{array}$ \\
\hline
\end{tabular}

Abbreviations: AE=Adverse Events; ANCOVA=Analysis of Covariance; CGI=Clinical Global Impressions Scale; CI=Confidence Interval; EPS=extrapyramidal symptoms; ESRS=Extrapyrimidal Symptom Rating Scale; GAS=Global Assessment Scale; MADRS=Montgomery-Asberg Depression Rating Scale; NR=Not reported; NS=not significant; OR=Odds Ratio; PMID=PubMed Identification Number; ROB=Risk of Bias; SAE=Serious Adverse Events; YMRS = Young Mania Rating Scale 
Appendix Table E42. Strength of evidence assessment: ziprasidone versus placebo for acute mania

\begin{tabular}{|c|c|c|c|c|c|c|c|c|}
\hline Comparison & Outcome & $\begin{array}{l}\text { \# Studies/ } \\
\text { Design } \\
\text { (n analyzed) }\end{array}$ & $\begin{array}{l}\text { Finding or } \\
\text { Summary } \\
\text { Statistic }\end{array}$ & $\begin{array}{c}\text { Study } \\
\text { Limitations }\end{array}$ & Consistency & Directness & Precision & $\begin{array}{c}\text { Overall } \\
\text { Gradel } \\
\text { Conclusion }\end{array}$ \\
\hline $\begin{array}{l}\text { Ziprasidone } \\
\text { vs. placebo }\end{array}$ & $\begin{array}{l}\text { Response } 3 \text { wks } \\
\text { YMRS } 3 \text { wks } \\
\text { CGI } 3 \text { wks }\end{array}$ & $\begin{array}{l}2 \text { RCTs } \\
(n=402)\end{array}$ & $\begin{array}{l}\text { Favors } \\
\text { Ziprasidone }\end{array}$ & Moderate & Consistent & Direct & Imprecise & Low \\
\hline
\end{tabular}

Abbreviations: CGI-BP=Clinical Global Impressions Scale for Bipolar Disorder; NS=not significant; RCT=randomized controlled trial; YMRS = Young Mania Rating Scale Notes:

1. Publication bias for antipsychotics, antidepressants, and behavioral interventions for depressive disorders is suspected.

2. Data were generally imprecise due to missing data from high attrition rates, which was commonly dealt with by Last Observation Carried Forward (LOCF). LOCF requires an assumption that the health status of patients who dropped out of the trial would not have changed had future observations been recorded, a strong assumption in the context of bipolar disorder research.

\section{Section 9. Aripiprazole Plus Mood Stabilizer}

Appendix Table E43. Characteristics of eligible studies: aripiprazole plus mood stabilizer drug treatments for acute mania

\begin{tabular}{|c|c|c|c|c|c|c|}
\hline $\begin{array}{c}\text { Study, Year } \\
\text { Design } \\
\text { Location } \\
\text { Funder } \\
\text { Risk of Bias } \\
\text { PMID }\end{array}$ & $\begin{array}{c}\text { \# Randomized } \\
\text { Age (mean) } \\
\text { Sex (\% Female) } \\
\text { Race (\% White) } \\
\text { Diagnosis } \\
\text { (\% BP I, II, NOS) } \\
\text { Setting }\end{array}$ & $\begin{array}{c}\text { Inclusions } \\
\text { Key Exclusions }\end{array}$ & $\begin{array}{l}\text { Intervention } \\
\text { Dosage }\end{array}$ & $\begin{array}{c}\text { Comparison } \\
\text { Dosage }\end{array}$ & $\begin{array}{c}\text { Follow-up } \\
\text { Duration }\end{array}$ & $\begin{array}{c}\text { Outcomes } \\
\text { Reported } \\
\begin{array}{c}\text { Withdrawal (\%) at } \\
\text { endpoint }\end{array}\end{array}$ \\
\hline $\begin{array}{l}\text { NCT00665366 } \\
2013 \\
\text { Unpublished RCT } \\
\text { Multiste } \\
3 \text { Continents } \\
\text { Industry } \\
\text { RoB NA }\end{array}$ & $\begin{array}{l}\mathrm{N}=370 \\
\text { Mean Age } 45 \\
\text { Female } 54 \% \\
\text { White } 95 \% \\
\text { BP I } 100 \% \\
\text { Setting NR }\end{array}$ & $\begin{array}{l}\text { Mania, manic or mixed } \\
\text { episode, with or without } \\
\text { psychotic features } \\
\text { Schizoaffective } \\
\text { Substance Abuse } \\
\text { Other Mental Health } \\
\text { Neurological disorders } \\
\text { Pregnant/Nursing } \\
\text { Labs/Other Conditions }\end{array}$ & $\begin{array}{l}\text { Aripiprazole } \\
5-15 \text { mg/day } \\
\text { Adjunctive to } \\
\text { valproate or lithium }\end{array}$ & $\begin{array}{l}\text { Placebo } \\
\text { Adjunctive to } \\
\text { valproate or } \\
\text { lithium }\end{array}$ & 12 weeks & $\begin{array}{l}\text { Response }(\geq 50 \% \\
\text { YMRS decrease) } \\
\text { Remission (YMRS } \leq \\
12) \\
\text { YMRS } \\
\text { CGI-BP } \\
\text { FAST } \\
\text { LIFE-RIFT } \\
\text { PGI-I } \\
\text { Weight change } \\
\text { Withdrawal } 32 \%\end{array}$ \\
\hline
\end{tabular}




\begin{tabular}{|c|c|c|c|c|c|c|}
\hline $\begin{array}{l}\text { Study, Year } \\
\text { Design } \\
\text { Location } \\
\text { Funder } \\
\text { Risk of Bias } \\
\text { PMID }\end{array}$ & $\begin{array}{c}\text { \# Randomized } \\
\text { Age (mean) } \\
\text { Sex (\% Female) } \\
\text { Race (\% White) } \\
\text { Diagnosis } \\
\text { (\% BP I, II, NOS) } \\
\text { Setting }\end{array}$ & $\begin{array}{l}\text { Inclusions } \\
\text { Key Exclusions }\end{array}$ & $\begin{array}{l}\text { Intervention } \\
\text { Dosage }\end{array}$ & $\begin{array}{c}\text { Comparison } \\
\text { Dosage }\end{array}$ & $\begin{array}{l}\text { Follow-up } \\
\text { Duration }\end{array}$ & $\begin{array}{c}\text { Outcomes } \\
\text { Reported } \\
\begin{array}{c}\text { Withdrawal (\%) at } \\
\text { endpoint }\end{array}\end{array}$ \\
\hline $\begin{array}{l}\text { Jeong, } 2012^{44} \\
\text { RCT } \\
\text { Multisite } \\
\text { South Korea } \\
\text { Industry } \\
22592508 \\
\text { RoB Low }\end{array}$ & $\begin{array}{l}\mathrm{N}=42 \\
\text { Mean Age } 37 \\
\text { Female 64\% } \\
\text { Race NR } \\
\text { BP I 100\% } \\
\text { Inpatient }\end{array}$ & $\begin{array}{l}\text { Manic; } \\
\text { DSM-IV-TR criteria } \\
\text { YMRS } \geq 20 \\
\text { Schizoaffective } \\
\text { Substance Abuse } \\
\text { Other Mental Health } \\
\text { Taking Other Meds } \\
\text { Labs/Other Conditions }\end{array}$ & $\begin{array}{l}\text { Aripiprazole } \\
\text { Flexible dosing } \\
\text { Mean } 20 \mathrm{mg} / \mathrm{day} \\
\\
\text { Adjunctive to } \\
\text { valproic acid (serum } \\
\text { level } 50-125 \mathrm{mg} / \mathrm{mL} \text { ) }\end{array}$ & $\begin{array}{l}\text { Haloperidol } \\
\text { Flexible dosing } \\
\text { Mean } 5 \mathrm{mg} / \text { day } \\
\\
\text { Adjunctive to } \\
\text { valproic acid } \\
\text { (serum level } 50- \\
125 \mathrm{mg} / \mathrm{mL})\end{array}$ & 8 weeks & \begin{tabular}{|l} 
Response $(\geq 50 \%$ \\
YMRS decrease) \\
Remission (YMRS $\leq$ \\
$12)$ \\
YMRS \\
CGI-S \\
Drug-induced \\
Extrapyramidal \\
Symptoms Scale \\
Weight gain \\
Withdrawal $7 \%$ \\
\end{tabular} \\
\hline $\begin{array}{l}\text { Vieta, } 2008^{45} \\
\text { RCT } \\
\text { Multisite/Not Disclosed } \\
\text { Industry } \\
\text { RoB Moderate } \\
18381903\end{array}$ & $\begin{array}{l}\mathrm{N}=384 \\
\text { Mean Age } 42 \\
\text { Female } 54 \% \\
\text { White } 91 \% \\
\text { BP I } 100 \% \\
\text { Outpatient }\end{array}$ & $\begin{array}{l}\text { Manic/Mixed episode; } \\
\text { Partial responders to } \\
\text { Lithium or Valproate; } \\
\text { YMRS } \geq 16 \text { with decrease } \\
\text { of } \leq 25 \% \text { after } 6 \text { weeks of } \\
\text { stabilization treatment } \\
\text { Substance Abuse } \\
\text { Other Mental Health }\end{array}$ & \begin{tabular}{|l|} 
Aripiprazole \\
15-30 mg/day \\
(19.0 mg/day ) \\
Adjunctive to lithium/ \\
divalproex/valproate
\end{tabular} & $\begin{array}{l}\text { Placebo } \\
\text { Adjunctive to } \\
\text { lithium/divalproex/ } \\
\text { valproate }\end{array}$ & 6 weeks & $\begin{array}{l}\text { YMRS } \\
\text { Response } \\
\text { Adverse Events } \\
\text { EPS } \\
\text { CGI-BP } \\
\text { Withdrawal 19\% }\end{array}$ \\
\hline
\end{tabular}

Abbreviations: AIMS=Abnormal Involuntary Movement Scale; ASI=Addiction Severity Index; BAS=Behavioral Approach System; BID=Twice a day; BP=bipolar disorder; BPRS=Brief Psychiatric Rating Scale; C=Comparison; CGI-BP=Clinical Global Impressions Scale, Bipolar; CGI-BP-S=Clinical Global Impressions, Bipolar, Severity Scale; CGI-I= Clinical Global Impressions-Improvement; CGI-S== Clinical Global Impressions-Severity Scale; DSM-IV-TR= Diagnostic and statistical manual, $4^{\text {th }}$ edition, Text Revision; EPS=extrapyramidal symptoms; FAST=Functional Assessment Short Test; GAF=General Assessment of Functioning Scale; GAS= Global Assessment Scale; HAMD=Hamilton Scale for Depression; HAMD-21=Hamilton Rating Scale for Depression (21-items); HDRS-21=Hamilton Depression Rating Scale (21=items); ISST=International Suicide Prevention Trial Scale for Suicidal Thinking; LIFE-RIFT=Longitudinal Interval Follow-up Evaluation-Rating Impaired Functioning Tool; MADRS=Montgomery-Asberg Depression Rating Scale; NOS=not otherwise specified; NR=not reported; OR=Odds Ratio; PANSS=Positive and Negative Syndrome Scale; PAS=Premornid Adjustment Scale; PGI-I=Patient Global Impression Improvement; PMID=PubMed Identification Number; QLS=Quality of Life Scale; RCT=randomized controlled trial; RDQ=Readiness to Discharge Questionnaire; ROB=risk of bias; SANS=Scale for the Assessment of Negative Symptoms; SAPS=Scale for the Assessment of Positive Symptoms; SAS=Simpson Angus Scale; SF-36=36-Item Short Form Health Survey; UKU=UKU rating scale; YMRS = Young Mania Rating Scale 
Appendix Table E44. Summary risk of bias assessments: arirprizaole plus mood stabilizers for acute mania

\begin{tabular}{|l|l|l|l|}
\hline \multicolumn{1}{|c|}{ Drug } & $\begin{array}{l}\text { Study } \\
\text { Funding Source } \\
\text { PMID }\end{array}$ & $\begin{array}{c}\text { Overall Risk of Bias } \\
\text { Assessment }\end{array}$ & \\
\hline Aripiprazole & $\begin{array}{l}\text { Jeong, 2012 } \\
\text { Industry } \\
22592508\end{array}$ & Low & Ray be underpowered and raters may not be blinded. $7 \%$ dropout. \\
\cline { 2 - 4 } & $\begin{array}{l}\text { Vieta } 2008^{45} \\
\text { Industry } \\
18381903\end{array}$ & Moderate & Randomization and blinding procedures not disclosed. \\
& & \\
\hline
\end{tabular}

Abbreviations: ITT=intention to treat; PMID=PubMed Identification Number 


\section{Antipsychotics Plus Mood Stabilizer Forest Plots}

Outcomes in studies assessed as having a high risk of bias, or low to moderate risk of bias but at least 40 percent attrition, are presented in grey tones. Both fixed-effect models and random-effects models are presented. We calculated fixed-effect models to provide a charitable estimate of the average effect among completed trials. However, we base our main conclusions on the random-effects models.

Appendix Figure E79. Adjunctive aripiprazole vs. placebo - 3 week response

Odds Ratio of Response ( $>50 \%$ Reduction in YMRS) at 3 Weeks

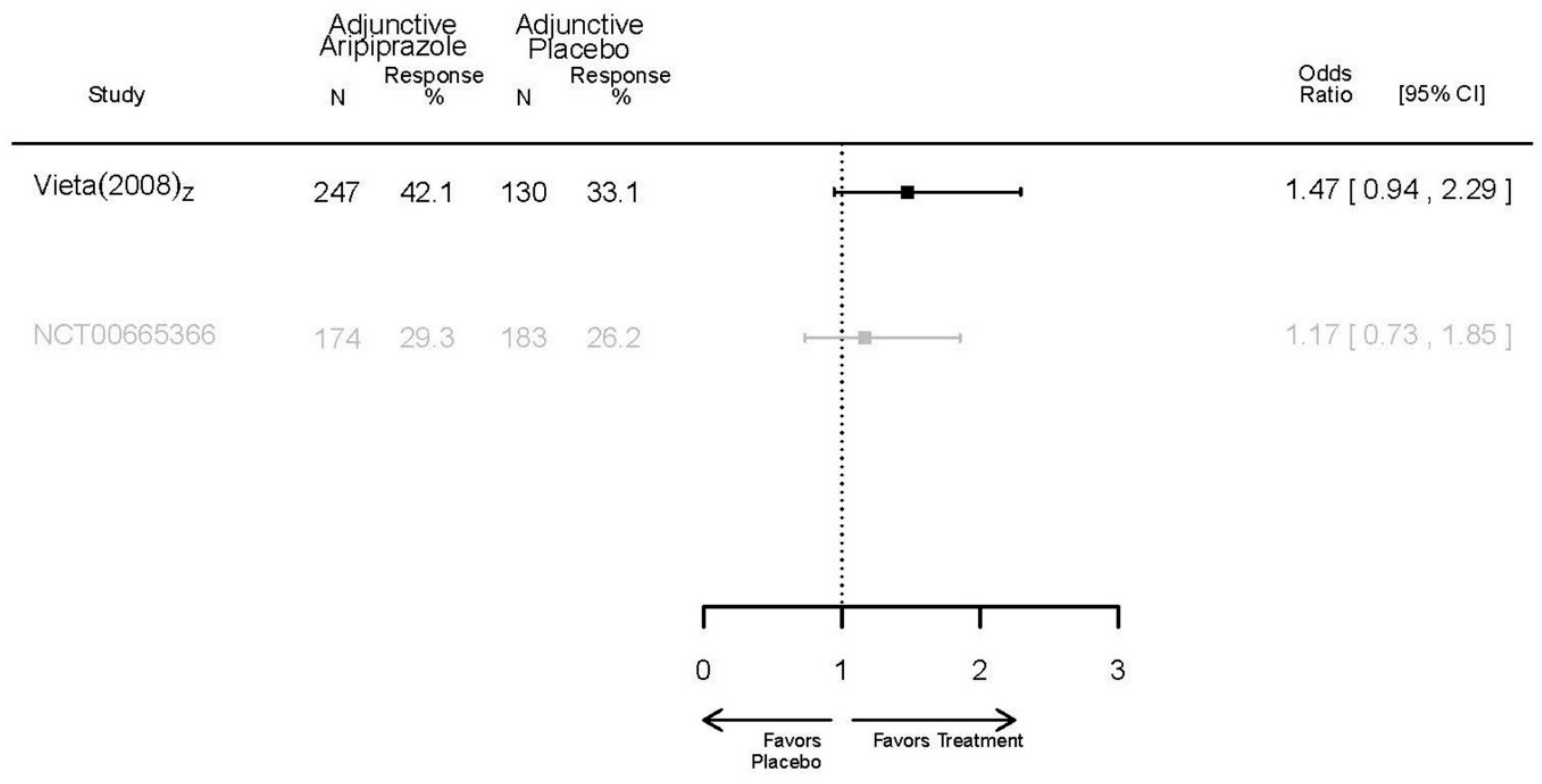


Appendix Figure E80. Adjunctive aripiprazole vs. placebo - last response

Odds Ratio of Response (> 50\% Reduction in YMRS) at Last Measurement

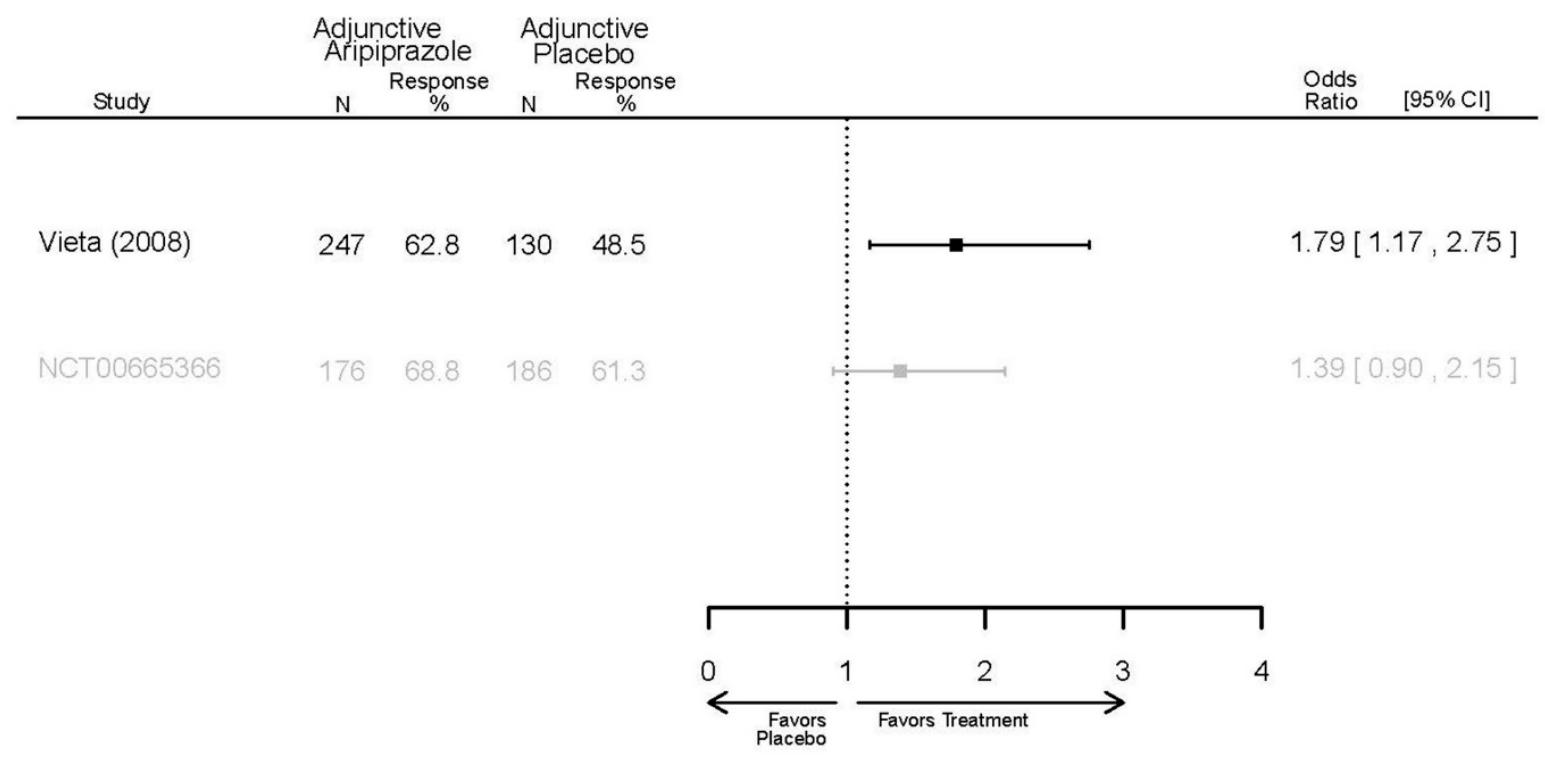

Appendix Figure E81. Adjunctive aripiprazole vs. placebo - 3 week remission Odds Ratio of Remission (YMRS $<=12$ ) at 3 Weeks

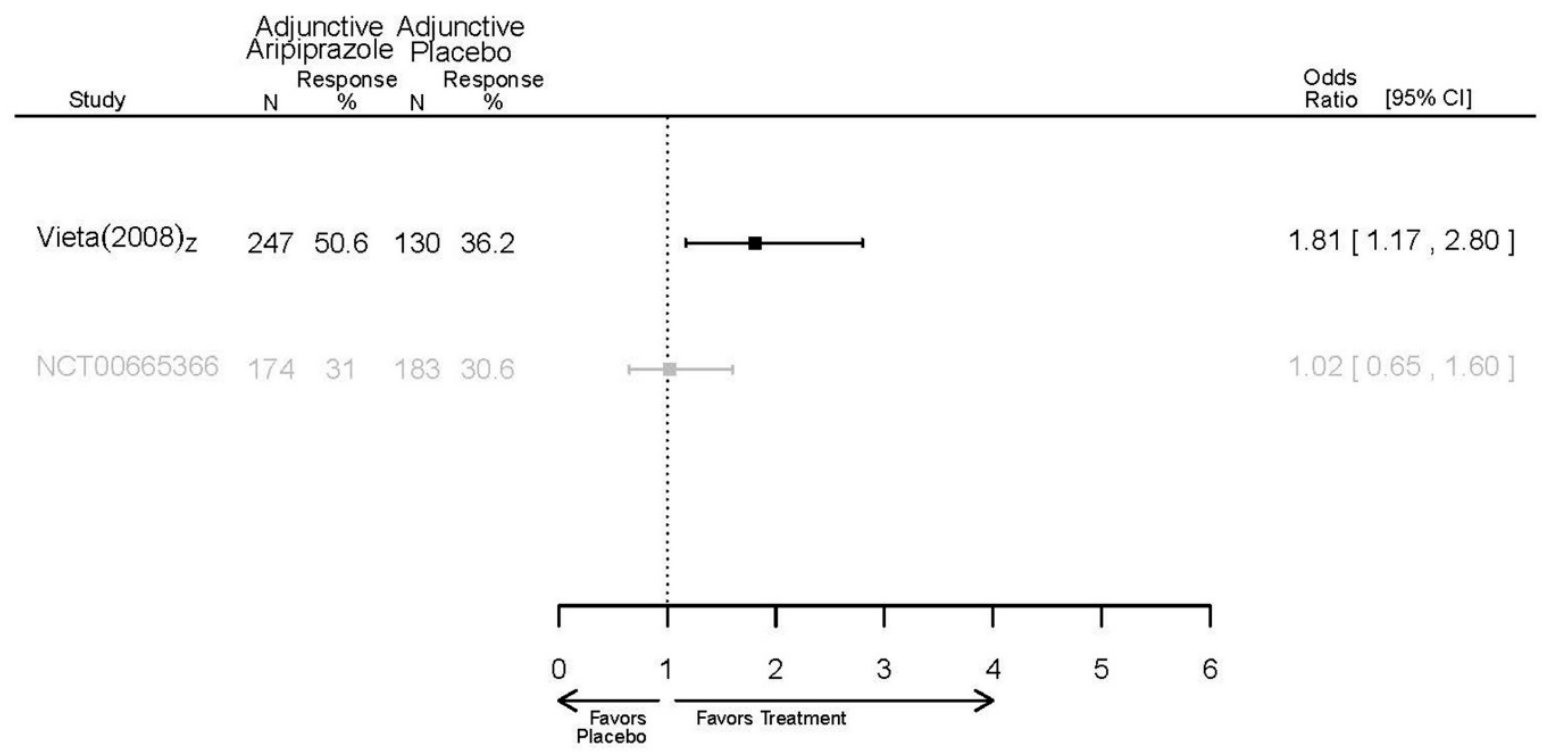


Appendix Figure E82. Adjunctive aripiprazole vs. placebo - last remission

Odds Ratio of Remission (YMRS $>=12$ ) at Last Measurement

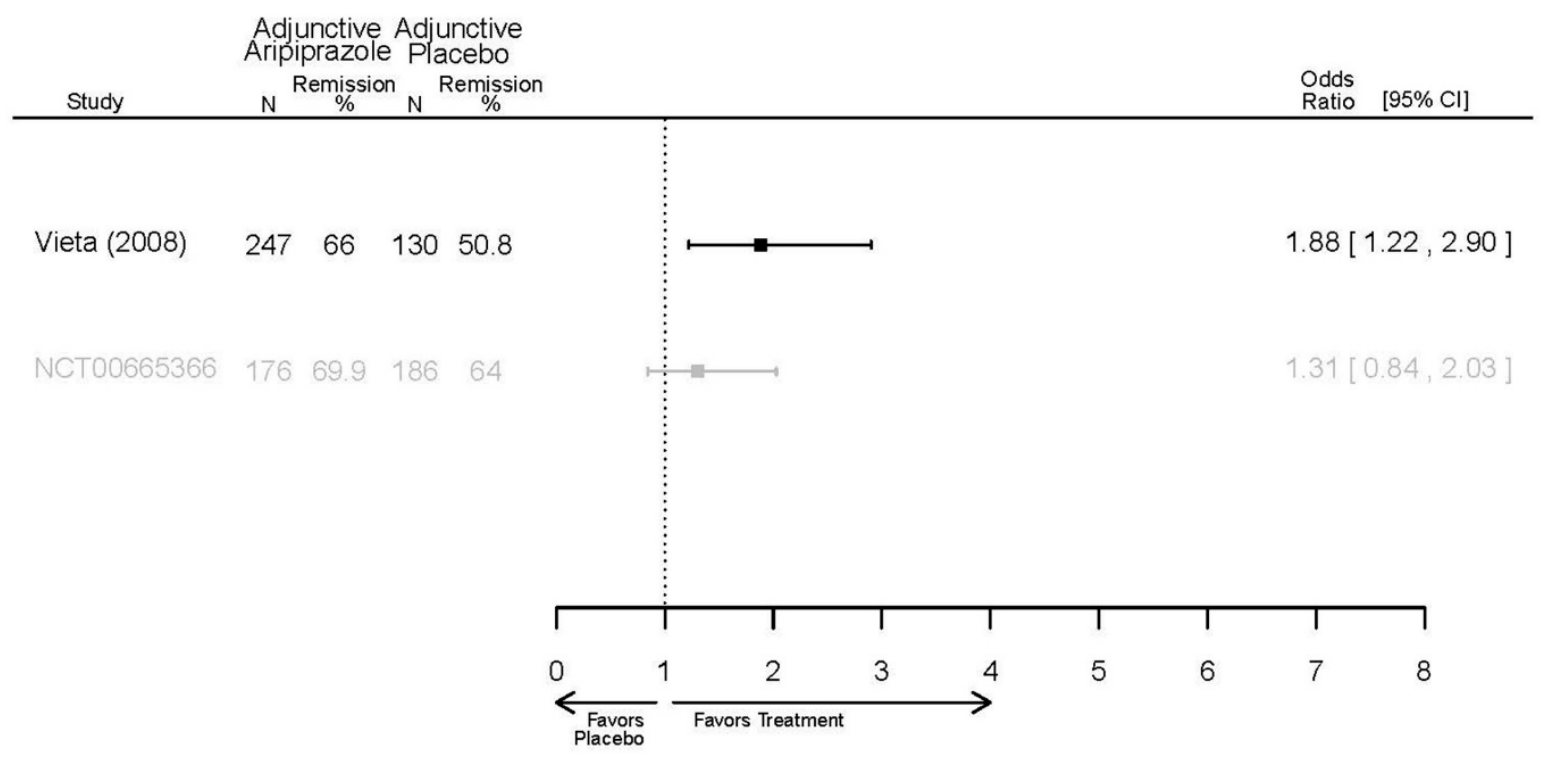

Appendix Figure E83. Adjunctive aripiprazole vs. placebo - YMRS

Difference in Mean Change in YMRS

from Baseline to Last Measurement

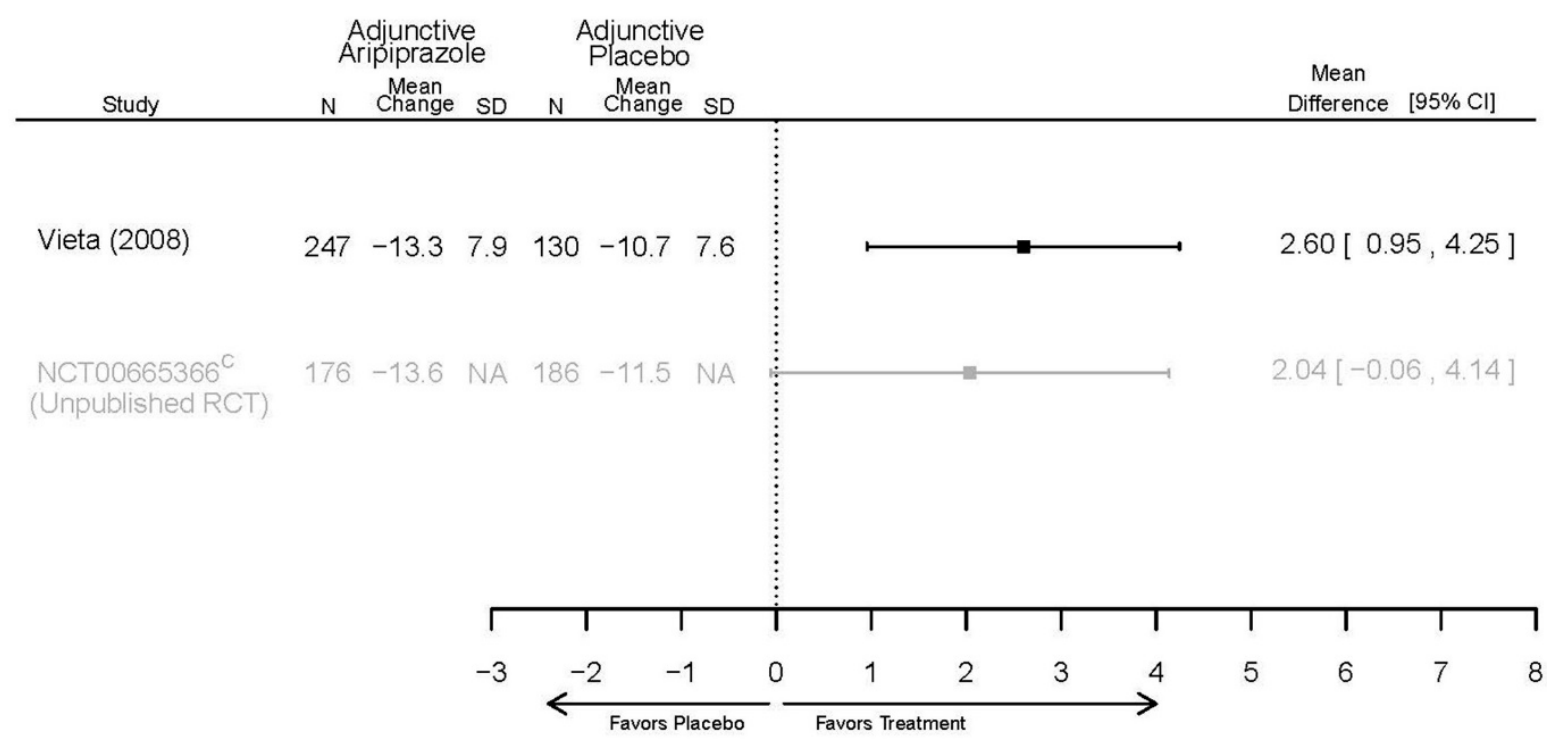


Appendix Figure E84. Adjunctive aripiprazole vs. placebo - CGI

Difference in Mean Change in CGI-BP-S (Overall)

from Baseline to Last Measurement

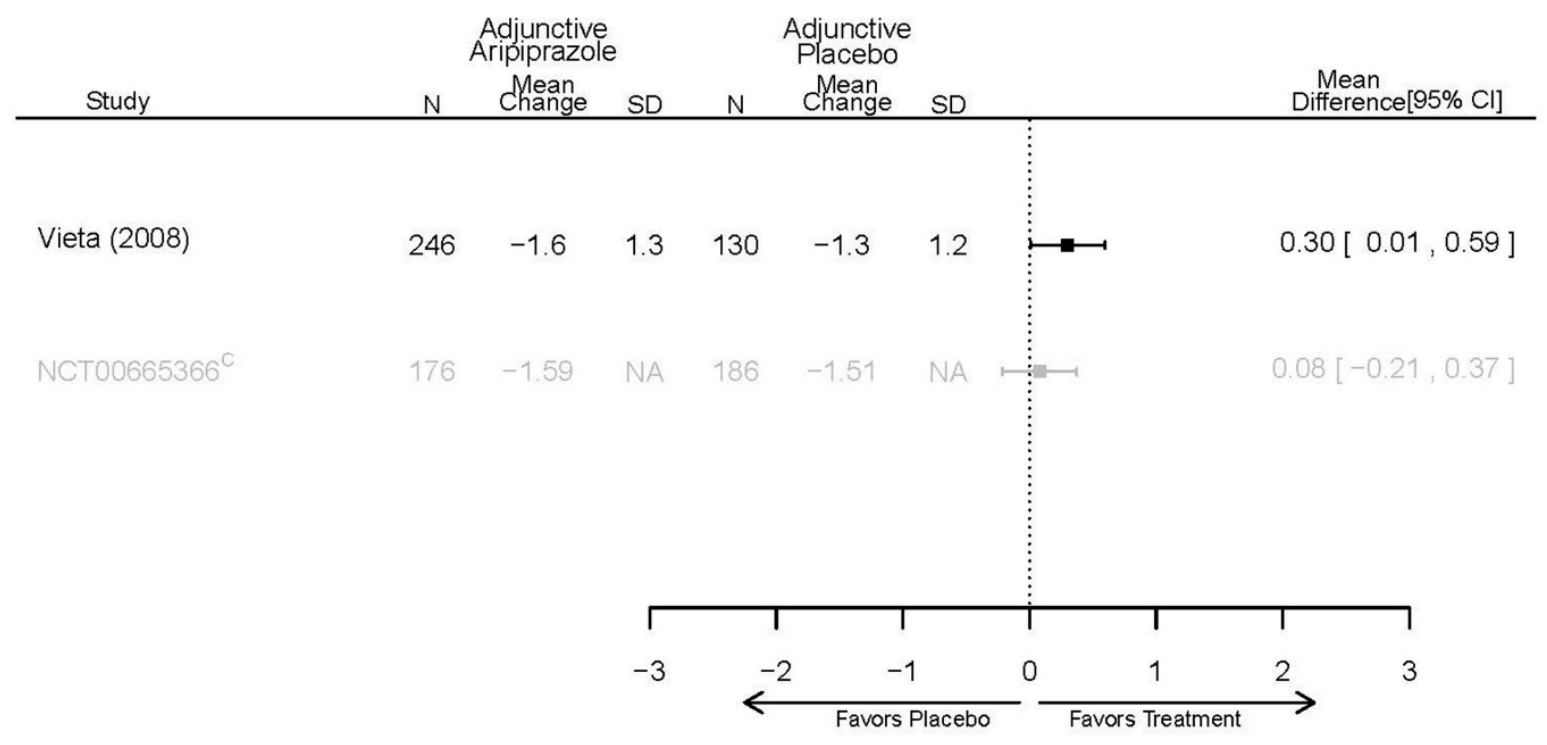

Appendix Figure E85. Adjunctive aripiprazole vs. placebo - overall withdrawal Odds Ratio of Withdrawal due to All Causes at Last Measurement

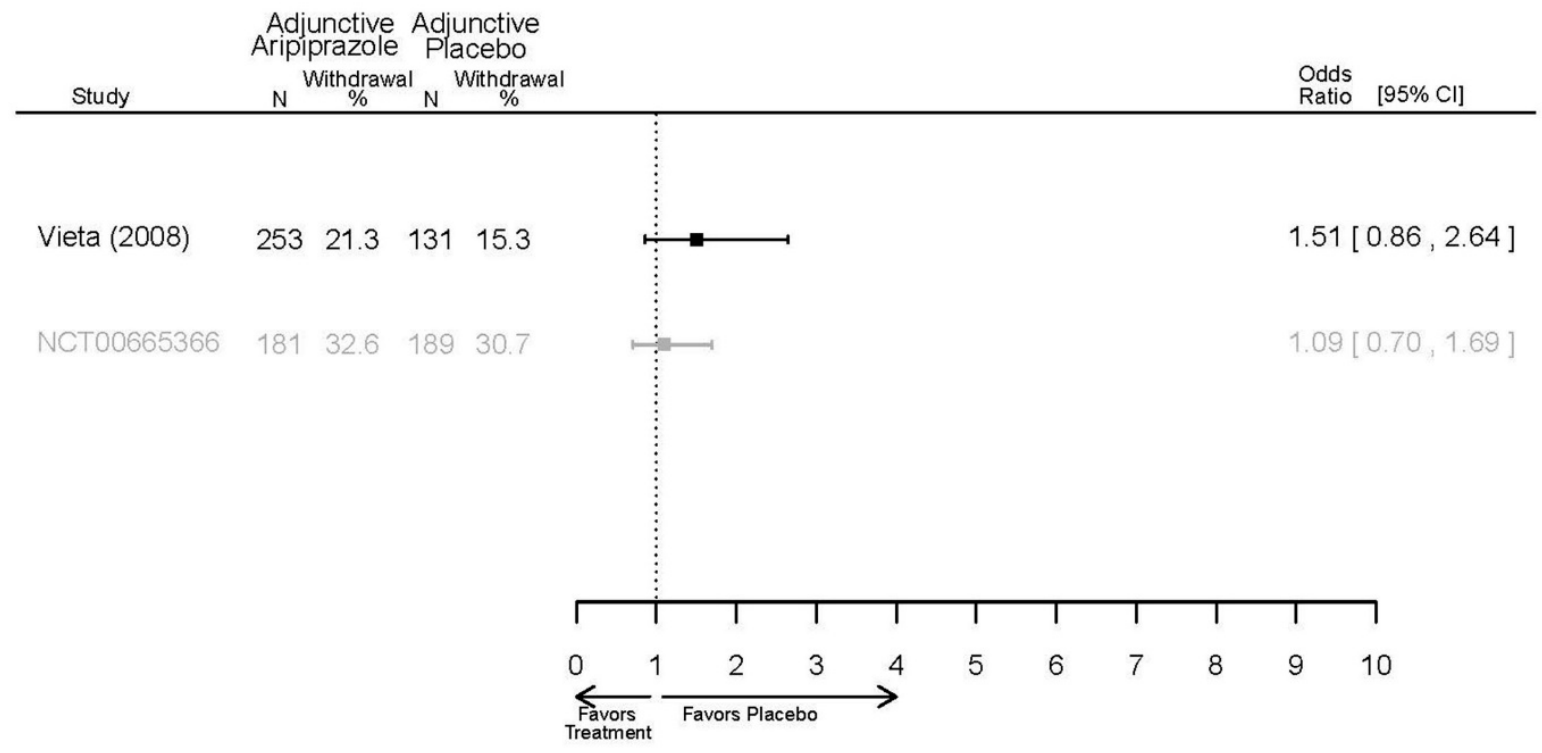


Appendix Figure E86. Adjunctive aripiprazole vs. placebo - withdrawal - lack of efficacy Odds Ratio of Withdrawal due to Lack of Efficacy at Last Measurement

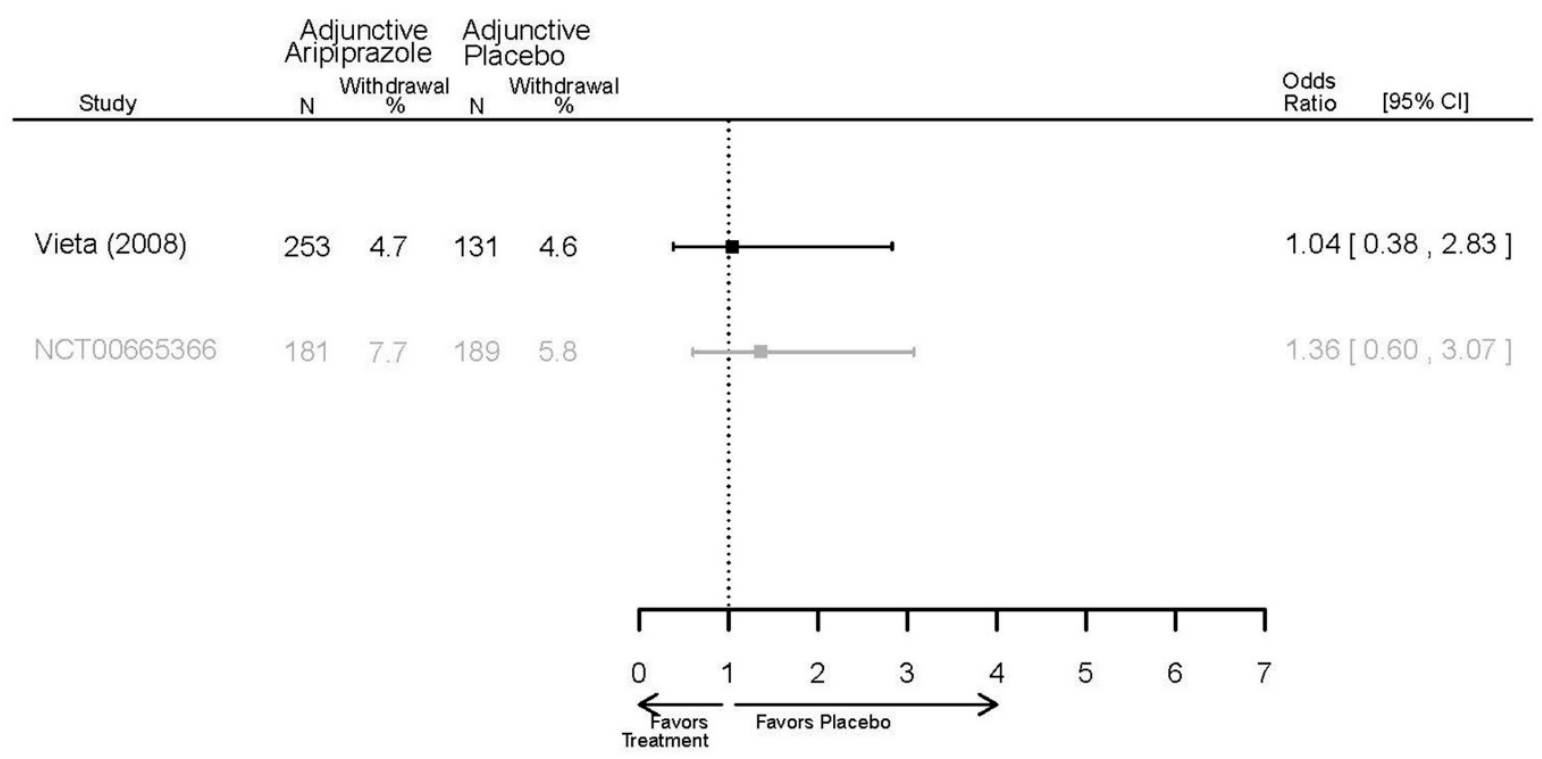

Appendix Figure E87. Adjunctive aripiprazole vs. placebo - withdrawal - adverse events Odds Ratio of Withdrawal due to Adverse Events at Last Measurement

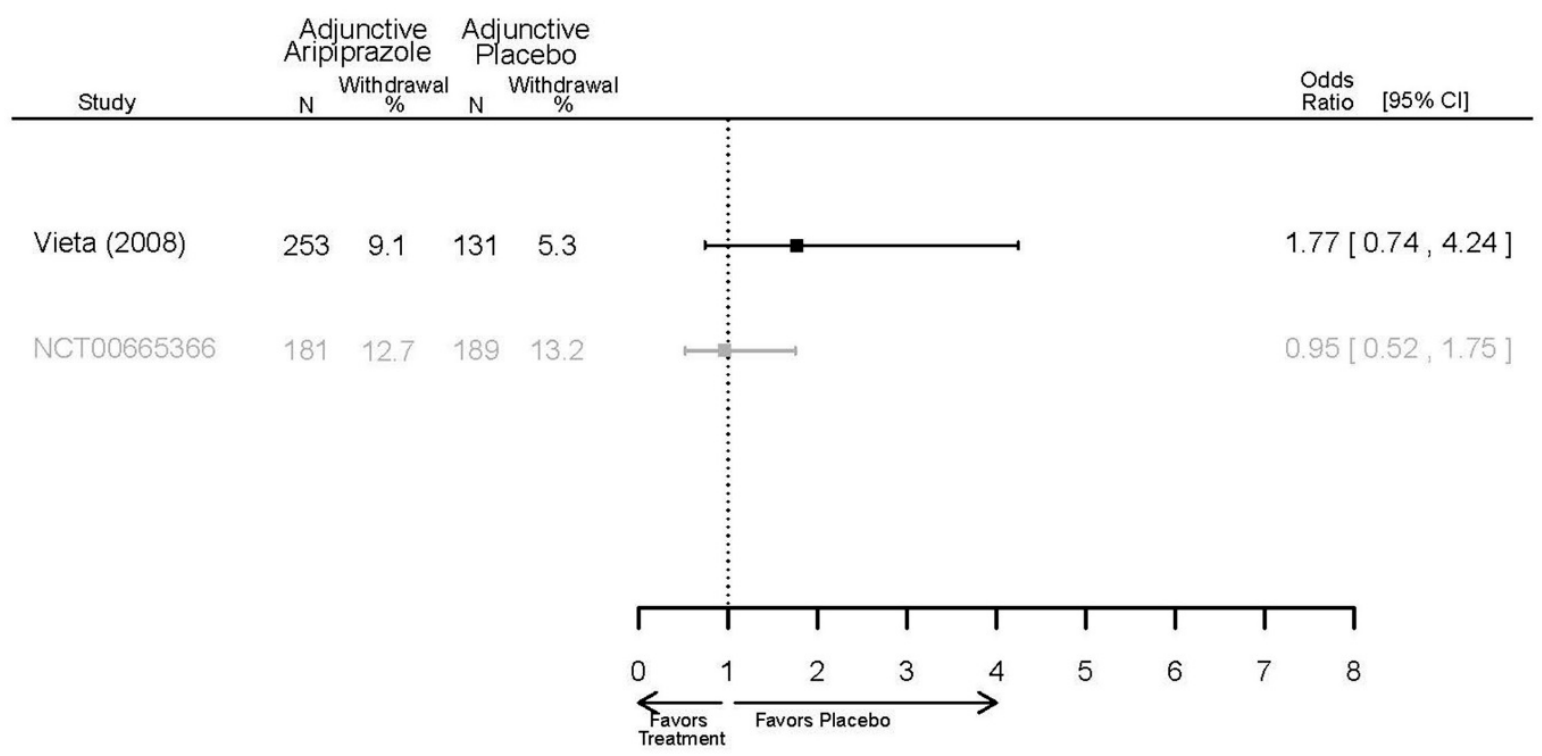


Appendix Figure E88. Adjunctive aripiprazole vs. placebo - serious adverse events Odds Ratio of Serious Adverse Event at Last Measurement

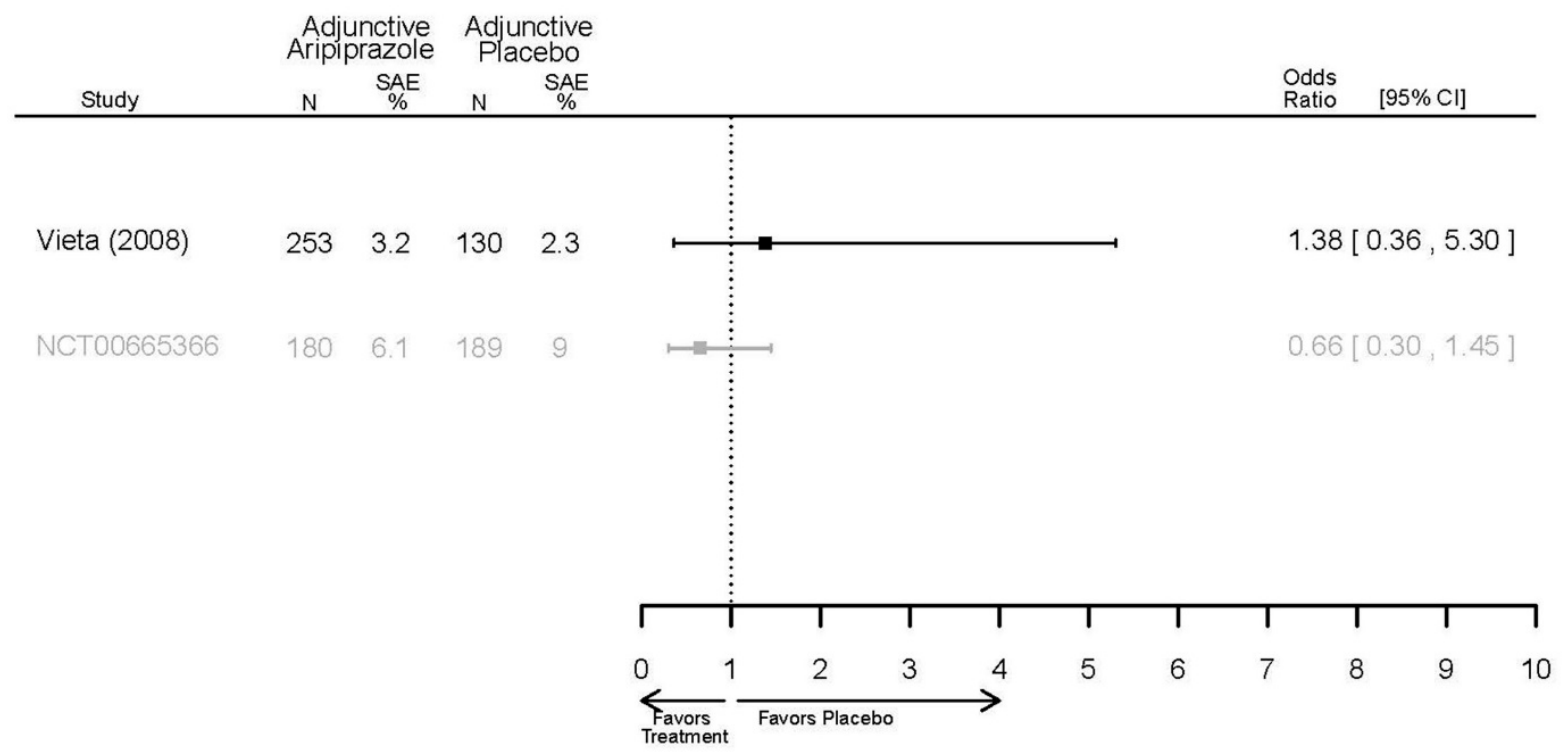


Appendix Figure E89. Adjunctive aripiprazole vs. placebo - akasthisia

\section{Odds Ratio of Akathisia}

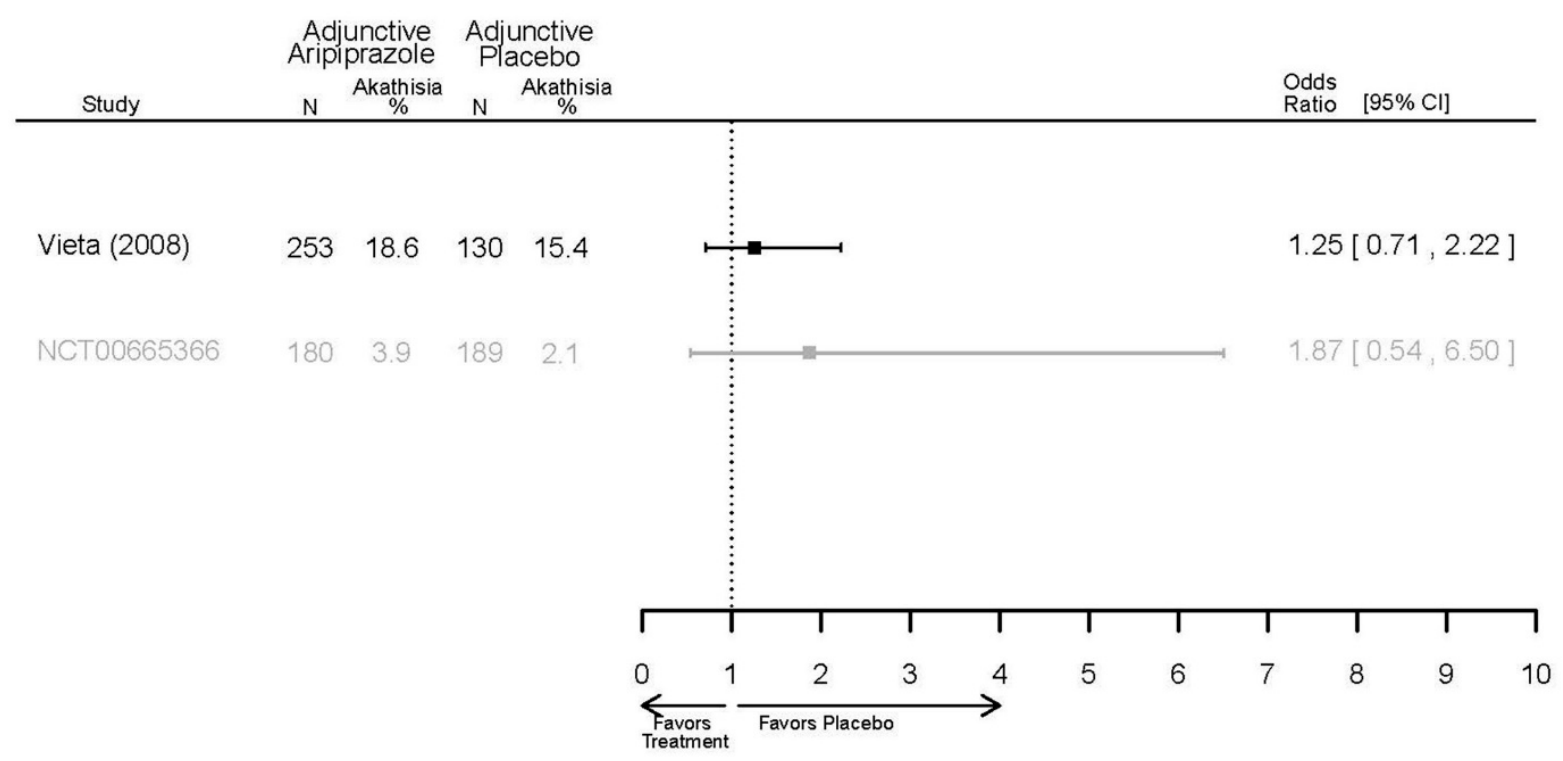

E-117 
Appendix Table E45. Outcomes summary: aripiprazole plus mood stabilizers versus placebo for acute mania

\begin{tabular}{|c|c|c|c|c|c|c|}
\hline Drug & $\begin{array}{l}\text { Study } \\
\text { PMID } \\
\text { RoB }\end{array}$ & $\begin{array}{l}\text { Responderl } \\
\text { Remitter }\end{array}$ & Symptom & Function & Other & $\mathbf{A E}$ \\
\hline $\begin{array}{l}\text { Aripiprazol } \\
\text { e } \\
\text { adjunctive } \\
\text { vs. placebo }\end{array}$ & $\begin{array}{l}\text { NCT00665366 } \\
2013\end{array}$ & $\begin{array}{l}\text { See forest plots } \\
\text { E79, E80, E81, and } \\
\text { E82 above. }\end{array}$ & $\begin{array}{l}\text { See forest plot E83 } \\
\text { above. }\end{array}$ & $\begin{array}{l}\text { See forest plot E84 } \\
\text { above for CGI }\end{array}$ & $\begin{array}{l}\text { See forest plot E85, } \\
\text { E86 and E87 above } \\
\text { for Withdrawals. }\end{array}$ & $\begin{array}{l}\text { See forest plot E88, E89, } \\
\text { and E90 above for adverse } \\
\text { events. } \\
\text { Very Serious AE } \\
1 \text { reported case of suicide } \\
\text { ideation related to } \\
\text { depression in the } \\
\text { aripiprazole arm } \\
1 \text { case of acute respiratory } \\
\text { failure in the Aripiprazole } \\
\text { arm } \\
\text { Cases of Severe } \\
\text { Depression } \\
12 \text { weeks } \\
\text { NS } \\
\text { Aripiprazole }=4 / 180 \\
\text { Placebo }=2 / 189 \\
\text { OR }=2.05(0.37,16.72) ; P \\
=0.38 \\
\text { Cases of Manic Reaction or } \\
\frac{\text { Relapse }}{12 \text { weeks }} \\
\text { NS } \\
\text { Aripiprazole }=2 / 180 \\
\text { Placebo }=7 / 189 \\
\text { OR }=0.31(0.04,1.34) ; P= \\
0.18\end{array}$ \\
\hline
\end{tabular}




\begin{tabular}{|c|c|c|c|c|c|c|}
\hline Drug & $\begin{array}{l}\text { Study } \\
\text { PMID } \\
\text { RoB }\end{array}$ & $\begin{array}{l}\text { Responderl } \\
\text { Remitter }\end{array}$ & Symptom & Function & Other & $\mathrm{AE}$ \\
\hline & $\begin{array}{l}\text { Vieta, } 2008^{45} \\
18381903 \\
\text { Moderate }\end{array}$ & $\begin{array}{l}\text { See forest plots } \\
\text { E79, E80, E81, and } \\
\text { E82 above. }\end{array}$ & $\begin{array}{l}\text { See forest plot E83 } \\
\text { above. }\end{array}$ & NR & $\begin{array}{l}\text { See forest plot E85, } \\
\text { E86 and E87 above } \\
\text { for Withdrawals. }\end{array}$ & $\begin{array}{l}\text { See forest plot above. } \\
\frac{\text { EPS }}{6 \text { weeks }} \\
\text { Favors Placebo } \\
\text { Aripiprazole }=71 / 253 \\
\text { Placebo }=18 / 130 \\
\text { OR }=2.41(1.39,4.37) ; \mathrm{P}= \\
0.002 \\
\\
\text { Normalized Weight Change } \\
\frac{(>7 \% \text { change })}{6 \text { weeks }} \\
\text { NS } \\
\text { Aripiprazole }=7 / 253 \\
\text { Placebo }=5 / 130 \\
\text { OR }=0.71(0.22,2.50) ; P= \\
0.57 \\
\text { Cases of Psychiatric } \\
\text { Disorder } \\
6 \text { weeks } \\
\text { NS } \\
\text { Aripiprazole }=6 / 253 \\
\text { Placebo }=3 / 130 \\
\text { OR }=1.00(0.25,5.11) ; P= \\
0.97\end{array}$ \\
\hline
\end{tabular}

Abbreviations: AE=Adverse Events; ASI=Addiction Severity Index; BPRS=Brief Psychiatric Rating Scale; CGI-Bp=Clinical Global Impresions Scale for Bipolar Disorder; CGIBP-S=Clinical Global Impressions, Bipolar, Severity Scale; CGI-S=Clinical Global Impressions, Severity Scale; CI=Confidence Interval; EPS=extrapyramidal symptoms;

GAF=General Assessment of Functioning Scale; HAM-D=Hamilton Scale for Depression; MADRS=Montgomery-Asberg Syndrome Scale; NR=not reported; NS=not significant; OR=Odds Ratio; PAS=Premorbid Adjustment Scale; PMID=PubMed Identification Number; QLS=Quality of Life Scale; ROB=risk of bias; SAE=Serious Adverse Events; $\mathrm{SE}=$ Standard Error; UKU=UKU rating scale;YMRS = Young Mania Rating Scale 
Appendix Table E46. Strength of evidence assessment: aripiprazole plus mood stabilizers versus placebo for acute mania

\begin{tabular}{|c|c|c|c|c|c|c|c|c|}
\hline Comparison & Outcome & $\begin{array}{l}\text { \# Studies/ } \\
\text { Design } \\
\text { (n analyzed) }\end{array}$ & $\begin{array}{l}\text { Finding or } \\
\text { Summary } \\
\text { Statistic }\end{array}$ & $\begin{array}{c}\text { Study } \\
\text { Limitations }\end{array}$ & Consistency & Directness & Precision & $\begin{array}{c}\text { Overall } \\
\text { Gradel } \\
\text { Conclusion }\end{array}$ \\
\hline $\begin{array}{l}\text { Aripiprazole } \\
\text { adjunctive vs. } \\
\text { placebo }\end{array}$ & $\begin{array}{l}\text { Response } 6 \text { wks } \\
\text { Remission } 6 \text { wks } \\
\text { YMRS } 6 \text { wks } \\
\text { CGI-BP } 6 \text { wks } \\
\text { Withdrawals }\end{array}$ & $\begin{array}{l}2 \text { RCTs } \\
(n=752)\end{array}$ & See forest plots & High & Unknown & Direct & Imprecise & Insufficient \\
\hline
\end{tabular}

Abbreviations: CGI-BP=Clinical Global Impressions Scale for Bipolar Disorder; NS=not significant; RCT=randomized controlled trial; YMRS = Young Mania Rating Scale Notes:

1. Publication bias for antipsychotics, antidepressants, and behavioral interventions for depressive disorders is suspected.

2. Data were generally imprecise due to missing data from high attrition rates, which was commonly dealt with by Last Observation Carried Forward (LOCF). LOCF requires an

assumption that the health status of patients who dropped out of the trial would not have changed had future observations been recorded, a strong assumption in the context of bipolar disorder research. 
Appendix Table E47. Outcomes summary: aripipirazole plus mood stabilizers versus active comparison for acute mania

\begin{tabular}{|c|c|c|c|c|c|c|}
\hline Drug & $\begin{array}{l}\text { Study } \\
\text { PMID }\end{array}$ & Responder/Remitter & Symptom & Function & Other & $\mathrm{AE}$ \\
\hline $\begin{array}{l}\text { Aripiprazole } \\
\text { adjunctive vs. } \\
\text { haloperidol } \\
\text { adjunctive }\end{array}$ & $\begin{array}{l}\text { Jeong, } 2012^{44} \\
22592508 \\
\text { RoB }\end{array}$ & $\begin{array}{l}\frac{\text { Response }}{3 \text { weeks }} \\
\text { NS } \\
\text { Aripiprazole= } 20(71.4 \%) \\
\text { Haloperidol= } 11(78.6 \%) \\
\text { No statistical test reported } \\
8 \text { weeks } \\
\text { NS } \\
\text { Aripiprazole=24 (85.7\%) } \\
\text { Haloperidol= } 13(92.9 \%) \\
\text { No statistical test reported } \\
\\
\text { Remission } \\
3 \text { weeks } \\
\text { NS } \\
\text { Aripiprazole= } 18(64.3 \%) \\
\text { Haloperidol= } 9(64.3 \%) \\
\text { No statistical test reported } \\
8 \text { weeks } \\
\text { NS } \\
\text { Aripiprazole= } 23(82.1 \%) \\
\text { Haloperidol= } 12(85.7 \%) \\
\text { No statistical test reported }\end{array}$ & $\begin{array}{l}\text { Change in YMRS } \\
8 \text { weeks } \\
\text { NS } \\
\text { Change (SE) } \\
\text { Aripiprazole }=-16.3(1.6) \\
\text { Haloperidol }=-17.5(2.3) \\
P=0.66\end{array}$ & $\begin{array}{l}\text { Change in CGI- } \\
\frac{\text { BP }}{8 \text { weeks }} \\
\text { NS } \\
\text { Change (SE) } \\
\text { Aripiprazole }=2.0 \\
(0.2) \\
\text { Haloperidol }=1.7 \\
(0.3) \\
P=0.41\end{array}$ & $\begin{array}{l}\text { Overall } \\
\text { Withdrawal } \\
8 \text { weeks } \\
\text { NS } \\
\text { Aripiprazole = } 2 \\
\text { (7.1\%) } \\
\text { Haloperidol = } 1 \\
\text { (7.1\%) } \\
\text { No statistical } \\
\text { test reported } \\
\text { Withdrawal due } \\
\text { to Lack of } \\
\text { Efficacy } \\
8 \text { weeks } \\
\text { NS } \\
\text { Aripiprazole =1 } \\
\text { (3.6\%) } \\
\text { Haloperidol =0 } \\
\text { (0\%) } \\
\text { No statistical } \\
\text { test reported } \\
\text { Withdrawal due } \\
\text { to AEs } \\
8 \text { weeks } \\
\text { NS } \\
\text { Aripiprazole = } 1 \\
(3.6 \%) \\
\text { Haloperidol = } 1 \\
(7.1 \%) \\
\text { No statistical } \\
\text { test reported }\end{array}$ & $\begin{array}{l}\text { Extrapyramidal } \\
\text { Symptoms } \\
8 \text { weeks } \\
\text { Favors Aripiprazole } \\
\text { Aripiprazole }=32.1 \% \\
\text { Haloperidol }=50 \% \\
\text { No statistical test } \\
\text { reported } \\
\text { Normalized Weight } \\
\text { Change }(>7 \% \text { change) } \\
8 \text { weeks } \\
\text { Favors Comparator } \\
\text { Aripiprazole }=18 / 28 \\
\text { Haloperidol }=4 / 14 \\
P=0.049 \\
\text { Emergent Mood Episode } \\
\frac{\text { (Depression) }}{8 \text { weeks }} \\
\text { Aripiprazole }=2 / 28 \\
\text { Haloperidol }=3 / 14 \\
\text { No statistical test } \\
\text { reported } \\
\text { Akathisia } \\
8 \text { weeks } \\
\text { Aripiprazole }=7 / 28 \\
\text { Haloperidol }=2 / 14 \\
\text { No statistical test } \\
\text { reported }\end{array}$ \\
\hline
\end{tabular}

Abbreviations: AE=Adverse Events; CGI-BP=Clinical Global Impressions Scale for Bipolar Disorder; CGI-BP-S-Clinical Global Impressions, Bipolar, Severity Scale; HAMD= Hamilton Scale for Depression; NS=not significant; PMID=PubMed Identification Number; ROB=risk of bias; SAE; Serious Adverse Events; SE=standard error; YMRS = Young Mania Rating Scale 
Appendix Table E48. Strength of evidence assessment: aripiprazole plus mood stabilizers versus active comparison for acute mania

\begin{tabular}{|c|c|c|c|c|c|c|c|c|}
\hline Comparison & Outcome & $\begin{array}{l}\text { \# Studies/ } \\
\text { Design } \\
\text { (n analyzed) }\end{array}$ & $\begin{array}{l}\text { Finding or } \\
\text { Summary } \\
\text { Statistic }\end{array}$ & $\begin{array}{c}\text { Study } \\
\text { Limitations }\end{array}$ & Consistency & Directness & Precision & $\begin{array}{c}\text { Overall } \\
\text { Gradel } \\
\text { Conclusion }\end{array}$ \\
\hline $\begin{array}{l}\text { Aripiprazole } \\
\text { adjunctive vs. } \\
\text { haloperidol } \\
\text { adjunctive }\end{array}$ & $\begin{array}{l}3 \text { weeks } \\
\text { Response } \\
\text { Remission } \\
\text { YMRS } \\
\text { CGI-BP } \\
\text { Withdrawal - } \\
\text { overall } \\
\text { Withdrawal - lack } \\
\text { of efficacy } \\
\text { Withdrawal - Aes }\end{array}$ & $\begin{array}{l}1 \mathrm{RCT} \\
(\mathrm{n}=42)\end{array}$ & See table above & Low & Unknown & Direct & Imprecise & Insufficient \\
\hline
\end{tabular}

Abbreviations: CGI-BP=Clinical Global Impressions Scale for Bipolar Disorder; NS=not significant; RCT=randomized controlled trial; YMRS = Young Mania Rating Scale Notes:

1. Publication bias for antipsychotics, antidepressants, and behavioral interventions for depressive disorders is suspected.

2. Data were generally imprecise due to missing data from high attrition rates, which was commonly dealt with by Last Observation Carried Forward (LOCF). LOCF requires an assumption that the health status of patients who dropped out of the trial would not have changed had future observations been recorded, a strong assumption in the context of bipolar disorder research. 


\section{Section 10. Asenapine Plus Mood Stabilizer}

Appendix Table E49. Characteristics of eligible studies: asenapine plus mood stabilizer drug treatments for acute mania

\begin{tabular}{|c|c|c|c|c|c|c|}
\hline $\begin{array}{c}\text { Study, Year } \\
\text { Design } \\
\text { Location } \\
\text { Funder } \\
\text { Risk of Bias } \\
\text { PMID }\end{array}$ & $\begin{array}{c}\text { \# Randomized } \\
\text { Age (mean) } \\
\text { Sex (\% Female) } \\
\text { Race (\% White) } \\
\text { Diagnosis } \\
\text { (\% BP I, II, NOS) } \\
\text { Setting }\end{array}$ & $\begin{array}{c}\text { Inclusions } \\
\text { Key Exclusions }\end{array}$ & $\begin{array}{c}\text { Intervention } \\
\text { Dosage }\end{array}$ & $\begin{array}{c}\text { Comparison } \\
\text { Dosage }\end{array}$ & $\begin{array}{c}\text { Follow-up } \\
\text { Duration }\end{array}$ & $\begin{array}{c}\text { Outcomes } \\
\text { Reported } \\
\begin{array}{c}\text { Withdrawal (\%) at } \\
\text { endpoint }\end{array}\end{array}$ \\
\hline $\begin{array}{l}\text { Szegedi, } 2012^{46} \\
\text { RCT } \\
\text { Multisite } \\
4 \text { Continents } \\
\text { Industry } \\
\text { RoB Moderate } \\
22198448\end{array}$ & $\begin{array}{l}\mathrm{N}=324 \\
\text { Mean Age } 39 \\
\text { Female } 43 \% \\
\text { White } 57 \% \\
\text { BP I } 100 \% \\
\text { Outpatient }\end{array}$ & $\begin{array}{l}\text { Mania; } \\
\text { YMRS } \geq 20 \\
\text { Current episode } \leq 3 \\
\text { months } \\
\\
\text { Substance Abuse } \\
\text { Other Mental Health } \\
\text { Neurological Disorders } \\
\text { Taking Other Meds } \\
\text { Pregnant/Nursing } \\
\text { Labs/Other Conditions } \\
\text { First Manic Episode }\end{array}$ & $\begin{array}{l}\text { Asenapine } \\
5-10 \mathrm{mg} \text { BID } \\
\text { (Mean } 11.8 \mathrm{mg} / \text { day) } \\
\text { Adjunctive to } \\
\text { Lithium } \\
0.6-1.2 \mathrm{mmol} / \mathrm{L} \\
\text { (mean } 12.8 \mathrm{mg} / \text { day) } \\
\text { OR } \\
\text { Valproate } \\
50-125 \mathrm{mcg} / \mathrm{mL} \\
\text { (mean } 11.0 \mathrm{mg} / \text { day) }\end{array}$ & $\begin{array}{l}\text { Placebo } \\
\text { Adjunctive to } \\
\text { Lithium } \\
0.6-1.2 \mathrm{mmol} / \mathrm{L} \\
\text { (mean } 12.8 \\
\text { mg/day) } \\
\text { OR } \\
\text { Valproate } \\
50-125 \mathrm{mcg} / \mathrm{mL} \\
\text { (mean } 11.0 \\
\text { mg/day) }\end{array}$ & $\begin{array}{l}3 \text { weeks } \\
(12 \text { week } \\
>50 \% \\
\text { attrition) }\end{array}$ & $\begin{array}{l}\text { YMRS } \\
\text { MADRS } \\
\text { CGI-BP } \\
\text { ISST } \\
\text { SF-36 } \\
\text { RDQ } \\
\text { Adverse Events } \\
\text { Withdrawal 37\% }\end{array}$ \\
\hline
\end{tabular}

Abbreviations: AIMS=Abnormal Involuntary Movement Scale; ASI=Addiction Severity Index; BAS=Behavioral Approach System; BID=Twice a day; BP=bipolar disorder; BPRS=Brief Psychiatric Rating Scale; C=Comparison; CGI-BP=Clinical Global Impressions Scale, Bipolar; CGI-BP-S=Clinical Global Impressions, Bipolar, Severity Scale; CGI-I= Clinical Global Impressions-Improvement; CGI-S== Clinical Global Impressions-Severity Scale; DSM-IV-TR= Diagnostic and statistical manual, $4^{\text {th }}$ edition, Text Revision; EPS=extrapyramidal symptoms; FAST=Functional Assessment Short Test; GAF=General Assessment of Functioning Scale; GAS= Global Assessment Scale; HAM$\mathrm{D}=$ Hamilton Scale for Depression; HAMD-21=Hamilton Rating Scale for Depression (21-items); HDRS-21=Hamilton Depression Rating Scale (21=items); ISST=International Suicide Prevention Trial Scale for Suicidal Thinking; LIFE-RIFT=Longitudinal Interval Follow-up Evaluation-Rating Impaired Functioning Tool; MADRS=Montgomery-Asberg Depression Rating Scale; NOS=not otherwise specified; NR=not reported; OR=Odds Ratio; PANSS=Positive and Negative Syndrome Scale; PAS=Premornid Adjustment Scale; PGI-I=Patient Global Impression Improvement; PMID=PubMed Identification Number; QLS=Quality of Life Scale; RCT=randomized controlled trial; RDQ=Readiness to Discharge Questionnaire; ROB=risk of bias; SANS=Scale for the Assessment of Negative Symptoms; SAPS=Scale for the Assessment of Positive Symptoms; SAS=Simpson Angus Scale; SF-36=36-Item Short Form Health Survey; UKU=UKU rating scale; YMRS = Young Mania Rating Scale 
Appendix Table E50. Summary risk of bias assessments: asenapine plus mood stabilizers for acute mania

\begin{tabular}{|l|l|l|l|}
\hline \multicolumn{1}{|c|}{ Drug } & $\begin{array}{c}\text { Study } \\
\text { Funding Source } \\
\text { PMID }\end{array}$ & $\begin{array}{c}\text { Overall Risk of Bias } \\
\text { Assessment }\end{array}$ & \multicolumn{1}{|c|}{ Rationale } \\
\hline Asenapine & $\begin{array}{l}\text { Szegedi, 2012 } \\
\text { Industry } \\
22198448\end{array}$ & $\begin{array}{l}\text { Moderate (3 week } \\
\text { outcomes) }\end{array}$ & $\begin{array}{l}\text { Statistically significant difference in the number of patients who complete the trial for both arms, } \\
\text { but 'if patients who had recurrence were also counted as completers, then completion rates } \\
\text { were 73.3\% for olanzapine and 67.3\% for lithium' -- which are similar but dropout rates are still } \\
\text { moderately high (30\%). }\end{array}$ \\
\hline
\end{tabular}

Abbreviations: ITT=intention to treat; PMID=PubMed Identification Number

Appendix Table E51. Outcomes summary: asenapine plus mood stabilizers versus placebo for acute mania

\begin{tabular}{|c|c|c|c|c|c|c|}
\hline Drug & $\begin{array}{c}\text { Study } \\
\text { PMID } \\
\text { RoB } \\
\end{array}$ & $\begin{array}{l}\text { Responderl } \\
\text { Remitter }\end{array}$ & Symptom & Function & Other & $\overline{A E}$ \\
\hline $\begin{array}{l}\text { Asenapine } \\
\text { adjunctive } \\
\text { vs. placebo }\end{array}$ & $\begin{array}{l}\text { Szegedi, } \\
2012^{46} \\
22198448 \\
\text { Moderate }\end{array}$ & $\begin{array}{l}\text { Response } \\
\text { No difference } \\
\text { between groups } \\
\text { OR } 1.14 \text { (0.71, } \\
1.84) \\
\frac{\text { Remission }}{\text { No difference }} \\
\text { between groups } \\
\text { OR } 1.4790 .90 \text {, } \\
2.42)\end{array}$ & $\begin{array}{l}\text { YRMS } \\
\text { Favors asenapine } \\
\text { Mean difference } 2.03 \\
(0.26,3.80)\end{array}$ & $\begin{array}{l}\text { CGI-BP-S } \\
\text { Favors asenapine } \\
\text { Mean difference } 0.30 \\
(0.08,0.52)\end{array}$ & NR at 3 weeks & $\begin{array}{l}\text { Serious Adverse Events } \\
52 \text { weeks } \\
1 \text { Asenapine } \\
\text { Deaths } \\
52 \text { weeks } \\
1 \text { Asenapine - suicide } \\
\text { EPS } \\
52 \text { weeks } \\
12 \% \text { placebo } \\
9.5 \% \text { Asenapine }\end{array}$ \\
\hline
\end{tabular}

Abbreviations: AE=Adverse Events; ASI=Addiction Severity Index; BPRS=Brief Psychiatric Rating Scale; CGI-Bp=Clinical Global Impresions Scale for Bipolar Disorder; CGIBP-S=Clinical Global Impressions, Bipolar, Severity Scale; CGI-S=Clinical Global Impressions, Severity Scale; CI=Confidence Interval; EPS=extrapyramidal symptoms; GAF=General Assessment of Functioning Scale; HAM-D=Hamilton Scale for Depression; MADRS=Montgomery-Asberg Syndrome Scale; NR=not reported; NS=not significant; OR=Odds Ratio; PAS=Premorbid Adjustment Scale; PMID=PubMed Identification Number; QLS=Quality of Life Scale; ROB=risk of bias; SAE=Serious Adverse Events;

$\mathrm{SE}=$ Standard Error; UKU=UKU rating scale; YMRS = Young Mania Rating Scale 


\section{Appendix Table E52. Strength of evidence assessment: asenapine plus mood stabilizers versus placebo for acute mania}

\begin{tabular}{|l|l|l|l|l|l|l|l|}
\hline Comparison & \multicolumn{1}{|c|}{ Outcome } & $\begin{array}{c}\text { \# Studies/ } \\
\text { Design } \\
\text { (n analyzed) }\end{array}$ & $\begin{array}{c}\text { Finding or } \\
\text { Summary } \\
\text { Statistic }\end{array}$ & $\begin{array}{c}\text { Study } \\
\text { Limitations }\end{array}$ & Consistency & $\begin{array}{c}\text { Overall } \\
\text { Gradel } \\
\text { Conclusion }\end{array}$ & Prectness \\
\hline $\begin{array}{l}\text { Asenapine } \\
\text { adjunctive vs. } \\
\text { placebo }\end{array}$ & $\begin{array}{l}\text { Response 3 wks } \\
\text { YMRS 3 wks } \\
\text { CGI-BP 3 wks }\end{array}$ & $\begin{array}{l}1 \mathrm{RCT} \\
(\mathrm{n}=324)\end{array}$ & NS & Moderate & Unknown & Direct \\
\hline
\end{tabular}

Abbreviations: CGI-BP=Clinical Global Impressions Scale for Bipolar Disorder; NS=not significant; RCT=randomized controlled trial; YMRS = Young Mania Rating Scale Notes:

1. Publication bias for antipsychotics, antidepressants, and behavioral interventions for depressive disorders is suspected.

2. Data were generally imprecise due to missing data from high attrition rates, which was commonly dealt with by Last Observation Carried Forward (LOCF). LOCF requires an assumption that the health status of patients who dropped out of the trial would not have changed had future observations been recorded, a strong assumption in the context of bipolar disorder research. 


\section{Section 11. Olanzapine Plus Mood Stabilizer}

Appendix Table E53. Characteristics of eligible studies: olanzapine plus mood stabilizer drug treatments for acute mania

\begin{tabular}{|c|c|c|c|c|c|c|}
\hline $\begin{array}{c}\text { Study, Year } \\
\text { Design } \\
\text { Location } \\
\text { Funder } \\
\text { Risk of Bias } \\
\text { PMID }\end{array}$ & $\begin{array}{c}\text { \# Randomized } \\
\text { Age (mean) } \\
\text { Sex (\% Female) } \\
\text { Race (\% White) } \\
\text { Diagnosis } \\
\text { (\% BP I, II, NOS) } \\
\text { Setting }\end{array}$ & $\begin{array}{c}\text { Inclusions } \\
\text { Key Exclusions }\end{array}$ & $\begin{array}{c}\text { Intervention } \\
\text { Dosage }\end{array}$ & $\begin{array}{c}\text { Comparison } \\
\text { Dosage }\end{array}$ & $\begin{array}{c}\text { Follow-up } \\
\text { Duration }\end{array}$ & $\begin{array}{c}\text { Outcomes } \\
\text { Reported } \\
\text { Withdrawal (\%) at } \\
\text { endpoint }\end{array}$ \\
\hline $\begin{array}{l}\text { Conus, } 2015^{47} \\
\text { RCT } \\
\text { Single-site } \\
\text { Australia } \\
\text { Industry } \\
\text { RoB Low } \\
26485297\end{array}$ & $\begin{array}{l}\mathrm{N}=83 \\
\text { Mean Age } 22 \\
\text { Female 32\% } \\
\text { Race NR } \\
\text { BP I 100\% } \\
\text { Inpatient }\end{array}$ & $\begin{array}{l}\text { First manic or mixed } \\
\text { episode; } \\
\text { YMRS } \geq 20 \\
\text { Other Mental Health } \\
\text { Neurological Disorders } \\
\text { Taking Other Meds } \\
\text { Pregnant/Nursing } \\
\text { Labs/Other Conditions }\end{array}$ & $\begin{array}{l}\text { Olanzapine } \\
5 \text { mg/day adjusted } \\
\text { by } 2.5 \text { mg/day } \\
\text { Adjunctive to lithium } \\
500 \text { mg/day } \\
\text { increased by } 500 \\
\text { mg/twice daily }\end{array}$ & $\begin{array}{l}\text { Chlorpromazine } \\
100 \text { mg/day } \\
\text { adjusted by } 50- \\
100 \text { mg/day } \\
\text { Adjunctive to } \\
\text { lithium } 500 \\
\text { mg/day increased } \\
\text { by } 500 \text { mg/twice } \\
\text { daily }\end{array}$ & 8 weeks & $\begin{array}{l}\text { Response ( } \geq 50 \% \\
\text { YMRS reduction) } \\
\text { Remission (YMRS } \leq \\
12 \text { ) } \\
\text { Symptomatic recovery } \\
\text { (YMRS } \leq 12 \text { and } \\
\text { HAMD-21 < 7) } \\
\text { Efficacy } \\
\text { YMRS } \\
\text { CGI-BP } \\
\text { BPRS } \\
\text { HAMD-21 } \\
\text { SAPS } \\
\text { SANS } \\
\text { ASI } \\
\text { PAS } \\
\text { GAF } \\
\text { QLS } \\
\text { Adverse events } \\
\text { UKU } \\
\text { Withdrawal 14\% }\end{array}$ \\
\hline
\end{tabular}




\begin{tabular}{|c|c|c|c|c|c|c|}
\hline $\begin{array}{l}\text { Study, Year } \\
\text { Design } \\
\text { Location } \\
\text { Funder } \\
\text { Risk of Bias } \\
\text { PMID }\end{array}$ & $\begin{array}{c}\text { \# Randomized } \\
\text { Age (mean) } \\
\text { Sex (\% Female) } \\
\text { Race (\% White) } \\
\text { Diagnosis } \\
\text { (\% BP I, II, NOS) } \\
\text { Setting }\end{array}$ & $\begin{array}{c}\text { Inclusions } \\
\text { Key Exclusions }\end{array}$ & $\begin{array}{l}\text { Intervention } \\
\text { Dosage }\end{array}$ & $\begin{array}{c}\text { Comparison } \\
\text { Dosage }\end{array}$ & $\begin{array}{l}\text { Follow-up } \\
\text { Duration }\end{array}$ & $\begin{array}{c}\text { Outcomes } \\
\text { Reported } \\
\begin{array}{c}\text { Withdrawal (\%) at } \\
\text { endpoint }\end{array}\end{array}$ \\
\hline $\begin{array}{l}\text { Xu, } 2015^{19} \\
\text { RCT } \\
\text { Single-site } \\
\text { China } \\
\text { Government } \\
\text { RoB Low } \\
\\
26060401\end{array}$ & $\begin{array}{l}\mathrm{N}=120 \\
\text { Mean Age } 31 \\
\text { Female } 52 \% \\
\text { Race NR } \\
\text { BP } 1100 \% \\
\text { Setting NR }\end{array}$ & $\begin{array}{l}\text { First manic; } \\
\text { YMRS } \geq 17 \\
\text { Substance Abuse } \\
\text { Neurological Disorders } \\
\text { Taking Other Meds } \\
\text { Pregnant/Nursing }\end{array}$ & $\begin{array}{l}\text { Olanzapine } \\
10 \text { mg/day }+ \\
\text { Valproate } 600 \\
\text { mg/day }\end{array}$ & $\begin{array}{l}\text { C1: Olanzapine } \\
10 \text { mg/day } \\
\text { Flexible dosing } \\
5-20 \text { mg/day } \\
\\
\text { C2: Valproate } 600 \\
\text { mg/day alone }\end{array}$ & 4 weeks & $\begin{array}{l}\text { Efficacy } \\
\text { YMRS } \\
\text { CGI-BP } \\
\text { Adverse events } \\
\text { Extrapyramidal } \\
\text { symptoms } \\
\text { SAS } \\
\text { Withdrawal 5\% }\end{array}$ \\
\hline $\begin{array}{l}\text { Houston, } 2009^{48} \\
\text { RCT } \\
\text { US and Puerto Rico } \\
\text { Industry } \\
\text { RoB High } \\
19778495\end{array}$ & $\begin{array}{l}\mathrm{N}=202 \\
\text { Mean Age } 39 \\
\text { Female 59\% } \\
\text { White 51\% } \\
\text { BP I 100\% } \\
\text { Outpatient }\end{array}$ & $\begin{array}{l}\text { Mixed Episode; } \\
\text { YMRS } \geq 16 \\
\text { HDRS-21 (inadequate } \\
\text { response to divalproex) } \\
\\
\text { First Manic Episode } \\
\text { Taking Other Meds } \\
\text { Labs/Other Conditions }\end{array}$ & $\begin{array}{l}\text { Olanzapine } \\
5-20 \mathrm{mg} / \text { day } \\
\\
\text { Adjunctive to } \\
\text { divalproex } \\
\text { (adjusted for blood } \\
\text { levels between } 75- \\
125 \mu \mathrm{g} / \mathrm{mL} \text { ) }\end{array}$ & $\begin{array}{l}\text { Divalproex alone } \\
\text { (adjusted for blood } \\
\text { levels between } 75- \\
125 \mu \mathrm{g} / \mathrm{mL} \text { ) }\end{array}$ & 6 weeks & $\begin{array}{l}\text { Response } \\
(\geq 50 \% \text { decrease } \\
\text { HDRS-21 and } \geq 25 \% \\
\text { decrease YMRS) } \\
\text { Remission } \\
\text { (YMRS } \leq 12 \text { and } \\
\text { HDRS-21 } \leq \text { ) } \\
\text { Efficacy } \\
\text { HDRS-21 } \\
\text { YMRS } \\
\text { CGI-BP } \\
\text { Adverse events } \\
\text { Extrapyramidal } \\
\text { symptoms } \\
\\
\text { Withdrawal 42\% }\end{array}$ \\
\hline
\end{tabular}




\begin{tabular}{|c|c|c|c|c|c|c|}
\hline $\begin{array}{l}\text { Study, Year } \\
\text { Design } \\
\text { Location } \\
\text { Funder } \\
\text { Risk of Bias } \\
\text { PMID }\end{array}$ & $\begin{array}{c}\text { \# Randomized } \\
\text { Age (mean) } \\
\text { Sex (\% Female) } \\
\text { Race (\% White) } \\
\text { Diagnosis } \\
\text { (\% BP I, II, NOS) } \\
\text { Setting }\end{array}$ & $\begin{array}{l}\text { Inclusions } \\
\text { Key Exclusions }\end{array}$ & $\begin{array}{l}\text { Intervention } \\
\text { Dosage }\end{array}$ & $\begin{array}{c}\text { Comparison } \\
\text { Dosage }\end{array}$ & $\begin{array}{l}\text { Follow-up } \\
\text { Duration }\end{array}$ & $\begin{array}{c}\text { Outcomes } \\
\text { Reported } \\
\begin{array}{c}\text { Withdrawal (\%) at } \\
\text { endpoint }\end{array}\end{array}$ \\
\hline $\begin{array}{l}\text { Tohen, } 2008 a^{49} \\
\text { RCT } \\
\text { Multisite } \\
3 \text { Continents } \\
\text { Industry } \\
\text { RoB Moderate } \\
18245032\end{array}$ & $\begin{array}{l}\mathrm{N}=118 \\
\text { Mean Age } 41 \\
\text { Female } 58 \% \\
\text { Race NR } \\
\text { BP I 100\% } \\
\text { Inpatient and Outpatient }\end{array}$ & $\begin{array}{l}\text { Manic or Mixed Episode; } \\
\text { YMRS } \geq 20 \\
\text { Labs/Other Conditions }\end{array}$ & $\begin{array}{l}\text { Olanzapine } \\
10-30 \mathrm{mg} / \text { day } \\
\\
\text { Adjunctive to } \\
\text { carbamazepine } \\
400-1200 \mathrm{mg} / \text { day }\end{array}$ & $\begin{array}{l}\text { Placebo } \\
\text { Adjunctive to } \\
\text { carbamazepine } \\
400-1200 \mathrm{mg} / \text { day }\end{array}$ & $\begin{array}{l}6 \text { weeks } \\
\text { blinded (26 } \\
\text { weeks open- } \\
\text { label } \\
\text { extension) }\end{array}$ & $\begin{array}{l}\text { Efficacy } \\
\text { YMRS } \\
\text { CGI (multiple } \\
\text { subscales) } \\
\text { MADRS } \\
\text { Adverse events } \\
\text { Extrapyramidal } \\
\text { symptoms } \\
\text { SAS } \\
\text { BAS } \\
\text { AIMS } \\
\text { Withdrawal 28\% at } 6 \\
\text { weeks } \\
\end{array}$ \\
\hline $\begin{array}{l}\text { Tohen, 2002a }{ }^{50} \\
\text { RCT } \\
\text { Multisite } \\
\text { US and Canada } \\
\text { Industry } \\
\text { RoB High } \\
\\
11779284 \\
15337326^{51} \\
15572737^{52}\end{array}$ & $\begin{array}{l}\mathrm{N}=344 \\
\text { Mean Age } 41 \\
\text { Female } 52 \% \\
\text { White } 85 \% \\
\text { Diagnosis NR } \\
\text { Outpatient }\end{array}$ & $\begin{array}{l}\text { Manic or Mixed Episode; } \\
\text { YMRS } \geq 16 \\
\text { First Manic Episode } \\
\text { Labs/Other Conditions }\end{array}$ & $\begin{array}{l}\text { Olanzapine } \\
10 \text { mg/day with } \\
\text { flexible dosing from } \\
5-20 \text { mg/day } \\
\text { Adjunctive to } \\
\text { ongoing open-label } \\
\text { valproate or lithium }\end{array}$ & $\begin{array}{l}\text { Placebo } \\
\text { Adjunctive to } \\
\text { ongoing open- } \\
\text { label valproate or } \\
\text { lithium }\end{array}$ & 6 weeks & \begin{tabular}{|l|} 
Efficacy \\
YMRS \\
HAM-D \\
PANSS \\
CGI \\
Remission \\
Adverse Events \\
Extrapyramidal \\
symptom \\
Withdrawal 30\%
\end{tabular} \\
\hline
\end{tabular}

Abbreviations: AIMS=Abnormal Involuntary Movement Scale; ASI=Addiction Severity Index; BAS=Behavioral Approach System; BID=Twice a day; BP=bipolar disorder; BPRS=Brief Psychiatric Rating Scale; C=Comparison; CGI-BP=Clinical Global Impressions Scale, Bipolar; CGI-BP-S=Clinical Global Impressions, Bipolar, Severity Scale; CGI-I= Clinical Global Impressions-Improvement; CGI-S== Clinical Global Impressions-Severity Scale; DSM-IV-TR= Diagnostic and statistical manual, $4^{\text {th }}$ edition, Text Revision; EPS=extrapyramidal symptoms; FAST=Functional Assessment Short Test; GAF=General Assessment of Functioning Scale; GAS= Global Assessment Scale; HAMD=Hamilton Scale for Depression; HAMD-21=Hamilton Rating Scale for Depression (21-items); HDRS-21=Hamilton Depression Rating Scale (21=items); ISST=International Suicide Prevention Trial Scale for Suicidal Thinking; LIFE-RIFT=Longitudinal Interval Follow-up Evaluation-Rating Impaired Functioning Tool; MADRS=Montgomery-Asberg Depression Rating Scale; NOS=not otherwise specified; NR=not reported; OR=Odds Ratio; PANSS=Positive and Negative Syndrome Scale; PAS=Premornid Adjustment Scale; PGI-I=Patient Global Impression Improvement; PMID=PubMed Identification Number; QLS=Quality of Life Scale; RCT=randomized controlled trial; RDQ=Readiness to 
Discharge Questionnaire; ROB=risk of bias; SANS=Scale for the Assessment of Negative Symptoms; SAPS=Scale for the Assessment of Positive Symptoms; SAS=Simpson Angus Scale; SF-36=36-Item Short Form Health Survey; UKU=UKU rating scale; YMRS = Young Mania Rating Scale

\section{Appendix Table E54. Summary risk of bias assessments: olanzapine plus mood stabilizers for acute mania}

\begin{tabular}{|c|c|c|c|}
\hline Drug & $\begin{array}{c}\text { Study } \\
\text { Funding Source } \\
\text { PMID }\end{array}$ & $\begin{array}{l}\text { Overall Risk of Bias } \\
\text { Assessment }\end{array}$ & Rationale \\
\hline \multirow[t]{5}{*}{ Olanzapine } & $\begin{array}{l}\text { Conus, } 201547 \\
\text { Industry } \\
26485297\end{array}$ & Low & Physician and patients may not be blinded. Dropout balanced and accounted for in analysis. \\
\hline & $\begin{array}{l}\text { Xu, } 201519 \\
\text { Government } \\
26060401\end{array}$ & Low & Well-constructed, described, and reported study. 5\% dropout. \\
\hline & $\begin{array}{l}\text { Houston, } 200948 \\
\text { Industry } \\
19778495 \\
\end{array}$ & High & Randomization and blinding procedures not described. $42 \%$ dropout. \\
\hline & $\begin{array}{l}\text { Tohen, 2008a49 } \\
\text { Industry } \\
18245032\end{array}$ & Moderate & Blinding procedure not described. $28 \%$ dropout. \\
\hline & $\begin{array}{l}\text { Tohen, 2002a50 } \\
\text { Industry } \\
11779284\end{array}$ & High & Randomization and blinding procedures not described. 30\% dropout. \\
\hline
\end{tabular}

Abbreviations: ITT=intention to treat; PMID=PubMed Identification Number 


\section{Antipsychotics Plus Mood Stabilizer Forest Plots}

Outcomes in studies assessed as having a high risk of bias, or low to moderate risk of bias but at least 40 percent attrition, are presented in grey tones. Both fixed-effect models and random-effects models are presented. We calculated fixed-effect models to provide a charitable estimate of the average effect among completed trials. However, we base our main conclusions on the random-effects models.

Appendix Figure E90. Adjunctive olanzapine vs. placebo - response

Odds Ratio of Response (> 50\% Reduction in YMRS) at Last Measurement ( 6 Weeks)

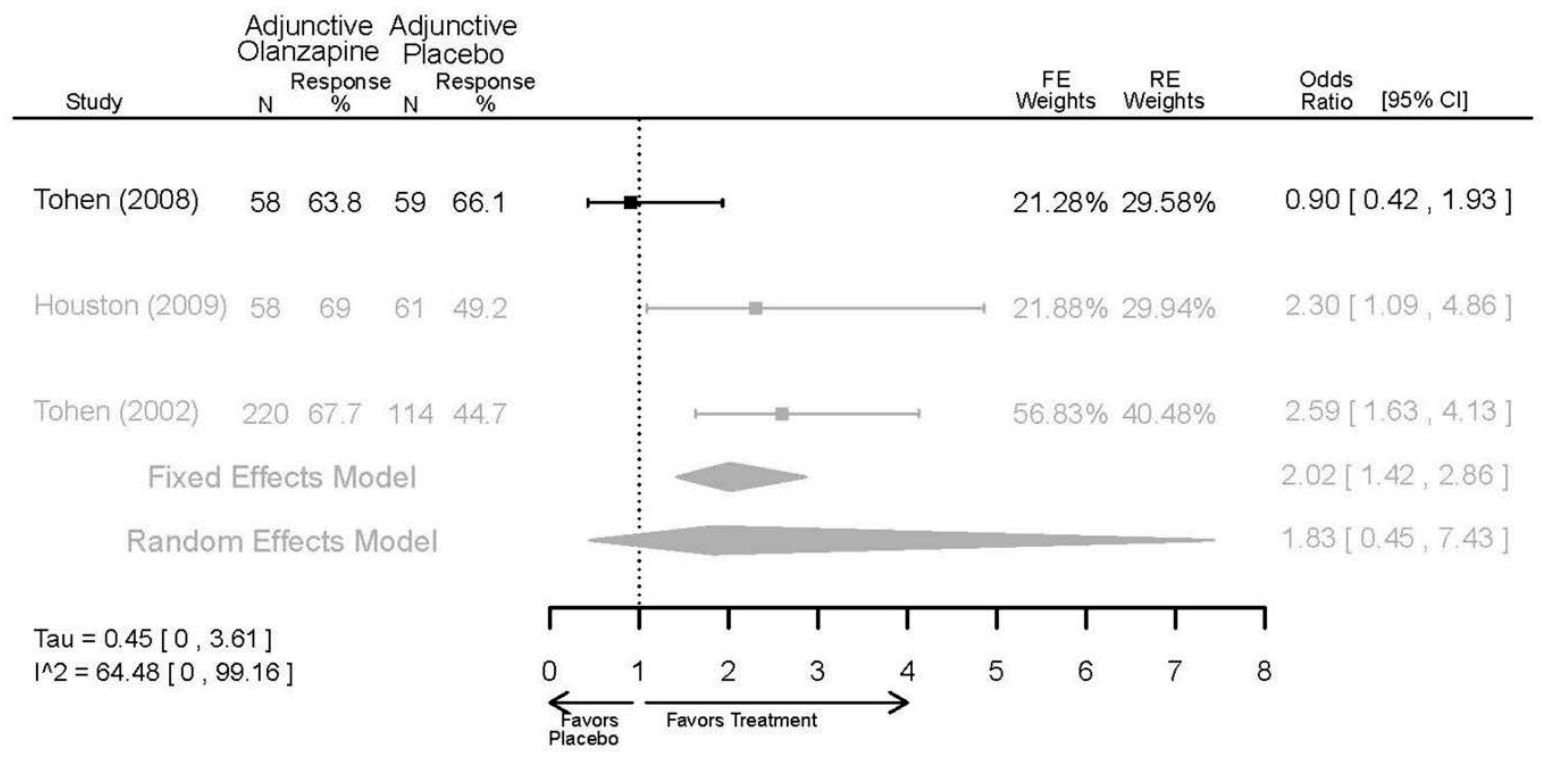


Appendix Figure E91. Adjunctive olanzapine vs. placebo - remission

\section{Odds Ratio of Remission (YMRS $=12$ ) at Last Measurement ( 6 Weeks)}

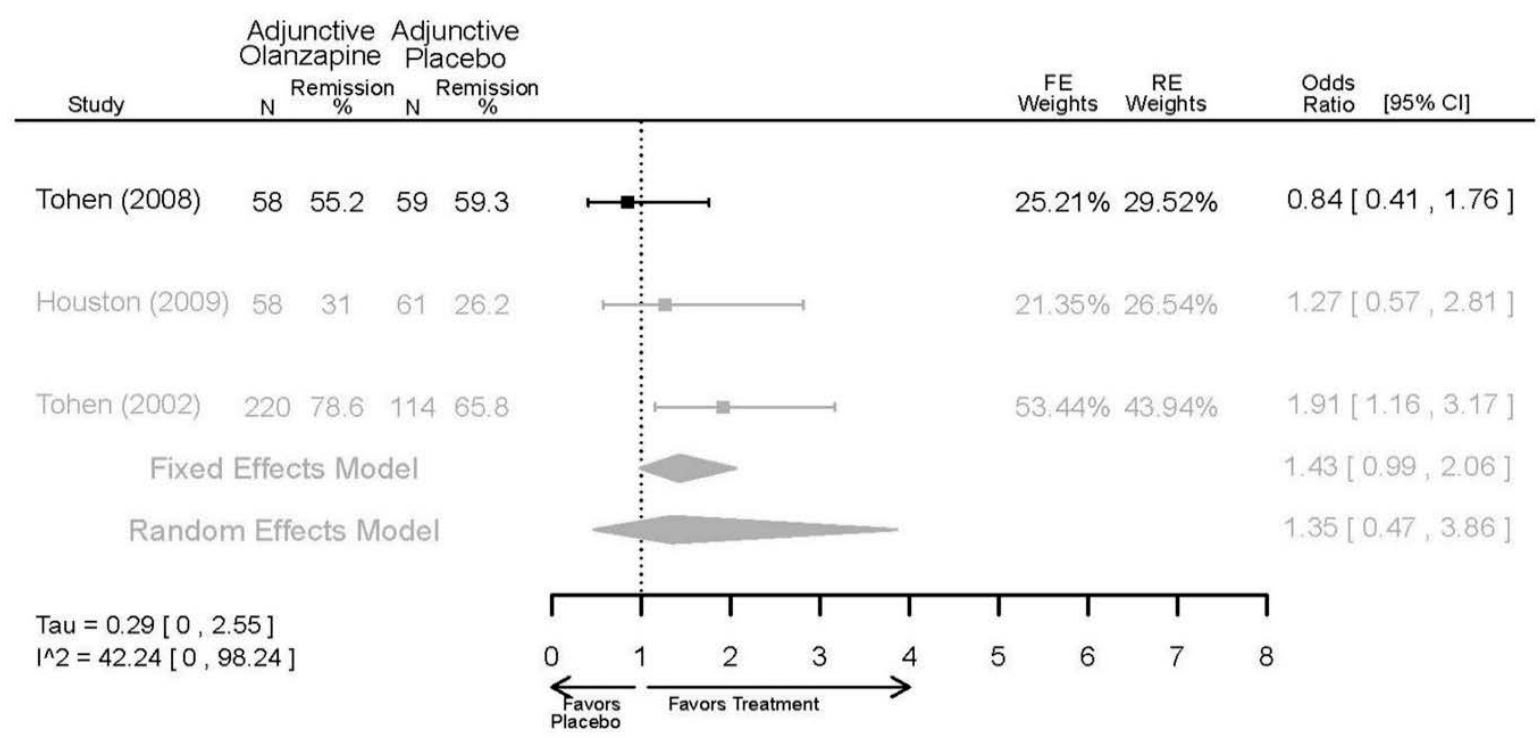

Appendix Figure E92. Adjunctive olanzapine vs. placebo - YMRS

Difference in Mean Change in YMRS

from Baseline to 6 Weeks

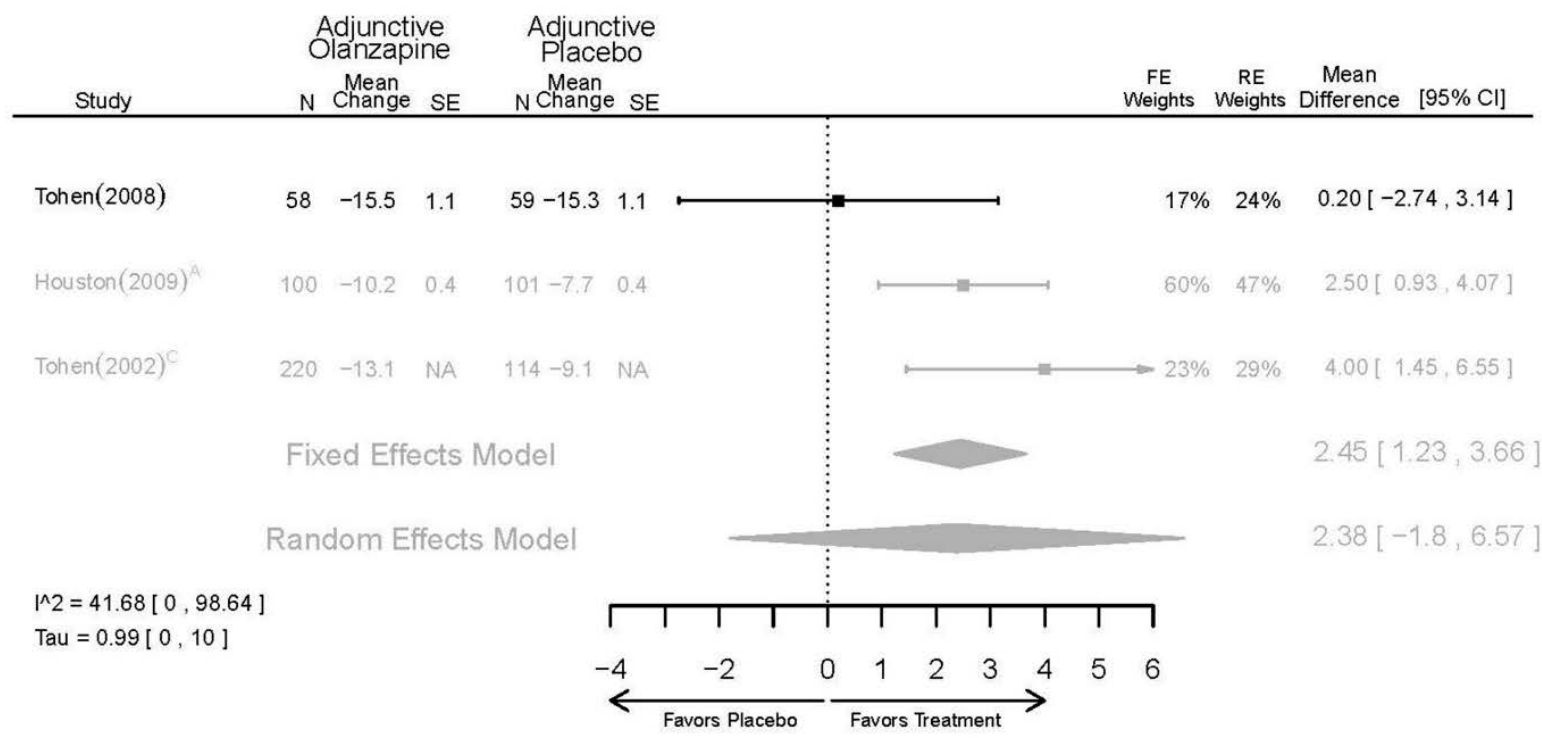


Appendix Figure E93. Adjunctive olanzapine vs. placebo - CGI

Difference in Mean Change in CGI-BP-S (Overall)

from Baseline to Last Measurement (6 Weeks)

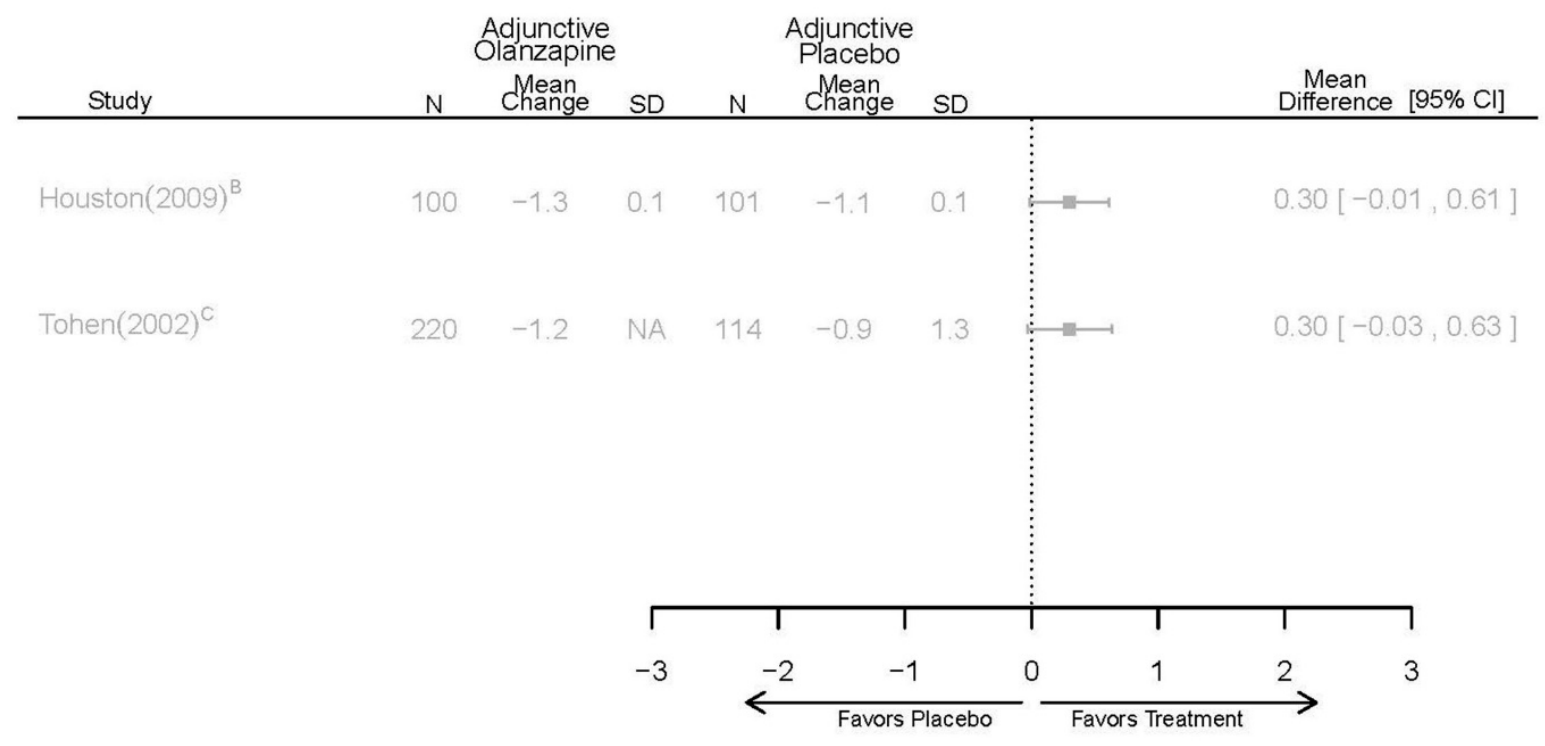

Appendix Figure E94. Adjunctive olanzapine vs. placebo - overall withdrawal

Odds Ratio of Withdrawal due to All Causes at Last Measurement (6 Weeks)

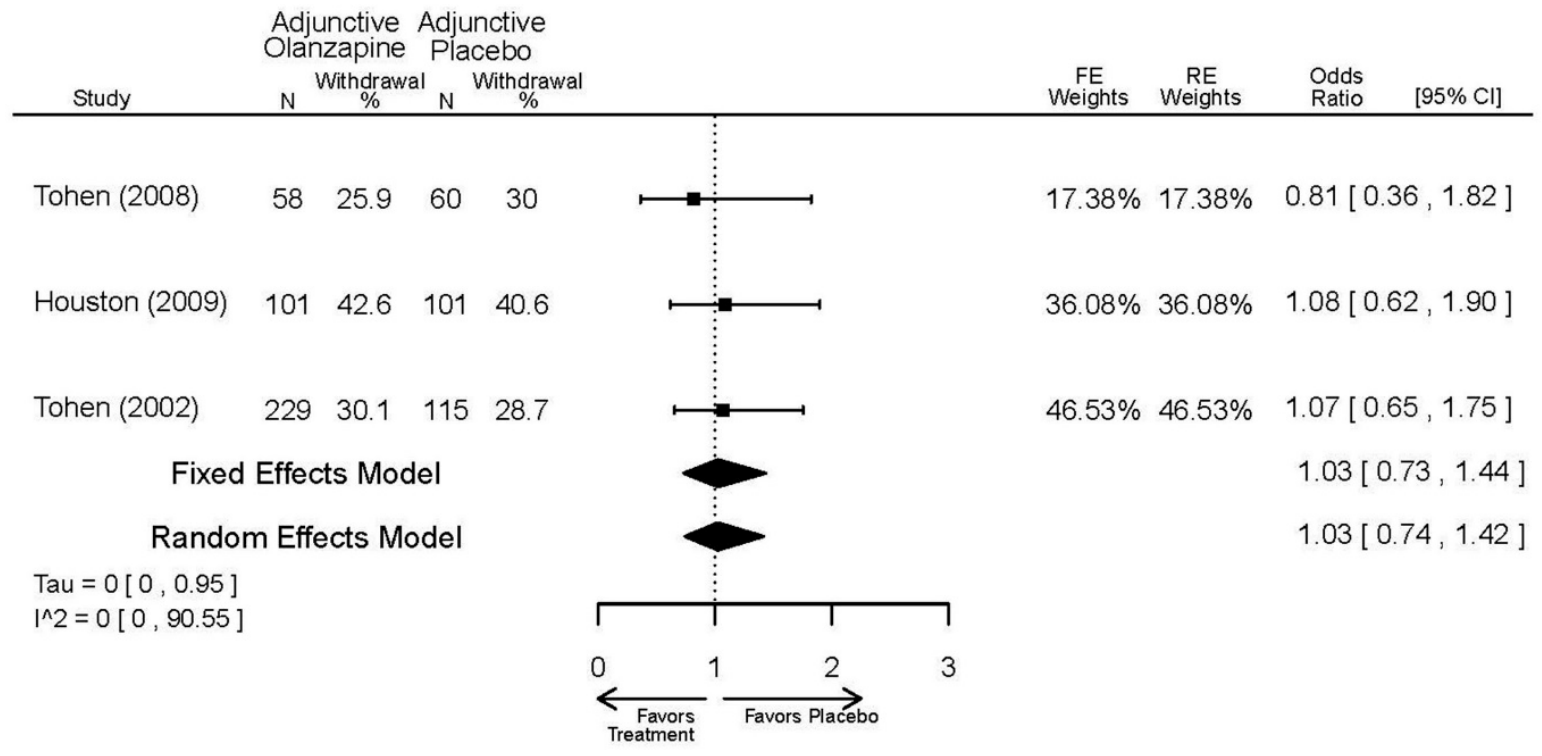


Appendix Figure E95. Adjunctive olanzapine vs. placebo - withdrawal - lack of efficacy

\section{Odds Ratio of Withdrawal due to Lack of Efficacy}

at Last Measurement (6 Weeks)

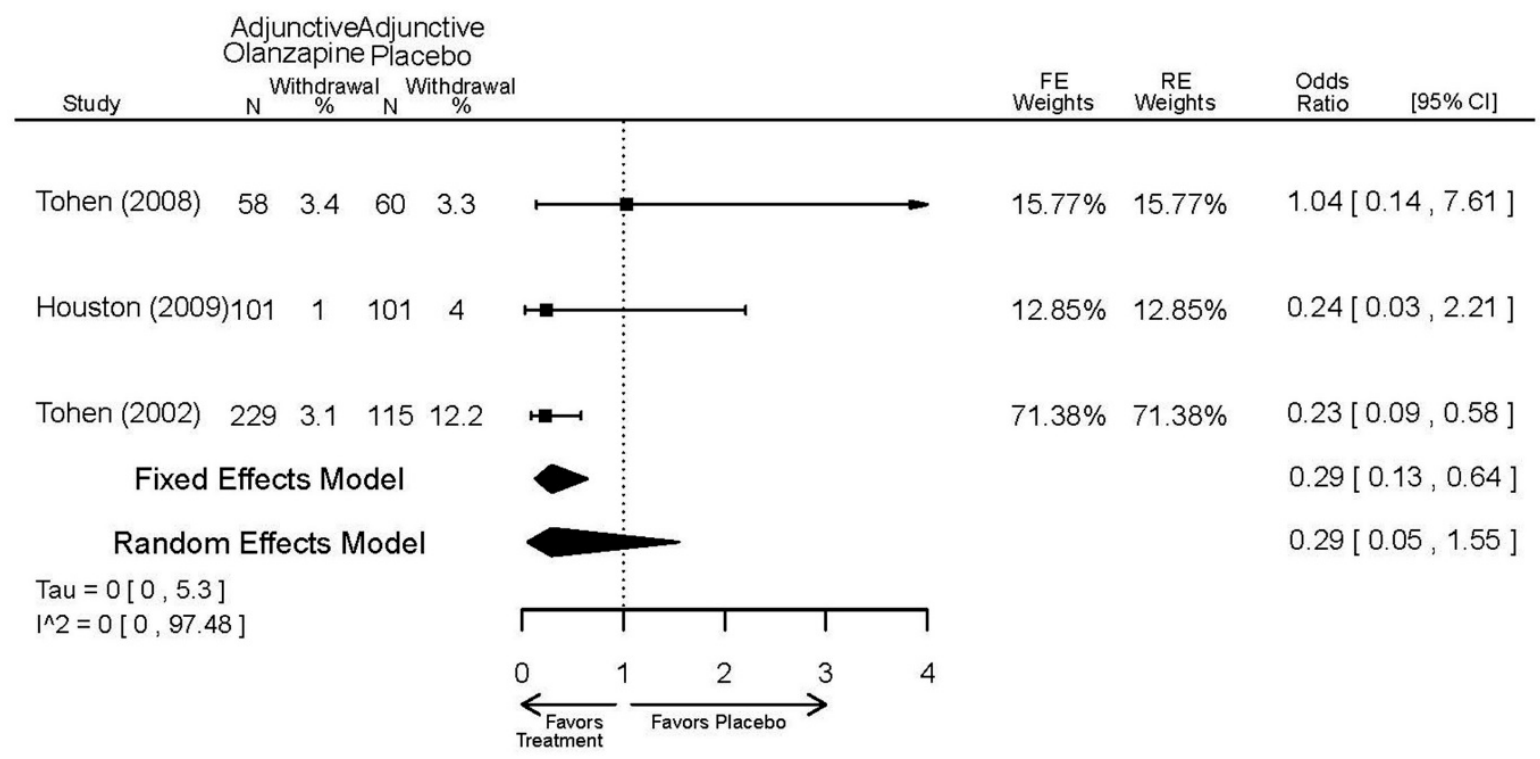

Appendix Figure E96. Adjunctive olanzapine vs. placebo - withdrawal - adverse events

Odds Ratio of Withdrawal due to Adverse Events at Last Measurement (6 Weeks)

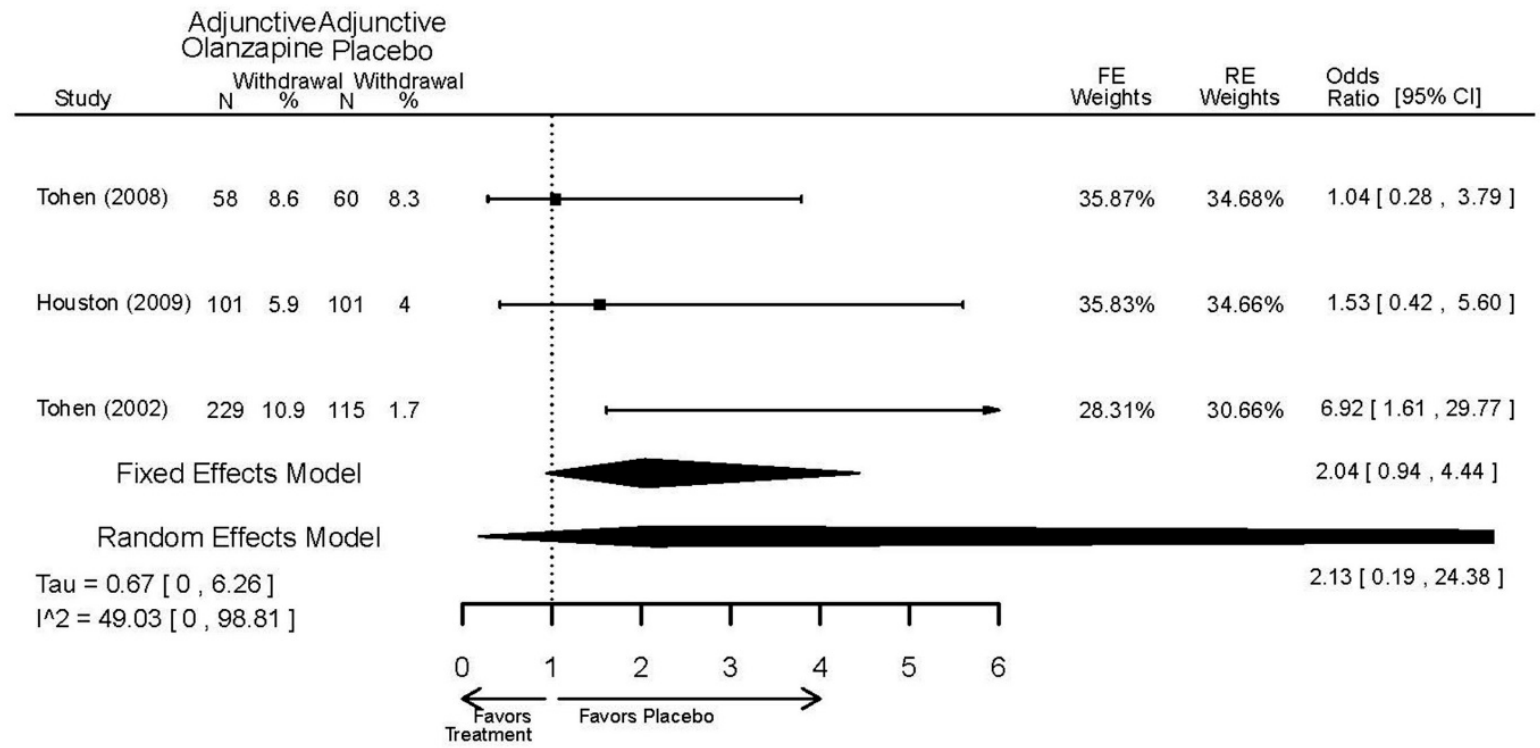


Appendix Figure E97. Adjunctive olanzapine vs. placebo - serious adverse events Odds Ratio of Serious Adverse Event at Last Measurement (6 Weeks)

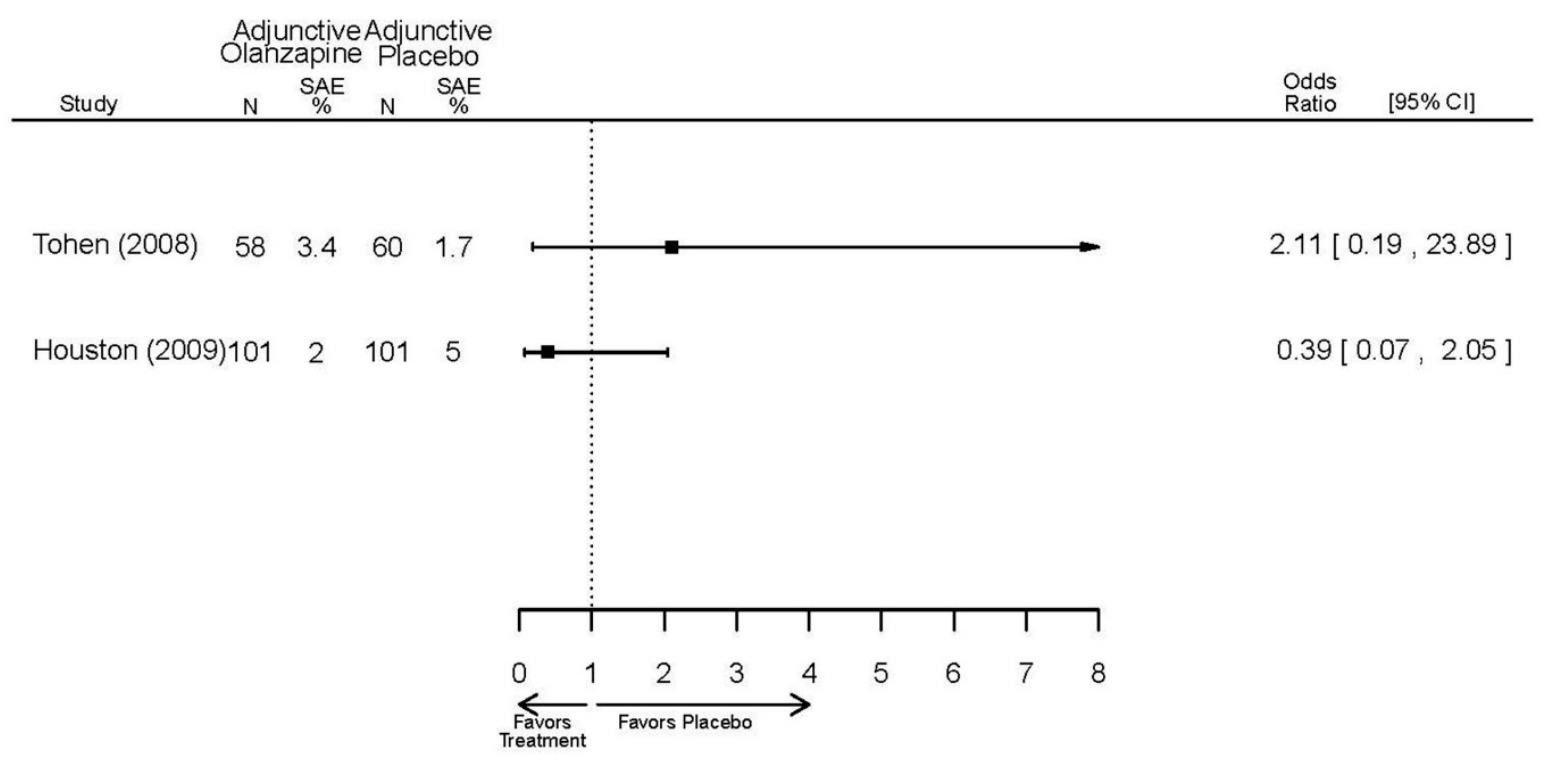

Appendix Figure E98. Adjunctive olanzapine vs. placebo - emergent depression last Odds Ratio of Emergent Depression

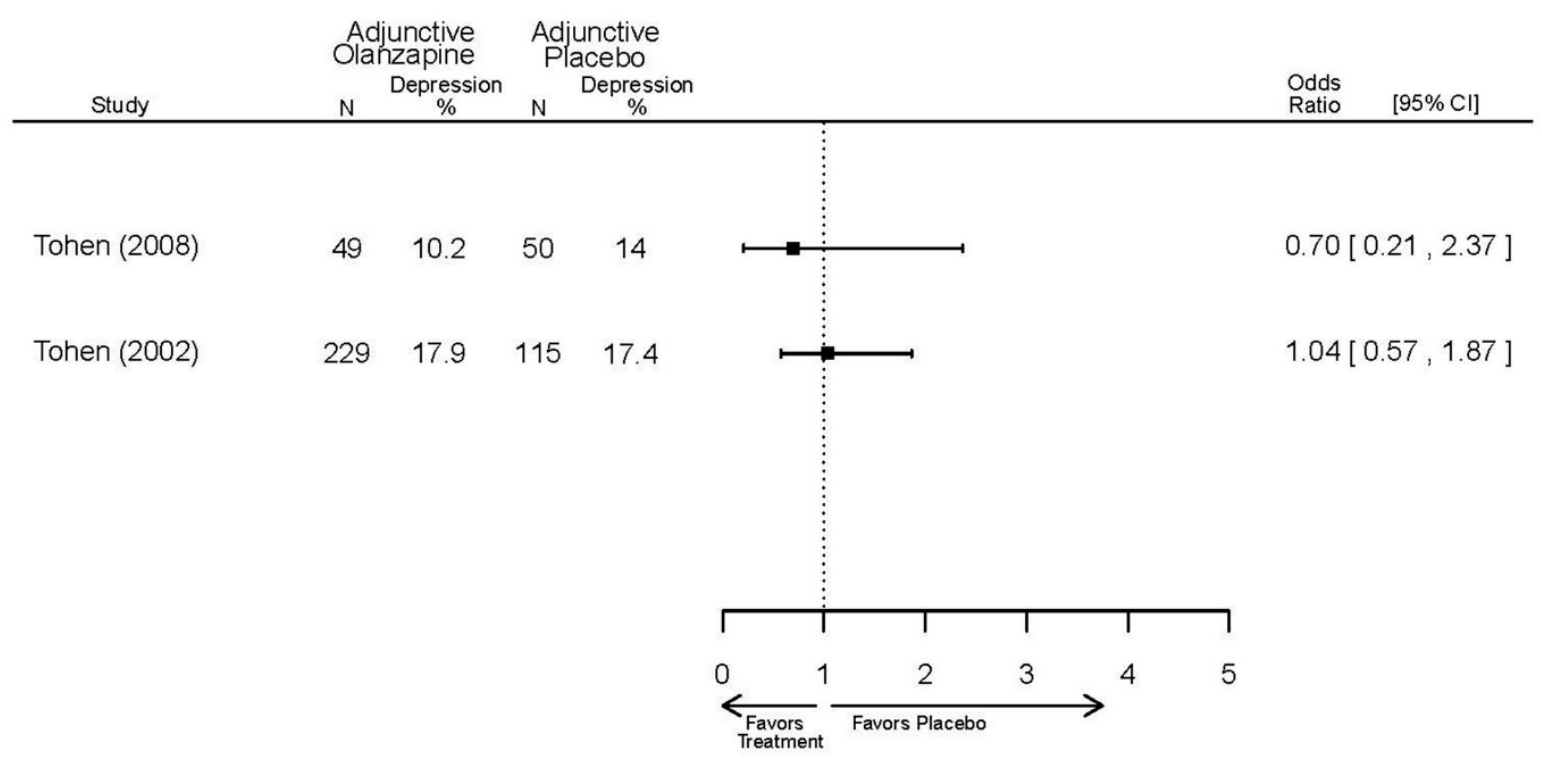


Appendix Figure E99. Adjunctive olanzapine vs. placebo - weight Odds Ratio of Normalized Weight Change ( $>=7 \%$ ) at Last Measurement (6 Weeks)

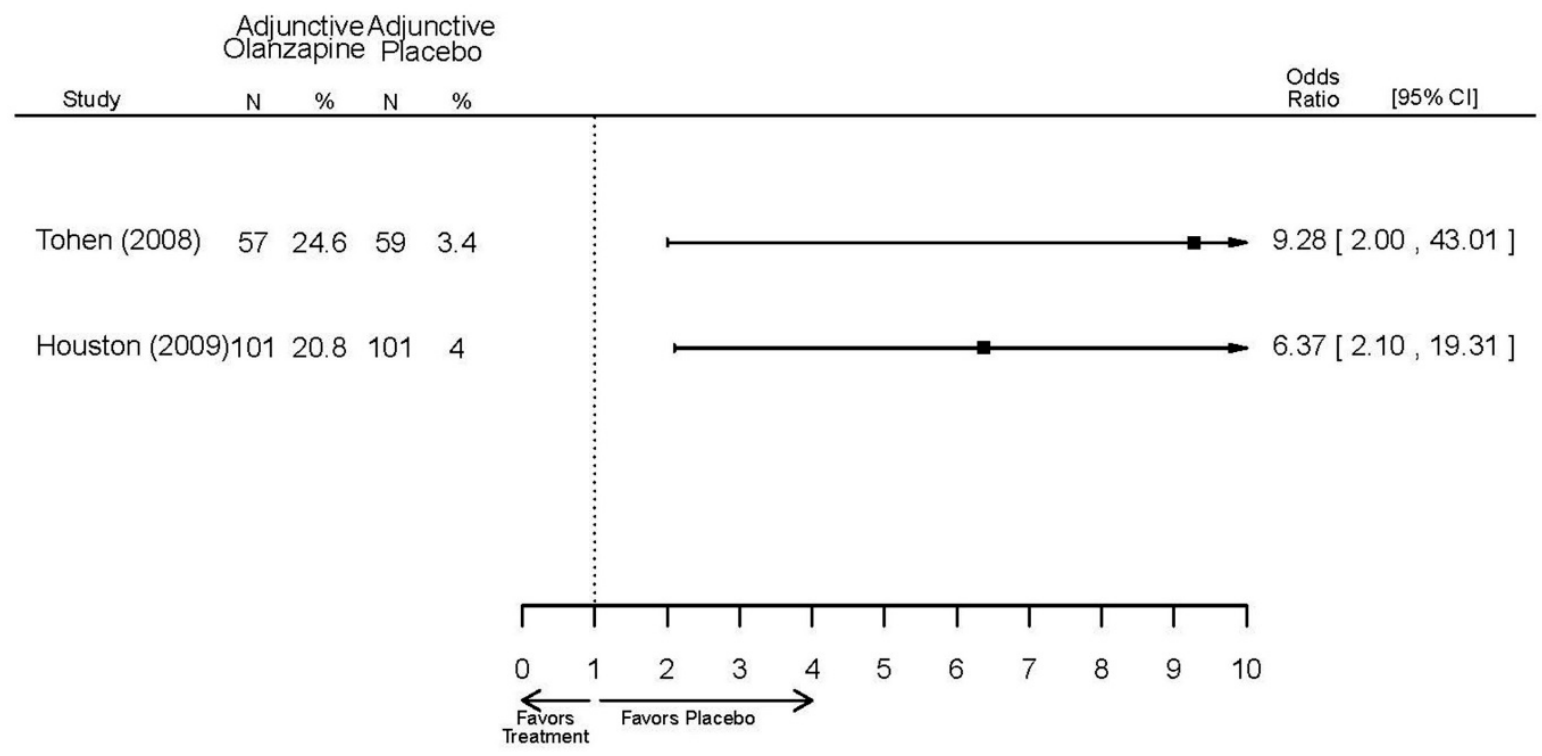




\begin{tabular}{|c|c|c|c|c|c|c|}
\hline Drug & $\begin{array}{l}\text { Study } \\
\text { PMID } \\
\text { RoB }\end{array}$ & $\begin{array}{l}\text { Responderl } \\
\text { Remitter }\end{array}$ & Symptom & Function & Other & $\mathrm{AE}$ \\
\hline $\begin{array}{l}\text { Olanzapine } \\
\text { adjunctive } \\
\text { vs. } \\
\text { chlorproma } \\
\text { zine }\end{array}$ & $\begin{array}{l}\text { Conus, } 2015^{47} \\
26485297 \\
\text { Low }\end{array}$ & $\begin{array}{l}\frac{\text { Symptomatic }}{\text { Recovery }} \\
8 \text { weeks } \\
\text { Olanzapine }=53.3 \% \\
\text { Chlorpromazine }=54 \\
.5 \% \\
\mathrm{P}=0.56 \\
\\
\text { Response } \\
\text { 8 weeks } \\
\text { Olanzapine }=96.7 \% \\
\text { Chlorpromazine }=84 \\
.8 \% \\
\mathrm{P}=0.12\end{array}$ & $\begin{array}{l}\frac{Y M R S}{8 \text { weeks }} \\
\text { Score } \leq 12 \\
\text { Olanzapine }=93.3 \% \\
\text { Chlorpromazine }=72.7 \\
\% \\
\mathrm{P}=0.03 \\
\text { Favors intervention } \\
\text { HAMD-21 } \\
8 \text { weeks } \\
\text { Score }<7 \\
\text { Olanzapine }=53.3 \% \\
\text { Chlorpromazine }=63.6 \\
\% \\
\mathrm{P}=0.28 \\
\mathrm{NS}\end{array}$ & $\begin{array}{l}\frac{\text { GAF }}{8 \text { weeks }} \\
\text { NS } \\
\text { No statistical test } \\
\text { reported } \\
\frac{\text { QLS }}{8 \text { weeks }} \\
\text { NS } \\
\text { No statistical test } \\
\text { reported } \\
\frac{\text { PAS }}{8 \text { weeks }} \\
\text { NS } \\
\text { No statistical test } \\
\text { reported } \\
\frac{\text { CGI-BP }}{8 \text { weeks }} \\
\text { NS } \\
\text { No statistical test } \\
\text { reported }\end{array}$ & $\begin{array}{l}\frac{\text { ASI }}{8 \text { weeks }} \\
\text { NS } \\
\text { No statistical test } \\
\text { reported } \\
\text { Weight Gain } \\
8 \text { weeks } \\
\text { NS; } p=0.22 \\
\text { Overall Withdrawal } \\
8 \text { weeks } \\
\text { Olanzapine }=4 / 42 \\
\text { Chlorpromazine }=5 / 41 \\
\text { NS }\end{array}$ & $\begin{array}{l}\frac{\mathrm{UKU}}{8 \text { weeks }} \\
\mathrm{NS} \\
\text { No statistical test reported } \\
\frac{\mathrm{AE} \text { Leading to Trial }}{\text { Medication Interruption }} \\
\text { 8 weeks } \\
\text { Olanzapine }=5.3 \% \\
\text { Chlorpromazine }=6.8 \% \\
\mathrm{NS} \\
\mathrm{P}=0.47\end{array}$ \\
\hline
\end{tabular}




\begin{tabular}{|c|c|c|c|c|c|c|}
\hline Drug & $\begin{array}{c}\text { Study } \\
\text { PMID } \\
\text { RoB }\end{array}$ & $\begin{array}{l}\text { Responderl } \\
\text { Remitter }\end{array}$ & Symptom & Function & Other & AE \\
\hline \multirow[t]{2}{*}{$\begin{array}{l}\text { Olanzapine } \\
\text { adjunctive } \\
\text { vs. placebo } \\
\text { or no } \\
\text { placebo }\end{array}$} & $\begin{array}{l}\text { Xu, } 2015^{19} \\
26060401 \\
\text { Low }\end{array}$ & NR & $\begin{array}{l}\frac{\text { YMRS }}{3 \text { weeks }} \\
\text { Favors Olanzapine } \\
\% \text { Reduction (SD) } \\
\text { Olanzapine+Valporate } \\
=86.5 \%(8.9 \%) \\
\text { Valproate }=55.2 \% \\
(5.7 \%) \\
P<0.01 \\
\text { CGI-BP } \\
3 \text { weeks } \\
\text { Difference in } \\
\text { Difference (SE) } \\
1.4 \text { (0.53) } \\
\text { Favors Olanzapine } \\
\text { p<0.01 }\end{array}$ & NR & $\begin{array}{l}\text { Overall Withdrawal } \\
3 \text { weeks } \\
\text { NS } \\
\text { Olanzapine+Valporat } \\
\text { e=2/40 } \\
\text { Valproate }=3 / 40 \\
\text { Withdrawal due to } \\
\frac{\text { AEs }}{3 \text { weeks }} \\
\text { NS } \\
\text { Olanzapine+Valporat } \\
\text { e=2/40 } \\
\text { Valproate }=0 / 40 \\
\text { Withdrawal, Lack of } \\
\text { Efficacy } \\
3 \text { weeks } \\
\text { NS } \\
\text { Olanzapine+Valporat } \\
\text { e=0/40 } \\
\text { Valproate=2/40 }\end{array}$ & $\begin{array}{l}\frac{\text { Severe Harms }}{3 \text { weeks }} \\
\text { NS } \\
\text { Olanzapine+Valporate }=1 / 38 \\
\text { Valproate }=0 / 37 \\
\text { Normalized Weight Change } \\
3 \text { weeks } \\
\text { Olanzapine+Valporate }=31 / 3 \\
8 \\
\text { Valproate }=21 / 37 \\
\text { Emergent Mood Episodes } \\
3 \text { weeks } \\
\text { NS } \\
\text { Olanzapine }+ \text { Valporate }=0 / 38 \\
\text { Valproate }=0 / 37\end{array}$ \\
\hline & $\begin{array}{l}\text { Houston, } \\
2009^{48} \\
19778495 \\
\text { High }\end{array}$ & $\begin{array}{l}\text { See forest plots } \\
\text { E91 and E92 } \\
\text { above }\end{array}$ & $\begin{array}{l}\text { YMRS } \\
\text { See forest plot E93 } \\
\text { above }\end{array}$ & $\begin{array}{l}\text { CGI-BP } \\
\text { See forest plot E94 } \\
\text { above }\end{array}$ & $\begin{array}{l}\text { See forest plots E95, } \\
\text { E96 and E97 above }\end{array}$ & $\begin{array}{l}\text { SAE } \\
\text { See forest plot E98 above } \\
\frac{\text { Deaths }}{1 \text { fatality from a car accident }} \\
\text { in the Olanzapine arm } \\
\frac{\text { Hepatic Failure }}{1 \text { case of acute hepatic }} \\
\text { failure in the Olanzapine } \\
\text { arm } \\
\text { Weight gain } \\
\text { See forest plot E100 above }\end{array}$ \\
\hline
\end{tabular}




\begin{tabular}{|c|c|c|c|c|c|c|}
\hline Drug & $\begin{array}{l}\text { Study } \\
\text { PMID } \\
\text { RoB }\end{array}$ & $\begin{array}{l}\text { Responderl } \\
\text { Remitter }\end{array}$ & Symptom & Function & Other & $\mathrm{AE}$ \\
\hline & $\begin{array}{l}\text { Tohen, } 2008^{49} \\
18245032 \\
\text { Moderate }\end{array}$ & $\begin{array}{l}\text { See forest plots } \\
\text { E91 and E92 } \\
\text { above }\end{array}$ & $\begin{array}{l}\text { YMRS } \\
\text { See forest plot E93 } \\
\text { above } \\
\text { MADRS } \\
6 \text { weeks } \\
\text { Change (SE) } \\
\text { Olanzapine }-1.22 \\
\text { (0.96) } \\
\text { Placebo -1.00 (0.96) } \\
\text { NS }\end{array}$ & NR & $\begin{array}{l}\text { See forest plots E95, } \\
\text { E96 and E97 above }\end{array}$ & $\begin{array}{l}\text { SAE } \\
\text { See forest plots E98 } \\
\text { aboveabove } \\
\text { Emergent depression } \\
\text { See forest plot E99 above } \\
\text { Weight gain } \\
\text { See forest plot E100 above }\end{array}$ \\
\hline & $\begin{array}{l}\text { Tohen, 2002 } \\
11779284 \\
\text { High } \\
\\
\text { Baker, 200452 } \\
15572737 \\
\text { High } \\
\\
\text { Namijoshi, } \\
2004^{51} \\
15337326 \\
\text { High } \\
\end{array}$ & $\begin{array}{l}\text { See forest plots } \\
\text { E91 and E92 } \\
\text { above }\end{array}$ & $\begin{array}{l}\text { YMRS } \\
\text { See forest plot E93 } \\
\text { above }\end{array}$ & $\begin{array}{l}\text { CGI-BP } \\
\text { See forest plot E94 } \\
\text { above }\end{array}$ & $\begin{array}{l}\text { See forest plots E95, } \\
\text { E96 and E97 above }\end{array}$ & $\begin{array}{l}\text { SAE } \\
\text { See forest plot E98 above } \\
\text { EPS } \\
\text { NS } \\
\text { Emergent depression } \\
\text { See forest plot E99 above }\end{array}$ \\
\hline
\end{tabular}

Abbreviations: AE=Adverse Events; ASI=Addiction Severity Index; BPRS=Brief Psychiatric Rating Scale; CGI-Bp=Clinical Global Impresions Scale for Bipolar Disorder; CGIBP-S=Clinical Global Impressions, Bipolar, Severity Scale; CGI-S=Clinical Global Impressions, Severity Scale; CI=Confidence Interval; EPS=extrapyramidal symptoms;

GAF=General Assessment of Functioning Scale; HAM-D=Hamilton Scale for Depression; MADRS=Montgomery-Asberg Syndrome Scale; NR=not reported; NS=not significant; OR=Odds Ratio; PAS=Premorbid Adjustment Scale; PMID=PubMed Identification Number; QLS=Quality of Life Scale; ROB=risk of bias; SAE=Serious Adverse Events; $\mathrm{SE}=$ Standard Error; UKU=UKU rating scale;YMRS = Young Mania Rating Scale 
Appendix Table E56. Strength of evidence assessment: olanzapine plus mood stabilizers versus placebo for acute mania

\begin{tabular}{|c|c|c|c|c|c|c|c|c|}
\hline Comparison & Outcome & $\begin{array}{l}\text { \# Studies/ } \\
\text { Design } \\
\text { (n analyzed) }\end{array}$ & $\begin{array}{l}\text { Finding or } \\
\text { Summary } \\
\text { Statistic }\end{array}$ & $\begin{array}{c}\text { Study } \\
\text { Limitations }\end{array}$ & Consistency & Directness & Precision & $\begin{array}{c}\text { Overall } \\
\text { Gradel } \\
\text { Conclusion }\end{array}$ \\
\hline $\begin{array}{l}\text { Olanzapine } \\
\text { vs. Placebo/ } \\
\text { adjunctive to } \\
\text { Lithium or } \\
\text { Valproate }\end{array}$ & $\begin{array}{l}6 \text { weeks } \\
\text { Remission } \\
\text { Response } \\
\text { YMRS } \\
\text { CGI-BP } \\
\text { Withdrawals }\end{array}$ & $\begin{array}{l}3 \text { RCTs } \\
(n=664)\end{array}$ & See table above & High & Consistent & Direct & Imprecise & Insufficient \\
\hline $\begin{array}{l}\text { Olanzapine } \\
\text { vs. Placebo/ } \\
\text { adjunctive to } \\
\text { Carbamazepin } \\
\text { e }\end{array}$ & $\begin{array}{l}\text { Response } \\
\text { Remission } \\
\text { YMRS } \\
\text { CGI-BP } \\
\text { Withdrawals }\end{array}$ & $\begin{array}{l}1 \mathrm{RCT} \\
(\mathrm{n}=118)\end{array}$ & See table above & Moderate & Unknown & Direct & Imprecise & Insufficient \\
\hline $\begin{array}{l}\text { Olanzapine } \\
\text { vs. No } \\
\text { Placebo/ } \\
\text { adjunctive to } \\
\text { Divalproex/ } \\
\text { Valproate }\end{array}$ & $\begin{array}{l}\text { Response } \\
\text { Remission } \\
\text { YMRS } \\
\text { CGI-BP } \\
\text { Withdrawals }\end{array}$ & $\begin{array}{l}1 \mathrm{RCT} \\
(\mathrm{n}=202)\end{array}$ & See table above & High & Unknown & Direct & Imprecise & Insufficient \\
\hline $\begin{array}{l}\text { Olanzapine } \\
\text { vs. No } \\
\text { Placebo/ } \\
\text { adjunctive to } \\
\text { Valproate }\end{array}$ & $\begin{array}{l}\text { YMRS } 3 \text { weeks } \\
\text { CGI-BP } 3 \text { weeks } \\
\text { Withdrawals }\end{array}$ & $\begin{array}{l}1 \mathrm{RCT} \\
(\mathrm{n}=80)\end{array}$ & See table above & Low & Unknown & Direct & Imprecise & Insufficient \\
\hline
\end{tabular}

Abbreviations: CGI-BP=Clinical Global Impressions Scale for Bipolar Disorder; NS=not significant; RCT=randomized controlled trial; YMRS = Young Mania Rating Scale Notes:

1. Publication bias for antipsychotics, antidepressants, and behavioral interventions for depressive disorders is suspected.

2. Data were generally imprecise due to missing data from high attrition rates, which was commonly dealt with by Last Observation Carried Forward (LOCF). LOCF requires an assumption that the health status of patients who dropped out of the trial would not have changed had future observations been recorded, a strong assumption in the context of bipolar disorder research. 
Appendix Table E57. Outcomes summary: olanzapine plus mood stabilizers versus active comparison for acute mania

\begin{tabular}{|c|c|c|c|c|c|c|}
\hline Drug & $\begin{array}{l}\text { Study } \\
\text { PMID }\end{array}$ & Responder/Remitter & Symptom & Function & Other & AE \\
\hline $\begin{array}{l}\text { Olanzapine } \\
\text { adjunctive vs. } \\
\text { chlorpromazine } \\
\text { adjunctive }\end{array}$ & $\begin{array}{l}\text { Conus, } 2015^{47} \\
26485297 \\
\text { Low }\end{array}$ & $\begin{array}{l}\text { Response } \\
8 \text { weeks } \\
\text { Olanzapine } 94.7 \% \\
\text { Chlorpromazine } 97.2 \% \\
\text { NS } \\
\text { Remission } \\
8 \text { weeks } \\
\text { Olanzapine } 89.5 \% \\
\text { Chlorpromazine } 88.9 \% \\
\text { NS }\end{array}$ & $\begin{array}{l}\text { YMRS } \\
8 \text { weeks } \\
\text { NS (data not reported) } \\
\text { HAMD } \\
8 \text { weeks } \\
\text { NS (data not reported) }\end{array}$ & $\begin{array}{l}\text { CGI-BP-S } \\
8 \text { weeks } \\
\text { NS (data not } \\
\text { reported) }\end{array}$ & $\begin{array}{l}\text { Withdrawal - } \\
\text { Aes } \\
\text { Olanzapine } \\
26.3 \% \\
\text { Chlorpromazine } \\
30.6 \% \\
\text { NS }\end{array}$ & $\begin{array}{l}\text { SAE } \\
3 \text { chlorpromazine } \\
\text { (extrapyramidal } \\
\text { symptoms) } \\
2 \text { olanzapine } \\
\text { (neutropenia, sedation) } \\
\text { Weight gain } \\
\text { NS }\end{array}$ \\
\hline
\end{tabular}

Abbreviations: AE=Adverse Events; CGI-BP=Clinical Global Impressions Scale for Bipolar Disorder; CGI-BP-S-Clinical Global Impressions, Bipolar, Severity Scale; HAMD= Hamilton Scale for Depression; NS=not significant; PMID=PubMed Identification Number; ROB=risk of bias; SAE; Serious Adverse Events; SE=standard error; YMRS = Young Mania Rating Scale

\section{Appendix Table E58. Strength of evidence assessment: olanzapine plus mood stabilizers versus active comparison for acute mania}

\begin{tabular}{|c|c|c|c|c|c|c|c|c|}
\hline Comparison & Outcome & $\begin{array}{l}\text { \# Studies/ } \\
\text { Design } \\
\text { (n analyzed) }\end{array}$ & $\begin{array}{l}\text { Finding or } \\
\text { Summary } \\
\text { Statistic }\end{array}$ & $\begin{array}{c}\text { Study } \\
\text { Limitations }\end{array}$ & Consistency & Directness & Precision & $\begin{array}{c}\text { Overall } \\
\text { Gradel } \\
\text { Conclusion }\end{array}$ \\
\hline $\begin{array}{l}\text { Olanzapine } \\
\text { adjunctive vs. } \\
\text { chlorpromazin } \\
\text { e adjunctive }\end{array}$ & $\begin{array}{l}\text { Response } \\
\text { Remission } \\
\text { YMRS } \\
\text { HAMD } \\
\text { Withdrawal AE }\end{array}$ & $\begin{array}{l}1 \mathrm{RCT} \\
(\mathrm{n}=83)\end{array}$ & See table above & Low & Unknown & Direct & Imprecise & linsufficient \\
\hline
\end{tabular}

Abbreviations: CGI-BP=Clinical Global Impressions Scale for Bipolar Disorder; NS=not significant; RCT=randomized controlled trial; YMRS = Young Mania Rating Scale Notes:

1. Publication bias for antipsychotics, antidepressants, and behavioral interventions for depressive disorders is suspected.

2. Data were generally imprecise due to missing data from high attrition rates, which was commonly dealt with by Last Observation Carried Forward (LOCF). LOCF requires an assumption that the health status of patients who dropped out of the trial would not have changed had future observations been recorded, a strong assumption in the context of bipolar disorder research. 


\section{Section 12. Quetiapine Plus Mood Stabilizer}

Appendix Table E59. Characteristics of eligible studies: quetiapine plus mood stabilizer drug treatments for acute mania by year then first author

\begin{tabular}{|c|c|c|c|c|c|c|}
\hline $\begin{array}{l}\text { Study, Year } \\
\text { Design } \\
\text { Location } \\
\text { Funder } \\
\text { Risk of Bias } \\
\text { PMID }\end{array}$ & $\begin{array}{c}\text { \# Randomized } \\
\text { Age (mean) } \\
\text { Sex (\% Female) } \\
\text { Race (\% White) } \\
\text { Diagnosis } \\
\text { (\% BP I, II, NOS) } \\
\text { Setting }\end{array}$ & $\begin{array}{c}\text { Inclusions } \\
\text { Key Exclusions }\end{array}$ & $\begin{array}{c}\text { Intervention } \\
\text { Dosage }\end{array}$ & $\begin{array}{c}\text { Comparison } \\
\text { Dosage }\end{array}$ & $\begin{array}{c}\text { Follow-up } \\
\text { Duration }\end{array}$ & $\begin{array}{c}\text { Outcomes } \\
\text { Reported } \\
\text { Withdrawal (\%) at } \\
\text { endpoint }\end{array}$ \\
\hline $\begin{array}{l}\text { Yatham, } 2007^{53} \\
\text { RCT } \\
\text { Multisite } \\
\text { 4 Continents } \\
\text { Industry } \\
\text { RoB High } \\
17519644\end{array}$ & $\begin{array}{l}\mathrm{N}=200 \\
\text { Mean Age 40 } \\
\text { Female 50\% } \\
\text { Race NR } \\
\text { BP I 100\% } \\
\text { Inpatient (1 week) } \\
\text { Outpatient (weeks 2-3, } \\
\text { subject to inspector } \\
\text { discretion) }\end{array}$ & $\begin{array}{l}\text { Mania; } \\
\text { YMRS } \geq 20 \text { AND } \geq 4 \text { on } \\
\text { at least } 2 \text { YMRS } \\
\text { subscales AND CGI-BP- } \\
\mathrm{S} \geq 4 \\
\\
\text { First Manic Episode } \\
\text { Taking Other Meds } \\
\text { Pregnant/Nursing }\end{array}$ & \begin{tabular}{|l} 
Quetiapine \\
$100-800 \mathrm{mg} /$ day \\
(455 mg/day mean) \\
Adjunctive to \\
Valproate \\
$50-100 \mathrm{mcg} / \mathrm{mL}$ \\
target \\
OR \\
Lithium \\
$0.7-1.0 \mathrm{mEq} / \mathrm{L}$ target
\end{tabular} & $\begin{array}{l}\text { Placebo } \\
\text { Adjunctive to } \\
\text { Valproate } \\
50-100 \mathrm{mcg} / \mathrm{mL} \\
\text { target } \\
\text { OR } \\
\text { Lithium } \\
0.7-1.0 \mathrm{mEq} / \mathrm{L} \\
\text { target }\end{array}$ & 3 weeks & $\begin{array}{l}\text { CGI-BP-S } \\
\text { Remission (Various } \\
\text { definitions) } \\
\text { Response (YMRS } \\
\text { 50\% decrease) } \\
\text { YMRS } \\
\text { Withdrawal 36\% }\end{array}$ \\
\hline $\begin{array}{l}\text { Yatham, } 2004^{54} \\
\text { RCT } \\
\text { Multisite } \\
\text { 4 Continents } \\
\text { Industry } \\
\text { RoB Moderate } \\
15538120\end{array}$ & $\begin{array}{l}\mathrm{N}=402 \\
\text { Mean Age } 40 \\
\text { Female 47\% } \\
\text { Race NR } \\
\text { BP I 100\% } \\
\\
\text { Inpatient }\end{array}$ & $\begin{array}{l}\text { Mania; } \\
\text { At least one manic or } \\
\text { mixed episode in } \\
\text { previous } 5 \text { years. } \\
\text { YMRS } \geq 20 \text {, including } \\
\text { score } \geq 4 \text { on two core } \\
\text { YMRS items; CGI-BP } \geq \\
4 \\
\text { First Manic Episode } \\
\text { Substance Abuse } \\
\text { Other Mental Health } \\
\text { Pregnant/Nursing }\end{array}$ & $\begin{array}{l}\text { Quetiapine } \\
100-800 \mathrm{mg} / \text { day } \\
\text { (492 mg/day mean) } \\
\text { Adjunctive to } \\
\text { Valproate } \\
50-100 \mathrm{mcg} / \mathrm{mL} \\
\text { target } \\
\text { OR } \\
\text { Lithium } \\
0.7-1.0 \mathrm{mEq} / \mathrm{L} \text { target }\end{array}$ & $\begin{array}{l}\text { Placebo } \\
\text { Adjunctive to } \\
\text { Valproate } \\
50-100 \mathrm{mcg} / \mathrm{mL} \\
\text { target } \\
\text { OR } \\
\text { Lithium } \\
0.7-1.0 \mathrm{mEq} / \mathrm{L} \\
\text { target }\end{array}$ & 6 weeks & $\begin{array}{l}\text { YMRS } \\
\text { CGI-BP } \\
\text { PANSS } \\
\text { MADRS } \\
\text { GAS } \\
\text { Adverse Events } \\
\text { Withdrawals 38\% }\end{array}$ \\
\hline
\end{tabular}

Abbreviations: AIMS=Abnormal Involuntary Movement Scale; ASI=Addiction Severity Index; BAS=Behavioral Approach System; BID=Twice a day; BP=bipolar disorder; BPRS=Brief Psychiatric Rating Scale; C=Comparison; CGI-BP=Clinical Global Impressions Scale, Bipolar; CGI-BP-S=Clinical Global Impressions, Bipolar, Severity Scale; CGI-I= Clinical Global Impressions-Improvement; CGI-S== Clinical Global Impressions-Severity Scale; DSM-IV-TR= Diagnostic and statistical manual, $4^{\text {th }}$ edition, Text Revision; EPS=extrapyramidal symptoms; FAST=Functional Assessment Short Test; GAF=General Assessment of Functioning Scale; GAS= Global Assessment Scale; HAM-

$$
\mathrm{E}-141
$$


D=Hamilton Scale for Depression; HAMD-21=Hamilton Rating Scale for Depression (21-items); HDRS-21=Hamilton Depression Rating Scale (21=items); ISST=International Suicide Prevention Trial Scale for Suicidal Thinking; LIFE-RIFT=Longitudinal Interval Follow-up Evaluation-Rating Impaired Functioning Tool; MADRS=Montgomery-Asberg Depression Rating Scale; NOS=not otherwise specified; NR=not reported; OR=Odds Ratio; PANSS=Positive and Negative Syndrome Scale; PAS=Premornid Adjustment Scale; PGI-I=Patient Global Impression Improvement; PMID=PubMed Identification Number; QLS=Quality of Life Scale; RCT=randomized controlled trial; RDQ=Readiness to

Discharge Questionnaire; ROB=risk of bias; SANS=Scale for the Assessment of Negative Symptoms; SAPS=Scale for the Assessment of Positive Symptoms; SAS=Simpson Angus Scale; SF-36=36-Item Short Form Health Survey; UKU=UKU rating scale; YMRS = Young Mania Rating Scale

\section{Appendix Table E60. Summary risk of bias assessments: quetiapine plus mood stabilizers for acute mania by year then first author}

\begin{tabular}{|c|c|c|c|}
\hline Drug & $\begin{array}{l}\text { Study } \\
\text { Funding Source } \\
\text { PMID }\end{array}$ & $\begin{array}{l}\text { Overall Risk of Bias } \\
\text { Assessment }\end{array}$ & Rationale \\
\hline \multirow[t]{2}{*}{ Quetiapine } & $\begin{array}{l}\text { Yatham, } 2007^{53} \\
\text { Industry } \\
17519644\end{array}$ & High & $\begin{array}{l}\text { Randomization and blinding not described. Improper definition of ITT "consisted of all the } \\
\text { patients who had received at least one dose of study drug and had undergone a baseline AND } \\
\text { at least one postbaseline efficacy assessment"; } 2 \text { patients missing from baseline quet. } \\
\text { Measures, } 9 \text { patients missing from baseline placebo measures. }\end{array}$ \\
\hline & $\begin{array}{l}\text { Yatham, } 2004^{54} \\
\text { Industry } \\
15538120\end{array}$ & Moderate & $\begin{array}{l}\text { Blinding and randomization not described; differential dropout rates between arms may alter } \\
\text { effectiveness of randomization }\end{array}$ \\
\hline
\end{tabular}

Abbreviations: ITT=intention to treat; PMID=PubMed Identification Number 


\section{Antipsychotics Plus Mood Stabilizer Forest Plots}

Outcomes in studies assessed as having a high risk of bias, or low to moderate risk of bias but at least 40 percent attrition, are presented in grey tones. Both fixed-effect models and random-effects models are presented. We calculated fixed-effect models to provide a charitable estimate of the average effect among completed trials. However, we base our main conclusions on the random-effects models.

Appendix Figure E100. Adjunctive Quetiapine vs. placebo - response

Odds Ratio of Response ( $>50 \%$ Reduction in YMRS) at 3 Weeks

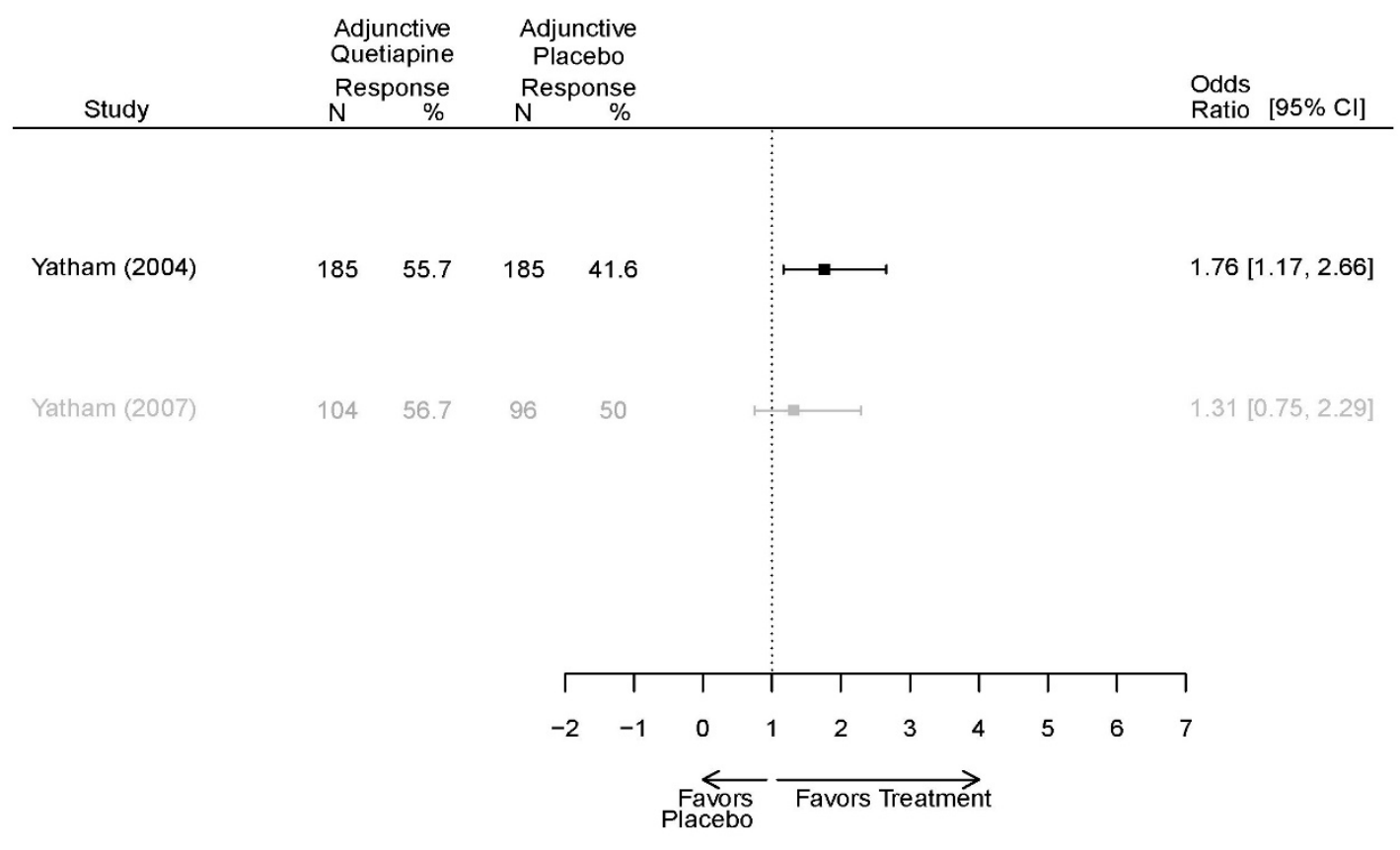


Appendix Figure E101. Adjunctive Quetiapine vs. placebo - remission

Odds Ratio of Remission (YMRS $<=12$ ) at 3 Weeks

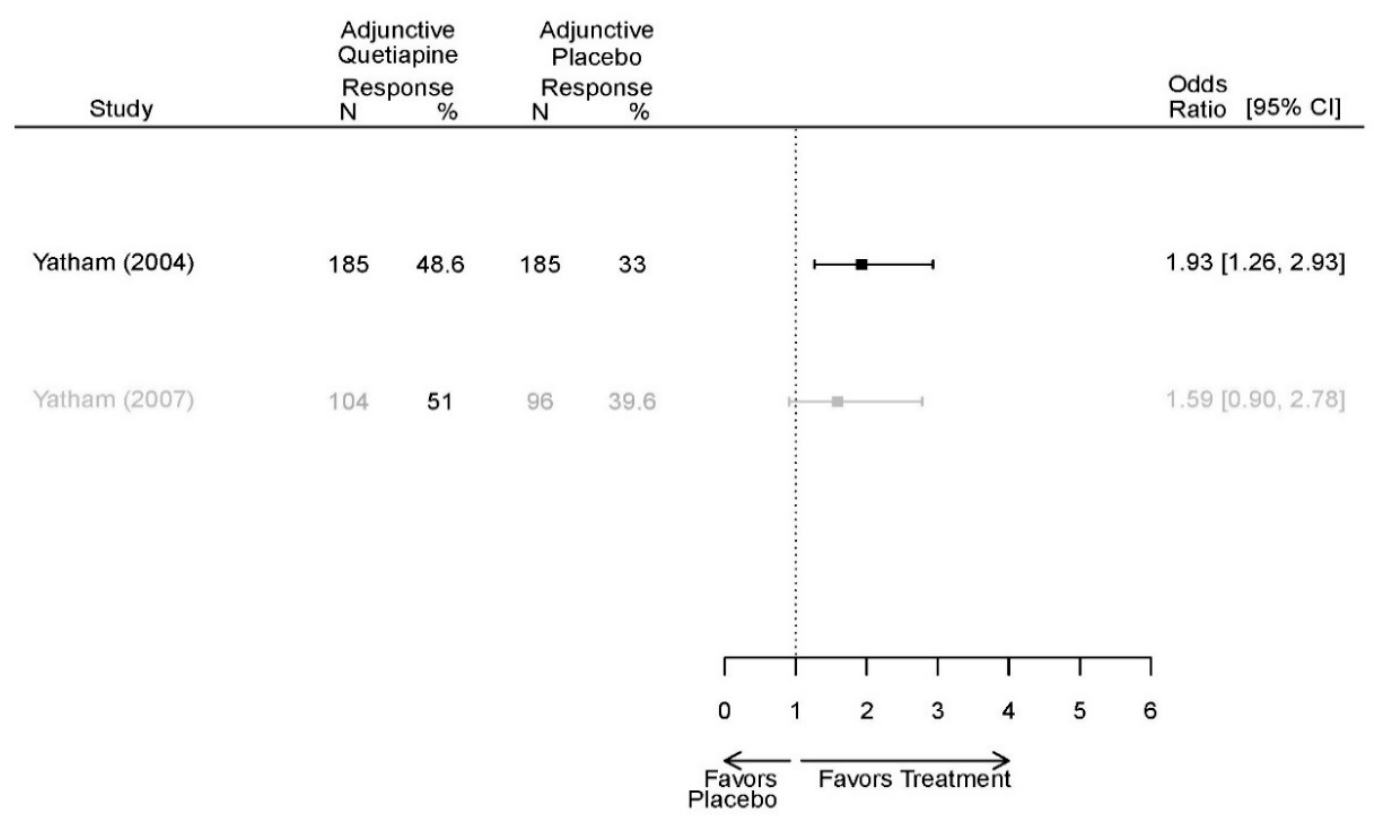

Appendix Figure E102. Adjunctive Quetiapine vs. placebo - YMRS

Difference in Mean Change in YMRS from Baseline to Last Measurement

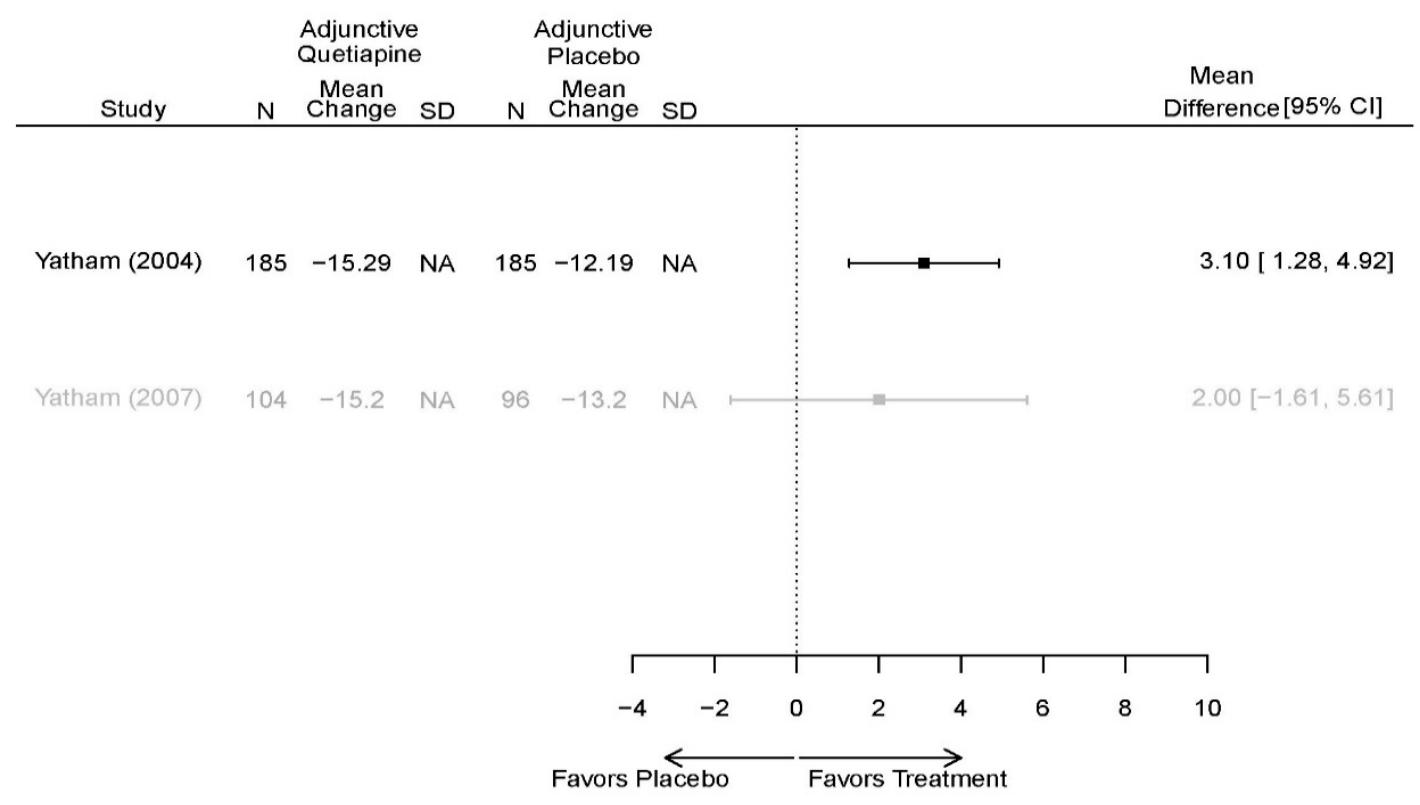


Appendix Figure E103. Adjunctive Quetiapine vs. placebo - CGI-BP-S

Difference in Mean Change in CGI-BP-S (Overall)

from Baseline to Last Measurement

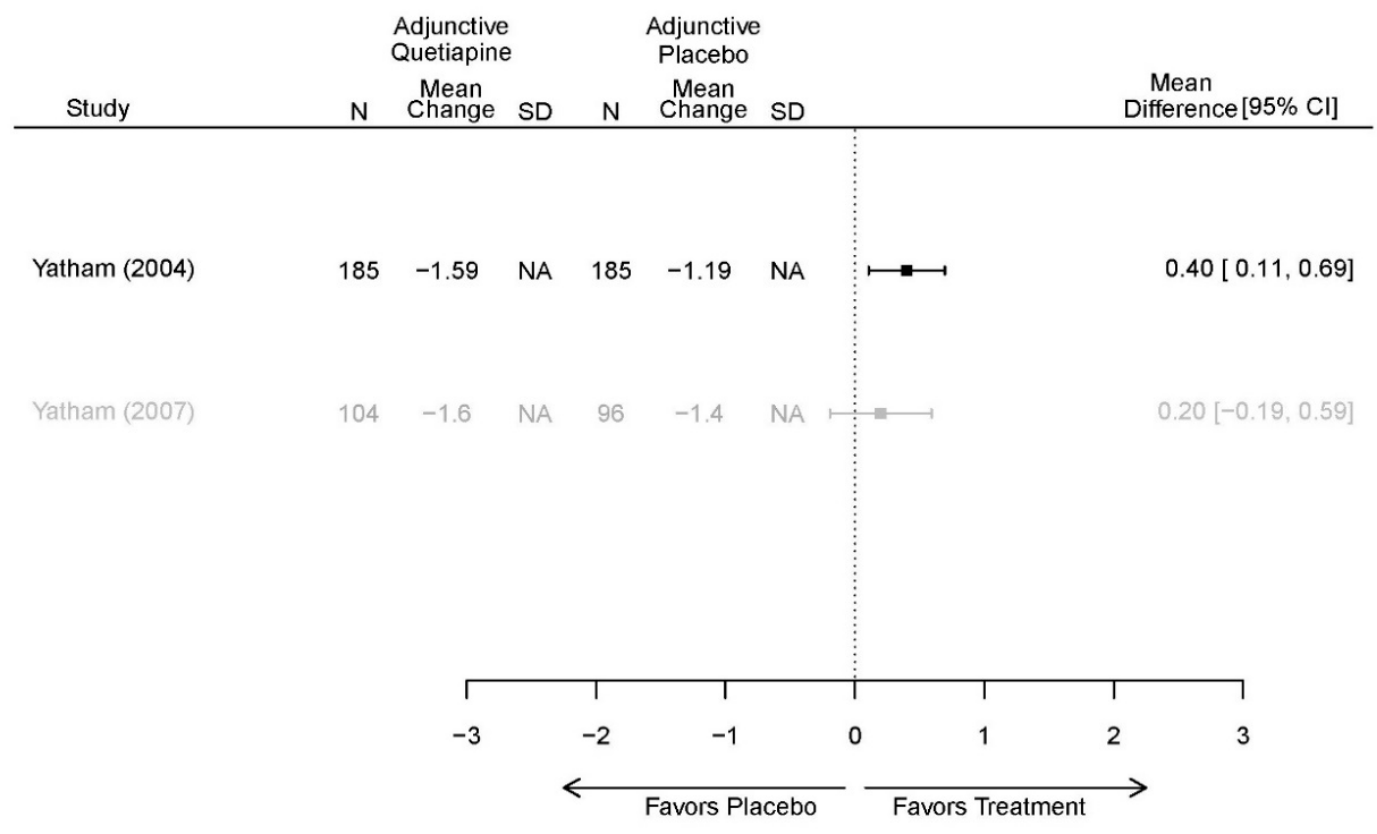

Appendix Figure E104. Adjunctive Quetiapine vs. placebo - withdrawal lack of efficacy Odds Ratio of Withdrawal due to Lack of Efficacy at 3 Weeks

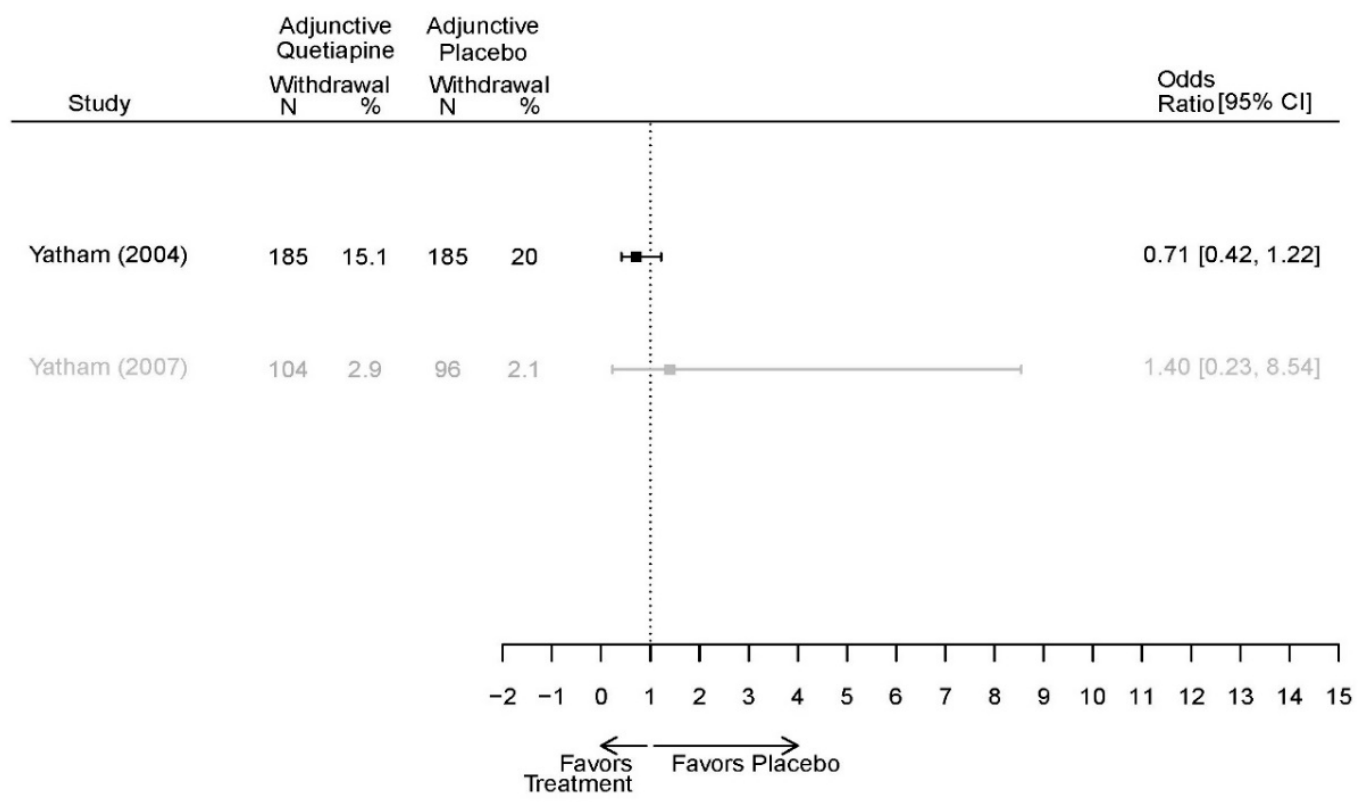


Appendix Figure E105. Adjunctive Quetiapine vs. placebo - withdrawal adverse events Odds Ratio of Withdrawal due to Adverse Events at 3 Weeks

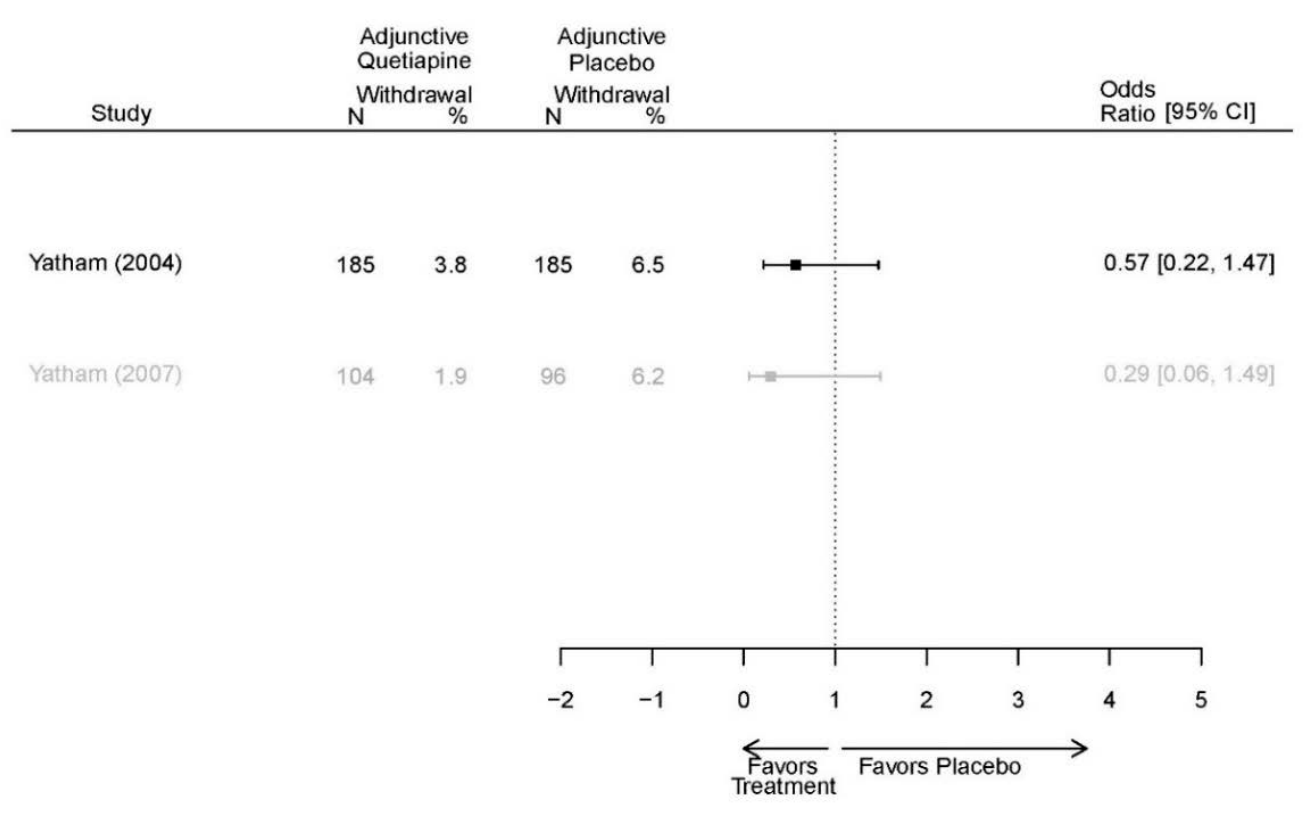

Appendix Figure E106. Adjunctive Quetiapine vs. placebo - overall withdrawal Odds Ratio of Overall Withdrawal

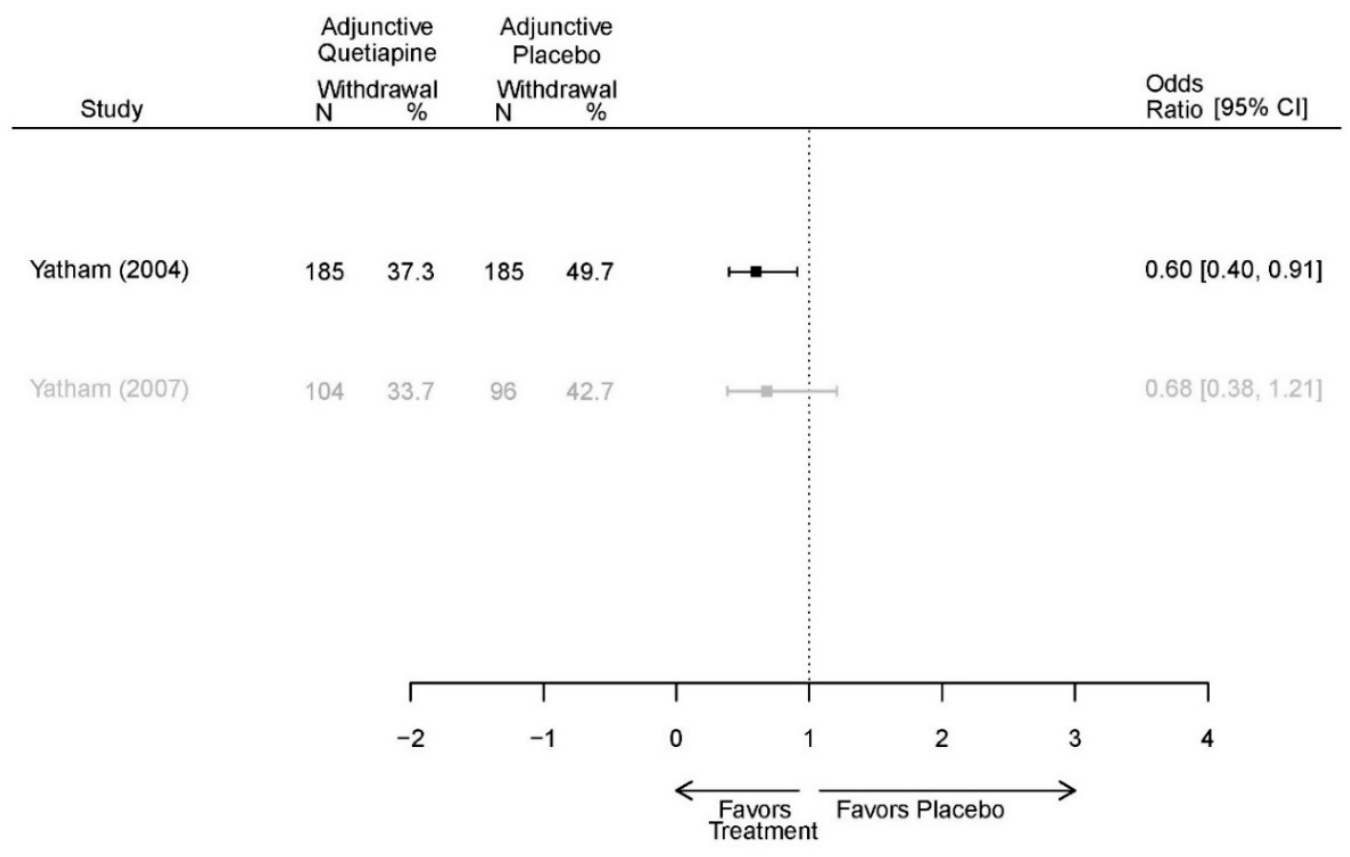




\begin{tabular}{|c|c|c|c|c|c|c|}
\hline Drug & $\begin{array}{c}\text { Study } \\
\text { PMID } \\
\text { RoB }\end{array}$ & $\begin{array}{l}\text { Responderl } \\
\text { Remitter }\end{array}$ & Symptom & Function & Other & $\mathrm{AE}$ \\
\hline \multirow[t]{2}{*}{$\begin{array}{l}\text { Quetiapine } \\
\text { adjunctive } \\
\text { vs. placebo }\end{array}$} & $\begin{array}{l}\text { Yatham, } \\
2007^{53} \\
17519644 \\
\text { High }\end{array}$ & $\begin{array}{l}\text { See forest plots } \\
\text { E101 and E102 } \\
\text { above }\end{array}$ & $\begin{array}{l}\text { See forest plot E103 } \\
\text { above }\end{array}$ & $\begin{array}{l}\text { See forest plot E104 } \\
\text { above }\end{array}$ & $\begin{array}{l}\text { See forest plots } \\
\text { E105, E106, and } \\
\text { E107 above }\end{array}$ & $\begin{array}{l}\text { Serious Adverse Events } \\
6 \text { weeks } \\
1 \text { placebo } \\
\frac{\text { Deaths }}{6 \text { weeks }} \\
1 \text { placebo } \\
\text { EPS } \\
6 \text { weeks } \\
29.2 \% \text { placebo } \\
17.9 \% \text { Quetiapine + Lithium }\end{array}$ \\
\hline & $\begin{array}{l}\text { Yatham, } \\
2004^{54} \\
15538120 \\
\text { Moderate }\end{array}$ & $\begin{array}{l}\text { See forest plots } \\
\text { E101 and E102 } \\
\text { above }\end{array}$ & $\begin{array}{l}\text { See forest plot E103 } \\
\text { above }\end{array}$ & $\begin{array}{l}\text { See forest plot E104 } \\
\text { above }\end{array}$ & $\begin{array}{l}\text { See forest plots } \\
\text { E105, E106, and } \\
\text { E107 above }\end{array}$ & $\begin{array}{l}\text { Serious Adverse Events } \\
6 \text { weeks } \\
0 \text { in all arms } \\
\text { Deaths } \\
6 \text { weeks } \\
0 \text { in all arms } \\
\text { EPS } \\
6 \text { weeks } \\
19.2 \% \text { placebo } \\
21.4 \% \text { Quetiapine + Lithium }\end{array}$ \\
\hline
\end{tabular}

Abbreviations: AE=Adverse Events; ASI=Addiction Severity Index; BPRS=Brief Psychiatric Rating Scale; CGI-Bp=Clinical Global Impresions Scale for Bipolar Disorder; CGIBP-S=Clinical Global Impressions, Bipolar, Severity Scale; CGI-S=Clinical Global Impressions, Severity Scale; CI=Confidence Interval; EPS=extrapyramidal symptoms;

GAF=General Assessment of Functioning Scale; HAM-D=Hamilton Scale for Depression; MADRS=Montgomery-Asberg Syndrome Scale; NR=not reported; NS=not significant; OR=Odds Ratio; PAS=Premorbid Adjustment Scale; PMID=PubMed Identification Number; QLS=Quality of Life Scale; ROB=risk of bias; SAE=Serious Adverse Events;

$\mathrm{SE}=$ Standard Error; UKU=UKU rating scale;YMRS = Young Mania Rating Scale 
Appendix Table E62. Strength of evidence assessment: quetiapine plus mood stabilizers versus placebo for acute mania

\begin{tabular}{|c|c|c|c|c|c|c|c|c|}
\hline Comparison & Outcome & $\begin{array}{l}\text { \# Studies/ } \\
\text { Design } \\
\text { (n analyzed) }\end{array}$ & $\begin{array}{l}\text { Finding or } \\
\text { Summary } \\
\text { Statistic }\end{array}$ & $\begin{array}{c}\text { Study } \\
\text { Limitations }\end{array}$ & Consistency & Directness & Precision & $\begin{array}{c}\text { Overall } \\
\text { Gradel } \\
\text { Conclusion }\end{array}$ \\
\hline $\begin{array}{l}\text { Quetiapine } \\
\text { adjunctive vs. } \\
\text { placebo }\end{array}$ & $\begin{array}{l}\text { Response } 3 \text { wks } \\
\text { Remission } 3 \text { wks } \\
\text { YMRS } 3 \text { wks } \\
\text { CGI-BP } 3 \text { wks } \\
\text { Withdrawals }\end{array}$ & $\begin{array}{l}2 \text { RCTs } \\
(n=570)\end{array}$ & $\begin{array}{l}\text { See forest plots } \\
\text { above }\end{array}$ & High & Consistent & Direct & Imprecise & Insufficient \\
\hline
\end{tabular}

Abbreviations: CGI-BP=Clinical Global Impressions Scale for Bipolar Disorder; NS=not significant; RCT=randomized controlled trial; YMRS = Young Mania Rating Scale Notes:

1. Publication bias for antipsychotics, antidepressants, and behavioral interventions for depressive disorders is suspected.

2. Data were generally imprecise due to missing data from high attrition rates, which was commonly dealt with by Last Observation Carried Forward (LOCF). LOCF requires an

assumption that the health status of patients who dropped out of the trial would not have changed had future observations been recorded, a strong assumption in the context of bipolar disorder research. 


\section{Section 13. Risperidone Plus Mood Stabilizer}

Appendix Table E63. Characteristics of eligible studies: risperidone plus mood stabilizer drug treatments for acute mania by year then first author

\begin{tabular}{|c|c|c|c|c|c|c|}
\hline $\begin{array}{c}\text { Study, Year } \\
\text { Design } \\
\text { Location } \\
\text { Funder } \\
\text { Risk of Bias } \\
\text { PMID }\end{array}$ & $\begin{array}{c}\text { \# Randomized } \\
\text { Age (mean) } \\
\text { Sex (\% Female) } \\
\text { Race (\% White) } \\
\text { Diagnosis } \\
\text { (\% BP I, II, NOS) } \\
\text { Setting }\end{array}$ & $\begin{array}{c}\text { Inclusions } \\
\text { Key Exclusions }\end{array}$ & $\begin{array}{c}\text { Intervention } \\
\text { Dosage }\end{array}$ & $\begin{array}{c}\text { Comparison } \\
\text { Dosage }\end{array}$ & $\begin{array}{c}\text { Follow-up } \\
\text { Duration }\end{array}$ & $\begin{array}{c}\text { Outcomes } \\
\text { Reported } \\
\text { Withdrawal (\%) at } \\
\text { endpoint }\end{array}$ \\
\hline $\begin{array}{l}\text { Yatham, } 2003^{55} \\
\text { RCT } \\
\text { Multisite } \\
4 \text { Continents } \\
\text { Industry } \\
\text { RoB Low } \\
12562742\end{array}$ & $\begin{array}{l}\mathrm{N}=151 \\
\text { Mean Age } 40 \\
\text { Female 58\% } \\
\text { Race NR } \\
\text { Inpatient }\end{array}$ & $\begin{array}{l}\text { Mania; } \\
\text { YMRS } \geq 20 \\
\text { Schizoaffective } \\
\text { Substance Abuse } \\
\text { Other Mental Health } \\
\text { Pregnant/Nursing } \\
\text { Labs/Other Conditions }\end{array}$ & $\begin{array}{l}\text { Risperidone } \\
\text { 2-6 mg/day (Mean } \\
4.0 \mathrm{mg} / \text { day) } \\
\\
\text { Adjunctive to } \\
\text { Lithium } \\
\text { or } \\
\text { Divalproex } \\
\text { or } \\
\text { Carbamazepine }\end{array}$ & $\begin{array}{l}\text { Placebo } \\
\text { Adjunctive to } \\
\text { Lithium } \\
\text { or } \\
\text { Divalproex } \\
\text { or } \\
\text { Carbamazepine }\end{array}$ & 3 weeks & $\begin{array}{l}\text { Response (YMRS } \\
50 \% \text { decrease) } \\
\text { BPRS } \\
\text { CGI } \\
\text { HAM-D } \\
\text { YMRS } \\
\text { Withdrawal 44\% }\end{array}$ \\
\hline
\end{tabular}

Abbreviations: AIMS=Abnormal Involuntary Movement Scale; ASI=Addiction Severity Index; BAS=Behavioral Approach System; BID=Twice a day; BP=bipolar disorder; BPRS=Brief Psychiatric Rating Scale; C=Comparison; CGI-BP=Clinical Global Impressions Scale, Bipolar; CGI-BP-S=Clinical Global Impressions, Bipolar, Severity Scale; CGI-I= Clinical Global Impressions-Improvement; CGI-S== Clinical Global Impressions-Severity Scale; DSM-IV-TR= Diagnostic and statistical manual, $4^{\text {th }}$ edition, Text Revision; EPS=extrapyramidal symptoms; FAST=Functional Assessment Short Test; GAF=General Assessment of Functioning Scale; GAS= Global Assessment Scale; HAM$\mathrm{D}=$ Hamilton Scale for Depression; HAMD-21=Hamilton Rating Scale for Depression (21-items); HDRS-21=Hamilton Depression Rating Scale (21=items); ISST=International Suicide Prevention Trial Scale for Suicidal Thinking; LIFE-RIFT=Longitudinal Interval Follow-up Evaluation-Rating Impaired Functioning Tool; MADRS=Montgomery-Asberg Depression Rating Scale; NOS=not otherwise specified; NR=not reported; OR=Odds Ratio; PANSS=Positive and Negative Syndrome Scale; PAS=Premornid Adjustment Scale; PGI-I=Patient Global Impression Improvement; PMID=PubMed Identification Number; QLS=Quality of Life Scale; RCT=randomized controlled trial; RDQ=Readiness to Discharge Questionnaire; ROB=risk of bias; SANS=Scale for the Assessment of Negative Symptoms; SAPS=Scale for the Assessment of Positive Symptoms; SAS=Simpson Angus Scale; SF-36=36-Item Short Form Health Survey; UKU=UKU rating scale; YMRS = Young Mania Rating Scale 
Appendix Table E64. Summary risk of bias assessments: risperidone plus mood stabilizers for acute mania

\begin{tabular}{|c|l|l|l|}
\hline Drug & $\begin{array}{c}\text { Study } \\
\text { Funding Source } \\
\text { PMID }\end{array}$ & $\begin{array}{c}\text { Overall Risk of Bias } \\
\text { Assessment }\end{array}$ & \multicolumn{1}{c|}{ Rationale } \\
\hline Risperidone & $\begin{array}{l}\text { Yatham, 200355 } \\
\text { Industry } \\
12562742\end{array}$ & Low & $\begin{array}{l}\text { A well described and reported study with some minor lack of disclosure in blinding procedure } \\
\text { and adverse events reporting. 44\% dropout. }\end{array}$ \\
\hline
\end{tabular}

Abbreviations: ITT=intention to treat; PMID=PubMed Identification Number

\section{Appendix Table E65. Outcomes summary: risperidone plus mood stabilizers versus placebo for acute mania}

\begin{tabular}{|c|c|c|c|c|c|c|}
\hline Drug & $\begin{array}{c}\text { Study } \\
\text { PMID } \\
\text { RoB } \\
\end{array}$ & $\begin{array}{l}\text { Responderl } \\
\text { Remitter }\end{array}$ & Symptom & Function & Other & $\mathrm{AE}$ \\
\hline $\begin{array}{l}\text { Risperidone } \\
\text { adjunctive } \\
\text { vs. placebo }\end{array}$ & $\begin{array}{l}\text { Yatham, } \\
2003^{55} \\
12562742 \\
\text { Low }\end{array}$ & $\begin{array}{l}\text { Response, Mean } \\
\text { Difference } \\
3 \text { weeks } \\
17.7 \%(95 \% \mathrm{Cl} 0.8- \\
33.5) ; \mathrm{p}<0.05 \\
\text { Favors intervention }\end{array}$ & $\begin{array}{l}\text { YMRS, Mean } \\
\text { Difference in Change } \\
\text { Score } \\
3 \text { weeks } \\
-4.2(95 \% \mathrm{Cl}-7.60, \\
0.53) ; p=0.09 \\
\text { NS } \\
\text { HAM-D } \\
3 \text { weeks } \\
\text { NS } \\
\text { No statistical test } \\
\text { reported } \\
\text { BPRS, Mean Change } \\
3 \text { weeks } \\
\text { Risperidone=-10.1 } \\
\text { Placebo=-4.8 } \\
\text { p=0.01 } \\
\text { Favors intervention }\end{array}$ & $\begin{array}{l}\text { CGI-BP, Mean } \\
\text { Difference in } \\
\text { Responders } \\
3 \text { weeks } \\
17.5 \%(95 \% \text { CI 1.1- } \\
33.9) \\
\text { Favors Intervention }\end{array}$ & $\begin{array}{l}\text { Overall Withdrawal } \\
3 \text { weeks } \\
\text { Risperidone }=36 \% \\
\text { Placebo }=52 \% \\
\text { Mean difference in } \\
\text { completion } \\
\text { rates }=16 \%(95 \% \mathrm{Cl} \\
0.32,31.68) \\
\text { Favors Intervention }\end{array}$ & $\begin{array}{l}\text { One or More AEs, Between } \\
\text { Group Difference } \\
3 \text { weeks } \\
6 \%(95 \% \mathrm{Cl}-9.9,21.9) \\
\text { NS } \\
\text { EPS Symptoms } \\
3 \text { weeks } \\
\text { Risperidone }=16 / 75 \\
\text { Placebo }=8 / 75 \\
P=0.013\end{array}$ \\
\hline
\end{tabular}

Abbreviations: AE=Adverse Events; ASI=Addiction Severity Index; BPRS=Brief Psychiatric Rating Scale; CGI-Bp=Clinical Global Impresions Scale for Bipolar Disorder; CGIBP-S=Clinical Global Impressions, Bipolar, Severity Scale; CGI-S=Clinical Global Impressions, Severity Scale; CI=Confidence Interval; EPS=extrapyramidal symptoms; GAF=General Assessment of Functioning Scale; HAM-D=Hamilton Scale for Depression; MADRS=Montgomery-Asberg Syndrome Scale; NR=not reported; NS=not significant; OR=Odds Ratio; PAS=Premorbid Adjustment Scale; PMID=PubMed Identification Number; QLS=Quality of Life Scale; ROB=risk of bias; SAE=Serious Adverse Events;

$\mathrm{SE}=$ Standard Error; UKU=UKU rating scale; YMRS = Young Mania Rating Scale 
Appendix Table E66. Strength of evidence assessment: risperidone plus mood stabilizers versus placebo for acute mania

\begin{tabular}{|c|c|c|c|c|c|c|c|c|}
\hline Comparison & Outcome & $\begin{array}{c}\text { \# Studies/ } \\
\text { Design } \\
\text { (n analyzed) }\end{array}$ & $\begin{array}{c}\text { Finding or } \\
\text { Summary } \\
\text { Statistic }\end{array}$ & $\begin{array}{c}\text { Study } \\
\text { Limitations }\end{array}$ & Consistency & Directness & Precision & $\begin{array}{c}\text { Overall } \\
\text { Gradel } \\
\text { Conclusion }\end{array}$ \\
\hline $\begin{array}{l}\text { Risperidone } \\
\text { adjunctive vs. } \\
\text { placebo }\end{array}$ & $\begin{array}{l}\text { Response } 3 \text { wks } \\
\text { YMRS } 3 \text { wks } \\
\text { CGI-BP } 3 \text { wks } \\
\text { Withdrawal - overall }\end{array}$ & $\begin{array}{l}1 \mathrm{RCT} \\
(\mathrm{n}=150)\end{array}$ & See table above & Low & Unknown & Direct & Imprecise & Insufficient \\
\hline
\end{tabular}

Abbreviations: CGI-BP=Clinical Global Impressions Scale for Bipolar Disorder; NS=not significant; RCT=randomized controlled trial; YMRS = Young Mania Rating Scale

Notes:

1. Publication bias for antipsychotics, antidepressants, and behavioral interventions for depressive disorders is suspected.

2. Data were generally imprecise due to missing data from high attrition rates, which was commonly dealt with by Last Observation Carried Forward (LOCF). LOCF requires an

assumption that the health status of patients who dropped out of the trial would not have changed had future observations been recorded, a strong assumption in the context of

bipolar disorder research. 


\section{Section 14. Ziprazidone Plus Mood Stabilizer}

Appendix Table E67. Characteristics of eligible studies: ziprasidone plus mood stabilizer drug treatments for acute mania

\begin{tabular}{|c|c|c|c|c|c|c|}
\hline $\begin{array}{c}\text { Study, Year } \\
\text { Design } \\
\text { Location } \\
\text { Funder } \\
\text { Risk of Bias } \\
\text { PMID }\end{array}$ & $\begin{array}{c}\text { \# Randomized } \\
\text { Age (mean) } \\
\text { Sex (\% Female) } \\
\text { Race (\% White) } \\
\text { Diagnosis } \\
\text { (\% BP I, II, NOS) } \\
\text { Setting }\end{array}$ & $\begin{array}{c}\text { Inclusions } \\
\text { Key Exclusions }\end{array}$ & $\begin{array}{l}\text { Intervention } \\
\text { Dosage }\end{array}$ & $\begin{array}{c}\text { Comparison } \\
\text { Dosage }\end{array}$ & $\begin{array}{c}\text { Follow-up } \\
\text { Duration }\end{array}$ & $\begin{array}{c}\text { Outcomes } \\
\text { Reported } \\
\text { Withdrawal (\%) at } \\
\text { endpoint }\end{array}$ \\
\hline $\begin{array}{l}\text { Sachs, } 2012^{56} \\
\text { RCT } \\
\text { Multisite } \\
\text { US } \\
\text { Industry } \\
\text { RoB High } \\
23218157\end{array}$ & $\begin{array}{l}\mathrm{N}=680 \\
\text { Mean Age } 41 \\
\text { Female } 50 \% \\
\text { White } 65 \% \\
\text { BP I } 100 \% \\
\text { Outpatient }\end{array}$ & $\begin{array}{l}\text { Mania; } \\
\text { YMRS } \geq 18 \text { with } 25 \% \\
\text { improvement between } \\
\text { screening and baseline; } \\
\text { current episode } \leq 3 \\
\text { months } \\
\\
\text { First manic episode } \\
\text { Schizoaffective } \\
\text { Substance Abuse } \\
\text { Other Mental Health } \\
\text { Conditions } \\
\text { Taking Other Meds } \\
\text { Labs/Other Conditions }\end{array}$ & 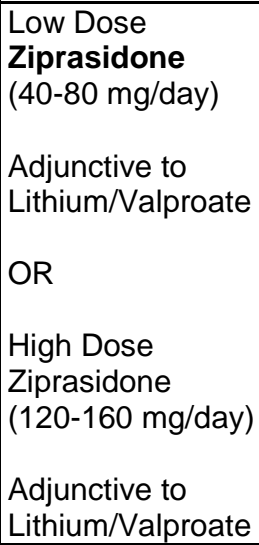 & $\begin{array}{l}\text { Placebo } \\
\text { Adjunctive to } \\
\text { Lithium/Valproate }\end{array}$ & 3 weeks & \begin{tabular}{|l} 
CGI-I \\
CGI-S \\
GAF \\
LIFE-RIFT \\
MADRS \\
PANSS \\
YMRS \\
Withdrawal 42\%
\end{tabular} \\
\hline
\end{tabular}

Abbreviations: AIMS=Abnormal Involuntary Movement Scale; ASI=Addiction Severity Index; BAS=Behavioral Approach System; BID=Twice a day; BP=bipolar disorder; BPRS=Brief Psychiatric Rating Scale; C=Comparison; CGI-BP=Clinical Global Impressions Scale, Bipolar; CGI-BP-S=Clinical Global Impressions, Bipolar, Severity Scale; CGI-I= Clinical Global Impressions-Improvement; CGI-S== Clinical Global Impressions-Severity Scale; DSM-IV-TR= Diagnostic and statistical manual, $4^{\text {th }}$ edition, Text Revision; EPS=extrapyramidal symptoms; FAST=Functional Assessment Short Test; GAF=General Assessment of Functioning Scale; GAS= Global Assessment Scale; HAM$\mathrm{D}=$ Hamilton Scale for Depression; HAMD-21=Hamilton Rating Scale for Depression (21-items); HDRS-21=Hamilton Depression Rating Scale (21=items); ISST=International Suicide Prevention Trial Scale for Suicidal Thinking; LIFE-RIFT=Longitudinal Interval Follow-up Evaluation-Rating Impaired Functioning Tool; MADRS=Montgomery-Asberg Depression Rating Scale; NOS=not otherwise specified; NR=not reported; OR=Odds Ratio; PANSS=Positive and Negative Syndrome Scale; PAS=Premornid Adjustment Scale; PGI-I=Patient Global Impression Improvement; PMID=PubMed Identification Number; QLS=Quality of Life Scale; RCT=randomized controlled trial; RDQ=Readiness to Discharge Questionnaire; ROB=risk of bias; SANS=Scale for the Assessment of Negative Symptoms; SAPS=Scale for the Assessment of Positive Symptoms; SAS=Simpson Angus Scale; SF-36=36-Item Short Form Health Survey; UKU=UKU rating scale; YMRS = Young Mania Rating Scale 
Appendix Table E68. Summary risk of bias assessments: ziprasidone plus mood stabilizers for acute mania

\begin{tabular}{|c|c|c|c|}
\hline Drug & $\begin{array}{c}\text { Study } \\
\text { Funding Source } \\
\text { PMID }\end{array}$ & $\begin{array}{l}\text { Overall Risk of Bias } \\
\text { Assessment }\end{array}$ & Rationale \\
\hline Ziprasidone & $\begin{array}{l}\text { Sachs, } 2012^{56} \\
\text { Industry } \\
23218157\end{array}$ & High & $\begin{array}{l}\text { Randomization and blinding not disclosed. Did not address how } 680 \text { were randomized but only } \\
656 \text { treated and analyzed. ITT noted, but they call this a modified ITT. }\end{array}$ \\
\hline
\end{tabular}

Abbreviations: ITT=intention to treat; PMID=PubMed Identification Number

\section{Appendix Table E69. Outcomes summary: ziprasidone plus mood stabilizers versus placebo for acute mania}

\begin{tabular}{|c|c|c|c|c|c|c|}
\hline Drug & $\begin{array}{c}\text { Study } \\
\text { PMID } \\
\text { RoB }\end{array}$ & $\begin{array}{l}\text { Responderl } \\
\text { Remitter }\end{array}$ & Symptom & Function & Other & $\mathrm{AE}$ \\
\hline $\begin{array}{l}\text { Ziprasidon } \\
\text { e } \\
\text { adjunctive } \\
\text { vs. placebo }\end{array}$ & $\begin{array}{l}\text { Sachs, } 2012^{56} \\
23218157 \\
\text { High }\end{array}$ & NR & $\begin{array}{l}\text { YMRS Change } \\
3 \text { week } \\
\text { Low dose NS } \\
\text { High dose NS }\end{array}$ & $\begin{array}{l}\frac{\text { CGI-S }}{3 \text { week }} \\
\text { Low dose NS } \\
\text { High dose NS } \\
\frac{\text { GAF }}{3 \text { week }} \\
\text { Low dose NS } \\
\text { High dose NS }\end{array}$ & $\begin{array}{l}\text { Overall Withdrawal } \\
\text { Low dose } 48 / 226 \\
\text { High dose } 62 / 232 \\
\text { Placebo } 38 / 222 \\
\text { Withdrawal Lack of } \\
\text { efficacy } \\
\text { Low dose } 6 / 226 \\
\text { High dose } 4 / 232 \\
\text { Placebo } 8 / 222 \\
\text { Withdrawal Adverse } \\
\text { event } \\
\text { Low dose } 15 / 226 \\
\text { High dose } 33 / 232 \\
\text { Placebo } 11 / 222\end{array}$ & $\begin{array}{l}\text { SAE } \\
11 \text { ziprasidone groups } \\
6 \text { placebo } \\
\text { EPS } \\
\text { Low dose } 1.9 \% \\
\text { High dose } 4.9 \% \\
\text { Placebo } 0.5 \%\end{array}$ \\
\hline
\end{tabular}

Abbreviations: AE=Adverse Events; ASI=Addiction Severity Index; BPRS=Brief Psychiatric Rating Scale; CGI-Bp=Clinical Global Impresions Scale for Bipolar Disorder; CGIBP-S=Clinical Global Impressions, Bipolar, Severity Scale; CGI-S=Clinical Global Impressions, Severity Scale; CI=Confidence Interval; EPS=extrapyramidal symptoms; GAF=General Assessment of Functioning Scale; HAM-D=Hamilton Scale for Depression; MADRS=Montgomery-Asberg Syndrome Scale; NR=not reported; NS=not significant; OR=Odds Ratio; PAS=Premorbid Adjustment Scale; PMID=PubMed Identification Number; QLS=Quality of Life Scale; ROB=risk of bias; SAE=Serious Adverse Events; $\mathrm{SE}=$ Standard Error; UKU=UKU rating scale;YMRS = Young Mania Rating Scale 
Appendix Table E70. Strength of evidence assessment: ziprasidone plus mood stabilizers versus placebo for acute mania

\begin{tabular}{|c|c|c|c|c|c|c|c|c|}
\hline Comparison & Outcome & $\begin{array}{l}\text { \# Studies/ } \\
\text { Design } \\
\text { (n analyzed) }\end{array}$ & $\begin{array}{l}\text { Finding or } \\
\text { Summary } \\
\text { Statistic }\end{array}$ & $\begin{array}{c}\text { Study } \\
\text { Limitations }\end{array}$ & Consistency & Directness & Precision & $\begin{array}{c}\text { Overall } \\
\text { Gradel } \\
\text { Conclusion }\end{array}$ \\
\hline $\begin{array}{l}\text { Ziprasidone } \\
\text { adjunctive vs. } \\
\text { placebo }\end{array}$ & $\begin{array}{l}\text { YMRS } 3 \text { wks } \\
\text { CGI-BP } 3 \text { wks } \\
\text { Withdrawal }\end{array}$ & $\begin{array}{l}1 \mathrm{RCT} \\
(\mathrm{n}=680)\end{array}$ & See table above & High & Unknown & Direct & Imprecise & Insufficient \\
\hline
\end{tabular}

Abbreviations: CGI-BP=Clinical Global Impressions Scale for Bipolar Disorder; NS=not significant; RCT=randomized controlled trial; YMRS = Young Mania Rating Scale Notes:

1. Publication bias for antipsychotics, antidepressants, and behavioral interventions for depressive disorders is suspected.

2. Data were generally imprecise due to missing data from high attrition rates, which was commonly dealt with by Last Observation Carried Forward (LOCF). LOCF requires an assumption that the health status of patients who dropped out of the trial would not have changed had future observations been recorded, a strong assumption in the context of bipolar disorder research. 


\section{References for Appendix E}

1. Kanba S, Kawasaki H, Ishigooka J, et al. A placebo-controlled, double-blind study of the efficacy and safety of aripiprazole for the treatment of acute manic or mixed episodes in Asian patients with bipolar I disorder (the AMAZE study). The World Journal of Biological Psychiatry. 2014 2014;15(2):113-21. doi: http://dx.doi.org/10.3109/15622975.2012.669047. PMID: 22540407.

2. Young AH, Oren DA, Lowy A, et al. Aripiprazole monotherapy in acute mania: 12week randomised placebo- and haloperidolcontrolled study. British Journal of Psychiatry. 2009 Jan;194(1):40-8. doi: http://dx.doi.org/10.1192/bjp.bp.108.049965. PMID: 19118324.

3. Sachs G, Bowden C, Calabrese JR, et al. Effects of lamotrigine and lithium on body weight during maintenance treatment of bipolar I disorder. Bipolar Disorders. 2006 Apr;8(2):175-81. PMID: 16542188.

4. Vieta E, Mullen J, Brecher M, et al. Quetiapine monotherapy for mania associated with bipolar disorder: combined analysis of two international, double-blind, randomised, placebo-controlled studies. Current Medical Research \& Opinion. 2005 Jun;21(6):923-34. PMID: 15969892.

5. Sachs G, Sanchez R, Marcus R, et al. Aripiprazole in the treatment of acute manic or mixed episodes in patients with bipolar I disorder: a 3-week placebo-controlled study. Journal of Psychopharmacology. 2006 Jul;20(4):536-46. PMID: 16401666.

6. Vieta E, Bourin M, Sanchez R, et al. Effectiveness of aripiprazole v. haloperidol in acute bipolar mania: double-blind, randomised, comparative 12-week trial. British Journal of Psychiatry. 2005 Sep;187:235-42. PMID: 16135860.

7. Landbloom RL, Mackle $\mathrm{M}, \mathrm{Wu} \mathrm{X}$, et al. Asenapine: Efficacy and safety of 5 and $10 \mathrm{mg}$ bid in a 3-week, randomized, double-blind, placebocontrolled trial in adults with a manic or mixed episode associated with bipolar I disorder. Journal of Affective Disorders. 2016 Jan 15;190:103-10. doi: http://dx.doi.org/10.1016/j.jad.2015.06.059. PMID: 26496015.
8. McIntyre RS. Aripiprazole for the maintenance treatment of bipolar I disorder: A review. Clinical Therapeutics. 2010;32 Suppl 1:S32-8. doi: http://dx.doi.org/10.1016/j.clinthera.2010.01.022. PMID: 20152551.

9. McIntyre RS, Cohen M, Zhao J, et al. A 3-week, randomized, placebo-controlled trial of asenapine in the treatment of acute mania in bipolar mania and mixed states.[Erratum appears in Bipolar Disord. 2010 May;12(3):350]. Bipolar Disorders. 2009 Nov;11(7):673-86. doi: http://dx.doi.org/10.1111/j.13995618.2009.00748.x. PMID: 19839993.

10. Calabrese JR, Keck PE, Jr., Starace A, et al. Efficacy and safety of low- and high-dose cariprazine in acute and mixed mania associated with bipolar I disorder: a double-blind, placebocontrolled study. Journal of Clinical Psychiatry. 2015 Mar;76(3):284-92. doi: http://dx.doi.org/10.4088/JCP.14m09081. PMID: 25562205.

11. Durgam S, Starace A, Li D, et al. The efficacy and tolerability of cariprazine in acute mania associated with bipolar I disorder: A phase II trial. Bipolar Disorders. 2015 Feb;17(1):63-75. doi: http://dx.doi.org/10.1111/bdi.12238. PMID: 25056368 (pubmed) 2014-30788-001(Psychinfo).

12. McIntyre RS, Cohen M, Zhao J, et al. Asenapine in the treatment of acute mania in bipolar I disorder: a randomized, double-blind, placebocontrolled trial. Journal of Affective Disorders. 2010 Apr;122(1-2):27-38. doi: http://dx.doi.org/10.1016/j.jad.2009.12.028. PMID: 20096936.

13. Sachs GS, Greenberg WM, Starace A, et al. Cariprazine in the treatment of acute mania in bipolar I disorder: a double-blind, placebocontrolled, phase III trial. Journal of Affective Disorders. 2015 Mar 15;174:296-302. doi: http://dx.doi.org/10.1016/j.jad.2014.11.018. PMID: 25532076.

14. McIntyre RS, Brecher M, Paulsson B, et al. Quetiapine or haloperidol as monotherapy for bipolar mania--a 12-week, double-blind, randomised, parallel-group, placebo-controlled trial. European Neuropsychopharmacology. 2005 Oct;15(5):573-85. PMID: 16139175. 
15. Smulevich AB, Khanna S, Eerdekens M, et al. Acute and continuation risperidone monotherapy in bipolar mania: a 3-week placebo-controlled trial followed by a 9-week double-blind trial of risperidone and haloperidol. European Neuropsychopharmacology. 2005 Jan;15(1):7584. PMID: 15572276.

16. McIntyre RS, Cucchiaro J, Pikalov A, et al. Lurasidone in the treatment of bipolar depression with mixed (subsyndromal hypomanic) features: post hoc analysis of a randomized placebocontrolled trial. Journal of Clinical Psychiatry. 2015 Apr;76(4):398-405. doi: http://dx.doi.org/10.4088/JCP.14m09410. PMID: 25844756.

17. Sachs GS, Grossman F, Ghaemi SN, et al. Combination of a mood stabilizer with risperidone or haloperidol for treatment of acute mania: a double-blind, placebo-controlled comparison of efficacy and safety. American Journal of Psychiatry. 2002 Jul;159(7):1146-54. PMID: 12091192.

18. Vieta E, Nuamah IF, Lim P, et al. A randomized, placebo- and active-controlled study of paliperidone extended release for the treatment of acute manic and mixed episodes of bipolar I disorder. Bipolar Disorders. 2010 May;12(3):23043. doi: http://dx.doi.org/10.1111/j.13995618.2010.00815.x. PMID: 20565430.

19. Xu L, Lu Y, Yang Y, et al. Olanzapine-valproate combination versus olanzapine or valproate monotherapy in the treatment of bipolar imania: A randomized controlled study in a chinese population group. Neuropsychiatric Disease and Treatment. 201525 May;11:1265-71. doi: http://dx.doi.org/10.2147/NDT.S81146. PMID: 26060401 (pubmed) 2015078440 (embase).

20. Katagiri H, Takita Y, Tohen M, et al. Efficacy and safety of olanzapine in the treatment of Japanese patients with bipolar I disorder in a current manic or mixed episode: a randomized, double-blind, placebo- and haloperidol-controlled study. Journal of Affective Disorders. 2012 Feb;136(3):476-84. doi: http://dx.doi.org/10.1016/j.jad.2011.10.045. PMID: 22134043.

21. Vieta E, Suppes T, Ekholm B, et al. Long-term efficacy of quetiapine in combination with lithium or divalproex on mixed symptoms in bipolar I disorder. Journal of Affective Disorders. 2012 Dec 15;142(1-3):36-44. doi: http://dx.doi.org/10.1016/j.jad.2012.04.014. PMID: 23062763.
22. Niufan G, Tohen M, Qiuqing A, et al. Olanzapine versus lithium in the acute treatment of bipolar mania: a double-blind, randomized, controlled trial. Journal of Affective Disorders. 2008 Jan;105(1-3):101-8. PMID: 17531327.

23. Tohen M, Vieta E, Goodwin GM, et al. Olanzapine versus divalproex versus placebo in the treatment of mild to moderate mania: a randomized, 12-week, double-blind study. Journal of Clinical Psychiatry. 2008 Nov;69(11):1776-89. PMID: 19014751.

24. Perlis RH, Welge JA, Vornik LA, et al. Atypical antipsychotics in the treatment of mania: a metaanalysis of randomized, placebo-controlled trials. Journal of Clinical Psychiatry. 2006 Apr;67(4):509-16. PMID: 16669715.

25. Revicki DA, Paramore LC, Sommerville KW, et al. Divalproex sodium versus olanzapine in the treatment of acute mania in bipolar disorder: health-related quality of life and medical cost outcomes. Journal of Clinical Psychiatry. 2003 Mar;64(3):288-94. PMID: 12716270.

26. Tohen M, Goldberg JF, Gonzalez-Pinto Arrillaga AM, et al. A 12-week, double-blind comparison of olanzapine vs haloperidol in the treatment of acute mania. Archives of General Psychiatry. 2003 Dec;60(12):1218-26. PMID: 14662554.

27. Shi L, Namjoshi MA, Zhang F, et al. Olanzapine versus haloperidol in the treatment of acute mania: clinical outcomes, health-related quality of life and work status. International Clinical Psychopharmacology. 2002 Sep;17(5):227-37. PMID: 12177585.

28. Tohen M, Baker RW, Altshuler LL, et al. Olanzapine versus divalproex in the treatment of acute mania.[Erratum appears in Am J Psychiatry. 2005 Feb;7(1):102]. American Journal of Psychiatry. 2002 Jun;159(6):1011-7. PMID: 12042191.

29. Tohen M, Jacobs TG, Grundy SL, et al. Efficacy of olanzapine in acute bipolar mania: a doubleblind, placebo-controlled study. The Olanzipine HGGW Study Group.[Erratum appears in Arch Gen Psychiatry 2002 Jan;59(1):91]. Archives of General Psychiatry. 2000 Sep;57(9):841-9. PMID: 10986547.

30. Berk M, Ichim L, Brook S. Olanzapine compared to lithium in mania: a double-blind randomized controlled trial. International Clinical Psychopharmacology. 1999 Nov;14(6):339-43. PMID: 10565800. 
31. Katagiri H, Takita Y, Tohen M, et al. Safety and efficacy of olanzapine monotherapy and olanzapine with a mood stabilizer in 18-week treatment of manic/mixed episodes for Japanese patients with bipolar I disorder. Current Medical Research \& Opinion. 2012 May;28(5):701-13. doi:

http://dx.doi.org/10.1185/03007995.2012.666961. PMID: 22356118.

32. Vieta E, Montgomery S, Sulaiman AH, et al. A randomized, double-blind, placebo-controlled trial to assess prevention of mood episodes with risperidone long-acting injectable in patients with bipolar I disorder. European

Neuropsychopharmacology. 2012

Nov;22(11):825-35. doi:

http://dx.doi.org/10.1016/j.euroneuro.2012.03.004 . PMID: 22503488.

33. Shafti SS. Olanzapine vs. lithium in management of acute mania. Journal of Affective Disorders. 2010 May;122(3):273-6. doi: http://dx.doi.org/10.1016/j.jad.2009.08.013. PMID: 19740546.

34. Perlis RH, Baker RW, Zarate CA, Jr., et al. Olanzapine versus risperidone in the treatment of manic or mixed States in bipolar I disorder: a randomized, double-blind trial. Journal of Clinical Psychiatry. 2006 Nov;67(11):1747-53. PMID: 17196055.

35. Zajecka JM, Weisler R, Sachs G, et al. A comparison of the efficacy, safety, and tolerability of divalproex sodium and olanzapine in the treatment of bipolar disorder. Journal of Clinical Psychiatry. 2002 Dec;63(12):1148-55. PMID: 12523875.

36. Cutler AJ, Datto C, Nordenhem A, et al. Extended-release quetiapine as monotherapy for the treatment of adults with acute mania: a randomized, double-blind, 3-week trial. Clinical Therapeutics. 2011 Nov;33(11):1643-58. doi: http://dx.doi.org/10.1016/j.clinthera.2011.10.002. PMID: 22054797.

37. McElroy SL, Martens BE, Winstanley EL, et al. Placebo-controlled study of quetiapine monotherapy in ambulatory bipolar spectrum disorder with moderate-to-severe hypomania or mild mania. Journal of Affective Disorders. 2010 Jul;124(1-2):157-63. doi: http://dx.doi.org/10.1016/j.jad.2009.11.014. PMID: 19963274.
38. Li H, Ma C, Wang G, et al. Response and remission rates in Chinese patients with bipolar mania treated for 4 weeks with either quetiapine or lithium: a randomized and double-blind study. Current Medical Research \& Opinion. 2008 Jan;24(1):1-10. PMID: 18028587.

39. Bowden CL, Grunze H, Mullen J, et al. A randomized, double-blind, placebo-controlled efficacy and safety study of quetiapine or lithium as monotherapy for mania in bipolar disorder. Journal of Clinical Psychiatry. 2005 Jan;66(1):111-21. PMID: 15669897.

40. Khanna S, Vieta E, Lyons B, et al. Risperidone in the treatment of acute mania. The British Journal of Psychiatry. 2005 2005;187(3):229-34. doi: http://dx.doi.org/10.1192/bjp.187.3.229. PMID: 16135859.

41. Segal J, Berk M, Brook S. Risperidone compared with both lithium and haloperidol in mania: a double-blind randomized controlled trial. Clinical Neuropharmacology. 1998 May-Jun;21(3):17680. PMID: 9617509.

42. Potkin SG, Keck PE, Jr., Segal S, et al. Ziprasidone in acute bipolar mania: a 21-day randomized, double-blind, placebo-controlled replication trial. Journal of Clinical Psychopharmacology. 2005 Aug;25(4):301-10. PMID: 16012271.

43. Keck PE, Jr., Versiani M, Potkin S, et al. Ziprasidone in the treatment of acute bipolar mania: a three-week, placebo-controlled, doubleblind, randomized trial. American Journal of Psychiatry. 2003 Apr;160(4):741-8. PMID: 12668364.

44. Jeong HG, Lee MS, Ko YH, et al. Combination treatment with aripiprazole and valproic acid for acute mania: an 8-week, single-blind, randomized controlled trial. Clinical Neuropharmacology. 2012 May-Jun;35(3):97-102. doi: http://dx.doi.org/10.1097/WNF.0b013e31825604 01. PMID: 22592508.

45. Vieta E, T'Joen C, McQuade RD, et al. Efficacy of adjunctive aripiprazole to either valproate or lithium in bipolar mania patients partially nonresponsive to valproate/lithium monotherapy: a placebo-controlled study. American Journal of Psychiatry. 2008 Oct;165(10):1316-25. doi: http://dx.doi.org/10.1176/appi.ajp.2008.07101560 . PMID: 18381903. 
46. Szegedi A, Calabrese JR, Stet L, et al. Asenapine as adjunctive treatment for acute mania associated with bipolar disorder: results of a 12-week core study and 40-week extension. Journal of Clinical Psychopharmacology. 2012 Feb;32(1):46-55. doi: http://dx.doi.org/10.1097/JCP.0b013e31823f872f. PMID: 22198448.

47. Conus P, Berk M, Cotton SM, et al. Olanzapine or chlorpromazine plus lithium in first episode psychotic mania: An 8-week randomised controlled trial. European Psychiatry: the Journal of the Association of European Psychiatrists. 2015 Nov;30(8):975-82. doi: http://dx.doi.org/10.1016/j.eurpsy.2015.09.009. PMID: 26485297.

48. Houston JP, Tohen M, Degenhardt EK, et al. Olanzapine-divalproex combination versus divalproex monotherapy in the treatment of bipolar mixed episodes: a double-blind, placebocontrolled study.[Erratum appears in J Clin Psychiatry. 2011 Aug;72(8):1157], [Erratum appears in J Clin Psychiatry. 2010 Jan;71(1):93]. Journal of Clinical Psychiatry. 2009 Nov;70(11):1540-7. doi: http://dx.doi.org/10.4088/JCP.08m04895yel. PMID: 19778495.

49. Tohen M, Bowden CL, Smulevich AB, et al. Olanzapine plus carbamazepine v. carbamazepine alone in treating manic episodes. British Journal of Psychiatry. 2008 Feb;192(2):135-43. doi: http://dx.doi.org/10.1192/bjp.bp.107.041301. PMID: 18245032.

50. Tohen M, Chengappa KN, Suppes T, et al. Efficacy of olanzapine in combination with valproate or lithium in the treatment of mania in patients partially nonresponsive to valproate or lithium monotherapy. Archives of General Psychiatry. 2002 Jan;59(1):62-9. PMID: 11779284.
51. Namjoshi MA, Risser R, Shi L, et al. Quality of life assessment in patients with bipolar disorder treated with olanzapine added to lithium or valproic acid. Journal of Affective Disorders. 2004;81(3):223-9. PMID: 15337326.

52. Baker RW, Brown E, Akiskal HS, et al. Efficacy of olanzapine combined with valproate or lithium in the treatment of dysphoric mania. British Journal of Psychiatry. 2004 Dec;185:472-8. PMID: 15572737.

53. Yatham LN, Vieta E, Young AH, et al. A double blind, randomized, placebo-controlled trial of quetiapine as an add-on therapy to lithium or divalproex for the treatment of bipolar mania. International Clinical Psychopharmacology. 2007 Jul;22(4):212-20. PMID: 17519644.

54. Yatham LN, Paulsson B, Mullen J, et al. Quetiapine versus placebo in combination with lithium or divalproex for the treatment of bipolar mania.[Erratum appears in J Clin Psychopharmacol. 2005 Apr;25(2):201], [Erratum appears in J Clin Psychopharmacol. 2005 Feb;25(1):103]. Journal of Clinical Psychopharmacology. 2004 Dec;24(6):599-606. PMID: 15538120.

55. Yatham LN, Grossman F, Augustyns I, et al. Mood stabilisers plus risperidone or placebo in the treatment of acute mania. International, double-blind, randomised controlled trial.[Erratum appears in Br J Psychiatry. 2003 Apr;182:369]. British Journal of Psychiatry. 2003 Feb;182:141-7. PMID: 12562742.

56. Sachs GS, Vanderburg DG, Edman S, et al. Adjunctive oral ziprasidone in patients with acute mania treated with lithium or divalproex, part 1: results of a randomized, double-blind, placebocontrolled trial. Journal of Clinical Psychiatry. 2012 Nov;73(11):1412-9. doi: http://dx.doi.org/10.4088/JCP.11m07388. PMID: 23218157. 


\section{Appendix F. Mood Stabilizers for Mania}

\section{Section 1. Carbamazepine}

Appendix Table F1. Characteristics of eligible studies: carbamazepine for acute mania

\begin{tabular}{|c|c|c|c|c|c|c|}
\hline $\begin{array}{l}\text { Study, Year } \\
\text { Design } \\
\text { Location } \\
\text { Funder } \\
\text { Risk of Bias } \\
\text { PMID }\end{array}$ & $\begin{array}{c}\text { \# Randomized } \\
\text { Age (mean) } \\
\text { Sex (\% Female) } \\
\text { Race (\% White) } \\
\text { Diagnosis } \\
\text { (\% BP I, II, NOS) } \\
\text { Setting }\end{array}$ & $\begin{array}{c}\text { Inclusions } \\
\text { Key Exclusions }\end{array}$ & $\begin{array}{c}\text { Intervention } \\
\text { Dosage }\end{array}$ & $\begin{array}{c}\text { Comparison } \\
\text { Dosage }\end{array}$ & $\begin{array}{c}\text { Follow-up } \\
\text { Duration }\end{array}$ & $\begin{array}{c}\text { Outcomes } \\
\text { Reported } \\
\text { Withdrawal (\%) at } \\
\text { endpoint }\end{array}$ \\
\hline $\begin{array}{l}\text { Weisler, } 20061 \\
2 \text { pooled RCTs } \\
\text { Multisite } \\
2 \text { Continents } \\
\text { Industry } \\
\text { RoB High } \\
16529527 \\
\text { (pooled 15766298 and } \\
15119909 \text { ) }\end{array}$ & $\begin{array}{l}\mathrm{N}=443 \\
\text { Mean Age } 38 \\
\text { Female } 38 \% \\
\text { White } 59 \% \\
\text { BP I 100\% } \\
\text { Inpatient or Outpatient }\end{array}$ & $\begin{array}{l}\text { Manic/Mixed; } \\
\text { YMRS } \geq 20 \\
\text { Taking Other Meds }\end{array}$ & $\begin{array}{l}\text { Carbamazepine ER } \\
200-1600 \text { mg/day } \\
\text { (642.6 mg/day } \\
\text { average) }\end{array}$ & Placebo & 3 weeks & $\begin{array}{l}\text { YMRS } \\
\text { CGI-S } \\
\text { CGI-I } \\
\text { HAM-D } \\
\text { Withdrawal 46\% }\end{array}$ \\
\hline $\begin{array}{l}\text { Vasudev, } 20002 \\
\text { RCT } \\
\text { Singlesite } \\
\text { India } \\
\text { Industry } \\
\text { RoB Moderate } \\
10867972\end{array}$ & $\begin{array}{l}\mathrm{N}=30 \\
\text { Age NR } \\
\text { Sex NR } \\
\text { Race NR } \\
\text { BP I 100\% } \\
\text { Outpatient }\end{array}$ & $\begin{array}{l}\text { Mania; } \\
\text { DSM-III criteria for BP } \\
\text { diagnosis } \\
\text { Substance Abuse } \\
\text { Neurological Disorders } \\
\text { Taking other meds } \\
\text { Pregnant/Nursing }\end{array}$ & \begin{tabular}{|l|} 
Carbamazepine \\
$800-1600 \mathrm{mg} / \mathrm{day}$ \\
$(22.05 \mathrm{mg} / \mathrm{kg} / \mathrm{day})$
\end{tabular} & \begin{tabular}{|l} 
Valproate \\
$800-2200 \mathrm{mg} /$ day \\
$(22.9 \mathrm{mg} / \mathrm{kg} / \mathrm{day})$
\end{tabular} & 4 weeks & $\begin{array}{l}\text { Response (50\% } \\
\text { decrease in YMRS) } \\
\text { YMRS } \\
\text { Withdrawal } 20 \%\end{array}$ \\
\hline
\end{tabular}




\begin{tabular}{|c|c|c|c|c|c|c|}
\hline $\begin{array}{l}\text { Study, Year } \\
\text { Design } \\
\text { Location } \\
\text { Funder } \\
\text { Risk of Bias } \\
\text { PMID }\end{array}$ & $\begin{array}{c}\text { \# Randomized } \\
\text { Age (mean) } \\
\text { Sex (\% Female) } \\
\text { Race (\% White) } \\
\text { Diagnosis } \\
\text { (\% BP I, II, NOS) } \\
\text { Setting }\end{array}$ & $\begin{array}{c}\text { Inclusions } \\
\text { Key Exclusions }\end{array}$ & $\begin{array}{c}\text { Intervention } \\
\text { Dosage }\end{array}$ & $\begin{array}{c}\text { Comparison } \\
\text { Dosage }\end{array}$ & $\begin{array}{c}\text { Follow-up } \\
\text { Duration }\end{array}$ & $\begin{array}{c}\text { Outcomes } \\
\text { Reported } \\
\text { Withdrawal (\%) at } \\
\text { endpoint }\end{array}$ \\
\hline $\begin{array}{l}\text { Small, } 19913 \\
\text { RCT } \\
\text { Singlesite } \\
\text { US } \\
\text { Government } \\
\text { RoB High } \\
1929761\end{array}$ & $\begin{array}{l}\mathrm{N}=48 \\
\text { Mean Age } 39 \\
\text { Female } 38 \% \\
\text { White 59\% } \\
\text { BP I 100\% } \\
\text { Inpatient }\end{array}$ & $\begin{array}{l}\text { Manic/Mixed; } \\
\text { YMRS } \geq 20 \\
\\
\text { First Manic Episode } \\
\text { Substance Abuse } \\
\text { Other Mental Health }\end{array}$ & $\begin{array}{l}\text { Carbamazepine } \\
700 \text { mg/day-1052 } \\
\text { mg/day (high and } \\
\text { low weekly mean } \\
\text { dose) (950.8 } \\
\text { mg/day mean } \\
\text { weekly dose) }\end{array}$ & $\begin{array}{l}\text { Lithium } \\
\text { 1035-1278 mg/day } \\
\text { (high and low mean } \\
\text { dose) (1207.5 } \\
\text { mean weekly dose) }\end{array}$ & 8 weeks & $\begin{array}{l}\text { SDMS-D } \\
\text { SDMS-M } \\
\text { YMRS } \\
\text { GAS } \\
\text { CGI-I } \\
\text { BCL } \\
\text { Withdrawal 42\% }\end{array}$ \\
\hline $\begin{array}{l}\text { Lerer, } 19874 \\
\text { RCT } \\
\text { Singlesite } \\
\text { US } \\
\text { Industry } \\
\text { RoB High } \\
3546274\end{array}$ & $\begin{array}{l}\mathrm{N}=34 \\
\text { Mean Age } 41 \\
\text { Female 54\% } \\
\text { Race NR } \\
\text { BP I 100\% } \\
\text { Inpatient }\end{array}$ & $\begin{array}{l}\text { Manic } \\
\text { Neurological Disorders }\end{array}$ & $\begin{array}{l}\text { Carbamazepine } \\
600-2600 \text { mg/day } \\
\text { (1250 mg/day mean } \\
\text { of the reported } \\
\text { weekly median) }\end{array}$ & $\begin{array}{l}\text { Lithium } \\
900-3900 \text { mg/day } \\
\text { (1650 mg/day mean } \\
\text { of the reported } \\
\text { weekly median) }\end{array}$ & 4 weeks & $\begin{array}{l}\text { Response (CGI } \\
\text { change 2+) } \\
\text { CGI } \\
\text { Withdrawal 18\% }\end{array}$ \\
\hline
\end{tabular}

Abbreviations: AE=Adverse Events; AIMS=Abnormal Involuntary Movement Scale; BAS=Behavioral Approach System; BCL= Shopsin-Gershon Social Behavior Checklist; BIS-11=Barratt Impulsiveness Scale; BP=bipolar disorder; BPRS=Brief Psychiatric Rating Scale; C=Comparision; CGI=Clinical Global Impressions Scale; CGI-BP =Clinical Global Impressions Scale for Bipolar Disorder; CGI-I= Clinical Global Impressions-Improvement; CGI-S=Clinical Global Impressions, Severity Scale; DSM=Diagnostic and Statistical Manual of Mental Disorders; DSM-IV= Diagnostic and statistical manual, $4^{\text {th }}$ edition; DSS=Depressive Syndrome Scale; ER=extended release; GAF=General Assessment of Functioning Scale; GAS= Global Assessment Scale; HAM-D=Hamilton Scale for Depression; LIFE-RIFT= Range of Impaired Functioning Tool;

MADRS=Montgomery-Asberg Depression Rating Scale; MSS=Manic Syndrome Scale; NOS=not otherwise specified; NR=not reported; PMID=PubMed Identification Number; RCT=randomized controlled trial; ROB=risk of bias; SADS-C=Schedule for Affective Disorders and Schizophrenia Change Version; SAS=Simpson Angus Scale;

SDMS=Symptoms of Depression and Mania Scale; YMRS = Young Mania Rating Scale 
Appendix Table F2. Summary risk of bias assessments: carbamazepine for acute mania

\begin{tabular}{|c|l|l|l|}
\hline \multicolumn{1}{|c|}{ Drug } & $\begin{array}{l}\text { Study } \\
\text { Funding Source } \\
\text { PMID }\end{array}$ & $\begin{array}{c}\text { Overall Risk of Bias } \\
\text { Assessment }\end{array}$ & \\
\hline Carbamazepine & $\begin{array}{l}\text { Weisler, 2006 } \\
\text { Industry } \\
16529527\end{array}$ & High & Rationale \\
\cline { 2 - 4 } & $\begin{array}{l}\text { Vasudev, 2000 } \\
\text { Industry } \\
10867972\end{array}$ & Moderate & $\begin{array}{l}\text { Protection of allocation not described, but blinding and randomization are well addressed as } \\
\text { are other aspects of the paper. Withdrawal 20\%. }\end{array}$ \\
\cline { 2 - 4 } & $\begin{array}{l}\text { Small, 19913 } \\
\text { Government } \\
1929761\end{array}$ & High & Randomization procedure and allocation masking not described. 42\% dropout. \\
\cline { 2 - 4 } & $\begin{array}{l}\text { Lerer, 1987 } \\
\text { Industry } \\
3546274\end{array}$ & High & $\begin{array}{l}\text { The researcher only included in the analysis those who completed the study. This is likely to } \\
\text { bias the results of the Lithium group who lost roughly 1/4 of the study population during the } \\
\text { four weeks. Randomization procedure and allocation masking not described. 18\% dropout. }\end{array}$ \\
\hline
\end{tabular}

Abbreviations: PMID=PubMed Identification Number

four weeks. Randomization procedure and allocation masking not described. $18 \%$ dropout. 


\section{Appendix Table F3. Outcomes summary: carbamezepine versus placebo for acute mania}

\begin{tabular}{|c|c|c|c|c|c|c|}
\hline Drug & $\begin{array}{l}\text { Study } \\
\text { PMID }\end{array}$ & Responder/Remitter & Symptom & Function & Other & $A E$ \\
\hline $\begin{array}{l}\text { Carbamazepine } \\
\text { vs. placebo }\end{array}$ & $\begin{array}{l}\text { Weisler, } \\
2006^{1} \\
16529527 \\
\\
2 \text { pooled } \\
\text { RCTs } \\
\text { (15766298 } \\
\text { and } \\
15119909) \\
\text { High }\end{array}$ & $\begin{array}{l}\text { Responders (>50\% } \\
\text { decrease YMRS) } \\
3 \text { weeks } \\
\text { Favors } \\
\text { carbamazepine } \\
\text { Carbamazepine } 52 \% \\
\text { Placebo } 26 \% \\
\text { p }<0.0001\end{array}$ & $\begin{array}{l}\text { YMRS decrease } \\
3 \text { weeks } \\
\text { Favors carbamazepine } \\
\text { Carbamazepine } 12.3 \\
\text { Placebo } 6.2 \\
p<0.0001 \\
\text { HAM-D decrease } \\
3 \text { weeks } \\
\text { Favors carbamazepine } \\
\text { Carbamazepine } 2.9 \\
\text { Placebo } 1.3 \\
p=0.01\end{array}$ & $\begin{array}{l}\text { CGI-S increase } \\
3 \text { weeks } \\
\text { Favors } \\
\text { carbamazepine } \\
\text { Carbamazepine } \\
1.2 \\
\text { Placebo } 0.5 \\
\text { p<0.0001 } \\
\text { CGI-I } \\
\text { improvement } \\
3 \text { weeks } \\
\text { Favors } \\
\text { carbamazepine } \\
\text { Carbamazepine } \\
55.6 \% \\
\text { Placebo } 28.4 \% \\
\text { p<0.0001 }\end{array}$ & $\begin{array}{l}\text { Overall Withdrawal } \\
\text { Carbamazepine } \\
93 / 223 \\
\text { Placebo 110/220 } \\
\text { NS } \\
\text { Withdrawal lack of effect } \\
\text { Carbamazepine } \\
22 / 223 \\
\text { Placebo 49/220 } \\
\text { Favors Carbamazepine } \\
\text { Withdrawal adverse events } \\
\text { Carbamazepine } \\
24 / 223 \\
\text { Placebo 12/220 } \\
\text { NR (EPC calculated favors } \\
\text { carbamazepine) } \\
\text { BMI Change } \\
\text { Favors placebo } \\
\geq 7 \% \text { gain } \\
\text { Carbamazepine } 5.3 \% \\
\text { Placebo 1\% } \\
\text { p=0.011 }\end{array}$ & $\begin{array}{l}\text { SAE } \\
\text { (from original studies) } \\
\text { Carbamazepine: } 8 \text { patients } \\
\text { Placebo: } 10 \text { patients } \\
\text { Severe Rash: } 11 \\
\text { carbamazepine patient } \\
1 \text { placebo patient attempted } \\
\text { suicide }\end{array}$ \\
\hline
\end{tabular}

Abbreviations: AE=Adverse Effects; BMI=Body Mass Index; CGI= Clinical Global Impressions; CGI-BP =Clinical Global Impressions Scale for Bipolar Disorder; CGI-BP-

S=Clinical Global Impressions, Bipolar, Severity Scale; CGI-I= Clinical Global Impressions-Improvement; CGI-S=CGI-Severity; CI= Confidence Interval; EPS=Extrapyramidal

Side Effects; GAS= Global Assessment Scale; HAM-D=Hamilton Scale for Depression; MADRS=Montgomery-Asberg Depression Rating Scale; NR=not reported; NS=not significant; OPT=Optimalized Personalized Treatment; OR=Odds Ratio; PMID=PubMed Identification Number; RCT=randomized controlled trial; SAE= Serious Adverse

Events; SD=Standard Deviation; SE=Standard Error; YMRS = Young Mania Rating Scale 


\section{Appendix Table F4. Strength of evidence assessment: carbamezepine versus placebo for acute mania}

\begin{tabular}{|c|c|c|c|c|c|c|c|c|}
\hline Comparison & Outcome & $\begin{array}{l}\text { \# Studies/ } \\
\text { Design } \\
\text { (n analyzed) }\end{array}$ & $\begin{array}{c}\text { Finding or } \\
\text { Summary } \\
\text { Statistic }\end{array}$ & $\begin{array}{c}\text { Study } \\
\text { Limitations }\end{array}$ & Consistency & Directness & Precision & $\begin{array}{c}\text { Overall } \\
\text { Gradel } \\
\text { Conclusion }\end{array}$ \\
\hline $\begin{array}{l}\text { Carbamazepine } \\
\text { vs. placebo }\end{array}$ & $\begin{array}{l}\text { Response } \\
\text { YMRS } \\
\text { CGI } \\
\text { Withdrawals }\end{array}$ & $\begin{array}{l}1 \mathrm{RCT}+1 \mathrm{IPD} \\
(\mathrm{n}=443)\end{array}$ & See table above & High & $\begin{array}{l}\text { Consistent } \\
\text { (based on } \\
\text { original RCTs) }\end{array}$ & Direct & Imprecise & Insufficient \\
\hline
\end{tabular}

Abbreviations: AE=Adverse Events; CGI= Clinical Global Impressions; CI=Confidence Interval; IPD=Individual patient data; MADRS=Montgomery-Asberg Depression Rating Scale; MD=Mean Difference; NS=Not Significant; RCT=randomized controlled trial; YMRS = Young Mania Rating Scale

Notes:

1. Publication bias for antipsychotics, antidepressants, and behavioral interventions for depressive disorders is suspected.

2. Data were generally imprecise due to missing data from high attrition rates, which was commonly dealt with by Last Observation Carried Forward (LOCF). LOCF requires an

assumption that the health status of patients who dropped out of the trial would not have changed had future observations been recorded, a strong assumption in the context of

bipolar disorder research. 
Appendix Table F5. Outcomes summary: carbamezapine versus active comparator for acute mania

\begin{tabular}{|c|c|c|c|c|c|c|}
\hline Drug & $\begin{array}{l}\text { Study } \\
\text { PMID }\end{array}$ & Responder/Remitter & Symptom & Function & Other & $A E$ \\
\hline \multirow[t]{2}{*}{$\begin{array}{l}\text { Carbamazepine } \\
\text { vs. lithium }\end{array}$} & $\begin{array}{l}\text { Small, 199133 } \\
1929761 \\
\text { High }\end{array}$ & NR & $\begin{array}{l}\frac{\text { YMRS }}{8 \text { weeks }} \\
\text { Carbamazepine }-8.5 \\
\text { Lithium -9.7 } \\
4 \% \text { difference between } \\
\text { groups } \\
\frac{\text { HAM-D }}{8 \text { weeks }} \\
\text { Carbamazepine }-2.5 \\
\text { Lithium } 0.5 \\
10 \% \text { difference between } \\
\text { groups } \\
\text { SDMS-D } \\
8 \text { weeks } \\
\text { Carbamazepine } 0 \\
\text { Lithium } 0.6 \\
18 \% \text { difference between } \\
\text { groups } \\
\text { SDMS-M } \\
\text { weeks } \\
\text { Carbamazepine }-3.4 \\
\text { Lithium -3.3 } \\
1 \% \text { difference between } \\
\text { groups }\end{array}$ & $\begin{array}{l}\text { CGI-I } \\
8 \text { weeks } \\
\text { Carbamazepine }-0.9 \\
\text { Lithium } 1.0 \\
1 \% \text { difference between } \\
\text { groups } \\
\frac{\text { GAS }}{8 \text { weeks }} \\
\text { Carbamazepine } 11.7 \\
\text { Lithium } 11.8 \\
3 \% \text { difference between } \\
\text { groups }\end{array}$ & $\begin{array}{l}\text { Overall } \\
\text { Withdrawal } \\
\text { Carbamazepine } \\
\text { 16/24 } \\
\text { Lithium 16/24 } \\
\text { NS }\end{array}$ & $\frac{\text { SAE }}{\text { None }}$ \\
\hline & $\begin{array}{l}\text { Lerer, } 1987^{4} \\
3546274 \\
\text { High }\end{array}$ & $\begin{array}{l}\text { Response (CGI } \\
\frac{\text { change } 2+)}{4 \text { weeks }} \\
\text { Favors lithium } \\
\text { Carbamazepine 4/14 } \\
\text { Lithium 11/14 } \\
p<0.05\end{array}$ & NR & $\begin{array}{l}\text { CGI } \\
\text { Carbamazepine } \\
\text { Baseline } 5.6(8.2) \\
4 \text { weeks } 4.1(1.51) \\
\text { Lithium } \\
\text { Baseline } 5.7(0.88) \\
4 \text { weeks } 3.1(1.5) \\
\text { ANOVA group effect NS }\end{array}$ & $\begin{array}{l}\text { Overall } \\
\text { Withdrawal } \\
\text { Carbamazepine } \\
1 / 15 \\
\text { Lithium 4/19 } \\
\text { NR } \\
\text { Withdrawal } \\
\text { adverse events } \\
\text { Carbamazepine } \\
\text { 1/15 } \\
\text { Lithium 2/19 } \\
\text { NR }\end{array}$ & $\begin{array}{l}\text { SAE } \\
\text { Carbamazepine } \\
4 / 15 \\
\text { Lithium } 1 / 19\end{array}$ \\
\hline
\end{tabular}




\begin{tabular}{|c|c|c|c|c|c|c|}
\hline Drug & $\begin{array}{l}\text { Study } \\
\text { PMID }\end{array}$ & Responder/Remitter & Symptom & Function & Other & $\mathrm{AE}$ \\
\hline $\begin{array}{l}\text { Carbamazepine } \\
\text { vs. valproate }\end{array}$ & $\begin{array}{l}\text { Vasudev, } 2000^{2} \\
10867972 \\
\text { Moderate }\end{array}$ & $\begin{array}{l}\frac{\text { Response }}{(50 \% \text { decrease in }} \\
\frac{\text { YMRS) }}{4 \text { weeks }} \\
\text { Carbamazepine 8/15 } \\
\text { Valproic acid 11/15 } \\
\text { NS }\end{array}$ & NR & NR & $\begin{array}{l}\text { Overall } \\
\text { Withdrawal } \\
\text { Carbamazepine } \\
3 / 15 \\
\text { Valproic acid } \\
\text { 3/15 } \\
\text { NS }\end{array}$ & $\begin{array}{l}\text { All AEs } \\
\text { Carbamazepine } \\
67 \% \\
\text { Valproic acid } 17 \% \\
\frac{\text { Tremors }}{\text { Carbamazepine }} \\
25 \% \\
\text { Valproic acid 8\% }\end{array}$ \\
\hline
\end{tabular}

Abbreviations: AE=Adverse Effects; BPRS=Brief Psychiatric Rating Scale; CGI= Clinical Global Impressions Scale; CGI-BP-S=Clinical Global Impressions, Bipolar, Severity Scale; CGI-I=Clinical Global Impressions Scale-Improvement; GAF=General Assessment of Functioning Scale; GAS=Global Assessment Scale; HAM-D=Hamilton Scale for Depression; MADRS=Montgomery-Asberg Syndrome Scale; MRS=mania rating scale; NR=not reported; NS=not significant; PMID=PubMed Identification Number; SAE= Serious Adverse Events; SD=standard deviation; SDMS-D=Symptoms of Depression and Mania Scale-Depression; YMRS = Young Mania Rating Scale

\section{Appendix Table F6. Strength of evidence assessment: carbamezapine versus active comparator for acute mania}

\begin{tabular}{|c|c|c|c|c|c|c|c|c|}
\hline Comparison & Outcome & $\begin{array}{c}\text { \# Studies/ } \\
\text { Design } \\
\text { (n analyzed) }\end{array}$ & $\begin{array}{c}\text { Finding or } \\
\text { Summary } \\
\text { Statistic }\end{array}$ & $\begin{array}{c}\text { Study } \\
\text { Limitations }\end{array}$ & Consistency & Directness & Precision & $\begin{array}{l}\text { Overall Gradel } \\
\text { Conclusion }\end{array}$ \\
\hline $\begin{array}{l}\text { Carbamazepine vs. } \\
\text { lithium }\end{array}$ & $\begin{array}{l}\text { Response } \\
\text { YMRS } \\
\text { CGI } \\
\text { Withdrawals - } \\
\text { overall } \\
\text { Withdrawal - } \\
\text { AEs } \\
\end{array}$ & $\begin{array}{l}2 \text { RCTs } \\
(n=82)\end{array}$ & See table above & High & Consistent & Direct & Imprecise & Insufficient \\
\hline $\begin{array}{l}\text { Carbamazepine vs. } \\
\text { valproate }\end{array}$ & $\begin{array}{l}\text { Response } \\
\text { Withdrawals - } \\
\text { overall } \\
\end{array}$ & $\begin{array}{l}1 \text { RCT } \\
(n=30)\end{array}$ & See table above & Moderate & Unknown & Direct & Imprecise & Insufficient \\
\hline
\end{tabular}

Abbreviations: AE=Adverse Event; CGI=Clinical Global Impressions Scale; CGI-BP-S=Clinical Global Impressions, Bipolar, Severity Scale; $\mathrm{n}=$ number; RCT=randomized controlled trial; YMRS = Young Mania Rating Scale

Notes:

1. Publication bias for antipsychotics, antidepressants, and behavioral interventions for depressive disorders is suspected.

2. Data were generally imprecise due to missing data from high attrition rates, which was commonly dealt with by Last Observation Carried Forward (LOCF). LOCF requires an assumption that the health status of patients who dropped out of the trial would not have changed had future observations been recorded, a strong assumption in the context of bipolar disorder research. 


\section{Section 2. Divalproex/Valproate}

Appendix Table F7. Characteristics of eligible studies: divalproex for acute mania

\begin{tabular}{|c|c|c|c|c|c|c|}
\hline $\begin{array}{l}\text { Study, Year } \\
\text { Design } \\
\text { Location } \\
\text { Funder } \\
\text { Risk of Bias } \\
\text { PMID }\end{array}$ & $\begin{array}{c}\text { \# Randomized } \\
\text { Age (mean) } \\
\text { Sex (\% Female) } \\
\text { Race (\% White) } \\
\text { Diagnosis } \\
\text { (\% BP I, II, NOS) } \\
\text { Setting }\end{array}$ & $\begin{array}{c}\text { Inclusions } \\
\text { Key Exclusions }\end{array}$ & $\begin{array}{c}\text { Intervention } \\
\text { Dosage }\end{array}$ & $\begin{array}{c}\text { Comparison } \\
\text { Dosage }\end{array}$ & $\begin{array}{c}\text { Follow-up } \\
\text { Duration }\end{array}$ & $\begin{array}{c}\text { Outcomes } \\
\text { Reported } \\
\text { Withdrawal (\%) at } \\
\text { endpoint }\end{array}$ \\
\hline $\begin{array}{l}\text { Tohen, } 2008 b^{5} \\
\text { RCT } \\
\text { Multisite } \\
3 \text { Continents } \\
\text { Industry } \\
\text { RoB Low } \\
19014751\end{array}$ & $\begin{array}{l}\mathrm{N}=521 \\
\text { Mean Age } 40 \\
\text { Female 49\% } \\
\text { Race NR } \\
\text { Diagnosis NR } \\
\text { Setting NR }\end{array}$ & $\begin{array}{l}\text { Manic or Mixed Episode; } \\
\text { YMRS 20-30 } \\
\text { CGI-BP mania 3-4 } \\
\text { Schizoaffective } \\
\text { Other Mental Health } \\
\text { Conditions } \\
\text { Pregnant/Nursing }\end{array}$ & \begin{tabular}{|l|} 
Divalproex \\
$500-2500 \mathrm{mg} / \mathrm{day}$
\end{tabular} & $\begin{array}{l}\text { C1: Placebo } \\
\text { C2: Olanzapine } \\
\text { 5-20 mg/day }\end{array}$ & 3 weeks & \begin{tabular}{|l} 
Response (YMRS \\
reduction $\geq 50 \%$ ) \\
Time to response \\
(days from baseline to \\
$\geq 50 \%$ YMRS \\
reduction) \\
Remission (YMRS $\leq$ \\
12 ) \\
Efficacy \\
YMRS \\
CGI (multiple \\
subscales) \\
MADRS \\
Adverse events \\
Extrapyramidal \\
symptoms \\
SAS \\
BAS \\
AIMS \\
Withdrawal 26\%
\end{tabular} \\
\hline
\end{tabular}




\begin{tabular}{|c|c|c|c|c|c|c|}
\hline $\begin{array}{l}\text { Study, Year } \\
\text { Design } \\
\text { Location } \\
\text { Funder } \\
\text { Risk of Bias } \\
\text { PMID }\end{array}$ & $\begin{array}{c}\text { \# Randomized } \\
\text { Age (mean) } \\
\text { Sex (\% Female) } \\
\text { Race (\% White) } \\
\text { Diagnosis } \\
\text { (\% BP I, II, NOS) } \\
\text { Setting }\end{array}$ & $\begin{array}{c}\text { Inclusions } \\
\text { Key Exclusions }\end{array}$ & $\begin{array}{c}\text { Intervention } \\
\text { Dosage }\end{array}$ & $\begin{array}{c}\text { Comparison } \\
\text { Dosage }\end{array}$ & $\begin{array}{c}\text { Follow-up } \\
\text { Duration }\end{array}$ & $\begin{array}{c}\text { Outcomes } \\
\text { Reported } \\
\text { Withdrawal (\%) at } \\
\text { endpoint }\end{array}$ \\
\hline $\begin{array}{l}\text { Bowden, } 2006^{6} \\
\text { RCT } \\
\text { Multisite } \\
\text { US } \\
\text { Industry } \\
\text { RoB High } \\
17107240\end{array}$ & $\begin{array}{l}\mathrm{N}=364 \\
\text { Mean Age } 38 \\
\text { Female } 43 \% \\
\text { White } 74 \% \\
\text { BP I } 100 \% \\
\text { Inpatient (0-15 days) } \\
\text { Outpatient (>15-21 days, } \\
\text { subject to clinical criteria) }\end{array}$ & $\begin{array}{l}\text { Mania; } \\
\text { Mania Rating Scale } \\
\text { (derived from SADS-C } \\
\text { interview) } \geq 18 \text { with at } \\
\text { least } 4 \text { item scores }>1 \text {. } \\
\\
\text { First Manic Episode; } \\
\text { Schizoaffective; } \\
\text { Substance Abuse; Other } \\
\text { Mental Health } \\
\text { Conditions; Taking other } \\
\text { Medications; }\end{array}$ & \begin{tabular}{|l|} 
Divalproex \\
$85-125$ \\
microgram/ml (2961 \\
mg/day average)
\end{tabular} & Placebo & 3 weeks & $\begin{array}{l}\text { Remission (YMRS } \leq \\
12) \\
\text { Response (50\% } \\
\text { decrease in YMRS) } \\
\text { YMRS } \\
\text { BIS } \\
\text { MSS } \\
\text { GAS } \\
\text { DSS } \\
\text { AEs } \\
\text { Weight gain } \\
\text { Withdrawal 45\% } \\
\end{array}$ \\
\hline $\begin{array}{l}\text { Xu, } 2015^{7} \\
\text { RCT } \\
\text { Single-site } \\
\text { China } \\
\text { Government } \\
\text { RoB Low } \\
26060401\end{array}$ & $\begin{array}{l}\mathrm{N}=120 \\
\text { Mean Age } 31 \\
\text { Female } 52 \% \\
\text { Race NR } \\
\text { BP } 1100 \% \\
\text { Setting NR }\end{array}$ & $\begin{array}{l}\text { First manic; } \\
\text { YMRS } \geq 17\end{array}$ & $\begin{array}{l}\text { Olanzapine } \\
10 \text { mg/day }+ \\
\text { Valproate } 600 \\
\text { mg/day }\end{array}$ & $\begin{array}{l}\text { C1: Olanzapine } \\
10 \text { mg/day } \\
\text { Flexible dosing } \\
5-20 \text { mg/day } \\
\\
\text { C2: Valproate } \\
600 \text { mg/day alone }\end{array}$ & 4 weeks & $\begin{array}{l}\text { Efficacy } \\
\text { YMRS } \\
\text { CGI-BP } \\
\text { Adverse events } \\
\text { Extrapyramidal } \\
\text { symptoms } \\
\text { SAS } \\
\text { Withdrawal 5\% }\end{array}$ \\
\hline
\end{tabular}

Abbreviations: AE=Adverse Events; AIMS=Abnormal Involuntary Movement Scale; BAS=Behavioral Approach System; BCL= Shopsin-Gershon Social Behavior Checklist; BIS-11=Barratt Impulsiveness Scale; BP=bipolar disorder; BPRS=Brief Psychiatric Rating Scale; C=Comparision; CGI=Clinical Global Impressions Scale; CGI-BP =Clinical Global Impressions Scale for Bipolar Disorder; CGI-I= Clinical Global Impressions-Improvement; CGI-S=Clinical Global Impressions, Severity Scale; DSM=Diagnostic and Statistical Manual of Mental Disorders; DSM-IV= Diagnostic and statistical manual, $4^{\text {th }}$ edition; DSS=Depressive Syndrome Scale; ER=extended release; GAF=General Assessment of Functioning Scale; GAS= Global Assessment Scale; HAM-D=Hamilton Scale for Depression; LIFE-RIFT= Range of Impaired Functioning Tool; MADRS=Montgomery-Asberg Depression Rating Scale; MSS=Manic Syndrome Scale; NOS=not otherwise specified; NR=not reported; PMID=PubMed Identification Number; RCT=randomized controlled trial; ROB=risk of bias; SADS-C=Schedule for Affective Disorders and Schizophrenia Change Version; SAS=Simpson Angus Scale; SDMS=Symptoms of Depression and Mania Scale; YMRS = Young Mania Rating Scale 
Appendix Table F8. Summary risk of bias assessments: dival/valproate for acute mania

\begin{tabular}{|l|l|l|l|}
\hline \multicolumn{1}{|c|}{ Drug } & $\begin{array}{c}\text { Appendix } \\
\text { Funding Source } \\
\text { PMID }\end{array}$ & $\begin{array}{c}\text { Overall Risk of Bias } \\
\text { Assessment }\end{array}$ & Rationale \\
\hline $\begin{array}{l}\text { Divalproex I } \\
\text { Valproate }\end{array}$ & $\begin{array}{l}\text { Tohen, 2008 } \\
\text { Industry } \\
19014751\end{array}$ & Low & No sources of bias identified. 26\% dropout at 3 weeks. \\
\cline { 2 - 4 } & $\begin{array}{l}\text { Bowden, 2006 } \\
\text { Industry } \\
17107240\end{array}$ & High & $\begin{array}{l}\text { Randomization and allocation procedures not described. Author notes, "We plan to report in a } \\
\text { separate article a detailed exploration of the site-related differences and the implications for } \\
\text { study design and execution" This statement infers that there is a difference caused by site that } \\
\text { is not addressed or controlled for in the paper. 45\% dropout. }\end{array}$ \\
\cline { 2 - 4 } & $\begin{array}{l}\text { Wu, 20157 } \\
\text { Government } \\
26060401\end{array}$ & Low & \\
\hline
\end{tabular}

Abbreviations: PMID=PubMed Identification Number

\section{Appendix Table F9. Outcomes summary: divalproex/valproate versus placebo for acute mania}

\begin{tabular}{|c|c|c|c|c|c|c|}
\hline Drug & $\begin{array}{l}\text { Study } \\
\text { PMID }\end{array}$ & Responder/Remitter & Symptom & Function & Other & $\mathrm{AE}$ \\
\hline $\begin{array}{l}\text { Divalproex vs. } \\
\text { placebo }\end{array}$ & $\begin{array}{l}\text { Bowden, } \\
2006^{6} \\
17107240 \\
\text { High }\end{array}$ & $\begin{array}{l}\frac{\text { Response }}{3 \text { weeks }} \\
\text { Favors divalproex } \\
\text { Divalproex 48\% } \\
\text { Placebo } 34 \% \\
\mathrm{p}=0.012 \\
\text { Remission } \\
3 \text { weeks } \\
\text { Favors divalproex } \\
\text { Divalproex 48\% } \\
\text { Placebo 35\% } \\
\mathrm{p}=0.015\end{array}$ & $\begin{array}{l}\text { MRS Change } \\
3 \text { weeks } \\
\text { Favors divalproex } \\
\text { Divalproex }-11.5 \\
\text { Placebo }-9.0 \\
p=0.013\end{array}$ & $\begin{array}{l}\frac{\mathrm{GAS}}{3 \text { weeks }} \\
\text { NS }\end{array}$ & $\begin{array}{l}\text { Overall Withdrawal } \\
\text { Divalproex } 108 / 187 \\
\text { Placebo 92/177 } \\
\text { NS } \\
\text { Withdrawal lack of effect } \\
\text { Divalproes } 24 / 187 \\
\text { Placebo 46/177 } \\
\text { Favors Divalproex } \\
\text { p=0.001 } \\
\text { Withdrawal adverse events } \\
\text { Divalproex } 19 / 187 \\
\text { Placebo 5/177 } \\
\text { Favors Placebo } \\
\text { p=0.003 }\end{array}$ & $\begin{array}{l}\text { Serious Adverse Events } \\
3 \text { weeks } \\
1 \text { in divalproex } \\
0 \text { in placebo } \\
\frac{\text { Deaths }}{3 \text { weeks }} \\
0 \text { in both arms }\end{array}$ \\
\hline
\end{tabular}




\begin{tabular}{|c|c|c|c|c|c|c|}
\hline Drug & $\begin{array}{l}\text { Study } \\
\text { PMID }\end{array}$ & Responder/Remitter & Symptom & Function & Other & $\mathrm{AE}$ \\
\hline & $\begin{array}{l}\text { Tohen, } \\
2008^{5} \\
19014751 \\
\text { Low }\end{array}$ & $\begin{array}{l}\frac{\text { Response }}{3 \text { weeks }} \\
\text { Divalproex } 40.3 \% \\
\text { Placebo } 31.3 \% \\
\text { NS } \\
\frac{\text { Remission }}{3 \text { weeks }} \\
\text { Divalproex } 40.3 \% \\
\text { Placebo } 35.4 \% \\
\text { NS }\end{array}$ & $\begin{array}{l}\text { YMRS Change } \\
3 \text { weeks } \\
\text { Divalproex }-8.2 \text { (SE } \\
0.62 \text { ) } \\
\text { Placebo }-7.4 \text { (SE 0.80) } \\
\text { NS }\end{array}$ & $\begin{array}{l}\text { CGI Overall } \\
\text { Change } \\
3 \text { weeks } \\
\text { Divalproex -0.6 } \\
\text { (SE 0.08) } \\
\text { Placebo -0.5 } \\
\text { (SE 0.10) } \\
\text { NS } \\
\\
\text { CGI Mania } \\
\text { Change } \\
\text { 3 weeks } \\
\text { Divalproex -0.8 } \\
\text { (SE 0.08) } \\
\text { Placebo -0.7 } \\
\text { (SE 0.11) } \\
\text { NS } \\
\text { CGI } \\
\text { Depression } \\
\text { Change } \\
\text { 3 weeks } \\
\text { Divalproex -0.2 } \\
\text { (SE 0.07) } \\
\text { Placebo -0.1 } \\
\text { (SE 0.09) } \\
\text { NS }\end{array}$ & $\begin{array}{l}\text { Withdrawal } \\
3 \text { weeks } \\
\text { Overall: } 25.5 \% \\
\text { Efficacy: } 2.0 \% \\
\text { AEs: } 2.3 \%\end{array}$ & $\begin{array}{l}\text { Serious Adverse Events } \\
3 \text { weeks } \\
1 \text { in divalproex } \\
1 \text { in placebo }\end{array}$ \\
\hline
\end{tabular}




\begin{tabular}{|c|c|c|c|c|c|c|}
\hline Drug & $\begin{array}{l}\text { Study } \\
\text { PMID }\end{array}$ & Responder/Remitter & Symptom & Function & Other & AE \\
\hline $\begin{array}{l}\text { Valproate } \\
\text { adjunctive vs. } \\
\text { placebo or no } \\
\text { placebo }\end{array}$ & $\begin{array}{l}\text { Xu, } 2015^{7} \\
26060401 \\
\text { Low }\end{array}$ & NR & $\begin{array}{l}\text { YMRS } \\
3 \text { weeks } \\
\text { Favors Valproate } \\
\% \text { Reduction (SD) } \\
\text { Olanzapine+Valporate= } \\
86.5 \%(8.9 \%) \\
\text { Olanzapine }=75.2 \% \\
(15.1 \%) \\
\text { P<0.01 } \\
\frac{\text { CGI-BP }}{3 \text { weeks }} \\
\text { Favors Valproate } \\
\text { p }<0.01\end{array}$ & NR & $\begin{array}{l}\frac{\text { Overall Withdrawal }}{3 \text { weeks }} \\
\text { NS } \\
\text { Olanzapine + Valporate }= \\
2 / 40 \\
\text { Olanzapine }=1 / 40 \\
\text { Withdrawal due to AEs } \\
3 \text { weeks } \\
\text { NS } \\
\text { Olanzapine+Valporate }=2 / 40 \\
\text { Olanzapine }=1 / 40 \\
\text { Withdrawal, Lack of } \\
\text { Efficacy } \\
3 \text { weeks } \\
\text { NS } \\
\text { Olanzapine }+ \text { Valporate }=0 / 40 \\
\text { Olanzapine }=0 / 40\end{array}$ & $\begin{array}{l}\frac{\text { Severe Harms }}{3 \text { weeks }} \\
\text { NS } \\
\text { Olanzapine+Valporate }=1 / 38 \\
\text { Olanzapine }=0 / 39 \\
\text { Normalized Weight Change } \\
3 \text { weeks } \\
\text { Olanzapine+Valporate }=31 / 38 \\
\text { Olanzapine }=29 / 39 \\
\text { Emergent Mood Episodes } \\
3 \text { weeks } \\
\text { NS } \\
\text { Olanzapine }+ \text { Valporate }=0 / 38 \\
\text { Olanzapine }=0 / 39\end{array}$ \\
\hline
\end{tabular}

Abbreviations: AE=Adverse Effects; BMI=Body Mass Index; CGI= Clinical Global Impressions; CGI-BP =Clinical Global Impressions Scale for Bipolar Disorder; CGI-BP-

S=Clinical Global Impressions, Bipolar, Severity Scale; CGI-I= Clinical Global Impressions-Improvement; CGI-S=CGI-Severity; CI= Confidence Interval; EPS=Extrapyramidal Side Effects; GAS= Global Assessment Scale; HAM-D=Hamilton Scale for Depression; MADRS=Montgomery-Asberg Depression Rating Scale; NR=not reported; NS=not significant; OPT=Optimalized Personalized Treatment; OR=Odds Ratio; PMID=PubMed Identification Number; RCT=randomized controlled trial; SAE= Serious Adverse

Events; SD=Standard Deviation; SE=Standard Error; YMRS = Young Mania Rating Scale 


\section{Appendix Table F10. Strength of evidence assessment: divalproex/valproate versus placebo for acute mania}

\begin{tabular}{|c|c|c|c|c|c|c|c|c|}
\hline Comparison & Outcome & $\begin{array}{l}\text { \# Studies/ } \\
\text { Design } \\
\text { (n analyzed) }\end{array}$ & $\begin{array}{c}\text { Finding or } \\
\text { Summary } \\
\text { Statistic }\end{array}$ & $\begin{array}{c}\text { Study } \\
\text { Limitations }\end{array}$ & Consistency & Directness & Precision & $\begin{array}{c}\text { Overall } \\
\text { Gradel } \\
\text { Conclusion }\end{array}$ \\
\hline $\begin{array}{l}\text { Divalproex vs. } \\
\text { placebo }\end{array}$ & $\begin{array}{l}\text { Relapse } \\
\text { Remission } \\
\text { YMRS } \\
\text { CGI } \\
\text { Withdrawals }\end{array}$ & $\begin{array}{l}2 \text { RCTs } \\
(n=670)\end{array}$ & See table above & Moderate & Inconsistent & Direct & Imprecise & Insufficient \\
\hline $\begin{array}{l}\text { Valproate vs. no } \\
\text { placebo }\end{array}$ & $\begin{array}{l}\text { YMRS } \\
\text { CGI } \\
\text { Withdrawals }\end{array}$ & $\begin{array}{l}1 \text { RCT } \\
(n=79)\end{array}$ & See table above & Low & Unknown & Direct & Imprecise & Insufficient \\
\hline
\end{tabular}

Abbreviations: AE=Adverse Events; CGI= Clinical Global Impressions; CI=Confidence Interval; IPD=Individual patient data; MADRS=Montgomery-Asberg Depression Rating Scale; MD=Mean Difference; NS=Not Significant; RCT=randomized controlled trial; YMRS = Young Mania Rating Scale Notes:

1. Publication bias for antipsychotics, antidepressants, and behavioral interventions for depressive disorders is suspected.

2. Data were generally imprecise due to missing data from high attrition rates, which was commonly dealt with by Last Observation Carried Forward (LOCF). LOCF requires an assumption that the health status of patients who dropped out of the trial would not have changed had future observations been recorded, a strong assumption in the context of bipolar disorder research. 


\section{Section 3. Lamotrigine}

Appendix Table F11. Characteristics of eligible studies: lamotrigine for acute mania

\begin{tabular}{|c|c|c|c|c|c|c|}
\hline $\begin{array}{c}\text { Study, Year } \\
\text { Design } \\
\text { Location } \\
\text { Funder } \\
\text { Risk of Bias } \\
\text { PMID }\end{array}$ & $\begin{array}{c}\text { \# Randomized } \\
\text { Age (mean) } \\
\text { Sex (\% Female) } \\
\text { Race (\% White) } \\
\text { Diagnosis } \\
\text { (\% BP I, II, NOS) } \\
\text { Setting }\end{array}$ & $\begin{array}{c}\text { Inclusions } \\
\text { Key Exclusions }\end{array}$ & $\begin{array}{l}\text { Intervention } \\
\text { Dosage }\end{array}$ & $\begin{array}{c}\text { Comparison } \\
\text { Dosage }\end{array}$ & $\begin{array}{c}\text { Follow-up } \\
\text { Duration }\end{array}$ & $\begin{array}{c}\text { Outcomes } \\
\text { Reported } \\
\text { Withdrawal (\%) at } \\
\text { endpoint }\end{array}$ \\
\hline $\begin{array}{l}\text { Ichim, } 2000^{8} \\
\text { RCT } \\
\text { Singlesite } \\
\text { South Africa } \\
\text { Industry } \\
\text { RoB Moderate } \\
10798820\end{array}$ & $\begin{array}{l}\mathrm{N}=30 \\
\text { Mean Age } 33 \\
\text { Female 47\% } \\
\text { Race NR } \\
\text { BP I 100\% } \\
\text { Inpatient }\end{array}$ & $\begin{array}{l}\text { Mania } \\
\text { Substance Abuse } \\
\text { Taking Other Meds } \\
\text { Pregnant/Nursing } \\
\text { Labs/Other Conditions }\end{array}$ & $\begin{array}{l}\text { Lamotrigine } \\
\text { Week } 1 \text { 25mg/day, } \\
\text { Week } 2 \text { 50mg/day, } \\
\text { Week } 3 \text { 100mg/day }\end{array}$ & $\begin{array}{l}\text { Lithium } \\
\text { 400mg twice/daily }\end{array}$ & 4 weeks & \begin{tabular}{|l} 
YMRS \\
BPRS \\
CGI \\
GAF \\
Withdrawal 17\%
\end{tabular} \\
\hline
\end{tabular}

Abbreviations: AE=Adverse Events; AIMS=Abnormal Involuntary Movement Scale; BAS=Behavioral Approach System; BCL= Shopsin-Gershon Social Behavior Checklist; BIS-11=Barratt Impulsiveness Scale; BP=bipolar disorder; BPRS=Brief Psychiatric Rating Scale; C=Comparision; CGI=Clinical Global Impressions Scale; CGI-BP =Clinical Global Impressions Scale for Bipolar Disorder; CGI-I= Clinical Global Impressions-Improvement; CGI-S=Clinical Global Impressions, Severity Scale; DSM=Diagnostic and Statistical Manual of Mental Disorders; DSM-IV= Diagnostic and statistical manual, $4^{\text {th }}$ edition; DSS=Depressive Syndrome Scale; ER=extended release; GAF=General Assessment of Functioning Scale; GAS= Global Assessment Scale; HAM-D=Hamilton Scale for Depression; LIFE-RIFT= Range of Impaired Functioning Tool;

MADRS=Montgomery-Asberg Depression Rating Scale; MSS=Manic Syndrome Scale; NOS=not otherwise specified; NR=not reported; PMID=PubMed Identification Number; RCT=randomized controlled trial; ROB=risk of bias; SADS-C=Schedule for Affective Disorders and Schizophrenia Change Version; SAS=Simpson Angus Scale; SDMS=Symptoms of Depression and Mania Scale; YMRS = Young Mania Rating Scale

\section{Appendix Table F12. Summary risk of bias assessments: lamotrigine for acute mania}

\begin{tabular}{|l|l|l|l|}
\hline \multicolumn{1}{|c|}{ Drug } & $\begin{array}{c}\text { Study } \\
\text { Funding Source } \\
\text { PMID }\end{array}$ & $\begin{array}{c}\text { Overall Risk of Bias } \\
\text { Assessment }\end{array}$ & Rationale \\
\hline Lamotrigine & $\begin{array}{l}\text { Ichim, } 20008 \\
\text { Industry } \\
10798820\end{array}$ & Moderate & $\begin{array}{l}\text { Randomization and blinding procedures not described. Initial difference between the two } \\
\text { groups identified on duration since index episode before hospitalization. }\end{array}$ \\
\hline
\end{tabular}

Abbreviations: PMID=PubMed Identification Number 
Appendix Table F13. Outcomes summary: lamotrigine versus active comparator for acute mania

\begin{tabular}{|c|c|c|c|c|c|c|}
\hline Drug & $\begin{array}{l}\text { Study } \\
\text { PMID }\end{array}$ & Responder/Remitter & Symptom & Function & Other & AE \\
\hline $\begin{array}{l}\text { Lamotrigine vs. } \\
\text { Lithium }\end{array}$ & $\begin{array}{l}\text { Ichim, } 2000^{8} \\
\text { Moderate } \\
10798820\end{array}$ & $\begin{array}{l}\text { Response } \\
4 \text { weeks } \\
\text { NS } \\
\text { Lamotrigine }=8 / 15 \\
\text { Lithium }=9 / 15\end{array}$ & $\begin{array}{l}\frac{\text { YMRS }}{4 \text { weeks }} \\
\text { NS } \\
\text { Lamotrigine }=14.3 \\
\text { Lithium }=13.2 \\
\frac{\text { BPRS }}{4 \text { weeks }} \\
\text { NS } \\
\text { Lamotrigine=30.2 } \\
\text { Lithium }=28.2\end{array}$ & $\begin{array}{l}\text { CGI-Severity } \\
4 \text { weeks } \\
\text { NS } \\
\text { Lamotrigine }=2.77 \\
\text { Lithium=2.87 } \\
\text { CGI-Improvement } \\
4 \text { weeks } \\
\text { NS } \\
\text { GAF } \\
4 \text { weeks } \\
\text { NS } \\
\text { Lamotrigine }=55.7 \\
\text { Lithium }=56.2\end{array}$ & $\begin{array}{l}\text { Overall } \\
\text { Withdrawal } \\
4 \text { weeks } \\
\text { NS } \\
17 \% \\
\text { Lamotrigine=2/15 } \\
\text { Lithium=3/15 }\end{array}$ & $\begin{array}{l}\text { Serious AEs } \\
4 \text { weeks } \\
\text { No serious AEs } \\
\text { reported in either } \\
\text { group and no } \\
\text { rashes were } \\
\text { reported in the } \\
\text { lamotrigine group. }\end{array}$ \\
\hline
\end{tabular}

Abbreviations: AE=Adverse Effects; BPRS=Brief Psychiatric Rating Scale; CGI= Clinical Global Impressions Scale; CGI-BP-S=Clinical Global Impressions, Bipolar, Severity -Clinical Global Impressions Scale-Improvement; GAF=General Assessment of Functioning Scale; GAS=Global Assessment Scale; HAM-D=Hamilton Scale for Serious Adverse Events; SD=standard deviation; SDMS-D=Symptoms of Depression and Mania Scale-Depression; YMRS = Young Mania Rating Scale

\section{Appendix Table F14. Strength of evidence assessment: lamotrigine versus active comparator for acute mania}

\begin{tabular}{|c|c|c|c|c|c|c|c|c|}
\hline Comparison & Outcome & $\begin{array}{l}\text { \# Studies/ } \\
\text { Design } \\
\text { (n analyzed) }\end{array}$ & $\begin{array}{c}\text { Finding or } \\
\text { Summary Statistic }\end{array}$ & $\begin{array}{c}\text { Study } \\
\text { Limitations }\end{array}$ & Consistency & Directness & Precision & $\begin{array}{l}\text { Overall Gradel } \\
\text { Conclusion }\end{array}$ \\
\hline $\begin{array}{l}\text { Lamotrigine vs. } \\
\text { lithium }\end{array}$ & $\begin{array}{l}\text { Response } \\
\text { Withdrawals - } \\
\text { overall }\end{array}$ & $\begin{array}{l}1 \mathrm{RCT} \\
(\mathrm{n}=30)\end{array}$ & See table above & Moderate & Unknown & Direct & Imprecise & Insufficient \\
\hline
\end{tabular}

Abbreviations: AE=Adverse Event; CGI=Clinical Global Impressions Scale; CGI-BP-S=Clinical Global Impressions, Bipolar, Severity Scale; n=number; RCT=randomized

controlled trial; YMRS = Young Mania Rating Scale

Notes:

1. Publication bias for antipsychotics, antidepressants, and behavioral interventions for depressive disorders is suspected.

2. Data were generally imprecise due to missing data from high attrition rates, which was commonly dealt with by Last Observation Carried Forward (LOCF). LOCF requires an assumption that the health status of patients who dropped out of the trial would not have changed had future observations been recorded, a strong assumption in the context of bipolar disorder research. 


\section{Section 4. Lithium}

Appendix Table F15. Characteristics of eligible studies: lithium for acute mania

\begin{tabular}{|c|c|c|c|c|c|c|}
\hline $\begin{array}{l}\text { Study, Year } \\
\text { Design } \\
\text { Location } \\
\text { Funder } \\
\text { Risk of Bias } \\
\text { PMID }\end{array}$ & $\begin{array}{c}\text { \# Randomized } \\
\text { Age (mean) } \\
\text { Sex (\% Female) } \\
\text { Race (\% White) } \\
\text { Diagnosis } \\
\text { (\% BP I, II, NOS) } \\
\text { Setting }\end{array}$ & $\begin{array}{c}\text { Inclusions } \\
\text { Key Exclusions }\end{array}$ & $\begin{array}{c}\text { Intervention } \\
\text { Dosage }\end{array}$ & $\begin{array}{c}\text { Comparison } \\
\text { Dosage }\end{array}$ & $\begin{array}{c}\text { Follow-up } \\
\text { Duration }\end{array}$ & $\begin{array}{c}\text { Outcomes } \\
\text { Reported } \\
\text { Withdrawal (\%) at } \\
\text { endpoint }\end{array}$ \\
\hline $\begin{array}{l}\text { Kushner, 20069 } \\
\text { RCT } \\
\text { Multisite } \\
\text { 4 Continents } \\
\text { Industry } \\
\text { RoB Low } \\
16411977\end{array}$ & $\begin{array}{l}\mathrm{N}=876 \text { (includes only } 400 \\
\text { mg/day topiramte arms } \\
\text { and placebo arms } \\
\\
\text { Mean Age } 41 \\
\text { Female (\%) } 51 \\
\text { White (\%) } 75 \\
\text { BP I (\%) } 100 \\
\\
\text { Inpatient (at least } 4 \text { days, } \\
\text { as clinically warranted) }\end{array}$ & $\begin{array}{l}\text { Mania; } \\
\text { YMRS } \geq 20 \\
\\
\text { Schizoaffective; } \\
\text { Substance Abuse; } \\
\text { Other Mental Health } \\
\text { Conditions; Taking Other } \\
\text { Medications; }\end{array}$ & $\begin{array}{l}\text { Topiramate } \\
\text { 400mg/day } \\
\text { (mean 313mg/day) } \\
\text { (only } 400 \text { mg/day } \\
\text { arms were common } \\
\text { across pooled } \\
\text { studies) }\end{array}$ & $\begin{array}{l}\text { C1: Placebo } \\
\mathrm{n}=427 \\
\\
\text { C2: Lithium } \\
300-1800 \mathrm{mg} / \mathrm{day} \\
(0.8-1.2 \mathrm{mEq} / \mathrm{L}) \\
\mathrm{n}=227\end{array}$ & 3 weeks & $\begin{array}{l}\text { YMRS } \\
\text { Weight } \\
\text { BPRS } \\
\text { CGI-S } \\
\text { GAS } \\
\text { Withdrawal 26\% }\end{array}$ \\
\hline $\begin{array}{l}\text { Bowden, } 2005^{10} \\
\text { RCT } \\
\text { Multisite } \\
2 \text { Continents } \\
\text { Industry } \\
\text { RoB High } \\
15669897\end{array}$ & $\begin{array}{l}\text { N=302 } \\
\text { Mean Age 39 } \\
\text { Female 42\% } \\
\text { Race NR } \\
\text { BP I 100\% } \\
\text { Inpatient (week 1) } \\
\text { Outpatient (weeks 2-12, } \\
\text { subject to inspector } \\
\text { discretion) }\end{array}$ & $\begin{array}{l}\text { Mania; } \\
\text { YMRS } \geq 20 \text { including } \\
\text { score of at least } 4 \text { on } 2 \\
\text { of the } 4 \text { double-weighted } \\
\text { items (irritability, speech, } \\
\text { content, and } \\
\text { disruptive/aggressive } \\
\text { behavior), CGI } \geq 4 \\
\\
\text { First manic episode; } \\
\text { Substance Use; } \\
\text { Taking other } \\
\text { medications; } \\
\text { Pregnant/Nursing; } \\
\text { Labs/Other Conditions }\end{array}$ & $\begin{array}{l}\text { Lithium } \\
0.6-1.4 \mathrm{mEq} / \mathrm{L} \\
\text { (mean } 0.80 \mathrm{mEq} / \mathrm{L} \text { ) }\end{array}$ & $\begin{array}{l}\text { C1:Placebo } \\
\text { C2: Quetiapine } \\
\text { 100-800mg/day }\end{array}$ & 12 weeks & $\begin{array}{l}\text { CGI-BP-S } \\
\text { GAS } \\
\text { Remission YMRS } \\
\leq 12) \\
\text { Response ( } \geq 50 \% \\
\text { YMRS decrease) } \\
\\
\text { Withdrawal 43\% }\end{array}$ \\
\hline
\end{tabular}




\begin{tabular}{|c|c|c|c|c|c|c|}
\hline $\begin{array}{l}\text { Study, Year } \\
\text { Design } \\
\text { Location } \\
\text { Funder } \\
\text { Risk of Bias } \\
\text { PMID }\end{array}$ & $\begin{array}{l}\text { \# Randomized } \\
\text { Age (mean) } \\
\text { Sex (\% Female) } \\
\text { Race (\% White) } \\
\text { Diagnosis } \\
\text { (\% BP I, II, NOS) } \\
\text { Setting }\end{array}$ & $\begin{array}{l}\text { Inclusions } \\
\text { Key Exclusions }\end{array}$ & $\begin{array}{l}\text { Intervention } \\
\text { Dosage }\end{array}$ & $\begin{array}{c}\text { Comparison } \\
\text { Dosage }\end{array}$ & $\begin{array}{l}\text { Follow-up } \\
\text { Duration }\end{array}$ & $\begin{array}{c}\text { Outcomes } \\
\text { Reported } \\
\text { Withdrawal (\%) at } \\
\text { endpoint }\end{array}$ \\
\hline $\begin{array}{l}\text { Segal, } 1998^{11} \\
\text { RCT } \\
\text { Singlesite } \\
\text { South Africa } \\
\text { Industry/ } \\
\text { University } \\
\text { RoB Moderate } \\
\\
9617509\end{array}$ & $\begin{array}{l}\mathrm{N}=45 \\
\text { Mean Age } 34 \\
\text { Female 78\% } \\
\text { Race NR } \\
\text { BP I 100\% } \\
\text { Inpatient }\end{array}$ & $\begin{array}{l}\text { Mania; } \\
\text { DSM-IV criteria for } \\
\text { Bipolar Manic Phase } \\
\text { Substance Abuse; } \\
\text { Taking other } \\
\text { Medications; } \\
\text { Pregnant/Nursing; } \\
\text { Abnormal Lab Results }\end{array}$ & \begin{tabular}{|l|} 
Lithium \\
$0.6-1.2 \mathrm{mmol} / \mathrm{L}$ \\
(Mean $0.72 \mathrm{mmol} / \mathrm{L}$ )
\end{tabular} & $\begin{array}{l}\text { C1: Placebo } \\
\text { C2: Haloperidol } \\
10 \text { mg/day }\end{array}$ & 4 weeks & $\begin{array}{l}\text { BPRS } \\
\text { CGI Unknown Scale - } \\
\text { Not reported whether } \\
\text { global improvement or } \\
\text { severity scale is being } \\
\text { reported } \\
\text { GAF } \\
\text { MRS } \\
\text { Seclusion - Hours } \\
\text { Seclusion - Proportion } \\
\text { of patients needing } \\
\text { SAS } \\
\text { Withdrawal 13\% }\end{array}$ \\
\hline $\begin{array}{l}\text { Bowden, } 2010^{12} \\
\text { RCT } \\
\text { Multisite } \\
2 \text { Continents } \\
\text { Industry } \\
\text { RoB Moderate } \\
20101186\end{array}$ & $\begin{array}{l}\mathrm{N}=270 \\
\text { Mean Age } 39 \\
\text { Female } 59 \% \\
\text { Race NR } \\
\text { BP I } 100 \% \\
\text { Inpatient and Outpatient }\end{array}$ & $\begin{array}{l}\text { Mania; } \\
\text { YMRS } \geq 18 \\
\text { First Manic Episode } \\
\text { Substand Abuse } \\
\text { Pregnant/Nursing } \\
\text { Labs/Other Conditions } \\
\text { Other Mental Health }\end{array}$ & \begin{tabular}{|l|} 
Lithium \\
$0.6-1.2 \mathrm{mmol} / \mathrm{L}$ \\
(mean $969 \mathrm{mg} /$ day)
\end{tabular} & $\begin{array}{l}\text { Valproate } \\
70-125 \mathrm{mcg} / \mathrm{ml} \\
\text { (mean 1394 } \\
\text { mg/day) }\end{array}$ & 12 weeks & $\begin{array}{l}\text { CGI-BP-S } \\
\text { Remission (YMRS } \\
\leq 12 \text { and decrease in } \\
\text { CGI-BP } \geq 2 \text { ) } \\
\text { Remission (YMRS } \leq \\
12 \text { and no increase in } \\
\text { MADRS total score } \\
\text { OR YMRS } \leq 12 \text { and } \\
\text { CGI } \leq 2 \text { points) } \\
\text { Response } \\
\text { (improvement in } \\
\text { YMRS } \geq 30 \% \text { ) } \\
\text { YMRS } \\
\\
\text { Withdrawal } 28 \% \\
\end{array}$ \\
\hline
\end{tabular}

Abbreviations: AE=Adverse Events; AIMS=Abnormal Involuntary Movement Scale; BAS=Behavioral Approach System; BCL= Shopsin-Gershon Social Behavior Checklist; BIS-11=Barratt Impulsiveness Scale; BP=bipolar disorder; BPRS=Brief Psychiatric Rating Scale; C=Comparision; CGI=Clinical Global Impressions Scale; CGI-BP =Clinical Global Impressions Scale for Bipolar Disorder; CGI-I= Clinical Global Impressions-Improvement; CGI-S=Clinical Global Impressions, Severity Scale; DSM=Diagnostic and Statistical Manual of Mental Disorders; DSM-IV= Diagnostic and statistical manual, $4^{\text {th }}$ edition; DSS=Depressive Syndrome Scale; ER=extended release; GAF=General Assessment of Functioning Scale; GAS= Global Assessment Scale; HAM-D=Hamilton Scale for Depression; LIFE-RIFT= Range of Impaired Functioning Tool; MADRS=Montgomery-Asberg Depression Rating Scale; MSS=Manic Syndrome Scale; NOS=not otherwise specified; NR=not reported; PMID=PubMed Identification Number; 
RCT=randomized controlled trial; ROB=risk of bias; SADS-C=Schedule for Affective Disorders and Schizophrenia Change Version; SAS=Simpson Angus Scale; SDMS=Symptoms of Depression and Mania Scale; YMRS = Young Mania Rating Scale

\section{Appendix Table F16. Summary risk of bias assessments: lithium for acute mania}

\begin{tabular}{|c|c|c|c|}
\hline Drug & $\begin{array}{l}\text { Study } \\
\text { Funding Source } \\
\text { PMID }\end{array}$ & $\begin{array}{l}\text { Overall Risk of Bias } \\
\text { Assessment }\end{array}$ & Rationale \\
\hline \multirow[t]{4}{*}{ Lithium } & $\begin{array}{l}\text { Bowden, } 2010^{12} \\
\text { Industry } \\
20101186\end{array}$ & Moderate & Randomization procedure not described, patients and raters may not be blinded. $28 \%$ dropout. \\
\hline & $\begin{array}{l}\text { Kushner, } 2006^{9} \\
\text { Industry } \\
16411977\end{array}$ & Low & No sources of bias identified \\
\hline & $\begin{array}{l}\text { Bowden, } 2005^{10} \\
\text { Industry } \\
15669897\end{array}$ & High & Randomization and blinding not described. Overall dropout of $43 \%$. \\
\hline & $\begin{array}{l}\text { Segal,199811 } \\
\text { Industry/ } \\
\text { University } \\
9617509\end{array}$ & Moderate & $\begin{array}{l}\text { Noted 'randomly and assigned consecutively to treatment with..." which is a pseudorandom } \\
\text { assignment technique. Blinding not described. }\end{array}$ \\
\hline
\end{tabular}

Abbreviations: PMID=PubMed Identification Number 


\section{Mood Stabilizer Forest Plots}

Outcomes in studies assessed as having a high risk of bias, or low to moderate risk of bias but at least 40 percent attrition, are presented in grey tones. Both fixed-effect models and random-effects models are presented. We calculated fixed-effect models to provide a charitable estimate of the average effect among completed trials. However, we base our main conclusions on the random-effects models.

Appendix Figure F1. Lithium vs. placebo - remission

Odds Ratio of Remission (YMRS 12 or Less) at 3 Weeks

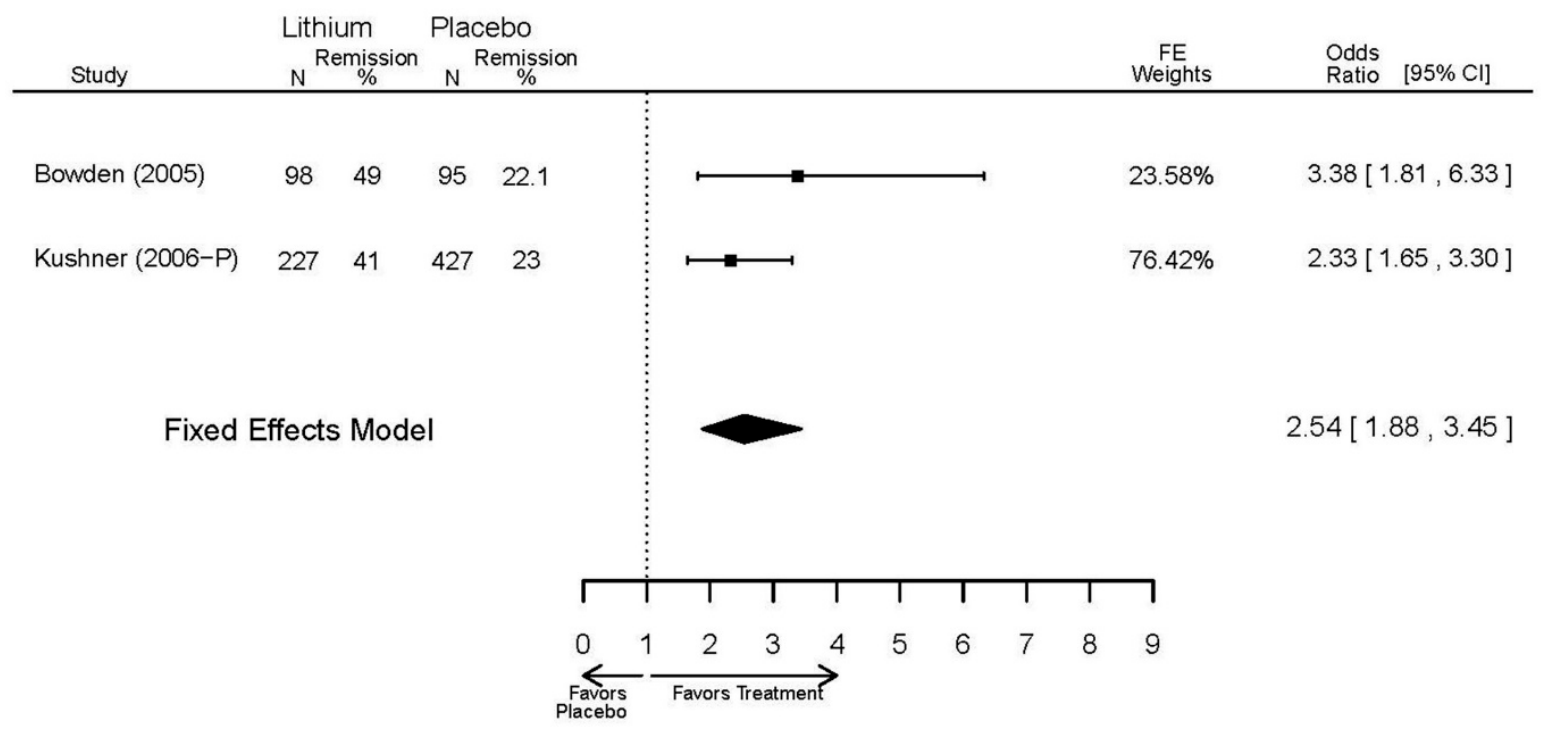


Appendix Figure F2. Lithium vs. placebo - response

Odds Ratio of Response (> 50\% Reduction in YMRS) at 3 Weeks

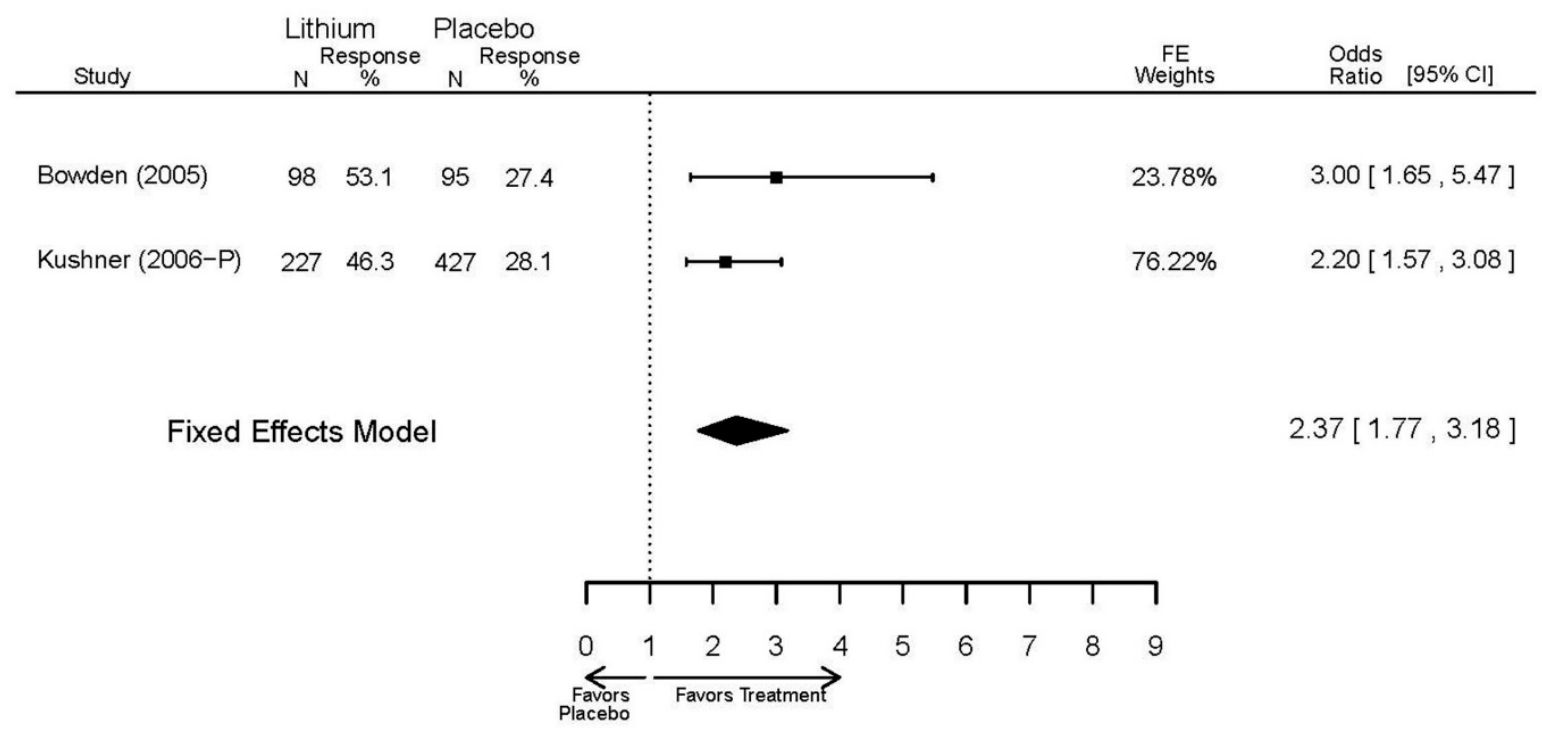

Appendix Figure F3. Lithium vs. placebo - YMRS

Difference in Mean Change in YMRS

from Baseline to 3 Weeks

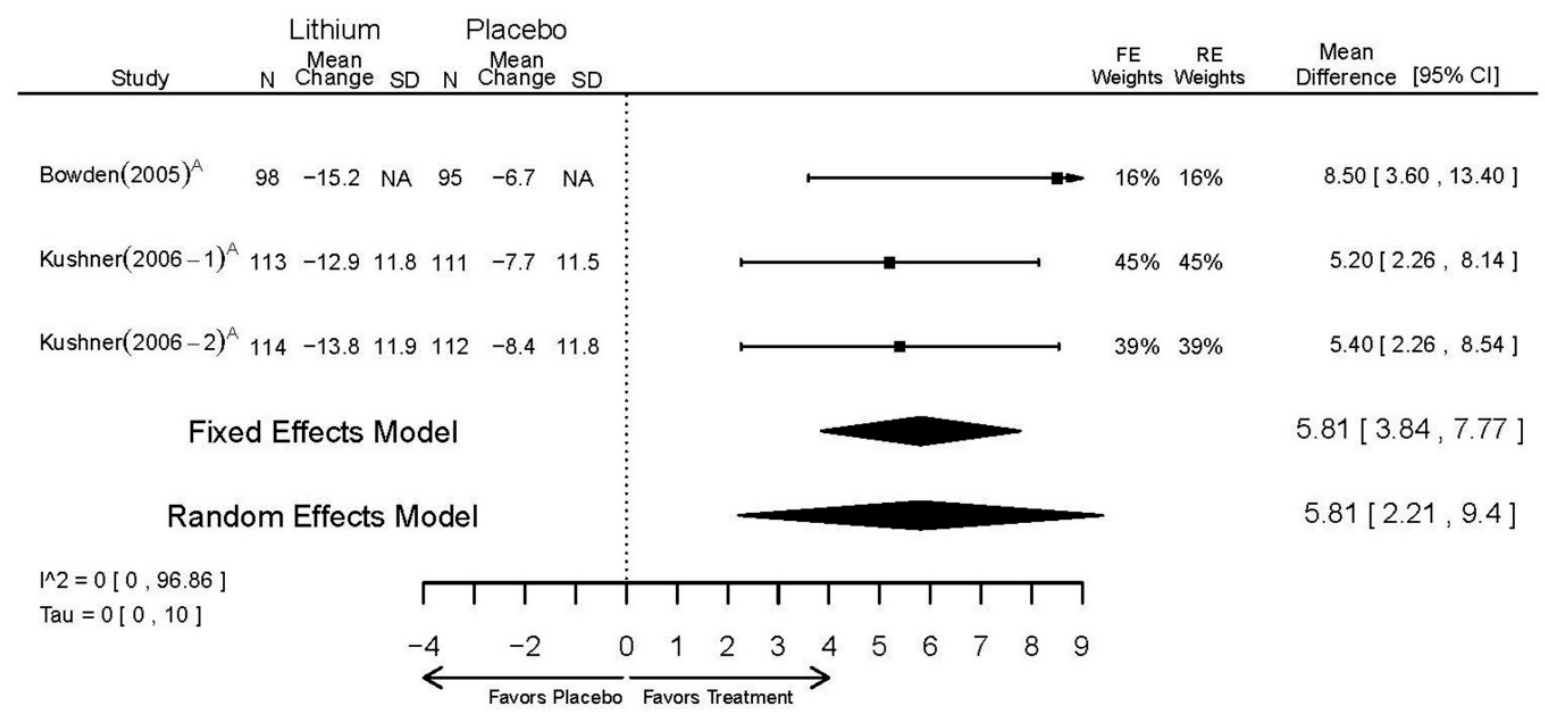


Appendix Figure F4. Lithium vs. placebo - overall withdrawal

Odds Ratio of Overall Withdrawal

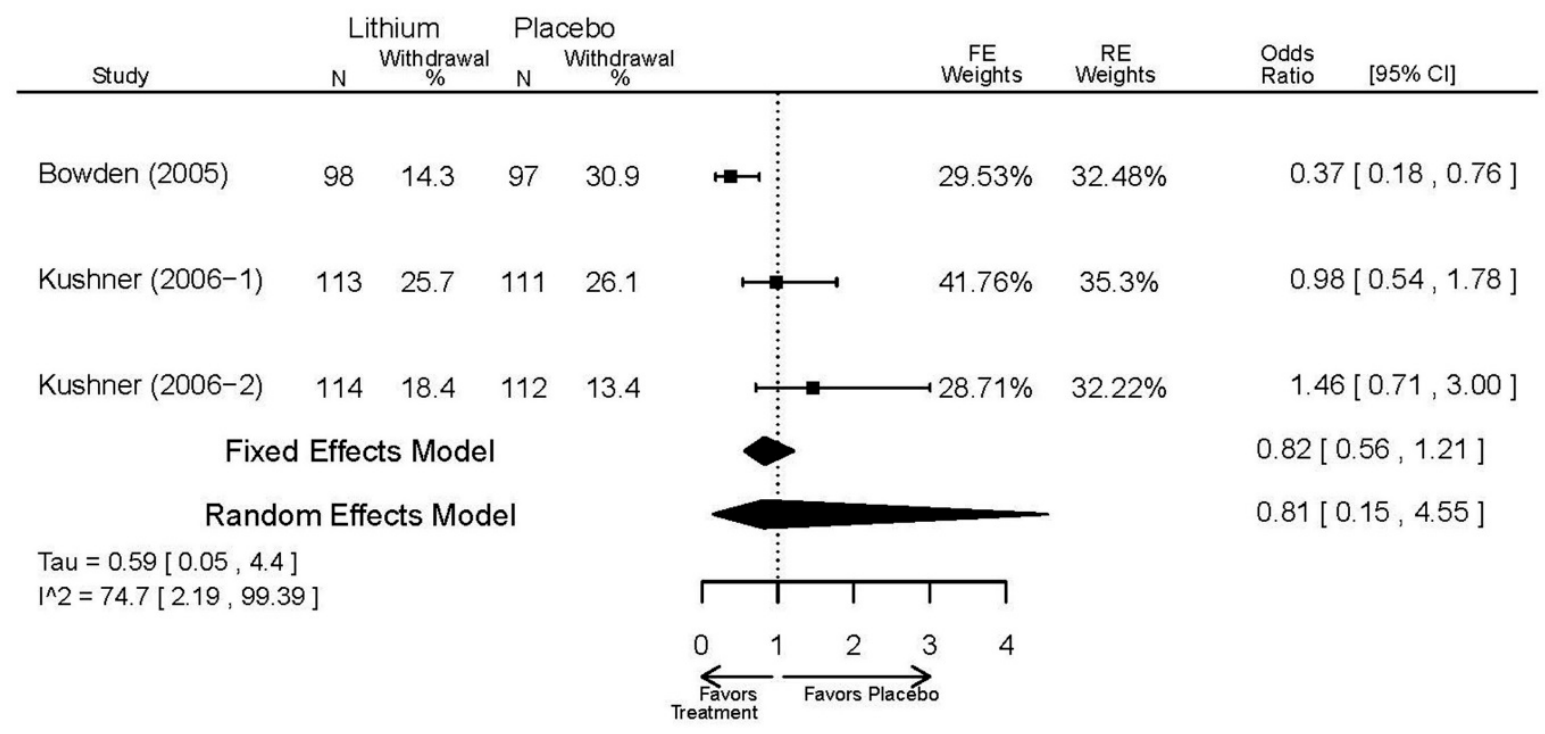

Appendix Figure F5. Lithium vs. placebo - withdrawal lack of efficacy

Odds Ratio of Withdrawal due to Lack of Efficacy at 3 Weeks

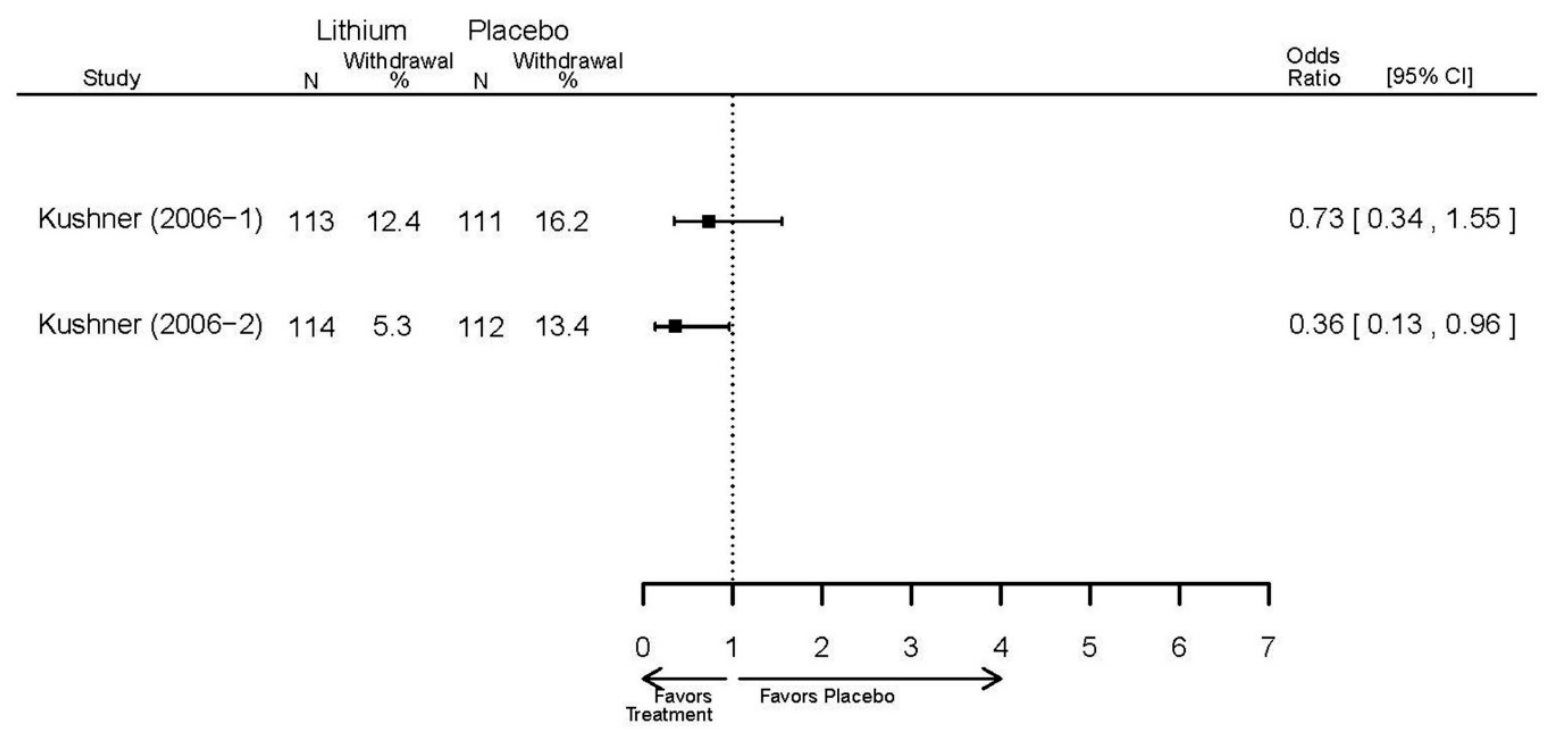


Appendix Figure F6. Lithium vs. placebo - withdrawal adverse events Odds Ratio of Withdrawal due to Adverse Events at 3 Weeks

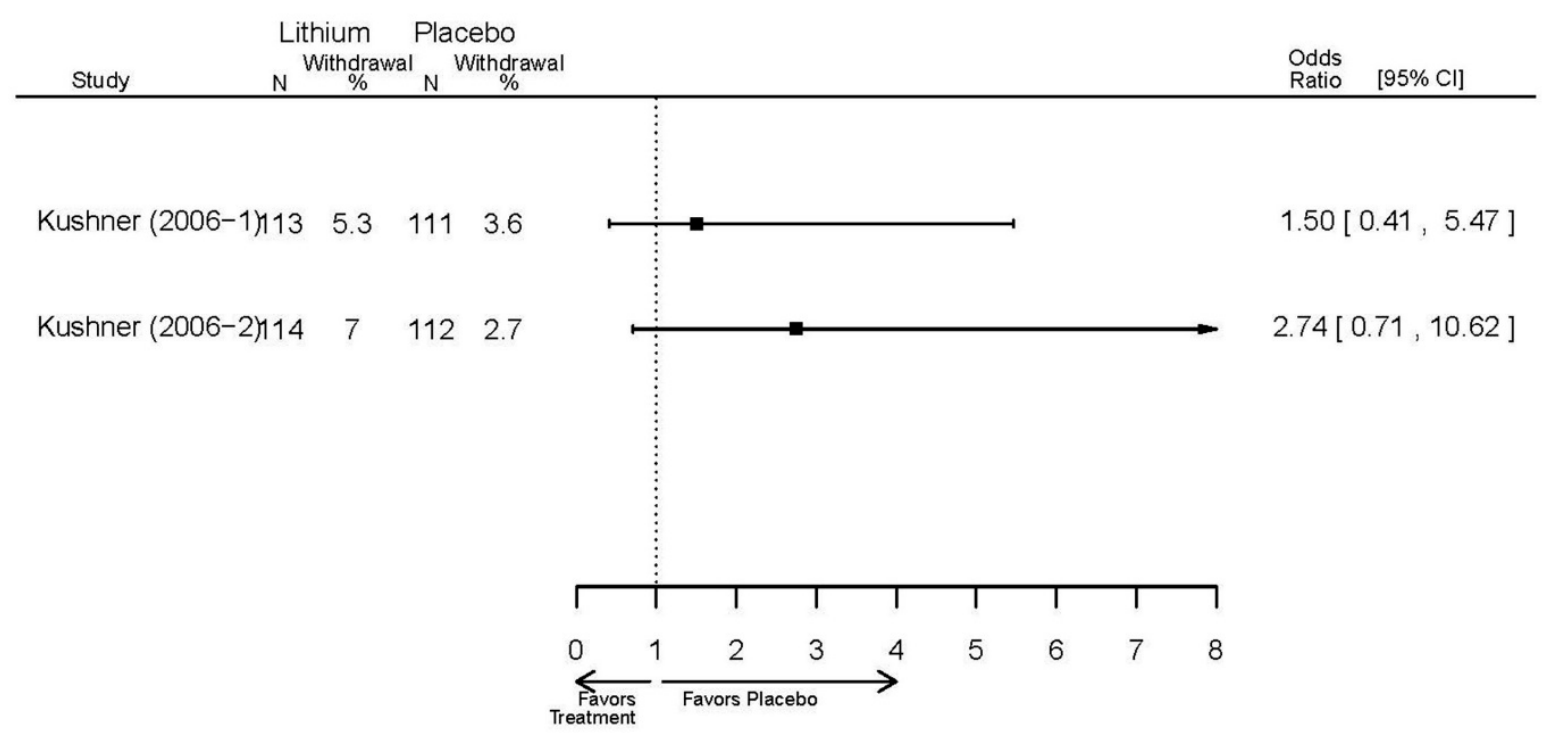


Appendix Table F17. Outcomes summary: lithium versus placebo for acute mania

\begin{tabular}{|c|c|c|c|c|c|c|}
\hline Drug & $\begin{array}{l}\text { Study } \\
\text { PMID }\end{array}$ & Responder/Remitter & Symptom & Function & Other & AE \\
\hline \multirow[t]{2}{*}{$\begin{array}{l}\text { Lithium vs. } \\
\text { placebo }\end{array}$} & $\begin{array}{l}\text { Bowden, } \\
2005^{10} \\
15669897 \\
\text { Moderate }\end{array}$ & $\begin{array}{l}\frac{\text { Response }}{\text { See forest plot F1 }} \text { above } \\
\frac{\text { Remission }}{\text { See forest plot F2 }} \\
\text { above }\end{array}$ & $\begin{array}{l}\text { YMRS } \\
\text { See forest plot F3 } \\
\text { above }\end{array}$ & $\begin{array}{l}\text { CGI-BP } \\
12 \text { weeks } \\
\text { Favors Lithium } \\
\text { Lithium }=-2.2 \\
\text { Placebo=-0.9 } \\
\text { Difference in } \\
\text { Difference } \\
(\mathrm{SD})=1.3 \\
(0.38) \\
\mathrm{p}<0.001\end{array}$ & $\begin{array}{l}\text { Overall Withdrawal } \\
\text { See forest plot F4 above } \\
\text { Withdrawal due to Lack of } \\
\text { Efficacy } \\
12 \text { weeks } \\
\text { Favors Lithium } \\
\text { Lithium }=12 / 98 \\
\text { Placebo=38/97 } \\
\text { OR }=0.22(95 \% \text { Cl } 0.10, \\
0.45) \\
\text { p }<0.0001 \\
\text { Withdrawal due to AEs } \\
12 \text { weeks } \\
\text { NS } \\
\text { Lithium }=6 / 98 \\
\text { Placebo }=4 / 97 \\
\text { OR }=1.49(95 \% \mathrm{Cl} 0.40, \\
6.26) \\
\text { p }=0.75\end{array}$ & $\begin{array}{l}\text { EPS } \\
12 \text { weeks } \\
\text { Lithium }=21 / 98^{*} \\
\text { Placebo }=35 / 97 \\
\text { *May be more cases, exact } \\
\text { number NR } \\
\text { Emergent Depression } \\
12 \text { weeks } \\
\text { Lithium }=8 / 95 \\
\text { Placebo }=3 / 97 \\
\text { Akathisia } \\
12 \text { weeks } \\
\text { Lithium }=3 / 98 \\
\text { Placebo }=6 / 97\end{array}$ \\
\hline & $\begin{array}{l}\text { Kushner, } \\
2006^{9} \\
16411977 \\
\text { Low }\end{array}$ & $\begin{array}{l}\frac{\text { Response }}{\text { See forest plot F1 }} \\
\text { above } \\
\frac{\text { Remission }}{\text { See forest plot F2 }} \\
\text { above }\end{array}$ & $\begin{array}{l}\frac{\text { YMRS }}{\text { See forest plot F3 }} \\
\text { above }\end{array}$ & NR & $\begin{array}{l}\frac{\text { Overall Withdrawal }}{\text { See forest plot F4 above }} \\
\frac{\text { Withdrawal Lack of Efficacy }}{\text { See forest plot F5 above }} \\
\frac{\text { Withdrawal due to AEs }}{\text { See forest plot F6 above }}\end{array}$ & $\begin{array}{l}\text { Severe AEs } \\
3 \text { weeks (Placebo) } \\
12 \text { weeks (Lithium) } \\
\text { Lithium }=4 / 227 \\
\text { Placebo }=9 / 427 \\
\text { Emergent Depression } \\
3 \text { weeks (Placebo) } \\
12 \text { weeks (Lithium) } \\
\text { Lithium }=16 / 227 \\
\text { Placebo }=51 / 427 \\
\text { Emergent Depression } \\
12 \text { weeks (Lithium) } \\
3 \text { weeks (Placebo) } \\
3 \text { cases of suicidal ideation } \\
\text { in placebo group. }\end{array}$ \\
\hline
\end{tabular}

Abbreviations: AE=Adverse Effects; BMI=Body Mass Index; CGI= Clinical Global Impressions; CGI-BP =Clinical Global Impressions Scale for Bipolar Disorder; CGI-BP-

S=Clinical Global Impressions, Bipolar, Severity Scale; CGI-I= Clinical Global Impressions-Improvement; CGI-S=CGI-Severity; CI= Confidence Interval; EPS=Extrapyramidal 
Side Effects; GAS= Global Assessment Scale; HAM-D=Hamilton Scale for Depression; MADRS=Montgomery-Asberg Depression Rating Scale; NR=not reported; NS=no significant; OPT=Optimalized Personalized Treatment; OR=Odds Ratio; PMID=PubMed Identification Number; RCT=randomized controlled trial; SAE= Serious Adverse Events; SD=Standard Deviation; SE=Standard Error; YMRS = Young Mania Rating Scale

Appendix Table F18. Strength of evidence assessment: lithium versus placebo for acute mania

\begin{tabular}{|c|c|c|c|c|c|c|c|c|}
\hline Comparison & Outcome & $\begin{array}{l}\text { \# Studies/ } \\
\text { Design } \\
\text { (n analyzed) }\end{array}$ & $\begin{array}{l}\text { Finding or } \\
\text { Summary } \\
\text { Statistic }\end{array}$ & $\begin{array}{c}\text { Study } \\
\text { Limitations }\end{array}$ & Consistency & Directness & Precision & $\begin{array}{c}\text { Overall } \\
\text { Gradel } \\
\text { Conclusion }\end{array}$ \\
\hline \multirow[t]{5}{*}{$\begin{array}{l}\text { Lithium vs. } \\
\text { placebo }\end{array}$} & $\begin{array}{l}\text { Remission } 3 \text { wks } \\
\text { Response } 3 \text { wks }\end{array}$ & $\begin{array}{l}1 \mathrm{RCT}+1 \text { IPD } \\
(\mathrm{n}=325)\end{array}$ & Favors Lithium & Moderate & Consistent & Direct & Imprecise & Low \\
\hline & YMRS 3 wks & $\begin{array}{l}3 \text { RCTs } \\
(n=325)\end{array}$ & $\begin{array}{l}\text { Favors Lithium } \\
\text { MD } 5.81(95 \% \\
\text { Cl } 2.21,9.4) \\
\end{array}$ & Moderate & Consistent & Direct & Imprecise & Low \\
\hline & $\begin{array}{l}\text { Withdrawal - } \\
\text { Overall } 3 \text { wks }\end{array}$ & $\begin{array}{l}3 \text { RCTs } \\
(n=325)\end{array}$ & NS & Moderate & Consistent & Direct & Imprecise & Low \\
\hline & $\begin{array}{l}\text { Withdrawal - } \\
\text { Lack of Efficacy, } \\
\text { AE }\end{array}$ & $\begin{array}{l}1 \text { IPD } \\
(n=450)\end{array}$ & NS & Moderate & Consistent & Direct & Imprecise & Low \\
\hline & CGI & $\begin{array}{l}1 \text { RCT } \\
(n=193)\end{array}$ & See table above & Moderate & Unknown & Direct & Imprecise & Insufficient \\
\hline
\end{tabular}

Abbreviations: AE=Adverse Events; CGI= Clinical Global Impressions; CI=Confidence Interval; IPD=Individual patient data; MADRS=Montgomery-Asberg Depression Rating Scale; MD=Mean Difference; NS=Not Significant; RCT=randomized controlled trial; YMRS = Young Mania Rating Scale

Notes:

1. Publication bias for antipsychotics, antidepressants, and behavioral interventions for depressive disorders is suspected.

2. Data were generally imprecise due to missing data from high attrition rates, which was commonly dealt with by Last Observation Carried Forward (LOCF). LOCF requires an assumption that the health status of patients who dropped out of the trial would not have changed had future observations been recorded, a strong assumption in the context of bipolar disorder research. 
Appendix Table F19. Outcomes summary: lithium active comparator for acute mania

\begin{tabular}{|c|c|c|c|c|c|c|}
\hline Drug & $\begin{array}{l}\text { Study } \\
\text { PMID }\end{array}$ & Responder/Remitter & Symptom & Function & Other & $\mathrm{AE}$ \\
\hline $\begin{array}{l}\text { Lithium vs. } \\
\text { haloperidol }\end{array}$ & $\begin{array}{l}\text { Segal, } 1998^{11} \\
9617509 \\
\text { Moderate }\end{array}$ & NR & $\begin{array}{l}\frac{\text { MRS }}{4 \text { weeks }} \\
\text { NS } \\
\text { Lithium }=-19.3 \\
\text { Haloperidol=-19.9 }\end{array}$ & $\begin{array}{l}\text { CGI-BP } \\
4 \text { weeks } \\
\text { NS } \\
\text { No additional data } \\
\text { reported }\end{array}$ & $\begin{array}{l}\frac{\text { Overall }}{\text { Withdrawal }} \\
4 \text { weeks } \\
\text { NS } \\
\text { Lithium }=1 / 15 \\
\text { Haloperidol=3/15 } \\
p=0.06\end{array}$ & NR \\
\hline $\begin{array}{l}\text { Lithium vs. } \\
\text { valproate }\end{array}$ & $\begin{array}{l}\text { Bowden, } 2010^{12} \\
20101186 \\
\text { Moderate }\end{array}$ & $\begin{array}{l}\frac{\text { Response }}{12 \text { weeks }} \\
\text { Valproate } 79.5 \% \\
\text { Lithium } 72.6 \% \\
\text { NS } \\
\text { Remission } \\
\text { (YMRS/MADRS) } \\
12 \text { weeks } \\
\text { Valproate } 71.3 \% \\
\text { Lithium } 65.9 \% \\
\text { NS } \\
\text { Remission } \\
\text { (YMRS/CGI) } \\
12 \text { weeks } \\
\text { Favors valproate } \\
\text { Valproate } 71.9 \% \\
\text { Lithium } 58.5 \% \\
\text { p=0.025 }\end{array}$ & $\begin{array}{l}\text { YMRS Change }(90 \% \mathrm{Cl}) \\
12 \text { weeks } \\
\text { Valproate }-17.3(9.4) \\
\text { Lithium }-15.8(5.3) \\
\text { NS }\end{array}$ & $\begin{array}{l}\text { CGI-BP-S Change } \\
12 \text { weeks } \\
\text { Valproate }-2.1(1.4) \\
\text { Lithium }-2.3(1.3) \\
\text { NS }\end{array}$ & $\begin{array}{l}\frac{\text { Overall }}{\text { Withdrawal }} \\
\text { Lithium } 38 / 135 \\
\text { Valproate } 34 / 122 \\
\text { Withdrawal Lack } \\
\text { of efficacy } \\
\text { Lithium } 13 / 135 \\
\text { Valproate } 13 / 122 \\
\text { Withdrawal AE } \\
\text { Lithium } 9 / 138 \\
\text { Valproate } 8 / 122\end{array}$ & $\begin{array}{l}\text { Serious Adverse } \\
\text { Events } \\
12 \text { weeks } \\
10 \text { in valproate } \\
5 \text { in lithium } \\
\\
\text { Weight gain }>7 \% \\
\text { Lithium } 9 \% \\
\text { Valproate } 7 \%\end{array}$ \\
\hline
\end{tabular}

Abbreviations: AE=Adverse Effects; BPRS=Brief Psychiatric Rating Scale; CGI= Clinical Global Impressions Scale; CGI-BP-S=Clinical Global Impressions, Bipolar, Severity Scale; CGI-I=Clinical Global Impressions Scale-Improvement; GAF=General Assessment of Functioning Scale; GAS=Global Assessment Scale; HAM-D=Hamilton Scale for Depression; MADRS=Montgomery-Asberg Syndrome Scale; MRS=mania rating scale; NR=not reported; NS=not significant; PMID=PubMed Identification Number; SAE= Serious Adverse Events; SD=standard deviation; SDMS-D=Symptoms of Depression and Mania Scale-Depression; YMRS = Young Mania Rating Scale 
Appendix Table F20. Strength of evidence assessment: lithium versus active comparator for acute mania

\begin{tabular}{|c|c|c|c|c|c|c|c|c|}
\hline Comparison & Outcome & $\begin{array}{l}\text { \# Studies/ } \\
\text { Design } \\
\text { (n analyzed) }\end{array}$ & $\begin{array}{c}\text { Finding or } \\
\text { Summary Statistic }\end{array}$ & $\begin{array}{c}\text { Study } \\
\text { Limitations }\end{array}$ & Consistency & Directness & Precision & $\begin{array}{c}\text { Overall Gradel } \\
\text { Conclusion }\end{array}$ \\
\hline $\begin{array}{l}\text { Lithium vs. } \\
\text { haloperidol }\end{array}$ & $\begin{array}{l}\text { YMRS } \\
\text { CGI }\end{array}$ & $\begin{array}{l}1 \mathrm{RCT} \\
(\mathrm{n}=30)\end{array}$ & See table above & Moderate & Unknown & Direct & Imprecise & Insufficient \\
\hline $\begin{array}{l}\text { Lithium vs. } \\
\text { divalproex }\end{array}$ & $\begin{array}{l}\text { Response } \\
\text { Remission } \\
\text { YMRS } \\
\text { CGI-BP-S } \\
\text { Withdrawals }\end{array}$ & $\begin{array}{l}1 \mathrm{RCT} \\
(\mathrm{n}=270)\end{array}$ & See table above & Moderate & Unknown & Direct & Imprecise & Insufficient \\
\hline
\end{tabular}

Abbreviations: AE=Adverse Event; CGI=Clinical Global Impressions Scale; CGI-BP-S=Clinical Global Impressions, Bipolar, Severity Scale; n=number; RCT=randomized controlled trial; YMRS = Young Mania Rating Scale

Notes:

1. Publication bias for antipsychotics, antidepressants, and behavioral interventions for depressive disorders is suspected.

2. Data were generally imprecise due to missing data from high attrition rates, which was commonly dealt with by Last Observation Carried Forward (LOCF). LOCF requires an

assumption that the health status of patients who dropped out of the trial would not have changed had future observations been recorded, a strong assumption in the context of

bipolar disorder research. 


\section{References for Appendix $F$}

1. Weisler RH, Hirschfeld R, Cutler AJ, et al. Extended-release carbamazepine capsules as monotherapy in bipolar disorder : pooled results from two randomised, double-blind, placebocontrolled trials. CNS Drugs. 2006;20(3):219-31. PMID: 16529527.

2. Vasudev K, Goswami U, Kohli K. Carbamazepine and valproate monotherapy: feasibility, relative safety and efficacy, and therapeutic drug monitoring in manic disorder.

Psychopharmacology. 2000 May;150(1):15-23. PMID: 10867972.

3. Small JG, Klapper MH, Milstein V, et al. Carbamazepine compared with lithium in the treatment of mania. Arch Gen Psychiatry. 1991 Oct;48(10):915-21. PMID: 1929761.

4. Lerer B, Moore N, Meyendorff E, et al. Carbamazepine versus lithium in mania: a double-blind study. J Clin Psychiatry. 1987 Mar;48(3):89-93. PMID: 3546274.

5. Tohen M, Vieta E, Goodwin GM, et al. Olanzapine versus divalproex versus placebo in the treatment of mild to moderate mania: a randomized, 12-week, double-blind study. Journal of Clinical Psychiatry. 2008 Nov;69(11):1776-89. PMID: 19014751.

6. Bowden CL, Swann AC, Calabrese JR, et al. A randomized, placebo-controlled, multicenter study of divalproex sodium extended release in the treatment of acute mania. Journal of Clinical Psychiatry. 2006 Oct;67(10):1501-10. PMID: 17107240.
7. Xu L, Lu Y, Yang Y, et al. Olanzapine-valproate combination versus olanzapine or valproate monotherapy in the treatment of bipolar imania: A randomized controlled study in a chinese population group. Neuropsychiatric Disease and Treatment. 201525 May;11:1265-71. doi: http://dx.doi.org/10.2147/NDT.S81146. PMID: 26060401 (pubmed) 2015078440 (embase).

8. Ichim L, Berk M, Brook S. Lamotrigine compared with lithium in mania: a double-blind randomized controlled trial. Annals of Clinical Psychiatry. 2000 Mar;12(1):5-10. PMID: 10798820.

9. Kushner SF, Khan A, Lane R, et al. Topiramate monotherapy in the management of acute mania: results of four double-blind placebo-controlled trials. Bipolar Disorders. 2006 Feb;8(1):15-27. PMID: 16411977.

10. Bowden CL, Grunze H, Mullen J, et al. A randomized, double-blind, placebo-controlled efficacy and safety study of quetiapine or lithium as monotherapy for mania in bipolar disorder. Journal of Clinical Psychiatry. 2005 Jan;66(1):111-21. PMID: 15669897.

11. Segal J, Berk M, Brook S. Risperidone compared with both lithium and haloperidol in mania: a double-blind randomized controlled trial. Clinical Neuropharmacology. 1998 May-Jun;21(3):17680. PMID: 9617509.

12. Bowden CL, Mosolov S, Hranov L, et al. Efficacy of valproate versus lithium in mania or mixed mania: a randomized, open 12-week trial. International Clinical Psychopharmacology. 2010 Mar;25(2):60-7. doi: http://dx.doi.org/10.1097/YIC.0b013e328333ac1b . PMID: 20101186. 


\section{Appendix G. Other Drugs for Acute Mania}

\section{Section 1. Allopurinol}

Appendix Table G1. Characteristics of eligible studies: allopurinol for acute mania

\begin{tabular}{|c|c|c|c|c|c|c|}
\hline $\begin{array}{c}\text { Study, Year } \\
\text { Design } \\
\text { Location } \\
\text { Funder } \\
\text { Risk of Bias } \\
\text { PMID }\end{array}$ & $\begin{array}{c}\text { \# Randomized } \\
\text { Age (mean) } \\
\text { Sex (\% Female) } \\
\text { Race (\% White) } \\
\text { Diagnosis } \\
\text { (\% BP I, II, NOS) } \\
\text { Setting }\end{array}$ & $\begin{array}{l}\text { Inclusions } \\
\text { Key Exclusions }\end{array}$ & $\begin{array}{c}\text { Intervention } \\
\text { Dosage }\end{array}$ & $\begin{array}{c}\text { Comparison } \\
\text { Dosage }\end{array}$ & $\begin{array}{c}\text { Follow-up } \\
\text { Duration }\end{array}$ & $\begin{array}{c}\text { Outcomes } \\
\text { Reported } \\
\text { Withdrawal (\%) at } \\
\text { endpoint }\end{array}$ \\
\hline $\begin{array}{l}\text { Jahangard, } 2014^{1} \\
\text { RCT } \\
\text { Singlesite } \\
\text { Iran } \\
\text { Nonprofit } \\
\text { RoB Low } \\
24953766\end{array}$ & $\begin{array}{l}\mathrm{N}=60 \\
\text { Mean Age NR } \\
\text { Female NR } \\
\text { Race NR } \\
\text { BP I 100\% } \\
\text { Inpatient }\end{array}$ & $\begin{array}{l}\text { Manic; YMRS } \geq 28 \\
\text { Schizoaffective } \\
\text { Substance Abuse } \\
\text { Other Mental Health } \\
\text { Pregnant/Nursing }\end{array}$ & $\begin{array}{l}\text { Allopurinol } \\
600 \text { mg/day } \\
+ \text { sodium valproate } \\
(15-20 \mathrm{mg} / \mathrm{kg}) \text { and } \\
\text { benzodiazepines }\end{array}$ & $\begin{array}{l}\text { Placebo } \\
+ \text { sodium } \\
\text { valproate (15-20 } \\
\text { mg/kg) and } \\
\text { benzodiazepines }\end{array}$ & 4 weeks & $\begin{array}{l}\text { YMRS } \\
\text { Remission (YMRS } \leq 7) \\
\text { CGI } \\
\text { Withdrawal 18\% }\end{array}$ \\
\hline $\begin{array}{l}\text { Weiser, } 2014^{2} \\
\text { RCT } \\
\text { Multisite } \\
\text { Romania } \\
\text { Nonprofit } \\
\text { RoB High } \\
24712840 \\
\end{array}$ & $\begin{array}{l}\mathrm{N}=180 \\
\text { Mean Age } 47 \\
\text { Female } 66 \% \\
\text { White } 100 \% \\
\text { BP I } 100 \% \\
\text { Inpatient or Outpatient }\end{array}$ & $\begin{array}{l}\text { Manic; Clinical Interview } \\
\text { in DSM-IV treated with } \\
\text { mood stabilizer or } \\
\text { neuroleptics for between } \\
3 \text { days and } 2 \text { weeks. } \\
\text { None Specified }\end{array}$ & $\begin{array}{l}\text { Allopurinol } \\
300 \text { mg/day } \\
+ \text { mood stabilizer } \\
\text { and/or antipsychotic }\end{array}$ & $\begin{array}{l}\text { Placebo+ mood } \\
\text { stabilizer and/or } \\
\text { antipsychotic }\end{array}$ & 6 weeks & $\begin{array}{l}\text { Response } \\
\text { (YMRS } \geq 50 \% \\
\text { improvement) } \\
\text { YMRS } \\
\text { CGI-BP } \\
\text { PANSS } \\
\text { AEs } \\
\text { Withdrawal } 17 \% \\
\end{array}$ \\
\hline $\begin{array}{l}\text { Fan, } 2012^{3} \\
\text { RCT } \\
\text { Singlesite } \\
\text { United States } \\
\text { Nonprofit } \\
\text { RoB Medium } \\
22420596\end{array}$ & $\begin{array}{l}\mathrm{N}=27 \\
\text { Mean Age } 43 \\
\text { Female } 50 \% \\
\text { White } 63 \% \\
\text { BP I } 100 \% \\
\text { Outpatient }\end{array}$ & $\begin{array}{l}\text { Manic; YMRS } \geq 14 \text { partial } \\
\text { response to lithium, } \\
\text { valproate, } \\
\text { carbamazepine, or } \\
\text { atypical antipsychotics } \\
\text { Substance Abuse } \\
\text { Other Mental Health } \\
\text { Pregnant/Nursing } \\
\text { Labs/Other Conditions }\end{array}$ & $\begin{array}{l}\text { Allopurinol } \\
600 \text { mg/day (300 } \\
\text { mg/day first week) } \\
\text { Current psychiatric } \\
\text { medications }\end{array}$ & $\begin{array}{l}\text { Placebo + current } \\
\text { psychiatric } \\
\text { medications }\end{array}$ & 6 weeks & $\begin{array}{l}\text { YMRS } \\
\text { HAM-D } \\
\text { CGI } \\
\text { SDS } \\
\text { Q-LES-Q } \\
\text { Withdrawal 15\% }\end{array}$ \\
\hline
\end{tabular}




\begin{tabular}{|c|c|c|c|c|c|c|}
\hline $\begin{array}{l}\text { Study, Year } \\
\text { Design } \\
\text { Location } \\
\text { Funder } \\
\text { Risk of Bias } \\
\text { PMID }\end{array}$ & $\begin{array}{c}\text { \# Randomized } \\
\text { Age (mean) } \\
\text { Sex (\% Female) } \\
\text { Race (\% White) } \\
\text { Diagnosis } \\
\text { (\% BP I, II, NOS) } \\
\text { Setting }\end{array}$ & $\begin{array}{c}\text { Inclusions } \\
\text { Key Exclusions }\end{array}$ & $\begin{array}{l}\text { Intervention } \\
\text { Dosage }\end{array}$ & $\begin{array}{c}\text { Comparison } \\
\text { Dosage }\end{array}$ & $\begin{array}{c}\text { Follow-up } \\
\text { Duration }\end{array}$ & $\begin{array}{c}\text { Outcomes } \\
\text { Reported } \\
\text { Withdrawal (\%) at } \\
\text { endpoint }\end{array}$ \\
\hline $\begin{array}{l}\text { Machado-Vieira, } 2008^{4} \\
\text { RCT } \\
\text { Brazil } \\
\text { Non-Profit } \\
\text { RoB Moderate } \\
18681754\end{array}$ & $\begin{array}{l}\mathrm{N}=180 \\
\text { Mean Age } 29.3 \\
\text { Female } 59 \% \\
\text { White NR } \\
\text { BP I 100\% } \\
\text { Not Disclosed }\end{array}$ & $\begin{array}{l}\text { Manic; } \\
\text { YMRS } \geq 22 \\
\text { Schizoaffective } \\
\text { Substance abuse } \\
\text { Other mental health } \\
\text { Taking other meds } \\
\text { Labs/other conditions }\end{array}$ & $\begin{array}{l}\text { T1: Allopurinol } \\
60 \text { mg/day } \\
\text { T2: Dipyridamole } \\
200 \mathrm{mg} / \text { day } \\
\\
\text { Lithium } \\
600-900 \mathrm{mg} / \text { day } \\
\text { serum level } \\
0.6-1.2 \mathrm{mmol} / \mathrm{L} \\
\text { (mean } 0.99 \mathrm{mmol} / \mathrm{L} \text { ) }\end{array}$ & $\begin{array}{l}\text { Placebo } \\
\text { Lithium } \\
600-900 \mathrm{mg} / \mathrm{day} \\
\text { serum level } \\
0.6-1.2 \mathrm{mmol} / \mathrm{L} \\
\text { (mean 0.95 } \\
\text { mmol/L) }\end{array}$ & 4 weeks & $\begin{array}{l}\text { CGI-S } \\
\text { Remission } \\
\text { (YMRS } \leq 7) \\
\text { (YMRS } \leq 12) \\
\text { Response } \\
\text { (50\% improved } \\
\text { YMRS) } \\
\text { Adverse Events } \\
\text { Lab Values } \\
\text { Withdrawal 20\% }\end{array}$ \\
\hline
\end{tabular}

Abbreviations: AE=Adverse Effects; BP=bipolar disorder; BPRS=Brief Psychiatric Rating Scale; CGI=Clinical Global Impressions; CGI-BP=Clinical Global Impressions Scale for Bipolar Disorder; CGI-BP-S=Clinical Global Impressions, Bipolar, Severity Scale; CGI-S=Clinical Global Impressions, Severity Scale; DSM-IV= Diagnostic and statistical manual, $4^{\text {th }}$ edition; EPS=Extrapyramidal Symptoms; GAF=General Assessment of Functioning Scale; GAS= Global Assessment Scale; HAM-D=Hamilton Depression Rating Scale; MADRS=Montgomery-Asberg Depression Rating Scale; NOS=not otherwise specified; NR= not reported; Q-LES-Q=Quality of Life Enjoyment and Satisfaction

Questionnaire; PANSS=Positive and Negative Syndrome Scale; PMID=PubMed Identification Number; RCT= Randomized Controlled Trial; RoB=risk of bias; SAE=Serious Adverse Events; SDS=Sheehan Disability Scale; T=Trial; YMRS = Young Mania Rating Scale 
Appendix Table G2. Summary risk of bias assessments: allopurinol for mania

\begin{tabular}{|c|c|c|c|}
\hline Drug & $\begin{array}{l}\text { Study } \\
\text { Funding Source } \\
\text { PMID }\end{array}$ & $\begin{array}{c}\text { Overall Risk of Bias } \\
\text { Assessment }\end{array}$ & Rationale \\
\hline \multirow[t]{4}{*}{ Allopurinol } & $\begin{array}{l}\text { Jahangard, } 2014^{1} \\
\text { No external } \\
\text { funding } \\
24953766\end{array}$ & Low & No sources of bias identified. \\
\hline & $\begin{array}{l}\text { Weiser, } 2014^{2} \\
\text { Non-Profit } \\
24712840\end{array}$ & High & $\begin{array}{l}\text { Randomization and blinding not described. The original study design allows for any } \\
\text { prescribed adjunctive medication so the medication effects cannot be localized to one drug. } \\
\text { These treatments are not measured as part of the baseline or endpoint characteristics to } \\
\text { ensure comparison group is similar to treatment group. }\end{array}$ \\
\hline & $\begin{array}{l}\text { Fan, } 2012^{3} \\
\text { Not reported } \\
22420596\end{array}$ & Moderate & Randomization and blinding procedures not described. \\
\hline & $\begin{array}{l}\text { Machado-Vieira, } \\
2008^{4} \\
\text { Non-Profit } \\
18681754\end{array}$ & Moderate & $\begin{array}{l}22 \%(39 / 180) \text { of patients randomized not included in results (censored due to } \\
\text { discontinuance), unclear how this group compares to general population. Dropout rates } \\
\text { appear similar. }\end{array}$ \\
\hline
\end{tabular}

Abbreviations: PMID=PubMed Identification Number; RCT=randomized controlled trial; 


\section{Allopurinol Forest Plots}

Outcomes in studies assessed as having a high risk of bias, or low to moderate risk of bias but at least 40 percent attrition, are presented in grey tones. Both fixed-effect models and random-effects models are presented. We calculated fixed-effect models to provide a charitable estimate of the average effect among completed trials. However, we base our main conclusions on the random-effects models.

Appendix Figure G1. Allopurinol vs. placebo - YMRS

\section{Difference in Mean Change in YMRS from Baseline to 3 Weeks}

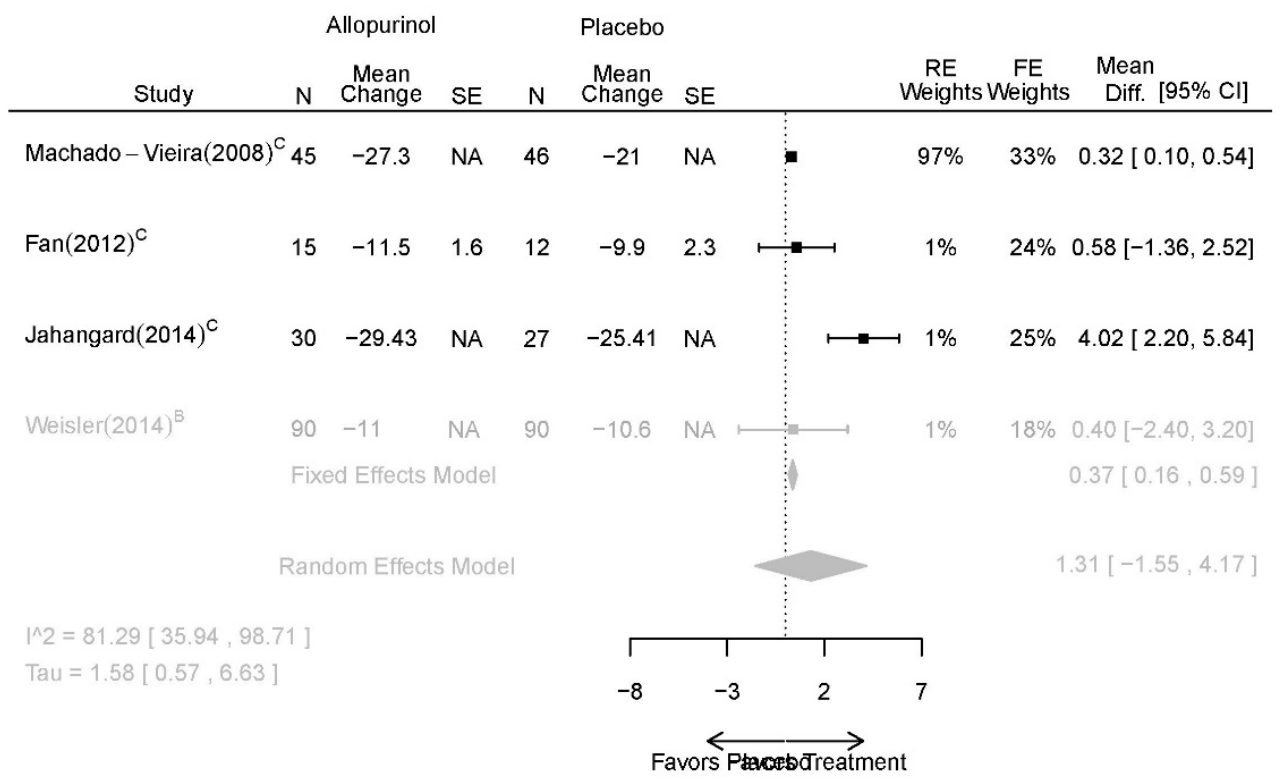


Appendix Figure G2. Allopurinol vs. placebo - CGI

Difference in Mean Change in CGI-BP-S (Overall) from Baseline to 3 Weeks

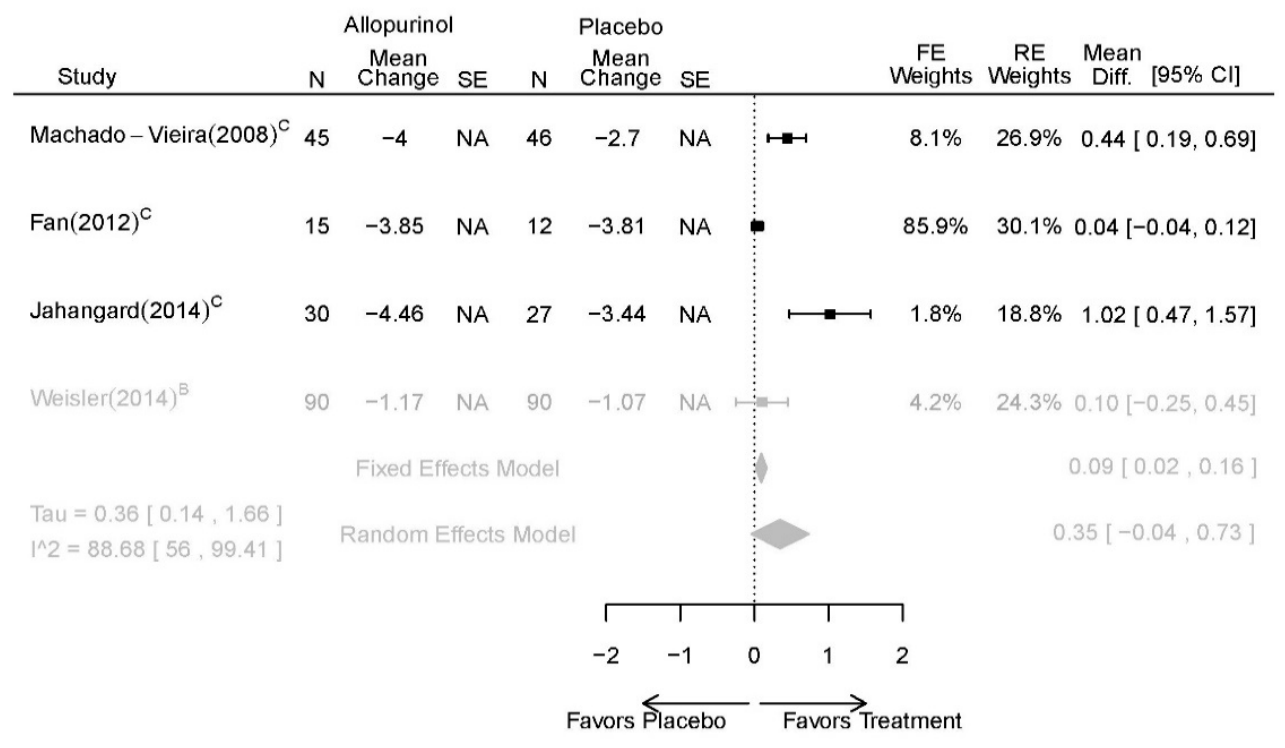

Appendix Figure G3. Allopurinol vs. placebo - overall withdrawal

\section{Odds Ratio of Overall Withdrawal}

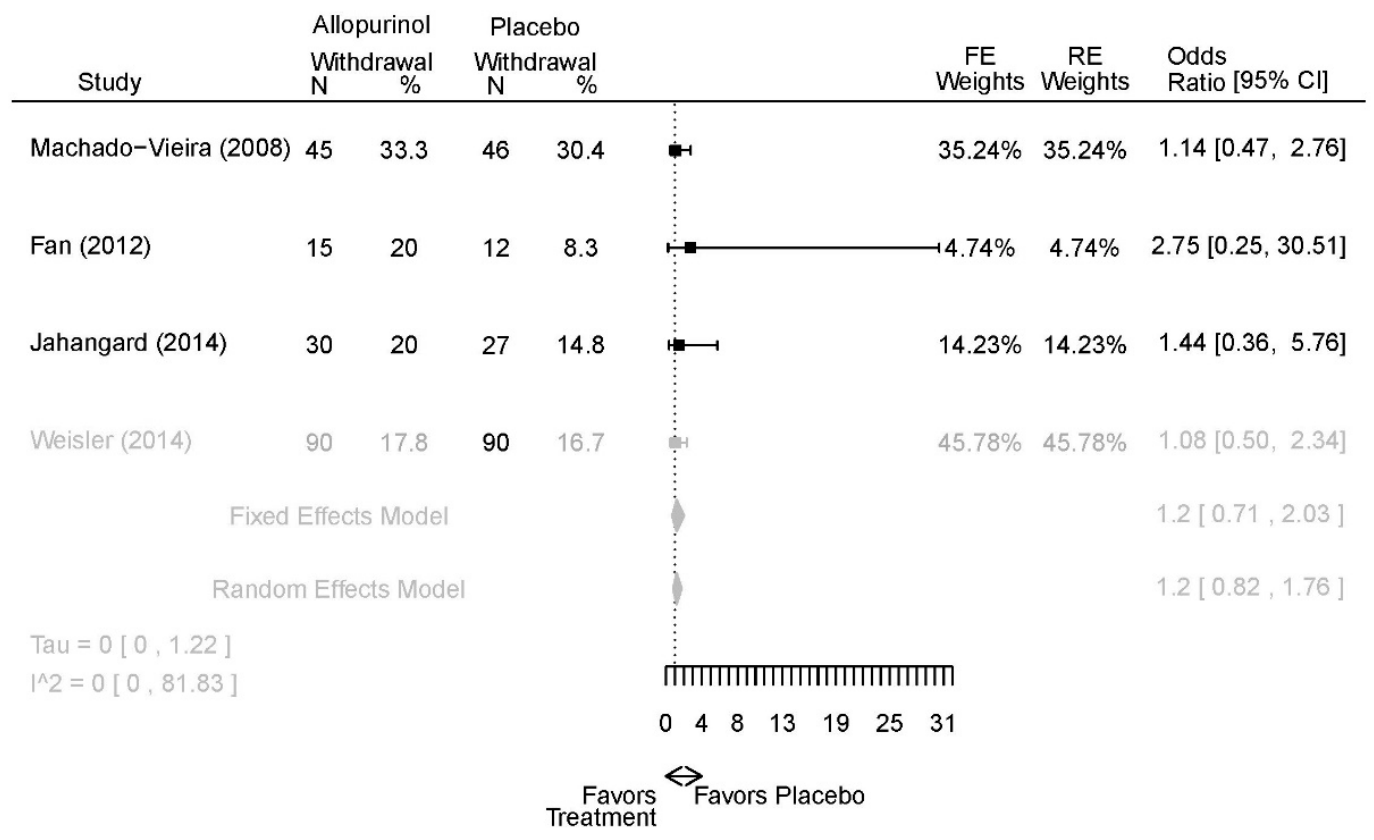




\begin{tabular}{|c|c|c|c|c|c|c|}
\hline Comparison & $\begin{array}{c}\text { Study } \\
\text { ROB } \\
\text { PMID }\end{array}$ & Responder/Remitter & Symptom & Function & Other & AE \\
\hline \multirow[t]{4}{*}{$\begin{array}{l}\text { Allopurinol + mood } \\
\text { stabilizers vs. } \\
\text { placebo + mood } \\
\text { stabilizers }\end{array}$} & $\begin{array}{l}\text { Jahangard, } \\
2014^{1} \\
\text { Low } \\
24953766\end{array}$ & $\begin{array}{l}\text { Remission } \\
\text { (YMRS } \leq 7) \\
4 \text { weeks } \\
\text { Allopurinol: } 24 / 30 \\
\text { Placebo: } 1 / 27 \\
\text { OR: } 9.46(1.19,81.57)\end{array}$ & $\begin{array}{l}\text { See forest plot G1 } \\
\underline{\text { above }}\end{array}$ & $\begin{array}{l}\text { See forest plot } \\
\underline{\text { G2 above }}\end{array}$ & $\begin{array}{l}\text { See forest plot G3 } \\
\underline{\text { above }} \\
\text { Withdrawal } \\
\text { Overall: } 17 \% \\
\text { Efficacy: NR } \\
\text { AEs: NR }\end{array}$ & No reported SAE \\
\hline & $\begin{array}{l}\text { Weiser, 2014² } \\
\text { High } \\
24712840\end{array}$ & $\begin{array}{l}\text { Response } \\
\text { (YMRS } \geq 50 \% \\
\text { decrease) } \\
4 \text { weeks } \\
\text { Allopurinol: } 34 / 90 \\
\text { Placebo: } 35 / 90 \\
\text { NS } \\
\text { OR } 0.95(0.52,1.74)\end{array}$ & $\begin{array}{l}\text { See forest plot G1 } \\
\underline{\text { above }}\end{array}$ & $\begin{array}{l}\text { See forest plot } \\
\underline{\text { G2 above }}\end{array}$ & $\begin{array}{l}\text { See forest plot G3 } \\
\text { above } \\
\text { Withdrawal } \\
\text { Overall: } 17 \% \\
\text { Efficacy: NR } \\
\text { AEs: NR }\end{array}$ & No reported SAE \\
\hline & $\begin{array}{l}\text { Fan, } 2012^{3} \\
\text { Moderate } \\
22420596\end{array}$ & $\underline{\mathrm{NR}}$ & $\begin{array}{l}\text { See forest plot G1 } \\
\underline{\text { above }}\end{array}$ & $\begin{array}{l}\text { See forest plot } \\
\underline{\text { G2 above }}\end{array}$ & $\begin{array}{l}\text { See forest plot G3 } \\
\underline{\text { above }} \\
\text { Withdrawal } \\
\text { Overall: 15\% } \\
\text { Efficacy: NR } \\
\text { AEs: NR }\end{array}$ & NR \\
\hline & $\begin{array}{l}\text { Machado-Vieira, } \\
2008^{4} \\
\text { Moderate } \\
18681754\end{array}$ & $\begin{array}{l}\frac{\text { Remission }}{(\text { YMRS } \leq 7)} \\
\text { 4 weeks } \\
\text { Allopurinol: } 32 / 45 \\
\text { Placebo: } 15 / 46 \\
\text { OR: } 5.09(2.09,12.41) \\
\text { Response } \\
\text { (YMRS } \geq 50 \% \\
\text { decrease) } \\
4 \text { weeks } \\
\text { Allopurinol: } 36 / 45 \\
\text { Placebo: } 29 / 56 \\
\text { NS } \\
2.34(0.91,6.03)\end{array}$ & $\begin{array}{l}\text { See forest plot G1 } \\
\underline{\text { above }}\end{array}$ & $\begin{array}{l}\text { See forest plot } \\
\text { G2 above } \\
\text { Linear mixed } \\
\text { model showed } \\
\text { drug main } \\
\text { effect was } \\
\text { significant } \\
\text { ( } p=0.004) \text {, and } \\
\text { mixed effects } \\
\text { with time were } \\
\text { significant } \\
\text { ( } p \leq 0.001)\end{array}$ & $\begin{array}{l}\text { NR } \\
\text { Withdrawal } \\
\text { Overall: } 22 \% \\
\text { Efficacy: } 5.6 \% \\
\text { AEs: } 5.56\end{array}$ & No reported SAE \\
\hline
\end{tabular}


Abbreviations: AE=Adverse Events; BMI=Body Mass Index; CI=Confidence Interval; CGI-BP-S=Clinical Global Impressions, Bipolar, Severity Scale; CGI-S= Clinical Global Impressions, Severity Scale; EPS=extrapyramidal symptoms; GAF=General Assessment of Functioning Scale; GAS=Global Assessment Scale; NR=not reported; NS=not significant; OR=Odds Ratio; PMID=PubMed Identification Number; RCT=randomized controlled trial; SAE=Serious Adverse Events; SD=standard deviation; YMRS = Young Mania Rating Scale

\section{Appendix Table G4. Strength of evidence assessment: allopurinol for mania vs. inactive control}

\begin{tabular}{|c|c|c|c|c|c|c|c|c|}
\hline Comparison & Outcome & $\begin{array}{l}\text { \# Studies/ } \\
\text { Design } \\
\text { (n analyzed) }\end{array}$ & $\begin{array}{l}\text { Finding or } \\
\text { Summary } \\
\text { Statistic }\end{array}$ & $\begin{array}{c}\text { Study } \\
\text { Limitations }\end{array}$ & Consistency & Directness & Precision & $\begin{array}{c}\text { Overall } \\
\text { Gradel } \\
\text { Conclusion }\end{array}$ \\
\hline \multirow{3}{*}{$\begin{array}{l}\text { Allopurinol + } \\
\text { lithium vs. } \\
\text { placebo + } \\
\text { lithium }\end{array}$} & Remission 4 wks & $\begin{array}{l}2 \mathrm{RCT} \\
(\mathrm{n}=96)\end{array}$ & See table above & Moderate & Inconsistent & Direct & Imprecise & Insufficient \\
\hline & Response 4 wks & $\begin{array}{l}2 \mathrm{RCT} \\
(\mathrm{n}=96)\end{array}$ & See table above & High & Consistent & Direct & Imprecise & Insufficient \\
\hline & $\begin{array}{l}\text { YMRS } 4 \text { wks } \\
\text { CGI } 4 \text { wks } \\
\text { Overall Withdrawal }\end{array}$ & $\begin{array}{l}4 \mathrm{RCT} \\
(\mathrm{n}=355)\end{array}$ & NS & Moderate & Consistent & Direct & Imprecise & Insufficient \\
\hline
\end{tabular}

Abbreviations: CGI= Clinical Global Impressions; CGI-S=Clinical Global Impressions, Severity Scale; IPD=Individual Patient Data; NS=not significant; RCT=randomized controlled trial; YMRS = Young Mania Rating Scale

Notes:

1. Publication bias for antipsychotics, antidepressants, and behavioral interventions for depressive disorders is suspected.

2. Data were generally imprecise due to missing data from high attrition rates, which was commonly dealt with by Last Observation Carried Forward (LOCF). LOCF requires an assumption that the health status of patients who dropped out of the trial would not have changed had future observations been recorded, a strong assumption in the context of bipolar disorder research. 
Appendix Table G5. Outcomes summary: allopurinol for mania vs. active control

\begin{tabular}{|c|c|c|c|c|c|c|}
\hline Drug & $\begin{array}{c}\text { Study } \\
\text { Comparison } \\
\text { PMID }\end{array}$ & $\begin{array}{l}\text { Responderl } \\
\text { Remitter }\end{array}$ & Symptom & Function & Other & $A E$ \\
\hline $\begin{array}{l}\text { Allopurinol + } \\
\text { lithium vs. } \\
\text { Dipyridamole + } \\
\text { lithium }\end{array}$ & $\begin{array}{l}\text { Machado-Vieira, } \\
2008^{4} \\
\text { Moderate } \\
18681754\end{array}$ & $\begin{array}{l}\frac{\text { Remission }}{\text { (YMRS } \leq 7)} \\
\text { Favors Allopurinol } \\
\mathrm{p}=0.03 \\
\frac{\text { Response }}{\text { (YMRS } \geq 50 \%} \\
\text { decrease) } \\
\text { NS }\end{array}$ & $\begin{array}{l}\text { YMRS } \\
4 \text { weeks } \\
\text { Mean change } \\
\text { Favors Allopurinol } \\
p<0.01\end{array}$ & $\begin{array}{l}\text { CGI-S } \\
\text { weeks } \\
\text { Linear mixed } \\
\text { model } \\
\text { Favors } \\
\text { Allopurinol } \\
d=0.29(0.09, \\
0.49)\end{array}$ & 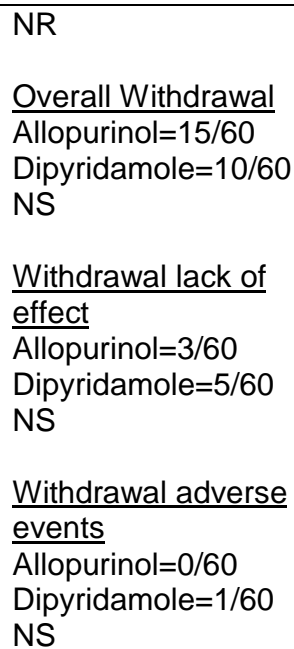 & $\begin{array}{l}\text { SAE } \\
1 \text { dipyridmol patient } \\
\text { severe skin rash }\end{array}$ \\
\hline
\end{tabular}

Abbreviations: AE=Adverse Events; BMI=Body Mass Index; CGI=Clinical Global Impressions; CGI-BP-S=Clinical Global Impressions, Bipolar, Severity Scale; CI=Confidence Interval; EPS=extrapyramidal symptoms; GAF=General Assessment of Functioning Scale; NR=not reported; NS=not significant; PMID=PubMed Identification Number;

RR=Risk Ratio; SAE=Serious Adverse Events; SD=standard deviation; YMRS = Young Mania Rating Scale

\section{Appendix Table G6. Strength of evidence assessment: allopurinol for mania vs. active control}

\begin{tabular}{|c|c|c|c|c|c|c|c|c|}
\hline Comparison & Outcome & $\begin{array}{l}\text { \# Studies/ } \\
\text { Design } \\
\text { (n analyzed) }\end{array}$ & $\begin{array}{l}\text { Finding or } \\
\text { Summary } \\
\text { Statistic }\end{array}$ & $\begin{array}{c}\text { Study } \\
\text { Limitations }\end{array}$ & Consistency & Directness & Precision & $\begin{array}{c}\text { Overall } \\
\text { Gradel } \\
\text { Conclusion }\end{array}$ \\
\hline $\begin{array}{l}\text { Allopurinol + } \\
\text { lithium vs. } \\
\text { Dipyridamole + } \\
\text { lithium }\end{array}$ & $\begin{array}{l}\text { Remission } 4 \text { wks } \\
\text { Response } 4 \text { wks } \\
\text { YMRS } 4 \text { wks } \\
\text { CGI } 4 \text { wks } \\
\text { Withdrawals }\end{array}$ & $\begin{array}{l}1 \mathrm{RCT} \\
(\mathrm{n}=120)\end{array}$ & See table above & Moderate & Unknown & Direct & Imprecise & Insufficient \\
\hline
\end{tabular}

Abbreviations: CGI= Clinical Global Impressions; CI=Confidence Interval; GAF=General Assessment of Functioning Scale; IPD=Individual Patient Data; NS=not significant;

RCT=randomized controlled trial; YMRS = Young Mania Rating Scale

Notes:

1. Publication bias for antipsychotics, antidepressants, and behavioral interventions for depressive disorders is suspected.

2. Data were generally imprecise due to missing data from high attrition rates, which was commonly dealt with by Last Observation Carried Forward (LOCF). LOCF requires an assumption that the health status of patients who dropped out of the trial would not have changed had future observations been recorded, a strong assumption in the context of bipolar disorder research. 


\section{Section 2. Dipyridamole}

Appendix Table G7. Characteristics of eligible studies: dipyridamole for acute mania

\begin{tabular}{|c|c|c|c|c|c|c|}
\hline $\begin{array}{l}\text { Study, Year } \\
\text { Design } \\
\text { Location } \\
\text { Funder } \\
\text { Risk of Bias } \\
\text { PMID }\end{array}$ & $\begin{array}{c}\text { \# Randomized } \\
\text { Age (mean) } \\
\text { Sex (\% Female) } \\
\text { Race (\% White) } \\
\text { Diagnosis } \\
\text { (\% BP I, II, NOS) } \\
\text { Setting }\end{array}$ & $\begin{array}{l}\text { Inclusions } \\
\text { Key Exclusions }\end{array}$ & $\begin{array}{l}\text { Intervention } \\
\text { Dosage }\end{array}$ & $\begin{array}{l}\text { Comparison } \\
\text { Dosage }\end{array}$ & $\begin{array}{l}\text { Follow-up } \\
\text { Duration }\end{array}$ & $\begin{array}{c}\text { Outcomes } \\
\text { Reported } \\
\text { Withdrawal (\%) at } \\
\text { endpoint }\end{array}$ \\
\hline $\begin{array}{l}\text { Machado-Vieira, } 2008^{4} \\
\text { RCT } \\
\text { Brazil } \\
\text { Non-Profit } \\
\text { RoB Moderate } \\
18681754\end{array}$ & $\begin{array}{l}\mathrm{N}=180 \\
\text { Mean Age 29.3 } \\
\text { Female 59\% } \\
\text { White NR } \\
\text { BP I 100\% } \\
\text { Not Disclosed }\end{array}$ & $\begin{array}{l}\text { Manic; } \\
\text { YMRS } \geq 22 \\
\text { Schizoaffective } \\
\text { Substance abuse } \\
\text { Other mental health } \\
\text { Taking other meds } \\
\text { Labs/other conditions }\end{array}$ & $\begin{array}{l}\text { T1: Allopurinol } \\
60 \mathrm{mg} / \text { day } \\
\\
\text { T2: Dipyridamole } \\
200 \mathrm{mg} / \text { day } \\
\\
\text { Lithium } \\
600-900 \mathrm{mg} / \mathrm{day} \\
\text { serum level } \\
0.6-1.2 \mathrm{mmol} / \mathrm{L} \\
\text { (mean } 0.99 \mathrm{mmol} / \mathrm{L} \text { ) }\end{array}$ & $\begin{array}{l}\text { Placebo } \\
\text { Lithium } \\
600-900 \mathrm{mg} / \text { day } \\
\text { serum level } \\
0.6-1.2 \mathrm{mmol} / \mathrm{L} \\
\text { (mean } 0.95 \\
\text { mmol/L) }\end{array}$ & 4 weeks & $\begin{array}{l}\text { CGI-S } \\
\text { Remission } \\
\text { (YMRS } \leq 7) \\
\text { (YMRS } \leq 12) \\
\text { Response } \\
\text { (50\% improved } \\
\text { YMRS) } \\
\text { Adverse Events } \\
\text { Lab Values } \\
\text { Withdrawal 20\% }\end{array}$ \\
\hline
\end{tabular}

Abbreviations: AE=Adverse Effects; BP=bipolar disorder; BPRS=Brief Psychiatric Rating Scale; CGI=Clinical Global Impressions; CGI-BP=Clinical Global Impressions Scale for Bipolar Disorder; CGI-BP-S=Clinical Global Impressions, Bipolar, Severity Scale; CGI-S=Clinical Global Impressions, Severity Scale; DSM-IV= Diagnostic and statistical manual, $4^{\text {th }}$ edition; EPS=Extrapyramidal Symptoms; GAF=General Assessment of Functioning Scale; GAS= Global Assessment Scale; HAM-D=Hamilton Depression Rating Scale; MADRS=Montgomery-Asberg Depression Rating Scale; NOS=not otherwise specified; NR= not reported; Q-LES-Q=Quality of Life Enjoyment and Satisfaction

Questionnaire; PANSS=Positive and Negative Syndrome Scale; PMID=PubMed Identification Number; RCT= Randomized Controlled Trial; RoB=risk of bias; SAE=Serious

Adverse Events; SDS=Sheehan Disability Scale; T=Trial; YMRS = Young Mania Rating Scale

\section{Appendix Table G8. Summary risk of bias assessments: dipyridamole for mania}

\begin{tabular}{|l|l|l|l|}
\hline \multicolumn{1}{|c|}{ Drug } & $\begin{array}{c}\text { Study } \\
\text { Funding Source } \\
\text { PMID }\end{array}$ & $\begin{array}{c}\text { Overall Risk of Bias } \\
\text { Assessment }\end{array}$ & Rationale \\
\hline Dipyridamole & $\begin{array}{l}\text { Machado-Vieira, } \\
2008^{4} \\
\text { Non-Profit } \\
18681754\end{array}$ & Moderate & $\begin{array}{l}22 \% \text { (39/180) of patients randomized not included in results (censored due to } \\
\text { discontinuance), unclear how this group compares to general population. Dropout rates } \\
\text { appear similar. }\end{array}$ \\
\hline
\end{tabular}

Abbreviations: PMID=PubMed Identification Number; RCT=randomized controlled trial 
Appendix Table G9. Outcomes summary: dipyridamole for mania vs. inactive control

\begin{tabular}{|c|c|c|c|c|c|c|}
\hline Comparison & $\begin{array}{c}\text { Study } \\
\text { ROB } \\
\text { PMID }\end{array}$ & Responder/Remitter & Symptom & Function & Other & $\mathrm{AE}$ \\
\hline $\begin{array}{l}\text { Dipyridamole + } \\
\text { lithium vs. placebo } \\
+ \text { lithium }\end{array}$ & $\begin{array}{l}\text { Machado-Vieira, } \\
2008^{4} \\
\text { Moderate } \\
18681754\end{array}$ & $\begin{array}{l}\frac{\text { Remission }}{(\text { YMRS } \leq 7)} \\
\text { NR } \\
\text { Response } \\
\text { (YMRS } \geq 50 \% \\
\text { decrease) } \\
\text { NR }\end{array}$ & $\begin{array}{l}\text { YMRS } \\
4 \text { weeks } \\
\text { NS } \\
\text { NR } \\
\text { Linear mixed model } \\
\text { showed drug main effect } \\
\text { was not significant } \\
(p=0.11)\end{array}$ & $\begin{array}{l}\frac{\text { CGI-S }}{4 \text { weeks }} \\
\text { NS } \\
p=0.13 \\
\text { Linear mixed } \\
\text { model showed } \\
\text { drug main } \\
\text { effect was } \\
\text { significant } \\
\text { ( } p=0.004) \text {, and } \\
\text { mixed effects } \\
\text { with time were } \\
\text { significant } \\
\text { ( } p \leq 0.001)\end{array}$ & $\begin{array}{l}\text { NR } \\
\text { Overall Withdrawal } \\
\text { Dipyridamole }=10 / 60 \\
\text { Placebo=14/60 } \\
\text { NS } \\
\text { Withdrawal lack of } \\
\text { efficacy } \\
\text { Dipyridamole=5/60 } \\
\text { Placebo=7/60 } \\
\text { NS } \\
\text { Withdrawal adverse } \\
\text { events } \\
\text { Dipyridamole }=1 / 60 \\
\text { Placebo=0/60 } \\
\text { NS }\end{array}$ & $\begin{array}{l}1 \text { dipyridamole } \\
\text { participant with } \\
\text { severe edverse } \\
\text { event skin rash }\end{array}$ \\
\hline
\end{tabular}

Abbreviations: AE=Adverse Events; BMI=Body Mass Index; CI=Confidence Interval; CGI-BP-S=Clinical Global Impressions, Bipolar, Severity Scale; CGI-S= Clinical Global Impressions, Severity Scale; EPS=extrapyramidal symptoms; GAF=General Assessment of Functioning Scale; GAS=Global Assessment Scale; NR=not reported; NS=not significant; OR=Odds Ratio; PMID=PubMed Identification Number; RCT=randomized controlled trial; SAE=Serious Adverse Events; SD=standard deviation; YMRS = Young Mania Rating Scale 
Appendix Table G10. Strength of evidence assessment: dipyridamole for mania vs. inactive control

\begin{tabular}{|c|c|c|c|c|c|c|c|c|}
\hline Comparison & Outcome & $\begin{array}{l}\text { \# Studies/ } \\
\text { Design } \\
\text { (n analyzed) }\end{array}$ & $\begin{array}{l}\text { Finding or } \\
\text { Summary } \\
\text { Statistic }\end{array}$ & $\begin{array}{c}\text { Study } \\
\text { Limitations }\end{array}$ & Consistency & Directness & Precision & $\begin{array}{c}\text { Overall } \\
\text { Gradel } \\
\text { Conclusion }\end{array}$ \\
\hline $\begin{array}{l}\text { Dipyridamole } \\
+ \text { lithium vs. } \\
\text { placebo + } \\
\text { lithium }\end{array}$ & $\begin{array}{l}\text { YMRS } 4 \text { wks } \\
\text { CGI-S } 4 \text { wks } \\
\text { Withdrawals }\end{array}$ & $\begin{array}{l}1 \text { RCT } \\
(n=120)\end{array}$ & See table above & Moderate & Unknown & Direct & Imprecise & Insufficient \\
\hline
\end{tabular}

Abbreviations: CGI= Clinical Global Impressions; CGI-S=Clinical Global Impressions, Severity Scale; IPD=Individual Patient Data; NS=not significant; RCT=randomized controlled trial; YMRS = Young Mania Rating Scale

Notes:

1. Publication bias for antipsychotics, antidepressants, and behavioral interventions for depressive disorders is suspected.

2. Data were generally imprecise due to missing data from high attrition rates, which was commonly dealt with by Last Observation Carried Forward (LOCF). LOCF requires an

assumption that the health status of patients who dropped out of the trial would not have changed had future observations been recorded, a strong assumption in the context of

bipolar disorder research. 


\section{Section 3. Celecoxib}

Appendix Table G11. Characteristics of eligible studies: celecoxib for acute mania

\begin{tabular}{|c|c|c|c|c|c|c|}
\hline $\begin{array}{c}\text { Study, Year } \\
\text { Design } \\
\text { Location } \\
\text { Funder } \\
\text { Risk of Bias } \\
\text { PMID }\end{array}$ & $\begin{array}{c}\text { \# Randomized } \\
\text { Age (mean) } \\
\text { Sex (\% Female) } \\
\text { Race (\% White) } \\
\text { Diagnosis } \\
\text { (\% BP I, II, NOS) } \\
\text { Setting }\end{array}$ & $\begin{array}{c}\text { Inclusions } \\
\text { Key Exclusions }\end{array}$ & $\begin{array}{l}\text { Intervention } \\
\text { Dosage }\end{array}$ & $\begin{array}{l}\text { Comparison } \\
\text { Dosage }\end{array}$ & $\begin{array}{c}\text { Follow-up } \\
\text { Duration }\end{array}$ & $\begin{array}{c}\text { Outcomes } \\
\text { Reported } \\
\text { Withdrawal (\%) at } \\
\text { endpoint }\end{array}$ \\
\hline $\begin{array}{l}\text { Arabzadeh, } 2015^{5} \\
\text { RCT } \\
\text { Iran } \\
\text { University } \\
\text { RoB Low } \\
26291962\end{array}$ & $\begin{array}{l}\mathrm{N}=48 \\
\text { Mean Age } 31.4 \\
\text { Female 35\% } \\
\text { White NR } \\
\text { BP I 100\% } \\
\text { Inpatient }\end{array}$ & $\begin{array}{l}\text { Manic; } \\
\text { YMRS } \geq 20 \\
\\
\text { Schizoaffective } \\
\text { Substance abuse } \\
\text { Other mental health } \\
\text { Taking other meds } \\
\text { Labs/other conditions }\end{array}$ & $\begin{array}{l}\text { Celecoxib } \\
400 \mathrm{mg} / \mathrm{day}\end{array}$ & Placebo & 6 weeks & $\begin{array}{l}\text { YMRS } \\
\text { HAM-D } \\
\text { Remission } \\
\text { (YMRS } \leq 7 \text { ) } \\
\text { Time to Remission } \\
\text { Response } \\
\text { (YMRS } \geq 50 \% \\
\text { decrease) } \\
\text { Adverse Events } \\
\\
\text { Withdrawal 4\% }\end{array}$ \\
\hline
\end{tabular}

Abbreviations: AE=Adverse Effects; BP=bipolar disorder; BPRS=Brief Psychiatric Rating Scale; CGI=Clinical Global Impressions; CGI-BP=Clinical Global Impressions Scale for Bipolar Disorder; CGI-BP-S=Clinical Global Impressions, Bipolar, Severity Scale; CGI-S=Clinical Global Impressions, Severity Scale; DSM-IV= Diagnostic and statistical manual, $4^{\text {th }}$ edition; EPS=Extrapyramidal Symptoms; GAF=General Assessment of Functioning Scale; GAS= Global Assessment Scale; HAM-D=Hamilton Depression Rating Scale; MADRS=Montgomery-Asberg Depression Rating Scale; NOS=not otherwise specified; NR= not reported; Q-LES-Q=Quality of Life Enjoyment and Satisfaction Questionnaire; PANSS=Positive and Negative Syndrome Scale; PMID=PubMed Identification Number; RCT= Randomized Controlled Trial; RoB=risk of bias; SAE=Serious Adverse Events; SDS=Sheehan Disability Scale; T=Trial; YMRS = Young Mania Rating Scale

\section{Appendix Table G12. Summary risk of bias assessments: celecoxib for mania}

\begin{tabular}{|l|l|l|l|}
\hline \multicolumn{1}{|c|}{ Drug } & $\begin{array}{c}\text { Study } \\
\text { Funding Source } \\
\text { PMID }\end{array}$ & $\begin{array}{c}\text { Overall Risk of Bias } \\
\text { Assessment }\end{array}$ & Rationale \\
\hline Celecoxib & $\begin{array}{l}\text { Arabzadeh, } 2015^{5} \\
\text { University } \\
26291962\end{array}$ & Low & No sources of bias identified \\
\end{tabular}

Abbreviations: PMID=PubMed Identification Number; RCT=randomized controlled trial; 
Appendix Table G13. Outcomes summary: celecoxib for mania vs. inactive control

\begin{tabular}{|c|c|c|c|c|c|c|}
\hline Comparison & $\begin{array}{c}\text { Study } \\
\text { ROB } \\
\text { PMID }\end{array}$ & Responder/Remitter & Symptom & Function & Other & $\mathrm{AE}$ \\
\hline $\begin{array}{l}\text { Celecoxib vs. } \\
\text { placebo }\end{array}$ & $\begin{array}{l}\text { Arabzadeh, } \\
2015^{5} \\
\text { Low } \\
26291962\end{array}$ & $\begin{array}{l}\text { Response } \\
\text { (YMRS } \geq 50 \% \\
\text { decrease) } \\
3 \text { weeks } \\
\text { NS } \\
(p=0.08) \\
6 \text { weeks } \\
\text { NS } \\
p=0.11 \\
\text { Remission } \\
\text { (YMRS } \leq 7) \\
3 \text { weeks } \\
\text { NS } \\
p=0.15 \\
6 \text { weeks } \\
\text { Favors celecoxib } \\
p=0.002\end{array}$ & $\begin{array}{l}\frac{Y M R S}{3 \text { weeks }} \\
\text { Favors celecoxib } \\
\text { Mean difference } \\
-5.17(-9.61,-0.74) \\
p=0.006 \\
6 \text { weeks } \\
\text { Favors celecoxib } \\
p<0.001\end{array}$ & NR & $\begin{array}{l}\text { NR } \\
\text { Withdrawal } \\
\text { Celecoxib }=1 / 24 \\
\text { Placebo=1/24 } \\
\text { NS }\end{array}$ & $\begin{array}{l}\frac{\text { Serious Adverse }}{\text { Events }} \\
6 \text { weeks } \\
0 \text { in both arms } \\
\frac{\text { Deaths }}{6 \text { weeks }} \\
0 \text { in both arms } \\
\frac{\text { EPS }}{\text { NR }}\end{array}$ \\
\hline
\end{tabular}

Abbreviations: AE=Adverse Events; BMI=Body Mass Index; CI=Confidence Interval; CGI-BP-S=Clinical Global Impressions, Bipolar, Severity Scale; CGI-S= Clinical Global Impressions, Severity Scale; EPS=extrapyramidal symptoms; GAF=General Assessment of Functioning Scale; GAS=Global Assessment Scale; NR=not reported; NS=not significant; OR=Odds Ratio; PMID=PubMed Identification Number; RCT=randomized controlled trial; SAE=Serious Adverse Events; SD=standard deviation; YMRS = Young Mania Rating Scale 
Appendix Table G14. Strength of evidence assessment: celecoxib for mania vs. inactive control

\begin{tabular}{|c|c|c|c|c|c|c|c|c|}
\hline Comparison & Outcome & $\begin{array}{l}\text { \# Studies/ } \\
\text { Design } \\
\text { (n analyzed) }\end{array}$ & $\begin{array}{l}\text { Finding or } \\
\text { Summary } \\
\text { Statistic }\end{array}$ & $\begin{array}{c}\text { Study } \\
\text { Limitations }\end{array}$ & Consistency & Directness & Precision & $\begin{array}{c}\text { Overall } \\
\text { Gradel } \\
\text { Conclusion }\end{array}$ \\
\hline $\begin{array}{l}\text { Celecoxib vs. } \\
\text { placebo }\end{array}$ & $\begin{array}{l}\text { Remission } 3 \text { wks } \\
\text { Response } 3 \text { wks } \\
\text { YMRS } 3 \text { wks } \\
\text { Withdrawals }\end{array}$ & $\begin{array}{l}1 \mathrm{RCT} \\
(\mathrm{n}=44)\end{array}$ & See table above & Low & Unknown & Direct & Imprecise & Insufficient \\
\hline
\end{tabular}

Abbreviations: CGI= Clinical Global Impressions; CGI-S=Clinical Global Impressions, Severity Scale; IPD=Individual Patient Data; NS=not significant; RCT=randomized controlled trial; YMRS = Young Mania Rating Scale

Notes:

1. Publication bias for antipsychotics, antidepressants, and behavioral interventions for depressive disorders is suspected.

2. Data were generally imprecise due to missing data from high attrition rates, which was commonly dealt with by Last Observation Carried Forward (LOCF). LOCF requires an

assumption that the health status of patients who dropped out of the trial would not have changed had future observations been recorded, a strong assumption in the context of

bipolar disorder research. 


\section{Section 4. Donepezil}

Appendix Table G15. Characteristics of eligible studies: donepezil for acute mania

\begin{tabular}{|c|c|c|c|c|c|c|}
\hline $\begin{array}{c}\text { Study, Year } \\
\text { Design } \\
\text { Location } \\
\text { Funder } \\
\text { Risk of Bias } \\
\text { PMID }\end{array}$ & $\begin{array}{c}\text { \# Randomized } \\
\text { Age (mean) } \\
\text { Sex (\% Female) } \\
\text { Race (\% White) } \\
\text { Diagnosis } \\
\text { (\% BP I, II, NOS) } \\
\text { Setting }\end{array}$ & $\begin{array}{c}\text { Inclusions } \\
\text { Key Exclusions }\end{array}$ & $\begin{array}{l}\text { Intervention } \\
\text { Dosage }\end{array}$ & $\begin{array}{c}\text { Comparison } \\
\text { Dosage }\end{array}$ & $\begin{array}{c}\text { Follow-up } \\
\text { Duration }\end{array}$ & $\begin{array}{c}\text { Outcomes } \\
\text { Reported } \\
\text { Withdrawal (\%) at } \\
\text { endpoint }\end{array}$ \\
\hline $\begin{array}{l}\text { Chen, } 2013^{6} \\
\text { RCT } \\
\text { China } \\
\text { Non-profit } \\
\text { RoB Moderate } \\
23807849\end{array}$ & $\begin{array}{l}\mathrm{N}=30 \\
\text { Mean Age } 34.1 \\
\text { Female 40\% } \\
\text { White (\%) NR } \\
\text { BP I 100\% } \\
\text { Inpatient }\end{array}$ & $\begin{array}{l}\text { Manic; } \\
\text { YMRS>20 } \\
\text { Schizoaffective } \\
\text { Substance abuse } \\
\text { Other mental health } \\
\text { Taking other meds } \\
\text { Pregnant/nursing } \\
\text { Labs/other conditions }\end{array}$ & $\begin{array}{l}\text { Donepezil } \\
10 \mathrm{mg} / \text { day } \\
\\
\text { Lithium } \\
600-900 \mathrm{mg} / \text { day } \\
\text { serum level } \\
0.8-1.2 \mathrm{mmol} / \mathrm{L} \\
\text { (mean } 0.83 \mathrm{mmol} / \mathrm{L} \text { ) }\end{array}$ & $\begin{array}{l}\text { Placebo } \\
\text { Lithium } \\
600-900 \mathrm{mg} / \text { day } \\
\text { serum level } \\
0.8-1.2 \mathrm{mmol} / \mathrm{L} \\
\text { (mean } 0.82 \\
\mathrm{mmol} / \mathrm{L})\end{array}$ & 4 weeks & $\begin{array}{l}\text { BPRS } \\
\text { YMRS } \\
\text { Response } \\
\text { (YMRS decrease } \\
\geq 50 \% \text { ) } \\
\text { Remission } \\
\text { (YMRS } \leq 12 \text { ) } \\
\text { Adverse Events } \\
\text { Withdrawal } 0 \%\end{array}$ \\
\hline
\end{tabular}

Abbreviations: AE=Adverse Effects; BP=bipolar disorder; BPRS=Brief Psychiatric Rating Scale; CGI=Clinical Global Impressions; CGI-BP=Clinical Global Impressions Scale for Bipolar Disorder; CGI-BP-S=Clinical Global Impressions, Bipolar, Severity Scale; CGI-S=Clinical Global Impressions, Severity Scale; DSM-IV= Diagnostic and statistical manual, $4^{\text {th }}$ edition; EPS=Extrapyramidal Symptoms; GAF=General Assessment of Functioning Scale; GAS= Global Assessment Scale; HAM-D=Hamilton Depression Rating Scale; MADRS=Montgomery-Asberg Depression Rating Scale; NOS=not otherwise specified; NR= not reported; Q-LES-Q=Quality of Life Enjoyment and Satisfaction

Questionnaire; PANSS=Positive and Negative Syndrome Scale; PMID=PubMed Identification Number; RCT= Randomized Controlled Trial; RoB=risk of bias; SAE=Serious Adverse Events; SDS=Sheehan Disability Scale; T=Trial; YMRS = Young Mania Rating Scale

\section{Appendix Table G16. Summary risk of bias assessments: donepezil for mania}

\begin{tabular}{|l|l|l|l|}
\hline \multicolumn{1}{|c|}{ Drug } & $\begin{array}{c}\text { Study } \\
\text { Funding Source } \\
\text { PMID }\end{array}$ & $\begin{array}{c}\text { Overall Risk of Bias } \\
\text { Assessment }\end{array}$ & Rationale \\
\hline Donepezil & $\begin{array}{l}\text { Chen, } 2013^{6} \\
\text { Nonprofit } \\
23807849\end{array}$ & Moderate & Randomization and blinding procedures not described. \\
& & & \\
\hline
\end{tabular}

Abbreviations: PMID=PubMed Identification Number; RCT=randomized controlled trial 
Appendix Table G17. Outcomes summary: donepezil for mania vs. inactive control

\begin{tabular}{|c|c|c|c|c|c|c|}
\hline Comparison & $\begin{array}{c}\text { Study } \\
\text { ROB } \\
\text { PMID }\end{array}$ & Responder/Remitter & Symptom & Function & Other & $\mathrm{AE}$ \\
\hline $\begin{array}{l}\text { Donepezil + lithium } \\
\text { vs. placebo + } \\
\text { lithium }\end{array}$ & $\begin{array}{l}\text { Chen, } 2013^{6} \\
\text { Moderate } \\
23807849\end{array}$ & $\begin{array}{l}\frac{\text { Remission }}{(Y M R S \leq 12)} \\
4 \text { weeks } \\
\text { NS } \\
p=0.27 \\
\text { Response } \\
\text { (YMRS } \geq 50 \% \\
\text { decrease) } \\
4 \text { weeks } \\
\text { NS } \\
p=1.0\end{array}$ & $\begin{array}{l}\text { YMRS Decrease } \\
4 \text { weeks } \\
\text { NS } \\
p=0.16\end{array}$ & NR & No withdrawals & $\begin{array}{l}\text { Reported no serious } \\
\text { adverse events }\end{array}$ \\
\hline
\end{tabular}

Abbreviations: AE=Adverse Events; BMI=Body Mass Index; CI=Confidence Interval; CGI-BP-S=Clinical Global Impressions, Bipolar, Severity Scale; CGI-S= Clinical Global Impressions, Severity Scale; EPS=extrapyramidal symptoms; GAF=General Assessment of Functioning Scale; GAS=Global Assessment Scale; NR=not reported; NS=not significant; OR=Odds Ratio; PMID=PubMed Identification Number; RCT=randomized controlled trial; SAE=Serious Adverse Events; SD=standard deviation; YMRS = Young Mania Rating Scale

\section{Appendix Table G18. Strength of evidence assessment: donepezil for mania vs. inactive control}

\begin{tabular}{|c|c|c|c|c|c|c|c|c|}
\hline Comparison & Outcome & $\begin{array}{l}\text { \# Studies/ } \\
\text { Design } \\
\text { (n analyzed) }\end{array}$ & $\begin{array}{l}\text { Finding or } \\
\text { Summary } \\
\text { Statistic }\end{array}$ & $\begin{array}{c}\text { Study } \\
\text { Limitations }\end{array}$ & Consistency & Directness & Precision & $\begin{array}{c}\text { Overall } \\
\text { Gradel } \\
\text { Conclusion }\end{array}$ \\
\hline $\begin{array}{l}\text { Donepezil + } \\
\text { lithium vs. } \\
\text { placebo + } \\
\text { lithium }\end{array}$ & $\begin{array}{l}\text { Response } 4 \text { wks } \\
\text { Remission } 4 \text { wks } \\
\text { YMRS } 4 \text { wks }\end{array}$ & $\begin{array}{l}1 \mathrm{RCT} \\
(\mathrm{n}=30)\end{array}$ & See table above & Moderate & Unknown & Direct & Imprecise & Insufficient \\
\hline
\end{tabular}

Abbreviations: CGI= Clinical Global Impressions; CGI-S=Clinical Global Impressions, Severity Scale; IPD=Individual Patient Data; NS=not significant; RCT=randomized

controlled trial; YMRS = Young Mania Rating Scale

Notes:

1. Publication bias for antipsychotics, antidepressants, and behavioral interventions for depressive disorders is suspected.

2. Data were generally imprecise due to missing data from high attrition rates, which was commonly dealt with by Last Observation Carried Forward (LOCF). LOCF requires an

assumption that the health status of patients who dropped out of the trial would not have changed had future observations been recorded, a strong assumption in the context of

bipolar disorder research. 


\section{Section 5. Endoxifen}

Appendix Table G19. Characteristics of eligible studies: endoxifen for acute mania

\begin{tabular}{|c|c|c|c|c|c|c|}
\hline $\begin{array}{c}\text { Study, Year } \\
\text { Design } \\
\text { Location } \\
\text { Funder } \\
\text { Risk of Bias } \\
\text { PMID }\end{array}$ & $\begin{array}{c}\text { \# Randomized } \\
\text { Age (mean) } \\
\text { Sex (\% Female) } \\
\text { Race (\% White) } \\
\text { Diagnosis } \\
\text { (\% BP I, II, NOS) } \\
\text { Setting }\end{array}$ & $\begin{array}{c}\text { Inclusions } \\
\text { Key Exclusions }\end{array}$ & $\begin{array}{c}\text { Intervention } \\
\text { Dosage }\end{array}$ & $\begin{array}{c}\text { Comparison } \\
\text { Dosage }\end{array}$ & $\begin{array}{c}\text { Follow-up } \\
\text { Duration }\end{array}$ & $\begin{array}{c}\text { Outcomes } \\
\text { Reported } \\
\text { Withdrawal (\%) at } \\
\text { endpoint }\end{array}$ \\
\hline $\begin{array}{l}\text { Ahmad, } 2016^{8} \\
\text { RCT } \\
\text { India } \\
\text { Industry } \\
\text { ROB Low } \\
27346789\end{array}$ & $\begin{array}{l}\mathrm{N}=66 \\
\text { Mean Age 33 } \\
\text { Female 52\% } \\
\text { Race NR } \\
\text { BP I 100\% } \\
\text { Inpatient }\end{array}$ & $\begin{array}{l}\text { Manic/Mixed; } \\
\text { YMRS } \geq 20 \text { and CGI-S } \geq \\
4 \\
\text { New diagnosis } \\
\text { Labs/other conditions } \\
\text { Pregnant/nursing }\end{array}$ & $\begin{array}{l}\text { Endoxifen } \\
\text { oral enteric coated } \\
\text { tablets at two fixed } \\
\text { doses } \\
\text { T1: } 4 \mathrm{mg} / \text { day } \\
\text { T2: } 8 \mathrm{mg} / \text { day) }\end{array}$ & \begin{tabular}{|l|} 
Divalproex \\
$1000 \mathrm{mg} / \mathrm{day}$
\end{tabular} & 3 weeks & \begin{tabular}{|l} 
Response (YMRS \\
decrease $\geq 50 \%)$ \\
CGI-S \\
YMRS \\
MADRS \\
Withdrawal $7 \%$
\end{tabular} \\
\hline
\end{tabular}

Abbreviations: AE=Adverse Effects; BP=bipolar disorder; BPRS=Brief Psychiatric Rating Scale; CGI=Clinical Global Impressions; CGI-BP=Clinical Global Impressions Scale for Bipolar Disorder; CGI-BP-S=Clinical Global Impressions, Bipolar, Severity Scale; CGI-S=Clinical Global Impressions, Severity Scale; DSM-IV= Diagnostic and statistical manual, $4^{\text {th }}$ edition; EPS=Extrapyramidal Symptoms; GAF=General Assessment of Functioning Scale; GAS= Global Assessment Scale; HAM-D=Hamilton Depression Rating Scale; MADRS=Montgomery-Asberg Depression Rating Scale; NOS=not otherwise specified; NR= not reported; Q-LES-Q=Quality of Life Enjoyment and Satisfaction

Questionnaire; PANSS=Positive and Negative Syndrome Scale; PMID=PubMed Identification Number; RCT= Randomized Controlled Trial; RoB=risk of bias; SAE=Serious Adverse Events; SDS=Sheehan Disability Scale; T=Trial; YMRS = Young Mania Rating Scale

Appendix Table G20. Summary risk of bias assessments: endoxifen for mania

\begin{tabular}{|l|l|l|l|}
\hline \multicolumn{1}{|c|}{ Drug } & $\begin{array}{c}\text { Study } \\
\text { Funding Source } \\
\text { PMID }\end{array}$ & $\begin{array}{c}\text { Overall Risk of Bias } \\
\text { Assessment }\end{array}$ & Rationale \\
\hline Endoxifen & $\begin{array}{l}\text { Ahmad, 2016 } \\
\text { Industry } \\
27346789\end{array}$ & Low & Well-disclosed and reported study. \\
\end{tabular}


Appendix Table G21. Outcomes summary table: endoxifen for mania vs. active control

\begin{tabular}{|c|c|c|c|c|c|c|}
\hline Drug & $\begin{array}{c}\text { Study } \\
\text { Comparison } \\
\text { PMID }\end{array}$ & $\begin{array}{l}\text { Responderl } \\
\text { Remitter }\end{array}$ & Symptom & Function & Other & $A E$ \\
\hline $\begin{array}{l}\text { Endoxifen vs. } \\
\text { divalproex }\end{array}$ & $\begin{array}{l}\text { Ahmad, } 2016^{8} \\
\text { Low } \\
27346789\end{array}$ & $\begin{array}{l}\frac{\text { Response }}{\text { (YMRS } \geq 50 \%} \\
\text { decrease) } \\
3 \text { weeks } \\
\text { NS } \\
\text { Endoxifen } 4 \mathrm{mg}: \\
44 \% \\
\text { Endoxifen } 8 \mathrm{mg} \text { : } \\
64 \% \\
\text { Divalproex:: } 72 \%\end{array}$ & $\begin{array}{l}\frac{\text { YMRS }}{3 \text { weeks }} \\
\text { NS } \\
\text { Mean change } \\
\text { Endoxifen } 4 \mathrm{mg}:-12.65 \\
\text { Endoxifen } 8 \mathrm{mg}:-16.21 \\
\text { Divalproex:: }-16.38\end{array}$ & $\begin{array}{l}\frac{\text { CGI-S }}{3 \text { week }} \\
\text { Reported NS } \\
\text { (details not } \\
\text { reported) }\end{array}$ & $\begin{array}{l}2 \text { patients withdrew } \\
\text { due to adverse } \\
\text { events } 4 \mathrm{mg} \text { arm }\end{array}$ & $\begin{array}{l}\text { Serious Adverse } \\
\text { Events } \\
3 \text { weeks } \\
24 \text { mg arm } \\
\text { (delusions) } \\
\text { No deaths }\end{array}$ \\
\hline
\end{tabular}

Abbreviations: AE=Adverse Events; BMI=Body Mass Index; CGI=Clinical Global Impressions; CGI-BP-S=Clinical Global Impressions, Bipolar, Severity Scale; CI=Confidence Interval; EPS=extrapyramidal symptoms; GAF=General Assessment of Functioning Scale; NR=not reported; NS=not significant; PMID=PubMed Identification Number; RR=Risk Ratio; SAE=Serious Adverse Events; SD=standard deviation; YMRS = Young Mania Rating Scale

\section{Appendix Table G22. Strength of evidence assessment: endoxifen for mania vs. active control}

\begin{tabular}{|c|c|c|c|c|c|c|c|c|}
\hline Comparison & Outcome & $\begin{array}{l}\text { \# Studies/ } \\
\text { Design } \\
\text { (n analyzed) }\end{array}$ & $\begin{array}{l}\text { Finding or } \\
\text { Summary } \\
\text { Statistic }\end{array}$ & $\begin{array}{c}\text { Study } \\
\text { Limitations }\end{array}$ & Consistency & Directness & Precision & $\begin{array}{c}\text { Overall } \\
\text { Gradel } \\
\text { Conclusion }\end{array}$ \\
\hline $\begin{array}{l}\text { Endoxifen vs. } \\
\text { divalproex }\end{array}$ & $\begin{array}{l}\text { Remission } 3 \text { wks } \\
\text { YMRS } 3 \text { weeks } \\
\text { CGI-S } 3 \text { weeks }\end{array}$ & $\begin{array}{l}1 \mathrm{RCT} \\
\text { (4 mg: } \mathrm{n}=42 \\
\text { (8 mg: } n=42)\end{array}$ & See table above & Low & Unknown & Direct & Imprecise & Insufficient \\
\hline
\end{tabular}

Abbreviations: CGI= Clinical Global Impressions; CI=Confidence Interval; GAF=General Assessment of Functioning Scale; IPD=Individual Patient Data; NS=not significant; RCT=randomized controlled trial; YMRS = Young Mania Rating Scale

Notes:

1. Publication bias for antipsychotics, antidepressants, and behavioral interventions for depressive disorders is suspected.

2. Data were generally imprecise due to missing data from high attrition rates, which was commonly dealt with by Last Observation Carried Forward (LOCF). LOCF requires an assumption that the health status of patients who dropped out of the trial would not have changed had future observations been recorded, a strong assumption in the context of

bipolar disorder research. 


\section{Section 6. Gabapentin}

Appendix Table G23. Characteristics of eligible studies: gabapentin for acute mania

\begin{tabular}{|c|c|c|c|c|c|c|}
\hline $\begin{array}{l}\text { Study, Year } \\
\text { Design } \\
\text { Location } \\
\text { Funder } \\
\text { Risk of Bias } \\
\text { PMID }\end{array}$ & $\begin{array}{c}\text { \# Randomized } \\
\text { Age (mean) } \\
\text { Sex (\% Female) } \\
\text { Race (\% White) } \\
\text { Diagnosis } \\
\text { (\% BP I, II, NOS) } \\
\text { Setting }\end{array}$ & $\begin{array}{c}\text { Inclusions } \\
\text { Key Exclusions }\end{array}$ & $\begin{array}{c}\text { Intervention } \\
\text { Dosage }\end{array}$ & $\begin{array}{c}\text { Comparison } \\
\text { Dosage }\end{array}$ & $\begin{array}{c}\text { Follow-up } \\
\text { Duration }\end{array}$ & $\begin{array}{c}\text { Outcomes } \\
\text { Reported } \\
\text { Withdrawal (\%) at } \\
\text { endpoint }\end{array}$ \\
\hline \begin{tabular}{|l} 
Astaneh, $2012^{7}$ \\
RCT \\
Single-site \\
Iran \\
University \\
RoB High \\
\\
22978083
\end{tabular} & $\begin{array}{l}\mathrm{N}=60 \\
\text { Mean Age NR } \\
\text { Female about 50\% } \\
\text { White NR } \\
\text { BP I NR } \\
\text { Inpatient }\end{array}$ & \begin{tabular}{|l|} 
Mania; \\
Not Defined \\
Substance abuse
\end{tabular} & $\begin{array}{l}\text { Gabapentin } \\
900 \text { mg/day } \\
\\
\text { Lithium } \\
\text { NR }\end{array}$ & $\begin{array}{l}\text { Placebo } \\
\text { Lithium } \\
\text { NR }\end{array}$ & 6 week & $\begin{array}{l}\text { YMRS } \\
\text { Withdrawal } 0\end{array}$ \\
\hline
\end{tabular}

Abbreviations: AE=Adverse Effects; BP=bipolar disorder; BPRS=Brief Psychiatric Rating Scale; CGI=Clinical Global Impressions; CGI-BP=Clinical Global Impressions Scale for Bipolar Disorder; CGI-BP-S=Clinical Global Impressions, Bipolar, Severity Scale; CGI-S=Clinical Global Impressions, Severity Scale; DSM-IV= Diagnostic and statistical manual, $4^{\text {th }}$ edition; EPS=Extrapyramidal Symptoms; GAF=General Assessment of Functioning Scale; GAS= Global Assessment Scale; HAM-D=Hamilton Depression Rating Scale; MADRS=Montgomery-Asberg Depression Rating Scale; NOS=not otherwise specified; NR= not reported; Q-LES-Q=Quality of Life Enjoyment and Satisfaction Questionnaire; PANSS=Positive and Negative Syndrome Scale; PMID=PubMed Identification Number; RCT= Randomized Controlled Trial; RoB=risk of bias; SAE=Serious Adverse Events; SDS=Sheehan Disability Scale; T=Trial; YMRS = Young Mania Rating Scale

\section{Appendix Table G24. Summary risk of bias assessments: gabapentin for mania}

Appendix Table G24. Summary risk of bias assessments: gabapentin for mania
\begin{tabular}{|l|l|l|l|}
\hline \multicolumn{1}{|c|}{ Drug } & $\begin{array}{c}\text { Study } \\
\text { Funding Source } \\
\text { PMID }\end{array}$ & $\begin{array}{c}\text { Overall Risk of Bias } \\
\text { Assessment }\end{array}$ & Rationale \\
\hline Gabapentin & $\begin{array}{l}\text { Astaneh, 2012 } \\
\text { University } \\
22978083\end{array}$ & High & $\begin{array}{l}\text { Clinical and demographic traits at baseline not reported or compared for similarity. Blinding of } \\
\text { staff and patients not addressed. Reporting is insufficient and may be misleading (e.g. } \\
\text { missing values in graphs, missing error bars in graphs, raw data not provided/only sum of } \\
\text { squares, asserts statistically meaningful improvement when improvement not shown } \\
\text { statistically). }\end{array}$ \\
\hline
\end{tabular}

Abbreviations: PMID=PubMed Identification Number; RCT=randomized controlled trial 
Appendix Table G25. Outcomes summary: gabapentin for mania vs. inactive control

\begin{tabular}{|c|c|c|c|c|c|c|}
\hline Comparison & $\begin{array}{c}\text { Study } \\
\text { ROB } \\
\text { PMID }\end{array}$ & Responder/Remitter & Symptom & Function & Other & $\mathrm{AE}$ \\
\hline $\begin{array}{l}\text { Gabapentin + } \\
\text { lithium vs. placebo } \\
+ \text { lithium }\end{array}$ & $\begin{array}{l}\text { Astaneh, } 2012^{7} \\
\text { High } \\
22978083\end{array}$ & NR & $\begin{array}{l}\text { YMRS } \\
\text { Reported favors } \\
\text { gabapentin. However, } \\
\text { baseline YMRS } \\
\text { gabapentin }=\sim 50 \text { while } \\
\text { control = } \sim 13 .\end{array}$ & NR & NR & NR \\
\hline
\end{tabular}

Abbreviations: AE=Adverse Events; BMI=Body Mass Index; CI=Confidence Interval; CGI-BP-S=Clinical Global Impressions, Bipolar, Severity Scale; CGI-S= Clinical Global Impressions, Severity Scale; EPS=extrapyramidal symptoms; GAF=General Assessment of Functioning Scale; GAS=Global Assessment Scale; NR=not reported; NS=not significant; OR=Odds Ratio; PMID=PubMed Identification Number; RCT=randomized controlled trial; SAE=Serious Adverse Events; SD=standard deviation; YMRS = Young Mania Rating Scale

\section{Appendix Table G26. Strength of evidence assessment: gabapentin for mania vs. inactive control}

\begin{tabular}{|c|c|c|c|c|c|c|c|c|}
\hline Comparison & Outcome & $\begin{array}{l}\text { \# Studies/ } \\
\text { Design } \\
\text { (n analyzed) }\end{array}$ & $\begin{array}{l}\text { Finding or } \\
\text { Summary } \\
\text { Statistic }\end{array}$ & $\begin{array}{c}\text { Study } \\
\text { Limitations }\end{array}$ & Consistency & Directness & Precision & $\begin{array}{c}\text { Overall } \\
\text { Gradel } \\
\text { Conclusion }\end{array}$ \\
\hline $\begin{array}{l}\text { Gabapentin + } \\
\text { lithium vs. } \\
\text { placebo + } \\
\text { lithium }\end{array}$ & YMRS 6 wks & $\begin{array}{l}1 \mathrm{RCT} \\
(\mathrm{n}=60)\end{array}$ & See table above & High & Unknown & Direct & Imprecise & Insufficient \\
\hline
\end{tabular}

Abbreviations: CGI= Clinical Global Impressions; CGI-S=Clinical Global Impressions, Severity Scale; IPD=Individual Patient Data; NS=not significant; RCT=randomized controlled trial; YMRS = Young Mania Rating Scale

Notes:

1. Publication bias for antipsychotics, antidepressants, and behavioral interventions for depressive disorders is suspected.

2. Data were generally imprecise due to missing data from high attrition rates, which was commonly dealt with by Last Observation Carried Forward (LOCF). LOCF requires an assumption that the health status of patients who dropped out of the trial would not have changed had future observations been recorded, a strong assumption in the context of bipolar disorder research. 


\section{Section 7. Paliperidone}

Appendix Table G27. Characteristics of eligible studies: paliperidone for acute mania

\begin{tabular}{|c|c|c|c|c|c|c|}
\hline $\begin{array}{c}\text { Study, Year } \\
\text { Design } \\
\text { Location } \\
\text { Funder } \\
\text { Risk of Bias } \\
\text { PMID }\end{array}$ & $\begin{array}{c}\text { \# Randomized } \\
\text { Age (mean) } \\
\text { Sex (\% Female) } \\
\text { Race (\% White) } \\
\text { Diagnosis } \\
\text { (\% BP I, II, NOS) } \\
\text { Setting }\end{array}$ & $\begin{array}{c}\text { Inclusions } \\
\text { Key Exclusions }\end{array}$ & $\begin{array}{c}\text { Intervention } \\
\text { Dosage }\end{array}$ & $\begin{array}{c}\text { Comparison } \\
\text { Dosage }\end{array}$ & $\begin{array}{l}\text { Follow-up } \\
\text { Duration }\end{array}$ & $\begin{array}{c}\text { Outcomes } \\
\text { Reported } \\
\text { Withdrawal (\%) at } \\
\text { endpoint }\end{array}$ \\
\hline $\begin{array}{l}\text { Berwaerts, } 2012 \mathrm{a}^{9} \\
\text { RCT } \\
\text { 3 Continents } \\
\text { Industry } \\
\text { ROB Moderate } \\
20624657\end{array}$ & $\begin{array}{l}\mathrm{N}=469 \\
\text { Mean Age } 40 \\
\text { Female } 47 \% \\
\text { White } 50 \% \\
\text { BP I } 100 \% \\
\text { Inpatient (at least } 1 \text { week) } \\
\text { Outpatient (weeks 2-3) }\end{array}$ & $\begin{array}{l}\text { Manic/Mixed; } \\
\text { YMRS } \geq 20 \text { with } 1 \text { manic } \\
\text { or mixed episode in past } \\
\text { three years } \\
\text { Schizoaffective; } \\
\text { Substance abuse; } \\
\text { Other Mental Health } \\
\text { Condition; } \\
\text { Taking other } \\
\text { medications; } \\
\text { Pregnant/Nursing }\end{array}$ & $\begin{array}{l}\text { Paliperdone } \\
\text { extended release } \\
\text { (separate 3,6,12 } \\
\text { mg/day arms) }\end{array}$ & Placebo & 3 weeks & $\begin{array}{l}\text { CGI-BP-S } \\
\text { GAF } \\
\text { MADRS } \\
\text { PANSS } \\
\text { Scale for Suicide } \\
\text { Ideation (SSI) } \\
\text { YMRS } \\
\text { SAE } \\
\text { EPS } \\
\text { Withdrawal 39\% }\end{array}$ \\
\hline $\begin{array}{l}\text { Berwaerts, } 2011^{10} \\
\text { RCT } \\
3 \text { Continents } \\
\text { Industry } \\
\text { ROB Moderate } \\
20947174\end{array}$ & $\begin{array}{l}n=300 \\
\text { Mean Age } 40 \\
\text { Female } 46 \% \\
\text { White } 77 \% \\
\text { BP I } 100 \% \\
\text { Inpatient (at least } 1 \text { week) } \\
\text { Outpatient (weeks 2-7) }\end{array}$ & $\begin{array}{l}\text { Manic/Mixed; } \\
\text { YMRS } \geq 20 \\
\text { First Manic Episode; } \\
\text { Schizoaffective; } \\
\text { Substance Abuse; } \\
\text { Other Mental Health } \\
\text { Conditions; } \\
\text { Neurological Disorders; } \\
\text { Taking other } \\
\text { medications; } \\
\text { Pregnant/Nursing }\end{array}$ & $\begin{array}{l}\text { Paliperidone } \\
\text { extended release } \\
\text { 3-12 mg/day } \\
\text { (mean } 8.1 \mathrm{mg} / \text { day) } \\
\\
\text { Lithium } \\
0.6-1.2 \mathrm{mEq} / \mathrm{L} \\
\text { (mean NR) } \\
\text { Or } \\
\text { Valproate } \\
50-125 \mathrm{mcg} / \mathrm{mL} \\
\text { (mean NR) }\end{array}$ & $\begin{array}{l}\text { Placebo } \\
\text { NA } \\
\text { Lithium } \\
0.6-1.2 \mathrm{mEq} / \mathrm{L} \\
\text { (mean NR) } \\
\text { Or } \\
\text { Valproate } \\
50-125 \mathrm{mcg} / \mathrm{mL} \\
\text { (mean NR) }\end{array}$ & 7 weeks & $\begin{array}{l}\text { CGI-BP-S } \\
\text { GAF } \\
\text { PANSS } \\
\text { YMRS } \\
\text { Response (YMRS } \\
\text { decrease } \geq 50 \% \text { ) } \\
\text { Remission } \\
\text { (YMRS } \leq 12 \text { ) } \\
\text { MADRS } \\
\text { Adverse Events } \\
\text { Withdrawal } 37 \%\end{array}$ \\
\hline
\end{tabular}




\begin{tabular}{|c|c|c|c|c|c|c|}
\hline $\begin{array}{l}\text { Study, Year } \\
\text { Design } \\
\text { Location } \\
\text { Funder } \\
\text { Risk of Bias } \\
\text { PMID }\end{array}$ & $\begin{array}{c}\text { \# Randomized } \\
\text { Age (mean) } \\
\text { Sex (\% Female) } \\
\text { Race (\% White) } \\
\text { Diagnosis } \\
\text { (\% BP I, II, NOS) } \\
\text { Setting }\end{array}$ & $\begin{array}{c}\text { Inclusions } \\
\text { Key Exclusions }\end{array}$ & $\begin{array}{c}\text { Intervention } \\
\text { Dosage }\end{array}$ & $\begin{array}{c}\text { Comparison } \\
\text { Dosage }\end{array}$ & $\begin{array}{c}\text { Follow-up } \\
\text { Duration }\end{array}$ & $\begin{array}{c}\text { Outcomes } \\
\text { Reported } \\
\text { Withdrawal (\%) at } \\
\text { endpoint }\end{array}$ \\
\hline $\begin{array}{l}\text { Vieta, } 2010^{11} \\
\text { RCT } 3 \text { weeks } \\
\text { Multisite } \\
4 \text { Continents } \\
\text { Industry } \\
\text { RoB Moderate } \\
20565430\end{array}$ & $\begin{array}{l}\mathrm{N}=493 \\
\text { Mean Age } 39 \\
\text { Female } 42 \% \\
\text { White } 68 \% \\
\text { BP I 100\% } \\
\text { Inpatient (at least } 1 \text { week) } \\
\text { Outpatient (weeks 2-3) }\end{array}$ & $\begin{array}{l}\text { Manic/Mixed; } \\
\text { YMRS } \geq 20 ; \\
\text { At least one episode } \\
\text { within three years prior } \\
\\
\text { First Manic Episode } \\
\text { Schizoaffective } \\
\text { Substance abuse } \\
\text { Other mental health } \\
\text { Neurological disorders } \\
\text { Labs/other conditions } \\
\end{array}$ & \begin{tabular}{|l} 
Paliperidone \\
extended release \\
3-12 $\mathrm{mg} / \mathrm{day}$ \\
(median/mode \\
dosage $9 \mathrm{mg}$ )
\end{tabular} & $\begin{array}{l}\text { C1: Placebo } \\
\text { C2: Quetiapine } \\
\text { 400-800 mg/day }\end{array}$ & $\begin{array}{l}3 \text { week } \\
\text { (12 week } \\
\text { excluded for } \\
\text { attrition) }\end{array}$ & $\begin{array}{l}\text { YMRS } \\
\text { GAF } \\
\text { PANSS } \\
\text { CGI-BP-S } \\
\text { Response (YMRS } \\
\text { decrease } \geq 50 \%) \\
\text { Remission } \\
\text { (YMRS } 12 \text { ) } \\
\text { Withdrawal } 21 \% \text { at } 3 \\
\text { weeks } \\
\end{array}$ \\
\hline $\begin{array}{l}\text { Berwaerts, } 2012^{12} \\
\text { RCT } \\
\text { Multisite } \\
5 \text { Continents } \\
\text { Industry } \\
\text { RoB Moderate } \\
22377512\end{array}$ & $\begin{array}{l}\mathrm{N}=766 \\
\text { Mean Age } 40 \\
\text { Female } 52 \% \\
\text { White } 62 \% \\
\text { BP I } 100 \% \\
\text { Outpatient }\end{array}$ & $\begin{array}{l}\text { Manic/Mixed; } \\
\text { YMRS } \geq 20 ; \\
2 \text { previous mood } \\
\text { episodes (1 of which } \\
\text { manic/mixed) within past } \\
3 \text { years; } \\
\\
\text { First manic episode; } \\
\text { Schizoaffective; } \\
\text { Other mental health; } \\
\text { Neurological disorders; } \\
\text { Taking other meds; } \\
\text { Pregnant/nursing; } \\
\text { Labs/Other conditions }\end{array}$ & $\begin{array}{l}\text { Paliperidone } \\
\text { extended release } \\
\text { 3-12 mg/day }\end{array}$ & $\begin{array}{l}\text { Olanzapine } \\
\text { 5-20 mg/day }\end{array}$ & 15 weeks & $\begin{array}{l}\text { Response } \\
(\geq 50 \% \text { reduction in } \\
\text { YMRS) } \\
\text { Remission } \\
\text { (YMRS and } \\
\text { MADRS } \leq 12) \\
\\
\text { Withdrawal } 49 \%\end{array}$ \\
\hline
\end{tabular}

Abbreviations: AE=Adverse Effects; BP=bipolar disorder; BPRS=Brief Psychiatric Rating Scale; CGI=Clinical Global Impressions; CGI-BP=Clinical Global Impressions Scale for Bipolar Disorder; CGI-BP-S=Clinical Global Impressions, Bipolar, Severity Scale; CGI-S=Clinical Global Impressions, Severity Scale; DSM-IV= Diagnostic and statistical manual, $4^{\text {th }}$ edition; EPS=Extrapyramidal Symptoms; GAF=General Assessment of Functioning Scale; GAS= Global Assessment Scale; HAM-D=Hamilton Depression Rating Scale; MADRS=Montgomery-Asberg Depression Rating Scale; NOS=not otherwise specified; NR= not reported; Q-LES-Q=Quality of Life Enjoyment and Satisfaction

Questionnaire; PANSS=Positive and Negative Syndrome Scale; PMID=PubMed Identification Number; RCT= Randomized Controlled Trial; RoB=risk of bias; SAE=Serious Adverse Events; SDS=Sheehan Disability Scale; T=Trial; YMRS = Young Mania Rating Scale 
Appendix Table G28. Summary risk of bias assessments: paliperidone for mania

Appendix Table G28. Summary risk of bias assessments: paliperidone for mania
\begin{tabular}{|l|l|l|l|}
\hline \multicolumn{1}{|c|}{ Drug } & $\begin{array}{c}\text { Study } \\
\text { Funding Source } \\
\text { PMID }\end{array}$ & $\begin{array}{c}\text { Overall Risk of Bias } \\
\text { Assessment }\end{array}$ & Rationale \\
\hline $\begin{array}{l}\text { Paliperidone } \\
\text { extended release }\end{array}$ & $\begin{array}{l}\text { Berwaerts, 2012 } \\
\text { Industry } \\
22377512\end{array}$ & High & Very large dropout rate among all study arms, across all time periods \\
\cline { 2 - 4 } & $\begin{array}{l}\text { Berwaerts, } \\
2012 a^{9} \\
\text { Industry } \\
20624657\end{array}$ & Moderate & Large dropout rate among all study arms; attrition 39\% \\
\cline { 2 - 4 } & $\begin{array}{l}\text { Vieta, } 2010^{11} \\
\text { Industry } \\
20565430\end{array}$ & Moderate & Moderate dropout rate among all study arms, across all time periods; raters may not be \\
\cline { 2 - 4 } & $\begin{array}{l}\text { Berwaerts, 2011 } \\
\text { Industry } \\
20947174\end{array}$ & Moderate & Large dropout rate among all study arms; attrition 37\% \\
\hline
\end{tabular}

Abbreviations: PMID=PubMed Identification Number; RCT=randomized controlled trial

\section{Appendix Table G29. Outcomes summary: paliperidone for mania vs. inactive control}

\begin{tabular}{|c|c|c|c|c|c|c|}
\hline Comparison & $\begin{array}{l}\text { Study } \\
\text { ROB } \\
\text { PMID }\end{array}$ & Responder/Remitter & Symptom & Function & Other & $\mathrm{AE}$ \\
\hline $\begin{array}{l}\text { Paliperidone vs. } \\
\text { placebo }\end{array}$ & $\begin{array}{l}\text { Vieta, } 2010^{11} \\
\text { Moderate } \\
20565430\end{array}$ & $\begin{array}{l}\text { Responders } \\
\text { (YMRS decrease } \\
\geq 50 \%) \\
3 \text { weeks } \\
\text { Favors Paliperidone } \\
\text { Paliperidone=106/190 } \\
\text { Placebo=36/104 } \\
\text { p }<0.001 \\
\text { Remission } \\
\text { (YMRS } \leq 12) \\
3 \text { weeks } \\
\text { Favors Paliperidone } \\
\text { Paliperidone=99/190 } \\
\text { Placebo=30/104 } \\
\text { p }<0.001\end{array}$ & $\begin{array}{l}\text { YMRS Change } \\
3 \text { weeks } \\
\text { Favors treatment } \\
\text { Least square mean } \\
\text { difference between } \\
\text { groups } \\
-5.5 \\
(95 \% \mathrm{Cl}-7.57,-3.35) \\
\mathrm{p}<0.001\end{array}$ & $\begin{array}{l}\frac{\text { CGI-BP-S }}{3 \text { week }} \\
\text { Paliperidone - } \\
2.0 \\
(95 \% \mathrm{Cl}-4,2) \\
\text { Placebo -0.5 } \\
(95 \% \mathrm{Cl}-4,2) \\
\text { Favors } \\
\text { Paliperidone } \\
\text { p<0.001 } \\
\text { GAF } \\
3 \text { weeks } \\
\text { Favors } \\
\text { treatment } \\
\text { Mean } \\
\text { difference } \\
\text { treatment: } 11.6 \\
\text { Placebo: } 12.2 \\
\text { p<0.001 }\end{array}$ & 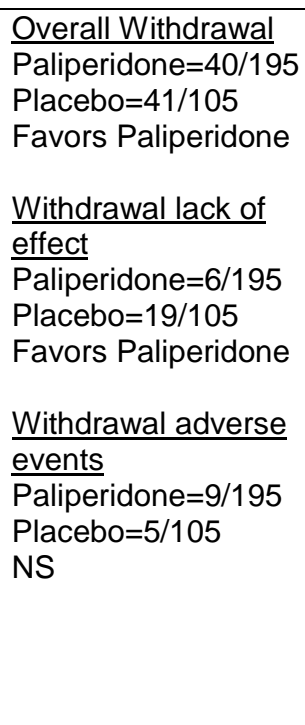 & $\begin{array}{l}\text { Serious AE } \\
\text { NR } \\
\frac{\text { EPS }}{\text { No serious events }} \\
\text { in any treatment } \\
\text { arm }\end{array}$ \\
\hline
\end{tabular}




\begin{tabular}{|c|c|c|c|c|c|c|}
\hline Comparison & $\begin{array}{l}\text { Study } \\
\text { ROB } \\
\text { PMID }\end{array}$ & Responder/Remitter & Symptom & Function & Other & AE \\
\hline & $\begin{array}{l}\text { Berwaerts, } \\
\text { 2012a }{ }^{9} \\
\text { Moderate } \\
20624657\end{array}$ & $\begin{array}{l}\text { Responders } \\
\text { (YMRS decrease } \\
\geq 50 \%) \\
3 \text { weeks } \\
\text { NS } \\
\frac{\text { Remission }}{\text { (YMRS } \leq 12)} \\
3 \text { weeks } \\
\text { NS }\end{array}$ & $\begin{array}{l}\text { YRMS Change } \\
3 \text { weeks } \\
\text { Least square mean } \\
\text { difference } \\
\text { Paliperidone } 12 \mathrm{mg}: \text { - } \\
13.5 \text { (9.17) } \mathrm{n}=109 \\
\text { Placebo: }-10.1 \text { (10.21) } \\
\text { Difference between } \\
\text { groups } 3.4 \\
\mathrm{n}=115 \\
\mathrm{p}=0.025 \\
\text { Favors Paliperidone } 12 \\
\text { mg (dose dependent) }\end{array}$ & $\begin{array}{l}\frac{\text { CGI-BP-S }}{3 \text { week }} \\
\text { NS } \\
\frac{\text { GAF }}{3 \text { weeks }} \\
\text { NS }\end{array}$ & $\begin{array}{l}\text { Overall Withdrawal } \\
\text { Paliperidone }=132 / 347 \\
\text { Placebo }=50 / 122 \\
\text { NS } \\
\text { Withdrawal lackof } \\
\frac{\text { effect }}{\text { Paliperidone }=31 / 347} \\
\text { Placebo }=24 / 122 \\
\text { Favors Paliperidone } \\
\text { Withdrawal adverse } \\
\text { events } \\
\text { Paliperidone }=25 / 347 \\
\text { Placebo }=6 / 122 \\
\text { NS }\end{array}$ & $\begin{array}{l}\text { Serious AE } \\
1 \text { death } \\
\text { Paliperidone } 6 \mathrm{mg} \\
\text { (deemed not } \\
\text { related) } \\
\text { EPS } \\
\text { Statistically } \\
\text { significantly more in } \\
12 \text { mg paliperidon } \\
\text { for akathisia and } \\
\text { dystonia } \\
\underline{\text { Treatment }} \\
\underline{\text { emergent }} \\
\underline{\text { depression: }} \\
\underline{\mathrm{NS}} \\
\underline{>7 \%} \text { weight gain } \\
\end{array}$ \\
\hline $\begin{array}{l}\text { Paliperidone + } \\
\text { mood stabilizers vs. } \\
\text { placebo + mood } \\
\text { stabilizers }\end{array}$ & $\begin{array}{l}\text { Berwaerts, } \\
2011^{10} \\
\text { Moderate } \\
20947174\end{array}$ & $\begin{array}{l}\text { Remission } \\
\text { (YMRS } \leq 12) \\
6 \text { weeks } \\
\text { NS } \\
\text { Paliperidone } 60 \% \\
\text { Placebo } 57 \% \\
\text { p=0.12 } \\
\text { Response } \\
\text { (YMRS } \geq 50 \% \\
\text { decrease) } \\
6 \text { weeks } \\
\text { NS } \\
\text { Paliperidone } 62 \% \\
\text { Placebo } 56 \% \\
\text { p=0.24 }\end{array}$ & $\begin{array}{l}\underline{\text { YMRS }} \\
6 \text { weeks } \\
\text { Least squares mean } \\
\text { difference } \\
\text { NS } \\
p=0.16\end{array}$ & $\begin{array}{l}\frac{\text { CGI-BP-S }}{6 \text { weeks }} \\
\text { NS } \\
p=0.26 \\
\text { GAF } \\
\text { N weeks } \\
p=0.71\end{array}$ & $\begin{array}{l}\frac{\text { Suicide Ideation }}{1 \text { in each group }} \\
\text { Overall Withdrawal } \\
\text { Paliperidon }=60 / 150 \\
\text { Placebo }=51 / 150 \\
\text { NS } \\
\text { Withdrawal lack of } \\
\text { effect } \\
\text { Paliperidone }=12 / 150 \\
\text { Placebo=18/150 } \\
\text { NS } \\
\text { Withdrawal adverse } \\
\frac{\text { events }}{\text { Paliperidone }=12 / 150} \\
\text { Placebo }=2 / 150 \\
\text { Favors Placebo }\end{array}$ & $\begin{array}{l}\text { SAE } \\
7 \text { in each group; } \\
\text { psychiatric } \\
\text { disorders most } \\
\text { common } \\
\text { Treament emergent } \\
\text { depression: 1\% in } \\
\text { each group } \\
\text { Akathesia 8\% vs. } \\
\text { 1\% Favored } \\
\text { placebo. } \\
\text { Weight } \\
\text { increase } \geq 7 \% \text { : } \\
\text { Paliperidone 15\% } \\
\text { Placebo 5\% }\end{array}$ \\
\hline
\end{tabular}

Abbreviations: AE=Adverse Events; BMI=Body Mass Index; CI=Confidence Interval; CGI-BP-S=Clinical Global Impressions, Bipolar, Severity Scale; CGI-S= Clinical Global Impressions, Severity Scale; EPS=extrapyramidal symptoms; GAF=General Assessment of Functioning Scale; GAS=Global Assessment Scale; NR=not reported; NS=not 
significant; OR=Odds Ratio; PMID=PubMed Identification Number; RCT=randomized controlled trial; SAE=Serious Adverse Events; SD=standard deviation; YMRS = Young Mania Rating Scale

\section{Appendix Table G30. Strength of evidence assessment: paliperidone for mania vs. inactive control}

\begin{tabular}{|c|c|c|c|c|c|c|c|c|}
\hline Comparison & Outcome & $\begin{array}{l}\text { \# Studies/ } \\
\text { Design } \\
\text { (n analyzed) }\end{array}$ & $\begin{array}{l}\text { Finding or } \\
\text { Summary } \\
\text { Statistic }\end{array}$ & $\begin{array}{c}\text { Study } \\
\text { Limitations }\end{array}$ & Consistency & Directness & Precision & $\begin{array}{c}\text { Overall } \\
\text { Gradel } \\
\text { Conclusion }\end{array}$ \\
\hline \multirow{3}{*}{$\begin{array}{l}\text { Paliperidone } \\
\text { vs. placebo }\end{array}$} & $\begin{array}{l}\text { Remission } 3 \text { wks } \\
\text { Response } 3 \text { wks } \\
\text { CGI } \\
\text { Withdrawal - } \\
\text { overall }\end{array}$ & $\begin{array}{l}2 \mathrm{RCT} \\
(\mathrm{n}=763)\end{array}$ & See table above & Moderate & Inconsistent & Direct & Imprecise & Insufficient \\
\hline & $\begin{array}{l}\text { Withdrawal - } \\
\text { adverse events }\end{array}$ & $\begin{array}{l}2 \text { RCT } \\
(n=763)\end{array}$ & NS & Moderate & Consistent & Direct & Imprecise & Low \\
\hline & $\begin{array}{l}\text { YMRS } 3 \text { wks } \\
\text { Withdrawal lack of } \\
\text { efficacy }\end{array}$ & $\begin{array}{l}2 \mathrm{RCT} \\
(\mathrm{n}=763)\end{array}$ & $\begin{array}{l}\text { Favors } \\
\text { Paliperidone } \\
\text { possible dose } \\
\text { response: } \mathrm{NS} \text { at } \\
3 \text { and } 6 \mathrm{mg}, \\
\text { benefit at } 12 \mathrm{mg} \\
\text { or median } \\
\text { dosage of } 9 \mathrm{mg}\end{array}$ & Moderate & Consistent & Direct & Imprecise & Low \\
\hline $\begin{array}{l}\text { Paliperidone + } \\
\text { mood } \\
\text { stabilizers vs. } \\
\text { placebo + } \\
\text { mood } \\
\text { stabilizers }\end{array}$ & $\begin{array}{l}\text { Remission } 6 \text { wks } \\
\text { Response } 6 \text { wks } \\
\text { YMRS } 6 \text { wks } \\
\text { CGI-S } 6 \text { wks } \\
\text { Withdrawals }\end{array}$ & $\begin{array}{l}1 \mathrm{RCT} \\
(\mathrm{n}=299)\end{array}$ & See table above & Moderate & Unknown & Direct & Imprecise & Insufficient \\
\hline
\end{tabular}

Abbreviations: CGI= Clinical Global Impressions; CGI-S=Clinical Global Impressions, Severity Scale; IPD=Individual Patient Data; NS=not significant; RCT=randomized controlled trial; YMRS = Young Mania Rating Scale

Notes:

1. Publication bias for antipsychotics, antidepressants, and behavioral interventions for depressive disorders is suspected.

2. Data were generally imprecise due to missing data from high attrition rates, which was commonly dealt with by Last Observation Carried Forward (LOCF). LOCF requires an assumption that the health status of patients who dropped out of the trial would not have changed had future observations been recorded, a strong assumption in the context of bipolar disorder research. 
Appendix Table G31. Outcomes summary: paliperidone for mania vs. active control

\begin{tabular}{|c|c|c|c|c|c|c|}
\hline Drug & $\begin{array}{c}\text { Study } \\
\text { Comparison } \\
\text { PMID }\end{array}$ & $\begin{array}{l}\text { Responderl } \\
\text { Remitter }\end{array}$ & Symptom & Function & Other & $A E$ \\
\hline $\begin{array}{l}\text { Paliperidone } \\
\text { extended release } \\
\text { vs. olanzapine }\end{array}$ & $\begin{array}{l}\text { Berwaerts, } 2012^{12} \\
\text { Moderate } \\
22377512\end{array}$ & $\begin{array}{l}\text { Response } \\
\text { (YMRS decrease } \\
\geq 50 \%) \\
15 \text { weeks } \\
\text { NS } \\
>70 \% \text { responded } \\
\text { overall } \\
\text { Remission } \\
\text { (YMRS } \leq 12) \\
15 \text { weeks } \\
\text { NS } \\
>60 \% \text { achieved } \\
\text { remission }\end{array}$ & $\begin{array}{l}\frac{\text { YMRS }}{\text { Least square mean }} \\
\text { difference } \\
15 \text { weeks } \\
\text { NS } \\
-0.3(-2.12,1.57)\end{array}$ & $\underline{\mathrm{NR}}$ & $\begin{array}{l}\text { Overall Withdrawal } \\
\text { 15 weeks } \\
\text { Paliperidone: 09/617 } \\
\text { Olanzapine: } 63 / 149 \\
\text { NS } \\
\text { Withdrawal lack of } \\
\text { effect } \\
\text { Paliperidone:30/617 } \\
\text { Olanzapine:5/149 } \\
\text { NS } \\
\text { Withdrawal adverse } \\
\text { events } \\
\text { Paliperidone:63/617 } \\
\text { Olanzapine:13/149 } \\
\text { NS }\end{array}$ & $\begin{array}{l}\text { SAE } \\
\text { Paliperidone: } \\
\text { 42/614 } \\
\text { Olanzapine: } 10 / 148 \\
\text { NS }\end{array}$ \\
\hline $\begin{array}{l}\text { Paliperidone } \\
\text { extended release } \\
\text { vs. quetiapine }\end{array}$ & $\begin{array}{l}\text { Vieta, } 2010^{11} \\
\text { Moderate } \\
20565430\end{array}$ & 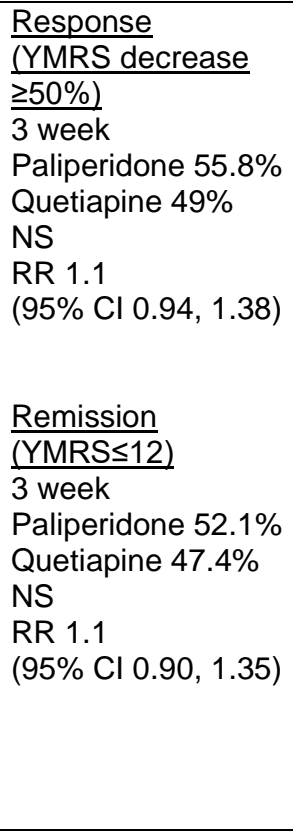 & $\begin{array}{l}\text { YMRS Change } \\
3 \text { week } \\
\text { LSM difference } \\
\text { (Quet-Pali) } 1.5 \\
\text { (95\% Cl -0.28, 3.22) } \\
\text { NS } \\
p=0.099\end{array}$ & $\begin{array}{l}\text { GAF Change }^{1} \\
3 \text { week } \\
\text { Paliperidone } 12.2 \\
\text { (sd 11.17) } \\
\text { Quetiapine } 11.6 \\
\text { (sd 11.96) } \\
\text { NS } \\
\text { p=NR } \\
\text { CGI-BP-S } \\
3 \text { week } \\
\text { Paliperidone -2.0 } \\
\text { (95\%Cl -4, 2) } \\
\text { Quetiapine -1.0 } \\
\text { (95\%Cl -4, 2) } \\
\text { NS } \\
\text { p=NR }\end{array}$ & $\begin{array}{l}\text { Switching } \\
3 \text { week } \\
\text { Paliperidone } 4.3 \% \\
\text { Quetiapine } 2.7 \% \\
\text { NS } \\
\text { p=NR } \\
\text { Overall Withdrawal } \\
\text { Paliperidone }=40 / 195 \\
\text { Quetiapine }=41 / 193 \\
\text { NS } \\
\text { Withdrawal lack of } \\
\frac{\text { effect }}{\text { Paliperidone }=6 / 195} \\
\text { Quetiapine=15/105 } \\
\text { Favors Paliperidone } \\
\text { Withdrawal adverse } \\
\frac{\text { events }}{\text { Paliperidone=9/195 }} \\
\text { Quetiapine }=4 / 193 \\
\text { NS }\end{array}$ & $\begin{array}{l}\frac{\mathrm{SAE}}{\mathrm{NR}} \\
\frac{\text { EPS }}{\text { No serious events }} \\
\text { in any treatment } \\
\text { arm }\end{array}$ \\
\hline
\end{tabular}


Abbreviations: AE=Adverse Events; BMI=Body Mass Index; CGI=Clinical Global Impressions; CGI-BP-S=Clinical Global Impressions, Bipolar, Severity Scale; CI=Confidence Interval; EPS=extrapyramidal symptoms; GAF=General Assessment of Functioning Scale; NR=not reported; NS=not significant; PMID=PubMed Identification Number;

RR=Risk Ratio; SAE=Serious Adverse Events; SD=standard deviation; YMRS = Young Mania Rating Scale

Appendix Table G32. Strength of evidence assessment: paliperidone for mania vs. active control

\begin{tabular}{|c|c|c|c|c|c|c|c|c|}
\hline Comparison & Outcome & $\begin{array}{l}\text { \# Studies/ } \\
\text { Design } \\
\text { (n analyzed) }\end{array}$ & $\begin{array}{l}\text { Finding or } \\
\text { Summary } \\
\text { Statistic }\end{array}$ & $\begin{array}{c}\text { Study } \\
\text { Limitations }\end{array}$ & Consistency & Directness & Precision & $\begin{array}{c}\text { Overall } \\
\text { Gradel } \\
\text { Conclusion }\end{array}$ \\
\hline $\begin{array}{l}\text { Paliperidone } \\
\text { extended } \\
\text { release vs. } \\
\text { olanzapine } \\
\end{array}$ & $\begin{array}{l}\text { Remission } 15 \text { wks } \\
\text { Response } 15 \text { wks } \\
\text { YMRS } 15 \text { wks } \\
\text { Withdrawals }\end{array}$ & $\begin{array}{l}1 \mathrm{RCT} \\
(\mathrm{n}=766)\end{array}$ & See table above & High & Unknown & Direct & Imprecise & Insufficient \\
\hline $\begin{array}{l}\text { Paliperidone } \\
\text { extended } \\
\text { release vs. } \\
\text { quetiapine }\end{array}$ & $\begin{array}{l}\text { Remission } 3 \text { wks } \\
\text { Response } 3 \text { wks } \\
\text { YMRS } 3 \text { wks } \\
\text { CGI } 3 \text { wks } \\
\text { GAF } 3 \text { wks } \\
\text { Withdrawals }\end{array}$ & $\begin{array}{l}1 \mathrm{RCT} \\
(\mathrm{n}=388)\end{array}$ & See table above & High & Unknown & Direct & Imprecise & Insufficient \\
\hline
\end{tabular}

Abbreviations: CGI= Clinical Global Impressions; CI=Confidence Interval; GAF=General Assessment of Functioning Scale; IPD=Individual Patient Data; NS=not significant; RCT=randomized controlled trial; YMRS = Young Mania Rating Scale

Notes:

1. Publication bias for antipsychotics, antidepressants, and behavioral interventions for depressive disorders is suspected.

2. Data were generally imprecise due to missing data from high attrition rates, which was commonly dealt with by Last Observation Carried Forward (LOCF). LOCF requires an assumption that the health status of patients who dropped out of the trial would not have changed had future observations been recorded, a strong assumption in the context of bipolar disorder research. 


\section{Section 8. Tamoxifen}

Appendix Table G33. Characteristics of eligible studies: tamoxifen for acute mania

\begin{tabular}{|c|c|c|c|c|c|c|}
\hline $\begin{array}{c}\text { Study, Year } \\
\text { Design } \\
\text { Location } \\
\text { Funder } \\
\text { Risk of Bias } \\
\text { PMID }\end{array}$ & $\begin{array}{c}\text { \# Randomized } \\
\text { Age (mean) } \\
\text { Sex (\% Female) } \\
\text { Race (\% White) } \\
\text { Diagnosis } \\
\text { (\% BP I, II, NOS) } \\
\text { Setting }\end{array}$ & $\begin{array}{c}\text { Inclusions } \\
\text { Key Exclusions }\end{array}$ & $\begin{array}{l}\text { Intervention } \\
\text { Dosage }\end{array}$ & $\begin{array}{c}\text { Comparison } \\
\text { Dosage }\end{array}$ & $\begin{array}{l}\text { Follow-up } \\
\text { Duration }\end{array}$ & $\begin{array}{c}\text { Outcomes } \\
\text { Reported } \\
\text { Withdrawal (\%) at } \\
\text { endpoint }\end{array}$ \\
\hline $\begin{array}{l}\text { Yildiz, } 2008^{13} \\
\text { RCT } \\
\text { Single-site } \\
\text { Turkey } \\
\text { Non-Profit } \\
\text { RoB Moderate } \\
18316672\end{array}$ & $\begin{array}{l}\mathrm{N}=66 \\
\text { Mean Age } 33 \\
\text { Female } 52 \% \\
\text { Race NR } \\
\text { BP I 100\% } \\
\text { Outpatient }\end{array}$ & $\begin{array}{l}\text { Mania; } \\
\text { YMRS } \geq 20 \\
\text { Schizoaffective } \\
\text { Substance abuse } \\
\text { Other mental health } \\
\text { Neurological disorders } \\
\text { Taking other meds } \\
\text { Pregnant/nursing }\end{array}$ & \begin{tabular}{|l} 
Tamoxifen \\
$20-80$ mg/twice daily
\end{tabular} & Placebo & 3 week & $\begin{array}{l}\text { CGI-BP-S } \\
\text { HAM-D } \\
\text { MADRS } \\
\text { PANSS } \\
\text { YMRS } \\
\text { Response (YMRS } \\
\text { decrease } \geq 50 \%) \\
\\
\text { Withdrawal } 24 \%\end{array}$ \\
\hline
\end{tabular}

Abbreviations: AE=Adverse Effects; BP=bipolar disorder; BPRS=Brief Psychiatric Rating Scale; CGI=Clinical Global Impressions; CGI-BP=Clinical Global Impressions Scale for Bipolar Disorder; CGI-BP-S=Clinical Global Impressions, Bipolar, Severity Scale; CGI-S=Clinical Global Impressions, Severity Scale; DSM-IV= Diagnostic and statistical manual, $4^{\text {th }}$ edition; EPS=Extrapyramidal Symptoms; GAF=General Assessment of Functioning Scale; GAS= Global Assessment Scale; HAM-D=Hamilton Depression Rating Scale; MADRS=Montgomery-Asberg Depression Rating Scale; NOS=not otherwise specified; NR= not reported; Q-LES-Q=Quality of Life Enjoyment and Satisfaction Questionnaire; PANSS=Positive and Negative Syndrome Scale; PMID=PubMed Identification Number; RCT= Randomized Controlled Trial; RoB=risk of bias; SAE=Serious Adverse Events; SDS=Sheehan Disability Scale; T=Trial; YMRS = Young Mania Rating Scale

Appendix Table G34. Summary risk of bias assessments: tamoxifen for mania

\begin{tabular}{|l|l|l|l|}
\hline \multicolumn{1}{|c|}{ Drug } & $\begin{array}{c}\text { Study } \\
\text { Funding Source } \\
\text { PMID }\end{array}$ & $\begin{array}{c}\text { Overall Risk of Bias } \\
\text { Assessment }\end{array}$ & Rationale \\
\hline Tamoxifen & $\begin{array}{l}\text { Yildiz, } 2008^{13} \\
\text { Non-Profit } \\
18316672\end{array}$ & Moderate & $\begin{array}{l}\text { Blinding of patients, staff, raters not described ; minor differences at baseline regarding } \\
\text { pretreatment drugs may create residual confounding }\end{array}$ \\
\hline
\end{tabular}

Abbreviations: PMID=PubMed Identification Number; RCT=randomized controlled trial; 
Appendix Table G35. Outcomes summary: tamoxifen for mania vs. inactive control

\begin{tabular}{|c|c|c|c|c|c|c|}
\hline Comparison & $\begin{array}{c}\text { Study } \\
\text { ROB } \\
\text { PMID }\end{array}$ & Responder/Remitter & Symptom & Function & Other & $\mathrm{AE}$ \\
\hline $\begin{array}{l}\text { Tamoxifen vs. } \\
\text { placebo }\end{array}$ & $\begin{array}{l}\begin{array}{l}\text { Yildiz, } 2008^{13} \\
\text { Moderate }\end{array} \\
18316672\end{array}$ & $\begin{array}{l}\text { Response } \\
\text { (YMRS decrease } \\
\geq 50 \%) \\
3 \text { weeks } \\
\text { Favors tamoxifen } \\
\text { Tamoxifen }=14 / 29 \\
\text { Placebo }=1 / 21 \\
p=0.003 \\
\text { Remission } \\
\text { (YMRS } \leq 12) \\
3 \text { weeks } \\
\text { Favors tamoxifen } \\
\text { Tamoxifen }=8 / 29 \\
\text { Placebo }=0 / 21 \\
p=0.03\end{array}$ & $\begin{array}{l}\text { YMRS Decrease Rate } \\
\text { Over } 3 \text { weeks } \\
\text { Favors tamoxifen } \\
\text { Linear mixed model } \\
\mathrm{p}<0.001 \\
\text { YMRS (Mean SD) } \\
\text { Week 0 } \\
\text { Tamoxifen } 38.6(5.0) \\
\text { Placebo } 37.2(6.6) \\
\text { Week } 3 \\
\text { Tamoxifen } 20.3(11.2) \\
\text { Placebo } 40.1(10.4)\end{array}$ & NR & $\begin{array}{l}\text { NR } \\
\text { Withdrawal } \\
\text { Tamoxifen=6/35 } \\
\text { Placebo=10/31 } \\
\text { NS }\end{array}$ & $\begin{array}{l}\text { Serious Adverse } \\
\text { Events } \\
1 \text { Tamoxifen - } \\
\text { Suicide Attempt } \\
1 \text { Placebo - Suicide } \\
\text { Attempt }\end{array}$ \\
\hline
\end{tabular}

Abbreviations: AE=Adverse Events; BMI=Body Mass Index; CI=Confidence Interval; CGI-BP-S=Clinical Global Impressions, Bipolar, Severity Scale; CGI-S= Clinical Global Impressions, Severity Scale; EPS=extrapyramidal symptoms; GAF=General Assessment of Functioning Scale; GAS=Global Assessment Scale; NR=not reported; NS=not significant; OR=Odds Ratio; PMID=PubMed Identification Number; RCT=randomized controlled trial; SAE=Serious Adverse Events; SD=standard deviation; YMRS = Young Mania Rating Scale

\section{Appendix Table G36. Strength of evidence assessment: tamoxifen for mania vs. inactive control}

\begin{tabular}{|c|c|c|c|c|c|c|c|c|}
\hline Comparison & Outcome & $\begin{array}{l}\text { \# Studies/ } \\
\text { Design } \\
\text { (n analyzed) }\end{array}$ & $\begin{array}{l}\text { Finding or } \\
\text { Summary } \\
\text { Statistic }\end{array}$ & $\begin{array}{c}\text { Study } \\
\text { Limitations }\end{array}$ & Consistency & Directness & Precision & $\begin{array}{l}\text { Overall } \\
\text { Gradel } \\
\text { Conclusion }\end{array}$ \\
\hline $\begin{array}{l}\text { Tamoxifen vs. } \\
\text { placebo }\end{array}$ & $\begin{array}{l}\text { Remission } 3 \text { wks } \\
\text { Response } 3 \text { wks } \\
\text { YMRS } 3 \text { wks } \\
\text { Withdrawals }\end{array}$ & $\begin{array}{l}1 \mathrm{RCT} \\
(\mathrm{n}=50)\end{array}$ & See table above & Low & Unknown & Direct & Imprecise & Insufficient \\
\hline
\end{tabular}

Abbreviations: CGI= Clinical Global Impressions; CGI-S=Clinical Global Impressions, Severity Scale; IPD=Individual Patient Data; NS=not significant; RCT=randomized controlled trial; YMRS = Young Mania Rating Scale

Notes:

1. Publication bias for antipsychotics, antidepressants, and behavioral interventions for depressive disorders is suspected.

2. Data were generally imprecise due to missing data from high attrition rates, which was commonly dealt with by Last Observation Carried Forward (LOCF). LOCF requires an assumption that the health status of patients who dropped out of the trial would not have changed had future observations been recorded, a strong assumption in the context of bipolar disorder research. 


\section{Section 9. Topiramate}

Appendix Table G37. Characteristics of eligible studies: topiramate for acute mania

\begin{tabular}{|c|c|c|c|c|c|c|}
\hline $\begin{array}{c}\text { Study, Year } \\
\text { Design } \\
\text { Location } \\
\text { Funder } \\
\text { Risk of Bias } \\
\text { PMID }\end{array}$ & $\begin{array}{c}\text { \# Randomized } \\
\text { Age (mean) } \\
\text { Sex (\% Female) } \\
\text { Race (\% White) } \\
\text { Diagnosis } \\
\text { (\% BP I, II, NOS) } \\
\text { Setting }\end{array}$ & $\begin{array}{c}\text { Inclusions } \\
\text { Key Exclusions }\end{array}$ & $\begin{array}{c}\text { Intervention } \\
\text { Dosage }\end{array}$ & $\begin{array}{c}\text { Comparison } \\
\text { Dosage }\end{array}$ & $\begin{array}{c}\text { Follow-up } \\
\text { Duration }\end{array}$ & $\begin{array}{c}\text { Outcomes } \\
\text { Reported } \\
\text { Withdrawal (\%) at } \\
\text { endpoint }\end{array}$ \\
\hline $\begin{array}{l}\text { Bahk, } 2005^{14} \\
\text { Open-label RCT } \\
\text { Multisite } \\
\text { South Korea } \\
\text { Industry } \\
\text { ROB High } \\
15610953\end{array}$ & $\begin{array}{l}\mathrm{N}=74 \\
\text { Mean Age } 37 \\
\text { Female 51\% } \\
\text { Race Asian } \\
\text { BP-I 100\% } \\
\text { Outpatient }\end{array}$ & $\begin{array}{l}\text { Mania } \\
\text { YMRS } \geq 20 \\
\text { Other mental health } \\
\text { Pregnant/nursing } \\
\text { Labs/other conditions } \\
\text { Taking other meds }\end{array}$ & $\begin{array}{l}\text { Topiramate } \\
\text { average } 220.6 \\
\text { mg/day }+ \\
\text { Risperidone } \\
\text { average } 3.4 \mathrm{mg} / \text { day }\end{array}$ & $\begin{array}{l}\text { Divalproex } \\
\text { average } 908.3 \\
\text { mg/day } \\
\text { Risperidone } \\
\text { average } 3.3 \\
\text { mg/day }\end{array}$ & 3 week & $\begin{array}{l}\text { YMRS } \\
\text { CGI } \\
\text { Adverse Events } \\
\text { Withdrawal 18\% }\end{array}$ \\
\hline $\begin{array}{l}\text { Chengappa, } 2006^{15} \\
\text { RCT } \\
\text { Multisite } \\
\text { US } \\
\text { Low } \\
\text { Industry } \\
\text { RoB Low } \\
17196048\end{array}$ & $\begin{array}{l}\mathrm{N}=287 \\
\text { Mean Age } 40 \\
\text { Female } 56 \% \\
\text { White } 84 \% \\
\text { BP I } 100 \% \\
\text { Outpatient }\end{array}$ & \begin{tabular}{|l} 
Manic/Mixed; \\
YMRS $\geq 18$ \\
Recevied lithium or \\
valproate at least 6 \\
weeks, including stable \\
dose 2 weeks prior to \\
screening within \\
specified serum levels \\
\\
Substance abuse \\
Other mental health \\
Neurological disorders \\
Taking other meds \\
Pregnant/nursing \\
Labs/other conditions
\end{tabular} & $\begin{array}{l}\text { T1: Topiramate } \\
50-400 \mathrm{mg} / \mathrm{day} \\
\text { (mean } 6.2 \mathrm{mcg} / \mathrm{mL} \\
\text { day } 42,7.8 \mathrm{mcg} / \mathrm{ml} \\
\text { day } 84 \text { ) } \\
\text { Lithium } \\
\text { mean } 0.7 \mathrm{mEq} / \mathrm{L} \\
\text { Valproate } \\
\text { mean } 69.8 \mathrm{mcg} / \mathrm{ml}\end{array}$ & $\begin{array}{l}\text { Placebo } \\
\text { Lithium } \\
\text { mean } 0.7 \mathrm{mEq} / \mathrm{L} \\
\text { Valproate } \\
\text { mean } 71.0 \mathrm{mcg} / \mathrm{ml}\end{array}$ & 12 week & $\begin{array}{l}\text { YMRS } \\
\text { CGI-S } \\
\text { BPRS } \\
\text { MADRS } \\
\text { GAS } \\
\text { Weight } \\
\text { Response ( } \geq 50 \% \\
\text { improvement in } \\
\text { YRMS) } \\
\text { Withdrawal 38\% }\end{array}$ \\
\hline
\end{tabular}




\begin{tabular}{|c|c|c|c|c|c|c|}
\hline $\begin{array}{l}\text { Study, Year } \\
\text { Design } \\
\text { Location } \\
\text { Funder } \\
\text { Risk of Bias } \\
\text { PMID }\end{array}$ & $\begin{array}{c}\text { \# Randomized } \\
\text { Age (mean) } \\
\text { Sex (\% Female) } \\
\text { Race (\% White) } \\
\text { Diagnosis } \\
\text { (\% BP I, II, NOS) } \\
\text { Setting }\end{array}$ & $\begin{array}{c}\text { Inclusions } \\
\text { Key Exclusions }\end{array}$ & $\begin{array}{l}\text { Intervention } \\
\text { Dosage }\end{array}$ & $\begin{array}{c}\text { Comparison } \\
\text { Dosage }\end{array}$ & $\begin{array}{c}\text { Follow-up } \\
\text { Duration }\end{array}$ & $\begin{array}{c}\text { Outcomes } \\
\text { Reported } \\
\text { Withdrawal (\%) at } \\
\text { endpoint }\end{array}$ \\
\hline $\begin{array}{l}\text { Kushner, } 2006^{16} \\
\text { Pooled Analysis of } 4 \\
\text { RCTs (3 week data) } \\
\text { Multisite } \\
6 \text { Continents } \\
\text { Industry } \\
\text { RoB Low } \\
16411977\end{array}$ & $\begin{array}{l}\mathrm{N}=876 \text { (includes only } 400 \\
\text { mg/day topiramte arms } \\
\text { and placebo arms } \\
\text { Mean Age } 41 \\
\text { Female } 51 \% \\
\text { White } 75 \% \\
\text { BP I } 100 \% \\
\text { Inpatient (at least } 4 \text { days, } \\
\text { as clinically warranted) }\end{array}$ & $\begin{array}{l}\text { Manic/Mixed; } \\
\text { YMRS } \geq 20 \\
\text { First Manic Episode } \\
\text { Schizoaffective } \\
\text { Substance abuse } \\
\text { Other mental health } \\
\text { Taking other meds } \\
\text { Pregnant/nursing } \\
\text { Labs/other conditions }\end{array}$ & $\begin{array}{l}\text { Topiramate } \\
\text { 400mg/day } \\
\text { (mean 313mg/day) } \\
\text { (only } 400 \text { mg/day } \\
\text { arms were common } \\
\text { across pooled } \\
\text { studies) }\end{array}$ & $\begin{array}{l}\text { C1: Placebo } \\
\text { C2: Lithium } \\
\text { 300-1800 mg/day } \\
(0.8-1.2 \mathrm{mEq} / \mathrm{L})\end{array}$ & 3 week & $\begin{array}{l}\text { Weight } \\
\text { YMRS } \\
\text { Withdrawal 27\% }\end{array}$ \\
\hline
\end{tabular}

Abbreviations: AE=Adverse Effects; BP=bipolar disorder; BPRS=Brief Psychiatric Rating Scale; CGI=Clinical Global Impressions; CGI-BP=Clinical Global Impressions Scale for Bipolar Disorder; CGI-BP-S=Clinical Global Impressions, Bipolar, Severity Scale; CGI-S=Clinical Global Impressions, Severity Scale; DSM-IV= Diagnostic and statistical manual, $4^{\text {th }}$ edition; EPS=Extrapyramidal Symptoms; GAF=General Assessment of Functioning Scale; GAS= Global Assessment Scale; HAM-D=Hamilton Depression Rating Scale; MADRS=Montgomery-Asberg Depression Rating Scale; NOS=not otherwise specified; NR= not reported; Q-LES-Q=Quality of Life Enjoyment and Satisfaction

Questionnaire; PANSS=Positive and Negative Syndrome Scale; PMID=PubMed Identification Number; RCT= Randomized Controlled Trial; RoB=risk of bias; SAE=Serious

Adverse Events; SDS=Sheehan Disability Scale; T=Trial; YMRS = Young Mania Rating Scale

\section{Appendix Table G38. Summary risk of bias assessments: topimarate for mania}

\begin{tabular}{|c|c|c|c|}
\hline Drug & $\begin{array}{c}\text { Study } \\
\text { Funding Source } \\
\text { PMID }\end{array}$ & $\begin{array}{c}\text { Overall Risk of Bias } \\
\text { Assessment }\end{array}$ & Rationale \\
\hline \multirow[t]{3}{*}{ Topiramate } & $\begin{array}{l}\text { Bahk, 2005 } \\
\text { Industry } \\
15610953 \\
\end{array}$ & High & Randomization and allocation concealment not specified, open label dosing \\
\hline & $\begin{array}{l}\text { Kushner, 2006 } \\
\text { Industry } \\
16411977\end{array}$ & Low & Well-disclosed and reported study (RCTs unique to this publication.) \\
\hline & $\begin{array}{l}\text { Chengappa, } \\
2006^{15} \\
\text { Industry } \\
17196048\end{array}$ & Low & Well-disclosed and reported study \\
\hline
\end{tabular}

Abbreviations: PMID=PubMed Identification Number; RCT=randomized controlled trial 
Appendix Table G39. Outcomes summary: topiramate for mania vs. inactive control

\begin{tabular}{|c|c|c|c|c|c|c|}
\hline Comparison & $\begin{array}{c}\text { Study } \\
\text { ROB } \\
\text { PMID }\end{array}$ & Responder/Remitter & Symptom & Function & Other & AE \\
\hline $\begin{array}{l}\text { Topiramate vs. } \\
\text { placebo }\end{array}$ & $\begin{array}{l}\text { Kushner, } 2006^{16} \\
\text { Low } \\
16411977\end{array}$ & NR & $\begin{array}{l}\text { YMRS Change } \\
3 \text { weeks } \\
\text { NS } \\
\text { Mean difference } \\
\text { (top-plac) } 0.60 \\
(95 \% \mathrm{Cl}-0.85,2.0 \text { ) } \\
\mathrm{p}=0.418 \\
\mathrm{n}=434 \mathrm{I}, \mathrm{n}=317 \mathrm{C}\end{array}$ & NR & $\begin{array}{l}\text { Overall Withdrawal } \\
\text { Topiramte }=123 / 331 \\
\text { Placebo }=85 / 317 \\
\text { Favors Placebo } \\
\text { Withdrawal lack of } \\
\text { effect } \\
\text { Topiramate }=52 / 331 \\
\text { Placebo }=39 / 317 \\
\text { NS } \\
\text { Withdrawal adverse } \\
\text { events } \\
\text { Topiramate }=20 / 331 \\
\text { Placebo }=9 / 317 \\
\text { Favors Placebo }\end{array}$ & $\begin{array}{l}\text { SAE } \\
\text { Topiramate } 3 \% \\
\text { Placebo } 2 \% \\
\text { Suicide Attempt } \\
3 \text { weeks } \\
\text { Topiramate 2/656 } \\
\text { Placebo 0/429 } \\
\text { (reported over } 4 \\
\text { pooled RCTs, not } \\
3 \text { monotherapy } \\
\text { tests) }\end{array}$ \\
\hline
\end{tabular}




\begin{tabular}{|c|c|c|c|c|c|c|}
\hline Comparison & $\begin{array}{c}\text { Study } \\
\text { ROB } \\
\text { PMID }\end{array}$ & Responder/Remitter & Symptom & Function & Other & $\mathrm{AE}$ \\
\hline $\begin{array}{l}\text { Topiramate+mood } \\
\text { stabilizer vs. } \\
\text { placebo+mood } \\
\text { stabilizer }\end{array}$ & $\begin{array}{l}\text { Chengappa, } \\
2006^{15} \\
\text { Low } \\
17196048\end{array}$ & $\begin{array}{l}\text { Response } \\
\text { (YMRS } \geq 50 \% \\
\text { decrease) } \\
12 \text { weeks } \\
\text { NS } \\
\text { Topiramate 39\% } \\
\text { Placebo } 38 \% \\
p=0.914\end{array}$ & $\begin{array}{l}\text { YMRS Change } \\
12 \text { weeks } \\
\text { NS } \\
\text { Topiramate }-10.1 \pm 8.7 \\
\text { Placebo }-9.6 \pm 8.2 \\
p=0.797\end{array}$ & $\begin{array}{l}\text { CGI-S Change } \\
12 \text { weeks } \\
\text { NS } \\
\text { Topiramate } \\
-0.9 \pm 1.1 \\
\text { Placebo - } \\
0.9 \pm 1.1 \\
\mathrm{p}=0.698 \\
\text { GAS Change } \\
12 \text { weeks } \\
\text { NS } \\
\text { Topiramate } \\
7.2 \pm 9.9 \\
\text { Placebo } \\
7.1 \pm 11.5 \\
\mathrm{p}=0.838\end{array}$ & $\begin{array}{l}\frac{\text { BMI Change }}{12 \text { weeks }} \\
\text { Favors Topiramate } \\
\text { Topiramate }-0.84 \pm 1.2 \\
\mathrm{~kg} / \mathrm{m}^{2} \\
\text { Placebo } 0.07 \pm 1.1 \\
\mathrm{~kg} / \mathrm{m}^{2} \\
\mathrm{p}<0.001 \\
\text { Suicide Ideation } \\
\text { Topiramate } 1 \text { patient } \\
\text { Placebo } 2 \text { patients } \\
\text { Overall Withdrawal } \\
\text { Topiramate }=57 / 143 \\
\text { Placebo=53/144 } \\
\text { NS } \\
\text { Withdrawal lack of } \\
\text { effect } \\
\text { Topiramate }=6 / 143 \\
\text { Placebo }=4 / 144 \\
\text { NS } \\
\text { Withdrawal adverse } \\
\text { events } \\
\text { Topiramate }=20 / 143 \\
\text { Placebo=10/144 } \\
\text { Favors Placebo }\end{array}$ & NR \\
\hline
\end{tabular}

Abbreviations: AE=Adverse Events; BMI=Body Mass Index; CI=Confidence Interval; CGI-BP-S=Clinical Global Impressions, Bipolar, Severity Scale; CGI-S= Clinical Global Impressions, Severity Scale; EPS=extrapyramidal symptoms; GAF=General Assessment of Functioning Scale; GAS=Global Assessment Scale; NR=not reported; NS=not significant; OR=Odds Ratio; PMID=PubMed Identification Number; RCT=randomized controlled trial; SAE=Serious Adverse Events; SD=standard deviation; YMRS = Young Mania Rating Scale 
Appendix Table G40. Strength of evidence assessment: topiramate for mania vs. inactive control

\begin{tabular}{|c|c|c|c|c|c|c|c|c|}
\hline Comparison & Outcome & $\begin{array}{l}\text { \# Studies/ } \\
\text { Design } \\
\text { (n analyzed) }\end{array}$ & $\begin{array}{l}\text { Finding or } \\
\text { Summary } \\
\text { Statistic }\end{array}$ & $\begin{array}{c}\text { Study } \\
\text { Limitations }\end{array}$ & Consistency & Directness & Precision & $\begin{array}{c}\text { Overall } \\
\text { Gradel } \\
\text { Conclusion }\end{array}$ \\
\hline \multirow{3}{*}{$\begin{array}{l}\text { Topiramate } \\
\text { vs. placebo }\end{array}$} & $\begin{array}{l}\text { YMRS } 3 \text { wks } \\
\text { Withdrawal - lack } \\
\text { of effect }\end{array}$ & $\begin{array}{l}4 \text { RCT (1 IPD) } \\
(n=876)\end{array}$ & NS & Low & Consistent & Direct & Imprecise & Low \\
\hline & $\begin{array}{l}\text { Withdrawals - } \\
\text { overall }\end{array}$ & $\begin{array}{l}4 \mathrm{RCT}(1 \mathrm{IPD}) \\
(\mathrm{n}=876)\end{array}$ & $\begin{array}{l}\text { Favors Placebo } \\
37.2 \% \text { vs. } \\
26.8 \%, p=0.005\end{array}$ & Low & Consistent & Direct & Imprecise & Low \\
\hline & $\begin{array}{l}\text { Withdrawals - } \\
\text { adverse events }\end{array}$ & $\begin{array}{l}4 \text { RCT (1 IPD) } \\
(n=876)\end{array}$ & $\begin{array}{l}\text { Favors Placebo } \\
6.04 \% \text { vs. } \\
2.84 \%, p=0.049\end{array}$ & Low & Consistent & Direct & Imprecise & Low \\
\hline $\begin{array}{l}\text { Topiramate } \\
\text { +mood } \\
\text { stabilizer vs. } \\
\text { placebo+moo } \\
\text { d stabilizer }\end{array}$ & $\begin{array}{l}\text { Response } 12 \text { wks } \\
\text { YMRS } 12 \text { wks } \\
\text { CGI-S } 12 \text { wks } \\
\text { Withdrawals }\end{array}$ & $\begin{array}{l}1 \mathrm{RCT} \\
(\mathrm{n}=287)\end{array}$ & See table above & Low & Unknown & Direct & Imprecise & Insufficient \\
\hline
\end{tabular}

Abbreviations: CGI= Clinical Global Impressions; CGI-S=Clinical Global Impressions, Severity Scale; IPD=Individual Patient Data; NS=not significant; RCT=randomized

controlled trial; YMRS = Young Mania Rating Scale

Notes:

1. Publication bias for antipsychotics, antidepressants, and behavioral interventions for depressive disorders is suspected.

2. Data were generally imprecise due to missing data from high attrition rates, which was commonly dealt with by Last Observation Carried Forward (LOCF). LOCF requires an

assumption that the health status of patients who dropped out of the trial would not have changed had future observations been recorded, a strong assumption in the context of

bipolar disorder research. 
Appendix Table G41. Outcomes summary: topiramate for mania vs. active control

\begin{tabular}{|c|c|c|c|c|c|c|}
\hline Drug & $\begin{array}{c}\text { Study } \\
\text { Comparison } \\
\text { PMID }\end{array}$ & $\begin{array}{l}\text { Responderl } \\
\text { Remitter }\end{array}$ & Symptom & Function & Other & $A E$ \\
\hline $\begin{array}{l}\text { Topiramate vs. } \\
\text { lithium }\end{array}$ & $\begin{array}{l}\text { Kushner, } 2006^{16} \\
\text { Low } \\
16411977\end{array}$ & NR & $\begin{array}{l}\text { YMRS Change } \\
3 \text { week } \\
\text { Mean difference } \\
\text { (top-lith) } 6.14 \\
\text { (95\% Cl 3.94, 8.34) } \\
\text { Favors Lithium } \\
\text { p<0.001 }\end{array}$ & NR & $\begin{array}{l}\frac{\text { Weight }}{3 \text { week }} \\
\text { Mean difference } \\
\text { (top-lith) }-1.82 \% \\
(95 \% \mathrm{Cl}-2.90 \%,- \\
0.74 \%) \\
\text { Favors Topiramate } \\
\text { p<0.001 } \\
\text { Overall Withdrawal } \\
\text { Topiramte }=47 / 226 \\
\text { Lithium }=51 / 227 \\
\text { NS } \\
\text { Withdrawal lack of } \\
\frac{\text { effect }}{\text { Topiramate }=23 / 226} \\
\text { Lithium }=19 / 227 \\
\text { NS } \\
\text { Withdrawal adverse } \\
\frac{\text { events }}{\text { Topiramate }=6 / 226} \\
\text { Lithium }=17 / 227 \\
\text { Favors Topiramate } \\
\text { p=.019 }\end{array}$ & $\begin{array}{l}\text { SAE } \\
\text { Topiramate 3\% } \\
\text { Lithium 1.5\% }\end{array}$ \\
\hline $\begin{array}{l}\text { Topiramate }+ \\
\text { risperidone vs. } \\
\text { divalproex }+ \\
\text { risperidone }\end{array}$ & $\begin{array}{l}\text { Bahk, } 2005^{14} \\
\text { High } \\
15610953\end{array}$ & $\begin{array}{l}\frac{\text { Response }}{\text { (YMRS decrease }} \\
\geq 50 \%) \\
15 \text { weeks } \\
\text { NS } \\
>70 \% \text { responded } \\
\text { overall } \\
\text { Remission } \\
\text { (YMRS } \leq 12) \\
15 \text { weeks } \\
\text { NS } \\
>60 \% \text { achieved } \\
\text { remission }\end{array}$ & $\begin{array}{l}\text { YMRS } \\
6 \text { weeks } \\
\text { NS } \\
\text { (Both groups improved } \\
\text { statistically significantly) }\end{array}$ & $\begin{array}{l}\text { CGI } \\
6 \text { weeks } \\
\text { NS } \\
\text { (Both groups } \\
\text { improved } \\
\text { statistically } \\
\text { significantly) }\end{array}$ & $\begin{array}{l}\text { BMI } \\
\text { Divalproex } 73 \% \\
\text { patients increase } \\
\text { Topiramate } 25 \% \\
\text { patients decreased } \\
\text { Overall Withdrawal } \\
\text { NS } \\
\text { Withdrawals } \\
\text { reasons not } \\
\text { reported by group }\end{array}$ & $\begin{array}{l}\text { Reported no SAEs } \\
\text { EPS } \\
\text { NS between groups } \\
\text { ("about } 1 / 3 \text { of } \\
\text { patients in both } \\
\text { groups") }\end{array}$ \\
\hline
\end{tabular}


Abbreviations: AE=Adverse Events; BMI=Body Mass Index; CGI=Clinical Global Impressions; CGI-BP-S=Clinical Global Impressions, Bipolar, Severity Scale; CI=Confidence Interval; EPS=extrapyramidal symptoms; GAF=General Assessment of Functioning Scale; NR=not reported; NS=not significant; PMID=PubMed Identification Number;

RR=Risk Ratio; SAE=Serious Adverse Events; SD=standard deviation; YMRS = Young Mania Rating Scale

\section{Appendix Table G42. Strength of evidence assessment: topiramate for mania vs. active control}

\begin{tabular}{|c|c|c|c|c|c|c|c|c|}
\hline Comparison & Outcome & $\begin{array}{l}\text { \# Studies/ } \\
\text { Design } \\
\text { (n analyzed) }\end{array}$ & $\begin{array}{l}\text { Finding or } \\
\text { Summary } \\
\text { Statistic }\end{array}$ & $\begin{array}{c}\text { Study } \\
\text { Limitations }\end{array}$ & Consistency & Directness & Precision & $\begin{array}{l}\text { Overall } \\
\text { Gradel } \\
\text { Conclusion }\end{array}$ \\
\hline \multirow{3}{*}{$\begin{array}{l}\text { Topiramate vs. } \\
\text { Lithium }\end{array}$} & YMRS 3 wks & $\begin{array}{l}2 \text { RCTs (1 IPD) } \\
(\mathrm{n}=453)\end{array}$ & $\begin{array}{l}\text { Favors Lithium } \\
\text { Mean difference } \\
6.14(95 \% \mathrm{Cl} \\
3.94,8.34) \\
\end{array}$ & Low & Consistent & Direct & Imprecise & Low \\
\hline & $\begin{array}{l}\text { Withdraw - } \\
\text { overall, lack of } \\
\text { effect }\end{array}$ & $\begin{array}{l}2 \text { RCTs (1 IPD) } \\
(n=453)\end{array}$ & NS & Low & Consistent & Direct & Imprecise & Low \\
\hline & $\begin{array}{l}\text { Withdraw - } \\
\text { adverse events }\end{array}$ & $\begin{array}{l}2 \text { RCTs (1 IPD) } \\
(n=453)\end{array}$ & $\begin{array}{l}\text { Favors } \\
\text { Topiramate } \\
2.65 \% \text { vs. } \\
7.49 \%, p=0.019\end{array}$ & Low & Consistent & Direct & Imprecise & Low \\
\hline $\begin{array}{l}\text { Topiramate + } \\
\text { risperidone vs. } \\
\text { divalproex }+ \\
\text { risperidone }\end{array}$ & $\begin{array}{l}\text { Remission } 6 \text { wks } \\
\text { Response } 6 \text { wks } \\
\text { YMRS } 6 \text { wks } \\
\text { CGI } 6 \text { wks } \\
\text { Withdrawals }\end{array}$ & $\begin{array}{l}1 \mathrm{RCT} \\
(\mathrm{n}=74)\end{array}$ & See table above & High & Unknown & Direct & Imprecise & Insufficient \\
\hline
\end{tabular}

Abbreviations: CGI= Clinical Global Impressions; CI=Confidence Interval; GAF=General Assessment of Functioning Scale; IPD=Individual Patient Data; NS=not significant; RCT=randomized controlled trial; YMRS = Young Mania Rating Scale

Notes:

1. Publication bias for antipsychotics, antidepressants, and behavioral interventions for depressive disorders is suspected.

2. Data were generally imprecise due to missing data from high attrition rates, which was commonly dealt with by Last Observation Carried Forward (LOCF). LOCF requires an assumption that the health status of patients who dropped out of the trial would not have changed had future observations been recorded, a strong assumption in the context of bipolar disorder research. 


\section{References for Appendix G}

1. Jahangard L, Soroush S, Haghighi M, et al. In a double-blind, randomized and placebo-controlled trial, adjuvant allopurinol improved symptoms of mania in in-patients suffering from bipolar disorder. European Neuropsychopharmacology. Jun. 2014 2, 2014(Pagination)doi: http://dx.doi.org/10.1016/j.euroneuro.2014.05.013 . PMID: 24953766.

2. Weiser M, Burshtein S, Gershon AA, et al. Allopurinol for mania: A randomized trial of allopurinol versus placebo as add-on treatment to mood stabilizers and/or antipsychotic agents in manic patients with bipolar disorder. Bipolar Disorders. 2014 2014;16(4):441-7. doi: http://dx.doi.org/10.1111/bdi.12202. PMID: 24712840.

3. Fan A, Berg A, Bresee C, et al. Allopurinol augmentation in the outpatient treatment of bipolar mania: a pilot study. Bipolar Disorders. 2012 Mar;14(2):206-10. doi: http://dx.doi.org/10.1111/j.13995618.2012.01001.x. PMID: 22420596.

4. Machado-Vieira R, Soares JC, Lara DR, et al. A double-blind, randomized, placebo-controlled 4week study on the efficacy and safety of the purinergic agents allopurinol and dipyridamole adjunctive to lithium in acute bipolar mania. Journal of Clinical Psychiatry. 2008 Aug;69(8):1237-45. PMID: 18681754.

5. Arabzadeh S, Ameli N, Zeinoddini A, et al. Celecoxib adjunctive therapy for acute bipolar mania: A randomized, double-blind, placebocontrolled trial. Bipolar Disorders. 201501 Sep;17(6):606-14. doi: http://dx.doi.org/10.1111/bdi.12324. PMID: 26291962 (pubmed) 2015329826 (embase).

6. Chen J, Lu Z, Zhang M, et al. A randomized, 4week double-blind placebo control study on the efficacy of donepezil augmentation of lithium for treatment of acute mania. Neuropsychiatric Disease and Treatment. 2013 17, 2013;9:839-45. PMID: 23807849.

7. Astaneh AN, Rezaei O. Adjunctive treatment with gabapentin in bipolar patients during acute mania. International Journal of Psychiatry in Medicine. 2012;43(3):261-71. PMID: 22978083.
8. Ahmad A, Sheikh S, Shah T, et al. Endoxifen, a New Treatment Option for Mania: A DoubleBlind, Active-Controlled Trial Demonstrates the Antimanic Efficacy of Endoxifen. Clinical and Translational Science. 201601 Oct;9(5):252-9. doi: http://dx.doi.org/10.1111/cts.12407. PMID: 27346789 PMID/611114504 Embase.

9. Berwaerts J, Xu H, Nuamah I, et al. Evaluation of the efficacy and safety of paliperidone extendedrelease in the treatment of acute mania: a randomized, double-blind, dose-response study. Journal of Affective Disorders. 2012 Jan;136(12):e51-60. doi:

http://dx.doi.org/10.1016/j.jad.2010.06.030. PMID: 20624657.

10. Berwaerts J, Lane R, Nuamah IF, et al. Paliperidone extended-release as adjunctive therapy to lithium or valproate in the treatment of acute mania: a randomized, placebo-controlled study. Journal of Affective Disorders. 2011 Mar;129(1-3):252-60. doi: http://dx.doi.org/10.1016/j.jad.2010.09.011. PMID: 20947174.

11. Vieta E, Nuamah IF, Lim P, et al. A randomized, placebo- and active-controlled study of paliperidone extended release for the treatment of acute manic and mixed episodes of bipolar I disorder. Bipolar Disorders. 2010 May;12(3):23043. doi: http://dx.doi.org/10.1111/j.13995618.2010.00815.x. PMID: 20565430.

12. Berwaerts J, Melkote R, Nuamah I, et al. A randomized, placebo- and active-controlled study of paliperidone extended-release as maintenance treatment in patients with bipolar I disorder after an acute manic or mixed episode. Journal of Affective Disorders. 2012 May;138(3):247-58. doi: http://dx.doi.org/10.1016/j.jad.2012.01.047. PMID: 22377512.

13. Yildiz A, Guleryuz S, Ankerst DP, et al. Protein kinase $C$ inhibition in the treatment of mania: a double-blind, placebo-controlled trial of tamoxifen. Archives of General Psychiatry. 2008 Mar;65(3):255-63. doi: http://dx.doi.org/10.1001/archgenpsychiatry.2007. 43. PMID: 18316672.

14. Bahk WM, Shin YC, Woo JM, et al. Topiramate and divalproex in combination with risperidone for acute mania: a randomized open-label study. Prog Neuropsychopharmacol Biol Psychiatry. 2005 Jan;29(1):115-21. doi: 10.1016/j.pnpbp.2004.10.013. PMID: 15610953. 
15. Chengappa RKN, Schwarzman LK, Hulihan JF, et al. Adjunctive topiramate therapy in patients receiving a mood stabilizer for bipolar I disorder: a randomized, placebo-controlled trial. Journal of Clinical Psychiatry. 2006 Nov;67(11):1698-706. PMID: 17196048.
16. Kushner SF, Khan A, Lane R, et al. Topiramate monotherapy in the management of acute mania: results of four double-blind placebo-controlled trials. Bipolar Disorders. 2006 Feb;8(1):15-27. PMID: 16411977. 


\section{Appendix H. Drug Treatments for Depression}

\section{Section 1. Sertraline vs. Lithium vs. Lithium + Sertraline}

Appendix Table H1. Characteristics of eligible studies: sertraline for depression

\begin{tabular}{|c|c|c|c|c|c|c|}
\hline $\begin{array}{c}\text { Study, Year } \\
\text { Design } \\
\text { Location } \\
\text { Funder } \\
\text { Risk of Bias } \\
\text { PMID }\end{array}$ & $\begin{array}{c}\text { \# Randomized } \\
\text { Age (mean) } \\
\text { Sex (\% Female) } \\
\text { Race (\% White) } \\
\text { Diagnosis } \\
\text { (\% BP I, II, NOS) } \\
\text { Setting }\end{array}$ & $\begin{array}{c}\text { Inclusions } \\
\text { Key Exclusions }\end{array}$ & $\begin{array}{c}\text { Intervention } \\
\text { Dosage }\end{array}$ & $\begin{array}{c}\text { Comparison } \\
\text { Dosage }\end{array}$ & $\begin{array}{l}\text { Followup } \\
\text { Duration }\end{array}$ & \begin{tabular}{|c|} 
Outcomes \\
Reported \\
$\begin{array}{c}\text { Withdrawal (\%) at } \\
\text { endpoint }\end{array}$
\end{tabular} \\
\hline $\begin{array}{l}\text { Altshuler, } 2017^{1} \\
\text { RCT } \\
\text { Multisite } \\
\text { US } \\
\text { Government } \\
\text { RoB High } \\
28135846\end{array}$ & $\begin{array}{l}\mathrm{N}=142 \\
\text { Mean Age } 39 \\
\text { Female } 54 \% \\
\text { White } 97 \% \\
\text { BP II } 100 \% \\
\text { Outpatient }\end{array}$ & $\begin{array}{l}\text { BPII; current major } \\
\text { depressive episode. } \\
\text { IDS-C } \geq 22 \text {; CGI-BP } \geq 3 \text { on } \\
\text { depression subscale; } \\
\text { YMRS } \leq 8 ; \text { CGI-BP }=1 \text { on } \\
\text { mania severity subscale } \\
\\
\text { Substance Abuse } \\
\text { Other Mental Health } \\
\text { (Nonresponsive to } \\
\text { Lithium or Sertraline) }\end{array}$ & $\begin{array}{l}\text { Sertraline } \\
\text { target } 100 \mathrm{mg} / \mathrm{day}\end{array}$ & $\begin{array}{l}\text { C1: Lithium } \\
\text { target } 900 \text { mg/day } \\
\text { C2: Sertraline+ } \\
\text { Lithium } \\
\text { target } 100 \\
\text { mg/day+900 } \\
\text { mg/day }\end{array}$ & 16 weeks & $\begin{array}{l}\text { Switch to } \\
\text { hypomania or } \\
\text { mania (YMRS } \geq 14 \\
+ \text { CGI-BP } \geq 4) \\
\text { Treatment } \\
\text { response } \\
\text { (decrease of } \geq 50 \% \\
\text { IDS-C OR } \\
\text { decrease } \geq 2 \text { points } \\
\text { CGI-BP } \\
\text { depression) } \\
\text { Adverse Events } \\
\\
\text { Withdrawal 56\% } \\
\text { (Non-relapse } \\
\text { withdrawal 32\%) }\end{array}$ \\
\hline
\end{tabular}

Abbreviations: BP=bipolar disorder; C=Comparison; CGI=Clinical Global Impressions Scale; CGI-BP=Clinical Global Impressions Scale for Bipolar Disorder; DSM-

IV=Diagnostic and Statistical Manual of Mental Disorders, $4^{\text {th }}$ Revision; GAF=General Assessment of Functioning Scale; HAM-D=Hamilton Scale for Depression;

HSRD=Hamilton Rating Scale for Depression; IDS-C=Inventory for Depressive Symptoms(Clinician-Rated); MADRS=Montgomery-Asberg Depression Rating Scale; NOS=not otherwise specified; NR=not reported; PMID=PubMed Identification Number; RCT=randomized controlled trial; ROB=risk of bias; SRS=symptom rating scale; SUM-

D=Symptom Subscale for Depression; SUM-ME=Symptom Subscale for Mood Elevation; YMRS = Young Mania Rating Scale 
Appendix Table H2. Summary risk of bias assessments: sertraline for depression

\begin{tabular}{|l|l|l|l|}
\hline \multicolumn{1}{|c|}{ Drug } & $\begin{array}{c}\text { Study } \\
\text { Funding Source } \\
\text { PMID }\end{array}$ & $\begin{array}{c}\text { Overall Risk of Bias } \\
\text { Assessment }\end{array}$ & Rationale \\
\hline $\begin{array}{l}\text { Sertraline vs. } \\
\begin{array}{l}\text { Lihtium vs. } \\
\text { Sithium }+\end{array}\end{array}$ & $\begin{array}{l}\text { Altshuler, 20171 } \\
\text { Government }\end{array}$ & High & $\begin{array}{l}\text { Overall attrition 56\%; only time to relapse or withdrawal outcomes used. Block randomization. } \\
\text { Allocation concealment described. Blinded assessors; unblinded treatment physicians in } \\
\text { communication with blinded physician. No discussion of missing data approaches for } \\
\text { generalized mixed modeling. Log rank test. }\end{array}$ \\
\hline
\end{tabular}

Abbreviations: PMID=PubMed Identification Number; YMRS=Young Mania Rating Scale

\section{Appendix Table H3. Outcomes summary: sertraline for depression}

\begin{tabular}{|l|l|l|l|l|l|}
\hline \multicolumn{1}{|c|}{ Drug } & \multicolumn{1}{|c|}{$\begin{array}{c}\text { Study } \\
\text { PMID }\end{array}$} & Responder/Remitter & Symptom & Function & Other \\
\hline Sertraline vs. & Altshuler, 20171 & Treatment response & NA & NA & Switching \\
Lihtium vs. & 28135846 & 16 weeks & NR & 16 weeks \\
Lithium + & & $62.7 \%$ overall & $14 \%$ overall \\
Sertraline & & NS across groups & & NS across \\
& & & & groups \\
\hline
\end{tabular}

Abbreviations: AE=Adverse Events; CGI=Clinical Global Impressions Scale; HAM-D=Hamilton Scale for Depression; MID=minimally important difference; NA=Not applicable; NR=not reported; NS=not significant; PMID=PubMed Identification Number; SAE=Serious Adverse Events; YMRS =Young Mania Rating Scale

Appendix Table H4. Strength of evidence assessment: sertraline for depression

\begin{tabular}{|c|c|c|c|c|c|c|c|c|}
\hline Comparison & Outcome & $\begin{array}{c}\text { \# Studies/ } \\
\text { Design } \\
\text { (n analyzed) }\end{array}$ & $\begin{array}{l}\text { Finding or } \\
\text { Summary } \\
\text { Statistic }\end{array}$ & $\begin{array}{c}\text { Study } \\
\text { Limitations }\end{array}$ & Consistency & Directness & Precision & $\begin{array}{c}\text { Overall } \\
\text { Gradel } \\
\text { Conclusion }\end{array}$ \\
\hline $\begin{array}{l}\text { Sertraline vs. } \\
\text { Lihtium vs. } \\
\text { Lithium + } \\
\text { Sertraline }\end{array}$ & $\begin{array}{l}\text { Response16 wks } \\
\text { Switching } 16 \text { wks }\end{array}$ & $\begin{array}{l}1 \mathrm{RCT} \\
(\mathrm{n}=142)\end{array}$ & See table above & High & Unknown & Direct & Imprecise & Insufficient \\
\hline
\end{tabular}

Abbreviations: CGI= Clinical Global Impressions; HAM-D=Hamilton Scale for Depression; $\mathrm{n}=$ number of subjects; RCT=randomized controlled trial

Notes:

1. Publication bias for antipsychotics, antidepressants, and behavioral interventions for depressive disorders is suspected.

2. Data were generally imprecise due to missing data from high attrition rates, which was commonly dealt with by Last Observation Carried Forward (LOCF). LOCF requires an

assumption that the health status of patients who dropped out of the trial would not have changed had future observations been recorded, a strong assumption in the context of

bipolar disorder research. 


\section{Section 2. Venlafaxine vs. Lithium}

Appendix Table H5. Characteristics of eligible studies: venlafaxine for depression

\begin{tabular}{|c|c|c|c|c|c|c|}
\hline $\begin{array}{c}\text { Study, Year } \\
\text { Design } \\
\text { Location } \\
\text { Funder } \\
\text { Risk of Bias } \\
\text { PMID }\end{array}$ & $\begin{array}{c}\text { \# Randomized } \\
\text { Age (mean) } \\
\text { Sex (\% Female) } \\
\text { Race (\% White) } \\
\text { Diagnosis } \\
\text { (\% BP I, II, NOS) } \\
\text { Setting }\end{array}$ & $\begin{array}{c}\text { Inclusions } \\
\text { Key Exclusions }\end{array}$ & $\begin{array}{l}\text { Intervention } \\
\text { Dosage }\end{array}$ & $\begin{array}{c}\text { Comparison } \\
\text { Dosage }\end{array}$ & $\begin{array}{l}\text { Followup } \\
\text { Duration }\end{array}$ & \begin{tabular}{|c|} 
Outcomes \\
Reported \\
Withdrawal (\%) at \\
endpoint
\end{tabular} \\
\hline $\begin{array}{l}\text { Amsterdam, } 2016^{2} \\
\text { RCT } \\
\text { Singlesite } \\
\text { US } \\
\text { Government } \\
\text { RoB Moderate } \\
\\
26892848 \\
26803764^{3}\end{array}$ & $\begin{array}{l}\mathrm{N}=129 \\
\text { Mean Age } 43 \\
\text { Female } 57 \% \\
\text { White } 73 \% \\
\text { BP II } 100 \% \\
\text { Outpatient }\end{array}$ & $\begin{array}{l}\text { BPII; current major } \\
\text { depressive episode. } \\
\text { HAM-D } \geq 16 \\
\text { Substance Abuse } \\
\text { Other Mental Health } \\
\text { Pregnant/Nursing } \\
\text { Taking Other Meds } \\
\text { Labs/Other Conditions } \\
\text { (Nonresponsive to } \\
\text { Venlafaxine or Lithium) }\end{array}$ & $\begin{array}{l}\text { Venlafaxine } \\
\max 375 \text { mg/day } \\
\min 75 \text { mg/day }\end{array}$ & $\begin{array}{l}\text { Lithium } \\
\text { serum level } 0.8 \text { to } \\
1.5 \mathrm{mmol} / \mathrm{L}\end{array}$ & 12 weeks & $\begin{array}{l}\text { Response (HSRD } \\
\text { reduction } \geq 50 \% \\
\text { plus CGI/S=1) } \\
\text { Remission } \\
\text { (HSRD } \leq 8 \text { plus } \\
\text { CGI/S=1 or 2) } \\
\text { HSRD } \\
\text { CGI/SRS } \\
\text { Adverse Events } \\
\text { Withdrawal } 29 \%\end{array}$ \\
\hline $\begin{array}{l}\text { Amsterdam, 2008 } \\
\text { Open label RCT } \\
\text { Singlesite } \\
\text { US } \\
\text { Government } \\
\text { RoB High } \\
18344727 \\
18486235^{5}\end{array}$ & $\begin{array}{l}\mathrm{N}=83 \\
\text { Mean Age } 37 \\
\text { Female } 57 \% \\
\text { White } 82 \% \\
\text { BP II } 100 \% \\
\text { Outpatient }\end{array}$ & $\begin{array}{l}\text { BPII; current major } \\
\text { depressive episode. } \\
\text { HAM-D } \geq 18 \\
\text { Substance Abuse } \\
\text { Other Mental Health } \\
\text { Pregnant/Nursing } \\
\text { Taking Other Meds } \\
\text { Labs/Other Conditions } \\
\text { (Nonresponsive to } \\
\text { Venlafaxine or Lithium) }\end{array}$ & $\begin{array}{l}\text { Venlafaxine } \\
\text { max } 375 \text { mg/day } \\
\text { min } 75 \text { mg/day }\end{array}$ & $\begin{array}{l}\text { Lithium } \\
\text { serum level } 0.8 \text { to } \\
1.5 \mathrm{mmol} / \mathrm{L}\end{array}$ & 12 weeks & $\begin{array}{l}\text { Response (HAM-D } \\
\text { reduction } \geq 50 \%) \\
\text { Remission (HAM-D } \\
\text { final } \leq 8) \\
\text { HAM-D } \\
\text { YMRS } \\
\text { CGI } \\
\text { Withdrawal } 40 \%\end{array}$ \\
\hline
\end{tabular}

Abbreviations: BP=bipolar disorder; C=Comparison; CGI=Clinical Global Impressions Scale; CGI-BP=Clinical Global Impressions Scale for Bipolar Disorder; DSMIV=Diagnostic and Statistical Manual of Mental Disorders, $4^{\text {th }}$ Revision; GAF=General Assessment of Functioning Scale; HAM-D=Hamilton Scale for Depression; HSRD=Hamilton Rating Scale for Depression; IDS-C=Inventory for Depressive Symptoms(Clinician-Rated); MADRS=Montgomery-Asberg Depression Rating Scale; NOS=not otherwise specified; NR=not reported; PMID=PubMed Identification Number; RCT=randomized controlled trial; ROB=risk of bias; SRS=symptom rating scale; SUM$\mathrm{D}=$ Symptom Subscale for Depression; SUM-ME=Symptom Subscale for Mood Elevation; YMRS = Young Mania Rating Scale 
Appendix Table H6. Summary risk of bias assessments: venlafaxine for depression

\begin{tabular}{|c|c|c|c|}
\hline Drug & $\begin{array}{c}\text { Study } \\
\text { Funding Source } \\
\text { PMID }\end{array}$ & $\begin{array}{c}\text { Overall Risk of Bias } \\
\text { Assessment }\end{array}$ & Rationale \\
\hline $\begin{array}{l}\text { Venlafaxine vs. } \\
\text { Lithium }\end{array}$ & $\begin{array}{l}\text { Amsterdam, } \\
20162 \\
\text { Government } \\
26892848 \\
268037643\end{array}$ & Moderate & $\begin{array}{l}\text { Overall attrition 29\%. Differential attrition between arms. No discussion of missing data } \\
\text { approaches for Fischers test or generalized estimating equations. }\end{array}$ \\
\hline & $\begin{array}{l}\text { Amsterdam, } \\
20084 \text { Government } \\
18344727 \\
184862355 \\
\end{array}$ & High & Open-label study. Attrition 40\%. Differential attrition. \\
\hline
\end{tabular}

Abbreviations: PMID=PubMed Identification Number; YMRS=Young Mania Rating Scale 
Appendix Table H7. Outcomes summary: venlafaxine for depression

\begin{tabular}{|c|c|c|c|c|c|c|}
\hline Drug & $\begin{array}{l}\text { Study } \\
\text { PMID }\end{array}$ & Responder/Remitter & Symptom & Function & Other & $\mathrm{AE}$ \\
\hline $\begin{array}{l}\text { Venlafaxine vs. } \\
\text { Lithium }\end{array}$ & $\begin{array}{l}\text { Amsterdam, } 20162 \\
26892848 \\
268037643\end{array}$ & $\begin{array}{l}\text { Remission } \\
12 \text { weeks } \\
\text { Venlafaxine 38/65 } \\
(58.5 \%) \\
\text { Lithium 18/64 (28.1\%) } \\
\text { Favors Venlafaxine } \\
\mathrm{p}=0.0007 \\
\\
\text { Response } \\
12 \text { weeks } \\
\text { Venlafaxine 44/65 } \\
\text { (67.7\%) } \\
\text { Lithium 22/64 (34.4\%) } \\
\text { Favors Venlafaxine } \\
\mathrm{p}=0.0002\end{array}$ & $\begin{array}{l}\text { HAM-D } \\
12 \text { weeks } \\
\text { Modeling Favors } \\
\text { Venlafaxine } \\
\text { p<0.0001 } \\
\text { (Not interpretable to MID) }\end{array}$ & $\begin{array}{l}\text { CGI/S } \\
12 \text { weeks } \\
\text { Modeling } \\
\text { Favors } \\
\text { Venlafaxine } \\
\text { p }<0.0001\end{array}$ & $\begin{array}{l}\text { Switching } \\
12 \text { weeks } \\
\text { NS }\end{array}$ & $\begin{array}{l}\text { Reported no serious } \\
\text { adverse events }\end{array}$ \\
\hline & $\begin{array}{l}\text { Amsterdam, } 2008^{4} \\
18344727 \\
18486235^{5}\end{array}$ & $\begin{array}{l}\frac{\text { Remission }}{12 \text { weeks }} \\
\text { Venlafaxine 28/43 } \\
(44.2 \%) \\
\text { Lithium } 13 / 40(7.5 \%) \\
\text { Favors Venlafaxine } \\
\text { p=0.0005 } \\
\text { Response } \\
12 \text { weeks } \\
\text { Venlafaxine 26/43 } \\
\text { (60.4\%) } \\
\text { Lithium 8/40 (20\%) } \\
\text { Favors Venlafaxine } \\
\text { p=0.0005 }\end{array}$ & $\begin{array}{l}\frac{\text { HAM-D } 17}{12 \text { weeks }} \\
\text { Modeling Favors } \\
\text { Venlafaxine } \\
-4.51(-8.36 \text { to }-0.66) \\
P=0.015 \\
\text { Less than MID of at least } \\
27.1 \% \text { improvement, or } \\
14 / 52\end{array}$ & $\begin{array}{l}\frac{\text { CGI/S }}{12 \text { weeks }} \\
\text { Modeling } \\
\text { Favors } \\
\text { Venlafaxine } \\
p<0.009\end{array}$ & $\begin{array}{l}\text { Switching } \\
12 \text { weeks } \\
\text { NS }\end{array}$ & $\begin{array}{l}\text { SAE } \\
1 \text { in lithium group (not } \\
\text { described) } \\
\text { NS for withdraw due } \\
\text { to adverse event }\end{array}$ \\
\hline
\end{tabular}

Abbreviations: AE=Adverse Events; CGI=Clinical Global Impressions Scale; HAM-D=Hamilton Scale for Depression; MID=minimally important difference; NA=Not applicable; NR=not reported; NS=not significant; PMID=PubMed Identification Number; SAE=Serious Adverse Events; YMRS =Young Mania Rating Scale 


\section{Appendix Table H8. Strength of evidence assessment: venlafaxine for depression}

\begin{tabular}{|c|c|c|c|c|c|c|c|c|}
\hline Comparison & Outcome & $\begin{array}{l}\text { \# Studies/ } \\
\text { Design } \\
\text { (n analyzed) }\end{array}$ & $\begin{array}{l}\text { Finding or } \\
\text { Summary } \\
\text { Statistic }\end{array}$ & $\begin{array}{c}\text { Study } \\
\text { Limitations }\end{array}$ & Consistency & Directness & Precision & $\begin{array}{c}\text { Overall } \\
\text { Gradel } \\
\text { Conclusion }\end{array}$ \\
\hline $\begin{array}{l}\text { Venlafaxine } \\
\text { vs. Lithium }\end{array}$ & $\begin{array}{l}\text { Response } 12 \text { wks } \\
\text { Remission } 12 \text { wks } \\
\text { Ham-D } 12 \text { wks } \\
\text { CGI/S } 12 \text { wks }\end{array}$ & $\begin{array}{l}2 \text { RCTs } \\
(n=212)\end{array}$ & See table above & Moderate & Consistent & Direct & Imprecise & $\begin{array}{l}\text { Insufficient } \\
\text { (weighted } \\
\text { toward single } \\
\text { moderate rob } \\
\text { study of 129) }\end{array}$ \\
\hline
\end{tabular}

Abbreviations: CGI= Clinical Global Impressions; HAM-D=Hamilton Scale for Depression; n=number of subjects; RCT=randomized controlled trial

Notes:

1. Publication bias for antipsychotics, antidepressants, and behavioral interventions for depressive disorders is suspected.

2. Data were generally imprecise due to missing data from high attrition rates, which was commonly dealt with by Last Observation Carried Forward (LOCF). LOCF requires an

assumption that the health status of patients who dropped out of the trial would not have changed had future observations been recorded, a strong assumption in the context of

bipolar disorder research. 


\section{Section 3. Memantine + Valproate vs. Placebo + Valproate}

Appendix Table H9. Characteristics of eligible studies: memantine for depression by year then first author

\begin{tabular}{|c|c|c|c|c|c|c|}
\hline $\begin{array}{c}\text { Study, Year } \\
\text { Design } \\
\text { Location } \\
\text { Funder } \\
\text { Risk of Bias } \\
\text { PMID }\end{array}$ & $\begin{array}{c}\text { \# Randomized } \\
\text { Age (mean) } \\
\text { Sex (\% Female) } \\
\text { Race (\% White) } \\
\text { Diagnosis } \\
\text { (\% BP I, II, NOS) } \\
\text { Setting }\end{array}$ & $\begin{array}{c}\text { Inclusions } \\
\text { Key Exclusions }\end{array}$ & $\begin{array}{c}\text { Intervention } \\
\text { Dosage }\end{array}$ & $\begin{array}{c}\text { Comparison } \\
\text { Dosage }\end{array}$ & $\begin{array}{l}\text { Followup } \\
\text { Duration }\end{array}$ & \begin{tabular}{|c}
$\begin{array}{c}\text { Outcomes } \\
\text { Reported }\end{array}$ \\
$\begin{array}{c}\text { Withdrawal (\%) at } \\
\text { endpoint }\end{array}$
\end{tabular} \\
\hline $\begin{array}{l}\text { Lee, } 2014^{6,7} \\
\text { RCT } \\
\text { Multisite } \\
\text { Asia } \\
\text { Government } \\
\text { RoB High } \\
\\
24103632 \text { I } \\
23870798\end{array}$ & \begin{tabular}{|l}
$\mathrm{N}=232$ \\
Mean Age 32 \\
Female 49\% \\
Race NR \\
BP II 100\% \\
Inpatient and/or Outpatient \\
(NR)
\end{tabular} & \begin{tabular}{|l} 
Modified BPII \\
(2-days hypomanic \\
versus 4-days used in \\
DSM-IV criteria); \\
HAM-D $\geq 18$ \\
\\
Schizoaffective \\
Substance abuse \\
Other mental health \\
Neurological disorders \\
Taking other medications
\end{tabular} & $\begin{array}{l}\text { Memantine } \\
5 \text { mg/ daily + } \\
\text { Valproate } \\
500-1000 \text { mg/day }\end{array}$ & $\begin{array}{l}\text { Placebo + } \\
\text { Valproate } \\
500-1000 \mathrm{mg} / \text { day }\end{array}$ & 12 weeks & $\begin{array}{l}\text { YMRS } \\
\text { HAM-D } \\
\text { Withdrawal 32\% }\end{array}$ \\
\hline
\end{tabular}

Abbreviations: BP=bipolar disorder; C=Comparison; CGI=Clinical Global Impressions Scale; CGI-BP=Clinical Global Impressions Scale for Bipolar Disorder; DSMIV=Diagnostic and Statistical Manual of Mental Disorders, $4^{\text {th }}$ Revision; GAF=General Assessment of Functioning Scale; HAM-D=Hamilton Scale for Depression; HSRD=Hamilton Rating Scale for Depression; IDS-C=Inventory for Depressive Symptoms(Clinician-Rated); MADRS=Montgomery-Asberg Depression Rating Scale; NOS=not otherwise specified; NR=not reported; PMID=PubMed Identification Number; RCT=randomized controlled trial; ROB=risk of bias; SRS=symptom rating scale; SUM$\mathrm{D}=$ Symptom Subscale for Depression; SUM-ME=Symptom Subscale for Mood Elevation; YMRS = Young Mania Rating Scale

\section{Appendix Table H10. Summary risk of bias assessments: memantine for depression}

\begin{tabular}{|l|l|l|l|}
\hline \multicolumn{1}{|c|}{ Drug } & $\begin{array}{c}\text { Study } \\
\text { Funding Source } \\
\text { PMID }\end{array}$ & $\begin{array}{c}\text { Overall Risk of Bias } \\
\text { Assessment }\end{array}$ & Rationale \\
\hline $\begin{array}{l}\text { Memantine }+ \\
\text { valproate vs. } \\
\text { placebo }+ \\
\text { valproate }\end{array}$ & $\begin{array}{l}\text { Lee, 20146, } 7 \\
\text { Government } \\
24103632\end{array}$ & High & $\begin{array}{l}\text { Randomization and blinding procedures not described; Inpatient and outpatient settings of } \\
\text { patients not described or included in analysis, creating a residual confounder; baseline YMRS } \\
\text { score not balanced between arms and not included in modelling analysis; handling of attrition } \\
\text { not described }\end{array}$ \\
\hline
\end{tabular}

Abbreviations: PMID=PubMed Identification Number; YMRS=Young Mania Rating Scale 
Appendix Table H11. Outcomes summary: memantine for depression

\begin{tabular}{|c|c|c|c|c|c|c|}
\hline Drug & $\begin{array}{l}\text { Study } \\
\text { PMID }\end{array}$ & Responder/Remitter & Symptom & Function & Other & $\mathrm{AE}$ \\
\hline $\begin{array}{l}\text { Memantine + } \\
\text { valproate vs. } \\
\text { placebo + } \\
\text { valproate }\end{array}$ & $\begin{array}{l}\text { Lee, } 2014^{6,7} \\
24103632\end{array}$ & NR & $\begin{array}{l}\frac{\text { HAM-D }}{12 \text { week }} \\
\text { NS } \\
p=0.363 \\
\frac{Y M R S}{12 \text { week }} \\
\text { NS } \\
p=0.115\end{array}$ & NR & NR & NR \\
\hline
\end{tabular}

Abbreviations: AE=Adverse Events; CGI=Clinical Global Impressions Scale; HAM-D=Hamilton Scale for Depression; MID=minimally important difference; NA=Not applicable; NR=not reported; NS=not significant; PMID=PubMed Identification Number; SAE=Serious Adverse Events; YMRS =Young Mania Rating Scale

\begin{tabular}{|c|c|c|c|c|c|c|c|c|}
\hline Comparison & Outcome & $\begin{array}{l}\text { \# Studies/ } \\
\text { Design } \\
\text { (n analyzed) }\end{array}$ & $\begin{array}{l}\text { Finding or } \\
\text { Summary } \\
\text { Statistic }\end{array}$ & $\begin{array}{c}\text { Study } \\
\text { Limitations }\end{array}$ & Consistency & Directness & Precision & $\begin{array}{c}\text { Overall } \\
\text { Gradel } \\
\text { Conclusion }\end{array}$ \\
\hline $\begin{array}{l}\text { Memantine + } \\
\text { valproate vs. } \\
\text { placebo + } \\
\text { valproate }\end{array}$ & HAM-D 12 wks & $\begin{array}{l}1 \mathrm{RCT} \\
(\mathrm{n}=232)\end{array}$ & See table above & High & Unknown & Direct & Imprecise & Insufficient \\
\hline
\end{tabular}

Abbreviations: CGI= Clinical Global Impressions; HAM-D=Hamilton Scale for Depression; n=number of subjects; RCT=randomized controlled trial

Notes:

1. Publication bias for antipsychotics, antidepressants, and behavioral interventions for depressive disorders is suspected.

2. Data were generally imprecise due to missing data from high attrition rates, which was commonly dealt with by Last Observation Carried Forward (LOCF). LOCF requires an assumption that the health status of patients who dropped out of the trial would not have changed had future observations been recorded, a strong assumption in the context of bipolar disorder research. 


\section{Section 4. Lamotrigine + Mood Stabilizers vs. Placebo + Mood Stabilizers}

Appendix Table H13. Characteristics of eligible studies: lamotrigine for depression

\begin{tabular}{|c|c|c|c|c|c|c|}
\hline $\begin{array}{c}\text { Study, Year } \\
\text { Design } \\
\text { Location } \\
\text { Funder } \\
\text { Risk of Bias } \\
\text { PMID }\end{array}$ & $\begin{array}{c}\text { \# Randomized } \\
\text { Age (mean) } \\
\text { Sex (\% Female) } \\
\text { Race (\% White) } \\
\text { Diagnosis } \\
\text { (\% BP I, II, NOS) } \\
\text { Setting }\end{array}$ & $\begin{array}{c}\text { Inclusions } \\
\text { Key Exclusions }\end{array}$ & $\begin{array}{l}\text { Intervention } \\
\text { Dosage }\end{array}$ & $\begin{array}{c}\text { Comparison } \\
\text { Dosage }\end{array}$ & $\begin{array}{l}\text { Followup } \\
\text { Duration }\end{array}$ & \begin{tabular}{|c|} 
Outcomes \\
Reported \\
Withdrawal (\%) at \\
endpoint
\end{tabular} \\
\hline $\begin{array}{l}\text { Kemp, } 2012^{8} \\
\text { RCT } \\
\text { Single } \\
\text { US } \\
\text { Gov't+nonprofit } \\
\text { ROB Moderate } \\
23107222\end{array}$ & $\begin{array}{l}\mathrm{N}=49 \\
\text { Mean Age } 50 \\
\text { Female } 56 \% \\
\text { White } 92 \% \\
\text { BP I } 55 \% \\
\text { BP II } 45 \%\end{array}$ & $\begin{array}{l}\text { BPI or II; rapid cycling in } \\
\text { previous } 12 \text { months; } \\
\text { MADRS } \geq 20 \\
\text { Substance Abuse } \\
\text { Other Mental Health } \\
\text { Pregnant/Nursing } \\
\text { Taking Other Meds } \\
\text { (nonresponsive to } \\
\text { lamotrigine previously) }\end{array}$ & $\begin{array}{l}\text { Lamotrigine } \\
15-200 \mathrm{mg} / \text { day }+ \\
\text { Lithium or divalproex }\end{array}$ & $\begin{array}{l}\text { Placebo + } \\
\text { Lithium or } \\
\text { divalproex }\end{array}$ & 12 weeks & \begin{tabular}{|l|} 
MADRS \\
CGI-S \\
YMRS \\
Response \\
(MADRS $\geq 50 \%$ \\
decrease) \\
Remission \\
(MADRS $\leq 10)$ \\
Bimodal response \\
(MADRS $\leq 19$, \\
YMRS $\leq 12$, GAF \\
$\geq 51$ ) \\
Withdrawal $17 \%$
\end{tabular} \\
\hline
\end{tabular}

Abbreviations: BP=bipolar disorder; C=Comparison; CGI=Clinical Global Impressions Scale; CGI-BP=Clinical Global Impressions Scale for Bipolar Disorder; DSMIV=Diagnostic and Statistical Manual of Mental Disorders, $4^{\text {th }}$ Revision; GAF=General Assessment of Functioning Scale; HAM-D=Hamilton Scale for Depression; HSRD=Hamilton Rating Scale for Depression; IDS-C=Inventory for Depressive Symptoms(Clinician-Rated); MADRS=Montgomery-Asberg Depression Rating Scale; NOS=not otherwise specified; NR=not reported; PMID=PubMed Identification Number; RCT=randomized controlled trial; ROB=risk of bias; SRS=symptom rating scale; SUM-

D=Symptom Subscale for Depression; SUM-ME=Symptom Subscale for Mood Elevation; YMRS = Young Mania Rating Scale

Appendix Table H14. Summary risk of bias assessments: lamotrigine for depression

Appendix Table H14. Summary risk of bias assessments: lamotrigine for depression
\begin{tabular}{|l|l|l|l|}
\hline \multicolumn{1}{|c|}{ Drug } & \multicolumn{1}{|c|}{$\begin{array}{c}\text { Study } \\
\text { Funding Source } \\
\text { PMID }\end{array}$} & $\begin{array}{c}\text { Overall Risk of Bias } \\
\text { Assessment }\end{array}$ & Rationale \\
\hline $\begin{array}{l}\text { Lamotrigine }+ \\
\text { mood stabilizers } \\
\begin{array}{l}\text { vs. placebo + } \\
\text { mood stabilizers }\end{array}\end{array}$ & $\begin{array}{l}\text { Kemp, 2012 } \\
\text { Gov't+nonprofit } \\
23107222\end{array}$ & Moderate & Randomization and blinding procedures not described. 16\% withdrawal. \\
\hline
\end{tabular}

Abbreviations: PMID=PubMed Identification Number; YMRS=Young Mania Rating Scale 
Appendix Table H15. Outcomes summary: lamotrigine for depression

\begin{tabular}{|c|c|c|c|c|c|c|}
\hline Drug & $\begin{array}{l}\text { Study } \\
\text { PMID }\end{array}$ & Responder/Remitter & Symptom & Function & Other & $\mathrm{AE}$ \\
\hline $\begin{array}{l}\text { Lamotrigine + } \\
\text { mood } \\
\text { stabilizers vs. } \\
\text { placebo + } \\
\text { mood } \\
\text { stabilizers }\end{array}$ & $\begin{array}{l}\text { Kemp, } 2012^{8} \\
\text { Gov't+nonprofit } \\
23107222\end{array}$ & $\begin{array}{l}\frac{\text { Remission }}{12 \text { weeks }} \\
\text { Lamotrigine: } 3 / 23 \\
\text { Placebo: } 8 / 26 \\
\text { NS } \\
\text { Response } \\
12 \text { weeks } \\
\text { Lamotrigine: } 2 / 23 \\
\text { Placebo: } 10 / 38 \\
\text { p=0.02 } \\
\text { Bimodal } \\
\text { 12 weeks } \\
\text { Lamotrigine: } 7 / 23 \\
\text { Placebo: } 8 / 26 \\
\text { NS }\end{array}$ & NR & NR & $\begin{array}{l}\text { Withdrawal for } \\
\text { nonresponse } \\
\text { or clinical } \\
\text { worsening: } \\
\text { Treat:4 } \\
\text { Placebo:4 }\end{array}$ & $\begin{array}{l}\text { Severe AE } \\
\text { NS } \\
\text { Lamotrigine: } 1 \\
\text { suicidality, } 1 \\
\text { depression } \\
\text { hospitalization } \\
\text { Placebo: none } \\
\text { reported } \\
\text { Switching: } 2 \text { placebo } \\
\text { patients }\end{array}$ \\
\hline
\end{tabular}

Abbreviations: AE=Adverse Events; CGI=Clinical Global Impressions Scale; HAM-D=Hamilton Scale for Depression; MID=minimally important difference; NA=Not applicable; NR=not reported; NS=not significant; PMID=PubMed Identification Number; SAE=Serious Adverse Events; YMRS =Young Mania Rating Scale

Appendix Table H16. Strength of evidence assessment: lamotrigine for depression

\begin{tabular}{|c|c|c|c|c|c|c|c|c|}
\hline Comparison & Outcome & $\begin{array}{l}\text { \# Studies/ } \\
\text { Design } \\
\text { (n analyzed) }\end{array}$ & $\begin{array}{l}\text { Finding or } \\
\text { Summary } \\
\text { Statistic }\end{array}$ & $\begin{array}{c}\text { Study } \\
\text { Limitations }\end{array}$ & Consistency & Directness & Precision & $\begin{array}{c}\text { Overall } \\
\text { Gradel } \\
\text { Conclusion }\end{array}$ \\
\hline $\begin{array}{l}\text { Lamotrigine + } \\
\text { mood } \\
\text { stabilizers vs. } \\
\text { placebo }+ \\
\text { mood } \\
\text { stabilizers }\end{array}$ & $\begin{array}{l}\text { Remission } 12 \text { wks } \\
\text { Response } 12 \text { wks } \\
\text { Bimodal } \\
\text { remission/response } \\
12 \text { wks }\end{array}$ & $\begin{array}{l}1 \text { RCT } \\
(n=133)\end{array}$ & See table above & Moderate & Unknown & Direct & Imprecise & Insufficient \\
\hline
\end{tabular}

Abbreviations: CGI= Clinical Global Impressions; HAM-D=Hamilton Scale for Depression; n=number of subjects; RCT=randomized controlled trial

Notes:

1. Publication bias for antipsychotics, antidepressants, and behavioral interventions for depressive disorders is suspected.

2. Data were generally imprecise due to missing data from high attrition rates, which was commonly dealt with by Last Observation Carried Forward (LOCF). LOCF requires an

assumption that the health status of patients who dropped out of the trial would not have changed had future observations been recorded, a strong assumption in the context of

bipolar disorder research. 


\section{Section 5. Antidepressants vs. Placebo}

Appendix Table H17. Characteristics of eligible studies: antidepressants for depression

\begin{tabular}{|c|c|c|c|c|c|c|}
\hline $\begin{array}{c}\text { Study, Year } \\
\text { Design } \\
\text { Location } \\
\text { Funder } \\
\text { Risk of Bias } \\
\text { PMID }\end{array}$ & $\begin{array}{c}\text { \# Randomized } \\
\text { Age (mean) } \\
\text { Sex (\% Female) } \\
\text { Race (\% White) } \\
\text { Diagnosis } \\
\text { (\% BP I, II, NOS) } \\
\text { Setting }\end{array}$ & $\begin{array}{c}\text { Inclusions } \\
\text { Key Exclusions }\end{array}$ & $\begin{array}{c}\text { Intervention } \\
\text { Dosage }\end{array}$ & $\begin{array}{c}\text { Comparison } \\
\text { Dosage }\end{array}$ & $\begin{array}{l}\text { Followup } \\
\text { Duration }\end{array}$ & \begin{tabular}{|c|} 
Outcomes \\
Reported \\
$\begin{array}{c}\text { Withdrawal (\%) a } \\
\text { endpoint }\end{array}$
\end{tabular} \\
\hline $\begin{array}{l}\text { Sachs, } 2007^{9} \\
\text { RCT } \\
\text { Multisite } \\
1 \text { Continent } \\
\text { Government } \\
\text { High } \\
17392295\end{array}$ & $\begin{array}{l}\mathrm{N}=366 \\
\text { Mean Age } 40 \\
\text { Female } 57 \% \\
\text { White } 90 \% \\
\text { BP I 68\% } \\
\text { BP-II 32\% } \\
\text { Outpatient }\end{array}$ & $\begin{array}{l}\text { Major Depressive } \\
\text { Episode } \\
\text { Substance Abuse } \\
\text { Other Mental Health } \\
\text { Taking Other Meds } \\
\text { Labs/Other Conditions }\end{array}$ & $\begin{array}{l}\text { Paroxetine } \\
\text { Initiated at } 10 \mathrm{mg} / \text { day } \\
\text { increased up to } \\
40 \mathrm{mg} / \text { day } \\
\\
\text { Bupropion } \\
\text { Initiated at } \\
150 \mathrm{mg} / \text { day increased } \\
\text { to maximum } \\
375 \mathrm{mg} . \text { day }\end{array}$ & Placebo & 26 weeks & $\begin{array}{l}\text { SUM-D } \\
\text { SUM-ME } \\
\text { MADRS } \\
\text { YMRS } \\
\text { GAF } \\
\text { CGI } \\
\text { Adverse Events } \\
\text { Withdrawal 44\% }\end{array}$ \\
\hline
\end{tabular}

Abbreviations: BP=bipolar disorder; C=Comparison; CGI=Clinical Global Impressions Scale; CGI-BP=Clinical Global Impressions Scale for Bipolar Disorder; DSMIV=Diagnostic and Statistical Manual of Mental Disorders, $4^{\text {th }}$ Revision; GAF=General Assessment of Functioning Scale; HAM-D=Hamilton Scale for Depression;

HSRD=Hamilton Rating Scale for Depression; IDS-C=Inventory for Depressive Symptoms(Clinician-Rated); MADRS=Montgomery-Asberg Depression Rating Scale; NOS=not otherwise specified; NR=not reported; PMID=PubMed Identification Number; RCT=randomized controlled trial; ROB=risk of bias; SRS=symptom rating scale; SUM-

D=Symptom Subscale for Depression; SUM-ME=Symptom Subscale for Mood Elevation; YMRS = Young Mania Rating Scale

\section{Appendix Table H18. Summary risk of bias assessments: Antidepressants for depression}

\begin{tabular}{|l|l|l|l|}
\hline \multicolumn{1}{|c|}{ Drug } & $\begin{array}{c}\text { Study } \\
\text { Funding Source } \\
\text { PMID }\end{array}$ & $\begin{array}{c}\text { Overall Risk of Bias } \\
\text { Assessment }\end{array}$ & Rationale \\
\hline $\begin{array}{l}\text { Antidepressives } \\
\text { vs. placebo }\end{array}$ & $\begin{array}{l}\text { Sachs, 20079 } \\
\text { Government } \\
17392295\end{array}$ & High & $\begin{array}{l}\text { Participants were switched to open label after a severe response and they remained in the } \\
\text { study, psychotherapies were included and not measured as a confounding effect, and they } \\
\text { pool results for all mood stabilizers and antidepressants. 44\% attrition }\end{array}$ \\
\hline
\end{tabular}

Abbreviations: PMID=PubMed Identification Number; YMRS=Young Mania Rating Scale 
Appendix Table H19. Outcomes summary: antidepressants for depression

\begin{tabular}{|c|c|c|c|c|c|c|}
\hline Drug & $\begin{array}{l}\text { Study } \\
\text { PMID }\end{array}$ & Responder/Remitter & Symptom & Function & Other & AE \\
\hline $\begin{array}{l}\text { Antidepressives } \\
\text { vs. placebo }\end{array}$ & $\begin{array}{l}\text { Sachs, } 2007^{9} \\
\text { Government } \\
17392295\end{array}$ & $\begin{array}{l}\text { Durable recovery } \\
26 \text { weeks } \\
\text { NS } \\
\text { Transient remission } \\
26 \text { weeks } \\
\text { NS }\end{array}$ & $\underline{\mathrm{NR}}$ & NR & $\begin{array}{l}\text { Withdrawal } \\
\text { due to clinical } \\
\text { worsening: } \\
\text { Treat: } 34.1 \% \\
\text { Placebo:33.7\% } \\
\text { Withdrawal } \\
\text { w/out reaching } \\
\text { clinical } \\
\text { outcome: } \\
\text { Treat:6.7\% } \\
\text { Placebo:7\% }\end{array}$ & $\begin{array}{l}\text { Severe AE } \\
\text { NS } \\
\text { Antidepressants: } 8 \\
\text { (4.5\%) } \\
\text { Placebo: } 10(5.3 \%) \\
\text { Included } \\
\text { (antidepressants, } \\
\text { placebo): medical } \\
\text { hospitalization( } 8,1) \text {, } \\
\text { medical illness }(0,2) \text {, } \\
\text { psychiatric } \\
\text { hospitalization for } \\
\text { depression or suicidal } \\
\text { ideation(6,6), or } \\
\text { nonbipolar } \\
\text { symptoms }(2,1), \text { or } \\
\text { increased suicidal } \\
\text { ideation without } \\
\text { hospitalization( } 0,1) \\
\text { Switching (10.1\%, } \\
\text { 10.7\%) } \\
\text { No reported deaths }\end{array}$ \\
\hline
\end{tabular}

Abbreviations: AE=Adverse Events; CGI=Clinical Global Impressions Scale; HAM-D=Hamilton Scale for Depression; MID=minimally important difference; NA=Not applicable; NR=not reported; NS=not significant; PMID=PubMed Identification Number; SAE=Serious Adverse Events; YMRS =Young Mania Rating Scale 
Appendix Table H20. Strength of evidence assessment: antidepressants for depression

\begin{tabular}{|c|c|c|c|c|c|c|c|c|}
\hline Comparison & Outcome & $\begin{array}{l}\text { \# Studies/ } \\
\text { Design } \\
\text { (n analyzed) }\end{array}$ & $\begin{array}{l}\text { Finding or } \\
\text { Summary } \\
\text { Statistic }\end{array}$ & $\begin{array}{c}\text { Study } \\
\text { Limitations }\end{array}$ & Consistency & Directness & Precision & $\begin{array}{l}\text { Overall } \\
\text { Gradel } \\
\text { Conclusion }\end{array}$ \\
\hline $\begin{array}{l}\text { Antidepressiv } \\
\text { es vs. placebo }\end{array}$ & $\begin{array}{l}\text { Durable recovery } \\
26 \text { wks } \\
\text { Transient remission } \\
26 \text { wks } \\
\text { Discontinuation } 26 \\
\text { wks }\end{array}$ & $\begin{array}{l}1 \mathrm{RCT} \\
(\mathrm{n}=366)\end{array}$ & See table above & High & Unknown & Direct & Imprecise & Insufficient \\
\hline
\end{tabular}

Abbreviations: CGI= Clinical Global Impressions; HAM-D=Hamilton Scale for Depression; n=number of subjects; RCT=randomized controlled trial

Notes:

1. Publication bias for antipsychotics, antidepressants, and behavioral interventions for depressive disorders is suspected.

2. Data were generally imprecise due to missing data from high attrition rates, which was commonly dealt with by Last Observation Carried Forward (LOCF). LOCF requires an assumption that the health status of patients who dropped out of the trial would not have changed had future observations been recorded, a strong assumption in the context of

bipolar disorder research. 


\section{Section 6. Lithium}

Appendix Table H21. Characteristics of eligible studies: lithium depression

\begin{tabular}{|c|c|c|c|c|c|c|}
\hline $\begin{array}{c}\text { Study, Year } \\
\text { Design } \\
\text { Location } \\
\text { Funder } \\
\text { Risk of Bias } \\
\text { PMID }\end{array}$ & $\begin{array}{c}\text { \# Randomized } \\
\text { Age (mean) } \\
\text { Sex (\% Female) } \\
\text { Race (\% White) } \\
\text { Diagnosis } \\
\text { (\% BP I, II, NOS) } \\
\text { Setting }\end{array}$ & $\begin{array}{l}\text { Inclusions } \\
\text { Key Exclusions }\end{array}$ & $\begin{array}{l}\text { Intervention } \\
\text { Dosage }\end{array}$ & $\begin{array}{c}\text { Comparison } \\
\text { Dosage }\end{array}$ & $\begin{array}{l}\text { Followup } \\
\text { Duration }\end{array}$ & $\begin{array}{c}\text { Outcomes } \\
\text { Reported } \\
\begin{array}{c}\text { Withdrawal (\%) at } \\
\text { endpoint }\end{array}\end{array}$ \\
\hline $\begin{array}{l}\text { Nierenberg, } 2013^{10} \\
\text { RCT } \\
\text { Multisite } \\
\text { US } \\
\text { Government } \\
\text { RoB Low } \\
23288387\end{array}$ & $\begin{array}{l}\mathrm{N}=283 \\
\text { Mean Age 39 } \\
\text { Female } 56.5 \% \\
\text { White } 75 \% \\
\text { African American } 17 \% \\
\text { BP I 76\% } \\
\text { BP II 24\% } \\
\text { Outpatient }\end{array}$ & \begin{tabular}{|l|} 
Currently symptomatic \\
(not defined), requiring a \\
change in medication \\
(Mean YMRS 12.5; \\
MADRS 22.5; CGI \\
severity 4.3) \\
Current lithium use \\
Need for hospitalization \\
Other Medical Conditions \\
Pregnancy
\end{tabular} & $\begin{array}{l}\text { Lithium } \\
600 \text { mg/day for first } 2 \\
\text { months; thereafter } \\
\text { adjusted as clinically } \\
\text { needed } \\
\text { + Optimized } \\
\text { Personalized } \\
\text { Treatment }\end{array}$ & $\begin{array}{l}\text { Optimized } \\
\text { Personalized } \\
\text { Treatment }\end{array}$ & 6 months & $\begin{array}{l}\text { CGI-BP-S } \\
\text { Necessary Clinical } \\
\text { Adjustments } \\
\text { (study-defined - all } \\
\text { medication } \\
\text { adjustments } \\
\text { needed to respond } \\
\text { to clinical need) } \\
\text { MADRS } \\
\text { YMRS } \\
\text { CGI-BP-S } \\
\text { LIFE-RIFT } \\
\text { Remission } \\
\\
\text { Withdrawal } 16 \%\end{array}$ \\
\hline
\end{tabular}

Abbreviations: BP=bipolar disorder; C=Comparison; CGI=Clinical Global Impressions Scale; CGI-BP=Clinical Global Impressions Scale for Bipolar Disorder; DSMIV=Diagnostic and Statistical Manual of Mental Disorders, $4^{\text {th }}$ Revision; GAF=General Assessment of Functioning Scale; HAM-D=Hamilton Scale for Depression;

HSRD=Hamilton Rating Scale for Depression; IDS-C=Inventory for Depressive Symptoms(Clinician-Rated); MADRS=Montgomery-Asberg Depression Rating Scale; NOS=not otherwise specified; NR=not reported; PMID=PubMed Identification Number; RCT=randomized controlled trial; ROB=risk of bias; SRS=symptom rating scale; SUM-

$\mathrm{D}=$ Symptom Subscale for Depression; SUM-ME=Symptom Subscale for Mood Elevation; YMRS = Young Mania Rating Scale

\section{Appendix Table H22. Summary risk of bias assessments: lithium for depression}

\begin{tabular}{|l|l|l|l|}
\hline \multicolumn{1}{|c|}{ Drug } & $\begin{array}{c}\text { Study } \\
\text { Funding Source } \\
\text { PMID }\end{array}$ & $\begin{array}{c}\text { Overall Risk of Bias } \\
\text { Assessment }\end{array}$ & \\
\hline Lithium & $\begin{array}{l}\text { Nierenberg, } \\
2013^{10} \\
\text { Government } \\
23288387\end{array}$ & Low & Open-label but rater blinded. \\
& & & \\
\hline
\end{tabular}

Abbreviations: PMID=PubMed Identification Number; YMRS=Young Mania Rating Scale 
Appendix Table H23. Outcomes summary: lithium for depression

\begin{tabular}{|c|c|c|c|c|c|c|}
\hline Drug & $\begin{array}{l}\text { Study } \\
\text { PMID }\end{array}$ & Responder/Remitter & Symptom & Function & Other & $A E$ \\
\hline $\begin{array}{l}\text { Lithium + } \\
\text { Optimalized } \\
\text { Personalized } \\
\text { Treatment } \\
\text { (OPT) vs. OPT }\end{array}$ & $\begin{array}{l}\text { Nierenberg, } 2013^{10} \\
\text { Low } \\
23288387\end{array}$ & $\begin{array}{l}\text { Remission } \\
6 \text { months } \\
\text { NS }\end{array}$ & $\begin{array}{l}\text { Necessary Clinical } \\
\text { Adjustment } \\
6 \text { months } \\
\text { NS } \\
\text { Lithium+OPT } 1.01 \\
\text { OPT } 0.99 \\
\text { YMRS } \\
6 \text { months } \\
\text { NS } \\
\text { Lithium+OPT -6.35 } \\
\text { OPT -5.79 } \\
\text { MADRS } \\
6 \text { months } \\
\text { NS } \\
\text { Lithium+OPT }-8.20 \\
\text { OPT -8.84 }\end{array}$ & $\begin{array}{l}\text { CGI-BP-S } \\
6 \text { months } \\
\text { NS } \\
\text { Lithium+OPT - } \\
1.2 \\
\text { OPT -1.5 }\end{array}$ & $\begin{array}{l}\text { Overall } \\
\text { Withdrawal } \\
\text { Lithium+OPT } \\
17.7 \% \\
\text { OPT } 14.8 \%\end{array}$ & $\begin{array}{l}\text { SAE } \\
\text { Reported no } \\
\text { difference between } \\
\text { groups } \\
\text { No deaths } \\
\text { Suicidal Ideation } \\
\text { No difference } \\
\text { between groups }\end{array}$ \\
\hline
\end{tabular}

Abbreviations: AE=Adverse Events; CGI=Clinical Global Impressions Scale; HAM-D=Hamilton Scale for Depression; MID=minimally important difference; NA=Not applicable; NR=not reported; NS=not significant; PMID=PubMed Identification Number; SAE=Serious Adverse Events; YMRS =Young Mania Rating Scale

\section{Appendix Table H24. Strength of evidence assessment: lithium for depression}

\begin{tabular}{|c|c|c|c|c|c|c|c|c|}
\hline Comparison & Outcome & $\begin{array}{l}\text { \# Studies/ } \\
\text { Design } \\
\text { (n analyzed) }\end{array}$ & $\begin{array}{l}\text { Finding or } \\
\text { Summary } \\
\text { Statistic }\end{array}$ & $\begin{array}{c}\text { Study } \\
\text { Limitations }\end{array}$ & Consistency & Directness & Precision & $\begin{array}{c}\text { Overall } \\
\text { Gradel } \\
\text { Conclusion }\end{array}$ \\
\hline $\begin{array}{l}\text { Lithium + OPT } \\
\text { vs. OPT alone }\end{array}$ & $\begin{array}{l}\text { CGI } \\
\text { Clinical Need } \\
\text { MADRS } \\
\text { YMRS } \\
\text { Remission } \\
\end{array}$ & $\begin{array}{l}1 \mathrm{RCT} \\
(\mathrm{n}=283)\end{array}$ & See table above & Low & Unknown & Direct & Imprecise & Insufficient \\
\hline
\end{tabular}

Abbreviations: CGI= Clinical Global Impressions; HAM-D=Hamilton Scale for Depression; n=number of subjects; RCT=randomized controlled trial

Notes:

1. Publication bias for antipsychotics, antidepressants, and behavioral interventions for depressive disorders is suspected.

2. Data were generally imprecise due to missing data from high attrition rates, which was commonly dealt with by Last Observation Carried Forward (LOCF). LOCF requires an

assumption that the health status of patients who dropped out of the trial would not have changed had future observations been recorded, a strong assumption in the context of

bipolar disorder research. 


\section{References for Appendix $\mathbf{H}$}

1. Altshuler LL, Sugar CA, McElroy SL, et al. Switch rates during acute treatment for Bipolar II depression with Lithium, Sertraline, or the two combined: A randomized double-blind comparison. American Journal of Psychiatry. 2017 March;174(3):266-76. doi: http://dx.doi.org/10.1176/appi.ajp.2016.15040558 . PMID: 28135846 PMID/614689619 Embase.

2. Amsterdam JD, Lorenzo-Luaces L, Soeller I, et al. Short-term venlafaxine v. lithium monotherapy for bipolar type II major depressive episodes: Effectiveness and mood conversion rate. British Journal of Psychiatry. 2016 April;208(4):359-65. doi: http://dx.doi.org/10.1192/bjp.bp.115.169375. PMID: 26892848 PMID/609914520 Embase.

3. Lorenzo-Luaces L, Amsterdam JD, Soeller I, et al. Rapid versus non-rapid cycling bipolar II depression: Response to venlafaxine and lithium and hypomanic risk. Acta Psychiatrica Scandinavica. 201601 Jun;133(6):459-69. doi: http://dx.doi.org/10.1111/acps.12557. PMID: 26803764 PMID/608565179 Embase.

4. Amsterdam JD, Shults J. Comparison of shortterm venlafaxine versus lithium monotherapy for bipolar II major depressive episode: a randomized open-label study. J Clin Psychopharmacol. 2008 Apr;28(2):171-81. doi: 10.1097/JCP.0b013e318166c4e6. PMID: 18344727.

5. Amsterdam JD, Wang CH, Shwarz M, et al. Venlafaxine versus lithium monotherapy of rapid and non-rapid cycling patients with bipolar II major depressive episode: a randomized, parallel group, open-label trial. Journal of Affective Disorders. 2009 Jan;112(1-3):219-30. doi: http://dx.doi.org/10.1016/j.jad.2008.03.029. PMID: 18486235.

6. Lee SY, Chen SL, Chang YH, et al. Genotype variant associated with add-on memantine in bipolar II disorder.[Erratum appears in Int J Neuropsychopharmacol. 2014 Jun;17(6):979 Note: Lu, Ru-Band [removed]; Hong, Jau-Shyong [added]]. International Journal of Neuropsychopharmacology. 2014 Feb;17(2):18997. doi: http://dx.doi.org/10.1017/S1461145713000825. PMID: 24103632.
7. Lee S-Y, Chen S-L, Chang Y-H, et al. Add-on memantine to valproate treatment increased HDLC in bipolar II disorder. Journal of Psychiatric Research. 2013 2013;47(10):1343-8. doi: http://dx.doi.org/10.1016/j.jpsychires.2013.06.017 . PMID: 23870798.

8. Kemp DE, Gao K, Fein EB, et al. Lamotrigine as add-on treatment to lithium and divalproex: lessons learned from a double-blind, placebocontrolled trial in rapid-cycling bipolar disorder. Bipolar Disorders. 2012 Nov;14(7):780-9. doi: http://dx.doi.org/10.1111/bdi.12013. PMID: 23107222.

9. Sachs GS, Nierenberg AA, Calabrese JR, et al. Effectiveness of adjuctive antidepressant treatment for bipolar depression. The New England Journal of Medicine. 2007 2007;356(17):1711-22. doi: http://dx.doi.org/10.1056/NEJMoa064135. PMID: 17392295.

10. Nierenberg AA, Friedman ES, Bowden CL, et al. Lithium treatment moderate-dose use study (LiTMUS) for bipolar disorder: a randomized comparative effectiveness trial of optimized personalized treatment with and without lithium. American Journal of Psychiatry. 2013 Jan 1;170(1):102-10. doi: http://dx.doi.org/10.1176/appi.ajp.2012.12060751 . PMID: 23288387. 


\section{Appendix I. Drug Treatments for Maintenance}

\section{Section 1. Lithium Monotherapy vs. Inactive Controls}

\begin{tabular}{|c|c|c|c|c|c|c|}
\hline $\begin{array}{c}\text { Study, Year } \\
\text { Design } \\
\text { Location } \\
\text { Funder } \\
\text { Risk of Bias } \\
\text { PMID }\end{array}$ & $\begin{array}{c}\text { \# Randomized } \\
\text { Age (mean) } \\
\text { Sex (\% Female) } \\
\text { Race (\% White) } \\
\text { Diagnosis } \\
\text { (\% BP I, II, NOS) } \\
\text { Setting }\end{array}$ & $\begin{array}{c}\text { Inclusions } \\
\text { Key Exclusions }\end{array}$ & $\begin{array}{l}\text { Intervention } \\
\text { Dosage }\end{array}$ & $\begin{array}{c}\text { Comparison } \\
\text { Dosage }\end{array}$ & $\begin{array}{l}\text { Followup } \\
\text { Duration }\end{array}$ & $\begin{array}{c}\text { Outcomes } \\
\text { Reported } \\
\text { Withdrawal (\%) at } \\
\text { endpoint }\end{array}$ \\
\hline $\begin{array}{l}\text { Bowden, } 2000^{1} \\
\text { RCT } \\
\text { Multisite } \\
\text { US } \\
\text { Industry } \\
\text { RoB High } \\
\\
10807488 \\
12784116^{2}\end{array}$ & $\begin{array}{l}\mathrm{N}=372 \\
\text { Mean Age } 39 \\
\text { Female } 51 \% \\
\text { White } 94 \% \\
\text { BP I } 100 \% \\
\text { Outpatient }\end{array}$ & $\begin{array}{l}\text { No Episode at } \\
\text { Randomization; Scores of } \\
\text { MRS } \leq 11, \text { DSS } \leq 13, \text { GAS } \\
>60 ; \text { At least one other } \\
\text { manic episode in past three } \\
\text { years. } \\
\\
\text { Substance Abuse; Other } \\
\text { Mental Health Conditions; } \\
\text { Taking Other Medications; } \\
\text { Pregnant/Nursing }\end{array}$ & \begin{tabular}{|l|} 
Divalproex \\
$71-125 \mathrm{mcg} / \mathrm{mL}$ \\
$($ Mean $84.8 \mathrm{mcg} / \mathrm{mL})$ \\
$\mathrm{N}=187$
\end{tabular} & $\begin{array}{l}\text { C1: Placebo } \\
n=92 \\
\text { C2: Lithium } \\
0.8-1.2 \mathrm{mEq} / \mathrm{L} \\
\text { (Mean } 0.9 \\
\text { mEq/L) } \\
n=90\end{array}$ & 52 week & $\begin{array}{l}\text { Time to recurrence, } \\
\text { any } \\
\text { Time to recurrence, } \\
\text { mania (MRS } \geq 16 \text { ) } \\
\text { Time to recurrence, } \\
\text { depression } \\
\text { (DSS } \geq 25) \\
\\
\text { Withdrawal } 69 \% \\
\text { (Time to recurrence } \\
\text { outcomes only } \\
\text { included) } \\
\text { Nonrelapse Withdrawal } \\
40 \%\end{array}$ \\
\hline
\end{tabular}




\begin{tabular}{|c|c|c|c|c|c|c|}
\hline $\begin{array}{l}\text { Study, Year } \\
\text { Design } \\
\text { Location } \\
\text { Funder } \\
\text { Risk of Bias } \\
\text { PMID }\end{array}$ & $\begin{array}{c}\text { \# Randomized } \\
\text { Age (mean) } \\
\text { Sex (\% Female) } \\
\text { Race (\% White) } \\
\text { Diagnosis } \\
\text { (\% BP I, II, NOS) } \\
\text { Setting }\end{array}$ & $\begin{array}{c}\text { Inclusions } \\
\text { Key Exclusions }\end{array}$ & $\begin{array}{c}\text { Intervention } \\
\text { Dosage }\end{array}$ & $\begin{array}{c}\text { Comparison } \\
\text { Dosage }\end{array}$ & $\begin{array}{l}\text { Followup } \\
\text { Duration }\end{array}$ & $\begin{array}{c}\text { Outcomes } \\
\text { Reported } \\
\text { Withdrawal (\%) at } \\
\text { endpoint }\end{array}$ \\
\hline $\begin{array}{l}\text { Amsterdam, } 2010^{3} \\
\text { RCT } \\
\text { Single-site } \\
\text { US } \\
\text { Gov't+nonprofit } \\
\text { RoB Moderate } \\
20360317\end{array}$ & $\begin{array}{l}\mathrm{N}=81 \\
\text { Mean Age } 38 \\
\text { Female 52\% } \\
\text { White NR } \\
\text { BP II 100\% } \\
\text { Outpatient }\end{array}$ & $\begin{array}{l}\text { Depression and } \\
\text { HAM-D } \geq 16 \text { initialy; then } \\
\text { HAM-D } \leq 8 \text { after } 12 \text { weeks of } \\
\text { initial Fluoxetine therapy at } \\
20-80 \mathrm{mg} / \text { day) } \\
\\
\text { Substance abuse } \\
\text { Neurological Disorders } \\
\text { Taking other medications } \\
\text { Pregnant/NursingLabs/Other } \\
\text { Conditions }\end{array}$ & $\begin{array}{l}\text { Fluoxetine }(\mathrm{n}=28) \\
10-40 \mathrm{mg} / \mathrm{day} \\
\text { (mean } 34.3 \mathrm{mg} / \text { day) } \\
\mathrm{N}=28\end{array}$ & $\begin{array}{l}\text { C1: Placebo } \\
(\mathrm{n}=27) \\
\\
\text { C2: Lithium } \\
(\mathrm{n}=26) \\
300-1200 \\
\mathrm{mg} / \mathrm{day} ; 0.5-1.5 \\
\mathrm{mmol} / \mathrm{L} \\
\text { (mean } 1027 \\
\text { mg/day; } 0.69 \\
\text { mmol/L) }\end{array}$ & 50 weeks & $\begin{array}{l}\text { YMRS } \\
\text { Relapse (HAM-D } \geq 14 \text { ) } \\
\text { Adverse Events } \\
\text { Lab Values } \\
\\
\text { Withdrawal } 72 \% \\
\text { (Time to recurrence } \\
\text { outcomes only } \\
\text { included) } \\
\text { Nonrelapse Withdrawal } \\
25 \%\end{array}$ \\
\hline $\begin{array}{l}\text { Bowden, } 2003^{4} \\
\text { RCT of responders } \\
\text { Multisite } \\
2 \text { continents } \\
\text { Industry } \\
\text { RoB Low } \\
12695317\end{array}$ & $\begin{array}{l}\mathrm{N}=175 \\
\text { Mean Age } 41 \\
\text { Female 47\% } \\
\text { Race NR } \\
\text { BP I 100\% } \\
\text { Outpatient }\end{array}$ & $\begin{array}{l}\text { Lamotrigine responders } \\
\text { (CGI-S } \leq 3 \text { for at least } 4 \\
\text { continuous weeks) } \\
\text { For open label period: } \\
\text { Manic; DSM-IV Criteria for } \\
\text { Mania or Hypomania } \\
\text { currently or within past } 60 \\
\text { days with previous episodes } \\
\text { in past } 3 \text { years } \\
\text { Other Mental Health } \\
\text { Conditions }\end{array}$ & $\begin{array}{l}\text { Lamotrigine } \\
100-400 \mathrm{mg} / \text { day } \\
(\text { Mean NR) } \\
\mathrm{N}=59\end{array}$ & $\begin{array}{l}\text { C1: Placebo } \\
\mathrm{N}=70 \\
\text { C2: Lithium } \\
\text { 0.8-1.1 mEq/L } \\
\text { Mean NR } \\
\mathrm{N}=59\end{array}$ & 18 Months & $\begin{array}{l}\text { Time to intervention for } \\
\text { mania, hypomania, } \\
\text { mixed, depression, and } \\
\text { any mood episodes } \\
\text { Time to early } \\
\text { discontinuation } \\
\text { Adverse Events I } \\
\\
\text { Withdrawal } 80 \% \\
\text { (Time to recurrence } \\
\text { outcomes only } \\
\text { included) } \\
\text { Nonrelapse Withdrawal } \\
20 \%\end{array}$ \\
\hline
\end{tabular}




\begin{tabular}{|c|c|c|c|c|c|c|}
\hline $\begin{array}{l}\text { Study, Year } \\
\text { Design } \\
\text { Location } \\
\text { Funder } \\
\text { Risk of Bias } \\
\text { PMID }\end{array}$ & $\begin{array}{c}\text { \# Randomized } \\
\text { Age (mean) } \\
\text { Sex (\% Female) } \\
\text { Race (\% White) } \\
\text { Diagnosis } \\
\text { (\% BP I, II, NOS) } \\
\text { Setting }\end{array}$ & $\begin{array}{c}\text { Inclusions } \\
\text { Key Exclusions }\end{array}$ & $\begin{array}{c}\text { Intervention } \\
\text { Dosage }\end{array}$ & $\begin{array}{c}\text { Comparison } \\
\text { Dosage }\end{array}$ & $\begin{array}{l}\text { Followup } \\
\text { Duration }\end{array}$ & $\begin{array}{c}\text { Outcomes } \\
\text { Reported } \\
\begin{array}{c}\text { Withdrawal (\%) at } \\
\text { endpoint }\end{array}\end{array}$ \\
\hline $\begin{array}{l}\text { Calabrese, } 2003^{5} \\
\text { RCT of responders } \\
\text { Multisite } \\
4 \text { Continents } \\
\text { Industry } \\
\text { RoB Moderate } \\
14628976\end{array}$ & $\begin{array}{l}\mathrm{N}=410 \\
\text { Mean Age } 43 \\
\text { Female 56\% } \\
\text { Race NR } \\
\text { BP I 100\% } \\
\text { Outpatient }\end{array}$ & $\begin{array}{l}\text { Lamotrigine responders } \\
\text { (CGI-S<3 for at least } 4 \\
\text { continuous weeks) } \\
\text { For open label period: } \\
\text { depression; DSM-IV criteria } \\
\text { for depression currently or } \\
\text { within past } 60 \text { days with } \\
\text { previous depression and } \\
\text { mania episodes in past } 3 \\
\text { years. } \\
\text { Other Mental Health } \\
\text { Conditions }\end{array}$ & $\begin{array}{l}\text { Lamotrigine } \\
50,200 \text {, or } 400 \\
\text { mg/day (in analysis } \\
50 \mathrm{mg} \text { group was } \\
\text { censored, } 200 \text { and } \\
400 \text { mg/day groups } \\
\text { were combined) } \\
\mathrm{N}=221\end{array}$ & $\begin{array}{l}\text { C1: Placebo } \\
\mathrm{N}=121 \\
\\
\text { C2: Lithium } \\
0.8-1.1 \mathrm{mEq} / \mathrm{L} \\
\text { (Mean } 0.8 \\
\mathrm{mEq} / \mathrm{L}) \\
\mathrm{N}=121\end{array}$ & 18 Months & $\begin{array}{l}\text { Time to intervention for } \\
\text { mania, hypomania, } \\
\text { depression, any mood } \\
\text { episode } \\
\text { Time to early } \\
\text { discontinuation } \\
\text { Adverse Events } \\
\\
\text { Withdrawal } 84.9 \% \\
\text { (Time to recurrence } \\
\text { outcomes only } \\
\text { included) } \\
\text { Nonrelapse Withdrawal } \\
34 \%\end{array}$ \\
\hline
\end{tabular}




\begin{tabular}{|c|c|c|c|c|c|c|}
\hline $\begin{array}{l}\text { Study, Year } \\
\text { Design } \\
\text { Location } \\
\text { Funder } \\
\text { Risk of Bias } \\
\text { PMID }\end{array}$ & $\begin{array}{c}\text { \# Randomized } \\
\text { Age (mean) } \\
\text { Sex (\% Female) } \\
\text { Race (\% White) } \\
\text { Diagnosis } \\
\text { (\% BP I, II, NOS) } \\
\text { Setting }\end{array}$ & $\begin{array}{c}\text { Inclusions } \\
\text { Key Exclusions }\end{array}$ & $\begin{array}{l}\text { Intervention } \\
\text { Dosage }\end{array}$ & $\begin{array}{c}\text { Comparison } \\
\text { Dosage }\end{array}$ & $\begin{array}{l}\text { Followup } \\
\text { Duration }\end{array}$ & $\begin{array}{c}\text { Outcomes } \\
\text { Reported } \\
\text { Withdrawal (\%) at } \\
\text { endpoint }\end{array}$ \\
\hline $\begin{array}{l}\text { Prien, } 1973^{6} \\
\text { RCT } \\
\text { Multisite } \\
\text { US } \\
\text { Government } \\
\text { RoB High } \\
4569674\end{array}$ & $\begin{array}{l}\mathrm{N}=205 \\
\text { Median Age } 44 \\
\text { Sex NR } \\
\text { Race NR } \\
\text { BP I 100\% } \\
\text { Outpatient }\end{array}$ & $\begin{array}{l}\text { No Episode at } \\
\text { Randomization; } \\
\text { Neurological Disorders; } \\
\text { Abnormal Lab Results }\end{array}$ & $\begin{array}{l}\text { Lithium } \\
0.5-1.4 \mathrm{mEq} / \mathrm{L} \\
\text { (Mean 0.7 mEq/L) }\end{array}$ & Placebo & 2 years & $\begin{array}{l}\text { Relapse, } \\
\text { type/proportion (manic, } \\
\text { mixed, schizoaffective, } \\
\text { depressive event } \\
\text { requiring } \\
\text { hospitalization 'severe' } \\
\text { or supplementary } \\
\text { drugs 'moderate') } \\
\text { Time to early } \\
\text { discontinuation } \\
\text { GAS } \\
\text { IMPS (Inpatient } \\
\text { Multidimensional } \\
\text { Psychiatric Scale) } \\
\text { Self-Report Mood } \\
\text { Scale } \\
\text { KAS (Katz Adjustment } \\
\text { Scale) } \\
\text { Adverse Events } \\
\\
\text { Withdrawal 42\% }\end{array}$ \\
\hline
\end{tabular}




\begin{tabular}{|c|c|c|c|c|c|c|}
\hline $\begin{array}{l}\text { Study, Year } \\
\text { Design } \\
\text { Location } \\
\text { Funder } \\
\text { Risk of Bias } \\
\text { PMID }\end{array}$ & $\begin{array}{c}\text { \# Randomized } \\
\text { Age (mean) } \\
\text { Sex (\% Female) } \\
\text { Race (\% White) } \\
\text { Diagnosis } \\
\text { (\% BP I, II, NOS) } \\
\text { Setting } \\
\end{array}$ & $\begin{array}{l}\text { Inclusions } \\
\text { Key Exclusions }\end{array}$ & $\begin{array}{c}\text { Intervention } \\
\text { Dosage }\end{array}$ & $\begin{array}{c}\text { Comparison } \\
\text { Dosage }\end{array}$ & $\begin{array}{l}\text { Followup } \\
\text { Duration }\end{array}$ & $\begin{array}{c}\text { Outcomes } \\
\text { Reported } \\
\begin{array}{c}\text { Withdrawal (\%) at } \\
\text { endpoint }\end{array}\end{array}$ \\
\hline $\begin{array}{l}\text { Weisler, } 2011^{7} \\
\text { RCT } \\
\text { Multisite } \\
5 \text { continents } \\
\text { Industry } \\
\text { RoB Moderate } \\
22054050\end{array}$ & $\begin{array}{l}\mathrm{N}=1226 \\
\text { Mean Age } 40 \\
\text { Female } 53 \% \\
\text { White } 63 \% \\
\text { BP } 1100 \% \\
\text { Outpatient }\end{array}$ & $\begin{array}{l}\text { Meeting stability criteria of } \\
\text { YMRS } \leq 12 \text { and MADRS } \leq \\
12 \text { after last episode of } \\
\text { depression/mania/mixed } \\
\text { episode at study entry or } \\
\text { within past two years } \\
\text { Substance Abuse } \\
\text { Other Mental Health } \\
\text { Conditions } \\
\text { Pregnant/Nursing } \\
\text { Labs/Other Conditions }\end{array}$ & $\begin{array}{l}\text { Quetiapine } \\
300-800 \text { mg/day } \\
\text { (543 mg/day mean) } \\
\mathrm{N}=404\end{array}$ & $\begin{array}{l}\text { C1: Placebo } \\
\mathrm{N}=404 \\
\\
\mathrm{C} 2: \text { Lithium } \\
600-1800 \\
\mathrm{mg} / \text { day } \\
0.6-1.2 \mathrm{mEq} / \mathrm{L} \\
(0.63 \mathrm{mEq} / \mathrm{L} \\
\text { mean) } \\
\mathrm{N}=364\end{array}$ & 104 weeks & $\begin{array}{l}\text { Time to recurrence any } \\
\text { mood (algorithm) } \\
\text { Time to manic event } \\
\text { Time to depressive } \\
\text { event } \\
\text { Time to all-cause } \\
\text { discontinuation } \\
\text { SDS } \\
\text { MOS-Cog } \\
\text { CGI-BP } \\
\text { PANNS-P } \\
\text { WPAI } \\
\text { TMT } \\
\\
\text { Withdrawal 55\% (Time } \\
\text { to recurrence } \\
\text { outcomes only } \\
\text { included) } \\
\text { Nonrelapse withdrawal } \\
22 \%\end{array}$ \\
\hline
\end{tabular}

Abbreviations: AIMS=Abnormal Involuntary Movement Scale; BARS=Barnes Akathisia Scale; BIS-11=Barratt Impulsiveness Scale; BMI=Body Mass Index; BP=bipolar disorder; C=Comparison; CGI-BP=Clinical Global Impressions Scale for Bipolar Disorder; CGI-BP-M=Clinical Global Impressions Scale-Bipolar-modified (for long-term followup); CGI-BP-S=Clinical Global Impressions, Bipolar, Severity Scale; CGI-EI=Clinical Global Impressions-Efficacy Index; CGI-I=Clinical Global Impressions Scale, Improvement; CGI-S=Clinical Global Impressions, Severity Scale; DSM=Diagnostic and Statistical Manual of Mental Disorders; DSS=Depressive Syndrome Scale;

EPS=extrapyramidal symptoms; ESRS=Extrapyramidal Symptom Rating Scale; GAF=General Assessment of Functioning Scale; GAS=Global Assessment Scale; HAM-

D=Hamilton Scale for Depression; IMPS=Inpatient Multidimensional Psychiatric Scale; KAS=Katz Adjustment Scale; LIFE-RIFT= Longitudinal Interval Follow-up EvaluationRange of Impaired Functioning Tool; MADRS=Montgomery-Asberg Depression Rating Scale; MOS-Cog=Medical Outcomes Study Cognitive Scale; MRS=Mania Rating Scale; NOS=not otherwise specified; NR=not reported; PANSS=Positive and Negative Syndrome Scale; PGWB=Psychological General Well-being Scale; PMID=PubMed Identification Number; PSQI=Pittsburgh Sleep Quality Index; QIDS-SR=Quick Inventory of Depressive Symptomatology (Self-reported); RCT=randomized controlled trial; ROB=risk of bias; SAS=Simpson Angus Scale; SDS=Sheehan Disability Scale; TMT=Trail Making Test; WPAI=Work Productivity and Activity Impairment Questionnaire; YMRS =Young Mania 
Appendix Table 12. Summary risk of bias assessments: Lithium monotherapy for maintenance

\begin{tabular}{|c|c|c|c|}
\hline Drug & $\begin{array}{l}\text { Study } \\
\text { Funding Source } \\
\text { PMID }\end{array}$ & $\begin{array}{l}\text { Overall Risk of Bias } \\
\text { Assessment }\end{array}$ & Rationale \\
\hline \multirow[t]{2}{*}{$\begin{array}{l}\text { Lamotrigine vs. } \\
\text { Lithium vs. } \\
\text { placebo }\end{array}$} & $\begin{array}{l}\text { Bowden, } 2003^{4} \\
\text { Industry } \\
12695317\end{array}$ & $\begin{array}{l}\text { Moderate } \\
\text { (High for log rank } \\
\text { test) }\end{array}$ & $\begin{array}{l}\text { Randomization and blinding procedures not described. Balanced traits among groups at } \\
\text { baseline. Efficacy data may be biased by dropout as endpoints are LOCF and differential } \\
\text { nonrelapse dropout rates that range from } 16-25 \% \text { of patients for each arm. }\end{array}$ \\
\hline & $\begin{array}{l}\text { Calabrese, } 2003^{5} \\
\text { Industry } \\
14628976\end{array}$ & $\begin{array}{l}\text { Moderate } \\
\text { (High for log rank } \\
\text { test) }\end{array}$ & $\begin{array}{l}\text { Randomization and allocation concealment not described. Balanced traits among groups at } \\
\text { baseline. Efficacy data may be biased by dropout as endpoints are LOCF. 34\% withdrawal. }\end{array}$ \\
\hline $\begin{array}{l}\text { Fluoxetine vs. } \\
\text { lithium vs. } \\
\text { placebo }\end{array}$ & $\begin{array}{l}\text { Amsterdam, } \\
2010^{3} \\
\text { Government/Non } \\
\text { profit } \\
20360317\end{array}$ & Moderate & $\begin{array}{l}\text { Randomization may not have been successful as it relates to a person's likelihood of relapse; } \\
\text { randomization, allocation, and blinding procedures are underdescribed. } 23 \% \text { dropout. }\end{array}$ \\
\hline $\begin{array}{l}\text { Divalproex vs. } \\
\text { lithium vs. } \\
\text { placebo }\end{array}$ & $\begin{array}{l}\text { Bowden, } 2000^{1} \\
\text { Industry } \\
10807488 \\
12784116^{2}\end{array}$ & High & Appears to be unblinded. Randomization not described. Nonrelapse dropout of $40 \%$. \\
\hline $\begin{array}{l}\text { Lithium vs. } \\
\text { placebo }\end{array}$ & $\begin{array}{l}\text { Prien, } 1973^{6} \\
\text { Government } \\
4569674\end{array}$ & High & Differential dropout rate is significant. Study didn't demonstrate allocation concealed. \\
\hline $\begin{array}{l}\text { Quetiapine vs. } \\
\text { lithium vs. } \\
\text { placebo + lithium }\end{array}$ & $\begin{array}{l}\text { Weisler, } 2011^{7} \\
\text { Industry } \\
22054050\end{array}$ & Moderate & $\begin{array}{l}\text { Generally a well conducted and reported study, however some sources of bias present } \\
\text { related to dropout rates. Overall } 21 \% \text { withdraw due to reasons unrelated to recurrence; } \\
\text { however by week } 16 \text { more than } 50 \% \text { of the placebo group has dropped for all causes, as well } \\
\text { as } 40 \% \text { of the lithium group and } 25 \% \text { of the quetiapine group) }\end{array}$ \\
\hline
\end{tabular}

Abbreviations: BP=bipolar; LOCF=Last observation carried forward; PMID=PubMed Identification Number; RCT=randomized controlled trial 
Appendix Table 13. Outcomes summary table: lithium monotherapy versus placebo for maintenance

\begin{tabular}{|c|c|c|c|c|c|c|}
\hline Drug & $\begin{array}{l}\text { Study } \\
\text { Comparison } \\
\text { PMID }\end{array}$ & Responder/Remitter & Symptom & Function & Other & $\mathrm{AE}$ \\
\hline \multirow[t]{3}{*}{$\begin{array}{l}\text { Lithium vs. } \\
\text { placebo }\end{array}$} & $\begin{array}{l}\text { Bowden, } 2000^{1} \\
10807488 \\
12784116^{2}\end{array}$ & $\begin{array}{l}\text { Time to any recurrence } \\
12 \text { months } \\
\text { NS } \\
\text { Time to mania recurrence } \\
12 \text { months } \\
\text { NS } \\
\text { Time to depression } \\
\text { recurrence } \\
12 \text { months } \\
\text { NS }\end{array}$ & $\begin{array}{l}\text { NA } \\
\text { (Exclude for attrition }=69 \%)\end{array}$ & $\underline{\mathrm{NR}}$ & $\underline{\mathrm{NR}}$ & $\begin{array}{l}\frac{\mathrm{SAE}}{\mathrm{NR}} \\
\frac{\text { Tremor }}{\text { Lithium } 42 \%(38 / 94)} \\
\text { Placebo 13\% (12/94) } \\
\text { Favors Placebo } \\
\text { p<0.001 } \\
\text { Akathisia } \\
\text { Lithium 4\% } \\
\text { (4/94)Placebo 1\% } \\
(1 / 94) \\
\text { NS }\end{array}$ \\
\hline & $\begin{array}{l}\text { Amsterdam, } \\
2010^{3} \\
20360317\end{array}$ & $\begin{array}{l}\text { Time to Relapse to } \\
\text { Depression } \\
50 \text { week } \\
\text { Log rank } \\
\text { NS }\end{array}$ & $\frac{\mathrm{NA}}{(\text { Exclude for attrition }=72 \%)}$ & NR & NR & $\begin{array}{l}\frac{\text { SAE }}{\text { Reported no events }} \\
\frac{\text { EPS }}{\text { Reported no events }}\end{array}$ \\
\hline & $\begin{array}{l}\text { Bowden, } 2003^{4} \\
1269531\end{array}$ & $\begin{array}{l}\text { Time to any recurrence } \\
18 \text { months } \\
\text { Log rank } \\
\text { Favors Lithium } \\
\mathrm{p}=0.001 \\
\frac{\text { Time to mania recurrence }}{18 \text { months }} \\
\text { Log rank } \\
\text { Favors Lithium } \\
\mathrm{p}=0.006 \\
\frac{\text { Time to depression }}{\text { recurrence }} \\
18 \text { months } \\
\text { Log rank } \\
\text { NS }\end{array}$ & $\begin{array}{l}\text { NA } \\
\text { (Exclude for attrition }=80 \%)\end{array}$ & NA & NA & $\begin{array}{l}\frac{\text { SAE }}{\text { No events reported }} \\
\text { Suicidality per HAM-D } \\
\text { NS between groups } \\
\frac{\text { Tremor }}{\text { Lithium } 42 \%(38 / 94)} \\
\text { Placebo 13\% (12/94) } \\
\text { Favors Placebo } \\
\text { P<0.001 } \\
\text { Akathisia } \\
\text { Lithium 4\% (4/94) } \\
\text { Placebo 1\% (1/94) } \\
\text { NS }\end{array}$ \\
\hline
\end{tabular}




\begin{tabular}{|c|c|c|c|c|c|c|}
\hline Drug & $\begin{array}{l}\text { Study } \\
\text { Comparison } \\
\text { PMID }\end{array}$ & Responder/Remitter & Symptom & Function & Other & AE \\
\hline & $\begin{array}{l}\text { Calabrese, } \\
2003^{5} \\
14628976\end{array}$ & $\begin{array}{l}\text { Time to any recurrence } \\
18 \text { months } \\
\text { Log rank } \\
\text { Favors Lithium } \\
\mathrm{p}=0.03 \\
\frac{\text { Time to mania recurrence }}{18 \text { months }} \\
\text { Log rank } \\
\text { NS } \\
\frac{\text { Time to depression }}{\text { recurrence }} \\
18 \text { months } \\
\text { Log rank } \\
\text { NS }\end{array}$ & $\begin{array}{l}\text { NA } \\
\text { (Exclude for attrition = 85\%) }\end{array}$ & NA & NA & $\begin{array}{l}\frac{\text { SAE }}{1 \text { death in lamotrigine }} \\
\text { groups } \\
\text { Suicidality per HAM-D } \\
\text { NS between groups } \\
\frac{\text { Tremor }}{\text { Lithium } 17 \%(20 / 120)} \\
\text { Placebo 5\% (6/121) } \\
\text { Favors Placebo } \\
\text { P<0.05 }\end{array}$ \\
\hline & $\begin{array}{l}\text { Prien, } 1973^{6} \\
\text { High } \\
4569674\end{array}$ & $\begin{array}{l}\text { Relapse, any episode } \\
\text { type (manic, mixed, } \\
\text { schizoaffective, } \\
\text { depressive event } \\
\text { requiring hospitalization } \\
\text { or supplementary drugs) } \\
\text { Lithium } 43 \%(43 / 101) \\
\text { Placebo } 80 \%(84 / 104) \\
\text { Favors Lithium } \\
\text { p }<0.001\end{array}$ & $\underline{N R}$ & NR & $\begin{array}{l}\text { Suicide } \\
\text { Placebo 1/104 } \\
\text { Overall Withdrawal } \\
\text { Lithium 27\% (27/101) } \\
\text { Placebo 57\% (59/104) } \\
\text { Favors lithium } \\
\text { p<0.001 } \\
\text { Withdrawal due to 'poor } \\
\text { clinical response' } \\
\text { (further participation } \\
\text { would seriously } \\
\text { jeopardize the patients } \\
\text { physical or mental } \\
\text { health) } \\
\text { Lithium 11\% (11/101) } \\
\text { Placebo 40\% (41/104) }\end{array}$ & $\begin{array}{l}\text { SAE } \\
\text { Lithium 24\% (24/101) } \\
\text { Placebo 0\% (assumed } \\
\text { - none reported) } \\
\text { Lithium: 3/101 - } \\
\text { Toxicity (1) } \\
\text { Hypothyroidism with } \\
\text { goiter, polyuria, } \\
\text { polydipsia (1) } \\
\text { Hypothyroidism w/o } \\
\text { goiter (3) } \\
\text { Leukocytosis (1) } \\
\text { Death } \\
\text { Lithium 1/101 } \\
\text { Placebo 1/104 }\end{array}$ \\
\hline
\end{tabular}




\begin{tabular}{|c|c|c|c|c|c|c|}
\hline Drug & $\begin{array}{c}\text { Study } \\
\text { Comparison } \\
\text { PMID }\end{array}$ & Responder/Remitter & Symptom & Function & Other & $\mathrm{AE}$ \\
\hline & $\begin{array}{l}\text { Weisler, } 2011^{7} \\
22054050\end{array}$ & $\begin{array}{l}\text { Time to recurrence of any } \\
\text { mood event } \\
\text { Favors Lithium } \\
\text { HR } 0.46(95 \% \mathrm{Cl} 0.36, \\
0.59), \mathrm{p}<0.0001 \\
\frac{\text { Time to recurrence of }}{\text { manic event }} \\
\text { Favors Lithium } \\
\text { HR } 0.37(95 \% \mathrm{Cl} 0.27, \\
0.53), \mathrm{p}<0.0001 \\
\text { Time to recurrence of } \\
\text { depression symptoms } \\
\text { Favors Lithium } \\
\text { HR 0.59 (95\% Cl 0.42, } \\
0.84), p<0.004\end{array}$ & $\begin{array}{l}\text { NA } \\
\text { (Exclude for attrition 55\%) }\end{array}$ & NR & $\underline{\mathrm{NR}}$ & $\begin{array}{l}\text { SAE } \\
10 \text { lithium } \\
11 \text { placebo } \\
\frac{\text { EPS }}{38 \text { lithium }} \\
18 \text { placebo } \\
\text { No deaths }\end{array}$ \\
\hline
\end{tabular}

Abbreviations: AE=Adverse Events; CGI-BP-S=Clinical Global Impressions, Bipolar, Severity Scale; CI=Confidence Interval; EPS=extrapyramidal symptoms; HAM-

$\mathrm{D}=$ Hamilton Scale for Depression; HR=Hazard Ratio; LSM=least-squares means; MADRS=Montgomery-Asberg Depression Rating Scale; NA=Not applicable; NR=not reported; NS=not significant; OR=Odds Ratio; PMID=PubMed Identification Number; SAE=Serious Adverse Events; YMRS = Young Mania Rating Scale 
Appendix Table 14. Strength of evidence assessment: lithium monotherapy versus placebo for maintenance

\begin{tabular}{|c|c|c|c|c|c|c|c|c|}
\hline Comparison & Outcome & $\begin{array}{l}\text { \# Studies/ } \\
\text { Design } \\
\text { (n analyzed) }\end{array}$ & $\begin{array}{c}\text { Finding or Summary } \\
\text { Statistic } \\
\text { Outcome Timing }\end{array}$ & $\begin{array}{c}\text { Study } \\
\text { Limitations }\end{array}$ & Consistency & Directness & Precision & $\begin{array}{c}\text { Overall } \\
\text { Gradel } \\
\text { Conclusion }\end{array}$ \\
\hline $\begin{array}{l}\text { Lithium vs. } \\
\text { placebo }\end{array}$ & $\begin{array}{l}\text { Time to overall } \\
\text { relapse } 1-2 \text { yrs }\end{array}$ & $\begin{array}{l}6 \mathrm{RCT} \\
(\mathrm{n}=1579)\end{array}$ & $\begin{array}{l}\text { Favors Lithium } \\
1 \text { to } 2 \text { years }\end{array}$ & Moderate & Consistent & Direct & Imprecise & $\begin{array}{l}\text { Low } \\
\text { (weighted by } \\
\text { moderate } \\
\text { study and } \\
\text { hazard ratio) }\end{array}$ \\
\hline $\begin{array}{l}\text { Lithium vs. } \\
\text { placebo }\end{array}$ & $\begin{array}{l}\text { Time to manic or } \\
\text { depressive } \\
\text { relapse } 1-2 \text { yrs }\end{array}$ & $\begin{array}{l}6 \text { RCT } \\
(n=1579)\end{array}$ & See table above & Moderate & Inconsistent & Direct & Imprecise & Insufficient \\
\hline
\end{tabular}

Abbreviations: MADRS=Montgomery-Asberg Depression Rating Scale; RCT=randomized controlled trial; YMRS = Young Mania Rating Scale

Notes:

1. Publication bias for antipsychotics, antidepressants, and behavioral interventions for depressive disorders is suspected.

2. Data were generally imprecise due to missing data from high attrition rates, which was commonly dealt with by Last Observation Carried Forward (LOCF). LOCF requires an

assumption that the health status of patients who dropped out of the trial would not have changed had future observations been recorded, a strong assumption in the context of

bipolar disorder research. 


\section{Section 2. Other Monotherapy}

Appendix Table 15. Characteristics of eligible studies: other monotherapy for maintenance by year then first author

\begin{tabular}{|c|c|c|c|c|c|c|}
\hline $\begin{array}{c}\text { Study, Year } \\
\text { Design } \\
\text { Location } \\
\text { Funder } \\
\text { Risk of Bias } \\
\text { PMID }\end{array}$ & $\begin{array}{c}\text { \# Randomized } \\
\text { Age (mean) } \\
\text { Sex (\% Female) } \\
\text { Race (\% White) } \\
\text { Diagnosis } \\
\text { (\% BP I, II, NOS) } \\
\text { Setting }\end{array}$ & $\begin{array}{c}\text { Inclusions } \\
\text { Key Exclusions }\end{array}$ & $\begin{array}{c}\text { Intervention } \\
\text { Dosage }\end{array}$ & $\begin{array}{c}\text { Comparison } \\
\text { Dosage }\end{array}$ & $\begin{array}{l}\text { Followup } \\
\text { Duration }\end{array}$ & $\begin{array}{c}\text { Outcomes } \\
\text { Reported } \\
\begin{array}{c}\text { Withdrawal (\%) at } \\
\text { endpoint }\end{array}\end{array}$ \\
\hline $\begin{array}{l}\text { Calabrese, } 2017^{8} \\
\text { RCT } \\
\text { Multisite } \\
4 \text { Continents } \\
\text { Industry } \\
\text { RoB Moderate } \\
28146613\end{array}$ & $\begin{array}{l}\mathrm{N}=266 \\
\text { Mean Age } 41 \\
\text { Female 58\% } \\
\text { Race White 54\% } \\
\text { Black/African American } \\
28 \% \\
\text { BP I 100\% } \\
\text { Inpatient/Outpatient }\end{array}$ & $\begin{array}{l}\geq 1 \text { previous manic or mixed } \\
\text { episode severity requiring } \\
\text { hospitalization or treatment } \\
\text { with mood stabilizer or } \\
\text { treatment with antipsychotic } \\
\text { agent. Current episode } \\
\text { YMRS } \geq 20 \text { but then met } \\
\text { YMRS } \leq 12, \text { MADRS } \leq 12, \text { no } \\
\text { active suicidality } \\
\text { Rapid Cycling } \\
\text { Refractory BP } \\
\text { First Manic Episode } \\
\text { Substance Abuse } \\
\text { Other Mental Health } \\
\text { Labs/Other Conditions }\end{array}$ & \begin{tabular}{|l} 
Aripiprazole \\
once-monthly \\
injections $400 \mathrm{mg}$
\end{tabular} & Placebo & 52 week & $\begin{array}{l}\text { Time to recurrence } \\
\text { (hospitalization or } \\
\text { YMRS>15; } \\
\text { MADRS>15; CGI- } \\
\text { BP>4; further } \\
\text { medication; suicidality) } \\
\text { Proportion meeting } \\
\text { recurrence } \\
\text { YMRS } \\
\text { MADRS } \\
\text { Withdrawal 61.7\% } \\
\text { (nonrelapse withdrawal } \\
\text { 23\%) }\end{array}$ \\
\hline $\begin{array}{l}\text { Keck, } 2006^{9} \\
\text { RCT } \\
\text { Multisite } \\
2 \text { Continents } \\
\text { Industry } \\
\text { Rob Moderate } \\
16669728\end{array}$ & $\begin{array}{l}\mathrm{N}=161 \\
\text { Mean Age 40; } \\
\text { Female 67\% } \\
\text { Race White 56\% } \\
\text { Hispanic/Latino 23\% } \\
\text { BP I 100\% } \\
\text { Outpatient }\end{array}$ & $\begin{array}{l}\text { Symptom stability: } \\
\text { YMRS } \leq 10 \text { and MADRS } \leq 13 \\
\text { for } 4 \text { consecutive visits over } \\
6 \text { weeks } \\
\text { Substance Abuse } \\
\text { Other Mental Health } \\
\text { Labs/Other Conditions } \\
\text { Pregnant/Nursing } \\
\text { Unresponsive to Clozapine } \\
\text { ECT in last } 2 \text { years }\end{array}$ & $\begin{array}{l}\text { Aripiprazaole } \\
15 \text { or } 30 \mathrm{mg} / \text { day } \\
\text { based on } \\
\text { investigator } \\
\text { discretion }\end{array}$ & Placebo & 26 weeks & $\begin{array}{l}\text { Time to relapse } \\
\text { (defined as lack of } \\
\text { efficacy) } \\
\text { YMRS } \\
\text { MADRS } \\
\text { PANSS } \\
\text { CGI-BP } \\
\\
\text { Withdrawal 58\% } \\
\text { (nonrelapse withdrawal } \\
\text { 24\%) } \\
\end{array}$ \\
\hline
\end{tabular}




\begin{tabular}{|c|c|c|c|c|c|c|}
\hline $\begin{array}{l}\text { Study, Year } \\
\text { Design } \\
\text { Location } \\
\text { Funder } \\
\text { Risk of Bias } \\
\text { PMID }\end{array}$ & $\begin{array}{c}\text { \# Randomized } \\
\text { Age (mean) } \\
\text { Sex (\% Female) } \\
\text { Race (\% White) } \\
\text { Diagnosis } \\
\text { (\% BP I, II, NOS) } \\
\text { Setting }\end{array}$ & $\begin{array}{c}\text { Inclusions } \\
\text { Key Exclusions }\end{array}$ & $\begin{array}{c}\text { Intervention } \\
\text { Dosage }\end{array}$ & $\begin{array}{c}\text { Comparison } \\
\text { Dosage }\end{array}$ & $\begin{array}{l}\text { Followup } \\
\text { Duration }\end{array}$ & $\begin{array}{c}\text { Outcomes } \\
\text { Reported } \\
\text { Withdrawal (\%) at } \\
\text { endpoint }\end{array}$ \\
\hline $\begin{array}{l}\text { Hartong, } 2003^{10} \\
\text { RCT } \\
\text { Multisite } \\
\text { Netherlands } \\
\text { Industry/Government } \\
\text { RoB Low } \\
12633122\end{array}$ & $\begin{array}{l}\mathrm{N}=98 \\
\text { Mean Age 42 } \\
\text { Female 54\% } \\
\text { Race NR } \\
\text { BP I 77\% } \\
\text { BP-II 23\% } \\
\text { Outpatient }\end{array}$ & $\begin{array}{l}\text { Remission from any Episode } \\
\text { Type; According to Bech } \\
\text { Rafaelsen Mania or } \\
\text { Melancholia Scales } \\
\text { First Manic Episode }\end{array}$ & \begin{tabular}{|l} 
Carbamazepine \\
6-10 mg/L $(6.8 \mathrm{mg} / \mathrm{L}$ \\
average)
\end{tabular} & $\begin{array}{l}\text { Lithium } \\
0.6-1 \mathrm{mmol} / \mathrm{L} \\
(0.75 \mathrm{mmol} / \mathrm{L} \\
\text { average })\end{array}$ & 2 years & $\begin{array}{l}\text { Remission (YMRS } \\
<=12 \text { ) } \\
\text { Response (50\% } \\
\text { decrease in YMRS) } \\
\text { Efficacy } \\
\text { YMRS } \\
\text { CGI-BP-S } \\
\text { MADRS } \\
\text { Adverse events } \\
\text { Extrapyramidal } \\
\text { symptoms } \\
\text { SAS } \\
\text { AIMS } \\
\text { BARS } \\
\\
\text { Withdrawal } 31 \% \\
\end{array}$ \\
\hline
\end{tabular}




\begin{tabular}{|c|c|c|c|c|c|c|}
\hline $\begin{array}{l}\text { Study, Year } \\
\text { Design } \\
\text { Location } \\
\text { Funder } \\
\text { Risk of Bias } \\
\text { PMID }\end{array}$ & $\begin{array}{c}\text { \# Randomized } \\
\text { Age (mean) } \\
\text { Sex (\% Female) } \\
\text { Race (\% White) } \\
\text { Diagnosis } \\
\text { (\% BP I, II, NOS) } \\
\text { Setting }\end{array}$ & $\begin{array}{c}\text { Inclusions } \\
\text { Key Exclusions }\end{array}$ & $\begin{array}{l}\text { Intervention } \\
\text { Dosage }\end{array}$ & $\begin{array}{l}\text { Comparison } \\
\text { Dosage }\end{array}$ & $\begin{array}{l}\text { Followup } \\
\text { Duration }\end{array}$ & $\begin{array}{c}\text { Outcomes } \\
\text { Reported } \\
\begin{array}{c}\text { Withdrawal (\%) at } \\
\text { endpoint }\end{array}\end{array}$ \\
\hline $\begin{array}{l}\text { Greil, } 1997^{11} \\
\text { RCT } \\
\text { Multisite } \\
\text { Germany } \\
\text { Government } \\
\text { RoB High } \\
\\
\text { 9165384 also } \\
9864077^{12} \\
10529070^{13} \\
10529071^{14} \\
11093063^{15}\end{array}$ & $\begin{array}{l}\text { N = 171 } \\
\text { Mean Age 40 } \\
\text { Female 57\% } \\
\text { Race NR } \\
\text { BP I 58\% } \\
\text { BP-NOS 33\% } \\
\text { Outpatient }\end{array}$ & $\begin{array}{l}\text { Remission from any Episode } \\
\text { Type; GAS }>70 \\
\text { First Manic Episode } \\
\text { Substance Abuse } \\
\text { Other Mental Health } \\
\text { Neurological Disorders }\end{array}$ & $\begin{array}{l}\text { Carbamazepine } 635 \\
\text { mg/day }\end{array}$ & $\begin{array}{l}\text { Lithium } \\
26.8 \mathrm{mmol} / \text { day }\end{array}$ & $\begin{array}{l}2 \text { years (130 } \\
\text { weeks?) }\end{array}$ & $\begin{array}{l}\text { Remission (YMRS } \\
<=12 \text { ) } \\
\text { Response (50\% } \\
\text { decrease in YMRS) } \\
\text { Efficacy } \\
\text { YMRS } \\
\text { CGI-BP-S } \\
\text { MADRS } \\
\text { Adverse events } \\
\text { Extrapyramidal } \\
\text { symptoms } \\
\text { SAS } \\
\text { AIMS } \\
\text { BARS } \\
\text { Withdrawal 23\% } \\
\end{array}$ \\
\hline $\begin{array}{l}\text { Calabrese, } 2005^{16} \\
\text { RCT of responders } \\
\text { US } \\
\text { Government } \\
\text { ROB Moderate } \\
16263857\end{array}$ & $\begin{array}{l}\text { N=60 } \\
\text { Mean Age } 37 \\
\text { Female 52\% } \\
\text { White NR } \\
\text { BP I 60\% } \\
\text { BP-II 40\% } \\
\text { Outpatient }\end{array}$ & $\begin{array}{l}\text { Responders to both drugs } \\
\text { Rapid cycling; mood } \\
\text { episode in previous } 3 \\
\text { months } \\
\text { Substance Use } \\
\text { Other Mental Health } \\
\text { Conditions } \\
\text { Pregnant/Nursing } \\
\text { Lab/other conditions } \\
\text { Intolerant of lithium }\end{array}$ & $\begin{array}{l}\text { Divalproex/valproate } \\
\text { mean divalproex } \\
\text { dose } 1571 \text { mg/day; } \\
\text { mean valproate } \\
\text { dose } 77\end{array}$ & $\begin{array}{l}\text { Lithium } \\
\text { mean lithium } \\
\text { dose } 1359 \\
\text { mg/day }\end{array}$ & 20 month & $\begin{array}{l}\text { Time to relapse (HAM- } \\
D \geq 20 \text { or YMRS } \geq 20 \text { for } \\
8 \text { weeks) } \\
\\
\text { Withdrawal } 88 \% \\
\text { (Time to recurrence } \\
\text { outcomes only } \\
\text { included) } \\
\text { Nonrelapse Withdrawal } \\
25 \%\end{array}$ \\
\hline
\end{tabular}




\begin{tabular}{|c|c|c|c|c|c|c|}
\hline $\begin{array}{l}\text { Study, Year } \\
\text { Design } \\
\text { Location } \\
\text { Funder } \\
\text { Risk of Bias } \\
\text { PMID }\end{array}$ & $\begin{array}{c}\text { \# Randomized } \\
\text { Age (mean) } \\
\text { Sex (\% Female) } \\
\text { Race (\% White) } \\
\text { Diagnosis } \\
\text { (\% BP I, II, NOS) } \\
\text { Setting }\end{array}$ & $\begin{array}{c}\text { Inclusions } \\
\text { Key Exclusions }\end{array}$ & $\begin{array}{c}\text { Intervention } \\
\text { Dosage }\end{array}$ & $\begin{array}{c}\text { Comparison } \\
\text { Dosage }\end{array}$ & $\begin{array}{l}\text { Followup } \\
\text { Duration }\end{array}$ & $\begin{array}{c}\text { Outcomes } \\
\text { Reported } \\
\begin{array}{c}\text { Withdrawal (\%) at } \\
\text { endpoint }\end{array}\end{array}$ \\
\hline $\begin{array}{l}\text { Bowden, } 2000^{1} \\
\text { RCT } \\
\text { Multisite } \\
\text { US } \\
\text { Industry } \\
\text { RoB High } \\
10807488 \\
12784116^{2}\end{array}$ & $\begin{array}{l}\mathrm{N}=372 \\
\text { Mean Age } 39 \\
\text { Female 51\% } \\
\text { White } 94 \% \\
\text { BP I 100\% } \\
\text { Outpatient }\end{array}$ & $\begin{array}{l}\text { No Episode at } \\
\text { Randomization; Scores of } \\
\text { MRS } \leq 11, \text { DSS } \leq 13, \text { GAS } \\
\text { > 60; At least one other } \\
\text { manic episode in past three } \\
\text { years. } \\
\text { Substance Abuse; Other } \\
\text { Mental Health Conditions; } \\
\text { Taking Other Medications; } \\
\text { Pregnant/Nursing }\end{array}$ & $\begin{array}{l}\text { Divalproex } \\
71-125 \mathrm{mcg} / \mathrm{mL} \\
(\text { Mean } 84.8 \mathrm{mcg} / \mathrm{mL}) \\
\mathrm{N}=187\end{array}$ & $\begin{array}{l}\text { C1: Placebo } \\
n=92 \\
\text { C2: Lithium } \\
0.8-1.2 \mathrm{mEq} / \mathrm{L} \\
\text { (Mean } 0.9 \\
\text { mEq/L) } \\
n=90\end{array}$ & 52 week & $\begin{array}{l}\text { Time to recurrence, } \\
\text { any } \\
\text { Time to recurrence, } \\
\text { mania (MRS } \geq 16) \\
\text { Time to recurrence, } \\
\text { depression } \\
(\mathrm{DSS} \geq 25) \\
\\
\text { Withdrawal } 69 \% \\
\text { (Time to recurrence } \\
\text { outcomes only } \\
\text { included) } \\
\text { Nonrelapse Withdrawal } \\
40 \% \\
\end{array}$ \\
\hline $\begin{array}{l}\text { Amsterdam, } 2010^{3} \\
\text { RCT } \\
\text { Single-site } \\
\text { US } \\
\text { Gov't+nonprofit } \\
\text { RoB Moderate } \\
20360317\end{array}$ & $\begin{array}{l}\mathrm{N}=81 \\
\text { Mean Age } 38 \\
\text { Female 52\% } \\
\text { White NR } \\
\text { BP II 100\% } \\
\text { Outpatient }\end{array}$ & $\begin{array}{l}\text { Depression and } \\
\text { HAM-D } \geq 16 \text { initialy; then } \\
\text { HAM-D } 88 \text { after } 12 \text { weeks of } \\
\text { initial Fluoxetine therapy at } \\
20-80 \text { mg/day) } \\
\text { Substance abuse } \\
\text { Neurological Disorders } \\
\text { Taking other medications } \\
\text { Pregnant/NursingLabs/Other } \\
\text { Conditions }\end{array}$ & $\begin{array}{l}\text { Fluoxetine }(\mathrm{n}=28) \\
\text { 10-40 mg/day } \\
\text { (mean } 34.3 \text { mg/day) } \\
\mathrm{N}=28\end{array}$ & \begin{tabular}{|l|} 
C1: Placebo \\
$(\mathrm{n}=27)$ \\
\\
C2: Lithium \\
$(\mathrm{n}=26)$ \\
$300-1200$ \\
mg/day; 0.5-1.5 \\
mmol/L \\
(mean 1027 \\
mg/day; 0.69 \\
mmol/L)
\end{tabular} & 50 weeks & $\begin{array}{l}\text { YMRS } \\
\text { Relapse (HAM-D } \geq 14 \text { ) } \\
\text { Adverse Events } \\
\text { Lab Values } \\
\\
\text { Withdrawal } 72 \% \\
\text { (Time to recurrence } \\
\text { outcomes only } \\
\text { included) } \\
\text { Nonrelapse Withdrawal } \\
25 \%\end{array}$ \\
\hline
\end{tabular}




\begin{tabular}{|c|c|c|c|c|c|c|}
\hline $\begin{array}{l}\text { Study, Year } \\
\text { Design } \\
\text { Location } \\
\text { Funder } \\
\text { Risk of Bias } \\
\text { PMID }\end{array}$ & $\begin{array}{c}\text { \# Randomized } \\
\text { Age (mean) } \\
\text { Sex (\% Female) } \\
\text { Race (\% White) } \\
\text { Diagnosis } \\
\text { (\% BP I, II, NOS) } \\
\text { Setting }\end{array}$ & $\begin{array}{c}\text { Inclusions } \\
\text { Key Exclusions }\end{array}$ & $\begin{array}{l}\text { Intervention } \\
\text { Dosage }\end{array}$ & $\begin{array}{c}\text { Comparison } \\
\text { Dosage }\end{array}$ & $\begin{array}{l}\text { Followup } \\
\text { Duration }\end{array}$ & $\begin{array}{c}\text { Outcomes } \\
\text { Reported } \\
\text { Withdrawal (\%) at } \\
\text { endpoint }\end{array}$ \\
\hline $\begin{array}{l}\text { Bowden, } 2003^{4} \\
\text { RCT of responders } \\
\text { Multisite } \\
2 \text { continents } \\
\text { Industry } \\
\text { RoB Low } \\
12695317\end{array}$ & $\begin{array}{l}\mathrm{N}=175 \\
\text { Mean Age } 41 \\
\text { Female 47\% } \\
\text { Race NR } \\
\text { BP I 100\% } \\
\text { Outpatient }\end{array}$ & $\begin{array}{l}\text { Lamotrigine responders } \\
\text { (CGI-S } \leq 3 \text { for at least } 4 \\
\text { continuous weeks) } \\
\text { For open label period: } \\
\text { Manic; DSM-IV Criteria for } \\
\text { Mania or Hypomania } \\
\text { currently or within past } 60 \\
\text { days with previous episodes } \\
\text { in past } 3 \text { years } \\
\text { Other Mental Health } \\
\text { Conditions }\end{array}$ & $\begin{array}{l}\text { Lamotrigine } \\
100-400 \mathrm{mg} / \text { day } \\
\text { (Mean NR) } \\
\mathrm{N}=59\end{array}$ & $\begin{array}{l}\text { C1: Placebo } \\
\mathrm{N}=70 \\
\\
\text { C2: Lithium } \\
0.8-1.1 \mathrm{mEq} / \mathrm{L} \\
\text { Mean NR } \\
\mathrm{N}=59\end{array}$ & 18 Months & $\begin{array}{l}\text { Time to intervention for } \\
\text { mania, hypomania, } \\
\text { mixed, depression, and } \\
\text { any mood episodes } \\
\text { Time to early } \\
\text { discontinuation } \\
\text { Adverse Events I } \\
\\
\text { Withdrawal 80\% } \\
\text { (Time to recurrence } \\
\text { outcomes only } \\
\text { included) } \\
\text { Nonrelapse Withdrawal } \\
20 \%\end{array}$ \\
\hline $\begin{array}{l}\text { Calabrese, } 2003^{5} \\
\text { RCT of responders } \\
\text { Multisite } \\
4 \text { Continents } \\
\text { Industry } \\
\text { RoB Moderate } \\
14628976\end{array}$ & $\begin{array}{l}\mathrm{N}=410 \\
\text { Mean Age } 43 \\
\text { Female 56\% } \\
\text { Race NR } \\
\text { BP I 100\% } \\
\text { Outpatient }\end{array}$ & $\begin{array}{l}\text { Lamotrigine responders } \\
\text { (CGI-S<3 for at least } 4 \\
\text { continuous weeks) } \\
\text { For open label period: } \\
\text { depression; DSM-IV criteria } \\
\text { for depression currently or } \\
\text { within past } 60 \text { days with } \\
\text { previous depression and } \\
\text { mania episodes in past } 3 \\
\text { years. } \\
\text { Other Mental Health } \\
\text { Conditions }\end{array}$ & $\begin{array}{l}\text { Lamotrigine } \\
50,200, \text { or } 400 \\
\text { mg/day (in analysis } \\
50 \mathrm{mg} \text { group was } \\
\text { censored, } 200 \text { and } \\
400 \text { mg/day groups } \\
\text { were combined) } \\
\mathrm{N}=221\end{array}$ & $\begin{array}{l}\text { C1: Placebo } \\
\mathrm{N}=121 \\
\\
\text { C2: Lithium } \\
0.8-1.1 \mathrm{mEq} / \mathrm{L} \\
\text { (Mean } 0.8 \\
\mathrm{mEq} / \mathrm{L}) \\
\mathrm{N}=121\end{array}$ & 18 Months & $\begin{array}{l}\text { Time to intervention for } \\
\text { mania, hypomania, } \\
\text { depression, any mood } \\
\text { episode } \\
\text { Time to early } \\
\text { discontinuation } \\
\text { Adverse Events } \\
\\
\text { Withdrawal } 84.9 \% \\
\text { (Time to recurrence } \\
\text { outcomes only } \\
\text { included) } \\
\text { Nonrelapse Withdrawal } \\
34 \%\end{array}$ \\
\hline
\end{tabular}




\begin{tabular}{|c|c|c|c|c|c|c|}
\hline $\begin{array}{l}\text { Study, Year } \\
\text { Design } \\
\text { Location } \\
\text { Funder } \\
\text { Risk of Bias } \\
\text { PMID }\end{array}$ & $\begin{array}{c}\text { \# Randomized } \\
\text { Age (mean) } \\
\text { Sex (\% Female) } \\
\text { Race (\% White) } \\
\text { Diagnosis } \\
\text { (\% BP I, II, NOS) } \\
\text { Setting }\end{array}$ & $\begin{array}{c}\text { Inclusions } \\
\text { Key Exclusions }\end{array}$ & $\begin{array}{c}\text { Intervention } \\
\text { Dosage }\end{array}$ & $\begin{array}{c}\text { Comparison } \\
\text { Dosage }\end{array}$ & $\begin{array}{l}\text { Followup } \\
\text { Duration }\end{array}$ & $\begin{array}{c}\text { Outcomes } \\
\text { Reported } \\
\begin{array}{c}\text { Withdrawal (\%) at } \\
\text { endpoint }\end{array}\end{array}$ \\
\hline $\begin{array}{l}\text { Calabrese, } 2000^{17} \\
\text { RCT of responders } \\
\text { Multisite } \\
\text { US, Canada } \\
\text { Industry } \\
\text { ROB High } \\
11105737\end{array}$ & $\begin{array}{l}\mathrm{N}=182 \\
\text { Mean Age } 38 \\
\text { Female 58\% } \\
\text { Race NR } \\
\text { BP I 70\% } \\
\text { BP-II 30\% } \\
\text { Outpatient }\end{array}$ & $\begin{array}{l}\text { Rapid cyclers, stabilized on } \\
\text { lamotrigine (no mood } \\
\text { episodes requiring other } \\
\text { drugs or ECT) } \\
\text { Other Mental Health } \\
\text { Conditions } \\
\text { Labs/Other conditions }\end{array}$ & $\begin{array}{l}\text { Lamotrigine } \\
100-500 \mathrm{mg} / \text { day } \\
\mathrm{N}=93\end{array}$ & $\begin{array}{l}\text { Placebo } \\
\mathrm{N}=89\end{array}$ & 26 weeks & $\begin{array}{l}\text { Time to addition drug } \\
\text { treatment } \\
\text { Time to overall } \\
\text { withdrawa } \\
\\
\text { Withdrawal } 67 \% \\
\text { (Time to recurrence } \\
\text { outcomes only } \\
\text { included) } \\
\text { Nonrelapse withdrawal } \\
15 \%\end{array}$ \\
\hline $\begin{array}{l}\text { Balance Investigators, } \\
2010^{18} \\
\text { RCT } \\
\text { Multisite } \\
\text { US and Europe } \\
\text { Industry } \\
\text { RoB Moderate } \\
20092882\end{array}$ & $\begin{array}{l}\mathrm{N}=330 \\
\text { Mean Age } 43 \\
\text { Female 49\% } \\
\text { Race NR } \\
\text { BP I 100\% } \\
\text { Outpatient }\end{array}$ & $\begin{array}{l}\text { Not having acute episode; } \\
\text { Not defined } \\
\text { Pregnant/Nursing }\end{array}$ & $\begin{array}{l}\text { Lithium } \\
0.4-1.0 \mathrm{mmol} / \mathrm{L} \\
\text { mean NR } \\
+ \text { Valproate } \\
750-1250 \text { or highest } \\
\text { tolerated } \\
\text { mean NR } \\
\mathrm{n}=110\end{array}$ & $\begin{array}{l}\text { C1: Lithium } \\
0.4-1.0 \text { mmol/L } \\
\text { mean NR } \\
\mathrm{N}=110 \\
\\
\text { C2: Valproate } \\
750-1250 \text { or } \\
\text { highest } \\
\text { tolerated } \\
\text { mean NR } \\
\mathrm{n}=110\end{array}$ & 24 months & $\begin{array}{l}\text { GAF } \\
\text { EuroQol (EQ-5D) } \\
\text { (quality of life) } \\
\text { Relapse } \\
\\
\text { Withdrawal } 20 \%\end{array}$ \\
\hline
\end{tabular}




\begin{tabular}{|c|c|c|c|c|c|c|}
\hline $\begin{array}{l}\text { Study, Year } \\
\text { Design } \\
\text { Location } \\
\text { Funder } \\
\text { Risk of Bias } \\
\text { PMID }\end{array}$ & $\begin{array}{c}\text { \# Randomized } \\
\text { Age (mean) } \\
\text { Sex (\% Female) } \\
\text { Race (\% White) } \\
\text { Diagnosis } \\
\text { (\% BP I, II, NOS) } \\
\text { Setting }\end{array}$ & $\begin{array}{c}\text { Inclusions } \\
\text { Key Exclusions }\end{array}$ & $\begin{array}{c}\text { Intervention } \\
\text { Dosage }\end{array}$ & $\begin{array}{c}\text { Comparison } \\
\text { Dosage }\end{array}$ & $\begin{array}{l}\text { Followup } \\
\text { Duration }\end{array}$ & $\begin{array}{c}\text { Outcomes } \\
\text { Reported } \\
\begin{array}{c}\text { Withdrawal (\%) at } \\
\text { endpoint }\end{array}\end{array}$ \\
\hline $\begin{array}{l}\text { Tohen, } 2006^{19} \\
\text { RCT } \\
\text { Multisite } \\
2 \text { Continents } \\
\text { Industry } \\
\text { RoB Moderate } \\
16449478\end{array}$ & $\begin{array}{l}\mathrm{N}=361 \\
\text { Mean Age } 41 \\
\text { Female 39\% } \\
\text { White } 87 \% \\
\text { BP I 100\% } \\
\text { Outpatient }\end{array}$ & $\begin{array}{l}\text { Remission; } \\
\text { YMRS } \leq 12 \\
\text { HAM-D } \leq 8 \\
\text { First Manic Episode }\end{array}$ & $\begin{array}{l}\text { Olanzapine } \\
\text { 5-20 mg/day }\end{array}$ & Placebo & 48 weeks & $\begin{array}{l}\text { Time to Relapse } \\
\text { Symptom Severity } \\
\text { YMRS } \\
\text { HAM-D } \\
\text { Adverse Events } \\
\\
\text { Withdrawal } 84 \% \\
\text { (Time to recurrence } \\
\text { outcomes only } \\
\text { included) } \\
\text { Nonrelapse Withdrawal } \\
25 \%\end{array}$ \\
\hline $\begin{array}{l}\text { Tohen, } 2003^{20} \\
\text { RCT } \\
\text { Multisite } \\
\text { US } \\
\text { Industry } \\
\text { ROB High } \\
12832240 \\
\text { Extension of Tohen, } \\
2002 b^{21} \\
12042191\end{array}$ & $\begin{array}{l}\mathrm{N}=251 \\
\text { Mean Age } 40 \\
\text { Female } 57 \% \\
\text { White } 82 \% \\
\text { BP-I 100\% } \\
\text { Outpatient }\end{array}$ & $\begin{array}{l}\text { YMRS }>19 \\
\text { Substance Use } \\
\text { Pregnant/Nursing } \\
\text { Labs/other conditions }\end{array}$ & \begin{tabular}{|l} 
Olanzapine \\
5-20 mg/day
\end{tabular} & $\begin{array}{l}\text { Divalproex } \\
500-2500 \\
\text { mg/day }\end{array}$ & 42 weeks & $\begin{array}{l}\text { Time to relapse } \\
\text { Withdrawal } 84 \% \text { (Time } \\
\text { to relapse only } \\
\text { included) } \\
\text { Nonrelapse withdrawal } \\
\text { unclear }\end{array}$ \\
\hline
\end{tabular}




\begin{tabular}{|c|c|c|c|c|c|c|}
\hline $\begin{array}{l}\text { Study, Year } \\
\text { Design } \\
\text { Location } \\
\text { Funder } \\
\text { Risk of Bias } \\
\text { PMID }\end{array}$ & $\begin{array}{c}\text { \# Randomized } \\
\text { Age (mean) } \\
\text { Sex (\% Female) } \\
\text { Race (\% White) } \\
\text { Diagnosis } \\
\text { (\% BP I, II, NOS) } \\
\text { Setting }\end{array}$ & $\begin{array}{c}\text { Inclusions } \\
\text { Key Exclusions }\end{array}$ & $\begin{array}{l}\text { Intervention } \\
\text { Dosage }\end{array}$ & $\begin{array}{c}\text { Comparison } \\
\text { Dosage }\end{array}$ & $\begin{array}{l}\text { Followup } \\
\text { Duration }\end{array}$ & $\begin{array}{c}\text { Outcomes } \\
\text { Reported } \\
\begin{array}{c}\text { Withdrawal (\%) at } \\
\text { endpoint }\end{array}\end{array}$ \\
\hline $\begin{array}{l}\text { Quiroz, } 2010^{22} \\
\text { RCT of Responders } \\
\text { Multisite } \\
3 \text { Continents } \\
\text { Industry } \\
\text { ROB Moderate } \\
20227682\end{array}$ & $\begin{array}{l}\mathrm{N}=303 \\
\text { Mean Age } 39 \\
\text { Female } 49 \% \\
\text { White } 80 \% \\
\text { BP-I } 100 \% \\
\text { Outpatient }\end{array}$ & $\begin{array}{l}\text { Responders to Phase III: } \\
\text { stable at CGI-BP-S <3 } \\
\text { Substand abuse } \\
\text { Taking other meds } \\
\text { Pregnant/nursing } \\
\text { Rapid cycling } \\
\text { Other mental health } \\
\text { Labs/other conditions }\end{array}$ & $\begin{array}{l}\text { Risperidone long- } \\
\text { acting injectable } \\
25,37.5 \text {, or } 50 \mathrm{mg} ; \\
77 \% \text { received } \\
25 \mathrm{mg} / 2 \text { weeks } \\
\mathrm{N}=154\end{array}$ & \begin{tabular}{|l|} 
Placebo \\
$\mathrm{N}=149$
\end{tabular} & 24 months & $\begin{array}{l}\text { Time to recurrence of } \\
\text { mood episode } \\
\text { Time to: } \\
\text { elevated mood } \\
\quad \text { depressive } \\
\text { early discontinue due } \\
\text { to medications } \\
\text { Efficacy } \\
\text { YMRS } \\
\text { MADRS } \\
\text { CGI-S } \\
\\
\text { Withdrawal 66\% (Time } \\
\text { to recurrence } \\
\text { outcomes only) } \\
\text { Nonrelapse withdrawal } \\
\text { 25\% }\end{array}$ \\
\hline
\end{tabular}




\begin{tabular}{|c|c|c|c|c|c|c|}
\hline $\begin{array}{l}\text { Study, Year } \\
\text { Design } \\
\text { Location } \\
\text { Funder } \\
\text { Risk of Bias } \\
\text { PMID }\end{array}$ & $\begin{array}{c}\text { \# Randomized } \\
\text { Age (mean) } \\
\text { Sex (\% Female) } \\
\text { Race (\% White) } \\
\text { Diagnosis } \\
\text { (\% BP I, II, NOS) } \\
\text { Setting }\end{array}$ & $\begin{array}{c}\text { Inclusions } \\
\text { Key Exclusions }\end{array}$ & $\begin{array}{l}\text { Intervention } \\
\text { Dosage }\end{array}$ & $\begin{array}{c}\text { Comparison } \\
\text { Dosage }\end{array}$ & $\begin{array}{l}\text { Followup } \\
\text { Duration }\end{array}$ & $\begin{array}{c}\text { Outcomes } \\
\text { Reported } \\
\text { Withdrawal (\%) at } \\
\text { endpoint }\end{array}$ \\
\hline $\begin{array}{l}\text { Vieta, } 2012^{23} \\
\text { RCT of Responders } \\
\text { Multisite } \\
3 \text { Continents } \\
\text { Industry } \\
\text { RoB High } \\
22503488\end{array}$ & $\begin{array}{l}\mathrm{N}=398 \\
\text { Mean Age } 36 \\
\text { Female } 52 \% \\
\text { White } 45 \% \\
\text { BP } 1100 \% \\
\text { Outpatient }\end{array}$ & $\begin{array}{l}\text { No recurrence event } \\
\text { (Responders from Phase II): } \\
\text { Not hypomanic, manic, } \\
\text { mixed, or depressive } \\
\text { episode; treatment with a } \\
\text { mood stabilizer, } \\
\text { antidepressant or prohibited } \\
\text { antipsychotic and } \\
\text { benzodiazepine usage; } \\
\text { hospitalization for a mood } \\
\text { episode; or CGI-S } \geq 4 \text { with } \\
\text { either YMRS }>12 \text { or } \\
\text { MADRS }>12 \\
\\
\text { First Manic Episode } \\
\text { Schizoaffective } \\
\text { Other Mental Health } \\
\text { Taking Other Meds } \\
\text { Pregnant/Nursing }\end{array}$ & $\begin{array}{l}\text { Risperidone long- } \\
\text { acting injectable } \\
1-6 \mathrm{mg} / \text { day } \\
\text { (fixed dose } \\
\text { distribution provided; } \\
25 \mathrm{mg} / 2 \text { weeks } 64 \% \text {, } \\
37.5 \mathrm{mg} / 2 \text { weeks } \\
32 \% ; 50 \mathrm{mg} / 2 \text { weeks } \\
4 \% \text { ) } \\
\mathrm{N}=131\end{array}$ & $\begin{array}{l}\text { C1: Placebo } \\
N=133 \\
\\
\text { C2: Olanzapine } \\
\text { 10mg/day } \\
N=130\end{array}$ & 18 months & $\begin{array}{l}\text { Response (YMRS } \leq \\
\text { 19) } \\
\text { Time to first recurrence } \\
\text { of mood symptoms } \\
\text { Efficacy } \\
\text { YMRS } \\
\text { CGI-S } \\
\text { MADRS } \\
\text { Adverse events } \\
\text { Extrapyramidal } \\
\text { symptoms } \\
\text { ESRS } \\
\text { Withdrawal 58\% (Time } \\
\text { to recurrence } \\
\text { outcomes only } \\
\text { included) } \\
\text { Nonrelapse withdrawal } \\
\text { 29\% }\end{array}$ \\
\hline $\begin{array}{l}\text { Tohen, } 2005^{24} \\
\text { RCT } \\
\text { Multisite } \\
5 \text { Continents } \\
\text { Industry } \\
\text { RoB Moderate } \\
15994710\end{array}$ & $\begin{array}{l}\mathrm{N}=431 \\
\text { Mean Age } 42 \\
\text { Female 53\% } \\
\text { White 99\% } \\
\text { BP NR } \\
\text { Outpatient }\end{array}$ & $\begin{array}{l}\text { Met remission criteria: } \\
\text { including YMRS } \leq 15 \text { and } \\
\text { HAM-D } \leq 8 \\
\text { After open-label period: } \\
\text { Manic or Mixed Episode } \\
\text { YMRS } \geq 20 \\
\\
\text { Substance Abuse } \\
\text { Other Mental Health } \\
\text { Neurological Disorders } \\
\text { Taking Other Meds } \\
\text { Labs/Other Conditions }\end{array}$ & $\begin{array}{l}\text { Olanzapine } \\
15 \text { mg/day } \\
\text { flexible dosing 5-20 } \\
\text { mg/day }\end{array}$ & $\begin{array}{l}\text { Lithium } \\
600 \mathrm{mg} / \text { day } \\
\text { flexible dosing } \\
\text { for blood level } \\
0.6-1.2 \\
\text { meq/liter }\end{array}$ & 52 weeks & $\begin{array}{l}\text { Time to Episode } \\
\text { Adverse Events } \\
\text { Extrapyramidal } \\
\text { Symptoms } \\
\\
\text { Withdrawal } 60 \% \\
\text { (Time to recurrence } \\
\text { outcomes only } \\
\text { included) } \\
\text { Nonrelapse Withdrawal } \\
30 \%\end{array}$ \\
\hline
\end{tabular}




\begin{tabular}{|c|c|c|c|c|c|c|}
\hline $\begin{array}{l}\text { Study, Year } \\
\text { Design } \\
\text { Location } \\
\text { Funder } \\
\text { Risk of Bias } \\
\text { PMID }\end{array}$ & $\begin{array}{c}\text { \# Randomized } \\
\text { Age (mean) } \\
\text { Sex (\% Female) } \\
\text { Race (\% White) } \\
\text { Diagnosis } \\
\text { (\% BP I, II, NOS) } \\
\text { Setting }\end{array}$ & $\begin{array}{c}\text { Inclusions } \\
\text { Key Exclusions }\end{array}$ & $\begin{array}{c}\text { Intervention } \\
\text { Dosage }\end{array}$ & $\begin{array}{c}\text { Comparison } \\
\text { Dosage }\end{array}$ & $\begin{array}{l}\text { Followup } \\
\text { Duration }\end{array}$ & $\begin{array}{c}\text { Outcomes } \\
\text { Reported } \\
\begin{array}{c}\text { Withdrawal (\%) at } \\
\text { endpoint }\end{array}\end{array}$ \\
\hline $\begin{array}{l}\text { Berwaerts, } 2012^{25} \\
\text { RCT } \\
\text { Multisite } \\
5 \text { Continents } \\
\text { Industry } \\
\text { RoB High } \\
22377512\end{array}$ & $\begin{array}{l}\mathrm{N}=383 \\
\text { Mean Age } 40 \\
\text { Female } 53 \% \\
\text { White } 60 \% \\
\text { BP I } 100 \% \\
\text { Outpatient }\end{array}$ & $\begin{array}{l}\text { Remission; } \\
\text { YMRS and MADRS } \leq 12 \text { for } \\
\text { last three weeks of acute } \\
\text { and continuation treatment } \\
\text { study phases } \\
\\
\text { First manic episode } \\
\text { Schizoaffective } \\
\text { Substance abuse } \\
\text { Other mental health } \\
\text { Neurological disorders } \\
\text { Labs/other conditions }\end{array}$ & $\begin{array}{l}\text { Paliperidone EX } \\
\text { 3-12 mg/day } \\
(n=152)\end{array}$ & $\begin{array}{l}\text { C1: Placebo } \\
(\mathrm{n}=148) \\
\\
\text { C2: Olanzapine } \\
\text { 5-20 mg/day - } \\
\text { few usable } \\
\text { outcomes } \\
\mathrm{N}=83\end{array}$ & $\begin{array}{l}\text { Up to } 3 \text { years } \\
\text { (until } \\
\text { recurrence) }\end{array}$ & $\begin{array}{l}\text { YMRS } \\
\text { Relapse (HAM-D } \geq 14 \text { ) } \\
\text { Adverse Events } \\
\text { Lab Values } \\
\text { Withdrawal } 38 \%\end{array}$ \\
\hline $\begin{array}{l}\text { Amsterdam, } 2015^{26} \\
\text { RCT extension of } \\
\text { responders } \\
\text { Single Site } \\
\text { US } \\
\text { Government } \\
\text { RoB High } \\
26143402\end{array}$ & $\begin{array}{l}\mathrm{N}=55 \\
\text { Mean Age } 42 \\
\text { Female } 54 \% \\
\text { White } 17 \% \\
\text { BP II } 100 \% \\
\text { Outpatient }\end{array}$ & $\begin{array}{l}\text { Responders to RCT phase: } \\
>50 \% \text { reduction in baseline } \\
\text { HAM-D + CGI-BP-S <3 } \\
\text { Substance abuse } \\
\text { Neurological disorders } \\
\text { Taking other meds } \\
\text { Pregnant/nursing } \\
\text { Labs/other conditions }\end{array}$ & $\begin{array}{l}\text { Venlafaxine } \\
75-375 \text { mg/day } \\
\text { (mean 253.9 } \\
\text { mg/day) }\end{array}$ & $\begin{array}{l}\text { Lithium } \\
300-1200 \\
\text { mg/day } \\
\text { (Serum level of } \\
0.8-1.5 \text { mEq/L) }\end{array}$ & 6 months & $\begin{array}{l}\text { Relapse (HAM-D } \\
>14+\text { CGI>3 for at least } \\
14 \text { days) } \\
\text { Relapse hazard } \\
\text { Time to relapse } \\
\text { YMRS } \\
\text { Withdrawal 18\% (after } \\
43 \% \text { attrition from } \\
\text { acute treatment) }\end{array}$ \\
\hline
\end{tabular}




\begin{tabular}{|c|c|c|c|c|c|c|}
\hline $\begin{array}{l}\text { Study, Year } \\
\text { Design } \\
\text { Location } \\
\text { Funder } \\
\text { Risk of Bias } \\
\text { PMID }\end{array}$ & $\begin{array}{c}\text { \# Randomized } \\
\text { Age (mean) } \\
\text { Sex (\% Female) } \\
\text { Race (\% White) } \\
\text { Diagnosis } \\
\text { (\% BP I, II, NOS) } \\
\text { Setting }\end{array}$ & $\begin{array}{l}\text { Inclusions } \\
\text { Key Exclusions }\end{array}$ & $\begin{array}{l}\text { Intervention } \\
\text { Dosage }\end{array}$ & $\begin{array}{l}\text { Comparison } \\
\text { Dosage }\end{array}$ & $\begin{array}{l}\text { Followup } \\
\text { Duration }\end{array}$ & $\begin{array}{c}\text { Outcomes } \\
\text { Reported } \\
\text { Withdrawal (\%) at } \\
\text { endpoint }\end{array}$ \\
\hline $\begin{array}{l}\text { Newport, } 2008^{27} \\
\text { Observational } \\
\text { Single Site } \\
1 \text { Continent } \\
\text { Government/Nonprofit } \\
\text { High } \\
18402631\end{array}$ & $\begin{array}{l}\mathrm{N}=26 \\
\text { Mean Age NR } \\
\text { Female 100\% } \\
\text { White 91\% } \\
\text { BP I 73\% } \\
\text { BP-II 23\% } \\
\text { BP-NOS 4\% } \\
\text { Outpatient }\end{array}$ & $\begin{array}{l}\text { Euthymic at conception } \\
\text { Labs/Other Conditions }\end{array}$ & $\begin{array}{l}\text { Lamotrigine } \\
\text { Average } 252 \mathrm{mg} / \text { day }\end{array}$ & $\begin{array}{l}\text { Mood Stabilizer } \\
\text { Discontinuation } \\
\text { Initial doses } \\
\text { Divalproex: } \\
1200 \text { mg/day } \\
\text { average } \\
\text { Lithium: } 825 \\
\text { mg/day } \\
\text { average } \\
\text { Lamotrigine: } \\
\text { 190 mg/day } \\
\text { average }\end{array}$ & 40 weeks & $\begin{array}{l}\text { Survival Time } \\
\text { Withdrawal NR }\end{array}$ \\
\hline
\end{tabular}




\begin{tabular}{|c|c|c|c|c|c|c|}
\hline $\begin{array}{l}\text { Study, Year } \\
\text { Design } \\
\text { Location } \\
\text { Funder } \\
\text { Risk of Bias } \\
\text { PMID }\end{array}$ & $\begin{array}{c}\text { \# Randomized } \\
\text { Age (mean) } \\
\text { Sex (\% Female) } \\
\text { Race (\% White) } \\
\text { Diagnosis } \\
\text { (\% BP I, II, NOS) } \\
\text { Setting }\end{array}$ & $\begin{array}{c}\text { Inclusions } \\
\text { Key Exclusions }\end{array}$ & $\begin{array}{c}\text { Intervention } \\
\text { Dosage }\end{array}$ & $\begin{array}{c}\text { Comparison } \\
\text { Dosage }\end{array}$ & $\begin{array}{l}\text { Followup } \\
\text { Duration }\end{array}$ & $\begin{array}{c}\text { Outcomes } \\
\text { Reported } \\
\begin{array}{c}\text { Withdrawal (\%) at } \\
\text { endpoint }\end{array}\end{array}$ \\
\hline $\begin{array}{l}\text { Weisler, } 2011^{7} \\
\text { RCT } \\
\text { Multisite } \\
5 \text { continents } \\
\text { Industry } \\
\text { RoB Moderate } \\
22054050\end{array}$ & $\begin{array}{l}\mathrm{N}=1226 \\
\text { Mean Age } 40 \\
\text { Female 53\% } \\
\text { White } 63 \% \\
\text { BP } 1100 \% \\
\text { Outpatient }\end{array}$ & $\begin{array}{l}\text { Meeting stability criteria of } \\
\text { YMRS } \leq 12 \text { and MADRS } \leq \\
12 \text { after last episode of } \\
\text { depression/mania/mixed } \\
\text { episode at study entry or } \\
\text { within past two years } \\
\\
\text { Substance Abuse } \\
\text { Other Mental Health } \\
\text { Conditions } \\
\text { Pregnant/Nursing } \\
\text { Labs/Other Conditions }\end{array}$ & $\begin{array}{l}\text { Quetiapine } \\
300-800 \mathrm{mg} / \text { day } \\
\text { (543 mg/day mean) } \\
\mathrm{N}=404\end{array}$ & $\begin{array}{l}\text { C1: Placebo } \\
\mathrm{N}=404 \\
\\
\mathrm{C} 2: \text { Lithium } \\
600-1800 \\
\mathrm{mg} / \mathrm{day} \\
0.6-1.2 \mathrm{mEq} / \mathrm{L} \\
(0.63 \mathrm{mEq} / \mathrm{L} \\
\text { mean) } \\
\mathrm{N}=364\end{array}$ & 104 weeks & $\begin{array}{l}\text { Time to recurrence any } \\
\text { mood (algorithm) } \\
\text { Time to manic event } \\
\text { Time to depressive } \\
\text { event } \\
\text { Time to all-cause } \\
\text { discontinuation } \\
\text { SDS } \\
\text { MOS-Cog } \\
\text { CGI-BP } \\
\text { PANNS-P } \\
\text { WPAI } \\
\text { TMT } \\
\text { Withdrawal 55\% (Time } \\
\text { to recurrence } \\
\text { outcomes only } \\
\text { included) } \\
\text { Nonrelapse withdrawal } \\
22 \%\end{array}$ \\
\hline
\end{tabular}

Abbreviations: AIMS=Abnormal Involuntary Movement Scale; BARS=Barnes Akathisia Scale; BIS-11=Barratt Impulsiveness Scale; BMI=Body Mass Index; BP=bipolar disorder; C=Comparison; CGI-BP=Clinical Global Impressions Scale for Bipolar Disorder; CGI-BP-M=Clinical Global Impressions Scale-Bipolar-modified (for long-term followup); CGI-BP-S=Clinical Global Impressions, Bipolar, Severity Scale; CGI-EI=Clinical Global Impressions-Efficacy Index; CGI-I=Clinical Global Impressions Scale, Improvement; CGI-S=Clinical Global Impressions, Severity Scale; DSM=Diagnostic and Statistical Manual of Mental Disorders; DSS=Depressive Syndrome Scale;

EPS=extrapyramidal symptoms; ESRS=Extrapyramidal Symptom Rating Scale; GAF=General Assessment of Functioning Scale; GAS=Global Assessment Scale; HAM-

D=Hamilton Scale for Depression; IMPS=Inpatient Multidimensional Psychiatric Scale; KAS=Katz Adjustment Scale; LIFE-RIFT= Longitudinal Interval Follow-up EvaluationRange of Impaired Functioning Tool; MADRS=Montgomery-Asberg Depression Rating Scale; MOS-Cog=Medical Outcomes Study Cognitive Scale; MRS=Mania Rating Scale; NOS=not otherwise specified; NR=not reported; PANSS=Positive and Negative Syndrome Scale; PGWB=Psychological General Well-being Scale; PMID=PubMed Identification Number; PSQI=Pittsburgh Sleep Quality Index; QIDS-SR=Quick Inventory of Depressive Symptomatology (Self-reported); RCT=randomized controlled trial; ROB=risk of bias; SAS=Simpson Angus Scale; SDS=Sheehan Disability Scale; TMT=Trail Making Test; WPAI=Work Productivity and Activity Impairment Questionnaire; YMRS =Young Mania Rating Scale 
Appendix Table 16. Summary risk of bias assessments: other monotherapy

\begin{tabular}{|c|c|c|c|}
\hline Drug & $\begin{array}{c}\text { Study } \\
\text { Funding Source } \\
\text { PMID }\end{array}$ & $\begin{array}{l}\text { Overall Risk of Bias } \\
\text { Assessment }\end{array}$ & Rationale \\
\hline $\begin{array}{l}\text { Long-activing } \\
\text { injectable } \\
\text { Aripiprazole vs. } \\
\text { placebo }\end{array}$ & $\begin{array}{l}\text { Calabrese, } 2017^{8} \\
\text { Industry } \\
28146613\end{array}$ & Moderate & $\begin{array}{l}\text { Allocation concealment unclear. Differential attrition between arms. } 61.7 \% \text { attrition rate, } 29 \% \\
\text { not due to relapse. Sensitivity testing for informative withdrawal }\end{array}$ \\
\hline $\begin{array}{l}\text { Aripiprazole vs. } \\
\text { placebo }\end{array}$ & $\begin{array}{l}\text { Keck, } 2006^{9} \\
\text { Industry } \\
16669728\end{array}$ & Moderate & $\begin{array}{l}\text { Randomization not described. Allocation concealment not described. Blinding of patients, } \\
\text { providers, outcome assessors not described. Attrition 58\% and differential drop-out. }\end{array}$ \\
\hline $\begin{array}{l}\text { Olanzapine vs. } \\
\text { placebo }\end{array}$ & $\begin{array}{l}\text { Tohen, } 2006^{19} \\
\text { Industry } \\
16449478 \\
\end{array}$ & Moderate & $\begin{array}{l}\text { Differential withdrawal rates (32\% olan, } 13 \% \text { plac) and high dropout of olanzapine group may } \\
\text { bias results. }\end{array}$ \\
\hline $\begin{array}{l}\text { Olanzapine vs. } \\
\text { placebo vs. } \\
\text { risperidone }\end{array}$ & $\begin{array}{l}\text { Vieta, } 2012^{23} \\
\text { Industry } \\
22503488 \\
\end{array}$ & High & $\begin{array}{l}\text { High - blinding and randomization procedures not well described. Period II results are biased } \\
\text { by the drug assignment being open label. Period three efficacy scores are likely to be biased } \\
\text { by the large attrition rate. }\end{array}$ \\
\hline $\begin{array}{l}\text { Risperidone vs. } \\
\text { placebo }\end{array}$ & $\begin{array}{l}\text { Quiroz, } 2010^{22} \\
\text { Industry } \\
20227682\end{array}$ & $\begin{array}{l}\text { Moderate } \\
\text { (High for log-ramk } \\
\text { test) }\end{array}$ & Randomization and blinding well described. Nonrelapse withdrawal 26\%. \\
\hline \multirow[t]{2}{*}{$\begin{array}{l}\text { Lamotrigine vs. } \\
\text { Lithium vs. } \\
\text { placebo }\end{array}$} & $\begin{array}{l}\text { Bowden, } 2003^{4} \\
\text { Industry } \\
12695317\end{array}$ & $\begin{array}{l}\text { Moderate } \\
\text { (High for log rank } \\
\text { test) }\end{array}$ & $\begin{array}{l}\text { Randomization and blinding procedures not described. Balanced traits among groups at } \\
\text { baseline. Efficacy data may be biased by dropout as endpoints are LOCF and differential } \\
\text { nonrelapse dropout rates that range from } 16-25 \% \text { of patients for each arm. }\end{array}$ \\
\hline & $\begin{array}{l}\text { Calabrese, } 2003^{5} \\
\text { Industry } \\
14628976\end{array}$ & $\begin{array}{l}\text { Moderate } \\
\text { (High for log rank } \\
\text { test) }\end{array}$ & $\begin{array}{l}\text { Randomization and allocation concealment not described. Balanced traits among groups at } \\
\text { baseline. Efficacy data may be biased by dropout as endpoints are LOCF. 34\% withdrawal. }\end{array}$ \\
\hline $\begin{array}{l}\text { Lamotrigine vs. } \\
\text { placebo (rapid } \\
\text { cyclers) }\end{array}$ & $\begin{array}{l}\text { Calabrese, } 2000^{17} \\
\text { Industry } \\
11105737\end{array}$ & $\begin{array}{l}\text { Moderate } \\
\text { (High for log rank } \\
\text { test) }\end{array}$ & $\begin{array}{l}\text { Randomization and allocation concealment not described. Balanced traits among groups at } \\
\text { baseline. ITT using LOCF. Log rank test. } 67 \% \text { attrition, 15\% nont related to relapse. }\end{array}$ \\
\hline $\begin{array}{l}\text { Fluoxetine vs. } \\
\text { lithium vs. } \\
\text { placebo }\end{array}$ & $\begin{array}{l}\text { Amsterdam, } \\
2010^{3} \\
\text { Government/Non } \\
\text { profit } \\
20360317 \\
\end{array}$ & Moderate & $\begin{array}{l}\text { Randomization may not have been successful as it relates to a person's likelihood of relapse; } \\
\text { randomization, allocation, and blinding procedures are underdescribed. } 23 \% \text { dropout. }\end{array}$ \\
\hline $\begin{array}{l}\text { Divalproex vs. } \\
\text { lithium (rapid } \\
\text { cyclers) }\end{array}$ & $\begin{array}{l}\text { Calabrese, } 2005^{16} \\
\text { Government } \\
16263857 \\
\end{array}$ & $\begin{array}{l}\text { Moderate } \\
\text { (High for log rank } \\
\text { test) }\end{array}$ & $\begin{array}{l}\text { Randomization and allocation not described. Balanced traits among groups at baseline. ITT. } \\
\text { Log rank test. } 25 \% \text { nonrelapse attrition }\end{array}$ \\
\hline
\end{tabular}




\begin{tabular}{|c|c|c|c|}
\hline Drug & $\begin{array}{l}\text { Study } \\
\text { Funding Source } \\
\text { PMID }\end{array}$ & $\begin{array}{c}\text { Overall Risk of Bias } \\
\text { Assessment }\end{array}$ & Rationale \\
\hline $\begin{array}{l}\text { Divalproex vs. } \\
\text { lithium vs. } \\
\text { placebo }\end{array}$ & $\begin{array}{l}\text { Bowden, } 2000^{1} \\
\text { Industry } \\
10807488 \\
12784116^{2}\end{array}$ & High & Appears to be unblinded. Randomization not described. Nonrelapse dropout of $40 \%$. \\
\hline \multirow[t]{2}{*}{$\begin{array}{l}\text { Carbamazepine } \\
\text { vs. lithium }\end{array}$} & $\begin{array}{l}\text { Hartong, } 2003^{10} \\
\text { Industry/Governm } \\
\text { ent } \\
12633122\end{array}$ & Low & No sources of bias identified. \\
\hline & $\begin{array}{l}\text { Greil, } 1997^{11} \\
\text { Government } \\
9165384 \\
9864077^{12} \\
10529070^{13} \\
10529071^{14} \\
11093063^{15}\end{array}$ & High & $\begin{array}{l}\text { Study is unblinded, there may be underlying differences between the groups in their likelihood } \\
\text { to recur because of differences baseline disease history. Dropout is inconsistent between } \\
\text { groups and no explanation is provided for why patients dropped. }\end{array}$ \\
\hline $\begin{array}{l}\text { Lithium }+ \\
\text { valproate vs. } \\
\text { valproate vs. } \\
\text { lithium }\end{array}$ & $\begin{array}{l}\text { Balance } \\
\text { Investigators, } \\
2010^{18} \\
\text { Industry } \\
20092882 \\
\end{array}$ & Moderate & Open label. Intention To Treat used, but handling of dropouts/missing data not described. \\
\hline $\begin{array}{l}\text { Olanzapine vs. } \\
\text { divalproex }\end{array}$ & $\begin{array}{l}\text { Tohen, } 2003^{20} \\
\text { Industry } \\
12832240 \\
\text { Extension of } \\
\text { Tohen, } 2002 b^{21} \\
12042191\end{array}$ & High & $\begin{array}{l}\text { Randomization and blinding procedures not described. Log rank test. Attrition 84\%, unclear } \\
\text { nonrelapse withdrawal. }\end{array}$ \\
\hline $\begin{array}{l}\text { Olanzapine vs. } \\
\text { lithium }\end{array}$ & $\begin{array}{l}\text { Tohen, } 2005^{24} \\
\text { Industry } \\
15994710\end{array}$ & High & $\begin{array}{l}\text { Well-constructed and reported study with high attrition rate (61\%). Time to recurrence only } \\
\text { includable outcome (log rank test). }\end{array}$ \\
\hline $\begin{array}{l}\text { Lamotrigine vs. } \\
\text { discontinue mood } \\
\text { stabilizers }\end{array}$ & $\begin{array}{l}\text { Newport, } 2008^{27} \\
\text { Gov't + nonprofit } \\
18402631\end{array}$ & High & Patients chose treatment assignment. Initial differences at baseline are noted. \\
\hline $\begin{array}{l}\text { Venlafaxine vs. } \\
\text { placebo }\end{array}$ & $\begin{array}{l}\text { Amsterdam, } \\
2015^{26} \\
\text { Government } \\
26143402 \\
\end{array}$ & $\begin{array}{l}\text { High (RCT extension } \\
\text { of responders) }\end{array}$ & $\begin{array}{l}\text { RCT extension. Did report baseline at maintenance phase; appeared balanced on measured } \\
\text { variables. } 43 \% \text { loss from initial randomization, further 18\% at maintenance }\end{array}$ \\
\hline
\end{tabular}




\begin{tabular}{|l|l|l|l|}
\hline \multicolumn{1}{|c|}{ Drug } & $\begin{array}{c}\text { Study } \\
\text { Funding Source } \\
\text { PMID }\end{array}$ & $\begin{array}{l}\text { Overall Risk of Bias } \\
\text { Assessment }\end{array}$ & Rationale \\
\hline $\begin{array}{l}\text { Paliperidone vs. } \\
\text { placebo vs. } \\
\text { olanzapine }\end{array}$ & $\begin{array}{l}\text { Berwaerts, 2012 } 25 \\
\text { Industry } \\
22377512\end{array}$ & $\begin{array}{l}\text { High (RCT } \\
\text { Extension) }\end{array}$ & $\begin{array}{l}\text { Large dropout rate among all study arms, across all time periods Did report baseline at } \\
\text { maintenance phase. }\end{array}$ \\
\hline $\begin{array}{l}\text { Quetiapine vs. } \\
\text { lithium vs. } \\
\text { placebo + lithium }\end{array}$ & $\begin{array}{l}\text { Weisler, 20117 } \\
\text { Industry } \\
22054050\end{array}$ & $\begin{array}{l}\text { Moderate } \\
\text { related to dropout rates. Overall 21\% withdraw due to reasons unrelated to recurrence; } \\
\text { however by week 16 more than 50\% of the placebo group has dropped for all causes, as well } \\
\text { as 40\% of the lithium group and 25\% of the quetiapine group) }\end{array}$ \\
\hline
\end{tabular}

Abbreviations: $\mathrm{BP}=$ bipolar; LOCF=Last observation carried forward; PMID=PubMed Identification Number; RCT=randomized controlled trial 
Appendix Table 17. Outcomes summary: other monotherapy versus placebo for maintenance

\begin{tabular}{|c|c|c|c|c|c|c|}
\hline Drug & $\begin{array}{l}\text { Study } \\
\text { Comparison } \\
\text { PMID }\end{array}$ & Responder/Remitter & Symptom & Function & Other & $A E$ \\
\hline $\begin{array}{l}\text { Long-acting } \\
\text { injectable } \\
\text { Aripiprazole vs. } \\
\text { placebo }\end{array}$ & $\begin{array}{l}\text { Calabrese, } \\
2017^{8}\end{array}$ & $\begin{array}{l}\text { Time to any recurrence } \\
52 \text { weeks } \\
\text { HR } 0.45 \text { (95\% Cl 0.30, } \\
0.68) \\
\text { Favors Aripiprazole } \\
\frac{\text { Any relapse }}{52 \text { weeks }} \\
\text { Aripiprazole } 35 / 132 \\
\text { Placebo 68/133 } \\
\text { Favors Aripiprazole } \\
\text { p<0.0001 } \\
\text { Manic relapse } \\
52 \text { weeks } \\
\text { Favors Aripiprazole } \\
\text { p<0.0001 } \\
\text { Depression relapse } \\
52 \text { weeks } \\
\text { NS }\end{array}$ & $\begin{array}{l}\text { NA } \\
\text { (Exclude for attrition }=62 \% \text { ) }\end{array}$ & $\underline{\mathrm{NR}}$ & $\begin{array}{l}\text { Weight gain }>7 \% \\
\text { Aripiprazole } 23 / 132 \\
\text { Placebo } 17 / 133\end{array}$ & $\begin{array}{l}\text { SAE }>1 \text { patient } \\
\text { Aripiprazole } 0.8 \% \\
\text { Placebo } 2.3 \% \\
1 \text { death reported } \\
\text { EPS } \\
\text { Aripiprazole } 36 / 132 \\
\text { Placebo } 22 / 133\end{array}$ \\
\hline $\begin{array}{l}\text { Aripiprazole vs. } \\
\text { placebo }\end{array}$ & $\begin{array}{l}\text { Keck, } 2006^{9} \\
16669728\end{array}$ & $\begin{array}{l}\frac{\text { Time to relapse }}{26 \text { weeks }} \\
\text { HR } 0.52 \text { (95\% Cl 0.30, } \\
0.91) \\
\text { Favors Aripiprazole } \\
\text { Time to manic relapse } \\
26 \text { weeks } \\
\text { HR } 0.31 \text { (95\% Cl 0.12, } \\
0.77) \\
\text { Favors Aripiprazole } \\
\frac{\text { Time to depression }}{\text { relapse }} \\
26 \text { weeks } \\
\text { NS }\end{array}$ & $\begin{array}{l}\text { NA } \\
\text { (Exclude for attrition = 58\% }\end{array}$ & $\begin{array}{l}\text { NA } \\
\text { (Exclude } \\
\text { for } \\
\text { attrition = } \\
58 \%\end{array}$ & $\underline{\mathrm{NR}}$ & $\begin{array}{l}1 \text { placebo group } \\
\text { patient attempted } \\
\text { suicide } \\
\text { EPS "more frequently } \\
\text { in the aripiprazole } \\
\text { group" } \\
7 \% \text { Weight gain } \\
\text { Aripiprazole } 13 \% \\
\text { Placebo } 0 \%\end{array}$ \\
\hline
\end{tabular}




\begin{tabular}{|c|c|c|c|c|c|c|}
\hline Drug & $\begin{array}{l}\text { Study } \\
\text { Comparison } \\
\text { PMID }\end{array}$ & Responder/Remitter & Symptom & Function & Other & AE \\
\hline $\begin{array}{l}\text { Divalproex vs. } \\
\text { placebo }\end{array}$ & $\begin{array}{l}\text { Bowden, } 2000^{1} \\
10807488 \\
12784116^{2}\end{array}$ & $\begin{array}{l}\text { Time to any recurrence } \\
12 \text { months } \\
\text { NS } \\
\text { Time to mania recurrence } \\
12 \text { months } \\
\text { NS } \\
\\
\text { Time to depression } \\
\text { recurrence } \\
12 \text { months } \\
\text { NS }\end{array}$ & $\begin{array}{l}\text { NA } \\
\text { (Exclude for attrition }=69 \%)\end{array}$ & $\mathrm{NR}$ & $\underline{\mathrm{NR}}$ & 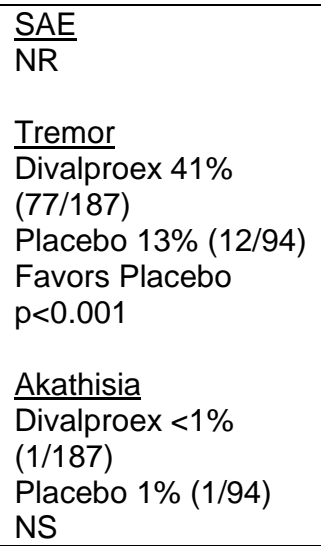 \\
\hline $\begin{array}{l}\text { Fluoxetine vs. } \\
\text { placebo }\end{array}$ & $\begin{array}{l}\text { Amsterdam, } \\
2010^{3} \\
20360317\end{array}$ & $\begin{array}{l}\text { Time to Relapse to } \\
\text { Depression } \\
50 \text { week } \\
\text { Log rank } \\
\text { Favors Fluoxetine } \\
\mathrm{p}=0.03\end{array}$ & $\frac{\mathrm{NA}}{(\text { Exclude for attrition }=72 \%)}$ & $\mathrm{NR}$ & NR & $\begin{array}{l}\text { SAE } \\
\text { No Events } \\
\text { Akathisia } \\
\begin{array}{l}\text { Fluoxetine } 1 \text { event/28 } \\
\text { patients }\end{array}\end{array}$ \\
\hline $\begin{array}{l}\text { Lamotrigine vs. } \\
\text { placebo }\end{array}$ & $\begin{array}{l}\text { Bowden, } 2003^{4} \\
12695317\end{array}$ & $\begin{array}{l}\frac{\text { Time to any recurrence }}{18 \text { months }} \\
\text { Log rank } \\
\text { Favors Lamotrigine } \\
\mathrm{p}=0.02 \\
\frac{\text { Time to mania recurrence }}{18 \text { months }} \\
\text { Log rank } \\
\text { NS } \\
\frac{\text { Time to depression }}{\text { recurrence }} \\
18 \text { months } \\
\text { Log rank } \\
\text { Favors Lamotrigine } \\
\mathrm{p}=0.002\end{array}$ & $\begin{array}{l}\text { NA } \\
(\text { Exclude for attrition }=80 \%)\end{array}$ & NA & NA & $\begin{array}{l}\frac{\text { SAE }}{1 \text { lamotrigine patient }} \\
\text { hospitzalized for rash } \\
1 \text { lamotrigine patient } \\
\text { attempted suicide } \\
\text { Suicidality per HAM-D } \\
\text { NS between groups }\end{array}$ \\
\hline
\end{tabular}




\begin{tabular}{|c|c|c|c|c|c|c|}
\hline Drug & $\begin{array}{l}\text { Study } \\
\text { Comparison } \\
\text { PMID }\end{array}$ & Responder/Remitter & Symptom & Function & Other & AE \\
\hline & $\begin{array}{l}\text { Calabrese, } \\
2003^{5} \\
14628976\end{array}$ & $\begin{array}{l}\frac{\text { Time to any recurrence }}{18 \text { months }} \\
\text { Log rank } \\
\text { Favors Lamotrigine } \\
\mathrm{p}=0.03 \\
\frac{\text { Time to mania recurrence }}{18 \text { months }} \\
\text { Log rank } \\
\text { NS } \\
\text { Time to depression } \\
\frac{\text { recurrence }}{18 \text { months }} \\
\text { Log rank } \\
\text { Favors Lamotrigine } \\
\text { p=0.047 }\end{array}$ & $\begin{array}{l}\text { NA } \\
\text { (Exclude for attrition = 85\%) }\end{array}$ & NA & NA & $\begin{array}{l}\text { SAE } \\
1 \text { death in lamotrigine } \\
\text { groups } \\
\text { Suicidality per HAM-D } \\
\text { NS between groups } \\
\text { Tremor } \\
\text { Lamotrigine 5\% } \\
\text { (9/169) } \\
\text { Placebo 5\% (6/121) } \\
\text { Lamotrigine vs. } \\
\text { Placebo } \\
\text { NS }\end{array}$ \\
\hline $\begin{array}{l}\text { Lamotrigine vs. } \\
\text { placebo (rapid } \\
\text { cyclers) }\end{array}$ & $\begin{array}{l}\text { Calabrese, } \\
2000^{17} \\
\text { Industry } \\
11105737\end{array}$ & $\begin{array}{l}\text { Time to new drug } \\
26 \text { weeks } \\
\text { Log rank } \\
\text { No difference by group, } \\
\text { or stratified by bipolar } \\
\text { type }\end{array}$ & $\begin{array}{l}\text { NA } \\
\text { (Exclude for attrition }=68 \% \text { ) }\end{array}$ & NA & $\begin{array}{l}\text { Time to overall } \\
\text { withdrawal } \\
\text { No difference by group. } \\
\text { BPII Lamotrigine group } \\
\text { more like remain in } \\
\text { study } \\
p=0.015\end{array}$ & $\begin{array}{l}\text { SAE } \\
\text { Lamotrigine: } 1 \\
\text { tachycardia } \\
\text { Placebo: } 1 \text { basal cell } \\
\text { carcinoma, 1benign } \\
\text { skull tumor } \\
\text { None reporte related } \\
\text { to treatment }\end{array}$ \\
\hline $\begin{array}{l}\text { Lamotrigine vs. } \\
\text { discontinued } \\
\text { mood } \\
\text { stabilizers }\end{array}$ & $\begin{array}{l}\text { Newport, } \\
2008^{27} \\
18402631\end{array}$ & $\begin{array}{l}\text { Relapse } \\
40 \text { weeks } \\
\text { Lamotrigine } 3 / 10 \\
\text { Discontinued 16/16 } \\
\text { Favors Lamotrigine }\end{array}$ & NR & NR & NR & NR \\
\hline
\end{tabular}




\begin{tabular}{|c|c|c|c|c|c|c|}
\hline Drug & $\begin{array}{l}\text { Study } \\
\text { Comparison } \\
\text { PMID }\end{array}$ & Responder/Remitter & Symptom & Function & Other & $\mathrm{AE}$ \\
\hline \multirow[t]{2}{*}{$\begin{array}{l}\text { Olanzapine vs. } \\
\text { placebo }\end{array}$} & $\begin{array}{l}\text { Tohen, } 2006^{19} \\
\text { Moderate } \\
16449478\end{array}$ & $\begin{array}{l}\text { Time to Relapse, Any } \\
\text { Mood Episode (Median) } \\
48 \text { weeks } \\
\text { Favors Olanzapine } \\
\text { HR 2.67 (95\% Cl 2.03, } \\
3.50), p<0.001 \\
\text { Time to relapse, Mania } \\
\frac{\text { (25th percentile) }}{\text { Favors Olanzapine }} \\
\text { HR 3.90 (95\% Cl 2.40, } \\
\text { 6.33), } p<0.001 \\
\text { Time to relapse, } \\
\text { depression (25th } \\
\text { percentile) } \\
\text { Favors Olanzapine } \\
\text { HR 2.10, (95\% Cl 1.46, } \\
\text { 3.02), } p<0.001\end{array}$ & $\frac{\text { NA }}{\text { (Exclude for attrition }=84 \%}$ & NA & NA & $\begin{array}{l}\text { SAE } \\
\text { Olanzapine 3\% } \\
\text { (7/225) } \\
\text { Placebo 7\% (10/136) } \\
\text { NS } \\
\text { EPS } \\
\text { Parkinsonism } \\
\text { Olanzapine 2\% } \\
\text { (5/206) } \\
\text { Placebo 0\% (0/118) } \\
\text { Favors Placebo } \\
\text { Absolute Risk } \\
\text { Reduction 0.02 (95\% } \\
\text { Cl 0.003, 0.045) } \\
\text { Akathisia } \\
\text { Olanzapine 5\% } \\
\text { (9/194) } \\
\text { Placebo 1\% (1/119) } \\
\text { NS } \\
\text { Dyskinesia } \\
\text { Olanzapine 0\% } \\
\text { (0/216) } \\
\text { Placebo 1\% (1/133) } \\
\text { NS }\end{array}$ \\
\hline & $\begin{array}{l}\text { Vieta, } 2012^{23} \\
22503488\end{array}$ & $\begin{array}{l}\text { Time to first recurrence of } \\
\text { mood symptoms } \\
18 \text { months } \\
\text { Log-rank (by region) } \\
\text { Favors Olanzapine } \\
p<0.001\end{array}$ & $\begin{array}{l}\mathrm{NA} \\
\text { (Due to attrition }=58 \% \text { ) }\end{array}$ & $\begin{array}{l}\text { NA } \\
\text { (Due to } \\
\text { attrition } \\
=58 \% \text { ) }\end{array}$ & NR & $\begin{array}{l}\text { Reported no SAE in } \\
\text { period III } \\
\text { (maintenance phase) }\end{array}$ \\
\hline
\end{tabular}




\begin{tabular}{|c|c|c|c|c|c|c|}
\hline Drug & $\begin{array}{l}\text { Study } \\
\text { Comparison } \\
\text { PMID }\end{array}$ & Responder/Remitter & Symptom & Function & Other & AE \\
\hline & $\begin{array}{l}\text { Berwaerts, } \\
2012^{25} \\
22377512\end{array}$ & $\begin{array}{l}\text { Time to Relapse to Any } \\
\text { Mood Episode } \\
\text { Favors Olanzapine } \\
\text { Post hoc } \\
\text { p }<0.001 \\
\text { (YMRS } \geq 15 \text {, CGI-BP-S for } \\
\text { mania } \geq 4 \text {; OR YMRS }<15 \text {, } \\
\text { MADRS } \geq 16 \text { and CGI-BP- } \\
\text { S for depression } \geq 4 \text {; } \\
\text { hospitalization; } \\
\text { therapeutic intervention } \\
\text { or other clinically relevant } \\
\text { indicators) } \\
\text { Paliperidone } \\
\text { Mean } 558 \text { days; } \\
\text { Placebo } \\
\text { Mean } 283 \text { days } \\
\text { Time to Relapse to Manic } \\
\text { Episode } \\
\text { Favors Olanzapine } \\
\text { Post hoc p }<0.001\end{array}$ & $\underline{\mathrm{NR}}$ & NR & $\begin{array}{l}\frac{\text { Death }}{\text { none }} \\
\text { Withdrawal for AE } \\
\text { Paliperidone 5 (3\%) } \\
\text { Placebo } 4(3 \%) \\
\text { Withdrawal for } \\
\text { Nonresponse } \\
\text { Paliperidone 1 (1\%) } \\
\text { Placebo } 2(1 \%)\end{array}$ & $\begin{array}{l}\text { SAE } \\
\text { up to } 3 \text { years } \\
\text { Olanzapine } 10 \% \\
\text { Placebo } 22 \% \\
\text { EPS } \\
\text { up to } 3 \text { years } \\
\text { Olanzapine } 1 \% \\
\text { Placebo } 1 \%\end{array}$ \\
\hline
\end{tabular}




\begin{tabular}{|c|c|c|c|c|c|c|}
\hline Drug & $\begin{array}{l}\text { Study } \\
\text { Comparison } \\
\text { PMID }\end{array}$ & Responder/Remitter & Symptom & Function & Other & AE \\
\hline $\begin{array}{l}\text { Paliparidone } \\
\text { vs. placebo }\end{array}$ & $\begin{array}{l}\text { Berwaerts, } \\
2012^{25} \\
22377512\end{array}$ & $\begin{array}{l}\text { Time to Relapse to Any } \\
\text { Mood Episode } \\
\text { Favors Paliperidone } \\
\text { HR } 1.43 \\
\text { (95\% Cl 1.03,1.98) } \\
\text { p=0.017 } \\
\text { (YMRS } \geq 15 \text {, CGI-BP-S for } \\
\text { mania } \geq 4 \text {; OR YMRS }<15 \text {, } \\
\text { MADRS } 16 \text { and CGI-BP- } \\
\text { S for depression } \geq 4 \text {; } \\
\text { hospitalization; } \\
\text { therapeutic intervention } \\
\text { or other clinically relevant } \\
\text { indicators) } \\
\text { Paliperidone } \\
\text { Mean } 558 \text { days; } \\
\text { Placebo } \\
\text { Mean } 283 \text { days } \\
\\
\text { Time to Relapse to Manic } \\
\text { Episode } \\
\text { Favors Paliperidone } \\
\text { HR 2.06 } \\
\text { (95\% Cl 1.32,3.22) } \\
\text { p<0.001 } \\
\text { Paliperidone } \\
\text { Mean 558 days; } \\
\text { Placebo } \\
\text { Mean 283 days }\end{array}$ & $\begin{array}{l}\frac{\text { YMRS Change }}{\text { up to } 3 \text { years }} \\
\text { Favors Paliperidone } \\
\text { LSM difference }-4.5(95 \% \\
\mathrm{Cl}-6.92,-1.98) \\
\text { MADRS Change } \\
\text { up to } 3 \text { years } \\
\text { NS } \\
\text { LSM difference } 0.3(95 \% \mathrm{Cl} \\
-1.87,2.55)\end{array}$ & NR & $\begin{array}{l}\text { Death } \\
\text { up to } 3 \text { years } \\
\text { Paliperidone } 2 \\
\text { (pneumonia, overdose) } \\
\text { Placebo } 0 \\
\text { Withdrawal for AE } \\
\text { Olanzapine } 7 / 83(8 \%) \\
\text { Placebo } 4 / 148(3 \%) \\
\text { Withdrawal for } \\
\text { Nonresponse } \\
\text { Olanzapine } 0 / 83(1 \%) \\
\text { Placebo } 2 / 148(1 \%)\end{array}$ & $\begin{array}{l}\text { SAE } \\
\text { up to } 3 \text { years } \\
\text { Paliperidone } 11 \% \\
\text { Placebo } 22 \% \\
\text { EPS } \\
\text { up to } 3 \text { years } \\
\text { Paliperidone } 1 \% \\
\text { Placebo } 1 \%\end{array}$ \\
\hline
\end{tabular}




\begin{tabular}{|c|c|c|c|c|c|c|}
\hline Drug & $\begin{array}{l}\text { Study } \\
\text { Comparison } \\
\text { PMID }\end{array}$ & Responder/Remitter & Symptom & Function & Other & AE \\
\hline $\begin{array}{l}\text { Quetiapine vs. } \\
\text { placebo }\end{array}$ & $\begin{array}{l}\text { Weisler, } 2011^{7} \\
22054050\end{array}$ & $\begin{array}{l}\text { Time to recurrence of any } \\
\text { mood event } \\
\text { Favors Quetiapine } \\
\text { HR 0.29 ( } 95 \% \mathrm{Cl} 0.23 \text {, } \\
0.38), p<0.0001 \\
\text { Time to recurrence of } \\
\text { manic event } \\
\text { Favors Quetiapine } \\
\text { HR 0.29 (95\% Cl } 0.21 \text {, } \\
0.40), p<0.0001 \\
\text { Time to recurrence of } \\
\text { depression symptoms } \\
\text { Favors Quetiapine } \\
\text { HR } 0.30 \text { (95\% Cl } 0.20 \text {, } \\
0.44), p<0.0001\end{array}$ & $\begin{array}{l}\underline{\mathrm{NA}} \\
\text { (Exclude for attrition } 55 \% \text { ) }\end{array}$ & NR & $\underline{N R}$ & $\begin{array}{l}\frac{\text { SAE }}{5 \text { quetiapine }} \\
11 \text { placebo } \\
\frac{\text { EPS }}{16 \text { quetiapine }} \\
18 \text { placebo } \\
\text { No deaths }\end{array}$ \\
\hline \multirow[t]{2}{*}{$\begin{array}{l}\text { Risperidone vs. } \\
\text { placebo }\end{array}$} & $\begin{array}{l}\text { Quiroz, } 2010^{22} \\
20227682\end{array}$ & $\begin{array}{l}\text { Time to first recurrence of } \\
\text { mood symptoms } \\
24 \text { months } \\
\text { Log-rank test } \\
\text { Favors Risperidone } \\
\text { HR } 0.40 \text { (95\% Cl } 0.27, \\
0.59) \\
p=0.001\end{array}$ & $\begin{array}{l}\text { NA } \\
\text { (Exclude for attrition }=64 \%)\end{array}$ & NA & $\begin{array}{l}\text { Time to withdrawal for } \\
\frac{\text { any reason }}{24 \text { months }} \\
\text { Log-rank test } \\
\text { Favors Risperidone } \\
\text { HR } 0.49 \text { (95\% Cl 0.36, } \\
0.67) \\
p=0.001\end{array}$ & $\begin{array}{l}\frac{\mathrm{SAE}}{1 \text { diabetes mellitus in }} \\
\text { Risperidone group } \\
\frac{\mathrm{EPS}}{3 \% \text { both groups }}\end{array}$ \\
\hline & $\begin{array}{l}\text { Vieta, } 2012^{23} \\
22503488\end{array}$ & $\begin{array}{l}\text { Time to first recurrence of } \\
\text { mood symptoms } \\
18 \text { months } \\
\text { Log-rank (by region) } \\
\text { Favors Risperidone } \\
p=0.03\end{array}$ & $\begin{array}{l}\mathrm{NA} \\
\text { (Exclude for attrition }=58 \% \text { ) }\end{array}$ & NA & NR & $\begin{array}{l}\text { Reported no SAE in } \\
\text { period III } \\
\text { (maintenance phase) }\end{array}$ \\
\hline
\end{tabular}

Abbreviations: AE=Adverse Events; CGI-BP-S=Clinical Global Impressions, Bipolar, Severity Scale; CI=Confidence Interval; EPS=extrapyramidal symptoms; HAM-

D=Hamilton Scale for Depression; HR=Hazard Ratio; LSM=least-squares means; MADRS=Montgomery-Asberg Depression Rating Scale; NA=Not applicable; NR=not reported; NS=not significant; OR=Odds Ratio; PMID=PubMed Identification Number; SAE=Serious Adverse Events; YMRS = Young Mania Rating Scale 
Appendix Table 18. Strength of evidence assessment: other monotherapy versus placebo for maintenance

\begin{tabular}{|c|c|c|c|c|c|c|c|c|}
\hline Comparison & Outcome & $\begin{array}{l}\text { \# Studies/ } \\
\text { Design } \\
\text { (n analyzed) }\end{array}$ & $\begin{array}{c}\text { Finding or Summary } \\
\text { Statistic } \\
\text { Outcome Timing }\end{array}$ & $\begin{array}{c}\text { Study } \\
\text { Limitations }\end{array}$ & Consistency & Directness & Precision & $\begin{array}{l}\text { Overall } \\
\text { Gradel } \\
\text { Conclusion }\end{array}$ \\
\hline $\begin{array}{l}\text { Long-acting } \\
\text { injectable } \\
\text { Aripiprazole } \\
\text { vs. placebo } \\
\end{array}$ & $\begin{array}{l}\text { Time to relapse } 52 \\
\text { wks } \\
\text { Relapse } 52 \text { wks }\end{array}$ & $\begin{array}{l}1 \mathrm{RCT} \\
(\mathrm{n}=266)\end{array}$ & See table above & Moderate & Unknown & Direct & Imprecise & Insufficient \\
\hline $\begin{array}{l}\text { Aripiprazole } \\
\text { vs. placebo }\end{array}$ & $\begin{array}{l}\text { Time to Relapse } \\
26 \text { wks }\end{array}$ & $\begin{array}{l}1 \mathrm{RCT} \\
(\mathrm{n}=161)\end{array}$ & See table above & Moderate & Unknown & Direct & Imprecise & Insufficient \\
\hline $\begin{array}{l}\text { Divalproex } \\
\text { vs. placebo }\end{array}$ & $\begin{array}{l}\text { Time to relapse } 52 \\
\text { wks }\end{array}$ & $\begin{array}{l}1 \mathrm{RCT} \\
(\mathrm{n}=281)\end{array}$ & See table above & High & Unknown & Direct & Imprecise & Insufficient \\
\hline $\begin{array}{l}\text { Fluoxetine vs. } \\
\text { placebo }\end{array}$ & $\begin{array}{l}\text { Time to relapse to } \\
\text { depression } 50 \text { wks }\end{array}$ & $\begin{array}{l}1 \text { RCT } \\
(n=55)\end{array}$ & See table above & Moderate & Unknown & Direct & Imprecise & Insufficient \\
\hline $\begin{array}{l}\text { Lamotrigine } \\
\text { vs. placebo }\end{array}$ & $\begin{array}{l}\text { Time to relapse } 18 \\
\text { mths }\end{array}$ & $\begin{array}{l}2 \mathrm{RCT} \\
(\mathrm{n}=471)\end{array}$ & See table above & $\begin{array}{l}\text { High (log rank } \\
\text { tests) }\end{array}$ & Consistent & Direct & Imprecise & Insufficient \\
\hline $\begin{array}{l}\text { Lamotrigine } \\
\text { vs. placebo } \\
\text { (rapid } \\
\text { cyclers) }\end{array}$ & $\begin{array}{l}\text { Time to new drug } \\
\text { treatment }\end{array}$ & $\begin{array}{l}1 \mathrm{RCT} \\
(\mathrm{n}=182)\end{array}$ & See table above & High & Unknown & Direct & Imprecise & Insufficient \\
\hline $\begin{array}{l}\text { Lamotrigine } \\
\text { vs. } \\
\text { discontinue } \\
\text { mood } \\
\text { stabilizers }\end{array}$ & Relapse 40 wks & $\begin{array}{l}1 \text { Observational } \\
(n=26)\end{array}$ & See table above & High & Unknown & Direct & Imprecise & Insufficient \\
\hline $\begin{array}{l}\text { Olanzapine } \\
\text { vs. placebo }\end{array}$ & $\begin{array}{l}\text { Time to relapse } 18 \\
\text { mths to } 3 \text { yrs }\end{array}$ & $\begin{array}{l}3 \mathrm{RCT} \\
(\mathrm{n}=855)\end{array}$ & 18 months to 3 years & High & Consistent & Direct & Imprecise & Insufficient \\
\hline $\begin{array}{l}\text { Paliperidone } \\
\text { vs. placebo }\end{array}$ & $\begin{array}{l}\text { Time to Relapse } 3 \\
\text { yrs } \\
\text { YMRS } 3 \text { yrs } \\
\text { MADRS } \\
\end{array}$ & $\begin{array}{l}1 \text { RCT extension } \\
(n=300)\end{array}$ & See table above & High & Unknown & Direct & Imprecise & Insufficient \\
\hline $\begin{array}{l}\text { Quetiapine } \\
\text { vs. placebo }\end{array}$ & $\begin{array}{l}\text { Time to relapse } \\
104 \text { wks }\end{array}$ & $\begin{array}{l}1 \mathrm{RCT} \\
(\mathrm{n}=808)\end{array}$ & See table above & Moderate & Unknown & Direct & Imprecise & Insufficient \\
\hline $\begin{array}{l}\text { Risperidone } \\
\text { vs. placebo }\end{array}$ & $\begin{array}{l}\text { Time to relapse } 52 \\
\text { wks }\end{array}$ & $\begin{array}{l}2 \mathrm{RCT} \\
(\mathrm{n}=353)\end{array}$ & See table above & $\begin{array}{l}\text { High (log rank } \\
\text { test) }\end{array}$ & Consistent & Direct & Imprecise & Insufficient \\
\hline
\end{tabular}

Abbreviations: MADRS=Montgomery-Asberg Depression Rating Scale; RCT=randomized controlled trial; YMRS = Young Mania Rating Scale

Notes: 
1. Publication bias for antipsychotics, antidepressants, and behavioral interventions for depressive disorders is suspected.

2. Data were generally imprecise due to missing data from high attrition rates, which was commonly dealt with by Last Observation Carried Forward (LOCF). LOCF requires an assumption that the health status of patients who dropped out of the trial would not have changed had future observations been recorded, a strong assumption in the context of bipolar disorder research. 
Appendix Table 19. Outcomes summary: other monotherapy versus active control for maintenance

\begin{tabular}{|c|c|c|c|c|c|c|}
\hline Drug & $\begin{array}{c}\text { Study } \\
\text { Comparison } \\
\text { PMID }\end{array}$ & Responder/Remitter & Symptom & Function & Other & $\mathrm{AE}$ \\
\hline \multirow[t]{2}{*}{$\begin{array}{l}\text { Carbamazepine } \\
\text { vs. lithium }\end{array}$} & $\begin{array}{l}\text { Hartong, } \\
2003^{10} \\
12633122\end{array}$ & $\begin{array}{l}\text { Time to relapse } \\
\text { Proportional hazard } \\
\text { assumption did not hold }\end{array}$ & NR & NR & NR & $\frac{\mathrm{SAE}}{\underline{\mathrm{NR}}}$ \\
\hline & $\begin{array}{l}\text { Greil, } 1999 \\
10529070^{13} \\
10529071^{14}\end{array}$ & $\begin{array}{l}\text { Time to clinical or } \\
\text { subclinical recurrence } \\
\text { BP-I } \\
2.5 \text { years } \\
\text { Favors lithium } \\
\mathrm{p}=0.034 \\
\mathrm{n}=114 \\
\text { Time to clinical or } \\
\text { subclinical recurrence } \\
\text { BP-II or NOS } \\
2.5 \text { years } \\
\mathrm{NS} \\
\mathrm{n}=57\end{array}$ & NR & NR & NR & $\begin{array}{l}1 \text { suicide, } 1 \text { attempted } \\
\text { suicide in carbazamepine } \\
\text { group }\end{array}$ \\
\hline $\begin{array}{l}\text { Divalproex vs. } \\
\text { lithium (rapid } \\
\text { cyclers) }\end{array}$ & $\begin{array}{l}\text { Calabrese, } \\
2005^{16} \\
\text { Government } \\
16263857\end{array}$ & $\begin{array}{l}\frac{\text { Time to treatment for }}{\text { mood episode, }} \\
\text { depression treatment, } \\
\text { elevated mood } \\
\frac{\text { treatment }}{26 \text { weeks }} \\
\text { No differences between } \\
\text { groups }\end{array}$ & $\begin{array}{l}\text { NA } \\
\text { (attrition 88\%) }\end{array}$ & NA & $\begin{array}{l}\text { Time to overall } \\
\frac{\text { withdrawal }}{26 \text { weeks }} \\
\text { No difference between } \\
\text { groups }\end{array}$ & $\begin{array}{l}\frac{\mathrm{SAE}}{\mathrm{NR}} \\
\text { Tremors/polyuria/polydipsia } \\
\text { "more common in those } \\
\text { assigned to lithium" }\end{array}$ \\
\hline $\begin{array}{l}\text { Paliparidone } \\
\text { vs. olanzapine }\end{array}$ & $\begin{array}{l}\text { Berwaerts, } \\
2012^{25} \\
22377512 \\
\end{array}$ & No usable outcomes & & & & \\
\hline
\end{tabular}




\begin{tabular}{|c|c|c|c|c|c|c|}
\hline Drug & $\begin{array}{c}\text { Study } \\
\text { Comparison } \\
\text { PMID }\end{array}$ & Responder/Remitter & Symptom & Function & Other & $\mathrm{AE}$ \\
\hline $\begin{array}{l}\text { Fluoxetine vs. } \\
\text { Lithium }\end{array}$ & $\begin{array}{l}\text { Amsterdam, } \\
2010^{3} \\
20360317\end{array}$ & $\begin{array}{l}\text { Time to Relapse to } \\
\text { Depression } \\
50 \text { week } \\
\text { Favors Fluoxetine } \\
\mathrm{p}=0.03 \\
\text { Relapse } \\
50 \text { week } \\
\text { Favors Fluoxetine } \\
\text { HR=0.04 (95\%Cl } \\
0.2,0.9)\end{array}$ & $\begin{array}{l}\text { YMRS Change } \\
50 \text { week } \\
\text { Fluoxetine }-6.3(95 \% \mathrm{Cl} \\
-47.5,34.9) \\
\text { Lithium } 7.2(95 \% \mathrm{Cl} \\
-33.3,53.8)\end{array}$ & NR & $\begin{array}{l}\text { Withdrawal for AE } \\
\text { Fluoxetine } 1 \\
\text { Lithium } 1\end{array}$ & $\frac{\text { SAE }}{\text { No Events }}$ \\
\hline $\begin{array}{l}\text { Lamotrigine vs. } \\
\text { Lithium }\end{array}$ & $\begin{array}{l}\text { Bowden, } \\
2003^{4} \\
12695317\end{array}$ & $\begin{array}{l}\frac{\text { Time to any recurrence }}{18 \text { months }} \\
\text { Log rank } \\
\text { NS } \\
\frac{\text { Time to mania }}{\text { recurrence }} \\
\frac{\text { Log months }}{\text { NS rank }} \\
\text { Time to depression } \\
\frac{\text { recurrence }}{18 \text { months }} \\
\text { Log rank } \\
\text { NS }\end{array}$ & $\begin{array}{l}\text { NA } \\
\text { (Exclude for attrition = } \\
80 \%)\end{array}$ & NA & NA & $\begin{array}{l}\frac{\text { SAE }}{1 \text { lamotrigine patient }} \\
\text { hospitzalized for rash } \\
1 \text { lamotrigine patient } \\
\text { attempted suicide } \\
\text { Suicidality per HAM-D NS } \\
\text { between groups }\end{array}$ \\
\hline
\end{tabular}




\begin{tabular}{|c|c|c|c|c|c|c|}
\hline Drug & $\begin{array}{c}\text { Study } \\
\text { Comparison } \\
\text { PMID }\end{array}$ & Responder/Remitter & Symptom & Function & Other & $\mathrm{AE}$ \\
\hline & $\begin{array}{l}\text { Calabrese, } \\
2003^{5} \\
14628976\end{array}$ & $\begin{array}{l}\frac{\text { Time to any recurrence }}{18 \text { months }} \\
\text { Log rank } \\
\text { NS } \\
\frac{\text { Time to mania }}{\text { recurrence }} \\
\frac{18 \text { months }}{\text { Log rank }} \\
\text { NS } \\
\frac{\text { Time to depression }}{\text { recurrence }} \\
\text { Log months } \\
\text { NS rank }\end{array}$ & $\begin{array}{l}\text { NA } \\
\text { (Exclude for attrition = } \\
85 \%)\end{array}$ & NA & NA & $\begin{array}{l}\frac{\text { SAE }}{1 \text { death in lamotrigine }} \text { groups } \\
\text { Suicidality per HAM-D NS } \\
\text { between groups }\end{array}$ \\
\hline $\begin{array}{l}\text { Lithium vs. } \\
\text { valproate }\end{array}$ & $\begin{array}{l}\text { Balance } \\
\text { Investigators, } \\
2010^{18} \\
20092882\end{array}$ & $\begin{array}{l}\text { Time to new intervention } \\
\text { for emerging mood } \\
\text { episode } \\
\text { 24 months } \\
\text { Hazard ratio } \\
\text { Favors Lithium } \\
\text { HR } 0.71(0.51,1.00) \\
p=0.047\end{array}$ & $\mathrm{NR}$ & $\begin{array}{l}\text { GAF } \\
\text { NS }\end{array}$ & $\begin{array}{l}\text { EuroQol (EQ-5D) } \\
\text { (quality of life) } \\
\text { NS } \\
\text { Overall Withdrawal } \\
\text { Lithium: } 23 / 110 \\
\text { Valproate: } 23 / 110 \\
\text { Withdrawal lack of } \\
\text { efficacy } \\
\text { NR } \\
\text { Withdrawal adverse } \\
\text { events } \\
\text { Lithium: } 6 / 110 \\
\text { Valproate: } 4 / 110\end{array}$ & $\begin{array}{l}\text { SAE } \\
\text { NS } \\
\text { Valproate: } 7 \text { SAE including } \\
3 \text { deaths } \\
\text { Lithium: } 5 \text { SAE including } 2 \\
\text { deaths }\end{array}$ \\
\hline
\end{tabular}




\begin{tabular}{|c|c|c|c|c|c|c|}
\hline Drug & $\begin{array}{c}\text { Study } \\
\text { Comparison } \\
\text { PMID }\end{array}$ & Responder/Remitter & Symptom & Function & Other & $\mathrm{AE}$ \\
\hline $\begin{array}{l}\text { Lithium vs. } \\
\text { divalproex }\end{array}$ & $\begin{array}{l}\text { Bowden, } \\
2000^{1} \\
10807488 \\
12784116^{2}\end{array}$ & $\begin{array}{l}\frac{\text { Time to any recurrence }}{12 \text { months }} \\
\text { NS } \\
\frac{\text { Time to mania }}{\frac{\text { recurrence }}{12 \text { months }}} \\
\text { NS } \\
\frac{\text { Time to depression }}{\text { recurrence }} \\
\text { NS months }\end{array}$ & $\begin{array}{l}\text { NA } \\
\text { (Exclude for attrition = } \\
69 \%)\end{array}$ & NR & NR & $\begin{array}{l}\frac{\text { SAE }}{\text { NR }} \\
\frac{\text { Tremor }}{\text { Divalproex } 41 \%(77 / 187)} \\
\text { Lithium 42\% (38/94) } \\
\text { Divalproex vs. Lithium } \\
\text { NS } \\
\text { Akathisia } \\
\text { Divalproex <1\% (1/187) } \\
\text { Lithium 4\% }(4 / 94) \\
\text { Divalproex vs. Lithium } \\
\text { Favors divalproex } \\
\text { p=0.04 }\end{array}$ \\
\hline $\begin{array}{l}\text { Olanzapine vs. } \\
\text { divalproex }\end{array}$ & $\begin{array}{l}\text { Tohen, } 2003^{20} \\
\text { Industry } \\
12832240 \\
\text { Extension of } \\
\text { Tohen, } \\
2002 \mathrm{~b}^{21} \\
12042191\end{array}$ & $\begin{array}{l}\text { Time to relapse } \\
42 \text { weeks } \\
\text { Log rank } \\
\text { NS }\end{array}$ & $\begin{array}{l}\text { NA } \\
\text { (attrition 84\%) }\end{array}$ & NA & NR & SAE not reported \\
\hline $\begin{array}{l}\text { Olanzapine vs. } \\
\text { Lithium }\end{array}$ & $\begin{array}{l}\text { Tohen, } 2005^{24} \\
\text { High } \\
15994710\end{array}$ & $\begin{array}{l}\text { Time to Relapse (YMRS } \\
\text { and/or HAM-D>15) } \\
52 \text { weeks } \\
\text { Log rank } \\
\text { NS } \\
p=0.07 \\
\text { Time to hospitalization } \\
52 \text { weeks } \\
\text { Log rank } \\
\text { Favors Olanzapine } \\
p=0.01\end{array}$ & $\begin{array}{l}\text { NA } \\
\text { (Exclude for attrition = } \\
61 \%)\end{array}$ & NR & $\begin{array}{l}\text { Overall Withdrawal } \\
\text { Olanzapine: } 116 / 217 \\
\text { Lithium: } 144 / 214\end{array}$ & $\begin{array}{l}\frac{\text { SAE }}{2 \text { deaths, lithium, } 1 \text { suicie, }} \\
1 \text { accident } \\
\text { EPS } \\
\text { NS }\end{array}$ \\
\hline
\end{tabular}




\begin{tabular}{|c|c|c|c|c|c|c|}
\hline Drug & $\begin{array}{c}\text { Study } \\
\text { Comparison } \\
\text { PMID }\end{array}$ & Responder/Remitter & Symptom & Function & Other & AE \\
\hline $\begin{array}{l}\text { Quetiapine vs. } \\
\text { Lithium }\end{array}$ & $\begin{array}{l}\text { Weisler, } \\
2011^{7} \\
22054050\end{array}$ & $\begin{array}{l}\text { Time to recurrence of } \\
\text { any mood event } \\
\text { Favors Quetiapine } \\
\text { HR } 0.66 \text { ( } 95 \% \mathrm{Cl} 0.49 \text {, } \\
0.88), p=0.005 \\
\text { Time to recurrence of } \\
\text { manic event } \\
\text { NS } \\
\text { Time to recurrence of } \\
\text { depression symptoms } \\
\text { Favors Quetiapine } \\
\text { HR } 0.54 \text { (95\% Cl } 0.35 \text {, } \\
0.84), p=0.006\end{array}$ & $\begin{array}{l}\text { NA } \\
\text { (Exclude for attrition } 55 \% \text { ) }\end{array}$ & NR & NR & $\begin{array}{l}\frac{\text { SAE }}{5 \text { quetiapine }} \\
10 \text { lithium } \\
\frac{\text { EPS }}{16 \text { quetiapine }} \\
38 \text { lithium } \\
\text { No deaths }\end{array}$ \\
\hline $\begin{array}{l}\text { Venlafaxine vs. } \\
\text { Lithium }\end{array}$ & $\begin{array}{l}\text { Amsterdam, } \\
2015^{26} \\
26143402\end{array}$ & $\begin{array}{l}\text { Time to depression } \\
\text { relapse } \\
6 \text { months } \\
\text { Log rank } \\
\text { NS }\end{array}$ & $\begin{array}{l}\text { YMRS } \\
\underline{\text { NS }}\end{array}$ & NR & $\begin{array}{l}\text { Withdrawal for } \mathrm{AE} \\
\text { Venlaxafine } 1 \\
\text { Lithium } 0\end{array}$ & NR \\
\hline $\begin{array}{l}\text { Risperidone vs. } \\
\text { olanzapine }\end{array}$ & $\begin{array}{l}\text { Vieta, } 2012^{23} \\
22503488\end{array}$ & $\begin{array}{l}\text { Time to first recurrence } \\
\text { of mood symptoms } \\
18 \text { months } \\
\text { Post-hoc Log-rank } \\
\text { Favors Olanzapine } \\
p=0.001\end{array}$ & $\begin{array}{l}\mathrm{NA} \\
\text { (Due to attrition }=58 \% \text { ) }\end{array}$ & $\begin{array}{l}\text { NA } \\
\text { (Due to } \\
\text { attrition } \\
=58 \% \text { ) }\end{array}$ & NR & $\begin{array}{l}\text { Reported no SAE in period } \\
\text { III (maintenance phase) }\end{array}$ \\
\hline
\end{tabular}

Abbreviations: AE=Adverse Events; $\mathrm{BP}=$ bipolar disorder; $\mathrm{CI}=$ Confidence Interval; EPS=extrapyramidal symptoms; GAF=General Assessment of Functioning Scale; HAM-

D=Hamilton Scale for Depression; HR=Hazard Ratio; NA= Not Applicable; NOS=Not Otherwise Specified; NR=not reported; NS=not significant; PMID=PubMed Identification Number; SAE=Serious Adverse Events; YMRS = Young Mania Rating Scale

\section{Appendix Table 110. Strength of evidence assessment: other monotherapy versus active control for maintenance}

\begin{tabular}{|l|l|l|l|l|l|l|}
\hline \multicolumn{1}{|c|}{ Comparison } & \multicolumn{1}{|c|}{ Outcome } & $\begin{array}{c}\text { \# Studies/ } \\
\text { Design } \\
\text { (n analyzed) }\end{array}$ & $\begin{array}{l}\text { Finding or Summary } \\
\text { Statistic } \\
\text { Outcome Timing }\end{array}$ & $\begin{array}{c}\text { Study } \\
\text { Limitations }\end{array}$ & Consistency & $\begin{array}{c}\text { Overall } \\
\text { Gradel } \\
\text { Conclusion }\end{array}$ \\
\hline $\begin{array}{l}\text { Carbamazepine } \\
\text { vs. Lithium }\end{array}$ & $\begin{array}{l}\text { Time to } \\
\text { recurrence 2.5 yrs }\end{array}$ & $\begin{array}{l}1 \text { RCT (Greil } \\
1999) \\
(n=171)\end{array}$ & See table above & Moderate & Unknown & Direct \\
\hline
\end{tabular}




\begin{tabular}{|c|c|c|c|c|c|c|c|c|}
\hline Comparison & Outcome & $\begin{array}{l}\text { \# Studies/ } \\
\text { Design } \\
\text { (n analyzed) }\end{array}$ & $\begin{array}{c}\text { Finding or Summary } \\
\text { Statistic } \\
\text { Outcome Timing }\end{array}$ & $\begin{array}{c}\text { Study } \\
\text { Limitations }\end{array}$ & Consistency & Directness & Precision & $\begin{array}{c}\text { Overall } \\
\text { Gradel } \\
\text { Conclusion }\end{array}$ \\
\hline $\begin{array}{l}\text { Divalproex vs. } \\
\text { lithium (rapid } \\
\text { cyclers) }\end{array}$ & $\begin{array}{l}\text { Time to treatment } \\
\text { for mood episode } \\
26 \text { weeks }\end{array}$ & $\begin{array}{l}1 \mathrm{RCT} \\
(\mathrm{n}=60)\end{array}$ & See table above & High & Unknown & Direct & Imprecise & Insufficient \\
\hline $\begin{array}{l}\text { Fluoxetine vs. } \\
\text { Lithium }\end{array}$ & $\begin{array}{l}\text { Time to relapse to } \\
\text { depression } 50 \\
\text { wks }\end{array}$ & $\begin{array}{l}1 \mathrm{RCT} \\
(\mathrm{n}=54)\end{array}$ & See table above & Moderate & Unknown & Direct & Imprecise & Insufficient \\
\hline $\begin{array}{l}\text { Lamotrigine vs. } \\
\text { Lithium }\end{array}$ & $\begin{array}{l}\text { Time to } \\
\text { recurrence } 18 \\
\text { mths }\end{array}$ & $\begin{array}{l}2 \text { RCTs } \\
(n=390)\end{array}$ & See table above & High & Consistent & Direct & Imprecise & Insufficient \\
\hline $\begin{array}{l}\text { Lithium vs. } \\
\text { Valproate* }\end{array}$ & $\begin{array}{l}\text { Time to new } \\
\text { intervention for } \\
\text { emerging mood } \\
\text { episode } 24 \text { mths } \\
\text { EuroQoL } 24 \text { mths }\end{array}$ & $\begin{array}{l}1 \mathrm{RCT} \\
(\mathrm{n}=220)\end{array}$ & See table above & Moderate & Unknown & Direct & Imprecise & Insufficient \\
\hline $\begin{array}{l}\text { Lithium vs. } \\
\text { divalproex* }\end{array}$ & $\begin{array}{l}\text { Time to } \\
\text { recurrence } 12 \\
\text { mths }\end{array}$ & $\begin{array}{l}1 \mathrm{RCT} \\
(\mathrm{n}=372)\end{array}$ & See table above & Moderate & Unknown & Direct & Imprecise & Insufficient \\
\hline $\begin{array}{l}\text { Olanzapine vs. } \\
\text { divalproex }\end{array}$ & $\begin{array}{l}\text { Time to } \\
\text { recurrence } \\
47 \text { weeks }\end{array}$ & $\begin{array}{l}1 \mathrm{RCT} \\
(\mathrm{n}=251)\end{array}$ & See table above & High & Unknown & Direct & Imprecise & Insufficient \\
\hline $\begin{array}{l}\text { Olanzapine vs. } \\
\text { Lithium }\end{array}$ & $\begin{array}{l}\text { Time to relapse } \\
104 \text { wks } \\
\text { time to } \\
\text { hospitalization } \\
104 \text { wks }\end{array}$ & $\begin{array}{l}1 \mathrm{RCT} \\
(\mathrm{n}=431)\end{array}$ & See table above & High & Consistent & Direct & Imprecise & Insufficient \\
\hline $\begin{array}{l}\text { Quetiapine vs. } \\
\text { Lithium }\end{array}$ & $\begin{array}{l}\text { Time to relapse } \\
104 \text { wks }\end{array}$ & $\begin{array}{l}1 \mathrm{RCT} \\
(\mathrm{n}=768)\end{array}$ & See table above & Moderate & Unknown & Direct & Imprecise & Insufficient \\
\hline $\begin{array}{l}\text { Paliperidone } \\
\text { vs. Olanzapine }\end{array}$ & $\begin{array}{l}\text { No usable } \\
\text { outcomes } 3 \text { yrs }\end{array}$ & $\begin{array}{l}1 \mathrm{RCT} \text { extension } \\
(\mathrm{n}=235)\end{array}$ & See table above & High & Unknown & Direct & Imprecise & Insufficient \\
\hline $\begin{array}{l}\text { Venlafaxine vs. } \\
\text { Lithium }\end{array}$ & $\begin{array}{l}\text { Time to Relapse } 6 \\
\text { mths } \\
\text { YMRS } 6 \text { mths }\end{array}$ & $\begin{array}{l}1 \mathrm{RCT} \text { extension } \\
(\mathrm{n}=55)\end{array}$ & See table above & High & Unknown & Direct & Imprecise & Insufficient \\
\hline
\end{tabular}




\begin{tabular}{|l|l|l|l|l|l|l|}
\hline \multicolumn{1}{|c|}{ Comparison } & \multicolumn{1}{|c|}{ Outcome } & $\begin{array}{c}\text { \# Studies/ } \\
\text { Design } \\
\text { (n analyzed) }\end{array}$ & $\begin{array}{l}\text { Finding or Summary } \\
\text { Statistic } \\
\text { Outcome Timing }\end{array}$ & $\begin{array}{c}\text { Study } \\
\text { Limitations }\end{array}$ & Consistency & $\begin{array}{c}\text { Overall } \\
\text { Gradel } \\
\text { Conclusion }\end{array}$ \\
\hline $\begin{array}{l}\text { Risperidone vs. } \\
\text { Olanzapine }\end{array}$ & $\begin{array}{l}\text { Time to relapse } \\
18 \text { mths }\end{array}$ & $\begin{array}{l}1 \mathrm{RCT} \\
(\mathrm{n}=263)\end{array}$ & See table above & High & Unknown & Direct \\
\hline
\end{tabular}

* If aggregating across lithium versus divalproex or valproate, strength of evidence remains insufficient due to inconsistency between study findings.

Abbreviations: RCT=randomized controlled trial; YMRS = Young Mania Rating Scale

Notes:

1. Publication bias for antipsychotics, antidepressants, and behavioral interventions for depressive disorders is suspected.

2. Data were generally imprecise due to missing data from high attrition rates, which was commonly dealt with by Last Observation Carried Forward (LOCF). LOCF requires an assumption that the health status of patients who dropped out of the trial would not have changed had future observations been recorded, a strong assumption in the context of bipolar disorder research.

\section{Section 3. Combination Therapy}

Appendix Table I11. Characteristics of eligible studies: combination therapy for maintenance

\begin{tabular}{|c|c|c|c|c|c|c|}
\hline $\begin{array}{l}\text { Study, Year } \\
\text { Design } \\
\text { Location } \\
\text { Funder } \\
\text { Risk of Bias } \\
\text { PMID }\end{array}$ & $\begin{array}{c}\text { \# Randomized } \\
\text { Age (mean) } \\
\text { Sex (\% Female) } \\
\text { Race (\% White) } \\
\text { Diagnosis } \\
\text { (\% BP I, II, NOS) } \\
\text { Setting }\end{array}$ & $\begin{array}{l}\text { Inclusions } \\
\text { Key Exclusions }\end{array}$ & $\begin{array}{l}\text { Intervention } \\
\text { Dosage }\end{array}$ & $\begin{array}{l}\text { Comparison } \\
\text { Dosage }\end{array}$ & $\begin{array}{l}\text { Followup } \\
\text { Duration }\end{array}$ & $\begin{array}{c}\text { Outcomes } \\
\text { Reported } \\
\begin{array}{c}\text { Withdrawal (\%) at } \\
\text { endpoint }\end{array}\end{array}$ \\
\hline $\begin{array}{l}\text { Balance Investigators, } \\
2010^{18} \\
\text { RCT } \\
\text { Multisite } \\
\text { US and Europe } \\
\text { Industry } \\
\text { RoB Moderate } \\
20092882\end{array}$ & $\begin{array}{l}\mathrm{N}=330 \\
\text { Mean Age } 43 \\
\text { Female 49\% } \\
\text { Race NR } \\
\text { BP I 100\% } \\
\text { Outpatient }\end{array}$ & $\begin{array}{l}\text { Not having acute episode; } \\
\text { Not defined } \\
\text { Pregnant/Nursing }\end{array}$ & $\begin{array}{l}\text { Lithium } \\
0.4-1.0 \mathrm{mmol} / \mathrm{L} \\
\text { mean NR } \\
+ \text { Valproate } \\
750-1250 \text { or highest } \\
\text { tolerated } \\
\text { mean NR } \\
n=110\end{array}$ & $\begin{array}{l}\text { C1: Lithium } \\
0.4-1.0 \text { mmol/L } \\
\text { mean NR } \\
\mathrm{N}=110 \\
\\
\text { C2: Valproate } \\
750-1250 \text { or } \\
\text { highest } \\
\text { tolerated } \\
\text { mean NR } \\
\mathrm{n}=110\end{array}$ & 24 months & $\begin{array}{l}\text { GAF } \\
\text { EuroQol (EQ-5D) } \\
\text { (quality of life) } \\
\text { Relapse } \\
\\
\text { Withdrawal 20\% }\end{array}$ \\
\hline
\end{tabular}




\begin{tabular}{|c|c|c|c|c|c|c|}
\hline $\begin{array}{l}\text { Study, Year } \\
\text { Design } \\
\text { Location } \\
\text { Funder } \\
\text { Risk of Bias } \\
\text { PMID }\end{array}$ & $\begin{array}{c}\text { \# Randomized } \\
\text { Age (mean) } \\
\text { Sex (\% Female) } \\
\text { Race (\% White) } \\
\text { Diagnosis } \\
\text { (\% BP I, II, NOS) } \\
\text { Setting }\end{array}$ & $\begin{array}{c}\text { Inclusions } \\
\text { Key Exclusions }\end{array}$ & $\begin{array}{c}\text { Intervention } \\
\text { Dosage }\end{array}$ & $\begin{array}{c}\text { Comparison } \\
\text { Dosage }\end{array}$ & $\begin{array}{l}\text { Followup } \\
\text { Duration }\end{array}$ & $\begin{array}{c}\text { Outcomes } \\
\text { Reported } \\
\begin{array}{c}\text { Withdrawal (\%) at } \\
\text { endpoint }\end{array}\end{array}$ \\
\hline $\begin{array}{l}\text { Marcus, } 2011^{28} \\
\text { RCT } \\
\text { Multisite/Not Disclosed } \\
\text { (8 countries) } \\
\text { Industry } \\
\text { RoB High } \\
21443567\end{array}$ & $\begin{array}{l}\mathrm{N}=337 \\
\text { Mean Age } 39 \\
\text { Female 55\% } \\
\text { White } 68 \% \\
\text { BP I } 100 \% \\
\text { Outpatient }\end{array}$ & $\begin{array}{l}\text { Manic/Mixed; } \\
\text { YMRS } \geq 16 \text { at study entry; } \\
\text { Current episode <2 years; } \\
\text { YMRS } \leq 12, \text { MADRS } \leq 12 \text { at } \\
\text { randomization after } 12 \\
\text { weeks of stabilization } \\
\text { treatment } \\
\text { First manic episode } \\
\text { Schizoaffective } \\
\text { Substance abuse } \\
\text { Other mental health } \\
\text { Neurological disorders } \\
\text { Taking other meds } \\
\text { Labs/other conditions } \\
\end{array}$ & $\begin{array}{l}\text { Aripiprazole } \\
\text { 10-30 mg/day } \\
\text { (15.8-16.9 mg/day) } \\
\text { + Lithium or valproic } \\
\text { acid }\end{array}$ & $\begin{array}{l}\text { Placebo } \\
+ \text { Lithium or } \\
\text { valproic acid }\end{array}$ & 52 weeks & $\begin{array}{l}\text { YMRS } \\
\text { MADRS } \\
\text { CGI-BP-S } \\
\text { Relapse } \\
\text { Adverse Events } \\
\text { EPS } \\
\text { Withdrawal 43\% }\end{array}$ \\
\hline $\begin{array}{l}\text { Carlson, } 2012^{29} \\
\text { RCT of responders } \\
\text { Multisite } \\
\text { US } \\
\text { Industry } \\
\text { RoB High } \\
22329471\end{array}$ & $\begin{array}{l}\mathrm{N}=351 \\
\text { Mean Age } 39 \\
\text { Female } 65 \% \\
\text { White } 90 \% \\
\text { BP I } 100 \% \\
\text { Outpatient }\end{array}$ & $\begin{array}{l}\text { Randomization after } \\
\text { stabilization; } 8 \text { weeks at } \\
\text { YMRS } \leq 12, \text { MADRS } \leq 12 . \\
\text { Study entry manic or mixed } \\
\text { YMRS } \geq 16 \text { in previous } 3 \\
\text { months with or without rapid } \\
\text { cycling (4 to } 7 \text { mood } \\
\text { episodes per year) } \\
\text { Substance abuse } \\
\text { Other Mental Health } \\
\text { Conditions } \\
\text { Pregnant/Nursing } \\
\text { Labs/other conditions } \\
\text { First manic episode } \\
\text { Treatment refractory } \\
\text { mania/mixed mania }\end{array}$ & $\begin{array}{l}\text { Aripiprazole } \\
\text { target } 15 \mathrm{mg} / \text { day } \\
\text { (range } 10-30 \\
\text { mg/day) } \\
+ \text { +Lamotrigine target } \\
200 \text { mg/day (range } \\
100-200 \text { mg/day) }\end{array}$ & \begin{tabular}{|l} 
Placebo \\
+Lamotrigine \\
target 200 \\
mg/day (range \\
$100-200$ \\
mg/day)
\end{tabular} & 52 weeks & $\begin{array}{l}\text { Time to relapse } \\
\text { (hospitalization; SAE or } \\
\text { lack of effect, including } \\
\text { YMRS }>14 \text { and } \\
\text { MADRS } \leq 16 \text { manic or } \\
\text { YMRS } \leq 14 \text { and MADRS } \\
>16 \text { ) } \\
\text { YMRS } \\
\text { MADRS } \\
\text { CGI-BP } \\
\text { Withdrawal } 66 \% \text { (Time } \\
\text { to recurrence } \\
\text { outcomes only } \\
\text { included) } \\
\text { Nonrelapse withdrawal } \\
\text {-40\% }\end{array}$ \\
\hline
\end{tabular}




\begin{tabular}{|c|c|c|c|c|c|c|}
\hline $\begin{array}{l}\text { Study, Year } \\
\text { Design } \\
\text { Location } \\
\text { Funder } \\
\text { Risk of Bias } \\
\text { PMID }\end{array}$ & $\begin{array}{c}\text { \# Randomized } \\
\text { Age (mean) } \\
\text { Sex (\% Female) } \\
\text { Race (\% White) } \\
\text { Diagnosis } \\
\text { (\% BP I, II, NOS) } \\
\text { Setting }\end{array}$ & $\begin{array}{c}\text { Inclusions } \\
\text { Key Exclusions }\end{array}$ & $\begin{array}{l}\text { Intervention } \\
\text { Dosage }\end{array}$ & $\begin{array}{c}\text { Comparison } \\
\text { Dosage }\end{array}$ & $\begin{array}{l}\text { Followup } \\
\text { Duration }\end{array}$ & $\begin{array}{c}\text { Outcomes } \\
\text { Reported } \\
\begin{array}{c}\text { Withdrawal (\%) at } \\
\text { endpoint }\end{array}\end{array}$ \\
\hline $\begin{array}{l}\text { Woo, } 2011^{30} \\
\text { RCT } \\
\text { Multisite/Asia } \\
\text { Industry } \\
\text { RoB High } \\
22134973\end{array}$ & $\begin{array}{l}\mathrm{N}=83 \\
\text { Mean Age } 38 \\
\text { Female 68\% } \\
\text { White NR } \\
\text { Japanese (32\%) } \\
\text { Korean/Chinese (43\%) } \\
\text { Other (25\%) } \\
\text { BP I 100\% } \\
\text { Outpatient }\end{array}$ & $\begin{array}{l}\text { Manic/Mixed initially with } \\
\text { YMRS } \geq 20 \text { at study entry; } \\
\text { then YMRS } \leq 12, \text { MADRS } \leq 13 \\
\text { at randomization after } 6 \\
\text { weeks of stabilization } \\
\text { treatment } \\
\text { Schizoaffective } \\
\text { Substance abuse } \\
\text { Neurological disorders } \\
\text { Taking other meds } \\
\text { Pregnant/nursing } \\
\text { Labs/other conditions }\end{array}$ & $\begin{array}{l}\text { Aripiprazole } \\
10-30 \mathrm{mg} / \text { day } \\
\text { (17.9 mg/day) } \\
+ \text { Lithium or valproic } \\
\text { acid }\end{array}$ & $\begin{array}{l}\text { Placebo } \\
+ \text { Lithium or } \\
\text { valproic acid }\end{array}$ & 24 weeks & $\begin{array}{l}\text { YMRS } \\
\text { MADRS } \\
\text { CGI-BP-S } \\
\text { Relapse } \\
\text { Adverse Events } \\
\text { EPS } \\
\text { Withdrawal 42\% }\end{array}$ \\
\hline $\begin{array}{l}\text { Kemp, } 2009^{31} \\
\text { RCT of responders } \\
\text { Single site } \\
\text { US } \\
\text { Gov't } \\
\text { RoB High } \\
19192457\end{array}$ & $\begin{array}{l}\mathrm{N}=31 \\
\text { Mean Age 36 } \\
\text { Female } 36 \% \\
\text { White } 82 \% \\
\text { BP I } 75 \% \\
\text { BP II } 25 \% \\
\\
\text { Outpatient }\end{array}$ & $\begin{array}{l}\text { Stable responders (HAM-D } \\
\text { score } \leq 20 \text {, YMRS score } \leq \\
12.5 \text { ) Rapid cycling, } \\
\text { substance use disorder as } \\
\text { ascertained by structured } \\
\text { interview; mood episode in } \\
\text { previous } 3 \text { months } \\
\\
\text { Labs/other conditions } \\
\text { Pregnant/nursing }\end{array}$ & $\begin{array}{l}\text { Divalproate } \\
250 \mathrm{mg} / \text { day (target } \\
\text { blood level } \\
50 \mathrm{mg} / \text { day+ Lithium }\end{array}$ & $\begin{array}{l}\text { Placebo + } \\
\text { Lithium }\end{array}$ & 26 weeks & $\begin{array}{l}\text { Time to treat mood } \\
\text { episode } \\
\text { Efficacy } \\
\text { YMRS } \\
\text { HAM-D } \\
\text { GAS } \\
\\
\text { Withdrawal 74\% (Time } \\
\text { to recurrence } \\
\text { outcomes only } \\
\text { included) } \\
\text { Nonrelapse withdrawal } \\
19 \%\end{array}$ \\
\hline
\end{tabular}




\begin{tabular}{|c|c|c|c|c|c|c|}
\hline $\begin{array}{l}\text { Study, Year } \\
\text { Design } \\
\text { Location } \\
\text { Funder } \\
\text { Risk of Bias } \\
\text { PMID }\end{array}$ & $\begin{array}{c}\text { \# Randomized } \\
\text { Age (mean) } \\
\text { Sex (\% Female) } \\
\text { Race (\% White) } \\
\text { Diagnosis } \\
\text { (\% BP I, II, NOS) } \\
\text { Setting }\end{array}$ & $\begin{array}{c}\text { Inclusions } \\
\text { Key Exclusions }\end{array}$ & $\begin{array}{l}\text { Intervention } \\
\text { Dosage }\end{array}$ & $\begin{array}{c}\text { Comparison } \\
\text { Dosage }\end{array}$ & $\begin{array}{l}\text { Followup } \\
\text { Duration }\end{array}$ & $\begin{array}{c}\text { Outcomes } \\
\text { Reported } \\
\begin{array}{c}\text { Withdrawal (\%) at } \\
\text { endpoint }\end{array}\end{array}$ \\
\hline $\begin{array}{l}\text { Tohen, } 2004^{32} \\
\text { RCT of Responders } \\
\text { Multisite } \\
\text { US } \\
\text { Industry } \\
\text { ROB High } \\
15056579 \\
\text { extension of Tohen, } \\
2002^{33} \\
11779284\end{array}$ & $\begin{array}{l}\mathrm{N}=99 \\
\text { Mean Age } 41 \\
\text { Female } 48 \% \\
\text { White } 85 \% \\
\text { BP-I 100\% } \\
\text { Outpatient }\end{array}$ & $\begin{array}{l}\text { Responders to olanzapine }+ \\
\text { lithium or valproate mania } \\
\text { and depression no worse } \\
\text { than mild; } \\
\text { First Manic Episode } \\
\text { Labs/Other Conditions }\end{array}$ & $\begin{array}{l}\text { Olanzapine } \\
10 \text { mg/day with } \\
\text { flexible dosing from } \\
5-20 \text { mg/day } \\
\\
\text { Adjunctive to } \\
\text { ongoing open-label } \\
\text { valproate or lithium } \\
n=51\end{array}$ & $\begin{array}{l}\text { Placebo } \\
\text { Adjunctive to } \\
\text { ongoing open- } \\
\text { label valproate } \\
\text { or lithium } \\
\mathrm{n}=48\end{array}$ & 18 months & $\begin{array}{l}\text { Time to any mood } \\
\text { episosde; } \\
\text { Withdrawal 78\% (Time } \\
\text { to recurrence } \\
\text { outcomes only } \\
\text { included) Unclear } \\
\text { nonrelapse withdrawal }\end{array}$ \\
\hline $\begin{array}{l}\text { Suppes, } 2009^{34} \\
\text { RCT } \\
\text { Multisite } \\
\text { US/Canada } \\
\text { Industry } \\
\text { RoB High } \\
19289454\end{array}$ & $\begin{array}{l}\mathrm{N}=623 \\
\text { Mean Age } 40 \\
\text { Female } 53 \% \\
\text { White } 82 \% \\
\text { BP I 100\% } \\
\text { Inpatient }\end{array}$ & $\begin{array}{l}\text { Mania at entry; Stable at } \\
\text { randomization after Lithium } \\
\text { or Valproate; YMRS and } \\
\text { MADRS } 12 \text { AND at least } 1 \\
\text { mood episode of any type in } \\
\text { past } 2 \text { years and another } 6 \\
\text { months prior to } \\
\text { randomization } \\
\text { First Manic Episode } \\
\text { Substance Abuse } \\
\text { Other Mental Health } \\
\text { Conditions } \\
\text { Pregnant/Nursing }\end{array}$ & $\begin{array}{l}\text { Quetiapine } \\
400-800 \mathrm{mg} / \text { day } \\
\text { (519 mg/day mean) } \\
+ \text { Valproate } \\
50-125 \mathrm{mcg} / \mathrm{mL} \\
\text { target } \\
\text { OR } \\
\text { Lithium } \\
0.5-1.2 \mathrm{mEq} / \mathrm{L} \text { target }\end{array}$ & $\begin{array}{l}\text { Placebo } \\
+ \text { Valproate } \\
50-125 \mathrm{mcg} / \mathrm{mL} \\
\text { target } \\
\text { OR } \\
\text { Lithium } \\
0.5-1.2 \mathrm{mEq} / \mathrm{L} \\
\text { target }\end{array}$ & $\begin{array}{l}104 \text { weeks } \\
\text { (only time to } \\
\text { occurrence } \\
\text { and } \\
\text { withdrawals } \\
\text { used due to } \\
\text { attrition) }\end{array}$ & $\begin{array}{l}\text { Recurrance } \\
\text { Adverse Events } \\
\\
\text { Withdrawal } 71 \% \text { (Time } \\
\text { to recurrence } \\
\text { outcomes only } \\
\text { included) } \\
\text { Nonrelapse withdrawal } \\
35 \%\end{array}$ \\
\hline
\end{tabular}




\begin{tabular}{|c|c|c|c|c|c|c|}
\hline $\begin{array}{l}\text { Study, Year } \\
\text { Design } \\
\text { Location } \\
\text { Funder } \\
\text { Risk of Bias } \\
\text { PMID }\end{array}$ & $\begin{array}{c}\text { \# Randomized } \\
\text { Age (mean) } \\
\text { Sex (\% Female) } \\
\text { Race (\% White) } \\
\text { Diagnosis } \\
\text { (\% BP I, II, NOS) } \\
\text { Setting }\end{array}$ & $\begin{array}{c}\text { Inclusions } \\
\text { Key Exclusions }\end{array}$ & $\begin{array}{l}\text { Intervention } \\
\text { Dosage }\end{array}$ & $\begin{array}{c}\text { Comparison } \\
\text { Dosage }\end{array}$ & $\begin{array}{l}\text { Followup } \\
\text { Duration }\end{array}$ & $\begin{array}{c}\text { Outcomes } \\
\text { Reported } \\
\text { Withdrawal (\%) at } \\
\text { endpoint }\end{array}$ \\
\hline $\begin{array}{l}\text { Vieta, } 2008^{35} \\
\text { RCT } \\
\text { Multisite } \\
4 \text { Continents } \\
\text { Industry } \\
\text { RoB } \\
18579216\end{array}$ & $\begin{array}{l}\mathrm{N}=706 \\
\text { Mean Age } 42 \\
\text { Female } 55 \% \\
\text { White } 97 \% \\
\text { BP I } 100 \% \\
\\
\text { Inpatient (1 week) } \\
\text { Outpatient (2-6 weeks, } \\
\text { subject to inspector } \\
\text { discretion) }\end{array}$ & $\begin{array}{l}\text { Mania, Depression, Mixed; } \\
\text { latest episode of any type } \\
\text { within } 26 \text { weeks, achived } \\
\text { clinical stability (YMRS and } \\
\text { MADRS } \leq 12 \text { ) prior to } \\
\text { randomization, subject to } \\
\text { specified time periods } \\
\\
\text { Substance Abuse; } \\
\text { Other Mental Health } \\
\text { Conditions; } \\
\text { Taking Other Meds; } \\
\text { Pregnant/Nursing }\end{array}$ & $\begin{array}{l}\text { Quetiapine } \\
400-800 \mathrm{mg} / \text { day } \\
\text { (497 mg/day mean) } \\
+ \text { Valproate } \\
50-125 \mathrm{mcg} / \mathrm{mL} \\
\text { target } \\
\text { OR } \\
\text { Lithium } \\
0.5-1.2 \mathrm{mEq} / \mathrm{L} \text { target }\end{array}$ & $\begin{array}{l}\text { Placebo } \\
+ \text { Valproate } \\
50-125 \mathrm{mcg} / \mathrm{mL} \\
\text { target } \\
\text { OR } \\
\text { Lithium } \\
0.5-1.2 \mathrm{mEq} / \mathrm{L} \\
\text { target }\end{array}$ & $\begin{array}{l}104 \text { weeks } \\
\text { (only time to } \\
\text { occurrence } \\
\text { and } \\
\text { withdrawals } \\
\text { used due to } \\
\text { attrition) }\end{array}$ & $\begin{array}{l}\text { Recurrance } \\
\text { CGI-BP } \\
\text { PANSS-P } \\
\text { SDS } \\
\text { PGWB } \\
\text { SAS } \\
\text { BARS } \\
\text { AIMS } \\
\text { Withdrawal 51\% (Time } \\
\text { to recurrence } \\
\text { outcomes only } \\
\text { included) } \\
\text { Nonrelapse withdrawal } \\
16 \%\end{array}$ \\
\hline $\begin{array}{l}\text { Bobo, } 2011^{36} \\
\text { RCT } \\
\text { Single-site } \\
\text { US } \\
\text { Industry } \\
\text { RoB High } \\
22104634\end{array}$ & $\begin{array}{l}\mathrm{N}=50 \\
\text { Mean Age } 40 \\
\text { Female } 67 \% \\
\text { White } 67 \% \\
\text { BP I } 73 \% \\
\text { BP II } 27 \% \\
\\
\text { Outpatient }\end{array}$ & $\begin{array}{l}\text { Any Phase } \\
\text { Schizoaffective; } \\
\text { Other Mental Health } \\
\text { Conditions; } \\
\text { Pregnant/Nursing }\end{array}$ & $\begin{array}{l}\text { Risperidone long- } \\
\text { acting injectable } \\
27 \pm 10.4 \text { mg every } \\
2 \text { weeks } \\
+ \text { Treatment as } \\
\text { Usual }\end{array}$ & $\begin{array}{l}\text { No Placebo + } \\
\text { Treatment as } \\
\text { Usual }\end{array}$ & 52 weeks & \begin{tabular}{|l|} 
AIMS \\
BARS \\
CGI-S \\
MADRS \\
Quick Inventory of \\
depressive symptoms \\
self-report (QIDS-SR) \\
SAS \\
YMRS \\
Withdrawal 25\%
\end{tabular} \\
\hline
\end{tabular}




\begin{tabular}{|c|c|c|c|c|c|c|}
\hline $\begin{array}{l}\text { Study, Year } \\
\text { Design } \\
\text { Location } \\
\text { Funder } \\
\text { Risk of Bias } \\
\text { PMID }\end{array}$ & $\begin{array}{c}\text { \# Randomized } \\
\text { Age (mean) } \\
\text { Sex (\% Female) } \\
\text { Race (\% White) } \\
\text { Diagnosis } \\
\text { (\% BP I, II, NOS) } \\
\text { Setting }\end{array}$ & $\begin{array}{c}\text { Inclusions } \\
\text { Key Exclusions }\end{array}$ & $\begin{array}{l}\text { Intervention } \\
\text { Dosage }\end{array}$ & $\begin{array}{c}\text { Comparison } \\
\text { Dosage }\end{array}$ & $\begin{array}{l}\text { Followup } \\
\text { Duration }\end{array}$ & $\begin{array}{c}\text { Outcomes } \\
\text { Reported } \\
\begin{array}{c}\text { Withdrawal (\%) at } \\
\text { endpoint }\end{array}\end{array}$ \\
\hline $\begin{array}{l}\text { Macfadden, } 2009^{37} \\
\text { RCT } \\
\text { Multisite } \\
2 \text { Continents } \\
\text { Industry } \\
\text { RoB High } \\
19922552\end{array}$ & $\begin{array}{l}\mathrm{N}=124 \\
\text { Mean Age } 39 \\
\text { Female } 28 \% \\
\text { White } 10 \% \\
\text { BP I } 100 \% \\
\text { Inpatient and outpatient }\end{array}$ & $\begin{array}{l}\text { Any Phase including } \\
\text { euthymic; } \\
4 \text { or more mood episodes in } \\
\text { past year } \\
\text { Substance Abuse; } \\
\text { Other Mental Health } \\
\text { Conditions; Taking other } \\
\text { Medications; } \\
\text { Abnormal Lab Results }\end{array}$ & $\begin{array}{l}\text { Risperidone long- } \\
\text { acting injectable } \\
25-30 \text { mg every } 2 \\
\text { weeks } \\
\text { +Treatment as } \\
\text { Usual }\end{array}$ & $\begin{array}{l}\text { Placebo + } \\
\text { Treatment As } \\
\text { Usual }\end{array}$ & 52 weeks & $\begin{array}{l}\text { CGI-BP-C } \\
\text { CGI-BP-S } \\
\text { MADRS } \\
\text { Relapse - Time to } \\
\text { (DSM diagnosis for } \\
\text { acute mood episode + } \\
\text { other complicated } \\
\text { criteria) } \\
\text { YMRS } \\
\text { Withdrawal 48\% }\end{array}$ \\
\hline $\begin{array}{l}\text { Bowden, } 2010^{38} \\
\text { RCT of responders } \\
\text { Multisite } \\
\text { 3 Continents } \\
\text { Industry } \\
\text { RoB High } \\
20122373 \text { (also } \\
22999893 \text { ) }\end{array}$ & $\begin{array}{l}\mathrm{N}=240 \\
\text { Mean Age } 39 \\
\text { Female } 54 \% \\
\text { White } 62 \% \\
\text { BP I } 100 \% \\
\text { Outpatient }\end{array}$ & $\begin{array}{l}\text { Mania; Initial inclusion: } \\
\text { YMRS } \geq 14 \text { with score } \geq 2 \\
\text { on at least four items at } \\
\text { screening and admission. } \\
\text { Extension inclusion: } \\
\text { stabilized (CGI-I } \leq 3 \text { at least } \\
2 \text { consecutive weeks } \\
\text { Substance Abuse; } \\
\text { Other Mental Health } \\
\text { Condition; } \\
\text { Pregnant/Nursing; } \\
\text { Labs/Other Conditions }\end{array}$ & \begin{tabular}{|l} 
Ziprasidone \\
(80-160 mg/day) \\
+ Lithium \\
$(0.6-1.2 \mathrm{mEq} / \mathrm{L})$ or \\
Valproate \\
$(50-125 \mathrm{mcg} / \mathrm{mL})$
\end{tabular} & \begin{tabular}{|l|} 
Placebo+ \\
Lithium \\
$(0.6-1.2 \mathrm{mEq} / \mathrm{L})$ \\
or \\
Valproate \\
$(50-125$ \\
$\mathrm{mcg} / \mathrm{mL})$
\end{tabular} & 26 weeks & $\begin{array}{l}\text { BMI or Weight } \\
\text { MADRS } \\
\text { Mania Rating Scale } \\
\text { (MRS) } \\
\text { Relapse - Relative } \\
\text { Risk of } \\
\text { Relapse - Time to } \\
\text { intervention for mood } \\
\text { episode } \\
\text { Withdrawal } 42 \%\end{array}$ \\
\hline
\end{tabular}




\begin{tabular}{|c|c|c|c|c|c|c|}
\hline $\begin{array}{l}\text { Study, Year } \\
\text { Design } \\
\text { Location } \\
\text { Funder } \\
\text { Risk of Bias } \\
\text { PMID }\end{array}$ & $\begin{array}{c}\text { \# Randomized } \\
\text { Age (mean) } \\
\text { Sex (\% Female) } \\
\text { Race (\% White) } \\
\text { Diagnosis } \\
\text { (\% BP I, II, NOS) } \\
\text { Setting }\end{array}$ & $\begin{array}{c}\text { Inclusions } \\
\text { Key Exclusions }\end{array}$ & $\begin{array}{c}\text { Intervention } \\
\text { Dosage }\end{array}$ & $\begin{array}{c}\text { Comparison } \\
\text { Dosage }\end{array}$ & $\begin{array}{l}\text { Followup } \\
\text { Duration }\end{array}$ & $\begin{array}{c}\text { Outcomes } \\
\text { Reported } \\
\text { Withdrawal (\%) at } \\
\text { endpoint }\end{array}$ \\
\hline $\begin{array}{l}\text { Vieta, } 2008^{39} \\
\text { RCT } \\
\text { Multisite } \\
\text { Spain } \\
\text { Industry } \\
\text { RoB Moderate } \\
18346292\end{array}$ & $\begin{array}{l}\mathrm{N}=55 \\
\text { Mean Age 44 } \\
\text { Female 65\% } \\
\text { White NR } \\
\text { BP I 76\% } \\
\text { BP II 24\% } \\
\text { Outpatient }\end{array}$ & $\begin{array}{l}\text { Euthymic; } \\
\text { YMRS } \leq 12 ; \\
\text { MADRS } \leq 20 ; \\
\text { 2+ episodes in past year; } \\
\geq 6 \text { months in remission; } \\
\text { Being treated with Lithium } \\
\text { ( } \geq 0.6 \text { meq/L) } \\
\text { Substance Abuse; } \\
\text { Other Mental Health } \\
\text { Conditions; } \\
\text { Taking Other Meds; } \\
\text { Pregnant/Nursing }\end{array}$ & $\begin{array}{l}\text { Oxcarbazepine } \\
1200 \mathrm{mg} / \mathrm{day}+ \\
\text { Lithium } \\
300-1200 \mathrm{mg} / \mathrm{d} \\
\text { (mean NR) }\end{array}$ & \begin{tabular}{|l} 
Placebo + \\
Lithium \\
$300-1200$ mg/d \\
$($ mean NR)
\end{tabular} & 52 weeks & $\begin{array}{l}\text { Relapse } \\
\text { (DSM-IV criteria } \\
\text { for manic, } \\
\text { hypomanic, } \\
\text { mixed or } \\
\text { depressive } \\
\text { episode; OR } \\
\text { YMRS } \geq 12 ; \text { OR } \\
\text { MADRS } \geq 20 \text { ) } \\
\text { CGI-BP-M } \\
\text { GAF } \\
\text { BIS-11 } \\
\text { Withdrawal } 36 \%\end{array}$ \\
\hline $\begin{array}{l}\text { Vieta, } 2006^{40} \\
\text { RCT } \\
\text { Multisite } \\
\text { Spain } \\
\text { Industry } \\
\text { RoB High } \\
16649836\end{array}$ & $\begin{array}{l}\mathrm{N}=25 \\
\text { Mean Age 49 } \\
\text { Female 72\% } \\
\text { White NR } \\
\text { BP I 76\% } \\
\text { BP II 24\% } \\
\text { Outpatient }\end{array}$ & $\begin{array}{l}\text { Euthymic; } \\
\text { CGI-BP-M } \geq 4 ; \\
\text { HAMD } \leq 8 \\
\text { YMRS } 4 \text {; } \\
\text { Treated with lithium for } 6 \\
\text { weeks; } \\
\text { Last episode within } 6 \text { mos; } \\
\text { Substance abuse } \\
\text { Pregnant/nursing } \\
\text { Labs/other conditions }\end{array}$ & $\begin{array}{l}\text { Gabapentin } \\
300-800 \mathrm{mg} / \mathrm{tid} \\
\text { (400mg/tid) } \\
+ \text { Lithium and/or } \\
\text { Valproate and/or } \\
\text { Carbamazepine } \\
\text { NR }\end{array}$ & $\begin{array}{l}\text { Placebo + } \\
\text { Lithium and/or } \\
\text { Valproate } \\
\text { and/or } \\
\text { Carbamazepine } \\
\text { NR }\end{array}$ & 52 week & $\begin{array}{l}\text { CGI-BP-M } \\
\text { YMRS } \\
\text { HAM-D } \\
\text { PQSI } \\
\text { Time to Relapse } \\
\text { Withdrawal 48\% }\end{array}$ \\
\hline
\end{tabular}




\begin{tabular}{|c|c|c|c|c|c|c|}
\hline $\begin{array}{l}\text { Study, Year } \\
\text { Design } \\
\text { Location } \\
\text { Funder } \\
\text { Risk of Bias } \\
\text { PMID }\end{array}$ & $\begin{array}{c}\text { \# Randomized } \\
\text { Age (mean) } \\
\text { Sex (\% Female) } \\
\text { Race (\% White) } \\
\text { Diagnosis } \\
\text { (\% BP I, II, NOS) } \\
\text { Setting }\end{array}$ & $\begin{array}{c}\text { Inclusions } \\
\text { Key Exclusions }\end{array}$ & $\begin{array}{c}\text { Intervention } \\
\text { Dosage }\end{array}$ & $\begin{array}{c}\text { Comparison } \\
\text { Dosage }\end{array}$ & $\begin{array}{l}\text { Followup } \\
\text { Duration }\end{array}$ & $\begin{array}{c}\text { Outcomes } \\
\text { Reported } \\
\begin{array}{c}\text { Withdrawal (\%) at } \\
\text { endpoint }\end{array}\end{array}$ \\
\hline $\begin{array}{l}\text { Zarate, } 2004^{41} \\
\text { Single-Site } \\
\text { RCT } \\
\text { US } \\
\text { Gov't+nonprofit } \\
\text { RoB High } \\
14702269\end{array}$ & $\begin{array}{l}\mathrm{N}=37 \\
\text { Mean Age } 34 \\
\text { Female 78\% } \\
\text { White NR } \\
\text { BP I 100\% } \\
\text { Setting NR }\end{array}$ & $\begin{array}{l}\text { Manic /Mixed at study entry; } \\
\text { DSM-IV criteria (Structured } \\
\text { Clinical Interview) } \\
\text { Euthymic by randomization } \\
\text { (week 10); } \\
\text { YMRS } 10 ; \\
\text { HAM-D } \leq 10 \\
\text { Schizoaffective } \\
\text { Substance abuse } \\
\text { Other mental health } \\
\text { Labs/other conditions }\end{array}$ & $\begin{array}{l}\text { Perphenazine } \\
\text { 4-64 mg/day } \\
+ \text { (Mood Stabilizers } \\
\text { Lithium 0.6-1.2 } \\
\text { meq/L } \\
\text { And/Or } \\
\text { Carbamazepine 4- } \\
12 \mathrm{mg} / \mathrm{L} \\
\text { And/Or } \\
\text { Valproate } 50-125 \\
\text { mg/L) }\end{array}$ & \begin{tabular}{|l|} 
Placebo+ \\
(Mood \\
Stabilizers \\
Lithium 0.6-1.2 \\
meq/L \\
And/Or \\
Carbamazepine \\
$4-12 \mathrm{mg} / \mathrm{L}$ \\
And/Or \\
Valproate 50- \\
125 mg/L)
\end{tabular} & 6 months & $\begin{array}{l}\text { Relapse } \\
\quad \text { (Not Defined) } \\
\text { HAM-D } \\
\text { Withdrawal 35\% }\end{array}$ \\
\hline $\begin{array}{l}\text { Nierenberg, } 2016^{42} \\
\text { RCT } \\
\text { Multisite } \\
\text { US } \\
\text { Government } \\
\text { RoB High } \\
26845264\end{array}$ & $\begin{array}{l}\mathrm{N}=482 \\
\text { Mean Age } 39 \\
\text { Female 59\% } \\
\text { White } 72 \% \\
\text { BP I } 68 \% \\
\text { BP II, NOS NR } \\
\text { Outpatient }\end{array}$ & $\begin{array}{l}\text { Any status } \\
\text { Pregnant/Nursing; } \\
\text { Labs/Other Conditions }\end{array}$ & $\begin{array}{l}\text { Quetiapine } \\
\text { 150-900 mg/day } \\
\text { (345 mg/day) } \\
\text { + Adjunctive } \\
\text { personalized } \\
\text { treatment } \\
\text { Texas Medication } \\
\text { Algorithm }\end{array}$ & $\begin{array}{l}\text { Lithium } \\
0.6-1.2 \mathrm{mEq} / \mathrm{L} \\
(0.6 \mathrm{mEg} / \mathrm{L}) \\
\text { + Adjunctive } \\
\text { personalized } \\
\text { treatment } \\
\text { Texas } \\
\text { Medication } \\
\text { Algorithm }\end{array}$ & 24 weeks & \begin{tabular}{|l|} 
CGI-El \\
MADRS \\
YMRS \\
Columbia-Suicide \\
Severity Rating Scale \\
\\
Withdrawal 25\%
\end{tabular} \\
\hline
\end{tabular}




\begin{tabular}{|c|c|c|c|c|c|c|}
\hline $\begin{array}{l}\text { Study, Year } \\
\text { Design } \\
\text { Location } \\
\text { Funder } \\
\text { Risk of Bias } \\
\text { PMID }\end{array}$ & $\begin{array}{c}\text { \# Randomized } \\
\text { Age (mean) } \\
\text { Sex (\% Female) } \\
\text { Race (\% White) } \\
\text { Diagnosis } \\
\text { (\% BP I, II, NOS) } \\
\text { Setting }\end{array}$ & $\begin{array}{c}\text { Inclusions } \\
\text { Key Exclusions }\end{array}$ & $\begin{array}{c}\text { Intervention } \\
\text { Dosage }\end{array}$ & $\begin{array}{c}\text { Comparison } \\
\text { Dosage }\end{array}$ & $\begin{array}{l}\text { Followup } \\
\text { Duration }\end{array}$ & $\begin{array}{c}\text { Outcomes } \\
\text { Reported } \\
\begin{array}{c}\text { Withdrawal (\%) at } \\
\text { endpoint }\end{array}\end{array}$ \\
\hline \begin{tabular}{|l|} 
Vieta, $2010^{43}$ \\
Observational (Partial \\
responders of earlier \\
RCT) \\
Multisite \\
Not Disclosed \\
Industry \\
RoB High \\
20429835 (Continuation \\
of 18381903 )
\end{tabular} & $\begin{array}{l}\mathrm{N}=283 \\
\text { Mean Age } 43 \\
\text { Female } 53 \% \\
\text { White } 93 \% \\
\text { BP I } 100 \% \\
\text { Outpatient }\end{array}$ & $\begin{array}{l}\text { Mania; } \\
\text { Mania Rating Scale (Spitzer, } \\
1978) \geq 14 \text { with score } \geq 2 \text { on } \\
\text { four items at screening and } \\
\text { admission } \\
\text { Other Mental Health } \\
\text { Conditions; Substance } \\
\text { Abuse }\end{array}$ & $\begin{array}{l}\text { Valproate } \\
500-2500 \mathrm{mg} / \text { day } \\
(1174.3 \mathrm{mg} / \text { day } \\
\text { average - last } 4 \\
\text { weeks) } \\
+ \text { Aripiprazole } \\
15-30 \mathrm{mg} / \text { day }(17.1- \\
18.5 \mathrm{mg} / \text { day } \\
\text { average) }\end{array}$ & \begin{tabular}{|l|} 
Lithium \\
$500-1500$ \\
mg/day \\
$(1105.5 \mathrm{mg} /$ day \\
average) \\
+ Aripiprazole \\
\\
$15-30 \mathrm{mg} /$ day \\
$(16.9-18.4$ \\
mg/day \\
average $)$
\end{tabular} & 46 weeks & $\begin{array}{l}\text { BMI or Weight } \\
\text { LIFE-RIFT } \\
\text { MADRS } \\
\text { Relapse - Emergent } \\
\text { Depression Incidence } \\
\text { (MADRS total score >= } \\
18 \text { and >= 4 point } \\
\text { increase in two } \\
\text { consecutive } \\
\text { assessments or last } \\
\text { observation) } \\
\text { Relapse (YMRS total } \\
\text { score <=12 and } \\
\text { MADRS total <=8 of } \\
\text { patients who achieved } \\
\text { remission at end of } \\
\text { week 6) } \\
\text { YMRS } \\
\text { Withdraw } 48 \%\end{array}$ \\
\hline
\end{tabular}

Abbreviations: AIMS=Abnormal Involuntary Movement Scale; BARS=Barnes Akathisia Scale; BIS-11=Barratt Impulsiveness Scale; BMI=Body Mass Index; BP=bipolar disorder; C=Comparison; CGI-BP=Clinical Global Impressions Scale for Bipolar Disorder; CGI-BP-M=Clinical Global Impressions Scale-Bipolar-modified (for long-term followup); CGI-BP-S=Clinical Global Impressions, Bipolar, Severity Scale; CGI-EI=Clinical Global Impressions-Efficacy Index; CGI-I=Clinical Global Impressions Scale, Improvement; CGI-S=Clinical Global Impressions, Severity Scale; DSM=Diagnostic and Statistical Manual of Mental Disorders; DSS=Depressive Syndrome Scale; EPS=extrapyramidal symptoms; ESRS=Extrapyramidal Symptom Rating Scale; GAF=General Assessment of Functioning Scale; GAS=Global Assessment Scale; HAM-

D=Hamilton Scale for Depression; IMPS=Inpatient Multidimensional Psychiatric Scale; KAS=Katz Adjustment Scale; LIFE-RIFT= Longitudinal Interval Follow-up EvaluationRange of Impaired Functioning Tool; MADRS=Montgomery-Asberg Depression Rating Scale; MOS-Cog=Medical Outcomes Study Cognitive Scale; MRS=Mania Rating Scale; NOS=not otherwise specified; NR=not reported; PANSS=Positive and Negative Syndrome Scale; PGWB=Psychological General Well-being Scale; PMID=PubMed Identification Number; PSQI=Pittsburgh Sleep Quality Index; QIDS-SR=Quick Inventory of Depressive Symptomatology (Self-reported); RCT=randomized controlled trial; ROB=risk of bias; SAS=Simpson Angus Scale; SDS=Sheehan Disability Scale; TMT=Trail Making Test; WPAI=Work Productivity and Activity Impairment Questionnaire; YMRS =Young Mania Rating Scale 
Appendix Table 112. Summary risk of bias assessments: oombination therapy for maintenance

\begin{tabular}{|c|c|c|c|}
\hline Drug & $\begin{array}{c}\text { Study } \\
\text { Funding Source } \\
\text { PMID }\end{array}$ & $\begin{array}{l}\text { Overall Risk of Bias } \\
\text { Assessment }\end{array}$ & Rationale \\
\hline $\begin{array}{l}\text { Lithium + } \\
\text { valproate vs. } \\
\text { valproate vs. } \\
\text { lithium }\end{array}$ & $\begin{array}{l}\text { Balance } \\
\text { Investigators, } \\
2010^{18} \\
\text { Industry } \\
20092882 \\
\end{array}$ & Moderate & Open label. Intention To Treat used, but handling of dropouts/missing data not described. \\
\hline \multirow{3}{*}{$\begin{array}{l}\text { Aripiprazole }+ \\
\text { mood stabilizer } \\
\text { vs. placebo }+ \\
\text { mood stabilizer }\end{array}$} & $\begin{array}{l}\text { Marcus, } 2011^{28} \\
\text { Industry } \\
21443567 \\
\end{array}$ & High & High Withdrawal rate (43\%) - maintenance study; blinding procedures not disclosed. \\
\hline & $\begin{array}{l}\text { Carlson, } 2012^{29} \\
\text { Industry } \\
22329471\end{array}$ & High & $\begin{array}{l}\text { High nonrelapse withdrawal rate (40\%). Overall attrition 66\%. Randomization, concealment, } \\
\text { and blinding not described. }\end{array}$ \\
\hline & $\begin{array}{l}\text { Woo, } 2011^{30} \\
\text { Industry } \\
22134973 \\
\end{array}$ & High & $\begin{array}{l}\text { High Withdrawal rate (42\%) - maintenance study; Randomization and blinding procedures } \\
\text { not disclosed. }\end{array}$ \\
\hline $\begin{array}{l}\text { Divalproex }+ \\
\text { lithium vs. } \\
\text { placebo + lithium } \\
\text { alone }\end{array}$ & $\begin{array}{l}\text { Kemp, } 2009^{31} \\
\text { Government } \\
19192457\end{array}$ & High & Randomization and allocation not reported. Overall 19\% withdraw due other than relapse. \\
\hline \multirow{2}{*}{$\begin{array}{l}\text { Quetiapine }+ \\
\text { mood stabilizers } \\
\text { vs. placebo }+ \\
\text { mood stabilizers }\end{array}$} & $\begin{array}{l}\text { Suppes, } 2009^{34} \\
\text { Industry } \\
19289454\end{array}$ & High & Blinding not described; differential dropout rates. High drop-out rates overall. \\
\hline & $\begin{array}{l}\text { Vieta, } 2008^{35} \\
\text { Industry } \\
18579216 \\
\end{array}$ & High & $\begin{array}{l}\text { Generally well reported with minor concerns related to blinding, subjective definition of } \\
\text { recurrence. High drop-out rates overall. }\end{array}$ \\
\hline $\begin{array}{l}\text { Risperidone long- } \\
\text { acting injectable+ } \\
\text { treatment as } \\
\text { usual vs. placebo } \\
+ \text { treatment as } \\
\text { usual }\end{array}$ & $\begin{array}{l}\text { Macfadden, } \\
2009^{37} \\
\text { Industry } \\
19922552\end{array}$ & High & $\begin{array}{l}\text { BPII patients enrolled, but removed from analysis. } 48 \% \text { dropout overall. Large differential } \\
\text { dropout with } 58 \% \text { placebo and } 40 \% \text { of treatment groups dropping. }\end{array}$ \\
\hline $\begin{array}{l}\text { Risperidone long- } \\
\text { acting injectable+ } \\
\text { treatment as } \\
\text { usual vs. } \\
\text { treatment as } \\
\text { usual }\end{array}$ & $\begin{array}{l}\text { Bobo, } 2011^{36} \\
\text { Industry } \\
22104634\end{array}$ & High & $\begin{array}{l}\text { No blinding. Treatment As Usual not well controlled. Treatment regimes of the two groups } \\
\text { prior to study were not tested for similarity and appear as though they may differ statistically } \\
\text { Results may be due to differences in TAU. } 25 \% \text { dropout. }\end{array}$ \\
\hline
\end{tabular}




\begin{tabular}{|c|c|c|c|}
\hline Drug & $\begin{array}{c}\text { Study } \\
\text { Funding Source } \\
\text { PMID }\end{array}$ & $\begin{array}{l}\text { Overall Risk of Bias } \\
\text { Assessment }\end{array}$ & Rationale \\
\hline $\begin{array}{l}\text { Olanzapine }+ \\
\text { mood stabilizer } \\
\text { vs. placebo }+ \\
\text { mood stabilizer }\end{array}$ & $\begin{array}{l}\text { Tohen, } 2004^{32} \\
\text { Industry } \\
15056579 \\
\text { extension of } \\
\text { Tohen, } 2002^{33} \\
11779284\end{array}$ & $\begin{array}{l}\text { Moderate } \\
\text { (High for log rank) }\end{array}$ & Allocation concealment not described. Log rank test. Unclear nonrelapse withdrawal. \\
\hline $\begin{array}{l}\text { Oxcarbazepine } \\
\text { lithium vs. } \\
\text { placebo + lithium }\end{array}$ & $\begin{array}{l}\text { Vieta, } 2008^{39} \\
\text { Industry } \\
18346292 \\
\end{array}$ & Moderate & Patients, staff, and raters may not be blinded; procedures not described \\
\hline $\begin{array}{l}\text { Gabapentin + } \\
\text { mood stabilizers } \\
\text { vs. placebo + } \\
\text { mood stabilizers }\end{array}$ & $\begin{array}{l}\text { Vieta, } 2006^{40} \\
\text { Industry } \\
16649836\end{array}$ & High & $\begin{array}{l}\text { Distribution of BP I and BP II patients differs between treatment arms, creating a residual } \\
\text { confounder. }\end{array}$ \\
\hline $\begin{array}{l}\text { Perphenazine + } \\
\text { mood stabilizers } \\
\text { placebo + mood } \\
\text { stabilizers }\end{array}$ & $\begin{array}{l}\text { Zarate, } 2004^{41} \\
\text { Gov't+nonprofit } \\
14702269\end{array}$ & High & $\begin{array}{l}\text { Randomization and blinding procedures not disclosed; numerical results of several measured } \\
\text { outcomes not presented; Relapse not defined }\end{array}$ \\
\hline $\begin{array}{l}\text { Ziprasidone + } \\
\text { mood stabilizers } \\
\text { vs. placebo + } \\
\text { mood stabilizers }\end{array}$ & $\begin{array}{l}\text { Bowden, } 2010^{38} \\
\text { Industry } \\
20122373 \text { (also } \\
\text { 22999893) }\end{array}$ & High & Randomization and blinding procedures not described. $40 \%+$ dropout. \\
\hline $\begin{array}{l}\text { Quetiapine }+ \\
\text { personalize } \\
\text { treatment vs. } \\
\text { Lithium }+ \\
\text { personalized } \\
\text { treatment }\end{array}$ & $\begin{array}{l}\text { Nierenberg, } \\
2016^{42} \\
\text { Government } \\
26845264\end{array}$ & High & $\begin{array}{l}\text { Does not report on adjunctive treatments received in results. Includes those who have 'off- } \\
\text { procedure' treatment deviations in analysis, who are people that have taken antipsychotics. } \\
\text { Also included are the roughly } 30 \% \text { of people in both treatment arms who have no adjunctive } \\
\text { treatment. None of these is accounted for in analysis as a possible confounding influence on } \\
\text { the underlying comparison of Quetiapine and Lithium. }\end{array}$ \\
\hline $\begin{array}{l}\text { Valproic acid + } \\
\text { Aripiprazole vs. } \\
\text { Lithium + } \\
\text { Aripiprazole }\end{array}$ & $\begin{array}{l}\text { Vieta, } 2010^{43} \\
\text { Industry } \\
20429835 \\
\text { (Continuation of } \\
\text { 18381903) }\end{array}$ & High & $\begin{array}{l}\text { Non-Randomized continuation study of partial responders, no blinding, initial baseline } \\
\text { measures of this group may not be similar, appears to be underpowered for the subgroup } \\
\text { analysis that is presented. } 48 \% \text { dropout. }\end{array}$ \\
\hline
\end{tabular}

Abbreviations: BP=bipolar; LOCF=Last observation carried forward; PMID=PubMed Identification Number; RCT=randomized controlled trial 
Appendix Table I13. Outcomes summary: combination therapy versus placebo for maintenance

\begin{tabular}{|c|c|c|c|c|c|c|}
\hline Drug & $\begin{array}{c}\text { Study } \\
\text { RoB } \\
\text { PMID }\end{array}$ & Responder/Remitter & Symptom & Function & Other & $\mathrm{AE}$ \\
\hline $\begin{array}{l}\text { Aripiprazole }+ \\
\text { mood stabilizer } \\
\text { vs. placebo }+ \\
\text { mood stabilizer }\end{array}$ & $\begin{array}{l}\text { Marcus, }{ }^{28} 2011 \\
\text { High } \\
21443567\end{array}$ & $\begin{array}{l}\text { Time to Relapse } \\
52 \text { weeks } \\
\text { Hazard Ratio } 0.54(0.33 \text {, } \\
0.89) \\
\text { Favors Aripiprazole }\end{array}$ & $\begin{array}{l}\text { YMRS } \\
52 \text { weeks } \\
\text { Mean change } \\
\text { Aripiprazole: }-0.1 \\
\text { Placebo: } 2.9 \\
\text { p<0.001 } \\
\text { Favors Aripiprazole } \\
\text { MADRS } \\
52 \text { weeks } \\
\text { Mean change } \\
\text { Aripiprazole: } 1.5 \\
\text { Placebo: } 2.5 \\
\text { p=0.02 } \\
\text { Favors Aripiprazole }\end{array}$ & $\begin{array}{l}\text { CGI-BP-S } \\
52 \text { weeks } \\
\text { Ari } n=162 \\
\text { Plc } n=164 \\
\text { Mean change } \\
\text { difference }-0.3 \text { (- } \\
0.62,-0.07) \\
\text { Favors } \\
\text { Aripiprazole } \\
\text { Less than } \mathrm{MID}=1\end{array}$ & $\begin{array}{l}\frac{\text { Weight gain }>7 \%}{\text { NS }} \\
\text { Overall Withdrawal } \\
\text { Aripiprazole } 65 / 168 \\
(38.7 \%) \\
\text { Placebo 80/169 (47.3\%) } \\
\text { NS } \\
\text { Withdrawal lack of } \\
\text { efficacy } \\
\text { Aripiprazole } 6 / 168 \\
\text { (8.3\%) } \\
\text { Placebo 31/169 (18.3\%) } \\
\text { p=0.007 } \\
\text { Withdrawal for AE } \\
\text { Aripiprazole } 19 / 168 \\
11.3 \% \\
\text { Placebo 15/169 (8.9\%) } \\
\text { NS }\end{array}$ & $\begin{array}{l}2 \text { deaths, } 1 \text { in each } \\
\text { arm; } 1 \text { suicide day } 83 \\
\text { deemed not due to } \\
\text { treatment } \\
1 \text { tardive dyskinesia } \\
\text { (placebo group) } \\
\text { SAE } \\
\text { Aripiprazole: } 11 \\
\text { (6.6\%) } \\
\text { Placebo: } 8(4.8 \%) \\
\text { NS }\end{array}$ \\
\hline
\end{tabular}




\begin{tabular}{|c|c|c|c|c|c|c|}
\hline Drug & $\begin{array}{c}\text { Study } \\
\text { RoB } \\
\text { PMID }\end{array}$ & Responder/Remitter & Symptom & Function & Other & $\mathrm{AE}$ \\
\hline & $\begin{array}{l}\text { Woo, } 2011^{30} \\
\text { High } \\
22134973\end{array}$ & $\begin{array}{l}\text { Time to Relapse } \\
6 \text { months } \\
\text { NS }\end{array}$ & $\begin{array}{l}\text { YMRS } \\
6 \text { months } \\
\text { NS } \\
\text { MADRS } \\
6 \text { months } \\
\text { NS }\end{array}$ & $\begin{array}{l}\text { CGI-CP-S } \\
6 \text { months } \\
\text { NS }\end{array}$ & $\begin{array}{l}\frac{\text { Weight gain }>7 \%}{\text { NS }} \\
\text { Overall Withdrawal } \\
\text { Aripiprazole } 17 / 40 \\
(38.7 \%) \\
\text { Placebo 18/43 (41.9\%) } \\
\text { NS } \\
\text { Withdrawal lack of } \\
\text { efficacy } \\
\text { Aripiprazole } 6 / 40(15 \%) \\
\text { Placebo } 8 / 43(18.6 \%) \\
\text { NS } \\
\text { Withdrawal for AE } \\
\text { Aripiprazole } 0 \% \\
\text { Placebo } 9.3 \% \\
\text { p=0.049 }\end{array}$ & $\begin{array}{l}\text { SAE } \\
\text { Aripiprazole: } 5 \% \\
\text { Placebo: } 11 \% \\
\text { Included } 1 \text { suicide } \\
\text { EPS } \\
\text { No discontinuation in } \\
\text { either group }\end{array}$ \\
\hline & $\begin{array}{l}\text { Carlson, } 2012^{29} \\
\text { High } \\
22329471\end{array}$ & $\begin{array}{l}\text { Time to Relapse } \\
52 \text { weeks } \\
\text { Hazard Ratio } 0.67(0.45 \text {, } \\
1.00) \\
\text { NS }\end{array}$ & $\begin{array}{l}\text { NA } \\
\text { (Attrition 66\%) }\end{array}$ & $\begin{array}{l}\text { NA } \\
\text { (Attrition 66\%) }\end{array}$ & $\begin{array}{l}\text { Withdraw for } \mathrm{AE} \\
\text { Aripiprazole } 14 / 176 \\
(8 \%) \\
\text { Placebo } 12 / 165(7.3 \%)\end{array}$ & $\begin{array}{l}\text { SAE } \\
\text { Aripiprazole 5/176 } \\
(2.8 \%) \\
\text { Placebo 9/165 (5.5\%) } \\
\text { No deaths or suicides } \\
\text { At least } 1 \text { EPS AE } \\
\text { Aripiprazole 28/176 } \\
\text { (15.9\%) } \\
\text { Placebo 15/165 } \\
\text { (9.1\%) } \\
\text { Weight gain >7\% } \\
\text { Aripiprazole } 11 \% \\
\text { Placebo 3.5\% } \\
\text { p=0.007 }\end{array}$ \\
\hline
\end{tabular}




\begin{tabular}{|c|c|c|c|c|c|c|}
\hline Drug & $\begin{array}{l}\text { Study } \\
\text { RoB } \\
\text { PMID }\end{array}$ & Responder/Remitter & Symptom & Function & Other & AE \\
\hline $\begin{array}{l}\text { Divalproex + } \\
\text { lithium vs. } \\
\text { placebo }+ \\
\text { lithium alone }\end{array}$ & $\begin{array}{l}\text { Kemp, } 2009^{31} \\
\text { High } \\
19192457\end{array}$ & $\begin{array}{l}\text { Time to Relapse } \\
52 \text { weeks } \\
\text { Hazard Ratio } 0.72(0.32, \\
1.65) \\
\text { NS }\end{array}$ & $\begin{array}{l}\text { NA } \\
\text { (Attrition 74\%) }\end{array}$ & $\begin{array}{l}\text { NA } \\
\text { (Attrition 74\%) }\end{array}$ & NA & $\begin{array}{l}\text { No SAEs mentioned. } \\
\frac{\text { EPS }}{\text { Tremors NS }}\end{array}$ \\
\hline $\begin{array}{l}\text { Olanzapine }+ \\
\text { mood stabilizer } \\
\text { vs. placebo }+ \\
\text { mood stabilizer }\end{array}$ & $\begin{array}{l}\text { Tohen, } 2004^{32} \\
\text { Industry } \\
15056579 \\
\text { extension of } \\
\text { Tohen, } 2002^{33} \\
11779284\end{array}$ & $\begin{array}{l}\text { Time to relapse } \\
18 \text { months } \\
\text { Log rank } \\
\text { NS }\end{array}$ & $\begin{array}{l}\text { NA } \\
\text { (Attrition 78\%) }\end{array}$ & NA & $\begin{array}{l}\text { Time to overall } \\
\text { withdrawal } \\
18 \text { month } \\
\text { Favors Olanzapine } \\
\text { Log rank } \\
p=0.049\end{array}$ & $\begin{array}{l}\text { SAE not reported } \\
\text { EPS } \\
\text { No difference between } \\
\text { groups }\end{array}$ \\
\hline $\begin{array}{l}\text { Oxcarbazepine } \\
+ \text { lithium vs. } \\
\text { placebo + } \\
\text { lithium }\end{array}$ & $\begin{array}{l}\text { Vieta, } 2008^{39} \\
\text { Moderate } \\
18346292\end{array}$ & $\begin{array}{l}\text { Time to relapse }{ }^{1} \\
52 \text { week } \\
\text { NS } \\
\text { Kaplan Meier log-rank } \\
\frac{\text { Relapse Rate }}{52 \text { week }} \\
\text { NS }\end{array}$ & $\begin{array}{l}\text { YMRS Change } \\
52 \text { week } \\
\text { NS } \\
\text { MADRS Change } \\
52 \text { week } \\
\text { NS }\end{array}$ & $\begin{array}{l}\text { CGI-BP-M } \\
52 \text { week } \\
\text { NS } \\
p=0.45\end{array}$ & $\begin{array}{l}\text { Weight Gain } \\
\geq 7 \% \text { of baseline } \\
\text { Oxcarbazapine } 19.2 \% \\
\text { Placebo } 6.9 \% \\
\text { Withdrawal for AE } \\
\text { Oxcarbazapine } 3 \\
\text { Placebo } 2 \\
\text { Withdrawal for lack of } \\
\text { efficacy } \\
\text { Oxcarbazapine } 0 \\
\text { Placebo } 2\end{array}$ & $\begin{array}{l}\text { Serious Adverse } \\
\text { Events } \\
52 \text { week } \\
3 \text { events - } \\
\text { Oxcarbazepine } \\
3 \text { events - Placebo }\end{array}$ \\
\hline $\begin{array}{l}\text { Gabapentin + } \\
\text { mood } \\
\text { stabilizers vs. } \\
\text { placebo + } \\
\text { mood } \\
\text { stabilizers }\end{array}$ & $\begin{array}{l}\text { Vieta, } 2006^{40} \\
\text { High } \\
16649836\end{array}$ & $\begin{array}{l}\text { Time to Relapse } \\
52 \text { week } \\
\text { NS } \\
\text { HR } 1.344\end{array}$ & $\begin{array}{l}\text { YMRS Change } \\
52 \text { week } \\
\text { NS } \\
\text { HAM-D } \\
52 \text { week } \\
\text { NS }\end{array}$ & $\begin{array}{l}\text { CGI-BP-M } \\
\text { Change } \\
52 \text { week } \\
\text { Favors } \\
\text { Gabapentin } \\
1.5 \%(95 \% \mathrm{Cl} \\
0.5,2.5) \\
p=0.0046\end{array}$ & $\begin{array}{l}\text { Withdrawal AE } \\
\text { Gabapentin } 1(8 \%) \\
\text { Placebo } 1(8 \%) \\
\text { Withdrawal lack of } \\
\text { efficacy } \\
\text { Gabapentin } 2(15 \%) \\
\text { Placebo } 1(8 \%)\end{array}$ & $\begin{array}{l}\text { Gabapentin: } 1 \\
\text { Myocardial Infarction } \\
\text { Placebo: No Events } \\
\text { Reported }\end{array}$ \\
\hline
\end{tabular}




\begin{tabular}{|c|c|c|c|c|c|c|}
\hline Drug & $\begin{array}{l}\text { Study } \\
\text { RoB } \\
\text { PMID }\end{array}$ & Responder/Remitter & Symptom & Function & Other & $\mathrm{AE}$ \\
\hline $\begin{array}{l}\text { Perphenazine } \\
+ \text { mood } \\
\text { stabilizers vs. } \\
\text { placebo + } \\
\text { mood } \\
\text { stabilizers }\end{array}$ & $\begin{array}{l}\text { Zarate, } 2004^{41} \\
\text { High } \\
14702269\end{array}$ & $\begin{array}{l}\frac{\text { Time to Relapse to }}{\text { Depression }} \\
\text { Favors placebo } \\
\text { Perphenazine: } 157 \text { days } \\
\text { (SE 10) } \\
\text { Placebo: None Occurred } \\
\text { p<0.03 } \\
\text { Depressive Relapse } \\
\text { Favors placebo } \\
\text { Perphenazine 21\% } \\
\text { Placebo 0\% } \\
\text { Manic Relapse } \\
\text { NS } \\
\text { Perphenazine } 5 \% \\
\text { Placebo 11\% }\end{array}$ & NR & NR & $\begin{array}{l}\text { Overall Withdrawal } \\
\text { Perphenazine } 10 \\
(52.6 \%) \\
\text { Placebo } 3(16.7 \%)\end{array}$ & NR \\
\hline \multirow[t]{2}{*}{$\begin{array}{l}\text { Quetiapine + } \\
\text { mood } \\
\text { stabilizers vs. } \\
\text { placebo + } \\
\text { mood } \\
\text { stabilizers }\end{array}$} & $\begin{array}{l}\text { Suppes, } 2009^{34} \\
\text { High } \\
19289454\end{array}$ & $\begin{array}{l}\text { Time to Recurrence of } \\
\text { mood event } \\
104 \text { weeks } \\
\text { Hazard Ratio } \\
0.32(0.24,0.42) \text { risk } \\
\text { reduction } 68 \% \\
\text { Favors Quetiapine } \\
\text { (not dependent on rapid } \\
\text { cycling) }\end{array}$ & $\begin{array}{l}70 \% \text { risk reduction in } \\
\text { time to recurrence of } \\
\text { mania } \\
67 \% \text { risk reduction in } \\
\text { time to recurrence of } \\
\text { depression }\end{array}$ & Not applicable & $\begin{array}{l}\text { Overall Withdrawal } \\
\text { Quetiapine 200/310 } \\
\text { Placebo } 247 / 313 \\
\text { Loss to followup and } \\
\text { Other categories greater } \\
\text { than adverse event or } \\
\text { lack of efficacy } \\
\text { categories }\end{array}$ & $\begin{array}{l}\text { SAE } \\
\text { Quetiapine: } 18 \text { (5.8\%) } \\
\text { Placebo: } 7(2.2 \%)\end{array}$ \\
\hline & $\begin{array}{l}\text { Vieta, } 2008^{35} \\
\text { High } \\
18579216\end{array}$ & $\begin{array}{l}\text { Time to Recurrence of } \\
\text { mood event } \\
104 \text { weeks } \\
\text { Hazard Ratio } \\
0.28(0.21,0.37) \text { risk } \\
\text { reduction } 72 \% \\
\text { Favors Quetiapine }\end{array}$ & $\begin{array}{l}\text { Time to Recurrence } \\
\text { of mania } \\
104 \text { weeks } \\
\text { Hazard Ratio } \\
0.30(0.20,0.44 \\
\text { Favors Quetiapine } \\
\\
\text { Time to Recurrence } \\
\text { of depression } \\
104 \text { weeks } \\
\text { Hazard Ratio } \\
0.26(0.17,0.41 \\
\text { Favors Quetiapine }\end{array}$ & Not applicable & $\begin{array}{l}\text { Overall Withdrawal } \\
\text { Quetiapine } 123 / 336 \\
\text { Placebo 233/367 } \\
\text { Loss to followup and } \\
\text { Other categories greater } \\
\text { than adverse event or } \\
\text { lack of efficacy } \\
\text { categories }\end{array}$ & $\begin{array}{l}\text { SAE } \\
\text { Quetiapine: } 5(1.5 \%) \\
\text { Placebo: } 20(5.4 \%)\end{array}$ \\
\hline
\end{tabular}




\begin{tabular}{|c|c|c|c|c|c|c|}
\hline Drug & $\begin{array}{l}\text { Study } \\
\text { RoB } \\
\text { PMID }\end{array}$ & Responder/Remitter & Symptom & Function & Other & AE \\
\hline $\begin{array}{l}\text { Risperidone } \\
\text { long acting } \\
\text { injectable + } \\
\text { treatment as } \\
\text { usual vs. } \\
\text { placebo + } \\
\text { treatment as } \\
\text { usual }\end{array}$ & $\begin{array}{l}\text { Macfadden, } \\
2009^{37} \\
\text { High } \\
19922552\end{array}$ & $\begin{array}{l}\text { Relapse - Time to (DSM } \\
\text { diagnosis for acute mood } \\
\text { episode + other } \\
\text { complicated criteria) } \\
52 \text { weeks } \\
\text { Log rank test } p=0.01 \\
\text { Favors Risperidone }\end{array}$ & $\begin{array}{l}\text { YMRS } \\
52 \text { weeks } \\
\text { Favors Risperidone } \\
\text { (only figure) } \\
\text { MADRS } \\
52 \text { weeks } \\
\text { NS }\end{array}$ & $\begin{array}{l}\text { CGI-BP-S } \\
52 \text { weeks } \\
\text { Favors } \\
\text { Risperidone } \\
\text { (only figure) }\end{array}$ & $\begin{array}{l}\text { Overall Withdrawal } \\
\text { Risperidone } 26 / 65 \\
(40 \%) \\
\text { Placebo } 34 / 59(57.6 \%) \\
\text { NS } \\
\text { Withdrawal lack of } \\
\text { efficacy } \\
\text { Risperidone } 13 / 65 \\
(20 \%) \\
\text { Placebo } 23 / 59(39 \%) \\
\text { p=0.02 } \\
\text { Withdrawal for AE } \\
\text { Risperidone } 3 / 65(4.6 \%) \\
\text { Placebo } 1 / 59(1.7 \% \\
\text { NS }\end{array}$ & $\begin{array}{l}\text { At least } 1 \text { SAE } \\
\text { Risperidone: } 9 \\
\text { Placebo: } 13 \\
\text { Deaths } \\
\text { Risperidone: } 1 \\
\text { Placebo: } 2 \text { (1 of } \\
\text { suicide } 3 \text { months after } \\
\text { study) } \\
\text { Suicide ideation: } \\
\text { Risperidone: } 1 \\
\text { Placebo: } 3 \\
\text { EPS } \\
\text { NS }\end{array}$ \\
\hline $\begin{array}{l}\text { Risperidone } \\
\text { long-acting } \\
\text { injectable + } \\
\text { treatment as } \\
\text { usual vs. } \\
\text { treatment as } \\
\text { usual }\end{array}$ & $\begin{array}{l}\text { Bobo, } 2011^{36} \\
\text { High } \\
22104634\end{array}$ & $\begin{array}{l}\text { Any cause relapse } \\
52 \text { weeks } \\
\text { NS }\end{array}$ & $\begin{array}{l}\text { YMRS } \\
52 \text { weeks } \\
\text { NS } \\
\text { MADRS } \\
52 \text { weeks } \\
\text { NS }\end{array}$ & $\begin{array}{l}\text { CGI-S } \\
52 \text { weeks } \\
\text { NS }\end{array}$ & $\begin{array}{l}\text { Overall Withdrawal } \\
\text { Risperidone } 4 / 25 \\
\text { Placebo } 6 / 25 \\
\text { NS } \\
\text { Withdrawal lack of } \\
\text { efficacy } \\
\text { Risperidone } 6 / 40(15 \%) \\
\text { Placebo } 8 / 43(18.6 \%) \\
\text { NS } \\
\text { Withdrawal for AE } \\
\text { Risperidone } 0 \% \\
\text { Placebo } 9.3 \% \\
p=0.049\end{array}$ & $\begin{array}{l}\text { No suicide attempts; } \\
\text { NS for suicide ideation } \\
\text { EPS } \\
\text { NS }\end{array}$ \\
\hline
\end{tabular}




\begin{tabular}{|c|c|c|c|c|c|c|}
\hline Drug & $\begin{array}{c}\text { Study } \\
\text { RoB } \\
\text { PMID }\end{array}$ & Responder/Remitter & Symptom & Function & Other & AE \\
\hline $\begin{array}{l}\text { Ziprasidone + } \\
\text { mood } \\
\text { stabilizers vs. } \\
\text { placebo + } \\
\text { mood } \\
\text { stabilizers }\end{array}$ & $\begin{array}{l}\text { Bowden, } \\
2010^{38} \\
\text { High } \\
20122373 \text { (also } \\
22999893 \text { ) }\end{array}$ & $\begin{array}{l}\text { Time to relapse } \\
26 \text { weeks } \\
\text { Log rank test } \\
p=0.01 \\
\text { Favors Ziprasidone }\end{array}$ & $\begin{array}{l}\text { YMRS } \\
26 \text { weeks } \\
\text { Least squares mean } \\
\text { difference } \\
-3.27(0.83) \\
\text { p }<0.001 \\
\text { Favors Ziprasidone } \\
\text { MADRS } \\
\text { NS }\end{array}$ & $\mathrm{NR}$ & $\begin{array}{l}\frac{\text { Weight gain }>7 \%}{\text { NS }} \\
\text { Overall Withdrawal } \\
\text { Ziprasidone 43/127 } \\
\text { Placebo 58/113 } \\
\mathrm{p}=0.007 \\
\text { Withdrawal lack of } \\
\text { efficacy } \\
\text { Ziprasidone 9/127 } \\
\text { Placebo 22/113 } \\
\mathrm{p}=0.004 \\
\text { Withdrawal for AE } \\
\text { Ziprasidone 11/127 } \\
\text { Placebo 15/113 } \\
\text { NS }\end{array}$ & $\begin{array}{l}\text { SAE } \\
\text { Ziprasidone: } 11 / 127 \\
8.7 \% \\
\text { Placebo: } 6 / 1125.4 \% \\
\text { No deaths }\end{array}$ \\
\hline
\end{tabular}

Abbreviations: AE=Adverse Events; CGI-BP-M=Clinical Global Impressions Scale-Bipolar-modified (for long-term follow-up); CGI-BP-S=Clinical Global Impressions, Bipolar, Severity Scale; CGI-S=Clinical Global Impressions, Severity Scale; CI=Confidence Interval; DSM=Diagnostic and Statistical Manual of Mental Disorders; EPS=extrapyramidal symptoms; HAM-D=Hamilton Scale for Depression; HR=Hazard Ratio; MADRS=Montgomery-Asberg Depression Rating Scale; MID=Minimally important difference; NA=Not applicable; NS= Not Significant; PMID=PubMed Identification Number; RoB=Risk of Bias; SAE=Serious Adverse Events; SE=standard error; YMRS = Young Mania Rating Scale;

\section{Appendix Table 114. Strength of evidence assessment: combination therapy versus placebo for maintenance}

\begin{tabular}{|c|c|c|c|c|c|c|c|c|}
\hline Comparison & Outcome & $\begin{array}{l}\text { \# Studies/ } \\
\text { Design } \\
\text { (n analyzed) }\end{array}$ & $\begin{array}{c}\text { Finding or Summary } \\
\text { Statistic } \\
\text { Outcome Timing }\end{array}$ & $\begin{array}{c}\text { Study } \\
\text { Limitations }\end{array}$ & Consistency & Directness & Precision & $\begin{array}{c}\text { Overall } \\
\text { Gradel } \\
\text { Conclusion }\end{array}$ \\
\hline $\begin{array}{l}\text { Aripiprazole }+ \\
\text { mood stabilizer } \\
\text { vs. placebo }+ \\
\text { mood stabilizer }\end{array}$ & $\begin{array}{l}\text { Time to Relapse } \\
52 \text { wks } \\
\text { YMRS } 6 \text { mths } \\
\text { MADRS } 6 \text { mths } \\
\text { CGI } 6 \text { mths } \\
\text { Withdrawals }\end{array}$ & $\begin{array}{l}2 \mathrm{RCT} \\
(\mathrm{n}=420)\end{array}$ & See table above & High & $\begin{array}{l}\text { Unknown } \\
\text { (over } 2 \text { time } \\
\text { periods; } \\
\text { Inconsistent if } \\
\text { combined) }\end{array}$ & Direct & Imprecise & Insufficient \\
\hline $\begin{array}{l}\text { Divalproex + } \\
\text { lithium vs. } \\
\text { placebo }+ \\
\text { lithium alone }\end{array}$ & $\begin{array}{l}\text { Time to relapse } \\
26 \text { weeks }\end{array}$ & $\begin{array}{l}1 \mathrm{RCT} \\
(\mathrm{n}=31)\end{array}$ & See table above & High & Unknown & Direct & Imprecise & Insufficient \\
\hline
\end{tabular}




\begin{tabular}{|c|c|c|c|c|c|c|c|c|}
\hline Comparison & Outcome & $\begin{array}{l}\text { \# Studies/ } \\
\text { Design } \\
\text { (n analyzed) }\end{array}$ & $\begin{array}{c}\text { Finding or Summary } \\
\text { Statistic } \\
\text { Outcome Timing }\end{array}$ & $\begin{array}{c}\text { Study } \\
\text { Limitations }\end{array}$ & Consistency & Directness & Precision & $\begin{array}{l}\text { Overall } \\
\text { Gradel } \\
\text { Conclusion }\end{array}$ \\
\hline $\begin{array}{l}\text { Olanzapine + } \\
\text { mood stabilizer } \\
\text { vs. placebo }+ \\
\text { mood stabilizer }\end{array}$ & $\begin{array}{l}\text { Time to relapse } \\
18 \text { months }\end{array}$ & $\begin{array}{l}1 \mathrm{RCT} \\
(\mathrm{n}=99)\end{array}$ & See table above & High & Unknown & Direct & Imprecise & Insufficient \\
\hline $\begin{array}{l}\text { Oxcarbazepine } \\
+ \text { lithium vs. } \\
\text { placebo + } \\
\text { lithium }\end{array}$ & $\begin{array}{l}\text { Time to relapse } \\
52 \text { wks } \\
\text { YMRS } 52 \text { wks } \\
\text { HAM-D } 52 \text { wks } \\
\text { CGI-BP-M } 52 \text { wks } \\
\end{array}$ & $\begin{array}{l}1 \mathrm{RCT} \\
(\mathrm{n}=55)\end{array}$ & See table above & Moderate & Unknown & Direct & Imprecise & Insufficient \\
\hline $\begin{array}{l}\text { Gabapentin + } \\
\text { mood } \\
\text { stabilizers vs. } \\
\text { placebo }+ \\
\text { mood } \\
\text { stabilizers }\end{array}$ & $\begin{array}{l}\text { Time to relapse } \\
52 \text { wks } \\
\text { YMRS } 52 \text { wks } \\
\text { HAM-D } 52 \text { wks } \\
\text { CGI-CP-M } 52 \text { wks }\end{array}$ & $\begin{array}{l}1 \mathrm{RCT} \\
(\mathrm{n}=25)\end{array}$ & See table above & High & Unknown & Direct & Imprecise & Insufficient \\
\hline $\begin{array}{l}\text { Perphenazine } \\
+ \text { mood } \\
\text { stabilizers vs. } \\
\text { placebo + } \\
\text { mood } \\
\text { stabilizers }\end{array}$ & $\begin{array}{l}\text { Time to } \\
\text { depression } \\
\text { relapse } 6 \text { mths }\end{array}$ & $\begin{array}{l}1 \mathrm{RCT} \\
(\mathrm{n}=37)\end{array}$ & See table above & High & Unknown & Direct & Imprecise & Insufficient \\
\hline $\begin{array}{l}\text { Quetiapine + } \\
\text { mood } \\
\text { stabilizers vs. } \\
\text { placebo + } \\
\text { mood } \\
\text { stabilizers }\end{array}$ & \begin{tabular}{|l|} 
Time to \\
recurrence any \\
mood,102 wks \\
time to mania 102 \\
wks \\
time to depression \\
102 wks
\end{tabular} & $\begin{array}{l}2 \mathrm{RCT} \\
(\mathrm{n}=1329)\end{array}$ & See table above & High & Consistent & Direct & Imprecise & Insufficient \\
\hline $\begin{array}{l}\text { Risperidone } \\
\text { long acting } \\
\text { injectable + } \\
\text { treatment as } \\
\text { usual vs. } \\
\text { placebo + } \\
\text { treatment as } \\
\text { usual }\end{array}$ & $\begin{array}{l}\text { Time to } \\
\text { recurrence } 52 \text { wks } \\
\text { YMRS } 52 \text { wks } \\
\text { MADRS } 52 \text { wks } \\
\text { CGI } 52 \text { wks } \\
\text { Withdrawals }\end{array}$ & $\begin{array}{l}2 \mathrm{RCT} \\
(\mathrm{n}=174)\end{array}$ & See table above & High & Inconsistent & Direct & Imprecise & Insufficient \\
\hline
\end{tabular}




\begin{tabular}{|c|c|c|c|c|c|c|c|c|}
\hline Comparison & Outcome & $\begin{array}{l}\text { \# Studies/ } \\
\text { Design } \\
\text { (n analyzed) }\end{array}$ & $\begin{array}{c}\text { Finding or Summary } \\
\text { Statistic } \\
\text { Outcome Timing }\end{array}$ & $\begin{array}{c}\text { Study } \\
\text { Limitations }\end{array}$ & Consistency & Directness & Precision & $\begin{array}{c}\text { Overall } \\
\text { Gradel } \\
\text { Conclusion }\end{array}$ \\
\hline $\begin{array}{l}\text { Ziprasidone + } \\
\text { mood } \\
\text { stabilizers vs. } \\
\text { placebo + } \\
\text { mood } \\
\text { stabilizers }\end{array}$ & $\begin{array}{l}\text { Relapse Risk } 26 \\
\text { wks } \\
\text { YMRS } 26 \text { wks } \\
\text { MADRS } 26 \text { wks } \\
\text { Withdrawals }\end{array}$ & $\begin{array}{l}1 \mathrm{RCT} \\
\text { Combination } \\
(\mathrm{n}=240)\end{array}$ & See table above & High & Unknown & Direct & Imprecise & Insufficient \\
\hline
\end{tabular}

Abbreviations: CGI=Clinical Global Impressions; CGI-BP-M=Clinical Global Impressions Scale-Bipolar-modified (for long-term follow-up); HAM-D=Hamilton Scale for Depression; MADRS=Montgomery-Asberg Depression Rating Scale; RCT=randomized controlled trial; YMRS = Young Mania Rating Scale Notes:

1. Publication bias for antipsychotics, antidepressants, and behavioral interventions for depressive disorders is suspected.

2. Data were generally imprecise due to missing data from high attrition rates, which was commonly dealt with by Last Observation Carried Forward (LOCF). LOCF requires an assumption that the health status of patients who dropped out of the trial would not have changed had future observations been recorded, a strong assumption in the context of bipolar disorder research

\begin{tabular}{|c|c|c|c|c|c|c|}
\hline Drug & $\begin{array}{c}\text { Study } \\
\text { Comparison } \\
\text { PMID }\end{array}$ & Responder/Remitter & Symptom & Function & Other & AE \\
\hline $\begin{array}{l}\text { Lithium + } \\
\text { valproate vs. } \\
\text { valproate vs. } \\
\text { lithium }\end{array}$ & $\begin{array}{l}\text { Balance } \\
\text { Investigators, } \\
2010^{18} \\
20092882\end{array}$ & $\begin{array}{l}\text { Time to new intervention } \\
\text { for emerging mood } \\
\frac{\text { episode }}{24 \text { months }} \\
\text { Hazard ratio } \\
\text { Favors } L+V \text { over } \\
\text { Valproate } \\
\text { NS for L+V vs. Lithium }\end{array}$ & NR & $\begin{array}{l}\text { GAF } \\
\text { NS }\end{array}$ & $\begin{array}{l}\text { EuroQol (EQ-5D) } \\
\text { (quality of life) } \\
\text { NS } \\
\text { Overall Withdrawal } \\
\text { L+V: } 21 / 110 \\
\text { Lithium: } 23 / 110 \\
\text { Valproate: } 23 / 110 \\
\text { Withdrawal lack of } \\
\text { efficacy } \\
\text { NR } \\
\text { Withdrawal adverse } \\
\text { events } \\
\text { L+V: } 11 / 110 \\
\text { Lithium: } 6 / 110 \\
\text { Valproate: } 4 / 110\end{array}$ & $\begin{array}{l}\text { SAE } \\
\text { NS } \\
\text { Valproate: } 7 \text { SAE } \\
\text { including } 3 \text { deaths } \\
\text { Lithium: } 5 \text { SAE } \\
\text { including } 2 \text { deaths } \\
\text { L+V: } 4 \text { SAE including } \\
1 \text { death } \\
\text { (only one deemed due } \\
\text { to study - did not } \\
\text { report which) }\end{array}$ \\
\hline
\end{tabular}




\begin{tabular}{|c|c|c|c|c|c|c|}
\hline Drug & $\begin{array}{c}\text { Study } \\
\text { Comparison } \\
\text { PMID }\end{array}$ & Responder/Remitter & Symptom & Function & Other & AE \\
\hline $\begin{array}{l}\text { Quetiapine + } \\
\text { personalize } \\
\text { treatment vs. } \\
\text { Lithium + } \\
\text { personalized } \\
\text { treatment }\end{array}$ & $\begin{array}{l}\text { Nierenberg, } \\
2016^{42} \\
26845264\end{array}$ & NR & $\begin{array}{l}\frac{\text { YMRS }}{24 \text { week }} \\
\text { NS (model-based effect) } \\
\frac{\text { MADRS }}{24 \text { week }} \\
\text { NS (model-based effect) }\end{array}$ & $\begin{array}{l}\text { CGI-El } \\
24 \text { weeks } \\
\text { NS } \\
\text { (model- } \\
\text { based } \\
\text { effect) }\end{array}$ & $\begin{array}{l}\text { Overall Withdrawa } \\
\text { Quetiapine: } 60 / 180 \\
24.8 \% \\
\text { Lithium: 58/182 24.2\% } \\
\text { Withdrawal lack of } \\
\frac{\text { efficacy }}{\text { NR }} \\
\text { Withdrawal serious } \\
\text { adverse events } \\
\text { Quetiapine: } 2 / 180 \\
\text { Lithium: } 0 / 18224\end{array}$ & $\begin{array}{l}\text { Death } \\
\text { Quetiapine: } 0 \\
\text { Lithium: } 2\end{array}$ \\
\hline $\begin{array}{l}\text { Valproic acid + } \\
\text { Aripiprazole vs. } \\
\text { Lithium }+ \\
\text { Aripiprazole }\end{array}$ & $\begin{array}{l}\text { Vieta, 201043 } \\
20429835\end{array}$ & $\begin{array}{l}\text { Remission (YMRS }<12, \\
\text { MADRS }<8) \\
\text { At least } 2 / 3 \text { patients in } \\
\text { both groups after } 40 \\
\text { weeks }\end{array}$ & $\begin{array}{l}\text { YMRS } \\
\text { 46 weeks } \\
\text { Mean change } \\
\text { + Lithium: -2.7 }(-4.5,-0.7) \\
\text { + Valproic: -5.8 }(-7.2,-4.3) \\
\text { MADRS } \\
\text { 46 weeks } \\
\text { Mean change } \\
\text { + Lithium: -0.8 }(-2.6,1.0) \\
\text { + Valproic: -1.2 }(-2.6,0.3)\end{array}$ & $\mathrm{NR}$ & $\begin{array}{l}\text { Overall Withdrawal } \\
\text { +Lithium: } 53 / 10849.1 \% \\
\text { +Valproic: } 84 / 17548 \% \\
\text { Withdrawal lack of } \\
\text { efficacy } \\
\text { +Lithium: } 1 / 1080.9 \% \\
\text { +Valproic: } 10 / 1755.7 \% \\
\text { Withdrawal adverse } \\
\text { events } \\
\text { +Lithium: } 20 / 10818.5 \% \\
\text { +Valproic: } 24 / 17513.7 \%\end{array}$ & $\begin{array}{l}\text { SAE } \\
\text { +Lithium: } 15(14.2 \%) \\
1 \text { patient suicidal } \\
\text { ideation } \\
1 \text { lithium overdose } \\
\text { death (50 days after } \\
\text { last study dose) } \\
\text { +Valproic: } 15(8.6 \%) \\
\text { EPS } \\
\text { +Lithium: } 24(22.6 \%) \\
\text { +Valproic: } 38(21.8 \%)\end{array}$ \\
\hline
\end{tabular}

Abbreviations: CGI-EI=Clinical Global Impressions-Efficacy Index; EPS=extrapyramidal symptoms; GAF=General Assessment of Functioning Scale; MADRS=MontgomeryAsberg Depression Rating Scale; NR=not reported; NS=not significant; PMID=PubMed Identification Number; SAE=Severe Adverse Events; YMRS = Young Mania Rating Scale 
Appendix Table I16. Strength of evidence assessment: combination therapy versus active control for maintenance

\begin{tabular}{|c|c|c|c|c|c|c|c|c|}
\hline Comparison & Outcome & $\begin{array}{l}\text { \# Studies/ } \\
\text { Design } \\
\text { (n analyzed) }\end{array}$ & $\begin{array}{c}\text { Finding or Summary } \\
\text { Statistic } \\
\text { Outcome Timing }\end{array}$ & $\begin{array}{c}\text { Study } \\
\text { Limitations }\end{array}$ & Consistency & Directness & Precision & $\begin{array}{c}\text { Overall } \\
\text { Gradel } \\
\text { Conclusion }\end{array}$ \\
\hline $\begin{array}{l}\text { Lithium + } \\
\text { valproate vs. } \\
\text { valproate vs. } \\
\text { lithium }\end{array}$ & $\begin{array}{l}\text { Time to Relapse } \\
24 \text { mths } \\
\text { GAF } 24 \text { mths } \\
\text { EuroQual } 24 \text { mths } \\
\text { Withdrawals }\end{array}$ & $\begin{array}{l}1 \mathrm{RCT} \\
(\mathrm{n}=330)\end{array}$ & See table above & Moderate & Unknown & Direct & Imprecise & Insufficient \\
\hline $\begin{array}{l}\text { Quetiapine + } \\
\text { personalize } \\
\text { treatment vs. } \\
\text { Lithium + } \\
\text { personalized } \\
\text { treatment }\end{array}$ & $\begin{array}{l}\text { YMRS } 24 \text { wks } \\
\text { MADRS } 24 \text { wks } \\
\text { CGI } 24 \text { wks } \\
\text { Withdrawals }\end{array}$ & $\begin{array}{l}1 \mathrm{RCT} \\
(\mathrm{n}=482)\end{array}$ & See table above & Moderate & Unknown & Direct & Imprecise & Insufficient \\
\hline $\begin{array}{l}\text { Valproic acid } \\
+ \text { Aripiprazole } \\
\text { vs. Lithium + } \\
\text { Aripiprazole }\end{array}$ & $\begin{array}{l}\text { Remission } 46 \text { wks } \\
\text { YMRS } 46 \text { wks } \\
\text { MADRS } 46 \text { wks } \\
\text { Withdrawals }\end{array}$ & $\begin{array}{l}1 \text { RCT open label } \\
\text { extension } \\
(n=28\end{array}$ & See table above & High & Unknown & Direct & Imprecise & Insufficient \\
\hline
\end{tabular}

Abbreviations: CGI= Clinical Global Impressions; GAF=General Assessment of Functioning Scale; MADRS=Montgomery-Asberg Depression Rating Scale; RCT=randomized controlled trial; YMRS = Young Mania Rating Scale

Notes:

1. Publication bias for antipsychotics, antidepressants, and behavioral interventions for depressive disorders is suspected.

2. Data were generally imprecise due to missing data from high attrition rates, which was commonly dealt with by Last Observation Carried Forward (LOCF). LOCF requires an assumption that the health status of patients who dropped out of the trial would not have changed had future observations been recorded, a strong assumption in the context of bipolar disorder research. 


\section{References for Appendix I}

1. Bowden CL, Calabrese JR, McElroy SL, et al. A randomized, placebo-controlled 12-month trial of divalproex and lithium in treatment of outpatients with bipolar I disorder. Divalproex Maintenance Study Group. Archives of General Psychiatry. 2000 May;57(5):481-9. PMID: 10807488.

2. Gyulai L, Bowden CL, McElroy SL, et al. Maintenance efficacy of divalproex in the prevention of bipolar depression. Neuropsychopharmacology. 2003 Jul;28(7):137482. PMID: 12784116.

3. Amsterdam JD, Shults J. Efficacy and safety of long-term fluoxetine versus lithium monotherapy of bipolar II disorder: a randomized, doubleblind, placebo-substitution study. American Journal of Psychiatry. 2010 Jul;167(7):792-800. doi:

http://dx.doi.org/10.1176/appi.ajp.2009.09020284 . PMID: 20360317.

4. Bowden CL, Calabrese JR, Sachs G, et al. A placebo-controlled 18-month trial of lamotrigine and lithium maintenance treatment in recently manic or hypomanic patients with bipolar I disorder.[Erratum appears in Arch Gen Psychiatry. 2004 Jul;61(7):680]. Archives of General Psychiatry. 2003 Apr;60(4):392-400. PMID: 12695317.

5. Calabrese JR, Bowden CL, Sachs G, et al. A placebo-controlled 18-month trial of lamotrigine and lithium maintenance treatment in recently depressed patients with bipolar I disorder. Journal of Clinical Psychiatry. 2003 Sep;64(9):1013-24. PMID: 14628976.

6. Prien RF, Point P, Caffey EM, et al. Prophylactic efficacy of lithium carbonate in manic-depressive illness: Report of the veterans administration and national institute of mental health collaborative study group. Archives of General Psychiatry. 1973;28(3):337-41. doi: 10.1001/archpsyc.1973.01750330035006. PMID: 4569674.

7. Weisler RH, Nolen WA, Neijber A, et al. Continuation of quetiapine versus switching to placebo or lithium for maintenance treatment of bipolar I disorder (Trial 144: a randomized controlled study). Journal of Clinical Psychiatry. 2011 Nov;72(11):1452-64. doi: http://dx.doi.org/10.4088/JCP.11m06878. PMID: 22054050.
8. Calabrese JR, Sanchez R, Jin N, et al. Efficacy and safety of aripiprazole once-monthly in the maintenance treatment of bipolar i disorder: A double-blind, placebo-controlled, 52-week randomized withdrawal study. Journal of Clinical Psychiatry. 2017 March;78(3):324-31. doi: http://dx.doi.org/10.4088/JCP.16m11201. PMID: 28146613 PMID/615221995 Embase.

9. Keck PE, Jr., Calabrese JR, McQuade RD, et al. A randomized, double-blind, placebo-controlled 26-week trial of aripiprazole in recently manic patients with bipolar I disorder. Journal of Clinical Psychiatry. 2006 Apr;67(4):626-37. PMID: 16669728.

10. Hartong EG, Moleman P, Hoogduin CA, et al. Prophylactic efficacy of lithium versus carbamazepine in treatment-naive bipolar patients. Journal of Clinical Psychiatry. 2003 Feb;64(2):144-51. PMID: 12633122.

11. Greil W, Ludwig-Mayerhofer W, Erazo N, et al. Lithium versus carbamazepine in the maintenance treatment of bipolar disorders--a randomised study. Journal of Affective Disorders. 1997 Apr;43(2):151-61. PMID: 9165384.

12. Greil W, Kleindienst N, Erazo N, et al. Differential response to lithium and carbamazepine in the prophylaxis of bipolar disorder. Journal of Clinical Psychopharmacology. 1998 Dec;18(6):455-60. PMID: 9864077.

13. Greil W, Kleindienst N. The comparative prophylactic efficacy of lithium and carbamazepine in patients with bipolar I disorder. International Clinical Psychopharmacology. 1999 Sep;14(5):277-81. PMID: 10529070.

14. Greil W, Kleindienst N. Lithium versus carbamazepine in the maintenance treatment of bipolar II disorder and bipolar disorder not otherwise specified. International Clinical Psychopharmacology. 1999 Sep;14(5):283-5. PMID: 10529071.

15. Kleindienst N, Greil W. Differential efficacy of lithium and carbamazepine in the prophylaxis of bipolar disorder: results of the MAP study. Neuropsychobiology. 2000;42 Suppl 1:2-10. PMID: 11093063.

16. Calabrese JR, Shelton MD, Rapport DJ, et al. A 20-month, double-blind, maintenance trial of lithium versus divalproex in rapid-cycling bipolar disorder. American Journal of Psychiatry. 2005 Nov;162(11):2152-61. PMID: 16263857. 
17. Calabrese JR, Suppes T, Bowden CL, et al. A double-blind, placebo-controlled, prophylaxis study of lamotrigine in rapid-cycling bipolar disorder. Lamictal 614 Study Group. Journal of Clinical Psychiatry. 2000 Nov;61(11):841-50. PMID: 11105737.

18. Balance investigators, Geddes JR, Goodwin GM, et al. Lithium plus valproate combination therapy versus monotherapy for relapse prevention in bipolar I disorder (BALANCE): a randomised open-label trial. Lancet. 2010 Jan 30;375(9712):385-95. doi: http://dx.doi.org/10.1016/S0140-6736(09)618286. PMID: 20092882.

19. Tohen M, Calabrese JR, Sachs GS, et al. Randomized, placebo-controlled trial of olanzapine as maintenance therapy in patients with bipolar I disorder responding to acute treatment with olanzapine. American Journal of Psychiatry. 2006 Feb;163(2):247-56. PMID: 16449478.

20. Tohen M, Ketter TA, Zarate CA, et al. Olanzapine versus divalproex sodium for the treatment of acute mania and maintenance of remission: a 47-week study. American Journal of Psychiatry. 2003 Jul;160(7):1263-71. PMID: 12832240.

21. Tohen M, Baker RW, Altshuler LL, et al. Olanzapine versus divalproex in the treatment of acute mania.[Erratum appears in Am J Psychiatry. 2005 Feb;7(1):102]. American Journal of Psychiatry. 2002 Jun;159(6):1011-7. PMID: 12042191.

22. Quiroz JA, Yatham LN, Palumbo JM, et al. Risperidone long-acting injectable monotherapy in the maintenance treatment of bipolar I disorder. Biological Psychiatry. 2010 Jul 15;68(2):156-62. doi:

http://dx.doi.org/10.1016/j.biopsych.2010.01.015. PMID: 20227682.

23. Vieta E, Montgomery S, Sulaiman AH, et al. A randomized, double-blind, placebo-controlled trial to assess prevention of mood episodes with risperidone long-acting injectable in patients with bipolar I disorder. European

Neuropsychopharmacology. 2012

Nov;22(11):825-35. doi:

http://dx.doi.org/10.1016/j.euroneuro.2012.03.004 . PMID: 22503488.
24. Tohen M, Greil W, Calabrese JR, et al. Olanzapine versus lithium in the maintenance treatment of bipolar disorder: a 12-month, randomized, double-blind, controlled clinical trial. American Journal of Psychiatry. 2005 Jul;162(7):1281-90. PMID: 15994710.

25. Berwaerts J, Melkote R, Nuamah I, et al. A randomized, placebo- and active-controlled study of paliperidone extended-release as maintenance treatment in patients with bipolar I disorder after an acute manic or mixed episode. Journal of Affective Disorders. 2012 May;138(3):247-58. doi: http://dx.doi.org/10.1016/j.jad.2012.01.047. PMID: 22377512.

26. Amsterdam JD, Lorenzo-Luaces L, Soeller I, et al. Safety and effectiveness of continuation antidepressant versus mood stabilizer monotherapy for relapse-prevention of bipolar II depression: A randomized, double-blind, parallelgroup, prospective study. Journal of Affective Disorders. 201506 Jul;185:31-7. doi: http://dx.doi.org/10.1016/j.jad.2015.05.070. PMID: 26143402 (pubmed) 2015175772 (embase).

27. Newport DJ, Stowe ZN, Viguera AC, et al. Lamotrigine in bipolar disorder: efficacy during pregnancy. Bipolar Disorders. 2008 May;10(3):432-6. doi: http://dx.doi.org/10.1111/j.13995618.2007.00565.x. PMID: 18402631.

28. Marcus R, Khan A, Rollin L, et al. Efficacy of aripiprazole adjunctive to lithium or valproate in the long-term treatment of patients with bipolar I disorder with an inadequate response to lithium or valproate monotherapy: a multicenter, doubleblind, randomized study. Bipolar Disorders. 2011 Mar;13(2):133-44. doi: http://dx.doi.org/10.1111/j.13995618.2011.00898.x. PMID: 21443567.

29. Carlson BX, Ketter TA, Sun W, et al. Aripiprazole in combination with lamotrigine for the long-term treatment of patients with bipolar I disorder (manic or mixed): a randomized, multicenter, double-blind study (CN138-392). Bipolar Disorders. 2012 Feb;14(1):41-53. doi: http://dx.doi.org/10.1111/j.13995618.2011.00974.x. PMID: 22329471. 
30. Woo YS, Bahk WM, Chung MY, et al. Aripiprazole plus divalproex for recently manic or mixed patients with bipolar I disorder: a 6-month, randomized, placebo-controlled, double-blind maintenance trial. Human Psychopharmacology. 2011 Dec;26(8):543-53. doi: http://dx.doi.org/10.1002/hup.1240. PMID: 22134973.

31. Kemp DE, Gao K, Ganocy SJ, et al. A 6-month, double-blind, maintenance trial of lithium monotherapy versus the combination of lithium and divalproex for rapid-cycling bipolar disorder and Co-occurring substance abuse or dependence. Journal of Clinical Psychiatry. 2009 Jan;70(1):113-21. PMID: 19192457.

32. Tohen M, Chengappa KN, Suppes T, et al. Relapse prevention in bipolar I disorder: 18month comparison of olanzapine plus mood stabiliser v. mood stabiliser alone. British Journal of Psychiatry. 2004 Apr;184:337-45. PMID: 15056579.

33. Tohen M, Chengappa KN, Suppes T, et al. Efficacy of olanzapine in combination with valproate or lithium in the treatment of mania in patients partially nonresponsive to valproate or lithium monotherapy. Archives of General Psychiatry. 2002 Jan;59(1):62-9. PMID: 11779284.

34. Suppes T, Vieta E, Liu S, et al. Maintenance treatment for patients with bipolar I disorder: results from a north american study of quetiapine in combination with lithium or divalproex (trial 127). American Journal of Psychiatry. 2009 Apr;166(4):476-88. doi: http://dx.doi.org/10.1176/appi.ajp.2008.08020189 . PMID: 19289454.

35. Vieta E, Suppes T, Eggens I, et al. Efficacy and safety of quetiapine in combination with lithium or divalproex for maintenance of patients with bipolar I disorder (international trial 126). Journal of Affective Disorders. 2008 Aug;109(3):251-63. doi: http://dx.doi.org/10.1016/j.jad.2008.06.001. PMID: 18579216.

36. Bobo WV, Epstein RA, Lynch A, et al. A randomized open comparison of long-acting injectable risperidone and treatment as usual for prevention of relapse, rehospitalization, and urgent care referral in community-treated patients with rapid cycling bipolar disorder. Clinical Neuropharmacology. 2011 Nov-Dec;34(6):22433. doi: http://dx.doi.org/10.1097/WNF.0b013e31823770 9a. PMID: 22104634.
37. Macfadden W, Alphs L, Haskins JT, et al. A randomized, double-blind, placebo-controlled study of maintenance treatment with adjunctive risperidone long-acting therapy in patients with bipolar I disorder who relapse frequently. Bipolar Disorders. 2009 Dec;11(8):827-39. doi: http://dx.doi.org/10.1111/j.13995618.2009.00761.x. PMID: 19922552.

38. Bowden CL, Vieta E, Ice KS, et al. Ziprasidone plus a mood stabilizer in subjects with bipolar I disorder: a 6-month, randomized, placebocontrolled, double-blind trial. Journal of Clinical Psychiatry. 2010 Feb;71(2):130-7. doi: http://dx.doi.org/10.4088/JCP.09m05482yel. PMID: 20122373.

39. Vieta E, Cruz N, Garcia-Campayo J, et al. A double-blind, randomized, placebo-controlled prophylaxis trial of oxcarbazepine as adjunctive treatment to lithium in the long-term treatment of bipolar I and II disorder. International Journal of Neuropsychopharmacology. 2008 Jun;11(4):44552. doi: http://dx.doi.org/10.1017/S1461145708008596. PMID: 18346292.

40. Vieta E, Manuel Goikolea J, Martinez-Aran A, et al. A double-blind, randomized, placebocontrolled, prophylaxis study of adjunctive gabapentin for bipolar disorder. Journal of Clinical Psychiatry. 2006 Mar;67(3):473-7. PMID: 16649836.

41. Zarate CA, Jr., Tohen M. Double-blind comparison of the continued use of antipsychotic treatment versus its discontinuation in remitted manic patients. American Journal of Psychiatry. 2004 Jan;161(1):169-71. PMID: 14702269.

42. Nierenberg AA, McElroy SL, Friedman ES, et al. Bipolar CHOICE (Clinical Health Outcomes Initiative in Comparative Effectiveness): a pragmatic 6-month trial of lithium versus quetiapine for bipolar disorder. Journal of Clinical Psychiatry. 2016 Jan;77(1):90-9. doi: http://dx.doi.org/10.4088/JCP.14m09349. PMID: 26845264.

43. Vieta E, Owen R, Baudelet C, et al. Assessment of safety, tolerability and effectiveness of adjunctive aripiprazole to lithium/valproate in bipolar mania: a 46-week, open-label extension following a 6-week double-blind study. Current Medical Research \& Opinion. 2010 Jun;26(6):1485-96. doi: http://dx.doi.org/10.1185/03007991003779380. PMID: 20429835. 


\section{Appendix J. Psychoeducation}

Appendix Table J1. Characteristics of eligible studies: psychoeducation vs. inactive comparators by year then first author

\begin{tabular}{|c|c|c|c|c|c|c|}
\hline $\begin{array}{c}\begin{array}{c}\text { Study, Year } \\
\text { Design } \\
\text { Location } \\
\text { Funder }\end{array} \\
\text { Risk of Bias } \\
\text { PMID }\end{array}$ & $\begin{array}{c}\text { Randomized (N) } \\
\text { Age (mean) } \\
\text { Sex (\% Female) } \\
\text { Race (\% White) } \\
\text { Diagnosis } \\
\text { (\% BP I, II, NOS) } \\
\text { Setting }\end{array}$ & $\begin{array}{c}\text { Inclusions } \\
\text { Key Exclusions }\end{array}$ & $\begin{array}{l}\text { Intervention } \\
\text { Description }\end{array}$ & $\begin{array}{l}\text { Comparison } \\
\text { Description }\end{array}$ & $\begin{array}{l}\text { Follow-up } \\
\text { Duration }\end{array}$ & $\begin{array}{c}\text { Outcomes } \\
\text { Reported } \\
\text { Withdrawal (\%) at } \\
\text { endpoint }\end{array}$ \\
\hline $\begin{array}{l}\text { Barnes, } 2015^{1} \\
\text { RCT } \\
\text { Australia } \\
\text { Non-Government } \\
\text { Moderate } \\
25554993\end{array}$ & $\begin{array}{l}\mathrm{N}=233 \\
\text { Age } 40(18-58) \\
\text { Female 72\% } \\
\text { White NR } \\
\text { BP I 88\% } \\
\text { BP II 12\% } \\
\text { Outpatient }\end{array}$ & $\begin{array}{l}\text { No current clinical state } \\
\text { excluded; Individuals with } \\
\text { BP I or II (DSM-IV) with } \\
\text { euthymia or a current } \\
\text { manic or depressive } \\
\text { episode and taking } \\
\text { medication for BP. } \\
\text { Labs/Other Conditions }\end{array}$ & $\begin{array}{l}\text { Internet-based } \\
\text { psychoeducation } \\
\text { (Road to Recovery } \\
\text { for Bipoloar } \\
\text { Disorder) } \\
\text { focused on } \\
\text { managing } \\
\text { symptoms, } \\
\text { medication, } \\
\text { psychological } \\
\text { approaches, } \\
\text { relationships, and } \\
\text { lifestyle. Participants } \\
\text { had access to } 10 \\
\text { sessions of } \\
\text { cognitive behavioral } \\
\text { therapy as } \\
\text { homework } \\
\\
-20 \text { online sessions, } \\
\text { first } 8 \text { sessions } \\
\text { weekly, } 9 \text { and } 10 \\
\text { every 2-week } \\
\text { period, and 11-20 } \\
\text { were monthly }\end{array}$ & \begin{tabular}{|l|} 
Internet-based \\
attention control \\
(Virtual Highway \\
for Bipolar \\
Disorder) \\
\\
-20 online \\
sessions, first 8 \\
sessions weekly, \\
9 and 10 every $2-$ \\
week period, and \\
$11-20$ were \\
monthly
\end{tabular} & 12 months & \begin{tabular}{|l|} 
Time to Relapse \\
Hospitalization \\
Relapse (Return of \\
significant symptoms \\
after a remission of at \\
least 8 weeks, DSM-IV) \\
Withdrawal 28\%
\end{tabular} \\
\hline
\end{tabular}




\begin{tabular}{|c|c|c|c|c|c|c|}
\hline $\begin{array}{l}\text { Study, Year } \\
\text { Design } \\
\text { Location } \\
\text { Funder } \\
\text { Risk of Bias } \\
\text { PMID }\end{array}$ & $\begin{array}{c}\text { Randomized (N) } \\
\text { Age (mean) } \\
\text { Sex (\% Female) } \\
\text { Race (\% White) } \\
\text { Diagnosis } \\
\text { (\% BP I, II, NOS) } \\
\text { Setting } \\
\end{array}$ & $\begin{array}{l}\text { Inclusions } \\
\text { Key Exclusions }\end{array}$ & $\begin{array}{l}\text { Intervention } \\
\text { Description }\end{array}$ & $\begin{array}{l}\text { Comparison } \\
\text { Description }\end{array}$ & $\begin{array}{l}\text { Follow-up } \\
\text { Duration }\end{array}$ & $\begin{array}{c}\text { Outcomes } \\
\text { Reported } \\
\begin{array}{c}\text { Withdrawal (\%) at } \\
\text { endpoint }\end{array}\end{array}$ \\
\hline $\begin{array}{l}\text { Gumus, } 2015^{2} \\
\text { RCT } \\
\text { Turkey } \\
\text { NR } \\
\text { High } \\
26001717\end{array}$ & $\begin{array}{l}\mathrm{N}=82 \\
\text { Age } 39(27-52) \\
\text { Female } 48 \% \\
\text { Race NR } \\
\text { BP I } 89 \% \\
\text { BP II } 11 \% \\
\text { Outpatient }\end{array}$ & $\begin{array}{l}\text { Euthymic; Individuals with } \\
\text { BP I or II (DSM-VI) who } \\
\text { received standard medical } \\
\text { treatment and were } \\
\text { euthymic (YMRS }>6 \text {, HDRS } \\
<17 \text { ) for at least } 3 \text { months. } \\
\text { Other Mental Health }\end{array}$ & $\begin{array}{l}\text { Psychoeducation } \\
\text { focused on illness } \\
\text { education, warning } \\
\text { signs, medication } \\
\text { and side effects, } \\
\text { and problem solving } \\
\text { skills as well as } \\
\text { standard clinical } \\
\text { monitoring } \\
\text { - } 60 \text { minute } \\
\text { sessions, once per } \\
\text { week, for } 4 \text { weeks } \\
\end{array}$ & $\begin{array}{l}\text { Standard clinical } \\
\text { follow up (not } \\
\text { described) } \\
\text {-Duration of } \\
\text { study }\end{array}$ & 12 months & $\begin{array}{l}\text { Hospitalization } \\
\text { Relapse (Emergency of } \\
\text { new clinical episode, } \\
\text { YMRS } \geq 20, H D R S \geq 17 \\
\text { or YMRS } \geq 20 \text { and } \\
\text { HDRS } \geq 12 \text { ) } \\
\text { Withdrawal } 5 \%\end{array}$ \\
\hline $\begin{array}{l}\text { de Barros Pellegrinelli, } \\
2013^{3} \\
\text { RCT } \\
\text { Brazil } \\
\text { Non-Government } \\
\text { High } \\
22943487\end{array}$ & $\begin{array}{l}\mathrm{N}=55 \\
\text { Age } 44(22-66) \\
\text { Female } 69 \% \\
\text { White NR } \\
\text { BP NR } \\
\text { Outpatient }\end{array}$ & $\begin{array}{l}\text { Euthymic/Maintenance; } \\
\text { Individuals diagnosed with } \\
\text { BP I or II (DSM-IV), in } \\
\text { remission for at least } 1 \\
\text { month (HDRS < } 7 \text { and } \\
\text { YMRS <6) } \\
\text { Schizoaffective; Substance } \\
\text { Abuse; Other Mental } \\
\text { Heath; Neurological } \\
\text { Disorders }\end{array}$ & $\begin{array}{l}\text { Psychoeducation } \\
\text { consisting of } 15 \mathrm{~min} \\
\text { introduction, } 30 \mathrm{~min} \\
\text { education, } 30 \mathrm{~min} \\
\text { discussion and } \\
\text { psychological } \\
\text { support, and } 15 \mathrm{~min} \\
\text { for conclusion } \\
\text {-16 twice-weekly 90- } \\
\text { minute sessions }\end{array}$ & \begin{tabular}{|l} 
Sessions \\
promoting \\
relaxation \\
consisting of \\
informal \\
conversation and \\
relaxation using \\
three different \\
types of \\
exercises \\
-16 twice-weekly \\
$90-$ minute \\
sessions
\end{tabular} & 12 months & $\begin{array}{l}\text { HDRS } \\
\text { YMRS } \\
\text { GAF } \\
\text { Withdrawal } 45 \%\end{array}$ \\
\hline
\end{tabular}




\begin{tabular}{|c|c|c|c|c|c|c|}
\hline $\begin{array}{l}\text { Study, Year } \\
\text { Design } \\
\text { Location } \\
\text { Funder } \\
\text { Risk of Bias } \\
\text { PMID }\end{array}$ & $\begin{array}{c}\text { Randomized (N) } \\
\text { Age (mean) } \\
\text { Sex (\% Female) } \\
\text { Race (\% White) } \\
\text { Diagnosis } \\
\text { (\% BP I, II, NOS) } \\
\text { Setting }\end{array}$ & $\begin{array}{c}\text { Inclusions } \\
\text { Key Exclusions }\end{array}$ & $\begin{array}{l}\text { Intervention } \\
\text { Description }\end{array}$ & $\begin{array}{l}\text { Comparison } \\
\text { Description }\end{array}$ & $\begin{array}{c}\text { Follow-up } \\
\text { Duration }\end{array}$ & $\begin{array}{c}\text { Outcomes } \\
\text { Reported } \\
\text { Withdrawal (\%) at } \\
\text { endpoint }\end{array}$ \\
\hline $\begin{array}{l}\text { Javadpour, } 2013^{4} \\
\text { RCT } \\
\text { Iran } \\
\text { Non-Government } \\
\text { High } \\
23642977\end{array}$ & $\begin{array}{l}\mathrm{N}=108 \\
\text { Age NR (18-60) } \\
\text { Female 51\% } \\
\text { White NR } \\
\text { BP NR } \\
\text { Outpatient }\end{array}$ & $\begin{array}{l}\text { Euthymic/Maintenance; } \\
\text { Individuals with BP with a } \\
\text { history of at least } 2 \\
\text { episodes of relapse in past } \\
2 \text { years or at least } 3 \\
\text { episodes in past } 5 \text { years, } \\
\text { and euthymic (HAM-D <8 } \\
\text { and BRMS <9) } \\
\text { First Manic Episode }\end{array}$ & $\begin{array}{l}\text { Psychoeducation } \\
\text { focusing on } \\
\text { understanding } \\
\text { bipolar, } \\
\text { familiarization with } \\
\text { symptoms } \\
\text { understanding signs } \\
\text { of an episodes, } \\
\text { awareness of } \\
\text { causes and } \\
\text { prognosis, } \\
\text { education about the } \\
\text { function, types and } \\
\text { adverse side effect } \\
\text { of mood stabilizer } \\
\text { medication, } \\
\text { functions, types and } \\
\text { adverse effects of } \\
\text { anti-manic and } \\
\text { antidepressant } \\
\text { medications, and } \\
\text { risks of } \\
\text { discontinuing } \\
\text { medications } \\
\text { - Eight } 50-\text { minute } \\
\text { weekly session } \\
\end{array}$ & \begin{tabular}{|l|} 
Standard \\
pharmacotherapy \\
(discretion of \\
treating \\
psychiatrist of \\
their choice)
\end{tabular} & 18 months & $\begin{array}{l}\text { Relapse (HAM-D >17 } \\
\text { or BRMS }>15) \\
\text { Hospitalizations } \\
\text { BRMS } \\
\text { HDRS } \\
\text { Withdrawal 20\% }\end{array}$ \\
\hline
\end{tabular}




\begin{tabular}{|c|c|c|c|c|c|c|}
\hline $\begin{array}{l}\text { Study, Year } \\
\text { Design } \\
\text { Location } \\
\text { Funder } \\
\text { Risk of Bias } \\
\text { PMID }\end{array}$ & $\begin{array}{c}\text { Randomized (N) } \\
\text { Age (mean) } \\
\text { Sex (\% Female) } \\
\text { Race (\% White) } \\
\text { Diagnosis } \\
\text { (\% BP I, II, NOS) } \\
\text { Setting }\end{array}$ & $\begin{array}{c}\text { Inclusions } \\
\text { Key Exclusions }\end{array}$ & $\begin{array}{l}\text { Intervention } \\
\text { Description }\end{array}$ & $\begin{array}{l}\text { Comparison } \\
\text { Description }\end{array}$ & $\begin{array}{l}\text { Follow-up } \\
\text { Duration }\end{array}$ & $\begin{array}{c}\text { Outcomes } \\
\text { Reported } \\
\text { Withdrawal (\%) at } \\
\text { endpoint }\end{array}$ \\
\hline $\begin{array}{l}\text { Smith, } 2011^{5} \\
\text { RCT } \\
\text { United Kingdom } \\
\text { Government } \\
\text { Low } \\
22017225\end{array}$ & $\begin{array}{l}\mathrm{N}=50 \\
\text { Age } 44(22-66) \\
\text { Female } 62 \% \\
\text { White } 98 \% \\
\text { BP I } 86 \% \\
\text { BP II } 12 \% \\
\text { BP NOS } 2 \% \\
\text { Outpatient }\end{array}$ & $\begin{array}{l}\text { Euthymic/Maintenance; } \\
\text { DSM-IV diagnosis of } \\
\text { bipolar disorder currently in } \\
\text { clinical remission, and not } \\
\text { fulfilling diagnostic criteria } \\
\text { for a depressive, manic or } \\
\text { mixed affective episode } \\
\text { during the preceding } 3 \\
\text { months } \\
\text { Neurological Disorders }\end{array}$ & $\begin{array}{l}\text { Internet-based } \\
\text { psychoeducation } \\
\text { focusing on causes, } \\
\text { role of medication, } \\
\text { lifestyle changes, } \\
\text { relapse prevention } \\
\text { and early } \\
\text { intervention, } \\
\text { psychological } \\
\text { approaches, } \\
\text { gender-specific } \\
\text { considerations, and } \\
\text { advice for family and } \\
\text { careers } \\
\text { - Initial face-to-face } \\
\text { meeting with } \\
\text { psychiatrist to learn } \\
\text { how to use program } \\
\text { followed by four } \\
\text { months of every- } \\
\text { other-week online } \\
\text { psychoeducation }\end{array}$ & \begin{tabular}{|l|} 
Treatment as \\
usual: Usual care \\
delivered in a \\
collaborative \\
model between \\
general \\
practitioners and \\
community \\
mental health \\
teams.
\end{tabular} & 6 months & $\begin{array}{l}\text { Relapse } \\
\text { MADRS } \\
\text { YMRS } \\
\text { GAF } \\
\text { FAST } \\
\text { WHO-QOL-bref } \\
\text { Withdrawal 26\% }\end{array}$ \\
\hline
\end{tabular}




\begin{tabular}{|c|c|c|c|c|c|c|}
\hline $\begin{array}{l}\text { Study, Year } \\
\text { Design } \\
\text { Location } \\
\text { Funder } \\
\text { Risk of Bias } \\
\text { PMID }\end{array}$ & $\begin{array}{c}\text { Randomized (N) } \\
\text { Age (mean) } \\
\text { Sex (\% Female) } \\
\text { Race (\% White) } \\
\text { Diagnosis } \\
\text { (\% BP I, II, NOS) } \\
\text { Setting }\end{array}$ & $\begin{array}{c}\text { Inclusions } \\
\text { Key Exclusions }\end{array}$ & $\begin{array}{l}\text { Intervention } \\
\text { Description }\end{array}$ & $\begin{array}{l}\text { Comparison } \\
\text { Description }\end{array}$ & $\begin{array}{l}\text { Follow-up } \\
\text { Duration }\end{array}$ & $\begin{array}{c}\text { Outcomes } \\
\text { Reported } \\
\begin{array}{c}\text { Withdrawal (\%) at } \\
\text { endpoint }\end{array}\end{array}$ \\
\hline $\begin{array}{l}\text { Sajatovic, } 2009^{6} \\
\text { RCT } \\
\text { United States } \\
\text { Government } \\
\text { Low } \\
19723732\end{array}$ & $\begin{array}{l}\mathrm{N}=164 \\
\text { Age } 41(18-76) \\
\text { Female } 68 \% \\
\text { White } 60 \% \\
\text { BP NR } \\
\text { Outpatient }\end{array}$ & $\begin{array}{l}\text { No current clinical state } \\
\text { excluded; Individuals with } \\
\text { type I or type II bipolar } \\
\text { disorder (MINI) } \\
\text { Other Conditions }\end{array}$ & \begin{tabular}{|l|} 
Group \\
psychoeducation \\
(Life Goals \\
Program) focusing \\
on illness education, \\
medication \\
adherence, \\
management, goal \\
setting, and problem \\
solving \\
-6 weekly sessions \\
followed by optional \\
monthly group \\
sessions
\end{tabular} & \begin{tabular}{|l|} 
Treatment as \\
usual: Treatment \\
at community \\
mental health \\
care including \\
medication \\
management and \\
psychosocial \\
therapy and \\
counseling
\end{tabular} & 12 months & $\begin{array}{l}\text { HAM-D } \\
\text { GAS } \\
\text { YMRS } \\
\text { Withdrawal 22\% }\end{array}$ \\
\hline $\begin{array}{l}\text { Colom, } 2009^{7} \\
\text { Colom, } 2003^{8} \text { Spain } \\
\text { Non-Government } \\
\text { Low } \\
12695318 \\
19252157\end{array}$ & $\begin{array}{l}\text { N = } 120 \\
\text { Age NR (18-65) } \\
\text { Female 63\% } \\
\text { White NR } \\
\text { BP I 83\% } \\
\text { BP II 17\% } \\
\text { Outpatient }\end{array}$ & $\begin{array}{l}\text { Euthymic/Maintenance; } \\
\text { Diagnosis of BP I or II, } \\
\text { euthymic (YMRS <6, HDRS } \\
<8 \text { ) for at least } 6 \text { months, } \\
\text { having sufficient data on } \\
\text { the prior course of illness } \\
\text { collected from a } \\
\text { prospective follow-up of at } \\
\text { least } 24 \text { months } \\
\text { Other Mental Health; } \\
\text { Neurological Disorders }\end{array}$ & $\begin{array}{l}\text { Group } \\
\text { psychoeducation } \\
\text { (and pharmacologic } \\
\text { treatment) } \\
\text { that focused on } \\
\text { illness awareness, } \\
\text { treatment } \\
\text { compliance, early } \\
\text { detection of } \\
\text { prodromal } \\
\text { symptoms and } \\
\text { recurrences, and } \\
\text { life-style regularity } \\
\\
-21 \text { weekly } 90- \\
\text { minute sessions }\end{array}$ & \begin{tabular}{|l|} 
Standard \\
pharmacologic \\
treatment and \\
group meetings \\
with \\
psychologists \\
without any \\
psychosocial \\
feedback (unless \\
necessary for \\
patient \\
interaction) \\
-20 weekly group \\
sessions
\end{tabular} & 5 years & $\begin{array}{l}\text { Relapse (DSM-IV } \\
\text { criteria for new acute } \\
\text { episode and one of } \\
\text { following: YMRS } \geq 20 \text { or } \\
\text { YMRS } \geq 12 \text { or } \\
\text { HDRS } \geq 17 \text {, or } Y M R S \geq \\
\text { and HDRS } \geq 20 \text { ) } \\
\text { Hospitalizations } \\
\text { Withdrawal } 18 \%\end{array}$ \\
\hline
\end{tabular}




\begin{tabular}{|c|c|c|c|c|c|c|}
\hline $\begin{array}{l}\text { Study, Year } \\
\text { Design } \\
\text { Location } \\
\text { Funder } \\
\text { Risk of Bias } \\
\text { PMID }\end{array}$ & $\begin{array}{c}\text { Randomized (N) } \\
\text { Age (mean) } \\
\text { Sex (\% Female) } \\
\text { Race (\% White) } \\
\text { Diagnosis } \\
\text { (\% BP I, II, NOS) } \\
\text { Setting }\end{array}$ & $\begin{array}{c}\text { Inclusions } \\
\text { Key Exclusions }\end{array}$ & $\begin{array}{l}\text { Intervention } \\
\text { Description }\end{array}$ & $\begin{array}{l}\text { Comparison } \\
\text { Description }\end{array}$ & $\begin{array}{l}\text { Follow-up } \\
\text { Duration }\end{array}$ & $\begin{array}{c}\text { Outcomes } \\
\text { Reported } \\
\begin{array}{c}\text { Withdrawal (\%) at } \\
\text { endpoint }\end{array}\end{array}$ \\
\hline $\begin{array}{l}\text { Colom, } 2003 b^{9} \\
\text { RCT } \\
\text { Spain } \\
\text { Non-Government } \\
\text { Low } \\
14628987\end{array}$ & $\begin{array}{l}\mathrm{N}=50 \\
\text { Age } 35(18-57) \\
\text { Female } 72 \% \\
\text { White NR } \\
\text { BP I 100\% } \\
\text { Outpatient }\end{array}$ & $\begin{array}{l}\text { Euthymic/Maintenance; A } \\
\text { lifetime diagnosis of BP I } \\
\text { (DSM-IV), euthymic (YMRS } \\
<6, \text { HAM-D <8) for at least } \\
6 \text { months, data on the prior } \\
\text { course of illness collected } \\
\text { from a prospective follow- } \\
\text { up of at least } 24 \text { months, } \\
\text { good treatment compliance } \\
\text { during at least } 6 \text { months } \\
\text { prior to enrollment. } \\
\text { Other Mental Health; } \\
\text { Neurological Disorders; } \\
\text { Taking Other Meds }\end{array}$ & \begin{tabular}{|l|} 
Group \\
psychoeducation \\
(and standard \\
treatment) \\
focused on illness \\
awareness, \\
treatment \\
compliance, \\
prodromal \\
symptoms and \\
relapse, lifestyle \\
regularity, symptom \\
monitoring, \\
treatment \\
adherence, and \\
illness management \\
skills. \\
-20 weekly group \\
sessions for 90 \\
minutes
\end{tabular} & $\begin{array}{l}\text { Standard } \\
\text { pharmacologic } \\
\text { treatment and } \\
\text { group meetings } \\
\text { with } \\
\text { psychologists } \\
\text { without any } \\
\text { psychosocial } \\
\text { feedback (unless } \\
\text { necessary for } \\
\text { patient } \\
\text { interaction). } \\
\text { Therapists } \\
\text { encouraged } \\
\text { communication } \\
\text { between } \\
\text { patients. } \\
\text {-20 weekly group } \\
\text { sessions }\end{array}$ & 2 years & $\begin{array}{l}\text { Relapse (DSM-IV } \\
\text { criteria and HAM-D or } \\
\text { YMRS } \geq 12 \text { ) } \\
\text { Hospitalizations } \\
\text { Withdrawal } 0 \%\end{array}$ \\
\hline
\end{tabular}




\begin{tabular}{|c|c|c|c|c|c|c|}
\hline $\begin{array}{l}\text { Study, Year } \\
\text { Design } \\
\text { Location } \\
\text { Funder } \\
\text { Risk of Bias } \\
\text { PMID }\end{array}$ & $\begin{array}{c}\text { Randomized (N) } \\
\text { Age (mean) } \\
\text { Sex (\% Female) } \\
\text { Race (\% White) } \\
\text { Diagnosis } \\
\text { (\% BP I, II, NOS) } \\
\text { Setting }\end{array}$ & $\begin{array}{c}\text { Inclusions } \\
\text { Key Exclusions }\end{array}$ & $\begin{array}{l}\text { Intervention } \\
\text { Description }\end{array}$ & $\begin{array}{l}\text { Comparison } \\
\text { Description }\end{array}$ & $\begin{array}{c}\text { Follow-up } \\
\text { Duration }\end{array}$ & $\begin{array}{c}\begin{array}{c}\text { Outcomes } \\
\text { Reported }\end{array} \\
\begin{array}{c}\text { Withdrawal (\%) at } \\
\text { endpoint }\end{array}\end{array}$ \\
\hline $\begin{array}{l}\text { Weiss, } 2000^{10} \\
\text { CCT } \\
\text { United States } \\
\text { Government } \\
\text { High } \\
10847311\end{array}$ & $\begin{array}{l}\mathrm{N}=45 \\
\text { Age } 36(18-54) \\
\text { Female } 49 \% \\
\text { White } 87 \% \\
\text { BP I 73\% } \\
\text { BP II 18\% } \\
\text { BP NOS 9\% } \\
\text { Outpatient }\end{array}$ & $\begin{array}{l}\text { No current clinical state } \\
\text { excluded; Current } \\
\text { diagnoses of BP and } \\
\text { substance dependence, } \\
\text { substance use within } 30 \\
\text { days, and taking a mood } \\
\text { stabilizer } \\
\text { Neurological Disorders; } \\
\text { Other Conditions (which } \\
\text { would preclude attendance) }\end{array}$ & $\begin{array}{l}\text { Psychoeducation } \\
\text { focused on } \\
\text { acceptance, self- } \\
\text { help, identifying and } \\
\text { fighting triggers, } \\
\text { medication } \\
\text { adherence, coping } \\
\text { skills, and } \\
\text { similarities between } \\
\text { recovery and } \\
\text { relapse for bipolar } \\
\text { and substance } \\
\text { abuse } \\
\text {-12-20 weekly group } \\
\text { therapy, } 60 \text { minutes } \\
\text { per session }\end{array}$ & $\begin{array}{l}\text { Treatment as } \\
\text { usual/No } \\
\text { treatment (not } \\
\text { described) with } 6 \\
\text { monthly } \\
\text { assessments }\end{array}$ & 6 months & $\begin{array}{l}\text { YMRS } \\
\text { HAM-D } \\
\text { Hospitalizations } \\
\text { Withdrawal } 47 \%\end{array}$ \\
\hline $\begin{array}{l}\text { Perry, 199911 } \\
\text { RCT } \\
\text { United Kingdom } \\
\text { Government } \\
\text { Moderate } \\
9888904\end{array}$ & $\begin{array}{l}\mathrm{N}=69 \\
\text { Age } 45(23-67) \\
\text { Female } 68 \% \\
\text { White } 91 \% \\
\text { BP I } 91 \% \\
\text { BP II 9\% } \\
\text { Outpatient }\end{array}$ & $\begin{array}{l}\text { Maintenance; A lifetime } \\
\text { diagnosis of bipolar } \\
\text { disorder elicited by a } \\
\text { trained research assistant } \\
\text { using a standardized } \\
\text { psychiatric interview and } \\
\text { two or more relapses, one } \\
\text { in the previous } 12 \text { months. } \\
\\
\text { Substance Abuse; } \\
\text { Neurological Disorders }\end{array}$ & $\begin{array}{l}\text { Psychoeducation } \\
\text { (and routine } \\
\text { treatment) involving } \\
12 \text { individual } \\
\text { treatment sessions } \\
\text { that focused on } \\
\text { identifying } \\
\text { prodromal } \\
\text { symptoms and } \\
\text { producing and } \\
\text { rehearsing an action } \\
\text { plan once } \\
\text { prodromes had } \\
\text { been recognized }\end{array}$ & $\begin{array}{l}\text { Treatment as } \\
\text { usual: Drug } \\
\text { treatment, } \\
\text { monitoring of } \\
\text { mood and } \\
\text { adherence to } \\
\text { treatment, } \\
\text { education about } \\
\text { BP, and inpatient } \\
\text { care if } \\
\text { necessary. }\end{array}$ & 18 months & $\begin{array}{l}\text { Relapse (Minimum of } \\
\text { five days of symptoms } \\
\text { of mania, hypomania, } \\
\text { mixed affective } \\
\text { disorder, or major } \\
\text { depression according } \\
\text { to the standardized } \\
\text { symptom criteria) } \\
\text { SPS } \\
\text { Withdrawal 14\% }\end{array}$ \\
\hline
\end{tabular}

Abbreviations: BP=bipolar disorder; BRMS = Bech-Rafaelsen Mania Scale; DSM=Diagnostic and Statistical Manual of Mental Disorders; GAF=General Assessment of

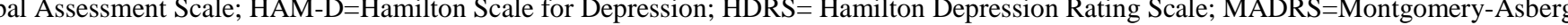
Depression Rating Scale; MINI=MINI International Nueropsychiatric Interview; NOS=not otherwise specified; NR=not reported; PMID=PubMed Identification Number; RCT=randomized controlled trial; SPS=Social Phobia Scale; WHO-QOL-bref= World Health Organization Quality of Life-short version; YMRS = Young Mania Rating Scale 
Appendix Table J2. Summary risk of bias assessments: psychoeducation vs. inactive comparators by year then first author

\begin{tabular}{|c|c|c|}
\hline $\begin{array}{l}\text { Study } \\
\text { Funder } \\
\text { PMID }\end{array}$ & $\begin{array}{c}\text { Overall Risk of Bias } \\
\text { Assessment }\end{array}$ & Rationale \\
\hline $\begin{array}{l}\text { Barnes, } 2015^{1} \\
\text { Non-Government } \\
25554993\end{array}$ & Moderate & Suspected bias due to incomplete reporting of outcomes (unable to separate outcomes by study arm). \\
\hline $\begin{array}{l}\text { de Barros } \\
\text { Pellegrinelli, } 2013^{3} \\
\text { Non-Government } \\
22943487\end{array}$ & High & Suspected bias due to high attrition rate (45\%). \\
\hline $\begin{array}{l}\text { Gumus, } 2015^{2} \\
\text { NR } \\
26001717\end{array}$ & High & Suspected bias due to procedures for randomization and unclear reporting of study attrition. \\
\hline $\begin{array}{l}\text { Javadpour, } 2013^{4} \\
\text { Non-Government } \\
23642977\end{array}$ & High & Suspected bias due to unclear reporting of outcomes (format of reporting makes it difficult to interpret results). \\
\hline $\begin{array}{l}\text { Smith, } 2011^{5} \\
\text { Government } \\
22017225\end{array}$ & Low & No significant suspected biases. \\
\hline $\begin{array}{l}\text { Sajatovic, } 2009^{6} \\
\text { Government } \\
19723732\end{array}$ & Low & No significant suspected biases. \\
\hline $\begin{array}{l}\text { Colom, } 2009^{7} \\
\text { Colom, 2003 } \\
\text { Non-Government } \\
12695318 \\
19252157 \\
\end{array}$ & Low & No significant suspected biases. \\
\hline $\begin{array}{l}\text { Colom, 2003b } \\
\text { Non-Government } \\
14628987\end{array}$ & Low & No significant suspected biases. \\
\hline $\begin{array}{l}\text { Weiss, } 2000^{10} \\
\text { Government } \\
10847311\end{array}$ & High & Suspected selection bias (subjects are not randomized) and unclear reporting of attrition and outcome data. \\
\hline $\begin{array}{l}\text { Perry, } 1999^{11} \\
\text { Government } \\
9888904\end{array}$ & Moderate & $\begin{array}{l}\text { Suspected bias due to lack of blinding. Assessors appeared to have access to full set of information/notes on } \\
\text { subjects. }\end{array}$ \\
\hline
\end{tabular}

Abbreviations: PMID=PubMed Identification Number 
Appendix Table J3. Outcomes summary: psychoeducation vs. inactive comparators

\begin{tabular}{|c|c|c|c|c|c|}
\hline $\begin{array}{l}\text { Study } \\
\text { PMID }\end{array}$ & Responder/Remitter & Symptom & Function & Other & $\mathrm{AE}$ \\
\hline $\begin{array}{l}\text { Barnes } 2015^{1} \\
25554993\end{array}$ & $\begin{array}{l}\text { Relapse } \\
12 \text { months, Any Type } \\
\text { NS at any threshold for } \\
\text { recurrence (low, moderate, } \\
\text { high). } \\
\text { Low: HR=0.86; } p=0.48 \\
\text { Moderate: HR= } 0.82 ; p=0.33 \\
\text { High: HR=0.91; } p=0.65 \\
\text { Time to Relapse } \\
12 \text { months, Any Type } \\
\text { NS at any threshold for } \\
\text { recurrence (low, moderate, } \\
\text { high). }\end{array}$ & NR & NR & $\begin{array}{l}\text { Hospitalizations } \\
12 \text { months } \\
\text { NS; } p=0.90\end{array}$ & NR \\
\hline $\begin{array}{l}\text { Gumus, } 2015^{2} \\
26001717\end{array}$ & $\begin{array}{l}\frac{\text { Relapse }^{*}}{12 \text { months, Any Type }} \\
\text { NS } \\
\text { OR=0.50 (95\% Cl 0.14, } \\
1.57) ; p=0.21\end{array}$ & NR & NR & $\begin{array}{l}\text { Hospitalizations } \\
12 \text { months } \\
\text { NS, Number of } \\
\text { Hospitalizations } \\
\text { Psychoeducation: } 0(0 \%) \\
\text { Comparator: } 3(7.3 \%)\end{array}$ & NR \\
\hline $\begin{array}{l}\text { de Barros } \\
\text { Pellegrinelli } \\
2013^{3} \\
22943487\end{array}$ & NR & $\begin{array}{l}\frac{\text { Depression }}{12 \text { months, HDRS }} \\
\text { No difference between groups } \\
E S=0.007 ; p=0.82 \\
\frac{\text { Mania }}{12 \text { months, YMRS }} \\
\text { NS } \\
\text { ES }=0.016 ; p=0.72\end{array}$ & $\begin{array}{l}\frac{\text { Global Function }}{12 \text { months, GAF }} \\
\text { NS } \\
\text { ES }=0.03 ; p=0.59\end{array}$ & NR & NR \\
\hline $\begin{array}{l}\text { Javadpour } \\
2013^{4} \\
23642977\end{array}$ & $\begin{array}{l}\text { Relapse } \\
18 \text { months, Any Type } \\
\text { Favors psychoeducation; } \\
p=0.00\end{array}$ & $\begin{array}{l}\frac{\text { Depression }}{18 \text { months, HDRS }} \\
\text { Favors psychoeducation; } \\
p=0.00 \\
\frac{\text { Mania }}{18 \text { months, BRMS }} \\
\text { Favors psychoeducation; } \\
p=0.00\end{array}$ & NR & $\begin{array}{l}\frac{\text { Hospitalizations }}{18 \text { months, Any Type }} \\
\text { Favors psychoeducation; } \\
p=0.00 \\
\text { Average Number of } \\
\text { Hospitalizations } \\
\text { Psychoeducation: } 0.22 \\
\text { Comparator: } 1.41\end{array}$ & NR \\
\hline
\end{tabular}




\begin{tabular}{|c|c|c|c|c|c|}
\hline $\begin{array}{l}\text { Study } \\
\text { PMID }\end{array}$ & Responder/Remitter & Symptom & Function & Other & $\mathrm{AE}$ \\
\hline $\begin{array}{l}\text { Smith } 2011^{5} \\
22017225\end{array}$ & $\begin{array}{l}\frac{\text { Relapse }^{\star}}{10 \text { months, Depressive }} \\
\text { NS } \\
\text { OR=1.75 ( } 95 \% \mathrm{Cl} 0.39 \text {, } \\
7.87) ; p=0.31 \\
10 \text { months, Manic } \\
\text { NS } \\
\text { OR=0.92 (95\% Cl 0.15, } \\
5.36) ; p=0.61\end{array}$ & $\begin{array}{l}\frac{\text { Depression* }}{10 \text { months, MADRS }} \\
\text { NS } \\
\text { ES }=-0.17(95 \%-0.82,0.48) \\
\frac{\text { Mania }^{*}}{10 \text { months, YMRS }} \\
\text { NS } \\
\text { ES }=-0.25(95 \%-0.90,0.40)\end{array}$ & $\begin{array}{l}\text { Global Function* } \\
10 \text { months, GAF } \\
\text { NS } \\
\text { ES=0.26 (95\% Cl - } \\
0.39,0.91) \\
\text { Global Function* } \\
10 \text { months, FAST } \\
\text { NS } \\
\text { ES=0.26 }(95 \% \mathrm{Cl} \text {, - } \\
0.39,0.91) \\
\text { Quality of Life* } \\
10 \text { months, WHO- } \\
\text { QOL-bref } \\
\text { NS } \\
\text { ES=-0.04 }(95 \% \mathrm{Cl}- \\
0.69,0.60)\end{array}$ & NR & NR \\
\hline $\begin{array}{l}\text { Sajatovic } 2009^{6} \\
19723732\end{array}$ & $\mathrm{NR}$ & $\begin{array}{l}\text { Depression* } \\
6 \text { months, HAM-D } \\
\text { NS } \\
\text { ES }=0.03(95 \% \text { Cl }-0.35,0.42) \\
\frac{\text { Mania }^{\star}}{6 \text { months, YMRS }} \\
\text { NS } \\
\text { ES }=-0.16(95 \% \mathrm{Cl}-0.54,0.23)\end{array}$ & $\begin{array}{l}\text { Global Function* } \\
6 \text { months, GAF } \\
\text { NS } \\
\text { ES }=-0.03(95 \% \mathrm{Cl}- \\
0.43,0.36)\end{array}$ & $\mathrm{NR}$ & $\mathrm{NR}$ \\
\hline
\end{tabular}




\begin{tabular}{|c|c|c|c|c|c|}
\hline $\begin{array}{l}\text { Study } \\
\text { PMID }\end{array}$ & Responder/Remitter & Symptom & Function & Other & $\mathrm{AE}$ \\
\hline $\begin{array}{l}\text { Colom } 2003^{8} \\
12695318 \\
\text { Colom } 2009^{7} \\
19252157\end{array}$ & $\begin{array}{l}\frac{\text { Relapse }^{\star}}{2 \text { years, Any Type }} \\
\text { Favors psychoeducation } \\
\text { OR }=0.18(95 \% \text { Cl } 0.05 \\
0.56) ; p=0.00\end{array}$ & NR & NR & $\begin{array}{l}\frac{\text { Hospitalizations }}{2 \text { Years }} \\
\text { NS; } p=0.24 \\
\text { Number of } \\
\text { Hospitalizations } \\
\text { Psychoeducation: } 14 \\
\text { (25\%) } \\
\text { Comparator: } 21 \text { (35\%) } \\
5 \text { Years } \\
\text { NS; } p=0.28 \\
\text { Number of } \\
\text { Hospitalizations } \\
\text { Psychoeducation: } 17 \\
\text { (30.4\%) } \\
\text { Comparator: } 24(40 \%)\end{array}$ & NR \\
\hline $\begin{array}{l}\text { Colom } 2003 b^{9} \\
14628987\end{array}$ & $\begin{array}{l}\text { Relapse }^{\star} \\
2 \text { years, Any Type } \\
\text { Favors psychoeducation } \\
\text { OR }=0.13(95 \% \text { Cl } 0.01 \\
0.77) ; p=0.02\end{array}$ & $\mathrm{NR}$ & $\overline{N R}$ & $\begin{array}{l}\text { Hospitalizations } \\
\text { 2 Years } \\
\text { Favors psychoeducation; } \\
\mathrm{p}=0.01 \\
\text { Number of } \\
\text { Hospitalizations } \\
\text { Psychoeducation: } 2 \\
(8.0 \%) \\
\text { Comparator: } 9(36 \%)\end{array}$ & $\mathrm{NR}$ \\
\hline $\begin{array}{l}\text { Weiss } 2000^{10} \\
10847311\end{array}$ & NR & $\begin{array}{l}\text { Depression } \\
6 \text { months, HAM-D } \\
\text { NS } \\
\text { Mania } \\
6 \text { months, YMRS } \\
\text { Favors psychoeducation; } \\
\text { p<0.04 }\end{array}$ & NR & $\begin{array}{l}\text { Hospitalizations } \\
6 \text { months } \\
\text { NS } \\
\text { Number of } \\
\text { Hospitalizations } \\
\text { Psychoeducation: } 8 \\
(38.1 \%) \\
\text { Comparator: } 10(41.7 \%)\end{array}$ & NR \\
\hline
\end{tabular}




\begin{tabular}{|c|c|c|c|c|c|}
\hline $\begin{array}{l}\text { Study } \\
\text { PMID }\end{array}$ & Responder/Remitter & Symptom & Function & Other & $\mathrm{AE}$ \\
\hline $\begin{array}{l}\text { Perry } 1999^{11} \\
9888904\end{array}$ & $\begin{array}{l}\frac{\text { Relapse}^{*}}{6 \text { months, Depressive }} \\
\text { NS } \\
\text { OR=1.44 (95\% Cl 0.45, } \\
4.73) ; p=0.60 \\
18 \text { months, Depressive } \\
\text { NS } \\
\text { OR=2.03 (95\% Cl 0.69, } \\
6.00) ; p=0.22 \\
6 \text { months, Manic } \\
\text { Favors psychoeducation } \\
\text { OR=0.14 (95\% Cl 0.01, } \\
0.75) ; p=0.01 \\
18 \text { months, Manic } \\
\text { Favors psychoeducation } \\
\text { OR=0.28 (95\% Cl 0.09, } \\
0.87) ; p=0.02\end{array}$ & NR & $\begin{array}{l}\text { Social Function } \\
6 \text { months, SPS } \\
\text { NS } \\
\text { Mean Difference }=0.44 \\
(95 \% \mathrm{Cl}-0.78,1.65) \\
18 \text { months, SPS } \\
\text { Favors } \\
\text { psychoeducation } \\
\text { Mean Difference }=1.97 \\
(95 \% \mathrm{Cl} 0.71,3.23)\end{array}$ & NR & NR \\
\hline
\end{tabular}

Abbreviations: AE=Adverse Events; BRMS=Bech-Rafaelsen Mania Scale; CI=Confidence Interval; ES=Effect Size; GAF=General Assessment of Functioning Scale; HAM$\mathrm{D}=$ Hamilton Scale for Depression; HDRS=Hamilton Depression Rating Scale; HR=Hazard Ratio; MADRS=Montgomery-Asberg Depression Rating Scale; NR=not reported; NS=not significant; OR= Odds Ratio; PMID=PubMed Identification Number; SPS=Social Phobia Scale; WHO-QOL-bref= World Health Organization Quality of Life-short version; YMRS = Young Mania Rating Scale 
Appendix Table J4. Summary of strength of evidence: psychoeducation vs. inactive comparators

\begin{tabular}{|c|c|c|c|c|c|c|c|c|}
\hline Outcome & Timing & $\begin{array}{l}\text { \# Studies/ Design } \\
\text { (n analyzed) }\end{array}$ & $\begin{array}{l}\text { Finding or } \\
\text { Summary } \\
\text { Statistic }\end{array}$ & $\begin{array}{c}\text { Study } \\
\text { Limitations }\end{array}$ & Consistency & Directness & $\begin{array}{c}\text { Precisio } \\
\text { n }\end{array}$ & $\begin{array}{l}\text { Overall Gradel } \\
\text { Conclusion }\end{array}$ \\
\hline Relapse & $\begin{array}{l}6 \text { months } \\
7-12 \\
\text { months } \\
12+ \\
\text { months } \\
\end{array}$ & 7 RCTs $(n=712)$ & $\begin{array}{l}\text { Mixed evidence } \\
\text { with no clear } \\
\text { direction of effect. } \\
\text { No pattern across } \\
\text { time periods. }\end{array}$ & Moderate & Inconsistent & Direct & Imprecise & Insufficient \\
\hline Depression & $\begin{array}{l}6 \text { months } \\
7-12 \\
\text { months } \\
12+ \\
\text { months } \\
\end{array}$ & 5 RCTs $(n=422)$ & $\begin{array}{l}\text { No difference } \\
\text { between groups } \\
\text { across a range of } \\
\text { outcome } \\
\text { timepoints. }\end{array}$ & High & Consistent & Direct & Imprecise & Insufficient \\
\hline Mania & $\begin{array}{l}6 \text { months } \\
7-12 \\
\text { months } \\
12+ \\
\text { months } \\
\end{array}$ & 5 RCTs $(n=422)$ & $\begin{array}{l}\text { Mixed evidence } \\
\text { with no clear } \\
\text { direction of effect. } \\
\text { No pattern across } \\
\text { time periods. }\end{array}$ & High & Inconsistent & Direct & Imprecise & Insufficient \\
\hline $\begin{array}{l}\text { Global } \\
\text { Function }\end{array}$ & $\begin{array}{l}6 \text { months } \\
12 \text { months }\end{array}$ & 3 RCTs $(n=269)$ & $\begin{array}{l}\text { No difference } \\
\text { between groups at } \\
6 \text { or } 12 \text { months. }\end{array}$ & Moderate & Consistent & Direct & Imprecise & Insufficient \\
\hline $\begin{array}{l}\text { Other } \\
\text { Measures } \\
\text { of Function }\end{array}$ & $\begin{array}{l}6 \text { months } \\
7-12 \\
\text { months } \\
12+ \\
\text { months } \\
\end{array}$ & 2 RCTs $(n=119)$ & $\begin{array}{l}\text { Mixed evidence } \\
\text { with no clear } \\
\text { direction of effect. } \\
\text { No pattern across } \\
\text { time periods. }\end{array}$ & Moderate & Inconsistent & Direct & Imprecise & Insufficient \\
\hline
\end{tabular}

Abbreviations: RCT=randomized controlled trial 


\begin{tabular}{|c|c|c|c|c|c|c|}
\hline $\begin{array}{l}\text { Study, Year } \\
\text { Design } \\
\text { Location } \\
\text { Funder } \\
\text { Risk of Bias } \\
\text { PMID }\end{array}$ & $\begin{array}{l}\text { Randomized (N) } \\
\text { Age (mean) } \\
\text { Sex (\% Female) } \\
\text { Race (\% White) } \\
\text { Diagnosis } \\
\text { (\% BP I, II, NOS) } \\
\text { Setting }\end{array}$ & $\begin{array}{l}\text { Inclusions } \\
\text { Key Exclusions }\end{array}$ & $\begin{array}{l}\text { Intervention } \\
\text { Description }\end{array}$ & $\begin{array}{l}\text { Comparison } \\
\text { Description }\end{array}$ & $\begin{array}{l}\text { Follow-up } \\
\text { Duration }\end{array}$ & $\begin{array}{l}\text { Outcomes } \\
\text { Reported } \\
\text { Withdrawal (\%) at } \\
\text { endpoint }\end{array}$ \\
\hline $\begin{array}{l}\text { Bilderbeck, } 2016^{12} \\
\text { RCT } \\
\text { United Kingdom } \\
\text { Government } \\
\text { Moderate } \\
27454410\end{array}$ & $\begin{array}{l}\mathrm{N}=121 \\
\text { Age } 44(16-76) \\
\text { Female } 73 \% \\
\text { White } 93 \% \\
\text { BP I } 65 \% \\
\text { BP II 35\% } \\
\text { Outpatient }\end{array}$ & $\begin{array}{l}\text { Maintenance; Individuals } \\
\text { with BP I or II (DSM-IV) but } \\
\text { not in a current mood } \\
\text { episode and without a need } \\
\text { for acute treatment } \\
\text { Labs/Other Conditions }\end{array}$ & $\begin{array}{l}\text { Therapist facilitated } \\
\text { psychoeducation via } \\
\text { manual focused on } \\
\text { identifying the relapse, } \\
\text { reviewing risk factors, } \\
\text { daily sleep regulation, } \\
\text { medications and } \\
\text { substance abuse; and } \\
\text { mood management } \\
\text { planning. } \\
-5 \text { face to face sessions } \\
\text { over } 12 \text { weeks }\end{array}$ & $\begin{array}{l}\text { Self-administered } \\
\text { psychoeducation via } \\
\text { manual focused on } \\
\text { identifying the } \\
\text { relapse, reviewing } \\
\text { risk factors, daily } \\
\text { sleep regulation, } \\
\text { medications and } \\
\text { substance abuse; and } \\
\text { mood management } \\
\text { planning. } \\
\text {-Manual access for } 12 \\
\text { weeks }\end{array}$ & 12 months & $\begin{array}{l}\text { Relapse (Intervention } \\
\text { for emergent mood } \\
\text { symptoms and/or } \\
\text { admission to inpatient } \\
\text { care or intensive } \\
\text { community treatment) } \\
\text { Hospitalization } \\
\text { QIDS-SR16 } \\
\text { ASRM } \\
\text { Withdrawal 31\% }\end{array}$ \\
\hline $\begin{array}{l}\text { Kallestad, } 2016^{13} \\
\text { RCT } \\
\text { Norway } \\
\text { NR } \\
\text { High } \\
27253214\end{array}$ & $\begin{array}{l}\mathrm{N}=85 \\
\text { Age } 38(19-64) \\
\text { Female 54\% } \\
\text { Race NR } \\
\text { BP I 47\% } \\
\text { BP II 53\% } \\
\text { Outpatient }\end{array}$ & $\begin{array}{l}\text { No current clinical state } \\
\text { excluded; Individuals with } \\
\text { BP I or II (DSM-IV) without } \\
\text { an elevated risk of suicide } \\
\\
\text { Labs/Other Conditions; } \\
\text { Neurological Disorders }\end{array}$ & $\begin{array}{l}\text { Group psychoeducation } \\
\text { focused on illness } \\
\text { education, symptoms, } \\
\text { early detection, sleep, } \\
\text { risk factors, stress } \\
\text { management, causes, } \\
\text { work, social } \\
\text { rights/welfare system } \\
\text { and law/regulations } \\
\text {-Ten initial 90-minute } \\
\text { sessions and } 8 \text { booster } \\
\text { sessions over next } 2 \\
\text { years at 3-montn } \\
\text { intervals }\end{array}$ & $\begin{array}{l}\text { Individual } \\
\text { psychoeducation } \\
\text { focused on } \\
\text { treatment, stress } \\
\text { management, sleep, } \\
\text { dysfunctional } \\
\text { cognitions, and other } \\
\text { psychosocial factors } \\
\text { associated with } \\
\text { increased risk of } \\
\text { relapse } \\
\text {-Three 1-hour weekly } \\
\text { sessions }\end{array}$ & 27 months & $\begin{array}{l}\text { Hospitalizations } \\
\text { Time to First Admission } \\
\text { Withdrawal } 11 \%\end{array}$ \\
\hline
\end{tabular}




\begin{tabular}{|c|c|c|c|c|c|c|}
\hline $\begin{array}{l}\text { Study, Year } \\
\text { Design } \\
\text { Location } \\
\text { Funder } \\
\text { Risk of Bias } \\
\text { PMID }\end{array}$ & $\begin{array}{l}\text { Randomized (N) } \\
\text { Age (mean) } \\
\text { Sex (\% Female) } \\
\text { Race (\% White) } \\
\text { Diagnosis } \\
\text { (\% BP I, II, NOS) } \\
\text { Setting }\end{array}$ & $\begin{array}{l}\text { Inclusions } \\
\text { Key Exclusions }\end{array}$ & $\begin{array}{l}\text { Intervention } \\
\text { Description }\end{array}$ & $\begin{array}{l}\text { Comparison } \\
\text { Description }\end{array}$ & $\begin{array}{l}\text { Follow-up } \\
\text { Duration }\end{array}$ & $\begin{array}{l}\text { Outcomes } \\
\text { Reported } \\
\text { Withdrawal (\%) at } \\
\text { endpoint }\end{array}$ \\
\hline $\begin{array}{l}\text { Morriss, } 2016^{14} \\
\text { RCT } \\
\text { United Kingdom } \\
\text { Government } \\
\text { Moderate } \\
27688021\end{array}$ & $\begin{array}{l}\mathrm{N}=304 \\
\text { Age } 45(33-57) \\
\text { Female 58\% } \\
\text { Race NR } \\
\text { BP I } 80 \% \\
\text { BP II 20\% } \\
\text { Outpatient }\end{array}$ & $\begin{array}{l}\text { Maintenance; Individuals } \\
\text { with BP I or II (DSM-IV) } \\
\text { with no current episode, but } \\
\text { with an increased risk of } \\
\text { relapse (occurrence of at } \\
\text { least one episode in the } \\
\text { past } 24 \text { months). } \\
\text { Labs/Other Conditions; } \\
\text { Other Mental Health }\end{array}$ & $\begin{array}{l}\text { Structured group } \\
\text { psychoeducation } \\
\text { focused on life charting, } \\
\text { recognition of early } \\
\text { warning signs, problem } \\
\text { solving, sleep hygiene, } \\
\text { and care planning } \\
\\
-21 \text { weekly sessions for } \\
2 \text { hours each over a } \\
\text { maximum of } 26 \text { weeks. }\end{array}$ & $\begin{array}{l}\text { Optimized } \\
\text { unstructured group } \\
\text { support where } \\
\text { participants set the } \\
\text { agenda at each } \\
\text { meeting } \\
-21 \text { weekly sessions } \\
\text { for } 2 \text { hours each over } \\
\text { a maximum of } 26 \\
\text { weeks }\end{array}$ & 96 weeks & $\begin{array}{l}\text { Relapse (LIFE, DSM- } \\
\text { IV) } \\
\text { Time to relapse } \\
\text { HAM-D } \\
\text { MAS } \\
\text { SOFAS } \\
\text { SAS } \\
\text { Withdrawal } 33 \%\end{array}$ \\
\hline
\end{tabular}

Abbreviations: BP=bipolar disorder; BRMS= Bech-Rafaelsen Mania Scale; DSM=Diagnostic and Statistical Manual of Mental Disorders; GAF=General Assessment of Functioning Scale; GAS=Global Assessment Scale; HAM-D=Hamilton Scale for Depression; HDRS= Hamilton Depression Rating Scale; MADRS=Montgomery-Asberg Depression Rating Scale; MINI=MINI International Nueropsychiatric Interview; NOS=not otherwise specified; NR=not reported; PMID=PubMed Identification Number; RCT=randomized controlled trial; SPS=Social Phobia Scale; WHO-QOL-bref= World Health Organization Quality of Life-short version; YMRS = Young Mania Rating Scale

\section{Appendix Table J6. Summary risk of bias assessments: psychoeducation vs. active comparators by year then first author}

Appendix Table J6. Summary risk of bias assessments: psychoeducation vs. active comparators by year then first author
\begin{tabular}{|l|l|l|}
\multicolumn{1}{|c}{$\begin{array}{c}\text { Study } \\
\text { Funder } \\
\text { PMID }\end{array}$} & \multicolumn{1}{c|}{$\begin{array}{c}\text { Overall Risk of Bias } \\
\text { Assessment }\end{array}$} & \\
\hline $\begin{array}{l}\text { Bilderbeck, } 2016^{12} \\
\text { Government } \\
27454410\end{array}$ & Moderate & Suspected bias due to possible selective reporting of outcome data (only summary statistics reported). \\
\hline $\begin{array}{l}\text { Kallestad, } 2016^{13} \\
\text { NR }\end{array}$ & High & \\
27253214 & & \\
\hline $\begin{array}{l}\text { Morriss, } 2016^{14} \\
\text { RCT }\end{array}$ & Moderate \\
Government & & Suspected bias due to attrition rate (33\%) and unclear reporting of loss to follow-up. \\
27688021 & & \\
\hline
\end{tabular}

Abbreviations: PMID=PubMed Identification Number 
Appendix Table J7. Outcomes summary: psychoeducation vs. active comparators

\begin{tabular}{|c|c|c|c|c|c|}
\hline $\begin{array}{l}\text { Study } \\
\text { PMID }\end{array}$ & Responder/Remitter & Symptom & Function & Other & $\mathrm{AE}$ \\
\hline $\begin{array}{l}\text { Bilderbeck, } \\
2016^{12} \\
27454410\end{array}$ & $\begin{array}{l}\text { Relapse } \\
12 \text { months } \\
\text { NS; } p>0.10 \\
N=88 \\
\text { Therapist-Administered } \\
\text { Psychoeducation=25 } \\
\text { Self-Administered } \\
\text { Psychoeducation }=25\end{array}$ & $\begin{array}{l}\frac{\text { Depression }}{12 \text { months, QIDS-SR16 }} \\
\text { NS } \\
\text { Adjusted Mean Difference }=0.17 \\
(95 \% \mathrm{Cl}-1.35,1.69) ; p=0.83 \\
\frac{\text { Mania }}{12 \text { months, ASRM (Score }>5)} \\
\text { NS } \\
\text { OR=0.71 (95\% Cl 0.35, 1.41); } \\
p=0.32\end{array}$ & NR & $\begin{array}{l}\text { Hospitalizations } \\
12 \text { months } \\
\text { NS } \\
\text { Therapist- } \\
\text { Administered } \\
\text { Psychoeducation=6 } \\
\text { Self-Administered } \\
\text { Psychoeducation=6 }\end{array}$ & NR \\
\hline $\begin{array}{l}\text { Kallestad, } 2016^{13} \\
27253214\end{array}$ & & & & $\begin{array}{l}\frac{\text { Hospitalizations }}{27 \text { months }} \\
\text { Group } \\
\text { Psychoeducation: } \\
581 . \% \\
\text { Individual } \\
\text { Psychoeducation: } \\
40.4 \% \\
\frac{\text { Time to First }}{\text { Admission }} \\
27 \text { months } \\
\text { Favors Group } \\
\text { Psychoeducation } \\
\text { p<0.01 }\end{array}$ & \\
\hline $\begin{array}{l}\text { Morriss, } 2016^{14} \\
27688021\end{array}$ & $\begin{array}{l}\frac{\text { Relapse* }}{96 \text { weeks, Any Type }} \\
\text { NS } \\
\text { OR=0.75 (95\% Cl 0.46, } \\
1.23) \text { ' } p=0.24 \\
\text { Time to Relapse } \\
96 \text { weeks } \\
\text { NS } \\
\text { HR }=0.83(95 \% \mathrm{Cl} 0.62 \text {, } \\
1.11) ; p=0.22\end{array}$ & $\begin{array}{l}\frac{\text { Mania* }}{96 \text { weeks, MAS* }} \\
\text { NS } \\
\text { ES }=0.01(95 \% \mathrm{Cl}-0.26,0.28) \\
\text { Depression* } \\
96 \text { weeks, HAM-D } \\
\text { NS } \\
\text { ES }=-0.10(95 \% \mathrm{Cl}-0.38,0.17)\end{array}$ & $\begin{array}{l}\text { Social and } \\
\text { Occupational } \\
\text { Functioning* } \\
96 \text { weeks, SOFAS } \\
\text { NS } \\
\text { ES }=0.16(95 \% \mathrm{Cl} \text { - } \\
0.11,0.44) \\
\text { Social Functioning* } \\
96 \text { weeks, SAS } \\
\text { NS } \\
\text { ES }=-0.26(95 \% \mathrm{Cl} \text { - } \\
0.54,0.01)\end{array}$ & NR & NR \\
\hline
\end{tabular}

Abbreviations: AE=Adverse Events; BRMS=Bech-Rafaelsen Mania Scale; CI=Confidence Interval; ES=Effect Size; GAF=General Assessment of Functioning Scale; HAMD=Hamilton Scale for Depression; HDRS=Hamilton Depression Rating Scale; HR=Hazard Ratio; MADRS=Montgomery-Asberg Depression Rating Scale; NR=not reported; 
NS=not significant; OR= Odds Ratio; PMID=PubMed Identification Number; SPS=Social Phobia Scale; WHO-QOL-bref= World Health Organization Quality of Life-short version; YMRS = Young Mania Rating Scale

\begin{tabular}{|c|c|c|c|c|c|c|c|c|}
\hline Outcome & Timing & $\begin{array}{l}\text { \# Studies/ Design } \\
\text { (n analyzed) }\end{array}$ & $\begin{array}{c}\text { Finding or Summary } \\
\text { Statistic }\end{array}$ & $\begin{array}{c}\text { Study } \\
\text { Limitations }\end{array}$ & Consistency & Directness & Precision & $\begin{array}{l}\text { Overall Gradel } \\
\text { Conclusion }\end{array}$ \\
\hline Relapse & $\begin{array}{l}12+ \\
\text { months }\end{array}$ & 2 RCTs $(n=425)$ & $\begin{array}{l}\text { No difference between } \\
\text { groups for two different } \\
\text { outcome time periods } \\
\text { (12 months and } 96 \\
\text { weeks). One RCT } \\
\text { compares } \\
\text { psychoeducation } \\
\text { formats. }\end{array}$ & Moderate & Consistent & Direct & Impercise & Insufficient \\
\hline Depression & $\begin{array}{l}12+ \\
\text { months }\end{array}$ & 2 RCTs $(n=425)$ & $\begin{array}{l}\text { No difference between } \\
\text { groups for two different } \\
\text { outcome time periods } \\
\text { (12 months and } 96 \\
\text { weeks). One RCT } \\
\text { compares } \\
\text { psychoeducation } \\
\text { formats. }\end{array}$ & Moderate & Consistent & Direct & Impercise & Insufficient \\
\hline Mania & $\begin{array}{l}12+ \\
\text { months }\end{array}$ & 2 RCTs $(n=425)$ & $\begin{array}{l}\text { No difference between } \\
\text { groups for two different } \\
\text { outcome time periods } \\
\text { (12 months and } 96 \\
\text { weeks). One RCT } \\
\text { compares } \\
\text { psychoeducation } \\
\text { formats. }\end{array}$ & Moderate & Consistent & Direct & Impercise & Insufficient \\
\hline $\begin{array}{l}\text { Global } \\
\text { Function }\end{array}$ & NR & - & - & - & - & - & - & - \\
\hline $\begin{array}{l}\text { Other } \\
\text { Measures } \\
\text { of Function }\end{array}$ & 96 weeks & $1 \operatorname{RCT}(n=121)$ & $\begin{array}{l}\text { No difference between } \\
\text { groups in two } \\
\text { measures of function at } \\
96 \text { weeks. }\end{array}$ & $\mathrm{Hgh}$ & Unclear & Direct & Impercise & Insufficient \\
\hline
\end{tabular}

Abbreviations: RCT=randomized controlled trial 


\section{References for Appendix $\mathbf{J}$}

1. Barnes CW, Hadzi-Pavlovic D, Wilhelm K, et al. A web-based preventive intervention program for bipolar disorder: outcome of a 12-months randomized controlled trial. Journal of Affective Disorders. 2015 Mar 15;174:485-92. doi: http://dx.doi.org/10.1016/j.jad.2014.11.038. PMID: 25554993.

2. Gumus F, Buzlu S, Cakir S. Effectiveness of individual psychoeducation on recurrence in bipolar disorder; a controlled study. Archives of psychiatric nursing. 201501 Jun;29(3):174-9. doi: http://dx.doi.org/10.1016/j.apnu.2015.01.005. PMID: 26001717.

3. de Barros Pellegrinelli K, de OCLF, Silval KI, et al. Efficacy of psychoeducation on symptomatic and functional recovery in bipolar disorder. Acta Psychiatrica Scandinavica. 2013 Feb;127(2):1538. doi: http://dx.doi.org/10.1111/acps.12007. PMID: 22943487.

4. Javadpour A, Hedayati A, Dehbozorgi GR, et al. The impact of a simple individual psychoeducation program on quality of life, rate of relapse and medication adherence in bipolar disorder patients. Asian Journal of Psychiatry. 2013 Jun;6(3):208-13. doi: http://dx.doi.org/10.1016/j.ajp.2012.12.005. PMID: 23642977.

5. Smith DJ, Griffiths E, Poole R, et al. Beating Bipolar: exploratory trial of a novel Internetbased psychoeducational treatment for bipolar disorder. Bipolar Disorders. 2011 Aug-Sep;13(56):571-7. doi: http://dx.doi.org/10.1111/j.13995618.2011.00949.x. PMID: 22017225.

6. Sajatovic M, Davies MA, Ganocy SJ, et al. A comparison of the life goals program and treatment as usual for individuals with bipolar disorder. Psychiatric Services. 2009

Sep;60(9):1182-9. doi: http://dx.doi.org/10.1176/appi.ps.60.9.1182. PMID: 19723732.

7. Colom F, Vieta E, Sanchez-Moreno J, et al. Group psychoeducation for stabilised bipolar disorders: 5-year outcome of a randomised clinical trial.[Erratum appears in Br J Psychiatry. 2009 Jun;194(6):571]. British Journal of Psychiatry. 2009 Mar;194(3):260-5. doi: http://dx.doi.org/10.1192/bjp.bp.107.040485. PMID: 19252157.
8. Colom F, Vieta E, Martinez-Aran A, et al. A randomized trial on the efficacy of group psychoeducation in the prophylaxis of recurrences in bipolar patients whose disease is in remission. Archives of General Psychiatry. 2003 Apr;60(4):402-7. PMID: 12695318.

9. Colom F, Vieta E, Reinares M, et al. Psychoeducation efficacy in bipolar disorders: beyond compliance enhancement. Journal of Clinical Psychiatry. 2003 Sep;64(9):1101-5. PMID: 14628987.

10. Weiss RD, Griffin ML, Greenfield SF, et al. Group therapy for patients with bipolar disorder and substance dependence: results of a pilot study. Journal of Clinical Psychiatry. 2000 May;61(5):361-7. PMID: 10847311.

11. Perry A, Tarrier N, Morriss R, et al. Randomised controlled trial of efficacy of teaching patients with bipolar disorder to identify early symptoms of relapse and obtain treatment. BMJ. 1999 Jan 16;318(7177):149-53. PMID: 9888904.

12. Bilderbeck AC, Atkinson LZ, McMahon HC, et al. Psychoeducation and online mood tracking for patients with bipolar disorder: A randomised controlled trial. Journal of Affective Disorders. 201615 Nov;205:245-51. doi: http://dx.doi.org/10.1016/j.jad.2016.06.064. PMID: 27454410 PMID/612460558 Embase.

13. Kallestad H, Wullum E, Scott J, et al. The longterm outcomes of an effectiveness trial of group versus individual psychoeducation for bipolar disorders. Journal of Affective Disorders. 2016;202:32-8. doi: http://dx.doi.org/10.1016/j.jad.2016.05.043. PMID: 27253214 PMID/611342382 Embase.

14. Morriss R, Lobban F, Riste L, et al. Clinical effectiveness and acceptability of structured group psychoeducation versus optimised unstructured peer support for patients with remitted bipolar disorder (PARADES): a pragmatic, multicentre, observer-blind, randomised controlled superiority trial. The Lancet Psychiatry. 201601 Nov;3(11):1029-38. doi: http://dx.doi.org/10.1016/S22150366\%2816\%2930302-9. PMID: 27688021. 


\section{Appendix K. Cognitive Behavioral Therapy}

Appendix Table K1. Characteristics of eligible studies: CBT vs. inactive comparators

\begin{tabular}{|c|c|c|c|c|c|c|}
\hline $\begin{array}{c}\text { Study, Year } \\
\text { Design } \\
\text { Location } \\
\text { Funder } \\
\text { Risk of Bias } \\
\text { PMID }\end{array}$ & $\begin{array}{c}\text { Randomized (N) } \\
\text { Age (mean) } \\
\text { Sex (\% Female) } \\
\text { Race (\% White) } \\
\text { Diagnosis } \\
\text { (\% BP I, II, NOS) } \\
\text { Setting }\end{array}$ & $\begin{array}{c}\text { Inclusions } \\
\text { Key Exclusions }\end{array}$ & $\begin{array}{l}\text { Intervention } \\
\text { Description }\end{array}$ & $\begin{array}{l}\text { Comparison } \\
\text { Description }\end{array}$ & $\begin{array}{c}\text { Follow-up } \\
\text { Duration }\end{array}$ & $\begin{array}{c}\text { Outcomes } \\
\text { Reported } \\
\text { Withdrawal (\%) at } \\
\text { endpoint }\end{array}$ \\
\hline $\begin{array}{l}\text { Jones, } 2015^{1} \\
\text { RCT } \\
\text { United Kingdom } \\
\text { Government } \\
\text { Moderate } \\
25213157\end{array}$ & $\begin{array}{l}\mathrm{N}=67 \\
39(18-65) \\
\text { Female } 70 \% \\
\text { White } 96 \% \\
\text { BP II } 21 \% \\
\text { Outpatient }\end{array}$ & $\begin{array}{l}\text { Euthymic/Maintenance; } \\
\text { DSM-IV diagnosis of } \\
\text { primary BP I or II (DSM-IV) } \\
\text { with onset in past } 5 \text { years } \\
\text { Schizoaffective }\end{array}$ & $\begin{array}{l}\text { Individual CBT } \\
\text { focused on recovery } \\
\text { approach, mood } \\
\text { functioning, } \\
\text { understanding of } \\
\text { diagnosis, recovery- } \\
\text { informed goals, } \\
\text { relationships } \\
\text { between mood and } \\
\text { progress towards } \\
\text { recovery goals, CBT } \\
\text { techniques to cope, } \\
\text { functioning issues in } \\
\text { relation to recovery, } \\
\text { development of } \\
\text { recovery plan, and } \\
\text { sharing lessons from } \\
\text { therapy with } \\
\text { stakeholders } \\
\text {-Total of } 18 \text { hours } \\
\text { over } 6 \text { months; } \\
\text { weekly or biweekly } \\
45-60 \text { minute } \\
\text { sessions }\end{array}$ & $\begin{array}{l}\text { Treatment as } \\
\text { usual: Routine } \\
\text { medication } \\
\text { (mood } \\
\text { stabilizers, } \\
\text { antipsychotics, } \\
\text { and } \\
\text { antidepressants) } \\
\text { and medical } \\
\text { care from } \\
\text { clinician and } \\
\text { community } \\
\text { mental health } \\
\text { team. }\end{array}$ & $\begin{array}{l}12 \text { months } \\
15 \text { months } \\
\text { (Relapse } \\
\text { Only) }\end{array}$ & $\begin{array}{l}\text { PSP } \\
\text { QoL.BD } \\
\text { BDI-II } \\
\text { Relapse (SCID-LIFE, } \\
\text { HRSD, MAS) } \\
\text { Time to Recurrence } \\
12 \text { months: } 33 \% \\
15 \text { months: } 54 \%\end{array}$ \\
\hline
\end{tabular}




\begin{tabular}{|c|c|c|c|c|c|c|}
\hline $\begin{array}{l}\text { Study, Year } \\
\text { Design } \\
\text { Location } \\
\text { Funder } \\
\text { Risk of Bias } \\
\text { PMID }\end{array}$ & $\begin{array}{c}\text { Randomized (N) } \\
\text { Age (mean) } \\
\text { Sex (\% Female) } \\
\text { Race (\% White) } \\
\text { Diagnosis } \\
\text { (\% BP I, II, NOS) } \\
\text { Setting }\end{array}$ & $\begin{array}{c}\text { Inclusions } \\
\text { Key Exclusions }\end{array}$ & $\begin{array}{l}\text { Intervention } \\
\text { Description }\end{array}$ & $\begin{array}{l}\text { Comparison } \\
\text { Description }\end{array}$ & $\begin{array}{c}\text { Follow-up } \\
\text { Duration }\end{array}$ & $\begin{array}{c}\text { Outcomes } \\
\text { Reported } \\
\begin{array}{c}\text { Withdrawal (\%) at } \\
\text { endpoint }\end{array}\end{array}$ \\
\hline $\begin{array}{l}\text { Perich, } 2013^{2} \\
\text { RCT } \\
\text { Australia } \\
\text { Government and } \\
\text { Non-Government } \\
\text { Moderate } \\
23216045\end{array}$ & $\begin{array}{l}\mathrm{N}=95 \\
\text { Age NR }(18+) \\
\text { Female } 65 \% \\
\text { White NR } \\
\text { BP I } 62 \% \\
\text { BP I 37\% } \\
\text { BP NOS 1\% } \\
\text { Outpatient }\end{array}$ & $\begin{array}{l}\text { Euthymic/Maintenance; BP } \\
\text { I or II (DSM-IV), on a mood } \\
\text { stabilizing medication for } \\
\text { the duration of study } \\
\text { treatment with at least one } \\
\text { bipolar disorder episode } \\
\text { (hypo/mania, depression, } \\
\text { mixed episode) over the } \\
\text { previous } 18 \text { months; and a } \\
\text { lifetime incidence of at least } \\
\text { three bipolar episodes } \\
\\
\text { Schizoaffective; Substance } \\
\text { Abuse; Other Mental } \\
\text { Health; Neurological } \\
\text { Disorders; Labs/Other } \\
\text { Conditions }\end{array}$ & $\begin{array}{l}\text { Group mindfulness- } \\
\text { based CBT } \\
\text { consisting of } \\
\text { mindfulness } \\
\text { meditation practice } \\
\text { and cognitive } \\
\text { therapy regarding } \\
\text { depression including } \\
\text { psycho- education } \\
\text { (education about } \\
\text { bipolar disorder, } \\
\text { depression, } \\
\text { hypo/mania, and } \\
\text { anxiety). } \\
\text {-8 weekly sessions, } \\
\text { each } 2 \text { to } 2.5 \text { hours }\end{array}$ & \begin{tabular}{|l|} 
Treatment as \\
usual: Weekly \\
handouts with \\
information \\
about bipolar \\
disorder via \\
email or mail. \\
Topics included \\
causes of \\
bipolar disorder, \\
available \\
treatments, and \\
common \\
symptoms.
\end{tabular} & 12 months & $\begin{array}{l}\text { MADRS } \\
\text { YMRS } \\
\text { Relapse (DSM-IV } \\
\text { major depressive, } \\
\text { hypomanic or manic } \\
\text { episode) } \\
38 \%\end{array}$ \\
\hline $\begin{array}{l}\text { Fava, } 2011^{3} \\
\text { RCT } \\
\text { Italy } \\
\text { Government and Non- } \\
\text { Government } \\
\text { Low } \\
21372621\end{array}$ & $\begin{array}{l}\mathrm{N}=62 \\
\text { Age } 40(18-65) \\
\text { Female 55\% } \\
\text { White NR } \\
\text { Cyclothymic 100\% } \\
\text { Outpatient }\end{array}$ & $\begin{array}{l}\text { No history of mania or } \\
\text { major depressive disorder; } \\
\text { Current diagnosis of } \\
\text { cyclothymic disorder } \\
\text { according to DSM-IV } \\
\\
\text { First Manic Episode; } \\
\text { Schizoaffective; Substance } \\
\text { Abuse; Other Mental } \\
\text { Health; Neurological } \\
\text { Disorders; Taking Other } \\
\text { Medications; } \\
\text { Pregnant/Nursing; } \\
\text { Labs/Other Conditions }\end{array}$ & $\begin{array}{l}\text { CBT and well-being } \\
\text { therapy focused on } \\
\text { patient's } \\
\text { symptomatology, } \\
\text { monitoring of } \\
\text { distress, strategies } \\
\text { for symptom } \\
\text { management, } \\
\text { psychotherapeutic } \\
\text { strategy for } \\
\text { enhancing well- } \\
\text { being } \\
\text {-10 sessions every } \\
\text { other week for 45- } \\
\text { minutes. }\end{array}$ & $\begin{array}{l}\text { Clinical } \\
\text { Management: } \\
\text { Reviewed the } \\
\text { patient's clinical } \\
\text { status and } \\
\text { provided the } \\
\text { patient with } \\
\text { support and } \\
\text { advice } \\
\text { according to } \\
\text { protocol } \\
\text {-10 sessions } \\
\text { every other } \\
\text { week for 45- } \\
\text { minutes. }\end{array}$ & 24 months & $\begin{array}{l}\text { CID } \\
\text { MAS } \\
18 \%\end{array}$ \\
\hline
\end{tabular}




\begin{tabular}{|c|c|c|c|c|c|c|}
\hline $\begin{array}{l}\text { Study, Year } \\
\text { Design } \\
\text { Location } \\
\text { Funder } \\
\text { Risk of Bias } \\
\text { PMID }\end{array}$ & $\begin{array}{c}\text { Randomized (N) } \\
\text { Age (mean) } \\
\text { Sex (\% Female) } \\
\text { Race (\% White) } \\
\text { Diagnosis } \\
\text { (\% BP I, II, NOS) } \\
\text { Setting }\end{array}$ & $\begin{array}{l}\text { Inclusions } \\
\text { Key Exclusions }\end{array}$ & $\begin{array}{l}\text { Intervention } \\
\text { Description }\end{array}$ & $\begin{array}{l}\text { Comparison } \\
\text { Description }\end{array}$ & $\begin{array}{c}\text { Follow-up } \\
\text { Duration }\end{array}$ & $\begin{array}{c}\text { Outcomes } \\
\text { Reported } \\
\text { Withdrawal (\%) at } \\
\text { endpoint }\end{array}$ \\
\hline $\begin{array}{l}\text { Gomes, } 2011^{4} \\
\text { RCT } \\
\text { Brazil } \\
\text { Government and Non- } \\
\text { Government } \\
\text { High } \\
21372622\end{array}$ & $\begin{array}{l}\mathrm{N}=50 \\
\text { Age } 39(18-60) \\
\text { Female } 76 \% \\
\text { White NR } \\
\text { BP I } 76 \% \\
\text { BP II } 24 \% \\
\\
\text { Outpatient }\end{array}$ & $\begin{array}{l}\text { Euthymic/Maintenance; BP } \\
\text { I or II (DSM-IV) with more } \\
\text { than } 5 \text { years of schooling, } \\
\text { and use of at least one } \\
\text { mood stabilizer or atypical } \\
\text { antipsychotic } \\
\text { YMRS <6 } \\
\text { HDRS <8 } \\
\text { Substance Abuse; } \\
\text { Neurological Disorders }\end{array}$ & $\begin{array}{l}\text { Group CBT focused } \\
\text { on information about } \\
\text { BP and stabilized } \\
\text { routine and } \\
\text { pharmacological } \\
\text { issues; use of mood } \\
\text { graphs and stress } \\
\text { vulnerability model, } \\
\text { cognitive and } \\
\text { behavioral strategies } \\
\text { to manage } \\
\text { depressive and } \\
\text { manic episodes; } \\
\text { specific problems in } \\
\text { BP; techniques to } \\
\text { improve relapse } \\
\text { prevention } \\
\text {-18 structured } \\
\text { sessions, } 90 \\
\text { minutes each }\end{array}$ & $\begin{array}{l}\text { Treatment as } \\
\text { usual: } \\
\text { Pharmacological } \\
\text { treatment }\end{array}$ & 24 months & $\begin{array}{l}\text { Relapse (Not Defined) } \\
\text { Time to relapse } \\
6 \%\end{array}$ \\
\hline
\end{tabular}




\begin{tabular}{|c|c|c|c|c|c|c|}
\hline $\begin{array}{l}\text { Study, Year } \\
\text { Design } \\
\text { Location } \\
\text { Funder } \\
\text { Risk of Bias } \\
\text { PMID }\end{array}$ & $\begin{array}{c}\text { Randomized (N) } \\
\text { Age (mean) } \\
\text { Sex (\% Female) } \\
\text { Race (\% White) } \\
\text { Diagnosis } \\
\text { (\% BP I, II, NOS) } \\
\text { Setting }\end{array}$ & $\begin{array}{c}\text { Inclusions } \\
\text { Key Exclusions }\end{array}$ & $\begin{array}{l}\text { Intervention } \\
\text { Description }\end{array}$ & $\begin{array}{l}\text { Comparison } \\
\text { Description }\end{array}$ & $\begin{array}{l}\text { Follow-up } \\
\text { Duration }\end{array}$ & $\begin{array}{c}\text { Outcomes } \\
\text { Reported } \\
\text { Withdrawal (\%) at } \\
\text { endpoint }\end{array}$ \\
\hline $\begin{array}{l}\text { Castle, } 2010^{5} \\
\text { RCT } \\
\text { Australia } \\
\text { Government and Non- } \\
\text { government } \\
\text { Low } \\
20435965\end{array}$ & \begin{tabular}{|l} 
N = 84 \\
Age $42(18-65)$ \\
Female $76 \%$ \\
White NR \\
BP I 74\% \\
BP II 25\% \\
BP NOS 1\% \\
Outpatient
\end{tabular} & $\begin{array}{l}\text { Euthymic/Maintenance; BP } \\
\text { I, BP II, or BP NOS (DSM- } \\
\text { IV); not in an acute episode } \\
\text { as defined by DSM-IV } \\
\text { criteria } \\
\text { Schizoaffective; } \\
\text { Neurological Disorders; } \\
\text { Labs/Other Conditions }\end{array}$ & $\begin{array}{l}\text { Group CBT focused } \\
\text { on monitoring mood } \\
\text { and activities, } \\
\text { assessing } \\
\text { prodromes, } \\
\text { preventing relapse, } \\
\text { and setting specific, } \\
\text { measurable, } \\
\text { achievable, realistic, } \\
\text { time-framed goals } \\
\text {-12 weekly group } \\
\text { sessions (90 } \\
\text { minutes) and } 3 \\
\text { monthly booster } \\
\text { sessions with } \\
\text { weekly telephone } \\
\text { calls }\end{array}$ & $\begin{array}{l}\text { Treatment as } \\
\text { usual (not } \\
\text { defined) and } \\
\text { weekly } \\
\text { telephone call }\end{array}$ & 12 months & $\begin{array}{l}\text { Relapse (DSM-IV-TR } \\
\text { criteria for any mood } \\
\text { episode) } \\
\text { MADRS } \\
\text { YMRS } \\
14 \%\end{array}$ \\
\hline
\end{tabular}




\begin{tabular}{|c|c|c|c|c|c|c|}
\hline $\begin{array}{l}\text { Study, Year } \\
\text { Design } \\
\text { Location } \\
\text { Funder } \\
\text { Risk of Bias } \\
\text { PMID }\end{array}$ & $\begin{array}{c}\text { Randomized (N) } \\
\text { Age (mean) } \\
\text { Sex (\% Female) } \\
\text { Race (\% White) } \\
\text { Diagnosis } \\
\text { (\% BP I, II, NOS) } \\
\text { Setting }\end{array}$ & $\begin{array}{c}\text { Inclusions } \\
\text { Key Exclusions }\end{array}$ & $\begin{array}{l}\text { Intervention } \\
\text { Description }\end{array}$ & $\begin{array}{l}\text { Comparison } \\
\text { Description }\end{array}$ & $\begin{array}{c}\text { Follow-up } \\
\text { Duration }\end{array}$ & $\begin{array}{c}\text { Outcomes } \\
\text { Reported } \\
\begin{array}{c}\text { Withdrawal (\%) at } \\
\text { endpoint }\end{array}\end{array}$ \\
\hline $\begin{array}{l}\text { Ball, } 2006^{6} \\
\text { RCT } \\
\text { Australia } \\
\text { Non-government and } \\
\text { industry } \\
\text { High } \\
16566624\end{array}$ & $\begin{array}{l}\mathrm{N}=52 \\
\text { Age } 42(23-77) \\
\text { Female 58\% } \\
\text { White NR } \\
\text { BP NR } \\
\text { Outpatient }\end{array}$ & $\begin{array}{l}\text { Without current episode of } \\
\text { severe depression or } \\
\text { mania; BP I or BP II (DSM- } \\
\text { IV) with at least } 1 \text { episode } \\
\text { of hypomania, mania, or } \\
\text { depression over prior } 18 \\
\text { months; able to maintain } \\
\text { usual mood stabilizing } \\
\text { medications for duration of } \\
\text { treatment. } \\
\text { BDI<30 } \\
\text { HAM-D-17 <15 } \\
\text { YMRS<20 } \\
\text { Schizoaffective; Other } \\
\text { Mental Health; Neurological } \\
\text { Disorders; Labs/Other } \\
\text { Conditions }\end{array}$ & \begin{tabular}{|l} 
CBT focused on \\
assessment, \\
psychoeducation, \\
identifying early \\
warning signs, \\
establishing stable \\
routines, identifying \\
and modifying \\
cognitions, \\
identifying and \\
modifying schemas \\
-20 weekly sessions, \\
60 minutes each
\end{tabular} & $\begin{array}{l}\text { Treatment as } \\
\text { usual: Regular } \\
\text { sessions as } \\
\text { prescribed by } \\
\text { patient's } \\
\text { medical } \\
\text { practitioner }\end{array}$ & 12 months & $\begin{array}{l}\text { Relapse (DSM-IV } \\
\text { hypo/manic, } \\
\text { depressive, or mixed } \\
\text { episodes at least } 2 \\
\text { months after } \\
\text { symptomatic remission) } \\
\text { MADRS } \\
\text { YMRS } \\
\text { GAF } \\
\text { SAS } \\
\text { ATQ-N } \\
\text { WHO-DAS } \\
37 \%\end{array}$ \\
\hline
\end{tabular}




\begin{tabular}{|c|c|c|c|c|c|c|}
\hline $\begin{array}{l}\text { Study, Year } \\
\text { Design } \\
\text { Location } \\
\text { Funder } \\
\text { Risk of Bias } \\
\text { PMID }\end{array}$ & $\begin{array}{c}\text { Randomized (N) } \\
\text { Age (mean) } \\
\text { Sex (\% Female) } \\
\text { Race (\% White) } \\
\text { Diagnosis } \\
\text { (\% BP I, II, NOS) } \\
\text { Setting }\end{array}$ & $\begin{array}{c}\text { Inclusions } \\
\text { Key Exclusions }\end{array}$ & $\begin{array}{l}\text { Intervention } \\
\text { Description }\end{array}$ & $\begin{array}{l}\text { Comparison } \\
\text { Description }\end{array}$ & $\begin{array}{c}\text { Follow-up } \\
\text { Duration }\end{array}$ & $\begin{array}{c}\text { Outcomes } \\
\text { Reported } \\
\text { Withdrawal (\%) at } \\
\text { endpoint }\end{array}$ \\
\hline $\begin{array}{l}\text { Scott, } 2006^{7} \\
\text { RCT } \\
\text { United Kingdom } \\
\text { Government } \\
\text { Low/High } \\
16582056\end{array}$ & $\begin{array}{l}\mathrm{N}=253 \\
\text { Age } 41(18-65) \\
\text { Female 65\% } \\
\text { White NR } \\
\text { BP I 94\% } \\
\text { BP II 6\% } \\
\text { Outpatient }\end{array}$ & $\begin{array}{l}\text { Depressed, Hypo/manic, or } \\
\text { Euthymic; BP I or BP II } \\
\text { (DSM-IV) with history of } \\
\text { two or more episodes of } \\
\text { illness meeting DSM-IV } \\
\text { criteria for mania, } \\
\text { hypomania, major } \\
\text { depressive disorder or } \\
\text { mixed affective disorder, } \\
\text { one of which must have } \\
\text { been within } 12 \text { months of } \\
\text { recruitment; and contact } \\
\text { with mental health services } \\
\text { within the past } 6 \text { months. } \\
\text { First Manic Episode; } \\
\text { Substance Abuse; Other } \\
\text { Mental Health; Neurological } \\
\text { Disorders }\end{array}$ & $\begin{array}{l}\text { CBT focused on } \\
\text { facilitating } \\
\text { acceptance of the } \\
\text { need for treatment, } \\
\text { reducing variability } \\
\text { in mood, managing } \\
\text { stressors, strategies } \\
\text { to cope with } \\
\text { depression, } \\
\text { identifying and } \\
\text { modifying } \\
\text { dysfunctional } \\
\text { automatic thoughts } \\
\text { and beliefs, improve } \\
\text { medication } \\
\text { adherence, tackling } \\
\text { substance misuse, } \\
\text { teaching early } \\
\text { recognition of } \\
\text { symptoms of } \\
\text { recurrence and } \\
\text { coping techniques } \\
\text { for symptoms } \\
\text {-Weekly sessions for } \\
15 \text { weeks with } \\
\text { reduction in } \\
\text { frequency from } \\
\text { week } 16-26 \text {. Two } \\
\text { booster sessions } \\
\text { week at } 32 \text { and } 38 .\end{array}$ & $\begin{array}{l}\text { Treatment as } \\
\text { usual: } \\
\text { Medication and } \\
\text { contact with key } \\
\text { mental health } \\
\text { professionals } \\
\text { when } \\
\text { appropriate. }\end{array}$ & 18 months & $\begin{array}{l}\text { Relapse } \\
\text { LIFE-II, Depression } \\
\text { LIFE-II, Mania } \\
26 \%\end{array}$ \\
\hline
\end{tabular}




\begin{tabular}{|c|c|c|c|c|c|c|}
\hline $\begin{array}{l}\text { Study, Year } \\
\text { Design } \\
\text { Location } \\
\text { Funder } \\
\text { Risk of Bias } \\
\text { PMID }\end{array}$ & $\begin{array}{c}\text { Randomized (N) } \\
\text { Age (mean) } \\
\text { Sex (\% Female) } \\
\text { Race (\% White) } \\
\text { Diagnosis } \\
\text { (\% BP I, II, NOS) } \\
\text { Setting }\end{array}$ & $\begin{array}{c}\text { Inclusions } \\
\text { Key Exclusions }\end{array}$ & $\begin{array}{l}\text { Intervention } \\
\text { Description }\end{array}$ & $\begin{array}{l}\text { Comparison } \\
\text { Description }\end{array}$ & $\begin{array}{l}\text { Follow-up } \\
\text { Duration }\end{array}$ & $\begin{array}{c}\text { Outcomes } \\
\text { Reported } \\
\begin{array}{c}\text { Withdrawal (\%) at } \\
\text { endpoint }\end{array}\end{array}$ \\
\hline $\begin{array}{l}\text { Lam, 2003, 20058, } \\
\text { RCT } \\
\text { England } \\
\text { NR } \\
\text { Low } \\
\text { Moderate } \\
12578431 \\
15677598\end{array}$ & $\begin{array}{l}\mathrm{N}=103 \\
\text { Age } 44(22-70) \\
\text { Female 56\% } \\
\text { White NR } \\
\text { BP I 100\% } \\
\text { Outpatient }\end{array}$ & \begin{tabular}{|l|} 
Euthymic/Maintenance; BP \\
I (DSM-IV) with prescribed \\
prophylactic medication at \\
an adequate dose \\
according to the British \\
National Formulary, with at \\
least 2 episodes in the last \\
2 years or 3 episodes in the \\
last 5 years, but currently \\
not fulfilling criteria for a \\
bipolar episode; \\
BDI $<30$ \\
BRMS $<9$ \\
First Manic Episode; \\
Schizoaffective; Substance \\
Abuse; Other Mental Health
\end{tabular} & $\begin{array}{l}\text { CBT focused on } \\
\text { traditional cognitive } \\
\text { therapy for } \\
\text { depression, } \\
\text { diathesis-stress } \\
\text { model and need for } \\
\text { pharmaceutical and } \\
\text { psychological } \\
\text { therapy, mood } \\
\text { monitoring and } \\
\text { prodromes, sleep } \\
\text { importance, and } \\
\text { targeting extreme } \\
\text { striving attitudes and } \\
\text { behavior } \\
-12 \text { to } 18 \text { individual } \\
60-\text { minute sessions } \\
\text { in the first } 6 \text { months } \\
\text { and } 2 \text { booster } \\
\text { sessions in the } \\
\text { second } 6 \text { months. }\end{array}$ & $\begin{array}{l}\text { Minimal } \\
\text { psychiatric care: } \\
\text { Mood stabilizers } \\
\text { (at appropriate } \\
\text { level) and } \\
\text { regular } \\
\text { outpatient } \\
\text { psychiatric } \\
\text { follow up }\end{array}$ & $\begin{array}{l}12 \text { months } \\
2.5 \text { years }\end{array}$ & $\begin{array}{l}\text { Relapse (DSM-IV } \\
\text { criteria for any bipolar } \\
\text { episode) } \\
\text { HDRS } \\
\text { BRMS } \\
\text { SPS } \\
\text { Hospitalizations } \\
16 \%\end{array}$ \\
\hline
\end{tabular}

Abbrevaitions: BDI=Beck Depression inventory; BP=bipolar disorder; BRMS=Bech-Rafaelsen Mania Scale; CBT=Cognitive Behavioral Therapy; CID=Clinical Interview for Depression; DSM=Diagnostic and Statistical Manual of Mental Disorders; GAF=General Assessment of Functioning Scale; HAM-D=Hamilton Scale for Depression;

HDRS=Hamilton Depression Rating Scale; LIFE=Longitudinal Interval Follow-up Evaluation; MADRS=Montgomery-Asberg Depression Rating Scale; MAS=Bech-Rafaelsen Mania Rating Scale; NOS=not otherwise specified; NR=not reported; PMID=PubMed Identification Number; PSP=Personal and Social Functioning Scale; QoL.BD=Quality of Life; RCT=randomized controlled trial; SCID=Sturctured Clinical Intervies for DSM Disorders; SPS=Social Phobia Scale; YMRS=Young Mania Rating Scale 


\section{Appendix Table K2. Summary risk of bias assessments: CBT vs. inactive comparators}

\begin{tabular}{|c|c|c|}
\hline $\begin{array}{l}\text { Study } \\
\text { Funding Source } \\
\text { PMID }\end{array}$ & $\begin{array}{c}\text { Overall Risk of Bias } \\
\text { Assessment }\end{array}$ & Rationale \\
\hline $\begin{array}{l}\text { Jones } 2015^{1} \\
\text { Government } \\
25213157\end{array}$ & Moderate & Potential bias due to rate of attrition at 12 months ( $33 \%)$ and differential rate of attrition between study arms. \\
\hline $\begin{array}{l}\text { Perich } 2013^{2} \\
\text { Government and } \\
\text { Non-government } \\
23216045\end{array}$ & Moderate & $\begin{array}{l}\text { Potential reporting bias due to unclear reporting of sample sizes by arm. Almost } 40 \% \text { lost to follow up at } \\
\text { outcome time points. }\end{array}$ \\
\hline $\begin{array}{l}\text { Fava } 2011^{3} \\
\text { Government and } \\
\text { Non-government } \\
21372621\end{array}$ & Low & No significant suspected biases. \\
\hline $\begin{array}{l}\text { Gomes } 2011^{4} \\
\text { Government and } \\
\text { Non-government } \\
21372622\end{array}$ & High & $\begin{array}{l}\text { Suspected bias due to attrition post-randomization in treatment arm with high differential attrition between } \\
\text { groups. }\end{array}$ \\
\hline $\begin{array}{l}\text { Castle } 2010^{5} \\
\text { Government and } \\
\text { Non-government } \\
20435965\end{array}$ & Low & No significant suspected biases. \\
\hline $\begin{array}{l}\text { Ball } 2006^{6} \\
\text { Non-government and } \\
\text { Industry } \\
16566624\end{array}$ & High & $\begin{array}{l}\text { Suspected bias due to unclear reporting of reasons for withdrawal by treatment arm. High differential attrition } \\
\text { between groups. }\end{array}$ \\
\hline $\begin{array}{l}\text { Scott } 2006^{7} \\
\text { Government } \\
16582056 \\
\end{array}$ & $\begin{array}{l}\text { Low/High (Post-hoc } \\
\text { analysis) }\end{array}$ & $\begin{array}{l}\text { No significant suspected biases related to pre-specified outcomes; however, there is a risk of bias due to post- } \\
\text { hoc analysis results. }\end{array}$ \\
\hline $\begin{array}{l}\text { Lam } 2003^{8} \\
\text { NR } \\
12578431 \\
\end{array}$ & Low/High & $\begin{array}{l}\text { No significant suspected biases for relapse outcomes; but there is a risk of bias due to unclear reporting of } \\
\text { symptom scores and time points. }\end{array}$ \\
\hline $\begin{array}{l}\text { Lam } 2005^{9} \\
\text { NR } \\
15677598 \\
\end{array}$ & Moderate & Suspected bias due to unclear reporting of attrition and sample size by arm. \\
\hline
\end{tabular}

Abbreviations: NR=not reported; PMID=PubMed Identification Number 
Appendix Table K3. Outcomes summary: CBT vs. inactive comparators

\begin{tabular}{|c|c|c|c|c|c|}
\hline $\begin{array}{c}\text { Study } \\
\text { ROB } \\
\text { PMID }\end{array}$ & Responder/Remitter & Symptom & Function & Other & $\mathrm{AE}$ \\
\hline $\begin{array}{l}\text { Jones, } 2015^{1} \\
\text { Moderate } \\
25213158\end{array}$ & $\begin{array}{l}\frac{\text { Relapse }^{*}}{\text { NS }} \\
15 \text { months, Any type } \\
\text { OR=0.32 (95\% Cl 0.09, } \\
1.06) ; p=0.06 \\
\text { Time to First Recurrence } \\
\text { Favors CBT } \\
\text { HR=0.38 (95\% Cl } 0.18, \\
0.78)\end{array}$ & $\begin{array}{l}\frac{\text { Depression* }}{\text { NS }} \\
6 \text { months, BDI } \\
\text { ES }=0.00(95 \% \mathrm{Cl}-0.58,0.58) \\
12 \text { months, BDI } \\
\text { ES }=0.02(95 \% \mathrm{Cl}-0.63,0.68)\end{array}$ & $\begin{array}{l}\text { Quality of Life* } \\
\text { NS } \\
6 \text { months, QoL.BD } \\
E S=-0.36(95 \% \mathrm{Cl} \text { - } \\
0.93,0.22) \\
12 \text { months, QoL.BD } \\
E S=-0.35(95 \% \mathrm{Cl}- \\
1.01,0.31 \\
\text { Social Function* } \\
\text { NS } \\
6 \text { months, PSP } \\
\text { ES }=-0.38(95 \% \mathrm{Cl}- \\
1.00,0.25) \\
12 \text { months, PSP } \\
\text { ES }=-0.35(95 \% \mathrm{Cl}- \\
0.75,0.60)\end{array}$ & NR & NR \\
\hline $\begin{array}{l}\text { Perich, } 2013^{2} \\
\text { Moderate } \\
23216045\end{array}$ & $\begin{array}{l}\frac{\text { Relapse }^{*}}{\text { NS }} \\
12 \text { months, Depression } \\
\text { OR= } 0.67(95 \% \mathrm{Cl} 0.19 \text {, } \\
2.24) ; p=0.59 \\
12 \text { months, Hypo/manic } \\
\text { OR= } 1.90(95 \% \mathrm{Cl} 0.59 \\
6.20) ; p=0.29\end{array}$ & $\begin{array}{l}\frac{\text { Depression* }}{\text { NS }} \\
6 \text { months, MADRS } \\
\text { ES }=0.05(95 \% \mathrm{Cl}-0.35,0.46) \\
12 \text { months, MADRS } \\
\text { ES }=0.23(95 \% \mathrm{Cl}-0.18,0.63) \\
\frac{\text { Mania* }}{\mathrm{NS}} \\
6 \text { months, YMRS } \\
\text { ES }=-0.27(95 \% \mathrm{Cl}-0.67,0.13) \\
12 \text { months, YMRS } \\
\text { ES }=0.06(95 \% \mathrm{Cl}-0.34,0.46)\end{array}$ & NR & NR & NR \\
\hline
\end{tabular}




\begin{tabular}{|c|c|c|c|c|c|}
\hline $\begin{array}{l}\text { Study } \\
\text { ROB } \\
\text { PMID }\end{array}$ & Responder/Remitter & Symptom & Function & Other & $\mathrm{AE}$ \\
\hline $\begin{array}{l}\text { Fava, } 2011^{3} \\
\text { Low } \\
21372621\end{array}$ & NR & $\begin{array}{l}\text { Depression* } \\
\text { Favors CBT } \\
6 \text { months, CID } \\
\text { ES }=-0.67(95 \% \mathrm{Cl}-1.18,-0.15) \\
12 \text { months, CID } \\
\text { ES }=-0.57(95 \% \mathrm{Cl}-1.08,-0.06) \\
\frac{\text { Mania }^{*}}{\text { Favors CBT }} \\
6 \text { months, BRMS } \\
\text { ES }=-0.74(95 \% \mathrm{Cl}-1.25,-0.22) \\
12 \text { months, BRMS } \\
\text { ES }=-0.94(95 \% \mathrm{Cl}-1.46,-0.41)\end{array}$ & NR & NR & NR \\
\hline $\begin{array}{l}\text { Gomes, 20114 } \\
\text { High } \\
21372622\end{array}$ & $\begin{array}{l}\frac{\text { Relapse }^{*}}{\text { NS }} \\
24 \text { months, Any type } \\
\text { OR=0.37 (95\% Cl 0.37, } \\
\text { 5.25); } p=0.77 \\
\text { Time to First Recurrence } \\
\text { Favors CBT } \\
\text { Median: } 31 \text { weeks CBT } \\
\text { vs. } 11.5 \text { weeks Usual } \\
\text { care; } p=0.01\end{array}$ & 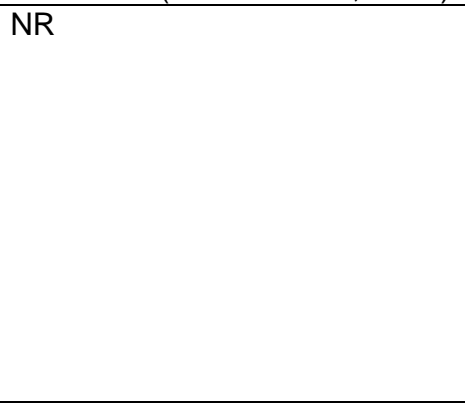 & NR & NR & NR \\
\hline $\begin{array}{l}\text { Castle, } 2010^{5} \\
\text { Low } \\
20435965\end{array}$ & $\begin{array}{l}\text { Relapse }^{*} \\
\text { Favors CBT } \\
12 \text { months, Any type } \\
\text { OR=0.32 (95\% Cl } 0.10, \\
0.95) ; p=0.03\end{array}$ & $\begin{array}{l}\text { Depression } \\
\text { NS } \\
12 \text { months, MADRS } \\
\text { ES }=0.41(95 \% \mathrm{Cl}-0.06,0.87) \\
\frac{\text { Mania }}{\text { NS }} \\
12 \text { months, YMRS } \\
\text { ES }=0.33(95 \% \mathrm{Cl}-0.14,0.80)\end{array}$ & NR & NR & NR \\
\hline
\end{tabular}




\begin{tabular}{|c|c|c|c|c|c|}
\hline $\begin{array}{l}\text { Study } \\
\text { ROB } \\
\text { PMID }\end{array}$ & Responder/Remitter & Symptom & Function & Other & $\mathrm{AE}$ \\
\hline $\begin{array}{l}\text { Ball, } 2006^{6} \\
\text { High } \\
16566624\end{array}$ & $\begin{array}{l}\frac{\text { Relapse }^{*}}{N S} \\
6 \text { months, Any type } \\
\text { OR }=0.50(95 \% \mathrm{Cl} 0.11, \\
2.07) ; p=0.36 \\
18 \text { months, Any type } \\
\text { OR=0.74 (95\% Cl } 0.22 \text {, } \\
2.56) ; p=0.78\end{array}$ & $\begin{array}{l}\frac{\text { Depression* }}{\text { Favors CBT at } 6 \text { months }} \\
6 \text { months, MADRS } \\
\text { ES }=-0.57(95 \% \mathrm{Cl}-1.12,--0.01) \\
18 \text { months, MADRS } \\
\text { ES }=-0.08(95 \%-0.62,0.47) \\
\frac{\text { Mania* }^{*}}{\text { NS }} \\
6 \text { months, YMRS } \\
\text { ES }=-0.02(95 \% \mathrm{Cl}-0.56,0.53) \\
18 \text { months, YMRS } \\
\text { ES }=-0.13(95 \%-0.67,0.42)\end{array}$ & $\begin{array}{l}\frac{\text { Function* }}{N S} \\
6 \text { months, GAF } \\
\text { ES=0.43 (95\% Cl - } \\
0.12,0.98) \\
18 \text { months, GAF } \\
\text { ES=0.24 (95\% Cl - } \\
0.30,0.79) \\
\text { Social Function* } \\
\text { NS } \\
6 \text { months, SAS } \\
\text { ES=-0.48 (95\% Cl - } \\
1.03,0.08) \\
18 \text { months, SAS } \\
\text { ES=-0.17 (95\% -0.71, } \\
0.38) \\
\text { Cognitive Function } \\
\text { NS } \\
6 \text { months, ATQ-N } \\
\text { ES=-0.37 (95\% Cl - } \\
0.91,0.18) \\
18 \text { months, ATQ-N } \\
\text { ES=0.22 (95\% Cl - } \\
0.32,0.77) \\
\text { Health and Disability* } \\
\text { Favors Intervention } \\
6 \text { months, WHO-DAS } \\
\text { ES=-0.58 (95\% Cl - } \\
1.13,-0.02) \\
\text { NS } \\
18 \text { months, WHO-DAS } \\
\text { ES=-0.40 (95\% Cl - } \\
0.95,0.15)\end{array}$ & NR & NR \\
\hline
\end{tabular}




\begin{tabular}{|c|c|c|c|c|c|}
\hline $\begin{array}{l}\text { Study } \\
\text { ROB } \\
\text { PMID }\end{array}$ & Responder/Remitter & Symptom & Function & Other & $\mathrm{AE}$ \\
\hline $\begin{array}{l}\text { Scott, } 2006^{7} \\
16582056\end{array}$ & $\begin{array}{l}\frac{\text { Relapse }^{\star}}{\text { NS }} \\
9 \text { months, Any type } \\
\text { OR }=0.99(95 \% \mathrm{Cl} 0.56, \\
1.75) ; p=0.97 \\
12 \text { months, Any type } \\
\text { OR=0.84 (95\% Cl 0.50, } \\
1.42) ; p=0.53\end{array}$ & $\begin{array}{l}\frac{\text { Depression* }}{18 \text { months, LIFE-II Depression }} \\
\text { NS } \\
\frac{\text { Mania }^{*}}{\text { NS }} \\
18 \text { months, LIFE-II Mania }\end{array}$ & NR & NR & NR \\
\hline $\begin{array}{l}\text { Lam, } 2003^{8} \\
12578431\end{array}$ & 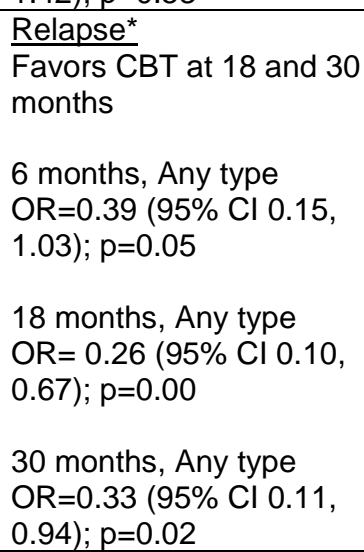 & $\begin{array}{l}\frac{\text { Depression }}{\text { NS }} \\
6 \text { months, HDRS } \\
\text { ES }=-0.17(95 \% \mathrm{Cl}-0.56,0.22) \\
12 \text { months, HDRS } \\
\text { ES }=-0.13(95 \% \mathrm{Cl}-0.52,0.26) \\
\frac{\text { Mania* }}{N S} \\
6 \text { months, BRMS } \\
\text { ES }=0.00(95 \% \mathrm{Cl}-0.39,0.39) \\
12 \text { months, BRMS } \\
\text { ES }=-0.32(95 \% \mathrm{Cl}-0.71,0.07)\end{array}$ & $\begin{array}{l}\text { Social Function } \\
\text { Favors CBT at } 6 \\
\text { months } \\
6 \text { months, SPS } \\
\text { ES }=-0.60(95 \% \mathrm{Cl}- \\
0.99,-0.20) \\
12 \text { months, SPS } \\
\text { ES }=0.00(-0.39,0.39)\end{array}$ & $\begin{array}{l}\text { Admissions for BP } \\
12 \text { months } \\
\text { Favors CBT } \\
\text { OR }=0.20(95 \% \mathrm{Cl} \\
0.06,0.61)\end{array}$ & NR \\
\hline
\end{tabular}

*=Self-calculated estimate based on reported data

Abbreviations: ATQ-N=Automatic Thoughts Questionnaire Negative Subscale; BP=Bipolar Disorder; BDI=Beck depression inventory; BRMS=Bech-Rafaelsen Mania Scale; CBT=Cognitive Behavioral Therapy; CI=Confidence Interval; ES=Effect Size; GAF=General Assessment of Functioning Scale; HR=Hazard Ratio; LIFE=Longitudinal Interval Follow-up Evaluation; MADRS=Montgomery-Asberg Depression Rating Scale; NR=not reported; NS=not significant; OR=Odds Ratio; PMID=PubMed Identification Number; QoL.BD=Quality of Life; ROB=risk of bias; SAS=Simpson Angus Scale; SPS=Social Phobia Scale; WHO-DAS=World Health Organization Disability Assessment Scale; YMRS

= Young Mania Rating Scale 
Appendix Table K4. Summary of strength of evidence: CBT vs. inactive comparators

\begin{tabular}{|c|c|c|c|c|c|c|c|c|}
\hline Outcome & Timing & $\begin{array}{l}\text { \# Studies/ Design } \\
\text { (n analyzed) }\end{array}$ & $\begin{array}{l}\text { Finding or } \\
\text { Summary } \\
\text { Statistic }\end{array}$ & $\begin{array}{c}\text { Study } \\
\text { Limitations }\end{array}$ & Consistency & Directness & Precision & $\begin{array}{l}\text { Overall Gradel } \\
\text { Conclusion }\end{array}$ \\
\hline Relapse & $\begin{array}{l}6 \text { months } \\
7-12 \\
\text { months } \\
12+ \\
\text { months } \\
\end{array}$ & 7 RCTs $(n=714)$ & $\begin{array}{l}\text { Mixed evidence } \\
\text { with no clear } \\
\text { direction of effect. } \\
\text { No pattern across } \\
\text { time periods. }\end{array}$ & Moderate & Inconsistent & Direct & Imprecise & Insufficient \\
\hline Depression & $\begin{array}{l}6 \text { months } \\
7-12 \\
\text { months } \\
12+ \\
\text { months } \\
\end{array}$ & 7 RCTs $(n=716)$ & $\begin{array}{l}\text { Mixed evidence } \\
\text { with no clear } \\
\text { direction of effect. } \\
\text { No pattern across } \\
\text { time periods. }\end{array}$ & Moderate & Inconsistent & Direct & Imprecise & Insufficient \\
\hline Mania & $\begin{array}{l}6 \text { months } \\
7-12 \\
\text { months } \\
12+ \\
\text { months } \\
\end{array}$ & 6 RCTs $(n=649)$ & $\begin{array}{l}\text { Mixed evidence } \\
\text { with no clear } \\
\text { direction of effect. } \\
\text { No pattern across } \\
\text { time periods. }\end{array}$ & Moderate & Inconsistent & Direct & Imprecise & Insufficient \\
\hline $\begin{array}{l}\text { Global } \\
\text { Function }\end{array}$ & $\begin{array}{l}6 \text { months } \\
12+ \\
\text { months }\end{array}$ & $1 \mathrm{RCT}(\mathrm{n}=52)$ & $\begin{array}{l}\text { No difference } \\
\text { between groups at } \\
6 \text { or } 18 \text { months. } \\
6 \text { months. GAF } \\
E S=0.43(95 \% \mathrm{Cl} \text { - } \\
0.12,0.98) \\
18 \text { months, GAF } \\
E S=0.24(95 \% \mathrm{Cl} \text { - } \\
0.30,0.79)\end{array}$ & Moderate & Unclear & Direct & Imprecise & Insufficient \\
\hline $\begin{array}{l}\text { Other } \\
\text { Measures } \\
\text { of Function }\end{array}$ & $\begin{array}{l}6 \text { months } \\
7-12 \\
\text { months } \\
12+ \\
\text { months }\end{array}$ & 3 RCTs (n=289) & $\begin{array}{l}\text { Mixed evidence } \\
\text { with no clear } \\
\text { direction of effect. } \\
\text { No pattern across } \\
\text { time periods. }\end{array}$ & Moderate & Inconsistent & Direct & Imprecise & Insufficient \\
\hline
\end{tabular}

Abbreviations: CBT=Cognitive Behavioral Therapy; CI=Confidence Interval; ES=Effect Size; GAF=General Assessment of Functioning Scale; RCT=randomized controlled trial 
Appendix Table K5. Characteristics of eligible studies: CBT vs. active comparators

\begin{tabular}{|c|c|c|c|c|c|c|}
\hline $\begin{array}{l}\text { Study, Year } \\
\text { Design } \\
\text { Location } \\
\text { Funder } \\
\text { Risk of Bias } \\
\text { PMID }\end{array}$ & $\begin{array}{c}\text { Randomized (N) } \\
\text { Age (mean) } \\
\text { Sex (\% Female) } \\
\text { Race (\% White) } \\
\text { Diagnosis } \\
\text { (\% BP I, II, NOS) } \\
\text { Setting }\end{array}$ & $\begin{array}{c}\text { Inclusions } \\
\text { Key Exclusions }\end{array}$ & $\begin{array}{l}\text { Intervention } \\
\text { Description }\end{array}$ & $\begin{array}{l}\text { Comparison } \\
\text { Description }\end{array}$ & $\begin{array}{c}\text { Follow-up } \\
\text { Duration }\end{array}$ & $\begin{array}{c}\text { Outcomes } \\
\text { Reported } \\
\text { Withdrawal (\%) at } \\
\text { endpoint }\end{array}$ \\
\hline $\begin{array}{l}\text { Harvey, } 2015^{10} \\
\text { RCT } \\
\text { United States } \\
\text { Government } \\
\text { Moderate } \\
25622197\end{array}$ & \begin{tabular}{|l}
$\mathrm{N}=58$ \\
Age 37 (18-62) \\
Female 62\% \\
White 64\% \\
BP I 100\% \\
Outpatient
\end{tabular} & \begin{tabular}{|l|} 
No current bipolar episode; \\
BP I(DSM-IV); interepisode \\
defined by YMRS score \\
$<12$ and an IDS-C score \\
$<24$ for the past week, with \\
general insomnia disorder \\
(International Classification \\
of Sleep Disorders, DSM- \\
IV-TR criteria for primary \\
insomnia) but without the \\
exclusion for mental \\
disorder, had a stable \\
medication regimen for the \\
past 4 weeks, had a \\
treating psychiatrist \\
\\
Substance Abuse; Other \\
Mental Health; Neurological \\
Disorders; \\
Pregnant/Nursing; \\
Labs/Other Conditions
\end{tabular} & $\begin{array}{l}\text { CBT for insomnia } \\
\text { focusing on stimulus } \\
\text { control, bed and } \\
\text { sleep associations, } \\
\text { regularizing sleep } \\
\text { and wake times, } \\
\text { sleep/circadian } \\
\text { education, relaxing } \\
\text { wind down, sleep- } \\
\text { enhancing activities, } \\
\text { and devising a } \\
\text { wake-up routine. } \\
\text { The module altered } \\
\text { unhelpful beliefs } \\
\text { about sleep, } \\
\text { bedtime worry, } \\
\text { rumination, and } \\
\text { vigilance } \\
-8 \text { weekly } 50-60 \\
\text { minute sessions with } \\
\text { behavioral module }\end{array}$ & \begin{tabular}{|l|} 
Psychoeducation \\
sessions that \\
provided \\
information but \\
no facilitation or \\
plan for behavior \\
change. \\
Sessions \\
focused on \\
mood regulation, \\
the etiology of \\
bipolar disorder, \\
symptoms, \\
prodromes, \\
medications, \\
substance use, \\
diet, physical \\
activity, stress \\
management, \\
relaxation, and \\
self-esteem and \\
sleep in a social \\
context \\
\\
-8 weekly $50-60$ \\
minute sessions \\
\end{tabular} & 6 months & $\begin{array}{l}\text { Relapse (emergence of } \\
\text { a new syndromal } \\
\text { DSM-IV-TR bipolar } \\
\text { episode) } \\
\text { YMRS } \\
\text { IDS-C } \\
\text { SDS-Mood } \\
\text { Q-LES-Q-SF } \\
29 \%\end{array}$ \\
\hline
\end{tabular}




\begin{tabular}{|c|c|c|c|c|c|c|}
\hline $\begin{array}{l}\text { Study, Year } \\
\text { Design } \\
\text { Location } \\
\text { Funder } \\
\text { Risk of Bias } \\
\text { PMID }\end{array}$ & $\begin{array}{c}\text { Randomized (N) } \\
\text { Age (mean) } \\
\text { Sex (\% Female) } \\
\text { Race (\% White) } \\
\text { Diagnosis } \\
\text { (\% BP I, II, NOS) } \\
\text { Setting }\end{array}$ & $\begin{array}{c}\text { Inclusions } \\
\text { Key Exclusions }\end{array}$ & $\begin{array}{l}\text { Intervention } \\
\text { Description }\end{array}$ & $\begin{array}{l}\text { Comparison } \\
\text { Description }\end{array}$ & $\begin{array}{c}\text { Follow-up } \\
\text { Duration }\end{array}$ & $\begin{array}{c}\text { Outcomes } \\
\text { Reported } \\
\text { Withdrawal (\%) at } \\
\text { endpoint }\end{array}$ \\
\hline $\begin{array}{l}\text { Meyer, } 2012^{11} \\
\text { RCT } \\
\text { Germany } \\
\text { Non-government } \\
\text { Low } \\
22099722\end{array}$ & $\begin{array}{l}\mathrm{N}=76 \\
\text { Age } 44(18-75) \\
\text { Female 50\% } \\
\text { White NR } \\
\text { BP 79\% } \\
\text { BP II 21\% } \\
\text { Outpatient }\end{array}$ & $\begin{array}{l}\text { Euthymic/Maintenance; } \\
\text { Primary diagnosis of BP } \\
\text { (DSM-IV), without a current } \\
\text { major effective episode, } \\
\text { and willingness to continue } \\
\text { current or start medication. } \\
\\
\text { Schizoaffective; Substance } \\
\text { Abuse; Other Mental } \\
\text { Health; Neurological } \\
\text { Disorders; Taking Other } \\
\text { Medications }\end{array}$ & $\begin{array}{l}\text { CBT focused on } \\
\text { understanding of } \\
\text { BP, identifying early } \\
\text { warning symptoms, } \\
\text { strategies for } \\
\text { management, } \\
\text { communication and } \\
\text { problem solving } \\
\text { skills } \\
\text {-20 sessions over } 9 \\
\text { months, 50-60 } \\
\text { minutes each }\end{array}$ & $\begin{array}{l}\text { Supportive } \\
\text { Therapy: Client- } \\
\text { centered focus; } \\
\text { whatever } \\
\text { problems the } \\
\text { patient } \\
\text { presented were } \\
\text { dealt with by } \\
\text { providing } \\
\text { emotional } \\
\text { support and } \\
\text { general advice } \\
\text {-20 sessions } \\
\text { over } 9 \text { months, } \\
50-60 \text { minutes } \\
\text { each. }\end{array}$ & 24 months & $\begin{array}{l}\text { Relapse (Any mood } \\
\text { episode that fulfilled } \\
\text { DSM-IV criteria) } \\
\text { BDI } \\
\text { BRMAS } \\
\text { GAS } \\
15 \%\end{array}$ \\
\hline
\end{tabular}




\begin{tabular}{|c|c|c|c|c|c|c|}
\hline $\begin{array}{l}\text { Study, Year } \\
\text { Design } \\
\text { Location } \\
\text { Funder } \\
\text { Risk of Bias } \\
\text { PMID }\end{array}$ & $\begin{array}{c}\text { Randomized (N) } \\
\text { Age (mean) } \\
\text { Sex (\% Female) } \\
\text { Race (\% White) } \\
\text { Diagnosis } \\
\text { (\% BP I, II, NOS) } \\
\text { Setting }\end{array}$ & $\begin{array}{c}\text { Inclusions } \\
\text { Key Exclusions }\end{array}$ & $\begin{array}{l}\text { Intervention } \\
\text { Description }\end{array}$ & $\begin{array}{l}\text { Comparison } \\
\text { Description }\end{array}$ & $\begin{array}{c}\text { Follow-up } \\
\text { Duration }\end{array}$ & $\begin{array}{c}\text { Outcomes } \\
\text { Reported } \\
\text { Withdrawal (\%) at } \\
\text { endpoint }\end{array}$ \\
\hline $\begin{array}{l}\text { Parikh, } 2012^{12} \\
\text { RCT } \\
\text { Canada } \\
\text { Government and Non- } \\
\text { Government } \\
\text { Low } \\
22795205\end{array}$ & $\begin{array}{l}\mathrm{N}=204 \\
\text { Age } 40.9(18-64) \\
\text { Female 58\% } \\
\text { White NR } \\
\text { BP I 72\% } \\
\text { BP II 28\% } \\
\text { Outpatient }\end{array}$ & $\begin{array}{l}\text { Euthymic/Maintenance; } \\
\text { Age 18-64; BP I or II (DSM- } \\
\text { IV) with at least } 2 \text { episodes } \\
\text { of significant symptoms or } \\
\text { full episodes within } \\
\text { previous } 3 \text { years; no } \\
\text { episode in month preceding } \\
\text { randomization } \\
\text { First Manic Episode; } \\
\text { Substance Abuse; Other } \\
\text { Mental Health; Neurological } \\
\text { Disorders; Labs/Other } \\
\text { Conditions }\end{array}$ & $\begin{array}{l}\text { CBT including } \\
\text { psychoeducation, } \\
\text { understanding of } \\
\text { personal warning } \\
\text { signs for onset and } \\
\text { action plan, and } \\
\text { cognitive } \\
\text { restructuring of } \\
\text { dysfunctional } \\
\text { thoughts and } \\
\text { assumptions } \\
\\
-20 \text { individual 50- } \\
\text { minute sessions }\end{array}$ & $\begin{array}{l}\text { Group } \\
\text { psychoeducation } \\
\text { using Life Goals } \\
\text { manual; focused } \\
\text { on illness } \\
\text { recognition, } \\
\text { treatment } \\
\text { approaches, and } \\
\text { coping strategies } \\
\text { and the creation } \\
\text { of Personal Care } \\
\text { Plan including } \\
\text { action plan for } \\
\text { both depression } \\
\text { and mania } \\
\\
\text {-6 sessions, } 90 \\
\text { minutes each } \\
\text { session }\end{array}$ & 18 months & $\begin{array}{l}\text { Relapse (Not } \\
\text { Described) } \\
\text { LIFE Depression } \\
\text { LIFE Mania } \\
38 \%\end{array}$ \\
\hline
\end{tabular}




\begin{tabular}{|c|c|c|c|c|c|c|}
\hline $\begin{array}{l}\text { Study, Year } \\
\text { Design } \\
\text { Location } \\
\text { Funder } \\
\text { Risk of Bias } \\
\text { PMID }\end{array}$ & $\begin{array}{c}\text { Randomized (N) } \\
\text { Age (mean) } \\
\text { Sex (\% Female) } \\
\text { Race (\% White) } \\
\text { Diagnosis } \\
\text { (\% BP I, II, NOS) } \\
\text { Setting }\end{array}$ & $\begin{array}{c}\text { Inclusions } \\
\text { Key Exclusions }\end{array}$ & $\begin{array}{l}\text { Intervention } \\
\text { Description }\end{array}$ & $\begin{array}{l}\text { Comparison } \\
\text { Description }\end{array}$ & $\begin{array}{c}\text { Follow-up } \\
\text { Duration }\end{array}$ & $\begin{array}{c}\text { Outcomes } \\
\text { Reported } \\
\text { Withdrawal (\%) at } \\
\text { endpoint }\end{array}$ \\
\hline $\begin{array}{l}\text { Weiss, } 2009^{13} \\
\text { RCT } \\
\text { United States } \\
\text { Government } \\
\text { Low } \\
19573999\end{array}$ & $\begin{array}{l}\mathrm{N}=61 \\
\text { Age } 38(18-58) \\
\text { Female } 41 \% \\
\text { White } 91.8 \% \\
\text { BP I 79\% } \\
\text { BP II } 15 \% \\
\text { BP NOS } 6 \% \\
\text { Outpatient }\end{array}$ & $\begin{array}{l}\text { Non-manic; Current } \\
\text { diagnosis of BP (DSM-IV) } \\
\text { and substance abuse other } \\
\text { than nicotine, substance } \\
\text { abuse within } 60 \text { days, a } \\
\text { mood stabilizer regimen for } \\
\text { more than } 2 \text { weeks, ability } \\
\text { to attend group sessions } \\
\text { and follow-up, without } \\
\text { current mania. } \\
\text { First Manic Episode; } \\
\text { Schizoaffective; Other } \\
\text { Mental Health; Labs/Other } \\
\text { Conditions }\end{array}$ & $\begin{array}{l}\text { Integrated group } \\
\text { CBT on the } \\
\text { cognitive-behavioral } \\
\text { relapse prevention } \\
\text { model which } \\
\text { focuses on the } \\
\text { similarities between } \\
\text { recovery and } \\
\text { relapse processes in } \\
\text { bipolar disorder and } \\
\text { substance abuse } \\
\text { and their interaction } \\
\\
-12 \text { weekly 60- } \\
\text { minute sessions }\end{array}$ & \begin{tabular}{|l|} 
Group Drug \\
Therapy: \\
Substance use \\
disorder therapy \\
sessions that \\
focused on \\
facilitating \\
abstinence, \\
encouraging \\
mutual support, \\
and teaching \\
new ways to \\
cope with \\
substance- \\
related problems \\
-12 weekly 60- \\
minute sessions
\end{tabular} & 6 months & $\begin{array}{l}\text { HAM-D } \\
\text { YMRS } \\
19.6 \%\end{array}$ \\
\hline $\begin{array}{l}\text { Weiss, } 2007^{14} \\
\text { RCT } \\
\text { United States } \\
\text { Government and Non- } \\
\text { Government } \\
\text { Moderate } \\
17202550\end{array}$ & $\begin{array}{l}\mathrm{N}=62 \\
\text { Age } 41.9(22-65) \\
\text { Female } 51.6 \% \\
\text { White } 93.5 \% \\
\text { BP I } 81 \% \\
\text { BP II } 16 \% \\
\text { BP NOS } 3 \% \\
\text { Outpatient }\end{array}$ & $\begin{array}{l}\text { Maintenance; A current } \\
\text { diagnosis of bipolar } \\
\text { disorder (DSM-IV) and } \\
\text { substance dependence } \\
\text { other than nicotine; } \\
\text { substance use within } 60 \\
\text { days; a mood stabilizer } \\
\text { regimen for } \geq 2 \text { weeks; and } \\
\text { age } \geq 18 \\
\text { First Manic Episode; } \\
\text { Schizoaffective; Other } \\
\text { Mental Health; Labs/Other } \\
\text { Conditions }\end{array}$ & $\begin{array}{l}\text { Integrated group } \\
\text { CBT on cognitive- } \\
\text { behavioral relapse } \\
\text { prevention model } \\
\text { which focuses on } \\
\text { the similarities } \\
\text { between recovery } \\
\text { and relapse } \\
\text { processes in bipolar } \\
\text { disorder and } \\
\text { substance abuse } \\
\text { and their interaction } \\
\text {-20 weekly 60- } \\
\text { minute sessions }\end{array}$ & $\begin{array}{l}\text { Group Drug } \\
\text { Therapy: } \\
\text { Focused on } \\
\text { facilitating } \\
\text { abstinence, } \\
\text { encouraging } \\
\text { mutual support, } \\
\text { and teaching } \\
\text { new ways to } \\
\text { cope with } \\
\text { substance- } \\
\text { related problems } \\
\text {-20 weekly 60- } \\
\text { minute sessions } \\
\end{array}$ & 8 months & $\begin{array}{l}\text { HAM-D } \\
\text { YMRS } \\
34 \%\end{array}$ \\
\hline
\end{tabular}

Abbreviations: BDI=Beck depression inventory; BP=bipolar disorder; BRMS=Bech-Rafaelsen Mania Scale; CBT=Cognitive Behavioral Therapy; DSM=Diagnostic and

Statistical Manual of Mental Disorders; GAS=Global Assessment Scale; HAM-D=Hamilton Scale for Depression; IDS=Inventory for Depressive Symptoms; LIFE=Longitudinal 
Interval Follow-up Evaluation; NOS=not otherwise specified; NR=not reported; PMID=PubMed Identification Number; Q-LES-Q-SF=Quality of Life Enjoyment and Satisfaction Questionnaire, Short Form; RCT=randomized controlled trial; SDS-Mood=Sheehan Disability Scale-Mood; YMRS = Young Mania Rating Scale

\section{Appendix Table K6. Summary risk of bias assessments: CBT vs. active comparators}

\begin{tabular}{|c|c|c|}
\hline $\begin{array}{c}\text { Study } \\
\text { Funding Source } \\
\text { PMID }\end{array}$ & $\begin{array}{c}\text { Overall Risk of Bias } \\
\text { Assessment }\end{array}$ & (2) \\
\hline $\begin{array}{l}\text { Harvey, } 2015^{10} \\
\text { Government } \\
25622197 \\
\end{array}$ & Moderate & Potential bias due to differential attrition rates between arms. \\
\hline $\begin{array}{l}\text { Meyer, } 2012^{11} \\
\text { Non-government } \\
22099722\end{array}$ & Low & No significant suspected biases. \\
\hline $\begin{array}{l}\text { Parikh, } 2012^{12} \\
\text { Government and } \\
\text { Non-Government } \\
22795205\end{array}$ & Low & No significant suspected biases. \\
\hline $\begin{array}{l}\text { Weiss, } 2009^{13} \\
\text { Government } \\
19573999\end{array}$ & Low & No significant suspected biases. \\
\hline $\begin{array}{l}\text { Weiss, } 2007^{14} \\
\text { Government and } \\
\text { Non-Government } \\
17202550\end{array}$ & Moderate & Potential bias due to incomplete outcome reported and unclear reporting of methods for analysis of data. \\
\hline
\end{tabular}

Abbreviations: PMID=PubMed Identification Number

\section{Appendix Table K7. Outcomes summary: CBT vs. active comparators}

\begin{tabular}{|c|c|c|c|c|c|}
\hline $\begin{array}{l}\text { Study } \\
\text { Risk of Bias } \\
\text { PMID }\end{array}$ & Responder/Remitter & Symptom & Function & Other & $\mathrm{AE}$ \\
\hline $\begin{array}{l}\text { Harvey, } 2015^{10} \\
\text { Moderate } \\
25622197\end{array}$ & $\begin{array}{l}\frac{\text { Relapse }}{6 \text { months, Favors CBT }} \\
\text { Hypo/manic } \\
4.6 \% \text { CBT vs. } 31.6 \% \\
\text { Psychoeducation, } \mathrm{p}= \\
.036 \\
\frac{\text { Relapse }}{\text { NS }} \\
6 \text { months, Depressive } \\
9.1 \% \text { CBT vs. } 21.1 \% \\
\text { Psychoeducation, } p= \\
0.39 .\end{array}$ & $\begin{array}{l}\text { Depression } \\
\text { NS } \\
6 \text { months, IDS-C } \\
E S=-0.30 ; p=0.33 \\
\frac{\text { Mania }}{\text { NS }} \\
6 \text { months, YMRS } \\
\text { ES }=-0.02 ; p=0.60\end{array}$ & $\begin{array}{l}\text { Quality of Life } \\
\text { NS } \\
6 \text { months, Q-LES-Q- } \\
\text { SF } \\
\text { ES }=-0.47(95 \% \mathrm{Cl} \text { - } \\
0.99,0.05) \\
\text { Disability } \\
\text { NS } \\
6 \text { months, SDS-Mood } \\
\text { ES }=0.24(95 \% \mathrm{Cl}- \\
0.27,0.76)\end{array}$ & NR & NR \\
\hline
\end{tabular}




\begin{tabular}{|c|c|c|c|c|c|}
\hline $\begin{array}{c}\text { Study } \\
\text { Risk of Bias } \\
\text { PMID }\end{array}$ & Responder/Remitter & Symptom & Function & Other & $\mathrm{AE}$ \\
\hline $\begin{array}{l}\text { Meyer, } 2012^{11} \\
\text { Low } \\
22099722\end{array}$ & $\begin{array}{l}\text { Relapse } \\
\text { NS } \\
9 \text { months, Any type } \\
\mathrm{OR}=0.42(95 \% \mathrm{Cl} 0.15, \\
1.16) ; p=0.10 \\
30 \text { months, Any type } \\
\text { OR=1.41 (95\% Cl } 0.50 \text {, } \\
4.05) ; p=0.63\end{array}$ & $\begin{array}{l}\text { Depression } \\
\mathrm{NS} \\
9 \text { months, BDI } \\
\text { No statistical test reported } \\
\text { Mania } \\
\mathrm{NS} \\
9 \text { months, BRMAS } \\
\mathrm{ES}=0.33(95 \% \mathrm{Cl}-0.16,0.82)\end{array}$ & $\begin{array}{l}\text { Global Function } \\
\text { NS } \\
9 \text { months, GAS } \\
\text { ES=-0.20 }(95 \% \mathrm{Cl}- \\
0.68,0.29)\end{array}$ & NR & NR \\
\hline $\begin{array}{l}\text { Parikh, } 2012^{12} \\
\text { Low } \\
22795205\end{array}$ & $\begin{array}{l}\text { Relapse } \\
\text { NS } \\
18 \text { months, } \\
\text { Hypomanic/manic } \\
p=0.46 \\
\text { Relapse } \\
\text { NS } \\
18 \text { months, Depressive } \\
p=0.76\end{array}$ & $\begin{array}{l}\text { Depression } \\
\text { NS } \\
18 \text { months, LIFE Depression } \\
p=0.89 \\
\text { Mania } \\
\text { NS } \\
18 \text { months, LIFE Mania } \\
p=0.96\end{array}$ & NR & NR & NR \\
\hline $\begin{array}{l}\text { Weiss, 200913 } \\
\text { Low } \\
19573999\end{array}$ & NR & $\begin{array}{l}\text { Depression } \\
\text { NS } \\
6 \text { months, HAM-D } \\
\text { No statistical test reported } \\
\text { Mania } \\
\text { NS } \\
6 \text { months, YMRS } \\
\text { No statistical test reported } \\
\text { ES }=-0.54(95 \% \mathrm{Cl}-1.05,-0.03)\end{array}$ & NR & NR & NR \\
\hline $\begin{array}{l}\text { Weiss, } 2007^{14} \\
17202550\end{array}$ & NR & $\begin{array}{l}\text { Depression } \\
\text { NS } \\
8 \text { months, HRSD } \\
\frac{\text { Mania }}{N S} \\
8 \text { months, YMRS }\end{array}$ & NR & NR & NR \\
\hline
\end{tabular}

Abbreviations: BDI=Beck depression inventory; CBT=Cognitive Behavioral Therapy; CI=Confidence Interval; ES=Effect Size; GAS=Global Assessment Scale; HAM-

$\mathrm{D}=$ Hamilton Scale for Depression; HRSD=Hamilton Rating Scale for Depression; IDS=Inventory for Depressive Symptoms; LIFE=Longitudinal Interval Follow-up Evaluation; NR=not reported; NS=not significant; OR=Odds Ratio; PMID=PubMed Identification Number; Q-LES-Q=Quality of Life Enjoyment and Satisfaction Questionnaire; SDS-

Mood=Sheehan Disability Scale-Mood; YMRS=Young Mania Rating Scale 
Appendix Table K8. Summary of strength of evidence: CBT vs. active comparators

\begin{tabular}{|c|c|c|c|c|c|c|c|c|}
\hline Outcome & Timing & $\begin{array}{l}\text { \# Studies/ Design } \\
\text { (n analyzed) }\end{array}$ & $\begin{array}{l}\text { Finding or } \\
\text { Summary } \\
\text { Statistic }\end{array}$ & $\begin{array}{c}\text { Study } \\
\text { Limitations }\end{array}$ & Consistency & Directness & Precision & $\begin{array}{l}\text { Overall Gradel } \\
\text { Conclusion }\end{array}$ \\
\hline Relapse & $\begin{array}{l}6 \text { months } \\
7-12 \\
\text { months } \\
12+ \\
\text { months } \\
\end{array}$ & $\begin{array}{l}3 \text { RCTs } \\
(n=338)\end{array}$ & $\begin{array}{l}\text { Mixed evidence } \\
\text { with no clear } \\
\text { direction of effect. } \\
\text { No pattern across } \\
\text { time periods. }\end{array}$ & Moderate & Inconsistent & Direct & Imprecise & Insufficient \\
\hline Depression & $\begin{array}{l}6 \text { months } \\
7-12 \\
\text { months } \\
12+ \\
\text { months }\end{array}$ & $\begin{array}{l}5 \text { RCTs } \\
(n=461)\end{array}$ & $\begin{array}{l}\text { No difference } \\
\text { between groups } \\
\text { across range of } \\
\text { time periods. }\end{array}$ & Moderate & Consistent & Direct & Imprecise* & $\begin{array}{l}\text { Low; No effect from } \\
\text { intervention }\end{array}$ \\
\hline Mania & $\begin{array}{l}6 \text { months } \\
7-12 \\
\text { months } \\
12+ \\
\text { months }\end{array}$ & $\begin{array}{l}5 \text { RCTs } \\
(n=461)\end{array}$ & $\begin{array}{l}\text { No difference } \\
\text { between groups } \\
\text { across range of } \\
\text { time periods }\end{array}$ & Moderate & Consistent & Direct & Imprecise* & $\begin{array}{l}\text { Low; No effect from } \\
\text { intervention }\end{array}$ \\
\hline $\begin{array}{l}\text { Global } \\
\text { Function }\end{array}$ & 9 months & $\begin{array}{l}1 \mathrm{RCT} \\
(\mathrm{n}=76)\end{array}$ & $\begin{array}{l}\text { No difference } \\
\text { between groups at } \\
9 \text { months } \\
\text { ES }=-0.20(95 \% \mathrm{Cl} \\
-0.68,0.29)\end{array}$ & Low & Unclear & Direct & Imprecise & Insufficient \\
\hline $\begin{array}{l}\text { Other } \\
\text { Measures } \\
\text { of Function }\end{array}$ & 6 months & $\begin{array}{l}1 \mathrm{RCT} \\
(\mathrm{n}=58)\end{array}$ & $\begin{array}{l}\text { No difference } \\
\text { between groups at } \\
6 \text { months in either } \\
\text { QoL or disability. } \\
\text { Q-LES-Q-SF } \\
\text { ES=-0.47 (95\% Cl } \\
-0.99,0.05) \\
\text { SDS-Mood } \\
\text { ES=0.24 (95\% Cl - } \\
0.27,0.76)\end{array}$ & Low & Unclear & Direct & Imprecise & Insufficient \\
\hline
\end{tabular}

*It is difficult to establish a level of precision that provides confidence of no effect. Due to the large number of comparisons with findings of no effect, we assessed strength of evidence cautiously when there was imprecision, only assigning low strength of evidence when there was sufficient sample size, low to moderate study limitations, and consistency

Abbreviations: CBT=Cognitive Behavioral Therapy; CI=Confidence Interval; ES=Effect Size; Q-LES-Q=Quality of Life Enjoyment and Satisfaction Questionnaire; RCT=randomized controlled trial; SDS-Mood=Sheehan Disability Scale-Mood 


\section{References for Appendix K}

1. Jones SH, Smith G, Mulligan LD, et al. Recovery-focused cognitive-behavioural therapy for recent-onset bipolar disorder: randomised controlled pilot trial.[Erratum appears in $\mathrm{Br} \mathrm{J}$ Psychiatry. 2015 Feb;206(2):169]. British Journal of Psychiatry. 2015 Jan;206(1):58-66. PMID 25213157.

2. Perich T, Manicavasagar V, Mitchell PB, et al. A randomized controlled trial of mindfulness-based cognitive therapy for bipolar disorder. Acta Psychiatrica Scandinavica. 2013 May;127(5):33343. PMID 23216045.

3. Fava GA, Rafanelli C, Tomba E, et al. The sequential combination of cognitive behavioral treatment and well-being therapy in cyclothymic disorder. Psychotherapy \& Psychosomatics. 2011;80(3):136-43. PMID 21372621.

4. Gomes BC, Abreu LN, Brietzke E, et al. A randomized controlled trial of cognitive behavioral group therapy for bipolar disorder. Psychotherapy \& Psychosomatics. 2011;80(3):144-50. PMID 21372622.

5. Castle D, White C, Chamberlain J, et al. Groupbased psychosocial intervention for bipolar disorder: randomised controlled trial. British Journal of Psychiatry. 2010 May;196(5):383-8. PMID 20435965.

6. Ball JR, Mitchell PB, Corry JC, et al. A randomized controlled trial of cognitive therapy for bipolar disorder: focus on long-term change. Journal of Clinical Psychiatry. 2006 Feb;67(2):277-86. PMID 16566624.

7. Scott J, Paykel E, Morriss R, et al. Cognitivebehavioural therapy for severe and recurrent bipolar disorders: randomised controlled trial. British Journal of Psychiatry. 2006 Apr;188:31320. PMID 16582056.
8. Lam DH, Watkins ER, Hayward P, et al. A randomized controlled study of cognitive therapy for relapse prevention for bipolar affective disorder: outcome of the first year. Archives of General Psychiatry. 2003 Feb;60(2):145-52. PMID 12578431.

9. Lam DH, Hayward P, Watkins ER, et al. Relapse prevention in patients with bipolar disorder: cognitive therapy outcome after 2 years. American Journal of Psychiatry. 2005 Feb;162(2):324-9. PMID 15677598.

10. Harvey AG, Soehner AM, Kaplan KA, et al. Treating insomnia improves mood state, sleep, and functioning in bipolar disorder: a pilot randomized controlled trial. J Consult Clin Psychol. 2015 Jun;83(3):564-77. PMID 25622197.

11. Meyer TD, Hautzinger M. Cognitive behaviour therapy and supportive therapy for bipolar disorders: relapse rates for treatment period and 2-year follow-up. Psychological Medicine. 2012 Jul;42(7):1429-39. PMID 22099722.

12. Parikh SV, Zaretsky A, Beaulieu S, et al. A randomized controlled trial of psychoeducation or cognitive-behavioral therapy in bipolar disorder: a Canadian Network for Mood and Anxiety treatments (CANMAT) study [CME]. Journal of Clinical Psychiatry. 2012 Jun;73(6):803-10. PMID 22795205.

13. Weiss RD, Griffin ML, Jaffee WB, et al. A "community-friendly" version of integrated group therapy for patients with bipolar disorder and substance dependence: a randomized controlled trial. Drug \& Alcohol Dependence. 2009 Oct 1;104(3):212-9. PMID 19573999.

14. Weiss RD, Griffin ML, Kolodziej ME, et al. A randomized trial of integrated group therapy versus group drug counseling for patients with bipolar disorder and substance dependence. American Journal of Psychiatry. 2007 Jan;164(1):100-7. PMID 17202550. 


\section{Appendix L. Systematic or Collaborative Care}

Appendix Table L1. Characteristics of eligible studies: systematic or collaborative care vs. inactive comparators

\begin{tabular}{|c|c|c|c|c|c|c|}
\hline $\begin{array}{l}\text { Study, Year } \\
\text { Design } \\
\text { Location } \\
\text { Funder } \\
\text { Risk of Bias } \\
\text { PMID }\end{array}$ & $\begin{array}{c}\text { Randomized (N) } \\
\text { Age (mean) } \\
\text { Sex (\% Female) } \\
\text { Race (\% White) } \\
\text { Diagnosis } \\
\text { (\% BP I, II, NOS) } \\
\text { Setting }\end{array}$ & $\begin{array}{l}\text { Inclusions } \\
\text { Key Exclusions }\end{array}$ & $\begin{array}{l}\text { Intervention } \\
\text { Description }\end{array}$ & $\begin{array}{l}\text { Comparison } \\
\text { Description }\end{array}$ & $\begin{array}{c}\text { Follow-up } \\
\text { Duration }\end{array}$ & $\begin{array}{c}\text { Outcomes } \\
\text { Reported } \\
\text { Withdrawal (\%) at } \\
\text { endpoint }\end{array}$ \\
\hline $\begin{array}{l}\text { van der Voort, 2015¹ } \\
\text { van der Voort, 2015b² } \\
\text { RCT } \\
\text { Netherlands } \\
\text { NR } \\
\text { Low } \\
25792695 \\
25841077\end{array}$ & $\begin{array}{l}\mathrm{N}=138 \\
\text { Age } 46 \text { (18-65) } \\
\text { Female 64\% } \\
\text { White NR } \\
\text { BP I 64\% } \\
\text { BP II 28\% } \\
\text { BP NOS 4\% } \\
\text { Outpatient }\end{array}$ & $\begin{array}{l}\text { Maintenance; BP I, II or } \\
\text { NOS (DSM-IV-TR) not } \\
\text { experiencing a severe } \\
\text { manic or depressive } \\
\text { episode (6 or } 7 \text { on CGI BP) } \\
\text { and stable enough to } \\
\text { function well with only low- } \\
\text { intensity treatment. } \\
\\
\text { Other Mental Health; } \\
\text { Labs/Other Conditions }\end{array}$ & $\begin{array}{l}\text { Collaborative care } \\
\text { including formation } \\
\text { of care team } \\
\text { (including a family } \\
\text { member with patient } \\
\text { consent), formation } \\
\text { of treatment plan } \\
\text { with needs } \\
\text { assessment, } \\
\text { psychoeducation, } \\
\text { problem solving } \\
\text { treatment, mood } \\
\text { charting, recognition } \\
\text { of early warning } \\
\text { signs and formation } \\
\text { of relapse } \\
\text { prevention, and } \\
\text { pharmacotherapy } \\
\text { and somatic care. } \\
\\
\text {-12 months of } \\
\text { collaborative care }\end{array}$ & $\begin{array}{l}\text { Treatment as } \\
\text { usual (not } \\
\text { described) }\end{array}$ & 12 months & \begin{tabular}{|l} 
QIDS \\
ASRM \\
FAST-NL-P \\
WHO-QOL-bref \\
Withdrawal 15\%
\end{tabular} \\
\hline
\end{tabular}




\begin{tabular}{|c|c|c|c|c|c|c|}
\hline $\begin{array}{l}\text { Study, Year } \\
\text { Design } \\
\text { Location } \\
\text { Funder } \\
\text { Risk of Bias } \\
\text { PMID }\end{array}$ & $\begin{array}{c}\text { Randomized (N) } \\
\text { Age (mean) } \\
\text { Sex (\% Female) } \\
\text { Race (\% White) } \\
\text { Diagnosis } \\
\text { (\% BP I, II, NOS) } \\
\text { Setting }\end{array}$ & $\begin{array}{c}\text { Inclusions } \\
\text { Key Exclusions }\end{array}$ & $\begin{array}{l}\text { Intervention } \\
\text { Description }\end{array}$ & $\begin{array}{l}\text { Comparison } \\
\text { Description }\end{array}$ & $\begin{array}{c}\text { Follow-up } \\
\text { Duration }\end{array}$ & $\begin{array}{c}\text { Outcomes } \\
\text { Reported } \\
\text { Withdrawal (\%) at } \\
\text { endpoint }\end{array}$ \\
\hline $\begin{array}{l}\text { Kessing, } 2013^{3} \\
\text { RCT } \\
\text { Denmark } \\
\text { Non-Government } \\
\text { Low/High } \\
23349295\end{array}$ & $\begin{array}{l}\text { N=158 } \\
\text { Age } 37(27-48) \\
\text { Female 54\% } \\
\text { White NR } \\
\text { BP NR } \\
\text { Outpatient }\end{array}$ & $\begin{array}{l}\text { No current clinical state } \\
\text { excluded; Individuals } \\
\text { discharged from first, } \\
\text { second, or third hospital } \\
\text { admission from inpatient } \\
\text { psychiatric hospital with } \\
\text { diagnosis of manic episode } \\
\text { of bipolar disorder. } \\
\\
\text { Neurological Disorders; } \\
\text { Other Mental Health; } \\
\text { Labs/Other Conditions }\end{array}$ & \begin{tabular}{|l|} 
Specialized \\
outpatient care \\
including a medical \\
evaluation, treatment \\
plan, \\
pharmacological \\
treatment, group \\
sessions consisting \\
of psychoeducation \\
and discussions \\
about subjects' \\
experiences and a \\
discharge group \\
focused on \\
identifying early \\
warning sings and \\
communication of \\
signs to clinicians. \\
\\
-Specialized care for \\
2 years including 12 \\
sessions of \\
psychoeducation \\
(1.5 hours per \\
session) and $3-6$ \\
months of discharge \\
group \\
\end{tabular} & $\begin{array}{l}\text { Treatment as } \\
\text { usual: } \\
\text { Standard } \\
\text { outpatient } \\
\text { mental health } \\
\text { services } \\
\text { included } \\
\text { treatment with } \\
\text { a general } \\
\text { practitioner, } \\
\text { psychiatrist, or } \\
\text { community } \\
\text { mental health } \\
\text { center. }\end{array}$ & 24 months & $\begin{array}{l}\text { Readmissions } \\
\text { Relapse } \\
\text { Withdrawal 35\% }\end{array}$ \\
\hline
\end{tabular}




\begin{tabular}{|c|c|c|c|c|c|c|}
\hline $\begin{array}{l}\text { Study, Year } \\
\text { Design } \\
\text { Location } \\
\text { Funder } \\
\text { Risk of Bias } \\
\text { PMID }\end{array}$ & $\begin{array}{c}\text { Randomized (N) } \\
\text { Age (mean) } \\
\text { Sex (\% Female) } \\
\text { Race (\% White) } \\
\text { Diagnosis } \\
\text { (\% BP I, II, NOS) } \\
\text { Setting }\end{array}$ & $\begin{array}{c}\text { Inclusions } \\
\text { Key Exclusions }\end{array}$ & $\begin{array}{l}\text { Intervention } \\
\text { Description }\end{array}$ & $\begin{array}{l}\text { Comparison } \\
\text { Description }\end{array}$ & $\begin{array}{c}\text { Follow-up } \\
\text { Duration }\end{array}$ & $\begin{array}{c}\text { Outcomes } \\
\text { Reported } \\
\text { Withdrawal (\%) at } \\
\text { endpoint }\end{array}$ \\
\hline $\begin{array}{l}\text { Kilbourne, } 2012^{4} \\
\text { RCT } \\
\text { US } \\
\text { Government } \\
\text { Low } \\
23203358\end{array}$ & $\begin{array}{l}\mathrm{N}=68 \\
\text { Age } 43(18-71) \\
\text { Female 61\% } \\
\text { White } 78 \% \\
\text { BP NR } \\
\text { Outpatient }\end{array}$ & $\begin{array}{l}\text { No current clinical state } \\
\text { excluded; BP I, II or NOS } \\
\text { with one or more } \\
\text { cardiometabolic risk factor. } \\
\\
\text { Neurological Disorders; } \\
\text { Labs/Other Conditions }\end{array}$ & $\begin{array}{l}\text { Life Goals } \\
\text { Collaborative Care } \\
\text { consisting of weekly } \\
\text { group self- } \\
\text { management } \\
\text { sessions (mixture of } \\
\text { motivational } \\
\text { interviewing and } \\
\text { cognitive behavioral } \\
\text { techniques) with } \\
\text { care management } \\
\text { by interventionist } \\
\text { and providers } \\
\\
\text {-Four 2-hour } \\
\text { sessions of self- } \\
\text { management, } 6 \\
\text { months of care } \\
\text { management }\end{array}$ & $\begin{array}{l}\text { Enhanced } \\
\text { Treatment as } \\
\text { Usual: Usual } \\
\text { care and } \\
\text { monthly } \\
\text { mailings on } \\
\text { mental health } \\
\text { care and } \\
\text { referrals to } \\
\text { primary care } \\
\text { services }\end{array}$ & 12 months & $\begin{array}{l}\text { ISS Depression } \\
\text { ISS Mania } \\
\text { SF-12 Mental } \\
\text { SF-12 Physical } \\
\text { WHO-DAS } \\
\text { Withdrawal 4\% }\end{array}$ \\
\hline
\end{tabular}




\begin{tabular}{|c|c|c|c|c|c|c|}
\hline $\begin{array}{l}\text { Study, Year } \\
\text { Design } \\
\text { Location } \\
\text { Funder } \\
\text { Risk of Bias } \\
\text { PMID }\end{array}$ & $\begin{array}{c}\text { Randomized (N) } \\
\text { Age (mean) } \\
\text { Sex (\% Female) } \\
\text { Race (\% White) } \\
\text { Diagnosis } \\
\text { (\% BP I, II, NOS) } \\
\text { Setting }\end{array}$ & $\begin{array}{c}\text { Inclusions } \\
\text { Key Exclusions }\end{array}$ & $\begin{array}{l}\text { Intervention } \\
\text { Description }\end{array}$ & $\begin{array}{l}\text { Comparison } \\
\text { Description }\end{array}$ & $\begin{array}{l}\text { Follow-up } \\
\text { Duration }\end{array}$ & $\begin{array}{c}\text { Outcomes } \\
\text { Reported } \\
\text { Withdrawal (\%) at } \\
\text { endpoint }\end{array}$ \\
\hline $\begin{array}{l}\text { Kilbourne, } 2008^{5} \\
\text { RCT } \\
\text { US } \\
\text { Government and } \\
\text { Industry } \\
\text { Moderate } \\
18586993\end{array}$ & $\begin{array}{l}\text { N=61 } \\
\text { Age } 55(39-71) \\
\text { Female } 9 \% \\
\text { White } 90 \% \\
\text { BP I } 76 \% \\
\text { BP II } 7 \% \\
\text { BP NOS 17\% } \\
\text { Outpatient }\end{array}$ & $\begin{array}{l}\text { No current clinical state } \\
\text { excluded; BP I, II or NOS } \\
\text { with cardiovascular } \\
\text { disease-related risk factor } \\
\text { Substance Abuse; Other } \\
\text { Mental Health; Labs/Other } \\
\text { Conditions }\end{array}$ & $\begin{array}{l}\text { Bipolar disorder } \\
\text { medical care model } \\
\text { consisting of self- } \\
\text { management } \\
\text { (adapted fro Life } \\
\text { Goals Program) } \\
\text { education, care } \\
\text { management via } \\
\text { nurse care manager } \\
\text { who coordinated } \\
\text { with providers } \\
\text { regarding medical } \\
\text { and psychiatric care, } \\
\text { and guideline } \\
\text { implementation } \\
\text { training for providers } \\
\text {-Three sessions (2 } \\
\text { hours) of self- } \\
\text { management } \\
\text { program; } 6 \text { months } \\
\text { of care management }\end{array}$ & $\begin{array}{l}\text { Treatment as } \\
\text { usual: Routine } \\
\text { care (as } \\
\text { selected by } \\
\text { provider) } \\
\text { without self- } \\
\text { management } \\
\text { or care } \\
\text { management }\end{array}$ & 6 months & $\begin{array}{l}\text { ISS Depression } \\
\text { ISS Mania } \\
\text { SF-12 Mental } \\
\text { SF-12 Physical } \\
\text { WHO-DAS } \\
\text { Withdrawal 5\% }\end{array}$ \\
\hline $\begin{array}{l}\text { Bauer, } 2006^{6} \\
\text { RCT } \\
\text { US } \\
\text { Government } \\
\text { Low } \\
16816277\end{array}$ & $\begin{array}{l}\mathrm{N}=330 \\
\text { Age } 47(26-66) \\
\text { Female } 28 \% \\
\text { White } 29 \% \\
\text { BP I } 87 \% \\
\text { BP II } 13 \% \\
\text { Inpatient/Outpatient }\end{array}$ & $\begin{array}{l}\text { No current clinical state } \\
\text { excluded; BP I, II or NOS } \\
\text { (DSM-IV) identified during } \\
\text { acute hospitalization for } \\
\text { bipolar disorder. } \\
\text { Neurological Disorders; } \\
\text { Labs/Other Conditions }\end{array}$ & $\begin{array}{l}\text { Bipolar Disorders } \\
\text { Program including } \\
\text { psychoeducation via } \\
\text { the Life Goals } \\
\text { Program and care } \\
\text { team consisting of } \\
\text { nurse care } \\
\text { coordinator and } \\
\text { psychiatrist } \\
\\
-3 \text { years of care via } \\
\text { the program }\end{array}$ & $\begin{array}{l}\text { Treatment as } \\
\text { usual: } \\
\text { Treatment } \\
\text { based on } \\
\text { psychiatrist } \\
\text { choice }\end{array}$ & 3 years & $\begin{array}{l}\text { SF-36 Mental } \\
\text { SF-36 Physical } \\
\text { PSR Depression } \\
\text { PSR Mania } \\
\text { Withdrawal 7\% }\end{array}$ \\
\hline
\end{tabular}




\begin{tabular}{|c|c|c|c|c|c|c|}
\hline $\begin{array}{l}\text { Study, Year } \\
\text { Design } \\
\text { Location } \\
\text { Funder } \\
\text { Risk of Bias } \\
\text { PMID }\end{array}$ & $\begin{array}{c}\text { Randomized (N) } \\
\text { Age (mean) } \\
\text { Sex (\% Female) } \\
\text { Race (\% White) } \\
\text { Diagnosis } \\
\text { (\% BP I, II, NOS) } \\
\text { Setting }\end{array}$ & $\begin{array}{c}\text { Inclusions } \\
\text { Key Exclusions }\end{array}$ & $\begin{array}{l}\text { Intervention } \\
\text { Description }\end{array}$ & $\begin{array}{l}\text { Comparison } \\
\text { Description }\end{array}$ & $\begin{array}{l}\text { Follow-up } \\
\text { Duration }\end{array}$ & $\begin{array}{c}\text { Outcomes } \\
\text { Reported } \\
\text { Withdrawal (\%) at } \\
\text { endpoint }\end{array}$ \\
\hline $\begin{array}{l}\text { Simon, } 2005^{7} \\
\text { Simon, } 2006^{8} \\
\text { RCT } \\
\text { US } \\
\text { Government } \\
\text { Low/High } \\
\\
15842025 \\
16651507\end{array}$ & $\begin{array}{l}\mathrm{N}=441 \\
\text { Age } 44(20-68) \\
\text { Female } 68 \% \\
\text { White } 88 \% \\
\text { BP I } 51 \% \\
\text { BP II } 49 \% \\
\\
\text { Outpatient }\end{array}$ & $\begin{array}{l}\text { No current clinical state } \\
\text { excluded; BP I or II (DSM- } \\
\text { IV or treating psychiatrist) } \\
\\
\text { Neurological Disorders; } \\
\text { Labs/Other Conditions }\end{array}$ & $\begin{array}{l}\text { Systematic care } \\
\text { consisting of } \\
\text { structured initial } \\
\text { assessment and } \\
\text { planning, telephone } \\
\text { monitoring, } \\
\text { coordinated mental } \\
\text { health treatment } \\
\text { team, and } \\
\text { psychoeducation. } \\
\text {-Services offered for } \\
24 \text { months post- } \\
\text { randomization }\end{array}$ & $\begin{array}{l}\text { Treatment as } \\
\text { usual: } \\
\text { Services that } \\
\text { are normally } \\
\text { available } \\
\text { without any } \\
\text { additional care }\end{array}$ & 24 months & $\begin{array}{l}\text { Hospitalizations } \\
\text { Relapse } \\
\text { PSR Depression } \\
\text { PSR Mania } \\
\text { Withdrawal 6\% }\end{array}$ \\
\hline
\end{tabular}

Abbreviations: ASRM=Altman Self-Rating Mania Scale; BP=bipolar disorder; CGI=Clinical Global Impressions; DSM=Diagnostic and Statistical Manual of Mental Disorders; FAST-NL-P=Functioning Assessment Short Test; ISS=Internal States Scale; NOS=not otherwise specified; NR=not reported; PMID=PubMed Identification Number;

PSR=Psychiatric Status Rating; QIDS=Quick inventory of depression symptomatology; RCT=randomized controlled trial; SF-12=12-Item Short Form Health Survey; SF-36=36Item Short Form Health Survey; WHO-DAS=World Health Organization Disability Assessment Scale; WHO-QOL-bref=World Health Organization Quality of Life-short version 
Appendix Table L2. Summary risk of bias assessments: systematic or collaborative care vs. inactive comparators

\begin{tabular}{|c|c|c|}
\hline $\begin{array}{c}\text { Study } \\
\text { Funding Source } \\
\text { PMID }\end{array}$ & $\begin{array}{c}\text { Overall Risk of Bias } \\
\text { Assessment }\end{array}$ & Rationale \\
\hline $\begin{array}{l}\text { van der Voort, } 2015^{1} \\
\text { van der Voort, } \\
2015 b^{2} \\
\text { NR } \\
25792695 \\
25841077\end{array}$ & Low & No significant suspected biases. \\
\hline $\begin{array}{l}\text { Kessing, } 2013^{3} \\
\text { Non-Government } \\
23349295 \\
\end{array}$ & Low/High & $\begin{array}{l}\text { No suspected risk of bias for primary outcome of hospitalizations; however suspected attrition bias for all other } \\
\text { outcomes. }\end{array}$ \\
\hline $\begin{array}{l}\text { Kilbourne, } 2012^{4} \\
\text { Government } \\
23203358\end{array}$ & Low & No significant suspected biases. \\
\hline $\begin{array}{l}\text { Kilbourne, } 2008^{5} \\
\text { Government and } \\
\text { Industry } \\
18586993\end{array}$ & Moderate & Suspected attrition bias due to attrition rate and incomplete outcome reporting. \\
\hline $\begin{array}{l}\text { Bauer, } 2006^{6} \\
\text { Government } \\
16816277\end{array}$ & Low & No significant suspected biases. \\
\hline $\begin{array}{l}\text { Simon, } 2005^{7} \\
\text { Simon, } 2006^{8} \\
\text { Government } \\
15842025 \\
16651507\end{array}$ & Low/High (by outcome) & Suspected biases due to reporting of primary outcome (symptom scores). \\
\hline
\end{tabular}

Abbreviations: NR=not reported; PMID=PubMed Identification Number 
Appendix Table L3. Outcomes summary: systematic or collaborative care vs. inactive comparators

\begin{tabular}{|c|c|c|c|c|c|}
\hline $\begin{array}{l}\text { Study } \\
\text { PMID }\end{array}$ & Responder/Remitter & Symptom & Function & Other & $\overline{A E}$ \\
\hline $\begin{array}{l}\text { Van der Voort } \\
20151,2 \\
25792695 \\
25841077\end{array}$ & NR & $\begin{array}{l}\text { Depression* } \\
6 \text { months, QIDS } \\
\text { NS; } p=0.20 \\
12 \text { months, QIDS } \\
\text { Favors Intervention } \\
P=0.004 ; \text { ES=0.40 } \\
\text { Mania* } \\
6 \text { months, ASRM } \\
\text { NS; } p=0.30 \\
12 \text { months, ASRM } \\
\text { NS; } p=0.80\end{array}$ & $\begin{array}{l}\text { Global Function* } \\
6 \text { months, FAST-NL-P } \\
\text { NS; } p=0.06 \\
12 \text { months, FAST-NL- } \\
\text { P } \\
\text { Favors Intervention } \\
p=0.01 \\
\text { Quality of Life* } \\
6 \text { months, WHO-Qol- } \\
\text { bref } \\
\text { NS } \\
\text { ES=-0.20 }(95 \% \mathrm{Cl} \text { - } \\
0.55,0.15) \\
12 \text { months, WHO-Qol- } \\
\text { bref } \\
\text { NS } \\
\text { ES=0.12 }(95 \% \mathrm{Cl}- \\
0.23,0.46)\end{array}$ & NR & NR \\
\hline $\begin{array}{l}\text { Kessing } 20133 \\
23349295\end{array}$ & $\begin{array}{l}\text { Relapse* }^{*} \\
2 \text { Years, Depressive } \\
\text { NS } \\
\text { OR=0.70 (95\% Cl 0.35, } \\
1.41) ; p=0.33 \\
2 \text { Years, Hypo/manic } \\
\text { NS } \\
\text { OR=1.26 (95\% Cl 0.63, } \\
2.51) ; p=0.52\end{array}$ & NR & NR & $\begin{array}{l}\text { Readmissions } \\
2-3 \text { years } \\
\text { Favors intervention } \\
\text { Number of } \\
\text { Readmissions (\%) } \\
\text { Intervention: } 26 \\
(36.1 \%), \text { Comparator: } \\
47(54.7 \%)\end{array}$ & NR \\
\hline
\end{tabular}




\begin{tabular}{|c|c|c|c|c|c|}
\hline $\begin{array}{l}\text { Study } \\
\text { PMID }\end{array}$ & Responder/Remitter & Symptom & Function & Other & $\mathrm{AE}$ \\
\hline $\begin{array}{l}\text { Kilbourne } 20124 \\
23203358\end{array}$ & NR & $\begin{array}{l}\text { Depression* } \\
6-12 \text { months, ISS Depression } \\
\text { NS; } p=0.15 \\
\text { Mania* } \\
6-12 \text { months, ISS Mania } \\
\text { NS; } p=0.68\end{array}$ & $\begin{array}{l}\text { Mental Function* } \\
6 \text { months, SF-12 } \\
\text { Mental } \\
\text { NS } \\
\text { ES }=0.13(95 \% \mathrm{Cl} \text { - } \\
0.36,0.62) \\
12 \text { months, SF-12 } \\
\text { Mental } \\
\text { NS } \\
\text { ES }=0.36(95 \% \mathrm{Cl} \text { - } \\
0.13,0.85) \\
\text { Physical Function* } \\
6 \text { months, SF-12 } \\
\text { Physical } \\
\text { NS } \\
\text { ES=-0.10 (95\% Cl - } \\
0.58,0.39) \\
12 \text { months, SF-12 } \\
\text { Physical } \\
\text { NS } \\
\text { ES=0.21 (95\% Cl - } \\
0.28,0.70) \\
\text { Health and Disability* } \\
6 \text { months, WHO-DAS } \\
\text { NS } \\
\text { ES=-0.44 (95\% Cl - } \\
0.93,0.06) \\
12 \text { months, WHO-DAS } \\
\text { Favors Intervention } \\
\text { ES=-0.56 (95\% Cl - } \\
1.05,-0.06)\end{array}$ & NR & NR \\
\hline
\end{tabular}




\begin{tabular}{|c|c|c|c|c|c|}
\hline $\begin{array}{l}\text { Study } \\
\text { PMID }\end{array}$ & Responder/Remitter & Symptom & Function & Other & $\mathrm{AE}$ \\
\hline $\begin{array}{l}\text { Kilbourne } 20085 \\
18586993\end{array}$ & NR & $\begin{array}{l}\text { Depression* } \\
6 \text { months, ISS Depression } \\
\text { NS } \\
\text { ES }=0.00(95 \% \mathrm{Cl}-0.52,0.52) \\
\text { Mania* }^{*} \\
6 \text { months, ISS Mania } \\
\text { NS } \\
\text { ES }=0.14(95 \% \mathrm{Cl}-0.38,0.66)\end{array}$ & $\begin{array}{l}\text { Mental Function* } \\
6 \text { months, SF-12 } \\
\text { Mental } \\
\text { NS } \\
\text { ES }=0.40(95 \% \mathrm{Cl} \text { - } \\
0.12,0.92) \\
\text { Physical Function* } \\
6 \text { months, SF-12 } \\
\text { Physical } \\
\text { NS } \\
\text { ES }=0.25(95 \% \mathrm{Cl} \text { - } \\
0.27,0.77) \\
\\
\text { Health and Disability* } \\
6 \text { months, WHO-DAS } \\
\text { NS } \\
\text { ES }=0.18 \text { (95\% Cl - } \\
0.33,0.70)\end{array}$ & NR & NR \\
\hline $\begin{array}{l}\text { Bauer } 20066 \\
16816277\end{array}$ & NR & $\begin{array}{l}\text { Depression } \\
3 \text { years, PSR Depression } \\
\text { NS; } p=0.23 \\
E S=0.17 \\
\text { Mania } \\
3 \text { years, PSR Mania } \\
\text { NS; } p=0.16 \\
E S=0.16\end{array}$ & $\begin{array}{l}\text { Mental Function* } \\
3 \text { years, SF-36 } \\
\text { Favors Intervention } \\
\text { ES=0.51 }(95 \% \mathrm{Cl} 0.29 \text {, } \\
0.73) \\
\\
\text { Physical Function* } \\
3 \text { years, SF-36 } \\
\text { NS } \\
\text { ES }=0.08(95 \% \mathrm{Cl}- \\
0.14,0.30)\end{array}$ & $\begin{array}{l}\text { Deaths } \\
\text { NS } \\
3 \text { years } \\
\text { Intervention: } 7 \% \\
\text { Control: } 5 \% \text { (one } \\
\text { suicide) }\end{array}$ & NR \\
\hline
\end{tabular}




\begin{tabular}{|c|c|c|c|c|c|}
\hline $\begin{array}{l}\text { Study } \\
\text { PMID }\end{array}$ & Responder/Remitter & Symptom & Function & Other & $\mathrm{AE}$ \\
\hline $\begin{array}{l}\text { Simon } 2005 \\
20067,8 \\
15842025 \\
16651507\end{array}$ & $\begin{array}{l}\text { Relapse } \\
12 \text { months, Depressive } \\
\text { NS } \\
\text { OR=1.00 (95\% Cl 0.60, } \\
1.67) ; p=1.00 \\
12 \text { months, Hypo/manic } \\
\text { NS } \\
\text { OR=0.64 (95\% Cl 0.39, } \\
1.06) ; p=0.07\end{array}$ & $\begin{array}{l}\text { Depression } \\
12 \text { months, PSR Depression } \\
\text { Favors intervention; } p=0.04 \\
24 \text { months, PSR Depression } \\
\text { NS; } p=0.52 \\
\text { Mania } \\
12 \text { months, PSR Mania } \\
\text { NS; } 0.70 \\
24 \text { months, PSR Mania } \\
\text { Favors intervention; } p=0.04\end{array}$ & NR & $\begin{array}{l}\text { Hospitalizations } \\
12 \text { months } \\
\text { NS; } p=0.29 \\
24 \text { months } \\
\text { NS; } p=0.91\end{array}$ & NR \\
\hline
\end{tabular}

Abbreviations: ASRM=Altman Self-Rating Mania Scale; CI=Confidence Interval; ES=effect size; FAST-NL-P=Functioning Assessment Short Test; ISS=Internal States Scale; NR=not reported; NS=not significant; OR=Odds Ratio; PMID=PubMed Identification Number; PSR= Psychiatric status rating; QIDS=Quick inventory of depression scores; SF12=12-item Short Form Health Survey; WHO-DAS=World Health Organization Disability Assessment Scale; WHO-QOL-bref=World Health Organization Quality of Life-Short version 
Appendix Table L4. Summary of strength of evidence: systematic or collaborative care vs. inactive comparators

\begin{tabular}{|c|c|c|c|c|c|c|c|c|}
\hline Comparator & Timing & $\begin{array}{l}\text { \# Studies/ } \\
\text { Design } \\
\text { (n analyzed) }\end{array}$ & $\begin{array}{l}\text { Finding or } \\
\text { Summary Statistic }\end{array}$ & $\begin{array}{c}\text { Study } \\
\text { Limitations }\end{array}$ & Consistency & Directness & Precision & $\begin{array}{l}\text { Overall Gradel } \\
\text { Conclusion }\end{array}$ \\
\hline Relapse & $\begin{array}{l}7-12 \text { months } \\
12+\text { months }\end{array}$ & 2 RCTs (n=599) & $\begin{array}{l}\text { No difference in } \\
\text { outcome between } \\
\text { groups across } \\
\text { different time } \\
\text { periods. }\end{array}$ & Moderate & Consistent & Direct & Imprecise & $\begin{array}{l}\text { Low; No effect from } \\
\text { the intervention }\end{array}$ \\
\hline Depression & $\begin{array}{l}6 \text { months } \\
7-12 \text { months } \\
12+\text { months }\end{array}$ & 5 RCTs $(n=1,038)$ & $\begin{array}{l}\text { Mixed evidence with } \\
\text { no clear direction of } \\
\text { effect. } \\
\text { No pattern across } \\
\text { time periods. }\end{array}$ & Moderate & Inconsistent & Direct & Imprecise & Insufficient \\
\hline Mania & $\begin{array}{l}6 \text { months } \\
7-12 \text { months } \\
12+\text { months }\end{array}$ & 5 RCTs $(n=1,038)$ & $\begin{array}{l}\text { No difference in } \\
\text { outcome between } \\
\text { groups across } \\
\text { different time } \\
\text { periods. }\end{array}$ & Moderate & Inconsistent & Direct & Imprecise & Insufficient \\
\hline $\begin{array}{l}\text { Global } \\
\text { Function }\end{array}$ & $\begin{array}{l}6 \text { months } \\
7-12 \text { months }\end{array}$ & $1 \mathrm{RCT}(\mathrm{n}=138)$ & $\begin{array}{l}\text { Mixed evidence with } \\
\text { no clear direction of } \\
\text { effect. } \\
\text { No pattern across } \\
\text { time periods. } \\
6 \text { months, FAST- } \\
\text { NL-P } \\
\text { NS; } p=0.06 \\
12 \text { months, FAST- } \\
\text { NL-P } \\
\text { Favors Intervention; } \\
\text { p=0.01 }\end{array}$ & Low & Unclear & Direct & Imprecise & Insufficient \\
\hline $\begin{array}{l}\text { Other } \\
\text { Measures of } \\
\text { Function }\end{array}$ & $\begin{array}{l}6 \text { months } \\
7-12 \text { months }\end{array}$ & 4 RCTs $(n=597)$ & $\begin{array}{l}\text { No difference in } \\
\text { outcome between } \\
\text { groups across } \\
\text { different time } \\
\text { periods. }\end{array}$ & Low & Inconsistent & Direct & Imprecise & Insufficient \\
\hline
\end{tabular}

* It is difficult to establish a level of precision that provides confidence of no effect. Due to the large number of comparisons with findings of no effect, we assessed strength of evidence cautiously when there was imprecision, only assigning low strength of evidence when there was sufficient sample size, low to moderate study limitations, and consistency

FAST-NL-P=Functiong Assessment Short Test; NS=not significant; RCT=randomized controlled trial 


\section{References for Appendix L}

1. van der Voort TY, van Meijel B, Goossens PJ, et al. Collaborative care for patients with bipolar disorder: randomised controlled trial. $\mathrm{Br} \mathrm{J}$ Psychiatry. 2015 Mar 19doi: 10.1192/bjp.bp.114.152520. PMID: 25792695.

2. van der Voort TY, van Meijel B, Hoogendoorn AW, et al. Collaborative care for patients with bipolar disorder: Effects on functioning and quality of life. J Affect Disord. 2015 Mar 11;179(1):14-22. doi: 10.1016/j.jad.2015.03.005. PMID: 25841077.

3. Kessing LV, Hansen HV, Hvenegaard A, et al. Treatment in a specialised out-patient mood disorder clinic v. standard out-patient treatment in the early course of bipolar disorder: randomised clinical trial. British Journal of Psychiatry. 2013 Mar;202(3):212-9. doi: http://dx.doi.org/10.1192/bjp.bp.112.113548. PMID: 23349295.

4. Kilbourne AM, Goodrich DE, Lai Z, et al. Life Goals Collaborative Care for patients with bipolar disorder and cardiovascular disease risk. Psychiatric Services. 2012 Dec;63(12):1234-8. doi: http://dx.doi.org/10.1176/appi.ps.201100528. PMID: 23203358.
5. Kilbourne AM, Post EP, Nossek A, et al. Improving medical and psychiatric outcomes among individuals with bipolar disorder: a randomized controlled trial. Psychiatric Services. 2008 Jul;59(7):760-8. doi:

http://dx.doi.org/10.1176/appi.ps.59.7.760. PMID: 18586993.

6. Bauer MS, McBride L, Williford WO, et al. Collaborative care for bipolar disorder: Part II. Impact on clinical outcome, function, and costs. Psychiatric Services. 2006 Jul;57(7):937-45. PMID: 16816277.

7. Simon GE, Ludman EJ, Unutzer J, et al. Randomized trial of a population-based care program for people with bipolar disorder. Psychological Medicine. 2005 Jan;35(1):13-24. PMID: 15842025.

8. Simon GE, Ludman EJ, Bauer MS, et al. Longterm effectiveness and cost of a systematic care program for bipolar disorder. Archives of General Psychiatry. 2006 May;63(5):500-8. PMID: 16651507. 


\section{Appendix M. Family or Partner Interventions}

Appendix Table M1. Characteristics of eligible studies: family or partner interventions vs. inactive comparator

\begin{tabular}{|c|c|c|c|c|c|c|}
\hline $\begin{array}{c}\text { Study, Year } \\
\text { Design } \\
\text { Location } \\
\text { Funder } \\
\text { Risk of Bias } \\
\text { PMID }\end{array}$ & $\begin{array}{c}\text { Randomized (N) } \\
\text { Age (mean) } \\
\text { Sex (\% Female) } \\
\text { Race (\% White) } \\
\text { Diagnosis } \\
\text { (\% BP I, II, NOS) } \\
\text { Setting }\end{array}$ & $\begin{array}{c}\text { Inclusions } \\
\text { Key Exclusions }\end{array}$ & $\begin{array}{l}\text { Intervention } \\
\text { Description }\end{array}$ & $\begin{array}{l}\text { Comparison } \\
\text { Description }\end{array}$ & $\begin{array}{l}\text { Follow-up } \\
\text { Duration }\end{array}$ & $\begin{array}{c}\text { Outcomes } \\
\text { Reported } \\
\begin{array}{c}\text { Withdrawal (\%) at } \\
\text { endpoint }\end{array}\end{array}$ \\
\hline $\begin{array}{l}\text { D'Souza, } 2010^{1} \\
\text { RCT } \\
\text { Australia } \\
\text { Non-Government } \\
\text { Low } \\
19428117\end{array}$ & $\begin{array}{l}\mathrm{N}=58 \\
\text { Age } 41(19-60) \\
\text { Female 52\% } \\
\text { White NR } \\
\text { BP I } 86 \% \\
\text { BP II 14\% } \\
\text { Outpatient }\end{array}$ & $\begin{array}{l}\text { Euthymic/Maintenance: } \\
\text { Recently remitted patients } \\
\text { with a YMRS score }<10 \\
\text { and a MADRS score }<8 \\
\text { recruited within one month } \\
\text { of discharge from hospital } \\
\text { for bipolar relapse (MINI). } \\
\\
\text { Substance Abuse; Other } \\
\text { Mental Health; Labs/Other } \\
\text { Conditions }\end{array}$ & \begin{tabular}{|l|} 
Patient/companion \\
group \\
psychoeducation \\
consisting of \\
discussion of \\
symptoms, \\
medications, and \\
warning signs, and \\
resources as well as \\
psychotherapy \\
-12 weekly \\
sessions, 90 \\
minutes each \\
session
\end{tabular} & $\begin{array}{l}\text { Treatment as } \\
\text { usual: Community } \\
\text { based case } \\
\text { management } \\
\text { involving weekly } \\
\text { review with a } \\
\text { mental health } \\
\text { clinician and a } \\
\text { monthly medical } \\
\text { review } \\
\text {-Weekly sessions } \\
\text { for } 45 \text { minutes }\end{array}$ & 15 months & \begin{tabular}{|l} 
MADRS \\
YMRS \\
Relapse (BP) \\
symptoms requiring \\
hospital admission or \\
intensive community \\
psychiatric \\
intervention) \\
Time to relapse \\
Withdrawal 22\%
\end{tabular} \\
\hline
\end{tabular}




\begin{tabular}{|c|c|c|c|c|c|c|}
\hline $\begin{array}{l}\text { Study, Year } \\
\text { Design } \\
\text { Location } \\
\text { Funder } \\
\text { Risk of Bias } \\
\text { PMID }\end{array}$ & $\begin{array}{c}\text { Randomized (N) } \\
\text { Age (mean) } \\
\text { Sex (\% Female) } \\
\text { Race (\% White) } \\
\text { Diagnosis } \\
\text { (\% BP I, II, NOS) } \\
\text { Setting }\end{array}$ & $\begin{array}{c}\text { Inclusions } \\
\text { Key Exclusions }\end{array}$ & $\begin{array}{l}\text { Intervention } \\
\text { Description }\end{array}$ & $\begin{array}{l}\text { Comparison } \\
\text { Description }\end{array}$ & $\begin{array}{l}\text { Follow-up } \\
\text { Duration }\end{array}$ & $\begin{array}{c}\text { Outcomes } \\
\text { Reported } \\
\begin{array}{c}\text { Withdrawal (\%) at } \\
\text { endpoint }\end{array}\end{array}$ \\
\hline $\begin{array}{l}\text { Miller } 2004^{2} \\
\text { Solomon } 2008^{3} \\
\text { Miller } 2008^{4} \\
\text { RCT } \\
\text { US } \\
\text { Government } \\
\text { High } \\
\text { High } \\
\text { Moderate } \\
15555694 \\
19032711 \\
18363424\end{array}$ & $\begin{array}{l}\mathrm{N}=92 \\
\text { Age } 39(18-65) \\
\text { Female } 57 \% \\
\text { White NR } \\
\text { BP I 100\% } \\
\text { Outpatient }\end{array}$ & $\begin{array}{l}\text { Current Episode: } \\
\text { Inpatients, partial } \\
\text { inpatients, or outpatients } \\
\text { with BP I (DSM-III), a } \\
\text { current episode (mania, } \\
\text { depression, mixed) without } \\
\text { alcohol or drug } \\
\text { dependence within the past } \\
12 \text { months and living or in } \\
\text { regular contact with a } \\
\text { relative or significant other } \\
\text { Substance abuse }\end{array}$ & \begin{tabular}{|l|} 
Individual or group \\
family therapy \\
consisting of semi- \\
structured family \\
interventions. \\
Individual therapy \\
was based on \\
McMaster Model of \\
Family Function and \\
group therapy \\
included sessions \\
focused on signs \\
and symptoms, \\
patient and family \\
perspectives, and \\
coping mechanisms. \\
-6 to 10 sessions of \\
family therapy, 50 \\
minutes per session \\
OR \\
-6 weekly group \\
sessions, 90 \\
minutes per session
\end{tabular} & $\begin{array}{l}\text { Pharmacotherapy: } \\
\text { Mood stabilizer } \\
\text { with other } \\
\text { medications as } \\
\text { necessary }\end{array}$ & 28 months & $\begin{array}{l}\text { Recovery (Two } \\
\text { consecutive months } \\
\text { with BRMS }<6 \text { and } \\
\text { HDRS }<7 \text { ) } \\
\text { Relapse (HDRS 17- } \\
\text { item score }>15 \text { or } \\
\text { BRMS score }>9 \text { after } \\
\text { recovery) } \\
\text { Time to recurrence } \\
\text { Hospitalizations } \\
\text { Withdrawal } 35 \%\end{array}$ \\
\hline
\end{tabular}

Abbreviations: BP=bipolar disorder; BRMS=Bech-Rafaelsen Mania Scale; DSM=Diagnostic and Statistical Manual of Mental Disorders; HDRS=Hamilton Depression Rating Scale; MADRS=Montgomery-Asberg Depression Rating Scale; MINI=MINI International Nueropsychiatric Interview; NOS=not otherwise specified; NR=not reported; PMID=PubMed Identification Number; RCT=randomized controlled trial; YMRS = Young Mania Rating Scale 
Appendix Table M2. Summary risk of bias assessments: family or partner interventions vs. inactive comparators

\begin{tabular}{|c|c|c|}
\hline $\begin{array}{l}\text { Study } \\
\text { Funder } \\
\text { PMID }\end{array}$ & $\begin{array}{c}\text { Overall Risk of Bias } \\
\text { Assessment }\end{array}$ & Rationale \\
\hline $\begin{array}{l}\text { D'Souza, } 2010^{1} \\
\text { Non-Government } \\
19428117\end{array}$ & Low & No significant suspected biases. \\
\hline $\begin{array}{l}\text { Miller } 2008^{4} \\
\text { Government } \\
15555694\end{array}$ & High & Suspected bias due to unclear reporting of randomization and attrition. \\
\hline $\begin{array}{l}\text { Solomon } 2008^{3} \\
\text { Government } \\
19032711 \\
\end{array}$ & High & Suspected bias due to unclear reporting of randomization and attrition. \\
\hline $\begin{array}{l}\text { Miller } 2004^{2} \\
\text { Government } \\
18363424 \\
\end{array}$ & Moderate & Suspected bias due to attrition rate of $35 \%$. \\
\hline
\end{tabular}

Abbreviations: PMID=PubMed Identification Number

Appendix Table M3. Outcomes summary: family or partner interventions vs. inactive comparators

\begin{tabular}{|c|c|c|c|c|c|}
\hline $\begin{array}{c}\text { Study } \\
\text { PMID } \\
\text { Risk of Bias }\end{array}$ & Responder/Remitter & Symptom & Function & Other & AE \\
\hline $\begin{array}{l}\text { D'Souza, } 2010^{2} \\
19428117 \\
\text { Low }\end{array}$ & $\begin{array}{l}\frac{\text { Relapse }^{*}}{15 \text { months, Any Type }} \\
\text { Favors FPI } \\
\text { OR=0.17 (95\% Cl 0.03, } \\
0.78) ; p=0.02\end{array}$ & $\begin{array}{l}\text { Depression* } \\
15 \text { months, MADRS } \\
\text { NS } \\
\text { ES }=-0.15(95 \% \mathrm{Cl}-0.66,0.37) \\
\frac{\text { Mania* }^{*}}{15 \text { months, YMRS }} \\
\text { Favors FPI } \\
\text { ES }=-0.78(95 \% \mathrm{Cl}-1.31,-0.24)\end{array}$ & NR & NR & NR \\
\hline
\end{tabular}




\begin{tabular}{|c|c|c|c|c|c|}
\hline $\begin{array}{c}\text { Study } \\
\text { PMID } \\
\text { Risk of Bias }\end{array}$ & Responder/Remitter & Symptom & Function & Other & $\mathrm{AE}$ \\
\hline $\begin{array}{l}\text { Miller } 20084 \\
19032711 \\
\text { High } \\
\text { Solomon } 20083 \\
18363424 \\
\text { Moderate } \\
\text { Miller } 20042 \\
15555694 \\
\text { High }\end{array}$ & $\begin{array}{l}\text { Relapse* } \\
15 \text { months, Any Type } \\
\text { NS } \\
\text { Individual Therapy } \\
\text { OR=1.32 (95\% Cl } 0.24 \text {, } \\
7.34) ; p=0.50 \\
\text { Group Therapy OR=0.98 } \\
\text { (95\% Cl 0.20, 4.51); } \\
\text { p=0.62 } \\
\text { Recovery } \\
28 \text { months } \\
\text { NS; } p=0.21 \\
\text { Number Recovered } \\
\text { Individual Therapy: } 16.0 \\
\text { Group Therapy: } 21.0 \\
\text { Comparator: } 16.0 \\
\text { Time to Recurrence } \\
28 \text { months } \\
\text { NS; } p=0.75 \\
\text { Months (Median) } \\
\text { Individual Therapy: } 6.0 \\
\text { Group Therapy: } 8.0 \\
\text { Comparator: } 12.0 \\
\text { Time to Recovery } \\
28 \text { months } \\
\text { NS; } p=0.55 \\
\text { Months (Median) } \\
\text { Individual Therapy: } 10 \\
\text { Group Therapy: } 7 \\
\text { Comparator: } 8\end{array}$ & NR & NR & $\begin{array}{l}\text { Hospitalizations } \\
28 \text { months } \\
\text { Favors FPI; } p=0.04 \\
\text { Number of Hospitalizations } \\
\text { Individual Therapy: } 5 \\
\text { Group Therapy: } 1 \\
\text { Comparator: } 6\end{array}$ & NR \\
\hline
\end{tabular}

Abbreviations: CI=Confidence Interval; ES=effect size; FPI=family and partner interventions; MADRS=Montgomery-Asberg Depression Rating Scale; NR=not reported; NS=not significant; OR=Odds Ratio; PMID=PubMed Identification Number; YMRS = Young Mania Rating Scale 
Appendix Table M4. Summary of strength of evidence: family or partner interventions vs. inactive comparators

\begin{tabular}{|c|c|c|c|c|c|c|c|c|}
\hline Outcome & Timing & $\begin{array}{l}\text { \# Studies/ Design } \\
\text { (n analyzed) }\end{array}$ & $\begin{array}{c}\text { Finding or } \\
\text { Summary } \\
\text { Statistic }\end{array}$ & $\begin{array}{c}\text { Study } \\
\text { Limitations }\end{array}$ & Consistency & Directness & Precision & $\begin{array}{l}\text { Overall Gradel } \\
\text { Conclusion }\end{array}$ \\
\hline Relapse & $\begin{array}{l}12+ \\
\text { months }\end{array}$ & $\begin{array}{l}2 \text { RCTs } \\
(n=150)\end{array}$ & $\begin{array}{l}\text { Mixed evidence } \\
\text { with no clear } \\
\text { direction of effect. } \\
\text { No pattern across } \\
\text { time periods. }\end{array}$ & Low & Inconsistent & Direct & Imprecise & Insufficient \\
\hline Depression & $\begin{array}{l}12+ \\
\text { months }\end{array}$ & $\begin{array}{l}1 \mathrm{RCT} \\
(\mathrm{n}=58)\end{array}$ & $\begin{array}{l}\text { No difference } \\
\text { between groups at } \\
15 \text { months. } \\
\text { MADRS } \\
\text { ES=-0.15 (95\% Cl } \\
-0.66,0.37)\end{array}$ & Low & Unclear & Direct & Imprecise & Insufficient \\
\hline Mania & $\begin{array}{l}12+ \\
\text { months }\end{array}$ & $\begin{array}{l}1 \mathrm{RCT} \\
(\mathrm{n}=58)\end{array}$ & $\begin{array}{l}\text { Favors FPI at } 15 \\
\text { months. } \\
\text { YMRS } \\
\text { ES=-0.78 (95\% Cl } \\
-1.31,-0.24)\end{array}$ & Low & Unclear & Direct & Imprecise & Insufficient \\
\hline $\begin{array}{l}\text { Global } \\
\text { Function }\end{array}$ & NR & - & - & - & - & - & - & - \\
\hline $\begin{array}{l}\text { Other } \\
\text { Measures } \\
\text { of Function }\end{array}$ & NR & - & - & - & - & - & - & - \\
\hline
\end{tabular}

Abbreviations: CI=Confidence Interval; ES=effect size; FPI=family and partner interventions; MADRS=Montgomery-Asberg Depression Rating Scale; NR=not reported;

RCT=Randomized Control Trial; YMRS = Young Mania Rating Scale 


\begin{tabular}{|c|c|c|c|c|c|c|}
\hline $\begin{array}{c}\text { Study, Year } \\
\text { Design } \\
\text { Location } \\
\text { Funder } \\
\text { Risk of Bias } \\
\text { PMID }\end{array}$ & $\begin{array}{c}\text { Randomized (N) } \\
\text { Age (mean) } \\
\text { Sex (\% Female) } \\
\text { Race (\% White) } \\
\text { Diagnosis } \\
\text { (\% BP I, II, NOS) } \\
\text { Setting }\end{array}$ & $\begin{array}{l}\text { Inclusions } \\
\text { Key Exclusions }\end{array}$ & $\begin{array}{l}\text { Intervention } \\
\text { Description }\end{array}$ & $\begin{array}{l}\text { Comparison } \\
\text { Description }\end{array}$ & $\begin{array}{l}\text { Follow-up } \\
\text { Duration }\end{array}$ & $\begin{array}{c}\text { Outcomes } \\
\text { Reported } \\
\text { Withdrawal (\%) at } \\
\text { endpoint }\end{array}$ \\
\hline $\begin{array}{l}\text { Wenze, } 2015^{5} \\
\text { RCT } \\
\text { US } \\
\text { Government and Non- } \\
\text { Government } \\
\text { Moderate } \\
26117247\end{array}$ & $\begin{array}{l}\mathrm{N}=30 \\
\text { Age } 47(24-68) \\
\text { Female } 50 \% \\
\text { White } 90 \% \\
\text { BP I } 77 \% \\
\text { BP II } 13 \% \\
\text { BP NOS 10\% } \\
\text { Inpatient/Outpatient }\end{array}$ & $\begin{array}{l}\text { No current clinical state } \\
\text { excluded; Inpatients or at- } \\
\text { risk outpatients diagnosed } \\
\text { with BP I, II, or NOS (DSM- } \\
\text { IV) and drug/alcohol abuse } \\
\text { disorder (DSM-IV) with a } \\
\text { current prescription for a } \\
\text { mood stabilizing medication } \\
\text { and access to a telephone } \\
\\
\text { Other Mental Health; } \\
\text { Pregnant/Nursing; } \\
\text { Labs/Other Conditions }\end{array}$ & $\begin{array}{l}\text { Integrated Treatment } \\
\text { Adherence Program } \\
\text { based on a cognitive } \\
\text { behavioral approach } \\
\text { focused on } \\
\text { transitioning patients } \\
\text { from acute to } \\
\text { maintenance care } \\
\text { using patient and } \\
\text { family or significant } \\
\text { other meetings in } \\
\text { person and via } \\
\text { telephone. } \\
\text {-3 individual in- } \\
\text { person sessions, } 60 \\
\text { minutes per session; } \\
\text { a } 60 \text { minute in- } \\
\text { person session with } \\
\text { family session, and } \\
11 \text { phone contacts } \\
\text { held separately with } \\
\text { subject and } \\
\text { designated family } \\
\text { member or } \\
\text { significant other }\end{array}$ & $\begin{array}{l}\text { Enhanced } \\
\text { Assessment } \\
\text { and Monitoring } \\
\text { consisting of } \\
\text { treatment as } \\
\text { usual with } \\
\text { enhanced } \\
\text { monitoring } \\
\text { (battery of } \\
\text { interview-rated } \\
\text { and self-report } \\
\text { assessments } \\
\text { followed by } \\
\text { feedback } \\
\text { letters) }\end{array}$ & 6 months & $\begin{array}{l}\text { QIDS-C } \\
\text { CARS-M } \\
\text { WHO-DAS } \\
\text { Hospitalizations } \\
\text { ER Visits } \\
\text { Withdrawal 27\% }\end{array}$ \\
\hline
\end{tabular}




\begin{tabular}{|c|c|c|c|c|c|c|}
\hline $\begin{array}{l}\text { Study, Year } \\
\text { Design } \\
\text { Location } \\
\text { Funder } \\
\text { Risk of Bias } \\
\text { PMID }\end{array}$ & $\begin{array}{c}\text { Randomized (N) } \\
\text { Age (mean) } \\
\text { Sex (\% Female) } \\
\text { Race (\% White) } \\
\text { Diagnosis } \\
\text { (\% BP I, II, NOS) } \\
\text { Setting }\end{array}$ & $\begin{array}{c}\text { Inclusions } \\
\text { Key Exclusions }\end{array}$ & $\begin{array}{l}\text { Intervention } \\
\text { Description }\end{array}$ & $\begin{array}{l}\text { Comparison } \\
\text { Description }\end{array}$ & $\begin{array}{c}\text { Follow-up } \\
\text { Duration }\end{array}$ & $\begin{array}{c}\text { Outcomes } \\
\text { Reported } \\
\text { Withdrawal (\%) at } \\
\text { endpoint }\end{array}$ \\
\hline $\begin{array}{l}\text { Miklowitz, } 2000^{6} \\
\text { Miklowitz, } 2003^{7} \\
\text { RCT } \\
\text { US } \\
\text { Government and Non- } \\
\text { Government } \\
\text { Low } \\
\text { Low } \\
11018229 \\
12963672\end{array}$ & $\begin{array}{l}\mathrm{N}=101 \\
\text { Age } 36(18-56) \\
\text { Female } 63 \% \\
\text { White NR } \\
\text { BP I 100\% } \\
\text { Inpatient/Outpatient }\end{array}$ & $\begin{array}{l}\text { No current clinical state } \\
\text { excluded: Inpatients with } \\
\text { BP I (DSM-III) who had } \\
\text { experienced a depressed, } \\
\text { manic, or mixed episode in } \\
\text { the past } 3 \text { months living } \\
\text { with or having regular } \\
\text { contact with close relatives } \\
\text { Substance Abuse; } \\
\text { Neurological Disorders; } \\
\text { Labs/Other Conditions }\end{array}$ & $\begin{array}{l}\text { Family-focused } \\
\text { therapy with } \\
\text { pharmacotherapy } \\
\text { consisting of } \\
\text { psychoeducation, } \\
\text { developing } \\
\text { communication } \\
\text { skills, and learning a } \\
\text { framework for } \\
\text { defining problems } \\
\text { and implementing } \\
\text { solutions. } \\
\\
\text {-Uo to } 21 \text { family or } \\
\text { martial sessions } \\
\text { over } 9 \text { months, } 60 \\
\text { minutes per session }\end{array}$ & $\begin{array}{l}\text { Family } \\
\text { education (2 } \\
\text { sessions) and } \\
\text { crisis } \\
\text { management } \\
\text { consisting of } \\
\text { treatment as } \\
\text { usual with } \\
\text { emergency } \\
\text { counseling } \\
\text { sessions as } \\
\text { needed and } \\
\text { monthly } \\
\text { telephone calls } \\
\text { with patient }\end{array}$ & 24 months & $\begin{array}{l}\text { SADS-C Depression } \\
\text { SADS-C Mania } \\
\text { Relapse (NR) } \\
\text { Withdrawal } 22 \%\end{array}$ \\
\hline
\end{tabular}




\begin{tabular}{|c|c|c|c|c|c|c|}
\hline $\begin{array}{l}\text { Study, Year } \\
\text { Design } \\
\text { Location } \\
\text { Funder } \\
\text { Risk of Bias } \\
\text { PMID }\end{array}$ & $\begin{array}{c}\text { Randomized (N) } \\
\text { Age (mean) } \\
\text { Sex (\% Female) } \\
\text { Race (\% White) } \\
\text { Diagnosis } \\
\text { (\% BP I, II, NOS) } \\
\text { Setting }\end{array}$ & $\begin{array}{c}\text { Inclusions } \\
\text { Key Exclusions }\end{array}$ & $\begin{array}{l}\text { Intervention } \\
\text { Description }\end{array}$ & $\begin{array}{l}\text { Comparison } \\
\text { Description }\end{array}$ & $\begin{array}{c}\text { Follow-up } \\
\text { Duration }\end{array}$ & $\begin{array}{c}\text { Outcomes } \\
\text { Reported } \\
\begin{array}{c}\text { Withdrawal (\%) at } \\
\text { endpoint }\end{array}\end{array}$ \\
\hline $\begin{array}{l}\text { Rea, } 2003^{8} \\
\text { RCT } \\
\text { US } \\
\text { Government and Non- } \\
\text { Government } \\
\text { Low } \\
12795572\end{array}$ & $\begin{array}{l}\text { N=53 } \\
\text { Age } 26(18-46) \\
\text { Female } 57 \% \\
\text { White } 60 \% \\
\text { BP I NR } \\
\text { Inpatient/Outpatient }\end{array}$ & $\begin{array}{l}\text { Manic; Inpatients with } \\
\text { bipolar disorder (DSM-III), } \\
\text { manic type currently taking } \\
\text { mood-regulating } \\
\text { medications with a close } \\
\text { family member that could } \\
\text { participate in intervention } \\
\text { with patient. } \\
\text { Substance Abuse; } \\
\text { Labs/Other Conditions }\end{array}$ & $\begin{array}{l}\text { Family-focused } \\
\text { treatment (with } \\
\text { medication } \\
\text { management) } \\
\text { consisting of } \\
\text { psychoeducation, } \\
\text { communication } \\
\text { enhancement } \\
\text { training, and } \\
\text { problem-solving } \\
\text { skills training } \\
\text {-21 therapy sessions } \\
\text { over } 9 \text { months (60 } \\
\text { minutes per session) } \\
\text { with } 1 \text { year of } \\
\text { medication } \\
\text { management }\end{array}$ & $\begin{array}{l}\text { Individual } \\
\text { treatment (with } \\
\text { medication } \\
\text { management) } \\
\text { consisting of } \\
\text { meeting a } \\
\text { therapist to } \\
\text { receive } \\
\text { education } \\
\text { about illness } \\
\text { and symptoms, } \\
\text { discuss } \\
\text { problem- } \\
\text { solving, and } \\
\text { establishing } \\
\text { goals. } \\
\text {-21 therapy } \\
\text { sessions over } \\
9 \text { months (30 } \\
\text { minutes per } \\
\text { session) with } 1 \\
\text { year of } \\
\text { medication } \\
\text { management }\end{array}$ & 24 months & $\begin{array}{l}\text { Relapse (6 or } 7 \text { on } \\
\text { BPRS/SADS-C core } \\
\text { symptoms of } \\
\text { depression, mania, or } \\
\text { psychosis, and at least } \\
\text { two ancillary } \\
\text { symptoms) } \\
\text { Withdrawal } 45 \%\end{array}$ \\
\hline
\end{tabular}




\begin{tabular}{|c|c|c|c|c|c|c|}
\hline $\begin{array}{l}\text { Study, Year } \\
\text { Design } \\
\text { Location } \\
\text { Funder } \\
\text { Risk of Bias } \\
\text { PMID }\end{array}$ & $\begin{array}{c}\text { Randomized (N) } \\
\text { Age (mean) } \\
\text { Sex (\% Female) } \\
\text { Race (\% White) } \\
\text { Diagnosis } \\
\text { (\% BP I, II, NOS) } \\
\text { Setting }\end{array}$ & $\begin{array}{l}\text { Inclusions } \\
\text { Key Exclusions }\end{array}$ & $\begin{array}{l}\text { Intervention } \\
\text { Description }\end{array}$ & $\begin{array}{l}\text { Comparison } \\
\text { Description }\end{array}$ & $\begin{array}{c}\text { Follow-up } \\
\text { Duration }\end{array}$ & $\begin{array}{c}\text { Outcomes } \\
\text { Reported } \\
\text { Withdrawal (\%) at } \\
\text { endpoint }\end{array}$ \\
\hline $\begin{array}{l}\text { SImoneau, 1999 } \\
\text { RCT } \\
\text { US } \\
\text { Government and Non- } \\
\text { Government } \\
\text { Moderate } \\
10609423\end{array}$ & $\begin{array}{l}\mathrm{N}=79 \\
\text { Age } 34(18-57) \\
\text { Female 54\% } \\
\text { White NR } \\
\text { BP I NR } \\
\text { Outpatient }\end{array}$ & $\begin{array}{l}\text { Depressive, Manic, or } \\
\text { Mixed Episode; Diagnosis } \\
\text { of BP (DSM-III) in a manic, } \\
\text { mixed, or depressed phase } \\
\text { in the } 3 \text { months prior } \\
\text { including month of study } \\
\text { entry, living or in close } \\
\text { contact with a relative for at } \\
\text { least } 1 \text { to } 3 \text { months prior to } \\
\text { study entry, and willing to } \\
\text { take mood stabilizing } \\
\text { medications } \\
\text { Substance Abuse; } \\
\text { Labs/Other Conditions }\end{array}$ & $\begin{array}{l}\text { Family-focused } \\
\text { therapy (with } \\
\text { medication } \\
\text { management) } \\
\text { consisting of } \\
\text { psychoeducation, } \\
\text { communication- } \\
\text { enhancement } \\
\text { training, and } \\
\text { problem-solving } \\
\text { skills training } \\
\text {-21 sessions over } 9 \\
\text { months }\end{array}$ & $\begin{array}{l}\text { Crisis } \\
\text { management } \\
\text { with } \\
\text { naturalistic } \\
\text { follow-up (with } \\
\text { medication } \\
\text { management) } \\
\text { consisting of } \\
\text { two sessions } \\
\text { of home-based } \\
\text { family } \\
\text { education, } \\
\text { crisis } \\
\text { intervention as } \\
\text { needed, } \\
\text { telephone } \\
\text { counseling and } \\
\text { individual } \\
\text { support } \\
\text { sessions as } \\
\text { needed, and } \\
\text { monthly } \\
\text { contacts. } \\
\text {-9 months of } \\
\text { management }\end{array}$ & 24 months & $\begin{array}{l}\text { SADS-C } \\
\text { Withdrawal 44\% }\end{array}$ \\
\hline
\end{tabular}

Abbreviations: BP=bipolar disorder; BPRS=Brief Psychiatric Rating Scale; CARS-M=Clinician-Administered Rating Scale for Mania; DSM=Diagnostic and Statistical Manual of Mental Disorders; NOS=not otherwise specified; NR=not reported; PMID=PubMed Identification Number; QIDS-C=Quick Inventory of Depression Scores; RCT=randomized controlled trial; SADS-C=Schedule for Affective Disorders and Schizophrenia-Change version; WHO-DAS=World Health Organization Disability Assessment Scale; 
Appendix Table M6. Summary risk of bias assessments: family or partner interventions vs. active comparators

\begin{tabular}{|c|c|c|}
\hline $\begin{array}{c}\text { Study } \\
\text { Funder } \\
\text { PMID }\end{array}$ & $\begin{array}{c}\text { Overall Risk of Bias } \\
\text { Assessment }\end{array}$ & Rationale \\
\hline $\begin{array}{l}\text { Wenze, } 2015^{5} \\
\text { Government and } \\
\text { Non-Government } \\
26117247\end{array}$ & Moderate & Suspected bias due to format of data reporting and incomplete reporting of outcomes. \\
\hline $\begin{array}{l}\text { Miklowitz, } 2003^{7} \\
\text { Miklowitz, } 2000^{6} \\
\text { Government and } \\
\text { Non-Government } \\
11018229 \\
12963672 \\
\end{array}$ & Low & No significant suspected biases. \\
\hline $\begin{array}{l}\text { Rea, } 2003^{8} \\
\text { Government and } \\
\text { Non-Government } \\
12795572\end{array}$ & Low & No significant suspected biases. \\
\hline $\begin{array}{l}\text { SImoneau. } 1999^{9} \\
\text { Government and } \\
\text { Non-Government } \\
10609423\end{array}$ & Moderate & Suspected bias due to differential attrition rate between study arms. \\
\hline
\end{tabular}

10609423

Abbreviations: PMID=PubMed Identification Number

Appendix Table M7. Outcomes summary: family or partner interventions vs. active comparators

\begin{tabular}{|c|c|c|c|c|c|}
\hline $\begin{array}{l}\text { Study } \\
\text { PMID }\end{array}$ & Responder/Remitter & Symptom & Function & Other & $\mathrm{AE}$ \\
\hline $\begin{array}{l}\text { Wenze } 2015^{5} \\
26117247\end{array}$ & NR & $\begin{array}{l}\frac{\text { Depression }}{6 \text { months, QIDS-C }} \\
\text { Favors FPI; } p<0.05 \\
\text { ES }=0.24 \\
\frac{\text { Mania }}{6 \text { months, CARS-M }} \\
\text { Favors FPI; } p<0.05 \\
E S=0.37\end{array}$ & $\begin{array}{l}\text { Health and Disability } \\
6 \text { months, WHO-DAS } \\
\text { Favors FPI; } p<0.05 \\
\text { ES }=0.12\end{array}$ & $\begin{array}{l}\text { Re-Hospitalizations } \\
6 \text { months } \\
\text { NS } \\
\text { Emergency Room } \\
\frac{\text { Visits }}{6 \text { months }} \\
\text { NS; } p<0.10\end{array}$ & NR \\
\hline
\end{tabular}




\begin{tabular}{|c|c|c|c|c|c|}
\hline $\begin{array}{l}\text { Study } \\
\text { PMID }\end{array}$ & Responder/Remitter & Symptom & Function & Other & $\mathrm{AE}$ \\
\hline $\begin{array}{l}\text { Miklowitz 20037 } \\
12963672 \\
\text { Miklowitz } 2000^{6} \\
11018229\end{array}$ & $\begin{array}{l}\frac{\text { Relapse }^{\star}}{12 \text { months, Any Type }} \\
\text { NS } \\
\text { OR=0.36 (95\% Cl } 0.11 \text {, } \\
1.09) ; p=0.05 \\
\text { Favors FPI } \\
24 \text { months, Any Type } \\
\text { OR=0.22 (95\% Cl 0.07, } \\
0.66) ; p=0.00\end{array}$ & $\begin{array}{l}\frac{\text { Depression }}{24 \text { months, SADS-C }} \\
\text { Depression } \\
\text { Favors FPI; } p=0.005 \\
\frac{\text { Mania }}{24 \text { months, SADS-C Mania }} \\
\text { NS; } p=0.06\end{array}$ & NR & NR & NR \\
\hline $\begin{array}{l}\text { Rea } 2003^{8} \\
12795572\end{array}$ & $\begin{array}{l}\text { Relapse } \\
12 \text { months } \\
\text { NS; } p>0.10 \\
\text { Family/Partner Therapy: } \\
46 \% \\
\text { Active Comparator: } 52 \% \\
\\
\text { 1-year Post-Treatment } \\
\text { Period ( } 24 \text { months) } \\
\text { Favors FPI; } p<0.05 \\
\text { FPI: } 28 \% \\
\text { Active Comparator: } 60 \%\end{array}$ & NR & NR & NR & NR \\
\hline $\begin{array}{l}\text { Simoneau } 1999^{9} \\
10609423\end{array}$ & NR & $\begin{array}{l}\text { Symptoms } \\
1 \text { year post-treatment, SADS-C } \\
\text { Favors FPI; } p<0.05\end{array}$ & NR & NR & NR \\
\hline
\end{tabular}

Abbreviations: CARS-M=Clinician-Administered Rating Scale for Mania; CI=Confidence Interval; FPI=Family or Partner Intervention; NR=not reported; NS=not significant; OR=Odds Ratio; PMID=PubMed Identification Number; QIDS-C=Quick Inventory of Depression Scores; SADS-C=Schedule for Affective Disorders and Schizophrenia- Change version; WHO-DAS=World Health Organization Disability Assessment Scale

\section{Appendix Table M8. Summary of strength of evidence: family or partner interventions vs. active comparators}

\begin{tabular}{|c|c|c|c|c|c|c|c|c|}
\hline Outcome & Timing & $\begin{array}{l}\text { \# Studies/ Design } \\
\text { (n analyzed) }\end{array}$ & $\begin{array}{l}\text { Finding or } \\
\text { Summary } \\
\text { Statistic }\end{array}$ & $\begin{array}{c}\text { Study } \\
\text { Limitations }\end{array}$ & Consistency & Directness & $\begin{array}{c}\text { Precisio } \\
n\end{array}$ & $\begin{array}{l}\text { Overall Gradel } \\
\text { Conclusion }\end{array}$ \\
\hline Relapse & $\begin{array}{l}7-12 \\
\text { months } \\
12+ \\
\text { months }\end{array}$ & 2 RCTs $(n=154)$ & $\begin{array}{l}\text { No difference } \\
\text { between groups at } \\
12 \text { months; } \\
\text { however FPI } \\
\text { groups experience } \\
\text { fewer relapses at } \\
24 \text { months. }\end{array}$ & Low & Consistent & Direct & Imprecise & Insufficient \\
\hline
\end{tabular}




\begin{tabular}{|c|c|c|c|c|c|c|c|c|}
\hline Outcome & Timing & $\begin{array}{l}\text { \# Studies/ Design } \\
\text { ( } \mathrm{n} \text { analyzed) }\end{array}$ & $\begin{array}{l}\text { Finding or } \\
\text { Summary } \\
\text { Statistic }\end{array}$ & $\begin{array}{c}\text { Study } \\
\text { Limitations }\end{array}$ & Consistency & Directness & $\begin{array}{c}\text { Precisio } \\
\mathrm{n}\end{array}$ & $\begin{array}{c}\text { Overall Grade } \\
\text { Conclusion }\end{array}$ \\
\hline Depression & $\begin{array}{l}6 \text { months } \\
12+ \\
\text { months }\end{array}$ & 2 RCTS (n=131) & $\begin{array}{l}\text { Favors FPI at } \\
\text { reported time } \\
\text { periods. }\end{array}$ & Moderate & Consistent & Direct & Imprecise & Insufficient \\
\hline Mania & $\begin{array}{l}6 \text { months } \\
12+ \\
\text { months }\end{array}$ & 2 RCTS ( $n=131)$ & $\begin{array}{l}\text { Mixed evidence } \\
\text { with no clear } \\
\text { direction of effect. } \\
\text { No pattern across } \\
\text { time periods. }\end{array}$ & Moderate & Inconsistent & Direct & Imprecise & Insufficient \\
\hline $\begin{array}{l}\text { Global } \\
\text { Function }\end{array}$ & NR & - & - & - & - & - & - & - \\
\hline $\begin{array}{l}\text { Other } \\
\text { Measures } \\
\text { of Function }\end{array}$ & 6 months & $1 \mathrm{RCT}(\mathrm{n}=30)$ & $\begin{array}{l}\text { Favors FPI at } 6 \\
\text { months. } \\
\text { WHO-DAS } \\
E S=0.12 ; p<0.05\end{array}$ & Moderate & Unclear & Direct & Imprecise & Insufficient \\
\hline
\end{tabular}

Abbreviations: ES=Effect Size; FPI=Family or Partner Intervention; NR=not reported; RCT=randomized controlled trial; WHO-DAS=World Health Organization Disability Assessment Scale 


\section{References for Appendix M}

1. D'Souza R, Piskulic D, Sundram S. A brief dyadic group based psychoeducation program improves relapse rates in recently remitted bipolar disorder: a pilot randomised controlled trial. Journal of Affective Disorders. 2010 Jan;120(1-3):272-6. doi: http://dx.doi.org/10.1016/j.jad.2009.03.018. PMID: 19428117.

2. Miller IW, Solomon DA, Ryan CE, et al. Does adjunctive family therapy enhance recovery from bipolar I mood episodes? Journal of Affective Disorders. 2004 Nov 1;82(3):431-6. PMID: 15555694.

3. Solomon DA, Keitner GI, Ryan CE, et al. Preventing recurrence of bipolar I mood episodes and hospitalizations: family psychotherapy plus pharmacotherapy versus pharmacotherapy alone. Bipolar Disorders. 2008 Nov;10(7):798-805. doi: http://dx.doi.org/10.1111/j.13995618.2008.00624.x. PMID: 19032711.

4. Miller IW, Keitner GI, Ryan CE, et al. Family treatment for bipolar disorder: family impairment by treatment interactions. Journal of Clinical Psychiatry. 2008 May;69(5):732-40. PMID: 18363424.

5. Wenze SJ, Gaudiano BA, Weinstock LM, et al. Adjunctive psychosocial intervention following Hospital discharge for Patients with bipolar disorder and comorbid substance use: A pilot randomized controlled trial. Psychiatry Res. 2015 Aug 30;228(3):516-25. doi: 10.1016/j.psychres.2015.06.005. PMID: 26117247.
6. Miklowitz DJ, Simoneau TL, George EL, et al. Family-focused treatment of bipolar disorder: 1year effects of a psychoeducational program in conjunction with pharmacotherapy. Biological Psychiatry. 2000 Sep 15;48(6):582-92. PMID: 11018229.

7. Miklowitz DJ, George EL, Richards JA, et al. A randomized study of family-focused psychoeducation and pharmacotherapy in the outpatient management of bipolar disorder. Archives of General Psychiatry. 2003 Sep;60(9):904-12. PMID: 12963672.

8. Rea MM, Tompson MC, Miklowitz DJ, et al. Family-focused treatment versus individual treatment for bipolar disorder: results of a randomized clinical trial. Journal of Consulting \& Clinical Psychology. 2003 Jun;71(3):482-92. PMID: 12795572.

9. Simoneau TL, Miklowitz DJ, Richards JA, et al. Bipolar disorder and family communication: effects of a psychoeducational treatment program. Journal of Abnormal Psychology. 1999 Nov;108(4):588-97. PMID: 10609423. 


\section{Appendix N. Interpersonal and Social Rhythm Therapy (IPSRT)}

Appendix Table N1. Characteristics of eligible studies: IPSRT vs. inactivecComparators

\begin{tabular}{|c|c|c|c|c|c|c|}
\hline $\begin{array}{l}\text { Study, Year } \\
\text { Design } \\
\text { Location } \\
\text { Funder } \\
\text { Risk of Bias } \\
\text { PMID }\end{array}$ & $\begin{array}{c}\text { Randomized (N) } \\
\text { Age (mean) } \\
\text { Sex (\% Female) } \\
\text { Race (\% White) } \\
\text { Diagnosis } \\
\text { (\% BP I, II, NOS) } \\
\text { Setting }\end{array}$ & $\begin{array}{c}\text { Inclusions } \\
\text { Key Exclusions }\end{array}$ & $\begin{array}{l}\text { Intervention } \\
\text { Description }\end{array}$ & $\begin{array}{l}\text { Comparison } \\
\text { Description }\end{array}$ & $\begin{array}{l}\text { Follow-up } \\
\text { Duration }\end{array}$ & $\begin{array}{c}\text { Outcomes } \\
\text { Reported } \\
\text { Withdrawal (\%) at } \\
\text { endpoint }\end{array}$ \\
\hline $\begin{array}{l}\text { Maintenance Therapies } \\
\text { in Bipolar Disorder } \\
\text { Frank, 1997 } \\
\text { Frank, 1999² } \\
\text { Rucci, 2002 } \\
\text { Frank, 2005 } \\
\text { Frank, 2008 } \\
\text { RCT } \\
\text { US } \\
\text { Government } \\
\text { High (All Publications) } \\
9171907 \\
10609422 \\
16143731 \\
18829872\end{array}$ & $\begin{array}{l}\mathrm{N}=38-175 \\
\text { Age } 35(18-55) \\
\text { Female 59\% } \\
\text { White } 94 \% \\
\text { BP I 93\% } \\
\text { Inpatient/Outpatient }\end{array}$ & $\begin{array}{l}\text { Depressive, Manic, or } \\
\text { Mixed; Individuals with BP I } \\
\text { or schizoaffective disorder, } \\
\text { manic type, (DSM-IV) and } \\
\text { in at least their third lifetime } \\
\text { affective episode. Severity } \\
\text { criteria: >15 on HDRS or } \\
>15 \text { on BRMS } \\
\text { Substance Abuse; Other } \\
\text { Mental Health; } \\
\text { Pregnant/Nursing; } \\
\text { Labs/Other Conditions }\end{array}$ & $\begin{array}{l}\text { IPSRT (acute, } \\
\text { maintenance, or } \\
\text { both) focused on } \\
\text { maintaining regular } \\
\text { daily routines, } \\
\text { identification and } \\
\text { management of } \\
\text { potential triggers and } \\
\text { interpersonal } \\
\text { psychotherapy. } \\
\text {-Acute weekly } \\
\text { treatment until } \\
\text { remission followed } \\
\text { by biweekly sessions } \\
\text { for } 12 \text { weeks and } \\
\text { monthly treatment to } \\
24 \text { months, } 45 \text { to } 55 \\
\text { minutes per session }\end{array}$ & \begin{tabular}{|l|} 
Clinical \\
management \\
(acute, \\
maintenance, \\
or both) \\
consisting of \\
medical \\
management \\
of bipolar \\
disorder \\
(education, \\
review of \\
symptoms, \\
management \\
of adverse \\
effects) \\
- Acute weekly \\
treatment until \\
remission \\
followed by \\
biweekly \\
sessions for 12 \\
weeks and \\
monthly \\
treatment to 24 \\
months, 20 to \\
25 minutes per \\
session
\end{tabular} & 24 months & $\begin{array}{l}\text { Risk of Recurrence } \\
\text { Remission } \\
\text { UCLA Social } \\
\text { Attainment Scale } \\
\text { HDRS } \\
\text { BRMS } \\
\text { Withdrawal } 47 \%\end{array}$ \\
\hline
\end{tabular}


Abbreviations: BP=bipolar disorder; BRMS=Bech-Rafaelsen Mania Scale; DSM-IV= Diagnostic and statistical manual, $4^{\text {th }}$ edition; HDRS=Hamilton Rating Scale for Depression; IPSRT=Interpersonal and Social Rhythm Therapy; NOS=not otherwise specified; PMID=PubMed Identification Number; RCT=randomized controlled trial;

\section{Appendix Table N2. Summary risk of bias assessments: IPSRT vs. inactive comparators}

\begin{tabular}{|l|l|l|}
\hline $\begin{array}{c}\text { Sunder } \\
\text { PMID }\end{array}$ & $\begin{array}{c}\text { Overall Risk of Bias } \\
\text { Assessment }\end{array}$ & \\
\hline Frank, $1997^{1}$ & High & Rationale \\
Frank, $1999^{2}$ & & \\
Rucci, $2002^{3}$ & \\
Frank, $2005^{4}$ & \\
Frank, 2008 & \\
Government & \\
9171907 & \\
10609422 & \\
16143731 & \\
18829872 & \\
\hline
\end{tabular}

Abbreviations: IPSRT=Interpersonal and Social Rhythm Therapy; PMID=PubMed Identification Number

\section{Appendix Table N3. Outcomes summary: IPSRT vs. inactive comparators}

\begin{tabular}{|c|c|c|c|c|c|}
\hline $\begin{array}{l}\text { Study } \\
\text { PMID }\end{array}$ & Responder/Remitter & Symptom & Function & Other & $\mathrm{AE}$ \\
\hline $\begin{array}{l}\text { Frank } 2008^{5} \\
18829872 \\
\text { Frank } 2005^{4} \\
16143731 \\
\text { Rucci } 2002^{3} \\
16143731 \\
\text { Frank } 1999^{2} \\
10609422\end{array}$ & $\begin{array}{l}\text { Risk of Recurrence } \\
12 \text { months } \\
\text { NS; } p=0.52 \\
\text { Time to Recurrence } \\
12 \text { months } \\
\text { Favors IPSRT } \\
\text { HR=0.34, } p=0.01 \\
\frac{\text { Remission }}{24 \text { months }} \\
\text { NS } \\
70 \% \text { ISPRT vs. } 72 \% \\
\text { Comparator }\end{array}$ & $\begin{array}{l}\frac{\text { Depression and Mania }}{24 \text { months, HDRS plus BRMS }} \\
\text { NS; } p=0.74\end{array}$ & $\begin{array}{l}\frac{\text { Occupational }}{\text { Functioning }} \\
24 \text { months } \\
\text { Favors IPSRT as } \\
\text { acute intervention; } \\
p=0.046\end{array}$ & $\begin{array}{l}\text { Suicide Attempts } \\
\text { NS (between groups) } \\
\text { Favors Intervention } \\
\text { (compared to rate prior } \\
\text { to study) } \\
24 \text { months } \\
\text { Base Rate: } 1.05 / 100 \\
\text { patient months } \\
\text { Acute Phase Rate: } \\
0.31 / 100 \text { patient } \\
\text { months; } p<0.02 \\
\text { Maintenance Phase } \\
\text { Rate: } 0.06 / 100 \text { patient } \\
\text { months; } p=0.004\end{array}$ & NR \\
\hline
\end{tabular}

Abbreviations: AE=Adverse Events; BRMS=Bech-Rafaelsen Mania Scale; HDRS=Hamilton Depression Rating Scale; HR=Hazard Ratio; IPSRT=Interpersonal and Social

Rhythm Therapy; NR=not reported; NS=not significant; PMID=PubMed Identification Number 
Appendix Table N4. Summary of strength of evidence: IPSRT vs. inactive comparators

\begin{tabular}{|c|c|c|c|c|c|c|c|c|}
\hline Outcome & Timing & $\begin{array}{l}\text { \# Studies/ Design } \\
\text { (n analyzed) }\end{array}$ & $\begin{array}{l}\text { Finding or } \\
\text { Summary } \\
\text { Statistic }\end{array}$ & $\begin{array}{c}\text { Study } \\
\text { Limitations }\end{array}$ & Consistency & Directness & $\begin{array}{c}\text { Precisio } \\
\mathbf{n}\end{array}$ & $\begin{array}{l}\text { Overall Gradel } \\
\text { Conclusion }\end{array}$ \\
\hline Relapse & 12 months & $\begin{array}{l}1 \mathrm{RCT} \\
(\mathrm{n}=82)\end{array}$ & $\begin{array}{l}\text { No difference } \\
\text { between groups at } \\
24 \text { months in risk of } \\
\text { recurrence; } p=0.52\end{array}$ & High & Unclear & Direct & Imprecise & Insufficient \\
\hline Depression & 24 months & $\begin{array}{l}1 \mathrm{RCT} \\
(\mathrm{n}=175)\end{array}$ & $\begin{array}{l}\text { No difference } \\
\text { between groups, at } \\
24 \text { months } \\
\text { HDRS and BRMS; } \\
\text { p=0.74 }\end{array}$ & High & Unclear & Direct & Imprecise & Insufficient \\
\hline Mania & 24 months & $\begin{array}{l}1 \mathrm{RCT} \\
(\mathrm{n}=175)\end{array}$ & $\begin{array}{l}\text { No difference } \\
\text { between groups, at } \\
24 \text { months } \\
\text { HDRS and BRMS; } \\
\text { p=0.74 }\end{array}$ & High & Unclear & Direct & Imprecise & Insufficient \\
\hline $\begin{array}{l}\text { Global } \\
\text { Function }\end{array}$ & NR & - & - & - & - & - & - & - \\
\hline $\begin{array}{l}\text { Other } \\
\text { Measures } \\
\text { of Function }\end{array}$ & 24 months & $\begin{array}{l}1 \mathrm{RCT} \\
(\mathrm{n}=125)\end{array}$ & $\begin{array}{l}\text { Favors IPSRT as } \\
\text { acute intervention } \\
\text { at } 24 \text { months } \\
\text { Occupational } \\
\text { Functioning; } \\
p=0.046\end{array}$ & High & Unclear & Direct & Imprecise & Insufficient \\
\hline
\end{tabular}

Abbrevaitions: BRMS=Bech-Rafaelsen Mania Scale; HDRS=Hamilton Depression Rating Scale; IPSRT=Interpersonal and Social Rhythm Therapy; NR=not reported; RCT=randomized controlled trial 
Appendix Table N5. Characteristics of eligible studies: IPSRT vs. active comparators

\begin{tabular}{|c|c|c|c|c|c|c|}
\hline $\begin{array}{l}\text { Study, Year } \\
\text { Design } \\
\text { Location } \\
\text { Funder } \\
\text { Risk of Bias } \\
\text { PMID }\end{array}$ & $\begin{array}{c}\text { Randomized (N) } \\
\text { Age (mean) } \\
\text { Sex (\% Female) } \\
\text { Race (\% White) } \\
\text { Diagnosis } \\
\text { (\% BP I, II, NOS) } \\
\text { Setting }\end{array}$ & $\begin{array}{c}\text { Inclusions } \\
\text { Key Exclusions }\end{array}$ & $\begin{array}{l}\text { Intervention } \\
\text { Description }\end{array}$ & $\begin{array}{l}\text { Comparison } \\
\text { Description }\end{array}$ & $\begin{array}{c}\text { Follow-up } \\
\text { Duration }\end{array}$ & $\begin{array}{c}\text { Outcomes } \\
\text { Reported } \\
\text { Withdrawal (\%) at } \\
\text { endpoint }\end{array}$ \\
\hline $\begin{array}{l}\text { Inder, 2016 } \\
\text { Inder, 2015 } \\
\text { New Zealand } \\
\text { Government } \\
\\
\text { Low } \\
\text { Low } \\
25346391 \\
26698820\end{array}$ & $\begin{array}{l}\mathrm{N}=100 \\
\text { Age } 27 \text { (15-36) } \\
\text { Female 76\% } \\
\text { White NR } \\
\text { BP I 78\% } \\
\text { BP II 17\% } \\
\text { BP NOS 5\% } \\
\text { Outpatient }\end{array}$ & $\begin{array}{l}\text { No current clinical state } \\
\text { excluded; Individuals with } \\
\text { BP I, II, or NOS (DSM-IV) } \\
\text { Substance Abuse }\end{array}$ & $\begin{array}{l}\text { IPSRT consisting of } \\
\text { interpersonal } \\
\text { psychotherapy with } \\
\text { a focus on social } \\
\text { routines and achieve } \\
\text { of goals. } \\
\text {-Weekly sessions for } \\
3 \text { months, fortnightly } \\
\text { for up to } 6 \text { months, } \\
\text { and then fortnightly } \\
\text { to monthly from } 6 \text { to } \\
18 \text { months } \\
\text { (frequency tailored } \\
\text { to patient needs) }\end{array}$ & \begin{tabular}{|l|} 
Specialist \\
supportive care \\
consisting of \\
supportive \\
psychotherapy \\
and \\
psychoeducation \\
-Weekly \\
sessions for 3 \\
months, \\
fortnightly for up \\
to 6 months, and \\
then fortnightly \\
to monthly from \\
6 to 18 months \\
(frequency \\
tailored to \\
patient needs)
\end{tabular} & 78 weeks & $\begin{array}{l}\text { LIFE Depression } \\
\text { LIFE Mania } \\
\text { SAS } \\
\text { Withdrawal 16\% }\end{array}$ \\
\hline
\end{tabular}

Abbreviations: BP=bipolar disorder; DSM-IV= Diagnostic and statistical manual, $4^{\text {th }}$ edition; IPSRT=Interpersonal and Social Rhythm Therapy; LIFE=Longitudinal Interval Follow-up Evaluation; NOS=not otherwise specified; PMID=PubMed Identification Number; SAS=Simpson Angus Scale 
Appendix Table N6. Summary risk of bias assessments: IPSRT vs. active comparators

\begin{tabular}{|c|c|c|}
\hline $\begin{array}{c}\text { Study } \\
\text { Funder } \\
\text { PMID }\end{array}$ & $\begin{array}{c}\text { Overall Risk of Bias } \\
\text { Assessment }\end{array}$ & Rationale \\
\hline $\begin{array}{l}\text { Inder, } 2016^{6} \\
\text { Inder, } 2015^{7} \\
\text { Government } \\
25346391 \\
26698820\end{array}$ & Low & No significant suspected biases. \\
\hline
\end{tabular}

Abbreviations: IPSRT=Interpersonal and Social Rhythm Therapy; PMID=PubMed Identification Number

\section{Appendix Table N7. Outcomes summary: IPSRT vs. active comparators}

\begin{tabular}{|c|c|c|c|c|c|}
\hline $\begin{array}{l}\text { Study } \\
\text { PMID }\end{array}$ & Responder/Remitter & Symptom & Function & Other & $\mathrm{AE}$ \\
\hline $\begin{array}{l}\text { Inder } 2016^{6} \\
\text { Inder } 2015^{7}\end{array}$ & NR & $\begin{array}{l}\frac{\text { Depression }}{6 \text { months, LIFE Depression }} \\
\text { NS } \\
1.9(95 \% \mathrm{Cl} 1.8-2.2) \text { IPSRT vs. } \\
1.8(95 \% \mathrm{Cl} 1.6-2.0) \text { Active } \\
\text { Comparator; } \mathrm{p}=0.25 \\
\\
\frac{\text { Mania }}{6 \text { months, LIFE Mania }} \\
\text { NS } \\
1.3(95 \% \mathrm{Cl} 1.2-1.4) \text { IPSRT vs. } \\
1.3(95 \% \mathrm{Cl} 1.2-1.4) \text { Active } \\
\text { Comparator; } p=0.64\end{array}$ & $\begin{array}{l}\text { Social Function } \\
6 \text { months, SAS } \\
\text { NS } \\
2.0 \text { (95\% Cl1.9-2.1) } \\
\text { IPSRT vs. } 1.9(95 \% \mathrm{Cl} \\
1.9-2.0) \text { Active } \\
\text { Comparator; } p=0.10\end{array}$ & $\begin{array}{l}\text { Suicide Attempts and } \\
\text { Other Self-Harm } \\
\text { Unclear } \\
\text { At } 78 \text { weeks there } \\
\text { were reductions in } \\
\text { attempts from baseline } \\
\text { rate, difference } \\
\text { between groups NR } \\
\\
\text { Baseline Suicide } \\
\text { Attempts: } 11 \% \\
78 \text { Week Suicide } \\
\text { Attempts: } 1 \% \\
\text { Baseline Self-Harm } \\
\text { Attempts: } 15 \% \\
78 \text { Week Self-Harm } \\
\text { Attempts: } 7 \%\end{array}$ & NR \\
\hline
\end{tabular}

Abbreviations: AE=Adverse Events; CI=Confidence Interval; IPSRT=Interpersonal and Social Rhythm Therapy; LIFE=Longitudinal Interval Follow-up Evaluation; NR=not reported; NS=not significant; PMID=PubMed Identification Number; SAS=Simpson Angus Scale 
Appendix Table N8. Summary of strength of evidence: IPSRT vs. active comparators

\begin{tabular}{|c|c|c|c|c|c|c|c|c|}
\hline Outcome & Timing & $\begin{array}{l}\text { \# Studies/ Design } \\
\text { (n analyzed) }\end{array}$ & $\begin{array}{l}\text { Finding or } \\
\text { Summary } \\
\text { Statistic }\end{array}$ & $\begin{array}{c}\text { Study } \\
\text { Limitations }\end{array}$ & Consistency & Directness & $\begin{array}{c}\text { Precisio } \\
\text { n }\end{array}$ & $\begin{array}{l}\text { Overall Gradel } \\
\text { Conclusion }\end{array}$ \\
\hline Relapse & NR & - & - & - & - & - & - & - \\
\hline Depression & 6 months & $\begin{array}{l}1 \mathrm{RCT} \\
(\mathrm{n}=100)\end{array}$ & $\begin{array}{l}\text { No difference } \\
\text { between groups at } \\
6 \text { months. } \\
\text { LIFE Depression } \\
1.9 \text { (95\% CI1.8- } \\
2.2) \text { IPSRT vs. } 1.8 \\
\text { (95\% Cl 1.6-2.0) } \\
\text { Active Comparator; } \\
p=0.25\end{array}$ & Low & Unclear & Direct & Imprecise & Insufficient \\
\hline Mania & 6 months & $\begin{array}{l}1 \mathrm{RCT} \\
(\mathrm{n}=100)\end{array}$ & $\begin{array}{l}\text { No difference } \\
\text { between groups at } \\
6 \text { months. } \\
\text { LIFE Mania } \\
1.3 \text { (95\% CI 1.2- } \\
1.4) \text { IPSRT vs. } 1.3 \\
(95 \% \text { CI1.2-1.4) } \\
\text { Active Comparator; } \\
p=0.64\end{array}$ & Low & Unclear & Direct & Imprecise & Insufficient \\
\hline $\begin{array}{l}\text { Global } \\
\text { Function }\end{array}$ & NR & - & - & - & - & - & - & - \\
\hline $\begin{array}{l}\text { Other } \\
\text { Measures } \\
\text { of Function }\end{array}$ & 6 months & $\begin{array}{l}1 \mathrm{RCT} \\
(\mathrm{n}=100)\end{array}$ & $\begin{array}{l}\text { No difference } \\
\text { between groups at } \\
6 \text { months. } \\
\text { SAS } \\
2.0 \text { (95\% CI1.9- } \\
2.1) \text { IPSRT vs. } 1.9 \\
\text { (95\% CI 1.9-2.0) } \\
\text { Active Comparator; } \\
p=0.10\end{array}$ & Low & Unclear & Direct & Imprecise & Insufficient \\
\hline
\end{tabular}

Abbreviations: CI=Confidence Interval; IPSRT=Interpersonal and Social Rhythm Therapy; LIFE=Longitudianl Interval Follow-up Evaluation; NR=not reported;

RCT=randomized controlled trial; SAS=Simpson Angus Scale 


\section{References for Appendix N}

1. Frank E, Hlastala S, Ritenour A, et al. Inducing lifestyle regularity in recovering bipolar disorder patients: results from the maintenance therapies in bipolar disorder protocol. Biological Psychiatry. 1997 Jun 15;41(12):1165-73. PMID: 9171907.

2. Frank E, Swartz HA, Mallinger AG, et al. Adjunctive psychotherapy for bipolar disorder: effects of changing treatment modality. Journal of Abnormal Psychology. 1999 Nov;108(4):579-87. PMID: 10609422.

3. Rucci P, Frank E, Kostelnik B, et al. Suicide attempts in patients with bipolar I disorder during acute and maintenance phases of intensive treatment with pharmacotherapy and adjunctive psychotherapy. American Journal of Psychiatry. 2002 Jul;159(7):1160-4. PMID: 12091194.

4. Frank E, Kupfer DJ, Thase ME, et al. Two-year outcomes for interpersonal and social rhythm therapy in individuals with bipolar I disorder. Archives of General Psychiatry. 2005 Sep;62(9):996-1004. PMID: 16143731.

5. Frank E, Soreca I, Swartz HA, et al. The role of interpersonal and social rhythm therapy in improving occupational functioning in patients with bipolar I disorder. American Journal of Psychiatry. 2008 Dec;165(12):1559-65. doi: http://dx.doi.org/10.1176/appi.ajp.2008.07121953 . PMID: 18829872.

6. Inder ML, Crowe MT, Luty SE, et al. Prospective rates of suicide attempts and nonsuicidal selfinjury by young people with bipolar disorder participating in a psychotherapy study. Australian \& New Zealand Journal of Psychiatry. 2016 Feb;50(2):167-73. doi: http://dx.doi.org/10.1177/0004867415622268. PMID: 26698820.

7. Inder ML, Crowe MT, Luty SE, et al. Randomized, controlled trial of Interpersonal and Social Rhythm Therapy for young people with bipolar disorder. Bipolar Disord. 2015 Mar;17(2):128-38. doi: 10.1111/bdi.12273. PMID: 25346391. 


\section{Appendix O. Combination Interventions}

Appendix Table 01. Characteristics of eligible studies: combination interventions vs. inactive comparators

\begin{tabular}{|c|c|c|c|c|c|c|}
\hline $\begin{array}{l}\text { Study, Year } \\
\text { Design } \\
\text { Location } \\
\text { Funder } \\
\text { Risk of Bias } \\
\text { PMID }\end{array}$ & $\begin{array}{c}\text { Randomized (N) } \\
\text { Age (mean) } \\
\text { Sex (\% Female) } \\
\text { Race (\% White) } \\
\text { Diagnosis } \\
\text { (\% BP I, II, NOS) } \\
\text { Setting }\end{array}$ & $\begin{array}{c}\text { Inclusions } \\
\text { Key Exclusions }\end{array}$ & $\begin{array}{l}\text { Intervention } \\
\text { Description }\end{array}$ & $\begin{array}{l}\text { Comparison } \\
\text { Description }\end{array}$ & $\begin{array}{l}\text { Followup } \\
\text { Duration }\end{array}$ & $\begin{array}{c}\text { Outcomes } \\
\text { Reported } \\
\text { Withdrawal (\%) at } \\
\text { endpoint }\end{array}$ \\
\hline $\begin{array}{l}\text { Gonzalez-Isasi, } 2014^{1} \\
\text { Gonzalez-Isasi, } 2010^{2} \\
\text { RCT } \\
\text { Spain } \\
\text { Non-Government } \\
\text { Low } \\
20444503 \\
23276524\end{array}$ & $\begin{array}{l}\mathrm{N}=40 \\
\text { Mean Age 41 (18-63) } \\
\text { Female 48\% } \\
\text { White NR } \\
\text { BP NR } \\
\text { Outpatient }\end{array}$ & $\begin{array}{l}\text { Euthymic or } \\
\text { Subsyndromal; BP I or II } \\
\text { (DSM-IV) for at least } 2 \\
\text { years, history of severe } \\
\text { or unfavorable } \\
\text { progression of disease, } \\
\text { euthymic or } \\
\text { subsyndromal } \\
\text { symptoms (BDI>7; } \\
\text { YMRS> 6), not receiving } \\
\text { any psychotherapy } \\
\text { Labs/Other Conditions }\end{array}$ & $\begin{array}{l}\text { Group psychoeducation and } \\
\text { CBT consisting of sessions } \\
\text { about their disorder, the } \\
\text { relationship between thoughts } \\
\text { and feelings, anxiety control } \\
\text { techniques, cognitive re- } \\
\text { structuring, problem-solving } \\
\text { and self-esteem, and social } \\
\text { skills. } \\
\text {-20 weekly sessions, } 90 \\
\text { minutes each }\end{array}$ & \begin{tabular}{|l} 
Standard \\
pharmacologic \\
treatment \\
(mood \\
stabilizers, \\
antipsychotics, \\
and/or \\
benzodiapines) \\
adjusted by \\
psychiatrist
\end{tabular} & 5 years & \begin{tabular}{|l|} 
BDI \\
YMRS \\
Hospitalizations \\
Withdrawal 5\%
\end{tabular} \\
\hline
\end{tabular}




\begin{tabular}{|c|c|c|c|c|c|c|}
\hline $\begin{array}{l}\text { Study, Year } \\
\text { Design } \\
\text { Location } \\
\text { Funder } \\
\text { Risk of Bias } \\
\text { PMID }\end{array}$ & $\begin{array}{c}\text { Randomized (N) } \\
\text { Age (mean) } \\
\text { Sex (\% Female) } \\
\text { Race (\% White) } \\
\text { Diagnosis } \\
\text { (\% BP I, II, NOS) } \\
\text { Setting }\end{array}$ & $\begin{array}{c}\text { Inclusions } \\
\text { Key Exclusions }\end{array}$ & $\begin{array}{l}\text { Intervention } \\
\text { Description }\end{array}$ & $\begin{array}{l}\text { Comparison } \\
\text { Description }\end{array}$ & $\begin{array}{l}\text { Followup } \\
\text { Duration }\end{array}$ & $\begin{array}{c}\text { Outcomes } \\
\text { Reported } \\
\text { Withdrawal (\%) at } \\
\text { endpoint }\end{array}$ \\
\hline $\begin{array}{l}\text { Todd, } 2014^{3} \\
\text { RCT } \\
\text { UK } \\
\text { Government and Non- } \\
\text { Government } \\
\text { Moderate } \\
25129531\end{array}$ & $\begin{array}{l}\mathrm{N}=122 \\
\text { Age } 43(21-65) \\
\text { Female } 72 \% \\
\text { White } 89 \% \\
\text { BP I 70\% } \\
\text { BP II 25\% } \\
\text { Rapid Cycling 5\% } \\
\text { Outpatient }\end{array}$ & $\begin{array}{l}\text { No current clinical state } \\
\text { excluded: Self-reported } \\
\text { BP I or II and scoring } \\
\text { above a threshold for } \\
\text { BP I or II on the MDQ } \\
\text { None }\end{array}$ & $\begin{array}{l}\text { Interactive, online recovery } \\
\text { informed self-management } \\
\text { intervention (Living with } \\
\text { Bipolar) based on both } \\
\text { psychoeducation and CBT. } \\
\text { Ten interactive modules to } \\
\text { help subjects learn more } \\
\text { about bipolar experiences, } \\
\text { increase self-esteem and self- } \\
\text { efficacy for managing bipolar, } \\
\text { increase ability to self- } \\
\text { manage, and develop } \\
\text { interpersonal skills. Modules } \\
\text { included case studies and } \\
\text { mood checking tools. } \\
\text {-Access to program for } 6 \\
\text { months }\end{array}$ & $\begin{array}{l}\text { Wait list control } \\
\text { receiving } \\
\text { treatment as } \\
\text { usual (general } \\
\text { practitioner } \\
\text { and/or } \\
\text { specialist } \\
\text { mental health } \\
\text { services). }\end{array}$ & 6 months & $\begin{array}{l}\text { ISS Depression } \\
\text { QoL.BD-Brief } \\
\text { WHO-QOL-bref } \\
\text { SASS } \\
\text { Withdrawal } 25 \%\end{array}$ \\
\hline
\end{tabular}




\begin{tabular}{|c|c|c|c|c|c|c|}
\hline $\begin{array}{l}\text { Study, Year } \\
\text { Design } \\
\text { Location } \\
\text { Funder } \\
\text { Risk of Bias } \\
\text { PMID }\end{array}$ & $\begin{array}{c}\text { Randomized (N) } \\
\text { Age (mean) } \\
\text { Sex (\% Female) } \\
\text { Race (\% White) } \\
\text { Diagnosis } \\
\text { (\% BP I, II, NOS) } \\
\text { Setting }\end{array}$ & $\begin{array}{c}\text { Inclusions } \\
\text { Key Exclusions }\end{array}$ & $\begin{array}{l}\text { Intervention } \\
\text { Description }\end{array}$ & $\begin{array}{l}\text { Comparison } \\
\text { Description }\end{array}$ & $\begin{array}{l}\text { Followup } \\
\text { Duration }\end{array}$ & $\begin{array}{c}\text { Outcomes } \\
\text { Reported } \\
\text { Withdrawal (\%) at } \\
\text { endpoint }\end{array}$ \\
\hline $\begin{array}{l}\text { Miklowitz } 2003^{4} \\
\text { Cohort } \\
\text { US } \\
\text { Government and Non- } \\
\text { Government } \\
\text { High } \\
12963672\end{array}$ & $\begin{array}{l}\mathrm{N}=100 \\
\text { Age } 36(18-55) \\
\text { Female 60\% } \\
\text { White } 89 \% \\
\text { BP NR } \\
\text { Outpatient }\end{array}$ & \begin{tabular}{|l|} 
No current clinical state \\
excluded; BP I or II \\
(DSM-IV) with a \\
hypo/manic, depressed, \\
or mixed episode within \\
the last 3 months, \\
willingness to be on \\
maintained drug \\
regimen, living with or in \\
regular contact with \\
close relatives \\
\\
Substance Abuse; \\
Neurological Disorders
\end{tabular} & $\begin{array}{l}\text { Individual IPSRT and family } \\
\text { (or partner) therapy. } \\
\text { Individual IPSRT consisted of } \\
\text { identifying interpersonal } \\
\text { problems, using Social } \\
\text { Rhythm Metric form, } \\
\text { managing symptoms and } \\
\text { identifying triggers, and } \\
\text { relapse prevention. Family } \\
\text { therapy involved education } \\
\text { about BP, identification of } \\
\text { triggers, communication } \\
\text { enhancement, and problem- } \\
\text { solving. } \\
\text {-25 sessions of individual } \\
\text { therapy and } 25 \text { sessions of } \\
\text { family-focused therapy } \\
\text { (frequency adapted to patient } \\
\text { needs) }\end{array}$ & $\begin{array}{l}\text { Treatment as } \\
\text { usual: Crisis } \\
\text { management } \\
\text { (not described, } \\
\text { comparison } \\
\text { group from } \\
\text { previous } \\
\text { clinical trial) }\end{array}$ & 12 months & $\begin{array}{l}\text { Relapse } \\
\text { SADS-C Depression } \\
\text { SADS-C Mania } \\
\text { Time to Recurrence } \\
\\
\text { Withdrawal } 28 \%\end{array}$ \\
\hline
\end{tabular}

Abbreviations: BDI=Beck depression inventory; BP=bipolar disorder; DSM-IV= Diagnostic and statistical manual, $4^{\text {th }}$ edition; ISS=Internal States Scale; MDQ=Mood Disorder Questionnaire; NOS=not otherwise specified; NR=not reported; PMID=PubMed Identification Number; QoL.BD-Brief=Quality of Life,Bipolar Disorder; RCT=randomized controlled trial; SADS-C= Schedule for Affective Disorders and Schizophrenia, change version; SASS=Simpson Angus Scale score; WHO-QOL-bref=World Health Organization Quality of Life -short version; YMRS = Young Mania Rating Scale 
Appendix Table 02. Summary risk of bias assessments: combination interventions vs. inactive comparators

\begin{tabular}{|c|c|c|}
\hline $\begin{array}{c}\text { Study } \\
\text { Funder } \\
\text { PMID }\end{array}$ & $\begin{array}{l}\text { Overall Risk of Bias } \\
\text { Assessment }\end{array}$ & Rationale \\
\hline $\begin{array}{l}\text { Gonzalez-Isasi, } \\
2014^{1} \\
\text { Gonzalez-Isasi, } \\
2010^{2} \\
\text { Non-Government } \\
20444503 \\
23276524 \\
\end{array}$ & Low & No significant suspected biases. \\
\hline $\begin{array}{l}\text { Todd, } 2014^{3} \\
\text { Government and } \\
\text { Non-Government } \\
25129531\end{array}$ & Moderate & $\begin{array}{l}\text { Suspected bias due to process for selection. Participant eligibility was based self-reported diagnosis and } \\
\text { online clinical questioonare. }\end{array}$ \\
\hline $\begin{array}{l}\text { Miklowitz } 2003^{4} \\
\text { Government and } \\
\text { Non-Government } \\
12963672\end{array}$ & High & $\begin{array}{l}\text { Suspected bias due to process for selection. Partcipants were not randomized to treatment or comparator } \\
\text { arm. Data used for comparison was from a previous study. }\end{array}$ \\
\hline
\end{tabular}

Abbreviations: PMID=PubMed Identification Number 
Appendix Table 03. Outcomes summary: combination interventions vs. inactive comparators

\begin{tabular}{|c|c|c|c|c|c|}
\hline $\begin{array}{l}\text { Study } \\
\text { PMID }\end{array}$ & Responder/Remitter & Symptom & Function & Other & $\mathrm{AE}$ \\
\hline $\begin{array}{l}\text { Gonzalez-Isasi } \\
2010^{2} \\
20444503 \\
\\
\text { Gonzalez-Isasi } \\
2014^{1} \\
23276524\end{array}$ & NR & $\begin{array}{l}\text { Depression* } \\
11 \text { months, BDI } \\
\text { Favors combination intervention } \\
\text { ES=-0.83 (95\% Cl -1.47, -0.18) } \\
17 \text { months, BDI } \\
\text { Favors combination intervention } \\
\text { ES=-1.21 (95\% Cl -1.89, -0.53) } \\
5 \text { years, BDI } \\
\text { Favors combination intervention } \\
\text { ES=-2.17 (95\% Cl -2.95, -1.37) } \\
\text { Mania* } \\
11 \text { months, YMRS } \\
\text { Favors combination intervention } \\
\text { ES=-1.0 (95\% Cl -1.60, -0.30) } \\
17 \text { months, YMRS } \\
\text { Favors combination intervention } \\
\text { ES=-1.5 (95\% Cl }-2.2,-0.80) \\
5 \text { years, YMRS } \\
\text { Favors combination intervention } \\
\text { ES=-1.10 (95\% Cl }-1.80,-0.40)\end{array}$ & NR & $\begin{array}{l}\text { Hospitalizations } \\
\text { Significant difference } \\
\text { between groups at } 17- \\
\text { months }(p=0.015) \text {. No } \\
\text { difference at } 11- \\
\text { months }(p=0.12) \text { or } 5- \\
\text { years }(p=0.11) \text {. }\end{array}$ & NR \\
\hline $\begin{array}{l}\text { Todd } 2014^{3} \\
25129531\end{array}$ & NR & $\begin{array}{l}\text { Depression* } \\
6 \text { months, ISS Depression } \\
\text { Favors combination intervention } \\
E S=-0.44(95 \% \mathrm{Cl}-0.83,-0.05)\end{array}$ & $\begin{array}{l}\frac{\text { Quality of Life* }}{6 \text { months, QoL.BD- }} \\
\text { Brief } \\
\text { Favors combination } \\
\text { intervention } \\
\text { ES }=0.42(95 \% \mathrm{Cl} 0.04 \text {, } \\
0.82) \\
\\
\text { Social Function* } \\
6 \text { months, SASS } \\
\text { Favors combination } \\
\text { intervention } \\
\text { ES=0.54 (95\% Cl 0.14, } \\
0.93)\end{array}$ & NR & NR \\
\hline
\end{tabular}




\begin{tabular}{|c|c|c|c|c|c|}
\hline $\begin{array}{l}\text { Study } \\
\text { PMID }\end{array}$ & Responder/Remitter & Symptom & Function & Other & $\mathrm{AE}$ \\
\hline $\begin{array}{l}\text { Miklowitz 20034 } \\
12963672\end{array}$ & $\begin{array}{l}\frac{\text { Relapse }}{12 \text { months, Any Type }} \\
\text { NS } \\
\text { OR=0.68 ( } 95 \% \text { Cl } 0.24 \text {, } \\
1.85) ; p=0.50 \\
\\
\text { Time to Recurrence } \\
12 \text { months } \\
\text { Favors combination } \\
\text { intervention } \\
\text { HR }=0.078, p<0.02 \\
42.5(2.2) \text { weeks IFIT vs. } \\
34.5(2.5) \text { weeks CM }\end{array}$ & $\begin{array}{l}\frac{\text { SADS-C Depression }}{12 \text { months }} \\
\text { Favors combination } \\
\text { intervention, } p<0.0001 \text {. } \\
\frac{\text { SADS-C Mania }}{12 \text { months }} \\
\text { NS, } p>0.10\end{array}$ & NR & NR & NR \\
\hline
\end{tabular}

Abbreviations: AE=Adverse Events; BDI=Beck depression inventory; CI=Confidence Interval; CM=Clinical Management; ES=Effect Size; HR=Hazard Ratio; IFIT=Integrated Family and Individual Therapy; ISS=Internal States Scale; NR=not reported; NS=not significant; OR=Odds Ratio; PMID=PubMed Identification Number; QoL.BD-Brief=Quality of Life,Bipolar Disorder; SADS-C=Schedule for Affective Disorders and Schizophrenia-Change version; SASS=Simpson Angus Scale score; YMRS = Young Mania Rating Scale 
Appendix Table 04. Summary of strength of evidence: combination intervention vs. inactive comparators

\begin{tabular}{|c|c|c|c|c|c|c|c|c|}
\hline Outcome & Timing & $\begin{array}{l}\text { \# Studies/ Design } \\
\text { (n analyzed) }\end{array}$ & $\begin{array}{l}\text { Finding or } \\
\text { Summary } \\
\text { Statistic }\end{array}$ & $\begin{array}{c}\text { Study } \\
\text { Limitations }\end{array}$ & Consistency & Directness & $\begin{array}{c}\text { Precisio } \\
\text { n }\end{array}$ & $\begin{array}{l}\text { Overall Gradel } \\
\text { Conclusion }\end{array}$ \\
\hline Relapse & 12 months & $\begin{array}{l}1 \text { Cohort Study } \\
(n=100)\end{array}$ & $\begin{array}{l}\text { No difference } \\
\text { between groups at } \\
12 \text { months. }\end{array}$ & High & Unclear & Direct & Imprecise & Insufficient \\
\hline Depression & $\begin{array}{l}7-12 \\
\text { months } \\
5 \text { years }\end{array}$ & $\begin{array}{l}3 \text { RCTs } \\
(n=262)\end{array}$ & $\begin{array}{l}\text { Favors } \\
\text { combination } \\
\text { intervention across } \\
\text { multiple time } \\
\text { periods. }\end{array}$ & Low & Consistent & Direct & Imprecise & Insufficient \\
\hline Mania & $\begin{array}{l}7-12 \\
\text { months } \\
5 \text { years }\end{array}$ & $\begin{array}{l}2 \text { RCTs } \\
(n=140)\end{array}$ & $\begin{array}{l}\text { Mixed evidence } \\
\text { with no clear } \\
\text { direction of effect. } \\
\text { No pattern across } \\
\text { time periods. }\end{array}$ & Low & Unclear & Direct & Imprecise & Insufficient \\
\hline $\begin{array}{l}\text { Global } \\
\text { Function }\end{array}$ & NR & - & - & - & - & - & - & - \\
\hline $\begin{array}{l}\text { Other } \\
\text { Measures } \\
\text { of Function }\end{array}$ & 6 months & $\begin{array}{l}1 \mathrm{RCT} \\
(\mathrm{n}=122)\end{array}$ & $\begin{array}{l}\text { Favors } \\
\text { combination } \\
\text { intervention at } 6 \\
\text { months. }\end{array}$ & Moderate & Unclear & Direct & Imprecise & Insufficient \\
\hline
\end{tabular}

Abbreviations: NR=not reported; RCT=randomized controlled trial 
Appendix Table 05. Characteristics of eligible studies: combination interventions vs. active comparators

\begin{tabular}{|c|c|c|c|c|c|c|}
\hline $\begin{array}{c}\text { Study, Year } \\
\text { Design } \\
\text { Location } \\
\text { Funder } \\
\text { Risk of Bias } \\
\text { PMID }\end{array}$ & $\begin{array}{c}\text { Randomized (N) } \\
\text { Age (mean) } \\
\text { Sex (\% Female) } \\
\text { Race (\% White) } \\
\text { Diagnosis } \\
\text { (\% BP I, II, NOS) } \\
\text { Setting }\end{array}$ & $\begin{array}{c}\text { Inclusions } \\
\text { Key Exclusions }\end{array}$ & $\begin{array}{c}\text { Intervention } \\
\text { Description }\end{array}$ & $\begin{array}{l}\text { Comparison } \\
\text { Description }\end{array}$ & $\begin{array}{l}\text { Followup } \\
\text { Duration }\end{array}$ & $\begin{array}{c}\text { Outcomes } \\
\text { Reported } \\
\text { Withdrawal (\%) at } \\
\text { endpoint }\end{array}$ \\
\hline $\begin{array}{l}\text { Fagiolini } 2009^{5} \\
\text { RCT } \\
\text { US } \\
\text { Government } \\
\text { Moderate } \\
19500091\end{array}$ & $\begin{array}{l}\mathrm{N}=463 \\
\text { Age } 41(12-75) \\
\text { Female } 61 \% \\
\text { White } 83 \% \\
\text { BP I } 68 \% \\
\text { BP II 19\% } \\
\text { BP NOS 11\% } \\
\text { Schizophrenia 2\% } \\
\text { Outpatient }\end{array}$ & $\begin{array}{l}\text { No current clinical state } \\
\text { excluded; BP I, II, or } \\
\text { NOS or schizoaffective } \\
\text { bipolar subtype disorder } \\
\text { (DSM-IV for adults, } \\
\text { KSADS-PL for } \\
\text { adolescents). } \\
\\
\text { Substance Abuse; Other } \\
\text { Mental Health; } \\
\text { Pregnant/Nursing; } \\
\text { Labs/Other Conditions }\end{array}$ & $\begin{array}{l}\text { Enhanced clinical } \\
\text { intervention and } \\
\text { specialized care for } \\
\text { bipolar disorder. } \\
\text { Enhanced clinical } \\
\text { intervention consisted of } \\
10 \text { basic elements plus } \\
\text { specific modules for } \\
\text { young, elderly, and } \\
\text { African American patients. } \\
\text { Elements consisted of } \\
\text { education (on disorder, } \\
\text { medications, sleep) and } \\
\text { management (review of } \\
\text { symptoms, discussion and } \\
\text { management of side } \\
\text { effects, discussion of early } \\
\text { waning signs). Additional } \\
\text { non-specific support } \\
\text { provided to both patient } \\
\text { and families. } \\
\text {-Weekly enhanced clinical } \\
\text { sessions for } 12 \text { weeks, } \\
\text { then every other week for } \\
8 \text { weeks, and then } \\
\text { monthly for remaining } \\
\text { time or until they achieved } \\
\text { recurrence }\end{array}$ & $\begin{array}{l}\text { Specialized care } \\
\text { for bipolar disorder } \\
\text { consisting of a } \\
\text { manualized system } \\
\text { of clinical } \\
\text { management } \\
\text { included } \\
\text { assessment of } \\
\text { quality of life, } \\
\text { standardized } \\
\text { assessments of } \\
\text { mood, } \\
\text { comprehensive } \\
\text { medical } \\
\text { evaluations, } \\
\text { frequent visits with } \\
\text { treatment team, } \\
\text { pharmacological } \\
\text { treatment and } \\
\text { tracking and } \\
\text { monitoring of visits. }\end{array}$ & 18 months & $\begin{array}{l}\text { CGI BP Depression } \\
\text { CGI BP Mania } \\
\text { GAF } \\
\text { QLESQ } \\
\text { Withdrawal } 30 \%\end{array}$ \\
\hline
\end{tabular}




\begin{tabular}{|c|c|c|c|c|c|c|}
\hline $\begin{array}{l}\text { Study, Year } \\
\text { Design } \\
\text { Location } \\
\text { Funder } \\
\text { Risk of Bias } \\
\text { PMID }\end{array}$ & $\begin{array}{c}\text { Randomized (N) } \\
\text { Age (mean) } \\
\text { Sex (\% Female) } \\
\text { Race (\% White) } \\
\text { Diagnosis } \\
\text { (\% BP I, II, NOS) } \\
\text { Setting }\end{array}$ & $\begin{array}{c}\text { Inclusions } \\
\text { Key Exclusions }\end{array}$ & $\begin{array}{l}\text { Intervention } \\
\text { Description }\end{array}$ & $\begin{array}{l}\text { Comparison } \\
\text { Description }\end{array}$ & $\begin{array}{l}\text { Followup } \\
\text { Duration }\end{array}$ & $\begin{array}{c}\text { Outcomes } \\
\text { Reported } \\
\text { Withdrawal (\%) at } \\
\text { endpoint }\end{array}$ \\
\hline $\begin{array}{l}\text { Zaretsky } 2008^{6} \\
\text { RCT } \\
\text { Canada } \\
\text { Government and Non- } \\
\text { Government } \\
\text { High } \\
18674402\end{array}$ & $\begin{array}{l}\text { N=79 } \\
\text { Age 41 (18-60) } \\
\text { Female NR } \\
\text { White NR } \\
\text { BP I 66\% } \\
\text { BP II 34\% } \\
\\
\text { Outpatient }\end{array}$ & $\begin{array}{l}\text { Euthymic/Maintenance; } \\
\text { BP I or II, not currently in } \\
\text { a full episode, taking a } \\
\text { standard mood stabilizer } \\
\text { regimen with no change } \\
\text { in regimen or prescribing } \\
\text { physician in month prior } \\
\text { to study entry. } \\
\\
\text { Substance Abuse; } \\
\text { Schizoaffective; Other } \\
\text { Mental Health; } \\
\text { Neurological Disorders; } \\
\text { Labs/Other Conditions }\end{array}$ & $\begin{array}{l}\text { Psychoeducation and } \\
\text { CBT. CBT was based on } \\
\text { Basco and Rush manual } \\
\text { and emphasized } \\
\text { collaborative goal setting, } \\
\text { cognitive restructuring, } \\
\text { problem-solving, and } \\
\text { enhancing interpersonal } \\
\text { communication. } \\
\text {-7 weekly, audiotaped } \\
\text { individual sessions of } \\
\text { psychoeducation and } 13 \\
\text { weekly, audiotaped } \\
\text { individual sessions of CBT }\end{array}$ & $\begin{array}{l}\text { Psychoeducation } \\
\text { based on the first } \\
\text { five chapters of the } \\
\text { Basco and Rush } \\
\text { CBT manual. } \\
\text {-7 weekly, } \\
\text { audiotaped } \\
\text { individual sessions }\end{array}$ & 6 months & $\begin{array}{l}\text { Relapse } \\
\text { HDRS } \\
\text { Withdrawal 42\% }\end{array}$ \\
\hline
\end{tabular}

Abbreviations: BP=bipolar disorder; CBT=Cognitive Behavioral Therapy; CGI=Clinical Global Impressions Scale; DSM-IV= Diagnostic and statistical manual, $4^{\text {th }}$ edition;

GAF=General Assessment of Functioning Scale; HDRS=Hamilton Depression Rating Scale; KSADS-PL=Kiddie Schedule for Affective Disorders and Schizophrenia-Present and Lifetime Version; NOS=not otherwise specified; NR=not reported; PMID=PubMed Identification Number; RCT=randomized controlled trial

Appendix Table O6. Summary risk of bias assessments: combination interventions vs. active comparators
\begin{tabular}{|l|l|l|}
\hline \multicolumn{1}{|c|}{$\begin{array}{c}\text { Study } \\
\text { Funder } \\
\text { PMID }\end{array}$} & \multicolumn{1}{|c|}{$\begin{array}{c}\text { Overall Risk of Bias } \\
\text { Assessment }\end{array}$} & \\
\hline $\begin{array}{l}\text { Fagiolini } 2009^{5} \\
\text { Government } \\
19500091\end{array}$ & Moderate & Suspected selection bias due to unclear reporting of randomization process. \\
\hline $\begin{array}{l}\text { Zaretsky } 2008^{6} \\
\text { Government and } \\
\text { Non-Government } \\
18674402\end{array}$ & High & $\begin{array}{l}\text { Suspected bias selection bias due to unclear reporting of randomization process and suspected bias due to } \\
\text { attrition rate of } 42 \% .\end{array}$ \\
\hline
\end{tabular}

Abbreviations: PMID=PubMed Identification Number 
Appendix Table 07. Outcomes Summary: combination interventions vs. active comparators

\begin{tabular}{|c|c|c|c|c|c|}
\hline $\begin{array}{l}\text { Study } \\
\text { PMID }\end{array}$ & Responder/Remitter & Symptom & Function & Other & $\overline{\mathrm{AE}}$ \\
\hline $\begin{array}{l}\text { Fagiolini } 2009^{5} \\
19500091\end{array}$ & NR & $\begin{array}{l}\frac{\text { Depression }}{18 \text { months, CGI }} \\
\text { Depression } \\
\text { NS } \\
\frac{\text { Mania }}{18 \text { months, CGI Mania }} \\
\text { NS }\end{array}$ & $\begin{array}{l}\frac{\text { Global Function }}{18 \text { months, GAF }} \\
\text { NS } \\
\text { Quality of Life } \\
18 \text { months, QLESQ } \\
\text { Favors combination } \\
\text { intervention. }\end{array}$ & NR & NR \\
\hline $\begin{array}{l}\text { Zaretsky } 2008^{6} \\
18674402\end{array}$ & $\begin{array}{l}\frac{\text { Relapse }^{*}}{12 \text { months, Any Type }} \\
\text { NS } \\
\text { OR=1.20 (95\% Cl 0.23, } \\
6.80) ; p=0.55\end{array}$ & $\begin{array}{l}\frac{\mathrm{HDRS}}{12 \text { months }} \\
\text { Favors combination } \\
\text { intervention, } p=0.055\end{array}$ & NR & NR & NR \\
\hline
\end{tabular}

Abbreviations: CGI=Clinical global impression scale; CI=Confidence Interval; GAF=General Assessment of Functioning Scale; HDRS=Hamilton Depression Rating Scale;

NR=Not Reported; NS=not significant; OR=Odds Ratio; PMID=PubMed Identification Number; Q-LES-Q=Quality of Life Enjoyment and Satisfaction Questionaire

\section{Appendix Table 08. Summary of strength of evidence: combination intervention vs. active comparators}

\begin{tabular}{|c|c|c|c|c|c|c|c|c|}
\hline Outcome & Timing & $\begin{array}{l}\text { \# Studies/ Design } \\
\text { (n analyzed) }\end{array}$ & $\begin{array}{l}\text { Finding or } \\
\text { Summary } \\
\text { Statistic }\end{array}$ & $\begin{array}{c}\text { Study } \\
\text { Limitations }\end{array}$ & Consistency & Directness & $\begin{array}{c}\text { Precisio } \\
\mathbf{n}\end{array}$ & $\begin{array}{l}\text { Overall Gradel } \\
\text { Conclusion }\end{array}$ \\
\hline Relapse & 12 months & $1 \mathrm{RCT}(\mathrm{n}=79)$ & $\begin{array}{l}\text { No difference } \\
\text { between groups at } \\
12 \text { months. }\end{array}$ & High & Unclear & Direct & Imprecise & Insufficient \\
\hline Depression & $\begin{array}{l}12 \text { months } \\
18 \text { months }\end{array}$ & 2 RCTs $(n=542)$ & $\begin{array}{l}\text { Mixed evidence } \\
\text { with no clear } \\
\text { direction of effect. } \\
\text { No pattern across } \\
\text { time periods. }\end{array}$ & High & Inconsistent & Direct & Imprecise & Insufficient \\
\hline Mania & 18 months & $1 \mathrm{RCT}(\mathrm{n}=463)$ & $\begin{array}{l}\text { No difference } \\
\text { between groups at } \\
18 \text { months. }\end{array}$ & High & Unclear & Direct & Imprecise & Insufficient \\
\hline $\begin{array}{l}\text { Global } \\
\text { Function }\end{array}$ & 18 months & $1 \mathrm{RCT}(\mathrm{n}=463)$ & $\begin{array}{l}\text { No difference } \\
\text { between groups at } \\
18 \text { months }\end{array}$ & Moderate & Unclear & Direct & Imprecise & Insufficient \\
\hline $\begin{array}{l}\text { Other } \\
\text { Measures } \\
\text { of Function }\end{array}$ & 18 months & $1 \mathrm{RCT}(\mathrm{n}=463)$ & $\begin{array}{l}\text { Favors } \\
\text { combination } \\
\text { intervention at } 18 \\
\text { months. }\end{array}$ & Moderate & Unclear & Direct & Imprecise & Insufficient \\
\hline
\end{tabular}

Abbreviations: RCT=randomized controlled trial 


\section{References for Appendix 0}

1. Gonzalez Isasi A, Echeburua E, Liminana JM, et al. Psychoeducation and cognitive-behavioral therapy for patients with refractory bipolar disorder: A 5-year controlled clinical trial. European Psychiatry. Dec. 2014 March 2014(Pagination)PMID 23276524.

2. Gonzalez-Isasi A, Echeburua E, Mosquera F, et al. Long-term efficacy of a psychological intervention program for patients with refractory bipolar disorder: a pilot study. Psychiatry Research. 2010 Apr 30;176(2-3):161-5. PMID 20096466.

3. Todd NJ, Jones SH, Hart A, et al. A web-based self-management intervention for Bipolar Disorder 'living with bipolar': a feasibility randomised controlled trial. J Affect Disord. 2014 Dec;169:21-9. PMID 25129531.
4. Miklowitz DJ, George EL, Richards JA, et al. A randomized study of family-focused psychoeducation and pharmacotherapy in the outpatient management of bipolar disorder. Archives of General Psychiatry. 2003 Sep;60(9):904-12. PMID 12963672.

5. Fagiolini A, Frank E, Axelson DA, et al. Enhancing outcomes in patients with bipolar disorder: results from the Bipolar Disorder Center for Pennsylvanians Study. Bipolar Disorders. 2009 Jun;11(4):382-90. PMID 19500091.

6. Zaretsky A, Lancee W, Miller C, et al. Is cognitive-behavioural therapy more effective than psychoeducation in bipolar disorder? Canadian Journal of Psychiatry - Revue Canadienne de Psychiatrie. 2008 Jul;53(7):441-8. PMID 18674402. 


\section{Appendix P. Systematic Treatment Enhancement Program for Bipolar Disorder (STEP-BD) Study and Other Psychosocial and Somatic Interventions}

\begin{tabular}{|c|c|c|c|c|c|c|}
\hline $\begin{array}{l}\text { Study, Year } \\
\text { Design } \\
\text { Location } \\
\text { Funder } \\
\text { Risk of Bias } \\
\text { PMID }\end{array}$ & $\begin{array}{c}\text { Randomized (N) } \\
\text { Age (mean) } \\
\text { Sex (\% Female) } \\
\text { Race (\% White) } \\
\text { Diagnosis } \\
\text { (\% BP I, II, NOS) } \\
\text { Setting }\end{array}$ & $\begin{array}{c}\text { Inclusions } \\
\text { Key Exclusions }\end{array}$ & $\begin{array}{l}\text { Intervention } \\
\text { Description }\end{array}$ & $\begin{array}{l}\text { Comparison } \\
\text { Description }\end{array}$ & $\begin{array}{l}\text { Followup } \\
\text { Duration }\end{array}$ & $\begin{array}{c}\text { Outcomes } \\
\text { Reported } \\
\text { Withdrawal (\%) at } \\
\text { endpoint }\end{array}$ \\
\hline $\begin{array}{l}\text { Depp, } 2015^{1} \\
\text { RCT } \\
\text { US } \\
\text { Government } \\
\text { Low } \\
25479050\end{array}$ & \begin{tabular}{|l}
$\mathrm{N}=104$ \\
Age 48 (22-74) \\
Female 59\% \\
White 70\% \\
BP I 88\% \\
BP II 12\% \\
Outpatient
\end{tabular} & $\begin{array}{l}\text { Without severe } \\
\text { symptoms; } \\
\text { Outpatients with BP } \\
\text { (DSM-IV) currently } \\
\text { prescribed } \\
\text { medications for } \\
\text { bipolar disorder } \\
\text { without severe } \\
\text { depressive (MADRS } \\
>32 \text { ) or manic } \\
\text { (YMRS> 20) } \\
\text { Substance Abuse; } \\
\text { Other Mental Health }\end{array}$ & $\begin{array}{l}\text { Psychoeducation followed by use } \\
\text { of a smart phone that delivered } \\
\text { interactive elements via a mobile } \\
\text { web-based program that delivered } \\
\text { questionnaires and responses } \\
\text { based on symptoms or early } \\
\text { warning signs } \\
-4 \text { sessions of psychoeducation } \\
\text { followed by smart intervention ( } 2 \\
\text { surveys per day) for } 10 \text { weeks }\end{array}$ & \begin{tabular}{|l|} 
Psychoeducation \\
followed by binder \\
with paper and \\
pencil mood charts. \\
Monitored remotely \\
via cell phone and \\
had to turn in \\
completed charts at \\
the end of study. \\
\\
-4 sessions of \\
psychoeducation \\
followed by mood \\
charts once per day \\
for 10 weeks
\end{tabular} & 6 months & $\begin{array}{l}\text { MADRS } \\
\text { YMRS } \\
\text { Withdrawal 22\% }\end{array}$ \\
\hline
\end{tabular}




\begin{tabular}{|c|c|c|c|c|c|c|}
\hline $\begin{array}{c}\text { Study, Year } \\
\text { Design } \\
\text { Location } \\
\text { Funder } \\
\text { Risk of Bias } \\
\text { PMID }\end{array}$ & $\begin{array}{c}\text { Randomized (N) } \\
\text { Age (mean) } \\
\text { Sex (\% Female) } \\
\text { Race (\% White) } \\
\text { Diagnosis } \\
\text { (\% BP I, II, NOS) } \\
\text { Setting }\end{array}$ & $\begin{array}{c}\text { Inclusions } \\
\text { Key Exclusions }\end{array}$ & $\begin{array}{l}\text { Intervention } \\
\text { Description }\end{array}$ & $\begin{array}{l}\text { Comparison } \\
\text { Description }\end{array}$ & $\begin{array}{l}\text { Followup } \\
\text { Duration }\end{array}$ & $\begin{array}{c}\text { Outcomes } \\
\text { Reported } \\
\text { Withdrawal (\%) at } \\
\text { endpoint }\end{array}$ \\
\hline $\begin{array}{l}\text { Faurholt-Jepsen, } \\
2015^{2} \\
\text { RCT } \\
\text { Denmark } \\
\text { Government and } \\
\text { Non-Government } \\
\text { Low } \\
26220802\end{array}$ & $\begin{array}{l}\mathrm{N}=78 \\
\text { Age 29 (18-60) } \\
\text { Female 82\% } \\
\text { White NR } \\
\text { BP I 82\% } \\
\text { Outpatient }\end{array}$ & $\begin{array}{l}\text { No current clinical } \\
\text { state excluded; } \\
\text { Individuals with a BP } \\
\text { diagnosis (ICD-10 } \\
\text { and Schedules for } \\
\text { Clinical Assessment } \\
\text { in Neuropsychiatry) } \\
\text { with a HDRS } \leq 17 \text { and } \\
\text { a YMRS } \leq 17 \\
\text { Other Mental Health; } \\
\text { Pregnant/Nursing; } \\
\text { Labs/Other } \\
\text { Conditions }\end{array}$ & $\begin{array}{l}\text { Smartphone with self-monitoring } \\
\text { system that documented mood, } \\
\text { sleep length, activity, medication } \\
\text { taken, irritability, cognitive } \\
\text { problems, alcohol consumption, } \\
\text { stress, menstruation, and early } \\
\text { warning signs. Patients could see } \\
\text { visual representations of data to } \\
\text { self-monitor. System included } \\
\text { feedback loop with clinic and } \\
\text { contact with study nurse. } \\
-6 \text { months of self-monitoring }\end{array}$ & $\begin{array}{l}\text { Smartphone without } \\
\text { self-monitoring } \\
\text { system and nurse } \\
\text { contact if needed. } \\
-6 \text { months of smart } \\
\text { phone access }\end{array}$ & 6 months & $\begin{array}{l}\text { HAMD-17 } \\
\text { YMRS } \\
\text { FAST } \\
\text { WHO-Qol-bref } \\
\text { Withdrawal 14\% }\end{array}$ \\
\hline
\end{tabular}




\begin{tabular}{|c|c|c|c|c|c|c|}
\hline $\begin{array}{l}\text { Study, Year } \\
\text { Design } \\
\text { Location } \\
\text { Funder } \\
\text { Risk of Bias } \\
\text { PMID }\end{array}$ & $\begin{array}{c}\text { Randomized (N) } \\
\text { Age (mean) } \\
\text { Sex (\% Female) } \\
\text { Race (\% White) } \\
\text { Diagnosis } \\
\text { (\% BP I, II, NOS) } \\
\text { Setting }\end{array}$ & $\begin{array}{c}\text { Inclusions } \\
\text { Key Exclusions }\end{array}$ & $\begin{array}{l}\text { Intervention } \\
\text { Description }\end{array}$ & $\begin{array}{l}\text { Comparison } \\
\text { Description }\end{array}$ & $\begin{array}{l}\text { Followup } \\
\text { Duration }\end{array}$ & $\begin{array}{c}\text { Outcomes } \\
\text { Reported } \\
\text { Withdrawal (\%) at } \\
\text { endpoint }\end{array}$ \\
\hline $\begin{array}{l}\text { Deckersbach } \\
2014^{3} \\
\text { Miklowitz } 2007^{4} \\
\text { Miklowitz } 2007 b^{5} \\
\text { RCT } \\
\text { US } \\
\text { Government and } \\
\text { Non-Government } \\
\text { High } \\
\text { Moderate } \\
\text { High } \\
24077657 \\
17728418 \\
17404119\end{array}$ & $\begin{array}{l}\mathrm{N}=293 \\
\text { Age } 40 \text { (18-62) } \\
\text { Female 59\% } \\
\text { White } 91 \% \\
\text { BP I } 67 \% \\
\text { BP II 31\% } \\
\text { BP NOS } 2 \% \\
\\
\text { Outpatient }\end{array}$ & $\begin{array}{l}\text { Major Depressive } \\
\text { Episode; BP I or II } \\
\text { (DSM-IV) with current } \\
\text { major depressive } \\
\text { episode (but no mixed } \\
\text { episode or depression } \\
\text { not otherwise } \\
\text { specified), currently } \\
\text { being treated with a } \\
\text { mood stabilizer (or } \\
\text { willing to initiate), not } \\
\text { currently undergoing } \\
\text { psychotherapy (or } \\
\text { willing to discontinue) } \\
\\
\text { Substance Abuse; } \\
\text { Other Mental Health; } \\
\text { Pregnant/Nursing; } \\
\text { Labs/Other } \\
\text { Conditions }\end{array}$ & $\begin{array}{l}\text { Intensive psychotherapy } \\
\text { consisting of one of the following: } \\
\text { 1) individual CBT consisting } \\
\text { psychoeducation, life events } \\
\text { scheduling, cognitive } \\
\text { restructuring, problem-solving, } \\
\text { strategies for early detection, and } \\
\text { interventions for comorbidities, 2) } \\
\text { IPSRT consisting of selecting a } \\
\text { primary problem area and } \\
\text { teaching patients about the Social } \\
\text { Rhythm Metric and interpersonal } \\
\text { problem resolution, or 3) family- } \\
\text { focused therapy which } \\
\text { encouraged patients and relatives } \\
\text { to develop a common } \\
\text { understanding, develop a relapse } \\
\text { prevention plan, participate in } \\
\text { communication enhancement } \\
\text { exercises, and identify and solve } \\
\text { problems related to illness or the } \\
\text { home environment. } \\
\text {-30 50-minute sessions over } 9 \\
\text { months }\end{array}$ & $\begin{array}{l}\text { Collaborative care } \\
\text { consisting of a } \\
\text { reviewing a } \\
\text { psychoeducational } \\
\text { videotape and } \\
\text { workbook and } \\
\text { developing a } \\
\text { treatment contract. } \\
\text { Worbook included } \\
\text { information about } \\
\text { BP, schedule } \\
\text { management and } \\
\text { mood charting, } \\
\text { improving } \\
\text { communication } \\
\text { skills, an developing } \\
\text { a treatment } \\
\text { contract. } \\
\text {-Three 50-minute } \\
\text { individual sessions }\end{array}$ & 12 months & $\begin{array}{l}\text { Recovery } \\
\text { LIFE-RIFT } \\
\text { Withdrawal 48\% }\end{array}$ \\
\hline
\end{tabular}

Test; HAMD-17= Hamilton Rating Scale for Depression (17-items); HDRS= Hamilton Depression Rating Scale; ICD-10= International Statistical Classification of Diseases and

Related Health Problems- $10^{\text {th }}$ Revision; IPSRT=Interpersonal and Social Rhythm Therapy; LIFE-RIFT= Longitudinal Interval Follow-up Evaluation-Range of Impaired Functioning Tool; MADRS=Montgomery-Asberg Depression Rating Scale; NOS=not otherwise specified; NR=not reported; PMID=PubMed Identification Number;

RCT=randomized controlled trial; WHO-QOL-bref= World Health Organization Quality of Life-short version; YMRS = Young Mania Rating Scale 
Appendix Table P2. Summary risk of bias assessments: STEP-BD study and other psychosocial interventions

\begin{tabular}{|c|c|c|}
\hline $\begin{array}{l}\text { Study } \\
\text { Funder } \\
\text { PMID }\end{array}$ & $\begin{array}{c}\text { Overall Risk of Bias } \\
\text { Assessment }\end{array}$ & Rationale \\
\hline $\begin{array}{l}\text { Depp, } 2015^{1} \\
\text { Government } \\
25479050\end{array}$ & Low & No significant suspected biases. \\
\hline $\begin{array}{l}\text { Faurholt-Jepsen, } \\
2015^{2} \\
\text { Government and } \\
\text { Non-Government } \\
26220802\end{array}$ & Low & No significant suspected biases. \\
\hline $\begin{array}{l}\text { Deckersbach } 2014^{3} \\
\text { Miklowitz 2007 } \\
\text { Miklowitz 2007b } \\
\text { Government and } \\
\text { Non-Government } \\
24077657 \\
17728418 \\
17404119\end{array}$ & $\begin{array}{l}\text { High } \\
\text { Moderate } \\
\text { High }\end{array}$ & $\begin{array}{l}\text { Suspected bias due to attrition rate of } 48 \% \text {. Part of analysis only includes subset of subjects from total study } \\
\text { population. }\end{array}$ \\
\hline
\end{tabular}

Abbreviations: PMID=PubMed Identification Number 


\section{Appendix Table P3. Outcomes summary: STEP-BD Study and other psychosocial interventions}

\begin{tabular}{|c|c|c|c|c|c|}
\hline $\begin{array}{l}\text { Study } \\
\text { PMID }\end{array}$ & Responder/Remitter & Symptom & Function & Other & $\mathrm{AE}$ \\
\hline $\begin{array}{l}\text { Depp, } 2015^{1} \\
25479050\end{array}$ & NR & $\begin{array}{l}\text { Depression } \\
6 \text { months, MADRS } \\
\text { NS } \\
E S=0.02 ; p=0.05 \\
\frac{\text { Mania }}{6 \text { months, YMRS }} \\
\text { NS } \\
E S=-0.09 ; p=0.26\end{array}$ & NR & NR & NR \\
\hline $\begin{array}{l}\text { Faurholt-Jepsen, } \\
2015^{2} \\
26220802\end{array}$ & NR & $\begin{array}{l}\text { Depression } \\
6 \text { months, HAMD-17 } \\
\text { NS } \\
\text { Adjusted Difference: } 0.96(95 \% \\
\mathrm{Cl}-4.36,6.28) ; p=0.72 \\
\text { Mania } \\
6 \text { months, YMRS } \\
\text { NS } \\
\text { Adjusted Difference }=-0.34 \\
(95 \% \mathrm{Cl}-1.14,0.47) ; p=0.41\end{array}$ & $\begin{array}{l}\text { Global Function } \\
6 \text { months, FAST } \\
\text { NS } \\
\text { Adjusted Difference= } \\
0.96(95 \% \mathrm{Cl}-4.36, \\
6.28) ; p=0.72 \\
\text { Quality of Life } \\
\text { NS } \\
6 \text { months, WHO-QOL- } \\
\text { bref } \\
\text { Adjusted Difference= } \\
1.24(95 \% \mathrm{Cl}-5.18, \\
2.70) ; p=0.54\end{array}$ & NR & NR \\
\hline $\begin{array}{l}\text { Deckersbach } \\
2014^{3} \\
24077657 \\
\text { Miklowitz } 2007^{4} \\
17728418 \\
\\
\text { Miklowitz 2007b } \\
17404119\end{array}$ & $\begin{array}{l}\text { Number Recovered } \\
1 \text { year } \\
\text { Favors intensive } \\
\text { psychosocial intervention } \\
\text { HR } 1.47 ; p=.01 \\
\text { Family Therapy: HR } 1.87 \\
\text { IPSRT: HR } 1.48 \\
\text { CBT: HR } 1.34\end{array}$ & NR & $\begin{array}{l}\frac{\text { Functional }}{\text { Impairement }} \\
9 \text { months, LIFT-RIFT } \\
\text { Favors intensive } \\
\text { psychosocial } \\
\text { intervention, } p=0.04 \\
\text { Mean Difference (SD) } \\
\text { Family Therapy: }-3.17 \\
\text { (3.06) } \\
\text { IPSRT: -1.63 (4.35) } \\
\text { CBT: -1.05 (4.77) } \\
\text { Collaborative Care: - } \\
\text { 0.94 (3.5) }\end{array}$ & NR & NR \\
\hline
\end{tabular}

Abbreviations: AE=Adverse Events; CBT= Cognitive Behavioral Theraphy; CI=Confidence Interval; ES=Effect Size; FAST= Functioning Assessment Short Test;

HAMD-17= Hamilton Rating Scale for Depression (17-items); HR=Hazard Ratio; LIFE-RIFT= Longitudinal Interval Follow-up Evaluation-Range of Impaired Functioning Tool; MADRS=Montgomery-Asberg Depression Rating Scale; IPSRT= Interpersonal and Social Rhythm Therapy; NR=not reported; NS=not significant; PMID=PubMed Identification Number; SD=standard deviation; WHO-QOL-bref= World Health Organization Quality of Life-short version; YMRS = Young Mania Rating Scale 
Appendix Table P4. Summary of strength of evidence: other psychosocial interventions, self-management interventions

\begin{tabular}{|c|c|c|c|c|c|c|c|c|}
\hline Outcome & Timing & $\begin{array}{l}\text { \# Studies/ Design } \\
\text { (n analyzed) }\end{array}$ & $\begin{array}{l}\text { Finding or } \\
\text { Summary } \\
\text { Statistic }\end{array}$ & $\begin{array}{c}\text { Study } \\
\text { Limitations }\end{array}$ & Consistency & Directness & Precision & $\begin{array}{l}\text { Overall Gradel } \\
\text { Conclusion }\end{array}$ \\
\hline Relapse & NR & - & - & - & - & - & - & - \\
\hline Depression & 6 months & 2 RCTs $(n=182)$ & $\begin{array}{l}\text { No difference } \\
\text { between groups in } \\
\text { MADRS at } 6 \\
\text { months. }\end{array}$ & Low & Consistent & Direct & Imprecise & Insufficient \\
\hline Mania & 6 months & 2 RCTs $(n=182)$ & $\begin{array}{l}\text { No difference } \\
\text { between groups in } \\
\text { YMRS at } 6 \text { months. }\end{array}$ & Low & Consistent & Direct & Imprecise & Insufficient \\
\hline $\begin{array}{l}\text { Global } \\
\text { Function }\end{array}$ & 6 months & $1 \mathrm{RCT}(\mathrm{n}=78)$ & $\begin{array}{l}\text { No difference } \\
\text { between groups at } \\
6 \text { months. } \\
\text { FAST } \\
\text { Adjusted } \\
\text { Difference }=0.96 \\
(95 \% \mathrm{Cl}-4.36 \text {, } \\
6.28) ; p=0.72\end{array}$ & Low & Unclear & Direct & Imprecise & Insufficient \\
\hline $\begin{array}{l}\text { Other } \\
\text { Measures } \\
\text { of Function }\end{array}$ & 6 months & $1 \mathrm{RCT}(\mathrm{n}=78)$ & $\begin{array}{l}\text { No difference } \\
\text { between groups at } \\
6 \text { months. } \\
\text { WHO-QOL-bref } \\
\text { Adjusted } \\
\text { Difference }=1.24 \\
(95 \% \mathrm{Cl}-5.18 \\
2.70) ; p=0.54\end{array}$ & Low & Unclear & Direct & Imprecise & Insufficient \\
\hline
\end{tabular}

Abbreviations: CI=Confidence Interval; FAST= Functioning Assessment Short Test; MADRS=Montgomery-Asberg Depression Rating Scale; NR=not reported;

RCT=randomized controlled trial; WHO-QOL-bref= World Health Organization Quality of Life-short version; YMRS = Young Mania Rating Scale 
Appendix Table P5. Characteristics of eligible studies: somatic therapy

\begin{tabular}{|c|c|c|c|c|c|c|}
\hline $\begin{array}{l}\text { Study, Year } \\
\text { Design } \\
\text { Location } \\
\text { Funder } \\
\text { Risk of Bias } \\
\text { PMID }\end{array}$ & $\begin{array}{c}\text { Randomized (N) } \\
\text { Age (mean) } \\
\text { Sex (\% Female) } \\
\text { Race (\% White) } \\
\text { Diagnosis } \\
\text { (\% BP I, II, NOS) } \\
\text { Setting }\end{array}$ & $\begin{array}{c}\text { Inclusions } \\
\text { Key Exclusions }\end{array}$ & $\begin{array}{l}\text { Intervention } \\
\text { Description }\end{array}$ & $\begin{array}{l}\text { Comparison } \\
\text { Description }\end{array}$ & $\begin{array}{c}\text { Follow-up } \\
\text { Duration }\end{array}$ & $\begin{array}{c}\text { Outcomes } \\
\text { Reported } \\
\begin{array}{c}\text { Withdrawal (\%) at } \\
\text { endpoint }\end{array}\end{array}$ \\
\hline $\begin{array}{l}\text { Fitzgerald, } 2016^{6} \\
\text { RCT } \\
\text { Australia } \\
\text { Government } \\
\text { Medium } \\
27016659\end{array}$ & $\begin{array}{l}\mathrm{N}=46 \\
\text { Age } 46(33-59) \\
\text { Female } 57 \% \\
\text { Race NR } \\
\text { BP I 63\% } \\
\text { BP II 37\% } \\
\text { Outpatient }\end{array}$ & $\begin{array}{l}\text { Depression; Individuals with } \\
\text { BP I or II (DSM-IV) with } \\
\text { persistent depressive } \\
\text { symptoms (HAM-D > 20) } \\
\text { who failed to respond to at } \\
\text { least two courses of anti- } \\
\text { depressants for at least } 6 \\
\text { weeks in current episode. } \\
\text { No increase or initiation of } \\
\text { new treatment in the four } \\
\text { weeks prior to rTMS. } \\
\text { Labs/Other Conditions; } \\
\text { Neurological Disorder }\end{array}$ & $\begin{array}{l}\text { Repetitive } \\
\text { transcranial magnetic } \\
\text { stimulation } \\
\text {-20 rTMS sessions } \\
\text { for four weeks }\end{array}$ & $\begin{array}{l}\text { Sham } \\
\text { stimulation } \\
\\
-20 \text { sham } \\
\text { sessions for } \\
\text { four weeks }\end{array}$ & 4 weeks & $\begin{array}{l}\text { HAM-D } \\
\text { Response } \\
\text { Remission (Not Defined) } \\
\text { Withdrawal 13\% }\end{array}$ \\
\hline
\end{tabular}

Abbreviations: BP=bipolar disorder; CBT= Cognitive Behavioral Therapy; DSM=Diagnostic and Statistical Manual of Mental Disorders; FAST= Functioning Assessment Short Test; HAMD-17= Hamilton Rating Scale for Depression (17-items); HDRS= Hamilton Depression Rating Scale; ICD-10= International Statistical Classification of Diseases and Related Health Problems- 10 ${ }^{\text {th }}$ Revision; IPSRT=Interpersonal and Social Rhythm Therapy; LIFE-RIFT= Longitudinal Interval Follow-up Evaluation-Range of Impaired

Functioning Tool; MADRS=Montgomery-Asberg Depression Rating Scale; NOS=not otherwise specified; NR=not reported; PMID=PubMed Identification Number; RCT=randomized controlled trial; WHO-QOL-bref= World Health Organization Quality of Life-short version; YMRS = Young Mania Rating Scale

Appendix Table P6. Summary risk of bias assessments: somatic therapy

\begin{tabular}{|l|l|l|}
\hline \multicolumn{1}{|c|}{$\begin{array}{c}\text { Study } \\
\text { Funder } \\
\text { PMID }\end{array}$} & \multicolumn{1}{c|}{$\begin{array}{c}\text { Overall Risk of Bias } \\
\text { Assessment }\end{array}$} & Rationale \\
\hline $\begin{array}{l}\text { Fitzgerald, } 2016^{6} \\
\text { Government } \\
27016659\end{array}$ & Moderate & Suspected bias due to unclear reporting of attrition/loss to followup. \\
\hline
\end{tabular}

Abbreviations: PMID=PubMed Identification Number 
Appendix Table P7. Outcomes summary: somatic therapy

\begin{tabular}{|c|c|c|c|c|c|}
\hline $\begin{array}{l}\text { Study } \\
\text { PMID }\end{array}$ & Responder/Remitter & Symptom & Function & Other & $\overline{A E}$ \\
\hline $\begin{array}{l}\text { Fitzgerald, } 2016^{6} \\
27016659\end{array}$ & $\begin{array}{l}\text { Response } \\
4 \text { weeks } \\
\text { NS; } p<0.05 \\
\text { rTMS }=3 \\
\text { Sham }=1 \\
\text { Remission } \\
4 \text { weeks } \\
\text { NS; } p<0.05 \\
\text { rTMS }=2 \\
\text { Sham }=0\end{array}$ & $\begin{array}{l}\text { Depression }{ }^{\star} \\
4 \text { weeks, HAM-D } \\
\text { NS } \\
\text { ES }=-0.04(95 \% \mathrm{Cl}-0.62,0.54)\end{array}$ & NR & NR & NR \\
\hline
\end{tabular}

Abbreviations: AE=Adverse Events; CI=Confidence Interval; ES=Effect Size; 


\section{References for Appendix $\mathbf{P}$}

1. Depp CA, Ceglowski J, Wang VC, et al. Augmenting psychoeducation with a mobile intervention for bipolar disorder: a randomized controlled trial. Journal of Affective Disorders. 2015 Mar 15;174:23-30. doi: http://dx.doi.org/10.1016/j.jad.2014.10.053. PMID: 25479050.

2. Faurholt-Jepsen M, Frost M, Ritz C, et al. Daily electronic self-monitoring in bipolar disorder using smartphones - the MONARCA I trial: a randomized, placebo-controlled, single-blind, parallel group trial. Psychological Medicine. 2015 Oct;45(13):2691-704. doi: http://dx.doi.org/10.1017/S0033291715000410. PMID: 26220802.

3. Deckersbach T, Peters AT, Sylvia L, et al. Do comorbid anxiety disorders moderate the effects of psychotherapy for bipolar disorder? Results from STEP-BD. American Journal of Psychiatry. 2014 Feb;171(2):178-86. doi: http://dx.doi.org/10.1176/appi.ajp.2013.13020225 . PMID: 24077657.
4. Miklowitz DJ, Otto MW, Frank E, et al. Intensive psychosocial intervention enhances functioning in patients with bipolar depression: results from a 9month randomized controlled trial. American Journal of Psychiatry. 2007 Sep;164(9):1340-7. PMID: 17728418.

5. Miklowitz DJ, Otto MW, Frank E, et al. Psychosocial treatments for bipolar depression: a 1-year randomized trial from the Systematic Treatment Enhancement Program. Archives of General Psychiatry. 2007 Apr;64(4):419-26. PMID: 17404119.

6. Fitzgerald PB, Hoy KE, Elliot D, et al. A negative double-blind controlled trial of sequential bilateral rTMS in the treatment of bipolar depression. Journal of Affective Disorders. 2016 01 Jul;198:158-62. doi: http://dx.doi.org/10.1016/j.jad.2016.03.052. PMID: 27016659 PMID/609157504 Embase. 


\section{Appendix Q. Harms Tables}

\begin{tabular}{|c|c|}
\hline Drug & Box Warning \\
\hline Aripiprazole & $\begin{array}{l}\text { Increased mortality in elderly patients with dementia related psychosis. IRisk of } \\
\text { suicide among adolescents. }\end{array}$ \\
\hline Asenapine & Increased mortality In elderly patients with dementia related psychosis. \\
\hline Carbamazepine & $\begin{array}{l}\text { Risk of suicide. } \\
\text { Serious, sometimes fatal dermatologic reactions reported, including toxic epidermal } \\
\text { necrolysis and Stevens-Johnson syndrome. Risk 10x greater in some Asian } \\
\text { countries; strong associated between risk and HLA-B*1502 allele, which is found } \\
\text { almost exclusively in Asian patients. } \\
\text { Transient or persistent decreased platelet or white blood cell counts not uncommon } \\
\text { with carbamazepine but majority of leukopenia cases do not progress to aplastic } \\
\text { anemia or agranulocytosis. Perform baseline and periodic hematological testing. } \\
\text { Consider discontinuing treatment if evidence of significant bone marrow depression. }\end{array}$ \\
\hline Cariprazine & Increased mortality In elderly patients with dementia related psychosis. \\
\hline Lamotrigine & $\begin{array}{l}\text { Risk of suicide. } \\
\text { Serious skin rashes. }\end{array}$ \\
\hline Lithium & $\begin{array}{l}\text { Lithium toxicity can occur at doses close to therapeutic levels. Facilities for prompt } \\
\text { and accurate serum lithium determinations should be available before initiating } \\
\text { therapy. }\end{array}$ \\
\hline Olanzapine & $\begin{array}{l}\text { Increased mortality In elderly patients with dementia related psychosis. When used in } \\
\text { combination with fluoxetine also warn against suicidality and antidepressant drugs. }\end{array}$ \\
\hline Quetiapine & $\begin{array}{l}\text { Increased mortality in elderly patients with dementia related psychosis. Risk of } \\
\text { suicide among adolescents. }\end{array}$ \\
\hline Risperidone & Increased mortality In elderly patients with dementia related psychosis. \\
\hline
\end{tabular}




\begin{tabular}{|c|c|}
\hline Drug & Box Warning \\
\hline $\begin{array}{l}\text { Valproic acid/ valproate/ } \\
\text { divalproex (same for all) }\end{array}$ & $\begin{array}{l}\text { Risk of suicide. } \\
\text { Serious or fatal hepatotoxicity has occurred, usually during first six months of } \\
\text { treatment. Patients < } 2 \text { years old are at increased risk, especially with the following } \\
\text { comorbidities: multiple anticonvulsant treatment, congenital metabolic disorder, } \\
\text { severe seizure disorder with mental retardation, or organic brain disorders. } \\
\text { Increased risk of acute liver failure and death in patients with hereditary } \\
\text { neurometabolic syndromes caused by mitochondrial DNA polymerase gamma gene } \\
\text { mutations (e.g. Alpers Huttenlocher Syndrome). } \\
\text { Fetal risk via major congenital malformations including neural tube defects and } \\
\text { decreased IQ scores after in utero exposure. } \\
\text { Life threatening pancreatitis including hemorrhagic cases with rapid progression from } \\
\text { initial symptoms to death reported in children and adults. }\end{array}$ \\
\hline Ziprasidone & Increased mortality In elderly patients with dementia related psychosis. \\
\hline Allopurinol & None \\
\hline Bupropion & $\begin{array}{l}\text { Risk of suicide among adolescents. } \\
\text { Serious neuropsychiatric events have been reported in patients taking bupropion for } \\
\text { smoking cessation. }\end{array}$ \\
\hline Celecoxib & $\begin{array}{l}\text { May cause an increased risk of serious cardiovascular thrombotic events, myocardial } \\
\text { infarction, and stroke. This risk may increase with duration of use. Patients with } \\
\text { cardiovascular disease or risk factors for cardiovascular disease may be at greater } \\
\text { risk. } \\
\text { Increased risk of serious gastrointestinal adverse events including bleeding, } \\
\text { ulceration, and perforation of the stomach or intestines, particularly in elderly } \\
\text { patients. }\end{array}$ \\
\hline Citalopram & Risk of suicide among adolescents. \\
\hline Dipyridamole & None \\
\hline Donepezil & None \\
\hline Fluoxetine & Risk of suicide among adolescents. \\
\hline Gabapentin & Risk of suicide. \\
\hline Haloperidol & Increased mortality In elderly patients with dementia related psychosis. \\
\hline Memantine & None \\
\hline Oxcarbazepine & Risk of suicide. \\
\hline Paliperidone & Increased mortality In elderly patients with dementia related psychosis. \\
\hline Paroxetine & Risk of suicide among adolescents. \\
\hline Perphenazine & Increased mortality In elderly patients with dementia related psychosis. \\
\hline Ramelteon & None \\
\hline
\end{tabular}




\begin{tabular}{|l|l|}
\hline \multicolumn{1}{|c|}{ Drug } & \multicolumn{1}{c|}{ Box Warning } \\
\hline Tamoxifen & $\begin{array}{l}\text { Women with ductal carcinoma in situ and at high risk for breast cancer at increased } \\
\text { risk of uterine malignancies, stroke and pulmonary embolism. }\end{array}$ \\
\hline Topiramate & Risk of suicide. \\
\hline Venlafaxine & Risk of suicide among adolescents. \\
\hline Verapamil & None \\
\hline
\end{tabular}

Sources:

www.fda.gov/

www.fda.gov/Drugs/DrugSafety/PostmarketDrugSafetyInformationforPatientsandProviders

Abbreviations: FDA=United States Food and Drug Administration

Appendix Table Q2. Previously reported side effects* of bipolar medications

\begin{tabular}{|c|c|}
\hline $\begin{array}{c}\text { Drug } \\
\text { Generic } \\
\text { Name } \\
\text { (Trade } \\
\text { Names) }\end{array}$ & Side Effects \\
\hline $\begin{array}{l}\text { Aripiprazole } \\
\text { (Ability) }^{1}\end{array}$ & 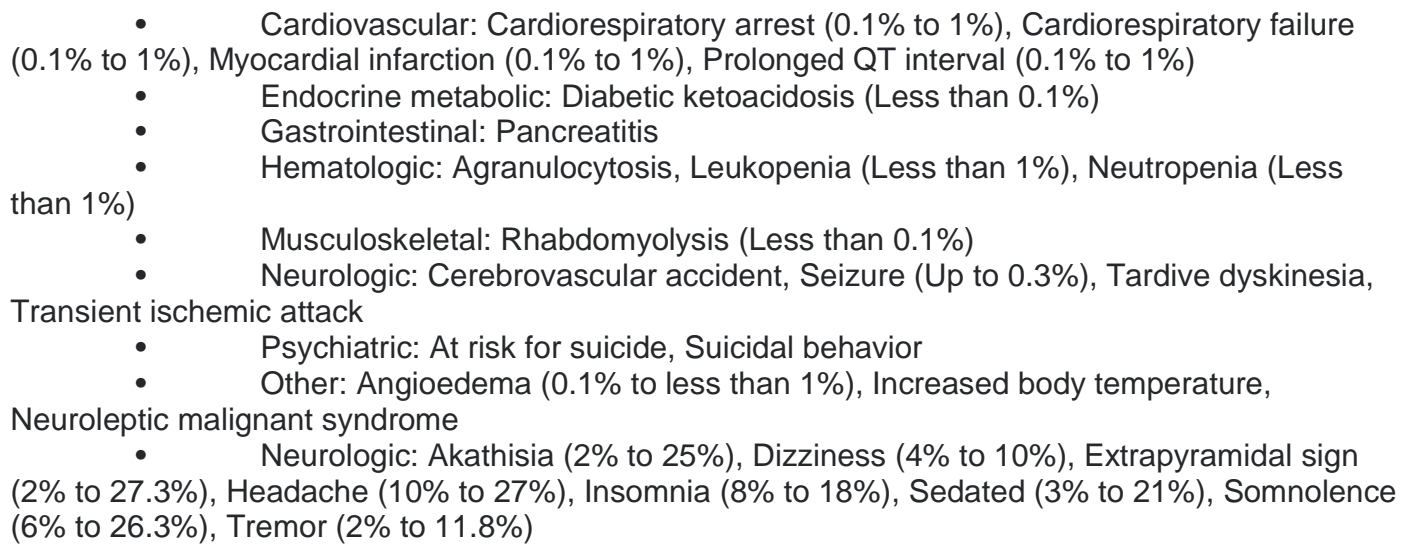 \\
\hline $\begin{array}{l}\text { Asenapine } \\
\text { (Saphris) }^{1}\end{array}$ & 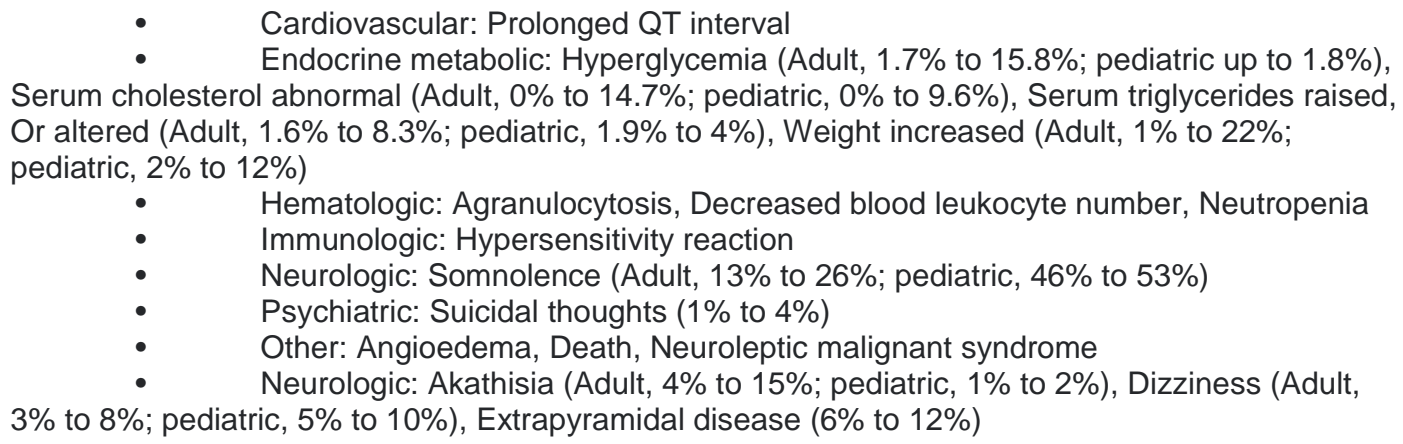 \\
\hline
\end{tabular}




\begin{tabular}{|c|c|}
\hline $\begin{array}{c}\text { Drug } \\
\text { Generic } \\
\text { Name } \\
\text { (Trade } \\
\text { Names) }\end{array}$ & Side Effects \\
\hline $\begin{array}{l}\text { Carbamaze } \\
\text { pine } \\
\text { (Carbetrol, } \\
\text { Epitol, } \\
\text { Equetro, } \\
\text { Tegretol, } \\
\text { Teril) }\end{array}$ & 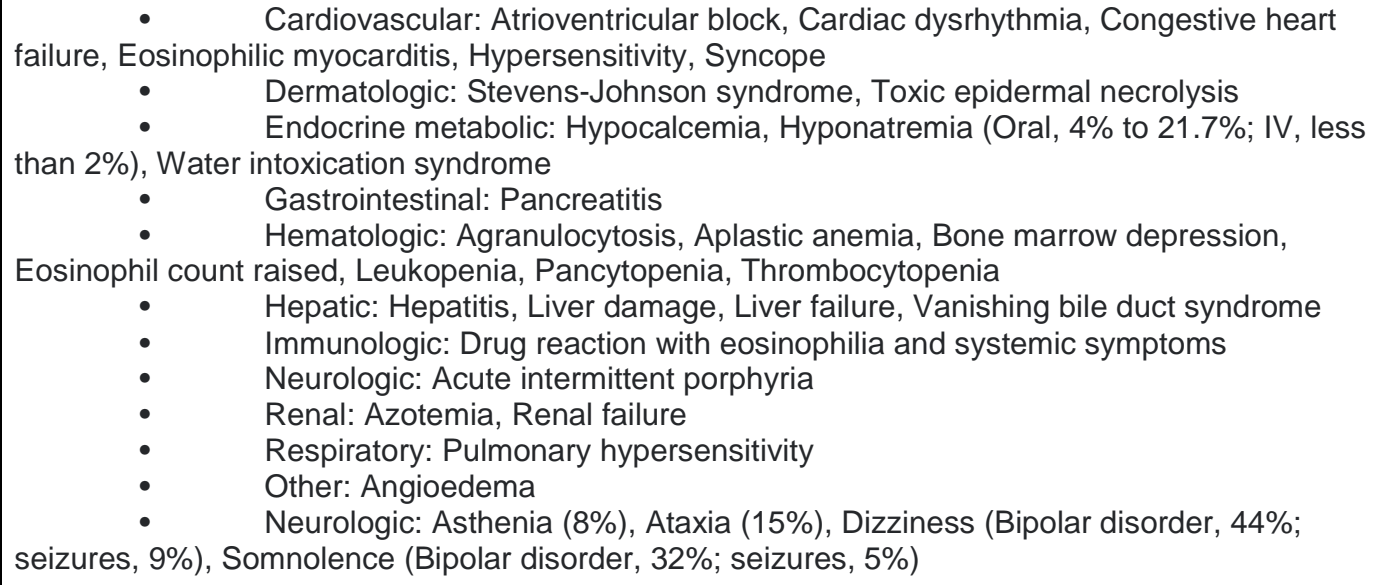 \\
\hline $\begin{array}{l}\text { Cariprazine } \\
\text { (Vraylar) }^{1}\end{array}$ & $\begin{array}{cl}\text { - } & \text { Cardiovascular: Ischemic stroke (Up to 0.1\%), Orthostatic hypotension } \\
& \text { Endocrine metabolic: Diabetes mellitus, Dyslipidemia, Hyperglycemia } \\
& \text { Gastrointestinal: Esophageal dysmotility } \\
\text { Hematologic: Leukopenia, Neutropenia } \\
\text { Musculoskeletal: Tardive dyskinesia } \\
\text { Neurologic: Seizure } \\
\text { Psychiatric: At risk for suicide (Up to 1\%), Loss of judgement } \\
\text { Respiratory: Pulmonary aspiration } \\
\text { malignant syndrome } \\
\text { Neurologic: Akathisia (Schizophrenia, } 9 \% \text {; bipolar, 20\%), Extrapyramidal sign } \\
\text { (Schizophrenia, 15\%; bipolar, 26\%), Somnolence (5\% to 8\%) }\end{array}$ \\
\hline $\begin{array}{l}\text { Lamotrigine } \\
\left({\text { Lamictal })^{1}}^{2}\right.\end{array}$ & 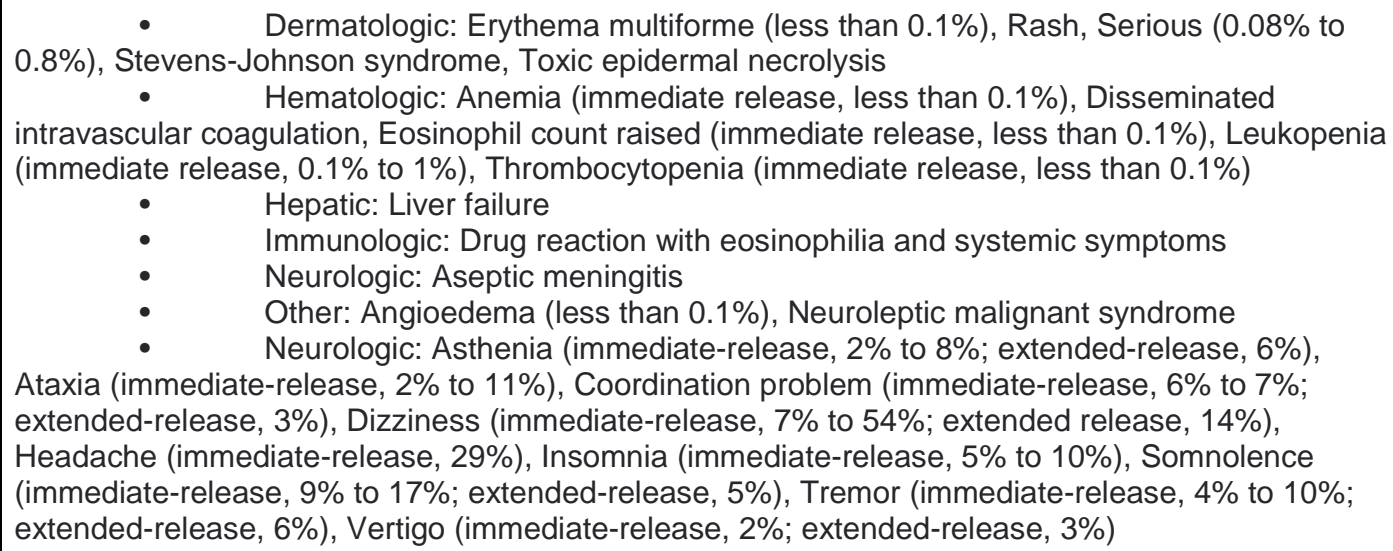 \\
\hline
\end{tabular}




\begin{tabular}{|c|c|}
\hline $\begin{array}{l}\text { Drug } \\
\text { Generic } \\
\text { Name } \\
\text { (Trade } \\
\text { Names) }\end{array}$ & Side Effects \\
\hline Lithium $^{3}$ & $\begin{array}{l}\text { Extensive side effects noted: for full list see } \\
\text { http://www.micromedexsolutions.com.ezp3.lib.umn.edu/micromedex2/librarian/PFDefaultActionld/evi } \\
\text { dencexpert.DolntegratedSearch } \\
\text { Black Box Warning: Lithium toxicity can occur at doses close to therapeutic levels. Keep all } \\
\text { appointments to check response to lithium. } \\
\text { unusual tiredness or weakness } \\
\text { excessive thirst } \\
\text { frequent urination } \\
\text { slow, jerky movements } \\
\text { movements that are unusual or difficult to control } \\
\text { blackouts } \\
\text { seizures } \\
\text { fainting } \\
\text { dizziness or lightheadedness } \\
\text { fast, slow, irregular, or pounding heartbeat } \\
\text { shortness of breath } \\
\text { chest tightness } \\
\text { confusion } \\
\text { hallucinations (seeing things or hearing voices that do not exist) } \\
\text { crossed eyes } \\
\text { painful, cold, or discolored fingers and toes } \\
\text { headache } \\
\text { pounding noises inside the head } \\
\text { swelling of the feet, ankles, or lower legs }\end{array}$ \\
\hline $\begin{array}{l}\text { Olanzapine } \\
(\text { (Zyprexa) })^{1}\end{array}$ & 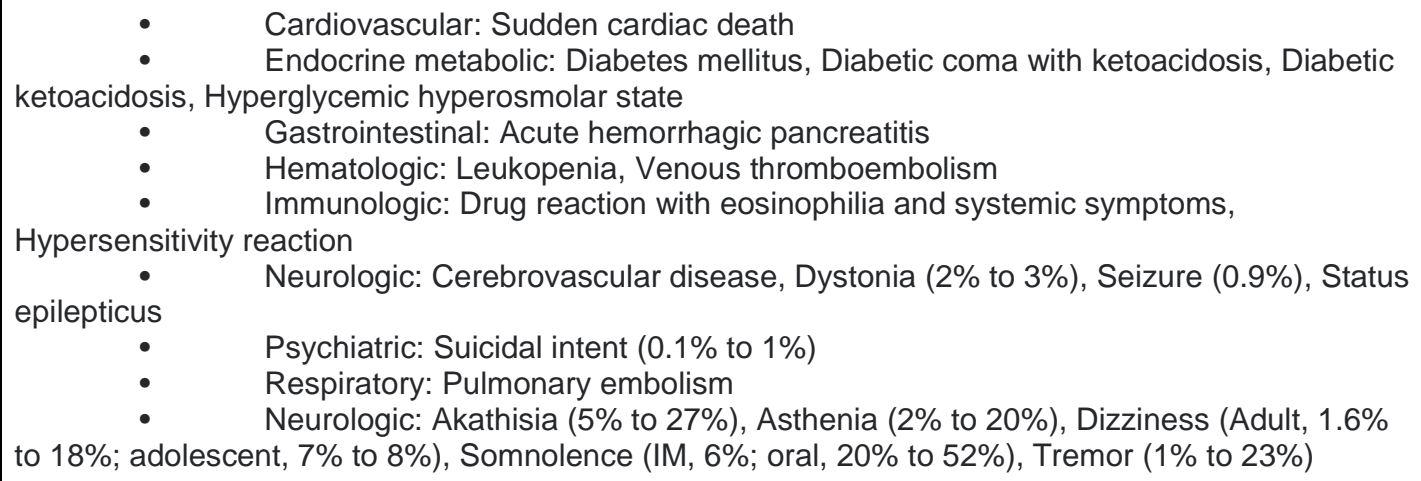 \\
\hline $\begin{array}{l}\text { Quetiapine } \\
\text { (Seroquel) }\end{array}$ & 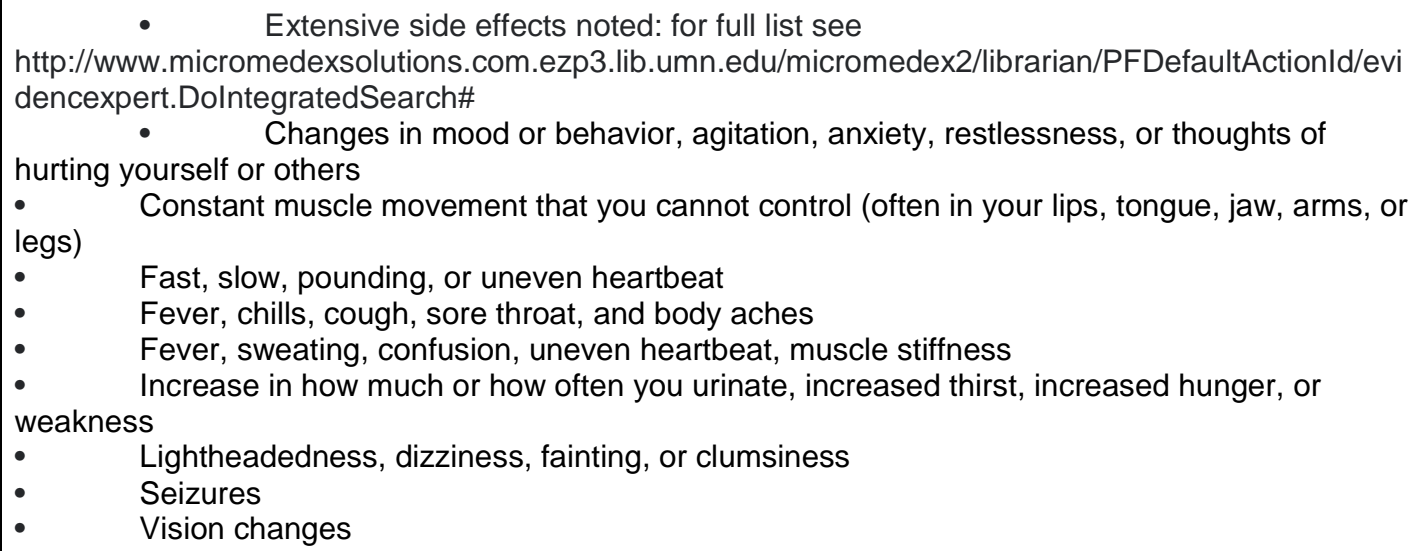 \\
\hline
\end{tabular}




\begin{tabular}{|c|c|}
\hline $\begin{array}{c}\text { Drug } \\
\text { Generic } \\
\text { Name } \\
\text { (Trade } \\
\text { Names) }\end{array}$ & Side Effects \\
\hline $\begin{array}{l}\text { Risperidone } \\
\text { (Risperdal) }^{1}\end{array}$ & 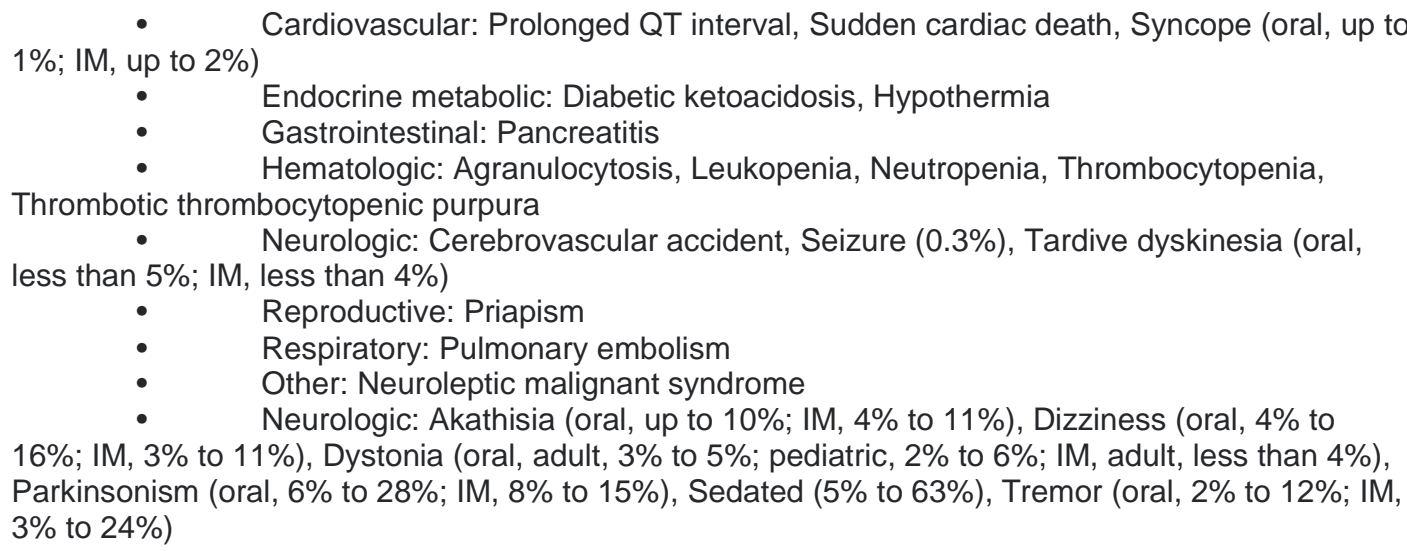 \\
\hline $\begin{array}{l}\text { Valproic } \\
\text { acid } \\
\text { (Depakene, } \\
\text { Stavzor, } \\
\text { Valproic) }^{1}\end{array}$ & 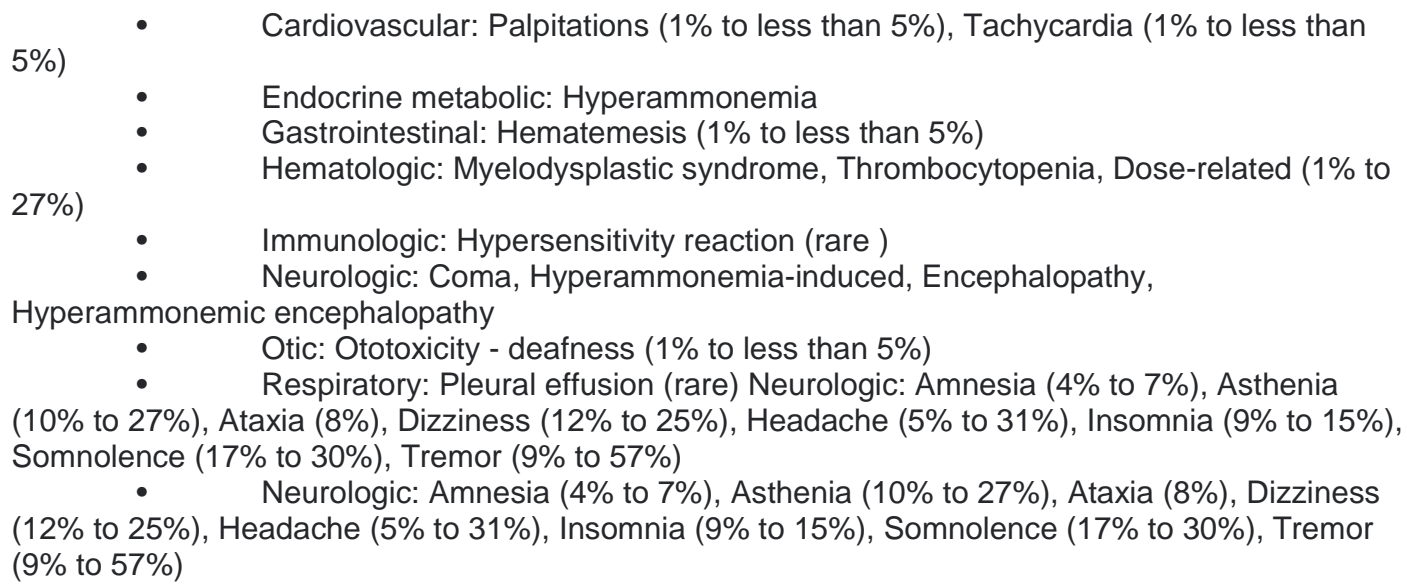 \\
\hline $\begin{array}{l}\text { Divalproex } \\
\text { (Depakote) }^{1}\end{array}$ & 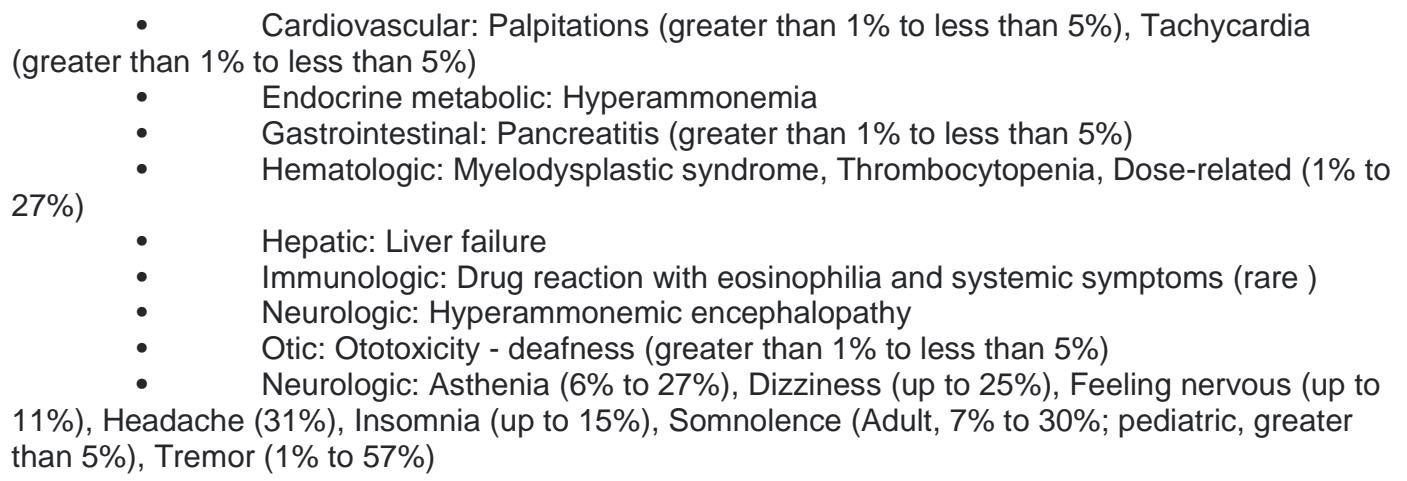 \\
\hline $\begin{array}{l}\text { Valproate } \\
\text { (Depacon) }^{1}\end{array}$ & $\begin{array}{ll}\text { - } & \text { Endocrine metabolic: Hyperammonemia } \\
\text { - } & \text { Gastrointestinal: Pancreatitis } \\
\text { - } & \text { Hematologic: Myelodysplastic syndrome, Thrombocytopenia (27\%) } \\
\text { - } & \text { Immunologic: Drug reaction with eosinophilia and systemic symptoms } \\
\text { Neurologic: Hyperammonemic encephalopathy }\end{array}$ \\
\hline
\end{tabular}




\begin{tabular}{|c|c|}
\hline $\begin{array}{c}\text { Drug } \\
\text { Generic } \\
\text { Name } \\
\text { (Trade } \\
\text { Names) }\end{array}$ & Side Effects \\
\hline $\begin{array}{l}\text { Ziprasidone } \\
3 \text { (Geodon) }\end{array}$ & $\begin{array}{l}\text { - } \quad \text { Extensive side effects noted: for full list see } \\
\text { http://www.micromedexsolutions.com.ezp3.lib.umn.edu/micromedex2/librarian/PFDefaultActionld/evi } \\
\text { dencexpert.DolntegratedSearch\# } \\
\text { - } \quad \text { unusual movements of your face or body that you cannot control } \\
\text { - } \quad \text { fast, irregular, or pounding heartbeat } \\
\text { - } \quad \text { ish or hives } \\
\text { itching } \\
\text { - } \quad \text { blisters or peeling of skin } \\
\text { - } \quad \text { mouth sores } \\
\text { - } \quad \text { fevollen glands } \\
\text { - } \quad \text { shaking } \\
\text { muscle stiffness } \\
\text { - } \quad \text { confusion } \\
\text { - } \quad \text { losweating } \\
\text { - } \quad \text { painful erection of the penis that lasts for hours } \\
\text { - }\end{array}$ \\
\hline $\begin{array}{l}\text { Allopurinol } \\
\text { (Aloprim, }^{1} \\
\text { Zyloprim) }\end{array}$ & 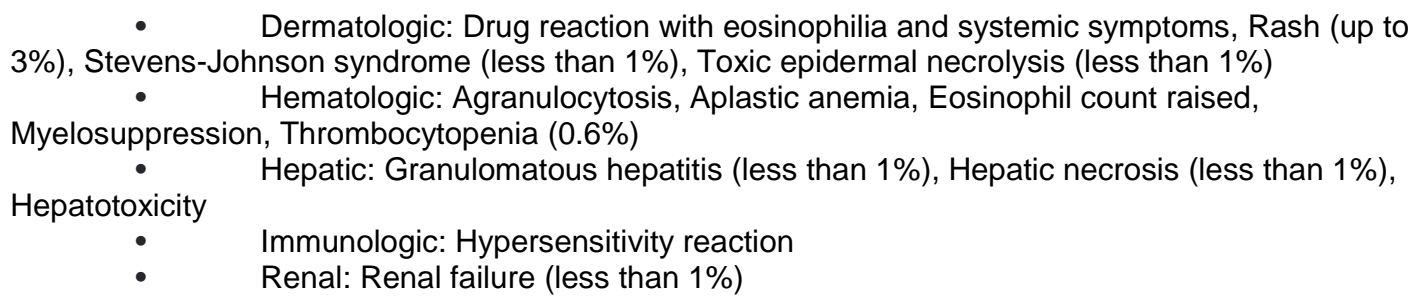 \\
\hline $\begin{array}{l}\text { Bupropion }{ }^{2} \\
\text { (Aplenzin, } \text { Wellbutrin, } \\
\text { Zyban) }\end{array}$ & $\begin{array}{l}\text { Extensive side effects noted: for full list see } \\
\text { http://www.micromedexsolutions.com.ezp3.lib.umn.edu/micromedex2/librarian/PFDefaultActionld/evi } \\
\text { dencexpert.DolntegratedSearch\# } \\
\text { - } \quad \text { Blistering, peeling, or red skin rash } \\
\text { - } \quad \text { Chest pain, trouble breathing, fast, slow, or uneven heartbeat } \\
\text { - } \quad \text { Suscle or joint pain, fever with rash } \\
\text { - } \quad \text { Seeing or hearing things that are not there, feeling like people are against you } \\
\text { - } \quad \text { Sudden increase in energy, racing thoughts, trouble sleeping } \\
\text { Thoughts of hurting yourself, worsening depression, severe agitation or confusion }\end{array}$ \\
\hline $\begin{array}{l}\text { Celecoxib } \\
(\text { Celebrex })^{1}\end{array}$ & 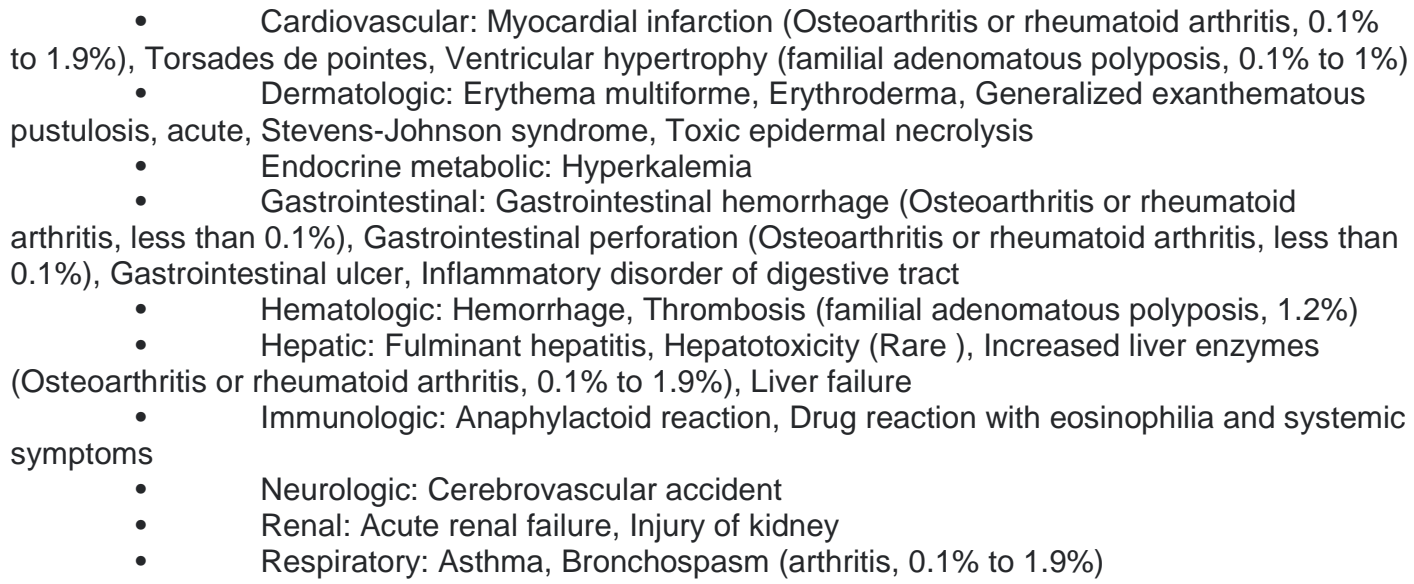 \\
\hline
\end{tabular}




\begin{tabular}{|c|c|}
\hline $\begin{array}{c}\text { Drug } \\
\text { Generic } \\
\text { Name } \\
\text { (Trade } \\
\text { Names) }\end{array}$ & Side Effects \\
\hline $\begin{array}{l}\text { Citalopram² } \\
\text { (Celexa) }\end{array}$ & $\begin{array}{l}\text { • Extensive side effects noted: for full list see } \\
\text { http://www.micromedexsolutions.com.ezp3.lib.umn.edu/micromedex2/librarian/PFDefaultActionld/evi } \\
\text { dencexpert.DolntegratedSearch\# } \\
\text { seeing or hearing things that are not there } \\
\text { Chest pain, trouble breathing } \\
\text { Confusion, weakness, and muscle twitching } \\
\text { - } \quad \text { Fast, pounding, or uneven heartbeat } \\
\text { - } \quad \text { Eeeling more excited or energetic than usual, trouble sleeping, racing thoughts } \\
\text { Eye pain, vision changes, seeing halos around lights } \\
\text { - } \quad \text { Lightheadedness, dizziness, fainting } \\
\text { Painful, prolonged erection of your penis } \\
\text { Thoughts of hurting yourself or others, unusual behavior } \\
\text { - } \\
\text { Unusual bleeding or bruising }\end{array}$ \\
\hline $\begin{array}{l}\text { Dipyridamol } \\
\text { e } \\
\text { (Persantine } \\
)^{1}\end{array}$ & $\begin{array}{cc}\text { • Cardiovascular: Angina pectoris, Cardiac arrest, Myocardial infarction (IV, } 0.1 \%), \\
\text { Myocardial ischemia, Ventricular fibrillation, Ventricular tachycardia (IV, 0.2\%) } \\
\text { • } & \text { Hepatic: Liver failure } \\
\text { - } & \text { Immunologic: Hypersensitivity reaction } \\
\text { - } & \text { Neurologic: Cerebrovascular accident, Seizure } \\
\text { Respiratory: Bronchospasm (IV, } 0.2 \%)\end{array}$ \\
\hline $\begin{array}{l}\text { Donepezil² } \\
\text { (Aricept) }\end{array}$ & $\begin{array}{l}\text { Extensive side effects noted: for full list see } \\
\text { http://www.micromedexsolutions.com.ezp3.lib.umn.edu/micromedex2/librarian/PFDefaultActionld/evi } \\
\text { dencexpert.DolntegratedSearch\# } \\
\text { • Allergic reaction: Itching or hives, swelling in your face or hands, swelling or } \\
\text { tingling in your mouth or throat, chest tightness, trouble breathing } \\
\text { Bloody or black, tarry stools } \\
\text { - Change in how much or how often you urinate } \\
\text { - } \quad \text { Chest pain, slow or uneven heartbeat, trouble breathing } \\
\text { - } \quad \text { Sightheadedness, dizziness, fainting } \\
\text { - } \quad \text { Seizures } \\
\text { - } \quad \text { Unusual bleeding, bruising, or weakness } \\
\text { - } \quad \text { Vomiting of blood or material that looks like coffee grounds }\end{array}$ \\
\hline $\begin{array}{l}\text { Fluoxetine } \\
\text { (Prozac, } \\
\text { Sarafem) }\end{array}$ & $\begin{array}{l}\text { • Extensive side effects noted: for full list see } \\
\text { http://www.micromedexsolutions.com.ezp3.lib.umn.edu/micromedex2/librarian/PFDefaultActionld/evi } \\
\text { dencexpert.DolntegratedSearch\# } \\
\text { • Anxiety, restlessness, fever, sweating, muscle spasms, nausea, vomiting, diarrhea, } \\
\text { seeing or hearing things that are not there } \\
\text { Confusion, weakness, and muscle twitching } \\
\text { Eye pain, trouble seeing, blurry vision } \\
\text { - } \quad \text { Fast, pounding, or uneven heartbeat, dizziness } \\
\text { Seizures } \\
\text { Skin rash, blisters, peeling, or redness } \\
\text { - } \quad \text { Trouble breathing } \\
\text { Unusual behavior, thoughts of hurting yourself or others, feeling more excited or energetic } \\
\text { than usual, trouble sleeping } \\
\text { - }\end{array}$ \\
\hline
\end{tabular}




\begin{tabular}{|c|c|}
\hline $\begin{array}{c}\text { Drug } \\
\text { Generic } \\
\text { Name } \\
\text { (Trade } \\
\text { Names) }\end{array}$ & Side Effects \\
\hline $\begin{array}{l}\text { Gabapentin } \\
\text { (Gralise, } \\
\text { Horizant, } \\
\text { Neurontin) }\end{array}$ & 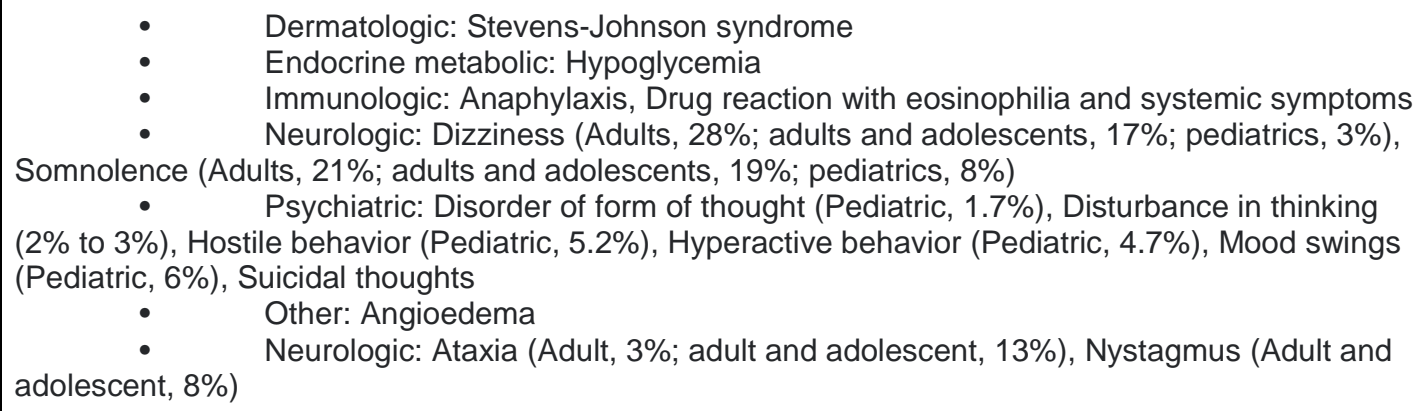 \\
\hline $\begin{array}{l}\text { Haloperidol } \\
(\text { Haldol })^{1}\end{array}$ & $\begin{aligned} \text { • } & \text { Cardiovascular: Prolonged QT interval, Sudden cardiac death, Torsades de } \\
\text { • } & \text { Gastrointestinal: Paralytic ileus } \\
\text { • } & \text { Hematologic: Agranulocytosis } \\
\text { dyskinesia } & \text { Neurologic: Dystonia, Neuroleptic malignant syndrome, Seizure, Tardive } \\
& \text { Reproductive: Priapism } \\
\text { - } & \text { Neurologic: Akathisia, Extrapyramidal disease (Frequent ), Somnolence }\end{aligned}$ \\
\hline $\begin{array}{l}\text { Memantine }{ }^{2} \\
\text { (Namenda) }\end{array}$ & $\begin{array}{l}\text { Extensive side effects noted: for full list see } \\
\text { http://www.micromedexsolutions.com.ezp3.lib.umn.edu/micromedex2/librarian/PFDefaultActionld/evi } \\
\text { dencexpert.DolntegratedSearch\# } \\
\text { Chest pain. } \\
\text { - } \\
\text { Lightheadedness, dizziness, or fainting. } \\
\text { Seeing or hearing things that are not there. } \\
\text { Severe sleepiness, restlessness, or confusion. } \\
\text { Sudden or severe headache. }\end{array}$ \\
\hline $\begin{array}{l}\text { Oxcarbaze } \\
\text { pine } \\
\text { (Trileptal) }^{1}\end{array}$ & 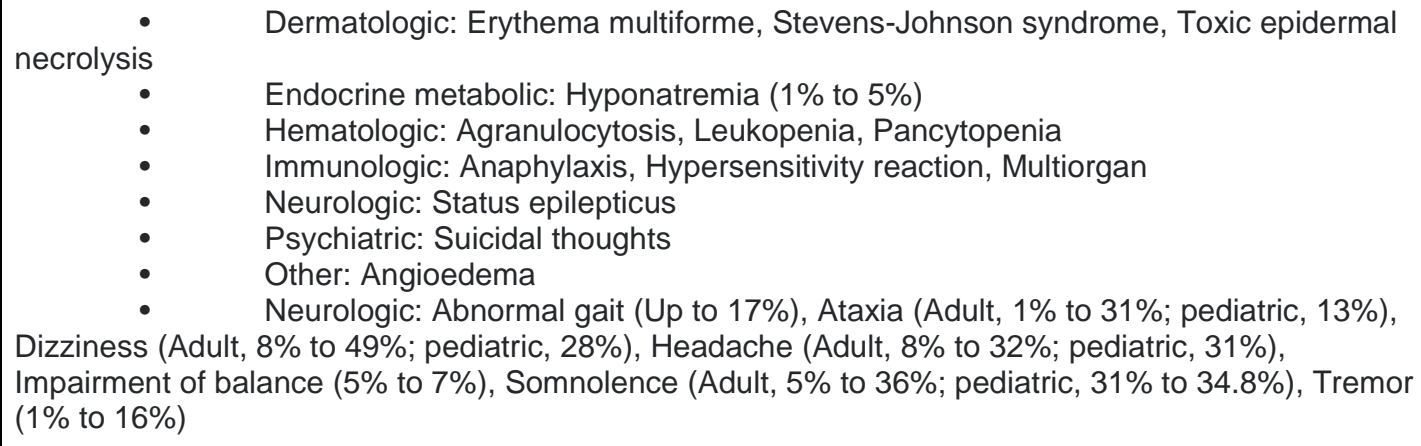 \\
\hline $\begin{array}{l}\text { Paliperidon } \\
\text { e (Invega) }\end{array}$ & $\begin{array}{ll} & \text { Cardiovascular: Prolonged QT interval (7\%) } \\
& \text { Hematologic: Agranulocytosis, Leukopenia } \\
& \text { Neurologic: Dysphagia, Tardive dyskinesia } \\
& \text { Reproductive: Priapism }\end{array}$ \\
\hline
\end{tabular}




\begin{tabular}{|c|c|}
\hline $\begin{array}{c}\text { Drug } \\
\text { Generic } \\
\text { Name } \\
\text { (Trade } \\
\text { Names) }\end{array}$ & Side Effects \\
\hline \begin{tabular}{|l} 
Paroxetine ${ }^{2}$ \\
(Brisdelle, \\
Paxil, \\
Pexeva)
\end{tabular} & $\begin{array}{l}\text { E Extensive side effects noted: for full list see } \\
\text { http://www.micromedexsolutions.com.ezp3.lib.umn.edu/micromedex2/librarian/PFDefaultActionld/evi } \\
\text { dencexpert.DolntegratedSearch\# } \\
\text { Anxiety, restlessness, fast heartbeat, fever, sweating, muscle spasms, nausea, } \\
\text { vomiting, diarrhea, seeing or hearing things that are not there } \\
\text { Bone pain, tenderness, swelling, or bruising } \\
\text { Changes in behavior, thoughts of hurting yourself or others } \\
\text { Confusion, weakness, and muscle twitching } \\
\text { Eye pain, vision changes, seeing halos around lights } \\
\text { Trouble keeping still, feeling restless and agitated, racing thoughts, excessive energy, } \\
\text { trouble sleeping } \\
\text { Unusual bleeding or bruising }\end{array}$ \\
\hline $\begin{array}{l}\text { Perphenazi } \\
\text { ne } \\
\text { (Trilafon) }^{1}\end{array}$ & 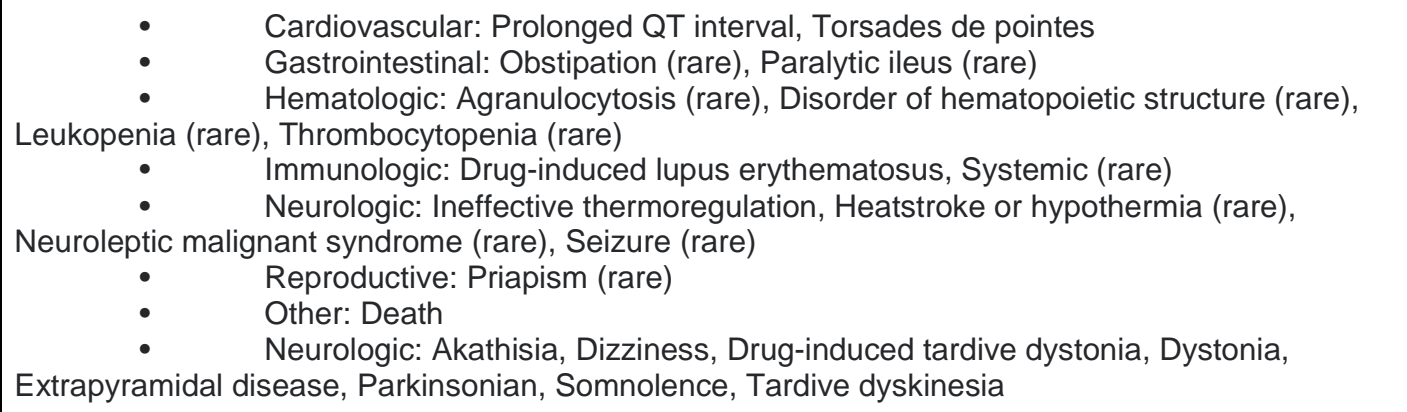 \\
\hline $\begin{array}{l}\text { Ramelteon } \\
(\text { Rozerem) }\end{array}$ & $\begin{array}{l}\text { - } \quad \text { Psychiatric: Depression, worsening, Hallucinations, Mania } \\
\text { Other: Angioedema (rare ) }\end{array}$ \\
\hline $\begin{array}{l}\text { Tamoxifen }{ }^{2} \\
\text { (Nolvadex, } \\
\text { Soltamox) }\end{array}$ & $\begin{array}{l}\text { - Extensive side effects noted: for full list see } \\
\text { http://www.micromedexsolutions.com.ezp3.lib.umn.edu/micromedex2/librarian/PFDefaultActionld/evi } \\
\text { dencexpert.DolntegratedSearch\# } \\
\text { - Chest pain, shortness of breath, or coughing up blood. } \\
\text { Dark-colored urine or pale stools. } \\
\text { - } \quad \text { Fever, chills, cough, sore throat, and body aches. } \\
\text { - } \\
\text { - Nausy or abnormal vaginal bleeding, pelvic pain or pressure. } \\
\text { New breast lumps. } \\
\text { - Numbness or weakness in your arm or leg, or on one side of your body. } \\
\text { Pain in your lower leg (calf). } \\
\text { Sudden or severe headache, or problems with vision, speech, or walking. } \\
\text { Swelling in your hands, ankles, or feet. } \\
\text { Unusual bleeding, bruising, or weakness. } \\
\text { Yellowing of your skin or the whites of your eyes. }\end{array}$ \\
\hline
\end{tabular}




\begin{tabular}{|c|c|}
\hline $\begin{array}{c}\text { Drug } \\
\text { Generic } \\
\text { Name } \\
\text { (Trade } \\
\text { Names) }\end{array}$ & Side Effects \\
\hline $\begin{array}{l}\text { Topiramate } \\
\text { (Qudexy, } \\
\text { Topamax) }\end{array}$ & 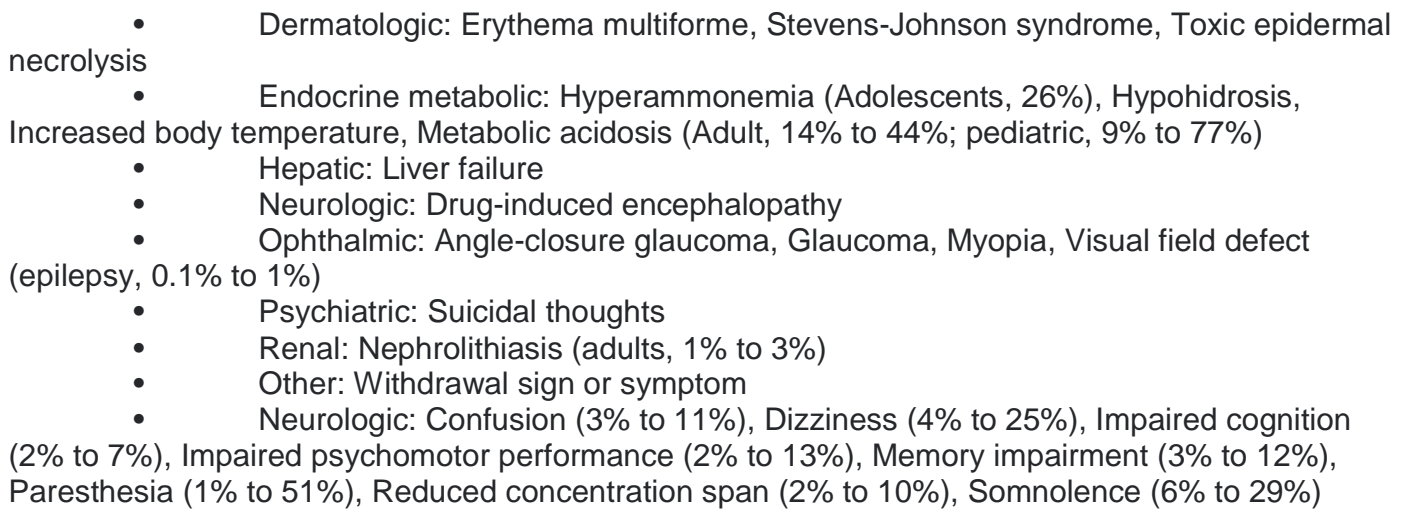 \\
\hline $\begin{array}{l}\text { Venlafaxine } \\
2 \text { (Effexor) }\end{array}$ & $\begin{array}{l}\text { Extensive side effects noted: for full list see } \\
\text { http://www.micromedexsolutions.com.ezp3.lib.umn.edu/micromedex2/librarian/PFDefaultActionld/evi } \\
\text { dencexpert.DolntegratedSearch\# } \\
\text { seeing or hearing things that are not there } \\
\text { Blistering, peeling, red skin rash } \\
\text { Chest pain, cough, trouble breathing } \\
\text { - } \quad \text { Confusion, weakness, and muscle twitching } \\
\text { - } \\
\text { Eye pain, vision changes, seeing halos around lights } \\
\text { - } \quad \text { Fast or pounding heartbeat } \\
\text { - } \\
\text { Heeling more excited or energetic than usual } \\
\text { - } \\
\text { Seizures, trouble concentrating, memory problems, unsteadiness } \\
\text { - } \\
\text { unusual behavior, thoughts of hurting yourself or others, trouble sleeping, nervousness, } \\
\text { - }\end{array}$ \\
\hline $\begin{array}{l}\text { Verapamil² } \\
\text { (Calan, } \text { Covera-HS, } \\
\text { Verelan) }\end{array}$ & $\begin{array}{l}\text { - Extensive side effects noted: for full list see } \\
\text { http://www.micromedexsolutions.com.ezp3.lib.umn.edu/micromedex2/librarian/PFDefaultActionld/evi } \\
\text { dencexpert.DolntegratedSearch\# } \\
\text { - } \quad \text { Chest pain } \\
\text { eyes } \quad \text { Dark urine or pale stools, nausea, vomiting, loss of appetite, stomach pain, yellow skin or } \\
\text { - } \\
\text { Fast, slow, uneven, or pounding heartbeat } \\
\text { - } \quad \text { Lightheadedness, dizziness, fainting } \\
\text { Rapid weight gain, swelling in your legs, feet, or ankles } \\
\text { Trouble breathing } \\
\text { Unusual tiredness or weakness }\end{array}$ \\
\hline
\end{tabular}

*We did not differentiate between mild/moderate versus serious side effects.

\section{Sources:}

${ }^{1}$ http://www.micromedexsolutions.com

${ }^{2}$ https://www.ncbi.nlm.nih.gov/pubmedhealth/

3https://medlineplus.gov/

Abbreviations: $\mathrm{BP}=$ bipolar disorder; FDA=United States Food and Drug Administration 